\title{
The LUX-ZEPLIN (LZ) Technical Design Report
}
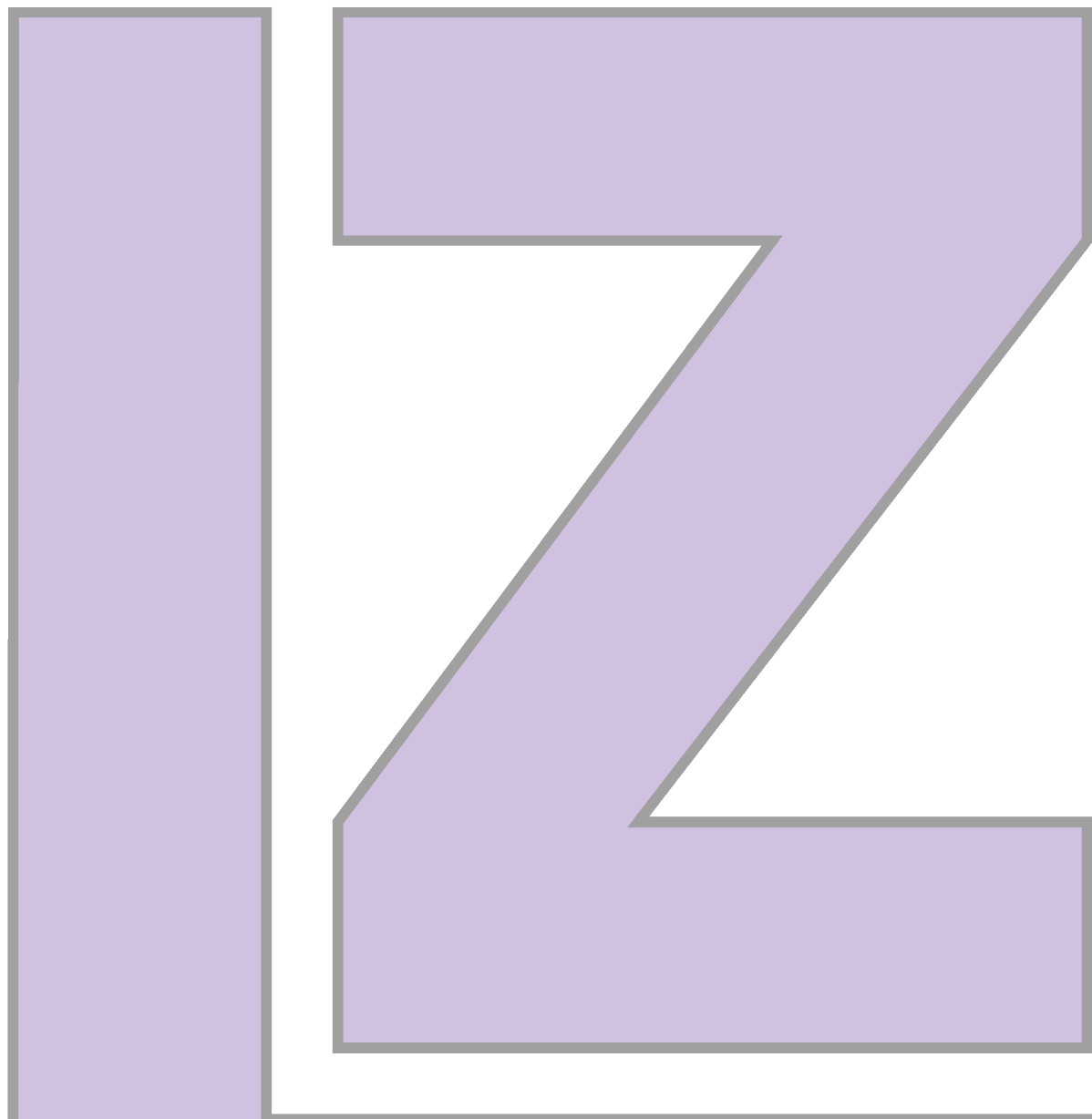

In this Technical Design Report (TDR) we describe the LZ detector to be built at the Sanford Underground Research Facility (SURF). The LZ dark matter experiment is designed to achieve sensitivity to a WIMPnucleon spin-independent cross section of $3 \times 10^{-48} \mathrm{~cm}^{2}$. 



\title{
The LUX-ZEPLIN (LZ) Collaboration
}

\author{
B.J. Mount
}

Black Hills State University, School of Natural Sciences, 1200 University Street, Spearfish, SD 57799-0002, USA

S. Hans, R. Rosero, M. Yeh

Brookhaven National Laboratory (BNL) P.O. Box 5000, Upton, NY 11973-5000, USA

C. Chan, R.J. Gaitskell, D.Q. Huang, J. Makkinje, D.C. Malling, ${ }^{1}$ M. Pangilinan, ${ }^{2}$ C.A. Rhyne, W.C. Taylor, J.R. Verbus ${ }^{3}$

Brown University, Department of Physics, 182 Hope Street, Providence, RI 02912-9037, USA

Y.D. Kim, H.S. Lee, J. Lee, D.S. Leonard, J. Li

IBS Center for Underground Physics (CUP), 70, Yuseong-daero 1689-gil, Yuseong-gu, Daejeon, Korea

J. Belle, ${ }^{4}$ A. Cottle, W.H. Lippincott, D.J. Markley, T.J. Martin, M. Sarychev, T.E. Tope, M. Utes, R. Wang, I. Young

Fermi National Accelerator Laboratory (FNAL), Batavia, IL 60510-0500, USA

H.M. Araújo, A.J. Bailey, ${ }^{5}$ D. Bauer, D. Colling, A. Currie, ${ }^{6}$ S. Fayer, F. Froborg, S. Greenwood, W.G. Jones, V. Kasey, M. Khaleeq, I. Olcina, B. López Paredes, A. Richards, T.J. Sumner, A. Tomás,

A. Vacheret

Imperial College London, Physics Department, Blackett Laboratory, Prince Consort Road, London, SW7 2BW, UK

P. Brás, A. Lindote, M.I. Lopes, F. Neves, J.P. Rodrigues, C. Silva, V.N. Solovov

Laboratório de Instrumentação e Física Experimental de Partículas (LIP), Department of Physics, University of

Coimbra, Rua Larga, 3004-516, Coimbra, Portugal

M.J. Barry, A. Cole, A. Dobi, ${ }^{7}$ W.R. Edwards, C.H. Faham, ${ }^{3}$ S. Fiorucci, N.J. Gantos, V.M. Gehman, ${ }^{8}$

M.G.D. Gilchriese, K. Hanzel, M.D. Hoff, K. Kamdin, ${ }^{9}$ K.T. Lesko, C.T. McConnell, K. O’Sullivan, ${ }^{10}$ K.C. Oliver-Mallory, ${ }^{9}$ S.J. Patton, J.S. Saba, P. Sorensen, K.J. Thomas, ${ }^{11,12}$ C.E. Tull, W.L. Waldron, M.S. Witherell ${ }^{9}$

Lawrence Berkeley National Laboratory (LBNL), 1 Cyclotron Road, Berkeley, CA 94720-8156, USA

A. Bernstein, K. Kazkaz, J. Xu

Lawrence Livermore National Laboratory (LLNL), 7000 East Avenue, Livermore, CA 94550-9698, USA

\footnotetext{
${ }^{1}$ Now at: Lincoln Laboratory, 244 Wood Street, Massachusetts Institute of Technology, Lexington, MA 02421-6426, USA

${ }^{2}$ Now at: Samba TV, 301 Brannan Street, Floor 6, San Francisco, CA 94107-3816, USA

${ }^{3}$ Now at: LinkedIn Corporation, 1000 West Maude Avenue, Sunnyvale, CA 94085, USA

${ }^{4}$ Also at: Belle Aerospace Corporation, 2237 Warrenville Ave., Wheaton, IL 60189, USA

${ }^{5}$ Now at: University of Valencia, Instituto de Física Corpuscular, Parc Científic de la Universitat de València, C/ Catedrático José Beltrán, 2, E-46980 Paterna, Spain

${ }^{6}$ Now at: HM Revenue and Customs, 100 Parliament Street, London, SW1A 2BQ, UK

${ }^{7}$ Now at: Zenysis Technology, 535 Mission Street Floor 14, San Franciso, CA 94105-3253, USA

${ }^{8}$ Now at: Cainthus, Otherlab, 701 Alabama Street, San Francisco, CA 94110-2022, USA

${ }^{9}$ Also at: University of California (UC), Berkeley, Department of Physics, 366 LeConte Hall MC 7300, Berkeley, CA 94720-7300, USA

${ }^{10}$ Now at: Insight Data Science, 260 Sheridan Avenue Suite 310, Palo Alto, CA 94306-2010, USA

${ }^{11}$ Also at: University of California (UC), Berkeley, Department of Nuclear Engineering, 4155 Etcheverry Hall MC 1730, CA 94720-1730, USA

${ }^{12}$ Now at: Lawrence Livermore National Laboratory (LLNL), 7000 East Avenue, Livermore, CA 94550-9698, USA
} 
D. Yu. Akimov, A.I. Bolozdynya, A.V. Khromov, A.M. Konovalov, A.V. Kumpan, V.V. Sosnovtsev National Research Nuclear University MEPhI (NRNU MEPhI), 31 Kashirskoe shosse, Moscow, 115409, RUS

$$
\text { C.E. Dahl, }{ }^{13} \text { D. Temples }
$$

Northwestern University, Department of Physics \& Astronomy, 2145 Sheridan Road, Evanston, IL 60208-3112, USA

M.C. Carmona-Benitez, L. de Viveiros

Pennsylvania State University, Department of Physics, 104 Davey Lab, University Park, PA 16802-6300, USA

D.S. Akerib ${ }^{b a}$ H. Auyeung, ${ }^{b}$ T.P. Biesiadzinski, ${ }^{b a}$ M. Breidenbach, ${ }^{b}$ R. Bramante, ${ }^{b a}$ R. Conley, ${ }^{b}$ W.W. Craddock, ${ }^{b}$ A. Fan, ${ }^{b a}$ A. Hau, ${ }^{b}$ C.M. Ignarra, ${ }^{b a}$ W. Ji, ${ }^{b a}$ H.J. Krebs, ${ }^{b}$ R. Linehan, ${ }^{b a}$ C. Lee ${ }^{b a, 14}$ S. Luitz, ${ }^{b}$ E. Mizrachi, ${ }^{b}$ M.E. Monzani, ${ }^{b a}$ F.G. O’Neill, ${ }^{b}$ S. Pierson, ${ }^{b}$ M. Racine, ${ }^{b}$ B.N. Ratcliff, ${ }^{b}$ G.W. Shutt, ${ }^{b}$ T.A. Shutt, ${ }^{b a}$ K. Skarpaas, ${ }^{b}$ K. Stifter, ${ }^{b a}$ W.H. To, ${ }^{b a, 15}$ J. Va'vra, ${ }^{b}$ T.J. Whitis, ${ }^{b a}$ W.J. Wisniewski ${ }^{b}$

${ }^{a}$ Kavli Institute for Particle Astrophysics and Cosmology (KIPAC), 452 Lomita Mall, Stanford University, Stanford, CA 94305, USA; ${ }^{b}$ SLAC National Accelerator Laboratory, 2575 Sand Hill Road, Menlo Park, CA 94205-7015, USA

X. Bai, R. Bunker, ${ }^{16}$ R. Coughlen, C. Hjemfelt, R. Leonard, E.H. Miller, E. Morrison, J. Reichenbacher, R.W. Schnee, M.R. Stark, ${ }^{17}$ K. Sundarnath, D.R. Tiedt, M. Timalsina

South Dakota School of Mines and Technology, 501 East Saint Joseph Street, Rapid City, SD 57701-3901, USA

P. Bauer, B. Carlson, M. Horn, M. Johnson, J. Keefner, C. Maupin, D.J. Taylor

South Dakota Science and Technology Authority (SDSTA), Sanford Underground Research Facility, 630 East Summit Street, Lead, SD 57754-1700, USA

S. Balashov, P. Ford, V. Francis, E. Holtom, A. Khazov, A. Kaboth, ${ }^{18}$ P. Majewski, J.A. Nikkel, J. O’Dell, R.M. Preece, M.G.D. van der Grinten, S.D. Worm ${ }^{19}$

STFC Rutherford Appleton Laboratory (RAL), Didcot, OX11 OQX, UK

R.L. Mannino, T.M. Stiegler, P.A. Terman, R.C. Webb

Texas A\&M University, Department of Physics and Astronomy, 4242 TAMU, College Station, TX 77843-4242, USA

$$
\text { C. Levy, J. Mock, }{ }^{20,21} \text { M. Szydagis }
$$

University at Albany (SUNY), Department of Physics, 1400 Washington Avenue, Albany, NY 12222-1000, USA

\section{J.K. Busenitz, M. Elnimr, ${ }^{22}$ J.Y-K. Hor, Y. Meng, A. Piepke, I. Stancu}

University of Alabama, Department of Physics \& Astronomy, 206 Gallalee Hall, 514 University Boulevard,

$$
\text { Tuscaloosa, AL 34587-0324, USA }
$$

\footnotetext{
${ }^{13}$ Also at: Fermi National Accelerator Laboratory (FNAL), Batavia, IL 60510-0500, USA

${ }^{14}$ Now at: IBS Center for Underground Physics (CUP), 70, Yuseong-daero 1689-gil, Yuseong-gu, Daejeon, Korea

${ }^{15}$ Now at: California State University, Stanislaus, Department of Physics, 1 University Circle, Turlock, CA 95382-3200, USA

${ }^{16}$ Now at: Pacific Northwest National Laboratory (PNNL), 902 Battelle Blvd., Richland WA 99354-1793, USA

${ }^{17}$ Now at: Northeastern University, 360 Huntington Ave. 111 Dana Research Center, Boston, MA 02115, USA

${ }^{18}$ Also at: Royal Holloway, University of London, Department of Physics, Egham Hill, Egham, Surry, TW20 0EX, UK

${ }^{19}$ Now at: University of Birmingham, Particle Physics Group, School of Physics and Astronomy, Edgbaston, Birmingham, B15 2TT, UK

${ }^{20}$ Also at: Lawrence Berkeley National Laboratory (LBNL), 1 Cyclotron Road, Berkeley, CA 94720-8156 USA

${ }^{21}$ Now at: Cosylab USA, Inc., 1530 Page Mill Road, Suite 200, Palo Alto, CA 94304-1140, USA

${ }^{22}$ Now at: University of California (UC), Irvine, Department of Physics \& Astronomy, 4129 Frederick Reines Hall, Irvine, CA 92697-4575, USA
} 


\section{Kreczko, B. Krikler, B. Penning \\ University of Bristol, School of Physics, Bristol, BS8 1TL, UK}

E.P. Bernard, ${ }^{20}$ R.G. Jacobsen, D.N. McKinsey, ${ }^{20}$ R. Watson ${ }^{20}$

University of California (UC), Berkeley, Department of Physics, 366 LeConte Hall MC 7300, Berkeley, CA

94720-7300, USA

J.E. Cutter, S. El-Jurf, R.M. Gerhard, D. Hemer, S. Hillbrand, B. Holbrook, B.G. Lenardo, ${ }^{23}$

A.G. Manalaysay, J.A. Morad, S. Stephenson, ${ }^{24}$ J.A. Thomson, M. Tripathi, S. Uvarov

University of California (UC), Davis, Department of Physics, One Shields Avenue, Davis, CA 95616-5270, USA

S.J. Haselschwardt, S. Kyre, C. Nehrkorn, H.N. Nelson, M. Solmaz, D.T. White

University of California (UC), Santa Barbara, Department of Physics, Broida Hall, Santa Barbara, CA 93106-9530, USA

M. Cascella, J.E.Y. Dobson, C. Ghag, X. Liu, L. Manenti, L. Reichhart, ${ }^{25}$ S. Shaw, ${ }^{26}$ U. Utku University College London (UCL), Department of Physics and Astronomy, Gower Street, London, WC1E 6BT, UK

P. Beltrame, T.J.R. Davison, M.F. Marzioni, A.St.J. Murphy, A. Nilima

University of Edinburgh, SUPA, School of Physics and Astronomy, Edinburgh, EH9 3FD, UK

B. Boxer, ${ }^{27}$ S. Burdin, A. Greenall, S. Powell, H.J. Rose, P. Sutcliffe

University of Liverpool, Department of Physics, Liverpool, L69 7ZE, UK

J. Balajthy, T.K. Edberg, C.R. Hall, J.S. Silk

University of Maryland, Department of Physics, College Park, MD 20742-4111, USA

\section{S. Hertel}

University of Massachusetts, Department of Physics, 1126 Lederle Graduate Research Tower (LGRT), Amherst, MA 01003-9337, USA

C.W. Akerlof, M. Arthurs, W. Lorenzon, K. Pushkin, M. Schubnell

University of Michigan, Randall Laboratory of Physics, 450 Church Street, Ann Arbor, MI 48109-1040, USA

K.E. Boast, C. Carels, T. Fruth, H. Kraus, F.-T. Liao, J. Lin, ${ }^{28}$ P.R. Scovell University of Oxford, Department of Physics, Oxford, OX1 3RH, UK

E. Druszkiewicz, D. Khaitan, M. Koyuncu, W. Skulski, F.L.H. Wolfs, J. Yin

University of Rochester, Department of Physics and Astronomy, Rochester, NY 14627-0171, USA

E.V. Korolkova, V.A. Kudryavtsev, P. Rossiter, D. Woodward

University of Sheffield, Department of Physics and Astronomy, Sheffield, S3 7RH, UK

\footnotetext{
${ }^{23}$ Also at: Lawrence Livermore National Laboratory (LLNL), 7000 East Avenue, Livermore, CA 94550-9698, USA

${ }^{24}$ Now at: Deepgram, 148 Townsend Street, San Francisco, CA, 94107-1919, USA

${ }^{25}$ Now at: IMS Nanofabrication AG, Wolfholzgasse 20-22, 2345 Brunn am Gebirge, AUT

${ }^{26}$ Now at: University of California (UC), Santa Barbara, Department of Physics, Broida Hall, Santa Barbara, CA 93106-9530, USA

${ }^{27}$ Also at: STFC Rutherford Appleton Laboratory (RAL), Didcot, OX11 0QX, UK

${ }^{28}$ Now at: University of California (UC), Berkeley, Department of Physics, 366 LeConte Hall MC 7300, Berkeley, CA 94720-7300, USA
} 
A.A. Chiller, C. Chiller, D.-M. Mei, L. Wang, W.-Z. Wei, M. While, C. Zhang

University of South Dakota, Department of Physics, 414 East Clark Street, Vermillion, SD 57069-2307, USA

S.K. Alsum, T. Benson, D.L. Carlsmith, J.J. Cherwinka, S. Dasu, G. Gregerson, B. Gomber, A. Pagac, K.J. Palladino, C.O. Vuosalo, Q. Xiao

University of Wisconsin-Madison, Department of Physics, 1150 University Avenue Room 2320, Chamberlin Hall, Madison, WI 53706-1390, USA

J.H. Buckley, V.V. Bugaev, M.A. Olevitch

Washington University in St. Louis, Department of Physics, One Brookings Drive, St. Louis, MO 63130-4862, USA

E.M. Boulton, ${ }^{9,20}$ W.T. Emmet, T.W. Hurteau, N.A. Larsen, ${ }^{29}$ E.K. Pease, ${ }^{9,20}$ B.P. Tennyson, L. Tvrznikova ${ }^{9,20}$

Yale University, Department of Physics, 217 Prospect Street, New Haven, CT 06511-8499, USA

\footnotetext{
${ }^{29}$ Now at: The University of Chicago, The Kavli Institute for Cosmological Physics, William Eckhardt Research Center (ERC) Suite 499, 5640 South Ellis Avenue, Chicago, IL 60637-1433, USA
} 


\section{Acknowledgements}

This work was partially supported by the U.S. Department of Energy (DOE) under award numbers DESC0012704，DE-SC0010010，DE-AC02-05CH11231，DE-SC0012161，DE-SC0014223，DE-FG0213ER42020, DE-FG02-91ER40674, DE-NA0000979, DE-SC0011702, DESC0012034, DESC0010072, DESC0006572, DE-SC0006605, DE-FG02-10ER46709, DE-AC02-07CH11359, DE-AC02-76SF00515, DE-AC52-07NA27344, DE-FG02-01ER41166, DE-FG02-91ER40628, DE-SC0015535, DE-SC0015708, and DE-SC0220187; by the U.S. National Science Foundation (NSF) under award numbers NSF PHY110447, NSF PHY-1506068, NSF PHY-1312561, NSF PHY-1406943, NSF PHY-1642619, and NSF PHY-1429544, by the U.K. Science \& Technology Facilities Council under award numbers ST/K006428/1, ST/M003655/1， ST/M003981/1， ST/M003744/1， ST/M003639/1， ST/M003604/1， ST/M003469/1, ST/M003779/1, and ST/N000250/1; by the Portuguese Foundation for Science and Technology (FCT) under award numbers CERN/FP/123610/2011 and PTDC/FIS-NUC/1525/2014; by the National Research Nuclear University MEPhI (Moscow Engineering Physics Institute) in the framework of the MEPhI Academic Excellence Project (contract 02.a03.21.0005, 27.08.2013); and by the Institute for Basic Science, Korea (budget numbers IBS-R016-D1, and IBS-R016-S1).

University College London and Lawrence Berkeley National Laboratory thank the U.K. Royal Society for travel funds under the International Exchange Scheme (IE141517). We acknowledge additional support from the Boulby Underground Laboratory in the U.K., the University of Wisconsin for grant UW PRJ82AJ, the GridPP Collaboration, in particular at Imperial College London, and from the South Dakota Science and Technology Authority, and the State of South Dakota. The University of Edinburgh is a charitable body, registered in Scotland, with the registration number SC005336.

\section{Disclaimers}

This document was prepared as an account of work sponsored by the United States Government. While this document is believed to contain correct information, neither the United States Government nor any agency thereof, nor the Regents of the University of California, nor any of their employees, makes any warranty, express or implied, or assumes any legal responsibility for the accuracy, completeness, or usefulness of any information, apparatus, product, or process disclosed, or represents that its use would not infringe privately owned rights. Reference herein to any specific commercial product, process, or service by its trade name, trademark, manufacturer, or otherwise, does not necessarily constitute or imply its endorsement, recommendation, or favoring by the United States Government or any agency thereof, or the Regents of the University of California. The views and opinions of authors expressed herein do not necessarily state or reflect those of the United States Government or any agency thereof or the Regents of the University of California.

\section{Copyright Notice}

This manuscript has been authored by an author at Lawrence Berkeley National Laboratory under Contract No. DE-AC02-05CH11231 with the U.S. Department of Energy. The U.S. Government retains, and the publisher, by accepting the article for publication, acknowledges, that the U.S. Government retains a non-exclusive, paid-up, irrevocable, world-wide license to publish or reproduce the published form of this manuscript, or allow others to do so, for U.S. Government purposes. 



\section{Contents}

1 Overview 1

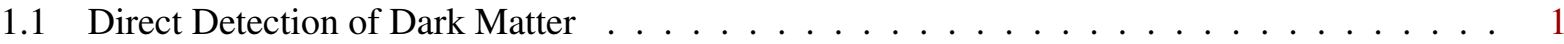

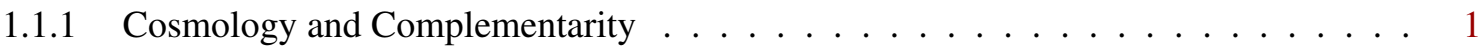

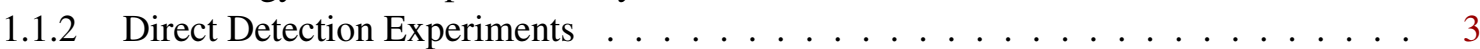

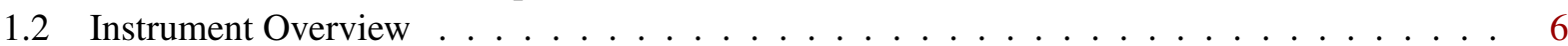

1.3 Design Drivers for WIMP Identification . . . . . . . . . . . . . . . . . 10

1.3.1 Overview of the Experimental Strategy . . . . . . . . . . . . . . . . . 10

1.3.2 Self-shielding in Liquid Xenon . . . . . . . . . . . . . . . . . . . . . 14

1.3.3 Low-energy Particle Detection in Liquid Xenon . . . . . . . . . . . . . . . . . . 16

1.3.3.1 Low Energy and Low Mass Sensitivity . . . . . . . . . . . . . . . . . . 18

1.3.4 Electron/Nuclear Recoil Discrimination . . . . . . . . . . . . . . . . . . . . 19

1.3.5 Outer Detector Systems _ . . . . . . . . . . . . . . . . . . . . 21

1.3.5.1 Xenon Skin Veto . . . . . . . . . . . . . . . . . 23

1.3.5.2 Scintillator Outer Detector . . . . . . . . . . . . . . . . . . 24

1.4 Internal Calibration with Dispersed Sources . . . . . . . . . . . . . . . . . . 25

1.5 Xenon Purity for Detector Performance . . . . . . . . . . . . . . . . . . . . 26

1.6 Dominant Backgrounds . . . . . . . . . . . . . . . . . . . . . . . . 27

1.6.1 Backgrounds from Material Radioactivity . . . . . . . . . . . . . . . 28

1.6.2 Surface Plating of Radon Progeny . . . . . . . . . . . . . . . . . . . . . . . 29

1.6.3 Intrinsic Backgrounds . . . . . . . . . . . . . . . . . . . 30

1.6.4 Cosmogenic Backgrounds . . . . . . . . . . . . . . . . . . 30

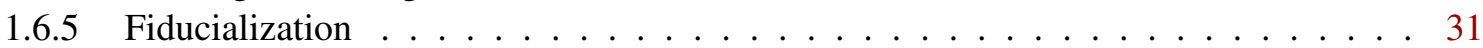

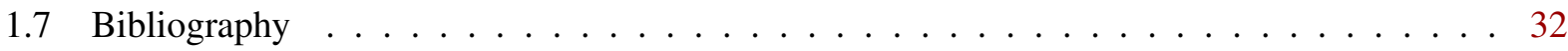

2 Scientific Performance $\quad 37$

2.1 WIMP Sensitivity and Discovery Potential . . . . . . . . . . . . . . 37

$2.1 .1 \quad \mathrm{~S} 1+\mathrm{S} 2$ Analysis . . . . . . . . . . . . . . . . . . . . 38

2.2 Neutrino Physics . . . . . . . . . . . . . . . . . . . . . . . . 39

2.2.1 Solar and Atmospheric Neutrinos . . . . . . . . . . . . . . . . . . . 40

2.2.1.1 Elastic Scattering of Solar Neutrinos . . . . . . . . . . . . . . . . . . 40

2.2.1.2 Coherent Nuclear Scattering of Solar Neutrinos . . . . . . . . . . . . . . 41

2.2.1.3 Atmospheric Neutrinos . . . . . . . . . . . . . . . . . 42

2.2.1.4 Neutrino Magnetic Moment . . . . . . . . . . . . . . . . . . . . . . 43

2.2.1.5 Other Neutrino Backgrounds . . . . . . . . . . . . . . . . . 43

2.2 .2 Double Beta Decay . . . . . . . . . . . . . . . . . . . . . . . 44

2.2.2.1 Neutrinoless Double Beta Decay . . . . . . . . . . . . . . . . . . . 44

2.2.2.2 Two Neutrino Double Beta Decay . . . . . . . . . . . . . . . . . . . . . 45

2.2 .3 Supernova Neutrinos . . . . . . . . . . . . . . . . . . . 45

2.2 .4 Sterile Neutrinos . . . . . . . . . . . . . . . . . . . . . . 45

2.3 New Physics Beyond Nuclear Recoils from WIMPs . . . . . . . . . . . . . . . . . . 47

2.3.1 Electrophilic WIMPs . . . . . . . . . . . . . . . . . . . . 47 
2.3.2 Axions and Axion-like Particles . . . . . . . . . . . . . . . . . 47

2.4 Physics with the Outer Detector . . . . . . . . . . . . . . . . . . . . 48

2.4.1 Muons and muon-induced neutrons . . . . . . . . . . . . . . . . . . 49

2.4 .2 Neutrinos . . . . . . . . . . . . . . . . . . . 50

2.4 .3 Exotic particles . . . . . . . . . . . . . . . . 50

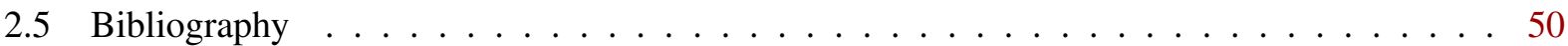

3 Xenon Detector System

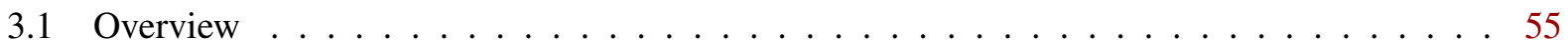

3.2 The Liquid Xenon Time Projection Chamber . . . . . . . . . . . . . . . . 58

3.2 .1 Drift Region . . . . . . . . . . . . . . . . . . . . 58

3.2 .2 Reverse-Field Region . . . . . . . . . . . . . . . . . . . . . . . . 59

3.2.3 Electroluminescence Region . . . . . . . . . . . . . . . . . . . . 61

3.2 .4 VUV Reflectors . . . . . . . . . . . . . . . . . . . . . . . 62

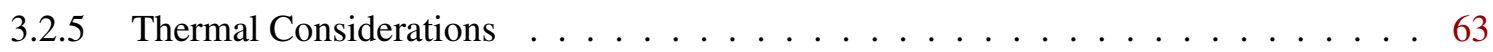

3.3 Cathode HV Delivery System . . . . . . . . . . . . . . . . . . 63

3.3.1 Cathode HV Requirements . . . . . . . . . . . . . . . . . . . 63

3.3 .2 HV System Overview . . . . . . . . . . . . . . . . . . . . 64

3.3.3 Cathode Supply and Cable Connection . . . . . . . . . . . . . . . 65

3.3 .4 Cathode Feedthrough . . . . . . . . . . . . . . . . . . . . . 65

3.3 .5 Cathode HV Umbilical . . . . . . . . . . . . . . . . . . . . . 65

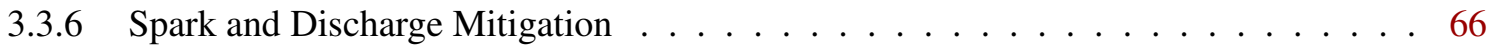

3.3.7 HV Connection to Cathode Ring . . . . . . . . . . . . . . . . . . . . . 67

3.3 .8 HV Safety Issues . . . . . . . . . . . . . . . . . . . . . . . . . . . . . . . . . . . . . . . . . . . . . . . . .

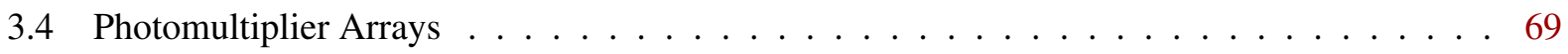

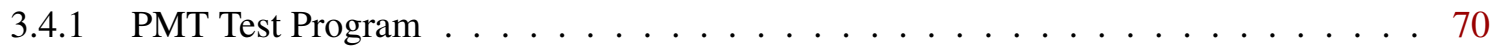

3.4 .2 PMT Radioactivity . . . . . . . . . . . . . . . . . . 70

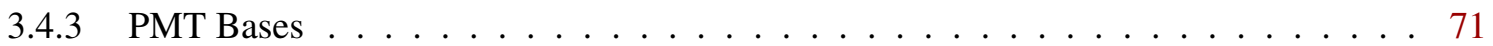

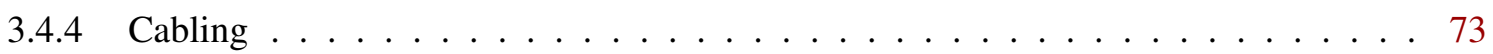

3.4 .5 Assembly and Integration with $\mathrm{TPC} \ldots \ldots \ldots \ldots . \ldots \ldots$

3.4 .6 PMT Calibration . . . . . . . . . . . . . . . . . . . . . 77

3.5 S1 Light Collection Design . . . . . . . . . . . . . . . . . . . . . . 77

3.5.1 Overview of Design and Optical Performance of the TPC . . . . . . . . . . . . 77

3.5 .2 TPC Optical Properties . . . . . . . . . . . . . . . . . . . . . 80

3.5 .3 PMT Arrays $\ldots \ldots \ldots \ldots \ldots \ldots \ldots \ldots$

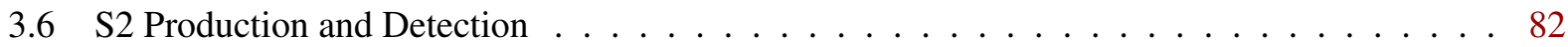

3.6 .1 S2 Photon Production $\ldots \ldots \ldots \ldots \ldots$

3.6 .2 S2 Photon Detection . . . . . . . . . . . . . . . . . 85

3.6.3 S2 Resolution and Electrode Configuration . . . . . . . . . . . . . . . . . 86

3.6.4 Design and Fabrication of the Grids . . . . . . . . . . . . . . . . . . . . . . . . . . . . . . . . . . .

3.6.5 Reconstruction of Peripheral Interactions . . . . . . . . . . . . . . . . . . 89

3.7 The Xe Skin Detector . . . . . . . . . . . . . . . . . . . . . . . . . . . . 90

3.7 .1 Skin performance . . . . . . . . . . . . . . . . . . 92

3.8 Internal Fluid System . . . . . . . . . . . . . . . . . . . . . . 93

3.9 Xenon System Monitoring _ . . . . . . . . . . . . . . . . . . . 97

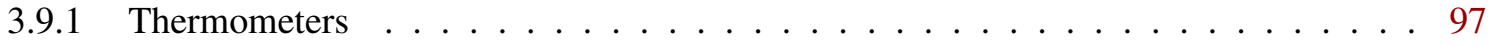

3.9.2 Liquid Level Sensors . . . . . . . . . . . . . . . . . . . . . . . . . . . . . 99 
3.9 .3 Acoustic Detection . . . . . . . . . . . . . . . . . . . . 100

3.9 .4 Loop Antennae for Discharge Detection . . . . . . . . . . . . . . . . . 100

3.9 .5 Position Sensors . . . . . . . . . . . . . . . . . . . . 100

3.10 Integrated System Testing . . . . . . . . . . . . . . . . . . . . . . 100

3.10 .1 Reflectivity Measurements . . . . . . . . . . . . . . . . . . . 101

3.10 .2 HV Studies in Small Two-Phase Chambers . . . . . . . . . . . . . . . . . 103

3.10 .3 TPC Design Testing . . . . . . . . . . . . . . . . . . . . . 106

3.10 .4 Full Scale Grid Testing at SLAC . . . . . . . . . . . . . . . . . 109

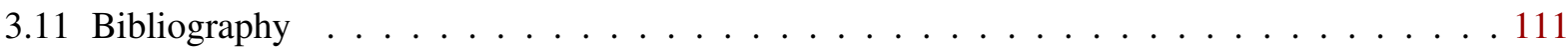

4 Outer Detector $\quad 115$

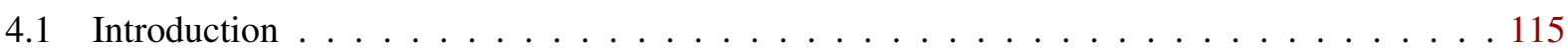

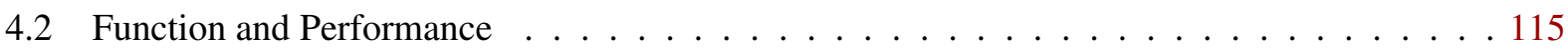

4.3 Outer Detector Overview . . . . . . . . . . . . . . . . . . . . . . . 117

4.4 Mechanical Systems . . . . . . . . . . . . . . . . . . . . . . . . . . 119

$4.4 .1 \quad$ Acrylic Vessels . . . . . . . . . . . . . . . . . . . . . . . . . 119

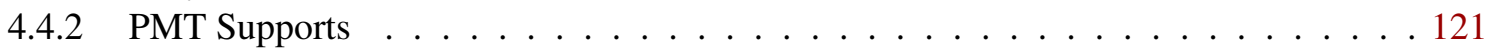

4.4 .3 Scintillator Distribution System . . . . . . . . . . . . . . . . 122

4.5 Liquid Scintillator . . . . . . . . . . . . . . . . . . . . . . 123

4.6 Light Detection . . . . . . . . . . . . . . . . . . . . . . 125

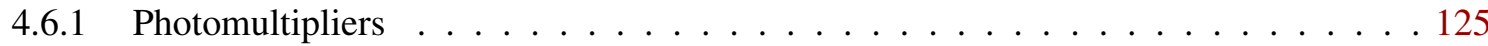

4.7 Optical Calibration System ～. . . . . . . . . . . . . . . . . . . . . 126

4.8 Threshold, singles rate, deadtime . . . . . . . . . . . . . . . . . 126

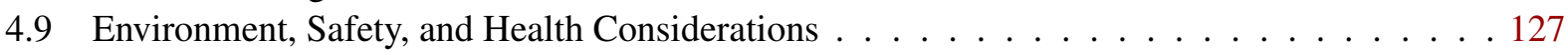

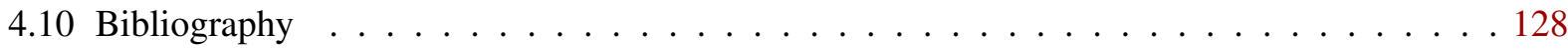

5 Cryostat 129

5.1 Material Search Campaign . . . . . . . . . . . . . . . . . . . . . . . . . 129

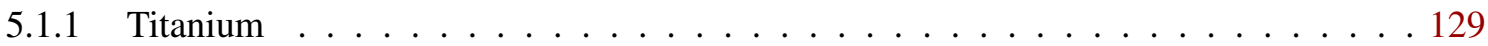

5.1 .2 Stainless Steel . . . . . . . . . . . . . . . . . . . . 130

5.2 Monte Carlo simulations of background from the cryostat . . . . . . . . . . . . . 133

5.3 Cryostat technical specification . . . . . . . . . . . . . . . . 133

5.4 Inner Cryostat Vessel . . . . . . . . . . . . . . . . . . . . . . . 135

5.5 Outer Cryostat Vessel . . . . . . . . . . . . . . . . . . . . . 137

5.6 Cryostat Support . . . . . . . . . . . . . . . . . . . . . . . 138

5.7 Cryostat Interfaces . . . . . . . . . . . . . . . . . . . . . . 138

5.8 Thermal Insulation $\ldots \ldots \ldots \ldots \ldots$

5.9 Fabrication, Cleanliness, Tests and Certifications . . . . . . . . . . . . . . . . 140

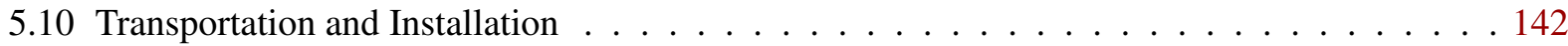

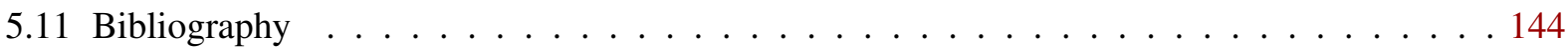

6 Xenon and Cryogenics Systems 147

6.1 Introduction and overview of requirements . . . . . . . . . . . . . . . . 147

6.2 Xenon purification and storage specifications . . . . . . . . . . . . . . . 148

6.2 .1 Air leaks during storage . . . . . . . . . . . . . . . . . . . . . 148

6.2 .2 One-time air contamination during operations. . . . . . . . . . . . . . . . . 148

6.2 .3 Continuous air leak during operations. . . . . . . . . . . . . . . . . . . . . 148

6.2.4 Cosmogenic activation of the Xe . . . . . . . . . . . . . . . . . . . 149 
6.3 Krypton Removal via Chromatography . . . . . . . . . . . . . . . . . . . . . . . . . . 149

6.4 Online Xenon Purification System ～. . . . . . . . . . . . . . . . . 156

6.4 .1 Gas recirculation system . . . . . . . . . . . . . . . . . . 157

6.4 .2 Liquid xenon tower . . . . . . . . . . . . . . . . . . . 161

6.4 .3 LXe transfer lines . . . . . . . . . . . . . . . . . . . . . . . . 164

6.4.4 SLAC System Test prototype of the circulation architecture . . . . . . . . . . . 165

6.4 .5 Radon removal . . . . . . . . . . . . . . . . . . . . . 166

6.4 .6 Slow controls . . . . . . . . . . . . . . . . . . . . . . . 169

6.5 Xenon Recovery . . . . . . . . . . . . . . . . . . . . . . . . . 170

6.6 Long-term xenon storage and transportation . . . . . . . . . . . . . . . . 175

6.7 Xenon Sampling and Assay ～. . . . . . . . . . . . . . . . . . 178

6.8 Cryogenics, vacuum services, and breakout boxes . . . . . . . . . . . . 181

6.9 Xenon Procurement . . . . . . . . . . . . . . . . . . . . . 186

6.10 Solubility and diffusion constants of common impurity gas species $\ldots \ldots$. . . . . . 186

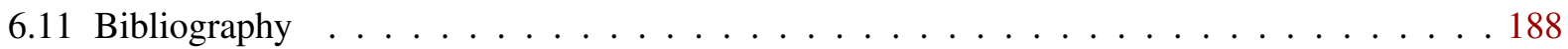

7 Calibration Systems $\quad 189$

7.1 Internal Radioisotope Sources _. . . . . . . . . . . . . . . . . . . . . . . . . 190

7.1 .1 Metastable Krypton $83\left({ }^{83 \mathrm{~m}} \mathrm{Kr}\right) \ldots \ldots \ldots \ldots$. . . . . . . . . . . . . . . . . . . . . . . . . . . . . . . . . . .

7.1.2 Metastable Xenon $131\left({ }^{131 \mathrm{~m}} \mathrm{Xe}\right) \ldots \ldots \ldots \ldots$

7.1.3 Tritium-Labeled Methane . . . . . . . . . . . . . . . . . . . . 191

7.1 .4 Radon $220\left({ }^{220} \mathrm{Rn}\right) \ldots \ldots \ldots \ldots$. . . . . . . . . . . . . . . . . . . . . . . . . . . . . . . . . . . . . . . . . . .

7.1.5 Internal Radioisotope Source Delivery . . . . . . . . . . . . . . . . . . . 192

7.2 External Radioisotope Source Delivery . . . . . . . . . . . . . . . . . . . . 193

7.2.1 Neutron sources . . . . . . . . . . . . . . . . . . . . . . 193

7.2.2 Gamma Sources for Calibration of the Active Xe TPC . . . . . . . . . . . . . . 194

7.2.3 Gamma Sources for Calibration of Xenon Skin and the Scintillator Veto . . . . . . . 195

7.2.4 Calibration Source Deployment (CSD) ～. . . . . . . . . . . . . . . . . 195

7.2.5 Radioisotope Capsule Design . . . . . . . . . . . . . . . . . . . 196

7.3 Photoneutron Sources ． . . . . . . . . . . . . . . . . . . . . . . . 197

7.3.1 Physics of Photoneutron Sources . . . . . . . . . . . . . . . . . . . . . 197

7.3.2 Photoneutron Source Deployment ． . . . . . . . . . . . . . . . . . . 198

7.4 Deuterium-Deuterium Neutron Source . . . . . . . . . . . . . . . . . . . . . . . . . . . 199

7.4.1 Physics motivation of Deuterium-Deuterium Neutron Sources . . . . . . . . . . . 199

7.4.1.1 NR Qy / Ly calibration of the LZ detector . . . . . . . . . . . . . . . . . 200

7.4.1.2 Calibrating LZ detector using Reflected Neutrons from D2(O) Target . . . 200

7.4.1.3 Calibrating LZ Detector with Neutron Events with no S1 Light . . . . . . 201

7.4.1.4 Direct Ly Measurement Using Double (Multiple) Scatter Events . . . . . 201

7.4 .2 DD Source Deployment and Neutron Rates . . . . . . . . . . . . . . . . . 202

7.5 Calibration Rates . . . . . . . . . . . . . . . . . . . . . . 203

7.5.1 Effect of $\mathrm{Gd}$ doping in liquid scintillator . . . . . . . . . . . . . . . . . . . . . . . . . . . . . . . .

7.5.2 Effect of gamma captures on useful neutron event rates . . . . . . . . . . . 203

7.5.3 Maximum useful calibration rates . . . . . . . . . . . . . . . . 203

7.6 Environment, Health and Safety Concerns _ . . . . . . . . . . . . . . . 205

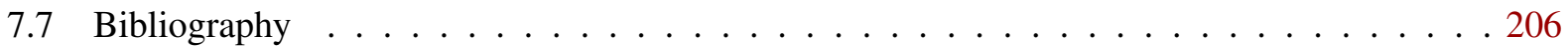

8 Electronics, DAQ, Controls, and Online Computing 207

8.1 Signal Processing . . . . . . . . . . . . . . . . . . . . . . 207 
8.2 Requirements . . . . . . . . . . . . . . . . . . . . 208

8.3 Analog Electronics . . . . . . . . . . . . . . . . . . . . . . . 210

8.3 .1 Design Criteria . . . . . . . . . . . . . . . . . . . 210

8.3.2 LZ Amplifier Prototype . . . . . . . . . . . . . . . . . . . . 214

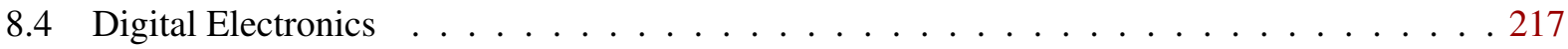

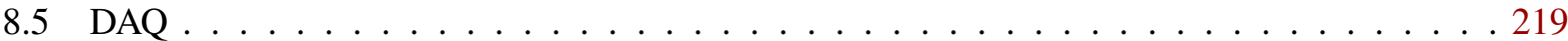

8.5.1 Data Extraction . . . . . . . . . . . . . . . . . . . 219

8.5 .2 Data Sparsification . . . . . . . . . . . . . . . . . . . 222

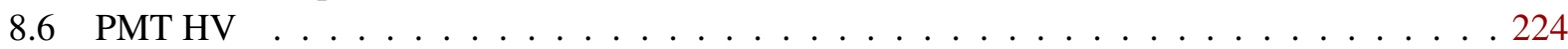

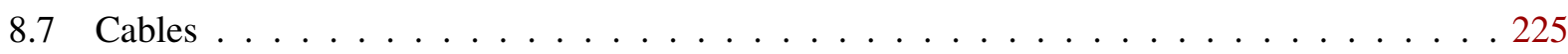

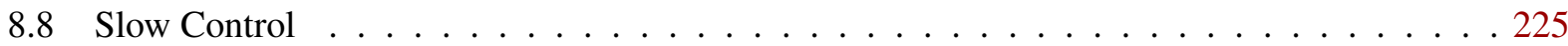

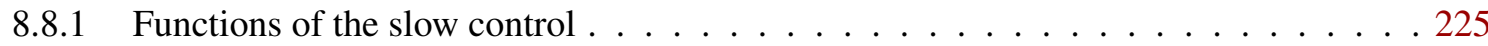

8.8 Requirements . . . . . . . . . . . . . . . . . . 226

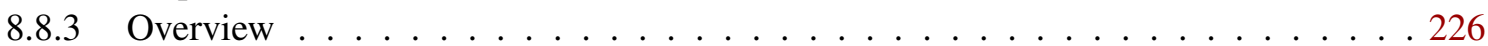

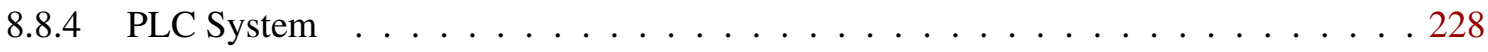

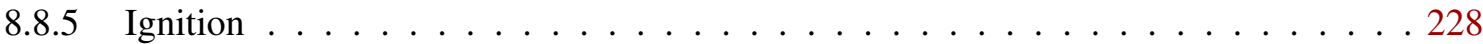

8.8 .6 Tags and device interfaces . . . . . . . . . . . . . . . . . . . . . 229

8.8.7 Historian, alarms and automation . . . . . . . . . . . . . . . . . . 229

8.8 .8 Graphical User Interface . . . . . . . . . . . . . . . . . . . . . 230

8.8 .9 Integration into experiment online IT $\ldots \ldots \ldots \ldots \ldots \ldots \ldots \ldots \ldots$

8.9 Online System . . . . . . . . . . . . . . . . . . . . . . . . . 231

8.10 Electronics Chain Test Facility . . . . . . . . . . . . . . . . . . . 234

8.10.1 Description and goals of the Electronics chain test facility . . . . . . . . . . . 234

8.10 .2 Single photoelectron pulse propagation . . . . . . . . . . . . 235

8.10 .3 PMT Base Saturation . . . . . . . . . . . . . . . . . . . . 237

8.10 .4 DAQ Code and Performance . . . . . . . . . . . . . . . . . . . 237

8.10 .5 Real Signals from LUX . . . . . . . . . . . . . . . . . . 239

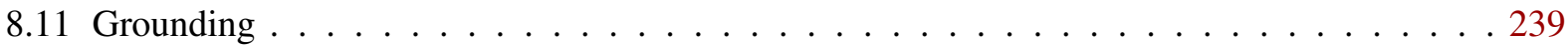

8.12 Network Infrastructure, Security, Remote Access _ . . . . . . . . . . . . . . . . 241

8.12 .1 Local Area Network . . . . . . . . . . . . . . . . . . . . . . . 241

8.12 .2 Reliability and Availability . . . . . . . . . . . . . . . . . . . 241

8.12 .3 Information Security . . . . . . . . . . . . . . . . . . . 241

8.12 .4 Remote Access . . . . . . . . . . . . . . . . . . . . . . . . . . . . . . . . . . . . . . . . . . . . . . . . . .

8.13 Installation and Commissioning . . . . . . . . . . . . . . . 243

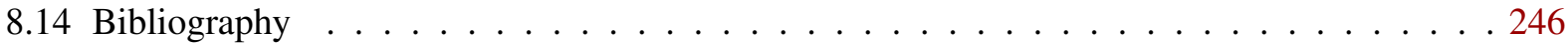

9 Material Screening $\quad 247$

9.1 Introduction . . . . . . . . . . . . . . . . . . . . . . . . 247

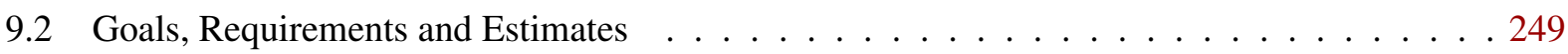

9.2 .1 Materials Table . . . . . . . . . . . . . . . . . . . 251

9.2 .2 Backgrounds Table . . . . . . . . . . . . . . . . . . . . 259

9.3 Techniques and Sensitivities . . . . . . . . . . . . . . . . . . . . 261

9.4 Intrinsic Contamination Techniques and Devices . . . . . . . . . . . . . . . . 264

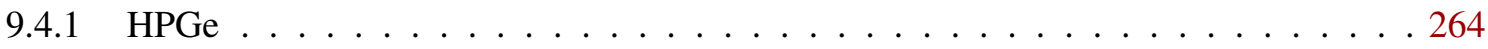

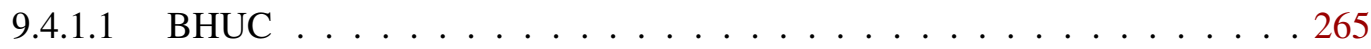

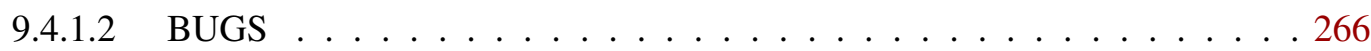

9.4.1.3 Surface HPGe Detectors . . . . . . . . . . . . . . . . . . . . . 267 


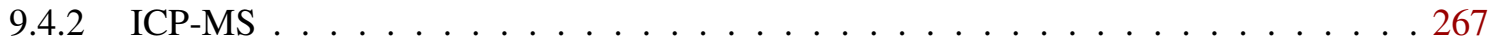

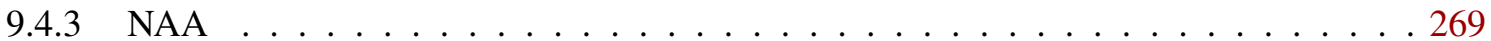

9.5 Radon Emanation . . . . . . . . . . . . . . . . . . . . . . . . . . . 270

9.6 Surface Cleanliness . . . . . . . . . . . . . . . . . . . . . . . . . . . 274

9.6 .1 Dust . . . . . . . . . . . . . . . . . . . . . . 274

9.6 .2 Radon Plate-out . . . . . . . . . . . . . . . . . . . . 277

9.7 Liquid Xenon Contamination ． . . . . . . . . . . . . . . . . . . . . . . . . . 280

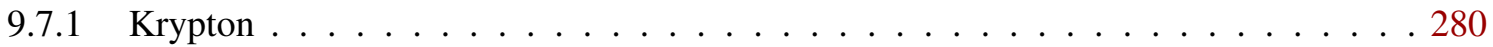

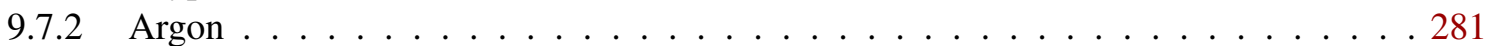

9.8 Laboratory and Cosmogenic Backgrounds . . . . . . . . . . . . . . . . . . 281

9.8.1 Laboratory Backgrounds . . . . . . . . . . . . . . . . . . . . . 282

9.8 .2 Cosmogenic Activation . . . . . . . . . . . . . . . . . . 283

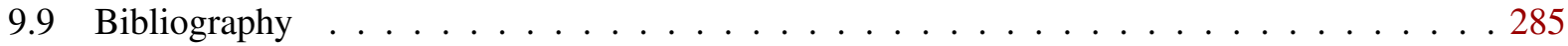

10 SURF Infrastructure, Assembly, and Integration $\quad 289$

10.1 Surface Infrastructure . . . . . . . . . . . . . . . . . . . . . 289

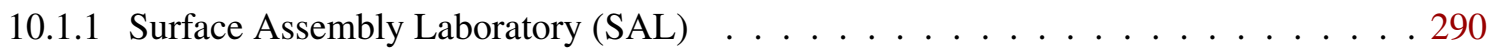

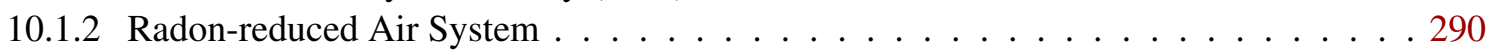

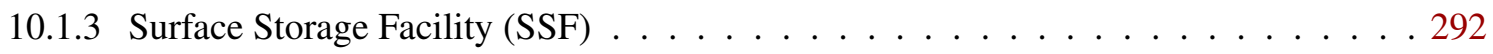

10.2 Yates Shaft Infrastructure and Custom Transport . . . . . . . . . . . . . . . . . 292

10.3 Underground Infrastructure ～. . . . . . . . . . . . . . . . . . . . . 294

10.4 Integration and Assembly . . . . . . . . . . . . . . . . . . . . . . 297

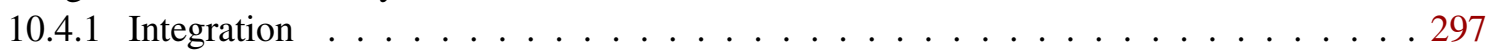

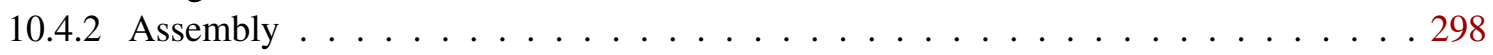

10.4.2.1 TPC Assembly (Steps SA2 - SA6) . . . . . . . . . . . . . 300

10.4.2.2 TPC Insertion into Cryostat with Fluid and Electrical Final Routing . . . . 302

10.4.2.3 Underground Outer Detector Tank Preparation / Staging (Steps U1 - U2) . 302

10.4.2.4 Cryostat Transportation and Underground Assembly (Steps U3 - U6) . . . 304

10.4.2.5 Utility Connection (Step U7) . . . . . . . . . . . . . . . . . . . . . . . . . 305

10.4.2.6 Outer Detector Assembly (Steps U8 - U9) . . . . . . . . . . . . . . 306

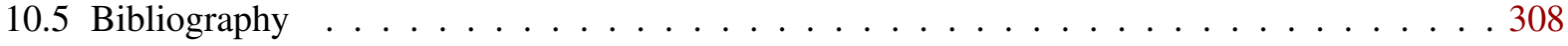

11 Offline Computing and Software $\quad 309$

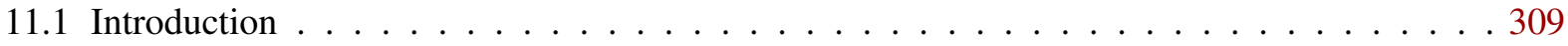

11.2 Data Volume, Data Processing, and Data Centers _ . . . . . . . . . . . . 310

11.2.1 The U.S. Data Center . . . . . . . . . . . . . . . . . . . . 311

11.2.2 The U.K. Data Center . . . . . . . . . . . . . . . . . . . . . 312

11.3 Analysis Framework . . . . . . . . . . . . . . . . 313

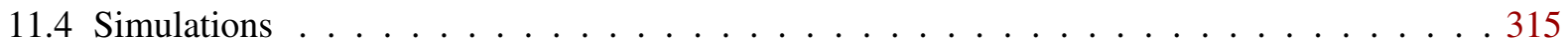

11.5 Software Infrastructure . . . . . . . . . . . . . . . . . 316

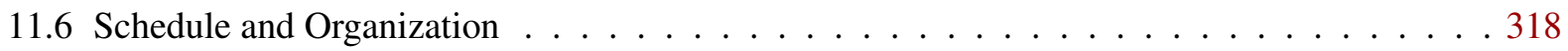

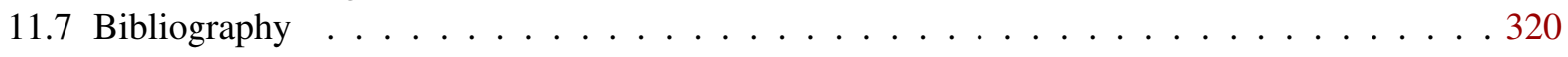

12 Simulations, Requirements, and Detector Performance 321

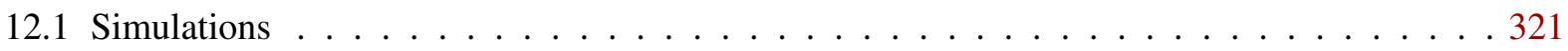

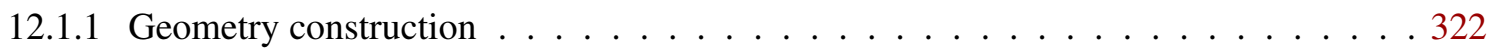

12.1.2 Event Generators . . . . . . . . . . . . . . . . . . . . . 323

12.1.2.1 Neutron production in detector materials . . . . . . . . . . . . . . . 324 
12.1.2.2 Muon-induced neutrons . . . . . . . . . . . . . . . . . . . 324

12.1.2.3 Gamma activity in large detector components . . . . . . . . . . . . 324

12.1.2.4 Gammas from the cavern rock . . . . . . . . . . . . . . . . . . 325

12.1.2.5 Benchmark points . . . . . . . . . . . . . . . . 325

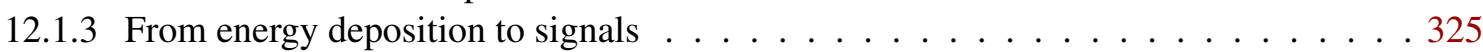

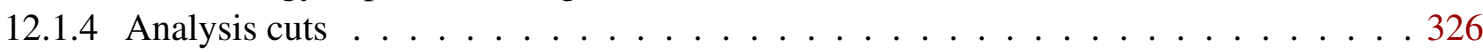

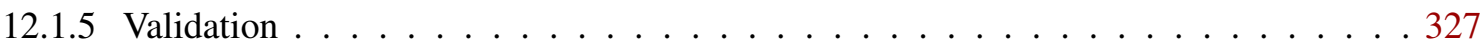

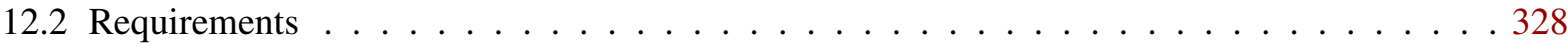

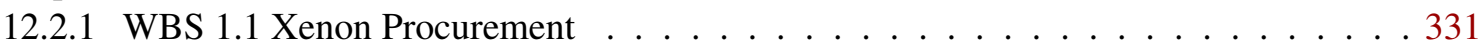

12.2.2 WBS 1.2 Xenon Vessel . . . . . . . . . . . . . . . . . . . 332

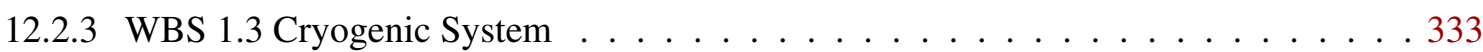

12.2.4 WBS 1.4 Xenon Purification . . . . . . . . . . . . . . . . . . 334

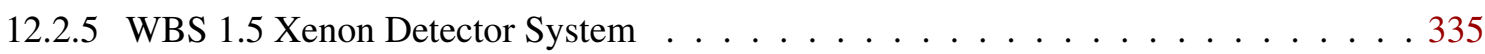

12.2.6 WBS 1.6 Outer Detector . . . . . . . . . . . . . . . . . . . 336

12.2.7 WBS 1.7 Calibration System . . . . . . . . . . . . . . . . . 336

12.2.8 WBS 1.8 Electronics, DAQ, Controls, and Computing . . . . . . . . . . . . 338

12.2.9 WBS 1.9 Integration and Installation . . . . . . . . . . . . . . . . . . 339

12.2.10 WBS 1.10 Cleanliness and Screening . . . . . . . . . . . . . . 340

12.2.11 WBS 1.11 Offline Computing . . . . . . . . . . . . . . . . . 341

12.3 Sensitivity and Detector Performance . . . . . . . . . . . . . . . . . 342

12.3.1 Profile Likelihood Ratio Method . . . . . . . . . . . . . . . . . . . 342

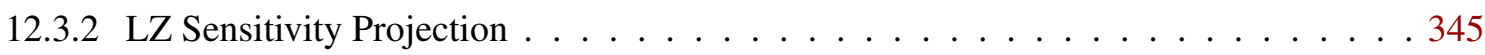

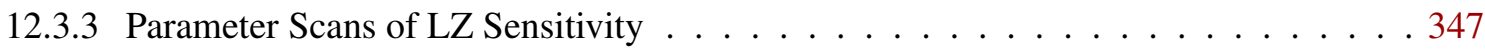

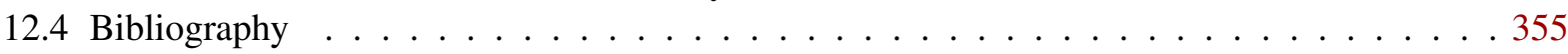

13 Management, Cost, and Schedule Summary 357

13.1 LZ Project Organization . . . . . . . . . . . . . . . . . . . 357

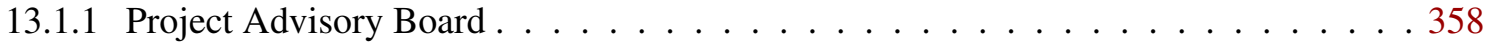

13.1.2 Project Management Office . . . . . . . . . . . . . . . . . 358

13.1.3 Project Work Breakdown Structure _ . . . . . . . . . . . . . . . . 359

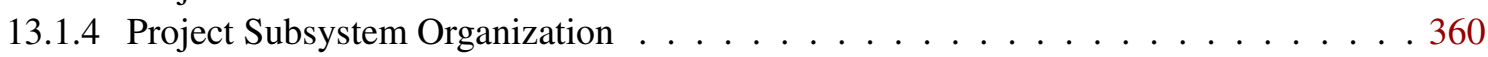

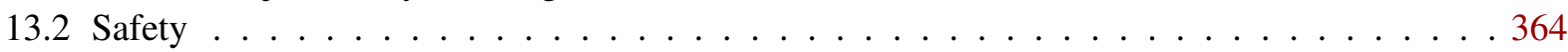

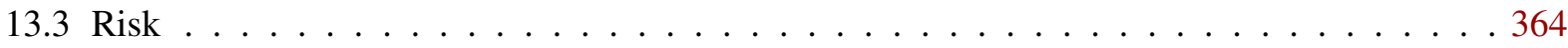

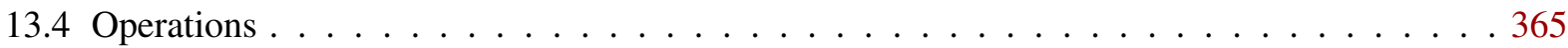

13.5 Cost and Schedule Summary . . . . . . . . . . . . . . . . . . 366

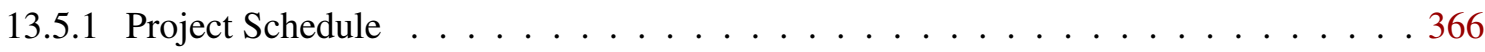

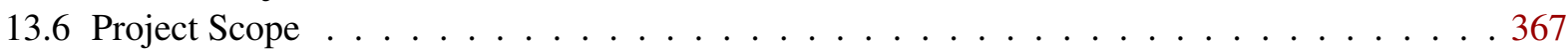

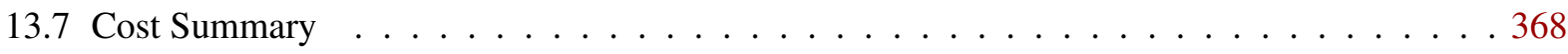





\section{List of Figures}

1.1.1 Scan of pMSSM parameter space and complementarity . . . . . . . . . . . 2

1.1.2 Compilation of current WIMP-nucleon SI cross-section upper limits (90\% CL) . . . . . . . . 3

1.1.3 Evolution of cross-section limits for 50-GeV WIMPs as function of time . . . . . . . . . 4

1.1.4 A compilation of WIMP-nucleon SI cross-section sensitivity . . . . . . . . . . . . 5

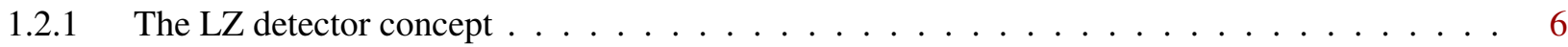

1.3.1 Integrated rates above threshold for various targets $\ldots \ldots \ldots \ldots \ldots \ldots$

1.3.2 Operating principle of the double-phase Xe TPC . . . . . . . . . . . . . . . 11

1.3.3 A double-scatter neutron event recorded in ZEPLIN-III . . . . . . . . . . . . . . . . 12

1.3.4 Mean interaction lengths for neutrons and gamma rays . . . . . . . . . . . . . . . . . . 14

1.3.5 Self-shielding of external neutrons and gamma rays in LXe . . . . . . . . . . . . . . . 15

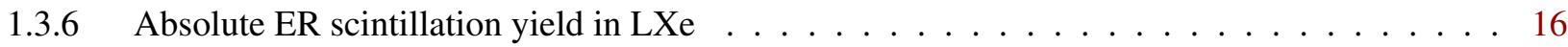

1.3 .7 ER ionization yield in $\mathrm{LXe} \ldots \ldots \ldots \ldots \ldots \ldots \ldots$

1.3.8 Absolute NR scintillation yield in LXe . . . . . . . . . . . . . . . . . . . 17

1.3.9 Absolute NR ionization yield in LXe . . . . . . . . . . . . . . . . . . . 18

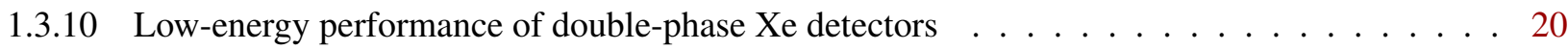

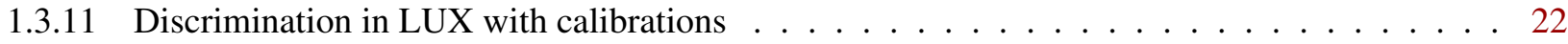

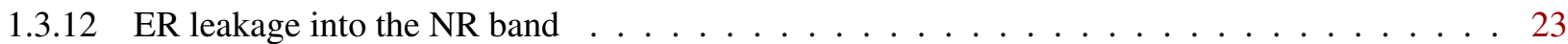

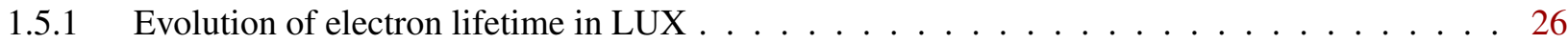

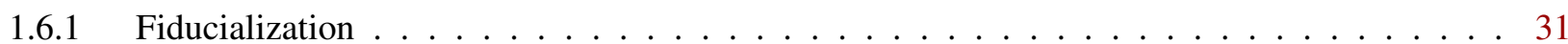

2.1 .1 The LUX WIMP search data $\ldots \ldots \ldots \ldots \ldots \ldots$

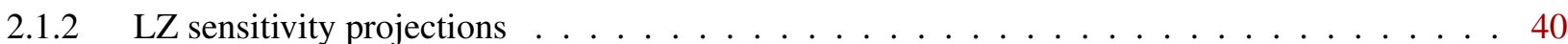

2.2.1 Calculated PDFs for NR from neutrinos . . . . . . . . . . . . . . . . . . . 42

2.2.2 Neutrino magnetic moment signal in LZ . . . . . . . . . . . . . . . . . . . . 43

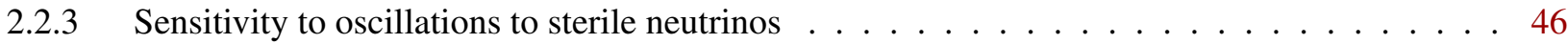

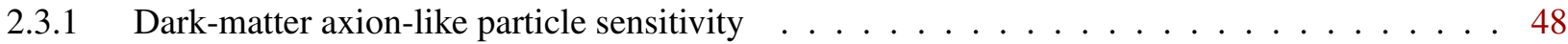

2.3.2 Solar axion-like particle sensitivity . . . . . . . . . . . . . . . . . . . . . 49

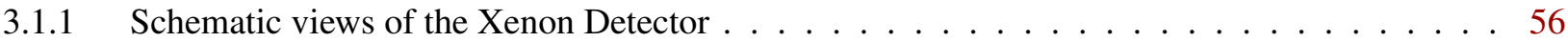

3.2 .1 TPC electric field regions $\ldots \ldots \ldots \ldots \ldots \ldots \ldots$

3.2 .2 TPC field non-uniformity $\ldots \ldots \ldots \ldots \ldots \ldots$

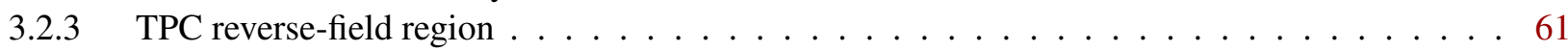

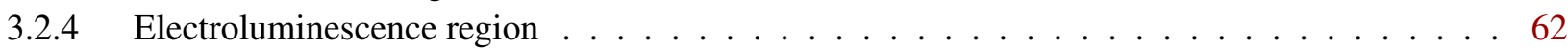

3.3.1 Cathode HV delivery . . . . . . . . . . . . . . . . . . . . . 65

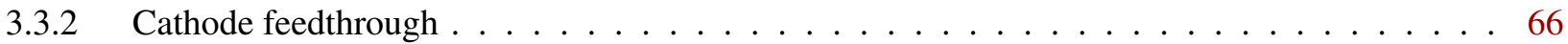

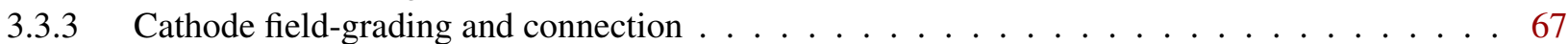

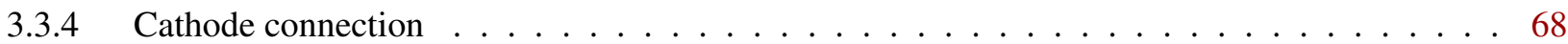

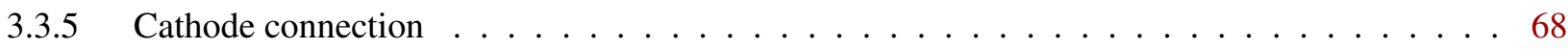

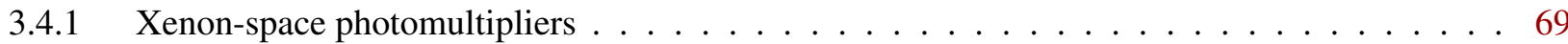

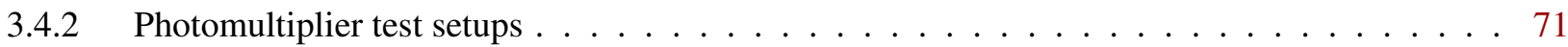

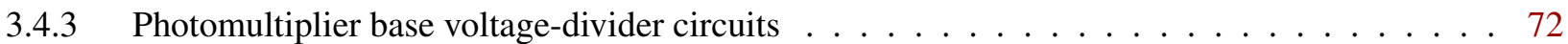




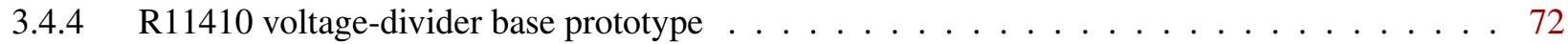

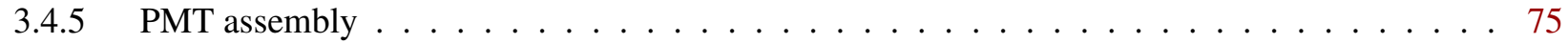

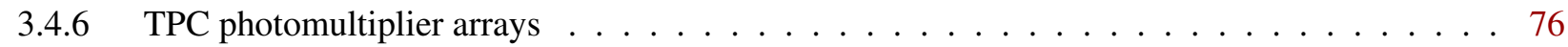

3.5.1 S1 photon detection efficiency scenarios . . . . . . . . . . . . . . . . 79

3.5.2 S1 PDE as a function of photon absorption length and PTFE reflectivity . . . . . . . . . . 80

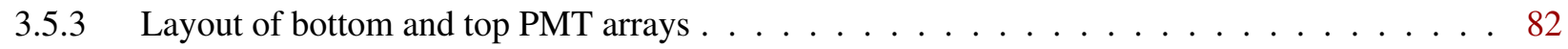

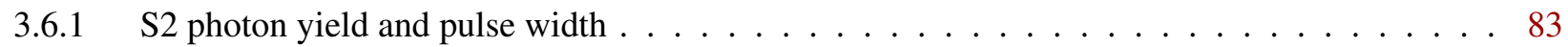

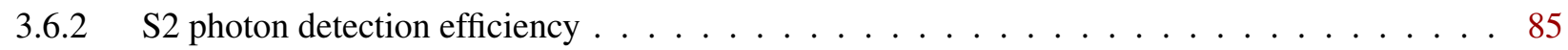

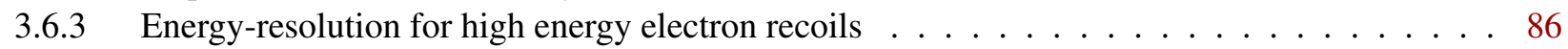

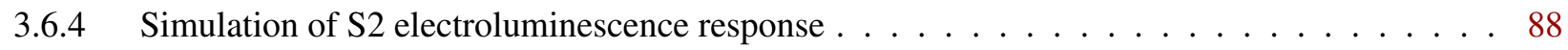

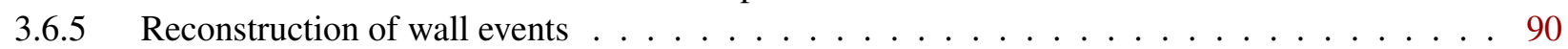

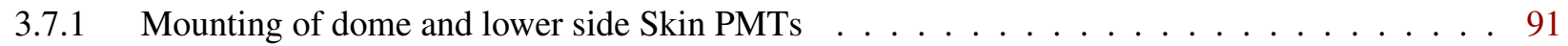

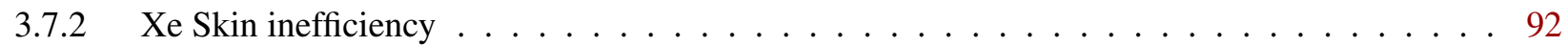

3.7.3 Light collection efficiency of Xe Skin detector . . . . . . . . . . . . . . . . . . 93

3.7.4 Skin performance as a function of PTFE reflectivity . . . . . . . . . . . . . 94

3.8 .1 Overview of internal circulation flow . . . . . . . . . . . . . . . . . 95

3.8.2 Details of fluid distribution pipes at the bottom of the vessel . . . . . . . . . . . 96

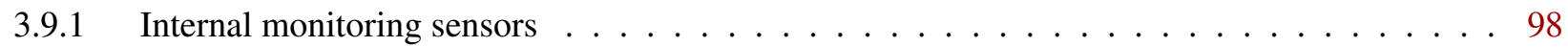

3.9.2 Xenon level sensors data . . . . . . . . . . . . . . . . . . . . . . . 99

3.10 .1 PTFE reflectivity measurements at LIP-Coimbra . . . . . . . . . . . . . . . . . . . 102

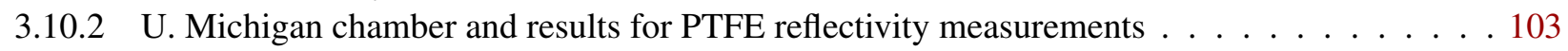

3.10.3 Imperial chamber to study high-field emission processes in thin wires . . . . . . . . . . . 104

3.10 .4 LBNL test chamber for small wire grids . . . . . . . . . . . . . . . . . 105

3.10 .5 SLAC System Test Phase-I TPC . . . . . . . . . . . . . . . . . . . . . . . 106

3.10 .6 LAr System Test at Yale . . . . . . . . . . . . . . . . . . . . . . . . 107

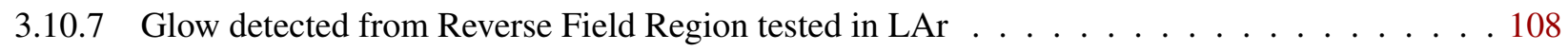

3.10 .8 LArTest . . . . . . . . . . . . . . . . . . . . . . . . . . . 109

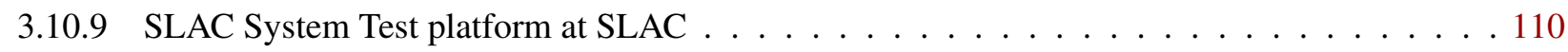

3.10 .10 SLAC Phase-I and Phase-II vessels . . . . . . . . . . . . . . . . . . . . . . 110

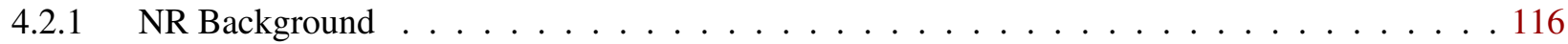

4.3.1 Layout of the LZ outer detector system . . . . . . . . . . . . . . . . . . 117

4.3 .2 Neutron Capture Times . . . . . . . . . . . . . . . . . . . . 118

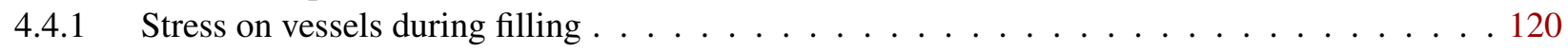

4.4.2 Two steps in the assembly sequence . . . . . . . . . . . . . . . . . . 121

4.4 .3 Plan view of the PMT support system . . . . . . . . . . . . . . . . . . . . 122

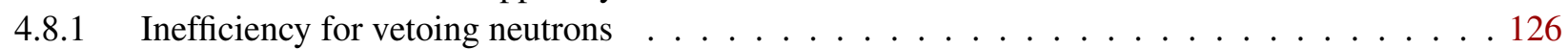

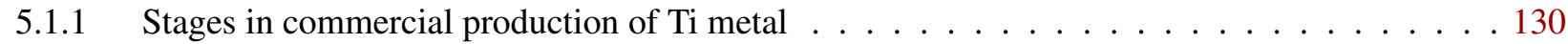

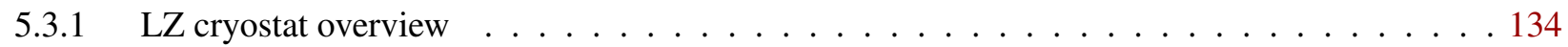

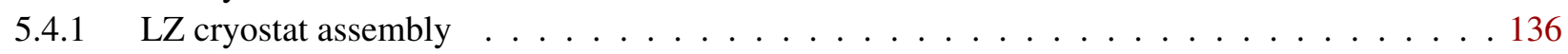

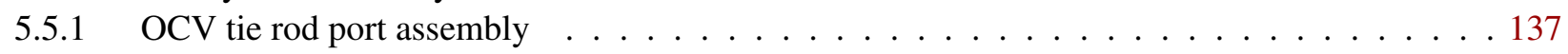

5.8 .1 Inner cryostat vessel thermal insulation . . . . . . . . . . . . . . . . . . . . 140

5.10 .1 Cryostat assembly . . . . . . . . . . . . . . . . . . . . . . 143

$6.3 .1 \quad \mathrm{Kr}$ removal $\mathrm{R} \& \mathrm{D}$ system . . . . . . . . . . . . . . . . . . . . . . . . . . . 149

$6.3 .2 \mathrm{Kr}$ removal time profiles of $\mathrm{Kr}$ and $\mathrm{Xe} \ldots \ldots \ldots \ldots \ldots$

$6.3 .3 \mathrm{Kr}$ removal simplified schematic . . . . . . . . . . . . . . . . . . . 152 
6.3.4 $\mathrm{Kr}$ removal condenser and $\mathrm{LN}$ system . . . . . . . . . . . . . . . . . . . . 153

$6.3 .5 \mathrm{Kr}$ removal physical layout $\ldots \ldots \ldots \ldots \ldots$

$6.3 .6 \mathrm{Kr}$ removal P\&ID . . . . . . . . . . . . . . . . . . . . . . . . 155

$6.4 .1 \quad$ Schematic diagram of $\mathrm{LZ}$ online purification system $\ldots \ldots \ldots \ldots \ldots$

6.4 .2 Xe Gas Recirculation system P\&ID . . . . . . . . . . . . . . . . . . . . . 158

6.4.3 Fluitron all-metal diaphragm compressor at SLAC . . . . . . . . . . . . . . . . . . 159

6.4.4 P\&ID of Detector and Xenon Handling Tie-Ins . . . . . . . . . . . . . . . . . . . . . . . 160

6.4 .5 Xe Tower with Internals . . . . . . . . . . . . . . . . . . . . . . . . . . . . 161

$6.4 .6 \quad$ P\&ID of the LXe Tower . . . . . . . . . . . . . . . . . . . . . . . . 162

6.4 .7 LXe Tower and LXe transfer lines . . . . . . . . . . . . . . . . . . . . . . . . . 164

6.4 .8 System Test LXe Tower . . . . . . . . . . . . . . . . . . . . . . . . . 166

6.4 .9 Breakthrough time . . . . . . . . . . . . . . . . . . . 168

6.4 .10 LZ radon removal system $\ldots \ldots \ldots \ldots 8$

6.4 .11 PLC Architecture . . . . . . . . . . . . . . . . . . . . . . . . . . . . . 169

6.5.1 Simplified flow schematic of LZ Xe Recovery System . . . . . . . . . . . . . . . . 171

6.5 .2 Xe system pressure threshold plot . . . . . . . . . . . . . . . . . . . 172

6.5.3 P\&ID of Recovery/Detector Tie-In . . . . . . . . . . . . . . . . . . . . . 173

6.5.4 Xe Delivery \& Recovery system P\&ID . . . . . . . . . . . . . . . . . . . . . . . . . 174

6.6 .1 Overview of LZ cylinder gas pack . . . . . . . . . . . . . . . . . . . . 176

6.6.2 Location of Xe Storage Room in the Davis campus . . . . . . . . . . . . . . . . . . . 178

6.6 .3 Xe Storage system P\&ID . . . . . . . . . . . . . . . . . . . . . . . . . 179

$6.7 .1 \quad \mathrm{Kr}$ pressure trace for $0.34 \mathrm{ppt}(\mathrm{g} / \mathrm{g}) \ldots \ldots \ldots \ldots \ldots \ldots$

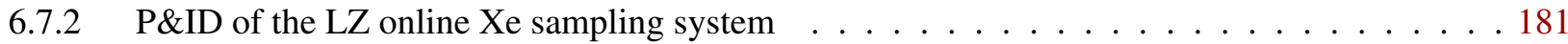

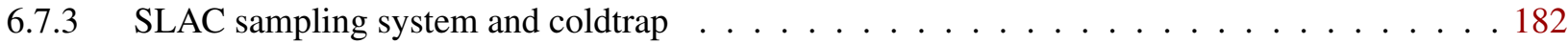

6.8.1 Cryogenics installation in the Davis Cavern . . . . . . . . . . . . . . . . . . . 183

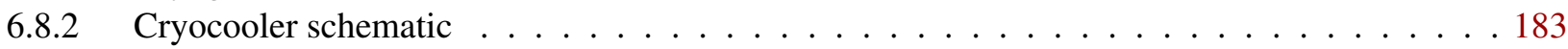

7.1.1 Illustrations of ${ }^{83 \mathrm{~m}} \mathrm{Kr}$ calibrations in LUX $\ldots \ldots \ldots \ldots 1 . \ldots \ldots \ldots$

7.1.2 Internal radioisotope source delivery system. . . . . . . . . . . . . . . . . . . . 193

7.2.1 Recoil spectra from neutron sources . . . . . . . . . . . . . . . . . . . . . . . . . . 194

7.2.2 Calibration of Xe Skin . . . . . . . . . . . . . . . . . . . . . 195

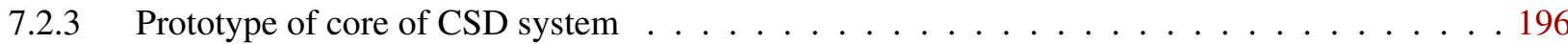

7.2.4 Prototype dummy source capsule . . . . . . . . . . . . . . . . . . . . . . . . . 197

7.3.1 S1/S2 spectra for ${ }^{8} \mathrm{~B}$ solar neutrino coherent scattering . . . . . . . . . . . . . . . . . 198

7.3.2 Photoneutron pig, various views . . . . . . . . . . . . . . . . . . . 199

7.4.1 Schematic setup for the utilization of a deuterium-based backscatter reflector . . . . . . . 200

7.4.2 LZ DD square wave neutron trigger pulse . . . . . . . . . . . . . . . . . 201

7.4.3 Solid model of LZ detector with angled neutron tube . . . . . . . . . . . . . . . . . 202

7.5.1 Event rate due to all gammas in the active xenon TPC from neutron captures on Gd . . . . 204

7.5.2 Event overlap probability as function of event rate . . . . . . . . . . . . . 205

8.1.1 TPC PMT signal processing schematics . . . . . . . . . . . . . . . . . . 207

8.1 .2 OD PMT signal processing schematics . . . . . . . . . . . . . . . . . . . 208

8.1 .3 A schematic of the data flow . . . . . . . . . . . . . . . . . . 209

8.3.1 Simulation of events with $>0.25$ PHE in two PMTs $\ldots \ldots \ldots \ldots \ldots \ldots$

$8.3 .2 \quad$ LZ amplifier . . . . . . . . . . . . . . . . . . . . . . . . 212

8.3.3 Simulation of $\mathrm{S} 2$ response for a $236-\mathrm{keV}$ Xe transition . . . . . . . . . . . . . . . . . 212

8.3.4 Simulation of $\mathrm{S} 2$ response for a 3-MeV Xe energy deposition . . . . . . . . . . . . . . . 212 
8.3.5 Dynamic range for $\mathrm{S} 2$ signals detected in the top and bottom PMTs . . . . . . . . . . . 213

8.3.6 Dynamic range for $\mathrm{S} 1$ signals detected in the bottom PMTs . . . . . . . . . . . . . 213

8.3 .7 Flanges and amplifier crate prototype . . . . . . . . . . . . . . . . . . . . . . . 214

8.3 .8 SPHE Waveforms . . . . . . . . . . . . . . . . . . . . 215

8.3 .9 Amplifier Noise . . . . . . . . . . . . . . . . . . . . . . . 215

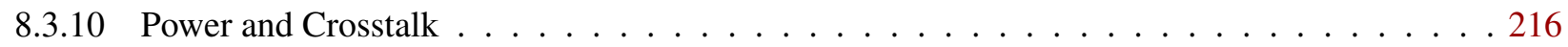

8.3 .11 Results of linearity measurements . . . . . . . . . . . . . . . . . . . . 216

8.3 .12 Lower Gain . . . . . . . . . . . . . . . . . . . . . . . . . . 217

8.4.1 Digital motherboard used to develop LZ digitizers . . . . . . . . . . . . . . . . . . 218

8.4.2 Prototype ADC daughter card with 32 channels . . . . . . . . . . . . . . . . . . 218

8.5.1 Diagram of the DAQ architecture . . . . . . . . . . . . . . . . . . . 219

8.5.2 Detailed depiction of interaction between DAQ elements . . . . . . . . . . . . . . . . 220

8.5.3 Proposed memory organization of POD waveform storage . . . . . . . . . . . . 220

8.5.4 Full digital waveform sum creation . . . . . . . . . . . . . . . . . . . . . . 223

8.8.1 Slow control functional diagram . . . . . . . . . . . . . . . . . . 227

8.8.2 An example of a low-level panel in the System Test slow control GUI . . . . . . . . . . . . . . 230

8.9.1 Primary interfaces between RC and other LZ subsystems . . . . . . . . . . . . . . . 231

8.9 .2 Run Control GUI screenshot . . . . . . . . . . . . . . . . . . . . 233

8.10.1 Diagram of the first phase of the Electronics Chain Test Facility . . . . . . . . . . . . 235

8.10 .2 Diagram of the Chain Test setup . . . . . . . . . . . . . . . . . . 235

8.10 .3 Single photoelectron intensity plots . . . . . . . . . . . . . . 236

8.10.4 Single photoelectron spectrum at estimated $1.9 \times 10^{6}$ and $3.25 \times 10^{6}$ gain . . . . . . . 236

8.10 .5 SPHE timing measurements . . . . . . . . . . . . . . . . . . 237

8.10 .6 PMT Base Pulse Shape Saturation . . . . . . . . . . . . . . . . . . . . . 238

8.10 .7 PMT Base Area Saturation f . . . . . . . . . . . . . . . . . . . . . 238

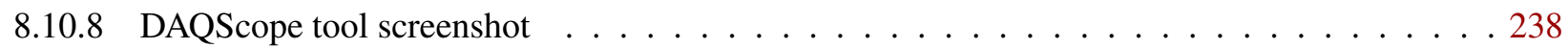

8.10 .9 Noise spectrum from LUX . . . . . . . . . . . . . . . . . . . . . . . 239

8.10 .10 SPHE spectrum obtained at LUX _ . . . . . . . . . . . . . . . . . . 240

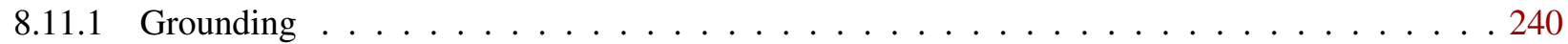

8.12 .1 The LZ Online network . . . . . . . . . . . . . . . . . . . . . . . . . 242

8.13 .1 Location of analog and digital electronics . . . . . . . . . . . . . . . . . . . 244

8.13.2 The top breakout box at the mezzanine level . . . . . . . . . . . . . . . . . . 244

8.13 .3 Details of one elements of the breakout system . . . . . . . . . . . . . . . . 245

8.13 .4 Layout of the LZ electronics racks . . . . . . . . . . . . . . . . . 245

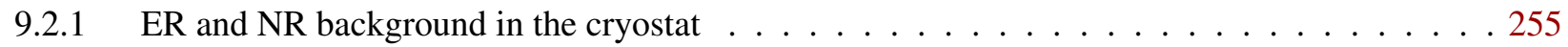

$10.1 .1 \quad$ SURF Aerial View . . . . . . . . . . . . . . . . . . . . . . . . . 289

10.1.2 The Surface Assembly Laboratory . . . . . . . . . . . . . . . . . . . . . . . . . 290

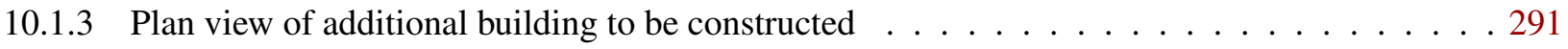

10.1.4 Plan view of RRS Building . . . . . . . . . . . . . . . . . . . . . 291

10.1.5 Building constructed for staging LZ detector components . . . . . . . . . . . . . . 292

10.2.1 LZ transport method from building to headframe . . . . . . . . . . . . . . . . . . . 293

10.3.1 Plan view of the Davis Campus at the 4850L . . . . . . . . . . . . . . . . . . . 294

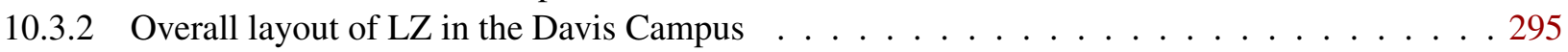

10.3.3 LZ and related support systems in the Davis Cavern . . . . . . . . . . . . . . . 296

10.4.1 LZ surface assembly sequence . . . . . . . . . . . . . . . . . . . . 301

10.4.2 Underground installation sequence in water tank . . . . . . . . . . . . . . . 303 


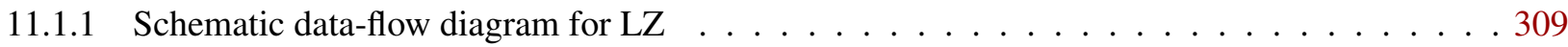

11.5.1 Parallel development workflow in GitLab . . . . . . . . . . . . . . . . . . 317

12.1.1 Engineering drawing and simulation geometry of the outer cryostat $\ldots \ldots \ldots 322$

12.1.2 Engineering drawing and simulation geometry of the TPC . . . . . . . . . . . . 323

12.1.3 Engineering drawing and simulation geometry of the bottom PMT array . . . . . . . . . 323

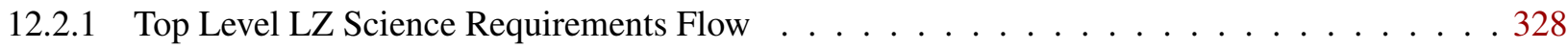

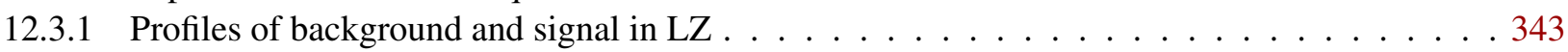

12.3.2 $\quad$ PLR discrimination statistic . . . . . . . . . . . . . . . . . . . . . . . 344

12.3.3 PLR technique for different masses . . . . . . . . . . . . . . . . . . . . 344

12.3.4 Acceptance and rejection for WIMP signals in LZ . . . . . . . . . . . . . . . . 345

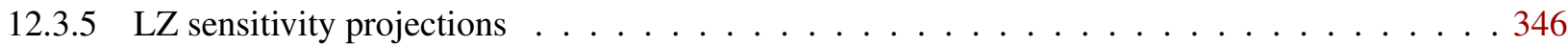

12.3.6 LZ discovery potential . . . . . . . . . . . . . . . . . . . . . . . 347

12.3.7 Example LZ exposure . . . . . . . . . . . . . . . . . . . . . . . . . . . . 348

12.3.8 LZ sensitivity projections for goal, baseline, and reduced parameters . . . . . . . . . . . 349

12.3.9 LZ sensitivity projections vs. radon concentration $\ldots \ldots \ldots \ldots \ldots$

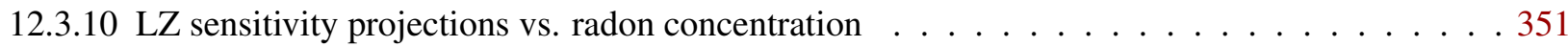

12.3.11 LZ sensitivity projections vs. atmospheric neutrino rate . . . . . . . . . . . . 351

12.3.12 LZ sensitivity projections vs. S1 photon detection efficiency . . . . . . . . . . . . . 352

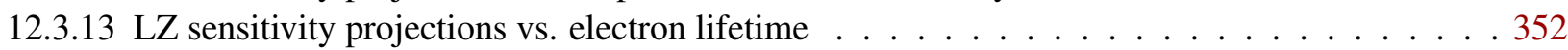

12.3.14 LZ sensitivity projections vs. electron extraction efficiency . . . . . . . . . . . . 353

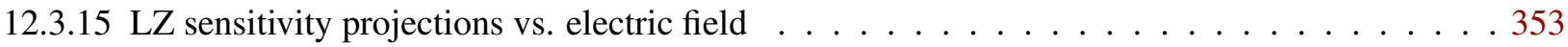

12.3.16 LZ sensitivity projections vs. trigger coincidence level . . . . . . . . . . . . . . 354

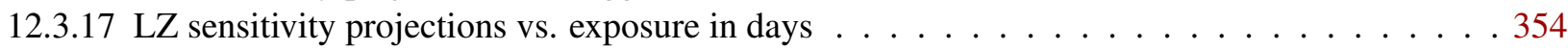

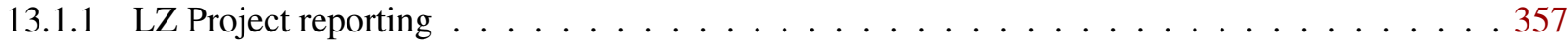

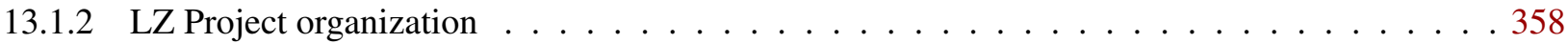

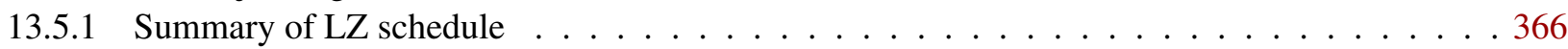





\section{List of Tables}

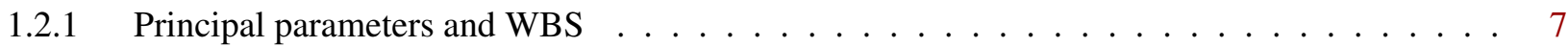

1.6.1 Summary of backgrounds in LZ . . . . . . . . . . . . . . . . . . . . 29

2.1.1 Key LZ and LUX Assumptions Compared . . . . . . . . . . . . . . . . . . . . . 39

3.1 .1 Xenon detector parameters $\ldots \ldots \ldots \ldots \ldots \ldots$

3.3.1 Dependence of TPC parameters on cathode HV . . . . . . . . . . . . . . . . . 64

3.3 .2 Safety factors . . . . . . . . . . . . . . . . . . . . . . 64

3.4 .1 Cabling in the xenon space $\ldots \ldots \ldots \ldots \ldots \ldots \ldots$

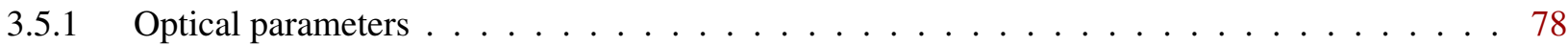

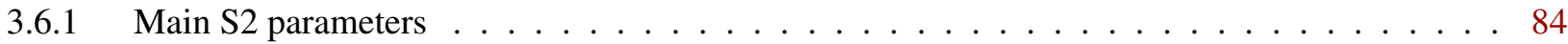

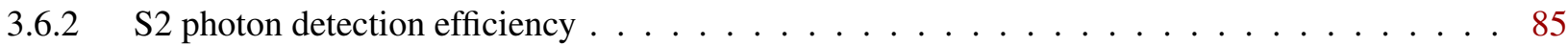

3.6 .3 TPC electrode grid parameters . . . . . . . . . . . . . . . . . . 87

3.9.1 Monitoring sensors in the xenon space . . . . . . . . . . . . . . . . . . . 97

3.10 .1 LZ System Test planning . . . . . . . . . . . . . . . . . . . . . . . 101

4.4.1 The volume and masses of the scintillator vessels . . . . . . . . . . . . . . . . . . . 120

4.5.1 Radioactive impurity goals in LAB-based Gd-LS . . . . . . . . . . . . . . . . . . . . . 124

4.6.1 Characteristics of the R5912 PMTs. . . . . . . . . . . . . . . . . . . . 125

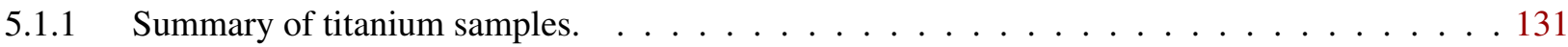

5.1.2 Summary of stainless steel samples. . . . . . . . . . . . . . . . . . . . 132

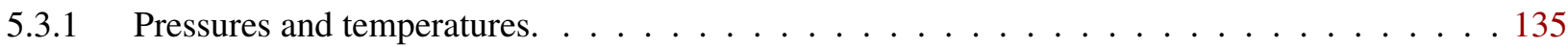

$5.4 .1 \quad$ Vessel-wall thicknesses. . . . . . . . . . . . . . . . . . . . . . 136

$5.7 .1 \quad$ External loads . . . . . . . . . . . . . . . . . . . . . . . . . 139

6.4.1 Radon emanation estimates . . . . . . . . . . . . . . . . . 167

6.8 .1 Heat load table. . . . . . . . . . . . . . . . . . . . . . . . 184

6.10 .1 Solubility and diffusion constants of common impurity species. . . . . . . . . . . . . 187

7.0.1 Baseline calibration sources for LZ . . . . . . . . . . . . . . . . . . . . . 189

7.5.1 Fraction of neutron scatters in the Xe TPC . . . . . . . . . . . . . . . . . . . 204

8.2.1 Properties of the PMTs . . . . . . . . . . . . . . . . . . . . . 209

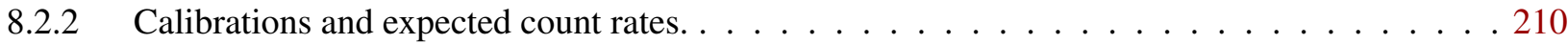

8.3.1 Summary of the number and type of the 1,359 analog signals. . . . . . . . . . . . . . . . 214

8.5.1 Key parameters of the prototype Data Collector. . . . . . . . . . . . . . . . . 221

8.5.2 Summary of the performances of the DAQ links . . . . . . . . . . . . . . . 221

8.5.3 Summary of data collectors expected peak storage and buffering capabilities . . . . . . . . 221

8.5.4 Summary of three major waveform-selection modes for the central TPC PMTs. . . . . . . . . 224

8.6.1 Details of the PMT HV system. . . . . . . . . . . . . . . . . . . . . . 224

8.7.1 Information on LZ signal, logic, HV, power, and network cables. . . . . . . . . . . . . 225 


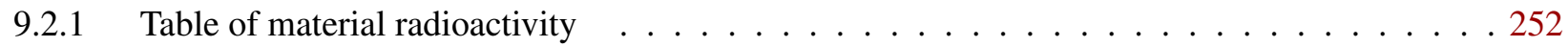

9.2.2 Results from the radio-assay of titanium sample TIMET HN3469 . . . . . . . . . . . . . 255

9.2.3 Assay results from R11410-20 PMT component materials . . . . . . . . . . . . . . . . 256

9.2.4 Assay results from 3-inch R11410 PMT base component materials . . . . . . . . . . . . . 257

9.2.5 Assay results from 1-inch R8520 skin PMT base component materials . . . . . . . . . . 258

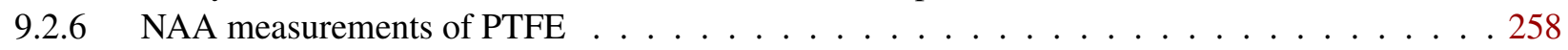

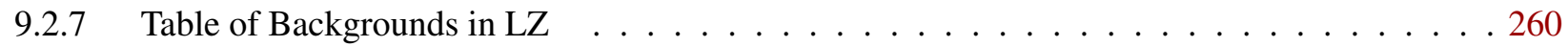

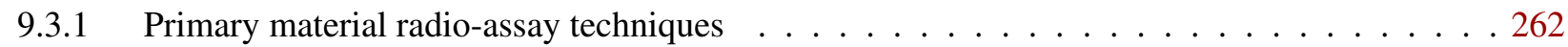

9.4.1 The LZ $\gamma$-ray spectroscopy detectors . . . . . . . . . . . . . . . . . . . 265

9.5.1 Radon emanation screening facilities available to LZ . . . . . . . . . . . . . . . . . 271

9.5.2 Materials in LZ to be assayed for radon emanation . . . . . . . . . . . . . . . . . 273

9.6.1 Expected NR background due to $(\alpha, n)$ reaction from plate-out activity . . . . . . . . . . . 278

9.6.2 Allowable exposure times (days) to reach radon daughter plate-out requirements . . . . . . 279

9.8.1 Background from laboratory, muon-induced neutrons, and cosmogenic activation . . . . . . 281

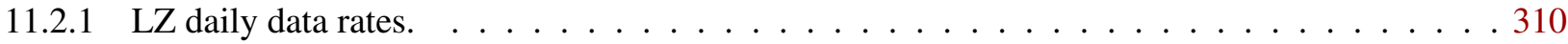

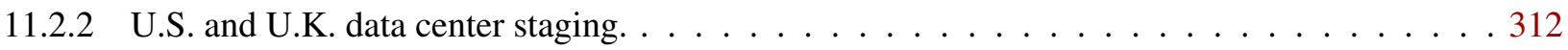

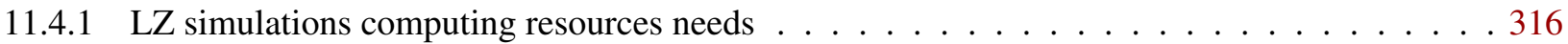

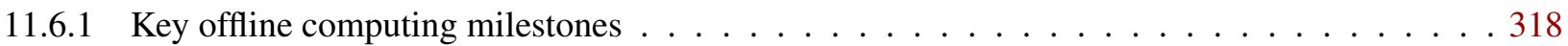

12.2.1 Top Level Science Requirements ～. . . . . . . . . . . . . . . . . . . . . . . . . . . . 329

12.2.2 Level 2 Requirements for WBS 1.2 Xenon Vessel . . . . . . . . . . . . . . . . . . 332

12.2.3 Level 2 Requirements for WBS 1.3 Cryogenic System . . . . . . . . . . . . . . . . . . . 333

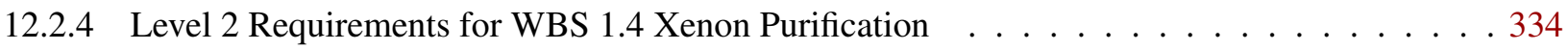

12.2.5 Level 2 Requirements for WBS 1.5 Xenon Detector System . . . . . . . . . . . . . . . 335

12.2.6 Level 2 Requirements for WBS 1.6 Outer Detector . . . . . . . . . . . . . . . . . 336

12.2.7 Level 2 Requirements for WBS 1.7 Calibration System . . . . . . . . . . . . . . . . 336

12.2.8 Level 2 Requirements for WBS 1.8 Electronics, DAQ, Controls, and Computing . . . . . . . 338

12.2.9 Level 2 Requirements for WBS 1.9 Integration and Installation . . . . . . . . . . . . . . 339

12.2.10 Level 2 Requirements for WBS 1.10 Cleanliness and Screening . . . . . . . . . . . . . . . . . . . . . . . . . . . . . . . .

12.2.11 Level 2 Requirements for WBS 1.11 Offline Computing . . . . . . . . . . . . . . . . . . . . 341

12.3.1 Backgrounds used in the profile likelihood analysis . . . . . . . . . . . . . . . . . . 342

12.3.2 Key parameters for reduced, baseline, and goal detector performance . . . . . . . . . . . 348

13.1.1 LZ Work Breakdown Structure . . . . . . . . . . . . . . . . . . . 359

13.1.2 LZ Work Breakdown Structure (WBS) shown at L2 . . . . . . . . . . . . . . . . . 361

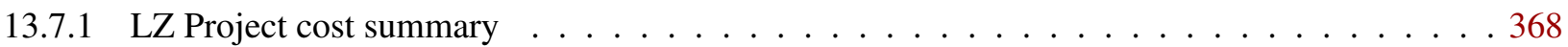




\section{Overview}

\subsection{Direct Detection of Dark Matter}

During the past two decades, a standard cosmological picture of the universe (the Lambda Cold Dark Matter or LCDM model) has emerged, which includes a detailed breakdown of the main constituents of the energy density of the universe. This theoretical framework is now on a firm empirical footing, given the remarkable agreement of a diverse set of astrophysical data [1,2]. Recent results by Planck largely confirm the earlier Wilkinson Microwave Anisotropy Probe (WMAP) conclusions and confirm that the universe is spatially flat, with an acceleration in the rate of expansion and an energy budget comprising approximately $5 \%$ baryonic matter, $26 \%$ cold dark matter (CDM), and roughly $69 \%$ dark energy [3-5]. With the generation-2 (G2) dark matter experiments, we are now in a position to identify this dark matter through sensitive terrestrial direct detection experiments. Failing to detect a signal in G2, or in subsequent generation (G3), experiments would rule out most of the natural parameter space that describes weakly interacting massive particles (WIMPs), forcing us to reassess the WIMP paradigm and look for new detection techniques. In the following sections, we introduce the cosmological and particle physics evidence pointing to the hypothesis that the dark matter is composed of WIMPs, detectable through nuclear recoil (NR) interactions in low-background experiments. We then give the motivation for a massive liquid xenon (LXe) detector as the logical next step in the direct detection of dark matter.

\subsubsection{Cosmology and Complementarity}

While the Large Hadron Collider (LHC) experiments continue to verify the Standard Model of particle physics to ever-greater precision, the nature of the particles and fields that constitute dark energy and dark matter remain elusive. The gravitational effects of dark matter are evident throughout the cosmos, dominating gravitational interactions of objects as small as dwarf satellites of the Milky Way, up to galaxy clusters and superclusters. Application of Kepler's laws leads to the inescapable conclusion that our own galaxy, and all others, are held together by the gravitational pull of a dark halo that outweighs the combined mass of stars and gas by an order of magnitude, and appears to form an extended halo beyond the distribution of luminous matter.

At the same time, very weakly interacting CDM, particles or compact objects that were moving nonrelativistically at the time of decoupling, appear to be an essential ingredient in the evolution of structure in the universe. N-body simulations of CDM can explain much of the structure, ranging from objects made of tens of thousands of stars to galaxy clusters. In the past few years, more realistic simulations, including both baryonic matter (gas and stars) and dark matter, are beginning to reveal how galaxy-like objects can arise from the primordial perturbations in the early universe $[6,7]$.

While we know much about the impact of dark matter on a variety of astrophysical phenomena, we know very little about its nature. An attractive conjecture is that dark matter particles were in equilibrium with ordinary matter in the hot early universe. We note, however, that there are viable dark matter candidates, including axions, where the conjecture of thermal equilibrium is not made [8]. Thermal equilibrium describes the balance between annihilation of dark matter into ordinary particle-antiparticle pairs, and vice versa. As the universe expanded and cooled, the reaction rates (the product of number density, cross section, and relative velocity) eventually fell below the level required for thermal equilibrium, leaving behind 
a relic abundance of dark matter. The lower the annihilation cross section of dark matter into ordinary matter, the higher the relic abundance of dark matter. An annihilation cross-section characteristic of the weak interaction results in a dark matter energy consistent with that observed by cosmological measurements [9].

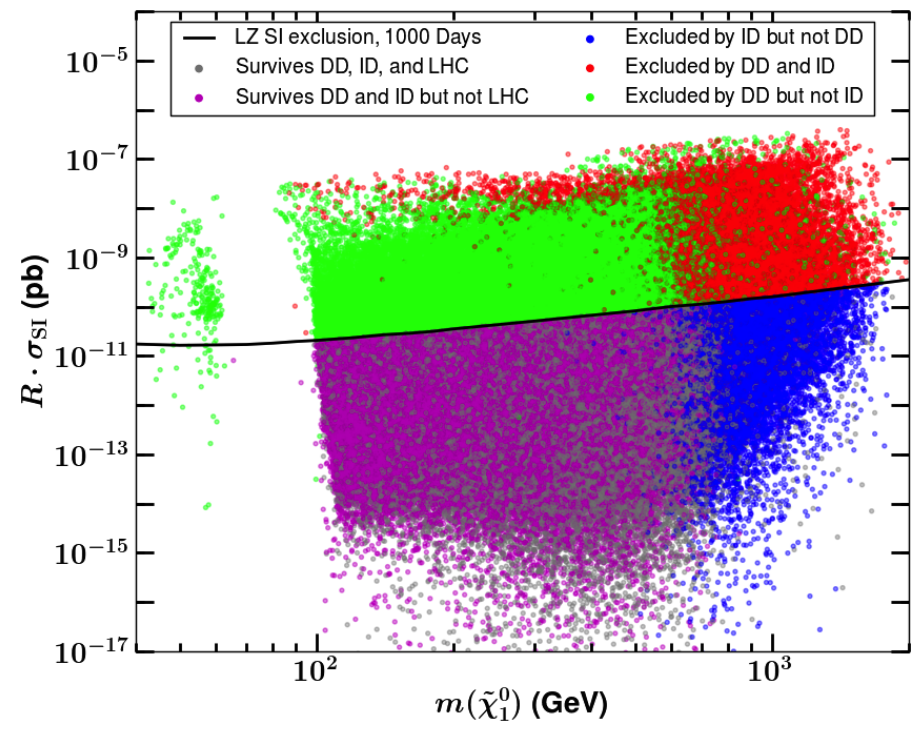

Figure 1.1.1: Scan of pMSSM parameter space and complementarity. Each point represents a SUSY model and is plotted as WIMP-nucleon scattering cross section scaled by abundance versus mass of the WIMP. The colors show models that can be tested by the three techniques of detection: direct detection (DD), LHC, and indirect detection (ID), and their combinations. The black curve shows the expected LZ sensitivity based on an early (2013) LZ estimate, weakened by a factor of four [10].

tal gravitational mass (from lensing) with the distribution of the dominant component of baryonic matter (evident in the X-ray-emitting cluster gas) one can see whether the dark mass follows the distribution of baryonic matter. For a number of galaxy clusters, in particular the Bullet cluster [13], the total gravitational mass (dominated by dark matter) follows the distribution of other non-interacting test particles (stars) rather than the dominant component of baryonic matter in the cluster gas. Combining this evidence with other observations of stellar distributions and velocity measurements for galaxies with a wide range of mass-tolight ratios, it appears that the total gravitational mass does not follow the distribution of baryonic matter as one would expect for modified gravity, but behaves like a second, dark component of relatively weakly interacting particles.

There are three complementary signals of WIMP dark matter. The dark matter of the Milky Way can interact with atomic nuclei, resulting in NRs that are the basis of direct detection (DD) experiments like LZ. At the LHC, the dark matter should be produced as a stable, non-interacting particle that appears as missing energy and momentum. Out in the cosmos, dark matter collects at the centers of galaxies and in the sun, where pairs of dark matter particles will annihilate with one another, if the dark matter is a Majorana particle, as expected in SUSY theories. The annihilations will produce secondary particles, including positrons, antiprotons, neutrinos, and gamma rays, providing the basis for "indirect" detection (ID) by gamma ray, cosmic ray, and neutrino telescopes. 
In Figure 1.1.1, we show the results of a recent analysis of the complementarity of the three signals from WIMP dark matter. The LHC has already provided constraints on the simplest SUSY parameter space, and the Higgs mass is in some tension with the most constrained versions of SUSY, requiring theorists to relax simplifying assumptions. One slightly less restrictive choice of parameters is the so-called phenomenological minimal supersymmetric standard model (pMSSM) model [14, 15]. In Figure 1.1.1, each point represents a choice of pMSSM parameters that satisfies all known physics and astrophysics constraints [10]. The color of the points show which experiments have adequate sensitivity to test whether that point is valid. The black curve shows the expected LZ sensitivity based on an early (2013) LZ estimate, weakened by a factor of four. The three experimental tests are: DD with the proposed LZ experiment, current LHC data, and ID from a proposed ID experiment; an enhanced version of the Cherenkov Telescope Array[16] with twice the number of telescopes compared with the current baseline. Each of the three experimental techniques tends to be most sensitive to one region in Figure 1.1.1, although there are regions of overlapping sensitivity.

The most important goal for the G2 program is to produce the best constraints over the natural mass range for dark matter. The LZ experiment accomplishes this goal. A broader goal of dark matter research is to use the three complementary approaches to establish the validity of any signal, and to identify the properties of the dark matter particle.

\subsubsection{Direct Detection Experiments}

The direct detection of dark matter in earthbound experiments depends on the local properties of the Milky Way's dark matter and on the properties of the dark matter particles themselves. The local properties of the Milky Way's dark halo are determined by astrophysical studies, and include the local dark matter mass density as well as the distribution of the velocities of dark matter particles. The conjecture that the dark matter particles are WIMPs implies that their scattering with nuclei is non-relativistic two-body scattering; in LZ, we seek to observe the xenon nuclei that recoil after having been struck by an incoming WIMP. The mass assumed for the WIMP determines the kinematics of the scattering, and the rate of WIMP-nucleus scatters seen in a WIMP detector further depends on the exposure defined as the product of the target mass and the live time, the WIMP-nucleus cross section, and the energy threshold for detection of the NR.

The dark matter halo of the Milky Way is harder to quantify than that of other galaxies. The density of all matter, including dark matter, in galaxies is quantified with rotation

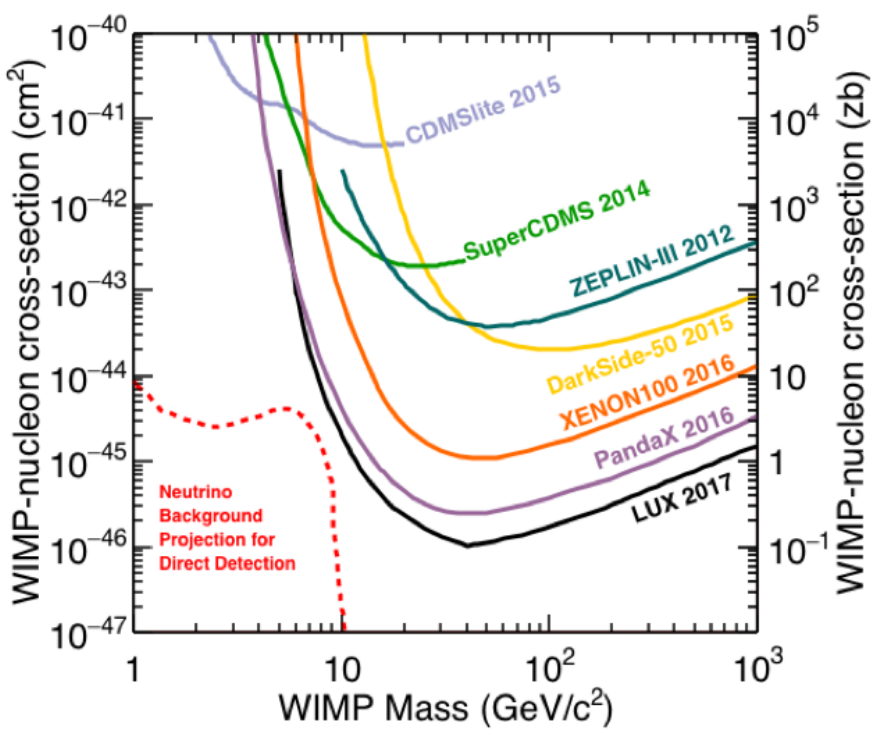

Figure 1.1.2: A compilation of current WIMP-nucleon SI cross-section upper limits at $90 \% \mathrm{CL}$ confidence level (solid colored curves, labeled by experiment names). The dotted region at the lower left indicates the region where solar neutrinos contribute background. 
velocity of matter in orbit about the galactic center as a function of radius from the galactic center. The rotation curve of the Milky Way for radii larger than $8.0 \mathrm{kpc}$, that of our sun, is a challenge to quantify. Estimates of the local dark matter density range from $(0.235 \pm 0.030) \mathrm{GeV} / \mathrm{cm}^{3}$ to $(0.389 \pm 0.025) \mathrm{GeV} / \mathrm{cm}^{3}[17-19]$. Most DD experiments adopt, for ease of inter-comparison, a standard value for the local dark matter mass density of $\rho_{0}=0.3 \mathrm{GeV} / \mathrm{cm}^{3}$. The experiments also adopt a standard distribution function for the velocity of dark matter particles, characterized by a Maxwell-Boltzmann distribution with solar circular velocity $v_{0}=220 \mathrm{~km} / \mathrm{s}$, which is cut off at the galactic escape velocity of $v_{\text {esc }}=544 \mathrm{~km} / \mathrm{s}$, and with proper accounting for the sun's peculiar velocity and the periodic annual motion of the Earth [20,21]. The minimum WIMP mass detectable with a particular DD experiment depends on the maximum velocity in the galactic WIMP spectrum, the atomic mass $A$ of the target nucleus, and the energy threshold $E_{\min }$ for NR detection in that experiment. Straightforward kinematics gives the minimum detectable WIMP mass as $M_{\mathrm{WIMP}}(\mathrm{GeV}) \approx(1 / 4) \sqrt{E_{\min }(\mathrm{keV}) A}$ for a maximum velocity of $v_{\mathrm{esc}}=544 \mathrm{~km} / \mathrm{s}$. This minimum $M_{\mathrm{WIMP}}$ is 1.6 GeV for the recent CDMSlite Ge-target result [22], and $4 \mathrm{GeV}$ for the recent LUX result [23] using LXe.

These results are shown in Figure 1.1.2, along with the current experimental situation for the spin-independent (SI) WIMP-nucleon cross section. We discuss the details of spin independence and other types of WIMP-nucleon interactions in Chapter 2. The SI cross section is the standard benchmark, and would result from a WIMP coupling to the Standard Model Higgs. As the presumed $M_{\text {WIMP }}$ rises above this value, the portion of the WIMP velocity distribution function that permits NR above the detectable threshold rises rapidly. This rapid rise drives the improvement in sensitivities as $M_{\mathrm{WIMP}}$ rises above $5 \mathrm{GeV}$, as shown in Figure 1.1.2. As $M_{\mathrm{WIMP}}$ approaches the mass of the atom used in the target, the kinematics of energy transfer to the target nuclei becomes most efficient, and experiments reach their maximum sensitivity.

This region, with $M_{\text {WIMP }}>10 \mathrm{GeV}$

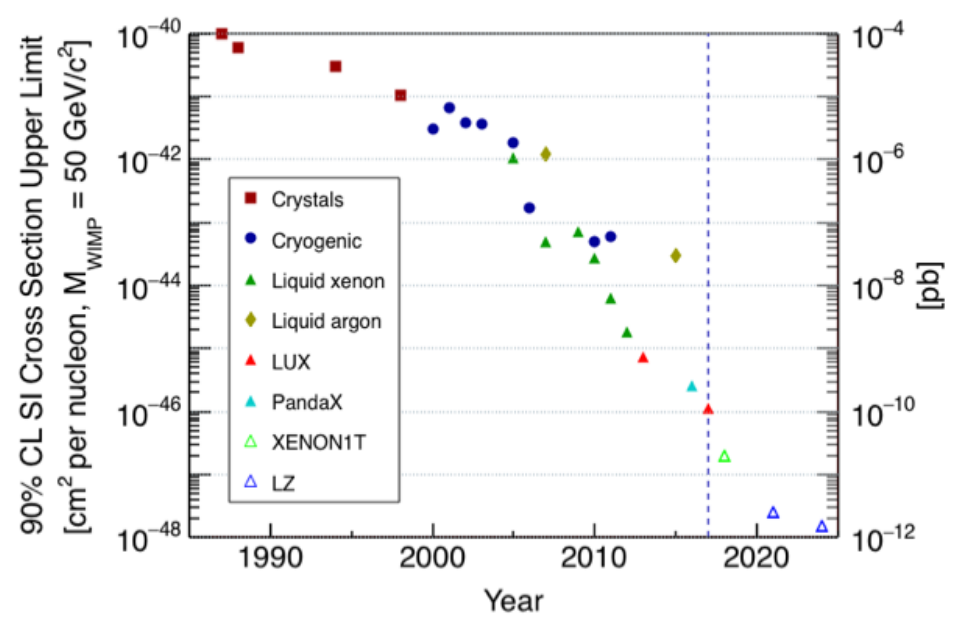

Figure 1.1.3: The evolution of cross-section limit for $50-\mathrm{GeV}$ WIMPs as a function of time. Past points are published results. Future points are from the Snowmass meetings [24]. roughly, is the region most likely for a WIMP, as other weak-interaction particles, including the $Z^{0}$, the $W$, and the Higgs particles, have masses in that region. As $E_{\min }$ grows yet larger, the number density of WIMPs implied by the astrophysical mass density, $\rho_{0}=\left(0.3 \mathrm{GeV} / M_{\mathrm{WIMP}}\right) / \mathrm{cm}^{3}$ falls, reducing the sensitivity of direct-detection experiments, as shown in Figure 1.1.2. The maximum WIMP mass consistent with thermal equilibrium in the early universe is $340 \mathrm{TeV} / c^{2}$, above which unitarity can no longer be satisfied [25].

The history of and future projections for WIMP sensitivity are shown in Figure 1.1.3. There are three distinct eras: (1) 1986-1996; (2) 2000-2010; and (3) post-2010. In the first era, lowbackground $\mathrm{Ge}$ and $\mathrm{NaI}$ crystals dominated. In these experiments, discrimination between the dominant background of electron recoils (ERs) from gamma rays and NRs was not available. The cross-section sensitivity per nucleon achieved, $10^{-41} \mathrm{~cm}^{2}$, was sufficient to rule out the most straightforward WIMP implementation: that the WIMP is a heavy Dirac neutrino, which was the original suggestion of Ref. [9]. In the second era, cryogenic Ge detectors with the ability to distinguish NR from the ER background dom- 
inated, and achieved a cross-section per nucleon sensitivity of $5 \times 10^{-44} \mathrm{~cm}^{2}$. This sensitivity began to probe dark matter that is a Majorana fermion, which couples to nucleons via the Higgs particle. In the third era, still under way, LXe time projection chambers (TPCs) have been most sensitive, and with the LUX experiment have achieved an upper limit for the WIMP-nucleon spin-independent cross section of $1.1 \times 10^{-46} \mathrm{~cm}^{2}\left(=1.1 \times 10^{-10} \mathrm{pb}\right)$ [26]. The LXe TPC has the ability to discriminate ER and NR, and can be expanded to large, homogeneous volumes. It is possible to make accurate predictions for the backgrounds in the central LXe TPC region, particularly with the added power of outer detectors that can characterize the radiation field in the TPC vicinity.

A more detailed portrayal of the variety of experiments, both from the past and projected into the future, is given in Figure 1.1.4 [24]. In the region $M_{\mathrm{WIMP}}>10 \mathrm{GeV} / c^{2}$, most likely for a WIMP due to proximity to the masses of the weak bosons, LZ will be the most sensitive experiment. Figure 1.1.4 also shows the "neutrino floor", where NRs from coherent neutrino scattering, a process that has not yet been observed, will greatly influence progress in sensitivity to WIMP interactions. A 1,000-day run of the LZ experiment will just begin to touch this background. Searches for direct interactions of dark matter with exposure substantially greater than LZ will see many candidate events from NRs in response to, primarily, atmospheric neutrinos.

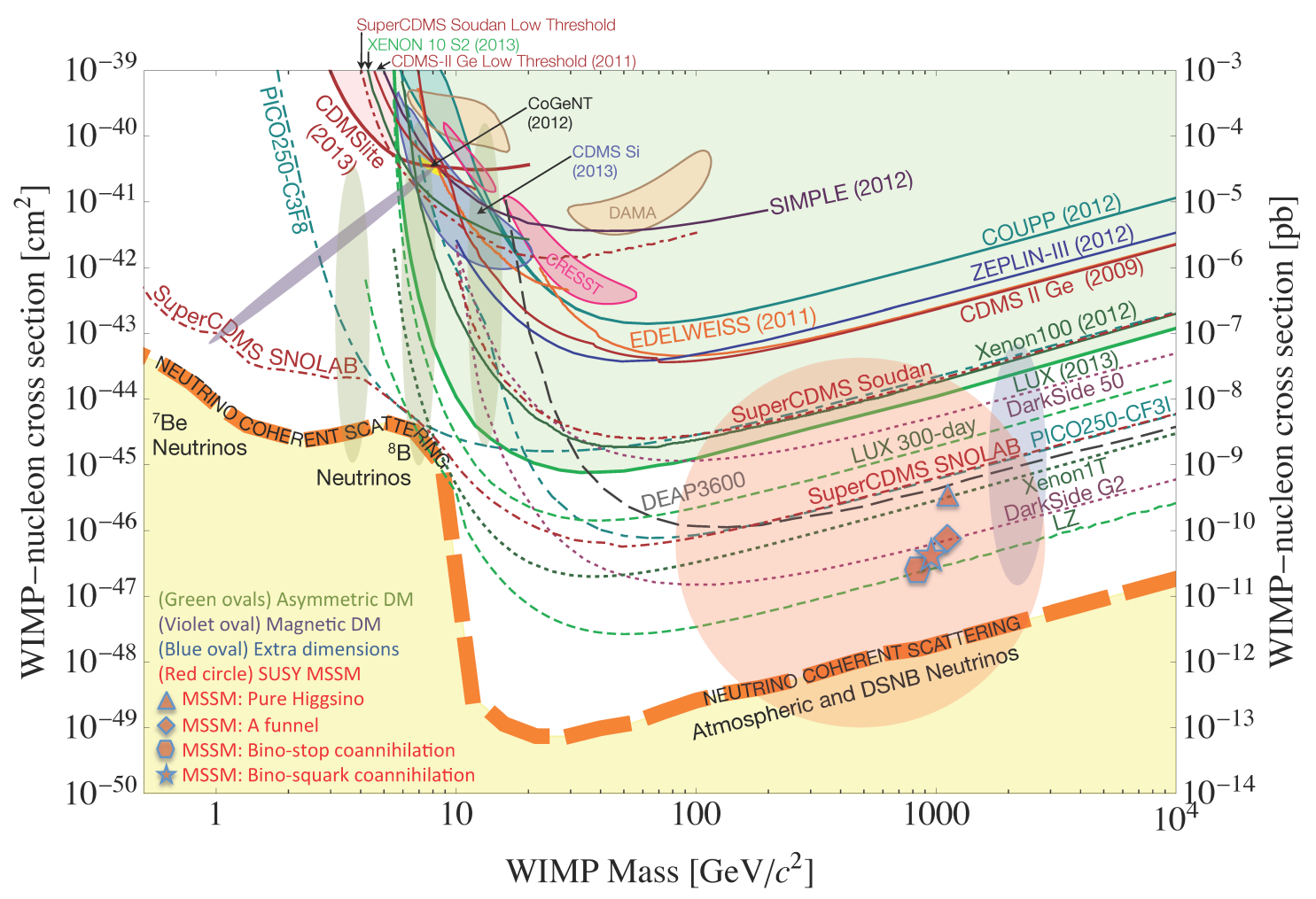

Figure 1.1.4: A compilation of WIMP-nucleon SI cross-section sensitivity (solid curves), hints for WIMP signals (shaded closed contours), and projections (dot and dot-dash curves) for DD experiments of the past and projected into the future. This figure captures the experimental situation as of the end of 2013 and was reported in the 2013 Snowmass CF1 summary. Also shown is an approximate band where the coherent nuclear scattering of ${ }^{8} \mathrm{~B}$ solar neutrinos, atmospheric neutrinos, and diffuse supernova neutrinos will limit the sensitivity of DD experiments to WIMPs. Finally, a suite of theoretical model predictions is indicated by the shaded regions, with model references included [24]. 


\subsection{Instrument Overview}

The core of the LZ experiment is a two-phase xenon (Xe) time projection chamber (TPC) containing 7 fully active tonnes of LXe. Scattering events in LXe create both a prompt scintillation signal (S1) and free electrons. Electric fields are employed to drift the electrons to the liquid surface, extract them into the gas phase above, and accelerate them to create a proportional scintillation signal (S2). Both signals are detected by arrays of photomultiplier tubes (PMTs) above and below the central region. The difference in time of arrival between the signals measures the position of the event in $z$, while the $x, y$ position is determined from the pattern of S2 light in the top PMT array. Events with an S2 signal but no S1 are also recorded. A 3-D model of the LZ detector located in a large water tank is shown in Figure 1.2.1. The water tank is located at the 4,850-foot level (4850L) of the Sanford Underground Research Facility (SURF). The heart of the LZ detector (including the inner titanium [Ti] cryostat) will be assembled on the surface at SURF, lowered in the Yates shaft to the 4850L of SURF, and deployed in the existing water tank in the Davis Cavern (where LUX is currently located). The LZ experiment's principal parameters are given in Table 1.2.1, along with the Work Breakdown Structure (WBS) for the LZ Project.

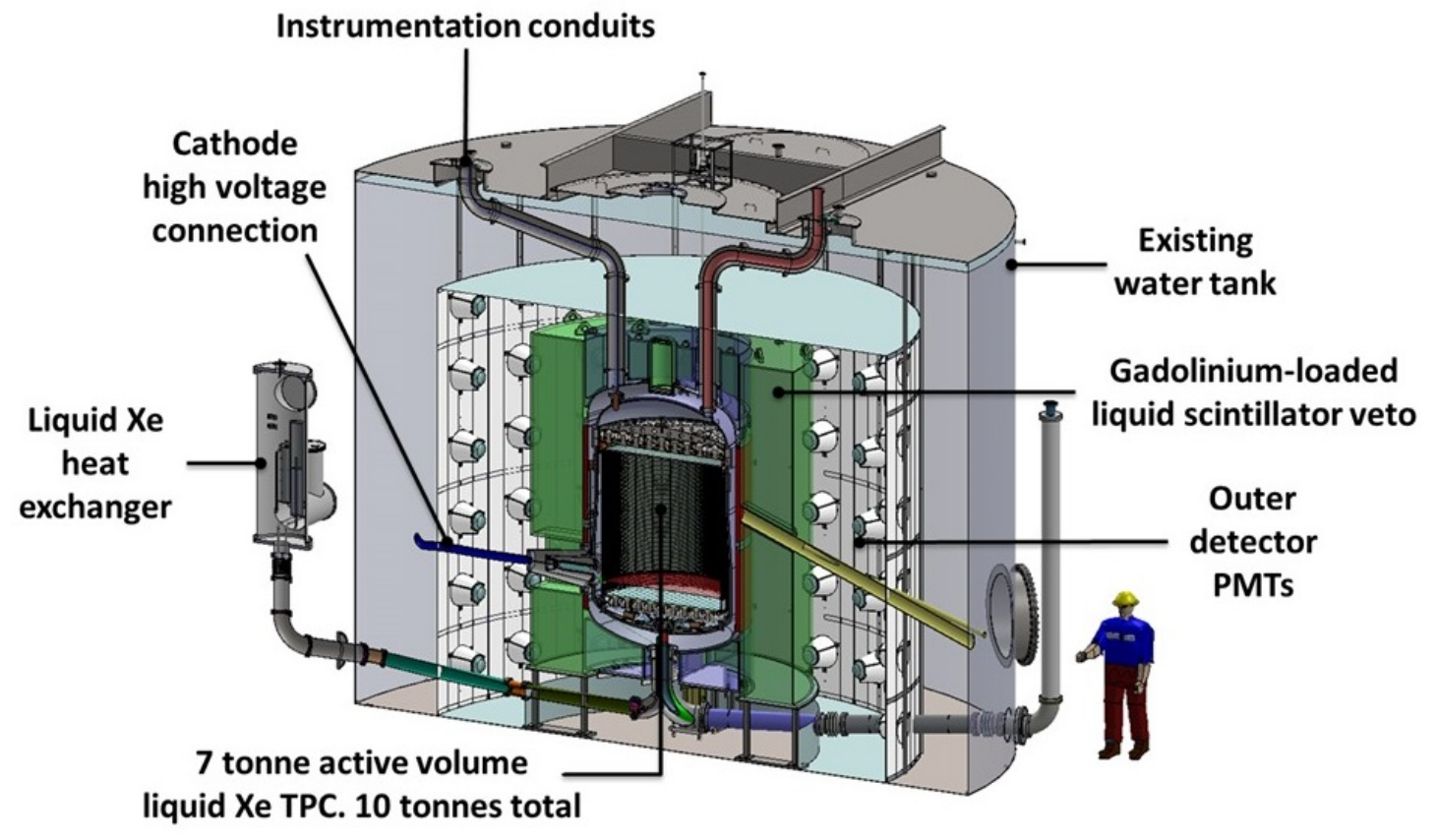

Figure 1.2.1: The LZ detector concept.

The LZ detector includes several added capabilities beyond the successfully demonstrated LUX and ZEPLIN designs. The most important addition is a nearly hermetic liquid organic scintillator (gadoliniumloaded linear alkyl benzene [LAB]) outer detector, which surrounds the central cryostat vessels and the TPC. The outer detector and the active Xe "skin" layer, the Xe between the inner cryostat wall and the outer wall of the TPC, operate as an integrated veto system, which has several benefits. The first is rejecting gammas and neutrons generated internally (e.g., in the PMTs) that scatter a single time in the fully active region of the TPC and would otherwise escape without detection; this could mimic a weakly interacting massive particle (WIMP) signal. As these internally generated backgrounds interact primarily at the outer regions of the detector, the veto thus allows an increase in the fiducial volume. 
Table 1.2.1: Principal parameters of the LZ detector organized to reflect the responsible work package under the Work Breakdown Structure (WBS).

\begin{tabular}{|c|c|c|}
\hline WBS & Description & Quantity \\
\hline \multirow[t]{4}{*}{1.1} & Xenon Procurement & \\
\hline & Approximate total mass & 10 tonnes \\
\hline & Mass inside TPC active region & 7 tonnes \\
\hline & Approximate fiducial mass & 5.6 tonnes \\
\hline \multirow[t]{4}{*}{1.2} & Xe Vessel(Cryostat) & \\
\hline & $\begin{array}{l}\text { Inner cryostat-inside diameter (tapered), height, } \\
\text { wall thickness }\end{array}$ & $1.58-1.66 \mathrm{~m}, 2.59 \mathrm{~m}, 8-11 \mathrm{~mm}$ \\
\hline & $\begin{array}{l}\text { Outer cryostat-inside diameter, height, wall thick- } \\
\text { ness }\end{array}$ & $1.83 \mathrm{~m}, 3.04 \mathrm{~m}, 7-14 \mathrm{~mm}$ \\
\hline & Approximate cryostat weights-inner, outer & 0.95 tonne, 1.12 tonne \\
\hline \multirow[t]{3}{*}{1.3} & Cryogenic System & \\
\hline & Cooling power & $1 \mathrm{~kW} @ 80 \mathrm{~K}$ \\
\hline & Input electrical power & $11 \mathrm{~kW}$ \\
\hline \multirow[t]{7}{*}{1.4} & Xenon Purification & \\
\hline & Krypton content & $<0.015$ ppt (g/g) \\
\hline & Allowed air content ( $\mathrm{Kr}$ equivalent) & $<40 \mathrm{cc}$ \\
\hline & ${ }^{222} \mathrm{Rn}$ content in active $\mathrm{Xe}$ & $<2 \mu \mathrm{Bq} / \mathrm{kg}$ \\
\hline & Recirculation rate & $500 \mathrm{slpm}$ \\
\hline & Electron lifetime & $>0.8 \mathrm{~ms}$ \\
\hline & Charge attenuation length & $>1.5 \mathrm{~m}$ \\
\hline \multirow[t]{7}{*}{1.5} & Xenon Detector & \\
\hline & Top (Bottom) TPC 3-inch PMT array & $253(241), 494$ total tubes \\
\hline & "Side skin" PMT and "dome" PMT & $\begin{array}{l}113 \text { and } 18,131 \text { (93 1-inch,38 2- } \\
\text { inch) total }\end{array}$ \\
\hline & Nominal (Design) cathode operating voltage & $50(100) \mathrm{kV}$ \\
\hline & Reverse field region (cathode to bottom tube shield) & $0.1375 \mathrm{~m}$ \\
\hline & TPC height (cathode to gate grid) & $1.456 \mathrm{~m}$ \\
\hline & TPC effective diameter & $1.456 \mathrm{~m}$ \\
\hline \multicolumn{3}{|c|}{ (continued on next page) } \\
\hline
\end{tabular}


Table 1.2.1: (continued)

\begin{tabular}{|c|c|c|}
\hline WBS & Description & Quantity \\
\hline \multirow[t]{8}{*}{1.6} & Outer Detector System & \\
\hline & Weight of Gd-loaded LAB scintillator & 17.5 tonnes \\
\hline & Number of acrylic vessels, total acrylic mass & 9 vessels, 3.1 tonnes \\
\hline & Number of 8-inch PMTs & 120 \\
\hline & Minimum thickness of scintillator & $0.61 \mathrm{~m}$ \\
\hline & Diameter of water tank & $7.62 \mathrm{~m}$ \\
\hline & Height of water tank & $5.92 \mathrm{~m}$ \\
\hline & Approximate weight of water & 228 tonnes \\
\hline \multirow[t]{4}{*}{1.7} & Calibration System & \\
\hline & Number of source deployment tubes & 3 \\
\hline & Number of neutron tubes & 3 \\
\hline & Other calibration tools & $\begin{array}{l}{ }^{83 \mathrm{~m}} \mathrm{Kr}, n \text {-generator, tritiated } \mathrm{CH}_{4}, \\
{ }^{37} \mathrm{Ar},{ }^{\mathrm{m}} \mathrm{Xe}\end{array}$ \\
\hline \multirow[t]{4}{*}{1.8} & Electronics, DAQ, Controls, and Computing & \\
\hline & Trigger rate (all energies, $0 \mathrm{keV}$ to $40 \mathrm{keV}$ ) & $40 \mathrm{~Hz}, 0.4 \mathrm{~Hz}$ \\
\hline & Average event size (noncalibration, uncompressed) & $0.2 \mathrm{MB}$ to $1.0 \mathrm{MB}$ \\
\hline & Data volume per year & 350 TB to 850 TB \\
\hline 1.9 & Integration and Installation & \\
\hline 1.10 & Cleanliness and Screening & \\
\hline 1.11 & Offline Computing & \\
\hline 1.12 & Project Management & \\
\hline
\end{tabular}

Additionally, this direct vetoing is an important means of risk mitigation against one of the detector components (e.g., the cryostat materials or the PMTs) having a higher-than-expected background. A second benefit of the outer detector is that the combination of outer detector and segmented Xe detector will form a nearly hermetic detection system for all internal radioactivity. This will not only directly measure the internal backgrounds with a very high level of detail and completeness, but also help provide an understanding of the detector's response to those backgrounds that can be included in our analysis for dark matter signals.

As shown in Figure 1.2.1, the liquid scintillator volume is confined in segmented, clear acrylic vessels encapsulating the Ti cryostat of the central LZ detector. PMTs mounted on ladders in the outer water shield simultaneously view the light from both the scintillator and inner water volumes. The design of the acrylic vessels is advanced and industrial fabrication of the vessels has started. The production of 
the low-radioactivity liquid scintillator is based on the successful production of a similar scintillator for the Daya Bay experiment, albeit with lower backgrounds. The layer of LXe located between the PTFE (polytetrafluoroethylene, or Teflon $($ ) ) structure that surrounds the fully active region and the cryostat wall, as well as the LXe region beneath the bottom PMT array forms the "skin" element of the veto system. A skin of some ( $\sim$ few $\mathrm{cm}$, more in the bottom region of the detector) thickness is difficult to avoid given the TPC geometry, the need for high-voltage (HV) standoff, and the strong mismatch in thermal expansion between the PTFE panels and Ti vessels. The skin readout alone has a limited veto efficiency, but has sufficient gamma-stopping power to augment the scintillator veto. The combination of skin readout and outer detector creates a highly efficient integrated veto system. Scintillation light from the skin region is observed by a sparse PMT array dedicated to this region. Thin PTFE panels are attached to the inner cryostat wall and bottom (dome) region to enhance light collection.

For detector calibration, neutron and gamma-ray sources will be brought next to the wall of the inner cryostat via an array of three source tubes that penetrate the water and organic scintillator. The principal calibrations, metastable krypton and xenon and tritiated methane, will be introduced directly into the LXe via the Xe gas-handling system to allow in situ calibration. An external neutron generator that produces neutrons through a deuterium-deuterium fusion reaction will also be employed, and the neutrons penetrate through the water and scintillator veto through dedicated tubes. Additional neutron calibration sources will also be employed.

Another key determinant of the sensitivity of the experiment is the level of discrimination of electron recoil (ER) backgrounds from nuclear recoils (NRs). This depends on the electric field established in the TPC. The nominal operating voltage (cathode-to-anode) is $50 \mathrm{kV}$ but all components will be designed to a voltage of $100 \mathrm{kV}$ (and in general tested to higher voltages) to have sufficient operating margin.

The LZ TPC detector will employ Hamamatsu R11410-20 3-inch-diameter PMTs with a demonstrated low level of radioactive contamination and high quantum efficiency. Materials for these PMTs have been procured and screened to demonstrate low radioactivity. Tube production and testing began in 2016. Care will be taken in the design of the highly reflective PTFE TPC structure to isolate light produced in the central volume from the skin region, and vice-versa. Additional 1-inch PMTs will be utilized in the cylindrical skin region as well as a few 2-inch tubes (from LUX) and 2-inch tubes used in the dome region.

The design of TPC mechanical and HV systems is advanced. Small prototypes of the high-electric field region of the TPC have been fabricated and tested in liquid argon (LAr) and successfully in a LXe test facility at SLAC National Accelerator Laboratory (SLAC). A scaled-prototype of the TPC and all grids that shape the electric fields is being tested at SLAC. HV systems, including the cathode HV delivery system, will be tested in LAr at LBNL in a dedicated facility. Assembly of the TPC structures and HV elements will occur primarily at LBNL-SLAC. The low-radioactivity Ti mechanical supports for the 3-inch PMTs will be industrially manufactured and the PMTs loaded and tested at Brown University.

LZ will employ an array of liquid nitrogen (LN)-cooled thermosyphons to control the detector temperature and minimize thermal gradients. The purification system is a scaled-up version of the LUX roomtemperature gas-phase purification system, and will exploit the liquid/gas heat-exchanger technology developed for LUX to minimize LN consumption. A cryocooler will be used underground to manufacture LN, and LN storage vessels used by LUX will be retained as backup. The Xe circulation and purification system is similar conceptually to that used in LUX but with a much higher capacity. The 10 tonnes of Xe will be circulated and purified in 2 to 3 days. The Xe purity will be monitored carefully by a dedicated system, expanding and improving on a similar system used in LUX. Radon will be removed from selected regions of the detector that contain Xe gas using a dedicated system underground, to lower the radon content.

The acquisition of the 10 tonnes of Xe has started. The Xe used in LUX and in other devices will be reused. Procurement began in 2015 and is planned to end by mid-2018. All gaseous Xe is to be delivered 
to SLAC, where krypton will be removed through a chromatographic process using scaled-up techniques already demonstrated successfully for LUX.

The electronics front-end, trigger and data acquisition, and slow controls are based on the LUX experience but will be significantly expanded and improved. A test of the PMT analog-amplifier-digitizer system, with full cable lengths, has been successfully completed. In addition, a few PMTs in the LUX experiment were operated successfully with prototype electronics systems after the completion of LUX data taking in mid2016. Software and computing systems for LZ are based on the successful operation of LUX, analysis of LUX data, and the experience of other experiments at LBNL and collaborating institutions. LZ data will be buffered locally at SURF and then transmitted to primary data storage at LBNL that will be mirrored in the U.K. The analysis framework to be used by the LZ collaboration has been defined and the simulation of the LZ detector and its response is already well advanced. The results are given in other chapters of this Technical Design Report.

Components of LZ will be manufactured at a number of locations and brought to SURF. Assembly of the Xe detector and the inner cryostat will occur in a dedicated and upgraded cleanroom at SURF that includes an augmented air handling system to reduce radon during the assembly process. The construction of the facilities to house the upgraded cleanroom began in September 2016. The reduced-radon air handling system is under construction as is a reduced-radon cleanroom. Work on improved surface facilities will be completed by mid-2017. A comprehensive system for tracking components, ensuring cleanliness and a very low dust environment, will be employed to minimize radioactive backgrounds. The inner cryostat with the Xe detector inside will be lowered as a unit down the Yates shaft and transported to the Davis Cavern water tank. Another system will be built underground to provide low-radon air to the water tank during the connection of the inner cryostat to other systems and for other uses. All other components will be staged at SURF, lowered via the Yates shaft, and similarly transported. This includes the segmented acrylic vessels for the outer detector system and other large components. The gaseous Xe will be stored in specially designed cylinders in a dedicated room underground close to the Davis Cavern.

\subsection{Design Drivers for WIMP Identification}

Having established the motivation to perform direct searches for WIMP dark matter, we introduced in the previous section the configuration of LZ. Searching for events that are rare $(\lesssim 0.1 / \mathrm{t} / \mathrm{d})$ and that involve very small energy transfers $(\lesssim 100 \mathrm{keV})$ is extremely challenging. This section focuses on the more salient features of the experiment and the detection medium, and how these will contribute to the identification of a galactic WIMP signal with low systematic uncertainty. The detailed design and its technical implementation are described in later sections; here, we address the key requirements that drive the technical design.

\subsubsection{Overview of the Experimental Strategy}

Xenon has long been recognized as a very attractive WIMP target material [27-29]. Its high atomic mass provides a good kinematic match to intermediate WIMP masses of $\mathrm{O}\left(100 \mathrm{GeV} / \mathrm{c}^{2}\right)$ and the largest spinindependent scattering cross section among the available detector technologies, as illustrated in Figure 1.3.1. Sensitivity to lighter WIMPs, with masses of $\mathrm{O}\left(10 \mathrm{GeV} / c^{2}\right)$, can be also be achieved, given the excellent low-energy scintillation and ionization yields in the liquid phase [30]. Xenon contains neither long-lived radioactive isotopes with troublesome decays nor activation products that remain significant after the first few months of underground deployment. It is also sensitive to spin-dependent interactions via the oddneutron isotopes ${ }^{129} \mathrm{Xe}$ and ${ }^{131} \mathrm{Xe}$, which account for approximately half of the natural isotopic abundance. If a WIMP discovery were made, the properties of the new particle could be studied by altering the isotopic composition of the target. This broad WIMP sensitivity confers maximum discovery potential to LZ. 

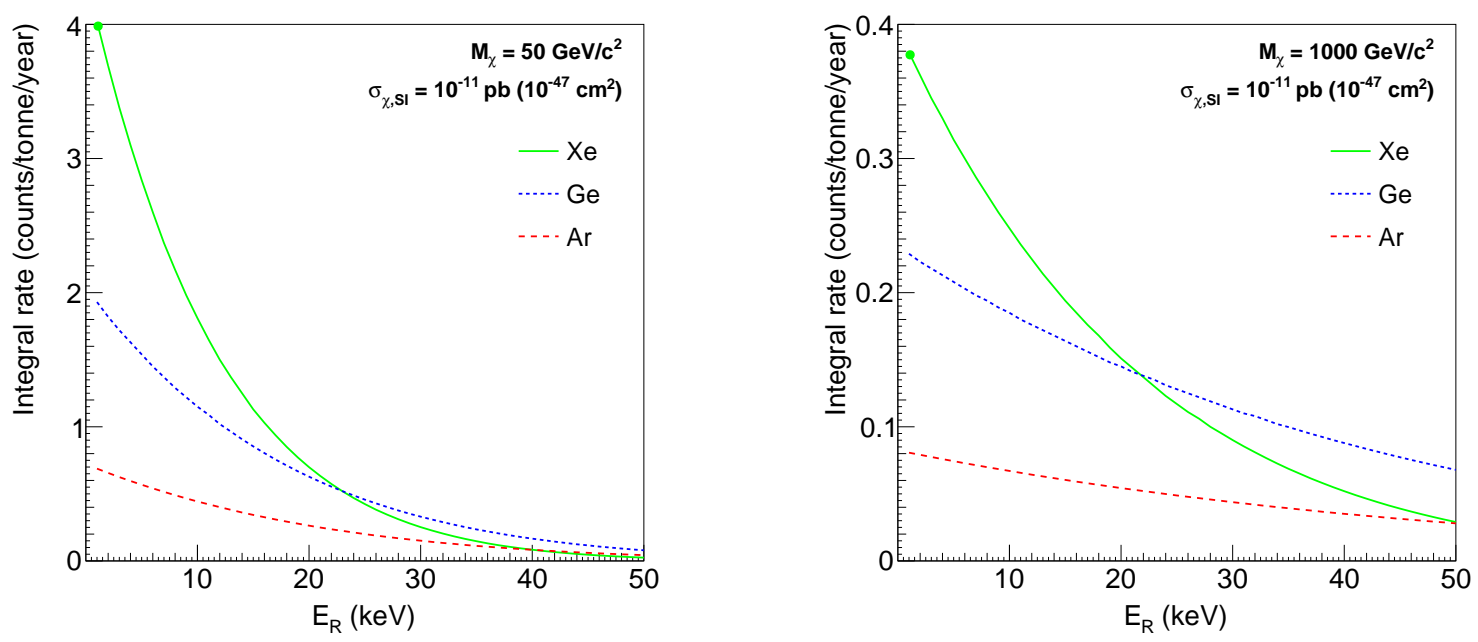

Figure 1.3.1: Integrated rate above threshold per tonne-year of exposure for WIMP elastic scattering on $\mathrm{Xe}, \mathrm{Ge}$, and Ar targets for $50 \mathrm{GeV} / c^{2}$ and $1 \mathrm{TeV} / c^{2}$ WIMP masses and $10^{-47} \mathrm{~cm}^{2}$ interaction cross section per nucleon. The green marker indicates the $1.1 \mathrm{keV}_{\mathrm{nr}}$ WIMP-search threshold in LUX with nominal ER/NR discrimination [30]. CDMS II searched above $\sim 5 \mathrm{keV}$ in their Ge target [31]; selected SuperCDMS detectors allowed a $1.6 \mathrm{keV}_{\mathrm{nr}}$ threshold with lower discrimination [32]; CDMSlite search for low-mass WIMPs with an electron recoil threshold down to $56 \mathrm{eV}$ [22]. In LAr, the DarkSide-50 experiment has recently conducted a WIMP search above $13 \mathrm{keV}_{\mathrm{nr}}$ [33].

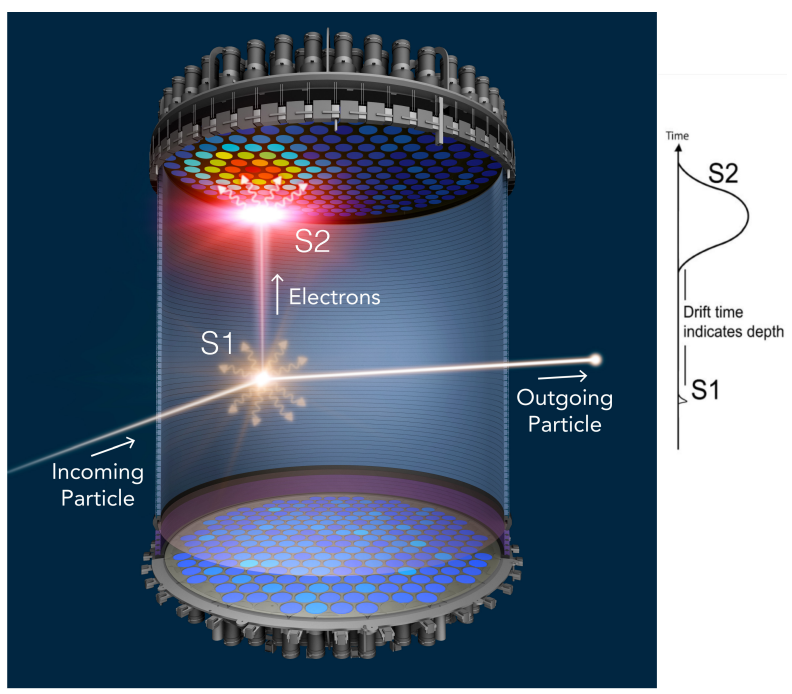

Figure 1.3.2: Operating principle of the double-phase Xe TPC. Each particle interaction in the LXe (the WIMP target) produces two signatures: one from prompt scintillation (S1) and a second, delayed one from ionization, via electroluminescence in the vapor phase (S2). This allows precise vertex location in three dimensions and discrimination between nuclear and electron recoils. 
The liquid phase is preferred over the gas phase due to its high density $\left(3 \mathrm{~g} / \mathrm{cm}^{3}\right)$ and high scintillation yield, and because its charge quenching of NRs provides a powerful particle ID mechanism. Early experiments such as ZEPLIN-I [34] exploited simple pulse shape discrimination (PSD) of the scintillation signal to reject electronic backgrounds; however, this achieved modest rejection efficiencies and only at relatively high recoil energies. When the first double-phase Xe detectors were deployed for dark matter searches, in the ZEPLIN-II/III [35, 36] and XENON10 [37] experiments, the increase in engineering complexity soon paid off in sensitivity, and this technique has been at the forefront of the field ever since. Comprehensive reviews on the application of the noble liquids to rare-event searches can be found in the literature [38, 39].

The TPC configuration at the core of double-phase detectors, illustrated in Figure 1.3.2, has several notable advantages for WIMP searches, in that two signatures are detected for every interaction: a prompt scintillation signal (S1) and the delayed ionization response, detected via electroluminescence in a thin gaseous phase above the liquid (S2). These permit precise event localization in three dimensions (to within a few $\mathrm{mm}$ [40]) and discrimination between electron and nuclear recoil events (potentially reaching $99.99 \%$ rejection [41]).

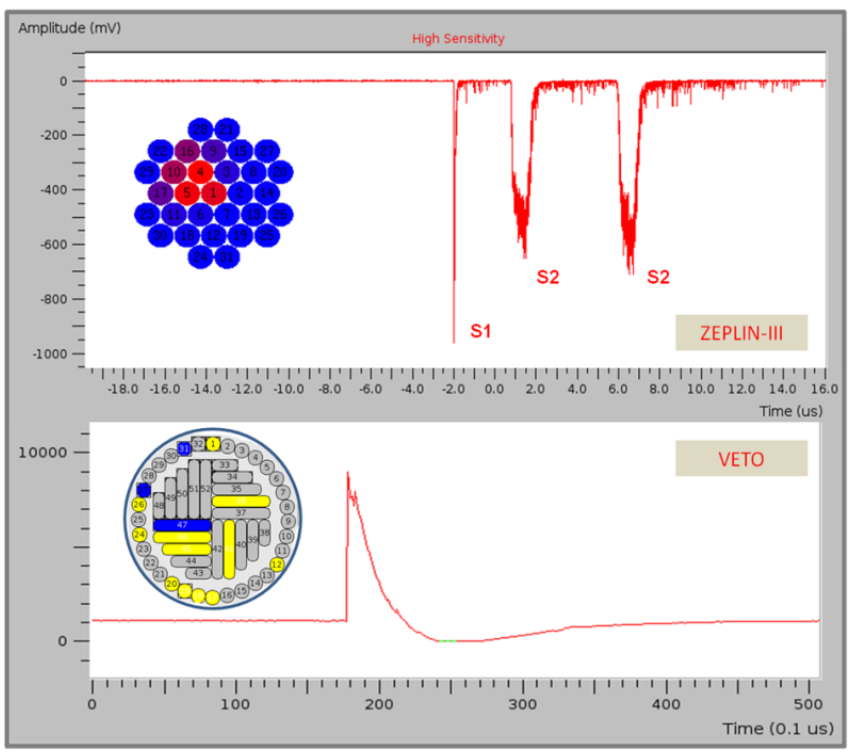

Figure 1.3.3: A double-scatter neutron event recorded in ZEPLIN-III. The upper panel shows two elastic vertices clearly resolved in drift time (two S2 pulses, representing different vertical coordinates), although both have similar horizontal positions. The lower panel shows the summed waveform from the 52-module veto detector which surrounded the main instrument, indicating radiative capture of this neutron some $17 \mu \mathrm{s}$ after interacting in the LXe target. Recording additional particle scatters (either in the WIMP target or in an ancillary veto detector) provides a powerful rejection of backgrounds.
Both channels are sensitive to very low NR energies. The S2 response enables detection of single ionization electrons extracted from the liquid surface due to the high photon yield that can be achieved with proportional scintillation in the gas $[42-$ 44]. In LUX we have demonstrated sufficient S1 light collection to achieve a NR energy threshold below $1.1 \mathrm{keV}_{\mathrm{nr}}$ [45]. The combination of accurate 3-D imaging capability within a monolithic volume of a readily purifiable, highly self-shielding liquid is nearly an ideal architecture for minimizing backgrounds. It allows optimal exploitation of the powerful attenuation of external gamma rays and neutrons into LXe, distinguishes multiplyscattered backgrounds from single-site signals, and precisely tags events on the surrounding surfaces. This latter feature is important, given the difficulty of achieving contamination-free surfaces. The low surface-to-volume ratio of the large, homogeneous TPC lowers surface backgrounds in comparison to signal, and stands in stark contrast to the high surface-to-volume ratio of segmented detectors.

These concepts are illustrated in Figure 1.3.3, which shows neutron interactions occurring just a few millimeters apart in the ZEPLIN-III detector. The S1 signals are essentially time-coincident, but the S2 pulses have different time delays correspond- 
ing to different vertical coordinates, making the rejection of such multiple scatters extremely efficient. The figure shows also a pulse observed in delayed coincidence in the surrounding veto detector, indicating radiative capture of this neutron on the gadolinium-loaded plastic installed around the WIMP target. LZ will utilize a similar anticoincidence detection technique to characterize the radiation environment around the $\mathrm{Xe}$ detector and to further reduce backgrounds.

Nevertheless, when the first tonne-scale Xe experiments were proposed just over a decade ago, it was unclear whether LXe technology could be monolithically scaled as now proposed for LZ, or if it would be necessary to replicate smaller devices with target masses of a few hundred kilograms each. The latter option, while conceptually simple, fails to fully exploit the power of self-shielding. Since then, several aspects of the double-phase TPC technique have been further developed to make LZ technologically feasible. First, the ionization and scintillation yields of LXe and their dependence on energy, electric field, and particle type have now been established down to a few $\mathrm{keV}$ by a comprehensive development program carried out around the globe, including substantial work by members of the LZ Collaboration. Second, good acceptance for the primary scintillation light must be maintained as the detector becomes larger, and the remarkably high reflectance $>95 \%$ of PTFE at the $178 \mathrm{~nm}$ LXe scintillation wavelength has made this practical. Third, considerable control over electronegative impurities is required to drift charge over a distance of a meter or more, and commercial purification technology and new screening and detection methods developed by LZ scientists have made this routinely achievable. Fourth, the extraordinary self-shielding of an LZ-class instrument requires the use of internal calibration sources, and these have now been developed and deployed within LUX by LZ groups. Fifth, radioactive impurities such as $\mathrm{Kr}$ must be reliably removed from the Xe, and other sources of internal radioactivity such as radon must be tightly controlled. Finally, a large detector requires a substantial cathode voltage, or else fluctuations in the charge recombination near the interaction site will degrade the recoil discrimination. Very recently, LUX has demonstrated a rejection efficiency of $99.6 \%$ (for $50 \% \mathrm{NR}$ acceptance) even at a modest field of $180 \mathrm{~V} / \mathrm{cm}$ [30], matching already the baseline assumption for LZ.

On the whole, the progressive nature of our program has contributed to an increase in the readiness level of this technology: LZ entails a twentyfold scale-up from LUX, the latter being also an order of magnitude or so larger than the ZEPLIN and XENON10 targets. Besides having the favorable properties of the WIMP target material and the proven sensitivity of the technology to small energy deposits, a successful experiment must achieve very low background rates over a significant fraction of its active medium. Indeed, it is worth noting that $\mathrm{LZ}$ will be a factor of $10^{4}$ times more sensitive than current limits from the EDELWEISS and Cryogenic Dark Matter Search (CDMS) experiments, which led in sensitivity only one decade ago $[46,47]$. This implies a corresponding reduction in the background rate. This is achieved to first order by the power of self-shielding of local radioactivity, in combination with an outer layer of instrumented LXe and a hermetic gadolinium-loaded scintillator "veto" shield capable of tagging neutrons and gamma rays with high efficiency. The construction of a veto instrument at the required scale builds on two decades of development work in the field of reactor neutrino physics, and its development within LZ is led by scientists with considerable expertise in this area. Three other important developments, again pioneered by LZ groups, have also made this possible: the development, in collaboration with Hamamatsu, of very-low-background PMTs compatible with LXe [48]; the identification via the LUX program and LZ R\&D program of radioclean titanium for cryostat fabrication [49,50]; and the development of krypton-removal and -screening technology capable of delivering sub-ppt concentrations [51, 52].

This strategy leads to a WIMP-search background of order 6 events in 1,000 days of live exposure for a 5.6-tonne fiducial mass. The remaining component is dominated by events from radon decay in the fiducial volume, a small fraction of which mimic NRs due to the finite S2/S1 discrimination power. Coherent scattering of atmospheric neutrinos from Xe nuclei (CNS) will constitute an even smaller, but irreducible, background. These rates are well understood and background expectations are calculable with small sys- 
tematic uncertainty (e.g., these events are spatially uniform and their energy spectra are well known). With its pioneering capability, $\mathrm{LZ}$ will be sensitive to these ultra-rare processes.

\subsubsection{Self-shielding in Liquid Xenon}
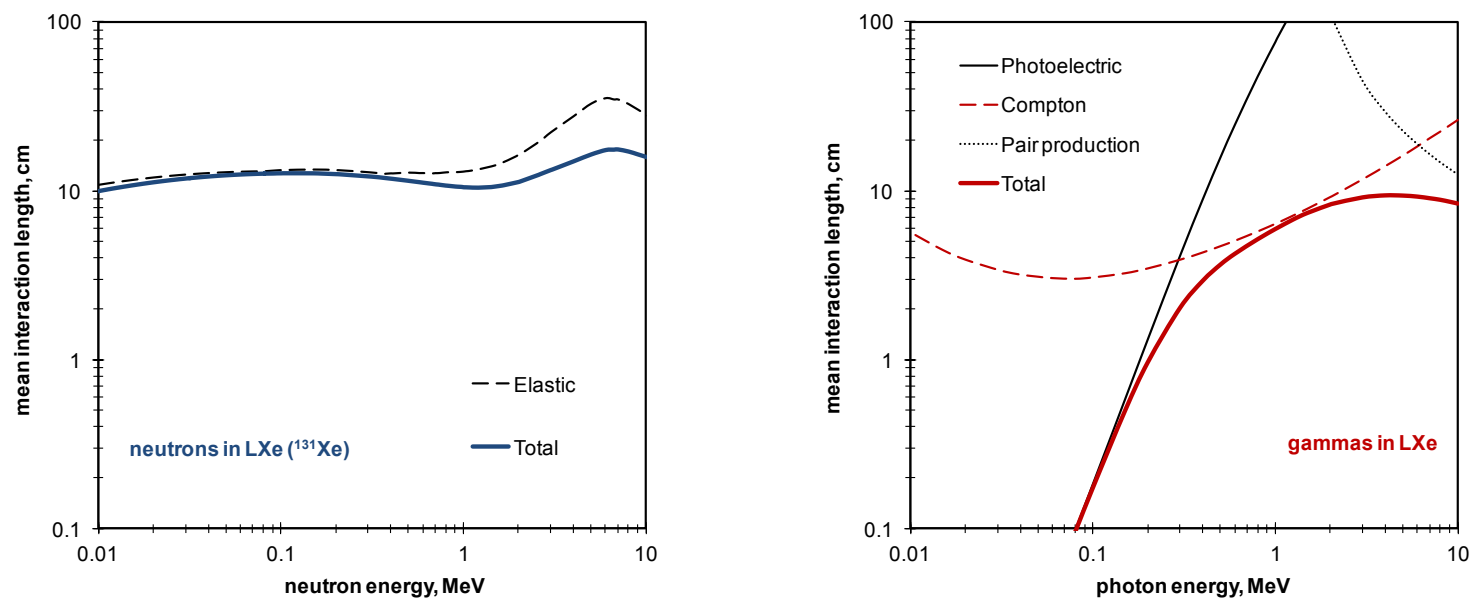

Figure 1.3.4: Mean interaction lengths for neutrons [53] and gamma rays [54] in LXe.

At the core of any WIMP search experiment is a substantial screening and materials-selection program (Chapter 9) that controls the trace radioactivity of the detector components. In the case of LZ, however, backgrounds from detector radioactivity will also be rejected to unprecedented levels by the combination of self-shielding of external particles and operation in anticoincidence with outer veto detectors (Chapter 4). This will render external gamma rays and neutrons less problematic than in other experiments.

The self-shielding strategy, in particular, relies on the combination of a large, dense, high- $Z$ and continuous detection medium with the ability to resolve interaction sites in three dimensions with high precision. An outer layer of the target can therefore be defined (in data analysis) that shields a fiducial region with extremely low background at the center of the active medium. The nonfiducial layer will be only a few centimeters thick. Because the size of the $\mathrm{LZ}$ detector is much larger than the interaction lengths for $\mathrm{MeV}$ gamma rays and neutrons, as shown in Figure 1.3.4, when these particles penetrate more than a few $\mathrm{cm}$ they will scatter multiple times and be rejected (Figure 1.3.5). X-rays, with energies similar to WIMP events, penetrate only a few mm into the LXe.

Double-phase Xe detectors implement this strategy very successfully, and this is reflected in their present dominance in WIMP sensitivity - with LUX being a prime example of this concept. In LZ, a fiducial mass of nearly 6 tonnes will be practically free of external gamma-ray or neutron backgrounds, which represents $85 \%$ of active mass within the TPC, compared to about $50 \%$ for LUX [30] and XENON100 [55]. In addition to the required high density and high- $Z$ of the detection medium, we highlight the importance of the precise spatial resolution that can be achieved in these detectors, which is of the order of $1 \mathrm{~cm}$ or better at threshold. 


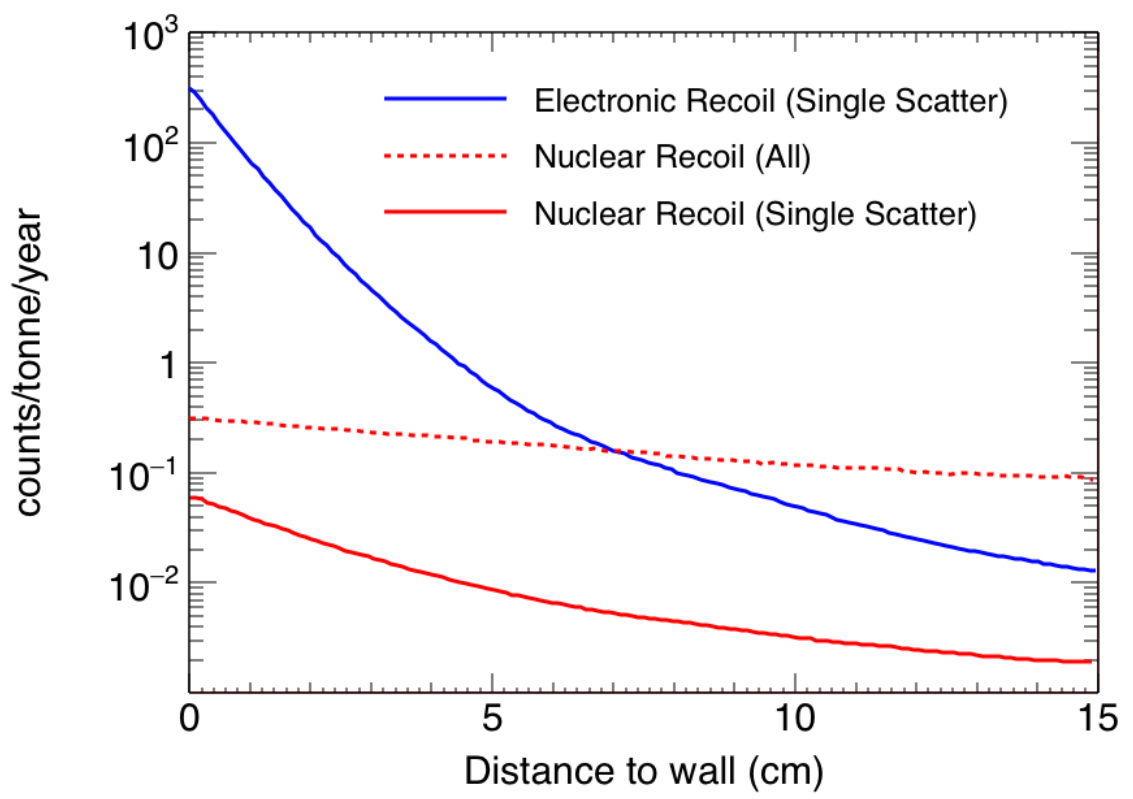

Figure 1.3.5: Self-shielding of external neutrons and gamma rays in LXe. The red lines indicate the number of elastic neutron scatters creating $6 \mathrm{keV}_{\mathrm{nr}}$ to $30 \mathrm{keV}_{\mathrm{nr}} \mathrm{NRs}$ as a function of distance to the lateral TPC wall; the continuous line shows single scatters only, while the dashed line includes all multiplicities adding up to the same total energy; the input spectrum is that from $(\alpha, n)$ neutron production in PTFE, an important background near the TPC walls. The blue line represents single-site ER interactions from $\mathrm{U} / \mathrm{Th}$ gamma rays from PTFE with energy $1.5 \mathrm{keV}_{\text {ee }}$ to $6.5 \mathrm{keV}_{\text {ee }}$. A tenfold decrease is achieved at $\approx 2 \mathrm{~cm}$ and $\approx 6 \mathrm{~cm}$ from the wall for gamma rays and neutrons, respectively. 


\subsubsection{Low-energy Particle Detection in Liquid Xenon}

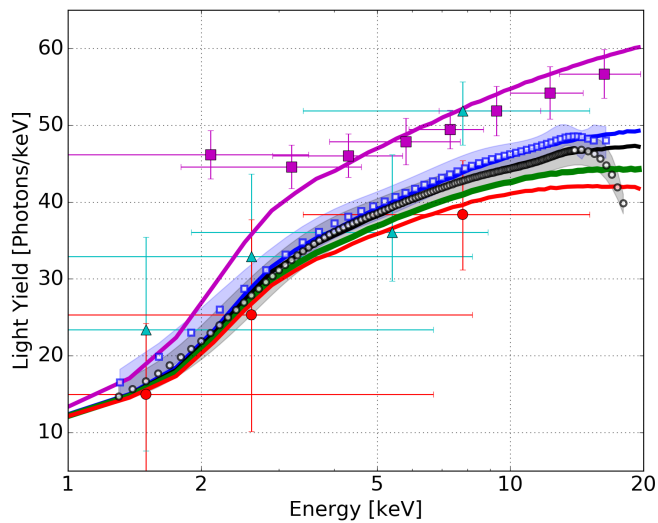

Figure 1.3.6: Absolute ER scintillation yield in LXe. Purple squares are from Comptonscattering measurements at Columbia [56] and cyan triangles from [57] - both at zero field. Blue/black squares/circles are from LUX tritium beta emission measurements in situ at 105 and $180 \mathrm{~V} / \mathrm{cm}$ respectively [58]. Red circles are from Compton-scattering measurements performed in Zürich at $450 \mathrm{~V} / \mathrm{cm}$ [57]. The NEST model (updated from $[59,60]$ but using the same framework and formulae still) is shown in purple, blue, black, green, and red for 0, 105, 180, 310 (LZ baseline), and $450 \mathrm{~V} / \mathrm{cm}$, respectively.

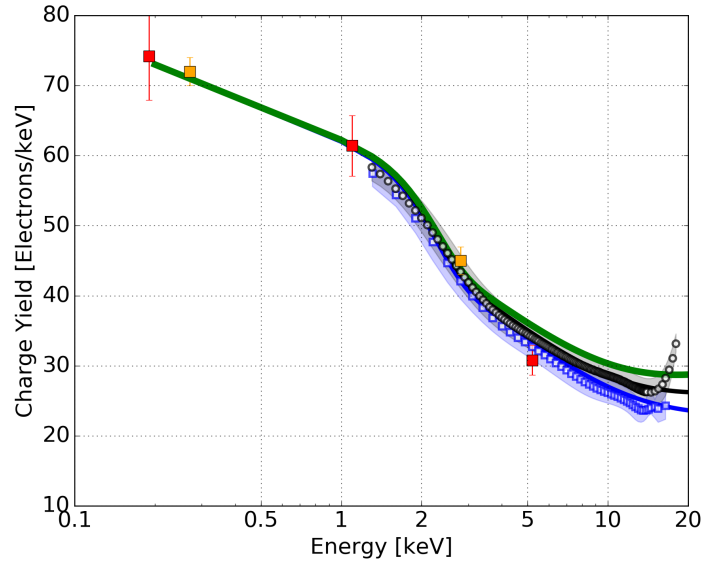

Figure 1.3.7: ER ionization yield in LXe. Data are as follows: blue squares from LUX tritium data beta spectrum matching at $105 \mathrm{~V} / \mathrm{cm}$ [58]; red and orange squares from ${ }^{127} \mathrm{Xe}$ activation lines in LUX and ${ }^{37} \mathrm{Ar}$ in PIXeY, respectively, all at $180 \mathrm{~V} / \mathrm{cm}[\mathrm{F}, \mathrm{G}]$; black circles from LUX at $180 \mathrm{~V} / \mathrm{cm}$ once again [58]. The NEST model is shown in blue, black, and green for 105, 180, and $310 \mathrm{~V} / \mathrm{cm}$ (LZ baseline), respectively. The green curve in each plot is what is used for all of the ER background modeling in LZ. Low-energy beta particles and gamma-rays are treated equivalently as both generating ER in the context of the simulation. Increasing the magnitude of the drift electric field reduces the recombination probability, thus raising the charge yield at the expense of light, as with NR.

The potential of this medium for particle detection was recognized in the mid-20th century, when the combination of good scintillation and ionization properties was first noted (see [38] and references therein). In the 1970s, the first double-phase detectors were demonstrated, originally using argon [61]. Initially, our understanding of the mechanisms involved in generating the scintillation and ionization responses in the noble liquids progressed slowly, especially regarding the response to low-energy nuclear and electron recoils. However, great steps have been taken in the past decade, with LZ collaborators taking a central role. This effort continues around the world.

In this section, we summarize those LXe properties that affect the detection of low-energy nuclear and electronic recoils via scintillation and ionization; the next section discusses how to discriminate between them. The response of LXe to electron and nuclear recoils is now well understood over the energy range of interest for "standard WIMP" searches $\gtrsim 1.1 \mathrm{keV}_{\mathrm{nr}}$ [45]. Significant progress has equally been made in modeling its behavior as a function of incident particle species, energy, and electric field, in order to optimize detector design and the physics analyses. Naturally, the increasing WIMP scattering rates with decreasing recoil energy and the need to probe lighter dark matter candidates mean that pushing further down in threshold is a perennial concern for any detection technology. 
Scintillation and ionization yields for ERs in LXe are shown in Figures 1.3.6 and 1.3.7, with predictions by the Noble Element Simulation Technique (NEST) model (see [56, 57, 60] and the brief description in Chapter 12); data for Compton electrons now reach down to $1.5 \mathrm{keV}_{\mathrm{ee}}[56,65]$, and NEST shows good agreement with these results [59]. LXe compares favorably to the best scintillators and is also a good ionization medium. For example, the maximum photon yield at a few tens of $\mathrm{keV}$ is some $40 \%$ higher than that of liquid argon. This is important for a number of reasons: It reduces the variance of the ER response, which is important for particle discrimination; it permits effective detector calibration; and it is directly relevant to some leptophilic dark matter searches.

As Figure 1.3.6 suggests, the scintillation yield is suppressed with increasing electric-field strength, while the ionization yield improves by the same amount. This behavior is also observed for individual events: A fraction of the photon yield comes from recombination luminescence, whereby VUV photons are generated from electron-ion recombination occurring near the interaction site, and therefore some electrons contribute either to scintillation (S1) or to ionization (S2), but not to both. This event-by-event anticorrelation of the two signatures can be exploited very effectively at higher energies in double-phase detectors to obtain good energy resolution for the spectroscopy of ERs, with application to gamma-ray background studies and searches for $0 v \beta \beta$ decay.

For NRs, both data and modeling have progressed markedly in recent years. The picture here is more complex than for electron interactions. Most of the energy deposited by electrons is shared between ionization and excitation of the medium, making much of it observable. In NR interactions, a larger fraction is spent in atomic collisions, which is dissipated as heat and not detected. However, data obtained by scattering experiments at neutron beams ex situ, e.g. [62,63], are now in good agreement with those from indirect in situ techniques [64], down to $\sim 3 \mathrm{keV}$.

As the NR scintillation yield declines gently at least down to $3 \mathrm{keV}$, the ionization yield increases accordingly, as shown in Figure 1.3.9, with the current state of the art for in-situ measurement coming from LUX. This measurement, for the first time, constrains the ionization yield below $1 \mathrm{keV}(0.7 \mathrm{keV})$ [23]. We note 


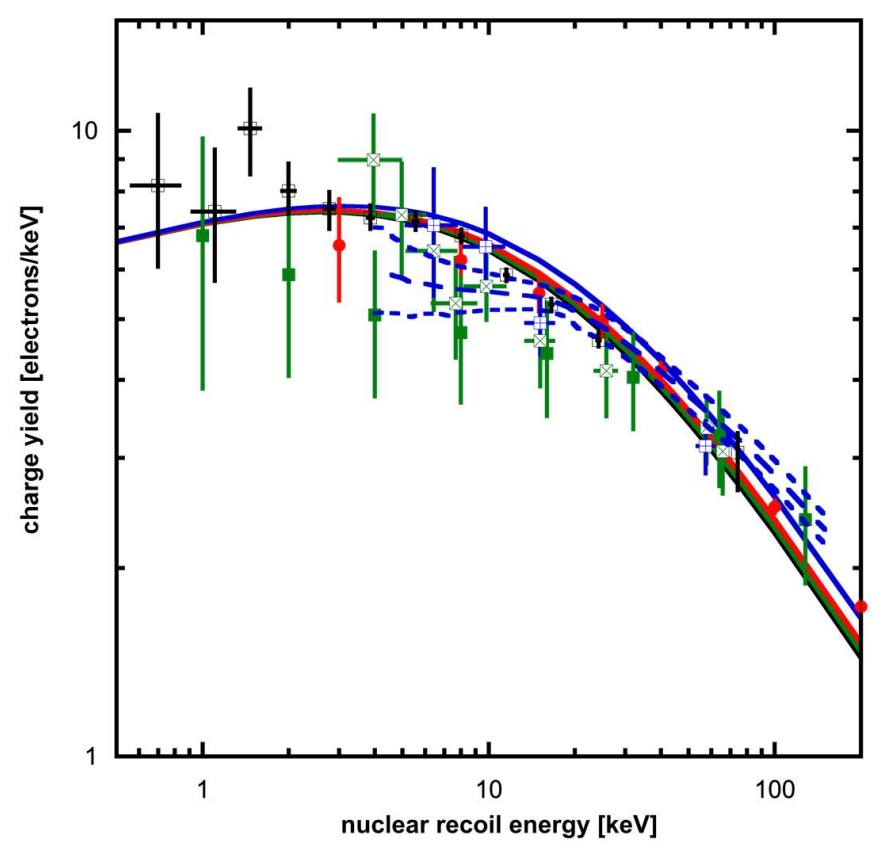

Figure 1.3.9: Absolute NR ionization yield in LXe. Data are as follows: blue and green hollow squares from neutron beam data from Yale at $4 \mathrm{kV} / \mathrm{cm}$ and $1 \mathrm{kV} / \mathrm{cm}$, respectively [62]; dashed blue curves from MC matching from ZEPLIN-III [64] at 3,650 V/cm; solid green squares from XENON10 at $730 \mathrm{~V} / \mathrm{cm}[66$ ]; red markers from XENON100 at $530 \mathrm{~V} / \mathrm{cm}$ [67]; black squares from LUX D-D neutron gun measurements in situ at $180 \mathrm{~V} / \mathrm{cm}$ [23]. The NEST model is shown in black, green, red, and blue for 180, 310 (LZ baseline), 530, and 3,650 V/cm, respectively. The green curve is used for LZ sensitivity calculations.

that the experimental data suggest a remarkably high yield even for 1-keV recoils, with several electrons being released per interaction. As with the scintillation yield, the electric field dependence is modest.

\subsubsection{Low Energy and Low Mass Sensitivity}

The high ionization yield allied to the ability to detect single electrons with high efficiency is a very attractive feature of this technology: Not only does it provide a low-threshold channel for light WIMP searches but, from a practical standpoint, it allows very high triggering efficiency (on S2) for the lowest-energy events, which are associated with very small S1 pulses.

Double-phase Xe detectors achieve the best NR energy threshold among the leading WIMP-search technologies, while maintaining discrimination and good vertex location. Of all such detectors operated so far, LUX can claim the lowest $50 \% \mathrm{NR}$ threshold of approximately $4 \mathrm{keV}$. WIMP masses down to $\sim 10 \mathrm{GeV} / \mathrm{c}^{2}$ are directly accessible to an instrument such as LZ operating in the "normal" TPC mode, requiring one S1 pulse and one $\mathrm{S} 2$ pulse. A low-energy ER interaction $\left(\approx 1.5 \mathrm{keV}_{\mathrm{ee}}\right)$ is shown in Figure 1.3.10 - 3-D position resolution and discrimination are fully effective even at these energies.

At the smallest NR energies $\left(\lesssim 4 \mathrm{keV}_{\mathrm{nr}}\right)$, it is clear that the $\mathrm{S} 1$ signal is often absent but $\mathrm{S} 2$ is still easily detectable, so that LZ can recover sensitivity in this regime by performing an "S2-only" analysis [68]. Discrimination based on S2/S1 ratio is not possible in this instance, but the detector retains the ability to reject edge events in $(x, y)$. A more limited but still useful degree of $z$ position reconstruction is possible based on the broadening of the S2 pulse due to longitudinal diffusion of electrons as they drift in the liquid. 
As a result, an S2-only search can still exploit the extremely radio-quiet inner region of the WIMP target, and place upper limits on the dark matter scattering cross section. Naturally, a thorough understanding of backgrounds is required for this type of analysis; several background mechanisms create single S2 electrons, while the two-electron random coincidence rate might still be significant. This technique is particularly applicable to particle masses lower than about $10 \mathrm{GeV} / c^{2}$.

One class of NR event that inevitably will be visible below the (3-phe) S1 threshold is due to coherent elastic scattering of ${ }^{8} \mathrm{~B}$ solar neutrinos off Xe nuclei. The electron counting technique (S2-only) was in fact suggested a decade ago to allow a first observation of this process [69]. Due to energy resolution broadening of the scintillation signal, some events will register both S1 and S2-from this and other neutrino fluxes. Despite significant interest in this signal per se, coherent neutrino-nucleus scattering is also a fundamental background for dark matter searches, which is quantified in Chapter 2.2.1.2.

\subsubsection{Electron/Nuclear Recoil Discrimination}

Discrimination of ER/NR is key to the positive identification of a WIMP signal, both by directly reducing the effect of the dominant electronic backgrounds in the detector, and by confirming a NR origin. The physical basis for discrimination is the difference in the ratio of ionization electrons to scintillation photons that emerge from the interaction site and subsequently create the measured S2 and S1 signals, respectively. In a plot of the logarithm of S2/S1 as a function of S1, as in Figure 1.3.11, electron and nuclear recoils each form a distinct band, with NRs having a lower average charge/light ratio.

A figure of merit for discrimination is commonly the ER leakage past the median of the NR population (i.e., retaining a flat $50 \%$ NR acceptance). Previous values are between $99.5 \%$ in XENON10 [37] and 99.99\% in ZEPLIN-III [41]. We assume a baseline discrimination value of $99.5 \%$, a conservative assumption given the performance already obtained in LUX, as discussed below.

Electron/nuclear recoil discrimination is determined by the separation of the bands as well as their widths, and in particular the "low tail" in $\log _{10}(\mathrm{~S} 2 / \mathrm{S} 1)$ of the ER band. Remarkably, the bands are mostly Gaussian when binned in slices of S1. Some skewness in the shape of the ER band has been observed in both the ZEPLIN-III and LUX experiments. In ZEPLIN-III, this skewness was observed using an external gammaray source whilst running at a high field [41]. Conversely, in LUX, the effect was seen when running an internal tritium calibration at a much lower electric field [58].

The physics determining both the position of the bands and their widths has been studied and we are increasingly able to model it successfully [71]. The overall separation of the bands is mostly due to NRs producing less initial ionization and more direct excitation (leading to scintillation) than do ERs. In turn, the band widths depend strongly on the physics of electron-ion recombination at the interaction site. A recombination episode generates an excited $\mathrm{Xe}$ atom that de-excites through scintillation (via the $\mathrm{Xe}_{2}^{*}$ state). Therefore, initial ionization is either measured as charge (via S2) or light (via S1), and event-by-event fluctuations in the amount of recombination are one of the primary sources of band broadening. These fluctuations increase with recoil energy over the range of interest.

At the lowest energies, however, the distributions "flare up" due to statistical fluctuations in the S1 signal. This broadening is therefore reduced in chambers with higher light yield, improving discrimination. In fact, ER rejection in this technology is better just above threshold ( $\sim 5 \mathrm{keV}$ to $15 \mathrm{keV})$, where WIMP-induced recoil rates are highest, than at intermediate energies $(\sim 15 \mathrm{keV}$ to $40 \mathrm{keV})$, and then improves dramatically beyond $\sim 40 \mathrm{keV}$. The excellent ER discrimination at low energies is due in part to a decrease of recombination fluctuations, but is also caused by the curvature of both bands at low energy, as shown in Figure 1.3.11: The bands are largely parallel to the direction of S1 fluctuations, sharply reducing their impact on discrimination.

In addition to light collection, the drift field is also expected to affect discrimination. Although largely determined by the amount of initial ionization, the positions of the band medians have a residual dependence 

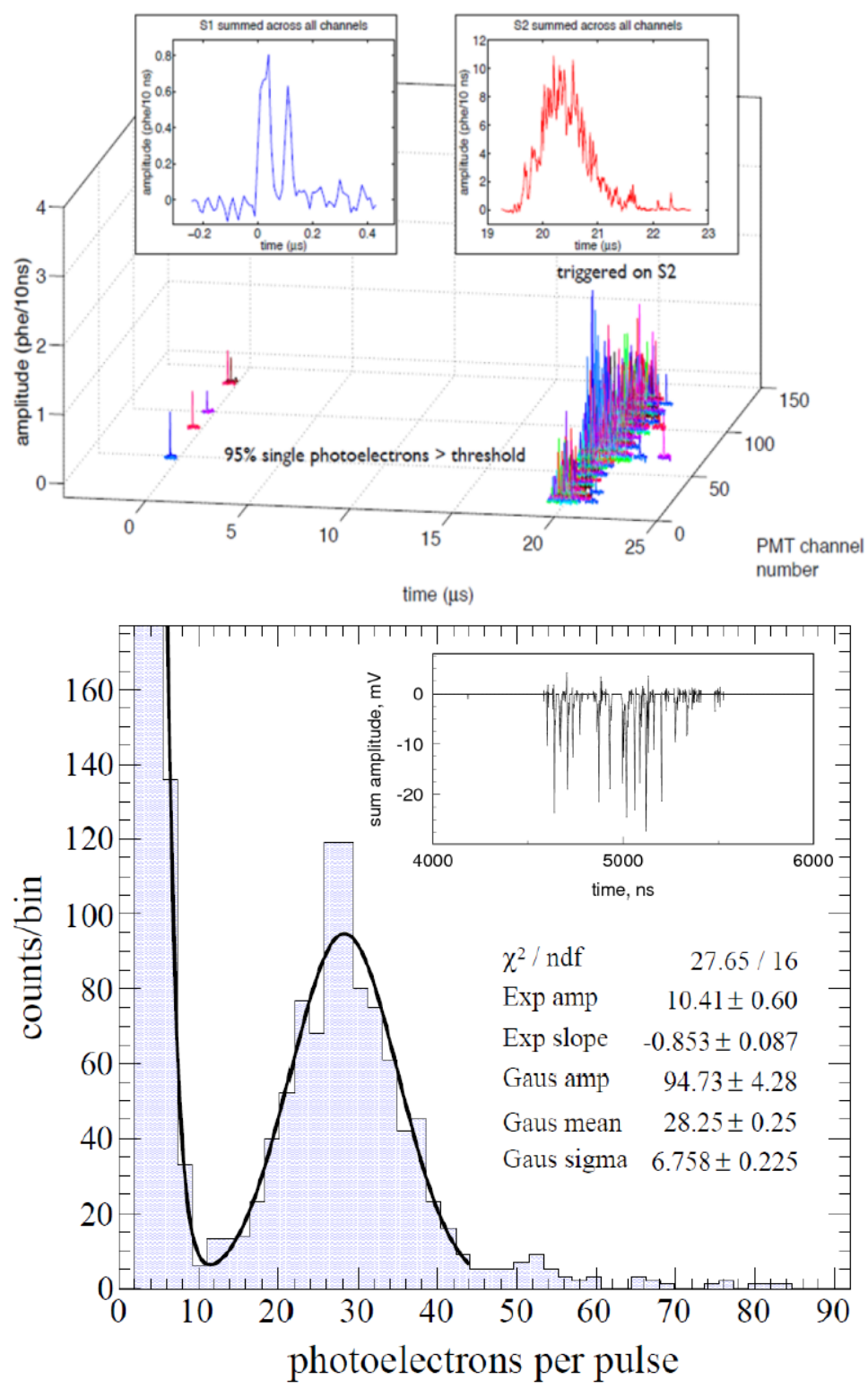

Figure 1.3.10: Low-energy performance of double-phase Xe detectors. Top: A $1.5 \mathrm{keV}_{\text {ee }}$ electron interaction in LUX [70], showing a fivefold coincidence for S1 and the corresponding (much larger) S2 delayed by $20 \mu \mathrm{s}$. Bottom: Pulse size distribution of single electrons measured by electroluminescence in ZEPLIN-III, showing a mean of 28 photoelectrons per emitted electron (one such waveform is shown in inset) [44]. 
on the different amounts of field-dependent recombination for the two recoil species, and their separation increases at higher fields [71]. This may explain the world-best discrimination observed in ZEPLIN-III, which operated with close to $4 \mathrm{kV} / \mathrm{cm}$ drift field, and is an important driver of the LZ design. The band width should, in principle, also have some field dependence, though this has not been well measured. The model developed in [71] has been incorporated in the NEST Monte Carlo package [60, 65], which has informed the sensitivity projections for this report. However, caution must be exercised when comparing values from different experiments, since instrumental effects other than electric field and light yield can impact discrimination very severely. A case in point is the degraded discrimination measured in the second science run (SSR) of ZEPLIN-III [72] relative to the first science run (FSR) [41], benchmarked essentially in the same detector and with the same software, but following upgrade of the TPC with underperforming photomultipliers.

The question of discrimination is of great importance in LZ, as its dominant background is ERs from ${ }^{222} \mathrm{Rn}$ and ${ }^{220} \mathrm{Rn}$ daughters as well as from solar neutrinos. To predict the LZ sensitivity, the electric field strength and the light-collection efficiency in the WIMP target are the main ingredients required. These are key performance parameters and we motivate their choice in two separate sections below; we also describe the steps we are taking to achieve the required performance. We use these parameters in conjunction with a full GEANT4 Monte Carlo simulation [73] based on the LUXSim package [74] and incorporating NEST.

The adopted values - $\mathrm{a}$ drift field of $310 \mathrm{~V} / \mathrm{cm}$ and an S1 photon detection efficiency (PDE) of $7.5 \%$ -motivate an average nominal discrimination of $99.5 \%$ for a flat ER spectrum such as that from ${ }^{222} \mathrm{Rn}$ and

${ }^{220} \mathrm{Rn}$ as well as from pp neutrinos (an ER leakage past the NR median of 1:200). This is supported by both NEST-based simulations and by XENON10 [37], which achieved that level of discrimination at similar field and light collection as proposed here. PANDA-X recorded $99.7 \%$ at $667 \mathrm{~V} / \mathrm{cm}$, for a PDE of $10.5 \%$ [75]. Significantly, LUX initially reported $99.6 \%$ discrimination at only $180 \mathrm{~V} / \mathrm{cm}$ in Run 3 [30], increasing to $99.8 \%$ in a subsequent reanalysis with improved algorithms [58]. Therefore, we are confident of reaching the $99.5 \%$ value assumed in this report.

In fact, Figure 1.3.12 shows that the discrimination generally improves for smaller $\mathrm{S} 1$ signals, as measured in LUX. Further, the signal from a light WIMP is not distributed symmetrically around the NR median: Upward fluctuations in S1 that cause low-energy events to exceed a given analysis threshold also induce a distribution in $\log _{10}(\mathrm{~S} 2 / \mathrm{S} 1)$ that is systematically lower than the NR median [76], as shown in Figure 2.2.1, Figure 12.3.1, and Figure 12.3.3. The acceptance of the ER rejection cut for light WIMPs is higher than the $50 \%$ one might surmise from the median of the average NR distribution.

Phenomenon like those discussed in the previous paragraph, combined with the high-statistics calibrations recently completed by the LUX collaboration $[45,58]$, have convinced us to replace the simple 'cut-andcount' technique, and a simple leakage figure of merit, with the profile likelihood ratio (PLR) technique described in Section 12.3.1.

\subsubsection{Outer Detector Systems}

A WIMP scatter would deposit a few $\mathrm{keV}$ in the central volume of the LXe TPC, with no simultaneous energy deposit in surrounding materials. Neutrons caused by radioactivity, which can fake WIMP interactions when they scatter elastically, are likely to interact again either within the TPC or nearby, and so it is broadly desirable to replace as much of the neighboring material as possible with additional radiation detectors. It also helps to minimize intervening material between the active LXe in the TPC and any such ancillary detectors, namely by decreasing the thickness of the field-cage and of the cryostat vessels. Active material surrounding the central LXe volume also permits assessment of the local radioactivity environment, and thus to infer additional information on the backgrounds in the WIMP search region. A persuasive WIMP discovery will require excellent understanding of all background sources, which is best done through the characterization of those sources in situ. 


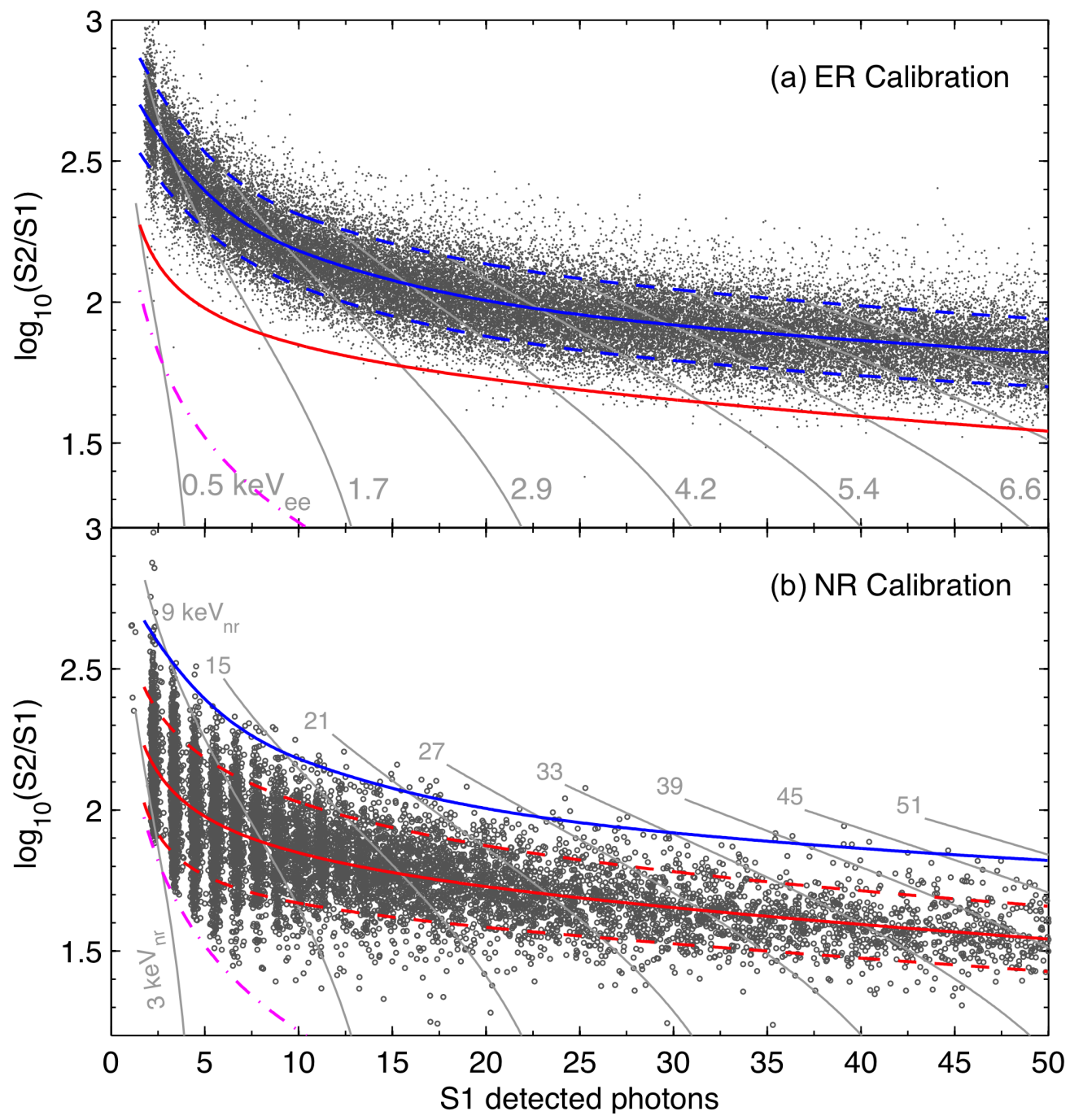

Figure 1.3.11: Discrimination parameter $\log _{10}(\mathrm{~S} 2 / \mathrm{S} 1)$ as a function of $\mathrm{S} 1$ signal obtained with LUX calibration [58]. (a) ER band calibrated with beta decays from a dispersed ${ }^{3} \mathrm{H}$ source; the median is shown in blue, with $80 \%$ population contours indicated by the dashed blue lines. (b) NR band populated by elastic neutron scattering from a D-D pulsed neutron source; the median (solid) and $80 \%$ band width (dotted) are indicated in blue and red, respectively. The mostly vertical gray lines are contours of constant energy deposition. For more information, see Chapter 7. 


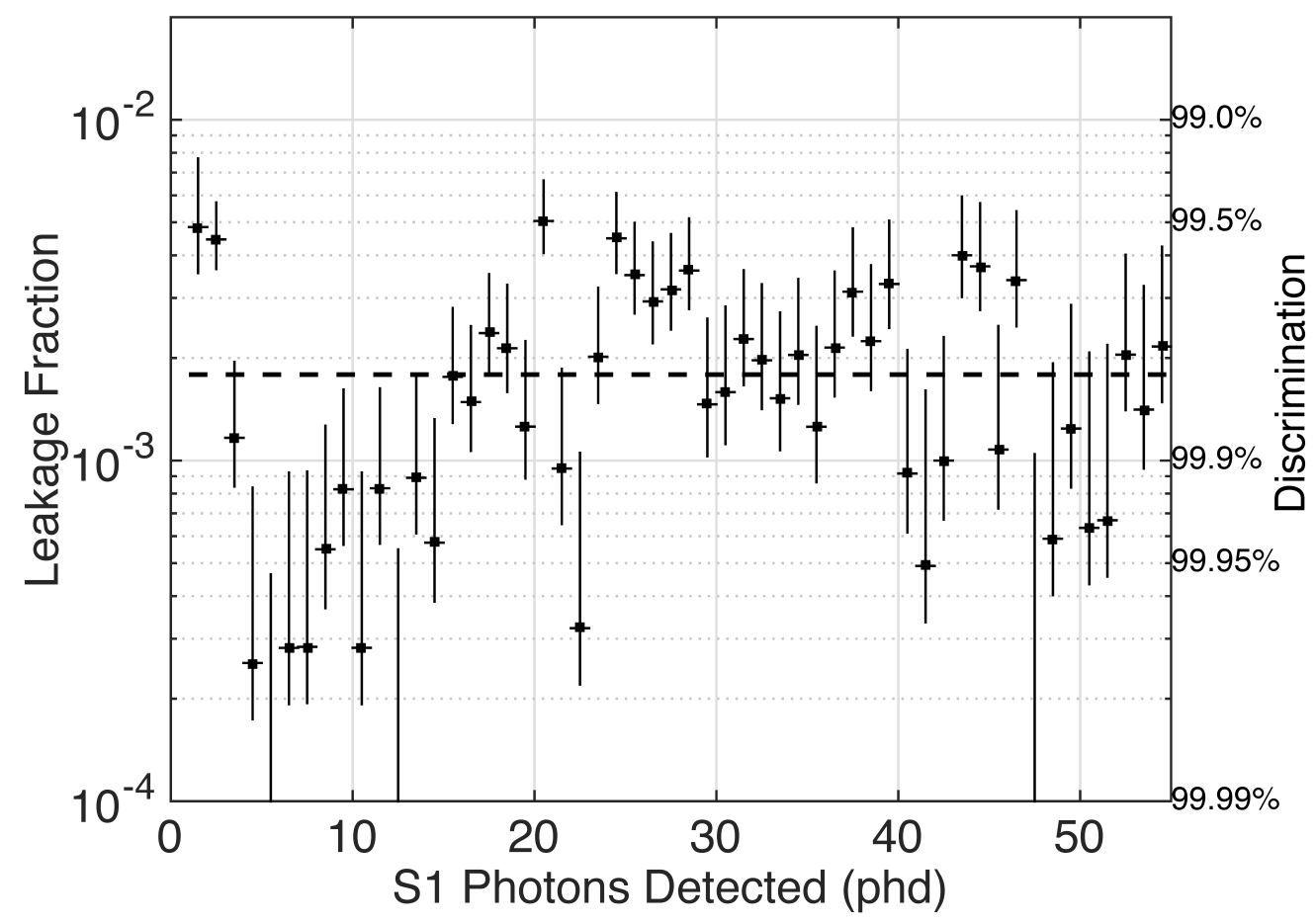

Figure 1.3.12: ER leakage fraction past the NR median line measured with tritium data in LUX. The dashed line indicates the average leakage of 0.002 (99.8\% discrimination) in the S1 range 2 phe to 50 phe. The general improvement of discrimination at low energies can be clearly seen, with the exception of the very lowest S1 data points where the ER band starts to flare up due to photoelectron statistics.

The LZ apparatus will feature two distinct regions where active material surrounds the LZ TPC. The first is a "skin" of LXe, formed by liquid between the field cage and the inner vessel of the cryostat. The second is surrounding detectors of liquid scintillator (LS), which is doped with a small amount of gadolinium, to enhance its capability for neutron detection. We expect a threshold of $100 \mathrm{keV}_{\mathrm{ee}}$ to $200 \mathrm{keV}_{\mathrm{ee}}$ for both systems, with the gadolinium-doped LS detecting neutrons more effectively and the skin detector performing best for internal gamma rays.

\subsubsection{Xenon Skin Veto}

The side skin region consists of an unavoidable $4 \mathrm{~cm}$ to $8 \mathrm{~cm}$ of LXe between the outer boundary of the field cage and the inner boundary of the cryostat. An even thicker LXe region, known as the dome skin, exists below the bottom PMT array. It is highly desirable to read out scintillation light generated in these regions for two main reasons:

1. They comprise anticoincidence detectors, where presence of a signal indicates that an interaction in the central LXe TPC is not from a WIMP.

2. They will also veto external LXe interactions where VUV photons could leak into the TPC and fake S1 light there.

Our approach is to maximize the optical isolation between inner and outer LXe volumes as far as practicable, and to instrument as much outer LXe as possible. Nevertheless, the sensitivity to deposited energy in the 
skin region will be far less than that of the central Xe TPC and, no ionization can be detected in the skin. Reasonable utility for the vetoing of gamma rays in the skin results when the threshold is a small fraction of the typical energy of an environmental gamma. To achieve this threshold, the side skin is equipped with 90 Hamamatsu R8520-406 PMT looking downwards and another 90 looking upwards to monitor the cylindrical shell between the sides of the TPC and the cryostat wall. The dome skin is viewed by 12 Hamamatsu R11410-22 PMT.

The PMT system achieves a threshold of about $100 \mathrm{keV}_{\text {ee }}$, sufficient to detect Compton recoils from MeV gamma rays from radiogenic backgrounds. The skin detector will have low sensitivity for neutron detection via elastic scattering due to the mismatch in mass between neutrons and Xe nuclei, but inelastic neutron interactions can be detected in the skin, as can gamma rays produced by neutron capture. The LXe skin veto has some advantages over the outer detector for gamma detection because some gamma rays do not penetrate the various vessels all the way to the OD.

\subsubsection{Scintillator Outer Detector}

The goal of the outer detector is to surround the LZ cryostat with a near-hermetic gamma-ray and neutron anticoincidence system. LZ will employ linear alkyl benzene (LAB), an LS solvent developed by the reactor neutrino community in the past decade [77, 78]. Small quantities of a standard fluor and wavelength shifter will be added to the solvent to provide the scintillation signal. A PMT system located in the water space outside of the clear acrylic tanks containing the LS will view this scintillation light.

To enhance neutron detection, $0.1 \%$ by weight of natural gadolinium will be dissolved in the LAB with a chelating agent to form Gd-loaded LS, or GdLS. Two isotopes of Gd, ${ }^{155} \mathrm{Gd}$ and ${ }^{157} \mathrm{Gd}$, have neutron-capture cross sections that are 61 and $254 \mathrm{~kb}$, respectively. Each of these isotopes constitutes about $15 \%$ of natural Gd and, at $0.1 \%$ concentration by weight, capture on Gd is about 1 order of magnitude more probable than is capture on hydrogen.

A neutron that can cause a Xe NR in the same energy range as the recoil from a WIMP will have an energy between about 0.5 and $5 \mathrm{MeV}$. The source of most of these neutrons will be from the $(\alpha, n)$ process from material around the edges of the Xe, and their energy spectrum will be toward the low end of this interval. A dangerous neutron will enter the LXe TPC and then scatter back out after one interaction. Many of those neutrons will traverse the intervening material and then thermalize and capture in the $\mathrm{Gd}$ in the GdLS. The length scale for thermalization and capture is a few centimeters and the typical capture time is $\sim 30 \mu$ s, which is small compared with the $670 \mu$ s maximum drift time of the TPC.

After capture on $\mathrm{Gd}$, a total energy of about $8 \mathrm{MeV}$ is emitted as a burst of several gamma rays, which then interact in the LS (or the skin, or the TPC). This large energy release separates neutron captures from the $\gamma$ rays from natural radioactivity, which die out above $3 \mathrm{MeV}$. The thickness of the GdLS layer is $61 \mathrm{~cm}$, determined by detailed GEANT4-based simulations and mechanical consderations.

For a fraction of $\gamma$-rays that deposit energy in the central LXe TPC, the outer detector system also functions as a $\gamma$-ray veto, for those that propagate through the LXe skin and cryostat; these share the same detection requirements as the capture gammas. To achieve good efficiency as a $\gamma$-ray veto, we require a threshold to Compton electrons near $200 \mathrm{keV}_{\mathrm{ee}}$, and we set a threshold goal of $100 \mathrm{keV}_{\mathrm{ee}}$

In reactor neutrino experiments, typically a single acrylic cylinder contains the Gd-loaded scintillator. Transport logistics preclude this solution for LZ, and instead segmentation of the volume into nine smaller tanks that can be transported through the shafts and drifts that lead to the Davis Cavern is the adopted solution. To enhance light collection, the outer surface of the LZ cryostat will be affixed with a diffuse

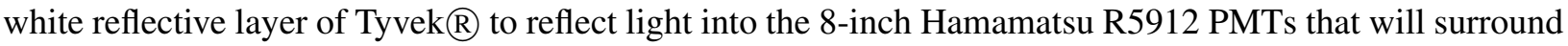

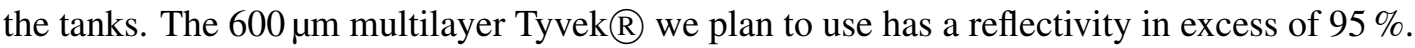


The goal is a sufficient PMT collecting power to achieve $100 \mathrm{keV}_{\text {ee }}$ threshold. Our preliminary studies

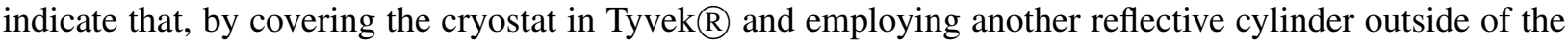
PMT system, the collecting power of 120 8-inch PMTs is sufficient to achieve the required threshold.

The GdLS tanks will be surrounded by ultrapure water, and the distance to the water-space PMT system that detects the LS scintillation light must be sufficient to attenuate gamma rays from PMT radioactivity (these are not low-background models). A distance of $80 \mathrm{~cm}$ from the scintillator tanks to the PMTs reduces the rate from this source to less than $5 \mathrm{~Hz}$.

The tightest specifications on radioactive impurities in the GdLS arise from considerations of deadtime caused by the outer detector system. Our goal is a false veto probability that does not exceed $5 \%$ over a $500 \mu$ s time window, which results in goals for radioactive impurities of $<1.3 \mathrm{ppt} \mathrm{U},<4.5 \mathrm{ppt} \mathrm{Th},<0.8 \mathrm{ppt}$

${ }^{40} \mathrm{~K}$, and $<13 \times 10^{-18} \mathrm{~g} / \mathrm{g}$ of ${ }^{14} \mathrm{C}$. Our requirements on impurities are $<10 \mathrm{ppt} \mathrm{U},<20 \mathrm{ppt} \mathrm{Th},<3 \mathrm{ppt}{ }^{40} \mathrm{~K}$, and $<15 \times 10^{-18} \mathrm{~g} / \mathrm{g}$ of ${ }^{14} \mathrm{C}$, which permit a $5 \%$ inefficiency at $5 \%$ false veto probability for a shorter $170 \mu \mathrm{s}$ time window.

While the LAB itself generally exceeds the impurity goals, the additives must be purified somewhat more completely than has been achieved in the reactor neutrino experiments. The purity achieved by the Borexino Collaboration greatly exceeds the $\mathrm{LZ}$ requirements for $\mathrm{U} / \mathrm{Th} / \mathrm{K}$. Borexino demonstrated a ${ }^{14} \mathrm{C}$ impurity slightly better than that needed for LZ [79]. Should the ${ }^{14} \mathrm{C}$ level be higher than our goal or requirement, we are able to set a threshold of $200 \mathrm{keV}_{\text {ee }}$, above the endpoint of ${ }^{14} \mathrm{C} \beta$ decay.

Chapter 7 details the implementation and performance of the outer detector more fully.

\subsection{Internal Calibration with Dispersed Sources}

The physics of self-shielding allows LZ to achieve its unprecedented sensitivity by reducing the rate of $\gamma$-ray scatters in the energy range of interest to a level of secondary importance. This is the central feature of the LZ detector design. Conversely, the same effect presents a challenge for a calibration program based solely on external $\gamma$-sources such as ${ }^{137} \mathrm{Cs}$, which range out in the first several centimeters.

LZ will use a suite of gaseous sources introduced into the xenon circulation path that will mix throughout the TPC active region and the xenon skin. In the active region we will rely primarily on the two sources that were developed and successfully used by LUX. ${ }^{83 \mathrm{~m}} \mathrm{Kr}$, a source of $9.4 \mathrm{keV}_{\mathrm{ee}}$ and $32.1 \mathrm{keV}_{\text {ee }}$ conversion electrons, separated in time by an average of $154 \mathrm{~ns}$, will be used to frequently calibrate spatial effects over the course of the run. However, owing to its complex decay structure and relatively high energy compared with the WIMP-search energy range, it is not suitable for calibrating the ER background.

The ${ }^{83 \mathrm{~m}} \mathrm{Kr}$ has a 1.8 hour half-life and will decay away quickly enough that no removal is required. However, because of the slow mixing of the xenon in LZ, that isotope may not populate the active region uniformly. Therefore, LZ will also use ${ }^{131 \mathrm{~m}} \mathrm{Xe}$, which has a 164-keV x-ray and an 11-day half-life, to occasionally make a homogeneous distribution of events to uniformly calibrate position-dependent effects. Its higher energy is also well suited to calibrating the xenon skin at the expected threshold of about $100 \mathrm{keV}_{\text {ee }}$. For calibrating the ER background in the WIMP-search energy range, we will use tritium $\left({ }^{3} \mathrm{H}\right)$, a $\beta^{-}$-emitter with a Q-value of $18.6 \mathrm{keV}_{\mathrm{ee}}$, in the form of tritiated methane. As we demonstrated in LUX, this molecule can be effectively removed by the purification system without leaving residual activity and its energy is ideally suited for modeling the ER band down to threshold.

Further calibration of the skin will use higher-energy events from ${ }^{220} \mathrm{Rn}$, a source of 6-MeV alpha particles. Unlike ${ }^{222} \mathrm{Rn}$, this isotope has no long-lived progeny and presents no background contamination concerns. Another benefit of ${ }^{220} \mathrm{Rn}$ is that some of its progeny should live long enough to deposit on the detector walls and produce a useful calibration of the S2 response there, which as we have seen in LUX, is different than in the bulk. 


\subsection{Xenon Purity for Detector Performance}

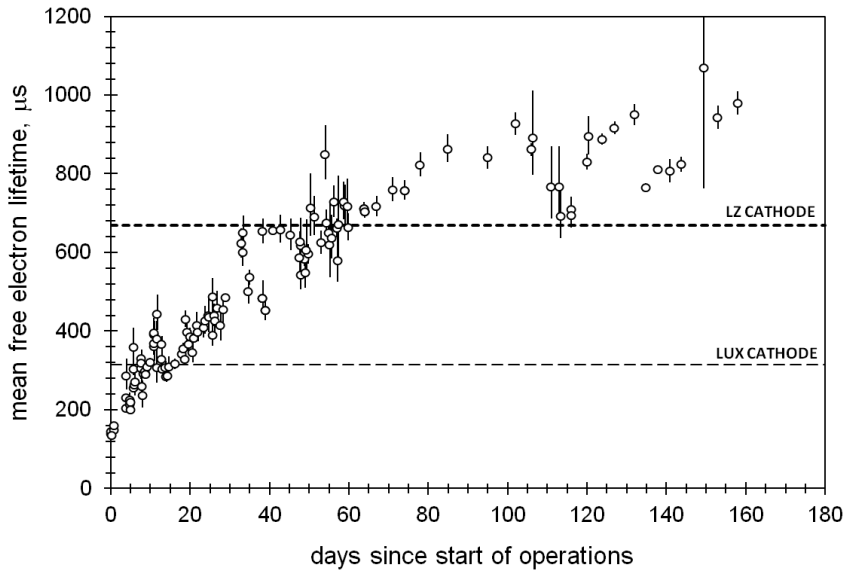

Figure 1.5.1: Evolution of the mean free electron lifetime in LUX during Run 3 . The electronegative purity required to observe signals from the LZ cathode $(\sim 670 \mu \mathrm{s}$ for the $147-\mathrm{cm}$ long TPC) has already been achieved in LUX.
The fluid nature of LXe provides an opportunity to manipulate the purity of the LZ target material. The previous section describes how this allows internal calibration sources to be temporarily introduced into the LXe. This section considers how the purity can be maximized for low-background physics running. We consider two classes of impurities: the radioactive noble gases ${ }^{85} \mathrm{Kr}$ and ${ }^{222} \mathrm{Rn}$ - although the latter is addressed fully only in Chapter 9 - and electronegative contaminants such as oxygen and water.

Electronegative impurities are introduced during operations by the outgassing of detector materials, and they must be continuously suppressed to the level of $\sim 0.1 \mathrm{ppb}$ by the purification system to ensure good charge and photon transport. Previous detectors such as ZEPLIN-III achieved this through clean construction techniques with low-outgassing materials (i.e., no plastics), which allowed these systems to maintain purity without recirculation [80]. In larger detectors such as LUX, the need to utilize large volumes of PTFE (see Chapter 3 for detail) demands active recirculation in the gas phase. The efficient removal of electronegative contaminants is made possible by purification technology developed for the semiconductor industry. The central elements of this technology are a heated zirconium getter for the removal of non-noble species, UHV-compatible plumbing and instrumentation, and a gas-circulation pump.

Despite the availability of this technology, until recently the achievement of good electronegative purity in a LXe TPC was considered a significant technical challenge. What was lacking was an economical and sensitive monitoring technique to allow the purification technology to be fully exploited. While free electron lifetime monitors for LXe were developed over 20 years ago, these devices cannot identify individual impurity species, are insensitive to noble gas impurities, and provide little guidance on the origin of any impurities.

LZ scientists have developed a mass spectrometry method that allows most electronegative and noble gas impurities to be individually monitored in real time [52]. Most crucially, the method provided, for the first time, information on the impurity source. For example, the presence of an air leak introduces $\mathrm{N}_{2}, \mathrm{O}_{2}$, and $\mathrm{Ar}$ in a characteristic ratio, while a large excess of $\mathrm{N}_{2}$ is a signature of a saturated purifier. Outgassing, on the other hand, leads to a uniform rate of increase of all common impurity species.

Experience with the mass spectrometry method, relevant to LZ, has been gained by applying it not only to LUX, but also to the EXO-200 $0 v \beta \beta$-decay experiment [81]. Both experiments achieved their purity goals with relative ease due in part to the effectiveness of this program, and this valuable experience can be brought to bear on LZ. We have learned, for example, that vendor-supplied Xe is often relatively pure of electro-negatives; that zirconium getters are effective for Xe purification but that their performance degrades at higher gas flow rates; and that purifier performance improves at elevated temperature. This experience 
is of direct relevance for the design of the LZ purification system. In LUX, electron lifetimes of the order of $1 \mathrm{~ms}$ have been demonstrated, as shown in Figure 1.5.1, which is already sufficient to drift charge from interactions near the LZ cathode.

An additional benefit is that the method is sensitive not only to electronegatives but also to trace quantities of noble gas impurities, which is of critical importance for control of krypton. Krypton is a particularly dangerous impurity for LZ because of the presence of the beta emitter ${ }^{85} \mathrm{Kr}$. This isotope, whose abundance at present is $\sim 2 \times 10^{-11}\left({ }^{85} \mathrm{Kr} /{ }^{\text {nat }} \mathrm{Kr}\right.$ ), presents the leading purification challenge for LZ because its noble nature makes it impervious to the zirconium getter technology. Vendor-supplied Xe typically contains about $100 \mathrm{ppb}$ of ${ }^{\text {nat }} \mathrm{Kr}$, which, if left untreated, would give rise to an ${ }^{85} \mathrm{Kr} \beta$ decay rate of $29 \mathrm{mBq} / \mathrm{kg}$ of Xe.

LZ will capitalize on the success of EXO-200 and LUX by integrating sensitive monitoring into its Xe handling program from the time of Xe procurement until the conclusion of the experiment. In addition, because the origin of impurities is now understood to be primarily due to outgassing, we have in place a comprehensive and extensive materials outgassing screening program.

While sensitive impurity monitoring will lay the groundwork for the LZ Xe purification program, the heart of the program to reduce long-lived noble-element radioisotopes will be the krypton removal program using the chromatographic technique developed by LZ scientists now at SLAC [51]. This program was successfully applied to LUX and will be scaled up in mass-throughput by a factor of 20 for LZ. The krypton concentration goal is $0.015 \mathrm{ppt}(\mathrm{g} / \mathrm{g})$, a factor of 300 below the LUX goal but only a factor of 10 worse than the upper limit of $0.2 \mathrm{ppt}(\mathrm{g} / \mathrm{g})$ demonstrated so far with this method. At $0.2 \mathrm{ppt}(\mathrm{g} / \mathrm{g})$, the rate of beta decays from ${ }^{85} \mathrm{Kr}$ in the region of interest is comparable to the pp neutrinos. In parallel, the krypton detection limit of the mass spectrometry method has been improved to $\sim 0.015 \mathrm{ppt}(\mathrm{g} / \mathrm{g})$. The ultimate source of krypton impurities in LZ will be the outgassing of detector materials, which we can control through careful materials selection and through our outgassing plan.

Similarly, ${ }^{222} \mathrm{Rn}$ must be controlled by limiting the emanation sources within the detector and the gas system via a careful screening program. In fact, the need to limit radon and krypton in the LZ Xe is a driving consideration in the choice of key Xe system components such as the gas-recirculation pump. More generally, the materials-screening program is occurring hand-in-hand with the design efforts of all systems whose materials touch $\mathrm{Xe}$, in order to ensure that systems meet both their technical and their background requirements on schedule.

\subsection{Dominant Backgrounds}

LZ has a clear background-control strategy with optimal exploitation of self-shielding to pursue an unprecedented science reach. These are the most salient features of this strategy: (1) underground operation within an instrumented water tank to mitigate cosmogenic backgrounds; (2) deployment of a target mass large enough to self-shield external radioactivity backgrounds, working in combination with outer, anticoincidence detectors; (3) construction from low-activity materials and purification of the target medium to render intrinsic backgrounds subdominant; and (4) rejection of the remaining ER backgrounds by S2/S1 discrimination.

The dominant fixed-contaminant radioactive backgrounds come from the Xe-space PMTs. This background is small when compared to that from radon. Our approach is to self-shield those sources over the first few centimeters of active liquid and thereby be sensitive to a population of ERs caused by elastic scattering of solar pp neutrinos - which can be further discriminated by their S2/S1 ratio. An irreducible but very small background of NRs will also arise from coherent neutrino-nucleus scattering. To achieve this we must reduce any intrinsic ER backgrounds contained within the Xe itself to a tolerable level, with ${ }^{85} \mathrm{Kr}$,

${ }^{39} \mathrm{Ar}$, and Rn progeny being a particular challenge. Electron recoils caused by the $2 v \beta \beta$ decay of ${ }^{136} \mathrm{Xe}$, now confirmed by the EXO-200 [82] and KamLAND-Zen [83] experiments, are subdominant below about 
$20 \mathrm{keV}_{\mathrm{ee}}$. Cosmogenic (muon-induced) backgrounds are not significant during operation due to the tagging capability of the instrumented water tank and the scintillator veto, but cosmogenic activation of detector materials prior to deployment (including the $\mathrm{Xe}$ ) must be addressed.

We describe briefly some of these background categories here (radioactivity external to the TPC, intrinsic contamination of the LXe, and cosmogenic backgrounds), as these are important LZ sensitivity drivers, but we offer a full discussion on their mitigation to Chapter 9. Neutrino backgrounds are explored further in Chapter 2. The solar pp neutrino scattering rate of $0.8 \times 10^{-5}$ events $/ \mathrm{kg} / \mathrm{d} / \mathrm{keV}$ (ee) is the benchmark against which other rates are assessed.

\subsubsection{Backgrounds from Material Radioactivity}

Radioactivity backgrounds have limited nearly all dark matter experiments so far and, in spite of the power of self-shielding, we are not complacent in addressing them in LZ. They impact the thickness of the sacrificial layer of LXe that shields the fiducial mass, and they may cause rare-event topologies that may be of consequence (from random coincidences, atypical surface interactions, or Cherenkov emission in PMT glasses, for example). It is important, therefore, to minimize the rate of $\alpha, \beta$, and $\gamma$ activity around the active volume, as well as neutron production from spontaneous fission of ${ }^{238} \mathrm{U}$ and from $(\alpha, n)$ reactions. In addition, the rate and spatial distribution of such backgrounds must be well characterized to build an accurate background model for the experiment. The LZ background model is derived from a high-fidelity simulation of the experiment in the LUXSim framework, which was successfully used for the LUX background model [84].

All materials to be used in LZ will be subject to stringent constraints as part of the comprehensive screening campaign described in Chapter 9, with $10 \%$ of the solar pp neutrino scattering rate and a maximum of $\sim 0.2$ NRs at $50 \%$ signal acceptance being the target for the total contribution from material radioactivity within the fiducial volume. The dominant rates come from the various PMT systems and the LZ cryostat, based on the large masses and close proximity to the active region of the detector. Table 1.6.1 summarizes the count rates from neutron and $\gamma$-ray emission expected from detector materials and other backgrounds, which are discussed in greater detail in Chapter 9. Here and hereafter count rates are given for an indicative 5.6-tonne fiducial mass, considering only single scatter events with no accompanying signal in either the LXe skin or the Outer detector veto systems. The region of interest for WIMP searches is defined as 1) the number of detected photons in the S1 signal to be greater than 0 and less than 20,2) 3-fold coincidence in $S 1$ between PMTs in the target volume, 3) the number of detected photons in the S2 signal is greater than 350. This region of interest approximately corresponds to energy deposits of $6 \mathrm{keV}$ to $30 \mathrm{keV}$ for nuclear recoils and $1.5 \mathrm{keV}$ to $6.5 \mathrm{keV}$ for electron recoils. The range of energy deposits is sometimes used to reference the region of interest throughout the text. A full description of the analysis cuts is given in Chapter 12.

The PMTs chosen for the LZ TPC are Hamamatsu R11410s, which have achieved very low radioactivity values; LZ scientists have been involved in a long campaign to establish their performance for dark matter experiments, working actively with the manufacturer to enable this [48]. The PTFE required to fabricate the TPC field cage, skin reflectors, and other internal components may also be an important source of neutron emission from the bulk material. We use upper limits on the contamination measured by EXO-200 in calculating its impact [85]. The cryostat is another dominant component, mostly owing to its large mass. For the titanium baseline design $(2,140 \mathrm{~kg})$, the total neutron emission rate is estimated at $0.6 \mathrm{n} /$ day based on recent titanium samples procured for LZ. As a result of a 2-year material search campaign, we were able to find titanium with U/Th contamination, which is a factor of 2 lower than that used in LUX [49] as explained in Section 5.1. 
Table 1.6.1: Summary of backgrounds in LZ, showing the number of counts expected in 1,000 live days in an indicative 5.6-tonne fiducial mass in the region of interest with all cuts applied. A comprehensive set of numbers can be found in Table 9.2.7.

\begin{tabular}{|l|c|c|}
\hline \multicolumn{1}{|c|}{ Item } & ER cts & NR cts \\
\hline Detector Componenents & 6.2 & 0.07 \\
\hline Dispersed radionuclides $(\mathrm{Rn}, \mathrm{Kr}, \mathrm{Ar})$ & 911 & - \\
\hline Laboratory and cosmogenic & 4.3 & 0.06 \\
\hline Fixed surface contamination & 0.19 & 0.37 \\
\hline 136 Xe $2 v \beta \beta$ & 67 & - \\
\hline Neutrinos $(v$-e, $v$-A) & 255 & 0.72 \\
\hline Total & 1244 & 1.22 \\
\hline Total (with 99.5\% ER discrimination, 50\% NR efficiency) & 6.22 & 0.61 \\
\hline Total ER+NR background events & 6.83 \\
\hline
\end{tabular}

\subsubsection{Surface Plating of Radon Progeny}

The noble gas radon consists solely of radioactive isotopes, of which four are found in nature: ${ }^{222} \mathrm{Rn}$ and ${ }^{218} \mathrm{Rn}$ produced in the ${ }^{238} \mathrm{U}$ decay chain, ${ }^{220} \mathrm{Rn}$ from the ${ }^{232} \mathrm{Th}$ decay chain, and ${ }^{219} \mathrm{Rn}$ from the ${ }^{227} \mathrm{Ac}$ series. As a result of its chemical inertness, radon exhibits long diffusion lengths in solids. ${ }^{222} \mathrm{Rn}$ is the most stable isotope $\left(\mathrm{T}_{1 / 2}=3.82\right.$ days $)$, and is present in air at levels of about ten to hundreds of $\mathrm{Bq} / \mathrm{m}^{3}$. Charged radon progeny - especially metallic species such as ${ }^{218} \mathrm{Po}$ - plate out onto macroscopic surfaces that are exposed to radon-laden air. A fraction will deposit and even implant into material surfaces during detector construction or installation [86].

Backgrounds from surface beta and gamma radioactivity, as well as recoiling nuclei (e.g., ${ }^{206} \mathrm{~Pb}$ from the alpha decay of ${ }^{210} \mathrm{Po}$ ), are largely mitigated by short half-lives and the self-shielding of LXe. However, $\alpha$ particles released in the decay chain, particularly from ${ }^{210} \mathrm{Po}$ - a granddaughter of the long-lived ${ }^{210} \mathrm{~Pb}\left(\mathrm{~T}_{1 / 2}\right.$ $=22.3$ years $)-$ result in neutron emission following $(\alpha, n)$ reactions. This is problematic for TPC materials with large $(\alpha, n)$ yields such as PTFE $\left(\sim 10^{-5} n / \alpha\right.$, due to the presence of fluorine). Additionally, because PTFE is produced in granular form before being sintered in molds, plate-out poses further risk because surface contamination of the granular form becomes bulk contamination when the granules are poured into molds.

A second concern relates to our ability to correctly reconstruct events at the TPC inner walls, since the imperfect reconstruction of these events leads to a background population leaking radially toward the fiducial volume [30, 35]. This concern drives the design of the top PMT array and places tight requirements on the plate-out of radon progeny on the TPC walls (see Section 3.4).

Controls to mitigate background from radon plate-out will include limiting the exposure of detector parts to radon-rich air; monitoring from point of production through transport and storage in Rn-proof materials; and employing surface cleaning techniques, such that neutron emission is negligible relative to material radioactivity from bulk uranium and thorium contamination. 


\subsubsection{Intrinsic Backgrounds}

We are confident that our requirements for intrinsic radioactive contamination from ${ }^{85} \mathrm{Kr}$ and ${ }^{222} \mathrm{Rn}$ can be met with the Xe-purification techniques described in Chapter 6, coupled with the radon emanation screening of Xe-wetted materials described in Chapter 9. We note that most of these backgrounds can be estimated with low systematic uncertainty. In addition to direct sampling, the ${ }^{85} \mathrm{Kr} \beta$ - decay spectrum is well understood and the decay rate can be measured during operation with delayed $\beta-\gamma$ coincidences.

Other delayed coincidence techniques as well as $\alpha$ spectroscopy allow precise estimation of radoninduced backgrounds. In fact, it is possible to follow dynamically the spatial distribution of these decays throughout the detector, which was done very successfully in LUX [87]. The two main concerns in this instance are a "weak" naked $\beta$-decay from ${ }^{214} \mathrm{~Pb}$ in the bulk of the TPC $\left(\mathrm{E}_{\max }=1,019 \mathrm{keV}, \mathrm{BR}=9.2 \%\right)$, and the possibility of $\gamma$-ray escape for peripheral events from the dominant ${ }^{214} \mathrm{~Pb}$ decay modes.

Our goal is to control each of these two backgrounds to $<10 \%$ of the solar pp neutrino rate, limiting the ${ }^{222} \mathrm{Rn}$ activity to $0.67 \mathrm{mBq}$ and the krypton concentration to $0.02 \mathrm{ppt}(\mathrm{g} / \mathrm{g})$. In a more conservative scenario, as requirements we allow the sum of these two components to be 3 times the ER background from pp neutrinos.

Trace quantities of argon are also a concern due to $\beta$-emitting ${ }^{39} \mathrm{Ar}$, with a 269 -year half-life and $565 \mathrm{keV}$ endpoint energy. This background is constrained to be less than $10 \%$ of ${ }^{85} \mathrm{Kr}$, resulting in a specification of $4.5 \times 10^{-10}(\mathrm{~g} / \mathrm{g})$ or $2.6 \mu \mathrm{Bq}$. The Kr-removal system, which also removes Ar, should easily achieve this.

\subsubsection{Cosmogenic Backgrounds}

A rock overburden of 4,300 m w. e. above the Davis Cavern at SURF reduces the muon flux by about a factor of $3 \times 10^{6}$ relative to the surface $[88,89]$. Muons crossing the water tank and/or liquid scintillator are readily detected via Cherenkov emission in water and/or scintillations, and any coincident energy deposition in LZ is similarly easily identified. However, neutron production in muon-induced electromagnetic and hadronic showers, in particular in high- $Z$ materials, may generate background events [90, 91]. The total muon-induced neutron flux at SURF from the surrounding rock is calculated to be about $0.5 \times 10^{-9} \mathrm{n} / \mathrm{cm}^{2} / \mathrm{s}$, with approximately half of this flux coming from neutrons above $10 \mathrm{MeV}$, and some $10 \%$ from energies above $100 \mathrm{MeV}[92,93]$.

Muon-induced neutrons generated in the water shield and liquid scintillator produce a similarly low rate, despite the several hundred tonnes of target mass, due to the low atomic number of water and scintillator and consequent low neutron yield $\left(\sim 2.5 \times 10^{-4} \mathrm{n} / \mathrm{muon} /\left(\mathrm{g} / \mathrm{cm}^{2}\right)\right)$, translating to a production rate of order of $\left.10^{-9} \mathrm{n} / \mathrm{kg} / \mathrm{s}\right)$.

Cosmogenic activation - radioisotopes production within materials, largely through spallation reactions of fast nucleons from cosmic rays while on the Earth's surface - can present electromagnetic background in LZ. ${ }^{46} \mathrm{Sc}$ produced in the titanium cryostat decays through emission of $889 \mathrm{keV}$ and $1,120 \mathrm{keV} \gamma$-rays (with $\mathrm{T}_{1 / 2}=84$ days). Cosmogenic ${ }^{60} \mathrm{Co}\left(\mathrm{T}_{1 / 2}=5.3\right.$ years) in copper components will produce gamma rays of $1,173 \mathrm{keV}$ and $1,332 \mathrm{keV}$.

Activation of the Xe itself during storage or transport generates several radionuclides, some of which are important, especially in the first few months of operation. Tritium $\left(\mathrm{T}_{1 / 2}=12.3\right.$ years and production rate of $\sim 15 / \mathrm{kg}$ /day at the Earth's surface [94]) was previously a concern; however, this is effectively removed through purification during operation. Production of Xe radioisotopes, such as ${ }^{127} \mathrm{Xe}\left(\mathrm{T}_{1 / 2}=36.4\right.$ days $)$, ${ }^{129 \mathrm{~m}} \mathrm{Xe}\left(\mathrm{T}_{1 / 2}=8.9\right.$ days $)$, and ${ }^{131 \mathrm{~m}} \mathrm{Xe}\left(\mathrm{T}_{1 / 2}=11.9\right.$ days $)$, are more problematic, as they cannot be mitigated through self-shielding or purification. In particular, atomic de-excitation of the $2 \mathrm{~s}$ and $3 \mathrm{~s}$ shells in ${ }^{127} \mathrm{Xe}$ generates 5.2 and $\leq 1.2 \mathrm{keV}$ energy deposits, respectively, which are an important background in the WIMP search energy region for certain event topologies [30]. These backgrounds soon reach negligible levels once Xe is moved underground. 


\subsubsection{Fiducialization}

The backgrounds from nearby materials cluster near the edges of the LZ central TPC, and they fall off according to an exponential distributions described in Fig. 1.3.5. The rapid fall off of backgrounds from material radioactivity suggests the definition of a fiducial volume, which is an inner region that is relatively free of background.

The fiducial volume is a simplification that allows intuitive understanding of the LZ background from nearby materials. Eventually, LZ will abandon the concept of a fiducial volume in favor of a PLR maximum likelihood fit which describes the spatial distributions of the various background components, like the LUX collaboration has recently done [23].

We define an LZ fiducial volume that starts $1.5 \mathrm{~cm}$ above the cathode at the bottom of the TPC, $4 \mathrm{~cm}$ inside of the reflective walls that surround the TPC, and $13.5 \mathrm{~cm}$ down from the gate grid at the top of the TPC. This fiducial volume encloses 5.6 tonnes of LXe, and the event numbers tabulated in Table 1.6.1 occur inside this fiducial volume in a 1,000 live day run.
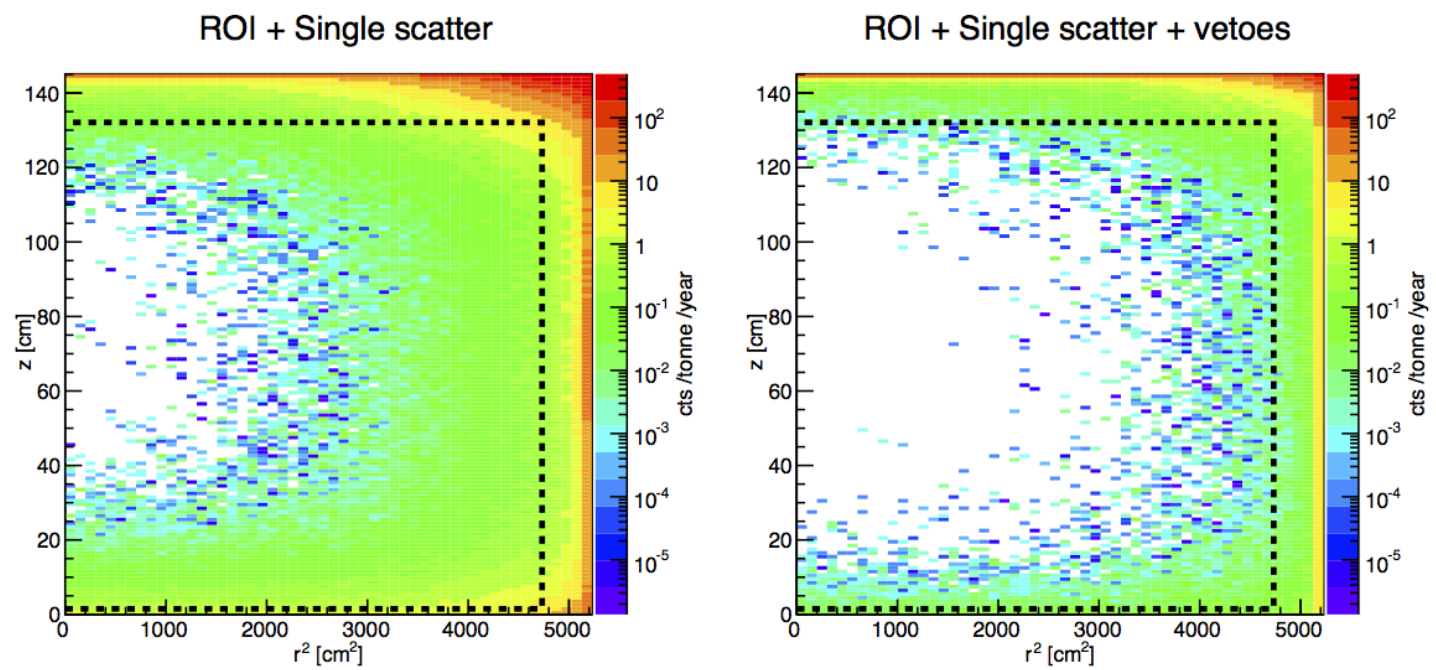

Figure 1.6.1: Total NR background plus ER leakage from material radioactivity for sources external to the $\mathrm{LXe}$ in the TPC, counted over a $6 \mathrm{keV}$ to $30 \mathrm{keV}$ acceptance region; a discrimination efficiency of $99.5 \%$ is applied to ERs from beta decays and gamma rays. Left: Single scatters only, no vetoing by the anti-coincidence systems. Right: Adding the combination of both the skin veto and the outer detector. The dashed line shows the boundary of the 5.6 tonnes fiducial mass.

Two main factors determine how far the fiducial boundary should lie from the lateral TPC walls. The prime consideration is to ensure a sufficiently thick layer of LXe to self-shield against the external radioactivity backgrounds. This is related to the mean attenuation length for those particles: Figure 1.3.5 confirmed that $\sim 2 \mathrm{~cm}$ of liquid decreases the $\gamma$-ray background tenfold, and as much as $\sim 6 \mathrm{~cm}$ is needed to mitigate neutrons by the same factor. However, the outer detector is very efficient for neutron tagging, which brings these two requirements closer together.

Secondly, it is essential that the reconstructed $(x, y)$ positions of low-energy interactions occurring near the TPC walls do not "leak" into the fiducial volume. As mentioned above (and discussed in Chapter 3) interactions from radon progeny plating the lateral PTFE are of particular concern: These can generate events with very small S2 signals due to trapping of charge drifting too close to the PTFE. If allied with poor position resolution, this can constitute a very challenging background [35]. In the vertical direction, 
only the former consideration arises. We point out that the reverse field region below the cathode will provide much of the required self-shielding $(>14 \mathrm{~cm})$, whereas at the top, the small thickness of liquid above the gate $(0.5 \mathrm{~cm})$ will have a limited impact.

Figure 1.6.1 shows the simulated background rate from material radioactivity in the WIMP region of interest $(6 \mathrm{keV}$ to $30 \mathrm{keV})$ as a function of radius squared and height above the cathode grid. Nuclear and electron recoil backgrounds were combined, with $50 \%$ acceptance applied to the former and $99.5 \%$ discrimination applied to the latter. The neutrino and dispersed background contributions listed in Table 1.6.1 are omitted from Figure 1.6.1 because they populate the figures uniformly.

The left panel of Figure 1.6.1 shows the rate of material backgrounds when neither of the outer detector systems, the LXe skin nor the outer detector, is utilized. The background-free region in the central TPC is small, and comprises about one-half of the 7 tonnes of active liquid xenon. The right panel shows the background rates after the application of the two outer detector systems, which enlarge considerably the background-free region. The event totals in Table 1.6.1 are computed from the events inside the fiducial volume enclosing 5.6 tonnes delineated by the dashed line in Fig. 1.6.1.

\subsection{Bibliography}

[1] D. N. Spergel et al. (WMAP), Astrophys. J. Suppl. 170, 377 (2007), arXiv:astro-ph/0603449 [astro$\mathrm{ph}]$.

[2] W. J. Percival et al., Astrophys. J. 657, 645 (2007), arXiv:astro-ph/0608636 [astro-ph].

[3] E. Komatsu et al. (WMAP), Astrophys. J. Suppl. 192, 18 (2011), arXiv:1001.4538 [astro-ph].

[4] P. A. R. Ade et al. (Planck), Astron. Astrophys. 571, A16 (2014), arXiv:1303.5076 [astro-ph].

[5] P. A. R. Ade et al. (Planck), Astron. Astrophys. 594, A13 (2016), arXiv:1502.01589 [astro-ph].

[6] C. S. Frenk and S. D. M. White, Ann. Phys. (Berlin) 524, 507 (2012), arXiv:1210.0544 [astro-ph].

[7] F. Governato, A. Zolotov, A. Pontzen, C. Christensen, S. H. Oh, A. M. Brooks, T. Quinn, S. Shen, and J. Wadsley, Mon. Not. R. Astron. Soc. 422, 1231 (2012), arXiv:1202.0554 [astro-ph].

[8] A. Kusenko and L. J. Rosenberg, in Planning the Future of U.S. Particle Physics, The Snowmass 2013 Proceedings, edited by N. A. Graf, M. E. Peskin, and J. L. Rosner (2013); Working Group Report: Non-WIMP Dark Matter, arXiv:1310.8642 [hep-ph].

[9] B. W. Lee and S. Weinberg, Phys. Rev. Lett. 39, 165 (1977).

[10] M. Cahill-Rowley, R. Cotta, A. Drlica-Wagner, S. Funk, J. Hewett, A. Ismail, T. Rizzo, and M. Wood, Phys. Rev. D91, 055011 (2015), arXiv:1405.6716 [hep-ph].

[11] G. Aad et al. (ATLAS), Phys. Lett. B716, 1 (2012), arXiv:1207.7214 [hep-ex].

[12] S. Chatrchyan et al. (CMS), Phys. Lett. B716, 30 (2012), arXiv:1207.7235 [hep-ex].

[13] D. Clowe, M. Bradac, A. H. Gonzalez, M. Markevitch, S. W. Randall, C. Jones, and D. Zaritsky, Astrophys. J. 648, L109 (2006), arXiv:astro-ph/0608407 [astro-ph].

[14] A. Djouadi et al. (MSSM Working Group), in GDR (Groupement de Recherche) - Supersymétrie Montpellier, France, April 15-17, 1998 (1998) arXiv:hep-ph/9901246 [hep-ph]. 
[15] C. F. Berger, J. S. Gainer, J. L. Hewett, and T. G. Rizzo, J. High Energy Phys. 2009, 023 (2009), arXiv:0812.0980 [hep-ph].

[16] B. S. Acharya et al. (CTA), Astropart. Phys. 43, 3 (2013).

[17] Y. Sofue, Publ. Astron. Soc. Jap. 64, 75 (2012), arXiv:1110.4431 [astro-ph].

[18] J. Bovy and S. Tremaine, Astrophys. J. 756, 89 (2012), arXiv:1205.4033 [astro-ph].

[19] R. Catena and P. Ullio, J. Cosmol. Astropart. Phys. 2010, 004 (2010), arXiv:0907.0018 [astro-ph].

[20] K. Freese, J. A. Frieman, and A. Gould, Phys. Rev. D37, 3388 (1988).

[21] C. Savage, K. Freese, and P. Gondolo, Phys. Rev. D74, 043531 (2006), arXiv:astro-ph/0607121 [astro$\mathrm{ph}]$.

[22] R. Agnese et al. (SuperCDMS), "WIMP-Search Results from the Second CDMSlite Run,” (2015), submitted to Phys. Rev. Lett., arXiv:1509.02448 [astro-ph].

[23] D. S. Akerib et al. (LUX), Phys. Rev. Lett. 116, 161301 (2016), arXiv:1512.03506 [astro-ph].

[24] P. Cushman et al., in Planning the Future of U.S. Particle Physics, The Snowmass 2013 Proceedings, edited by N. A. Graf, M. E. Peskin, and J. L. Rosner (2013); Working Group Report:WIMP Dark Matter Direct Detection, arXiv:1310.8327 [hep-ex].

[25] K. Griest and M. Kamionkowski, Phys. Rev. Lett. 64, 615 (1990).

[26] D. S. Akerib et al. (LUX), Phys. Rev. Lett. 118, 021303 (2017), arXiv:1608.07648 [astro-ph.CO].

[27] P. Belli, R. Bernabei, S. D’Angelo, A. Incicchitti, and D. Prosperi, Nuovo Cim. A103, 767 (1990).

[28] G. J. Davies, W. G. Jones, J. D. Davies, J. D. Lewin, and P. F. Smith, Phys. Lett. B320, 395 (1994).

[29] P. Benetti et al., Nucl. Instrum. Meth A327, 203 (1993).

[30] D. S. Akerib et al. (LUX), Phys. Rev. Lett. 112, 091303 (2014), arXiv:1310.8214 [astro-ph].

[31] R. Agnese et al. (SuperCDMS), Phys. Rev. D92, 072003 (2015), arXiv:1504.05871 [hep-ex].

[32] R. Agnese et al. (SuperCDMS), Phys. Rev. Lett. 112, 241302 (2014), arXiv:1402.7137 [hep-ex].

[33] P. Agnes et al. (DarkSide), Phys. Rev. D93, 081101 (2016), arXiv:1510.00702 [astro-ph].

[34] G. J. Alner et al. (ZEPLIN-I), Astropart. Phys. 23, 444 (2005).

[35] G. J. Alner et al. (ZEPLIN-II), Astropart. Phys. 28, 287 (2007), arXiv:astro-ph/0701858 [astro-ph].

[36] D. Yu. Akimov et al. (ZEPLIN-III), Astropart. Phys. 27, 46 (2007), arXiv:astro-ph/0605500 [astro-ph].

[37] E. Aprile et al. (XENON10), Astropart. Phys. 34, 679 (2011), arXiv:1001.2834 [astro-ph].

[38] V. Chepel and H. Araújo, J. Instrum. 8, R04001 (2013), arXiv:1207.2292 [physics.ins-det].

[39] E. Aprile and T. Doke, Rev. Mod. Phys. 82, 2053 (2010), arXiv:0910.4956 [physics.ins-det].

[40] V. N. Solovov et al. (ZEPLIN-III), IEEE Trans. Nucl. Sci. 59, 3286 (2012), arXiv:1112.1481 [physics.ins-det]. 
[41] V. N. Lebedenko et al. (ZEPLIN-III), Phys. Rev. D80, 052010 (2009), arXiv:0812.1150 [astro-ph].

[42] B. Edwards et al. (ZEPLIN-II), Astropart. Phys. 30, 54 (2008), arXiv:0708.0768 [physics.ins-det].

[43] P. F. Sorensen, A Position-Sensitive Liquid Xenon Time-Projection Chamber for Direct Detection of Dark Matter: The XENON10 Experiment, Ph.D. thesis, Brown U., Phys. Dept. (2008).

[44] E. Santos et al. (ZEPLIN-III), J. High Energy Phys. (Online) 2011, 115 (2011), arXiv:1110.3056 [physics.ins-det].

[45] D. S. Akerib et al. (LUX), "Low-energy (0.7-74 keV) nuclear recoil calibration of the LUX dark matter experiment using D-D neutron scattering kinematics," (2016), submitted to Phys. Rev. C, arXiv:1608.05381 [physics.ins-det].

[46] A. Benoit et al. (EDELWEISS), Phys. Lett. B513, 15 (2001), arXiv:astro-ph/0106094 [astro-ph].

[47] D. Abrams et al. (CDMS), Phys. Rev. D66, 122003 (2002), arXiv:astro-ph/0203500 [astro-ph].

[48] D. S. Akerib et al. (LUX), Nucl. Instrum. Meth. A703, 1 (2013), arXiv:1205.2272 [physics.ins-det].

[49] D. S. Akerib et al. (LUX), "Radio-assay of Titanium samples for the LUX Experiment," (2011), (unpublished), arXiv:1112.1376 [physics.ins-det].

[50] D. S. Akerib et al. (LZ), Submitted to: Astropart. Phys. (2017), arXiv:1702.02646 [physics.ins-det].

[51] A. I. Bolozdynya, P. P. Brusov, T. Shutt, C. E. Dahl, and J. Kwong, in Proceedings of the 11th Symposium on Radiation Measurements and Applications Ann Arbor, MI, USA 23-26 May 2006, Nucl. Instrum. Meth., Vol. A579 (2007) pp. 50-53, A chromatographic system for removal of radioactive ${ }^{85}$ Kr from xenon.

[52] D. S. Leonard, A. Dobi, C. Hall, L. Kaufman, T. Langford, S. Slutsky, and Y.-R. Yen, Nucl. Instrum. Meth. A621, 678 (2010), arXiv:1002.2742 [physics.ins-det].

[53] M. B. Chadwick et al., Nucl. Data Sheets 112, 2887 (2011), special issue on ENDF/B-VII.1 library.

[54] M. J. Berger et al., XCOM: Photon Cross Section Database (version 1.5) (2010), National Institute of Standards and Technology, 100 Bureau Drive, Stop 1070, Gaithersburg, MD 20899-1070.

[55] E. Aprile et al. (XENON100), Phys. Rev. Lett. 109, 181301 (2012), arXiv:1207.5988 [astro-ph].

[56] E. Aprile et al., Phys. Rev. D86, 112004 (2012), arXiv:1209.3658 [astro-ph].

[57] L. Baudis, H. Dujmovic, C. Geis, A. James, A. Kish, A. Manalaysay, T. M. Undagoitia, and M. Schumann, Phys. Rev. D87, 115015 (2013), arXiv:1303.6891 [astro-ph].

[58] D. S. Akerib et al. (LUX), Phys. Rev. D93, 072009 (2016), arXiv:1512.03133 [physics.ins-det].

[59] M. Szydagis, N. Barry, K. Kazkaz, J. Mock, D. Stolp, M. Sweany, M. Tripathi, S. Uvarov, N. Walsh, and M. Woods (NEST), J. Instrum. 6, P10002 (2011), arXiv:1106.1613 [physics.ins-det].

[60] M. Szydagis, A. Fyhrie, D. Thorngren, and M. Tripathi (NEST), Proceedings, LIght Detection In Noble Elements (LIDINE2013), J. Instrum. 8, C10003 (2013), arXiv:1307.6601 [physics.ins-det].

[61] B. A. Dolgoshein, V. A. Lebedenko, and B. U. Rodionov, JETP Lett. 11, 351 (1970). 
[62] A. Manzur, A. Curioni, L. Kastens, D. N. McKinsey, K. Ni, and T. Wongjirad, Phys. Rev. C81, 025808 (2010), arXiv:0909.1063 [physics.ins-det].

[63] G. Plante, E. Aprile, R. Budnik, B. Choi, K. L. Giboni, L. W. Goetzke, R. F. Lang, K. E. Lim, and A. J. Melgarejo Fernandez, Phys. Rev. C84, 045805 (2011), arXiv:1104.2587 [nucl-ex].

[64] M. Horn et al. (ZEPLIN-III), Phys. Lett. B705, 471 (2011), arXiv:1106.0694 [physics.ins-det].

[65] B. Lenardo, K. Kazkaz, A. Manalaysay, J. Mock, M. Szydagis, and M. Tripathi (NEST), IEEE Trans. Nucl. Sci. 62, 3387 (2015), arXiv:1412.4417 [astro-ph.IM].

[66] P. Sorensen et al., in Proceedings, 8th International Workshop on The Identification of Dark Matter (IDM 2010), Proc. of Science, Vol. IDM2010 (2011) p. 017, Lowering the low-energy threshold of xenon detectors, arXiv:1011.6439 [astro-ph].

[67] E. Aprile et al. (XENON100), Phys. Rev. D88, 012006 (2013), arXiv:1304.1427 [astro-ph].

[68] J. Angle et al. (XENON10), Phys. Rev. Lett. 107, 051301 (2011), [Erratum: Phys. Rev. Lett. 110, 249901 (2013)], arXiv:1104.3088 [astro-ph].

[69] C. Hagmann and A. Bernstein, in Proceedings, 2003 IEEE Nuclear Science Symposium, RoomTemperature Semiconductor Detector Workshop, and Symposium on Nuclear Power Systems (2003 NSS/RTSD/SNPS), IEEE Trans. Nucl. Sci., Vol. 51 (2004) pp. 2151-2155, Two-phase emission detector for measuring coherent neutrino-nucleus scattering, arXiv:nucl-ex/0411004 [nucl-ex].

[70] D. S. Akerib et al. (LUX), Astropart. Phys. 45, 34 (2013), arXiv:1210.4569 [astro-ph].

[71] C. E. Dahl, The physics of background discrimination in liquid xenon, and first results from Xenon10 in the hunt for WIMP dark matter, Ph.D. thesis, Princeton U. (2009).

[72] D. Yu. Akimov et al. (ZEPLIN-III), Phys. Lett. B709, 14 (2012), arXiv:1110.4769 [astro-ph].

[73] S. Agostinelli et al. (GEANT4), Nucl. Instrum. Meth. A506, 250 (2003).

[74] D. S. Akerib et al. (LUX), Nucl. Instrum. Meth. A675, 63 (2012), arXiv:1111.2074 [physics.data-an].

[75] M. Xiao et al. (PandaX), Sci. China Phys. Mech. Astron. 57, 2024 (2014), arXiv:1408.5114 [hep-ex].

[76] P. Sorensen, Phys. Rev. D86, 101301 (2012), arXiv:1208.5046 [astro-ph].

[77] X. Guo et al. (Daya Bay), "A Precision measurement of the neutrino mixing angle $\theta_{13}$ using reactor antineutrinos at Daya-Bay," (2007), proposal, arXiv:hep-ex/0701029 [hep-ex].

[78] F. P. An et al. (Daya Bay), Nucl. Instrum. Meth. A685, 78 (2012), arXiv:1202.6181 [physics.ins-det].

[79] G. Alimonti et al. (Borexino), Nucl. Instrum. Meth. A600, 568 (2009), arXiv:0806.2400 [physics.insdet].

[80] P. Majewski et al. (ZEPLIN-III), in Position sensitive detectors. Proceedings, 9th International Conference, PSD9, Aberystwyth, UK, September 12-16, 2011, J. Instrum. 7, C03044 (2012), arXiv:1112.0080 [physics.ins-det].

[81] A. Dobi et al., Nucl. Instrum. Meth. A675, 40 (2012), arXiv:1109.1046 [physics.ins-det].

[82] N. Ackerman et al. (EXO-200), Phys. Rev. Lett. 107, 212501 (2011), arXiv:1108.4193 [nucl-ex]. 
[83] A. Gando et al. (KamLAND-Zen), Phys. Rev. C85, 045504 (2012), arXiv:1201.4664 [hep-ex].

[84] D. S. Akerib et al. (LUX), Astropart. Phys. 62, 33 (2015), arXiv:1403.1299 [astro-ph].

[85] D. S. Leonard et al., Nucl. Instrum. Meth. A591, 490 (2008), arXiv:0709.4524 [physics.ins-det].

[86] V. E. Guiseppe, S. R. Elliott, A. Hime, K. Rielage, and S. Westerdale, in Topical Workshop on Low Radioactivity Techniques: LRT-2010, AIP Conf. Proc., Vol. 1338, edited by R. Ford (2011) pp. 95-100, A Radon Progeny Deposition Model, Conference Presentation, arXiv:1101.0126 [nucl-ex].

[87] A. W. Bradley, LUX Thermosyphon Cryogenics and Radon-Related Backgrounds for the First WIMP Result, Ph.D. thesis, Case Western Reserve U. (2014).

[88] D.-M. Mei, C. Zhang, K. Thomas, and F. Gray, Astropart. Phys. 34, 33 (2010), arXiv:0912.0211 [nucl-ex].

[89] V. A. Kudryavtsev, Comput. Phys. Commun. 180, 339 (2009), (MUSIC), arXiv:0810.4635 [physics.comp-ph].

[90] L. Reichhart et al. (ZEPLIN-III), Astropart. Phys. 47, 67 (2013), arXiv:1302.4275 [physics.ins-det].

[91] F. E. Gray, C. Ruybal, J. Totushek, D.-M. Mei, K. Thomas, and C. Zhang, Nucl. Instrum. Meth. A638, 63 (2011), arXiv:1007.1921 [nucl-ex].

[92] D.-M. Mei and A. Hime, Phys. Rev. D73, 053004 (2006), arXiv:astro-ph/0512125 [astro-ph].

[93] A. Lindote, H. M. Araujo, V. A. Kudryavtsev, and M. Robinson, Astropart. Phys. 31, 366 (2009), arXiv:0810.1682 [hep-ex].

[94] D.-M. Mei, Z.-B. Yin, and S. R. Elliott, Astropart. Phys. 31, 417 (2009), arXiv:0903.2273 [nucl-ex]. 


\section{Scientific Performance}

The LZ detector system described in the previous and subsequent chapters is highly sensitive to a variety of physics signals. The principal signal we seek is that of NRs distributed uniformly throughout the LXe TPC volume, in response to an impinging flux of nonrelativistic WIMPs that are gravitationally bound to the Milky Way galaxy. In the first sections of this chapter, we describe the sensitivity to various WIMP-particle cross sections.

The first step in selecting the sample of WIMP candidates is to define a search region in the two variables: S1 (the prompt scintillation light) and S2 (the delayed electroluminescence light, a measure of primary ionization). The use of both variables allows the distinction of NRs from the much more numerous ERs.

The LUX collaboration has recently completed extensive calibrations of the response of liquid xenon to NRs [1] and ERs [2], as shown in Figure 1.3.11. These high-statistics data permit us to employ the detailed shapes of the response probability distribution functions (PDFs) in a profile likelihood ratio (PLR) fit to estimate the LZ sensitivity to NRs from WIMPS.

We first describe our sensitivity and discovery potential for the spin-independent (SI) WIMP-nucleon interaction. We then discuss interpretations involving more general forms of the WIMP-nucleon interaction.

Should LZ see a WIMP signal, the distribution of that signal in NR energy will allow constraints on the WIMP-Xe scattering cross section, the WIMP-Xe reduced mass, and on the velocity distribution of galactic WIMPs [3].

A variety of other physics processes can be probed by selective detection of NRs and ERs as defined with S1 and S2. The central fiducial region of the LZ detector will be an extraordinarily quiet laboratory for processes that deposit energy. Among the physics processes that can be probed are:

1. Solar neutrino detection.

2. A neutrino magnetic moment.

3. Double beta decay.

4. Neutrinos from supernovae.

5. Sterile neutrinos.

6. Interaction of WIMPs with atomic electrons.

7. Solar and certain dark-matter axion-like particles (ALPs).

8. Exotic particles that interact in the LZ outer detector.

\subsection{WIMP Sensitivity and Discovery Potential}

The principal physics analyses of the LZ experiment will be searches for the recoils of Xe atoms caused by the interaction of WIMPs with the Xe nucleus. As discussed above, two types of signal are formed in the LXe response to the recoils: S1 and S2. In the principal LZ search, the energy of the recoil is reconstructed from a combination of $\mathrm{S} 1$ and $\mathrm{S} 2$, and the ratio $\mathrm{S} 2 / \mathrm{S} 1$ provides discrimination of NRs from the background of ERs. The value of the reconstructed energy depends on whether the event is an NR or an ER. 


\subsubsection{S1+S2 Analysis}

The S1+S2 analysis in LZ will follow the general framework of the recently published LUX search for NRs in response to WIMPs $[4,5]$. We define a search region in the plane of $\log _{10}(\mathrm{~S} 2)$ versus $\mathrm{S} 1$, shown in Figure 2.1.1. ${ }^{1}$ LUX determines of the sensitivity to WIMP-nucleon scattering with a multi-dimensional PLR fit in that plane, which is also how the LZ sensitivity is determined.

A comparison of the key performance assumptions for LZ as well as the comparable achievements in LUX are given in Table 2.1.1. The baseline detector performance assumed for LZ is in many aspects more conservative than that achieved by LUX. The most prominent exception in Table 2.1.1 is the liquid/gas emission probability, where we presume that the limitations of the LUX electric field will be removed in the LZ experiment.

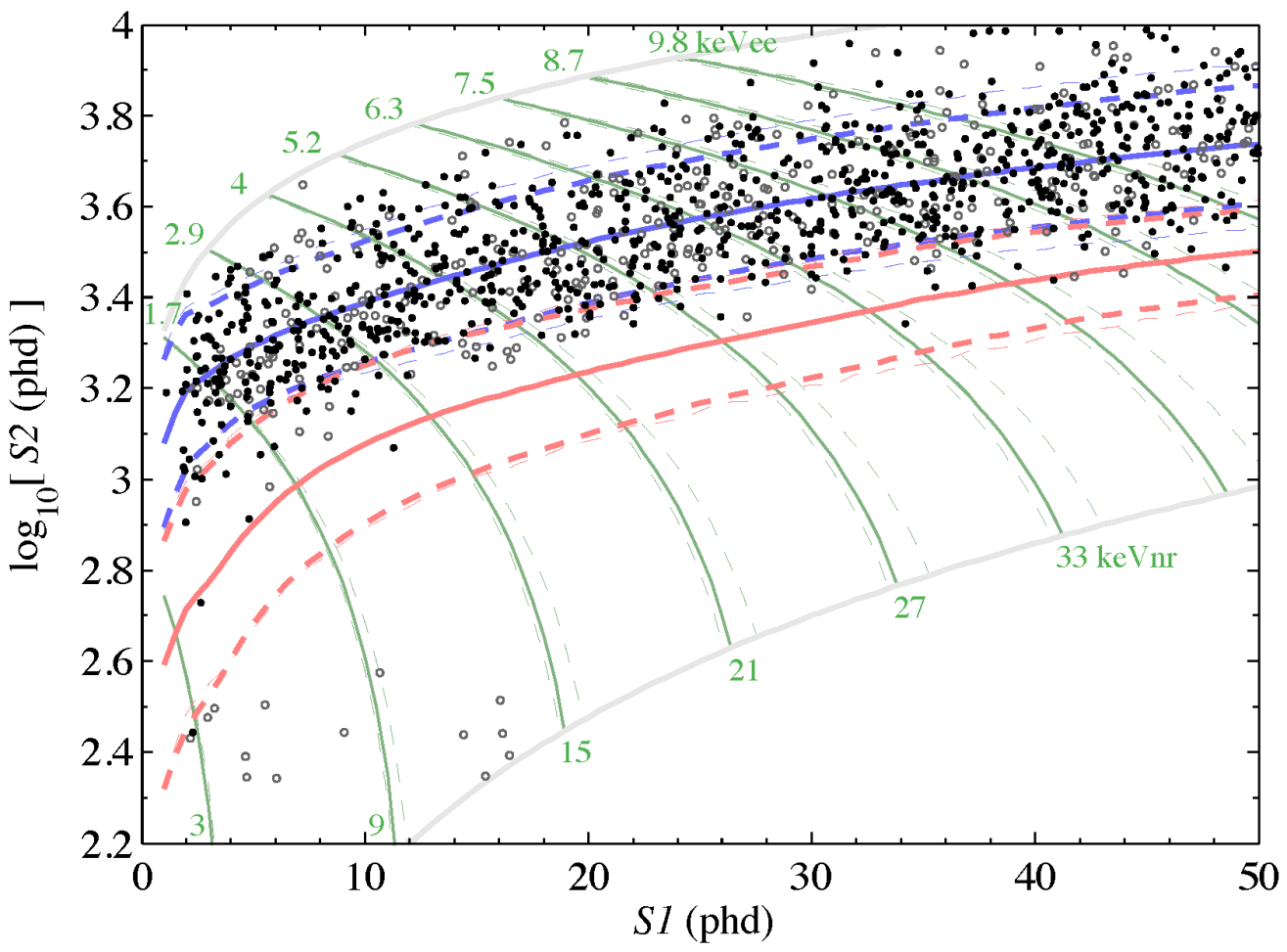

Figure 2.1.1: The LUX WIMP search data [5]. Shown is all data after selection criteria for the 332 live days of the LUX 2014-2016 run. The logarithm of S2 is plotted versus S1, after spatial corrections. Filled black circles are in the detector central region (radius $<18 \mathrm{~cm}$ ) and the edge of the detector (radius $18 \mathrm{~cm}$ to $20 \mathrm{~cm}$ ) in grey open circles. The centroid (solid) and search region boundaries (dotted) are red for the signal (NR) region or "band", and corresponding lines in blue describe the primary background (ER) band. The dotted lines are $\pm 1.28 \sigma$ around the centroid. Contours of equal recoil energy for $N R\left(\mathrm{keV}_{\mathrm{nr}}\right)$ and ER $\left(\mathrm{keV}_{\mathrm{ee}}\right)$ interpretations are shown in grey. The unit "phd" is photons detected, and results from correcting the photoelectrons detected for the probability of one UV photon inducing two photoelectrons. The data is consistent with a background of ERs and wall-induced events.

The benchmark process we will use to interpret NRs will be the interaction of WIMPs via an SI process, such as exchange of a Higgs particle [6], with the gluons in the nucleons in the Xe nucleus [7]. This process produces a WIMP-nucleus scattering rate that is independent of the identity, neutron or proton, of the nucleon in the nucleus. For the low-momentum transfers of typical WIMP interactions, the scattering

\footnotetext{
${ }^{1}$ Sometimes, the equivalent plane of $\log _{10}(\mathrm{~S} 2 / \mathrm{S} 1)$ versus $\mathrm{S} 1$ is utilized to display the same data.
} 
Table 2.1.1: Key LZ and LUX Assumptions Compared

\begin{tabular}{|c|c|c|c|}
\hline Quantity & Units & $\begin{array}{c}\text { LZ } \\
\text { Assumption }\end{array}$ & LUX [5] \\
\hline Recoil threshold, 50\% efficiency & keV $_{\mathrm{nr}}$ & 6 & 3.3 \\
\hline S1 range & $\begin{array}{c}\text { Photons } \\
\text { detected }\end{array}$ & $3-30$ & $2-50$ \\
\hline S2 range & $\begin{array}{l}\text { Photons } \\
\text { detected }\end{array}$ & $>350$ & $>200$ \\
\hline S1 light-collection efficiency & Absolute & $7.5 \%$ & $14 \%$ \\
\hline Photocathode efficiency & Absolute & $25 \%$ & $30 \%$ \\
\hline Liquid/gas emission probability & Absolute & $95 \%$ & $73 \%$ \\
\hline ER discrimination & Absolute & $99.5 \%$ & $99.8 \%$ \\
\hline
\end{tabular}

amplitude is proportional to A, the number of nucleons in the nucleus. The scattering cross section includes the density of states, which also favors larger A, while the threshold for energy detection favors smaller A. The nuclear form factor is employed to account for quantum-mechanical interference attributable to the non-zero nuclear size [8], and the standard halo model (SHM) of the distribution of WIMP velocities in the Milky Way is used [9].

The backgrounds expected for LZ are described in detail in Chapter 9 and summarized in Table 1.6.1 and in Table 12.3.1. In the LZ Conceptual Design Report (CDR) [10], we performed a simple cut-and-count estimate of the LZ sensitivity, under the assumption that $0.5 \%$ of the ER events would contaminate an NR signal region defined to retain $50 \%$ of NR events. The vastly improved understanding of the PDFs of ERs and NRs in S1 and S2 achieved by LUX have caused us to utilize the more advanced PLR statistical technique for estimates of WIMP sensitivity in this report[11]. In general this technique substantially reduces the fraction of ER events that contaminate the NR signal region, as discussed in Section 12.3.1. A consequence is the extremely stringent requirements in the LZ CDR on the permissible rate of radon decays in liquid xenon are substantially relaxed in this report, to a level of $\approx 20 \mathrm{mBq}$ for the 10 tonnes $\mathrm{LZ}$ total volume. This rate of radon decays is commensurate with the achievements of LUX and other existing liquid xenon experiments. However, at a radon decay rate of $\approx 20 \mathrm{mBq}$ ERs from the quiet beta decays of radon daughters outnumber ERs from solar pp neutrinos by a factor of about 3.5.

The resulting sensitivity plot is shown in Figure 2.1.2, along with LUX and ZEPLIN limits. For the baseline assumptions described in this report, $\mathrm{LZ}$ achieves a median sensitivity at a mass of approximately $40 \mathrm{GeV} / c^{2}$ of $2.3 \times 10^{-48} \mathrm{~cm}^{2}$.

The project LZ sensitivity for low WIMP masses is considerably improved in this report, compared to the CDR. The improvement is due to the utilization in this report of the improved calibrations reported by LUX[1, 2], which document higher S1 and S2 responses to low energy NR than were assumed in the CDR. Further improvements in sensitivity for low WIMP masses is possible through an "S2-only" analysis[12], or through the detection of bremmstrahlung from the nuclear recoil[13].

\subsection{Neutrino Physics}

The LZ detector will have a sufficiently large mass and low background that several types of neutrino interactions will be visible. These events will be uniform throughout the liquid xenon volume and cannot be shielded. We have studied the sensitivity of LZ to solar, atmospheric, astrophysical, reactor, and geophys- 


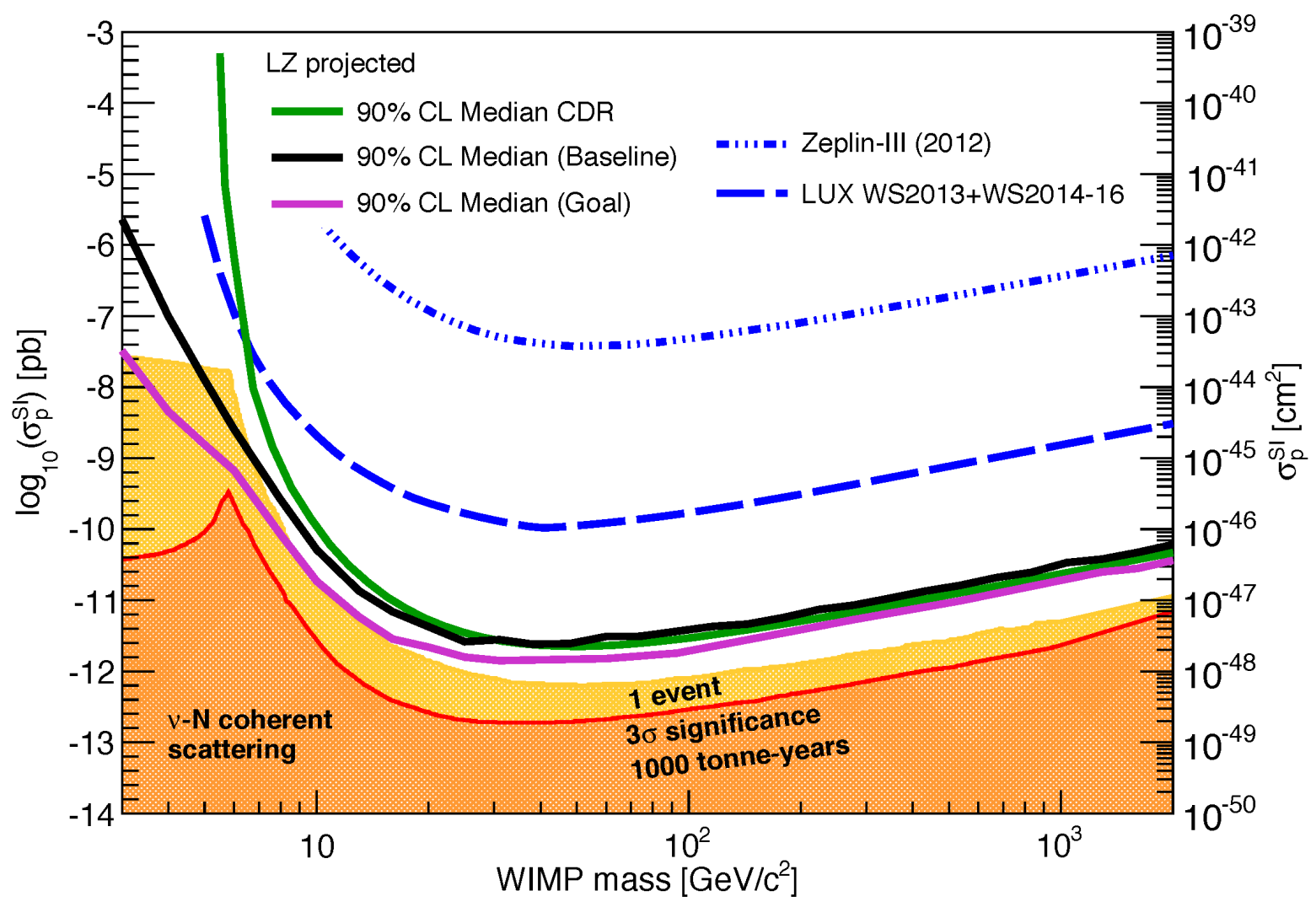

Figure 2.1.2: LZ sensitivity projections. The baseline $L Z$ assumptions described in this Technical Design Report give the solid black curve. LUX and ZEPLIN results are shown in broken blue lines. If LZ achieves the design goals listed in Table 12.3.2, the sensitivity would improve, resulting in the pink sensitivity curve. The gray line shows the projected sensitivity in the LZ Conceptual Design Report (CDR) [10] (see text for details of the changes from the CDR to this report). The shaded regions show regions where background NRs from cosmic neutrinos emerge [14].

ical neutrinos. In particular, solar neutrinos have been considered as both an interesting signal and as an irreducible background to a WIMP search.

LZ will observe the pp fusion chain of our sun in real time via elastic $v e \rightarrow v e$ scattering, in a lower energy regime than the only other real-time measurement to date, and will most likely detect neutrinos from ${ }^{8} \mathrm{~B}$ via coherent nuclear scattering. The coherent neutrino signal from a nearby supernova would be a unique, flavor-independent probe of the neutrino flux.

We have also estimated the potential of LZ to observe neutrinoless double-beta decay $(0 v \beta \beta)$ from ${ }^{136} \mathrm{Xe}$, and considered the impact on the reactor/source neutrino anomaly and on searches for a neutrino magnetic moment of a prolonged exposure of $\mathrm{LZ}$ to a nearby ${ }^{51} \mathrm{Cr}$ neutrino source.

\subsubsection{Solar and Atmospheric Neutrinos}

\subsubsection{Elastic Scattering of Solar Neutrinos}

A prominent background for WIMP dark matter searches in LZ will come from the elastic scattering of solar neutrinos from the pp fusion chain [15] with the atomic electrons in xenon. Our calculations of the rate of these scatters agree with those of [16] under the same assumptions. The calculations in this report, how- 
ever, use updated neutrino mixing parameters [17] and solar fluxes obtained from a luminosity-constrained analysis of Borexino data (cf. Table 2 of [18]). Our projections assume the standard LZ fiducial target mass of 5.6 tonnes and an exposure of 1,000 live days. For electron recoil events with energies between 1.5 and $20 \mathrm{keV}_{\text {ee }}$, we expect 838 observable pp events, 69 events from ${ }^{7} \mathrm{Be}$ and $<10$ events from ${ }^{13} \mathrm{~N}$. For electron recoil energies above $20 \mathrm{keV}_{\mathrm{ee}}, 2 v \beta \beta$ events from ${ }^{136} \mathrm{Xe}$ are expected to dominate the counting rate.

In the WIMP dark matter search window between 1.5 and $6.5 \mathrm{keV}_{\text {ee }}$, the corresponding calculated numbers are 233, 19 and 3 for a total of 255 electron recoil background events from solar neutrinos. Our calculation has neglected atomic binding effects on the scattering process. Inclusion these effects will result in a suppression of result in a suppression of $24 \%$ to $28 \%$ [19].

The LZ experiment would add an interesting data point to the existing world experimental sample on pp solar neutrinos, in the context of the MSW-LMA explanation to the observed solar neutrino flux. Existing data to support this model in the low-energy regime are from the SAGE experiment [20] and from Borexino [21]. The 50 tonnes gallium target in SAGE inferred 854 inverse beta decay events attributed to pp solar neutrinos over 18 years of operations. The neutrino energy threshold for that measurement was $233 \mathrm{keV}$. More recently, the Borexino Collaboration made the first real-time detection of the pp solar neutrinos via the elastic scattering process with atomic electrons. The Borexino neutrino energy threshold was $\approx 300 \mathrm{keV}$, while LZ will be uniquely sensitive, with a neutrino energy threshold of a few tens of keV.

Although the LZ experiment will open up new experimental territory in the study of pp solar neutrinos, the current consensus in the solar neutrino community is that the accuracy of an pp solar neutrino measurement must be better than $1 \%$ to improve understanding of solar neutrinos [18]. To achieve $1 \%$ accuracy, LZ would need to observe several tens of thousands of pp neutrino-induced ER events, and also control systematics at a sub- $1 \%$ level. The elimination of the ${ }^{136} \mathrm{Xe}$ isotope and a live time of 2,000 to 4,000 days would allow the accuracy of an LZ measurement of pp solar neutrinos to approach $1 \%$.

\subsubsection{Coherent Nuclear Scattering of Solar Neutrinos}

Neutrinos are expected to elastically scatter coherently across nucleons in the nucleus [22,23]. This process has yet to be observed. Dedicated experiments aim to measure the process in the laboratory. The energy transferred to the nucleus from coherent neutrino scattering is typically suppressed by $\approx m_{e} / m_{N}$ relative to the elastic electron scattering process, so signals from the coherent scattering of solar pp neutrinos will fall well below the LZ S1+S2 detection threshold.

Neutrinos from ${ }^{8} \mathrm{~B}$ decay, which occur at the end of the pp chain about $0.1 \%$ of the time, range in energy up to $\approx 15 \mathrm{MeV}$. This energy to is sufficient to transfer up to a few $\mathrm{keV}$ of energy to a xenon nucleus via coherent scatter, and so these events are expected to fall at the threshold of detectability in LZ. Figure 2.2.1 (left) shows the expected rate and signal distribution of these events. Our calculations agree with those of [24] if we make the same assumptions. For the calculations in this report, we assume a ${ }^{8} \mathrm{~B}$ neutrino flux of $5.25 \times 10^{-6} \mathrm{~cm}^{-2} \mathrm{~s}^{-1}$ as measured by SNO [25], with a total uncertainty of $<5 \%$. The largest uncertainty in the number of detected ${ }^{8} \mathrm{~B}$ neutrinos is due to the signal yield in the liquid xenon. We assume the latest results obtained by the LUX Collaboration [4]. Assuming LZ baseline detector parameters we expect 7 events from ${ }^{8} \mathrm{~B}$ coherent neutrino scatter in the full 5,600 tonne-day LZ exposure. Systematic uncertainty, due primarily to photon collection and liquid xenon signal yields) is comparable to statistical uncertainty in this case.

The measurement of the flux of ${ }^{8} \mathrm{~B}$ neutrinos through coherent neutrino scattering is sensitive to all neutrino flavors, forming an interesting result in its own right. From a dark matter perspective, these neutrinos are an irreducible background which looks very similar to a $6 \mathrm{GeV}$ WIMP. The distinct and complimentary calibrations planned in LZ, described in Chapter 7 will allow a thorough mapping of the ${ }^{8} \mathrm{~B}$ neutrino signal region. Combined with the distinctive, soft spectrum of ${ }^{8} \mathrm{~B}$ neutrino events, $\mathrm{LZ}$ will be able to in essence fit and subtract out the ${ }^{8} \mathrm{~B}$ neutrinos from the WIMP search. 

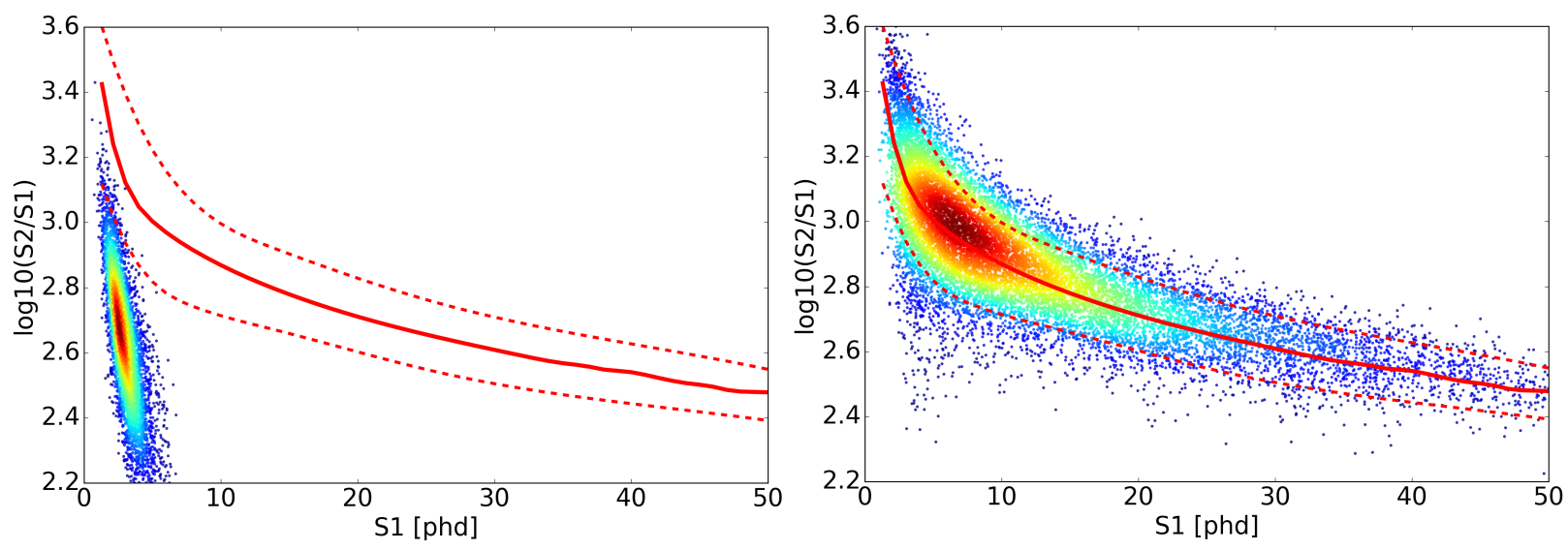

Figure 2.2.1: Calculated high-statistics probability distribution functions (PDFs) for ${ }^{8} \mathrm{~B}$ (left panel) and atmospheric (right panel) coherent neutrino-nucleus scattering events. The solid curve is the centroid of the nuclear recoil band, and the dotted lines define $a \pm 3 \sigma$ band. These lines are defined for a spectrum flat in nuclear recoil energy. The recoils from ${ }^{8} \mathrm{~B}$ on the left fall outside the band because the bulk of the PDF is under the threshold, and correlated fluctuations must occur for events to enter this plot. In 5,600 tonne-day of LZ exposure, we expect a mean of 7 and $0.5 \mathrm{NR}$ events from these neutrino sources to meet selection requirements.

In contrast to the atmospheric neutrino signal (Fig. 2.2.1, right panel), the ${ }^{8} \mathrm{~B}$ signal shown (Fig. 2.2.1, left panel) appears below the nuclear recoil band. This is true despite the fact that both distributions are due to nuclear recoils from coherent neutrino nucleus scattering. The primary reason is an artifact of the signal detection threshold. Because LZ will not be able to detect fewer than one S1-induced detected photon, the ${ }^{8} \mathrm{~B}$ signal consists of the tail of upward fluctuations in the number of scintillation photons produced. This comes at the expense of the number of ionized electrons in the S2 signal. Therefore, the ratio S2/S1 for these events is systematically biased below the nuclear recoil band, which is defined for a flat distribution in recoil energy.

\subsubsection{Atmospheric Neutrinos}

Atmospheric neutrinos result from muon and pion decay in the atmosphere. Historically, they were considered as a background for nucleon decay experiments, and then exhibited a surprising flavor-mixing phenomenon that has now been verified in accelerator-based experiments.

Consequently, the literature shows measurements or calculations of the atmospheric neutrino flux for energies $\gtrsim 1 \mathrm{GeV}$. The flux of atmospheric neutrinos is not a significant background for LZ in the elastic neutrino-electron scattering channel. However, coherent neutrino-nucleus scattering events present a serious background concern. This is because the hard energy spectrum of the neutrinos results in a recoil spectrum which is essentially indistinguishable from a typical WIMP. The PDF for the expected spectrum is shown in Figure 2.2.1 (right).

There is a detector-dependent sweet spot in neutrino energy for detection of coherent neutrino nucleus scattering. For $\mathrm{LZ}$ this is in the range of a few tens of MeV. Lower energies cannot register a signal, and higher energies begin to suffer a nuclear form factor suppression from the loss of coherence. Therefore LZ is singular in its need to understand the atmospheric neutrino flux for energies $\lesssim 100 \mathrm{MeV}$. A single calculation exists for the flux in this energy region [26], and it is tailored to two particular experimental sites: Kamioka, and Gran Sasso. The latitude of the site is important because the largest uncertainty is attributed to the 
geomagnetic cutoff, which limits the penetration of the primary cosmic rays into the atmosphere. Previous work [24] assumed the flux values for Kamioka, which are about $30 \%$ lower than the flux at Gran Sasso.

Our event rate calculations assume the Gran Sasso flux values tabulated in [26], with a single-sided uncertainty of $50 \%$. This uncertainty was estimated by comparing the Kamioka, Gran Sasso and SURF locations with a vertical cutoff rigidity map [27]. In 5,600 tonne-days, LZ expects to observe 0.5 signallike events from atmospheric neutrinos, distributed in S2 and S1 very much like the expected signal from a high-mass WIMP. This PDF shown in Fig. 2.2.1 (right panel).

\subsubsection{Neutrino Magnetic Moment}

It is not known if the neutrino has a small magnetic moment, and upper limits exist on its possible magnitude. The strongest direct particle physics upper limit is $5.4 \times 10^{-11} \mu_{\mathrm{B}}$ from Borexino [28], while analysis of supernovae provide a stronger upper limit of $5 \times 10^{-13} \mu_{\mathrm{B}}$ [29].

The effect of a neutrino magnetic moment is an increased scattering rate with electrons. A larger magnetic moment shifts the turn-on of the increase to higher energy. The $\approx 1 \mathrm{keV}$ energy threshold of LZ suggests an order of magnitude improvement in sensitivity, relative to Borexino. This is shown in Fig. 2.2.2, assuming $\mu_{v}=5 \times 10^{-12} \mu_{\mathrm{B}}$, in which case an increase in the scattering rate at threshold would be just barely observable.

\subsubsection{Other Neutrino Backgrounds}

One other possible source of signal-like events arises from coherent neutrino-nucleus scattering of the diffuse supernova neu-

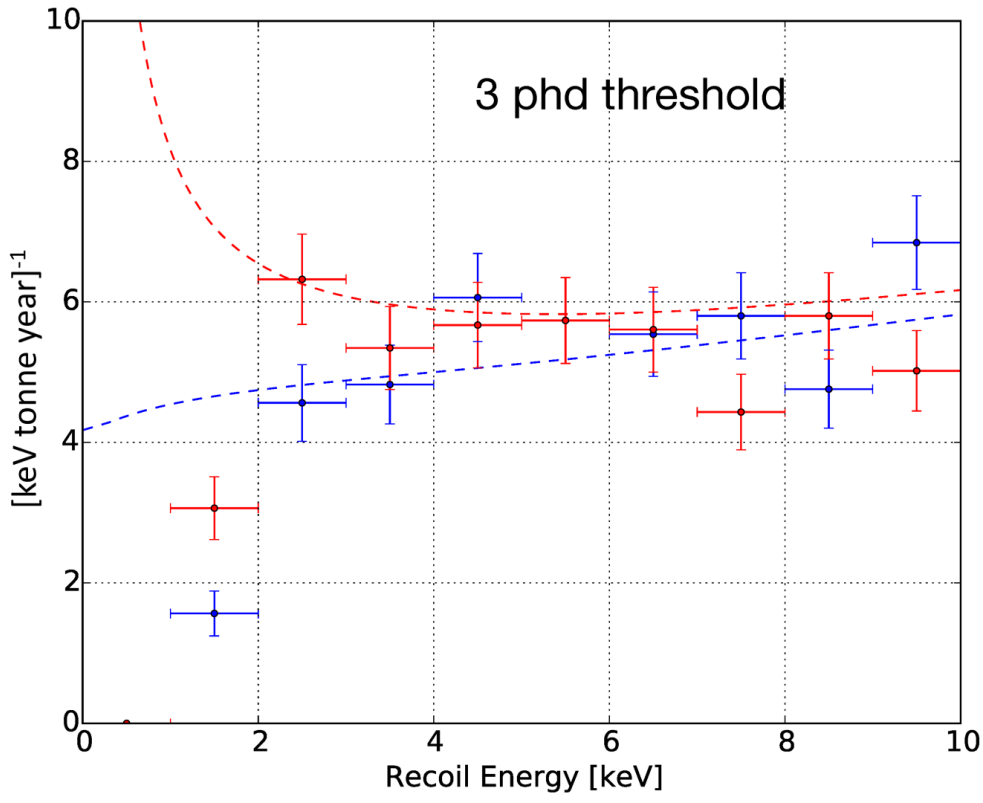

Figure 2.2.2: Predicted neutrino-dominated electron recoil background rate in LZ, for no magnetic moment (blue) and a magnetic moment $5 \times 10^{-12} \mu_{\mathrm{B}}$ (red). trino background (DSNB). We estimate this background in the NR search region to be 0.05 (DSNB) for the LZ fiducial mass of 5.6 tonnes and run duration of 1,000 days.

Geophysical neutrinos from ${ }^{238} \mathrm{U}$ and ${ }^{232}$ Th decays have been seen by the KamLAND [30-32] and Borexino [33] detectors. Those detectors have an energy threshold for neutrinos of about $1.8 \mathrm{MeV}$. They are unable to detect neutrinos from the decay of ${ }^{40} \mathrm{~K}$, which have an energy just below $1.5 \mathrm{MeV}$. Using the Reference Earth Model and neutrino flux calculations from the KamLAND work, we estimate for LZ 1.5 ER events/year from ${ }^{40} \mathrm{~K}$ decay, $0.3 \mathrm{ER}$ events/year from ${ }^{238} \mathrm{U}$ decay, and $0.2 \mathrm{ER}$ events/year from ${ }^{232} \mathrm{Th}$ decay. With the ability to distinguish ER and NR in LZ, these signals provide negligible backgrounds for the dark-matter search.

It is possible for neutrinos to capture on xenon nuclei. This process, $\mathrm{Xe}\left(v, e^{-}\right) \mathrm{Cs}$, is analogous to that employed in the Davis experiment at Homestake. The Feynman diagram for this process results from cross- 
ing the electron capture process, so we expect the electron capture Q value to set the threshold for neutrino capture proceed. Only ${ }^{131} \mathrm{Xe}$ has a sufficiently low $\mathrm{Q}$ value $(352 \mathrm{keV})$ to exhibit sensitivity to the pp neutrinos. A single calculation of the event rate exists [34], from which we estimate $<1$ events/year in LZ, in the $1.5 \mathrm{keV}$ to $6.5 \mathrm{keV}$ energy window.

The nearest power reactors are about $800 \mathrm{~km}$ away, in Fort Calhoun, NE $(0.5 \mathrm{GWe})$, and Cooper, NE $(0.8 \mathrm{GWe})$. The power/distance ${ }^{2}$ distribution shows a broad peak for reactors in Illinois and Wisconsin. The net flux is small enough, however, that we expect negligible detected events from power-reactor neutrinos in $\mathrm{LZ}$.

\subsubsection{Double Beta Decay}

\subsubsection{Neutrinoless Double Beta Decay}

If the electron neutrino is its own anti-particle, this would allow for the possibility of a process whereby double beta decay occurs but the two neutrinos annihilate. This process is referred to as neutrinoless double beta decay $(0 v \beta \beta)$. In this case, all decay energy goes into the two electrons. Thus, the signature is a monoenergetic, single-site event at the Q-value of the decay. Observation of this process would imply discovery of

1. fermions which are their own anti-particles (so called Majorana particles).

2. Lepton number violation.

3. Violation of conservation of the net difference between baryon and lepton number.

Currently the best lower limit on the half-life for $0 v \beta \beta$ of ${ }^{136} \mathrm{Xe}$ comes KamLAND-ZEN results, $1.06 \times 10^{26}$ y [35] at $90 \%$ confidence. Searches involving different xenon isotopes and related processes are discussed in Ref. [36].

Any search for $0 v \beta \beta$ needs low backgrounds, a large amount of the relevant isotope, and good energy resolution. Note that LZ requirement R-150004 implies a resolution of better than $2.0 \% \sigma / E$ at $2.5 \mathrm{MeV}$. The criteria for a $0 v \beta \beta$ search are very similar to those for a competitive dark matter experiment, however traditionally building an experiment which is competitive for both tends to be quite difficult. The large mass and exceptionally low backgrounds make this search possible in LZ. Typically searches for $0 v \beta \beta$ use enriched ${ }^{136} \mathrm{Xe}$ to enhance their signal. The 7 tonnes natural xenon implies almost $623 \mathrm{~kg}$ of ${ }^{136} \mathrm{Xe}$, which is more than previous $0 v \beta \beta$ searches.

The main backgrounds at the ${ }^{136} \mathrm{Xe} \mathrm{Q}$-value are the $2,447.7 \mathrm{keV} \gamma$-line from ${ }^{214} \mathrm{Bi}$ in the uranium chain and the $2,614.5 \mathrm{keV} \gamma$-line from ${ }^{210} \mathrm{Tl}$ from the thorium chain. Unlike in the many background that are distributed uniformly throughout the active xenon mass in the WIMP-search analysis, these backgrounds are completely from external detector components, and the size of LZ afford substantial screening of these background.

The same background simulations used to estimate the sensitivity of LZ to WIMPs are used to project the sensitivity to $0 v \beta \beta$. The analysis for $0 v \beta \beta$ is slightly different because the result is more dependent on the input assumptions. The energy resolution at the Q-value affects the experiment's ability to reject backgrounds from the penetrating $2,614.5 \mathrm{keV}^{210} \mathrm{Tl}$ line. Major background contributions include the TPC PMTs, the xenon vessel, and the resistors. There is some contribution from the radioactivity of the Davis cavern walls that is still under investigation, but at worst some additional shielding may be necessary above and below the xenon vessel, but not on the sides. The sensitivity estimate also depends on the minimal vertex separation needed to identify a multiple scatter and the energy resolution at the Q-value $(\sigma / E)$. In previous work by [16], it was assumed multiple scatters could be rejected down to $3 \mathrm{~mm}$ separations, and we make the same assumption. The choice of fiducial volume for the $0 v \beta \beta$ search is also different than that 
for the WIMP search, because of the penetrating nature of the ${ }^{210} \mathrm{Tl}$ line and the fact that its signal cannot be distinguished by the S1 and S2 signals. Smaller fiducial volumes have less total background due to the self-shielding effect of xenon.

For the purposes of these projections a fiducial volume of $1,000 \mathrm{~kg}$ was chosen as a proper tradeoff between backgrounds and exposure. A Feldman-Cousins cut-and-count analysis is used with a $2 \sigma$ region-ofinterest and $\mathrm{Q}_{\beta \beta}$. LZ has the potential to a sensitivity to a $90 \%$ median expected C.L. limit on the $0 v \beta \beta$ halflife of of ${ }^{136} \mathrm{Xe}$ of $1.2 \times 10^{26} \mathrm{y}$ with an energy resolution $(\sigma / \mathrm{E})$ of $1.0 \%$ or better. For comparison, at $90 \%$ confidence, the half-life limit from EXO 200 [37] is $1.1 \times 10^{25} \mathrm{y}$, that from GERDA [38] is $5.3 \times 10^{25} \mathrm{y}$, and KamLAND-Zen has achieved $1.06 \times 10^{26}$ y [35].

\subsubsection{Two Neutrino Double Beta Decay}

Two-neutrino double beta decay $(2 v \beta \beta)$ is a standard model decay which has been observed in several isotopes which occurs when single beta decay is energetically forbidden. For example, ${ }^{136} \mathrm{Xe}$ is lighter than ${ }^{136} \mathrm{Cs}$, so conservation of energy makes single beta decay of ${ }^{136} \mathrm{Xe}$ impossible. However, ${ }^{136} \mathrm{Xe}$ can undergo two simultaneous beta decays, emitting two electrons and two anti-electron neutrinos. This process has been observed in many different isotopes and the half-lives are always greater than $10 \times 10^{18} \mathrm{y}$.

For example, ${ }^{136} \mathrm{Xe}$ has a half-life due to $2 v \beta \beta$ of $2.2 \times 10^{21}$ y [37] and a Q-value of $2,456 \mathrm{keV} \mathrm{[39].} \mathrm{LZ}$ should observed $3 \times 10^{6}$ double beta decays over 1,000 live days.

The isotope ${ }^{134} \mathrm{Xe}$ is also believed to undergo $2 v \beta \beta$ with a Q-value of $826 \mathrm{keV}$ [40].

Although $2 v \beta \beta$ of ${ }^{136} \mathrm{Xe}$ has been observed by both EXO-200 and KamLAND-ZEN, both had analysis thresholds at or above the peak of the spectrum from $2 v \beta \beta$ of ${ }^{134} \mathrm{Xe}$ near $800 \mathrm{keV}$. LZ will be in a position to measure the full spectrum of ${ }^{134} \mathrm{Xe} 2 v \beta \beta$ down to $1 \mathrm{keV}_{\text {ee }}$ and see the turnover at the peak of the spectrum.

\subsubsection{Supernova Neutrinos}

Should a supernova occur in our galaxy during LZ operation, neutrinos emitted from the supernova would be detected via coherent neutrino-nucleus scattering, which is blind with respect to neutrino flavor. The energy spectrum of neutrinos emitted from a typical supernova peaks near $10 \mathrm{MeV}$, and has a tail that extends above $50 \mathrm{MeV}$, which causes NRs above the LZ threshold [41]. Coherent neutrino-nucleus scattering is mediated by the weak neutral current, and thus provides important information on the flux and spectrum of muon and tau neutrinos from supernovae, complementary to the signals that would be seen in other detectors. From a supernova in our own galaxy at distance $10 \mathrm{kpc}$ from Earth, LZ would see $\sim 50 \mathrm{NR}$ events of energy greater than $6 \mathrm{keV}_{\mathrm{nr}}$ in a rapid 10 -sec burst $[42,43]$.

The NR recoil spectrum increases as the recoil energy decreases; a threshold of $3 \mathrm{keV}_{\mathrm{nr}}$ would allow detection of $\sim 100$ supernova neutrino-induced NR events. The current world sample of 19 supernova neutrinoinduced events were detected from supernova 1987a, $50 \mathrm{kpc}$ from Earth, by detectors with total mass 1,200 times greater than LZ. A supernova $10 \mathrm{kpc}$ from Earth would cause about 7,000 neutrino-induced events in the 32,000 tonnes of water in the Super-Kamiokande detector [41].

The response of large, liquid xenon detectors to supernova signals has been recently reviewed[44].

\subsubsection{Sterile Neutrinos}

There are long-standing anomalies arising from the detailed study of antineutrinos from reactors, and from source-calibration of solar neutrino experiments [45]. A recent study has evaluated the capabilities of deployment of a $5 \mathrm{MCi}{ }^{51} \mathrm{Cr}$ electron neutrino source near to the LZ detector [46]. The excellent spatial resolution of the LZ liquid xenon TPC allows the spatial pattern of electron neutrino oscillation into a sterile neutrino to be detected. A neutrino source experiment with LZ would not be part of the principal LZ science 
goal, which is the WIMP search, and could constitute a distinct follow-on experiment after the WIMP search had achieved significant results.

The sensitivity achievable by five source deployments of a $5 \mathrm{MCi}{ }^{51} \mathrm{Cr}$ source near LZ is shown in Figure 2.2.3. Numerous proposals are underway to probe the origin of the reactor/source anomalies [47], but the potential LZ advantage is a diminished need to control the source normalization due to LZ's excellent spatial resolution. In addition, a source deployment near LZ will bring sensitivity to an electron neutrino magnetic moment that is close to the limits deduced from astrophysical considerations [46].

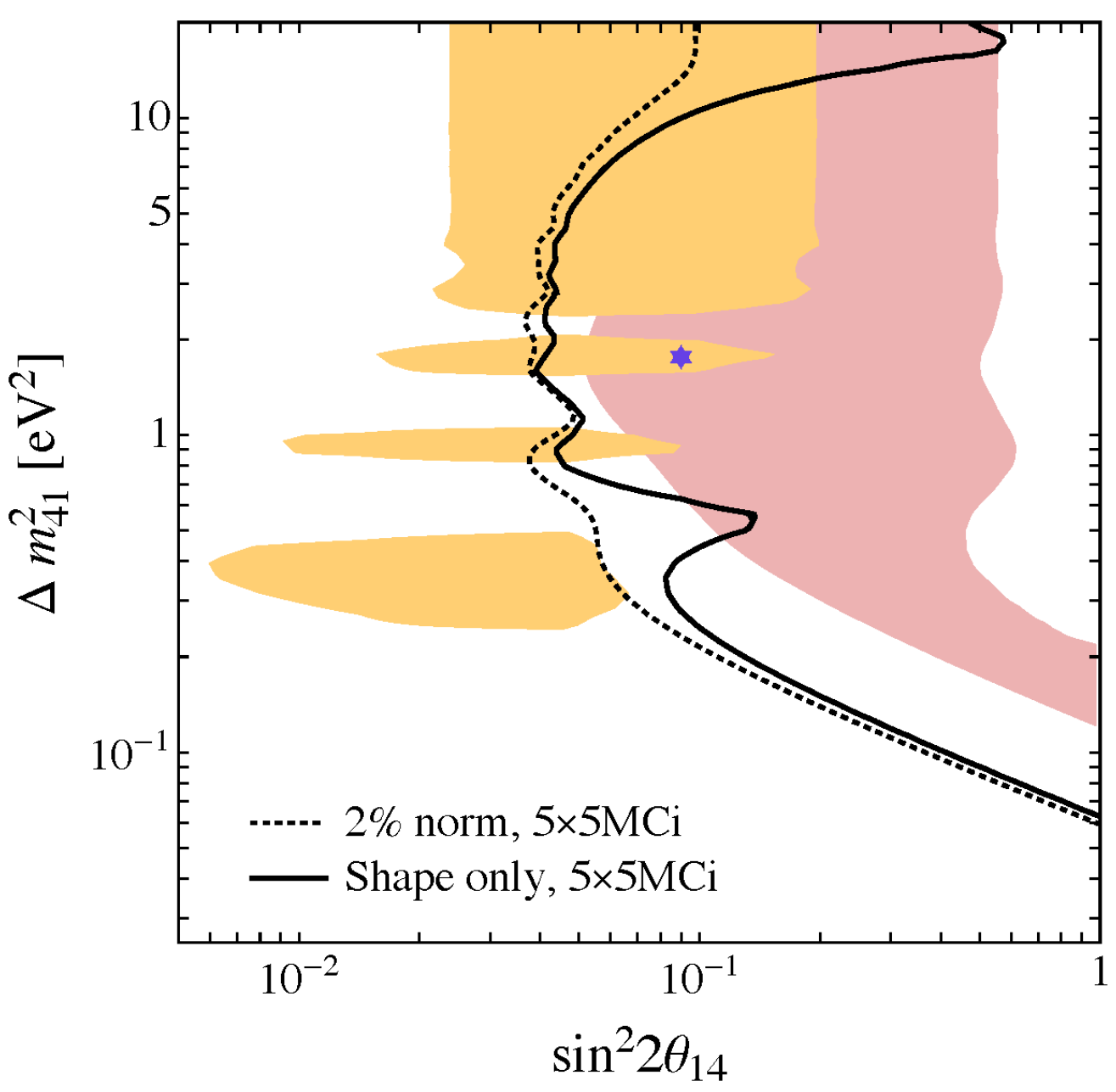

Figure 2.2.3: Sensitivity to sterile neutrino oscillations as a function of mass-difference and mixing angle. The parameter space to the right of each line would be excluded at $95 \% \mathrm{CL}$. The shaded areas show the $95 \% \mathrm{CL}$ allowed regions for source (pink) and reactor (yellow) anomalies. The blue star is the joint best fit. The black solid line shows the expected contours for five $100 \mathrm{~d}$ deployments of a $5 \mathrm{MCi}{ }^{51} \mathrm{Cr}$ source next to $L Z$, without use of the source normalization. The dotted line shows the contour if a $2 \%$ normalization of the source is available. From Ref. [46]. 


\subsection{New Physics Beyond Nuclear Recoils from WIMPs}

\subsubsection{Electrophilic WIMPs}

One type of WIMP-matter coupling that does not cause NRs, at least at tree-level, is the coupling of a WIMP to a charged lepton. A WIMP-charged lepton vector coupling induces a WIMP-nucleon interaction at one loop in perturbation theory, where the charged lepton loop interacts with the nucleon via photon exchanges [48]. This interaction is surprisingly sensitive. The WIMP-nucleon SI cross-section sensitivity of $2.3 \times 10^{-48} \mathrm{~cm}^{2}$ achievable by LZ at a WIMP mass of $40 \mathrm{GeV} / \mathrm{c}^{2}$ corresponds, when converted via a oneloop calculation, to a WIMP-electron cross section of $1 \times 10^{-50} \mathrm{~cm}^{2}$. Should the interaction be exclusively WIMP-muon, the LZ sensitivity at $40 \mathrm{GeV} / c^{2}$ corresponds to a vector-mediated WIMP-muon cross section of $5 \times 10^{-50} \mathrm{~cm}^{2}$; for a tau, the corresponding WIMP-tau cross section is $4 \times 10^{-49} \mathrm{~cm}^{2}$.

If the WIMP is a Majorana particle, all its vector couplings vanish, but an SD axial-vector coupling is still possible. The axial-vector coupling does not induce an interaction at higher order in perturbation theory with the nucleus; the only observable consequence in LZ of an axial-vector coupling of a WIMP to an electron is WIMP-electron scattering.

The electron motion is crucial for the appropriate treatment of WIMP-electron scattering. It is the very highest momentum tails of the electron wavefunction that determine the cross section for an impinging WIMP to ionize a Xe atom. The resulting events are ERs, and their energy spectrum rises very quickly as the energy deposition falls. Limits on axial-vector WIMP-electron scattering depend critically on the low energy threshold [48].

Interpretations of the DAMA [49] event excess as axial-vector WIMP-electron scattering imply a W IMP-electron cross section of $2 \times 10^{-32} \mathrm{~cm}^{2}$ at a WIMP mass $50 \mathrm{GeV} / \mathrm{c}^{2}$. The $\mathrm{LZ}$ experiment is likely to observe an ER background primarily from ${ }^{219} \mathrm{Rn}$ daughters, about 4 orders of magnitude lower than DAMA backgrounds, so LZ should achieve a limit, assuming background subtraction, of approximately $6 \times 10^{-38} \mathrm{~cm}^{2}$. This sensitivity is comparable to the indirect astrophysical limits on the SD WIMP-electron scattering cross sections deduced from Super-Kamiokande data [50].

\subsubsection{Axions and Axion-like Particles}

The axion was introduced to describe the absence of CP-violation in the strong interaction. These particles, known as QCD axions, have a specific relationship between their mass and their coupling to fermions [5153]. A particle with properties similar to the axion, but without the relationship between mass and fermion coupling, is known as an axion-like particle (ALP) [54].

The LZ experiment will be sensitive to axions and ALPs via the axioelectric effect, where an axion is absorbed and an atomic electron is ejected [55]. In contrast to the photoelectric effect, the mass of the axion or ALP is available for transfer to the atomic electron.

Two sources of axions or ALPs contribute to a possible signal in LZ [56]:

1. Nonrelativistic ALPs that might constitute the dark matter of our galaxy could cause signals in LZ, if their masses are sufficient to provide enough energy to ionize a Xe atom.

2. Axions or ALPs with a mass less than about $15 \mathrm{keV}$ emitted by bremsstrahlung, Compton scattering, or other atomic processes in the sun also can ionize the Xe atoms in LZ [57].

Events caused by axions or ALPs in LZ would be ERs with energy up to a few tens of $\mathrm{keV}_{\mathrm{ee}}$. The signal identification relies on the distinct shape of the energy spectrum of the axion or ALP signal.

The signal for a galactic dark-matter ALP would be a peak in ERs with energy at the mass of the particle. Our studies indicate that the LZ sensitivity to the coupling between electrons and galactic dark-matter ALPs 
ranges from a coupling constant $g_{A e}$ of $10^{-14}$ to one of $10^{-13}$ for masses between $1 \mathrm{keV} / \mathrm{c}^{2}$ and $20 \mathrm{keV} / \mathrm{c}^{2}$, as shown in Figure 2.3.1.

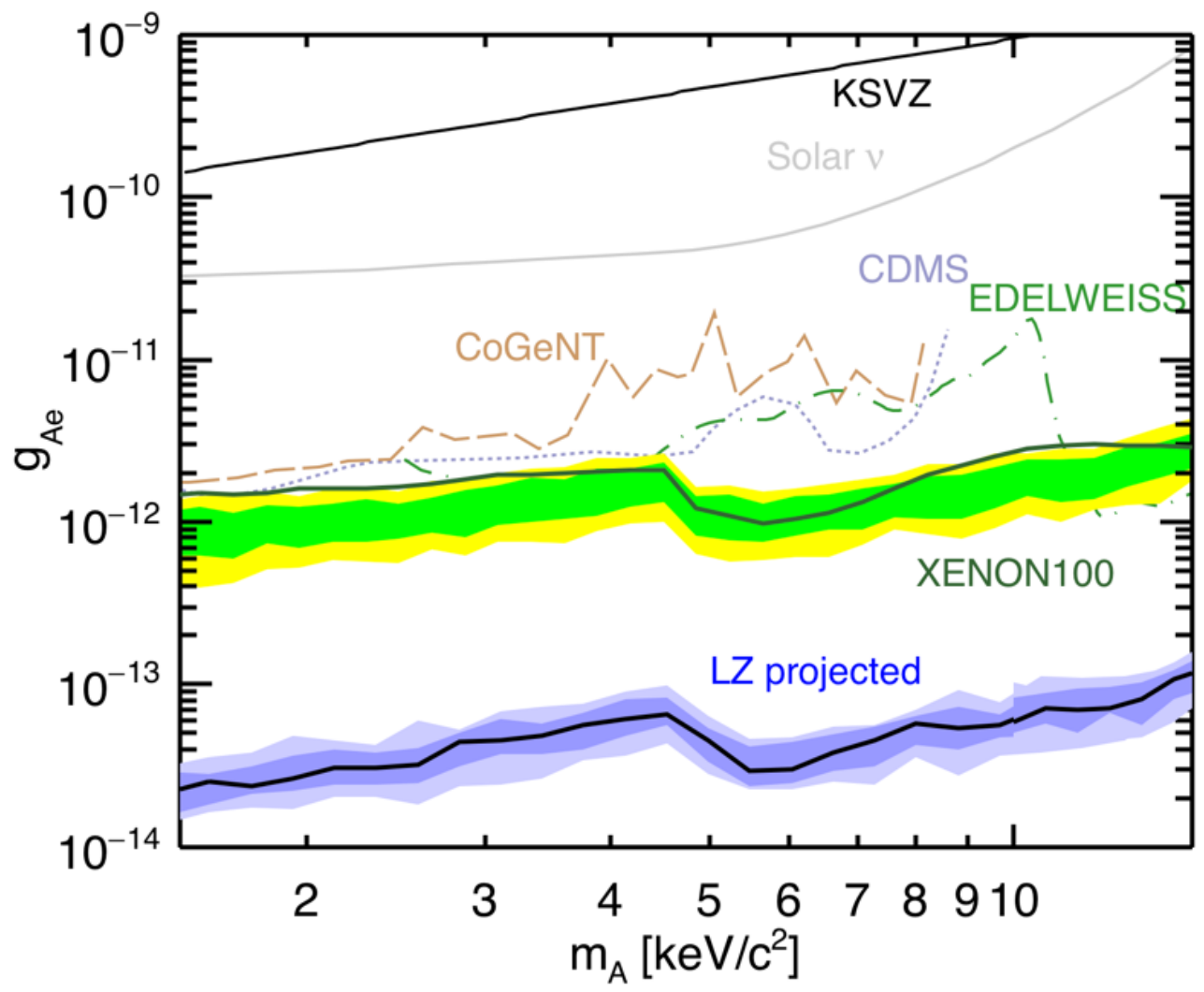

Figure 2.3.1: Dark-matter axion-like particle sensitivity. The LZ projected sensitivity for ALPs at $90 \% \mathrm{CL}$ is shown by the dark/light blue bands, which show the $68 \%(1 \sigma)$ and $95 \%(2 \sigma)$ bands for that sensitivity. The line that defines $\mathrm{KSVZ}$ axions [58, 59], an astrophysical upper limit from solar neutrinos [60], is shown. Upper limits by the experiments CDMS [61], EDELWEISS [62], CoGeNT [63], and XENON100 [64] are also shown.

The signal for solar ALPs is a broad thermal spectrum caused principally by bremsstrahlung and the Compton effect in the sun convolved with the axioelectric cross section. Our studies indicate that LZ is sensitive to a coupling constant $g_{A e}$ between solar ALPs and the electron of about $1.3 \times 10^{-12}$ for masses between $0 \mathrm{keV} / \mathrm{c}^{2}$ and approximately $1 \mathrm{keV} / \mathrm{c}^{2}$, as shown in Figure 2.3.2.

\subsection{Physics with the Outer Detector}

The primary goal of the LZ Outer Detector (OD) consisting of Gd-loaded liquid scintillator (approximately $20 t$ ) surrounded by water is to efficiently veto events in the LXe TPC which have additional energy depositions in the OD. These events are background to the WIMP search. However, the OD could be used for additional physics analyses on its own or together with the LXe. A better understanding of possible background induced by muons requires dedicated studies with the OD. The OD is also sensitive to neutrino and weak signals from exotic particles which are dark matter candidates. 


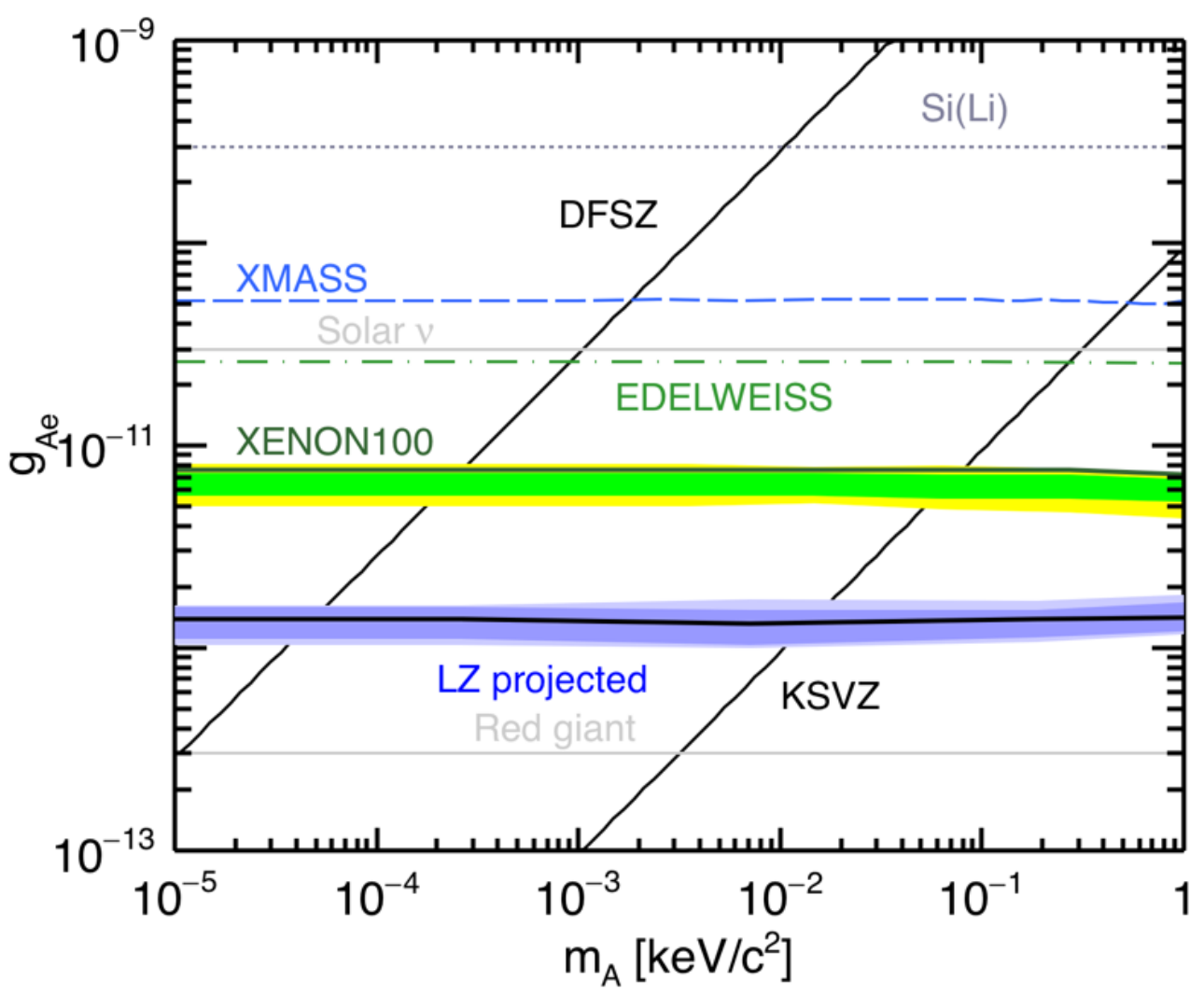

Figure 2.3.2: Solar axion-like particle sensitivity. Horizontal lines all extend down to $m_{A}=0$. The LZ projected sensitivity for ALPs at $90 \% \mathrm{CL}$ is shown by the dark/light blue bands, which show the $68 \%$ $(1 \sigma)$ and $95 \%(2 \sigma)$ bands for that sensitivity. The lines that define DFSZ axions $[65,66]$ and KSVZ axions [58, 59], neutrinos [60], and from red giants [67], are shown. Upper limits by the experiments XMASS [68], EDELWEISS [62], and XENON100 [64] are also shown.

\subsubsection{Muons and muon-induced neutrons}

A few potential physics topics to be addressed by the OD of LZ are linked to muons and muon-induced neutrons. The muon flux at the Davis Campus at SURF has been calculated as $6.2 \times 10^{-5} \mathrm{~m}^{-2} \mathrm{~s}^{-1}$, giving the muon event rate in the OD (water Cherenkov plus liquid organic scintillator) of about 300 per day. Most of these events will be single muons with multiple muons contributing a small fraction $(<1 \%)$.

There will also be a few hundred of stopping muons detected in scintillator and/or LXe in 1,000 days of running time. Stopping muons can be identified by a delayed signal from either muon decay or absorption on a nucleus LZ will be able to measure the rate of stopping muon signals and the life-time of muons, although no separation between positive and negative muons is possible, apart from detecting neutron capture from absorption of negative muons.

The high probability of neutron detection in the OD having a delayed coincidence with a muon signal allows the identification of a negative muon absorption. Nuclear recoils in LXe will be delayed by a few microseconds with respect to the muon ionization signal, providing a measurements of the negative muon life time in xenon. These measurements require operation of the OD and LXe in coincidence and an independent trigger from the OD. 
The detection of neutron capture events will also allow the measurements of neutron yields from this specific process. The accurate interpretation of the results will require the full Monte Carlo simulations of all processes involved including detector response.

Probably the most important measurements that can be carried out by the OD (also in combination with LXe target) are muon-induced neutrons. There have been a number of measurements of neutron production by muons including muon-induced cascades, come carried out by the dark matter search experiments. [6971]

Neutrons are usually identified via their capture on hydrogen or other elements (for instance, Gd) in active veto systems containing a liquid organic scintillator. With a very large OD (water and scintillator), LZ will detect many thousands of neutrons within its expected 1,000 days of running time. These neutron events will be efficiently rejected in dark matter analysis using various cuts but can be studied for the purpose of better understanding their production, transport and detection. This is particularly important for designing 3rd generation dark matter experiments, especially if a dark matter signal is found, as well as for other rare event searches.

In a conventional analysis, the muon trigger can be provided by either the LXe target or the OD system, and neutrons can be produced in a number of materials in the LZ setup (xenon, titanium, steel, scintillator, water), moderated by hydrogen in scintillator or water and captured predominantly on Gd (or hydrogen) in scintillator.

In addition, with a large mass of LXe, we expect to have hundreds of events with NRs without a muon in LXe allowing studies of NR rate, multiplicities and separation from primary muons (also in delayed coincidences with neutron capture signals).

\subsubsection{Neutrinos}

The possible sources of neutrino signals in the LZ OD include solar neutrinos, geoneutrinos, supernova bursts and neutrinos from LBNF. In general the LZ OD is not competitive with dedicated neutrino detectors due to its small mass. The supernova burst similar to the SN1987A would give approximately 10 events in the OD. The rate of geoneutrinos is approximately 1 per year. Our estimates of signals from the LBNF neutrinos give 9 events per year for the low energy beam and 25 events per year for the medium energy beam.

\subsubsection{Exotic particles}

Models of the exotic candidates to be the dark matter include the ones where an excitation can happen in the LXe and deexcitation in the OD. The standard approach would veto such events. A method the discover such interactions would be to measure the spectrum of energy depositions in the OD as it is expected that it should be monochromatic. Another exotic candidate for DM is a fractionally charged particle. The OD could be sensitive to particles with charges down to a fraction of 0.025 of the elementary charge, and coincidences with the LXe TPC provide an interesting cross check. Given the relatively large mass, quietness of the detector, and long exposure time the OD can contribute important capability to these searches.

\subsection{Bibliography}

[1] D. S. Akerib et al. (LUX), "Low-energy (0.7-74 keV) nuclear recoil calibration of the LUX dark matter experiment using D-D neutron scattering kinematics," (2016), submitted to Phys. Rev. C, arXiv:1608.05381 [physics.ins-det].

[2] D. S. Akerib et al. (LUX), Phys. Rev. D93, 072009 (2016), arXiv:1512.03133 [physics.ins-det]. 
[3] M. Pato, L. E. Strigari, R. Trotta, and G. Bertone, J. Cosmol. Astropart. Phys. 2013, 041 (2013), arXiv:1211.7063 [astro-ph].

[4] D. S. Akerib et al. (LUX), Phys. Rev. Lett. 116, 161301 (2016), arXiv:1512.03506 [astro-ph].

[5] D. S. Akerib et al. (LUX), Phys. Rev. Lett. 118, 021303 (2017), arXiv:1608.07648 [astro-ph.CO].

[6] R. Barbieri, M. Frigeni, and G. F. Giudice, Nucl. Phys. B313, 725 (1989).

[7] M. A. Shifman, A. I. Vainshtein, and V. I. Zakharov, Phys. Lett. B78, 443 (1978).

[8] J. D. Lewin and P. F. Smith, Astropart. Phys. 6, 87 (1996).

[9] C. McCabe, Phys. Rev. D82, 023530 (2010), arXiv:1005.0579 [hep-ph].

[10] D. S. Akerib et al. (LZ), (2015), Conceptual Design Report; LBNL-190005, FERMILAB-TM-2621AE-E-PPD, arXiv:1509.02910 [physics.ins-det].

[11] G. Cowan, K. Cranmer, E. Gross, and O. Vitells, Eur. Phys. J. C71, 1554 (2011), [Erratum: Eur. Phys. J.C73,2501(2013)], arXiv:1007.1727 [physics.data-an].

[12] P. Sorensen et al., in Proceedings, 8th International Workshop on The Identification of Dark Matter (IDM 2010), Proc. of Science, Vol. IDM2010 (2011) p. 017, Lowering the low-energy threshold of xenon detectors, arXiv:1011.6439 [astro-ph].

[13] C. McCabe, (2017), arXiv:1702.04730 [hep-ph].

[14] J. Billard, L. Strigari, and E. Figueroa-Feliciano, Phys. Rev. D89, 023524 (2014), arXiv:1307.5458 [hep-ph]; F. Ruppin, J. Billard, E. Figueroa-Feliciano, and L. Strigari, Phys. Rev. D90, 083510 (2014), arXiv:1408.3581 [hep-ph].

[15] J. N. Bahcall and C. Peña-Garay, New J. Phys. 6, 63 (2004), arXiv:hep-ph/0404061 [hep-ph].

[16] L. Baudis, A. Ferella, A. Kish, A. Manalaysay, T. Marrodan Undagoitia, and M. Schumann, J. Cosmol. Astropart. Phys. 2014, 044 (2014), arXiv:1309.7024 [physics.ins-det].

[17] J. Beringer et al. (Particle Data Group), Phys. Rev. D86, 010001 (2012).

[18] W. C. Haxton, R. G. Hamish Robertson, and A. M. Serenelli, Ann. Rev. Astron. Astrophys. 51, 21 (2013), arXiv:1208.5723 [astro-ph].

[19] J.-W. Chen, H.-C. Chi, C. P. Liu, and C.-P. Wu, (2016), arXiv:1610.04177 [hep-ex]; V. L. Morgunov and I. O. Pilugin, "Low-energy neutrino weak and electromagnetic scattering cross-sections on atomic electrons,” (1996), ITEP-28-96.

[20] J. N. Abdurashitov et al. (SAGE), Phys. Rev. C80, 015807 (2009), arXiv:0901.2200 [nucl-ex].

[21] G. Bellini et al. (Borexino), Nature 512, 383 (2014).

[22] D. Z. Freedman, Phys. Rev. D9, 1389 (1974).

[23] B. Cabrera, L. M. Krauss, and F. Wilczek, Phys. Rev. Lett. 55, 25 (1985).

[24] L. E. Strigari, New J. Phys. 11, 105011 (2009), arXiv:0903.3630 [astro-ph]. 
[25] B. Aharmim et al. (SNO), Phys. Rev. C88, 025501 (2013), arXiv:1109.0763 [nucl-ex].

[26] G. Battistoni, A. Ferrari, T. Montaruli, and P. R. Sala, Astropart. Phys. 23, 526 (2005).

[27] D. Smart and M. Shea, Adv. Space Res. 36, 2012 (2005), solar Wind-Magnetosphere-Ionosphere Dynamics and Radiation Models.

[28] C. Arpesella et al. (Borexino), Phys. Rev. Lett. 101, 091302 (2008), arXiv:0805.3843 [astro-ph].

[29] J. M. Lattimer and J. Cooperstein, Phys. Rev. Lett. 61, 23 (1988).

[30] E. Sanshiro, Neutrino Geophysics and Observation of Geo-Neutrinos at KamLAND, Ph.D. thesis, Tohoku University (2005).

[31] T. Araki et al., Nature 436, 499 (2005).

[32] S. Enomoto, E. Ohtani, K. Inoue, and A. Suzuki, Earth Planet. Sc. Lett. 258, 147 (2007).

[33] G. Bellini et al. (Borexino), Phys. Lett. B687, 299 (2010), arXiv:1003.0284 [hep-ex].

[34] A. S. Georgadze, H. V. Klapdor-Kleingrothaus, H. Pas, and Yu. G. Zdesenko, 4th International Solar Neutrino Conference Heidelberg, Germany, April 8-11, 1997, Astropart. Phys. 7, 173 (1997), [,283(1997)], arXiv:nucl-ex/9707006 [nucl-ex].

[35] A. Gando et al. (KamLAND-Zen), Phys. Rev. Lett. 117, 082503 (2016), [Addendum: Phys. Rev. Lett.117,no.10,109903(2016)], arXiv:1605.02889 [hep-ex].

[36] N. Barros, J. Thurn, and K. Zuber, J. Phys. G41, 115105 (2014), arXiv:1409.8308 [nucl-ex].

[37] J. B. Albert et al. (EXO-200), Nature 510, 229 (2014), arXiv:1402.6956 [nucl-ex].

[38] M. Agostini et al. (GERDA), (2017), arXiv:1703.00570 [nucl-ex].

[39] M. Redshaw, E. Wingfield, J. McDaniel, and E. G. Myers, Phys. Rev. Lett. 98, 053003 (2007).

[40] M. Wang, G. Audi, A. Wapstra, F. Kondev, M. MacCormick, X. Xu, and B. Pfeiffer, Chin. Phys. C36, 1603 (2012).

[41] K. Scholberg, Ann. Rev. Nucl. Part. Sci. 62, 81 (2012), arXiv:1205.6003 [astro-ph].

[42] C. J. Horowitz, K. J. Coakley, and D. N. McKinsey, Phys. Rev. D68, 023005 (2003), arXiv:astro$\mathrm{ph} / 0302071$ [astro-ph].

[43] S. Chakraborty, P. Bhattacharjee, and K. Kar, Phys. Rev. D89, 013011 (2014), arXiv:1309.4492 [astro$\mathrm{ph}]$.

[44] R. F. Lang, C. McCabe, S. Reichard, M. Selvi, and I. Tamborra, Phys. Rev. D94, 103009 (2016), arXiv:1606.09243 [astro-ph.HE].

[45] K. N. Abazajian et al., "Light Sterile Neutrinos: A White Paper," (2012), (unpublished), arXiv:1204.5379 [hep-ph].

[46] P. Coloma, P. Huber, and J. M. Link, J. High Energy Phys. (online) 2014, 042 (2014), arXiv:1406.4914 [hep-ph]. 
[47] B. Caccianiga, in XXVI INTERNATIONAL CONFERENCE ON NEUTRINO PHYSICS AND ASTROPHYSICS: Neutrino 2014, AIP Conf. Proc., Vol. 1666 (2015) p. 180002, Future short baseline neutrino searches with nuclear decays; D. Lhuillier, in XXVI INTERNATIONAL CONFERENCE ON NEUTRINO PHYSICS AND ASTROPHYSICS: Neutrino 2014, Vol. 1666 (2015) p. 180003, Future short-baseline sterile neutrino searches with reactors.

[48] J. Kopp, V. Niro, T. Schwetz, and J. Zupan, Phys. Rev. D80, 083502 (2009), arXiv:0907.3159 [hep$\mathrm{ph}]$.

[49] R. Bernabei et al. (DAMA), Nuclear Physics in Astrophysics. Proceedings, $17^{\text {th }}$ International Conference, NPDC 17, Debrecen, Hungary, September 30 to October 4, 2002, Nucl. Phys. A719, 257 (2003), Searching for the dark universe by the DAMA experiment.

[50] T. Tanaka et al. (Super-Kamiokande), Astrophys. J. 742, 78 (2011), arXiv:1108.3384 [astro-ph].

[51] R. D. Peccei and H. R. Quinn, Phys. Rev. Lett. 38, 1440 (1977).

[52] S. Weinberg, Phys. Rev. Lett. 40, 223 (1978).

[53] F. Wilczek, Phys. Rev. Lett. 40, 279 (1978).

[54] K. A. Olive et al. (Particle Data Group), Chin. Phys. C38, 090001 (2014), Review of Particle Physics: Gauge and Higgs Bosons, A. Ringwald, L.J Rosenberg, and G. Rybka, Axions and Other Similar Particles, pp. 626-635.; J. Beringer et al. (Particle Data Group), Phys. Rev. D 86, 010001 (2012).

[55] S. Dimopoulos, G. D. Starkman, and B. W. Lynn, Phys. Lett. B168, 145 (1986).

[56] K. Arisaka, P. Beltrame, C. Ghag, J. Kaidi, K. Lung, A. Lyashenko, R. D. Peccei, P. Smith, and K. Ye, Astropart. Phys. 44, 59 (2013), arXiv:1209.3810 [astro-ph].

[57] J. Redondo, J. Cosmol. Astropart. Phys. 2013, 008 (2013), arXiv:1310.0823 [hep-ph].

[58] J. E. Kim, Phys. Rev. Lett. 43, 103 (1979).

[59] M. A. Shifman, A. I. Vainshtein, and V. I. Zakharov, Nucl. Phys. B166, 493 (1980).

[60] P. Gondolo and G. Raffelt, Phys. Rev. D79, 107301 (2009), arXiv:0807.2926 [astro-ph].

[61] Z. Ahmed et al. (CDMS-II), Phys. Rev. Lett. 103, 141802 (2009), arXiv:0902.4693 [hep-ex].

[62] E. Armengaud et al. (EDELWEISS-II), J. Cosmol. Astropart. Phys. 2013, 067 (2013), arXiv:1307.1488 [astro-ph].

[63] C. E. Aalseth et al. (CoGeNT), Phys. Rev. Lett. 101, 251301 (2008), [Erratum: Phys. Rev. Lett. 102,109903(2009)], arXiv:0807.0879 [astro-ph].

[64] E. Aprile et al. (XENON100), Phys. Rev. D90, 062009 (2014), arXiv:1404.1455 [astro-ph].

[65] A. R. Zhitnitsky, Sov. J. Nucl. Phys. 31, 260 (1980), [Yad. Fiz. 31, 497 (1980)].

[66] M. Dine, W. Fischler, and M. Srednicki, Phys. Lett. B104, 199 (1981).

[67] G. G. Raffelt, in Axions: Theory, Cosmology, and Experimental Searches, Proceedings, 1st Joint ILIAS-CERN-CAST Axion Training, Geneva, Switzerland, November 30-December 2, 2005, Lect. Notes Phys., Vol. 741 (2008) pp. 51-71, Astrophysical Axion Bounds, arXiv:hep-ph/0611350 [hep$\mathrm{ph}]$. 
[68] K. Abe et al. (XMASS), Phys. Lett. B724, 46 (2013), arXiv:1212.6153 [astro-ph].

[69] H. M. Araujo et al., Astropart. Phys. 29, 471 (2008), arXiv:0805.3110 [hep-ex].

[70] L. Reichhart et al. (ZEPLIN-III), Astropart. Phys. 47, 67 (2013), arXiv:1302.4275 [physics.ins-det].

[71] B. Schmidt et al. (EDELWEISS), Astropart. Phys. 44, 28 (2013), arXiv:1302.7112 [astro-ph.CO]. 


\section{Xenon Detector System}

\subsection{Overview}

The direct observation of WIMP scattering within a radiation detector is a significant experimental challenge. Searches for these elusive particles require an extremely sensitive, low-background instrument able to separate NR events at the few-keV energy from a dominant background of ER interactions, some created by particles external to the WIMP target and others arising within it from radioactive contaminants. The LZ experiment addresses part of the background issue by operating deep underground, and by surrounding the instrument with large ultra-pure water and liquid scintillator shields. However, observing these small energy depositions in space and in time in the central LXe target requires a highly instrumented doublephase (liquid/vapor) Time Projection Chamber (TPC) assembled from high-performance, low-radioactivity components operating in a cryogenic environment. An additional constraint is that the detector must operate as conveyed underground, which demands stringent quality requirements of its components.

While LXe is inherently a very radio-quiet detector material, with high enough density and atomic number to very effectively self-shield against external backgrounds, the design of this new detector requires nonetheless that attention be paid to the radiopurity of a number of significant detector elements, such as the PMTs, their voltage-divider bases, cabling, support structures, and reflecting surfaces. This imposes serious constraints on material composition and their location, adding significant complication to the design of the instrument. The details of these developments will be described in the related sections below.

The Xenon Detector System includes the LXe TPC and ancillary systems required for its readout, monitoring and control. An additional anti-coincidence detector is formed by a layer of LXe enveloping the TPC, which we term the "Xe Skin" detector. The main components of these two instruments are described in this chapter: the TPC, including HV delivery, PMT systems, and internal liquid flow and monitoring instrumentation; and the Skin detector and its readout. We describe at the end of this Chapter our multi-scale System Test effort which is working to validate the performance of many detector components before their assembly into LZ.

An overview of the xenon detector system is shown in Figure 3.1.1. The LZ TPC diameter is determined by the maximum cryostat width that can be conveyed underground and the matching TPC length provides a compact form factor to minimize external radioactivity backgrounds. The TPC itself has a three-electrode configuration: a cathode grid at the bottom, a gate grid just below the liquid surface, and an anode grid just above the liquid surface. It features two arrays of PMTs, one immersed in the LXe viewing up (241 tubes), and the other in the gas phase viewing down (253 tubes). The WIMP target contains 7 tonnes of active LXe, located vertically between the cathode and gate grids and enclosed laterally by a cylindrical arrangement of PTFE reflector panels. These embed a resistive electrical ladder which grades provides an approximately vertical electric field inside the TPC. Interactions in this region generate prompt vacuum ultraviolet (VUV) scintillation light detected by the PMTs (S1 pulse). The applied electric field sweeps the ionization charge liberated at the interaction site and drifts it upward to the liquid surface past the gate electrode; these electrons are extracted into the vapor phase, where they generate electroluminescence-which is again detected by the same two PMT arrays (S2 pulse). This double-phase TPC technique, which generates two optical pulses per interaction, resolves the energy deposition sites with great spatial accuracy down to very low ener- 


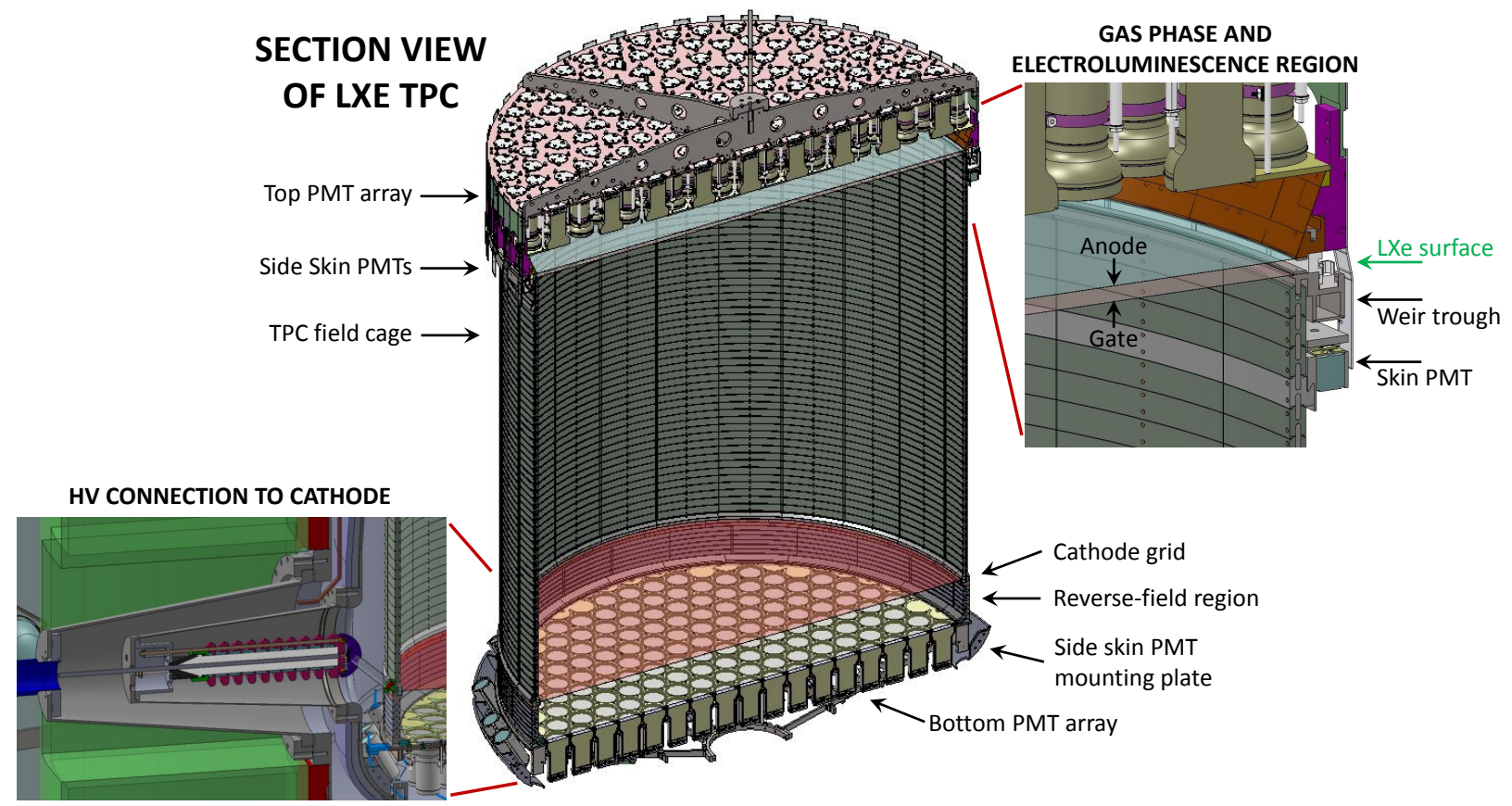

Figure 3.1.1: Schematic views of the Xenon Detector. The 7-tonne active region is contained within the TPC field cage between cathode and gate electrodes, viewed by "top" and "bottom" PMT arrays in the vapor and liquid phases, respectively. S2 signal generation occurs between the liquid surface and the anode (right inset). The HV connection to the cathode (left inset) uses a dedicated conduit leading from outside of the water tank. Below the TPC, a reverse-field region grades the cathode potential to low voltage at the bottom PMT array. The Side Skin PMT readout is shown outside of the TPC field cage.

gies, allowing identification of multiple scatter events and, as described in the previous Chapter, it provides discrimination between ER and NR interactions.

Table 3.1.1 lists the key design parameters of the Xenon Detector System needed to meet the LZ scientific requirements. An important enhancement beyond LUX is the treatment of the Skin layer of LXe located between the PTFE-clad field cage and the cryostat inner wall, as well as the region beneath the bottom PMT array. A high-quality dielectric standoff is needed between the high electric field regions of the field cage and the grounded vessel wall. A few-cm-thick layer of LXe performs this role, with the added advantage of allowing measurement of any energy deposited in this layer, from which we read out the scintillation light. Operated as a stand-alone veto, this layer is too thin to have high efficiency. However, the combination of this Skin Detector and the liquid scintillator Outer Detector is highly efficient at tagging internal and external backgrounds. The efficiency is further enhanced by the overall minimization of inert materials that can absorb gamma rays and neutrons: The TPC is constructed of the minimum needed mass of PTFE and field-shaping rings, and the vessels and PMT support structures are made of titanium. Both PTFE and Ti have low density and atomic number, and are thus quite transparent to gamma rays. Important design drivers for the Skin are its optical decoupling from the TPC, and compatibility between the Skin readout and the TPC HV design.

Another area of major difference between the device described here and the previous LUX and ZEPLIN detectors is the side-entry method to deliver the high-voltage connection to the cathode, and the relatively short "reverse-field" region (RFR) between the cathode and the lower PMT array. The RFR is especially 
Table 3.1.1: Main parameters of the Xenon Detector System.

\begin{tabular}{|c|c|}
\hline Parameter & Value \\
\hline \multicolumn{2}{|l|}{ Liquid xenon } \\
\hline TPC active mass & $7,000 \mathrm{~kg}$ \\
\hline Skin mass (side+dome) & $2,000 \mathrm{~kg}$ \\
\hline Total mass within cryostat & $9,600 \mathrm{~kg}$ \\
\hline \multicolumn{2}{|l|}{ Photomultipliers } \\
\hline TPC (Hamamatsu R11410-22) & 253 (top) +241 (bottom) \\
\hline Top Skin (Hamamatsu R8520-406) & 93 \\
\hline Bottom Skin (Hamamatsu R8778) & 20 (side) +18 (dome) \\
\hline \multicolumn{2}{|l|}{ Vertical dimensions (cold) } \\
\hline Electroluminescence region (gate-anode) & $13 \mathrm{~mm}$ (8 mm gas) \\
\hline Drift region (cathode-gate) & $1,456 \mathrm{~mm}$ \\
\hline Reverse-field region (sub-cathode) & $137.5 \mathrm{~mm}$ \\
\hline \multicolumn{2}{|l|}{ Transverse dimensions (cold) } \\
\hline TPC inner diameter & $1,456 \mathrm{~mm}$ \\
\hline Field cage thickness & $15 \mathrm{~mm}$ \\
\hline Skin thickness at surface (at cathode) & $40(80) \mathrm{mm}$ \\
\hline \multicolumn{2}{|l|}{ Electric fields } \\
\hline Electroluminescence field (GXe) & $10.2 \mathrm{kV} / \mathrm{cm}$ \\
\hline Drift field baseline (goal) & $0.31(0.65) \mathrm{kV} / \mathrm{cm}$ \\
\hline Reverse field baseline (goal) & $2.9(5.9) \mathrm{kV} / \mathrm{cm}$ \\
\hline Drift (reverse-field) stages & $57(7)$ \\
\hline \multicolumn{2}{|l|}{ Operating conditions } \\
\hline Operating pressure (range) & 1.8 (1.6 to 2.2$)$ bar(a) \\
\hline Equilibrium temperature & $175.8 \mathrm{~K}\left(-97.4^{\circ} \mathrm{C}\right)$ \\
\hline
\end{tabular}

challenging because of the very high electric field there, which results from balancing a high cathode voltage and minimizing the mass of S2-inactive LXe below the cathode. Our approach to these issues is described below in separate sections on the reverse-field region and cathode HV delivery system.

By design, the structures surrounding the central LXe volume are as lightweight as possible for transparency to gamma rays and neutrons; this also helps to keep their total radioactivity low. The most challenging requirements on the intrinsic radioactivity (i.e., radioactivity per mass or area) are in the largest or most massive components - the PTFE walls and field-shaping rings, and the PMTs with their bases and cables. This Chapter discusses the approach to obtaining the needed radioactivity levels for a number of these major items. However, the absolute level of radioactivity surrounding the detector must be held at acceptable levels, so all components must be carefully selected and screened. The screening program that ensures this is discussed in Chapter 9. 


\subsection{The Liquid Xenon Time Projection Chamber}

At the heart of the LXe TPC are the field cage embedded in the reflective PTFE panels and the various electrode grids. The grids and field cage create the set of electric fields that drift the electrons to create the S2 signal, and the highly reflective PTFE panels are essential for efficient measurement of the initial S1 scintillation signal. In this section we describe the electrostatic design of the TPC, and then highlight optical and thermal considerations that shape this design.

The electric field configuration inside the TPC volume defines three distinct regions: (1) the drift region, (2) the reverse-field region, and (3) the electroluminescence region. We describe these in turn, with help from Figure 3.1.1 which overviews the detector geometry, and Figure 3.2.1 which illustrates the field distributions.
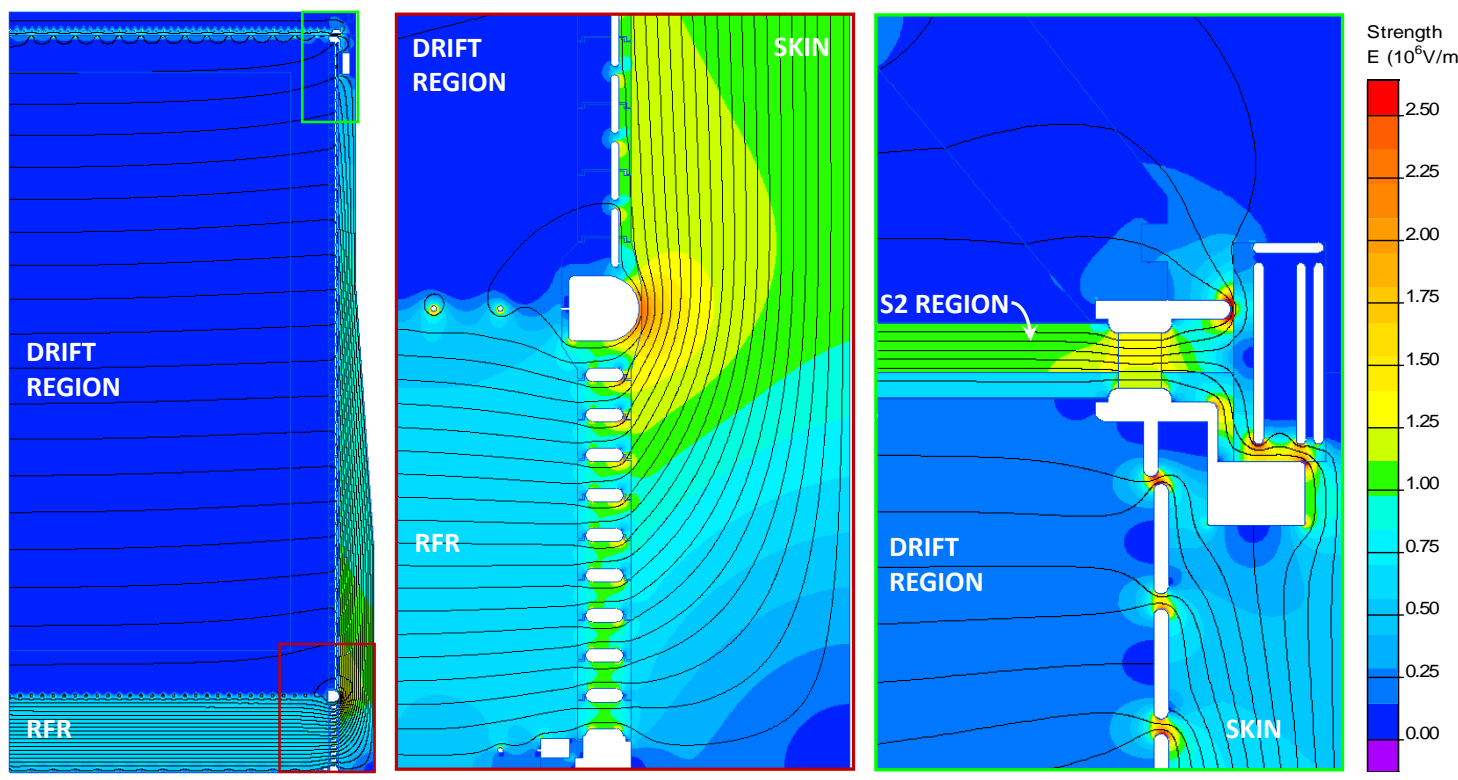

Figure 3.2.1: Electrostatic modeling of the various electric field regions of the TPC; applied voltages are design goals rather than nominal in this figure (cathode: $-100 \mathrm{kV}$, gate: $-7 \mathrm{kV}$, anode: $+7 \mathrm{kV}$ ); the field on cathodic surfaces in the liquid phase is kept below the $50 \mathrm{kV} / \mathrm{cm}$ allowable field. Left: General cross-section showing the drift field in the active region, the side Skin, the reverse-field region below the cathode, and the electroluminescence (S2) region at the top. Center: Detail of the drift and reverse-field regions with field-shaping structures embedded in the PTFE walls (expanding region in red in the left panel). Right: Detail of the electroluminescence region in the TPC top corner (in green in the left panel). In all panels the right-most surface is the grounded inner cryostat wall.

\subsubsection{Drift Region}

The region between the cathode and gate contains the active volume (WIMP target) and is therefore the most important region of the detector. This is where electrons are removed from the particle interaction site and drifted up to be detected in the vapor layer above the surface. Hence, the electric field strength and uniformity in this region have a major impact on the ability to identify and locate interactions in the detector. It is important that the electric field in this region is as uniform as possible, with field lines parallel to the PTFE reflector walls, so that S2 pulses can be generated for all interactions in this region. The electric field 
requirement for $\mathrm{LZ}$ is $0.3 \mathrm{kV} / \mathrm{cm}$, with a design goal of $0.6 \mathrm{kV} / \mathrm{cm}$. This range will provide the required level of ER/NR discrimination of $99.5 \%$ or better.

An additional requirement in our design is that all cathodic surfaces immersed in the LXe must comply with a maximum allowable field of $50 \mathrm{kV} / \mathrm{cm}$. As described in the LZ Conceptual Design Report (Section 3.4.2 in [1]], is can be problematic to sustain higher fields in particular on practical stainless steel surfaces.

To produce a uniform electric field between the cathode and the gate electrodes a set of 57 equally-spaced field rings is embedded within the PTFE and connected by pairs of $2 \mathrm{G} \Omega$ high-voltage resistors. The rings will be made from titanium from the same source as that used for the cryostat. The details of the design can be seen in Figure 3.2.1. The rings are "I"-shaped to help maintain the uniform field pattern needed within the TPC region by keeping the equipotential surfaces nearly normal to the inner surface of the PTFE ring. The field-shaping rings are embedded in vertically-segmented rings of PTFE that have been precision machined and then assembled in a stack to produce the completed field cage. In areas where the large difference in thermal contraction between PTFE and the metal field-shaping rings would cause problems, the PTFE rings are additionally segmented in the circumferential direction to allow the PTFE segments to slide along the field rings when the detector is cooled. The field cage structure will be mounted to the lower reverse-field region and lower PMT support, which in turn is supported from the bottom of the cryostat inner vessel.

All electrodes consist of woven meshes made from thin stainless steel wires-these are described in detail in Section 3.6.4. The cathode is at the highest potential and the design of its holding ring must be optimized to avoid high-field regions (see Figure 3.2.1 center).

The issue of field uniformity is important. In LUX it was observed that field lines near the lateral edges of the TPC, particularly near the top and bottom, are not fully parallel to the PTFE surfaces. We have come to understand this as being intrinsic to its design: The overall fields resulting from the grids and field cage structure were designed using 2-D electrostatics calculations that treated the grids as continuous conducting sheets. It is well known $[2,3]$ that the 3-D stretched-wire grids have an electrostatic "transparency" such that the bulk electric fields are somewhat $(\sim 10 \%)$ different to the values calculated assuming the grids are conducting planes, and this effect was taken into account in establishing the LZ operating fields. A subtler additional effect happens at the top and bottom of the TPC cylinder, where the transparency of the grids causes some bleed-through of the concentrated fields that terminate on the vessel and other grounded structures just outside the main part of the TPC. A more complete calculation using transparent grids reproduces the observed pattern in LUX. Such an effect was in fact previously observed in XENON100 and understood as described above [4].

In LUX, this effect caused electrons at the bottom edge of the detector to deflect $2 \mathrm{~cm}$ to $3 \mathrm{~cm}$ inward as they followed distorted field lines. This did not pose a fundamental problem for the science data, since the effect could be readily corrected for in analysis. Nonetheless, we will seek to better control the fields in LZ. Based on preliminary electrostatic calculations, we believe that this effect can be mitigated, for example by adjusting the values of the last few resistors at the top and bottom of the field cage (this is not assumed in this report). Another design change over LUX is the vertically-segmented design of the PTFE field cage walls. The essentially uninterrupted PTFE surfaces of the field cage are necessary for good light collection, but not ideal from the point of view of good high-voltage design practice, because insulating surfaces can at least in principle accumulate charge that distorts fields. LUX was constructed from single, continuous slabs of PTFE in the vertical direction, whereas the $2.5-\mathrm{cm}$-tall segments in LZ provide much shorter paths to the conducting field rings from any location on the PTFE walls.

\subsubsection{Reverse-Field Region}

Located between the cathode grid and the bottom PMT shield grid, the RFR is a significant challenge for constructing the LZ TPC because of the high fields involved $(3 \mathrm{kV} / \mathrm{cm}$ to $6 \mathrm{kV} / \mathrm{cm})$. The cathode voltage 

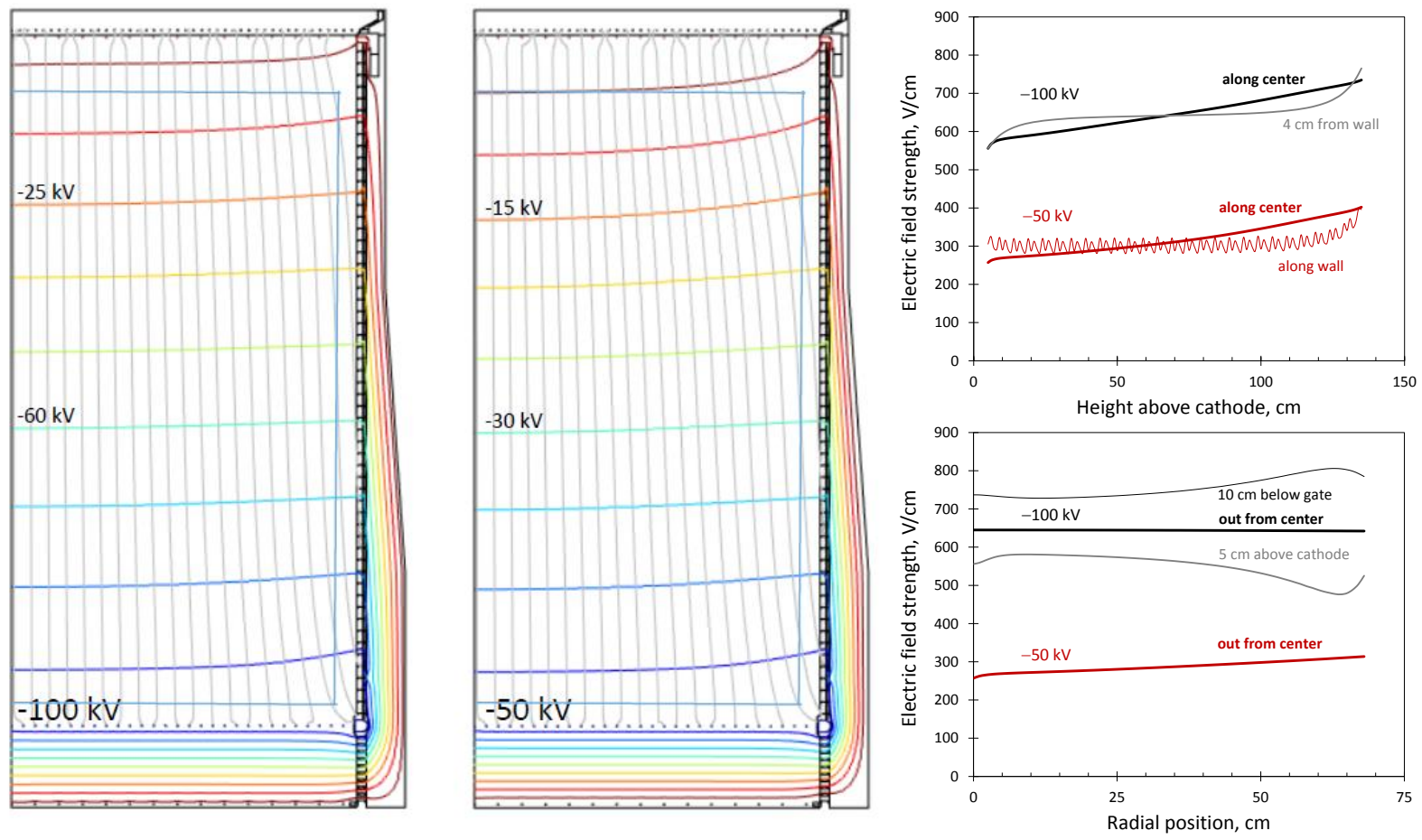

Figure 3.2.2: TPC field uniformity. Left and center: Equipotential lines and electron trajectories for $-100 \mathrm{kV}$ and $-50 \mathrm{kV}$ cathode $\mathrm{HV}$. In both cases a preliminary 5.6-tonne fiducial volume is shown which extends from $5 \mathrm{~cm}$ above the cathode to $10 \mathrm{~cm}$ below the gate, out to $4 \mathrm{~cm}$ from the TPC tall. Right, top: Field magnitude vertically along the center of the fiducial volume as well as along the TPC walls; Right, bottom: Field strength horizontally out from the center at the bottom, middle and top of the fiducial volume.

must be graded to near-ground while keeping all surfaces in this region below the $50 \mathrm{kV} / \mathrm{cm}$ allowable field. At the same time, this space must be kept as small as possible, both to reduce the amount of expensive LXe in this region, and to reduce the rate of events that scatter in both the reverse-field region and the active volume of the TPC. Such events are a class of background that can mimic WIMP signals; however, they have an acceptably low rate for the design presented here. In the LUX detector, due to the much lower cathode voltages and the shorter drift region in the TPC, this was handled with a $4 \mathrm{~cm}$ spacing and no field grading between the cathode and PMT shield grids, along with a near-zero field region of $2 \mathrm{~cm}$ between the shield and the PMT front surfaces. For the LZ configuration, we have chosen a voltage-grading structure similar to that in the drift region. This better defines the fields, and is a more robust approach to the more challenging LZ voltage requirements.

The RFR design, shown in Figure 3.2.3 (left), is composed of a stack of eight PTFE "rings", each $\approx 1.5 \mathrm{~cm}$ high and embedded with titanium field-shaping rings. These conducting rings are optimized for field uniformity above the PMTs - to control the electrical environment near their input optics-while keeping the region between the TPC and the grounded cryostat below $50 \mathrm{kV} / \mathrm{cm}$. The central panel in Figure 3.2.1 shows the fields in this region. The smooth oval shape of the RFR rings, compared with the "I"-shape in the drift region, creates lower surface fields on these elements at the expense of a less-uniform field in the central LXe region. This is not problematic since the uniformity requirement is not strong in the reverse-field region.

The voltages between each of the field rings are graded down from the cathode potential using a set of series resistors, similar to those used in the drift region, but with a higher resistance value between each 

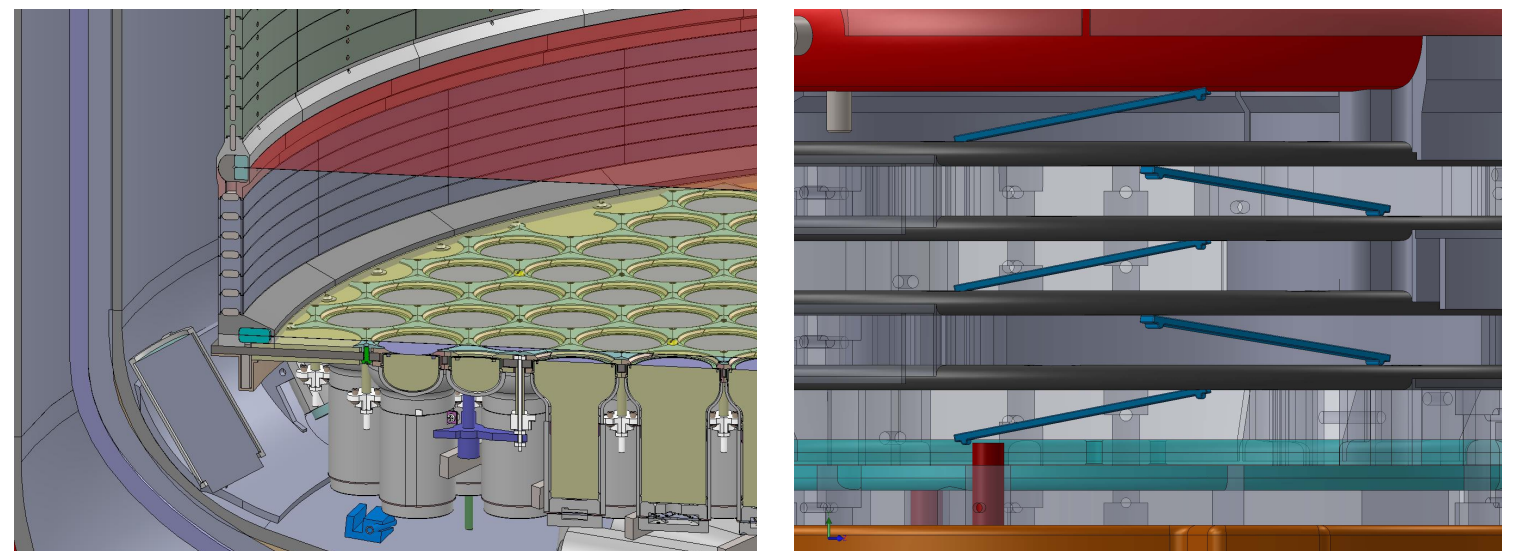

Figure 3.2.3: Reverse-field region design. Left: The RFR, with the cathode and bottom shield grids visible, and the oval field-shaping rings used here. Right: Placement of the RFR field-grading resistors, which are embedded inside the PTFE ring structure and attached to successive field-shaping rings.

ring to accomplish this stronger field grading. The resistors in the reverse-field region are more challenging for radioactivity than those in the drift region because they are larger. The main radioactive challenge in electronic components is alumina ceramics, which in all standard (non-"synthetic") forms is very high in both gamma activity and neutron yield. We use standard surface-mount resistors with the exception of getting the manufacturer to make them with a thin protective glass coating instead of potting them in epoxy. The resistors have the smallest available ceramic mass for the required voltage rating. Figure 3.2.3 (right) shows the current design and location of these grading resistors inside the PTFE spacers. The lowest PTFE "ring" will be attached to the top of the lower PMT shield grid and this grid will be spaced approximately $2 \mathrm{~cm}$ above the PMT surfaces, also using a PTFE spacer ring. The entire assembly will in turn be attached to the lower PMT support structure, which will then be fixed to the cryostat for final mechanical support. Electrostatic and mechanical studies of this region have been carried out, and are part of the System Test program described at the end of this Chapter.

\subsubsection{Electroluminescence Region}

In the region above the gate grid, drifting electrons are emitted across the liquid surface and produce electroluminescence (S2) photons while traveling through the gaseous phase on their way to the anode. The separation between these electrodes is merely $13 \mathrm{~mm}$, since only a narrow gas gap $(8 \mathrm{~mm})$ is required to provide enough S2 gain. Fields here are necessarily high $(\approx 10 \mathrm{kV} / \mathrm{cm}$ in the gas phase $)$ demanded by both the cross-phase electron emission process and the $\mathrm{S} 2$ photon production. The field in the liquid above the gate is approximately half that in the gas above the surface due to the relative permittivity of the liquid phase $\left(\varepsilon_{r}=1.96\right)$.

The optimization of the grids to create the S2 signal requires care, as is discussed in detail in Section 3.6; the mechanics of having gate and anode grids with very low deflection from the large electric fields is challenging; both the Skin PMTs (Section 3.7) and the weir structure (Section 3.8) must be accommodated in a tight space; and the overall structure must maintain a very low level of distortion in the rings supporting those grids, so that a parallel arrangement of electrodes and liquid surface can be obtained (tip-tilt adjustment of the detector to assure parallelism of grids and liquid surface is discussed in Chapter 5). A close-up view of this region is shown in Figure 3.2.4.

The field in the liquid above the gate must be significantly stronger than the field in the drift region: a $\approx 5-\mathrm{kV} / \mathrm{cm}$ "extraction" field is needed below the liquid surface in order to give most electrons sufficient 


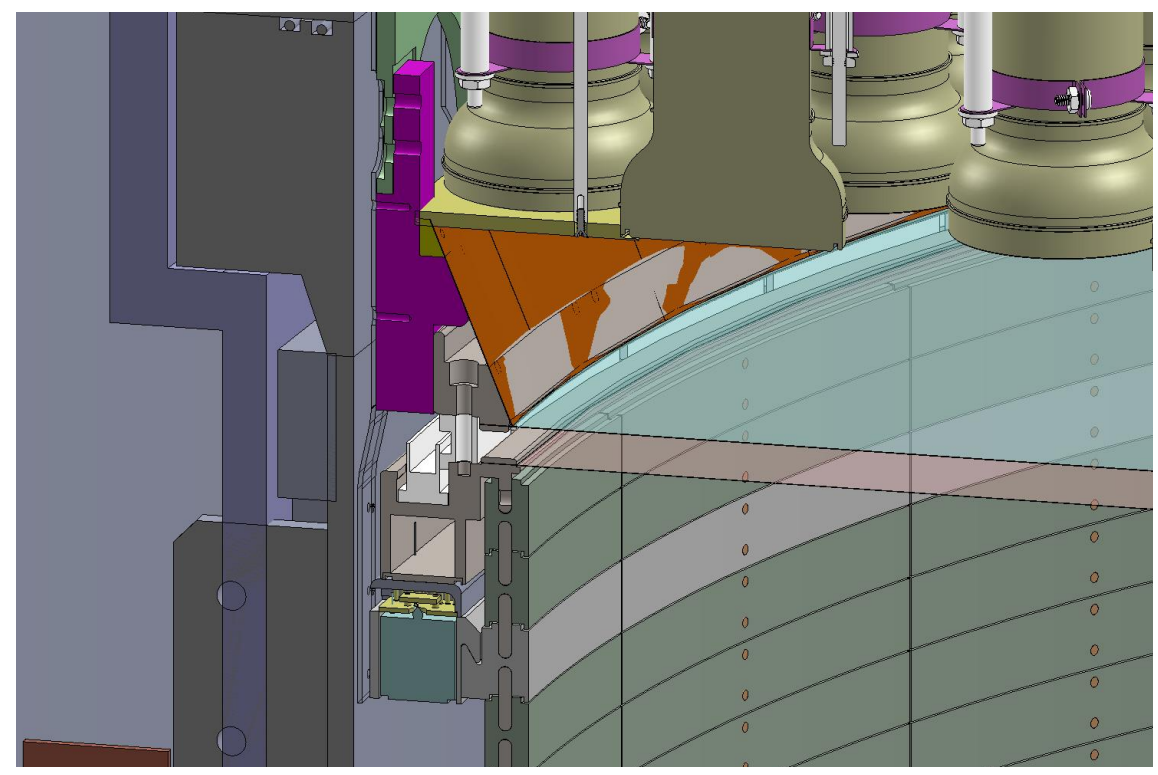

Figure 3.2.4: The electroluminescence region, with the gate and anode grids shown, along with the weir and top Skin PMTs.

kinetic energy to overcome the energy barrier at the liquid surface $(0.61 \mathrm{eV})$ and be extracted into the gas phase with high probability [5]. Once electrons enter the gas phase, where the field is approximately twice as strong, they are accelerated and produce electroluminescence photons in the 8-mm drift distance until they are collected on the anode grid. The photon yield is $\approx 800$ photons per emitted electron at 1.8-bar operating pressure, with $10 \mathrm{kV} / \mathrm{cm}$ in the gas [6]. For these operating conditions, the electron transit time to the anode is $\approx 1.2 \mu$ s which, along with diffusion while the electrons travel in the drift region, determines the width of the $\mathrm{S} 2$ pulse.

Because the S2 signal develops as the electrons drift from the liquid surface to the anode electrode, it is essential to minimize the variance of $\mathrm{S} 2$ photon production for different electron emission points, as this relates directly to the energy resolution achieved in the $\mathrm{S} 2$ channel. This imposes requirements on grid deflection as well as micro-uniformity of electric fields. The intricacies of this design are described in Section 3.6. Additional constraints come from the need to attach other elements to the side of this region of high field, such as the weirs that control the liquid level and the side Skin PMTs-this can be appreciated from Figure 3.2.1 (right). The "top corner" of the TPC is a crowded region and required significant design effort.

\subsubsection{VUV Reflectors}

In the same way that the electrostatic design of the TPC optimizes the detection of ionization electrons extracted from particle interactions, the optical design must accomplish the harder task of maximizing the detection of scintillation photons. Although the numbers of each quanta produced initially are comparable, it is easier to detect electrons efficiently than it is to detect photons. The energy threshold of the TPC is therefore determined primarily by its ability to detect prompt scintillation (S1).

PTFE is the VUV reflector of choice for this purpose, possessing good mechanical properties, low outgassing, and-crucially_achieving hemispherical reflectances in the liquid xenon as high as $\approx 97 \%$ at the xenon scintillation wavelength. The experience of the LUX experiment in optimizing light collection within the TPC volume was very successful, translating to a very low NR detection threshold of below $4 \mathrm{keV}$. We 
plan to use machined "segments" of high-purity PTFE approximately $1.5 \mathrm{~cm}$ thick and $2.5 \mathrm{~cm}$ tall to form the inner reflecting surface in the TPC region as well as the outer reflecting surface between the TPC and the cryostat wall, which itself will have a one-mm-thick segmented lining of PTFE.

There are many types of PTFE exhibiting different reflectivity both in the liquid and gas phases [7, 8]. It is essential that the best material is selected for this purpose since this is a leading parameter that determines the performance of both the TPC and the Skin detectors. The topic of S1 photon detection is discussed in some detail in Section 3.5; the PTFE reflectivity is such a critical parameter that we devote part of our System Test program to this topic, as described in Section 3.10.

The radioactivity of this material must be held extremely low both because of direct gamma-ray production but, more importantly, neutron production from $(\alpha, n)$ reactions on $\mathrm{F}$ from $\alpha$ decays in the $\mathrm{U}$ and Th chains. The raw precursor material for structural PTFE is a powder form produced by only a few suppliers, and is expected to be extremely radio-pure because it is synthesized from gas. A large number of smaller manufacturers produce structural shapes from these powders, and the final material can be very low in radioactivity if there is sufficient care in controlling contamination (e.g., from dust) in this second manufacturing step. In this topic we benefited from the experience of the EXO-200 collaboration, who achieved excellent radiopurity, rendering this critical material sub-dominant in terms of background in LZ, as detailed in Chapter 9.

\subsubsection{Thermal Considerations}

Given that the inner detector region is primarily made from PTFE, stainless steel and titanium parts, attention must be paid to the differential thermal contraction as the detector is cooled to LXe temperatures. The PTFE that makes up the majority of the surface area of the TPC is expected to shrink by $\approx 1.5 \%$ linearly $[9,10]$, or about $7 \mathrm{~cm}$ in circumference and $1 \tilde{\mathrm{cm}}$ in TPC radius when cooled from room temperature to $\approx 170 \mathrm{~K}$. Stainless steel, by contrast, contracts only $\approx 0.2 \%$ over the same temperature range, and titanium even less. Where this difference would result in destructive forces in the assembly, the metallic field cage rings will be constructed as solid parts, while the PTFE parts are segmented both horizontally (i.e., into rings) and vertically, so that each ring is itself composed of several segments. These segments contract and slide circumferentially along the solid metal field cage rings. In this way, the overall diameter of the TPC is determined by the metal field cage rings, and thus undergoes a relatively small thermal contraction. As the PTFE shrinks, the seams between the segments open, but the design has overlaps so there will continue to be a reflecting surface in the exposed gaps. In the vertical direction, the dimension of the field cage is determined by a combination of PTFE panels and metal rings. There is an overall height (top PMT array to bottom PMT array) contraction of $\approx 1 \mathrm{~cm}$. To minimize the movement in the critical region where the HV connection to the cathode is made (discussed in Section 3.3), the entire TPC assembly will be supported from the bottom PMT array, which will be connected to the cryostat vessel. This means that the top PMT array will move downward during cool-down, increasing the clearance in the top dome.

\subsection{Cathode HV Delivery System}

\subsubsection{Cathode HV Requirements}

The cathode HV is a key performance parameter that will directly affect the science reach of the instrument because of its impact on ER rejection and other factors. Table 3.3.1 summarizes the HV requirement and some detector parameters it determines. The LZ nominal operating voltage is $-50 \mathrm{kV}$, which establishes a drift field of $\approx 300 \mathrm{~V} / \mathrm{cm}$ and allows $\mathrm{LZ}$ to meet its baseline sensitivity. The design goal is $-100 \mathrm{kV}$-the maximum operating voltage for the system. Introduction of HV into the Xe space is challenging because of 
possible charge buildup and sparking, and since high-field regions can produce spurious electroluminescence that blinds the detector to the minute flashes of light produced by WIMP interactions.

Table 3.3.1: Dependence of TPC parameters on cathode HV.

\begin{tabular}{|lcccl|}
\hline Parameter & $\begin{array}{c}-30 \mathrm{kV} \\
(\mathrm{LUX})\end{array}$ & $\begin{array}{c}-50 \mathrm{kV} \\
(\text { Base })\end{array}$ & $\begin{array}{c}-100 \mathrm{kV} \\
(\text { Goal })\end{array}$ & Comments \\
\hline TPC drift field, $\mathrm{kV} / \mathrm{cm}$ & 0.17 & 0.31 & 0.65 & Gate $-5.5 \mathrm{kV}$ \\
\hline ER/NR discrimination & $99.6 \%$ & $99.7 \%$ & $99.7 \%$ & NEST LZ04 \\
\hline Electron drift velocity, $\mathrm{mm} / \mu \mathrm{s}$ & 1.5 & 1.8 & 2.2 & {$[11]$} \\
Maximum drift time, $\mu \mathrm{s}$ & 970 & 806 & 665 & Interactions at cathode \\
\hline Longitudinal diffusion, $\mu \mathrm{s}$ & 2.4 & 2.2 & 2.0 & FWHM, cathode events \\
Transverse diffusion, $\mathrm{mm}$ & 2.4 & 1.8 & 1.4 & FWHM, cathode events \\
\hline Gate wire field, $\mathrm{kV} / \mathrm{cm}$ & -64 & -62 & -58 & \\
Cathode wire field, $\mathrm{kV} / \mathrm{cm}$ & -18 & -31 & -63 & \\
\hline
\end{tabular}

The LZ operating and design voltages were determined through a combination of task-force activity, evaluation of WIMP sensitivity, and project cost and risk. At the nominal voltage, an ER rejection efficiency of $99.5 \%$ is expected at $50 \% \mathrm{NR}$ acceptance, as demonstrated in previous double-phase Xe detectors and modeled through the Noble Element Simulation Technique (NEST) simulation package. The LZ cathode is required to operate up to $-50 \mathrm{kV}$; in addition to allowing adequate discrimination power, this voltage further minimizes drift time, event pileup, charge cloud diffusion, and optimized energy resolution. All subsystems in LZ will be designed to withstand higher voltages to help ensure that a $-50 \mathrm{kV}$ operational voltage can be met. High voltage tests of LZ prototypes and final components will also be done to ensure this voltage capability. The planned design safety factors (DSFs) and test safety factors (TSFs) are summarized in Table 3.3.2.

Table 3.3.2: Design safety factors (DSFs) and test safety factors (TSFs) for the cathode high voltage delivery system. The DSFs and TSFs are defined as percentages above $-50 \mathrm{kV}$ (e.g. a $100 \%$ safety factor implies a voltage of $-100 \mathrm{kV})$.

\begin{tabular}{|lcc|}
\hline Item & Design Safety Factor & Test Safety Factor \\
\hline Cathode power supply & $140 \%$ & $140 \%$ \\
Warm feedthrough & $200 \%$ & $200 \%$ \\
Cable & $200 \%$ & $200 \%$ \\
Grading region & $100 \%$ & $140 \%$ \\
Cathode connection region & $100 \%$ & $140 \%$ \\
\hline
\end{tabular}

\subsubsection{HV System Overview}

A schematic overview of the cathode HV system is shown in Figure 3.3.1. The baseline LZ design places the cathode HV feedthrough (from air into Xe space) outside the shield at room temperature, at the end of a long vacuum-insulated, Xe-filled umbilical. The cathode high voltage cable has been chosen to be Dielectric Sciences model SK160318. It is rated to $150 \mathrm{kV}$, and has a conductive polyethylene core, polyethylene 
insulator, and conductive polyethylene sheath. With the dominant cable material being polyethylene, Rn emanation is minimized. Polyethylene is also known to be a safe material in LXe, mitigating concerns about emanation of electronegative contaminants. With the feedthrough at room temperature and far away from the active LXe, there are no concerns of thermal contraction compromising a leak-tight seal to the Xe space, and no concerns about feedthrough radioactivity. A feedthrough at the warm end of the umbilical allows a commercial polyethylene-insulated cable to pass from a commercial power supply, through a double O-ring seal, and into the gaseous Xe. The cable then travels through the center of the umbilical and routes the HV through LXe and to a field-graded connection to the cathode.

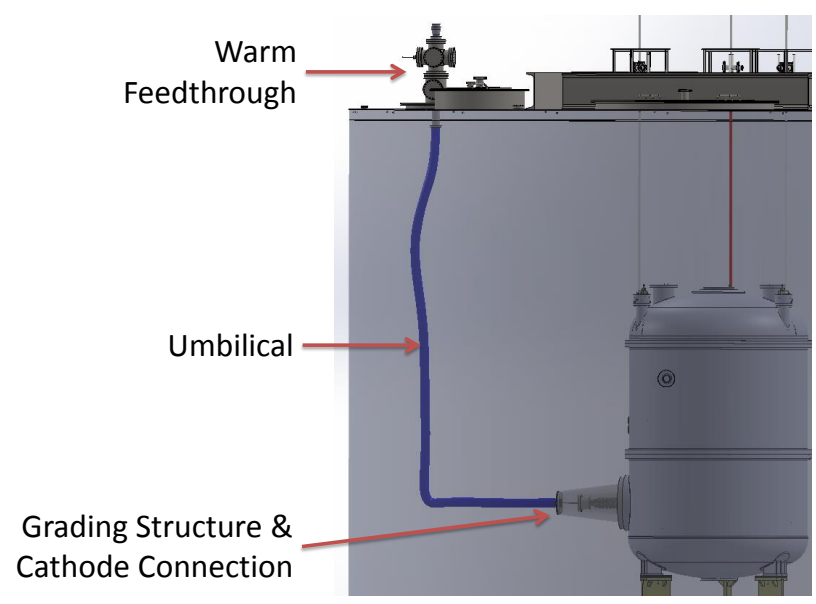

Figure 3.3.1: Schematic overview of the cathode HV delivery system.

\subsubsection{Cathode Supply and Cable Connection}

The cathode grid power supply (Spellman SL120N10) is rated at $-120 \mathrm{kV}$ and is limited to a maximum current of $100 \mu \mathrm{A}$. This power supply has an adjustable current trip feature which can be used to shut the power supply off in the event of an over-current condition such as HV breakdown to protect the load. The output cable leads directly into the warm feedthrough, and from there into the xenon space and cathode connection region.

\subsubsection{Cathode Feedthrough}

The warm cathode HV feedthrough, shown in Figure 3.3.2, allows high voltage to be passed from outside the LZ detector into the top of the Xe-filled umbilical space. The HV cable passes continuously through the feedthrough, with electric field confined to the cable insulation. The feedthrough includes 2 O-rings that seal to the outside of the conducting outer sheath of the HV cable. Between the O-rings is a vacuum space, maintained with a turbopump and monitored with a residual gas analyzer. The first O-ring allows the cable to pass between air and vacuum, and the second O-ring allows the cable to pass from vacuum to xenon gas in the umbilical. The cable is supported mechanically, both in air before the first O-ring, and in the xenon gas space after the second O-ring.

\subsubsection{Cathode HV Umbilical}

The cathode HV umbilical is designed to carry the Dielectric Sciences HV cable from the warm feedthrough to the cathode of the detector. The umbilical is a nested pair of tubes that protrude from the side of the 


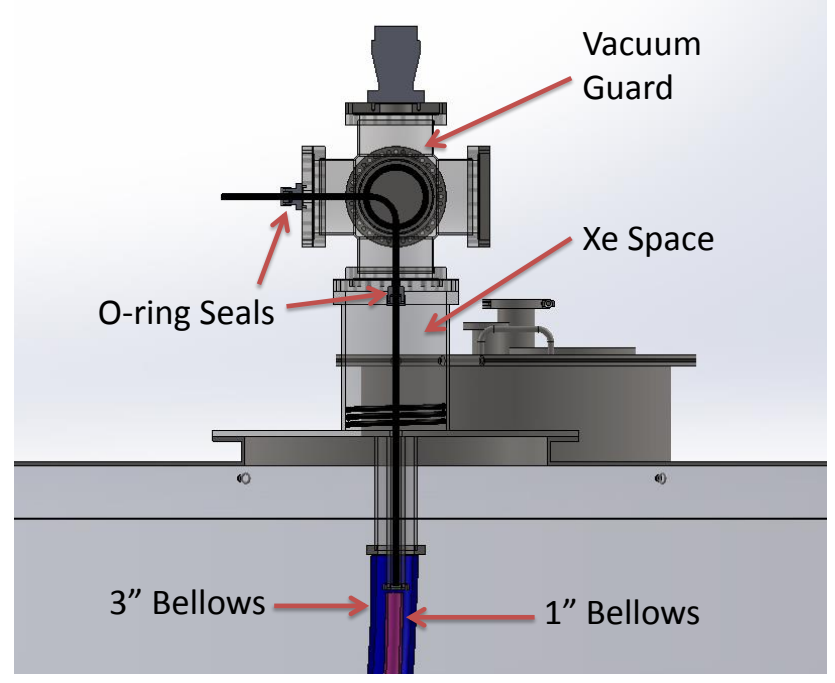

Figure 3.3.2: Warm feedthrough detail for the HV connection to the cathode.

detector at about the height of the cathode. The tubes include formed bellows for flexibility, and after leaving the cryostat horizontally, bend upward to the top of the water tank. The HV cable is also flexible, allowing it to turn with the umbilical.

The 1-inch-diameter inner tube of the umbilical is connected to the Xe space and is joined to a stainless steel cone that makes a seal to the inner vessel of the detector. To avoid gas bubbles within the cone that might cause electrical discharge, the cone is cooled through a conducting strap in the vacuum space that connects to a detector thermosyphon. The 3-inch-diameter outer tube of the umbilical contains vacuum and is similarly connected to the outer vessel of the detector.

The outside of the outer tube is immersed in the water of the tank. The evacuated space between the tubes contains super-insulation reflective wrap and acts to thermally isolate the Xe space from the water. This allows LXe to fill the inner tube of the umbilical until it reaches a height equal to the level of the Xe surface inside the detector. Thus the lower part of the umbilical is filled with LXe, while the upper part contains Xe gas. The long length of LXe is necessary to accommodate the field-grading region of the HV cable.

A gas return to the circulation pump connects to a tube that leads inside the xenon-filled inner umbilical to just above the liquid surface. This port allows control over the flow of Xe boiling in the lower umbilical. A second port connects to the high end of the umbilical, to slowly purge xenon exposed to the warm cable directly to the Rn scrubber system. Finally, the high end of the umbilical connects to the warm HV feedthrough. The feedthrough and umbilical are supported by the top of the water tank.

\subsubsection{Spark and Discharge Mitigation}

The field-grading structure at the cold end of the HV cable, shown in Figure 3.3.3, allows for the ground braid of the cable to terminate while the polyethylene insulation and conductive center of the cable continue. The departure of the cable ground braid from the cable surface is gradual, and connected to a stress cone made of conductive polyethylene. The stress cone is cryofitted within a xenon-displacing polyethylene wedge, which expands to a polyethylene tube that tightly surrounds the cable insulation. As this tube is larger in outer radius that the commercial cable, the field within the LXe is reduced in comparison with a design that only relied upon the cable insulation. This polyethylene structure is long in order to minimize the electric field parallel to the surface of the cable, and is surrounded by 20 field rings made of conductive plastic. These rings enclose coil springs that grip the cable circumferentially and provide electrical contact 
to its surface. The field rings are connected in series by small resistors to establish a uniform voltage grading between them. The highest potential ring is connected to the center conductor of the cable, while the lowest potential ring is connected to the cable ground braid. The surfaces of the rings are heavily rounded, and the resistors are nested between them. This minimizes the field within the LXe that surrounds the grading structure and separates it from the grounded wall of the inner tube of the umbilical. The grading ring structure is supported by three rods entirely from its grounded end, which is attached to the inside of the umbilical. The rods are pulled by springs to the ground end, and the rods compress the ring structure for rigidity. The entire grading structure is immersed within the LXe; all sections of the cable within Xe gas have an intact ground shield. The end of the grading structure connects to a rounded mushroom head, also made of conductive polyethylene, which captures the spring connection to the cathode.

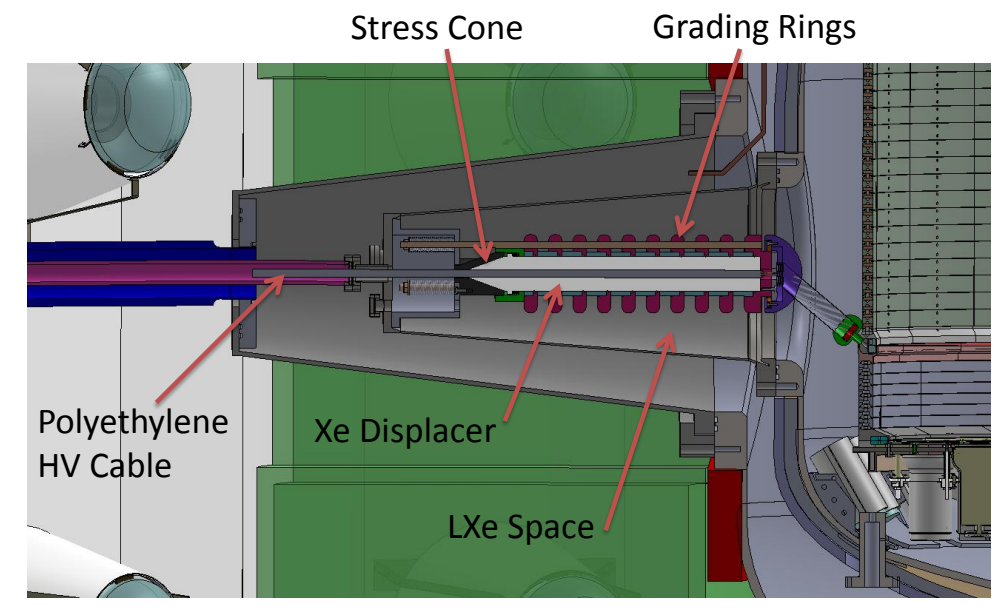

Figure 3.3.3: Schematic of the flexible HV connection to the cathode grid, showing details of the field-grading structures on the incoming HV cable required to keep the fields in the LXe below $50 \mathrm{kV} / \mathrm{cm}$.

\subsubsection{HV Connection to Cathode Ring}

A schematic of the HV connection to the cathode is shown in Figure 3.3.4, and the simulated electric fields in this region are shown in Figure 3.3.5. Because the TPC (including the cathode grid) is supported from the bottom of the vessel, the cathode grid moves down approximately $2 \mathrm{~mm}$ as the PTFE TPC components contract when the system is brought from room temperature to operating temperature $(\approx 172 \mathrm{~K})$. To account for this movement, there is a compliant spring connection between the end of the grading structure and the cathode grid ring.

The grading structure mushroom head is attached to the spring connection prior to installation into the detector. The lower end of the spring is captured by a rounded cup that has a through hole at its lower end, so that the mushroom head, spring and cup are all one assembly. A loose screw is captured inside the rounded cup. This screw will make the connection to the cathode ring. This screw is captive and cannot fall out of the assembly. The threaded end of this screw sticks out of the lower end of the cup.

During underground installation, the outer and inner cryostat ports are opened. A long narrow screwdriver is inserted through the mushroom head and into the spring, where it engages the head of the captive screw. The end of this screw emerges from the lower end of the cup. The screw inserted into the socket in the cathode ring and tightened. This mechanically and electrically connects the mushroom head to the cathode ring. A disk spring held captive under the screw head provides a constant force that prevents unscrewing.

The end of the cold grading structure is then brought close to the mushroom head. The mushroom head is grasped by hand and pulled toward the end of the cold grading structure, and rotated counter clockwise. 


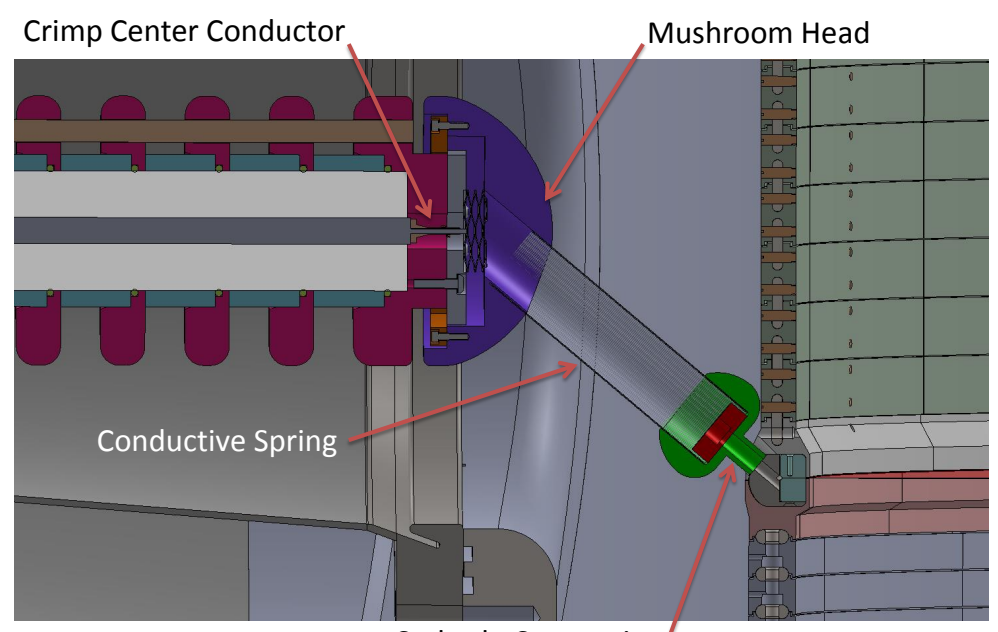

Cathode Connection?

Figure 3.3.4: Schematic of the cathode connection region.

This stretches the spring. The mushroom head is then pushed into the cold grading structure and rotated clockwise. This compresses a wave spring that is held captive on the end of the cold grading structure. The wave spring makes an electrical connection between the cold grading structure and the mushroom head. It also engages detents in the blue cap and provides a force that prevents the unscrewing of the blue cap. The mechanical locking of the mushroom head to the cathode grading structure resembles that of a bayonet mount.

At this point the spring is in its extended position. The spring is relaxed when the inner CHV cone is brought to meet the face of the cryostat. Ports in the back of the inner CHV allow for inspection of the spring to make sure it is located correctly. After the correct position is verified and electrical continuity to the cathode is verified, the bolts connecting inner $\mathrm{CHV}$ cone are tightened, making the seal between the inner cone and the inner cryostat.

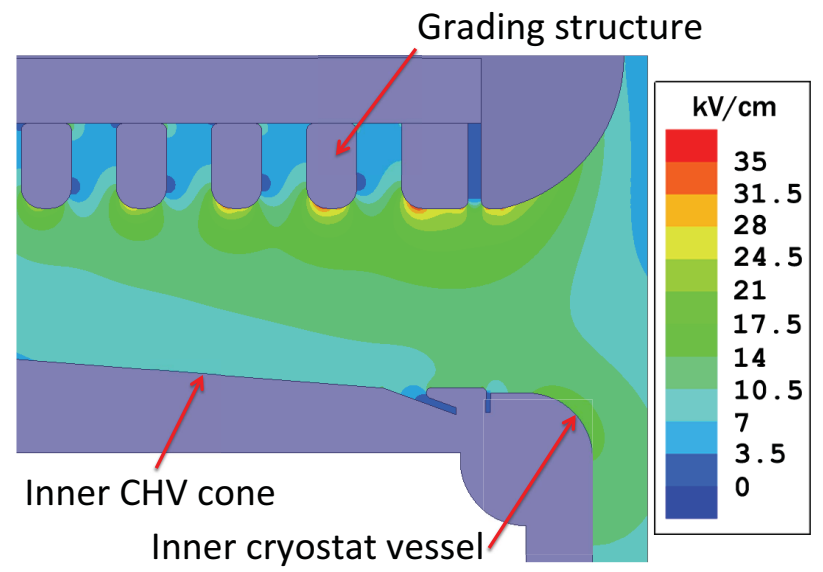

Figure 3.3.5: Simulation of the electric fields in the cathode connection region, assuming an applied cathode voltage of $-100 \mathrm{kV}$. 


\subsubsection{HV Safety Issues}

The combined stored energy from the cathode power supply output capacitance, output cable capacitance, warm feedthrough and umbilical capacitance, and TPC capacitance is $8 \mathrm{~J}$ at the maximum operating voltage of $-100 \mathrm{kV}$ and is classified as a 3.2C [12] shock hazard per the DOE Electrical Safety Handbook [12]. This shock hazard class indicates that injury or death could occur by contact. To mitigate this hazard, engineering controls are required for operation, and administrative controls are required for electrical work. Specific "lockout/tagout" and grounding procedures will be implemented for various operations such as unplugging the output cable and accessing the internals of the warm feedthrough, umbilical, and the TPC. Each worker who is authorized to perform these tasks will have specific high voltage and capacitor safety training.

\subsection{Photomultiplier Arrays}

To reach the performance specifications described previously, the Xe detector is equipped with top and bottom arrays of 3-inch-diameter PMTs (Hamamatsu R11410-22) to view the active region of the TPC. A top ring of smaller, 1-inch-square PMTs (R8520) view the scintillation light emitted in the side Xe Skin, with additional 2-inch PMTs (R8778) recycled from LUX complementing the Skin readout at the bottom of the detector. All three PMT types, shown in Figure 3.4.1, have been developed to meet important performance requirements in the field, including good spectral response in the VUV, good single-photoelectron definition, low dark noise, and the ability to operate at LXe temperature-in addition to having ultra-low levels of radioactivity: of order $\mathrm{mBq} / \mathrm{unit}$ in $\mathrm{U} / \mathrm{Th} /{ }^{60} \mathrm{Co} /{ }^{40} \mathrm{~K}$ for the 3-inch and 1-inch tubes, and a few times higher for the 2-inch units. This section details the properties and deployment of the PMTs for the TPC and Skin. Subsequent sections discuss the design and optimization of S1, S2, and Skin optical signals.

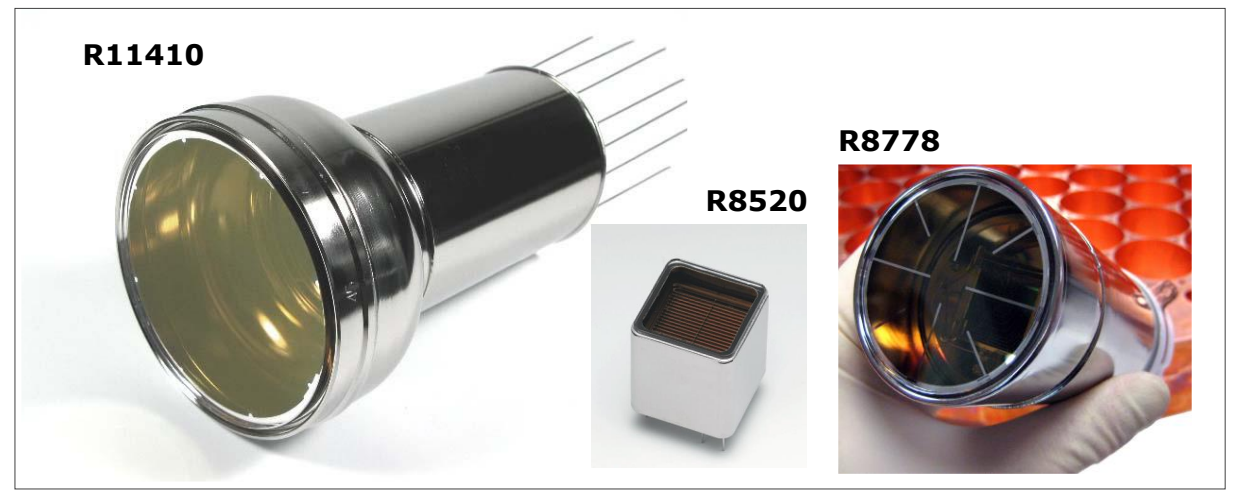

Figure 3.4.1: Photographs of xenon-space photomultipliers in LZ.

The LZ Collaboration has been pursuing the development of low-background PMTs tailored specifically for use in LXe with a radioactivity goal of $1 / 1 \mathrm{mBq}$ per unit in for U/Th and QE $>30 \%$ at $175 \mathrm{~nm}$ wavelength [13]. The detector configuration requires $\approx 5003$-inch tubes. Because of its outstanding radiopurity, the 3-inch Hamamatsu R11410-22 model has been adopted; this tube contains 1,000 times less radioactivity than a standard off-the-shelf item and is the result of a coordinated development with the manufacturer and a rigorous screening campaign of sub-components before the items are even manufactured.

The dynode optics in the R11410 are electrically identical to those used in LUX (2-inch R8778), exhibiting similar gain and single photoelectron response. The photocathode diameter is $64 \mathrm{~mm}$. This tube has 12 dynodes and provides a minimum gain of $2 \times 10^{6}$ at $1,500 \mathrm{~V}$ bias voltage. The PMTs are assembled to passive voltage-divider bases and will be negatively biased so that the signal can be collected by directly 
coupling the front-end electronics at near-ground potential. High peak-to-valley ratios $(>2)$ are obtained for the single photoelectron response, which is a key parameter to ensure high detection efficiency for the smallest S1 signals that are composed of single photoelectrons appearing in multiple PMTs.

Aside from good VUV sensitivity, these quartz-windowed PMTs are designed to be operated at LXe temperature featuring a special low-temperature bialkali photocathode with low surface resistivity. This obviates the need for metallic underlayers or conductive fingers [14]. They also have a pressure rating of $4 \mathrm{~atm}$ absolute for operation in liquid xenon. Nominal performance will be verified for every unit through a comprehensive low-temperature test program to confirm optical and electrical parameters during and after thermal cycling.

\subsubsection{PMT Test Program}

The full order of the R11410 PMTs is split between the U.S. (2/3) and the U.K. (1/3), delivered in batches of 40 per month. It is crucial to characterize every PMT before they are installed, and a detailed testing program is designed to test and radio-assay all the PMTs. This is the testing sequence that will be carried out at Brown University for all tubes (except for the confirmatory radioactivity screening, as explained below):

1. Warm test \#1

2. Cryogenic test

3. Radioactivity screening

\section{Warm test \#2}

In the first warm test the after-pulsing, gain, single photoelectron resolution, dark count rate and linearity will be measured. These measurements are categorized as "standard testing" and they will identify any PMT that fails to meet agreed specifications for room temperature operation. In the cryogenic test PMTs will be thermal-cycled from $300 \mathrm{~K}$ to $160 \mathrm{~K}$ and the standard testing measurements will be repeated, plus some exceptional studies such as photocathode uniformity and long-term stability monitoring. The Brown PMT test chamber known as PATRIC, shown schematically in Figure 3.4.2 (left), is designed to hold 14 R11410 PMTs for batch testing. After radioactivity screening (carried out at SURF and Boulby underground laboratories) the PMTs will undergo a second warm test to establish stability over time.

The U.K.-procured PMTs will go through the same test sequence, but a sample of 30 units will undergo characterization of quantum efficiency $(\mathrm{QE})$ and dual-photoelectron emission probability at room temperature and at LXe temperature at Imperial College London. The main goal of the QE test is to establish a statistical correction function to relate the QE at $175 \mathrm{~nm}$ obtained by Hamamatsu at room temperature and the relative change of QE upon cooling [15]. The PMT test chamber at Imperial, shown in Figure 3.4.2 (right), uses a xenon scintillation cell maintained at room temperature to shine $175 \mathrm{~nm}$ scintillation light into a cryostat containing 7 PMTs (6 under test plus a reference tube) which are cooled to LXe temperature. Correlation of QE data with PMT gains, dark counts and the QE values provided by Hamamatsu will also be studied.

\subsubsection{PMT Radioactivity}

Due to their complexity, total mass $(\approx 100 \mathrm{~kg})$, and proximity to the active volume, the Xe-space PMTs are a significant source of radioactivity background in LZ. For this reason, they will be subject to a thorough 

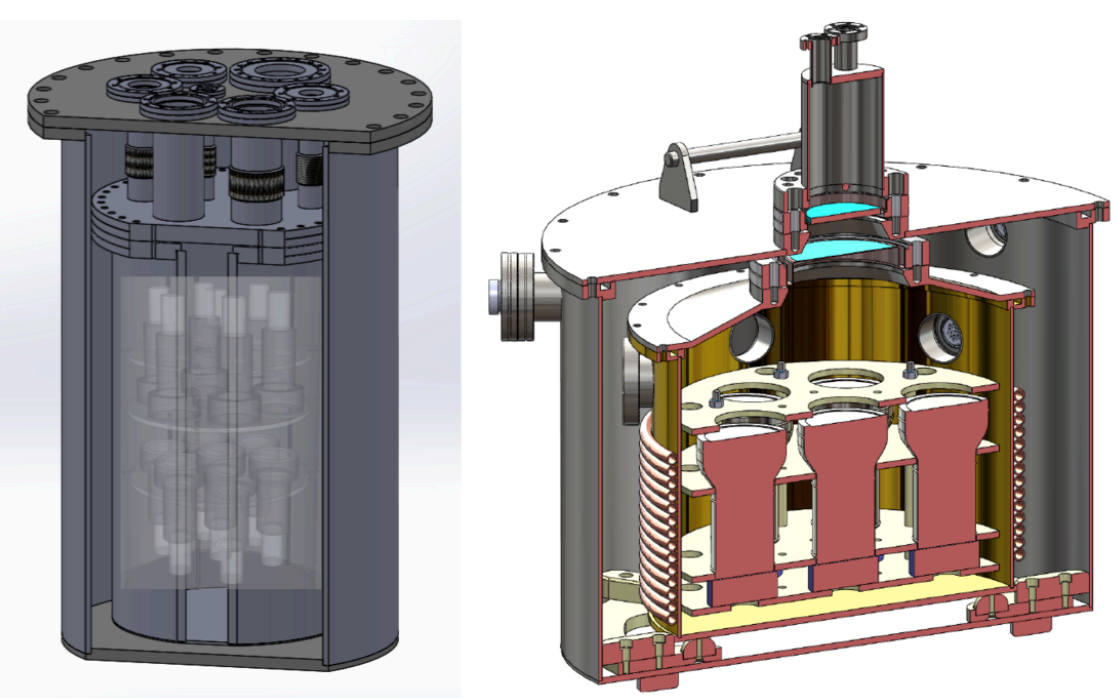

Figure 3.4.2: PMT test setups. The Brown PMT Array Test Rig In Cryogens (PATRIC), shown on the left, is a pressure vessel that can test 14 PMTs at LXe temperature and pressure. After the R11410 PMT testing is completed, the chamber will be modified to test R8520 PMTs. The Imperial setup on the right uses a xenon scintillation cell on top of a vacuum cryostat, shining onto 7 PMTs located in cold nitrogen gas.

screening campaign using HPGe detectors (see Chapter 9). Screening of fabrication materials and subcomponents has taken place prior to PMT manufacture, and every assembled PMT will again be screened after delivery from Hamamatsu.

The 3-inch R11410 has been delivered in part through a 4-year NSF S4 development program with Hamamatsu, which achieved unprecedented radioactivity performance compared with previous generation tubes $[13,16]$. This same model has also been advanced by other collaborations, notably XENON1T with variant R11410-21. Comprehensive radioactive screening results for 216 of these PMTs are publicly available [17]. Screening results from our prototype tubes are in broad agreement, and the pre-screening of construction materials also yielded similar results to those obtained by XENON1T—as detailed in Chapter 9. LZ will procure the most recent (-22) version of these tubes.

The 1-inch R8520 PMTs used in the top side of the Skin Detector have radioactivity levels that are well understood thanks to their wide use in previous detectors. Nevertheless, they will follow the same screening procedures as the larger R11410 model. The contribution of the 93 Skin PMTs to the background of the instrument is sub-dominant given their comparable specific activity but more peripheral location and smaller number.

The 2-inch R8778 PMTs used in the bottom of the Skin Detector will be reused from the LUX experiment and are also well understood from both a radioactivity and performance perspective. Although they have somewhat higher radioactivity per PMT relative to the R11410 PMTs, their background contribution is also sub-dominant given their peripheral location and small number.

\subsubsection{PMT Bases}

Individual voltage-divider bases connected to two coaxial cables (for HV bias and signal) are attached to each PMT. Given their locations, these components are under detailed scrutiny as part of the radioactivity and radon-emanation screening programs, and they must meet also demanding requirements related to operational reliability and xenon poisoning. 

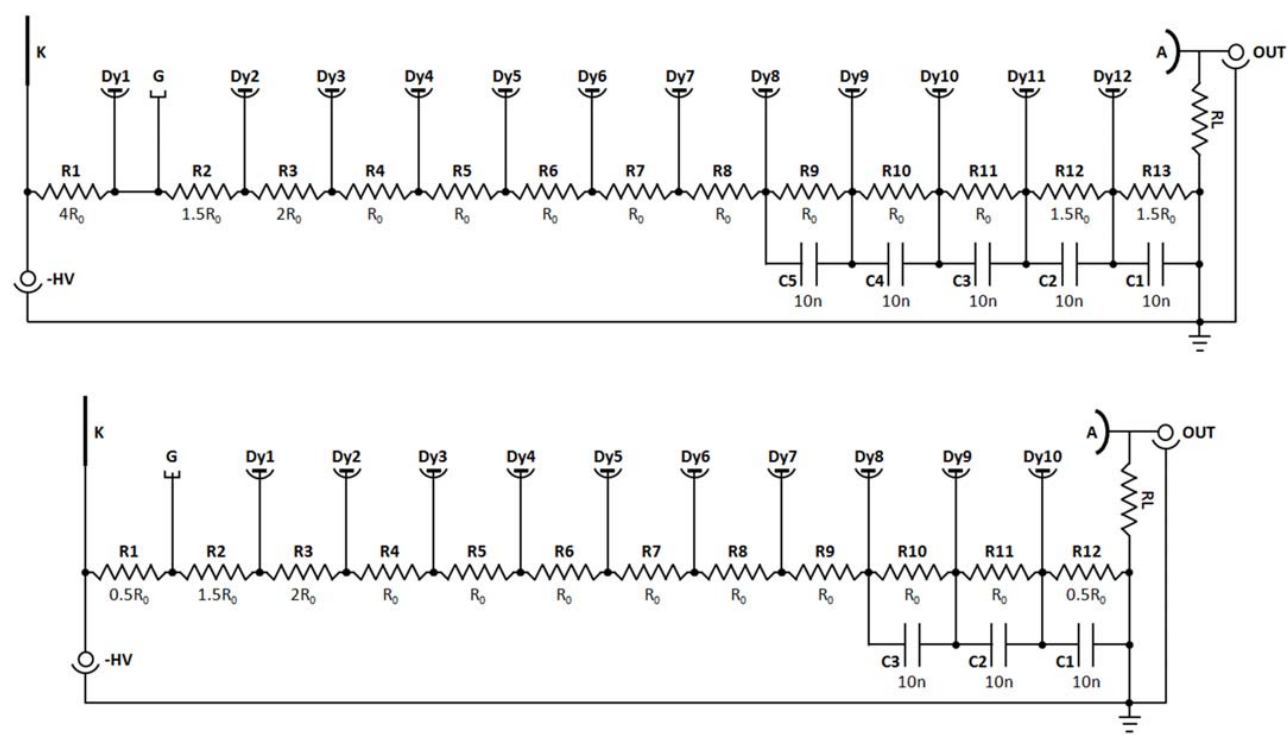

Figure 3.4.3: Photomultiplier base voltage-divider circuits for the R11410 PMT (upper panel) and R8520 PMT. In both cases the voltage distribution recommended by Hamamatsu was adopted, with $R_{0}=4.99 \mathrm{M} \Omega . \quad R_{L}=49.9 \Omega$ for the R11410 base and $R_{L}=100 \mathrm{k} \Omega$ for the R8520 base. The 2-inch R8778 Skin PMT bases will employ the same circuit design as the 3-inch R11410 units.

The electrical circuits are shown in Figure 3.4.3 for the 3-inch and 1-inch tubes (the 2-inch R8778 base is similar to the former), and a prototype base for the R11410 PMT is shown in Figure 3.4.4. The voltage distributions are those recommended by Hamamatsu. High-resistance chains are used for low power dissipation in the LXe: $24.3 \mathrm{~mW} /$ unit at the nominal $1,500 \mathrm{~V}$ for the 3-inch PMTs, and $10.3 \mathrm{~mW}$ at $800 \mathrm{~V}$ for the 1-inch PMTs. This is required for minimal impact on the thermal design of the detector and to prevent localized bubbling of the liquid. We have confirmed the bubble nucleation threshold of liquid xenon as $\approx 20 \mathrm{~mW} / \mathrm{mm}^{2}$, in agreement with Ref. [18]; all resistors operate far from this value. Still, the divider current is much higher $(\approx 100 \times)$ than the average PMT anode current expected during calibration, which is required for response linearity. Anode pulse linearity is also addressed by charge supply capacitors $(10 \mathrm{nF}$, de-rating to $\approx 8 \mathrm{nF}$ at LXe temperature) which are added to the last few dynodes. Our main requirement ensures accurate response to ${ }^{83 \mathrm{~m}} \mathrm{Kr}$ calibration events, translating to percent-level non-linearity in top-array channels for S2 signals from this key calibration source. This determines a minimum of five decoupling capacitors for the TPC PMTs. These S2 signals also approach the $\pm 5 \%$ pulse non-linearity expected in these PMTs above $\approx 20 \mathrm{~mA}$ anode current.

The bases are made from thick polyimide $\left(\right.$ Cirlex $\left.^{\mathrm{TM}}\right)$ PCBs with surface-mounted passive components. Cirlex is a good material for this purpose, having very high dielectric strength, a low thermal expansion coefficient, high tensile strength and low internal stress. It is, however, prone to delamination and fiber release at the edges, demanding careful quality control. The Cirlex is patterned with standard photo-lithography and the cutout and drill-holes are made with a PCB router-all processes are conducted in-house to ensure strict con-

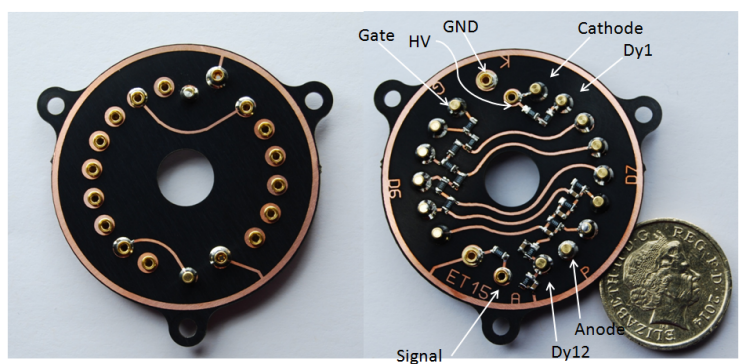

Figure 3.4.4: Prototype of the R11410 PMT voltage-divider base. 
trol of radioactivity and cleanliness. Candidate components and full prototypes were tested in gaseous and liquid xenon for electrical resilience and outgassing as well as for spurious optical photon emission. All bases will be thermal-cycled in $\mathrm{LN}_{2}$ and tested at ambient temperature for 5 days above their maximum allowable voltage. Final inspection, cleaning and radon-tight packaging are conducted in a class 1,000 cleanroom.

The radioactivity performance of the PMT bases is of special concern, both from the point of view of neutron/gamma emission and from radon emanation. Although Cirlex is intrinsically a radio-clean material and a good $\mathrm{Pb}$-free solder has been identified, the discrete components have significant U/Th contributions in spite of the small masses employed. The X7R ceramic capacitors, the thick-film ceramic-chip resistors, and the PMT-pin receptacles all posed significant challenges and we radio-assayed some 25 components in order to select a viable set meeting our radioactivity goal- to be sub-dominant to the PMTs. Ceramic capacitors have typically high uranium content, with those selected (Kemet, 0603 package) showing the lowest levels $(11.5 \mathrm{mBq} / \mathrm{g})$; fortunately, the XR7 ceramic is barium titanate (confirmed through SEM/EDX elemental analysis) which has an order of magnitude lower intrinsic neutron yield than alumina, making these components viable - although they pose some radon emanation risk as discussed below. The selected resistors (Vishay, 0805 package) showed acceptable levels of $\mathrm{U} / \mathrm{Th}(<1 \mathrm{mBq} / \mathrm{g})$, but high levels of ${ }^{210} \mathrm{~Pb}$ (found in all models tested); the resulting $(\alpha, n)$ yield on the alumina becomes a significant contribution to the neutron yield from the bases. Finally, the greatest challenge came from the receptacles that mate to the PMT contact pins and to the cable connectors. These contain BeCu alloy spring clips which are very "hot" in early uranium $(\approx 100 \times$ out of equilibrium). Avoiding the use of these sockets by directly soldering to the PMT pins and cables would have cleanliness implications that would delay the integration sequence of the PMT arrays, and was thus not considered an attractive option. We identified suitable sockets from Harwin which feature $\mathrm{CuNi}_{2} \mathrm{Be}$ alloy spring clips within a brass shell; these have lower Be content $(0.4 \%)$ and lower radioactivity than the other models we assayed (note that $\approx 50 \%$ of ${ }^{9} \mathrm{Be}(\alpha, n)$ reactions can be vetoed due to the $4.4 \mathrm{MeV}$ gamma ray [19]; this was considered in the background calculations). In conclusion, we were able to obtain a suitably low radioactivity content, where no single component dominates too significantly, balanced against practical considerations such as robustness and ease of integration. The bottom-up radioactivity estimates are: 390/140 $\mu \mathrm{Bq} / \mathrm{unit}$ in mid-U/Th for the R11410 and R8778 base (0.06 n/yr) and $230 / 80 \mu \mathrm{Bq} / \mathrm{unit}$ for the R8520 base (0.04 n/yr), respectively.

Radon emanation from the bases was a concern, prompted especially by the total ${ }^{226} \mathrm{Ra}$ content observed in the capacitors. Fortunately, the ${ }^{222} \mathrm{Rn}$ emanation rate measured from a very large number of these components was found to be below $1 \%$ relative to production even at room temperature. The emanation from all bases in the xenon space is expected to be $\approx 1 \mathrm{mBq}$ at ambient conditions; a modest reduction with cooling is expected from the resistors and capacitors, while the Cirlex should exhibit a significant decrease. Therefore, expect the bases to make a limited contribution to the LZ radon budget (and to confortably meet the radon budget requirement).

\subsubsection{Cabling}

The PMT signals and HV supplies are carried separately between the PMT bases and the warm breakout interface by low-radioactivity coax cables. The baseline design is to use Gore 3007 Coax with no outer jacket, the same cable that was used in this role for LUX. The $50 \Omega$ characteristic impedance cable uses an AWG 30, silver-plated, Cu-clad steel, surrounded by an AWG 40 stainless steel braid. The cables from the PMTs associated with the upper and lower parts of the TPC are housed in separate conduits, so that no cabling is routed through the side Skin region (these conduits can be seen in Figure 1.2.1 in Chapter 1). This could interfere with the ability to hold a high voltage on the cathode and it would degrade the light collection efficiency in the Skin. 
Table 3.4.1: Coaxial cables in the xenon space routed via upper and lower conduits.

\begin{tabular}{|lrr|}
\hline & Upper & Lower \\
\hline TPC PMTs & 506 & 482 \\
Skin PMTs & 186 & 76 \\
LED calibration & 39 & 45 \\
Dummy channels & 11 & 11 \\
Monitoring sensors & 59 & 11 \\
\hline Total & 801 & 625 \\
\hline
\end{tabular}

The lengths of typical top and bottom cables are $12.8 \mathrm{~m}$ and $11.6 \mathrm{~m}$, respectively. The upper routing carries 801 cables and the lower routing consists of 625 cables, as listed in Table 3.4.1. The total heat load calculated from the cables is less than $6 \mathrm{~W}$, which is a sub-dominant contribution to the thermal model. The total cable length within the xenon space is $\approx 17 \mathrm{~km}$, weighing some $72 \mathrm{~kg}$.

A screening program has been initiated to measure the radon emanation from the baseline cabling, as well as possible alternatives, to ensure that the finally selected model will meet the overall Rn requirements discussed in Chapter 9. Rn emanation from cables is expected to be dominated by the warm region, which is $\approx 8 \mathrm{~m}$ long for the upper routing, and only $\approx 1 \mathrm{~m}$ for the lower routing. To mitigate excessive $\mathrm{Rn}$ emanation due both to dust and radioactivity of the cable materials, we are evaluating the benefit of having the cables sheathed in a $150 \mu \mathrm{m}$ thick coat of FEP Teflon to reduce the rate of Rn reaching the active detector volume. The MJD and GERDA collaborations have observed that including such a FEP jacket provides a suitable Rn barrier in their detectors. Emanation from the feedthroughs (which were previously used in the LUX experiment) will also be measured. Finally, we are planning to add a radon trap to the gas purification system, as discussed in Section 6.4.5, to address emanation from the cables and room temperature feedthroughs.

The Gore 3007 cable has been tested and shown to support $2 \mathrm{kV}$, comfortably meeting the HV requirements of all PMTs. The way the cable affects the signal characteristics can be seen in Figure 8.3.8 in Chapter 8, which shows the effect of a full cable run (internal and external to the detector) on the single photoelectron response of a R11410 PMT. For these signals there is an amplitude reduction of $47 \%$ and a pulse area loss of $20 \%$.

\subsubsection{Assembly and Integration with TPC}

The 253/241 PMTs per top/bottom array will be assembled onto titanium support frames. The PMTs will be held in position using cobalt-free metal belts fabricated by Hamamatsu from the same material used for the PMT body production. Three PTFE columns will then be used to hold the collar to the PMT mounting plates, as shown in Figure 3.4.5. This mounting system is specified to hold the PMTs in place in the Ti mounting plate both when the plate is in the vertical and horizontal orientations (during assembly and transport).

The PMT arrays are shown in Figure 3.4.6. The support frames consist of a flat titanium plate with supporting truss-work. The loads on this structure are substantial, particularly in the case of the lower array. For the submerged PMTs, the buoyancy force far exceeds the gravitational force: the net upward load is approximately $8 \mathrm{~N}$ per PMT, and collectively the total load for the bottom array is approximately 2,200 N. Many configurations of the support frame were considered and simulated using finite element analysis. Starting with a bare plate (no truss-work), a single 6 to $7-\mathrm{mm}$ thick Ti plate deflected upward approximately $19 \mathrm{~mm}$. Other options include successively thicker plates, double plates, curved plates, honeycomb reinforcement, and truss reinforcement. The truss reinforcement had the best overall performance when trying to limit deflection, minimize mass (and therefore background radiation), and provide a relatively open volume for 


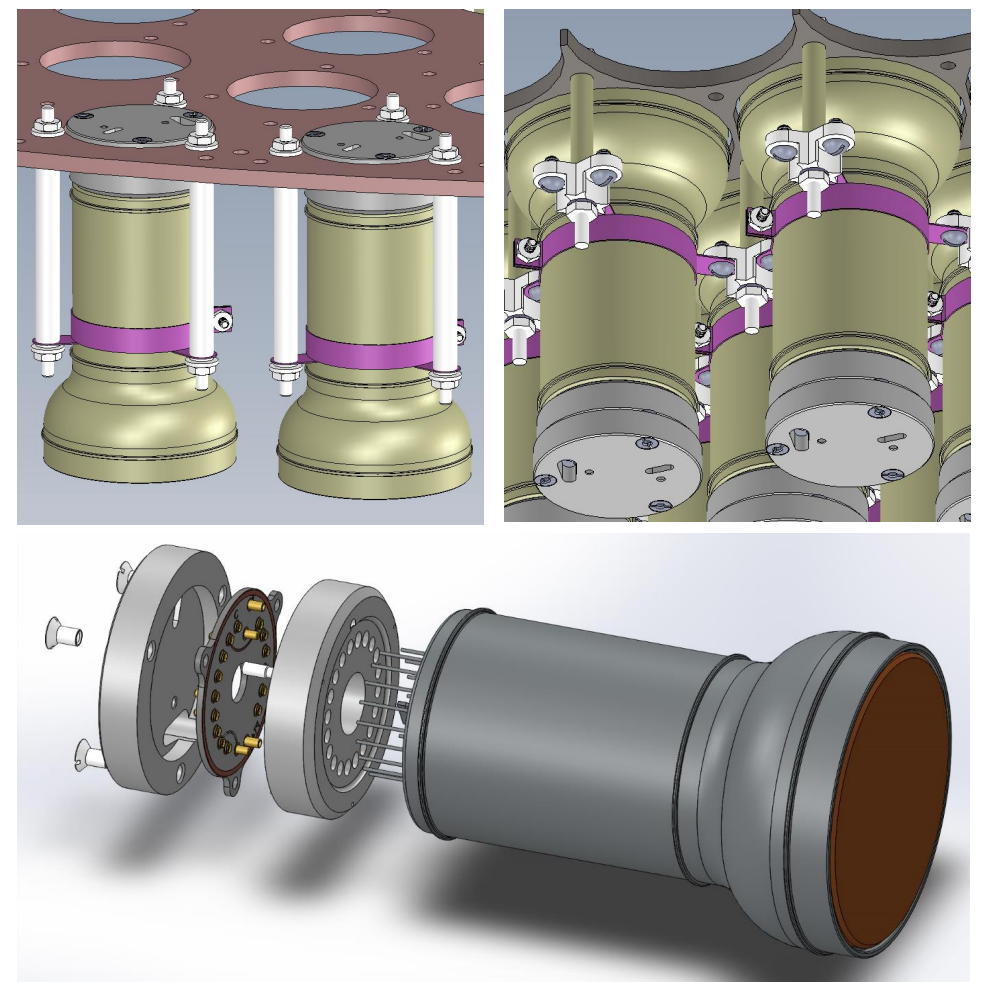

Figure 3.4.5: PMT assembly to the top and bottom array supports(upper right and left, respectively). The lower array PMTs are sleeved in PTFE and attached to the Ti plate with three PTFE rods. The lower panel shows the assembly to the base circuit, which is encased in PTFE caps; that at the back provides high reflectivity for the Skin detector and is used to strain-relive the cables.

scintillation light in the dome Skin to find its way to the Skin PMTs. The baseline lower PMT support frame is expected to deflect approximately $1 \mathrm{~mm}$ upward in operation. The upper PMT support frame (in the gas phase) will have a similar design, but the expected downward deflection is only $\approx 0.3 \mathrm{~mm}$ for that array.

The Ti surfaces surrounding the front faces of the PMTs, in both the top and bottom arrays, will be covered by PTFE pieces designed for photon recycling, and so increase photon detection efficiency in the main chamber, as discussed in Section 3.5. The pieces are designed to provide at least $95 \%$ coverage of the Ti structural elements, while accommodating the differential thermal contraction coefficients of the PTFE and the Ti mount.

The lower LXe region, below the bottom PMT array, forms part of the Xe Skin detector in which the goal is to maintain over $50 \%$ detection efficiency for ER events above $100 \mathrm{keV}$ in more than $95 \%$ of the Skin volume-see Section 3.7. The rear of the bottom PMTs, which project into this volume, are also sleeved in PTFE in order to increase photon recycling in the LXe below the array-this includes both a PTFE sleeve for the PMT body, and end-caps to cover the PMT bases. The PTFE base covers also prevent stray light leaking into the PMT envelope, and avoid pin short-circuits. The underside of the PMT mounting structure and braces will also be covered in PTFE reflectors where required, to increase the overall photon detection efficiency in the Skin region.

The PTFE components will be fabricated from material that has been pre-screened to achieve the intrinsic activity budget with respect to both gamma and neutron emission, as discussed in Chapter 9. During machining of the components and the assembly of the PMT arrays, the PTFE components will be maintained in 

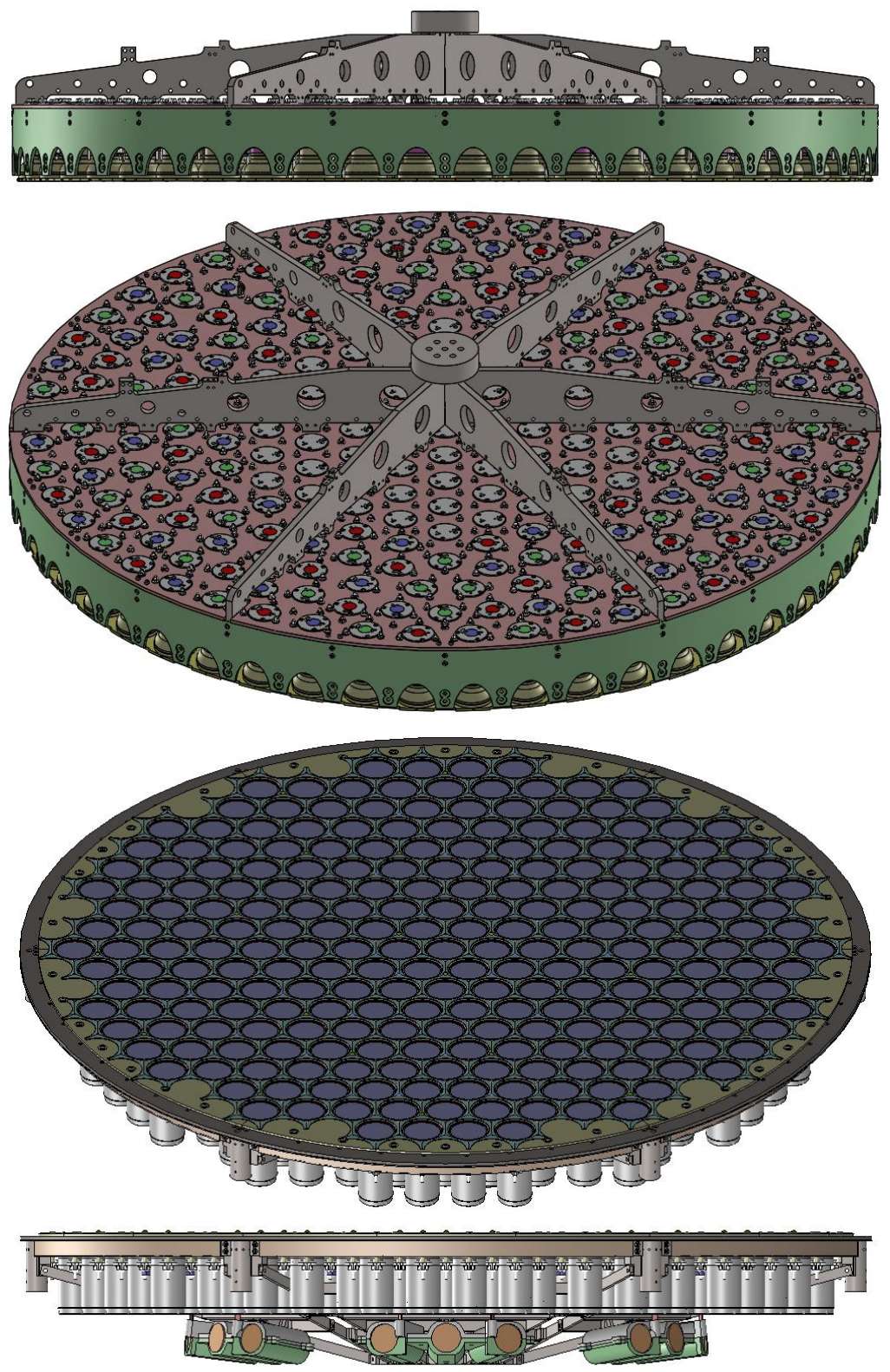

Figure 3.4.6: The bottom PMT array has 241 3-inch PMTs arranged in hexagonal configuration. The top PMT array accommodates 253 PMTs in a hexagonal-circular hybrid configuration.

purge boxes to reduce the plating of alpha emitters associated with airborne Rn, and ensure that additional $(\alpha, n)$ neutron generation is significantly below the intrinsic neutron emission goals.

PMTs passing all the testing and screening procedures will be installed onto their arrays inside a dedicated cleanroom at Brown University. Two PMT Array Lifting And Commissioning Enclosures (PALACE) will be built for this purpose. The arrays will be hanged vertically so that all PMT slots can be easily accessed during assembly. PALACE is also designed to be an airtight vessel so that the whole array and PMTs are under nitrogen purge when there is no assembly work on-going. With appropriate high voltage and signal feedthroughs, PALACE will also be used as dark chambers for final testing before TPC integration. Since each PALACE will hold the PMT array and over 240 PMTs, using PALACE as a radon emanation chamber 
will help understand the radon backgrounds in the detector; we are pursuing the goal of building PALACE as a radon emanation chamber with a target sensitivity of $0.3 \mathrm{mBq}$.

To meet these design goals, PALACE will consist of a stainless steel frame creating a $6 \times 6 \times 1.5$-foot ${ }^{3}$ space to hold the PMT array. The nitrogen purge system will feed a $10 \mathrm{slpm}$ flow from $\mathrm{LN}_{2}$ boil-off into the chamber. All materials will be chosen so they emanate less than $0.1 \mathrm{mBq}$ radon, and the vessel will be welded and electropolished to help achieve this. For the top lid a 1-inch thick plastic plate would be a sufficient radon barrier; we are investigating the best sealing technique for this purpose.

After PMTs are installed and the final electronics checkout is complete, PALACE is ready to be transported to SURF. The chambers will be sealed, purged and triple-bagged. During shipment PALACE will remain airtight, with the PMT arrays sitting in a nitrogen environment. Before the actual shipment happens test shipments with empty crates and accelerometers will be carried out to verify that PALACE is well taken care of under a medical equipment shipping company. After PALACE arrives at SURF it will be unwrapped and placed in the SURF cleanroom. PMTs will also be checked repeatedly to ensure their stability.

\subsubsection{PMT Calibration}

Three calibration techniques will be employed to monitor the PMT performance during LZ operation. The first one entails flashing $470 \mathrm{~nm}$ LEDs producing $\mathrm{O}(100)$ photons per pulse to measure after-pulsing, providing a direct indication of vacuum integrity of each tube. It is caused by residual gas ions inside the PMT body hitting the photocathode, creating a secondary signal appearing at a certain time after the main pulse. The after-pulse from $\mathrm{Xe}^{+}$ions would appear around $3 \mu$ s after the main pulse [20]. The second calibration is of the single photoelectron response, i.e. the absolute gain. The LEDs can be pulsed at a very low voltage such that signals seen by the PMTs come from single photons with high probability. The LZ LED calibration system is an evolution on the system developed for LUX. Calibration LEDs will be mounted on the face of each array to shine onto the PMTs opposite under individual DAQ control. A synchronized trigger is also fed into the DAQ system so the LED pulse timing is recorded along with the calibration data.

While the LED measurements monitor PMT gain (at $470 \mathrm{~nm}$ ) and after-pulsing stability, the VUV response will be measured in-situ as the third calibration technique. In LUX this was achieved by extracting single photoelectron signals from tritium beta decay calibration data. The same calibration will be done in LZ (see Chapter 7). It was understood from LUX experience that when a PMT sees a $175 \mathrm{~nm}$ VUV photon, there is $\mathrm{a} \approx 20 \%$ probability that a second photoelectron is emitted in coincidence [21]. This double-photoelectron emission phenomenon has very important consequences-for understanding the optical performance of the detector, and on its energy resolution; therefore, it must be well characterized for all PMTs in operating conditions. The combination of the LED calibration, which produces the true single photoelectron response, with analysis of the response induced by xenon scintillation photons will allow us to obtain this information.

\subsection{S1 Light Collection Design}

\subsubsection{Overview of Design and Optical Performance of the TPC}

Achieving the highest possible collection of scintillation photons is a design priority: It leads to the lowest energy thresholds in the S1 and S2 channels and, by reducing statistical fluctuations in the S1 signal, it improves ER/NR discrimination. It also improves energy resolution, which is important for identifying gamma-ray background lines and for $0 v \beta \beta$ sensitivity. The scintillation yields for electron and nuclear recoils depend on both energy and electric field. For reference, at the LZ field design goal 445 scintillation photons are expected to be emitted from a $10 \mathrm{keV}$ ER track and 84 photons from a $10 \mathrm{keV}$ NR interaction. The experimental challenge is to maximize how many are recorded as photoelectrons (phe) in the PMT 
arrays. In comparison, the high electroluminescence yield in saturated Xe vapor (typically $\approx 1,000$ photons/cm per emitted electron [6]) leads to very large S2 signals such that single electrons are readily measured and sub-keV detection thresholds are obtained. The LXe scintillation emission is centered at $178 \mathrm{~nm}$, with FWHM $=14 \mathrm{~nm}$ [22]. Light from electroluminescence in Xe gas has a similar spectrum, but not quite identical [23]. Wavelength shifting is not required since the Xe luminescence spectrum is compatible with quartz-windowed photomultipliers. The basic optical properties of LXe are established: The refractive index for scintillation light is $n=1.67$ [24], which is well matched to that of quartz $(n=1.57)$. This allows good optical coupling to the PMTs immersed in the liquid phase. The Rayleigh scattering length is $30 \mathrm{~cm}$ to $50 \mathrm{~cm}$ (see [23] and references therein), which must be considered in optical simulations.

The key issue is then to maximize the $\mathrm{S} 1$ photon-detection efficiency, $\alpha_{1}$, defined as the number of detected photoelectrons per emitted scintillation photon from the event site. The main factors affecting $\alpha_{1}$ in LZ are: (1) the VUV reflectivity of internal surfaces made from PTFE, especially those in the liquid; (2) the photon absorption length in the liquid bulk; (3) the geometric transparency and reflectivity of all grids; (4) the PMT photocathode coverage fraction; and (5) the PMT optical performance.

Most of these VUV optical properties have uncertainties that can result in significant differences in overall light collection. The factors affecting each of these properties are discussed in some detail below. In order to assess the detector performance we have adopted three sets of properties for light collection simulations which should bracket the final LZ response. The first is a realistic "baseline" set, which in most cases matches or only slightly exceeds the design requirements for individual optical parameters. The model which feeds into the estimation of the baseline LZ performance is slightly more conservative $(\approx 10 \%$ lower $\alpha_{1}$ ) than this baseline optical model, to ensure that the baseline cross-section sensitivity is achieved with high probability. There is also a more optimistic (but still plausible) set of values which feed into the more ambitious sensitivity goal. Finally, for reference, there is a set of pessimistic values which are each somewhat unlikely, and highly unlikely to all occur together. These three sets of properties are listed in Table 3.5.1.

Table 3.5.1: Baseline as well as optimistic and pessimistic sets of optical properties considered for the LZ detector. The baseline LZ sensitivity is calculated using an optical model which is slightly more conservative than the optical baseline model (yielding a fiducial volume (FV) averaged $\alpha_{1}$ of $7.5 \%$ ). The LZ sensitivity goal assumes the optimistic model.

\begin{tabular}{|lccl|}
\hline Property & Pessimistic & Baseline & Optimistic \\
\hline PTFE - in liquid & $93 \%$ & $95 \%$ & $97 \%$ \\
PTFE - in gas & $75 \%$ & $80 \%$ & $85 \%$ \\
Average PMT QE & $22 \%$ & $25 \%$ & $28 \%$ \\
Grid reflectivity (liquid and gas) & $0 \%$ & $20 \%$ & $40 \%$ \\
Absorption length in liquid & $15 \mathrm{~m}$ & $30 \mathrm{~m}$ & $100 \mathrm{~m}$ \\
\hline FV-averaged S1 PDE $\left(\alpha_{1}\right)$ & $5.5 \%$ & $8.5 \%$ & $13.3 \%$ \\
\hline
\end{tabular}

In Figure 3.5.1 we show the photon detection efficiency (PDE, or $\alpha_{1}$ ) as a function of depth for the three sets of properties, and its partitioning into the top and bottom PMT arrays for the baseline properties. This is defined as the probability of a photon from the interaction site reaching a PMT and creating a photoelectron. The resulting S1 PDE averaged over a preliminary 5.6-tonne fiducial volume is also given in Table 3.5.1.

We effectively assume here that there is zero probability for single photons to create two photoelectrons. However, for the baseline we adopt an average PMT QE of $25 \%$; while typical QEs reported by the manufacturer for LXe scintillation are more like 30\%, this conservative QE matches the manufacturer 
specification - and it also allows for the $20 \%$ fraction of double-photoelectron emission, not captured by the QE specification, that has been observed in these tubes for LXe scintillation [21]. Note that there will be an additional $92 \%$ efficiency for triggering on single photoelectrons, as discussed in Chapter 8 . This is applied for LZ performance estimates, but is not included here.
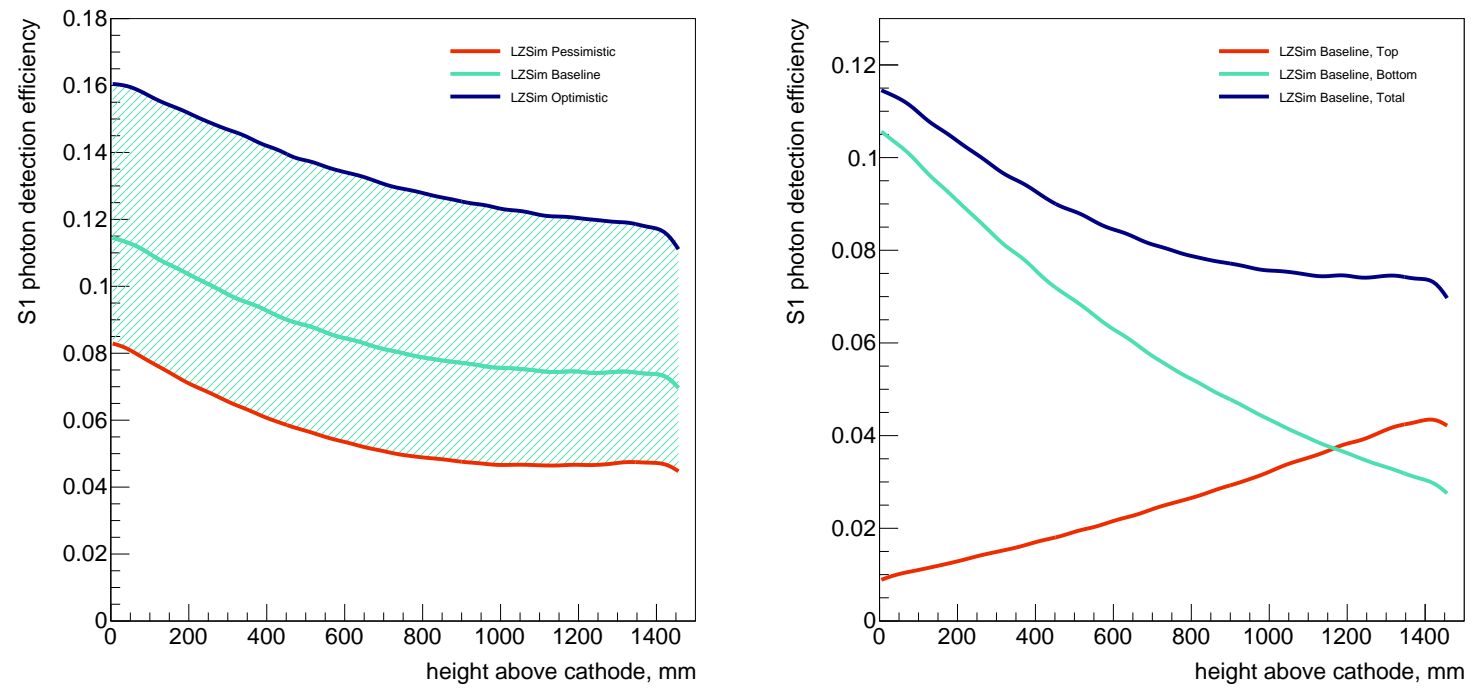

Figure 3.5.1: Left: Photon detection efficiency $\left(\alpha_{1}\right)$ versus interaction height above the cathode for the three optical scenarios listed in Table 3.5.1 (fiducial volume averages are also given in the table). Right: contributions from top and bottom arrays for the baseline optical model.

The baseline PDE of $8.5 \%$ translates to an $\mathrm{S} 1$ response of 5.3 phe/keV at zero field for ${ }^{57} \mathrm{Co} \gamma$-rays (a traditional measure of light yield in LXe chambers)—cf. 8.8 phe/keV in LUX [25], 6.6 in XENON10 [26, 27], 6.0 in PandaX-I [28], 5.0 in ZEPLIN-III [29], 4.3 in XENON100 [30], and 1.1 in ZEPLIN-II [31]. According to NEST, the corresponding NR energy threshold is $\approx 5.3 \mathrm{keV}$ for a 3 -phe coincidence requirement (while we adopt $5.8 \mathrm{keV}$ for sensitivity estimates). Note that a lower, 2-fold coincidence may be possible (as in LUX) that would lower this threshold.

There are several basic features of the light collection response shown in Figure 3.5.1. The first, obvious from the figure, is that for most depths more light is collected in the bottom array that the top. This is due to the strong total internal reflection at the liquid surface due to the mismatch in refractive index, giving a critical angle for total internal reflection of $36^{\circ}$, and also the good match in VUV refractive indices between the quartz in the PMT windows and LXe. The second is the high degree of scattering from PFTE surfaces. This can be seen by considering the $8.5 \%$ mean PDE (baseline scenario), which, with a $25 \%$ PMT QE, implies a $34 \%$ probability of a photon striking a photocathode, while the photocathode coverage is only $\approx 14 \%$ of the total TPC internal surface. In the simulations the average number of scatters on PTFE is a depth dependent value between about 3 and 5. Thus, the value of PTFE reflectivity is quite important: the detector is effectively a "mirrored box" in which the value of light collection is the result of a competition between a photon being detected at a photocathode, and absorption that occurs with low probability but a high number of chances as the photon scatters around the detector. Finally, though not obvious in the figure, is that the mean path length traveled is a depth-dependent value between 3 and $5 \mathrm{~m}$. Rayleigh scattering is also strong (with $30 \mathrm{~cm}$ length, the mean number of Rayleigh scatters is between 10 and 20), but does not significantly alter either the pattern or amount of light collection. 


\subsubsection{TPC Optical Properties}

Here we discuss the reflectivity of PTFE in LXe, the absorption length in LXe, and the optical properties of the grids. The values shown in Table 3.5.1 are based on our best understanding, but, remarkably, they also happen to result in roughly equal loss of photons to absorption in the PTFE, LXe, and the grids. Somewhat modest gains (or losses) can be achieved by maximizing (or doing less well in) any one of these parameters, while the combined effect of improving (or doing worse in) all three could result in perhaps doubling (or near halving) of the overall light collection.

The photon absorption length in the bulk LXe depends on the purity of the liquid with respect to trace amounts of contaminants with absorption bands overlapping the LXe scintillation spectrum, mostly $\mathrm{H}_{2} \mathrm{O}$ and $\mathrm{O}_{2}$. For the tight purity requirements for those electronegative species ( $0.1 \mathrm{ppb}$, see Chapter 6), values in excess of $100 \mathrm{~m}$ can be expected (see Figure 15 in [23]). We have adopted a somewhat conservative $30 \mathrm{~m}$ baseline, along with $15 \mathrm{~m}$ and $100 \mathrm{~m}$ as pessimistic and optimistic values, respectively. The S1 PDE depth profile is shown in Figure 3.5.2 (left) for several values of this parameter. In the baseline and pessimistic scenarios, absorption is a comparable loss term to absorption on PTFE and grids, but becomes sub-dominant with the optimistic $100 \mathrm{~m}$ length and achieving even longer lengths gives diminishing benefit.
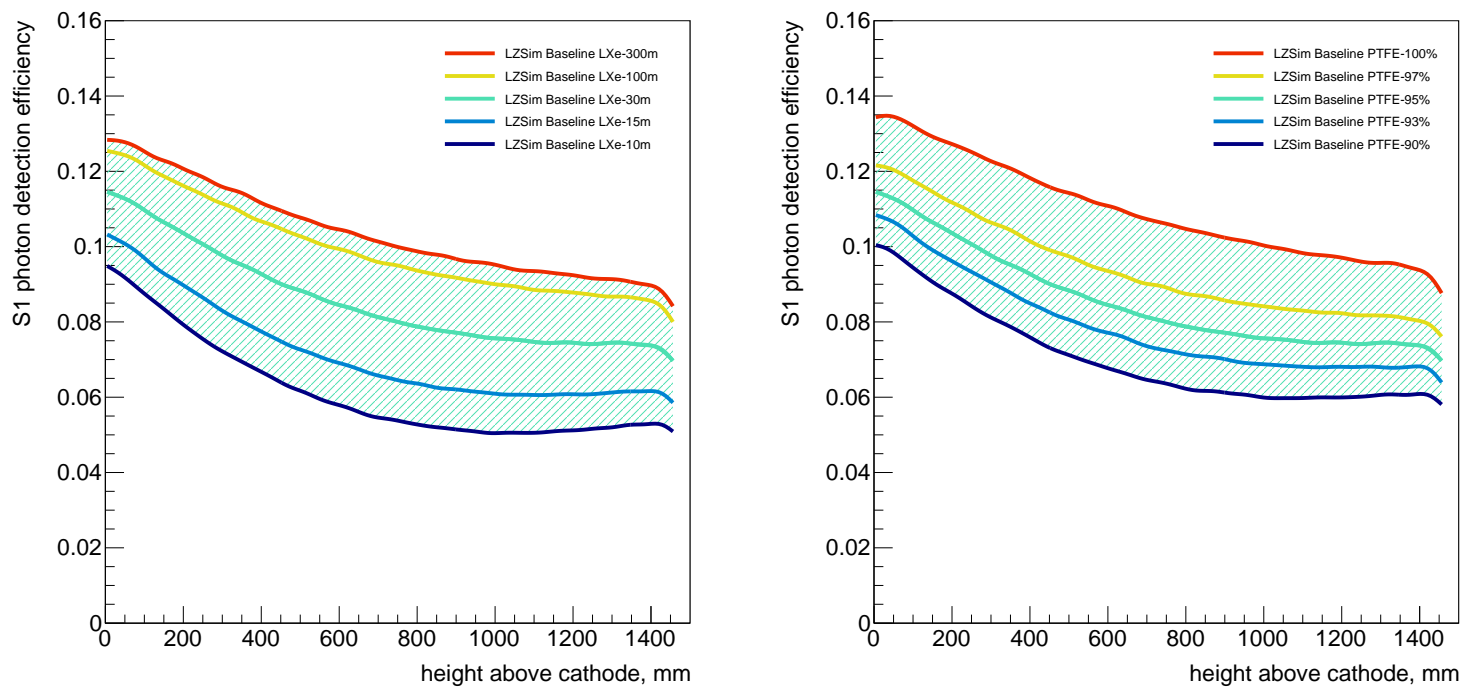

Figure 3.5.2: Left: S1 PDE $\left(\alpha_{1}\right)$ as a function of the photon absorption length in the liquid; Right: S1 PDE as a function of the PTFE/LXe interface reflectivity.

The four electrode grids in the LZ TPC all affect light collection through obscuration and wire reflectivity and, in a chamber where other sources of optical extinction have been minimized, these grids can have a significant effect on the light yield. The limited literature on reflectivity for metals at $178 \mathrm{~nm}$ indicates that very high values are unlikely, hence we have adopted values between $0 \%$ and $40 \%$. This range is motivated by expectation that the specific surface treatment of the stainless steel grid material might have an effect on reflectivity, and that the final electropolishing step that we take in grid production could be beneficial. It is conceivable that a (conductive) reflective coating could be deployed (e.g., Al). The grid opacities shown (for normal incidence) in Table 3.6.3 are selected as a compromise between minimal opacity and minimizing the electric field at the wire surfaces, especially for the cathode and the gate grid, which is also cathodic. It is possible that the ongoing HV tests of grids (Section 3.10) may allow to deploy grids with higher geometrical transparency, which has a similar effect as increasing the reflectivity. Because light is partially trapped in the liquid phase by total internal reflection at the liquid surface, the grids in liquid have a relatively larger 
effect than the anode in the gas. This is fortunate, because considerations of generating uniform S2 light, discussed in Section 3.6, lead to the anode being the least transparent of the grids.

Perhaps the most important optical parameter is the reflector used in constructing the TPC. Based on a decade of experience, PTFE is indeed the best reflector for LXe scintillation, and also for properties other than optical: The manufacturing process yields very radiopure material ( $\sim 1 \mathrm{ppt}$ in U/Th); it has good mechanical properties (despite the $1.4 \%$ to $1.5 \%$ thermal contraction to LXe temperatures [9, 10]); and outgassing rates are relatively low. The reflectivity values we have adopted are based on optical simulations of the performance of LUX, similar analysis of other detectors, and recent results from small chamber measurements ongoing at LIP-Coimbra and recently starting up at Michigan. These test studies are discussed in Section 3.10. The effect of this parameter on the S1 PDE is shown in Figure 3.5.2 (right).

To reduce the dead volume around the active LXe, as well as outgassing and potential backgrounds, it is desirable to minimize the thickness of the PTFE walls of the TPC and Skin detectors. A lower limit is established by the transmittance of PTFE to Xe scintillation light, and the need for optical isolation between the TPC and Skin regions as well as between these and any dead regions containing LXe. The transmittance of the PTFE used in LUX was measured at LIP-Coimbra as a function of thickness and for different wavelengths: Xe gas scintillation $(178 \mathrm{~nm})$ as well as $255 \mathrm{~nm}, 340 \mathrm{~nm}$ and $470 \mathrm{~nm}$. Results for Xe scintillation light show a transmittance $<0.1 \%$ for $1.5 \mathrm{~mm}$ PTFE thickness (but rising significantly to as much as $10 \%$ for $5 \mathrm{~mm}$ in the case of $470 \mathrm{~nm}$ blue light). The TPC wall thickness in LZ is $15 \mathrm{~mm}$, while the covering tiles of the vessel in the skin region is $1 \mathrm{~mm}$ and a similar though non-uniform thickness is used on the PMT arrays.

\subsubsection{PMT Arrays}

The two PMT arrays, shown in Figures 3.4.6 an 3.5.3, are similar, but not identical. Since most light is collected in the bottom array, it is a closed-packed hexagonal array of 241 PMTs, optimized purely for maximum photocathode coverage within the mechanical constraints of the low-mass structure that holds the PMTs. The mechanics of the array and PMT mounting are discussed in Section 3.4.5. In the bottom array the $76 \mathrm{~mm}$ diameter tubes are housed in $80 \mathrm{~mm}$-diameter holes on an $82.5 \mathrm{~mm}$ center-to-center spacing, resulting in a $54 \%$ coverage fraction of the $64 \mathrm{~mm}$ diameter photocathode surfaces. The spaces between the photocathode surfaces are fully covered with PTFE.

By contrast, while the top array collects a significant fraction of S1 light for events near the top of the detector, this array has the crucial role of reconstructing the $x, y$ location of events from the S2 signal. Especially critical is the accuracy of reconstructing the position of "wall events" that result from interactions near the vertical cylindrical surface of the PTFE that defines the TPC. A particular concern is the population of decays from radon progeny plated out on the TPC walls. These consist of ER interactions from the beta decays and gamma-ray emission from the ${ }^{210} \mathrm{~Pb}$ sub-chain, and from alpha particles and $\approx 100 \mathrm{keV}{ }^{206} \mathrm{~Pb}$ nuclear recoils from the alpha decay of ${ }^{210} \mathrm{Po}$. All of these, due to both energy and charge loss at the wall, lead to a broad distribution of low-energy signals that in part overlaps with the NR signal region in S2-S1 space. Therefore, it is critical to minimize the leakage of these events towards the center of the detector from errors in the reconstruction algorithm in order not to compromise the fiducial mass at low energy. Here the placement of the outer few PMT rows is critical. In contrast to the bottom array design, which is fully contained within the TPC diameter, the top array must overhang the edge of the TPC or all the reconstruction bias will point inward. Ideally, at least a full row of tubes would be located beyond the inner radius of the chamber. This is not possible due to the proximity of the inner cryostat vessel, and instead we locate the outermost circle of tubes at the largest-possible radius, which aligns the PMT centers above the TPC wall. From among a study of several layouts we adopted a "hybrid" array of 253 PMTs which has two nearly circular rows of PMTs at the perimeter but transitions to an hexagonal pattern in the center. The two outer circular rows maximize uniformity at the edge of the detector, which improves the uniformity 

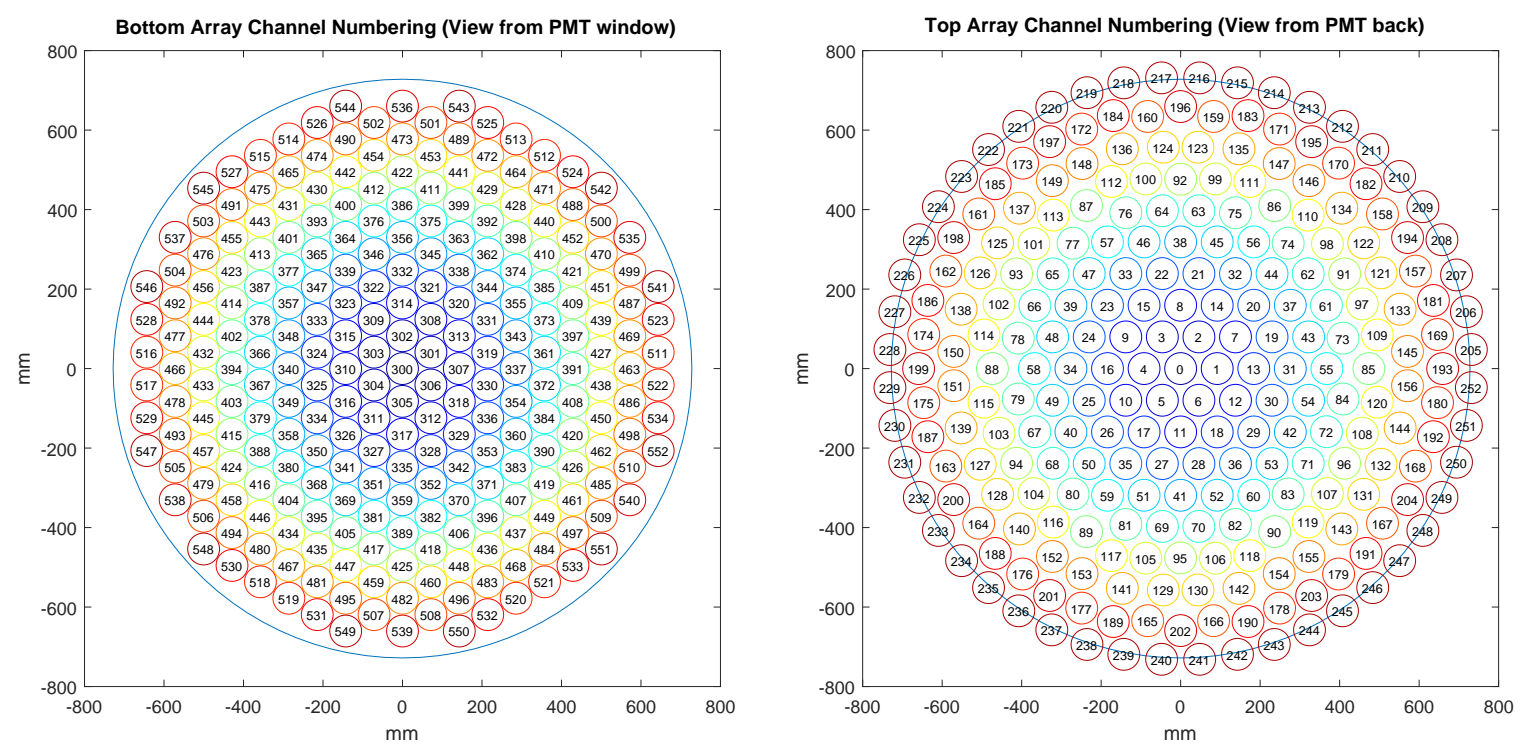

Figure 3.5.3: Layout of the bottom (left) and top (right) PMT arrays viewed from above. The channel numbering is done by radial position, with similar distances to the center indicated by color.

of the $x, y$ response near the wall and minimizes inward leakage. The larger overall diameter leads to the larger number of tubes, and the array is slightly less compact it the central hexagonal region, with a $93 \mathrm{~mm}$ center-to-center spacing (with the same $80 \mathrm{~mm}$ holes as the bottom array). We return to this optimization in Section 3.6.5.

\subsection{S2 Production and Detection}

Electrons escaping the interaction site are drifted under the influence of the "drift" electric field in the central part of the TPC to the electroluminescence region where they create the S2 signal. In this section we discuss the design of this region, including that of the electrode grids that create the drift and extraction/electroluminescence fields. The field cage that works in conjunction with the grids to generate the drift field is described in Section 3.2.

The electroluminescence region of the TPC is located at the top of the field cage, with the gate and anode electrodes (nominally $13 \mathrm{~mm}$ apart) straddling the liquid surface. The liquid level is controlled by a weir system at the edge of the TPC, as detailed in Section 3.8. This region controls the emission of the drifting electrons into the vapor phase and the subsequent production of electroluminescence photons, in proportion to the number of ionization electrons drifted away from the interaction site. This response channel readily provides sensitivity to single ionization electrons emitted from the liquid.

The S2 signal is also used for spatial localization of the interactions. In particular, the accurate reconstruction of "wall events" drives both the gain of the S2 response (involving the optimization of both photon production and collection efficiency) and of the layout of the top PMT array; this optimization is discussed below too.

Three main parameters characterize the S2 response: (1) the photoelectron yield, which depends itself on the cross-surface extraction probability for ionization electrons, the electroluminescence gain, and the efficiency of light collection for photons generated in the electroluminescence region $\left(\alpha_{2}\right)$; (2) the S2 pulse width, which is proportional to the electron transit time in the gas phase to first order; and (3) the resolution of the S2 signal, which depends on the detailed electric field distribution near the grid wires. Once the 
gate-anode separation is fixed (and therefore the gas gap $L_{g}$ ), these characteristics depend on operating parameters such as the xenon vapor pressure $P$ and the voltage applied to those electrodes $\Delta V$.

The S2 performance is affected by other electrostatic considerations (such as the maximum fields that can be sustained at the wire surfaces), and by mechanical considerations, such as the manufacture feasibility of large and densely-meshed wire grids, grid deflection and non-parallelism, etc. In the following sections we highlight the baseline design and the design goal, and describe how the S2 response depends on these operating conditions.

\subsubsection{S2 Photon Production}

For the smallest S2 signals, generated by one to a few ionization electrons, the main S2 requirements are: (1) definition of the single-electron response with a high signal-to-noise ratio, to allow absolute calibration of the ionization channel and to enable physics searches down to S2 signals as small as a few electrons; and (2) sufficiently large S2 signal for accurate reconstruction of the $(x, y)$ location of peripheral interactions, such as those arising from contamination on the TPC walls. This motivates a photon yield of at least $\approx 50$ photoelectrons per emitted electron, especially at the edge of the TPC. More details on how the S2 pulse size affects the reconstruction of wall events was given in LZ CDR [1]. Key parameters related to S2 photon production in LZ are presented in Table 3.6.1.

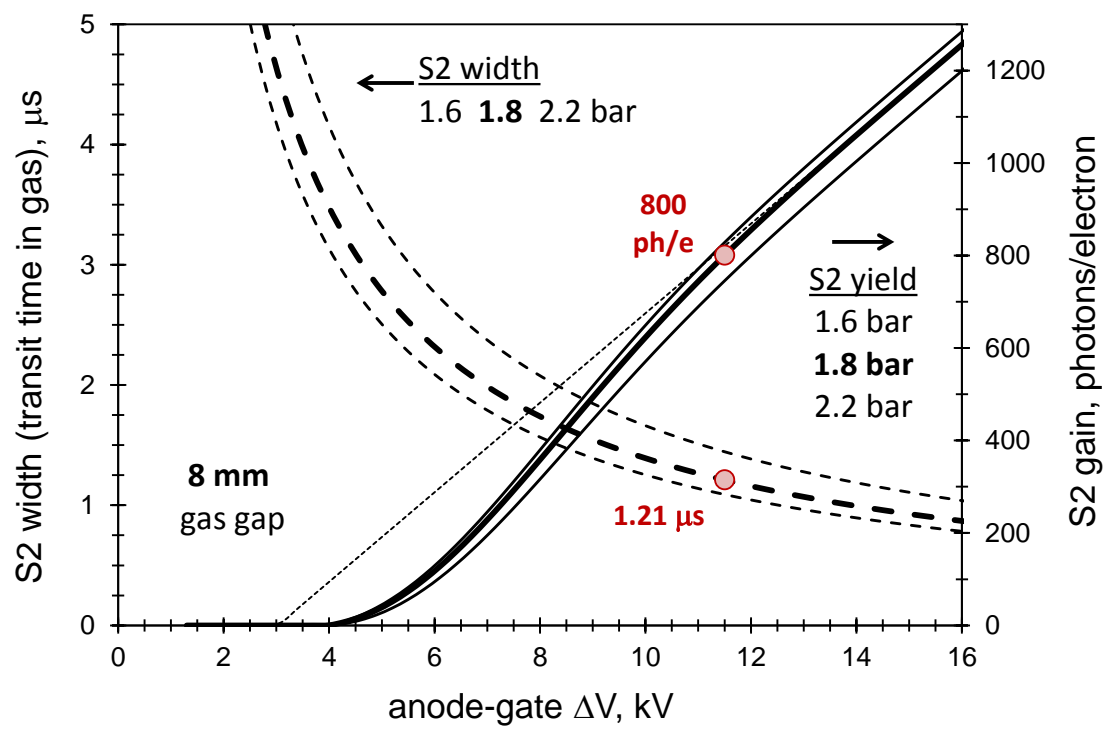

Figure 3.6.1: Dependence of the S2 photon yield and mean S2 pulse width (ignoring longitudinal diffusion in the liquid) on the voltage between anode and gate electrodes. The nominal photon yield [6], including the electron emission probability [5], and the electron transit time in the gas phase (S2 pulse width) [32], are indicated at the nominal $\Delta V=11.5 \mathrm{kV}$, for operating pressures around the $1.8 \mathrm{bar}$ nominal and a gas gap of $8 \mathrm{~mm}$ ).

Considering an S2 photon detection efficiency of $\approx 5 \%$ for the top array for peripheral interactions, predicted by simulation as presented below, the above photoelectron yield implies a minimum of 800 photons generated per emitted electron. For a gate-anode distance of $13 \mathrm{~mm}$ with $L_{g}=8 \mathrm{~mm}$, this is achieved with $\Delta V=11.5 \mathrm{kV}$ at the operating pressure $P=1.8 \mathrm{bar}$, as shown in Figure 3.6.1. A voltage of $+5.75 \mathrm{kV}$ will be applied to the anode and $-5.75 \mathrm{kV}$ to the gate, leaving the liquid surface near $-2.7 \mathrm{kV}$. Note, however, that the total gate-anode voltage is the real requirement, which gives the flexibility to trade-off between anode 
and gate voltages in case the nominal division becomes problematic (the anode sits in the gas phase, which is less resilient electrically and can give rise to spurious electroluminescence, but the gate is a cathodic electrode, and therefore susceptible to spurious electron emission).

All of these parameters are intimately connected to S2 light production: Both the electroluminescence yield and the electron drift velocity in the gas are determined by the reduced electric field in that region, $E / P$; in addition to the applied voltages, the electric field depends on both $L$ and $L_{g}$. Therefore, these parameters must be studied together and their optimization is subtle. We described two viable configurations in the CDR and the one described here is intermediate between them in terms of gas gap and photon yield. The gas gap is sufficiently large to provide high gain, low variance of S2 photon production, and allowance for electrostatic deflection during operation (which will force the grids closer together at the detector center), but small enough to preserve the required dynamic range of the optical readout.

Table 3.6.1: Main S2-related parameters. The predicted S2 response is indicated for the parallel-field model assumed in Figure 3.6.1, as well as for the more detailed modeling illustrated in Figure 3.6.4. A nominal yield of $800 \mathrm{ph} / \mathrm{e}$ is used for sensitivity calculations (this includes a non-unity emission probability.

\begin{tabular}{|lr|}
\hline Parameter & value \\
\hline Gate-Anode separation (and tolerance) & $13.0 \mathrm{~mm}( \pm 0.2 \mathrm{~mm})$ \\
Gas gap (and tolerance) & $8.0 \mathrm{~mm}( \pm 0.2 \mathrm{~mm})$ \\
Field in LXe (GXe) & $5.2 \mathrm{kV} / \mathrm{cm}(10.2 \mathrm{kV} / \mathrm{cm})$ \\
\hline Electron emission probability & $97.6 \%$ \\
\hline S2 photon yield & $820 \mathrm{ph} / \mathrm{e}$ \\
S2 width FWHM & $1.2 \mu \mathrm{s}$ \\
\hline Detailed modeling & \\
\hline S2 photon yield & $910 \mathrm{ph} / \mathrm{e}$ \\
S2 photon rms & $2.0 \%$ \\
S2 width FWHM & $1.0 \mu$ to $2.0 \mu \mathrm{s}^{a}$ \\
\hline
\end{tabular}

${ }^{a}$ The larger value is for diffusion-broadened S2 pulses from interactions near the cathode (see Figure 3.6.4).

Nonetheless, the choice of higher voltages across a longer gap has some disadvantages which must be mitigated. The electron emission probability at the liquid surface decreases rapidly when the field in the gas drops below $10 \mathrm{kV} / \mathrm{cm}$ [5]. In Figure 3.6.1, the S2 yield assuming full extraction efficiency is represented by the dotted line, while the continuous lines include the field-dependent extraction probability. For our nominal parameters, that probability is close to unity, but poor extraction efficiency may result if nominal voltages fail to be achieved. We mitigate this with Phase-II of our System Test program described in Section 3.10.4-this includes testing the final gate-anode assembly to ensure that the design voltages can be realized.

Longer electron transit times in the gas also hide the effect of electron diffusion in the liquid, which encodes (modest) interaction-depth information on the $\mathrm{S} 2$ pulse shape. This information allows some coarse fiducialization, which is important for an "S2-only" analysis. The mean S2 pulse width is $1.2 \mu$ s, which will make the precise measurement of diffusion-broadening of the $\mathrm{S} 2$ response more difficult. If required, this may be mitigated with operation at lower drift field (see Table 3.3.1). 


\subsubsection{S2 Photon Detection}

The LZSim model was used to obtain the baseline photon detection efficiency for the S2 response $\left(\alpha_{2}\right)$, representing the fraction of photons which are detected by the PMT arrays. This parameter multiplies the photon yield per electron discussed in Section 3.6.2 and the number of ionization electrons drifted away from the interaction site to yield the total $\mathrm{S} 2$ response (assuming no loss to electronegative impurities). The dependence of $\alpha_{2}$ on radius is shown in Figure 3.6.2 and values for central and peripheral locations are summarized in Table 3.6.2.
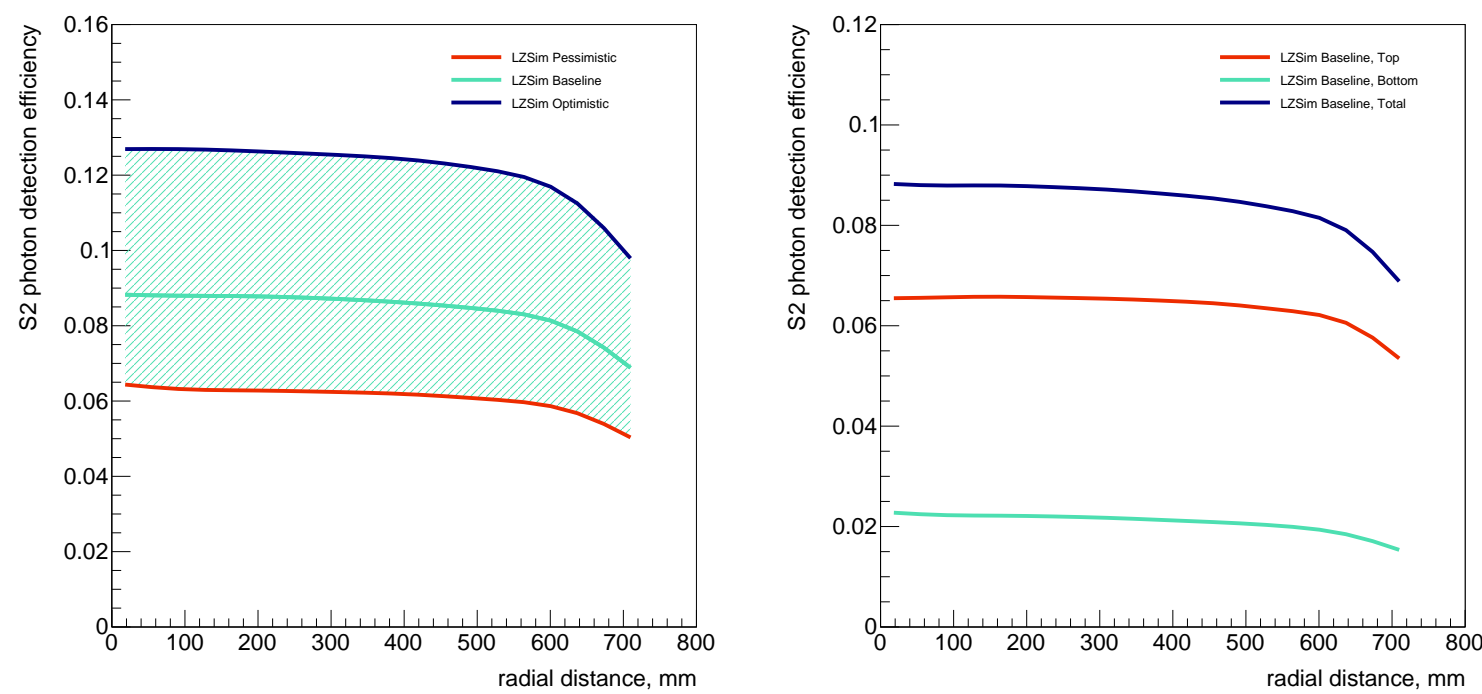

Figure 3.6.2: Left: Radial dependence of S2 photon detection efficiency for several scenarios; the nominal optical model averages $\alpha_{2}=12.0 \%$ and is used to calculate the LZ baseline performance; Right: Contributions from top and bottom arrays for baseline optical model.

The optical parameters are as assumed for the $\alpha_{1}$ simulation described in Section 3.5.2. In this instance, the top array makes the largest contribution to $\alpha_{2}$ as expected, partly due to the optical mismatch between the gas and liquid phases. Other driving parameters are the anode transparency and the reflectivity of PTFE in the gas phase (trefoil structures surrounding the PMT windows).

Table 3.6.2: S2 photon detection efficiency $\left(\alpha_{2}\right)$ and photoelectron yield for single electron signals (in brackets, assuming $800 \mathrm{ph} / \mathrm{e}$ ) for central and TPC-wall interactions.

\begin{tabular}{|lcc|}
\hline PMT array & Center & Edge \\
\hline Top & $6.6 \%(52 \mathrm{phe} / \mathrm{e})$ & $5.4 \%$ (43 phe) \\
Bottom & $2.2 \%(18 \mathrm{phe} / \mathrm{e})$ & $1.5 \%$ (12 phe) \\
\hline Top+Bottom & $8.8 \%(70 \mathrm{phe} / \mathrm{e})$ & $6.9 \%$ (55 phe) \\
\hline
\end{tabular}

Towards the center of the TPC $\alpha_{2}$ exceeds $8 \%$, decreasing to below $7 \%$ for wall events, which enables a single electron response comfortably above 50 phe/e everywhere in the detector. For wall events the top array detects some 40 phe/e, which is sufficient for the effective reconstruction of plateout backgrounds as discussed later in this section.

The bottom array converts just over $2 \%$ of the S2 photons, with each PMT contributing a fairly constant $0.01 \%$ to the overall detection efficiency in that array. This allows LZ to reconstruct large S2 pulses which 
will saturate many channels in the top array, where an individual PMT located just above the S2 vertex can convert $2 \%$ of the S2 photons - as much as the whole of the bottom array.

Using instead the optimistic optical parameters presented in Section 3.5.2-which bring the baseline $\alpha_{1}$ from $8.5 \%$ to $13.3 \%$-the effect on $\alpha_{2}$ is comparable: the baseline S2 detection efficiency increases from $8.8 \%$ to $12.7 \%$ for central interactions, with roughly equal gains in both arrays in absolute terms.

\subsubsection{S2 Resolution and Electrode Configuration}

In addition to appropriate S2 gain and pulse width, we must ensure that the overall energy resolution of the TPC is good. At low recoils energies (ER and NR) this is dominated by statistical fluctuations on the small number of detected S1 and S2 quanta (including significant recombination fluctuations) and the quality of the S2 design is unlikely to be a dominant factor for WIMP searches. However, for MeV electron-recoil energies, instrumental effects eventually dominate the overall resolution since a combined energy scale using both S1 and S2 can eliminate the recombination fluctuations, and the number of S1 and S2 quanta is large.

This demands a low dispersion of photon production from the electroluminescence region, which in turn drives the detailed design of the gate and especially the anode grids. This is intimately related to the characterization of detector backgrounds, in particular enabling high-quality spectroscopy of radioactivity gamma-rays generating up to $\sim 10^{5}$ electrons-see Figure 3.6.3. These are important to understand lowenergy, external ER backgrounds, but especially to constrain radioactivity neutrons. Clearly, fine energy resolution is also required if $\mathrm{LZ}$ is to attempt the detection of $0 v \beta \beta$-decay in ${ }^{136} \mathrm{Xe}$. We quantify our resolution requirement at the respective Q-value of $2,458 \mathrm{keV}$, and we designed to achieve $2 \%$ in the combined energy scale ( $1.5 \%$ goal).

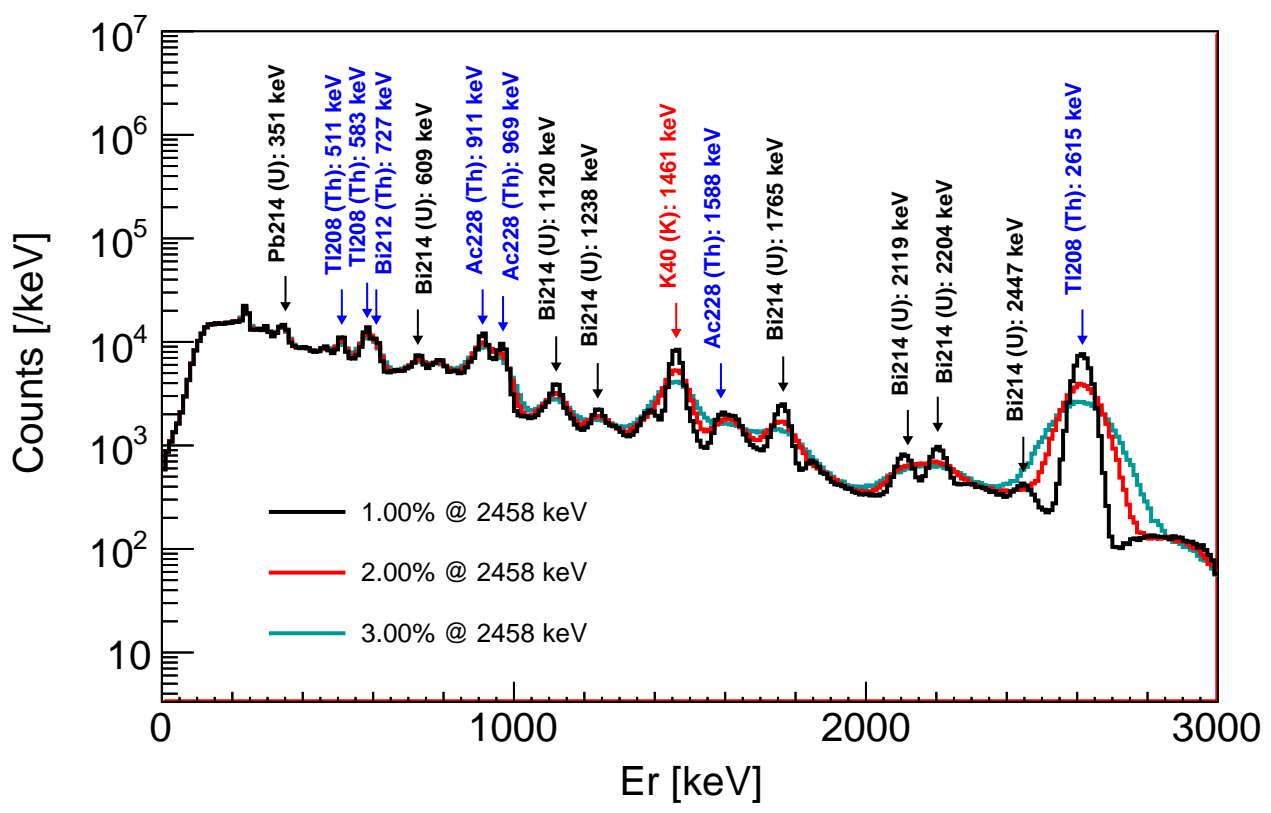

Figure 3.6.3: Energy-resolution for high energy electron recoils (S1-S2 combined energy scale). Spectrum is simulated ER background from the titanium cryostat in the full active volume in 1,000 days.

Therefore, fluctuations related to S2 photon production and detection must remain small, at $\sim 1 \%$ level. This motivated a detailed study of electroluminescence and electrode grid configuration presented in the LZ CDR, and subsequently the selection of the electrode grids listed in Table 3.6.3. Other factors contributing to the S2 resolution are the uniformity of response in the horizontal plane over the whole TPC diameter 
(e.g., wire sagging and electrostatic deflection), although those can be calibrated and hence removed to first order.

Aside from diffusion (in both gas and liquid phases), drifting electrons follow electric field lines and therefore their length and the field strength close to the wires must be carefully controlled to avoid substantial dispersion or even the possibility of significant charge multiplication (the first Townsend coefficient for cold $\mathrm{Xe}$ vapor at the 1.8 bar operating pressure reaches $1 \mathrm{e} / \mathrm{mm}$ at $35 \mathrm{kV} / \mathrm{cm}[32,33])$. Two additional concerns, which are intimately related, are the ability of the electrodes to hold HV and their VUV reflectivity, which depend strongly on the wire material and surface properties; we have investigated these issues through the dedicated R\&D activities described in Section 3.10.2.

Table 3.6.3: TPC electrode grid parameters (all $90^{\circ}$ woven meshes). Surface fields are wire-average for $-100 \mathrm{kV}$ cathode voltage. The geometric opacity is given at normal incidence.

\begin{tabular}{|lrrcrc|}
\hline Electrode & Voltage & Wire diameter/pitch & Number & Wire field & Opacity \\
\hline Anode & $+5.5 \mathrm{kV}$ & $100 \mu \mathrm{m} / 2.5 \mathrm{~mm}$ & 1,184 & $+55 \mathrm{kV} / \mathrm{cm}$ & $8.0 \%$ \\
Gate & $-5.5 \mathrm{kV}$ & $75 \mu \mathrm{m} / 5.0 \mathrm{~mm}$ & 592 & $-62 \mathrm{kV} / \mathrm{cm}$ & $3.0 \%$ \\
Cathode & $-50 \mathrm{kV}$ & $100 \mu \mathrm{m} / 5.0 \mathrm{~mm}$ & 592 & $-31 \mathrm{kV} / \mathrm{cm}$ & $4.0 \%$ \\
Bottom & $-1.5 \mathrm{kV}$ & $75 \mu \mathrm{m} / 5.0 \mathrm{~mm}$ & 592 & $+34 \mathrm{kV} / \mathrm{cm}$ & $3.0 \%$ \\
\hline
\end{tabular}

The optimization of the anode geometry involves a compromise between optical, electrostatic, mechanical, and electroluminescence properties. The latter were assessed through full electron transport modeling, in particular examining the $\mathrm{S} 2$ photon production statistics from single electron drifts in the gas phase of several candidate geometries. This method has been validated by comparison of simulated and actual signals in the LUX detector [34]. Subsequently we applied this methodology to the baseline gate-anode electrode configuration, and some results are shown in Figure 3.6.4. This involved the detailed modeling of electrostatic fields in the electroluminescence region using the Elmer solver [35] and the meshing tool Gmsh [36], followed by simulation of electron transport and photon production with Garfield++ [37] using xenon transport parameters from Magboltz [33].

As discussed below, all of the grids are woven meshes. Increasing the density (smaller pitch and/or larger diameter wires) decreases the electric field near the wire surfaces, reducing spurious electron emission from the gate and charge multiplication near the anode. It also provides more uniform field lines, especially near the anode, which is important for reducing dispersion of the produced S2 signal. However, decreasing the opacity increases $\alpha_{1}$ and $\alpha_{2}$, and increases the ease of manufacture. The gate pitch is half that of the anode pitch, and the two grids are aligned such that an anode wire crossing sits directly above the center of each open square in the gate grid. As the electrons pass through the gate they are focused towards the center of each square, and hence towards this crossing. This alignment gives smallest spread in path lengths traversed from the liquid to the anode, and hence smallest dispersion in the generated S2 signal.

Details of the simulated performance of the baseline gate-anode design are shown in Figure 3.6.4. This study assumes that electrons start evenly spread in $(x, y)$ below the gate grid. The $\mathrm{S} 2$ signal resolution and the timing performance are those reported in Table 3.6.1 above. Without diffusion the pulse would have a "box" shape if the anode were a plane; the spike at the end of the pulse (shown in d) is from electrons moving through the short region of enhanced field near the anode wires. The predicted photon yield is slightly higher than suggested by the parallel-plate calculation presented in Figure 3.6.1 for this reason. Conversely, some electrons move near the center of the unit cell, experiencing lower average fields, and this motivates the occasional lower yield visible in panel c) of the same figure. Those electrons also arrive slightly later, and form the tail above $1 \mu$ s shown in d). Sparser grids aggravate both of these effects very quickly. 
a)

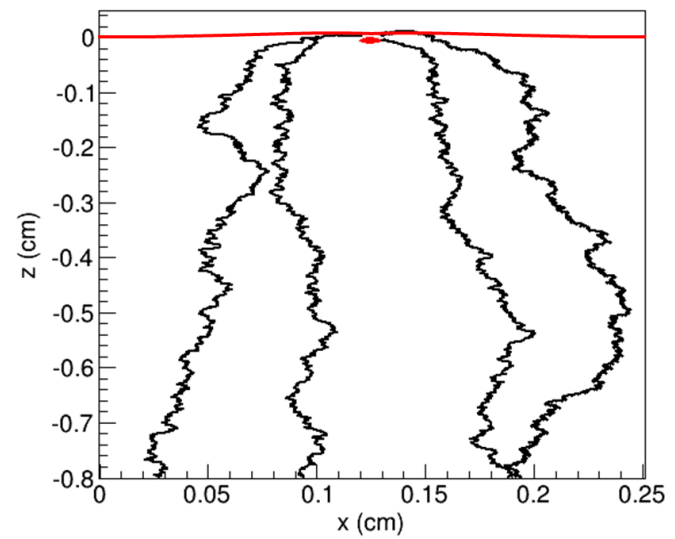

c)

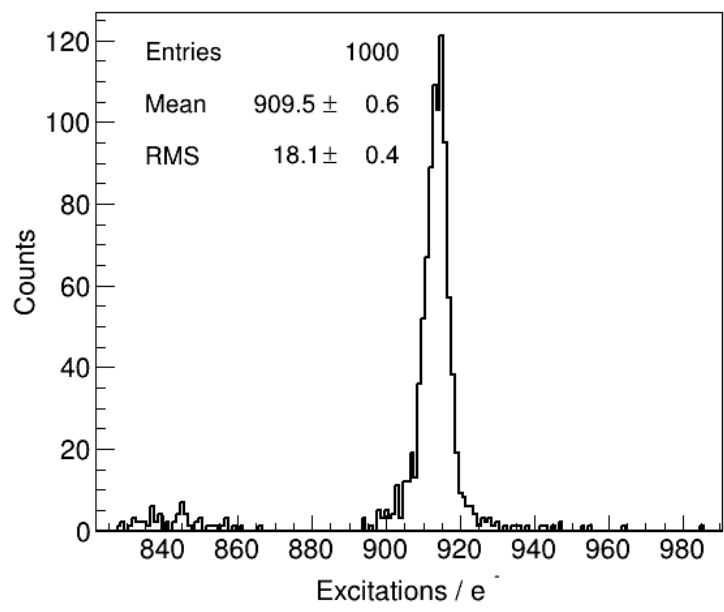

b)
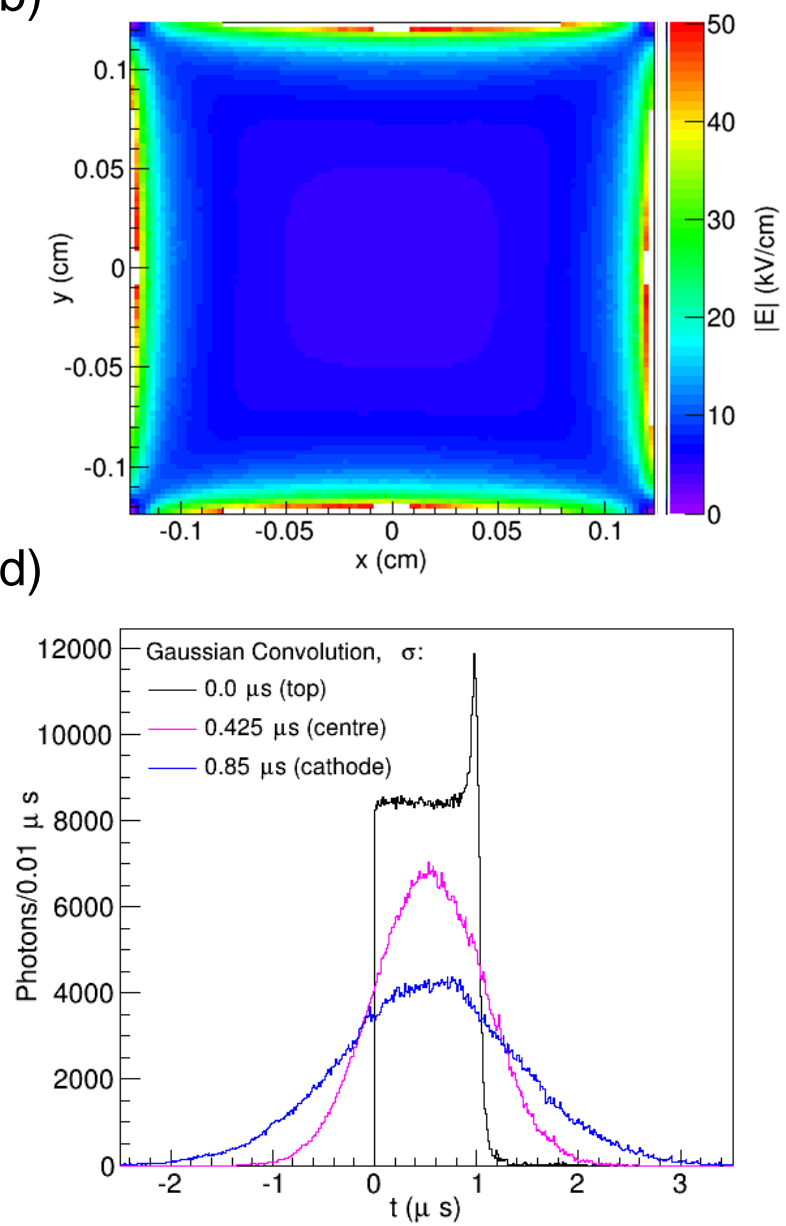

Figure 3.6.4: Simulated S2 electroluminescence response of 1,000 electrons for the baseline gate-anode design [34]. a) Examples of electron drifts from the liquid surface (at $z=-0.8 \mathrm{~cm}$ ) until their collection at the anode mesh wires (shown in red); b) Electrostatic field strength through the anode plane; c) Photon yield (number of excitations), confirming $2 \% \mathrm{rms}$ for electrons distributed evenly in the liquid xenon bulk; d) S2 simulated pulse shape of the electron signal, without longitudinal electron diffusion in the liquid (black), and the same distribution convolved with the amount of Gaussian diffusion expected for drift from the center (magenta) and bottom of the detector (blue).

\subsubsection{Design and Fabrication of the Grids}

The four grids, whose basic parameters are list in Table 3.6.3, are all of a crossed mesh design. They are fabricated by weaving and individually tensioning a set of series 300 stainless steel wires, and held by a set of low mass grid stainless steel rings housed within the field cage. The crossed mesh design has several benefits. Compared to a parallel set of wires, it presents a more uniform load on the ring, allowing it to be smaller in mass. The crossed wire mesh is also more mechanically robust than free-standing wires, and the fields are more uniform. Note that electroformed grids are not readily available at the size of LZ, and also do not naturally have the minimum-surface field producing the profile of a round wire. The wires are chosen to have the highest available surface smoothness. 
Large, attractive electrostatic forces exist between the gate and anode grids because of the strong electroluminescence field and, to a lesser extent, between the cathode and bottom grids due to the high field in the reverse field region. The tension on the wires in these grids, nominally $0.25 \mathrm{~kg}$ on the anode and $0.5 \mathrm{~kg}$ on the others, should achieve less than $2 \mathrm{~mm}$ combined maximum deflection between the gate and anode, when the grids are treated as individual wires. These loads are well within the yield strength of available stainless steel wires, but nonetheless represents an important mechanical requirement on the assembly. Evaluation of the mesh grid is much more difficult, though approximate treatments may be possible. We expect that the resulting deflection will be smaller than that estimated from the individual wire calculation, possibly allowing a reduction in tension. The actual tension in full-size prototype grids will be directly measured under field. Note the tension needed to minimize this overall deflection is larger than the minimum tension needed to prevent the well-known wire-to-wire "saw-tooth" instability encountered in a single plane of wires in a wire chamber.

The technique for weaving the wires is analogous to the process used in the textile industry for making woven cloth with a loom. Initially, the wire forces are supported by the loom. The technique for anchoring the grid wires is to capture them between two support rings that are epoxied together. The epoxy locks the grid wires in the space between the support rings. Once the epoxy is fully cured, grid wires outside the support rings are trimmed off, and the wire load is solely carried by the rings. The process for making all the grids is the same as the cathode, albeit with different diameter wires and spacings. After production, the entire grid assemblies will be electropolished and passivated chemically to achieve the highest possible surface quality. Such a treatment has been shown to be beneficial on single wire samples as described in Section 3.10.2. We have demonstrated the technique of electropolishing completed grids for the Phase-I System Test TPC prototype which is described in Section 3.10.3.

\subsubsection{Reconstruction of Peripheral Interactions}

The mis-reconstruction to smaller radii of peripheral background events— such as those arising from radon progeny plateout on the inner field cage walls - can be a leading source of background in double-phase xenon TPCs (e.g. ZEPLIN-II [31] and LUX [25] were so affected). This must be addressed by both lowering plateout rates and ensuring good quality spatial reconstruction for the remaining decays. The layout of the top PMT array, and in particular of the peripheral tubes, is therefore of great importance. We considered five array configurations in our initial optimization and this was discussed in the LZ CDR (Section 6.5.3 in Ref. [1]). An additional study was carried out to optimize the hybrid configuration selected previously, based on further considerations including electric field between PMTs, S2 light collection uniformity, and mechanical feasibility.

Based on this new study we adopted for the top array a circular/hexagonal hybrid layout containing 253 PMTs, transitioning from a close-packed hexagonal core to a 48-unit circular outer row (see Figure 3.5.3). The minimum PMT separation is $86.3 \mathrm{~mm}$. Our methodology involved extensive optical Monte Carlo using the code ANTS2 [38] coupled to the Mercury vertex reconstruction algorithm [39]. This provided a realistic assessment of the position resolution of the chamber for small S2 signals and, in particular, the fraction of peripheral events that is misreconstructed into the TPC volume. This "leakage" fraction was the main design criterion used to select the best array configuration. In addition to the position of the outer PMTs, two other design parameters influence the peripheral position resolution: the distance between the anode grid and the PMT windows, and the reflectivity of the lateral wall in the gas. Regarding the latter issue, a low-reflectance material is desirable so as not to overly distort the spatial response of the outer PMTs. Titanium has $16 \%$ reflectance at $178 \mathrm{~nm}$, but its oxides can be more reflective in the VUV [40], and a nonconducting material is preferred to minimize fields. Thus, that region will be covered by Kapton ${ }^{\mathrm{TM}}$ foil instead. 


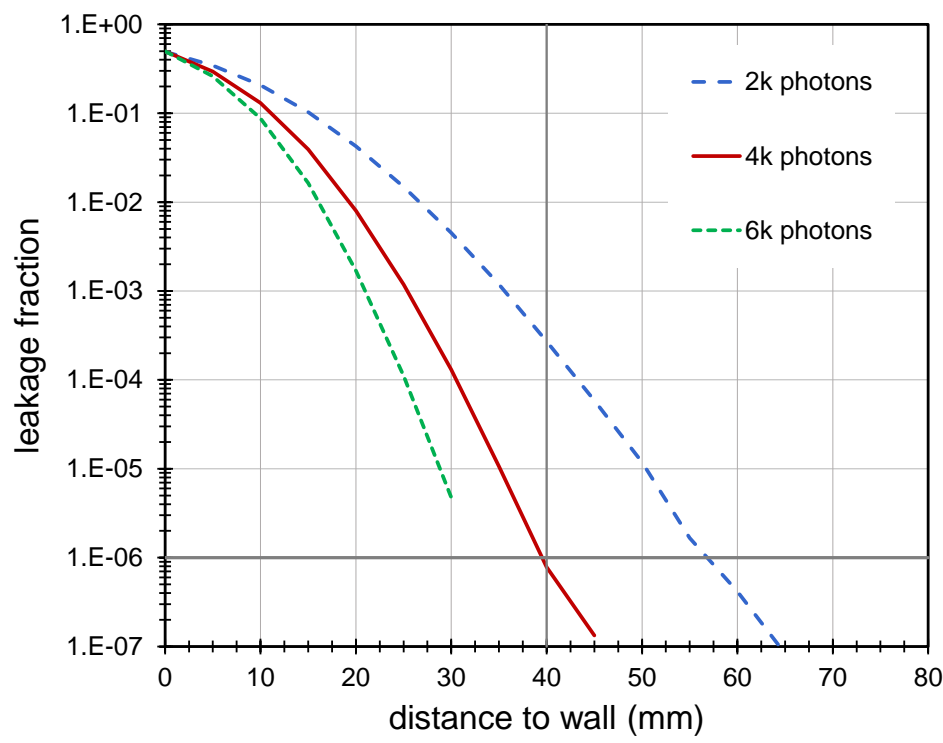

Figure 3.6.5: Simulated reconstruction of wall events as a function of signal size. For 4,000-photon S2 signals $\left(\approx 5\right.$ electrons emitted) a leakage of $10^{-6}$ is achievable at the edge of our preliminary fiducial volume which is located at $40 \mathrm{~mm}$ from the wall.

Figure 3.6.5 summarizes the results from a high-statistics study of the leakage past various reconstructed radii, with $40-\mathrm{mm}$ being the nominal distance to our preliminary 5.6-tonne fiducial volume. This leakage is indicated as a function of S2 signal size (with 4,000 S2 photons corresponding to about 5 emitted electrons). This confirms that we can achieve a very small leakage into the fiducial volume above the nominal S2 threshold.

It should be noted that the ionization removed from wall interactions tends to be pushed into the TPC as it drifts and ends up being emitted up to several centimeters away from the TPC edge (as shown in Fig. 3.2.2), which will help mitigate this background. We do not take this field distortion into account in this calculation.

\subsection{The Xe Skin Detector}

The region between the outer walls of the TPC and the inner cryostat vessel is called the "Xe Skin". This region provides necessary mechanical clearance to allow for detector assembly, houses instrumentation including PMTs and other sensors, and provides a standoff between biased TPC components and the electricallygrounded inner vessel. The Xe Skin contains more than 2 tonnes of LXe, and it is divided into two primary functional regions: a cylindrical, "side Skin" region outside of the main TPC field rings, and a "dome Skin" region underneath the TPC.

Because of the high density of LXe, gamma rays (and, to a lesser extent, neutrons) have a high probability of interacting or being absorbed in the Skin region. This poses a problem for the efficiency of the outer LS veto detector, a key element in reducing backgrounds in LZ, as secondary scatters that would otherwise be tagged in the Outer Detector are lost in the Skin. Additionally, for events that deposit energy in both the main TPC and the Xe Skin, leakage of light from the Skin to the TPC can compromise the rejection of the dominant ER background by increasing the observed S1 signal and lowering the S2/S1 ratio used for particle discrimination. To counter these effects, we instrument the Xe Skin region with 93 dedicated 1-inch R8520 PMTs in the top half of the side Skin, 20 2-inch R8778 PMTs in bottom half of the side skin, and 18 more 2-inch R8778 PMTs in the dome, turning the Xe Skin into a second component of the 
LZ veto strategy to clearly identify events with scattering vertices in the Skin. Instrumenting the Xe Skin also increases the amount of information available about the background environment of the WIMP target, improving the background model and the efficiency of the data analysis. The goal for the Xe Skin detector design is to achieve an energy threshold for observing ER scatters produced by gamma-ray backgrounds or radiative neutron capture on detector materials of $100 \mathrm{keV}_{\mathrm{ee}}$ in over $95 \%$ of the volume of the Xe Skin. A second goal is to minimize light leakage between the Xe Skin and the TPC. These are related goals: Since optical isolation is never perfectly realized, any scintillation emitted in "S2-inactive" regions of the detector must be read out to prevent the dangerous background topologies described above.

The side Skin is $4 \mathrm{~cm}$ wide near the top of the TPC, increasing to $8 \mathrm{~cm}$ in the lower half due to the tapered vessel shape. This region is instrumented with 93 1-inch R8520 PMTs viewing down located just below the LXe surface and a further 20 2-inch R8778 PMTs looking up. 18 of the R8778 tubes are arranged symmetrically around the bottom of the TPC, with 2 extra PMTs looking specifically at the region where the cathode HV feedthrough enters the detector. Light emission from surfaces is often the first sign of issues maintaining high voltage, and photons produced near the cathode region, whether from radioactivity or from voltage breakdown, have a high probability of entering the feedthrough umbilical and being absorbed. The additional PMTs increase the photocathode coverage in the area to mitigate such photon losses.

The Skin PMTs mount to the weir system as shown in Figure 3.1.1 on page 56, and to the bottom array as depicted in Figure 3.7.1. The inside surface of the inner cryostat vessel is lined with thin PTFE sheets for improved light collection, and the outer surface of the TPC walls also provides a PTFE reflector. The top side Skin region uses 1-inch PMTs, rather than the larger model in the TPC due to mechanical constraints. The Hamamatsu R8520 is specifically designed for LXe operation and was the primary PMT used, for example, in the XENON10 and XENON100 detectors $[26,30]$. The R8520 is a compact 1-inch, square PMT with quartz window and bialkali photocathode with a typical QE of $30 \%$ at $175 \mathrm{~nm}$. A gain of $10^{6}$ is provided by an 11-stage metal channel dynode chain. We use a smaller number of 2-inch R8778 tubes in the bottom side skin and in the dome also for mechanical constraints, as R11410 PMTs would not fit in either region. The R8778 PMTs will be recovered from the LUX experiment, where they have operated for several years in a low-background, LXe environment and have been well characterized.

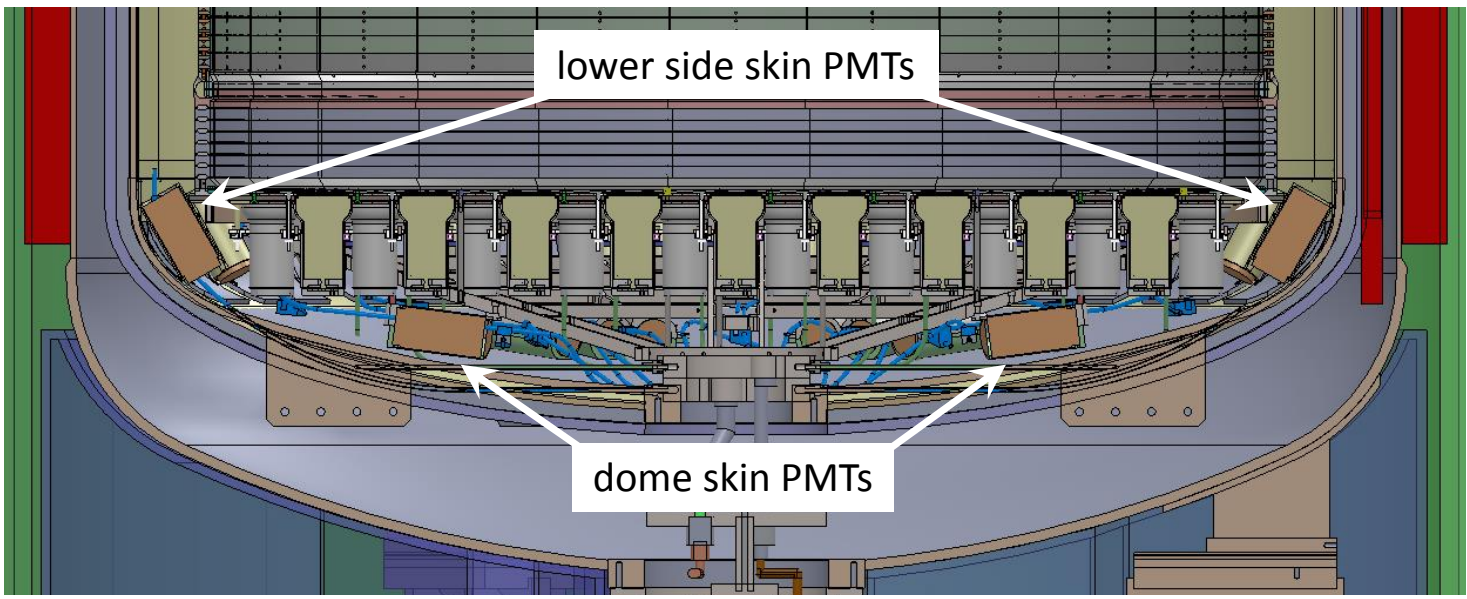

Figure 3.7.1: Mounting of the Skin PMTs in the dome and lower side regions. The upper Skin PMTs mount to the weir system as shown in Figure 3.1.1.

Similar to the inside surface of the TPC, PTFE will be used to cover the underside of the TPC PMT support structure facing the Skin. Both the main TPC PMTs and the R8778 PMTs will have reflective PTFE sleeves to reduce photon absorption on their metal envelopes. The dome Skin PMTs will be mounted to to the lower truss of the TPC structure as depicted in Figure 3.7.1. 


\subsubsection{Skin performance}

Design studies for this region begin by assessing the combined Xe Skin and Outer Detector veto performance as a function of threshold in the Skin. As described in Chapter 12, a comprehensive model of the LZ detector is implemented in GEANT4, and the effect of radioactivity in various detector components is simulated. The key performance metric of the combined anti-coincidence system is the veto inefficiency, defined as the number of unvetoed single scatter events in a detector divided by the total number of single scatters in that volume. This inefficiency varies with threshold in both veto regions. Figure 3.7.2 shows the veto inefficiency for single scatters caused by gamma-rays as a function of Skin threshold, showing a $50 \%$ increase as the threshold varies from $100 \mathrm{keV}_{\text {ee }}$ to $200 \mathrm{keV}_{\mathrm{ee}}$. In other words, the un-rejected background rate increases by that factor as the Skin threshold is increased. The requirement that the number of electron recoil events produced by detector materials be less than $10 \%$ of the predicted rate of neutrino-electron scatters therefore drives the requirement that the energy threshold in the Skin must be $100 \mathrm{keV}_{\mathrm{ee}}$. As the light collection efficiency in the Xe Skin is very non-uniform (comparing, for example, the side and dome regions), we include in the requirement the demand that $95 \%$ of the volume in the Skin meets the $100 \mathrm{keV}_{\text {ee }}$ threshold target.

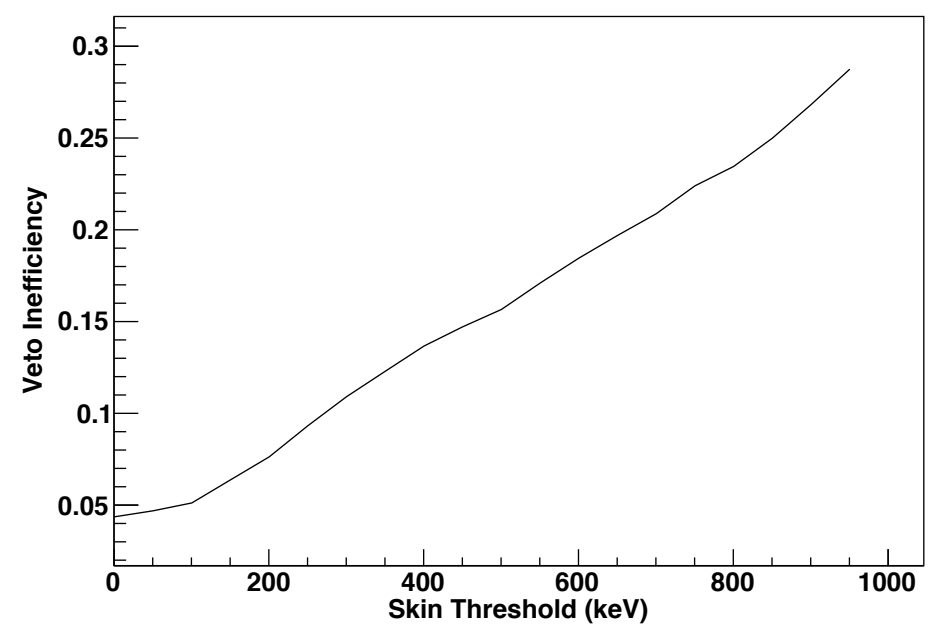

Figure 3.7.2: Skin veto inefficiency for gamma backgrounds, defined as the number of unvetoed singlescatter events divided by the total number of single-scatter events as a function of energy threshold in the $\mathrm{Xe}$ Skin. The inefficiency increases from $5.1 \%$ to $7.6 \%$ as the threshold rises from $100 \mathrm{keV}_{\text {ee }}$ to $200 \mathrm{keV}_{\text {ee }}$, representing a $50 \%$ increase in gamma backgrounds from internal radioactivity. The background requirements for LZ demand that an energy threshold of $100 \mathrm{keV}_{\text {ee }}$ be achieved in over $95 \%$ of the Xe Skin volume.

The next step of the design is to create an optical model for the Skin to understand how much photocathode coverage and surface reflectivity are needed. We use the same simulation geometry developed for the background studies, taking care to define the optical interfaces to understand the effect of surface reflectivity. We first divide the Xe Skin into $1 \mathrm{~cm} \times 1 \mathrm{~cm} \times 1 \mathrm{~cm}$ pixels. The electric field in each pixel is calculated from an electrostatic model, and we use the NEST package to find the mean number of photons produced by a $100 \mathrm{keV}$ electron recoil in each pixel, accounting for suppression in scintillation yield caused by the high electric field. Photons are then generated in each pixel and propagated through the optical model, and the photon collection efficiency (PDE) is defined as the number of observed photoelectrons per generated photon, including PMT effects such as quantum efficiency (as for the TPC). Finally, a pixel is deemed "good" if the PDE is high enough that $50 \%$ of $100 \mathrm{keV}_{\mathrm{ee}}$ events produce at least 3 detected photo- 
electrons. Figure 3.7.3 shows the PDE in the Skin as a function of position (averaged over azimuth) for the design described here. Over $97 \%$ of the Skin region achieves an energy threshold of $100 \mathrm{keV}_{\mathrm{ee}}$, meeting the requirement.

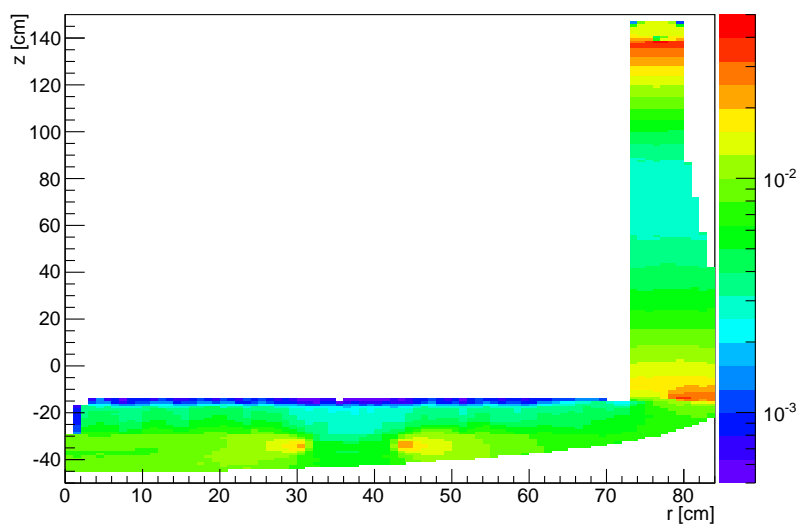

Figure 3.7.3: Photon detection efficiency (PDE) in the Skin region as a function of position (averaged over the azimuth) for the design described in the text. Over $97 \%$ of the Skin region achieves an energy threshold of $100 \mathrm{keV}_{\mathrm{ee}}$, meeting the requirement.

The Skin PDE is highly dependent on the wall reflectivity, especially in the side Skin, and the left panel of Figure 3.7.4 shows how this parameter varies as a function of $z$ in the side Skin for different reflectivity assumptions. Given the strong dependence, we set a requirement on the PTFE reflectivity of the cryostat liner of $95 \%$, the same as for the TPC walls. The final model described above assumes that only $98 \%$ of the wall is covered, allowing for joints or other imperfections in the liner. The threshold requirements are met in the dome region without any additional PTFE beyond that covering the PMTs.

The PTFE liner will be supported by metal "buttons" that are bonded to the cryostat walls using cryogenic epoxy. Simulations of the field in the skin show that metal buttons can be used without causing excessive surface fields, even near the cathode ring. A schematic showing how the buttons will hold PTFE tiles in place can be seen in the right panel of Figure 3.7.4. Holes will be cut into the liner to go around the buttons, and PTFE screws will hold PTFE washers above the liner, capturing it in place.

\subsection{Internal Fluid System}

Efficient purification of LXe is a significant challenge, and is especially important given the large size of LZ and the resultant long electron drift lengths and long photon path lengths involved. Purification is discussed in detail in Chapter 6, and the overall internal flow diagram is shown schematically in Figure 3.8.1. Liquid in the detector is continuously circulated to a purification tower located outside of the water tank, where it is evaporated in a two-phase heat exchanger and passed to a gas purification system. There, it is purified by a commercial heated getter. While the getter is highly efficient in a single pass, continuous purification has proved necessary in most previous such detectors, primarily because of the large amount of PTFE and other plastics especially in cables that serve as a long-term source of outgassing. After passing through the getter, the Xe returns to the liquid tower, where it is recondensed in the two-phase heat exchanger, degassed and subcooled, and then passed back to the detector. In addition, separate gas flow through the external purification system purges the space above the liquid, and the conduits. The overall flow rate is $500 \mathrm{slpm}$ of gas, which is $16 \mathrm{~cm}^{3} / \mathrm{s}$ of liquid flow, $2.8 \mathrm{~kg} / \mathrm{min}$, and fully processes 10 tonnes of Xe in $2.5 \mathrm{~d}$. 

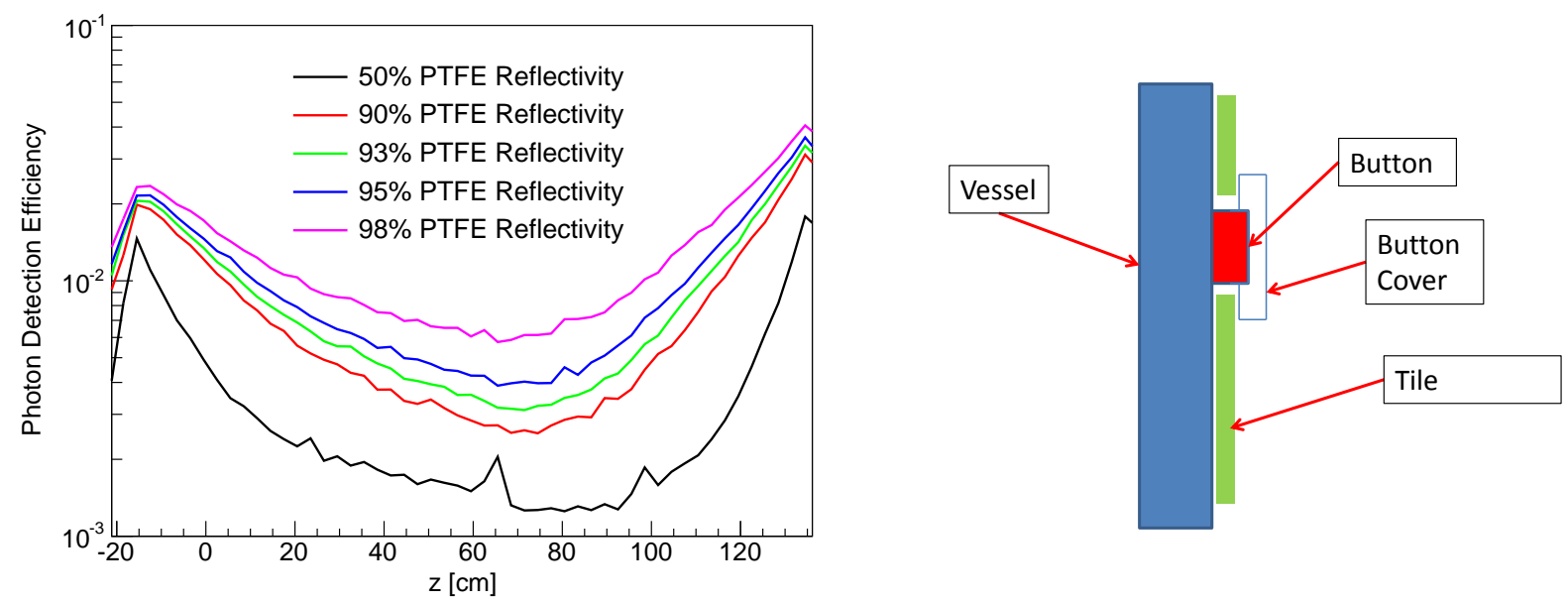

Figure 3.7.4: Left: Photon detection efficiency as a function of $z$ in the side Skin region for different values of PTFE reflectivity (in percent), where the inner wall of the cryostat is lined with PTFE and the outer wall of the TPC is made of PTFE. The LZ requirement is $95 \%$ reflectivity for the PTFE/LXe interface. Right: A schematic showing how buttons bonded to the cryostat wall will hold PTFE tiles in place to line the cryostat with reflective material.

While much of the functionality and complexity of the system shown in Figure 6.4.1 is external to the detector and is described in Chapter 6, the "internal circulation system" that resides within the detector has important design considerations too. Primarily, the system must purify the liquid while sweeping all regions of LXe to avoid stagnant fluid, which is especially important when using the dissolved tritium calibration source. It must do this while maintaining a stable liquid surface with no boiling throughout the volume, and allowing operation within the 1.6 bar to 2.2 bar (a) pressure range. Along with the cryogenics system, it provides cooling to counter the various heat loads in the system. Finally, it should allow, to the extent possible, operation in two modes: one where the liquid is convectively mixed on a time scale that is much shorter than the 2.5-day circulation time, and one where the fluid has a stable thermal gradient with minimal mixing.

The overall flow path is shown in Figure 3.8.1. No plumbing is routed near or through any of the challenging high-field regions of the detector. This limits locations where fluid lines can access the central TPC volume to the bottom PMT array, and to the perimeter of the TPC near the liquid surface, both of which regions have voltages relatively close to ground. Accordingly two streams of liquid enters the detector from the bottom conduit, one of which is distributed into the dome Skin, and one of which is distributed through the bottom PMT plate and into the TPC. The flow rates into the Skin and TPC are separately controlled. The overall movement is then upward, with LXe spilling over a set of six evenly spaced level-defining weirs for each of the skin and TPC regions. These drain into a triply-segmented drain collection trough, and out through drain tubes. These penetrate the vessel walls to avoid the high field region of the skin near the cathode, and then back to the bottom conduit and over to the purification tower. All tubing and associated fittings housed in the skin will be made from high-reflectivity PTFE so as to minimize absorption of scintillation photons.

The entering fluid is distributed in the dome region, and through the bottom of the TPC through a set of some 20 each small diameter distribution tubes, as seen in Figure 3.8.2. The weirs are housed just outside the gate anode rings, a challenging region with confined mechanical space and relatively high fields created by the the two different voltages of the gate and anode grids rings. Details of the weir assembly are shown in 


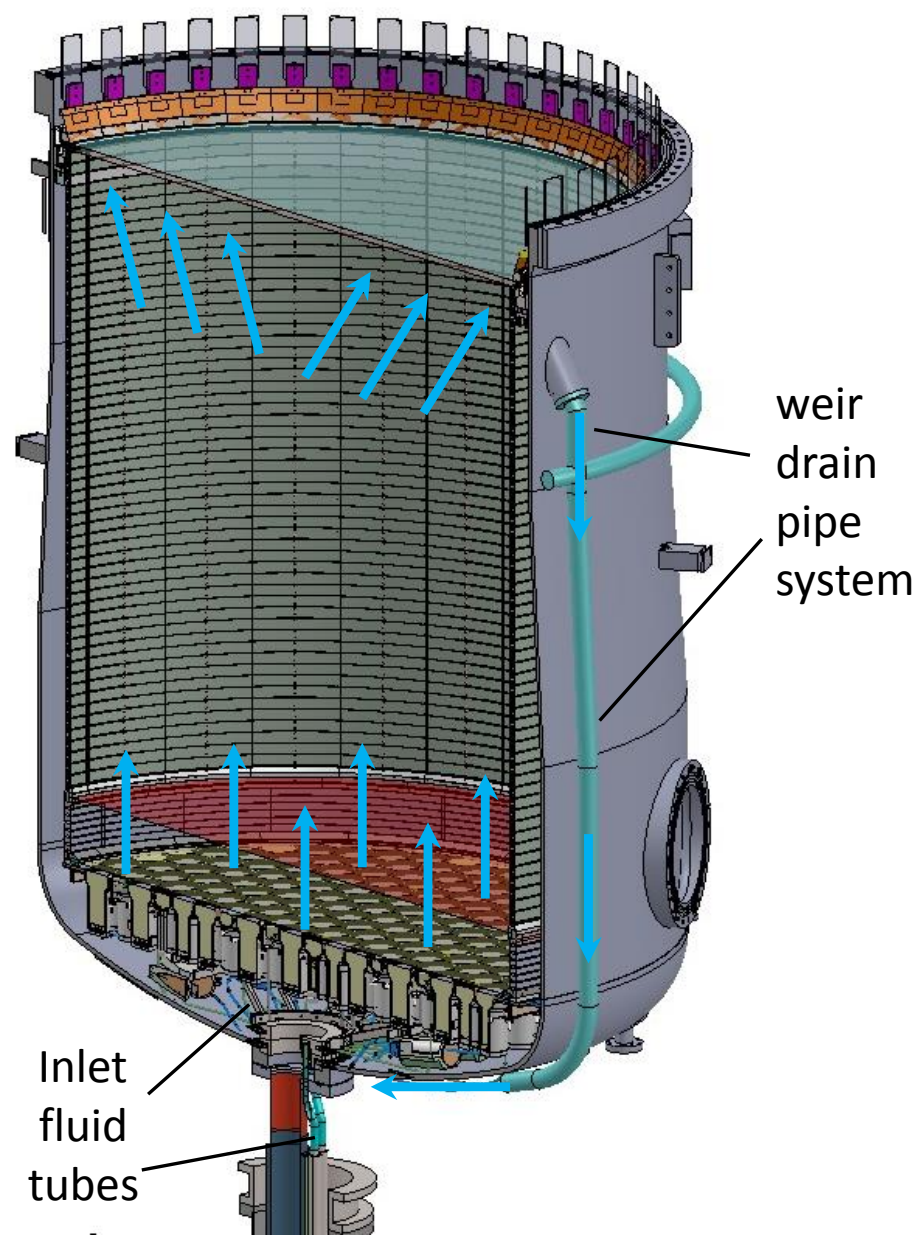

Figure 3.8.1: Cutaway of the Xe detector system, with the internal circulation system elements and flow paths indicated. Purified LXe in two separate tubing sets is distributed into the bottom of the TPC and the bottom of the dome Skin region. The flow is then up to the liquid surface and over a set of liquid level-maintaining weirs, into a set of thee collection troughs and drain pipes, and then return via the bottom conduit to the external system.

Figure 3.2.4. When the liquid flows over the weir, there is a small height of liquid that depends on the flow rate. This design allows a long weir length, and the weir location to be well registered to the grids. This is important because the S2 light production depends directly on the relative levels of the gate and anode grids and the liquid height.

LXe in the bottom conduit and the HV feedthrough conduit is slowly circulated outward by pumping on the gas space at the ends of these conduits while also applying heat to the LXe. The conduits must be purged because of the outgassing from the plastics in the cables in them, especially at their warm ends for which outgassing is orders of magnitude higher than from cold plastics. A second potential issue is Rn emanation, especially from the cables in the conduits, some portion of which is warm, especially for the top conduit. The flow from the conduits is thus passed through a Rn removal system described in Section 6.4.5, before it is passed through the main purification system. None of these flows are routed through the heat exchange system. Finally, there is separate capability to pump the gas directly above the TPC (i.e., in the "S2" space) at a low rate, using a set of tubes distributed in the top TPC array much like those in the bottom array. 
A central goal in all of these flows is to eliminate as far as practical any stagnant "dead" regions-the prime example of which would be the conduits if they were not purged. Such dead spaces, once impure, serve as a slow source of diffusively-driven impurities that can greatly complicate purification. This is an issue not only for purity that affects charge and light collection, but, critically, following the use of radioactive tritium introduced as a calibration source (see Chapter 7), which must be subsequently removed.

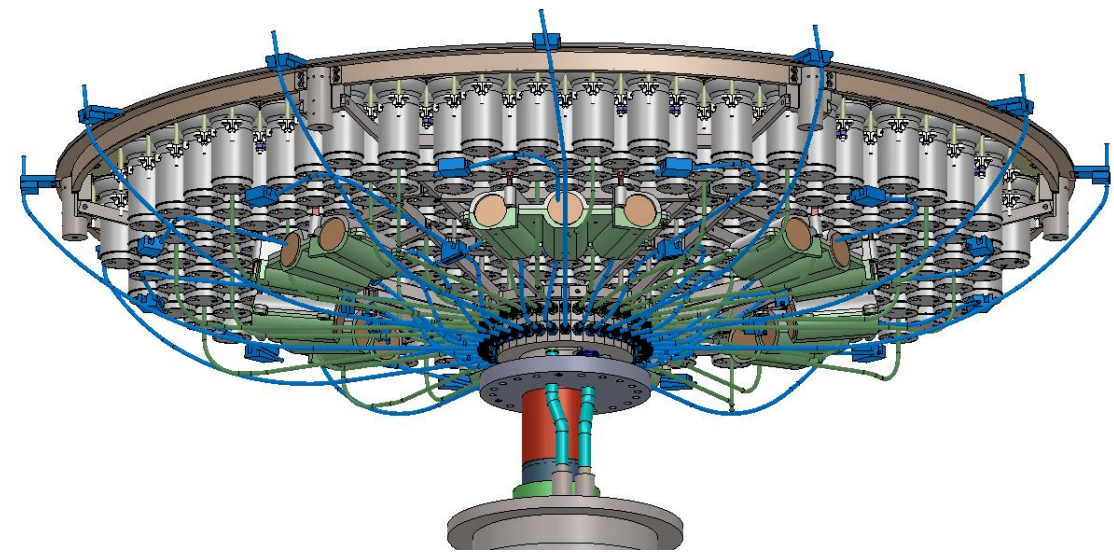

Figure 3.8.2: Fluid distribution in the bottom dome region. Fluid tubes in blue distribute flow into the dome and wall skin regions, while tubes in green distribute fluid into the TPC.

Both the LXe circulation system and the cryogenic system (Section 6.8) must work together to control the overall thermal environment of the detector and also the degree of convection, if any, of the fluid in the TPC. Most cooling of the detector while running will be provided by a set of six thermosyphons attached to the vessel at the level of the liquid surface. The total heat load internal to the vessel (not including cables, whose load is in the conduits) is roughly $40 \mathrm{~W}$, and we plan to deploy up to $30 \mathrm{~W}$ from heaters in the bottom PMT array (see below). Most of this heat is applied to the Skin, and so a set of six thermosyphon heads will be deployed around the vessel at the level of the liquid surface. Liquid cooled by these heads and liquid warmed by the radiative load on the wall and the bottom PMT bases in the Skin should set up convective currents that lead to a fairly uniform temperature in the Skin.

The interior of the TPC has a much smaller heat load than the Skin, with only a fraction of the $8 \mathrm{~W}$ from the bottom PMTs being transmitted into the TPC (ditto for the $1.4 \mathrm{~W}$ from the resistor chains embedded in the TPC field cage). The temperature of the fluids returning to the detector will be set in the purification tower, and so with the fluid entering the TPC can be set to a lower temperature than that in the TPC; this fluid should counter the heat loads from the PMT bases.

In this mode a fairly stable fluid should be achieved in the TPC. Running with a stable, non-mixing fluid has several advantages. It should allow much faster purification because impurities are mostly directly swept out of the detector in the 2.5-day time scale for circulating the fluid. This is termed "batch mode" purification in chemical engineering, as opposed to the "mixed tank" mode obtained with strong mixing in which purification reduces only exponentially with the circulation time as the time constant. Another advantage of a nearly static fluids is that it may be possible to reduce the effect of Rn events by tagging the position of the various daughters and in particular ${ }^{214} \mathrm{Bi}$, the decay of which produces an important ER background.

However, the use of the dissolved source ${ }^{83 \mathrm{~m}} \mathrm{Kr}$ argues for convective mixing that is at least as fast as its $1.83 \mathrm{~h}$ half-life. This source provided the main calibration of position response of S1 and S2 signals in LUX, including the crucial measurement of the electron drift length, which varies over time. Because it is outside (but relatively near) the WIMP-search energy window it was used on a frequent basis, and it would be highly desirable to do the same for LZ. The basic plan for achieving mixing in the TPC is to use heaters 
installed just inside the outlets of the fluid tubes at the bottom of the TPC, so that fluid enters in a heated state. The amount of power planned to use is up to $30 \mathrm{~W}$. We plan to remove this heat by evaporating liquid from the LXe surface via a set of tubes in the top array that mirror those in the bottom array, and a flow that is limited to rates that do not induce boiling. The combination of cooling at the top and heating below should induce convection motion. It is challenging to fully predict the motion of the fluids, though we plan some studies to analyze design using computational fluid dynamics. The goal of the system described here is to support operations with the fluid in either static or convective states.

Initial cooling of the detector must be done with care in order to avoid large thermal gradients in the TPC structure, which has no obvious thermal anchor point. We thus plan to circulate Xe gas, cooled in the purification tower, to slowly cool the entire system at a controlled rate. We will make use of the separate flow streams in the TPC and Skin, as well as the gas purge in the top dome area, and are using computational fluid dynamics (CFD) to plan the rate of cooling. Sensors described in the next section will be used to monitor this process. We will also attach a thermosyphon head to the bottom of the vessel to allow us to cool the bottom of the detector.

\subsection{Xenon System Monitoring}

Monitoring sensors are required for controllable and reliable operation of LZ; this Section describes the monitoring systems contained in the xenon space. Some of the data generated by these devices can also be used to identify temporary departures from optimal operating conditions and thus provide additional information for the science analyses. For example, achieving good resolution of the S2 signal relies on a quiet liquid surface, which we will monitor through precision level sensors and acoustic bubble sensors. The thermal profile of the detector is an important aspect of liquid circulation and the stability of the liquid surface, and this is measured by an array of thermometers. The ability of the system to sustain high voltages is very important and a set of loop antennae will measure discharges but may also detect any precursor signals and thus enable mitigation. Bubbles encountering a high-field surface can also lead to discharge and spurious light emission, and thus detection of bubbles is an important aspect of achieving high voltages. Finally, it is important to confirm that the very significant thermal contraction of the plastic field-cage system behaves as expected, and so this motion will be monitored by a set of position sensors. This section discusses the monitoring systems in detail, which are summarized in Table 3.9.1.

Table 3.9.1: Monitoring sensors located in the LZ xenon space.

\begin{tabular}{|lccll|}
\hline Sensor & Acronym & Number & Location \\
\hline Thermometers & - & 101 & 66 in Xe space, 35 in vacuum space \\
Weir Precision Sensors & WPS & 6 & Surface level \\
Long Level Sensors & LLS & 4 & Surface level, dome Skin \\
Position Sensors & POS & 6 & On top PMT truss \\
Loop Antennae & LA & 8 & On PMT trusses and near HV feedthrough \\
Acoustic Sensors & AS & 8 & Attached to IVC outer wall \\
\hline
\end{tabular}

\subsubsection{Thermometers}

Temperatures need to be monitored at approximately 100 locations throughout the Xe detector and nearby. The chosen temperature sensor is a platinum (PT100-type) resistor. The readout method is 4-wire throughout 


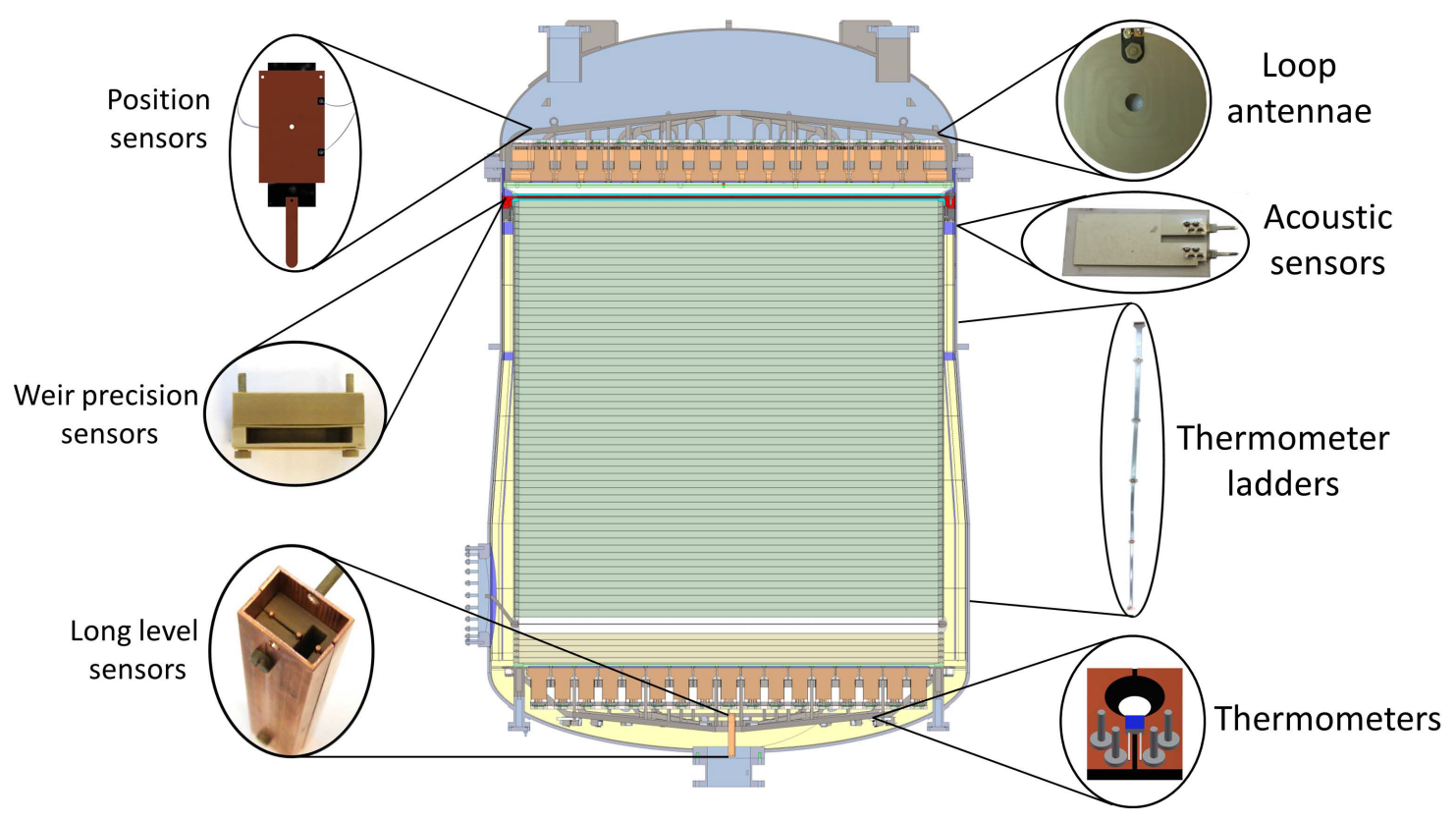

Figure 3.9.1: Schematic diagram of the internal monitoring sensors in the xenon detector.

and the cabling used is twisted wire bundles in the xenon space, while in the vacuum space of the LZ cryostat it is a semi-rigid polyimide-stainless steel layered composite structure; the latter features parallel strip-line pairs sandwiched between shielding/ground planes on the outside. The design of the semi-rigid cabling is individual to each group of sensors, with a maximum length of a single section being $1.5 \mathrm{~m}$, which is sufficient to cover the height of the ICV. There is a connector block at one end of the semi-rigid cabling at which the signals transition to conventional shielded 4-core wiring to cover the long stretch towards the electronics "breakout boxes", where mechanical robustness and threading capability are important. At the vacuum barriers of the breakout boxes, standard DB25 connectors are used, with 100 thermometers (400 wires) requiring at least 16 such connectors. The provision of DB25 connectors marks the interface to the LZ Slow Control system (Section 8.8). Calibration of the platinum resistors is via a generic table available for these, where precision requirements are less stringent, or from individual calibrations and corresponding look-up tables or polynomial corrections (within the Slow Control), where higher precision is required. At positions where the potential effects of intrinsic component radioactivity is minor, commercial pin headers and sockets can be used. Closer to the center of the detector, a combination of pins and clean PTFE, PEEK, or Delrin connector bodies will be used. All materials used are checked for compatible radiopurity (see Chapter 9).

Cryogenic, laminated, layered semi-rigid cabling will be used for reading out the bulk of the thermometers in the vacuum. This cabling and the low-radioactivity connectors (where needed) will be made in-house to individual designs for easy installation on site. The cable is based on polyimide/Kapton, to which stainless steel is laminated. These raw materials are etched to produce individual cabling and are laminated to receive shielding and ground layers on either side. A minimum track width of $150 \mu \mathrm{m}$ and pitch of $300 \mu \mathrm{m}$ have been demonstrated. The electronic capacitance between wire pairs is $\approx 80 \mathrm{pF} / \mathrm{m}$, depending on detailed geometry and operating temperature (via the temperature-dependent permittivity of Kapton). Thermometer mounting blocks in the xenon space will be based on Cirlex boards. 


\subsubsection{Liquid Level Sensors}

For level sensors, two main designs are needed: a parallel-plate type for precision surface sensing, and long coaxial types. The precision surface sensor (or Weir Precision Sensor, WPS) has the plates installed horizontally, straddling the boundary between the LXe and the electroluminescence region to measure the liquid level with high precision, allowing any tilt of the detector to be readily measured, and seeing variations in the liquid surface-for example from bubbles or surface waves. Six WPS sensors are installed at the weir overflow openings. The coaxial Long Level Sensors (LLS) monitor primarily the liquid levels during filling and emptying of the TPC. There are three such sensors, that will span the height of the weir trough and the full 13-mm gate-anode distance.

The sensors will be read out with high bandwidth (up to $200 \mathrm{kHz}$ ) aiming to monitor the condition of the Xe surface (level, ripples, waves) and, as such, will have a precision of $\sim 10 \mu \mathrm{m}$. Further sensors will monitor the bottom Skin region during filling and emptying, and a LLS will be used inside the PMT cabling standpipe to monitor the filling process. A pressure sensor at the bottom of the cryostat vessel measures the head of the liquid and provides further information about the Xe level during filling and emptying. The readout method is via determination of capacitance with respect to a reference capacitance (all sensors employ three electrodes and a feedback readout circuit). This arrangement greatly reduces systematic effects arising from the long cabling in LZ and its variable capacitance (mechanical and thermal effects). The feedback readout circuit is based on modulated readout with a minimum number of analogue components and the bulk of front-end complexity absorbed into the firmware of a field-programmable gate array (FPGA). For feedthroughs at the vacuum barrier, a standard flange with DB25 connectors is foreseen into which the 8channel readout boards plug directly without need for external cabling. Figure 3.9.2 shows results obtained in a liquid xenon test chamber. The achieved precision satisfied the requirements and features visible in the data are understood.
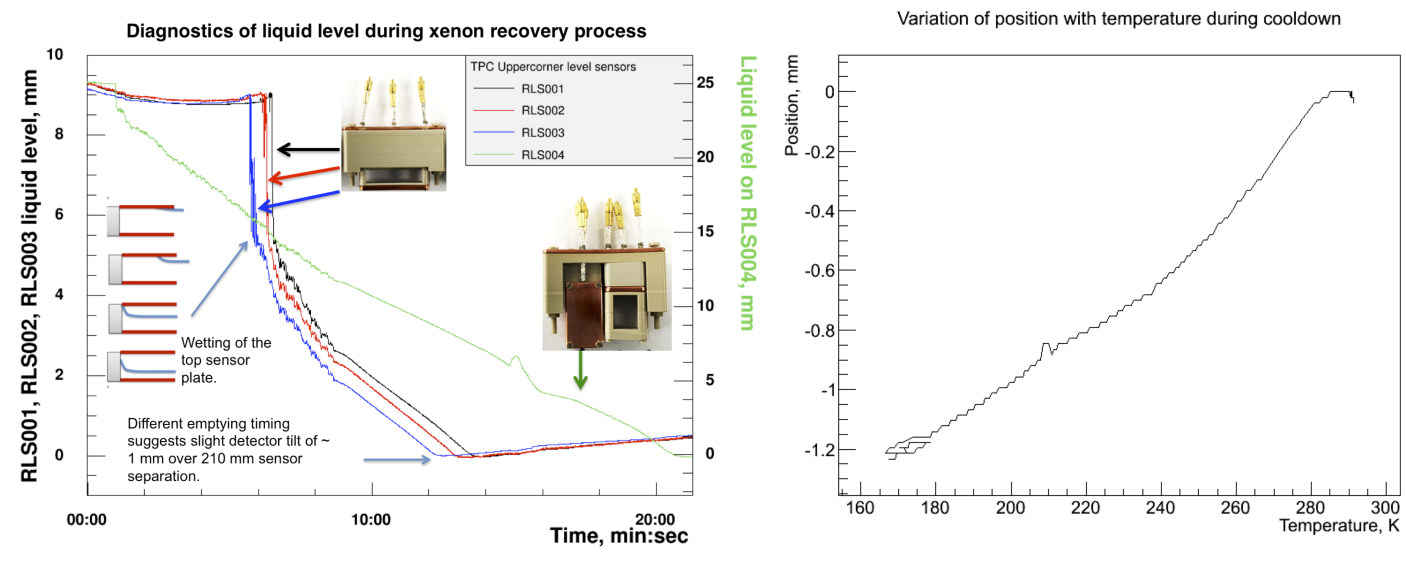

Figure 3.9.2: Left: Data from a Weir Precision Sensor (WPS) and a Long Level Sensor (LLS), installed inside a liquid xenon test chamber, showing xenon being emptied. The shapes of the graphs are well understood; for example, regions pointed to by the arrows can be linked to xenon gradually overflowing level sensor plates, capillary effect, or known fringe fields. Right: Data from a position sensor, showing the contraction of the TPC as it cools. 


\subsubsection{Acoustic Detection}

Acoustic sensor (AS) heads based on a polymer film (PVDF) have been chosen due to constraints on radioactivity levels. The sensors will be installed in direct contact with the outside of the inner cryostat vessel to pick up internal sound. Eight sensors are foreseen, with three at $120^{\circ}$ spacing near the lower cylindrical section of the cryostat, three near the top of that section with the same spacing, and one each on the top and bottom domes. The vacuum-barrier electrical connection is via a standard flange with a DB25 connector, interfacing to a dedicated 8-channel differential readout front-end board. Connection to Slow Control is via a standard ModBus interface, allowing to query minimal information such as when an acoustic signal (above trigger) occurred, along with its magnitude and a crude classification of the likely nature of the sound. Simultaneously, via a different Ethernet readout channel, complete acoustic recordings from the sensors, sampled at rates up to $200 \mathrm{kS} / \mathrm{s}$ will be available for feeding into the fast data stream and for subsequent off-line analysis.

\subsubsection{Loop Antennae for Discharge Detection}

To monitor for onsets or occurrence of HV breakdown events, loop antennae (LA) capable of picking these up will be installed in critical positions. Eight such antennae will be assembled on the top and bottom PMTarray trusses, sufficiently far from high-field regions to ensure that the presence of the metal aerials does not interfere. With such a set of LA sensors, insight into locations of possible HV breakdowns and types should be possible. The feedthrough at the xenon space to the external environment will be through a DB25 connector, similar to the PMT readout. Two sets of fast 4-channel readout electronics with a sampling rate of up to $200 \mathrm{MS} / \mathrm{s}$ will be used. The readout board can be polled by the Slow Control through a standard ModBus interface. It will provide a fast digital signal for alerting the HV power supplies to situations when a quick shut-down is required, and there will be capability for reading out triggered fast signals for inclusion in the data stream and further offline analysis.

\subsubsection{Position Sensors}

Position Sensors (POS) capable of measuring linear displacements of up to about $2 \mathrm{~cm}$ will be fitted on and around the top PMT array to monitor the thermal contraction and expansion of the TPC during cooling and warming, allowing to apply countermeasures, if needed, to ensure uniform cooling or warming of the TPC. These sensors will also give important information on any lateral displacements that would alter the Skin region gap, and the alignment of the top PMT array with respect to the TPC anchor points. The operating principle of the displacement sensors is based on picking up linear travel via a slider that moves a dielectric between two adjoining parallel plate capacitors. The position sensors are of simple and robust design and made from radiopure materials. The electronic readout is based on the same feedback circuit used in the level sensors, which acts to minimize the effect of cable capacitance on the sensor output. Eight sensors (three for vertical movement, three for horizontal movement, and two for helical movement) will be installed.

\subsection{Integrated System Testing}

A vital part of the preparation for LZ is the testing of key design aspects and critical components of the Xe Detector and integrated "System Testing" of the TPC and associated systems. Several small LXe test chambers, with less than $10 \mathrm{~kg}$ of LXe, are available at LZ member institutions, targeting studies in specific areas (spurious electron and photon emission from grid wires, VUV reflectivity measurements, small-component QA testing). Additionally, a larger System Test platform has been developed at SLAC which can operate 
with over $100 \mathrm{~kg}$ of LXe, conducting integrated tests to answer broader HV performance questions, and involving full-scale sub-systems in some cases. This coordinated, multi-scale approach is a key part of the risk mitigation strategy in LZ. We outline the tests being performed at collaborating institutions in Table 3.10.1.

Table 3.10.1: High priority studies and tests to be performed with small LXe chambers or the SLAC System Test facility. Note that SLAC I (II) denotes SLAC (Phase-I) or (Phase-II). Timing of results is indicated (calender years).

\begin{tabular}{|lllc|}
\hline Studies/Tests & Topics & Groups Involved & Results \\
\hline Reflectivity & PTFE reflectivity in LXe & Coimbra / U-M & 2016 \\
& PTFE reflectivity vs thickness & Coimbra / U-M & $2016-2017$ \\
\hline Wire tests & Electron/photon emission studies & Imperial & 2015 \\
& Small wire-grid testing & LBNL & 2016 \\
& Effect of electropolishing/passivation & Imperial & 2016 \\
\hline HV studies & Cathode ring: large area at HV & UC Berkeley & 2016 \\
& Effects of surface treatment & SLAC I & $2015-2017$ \\
\hline TPC prototype tests & Reverse field region (RFR) & Yale/SLAC I & $2014-2015$ \\
& HV performance & SLAC I & 2016 \\
& Skin optics and performance & SLAC I & 2016 \\
& Handling/cleanliness protocols & SLAC I/SLAC II & $2016-2017$ \\
\hline Full scale grid tests & Electroluminescence region & SLAC II & 2017 \\
& RFR with full surface fields & SLAC II & 2017 \\
\hline
\end{tabular}

\subsubsection{Reflectivity Measurements}

The performance of both the TPC and the Skin detectors is strongly dependent on their ability to collect xenon scintillation light, and this is in great measure driven by the reflectance of the surfaces that surround these LXe volumes. The optical performance of these systems has been described in Sections 3.5 and 3.7. PTFE is the material of choice in LZ and past LXe detectors, used due to its surprisingly high bi-hemispherical reflectance. However, there remains some uncertainty as to the precise value and angular distribution of this reflectance, and its dependence on precise material provenance and surface properties. In fact, high-quality optical data is lacking in the VUV range for many materials of interest. As was shown in Figure 3.5.2, a few percent difference in PTFE reflectivity has a noticeable impact on the performance of the detector.

At LIP-Coimbra, Portugal, a dedicated chamber is operated for the measurement of PTFE reflectance in LXe, which follows on from earlier studies of angular profile and total reflectance of different PTFE samples for the xenon scintillation wavelength at room temperature $[7,8]$. The chamber used for the new measurements is represented schematically in Figure 3.10.1 (left and center). A volume of LXe enclosed by PTFE walls is topped by a PTFE "plunger"; the chamber is $10 \mathrm{~mm} \times 10 \mathrm{~mm}$ in cross section and up to $150 \mathrm{~mm}$ in height. The scintillation light is provided by an ${ }^{241} \mathrm{Am}$ source deposited onto a small stainless steel holder and attached to inner surface of the movable wall. After traveling across the LXe volume and bouncing between the PTFE walls, the scintillation light is detected by a single PMT which makes up the bottom of the chamber. 

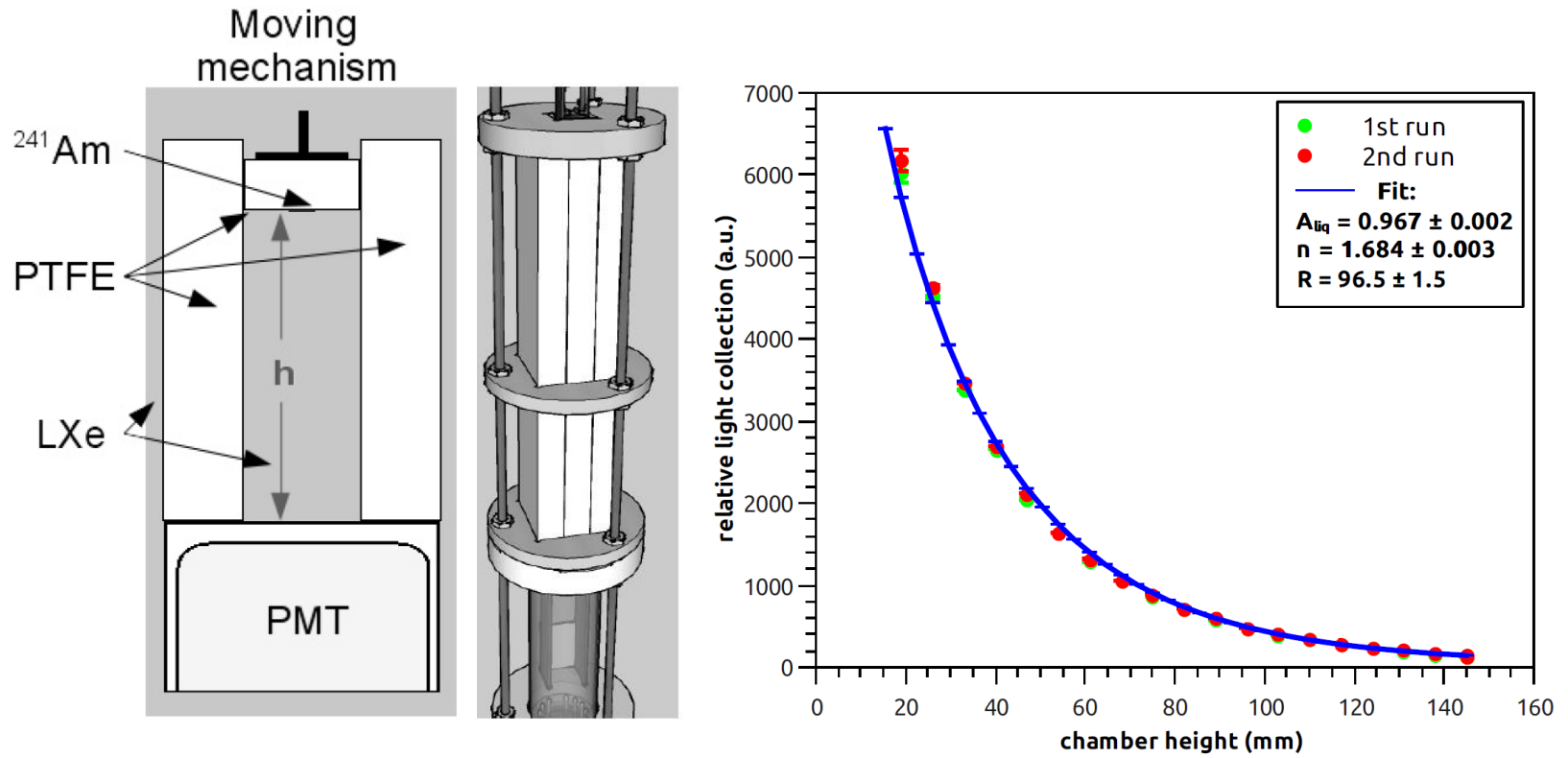

Figure 3.10.1: Measurements of PTFE/LXe reflectivity at LIP-Coimbra. The PTFE-built chamber is shown schematically on the left and center. The height of the chamber $(h)$ is varied with a "plunger" mechanism as an alpha source generates scintillation in the LXe; this is detected with a PMT at the bottom. The relative optical response as a function of the chamber height is fitted by the optical model described in Ref. [7] with three free parameters, from which the reflectivity, $R$, is derived. Preliminary results are shown on the right for two independent runs with the same PTFE sample (the error bars are smaller than the markers); the fit result indicates $R=(96.5 \pm 1.5) \%$ for this sample.

Preliminary experimental results obtained for one PTFE sample (APT \#807NX, a possible material for LZ) are shown in Figure 3.10.1 (right), overlaid with the corresponding fit. The PTFE/LXe reflectivity $(R)$ is obtained by fitting the measured relative light collection at different LXe heights with the prediction of the detailed simulation model described in Ref. [7]. The Monte Carlo framework for this model is the ANTS2 package [38]. The free fitting parameters were the PTFE albedo in LXe $\left(A_{\text {liq }}\right)$, the PTFE refractive index $(n)$, and the attenuation length $(\lambda)$ for the scintillation light in LXe (these are indicated in the figure, and $\lambda>2 \mathrm{~m}$ ). The LXe refractive index and the mean free path for Rayleigh scattering were fixed and set to 1.69 and $29 \mathrm{~cm}$, respectively.

These optical measurements are challenging and prone to systematic uncertainty and, due to the importance of PTFE to our design, additional reflectivity studies are being conducted with the MiX detector at the University of Michigan. These utilize a cylindrical PTFE structure with a PMT on the bottom, a circular PTFE disc on the top that floats on the LXe, and a ${ }^{210} \mathrm{Po}$ alpha source on the circular disc facing down, as shown schematically in Figure 3.10.2 (left). As the chamber is filled, the floating circular PTFE disc rises and the fractional area, defined as the PMT photocathode area divided by the total surface area of the chamber, diminishes.

It is desirable to minimize the thickness of any PTFE panels in the LZ TPC and Skin detectors, in order to reduce dead volumes around the active LXe, outgassing and potential backgrounds. A lower limit on this thickness is established by the PTFE transmittance to xenon scintillation light, and the need for optical isolation between the TPC and Skin regions-as well as between these and any dead regions containing LXe. Preliminary measurements have been carried out for APT \#807NX PTFE that cover chamber wall 

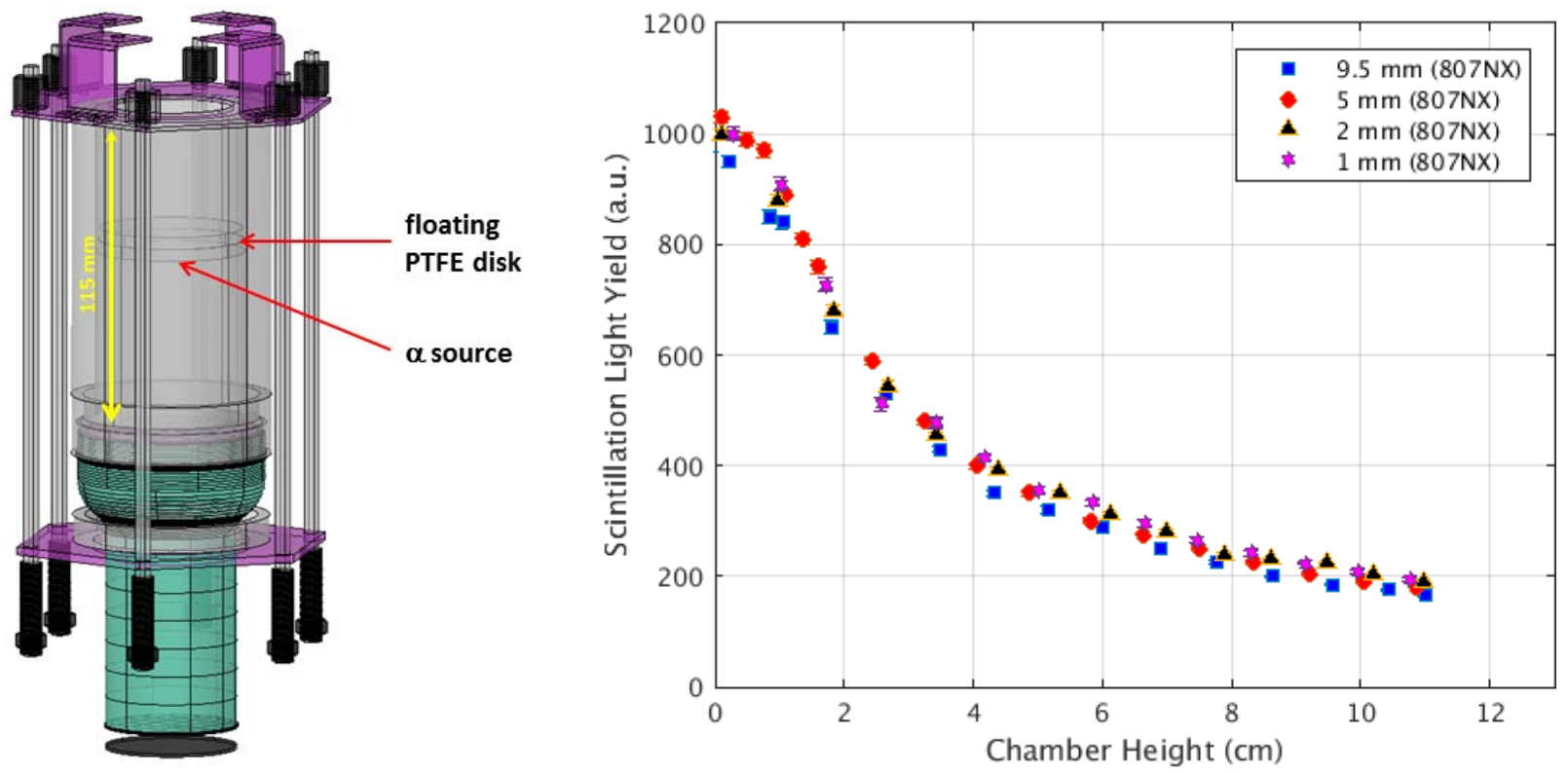

Figure 3.10.2: Left: Schematic view of the U-M PTFE reflectivity setup which is based on the MiX chamber [41]. It consists of a 3-inch Hamamatsu R11410 PMT which covers the bottom part of the 4.6-inch high and 2.45-inch ID PTFE cylinder, and a Po-210 source to provide a mono-energetic light source. The fractional area in the setup is varied as the floating disc raises or descends inside the PTFE cylinder. Right: Preliminary results for light yield as a function of PTFE wall thickness for APT \#807NX PTFE. Draft figure, will be updated soon

thicknesses from $1 \mathrm{~mm}$ to $9.5 \mathrm{~mm}$, as shown in Figure 3.10.2 (right), to verify the minimum panel thickness required in LZ. These data suggest that decreasing the PTFE wall thickness in this range does not appear to change reflectance. Further measurements will be carried out for various surface treatments and PTFE materials at thicknesses from $1 \mathrm{~mm}$ to $9.5 \mathrm{~mm}$. Following the PTFE measurements, these chambers will go on to study the optical properties of grid wires and other materials (e.g., the non-reflective PEEK used at the perimeter of the gas region).

\subsubsection{HV Studies in Small Two-Phase Chambers}

To ensure the successful delivery of HV to the LZ TPC we take a comprehensive approach, beginning with an experimental study of the physics processes involved in the electric breakdown of individual cathode wires at a microscopic (quantum) level. Chambers at Imperial College London and Lawrence Berkeley National Lab have been built and operated for this purpose.

Electron and photon emission are observed from cathodic surfaces in LXe at relatively low electric fields: $\sim 10 \mathrm{kV} / \mathrm{cm}$, a factor of 100 to 1,000 lower than expected for standard (cold cathode) field emission from metals. These have caused serious limitations to the operating voltage of previous LXe TPCs, almost without exception. These emissions can be caused by local enhancement of the electric field combined with the presence of thin insulating layers, surface defects, or other effects that result in a lower effective work function. This may be accompanied by simultaneous photon emission. We must understand the physical origin of these emissions and then develop effective mitigation in time to inform the production of the LZ wire grids. The extraordinary sensitivity of the S2 response, discussed in Section 3.6, means that electron emission must be prevented at all cost up to the $50 \mathrm{kV} / \mathrm{cm}$ maximum field which we allow for cathodic surfaces in LXe (e.g., the cathode and gate grids and their connections). 
At Imperial, a small two-phase chamber was developed to study individual cathode wire samples installed at the bottom of the liquid region, where a cathode grid would normally exist-see Figure 3.10.3. This configuration ensures that high electric fields can be achieved at the wire surface by applying modest voltages (e.g. $\approx 150 \mathrm{kV} / \mathrm{cm}$ for a $100-\mu \mathrm{m}$ sample and $\approx 400 \mathrm{kV} / \mathrm{cm}$ for $40 \mu \mathrm{m}$ wire for a cathode voltage of $5.5 \mathrm{kV}$ ). This study focuses on the phenomenology associated with the onset of electron and/or photon emission. In particular, we are exploring its dependence on electric-field strength, wire material, surface quality, history, as well as the effectiveness of mitigation steps such as conditioning, electropolishing, and chemical passivation.

The chamber has a single internal PMT viewing down from the gas phase to detect both photon and electron emission from the wire sample. The electroluminescence response has single-electron (SE) sensitivity, allowing to measure minute currents $\left(\sim 100 \mathrm{e} / \mathrm{s}\right.$, or $\left.10^{-17} \mathrm{~A}\right)$. The gate and anode grids, $14 \mathrm{~mm}$ apart, straddle the liquid surface, ensuring S2 yields that are mostly independent of the cathode voltage and sufficiently high for efficient cross-phase extraction and SE detection. The 130-mm-long cathode wire is mounted $25 \mathrm{~mm}$ below the gate, stretched between two feedthroughs that deliver up to $-5.5 \mathrm{kV}$ to the liquid. Electric field lines radiating from the upper surface of the sample guide emitted electrons to the electroluminescence region
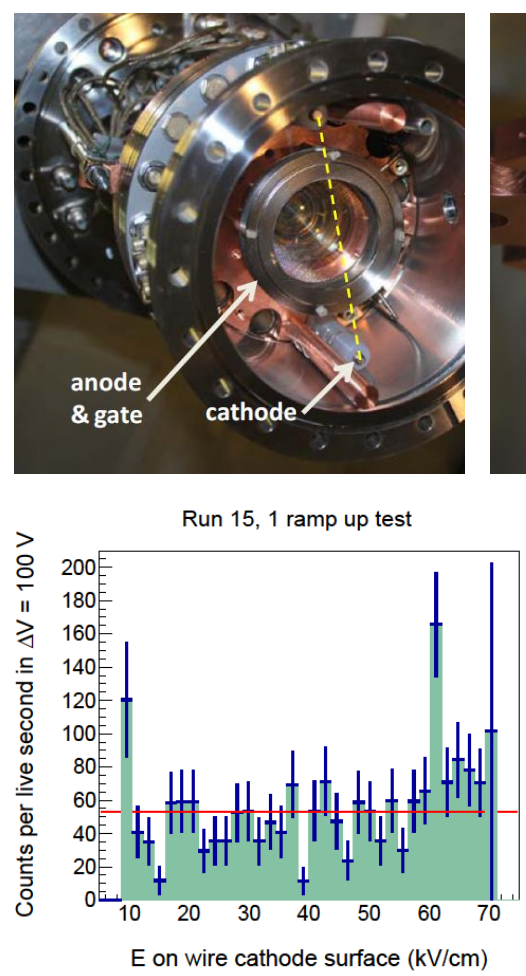
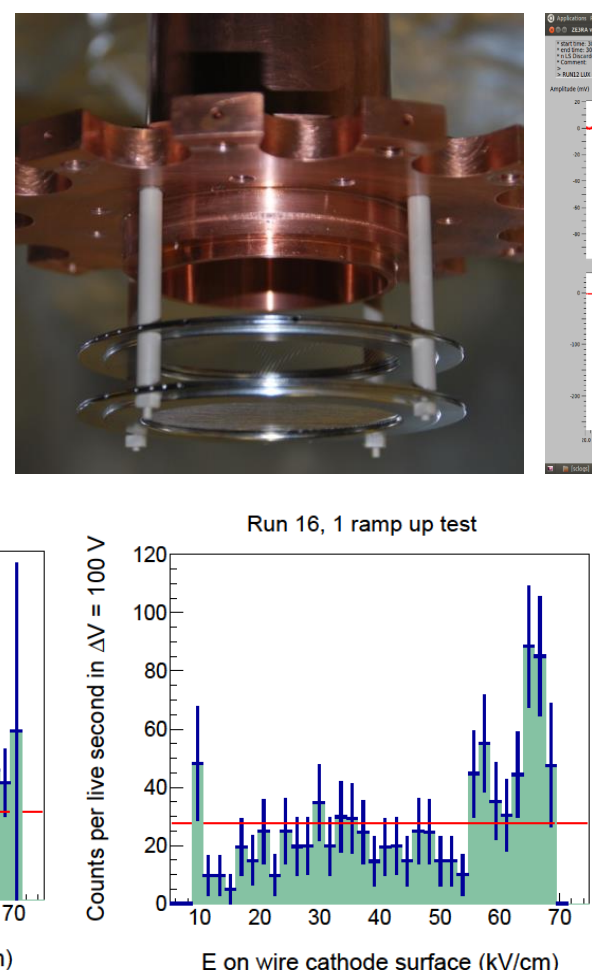
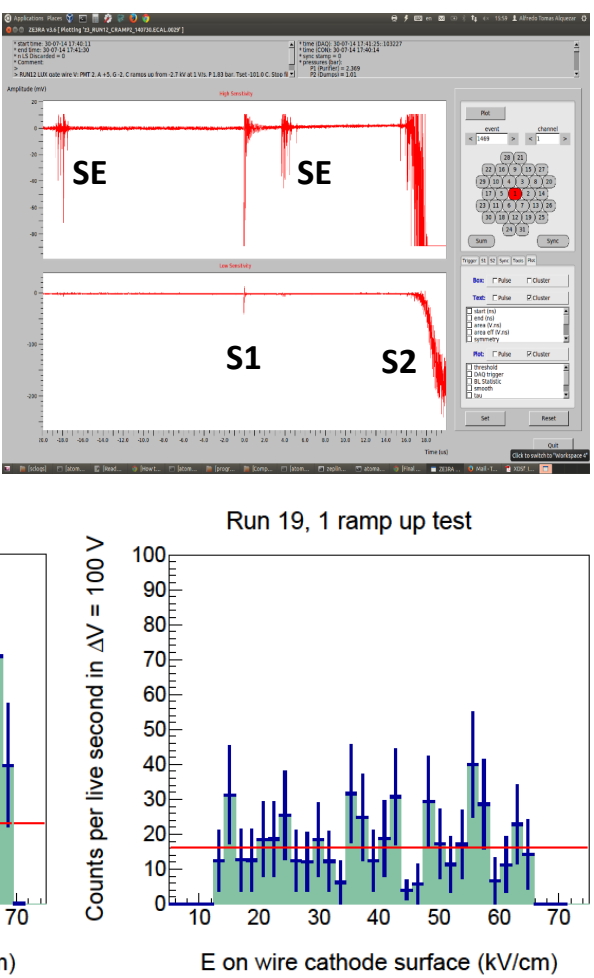

Figure 3.10.3: Imperial chamber to study electron and photon emission from thin wires; Top from left: Photograph of upward view of the chamber from the bottom, indicating the location of the cathode wire and the gate-anode system (a PMT window can be seen through those grids); Photograph of side view of the gate and anode grids, $14 \mathrm{~mm}$ apart; An example event showing S1 and S2 pulses (in dual dynamic range) as well as two single electron (SE) pulses; we search for candidate SE pulses unrelated to any particle interactions producing S1 and S2. Bottom: Evidence for electron emission from a $200 \mu \mathrm{m}$ stainless steel wire (same type as used in LUX) before treatment (two samples shown left and center) and after electropolishing (right). 
in the gas where they are detected with high efficiency. Most subsystems required to operate the chamber were inherited from ZEPLIN-III. The ZE3RA analysis software allows full exploitation of the 2-ns-sampled waveforms [42], which are recorded in high- and low-sensitivity channels to cover both very small signals and larger S1 and S2 pulses (Figure 3.10.3, top right). The voltage applied to the wire sample is ramped up slowly ( $1 \mathrm{~V} / \mathrm{s})$ to several $\mathrm{kV}$, with PMT signals being digitized simultaneously. Electron emission from the cathode can, if accompanied by prompt light, be reconstructed to the cathode depth by electron drift time.

We find such emission occurring from fields as low as $\approx 10 \mathrm{kV} / \mathrm{cm}$ in some wires (e.g. $200 \mu \mathrm{m}$ stainless steel), while at least one other could withstand hundreds of $\mathrm{kV} / \mathrm{cm}$ without any discernible effect $(40 \mu \mathrm{m}$ $\mathrm{BeCu}$ ). Emission episodes are consistent with average SE rates of $100 \mathrm{~Hz}$ to $1,000 \mathrm{~Hz}$ (faster, "impulsive" structure can be observed within these). Two examples are shown in Figure 3.10 .3 (bottom left and center) for samples from the $200 \mu \mathrm{m}$ LUX cathode wire, the least resilient tested so far. In some instances we have evidence of simultaneous photon emission, which suggest that the local electric field is higher than the electroluminescence threshold of LXe [43]. Emitters are (probably) localized in field, and they are rarer and fainter on a second ramping test, suggesting a partial conditioning effect. Significantly, they subside at higher fields and are not reproducible subsequently. We tested electropolished samples of the same wire and obtained significantly improved results, as shown in the same figure (bottom right). Finally, we will explore chemical passivation. Both of these measures can be applied to the LZ grids.

At LBNL, the test bed pictured in Figure 3.10.4 is operated which is functionally similar to that at Imperial, but devoted to evaluate small cathode wire planes consisting of a stainless steel frame and stretched wires. A key question is whether this performance can be simply inferred from the single-wire studies, or if the length of wire is in fact a critical parameter. A complete cathode grid frame will allow an approximate 10-fold increase in wire length compared with a single wire. This chamber has also improved electric field uniformity as well as higher light collection, allowing a better understanding of the correlation between electron and photon emission. The baseline gate-anode design can be studied in detail at this scale. Other studies with this chamber will include understanding more rigorously the cross-phase electron emission process at the liquid surface.
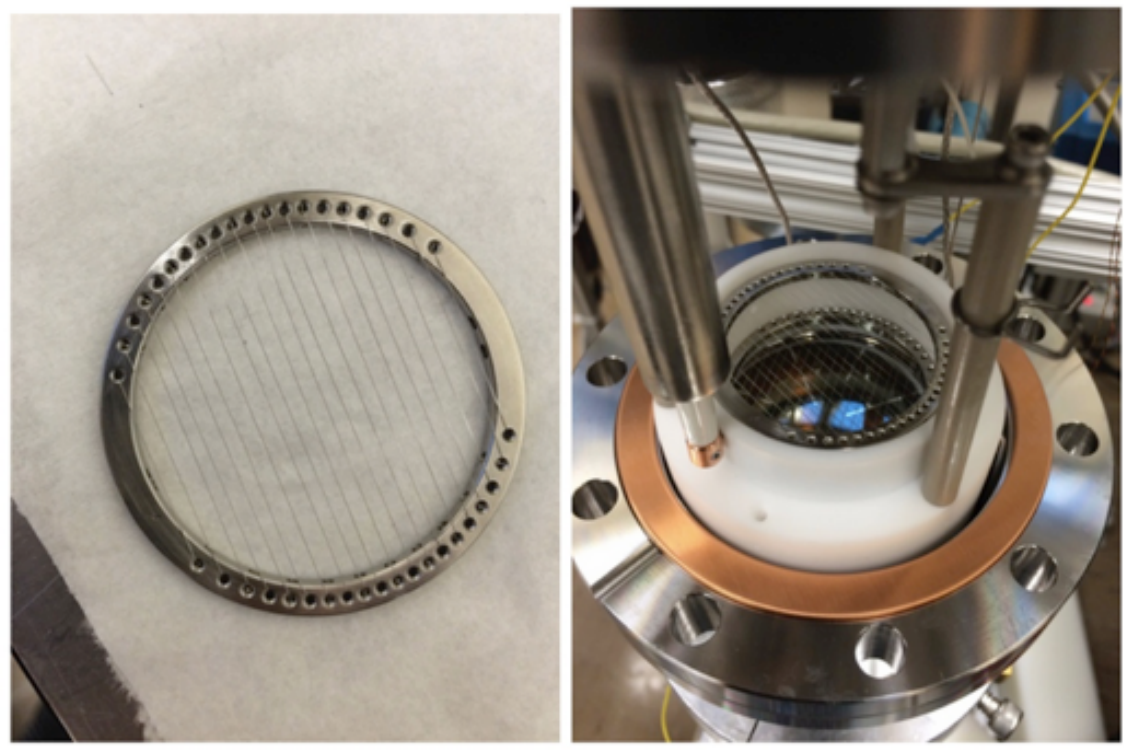

Figure 3.10.4: Left: A wire grid that will be tested in the small LBNL test chamber. Right: Photograph of the LBNL small grid test chamber. 


\subsubsection{TPC Design Testing}

Larger, scaled components of the LZ TPC must also be tested to assure high voltage performance. It is critical that these assemblies sustain the applied cathode voltage without producing light from electrical discharges across their components or to the inner wall of the cryostat. The scaled Phase-I TPC design for testing has a 6-inch inner diameter, and with appropriate scaling of the vessel, achieves the same surface and bulk fields as LZ with the application of half the desired cathode voltage, i.e. $-50 \mathrm{kV}$ in $\mathrm{LZ}$ is equivalent to $-25 \mathrm{kV}$ in the System Test.
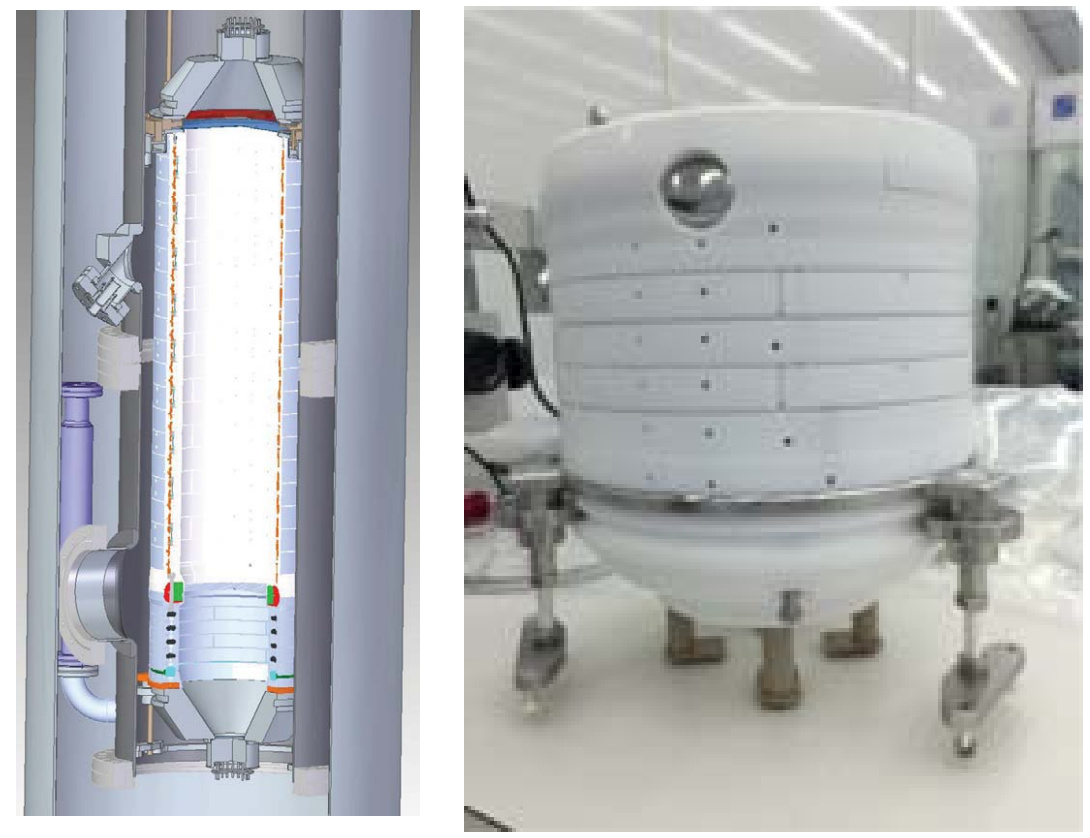

Figure 3.10.5: Left: Schematic CAD representation of the full SLAC Phase-I TPC; Right: Photograph of the Phase-I reverse-field region.

The design of the Phase-I TPC is shown in Figure 3.10.5. It follows closely that of the LZ TPC, being roughly divided into three functional sections. The first is the reverse-field region between the cathode and bottom grids, with five pairs of resistors grading down to ground with a total $12.5 \mathrm{G} \Omega$ impedance set between four field-shaping rings enclosed in PTFE. Above that, the drift field region consists of 20 resistor pairs running from the cathode to the gate grid between field shaping electrodes encased in PTFE rings within the LXe. The final section is the electroluminescence region, which consists of the gate grid, weir manifold, and anode grid, and spans the liquid surface. PMTs, located at the bottom and top of the TPC, provide the primary data on emission, supplemented by optical fibers running to an external camera for data on electrical breakdown.

A scaled reverse field region, from the cathode to the bottom grid, was first tested in liquid argon (LAr) at Yale University in 2014. A schematic view of the setup is shown in Figure 3.10.6 (left) and dewar and HV connection (right).

The HV tests were performed within a 240-liter cryogenic dewar of 16-inch bore. The bottom of the tested assembly was grounded by the platform; the top was connected to HV that can be ramped to $200 \mathrm{kV}$ to simulate the LZ cathode. The HV is delivered through a polyethylene cable that originates at a feedthrough located 8 foot above the top of the dewar. The feedthrough is connected to a DC power supply from Glassman High Voltage. A controlled electrostatic environment was maintained around the test assembly by surrounding it with a highly transparent grounded metal mesh that shields any nearby structures. Seven 

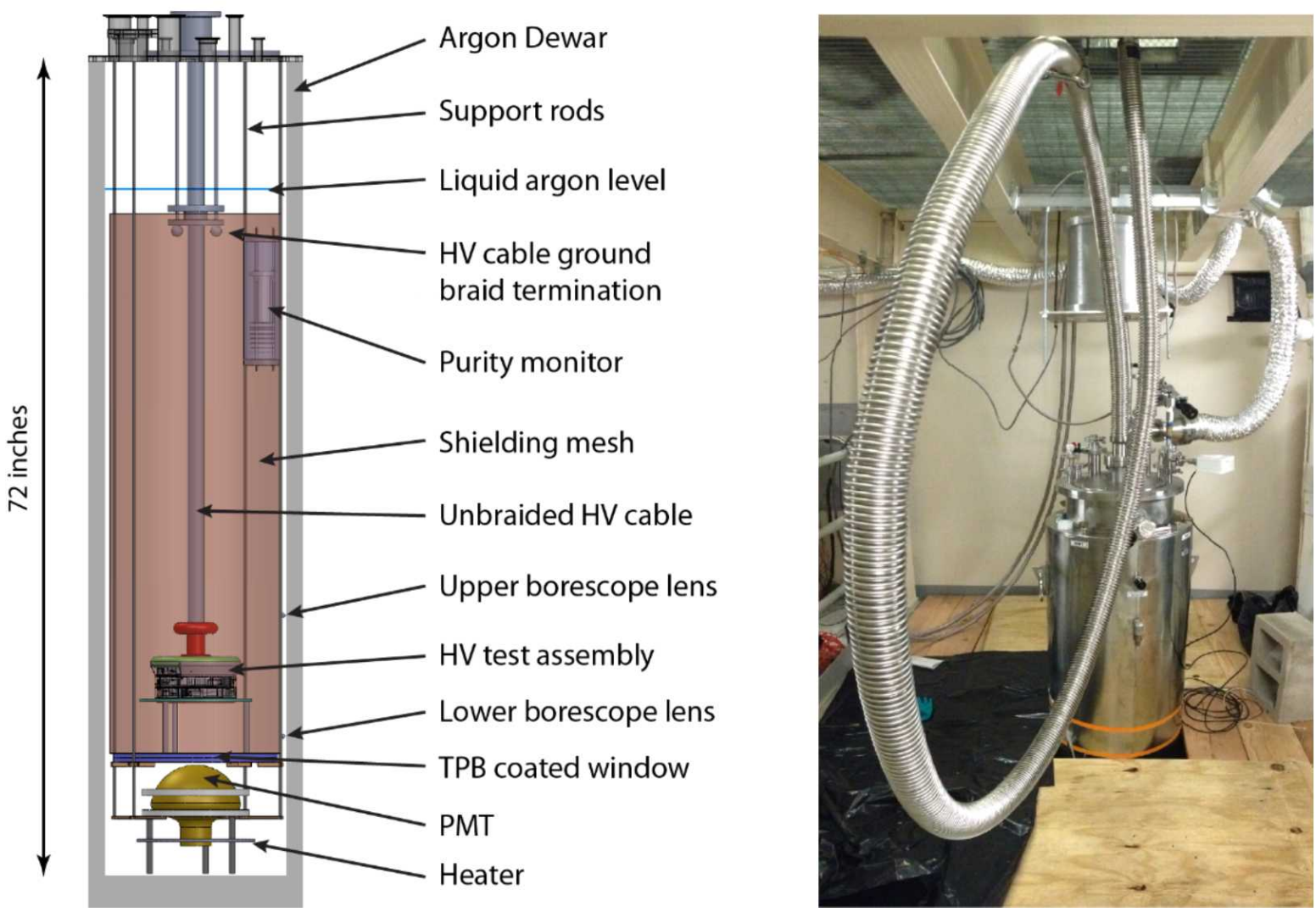

Figure 3.10.6: Left: Side view showing the internal components of the liquid argon test system. Note that the vertical support rods (shown out-of-plane in this view) are positioned outside the shielding mesh. For clarity, only two of the seven borescope lenses are shown. The HV test assembly shown here is a portion of the grading structure of the reverse- field-region of the detector. Right: The liquid argon Dewar and HV cable conduit. The HV feedthrough sits above the square hole in the steel grating at the top of the image.

lenses at various locations viewed the assembly from just outside the mesh. These were connected to fiber bundles that routed the images to a charge-coupled device (CCD) camera located just above the top flange of the LAr dewar, providing a real-time view of any electrical discharges that occurred during testing. Below the tested assembly was a quartz window coated with fluorescent tetraphenyl butadiene (TPB) wavelength shifter. This window was viewed by an 8-inch PMT, giving efficient detection of ultraviolet light with single-photon sensitivity.

The tests completed at Yale identified the installed RFR resistors as a weak point when oriented vertically (required by the small radius of curvature of the Phase-I TPC) and with resistors connected in series together between field shaping rings. The camera system identified light produced at the resistor locations during breakdown, as shown in Figure 3.10.7 at fields equivalent to $200 \mathrm{kV}$ applied in LZ, and this guided us to the current design of more field shaping rings in the reverse field region with resistors oriented horizontally and not connected directly to each other.

Using this system, future $\mathrm{HV}$ tests are planned for the cathode connection region. The cathode connection grading structure and the connection itself will undergo testing at voltages up to $-150 \mathrm{kV}$, while viewed by a PMT and CCD camera as in previous tests. Any glow of these components under applied voltage, or electrical discharges from high voltage to ground will be immediately visible. Purity of the LAr will be 


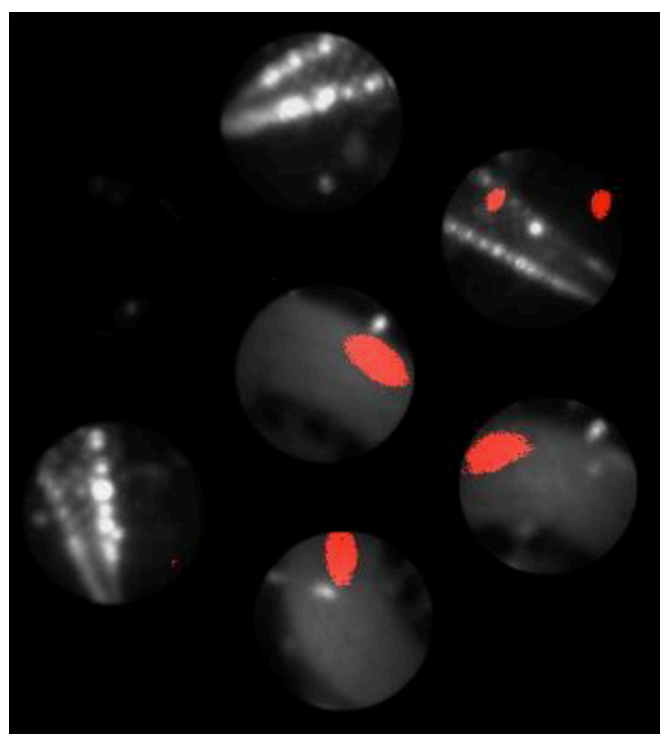

Figure 3.10.7: Image from 7 fibers from the RFR tests at Yale. Light preceding breakdown at the locations of the resistors is shown in red, overlaid on the images of the RFR under LED illumination.

monitored using a small TPC with Au photocathode to ensure that electron lifetime is sufficiently high that impurities do not suppress electrical breakdown. A schematic of this test setup may be seen in Figure 3.10.8.

The Phase-I TPC will undergo testing in liquid xenon at the SLAC System test platform. The staging at SLAC is shown in Figure 3.10.9. The former BaBar counting room and surrounding space in the IR2 experimental hall was renovated for the purpose of hosting this system, with ample room for expansion. A soft-wall clean area with HEPA units surrounds the vessels. The support systems for these test vessels, partially visible in Figure 3.10.9, are extensive. They build on developments from LUX and ZEPLIN and serve as prototypes of what will be used on LZ. Cryogenics for both phases are supplied by a thermosyphon system, which consists of a multi-port $\mathrm{LN}_{2}$ dewar capable of providing more than 12 separate PID-controlled cooling heads.

The system for online purification through a hot getter uses highly automated gas-handling panels based on 1/2-inch-diameter tubing that will accommodate flow rates well in excess of $100 \mathrm{slpm}$. The system also features a high-flow capacity metal diaphragm compressor as the circulation pump. This technology allows very high flow rates and has been identified (Chapter 6) as the technology for LZ, but to our knowledge it has not been used in any previous similar Xe experiment. Thus, the system will provide an important test of these pumps.

Critical elements of control and fail-safe recovery of the Xe have also been developed as part of the system test platform, including integration of process loop controllers (PLCs) for essential systems and integration with larger slow-control system development. Phase-I Xe recovery uses a thermosyphon-driven storage and recovery vessel patterned on a similar device used for LUX. Elements of the planned LZ online and slowcontrol systems have been developed for the system test to allow a high degree of test automation. The gas system is also designed to be closely integrated with an automated, high-sensitivity purity-monitoring system initially developed by the Maryland group. This will be important in order to achieve purity for successful HV testing; it also allows us to check our understanding of various parameters related to purification that will be important for LZ. Finally, the system is designed to accommodate the range of gaseous radioactive calibration sources deployed in LUX and planned for LZ.

The SLAC Phase-I vessel set is shown in Figure 3.10.10 (left) with the primary Xe vessel on the left, and the purification tower on the right of the image. The purification tower includes prototypes of the 


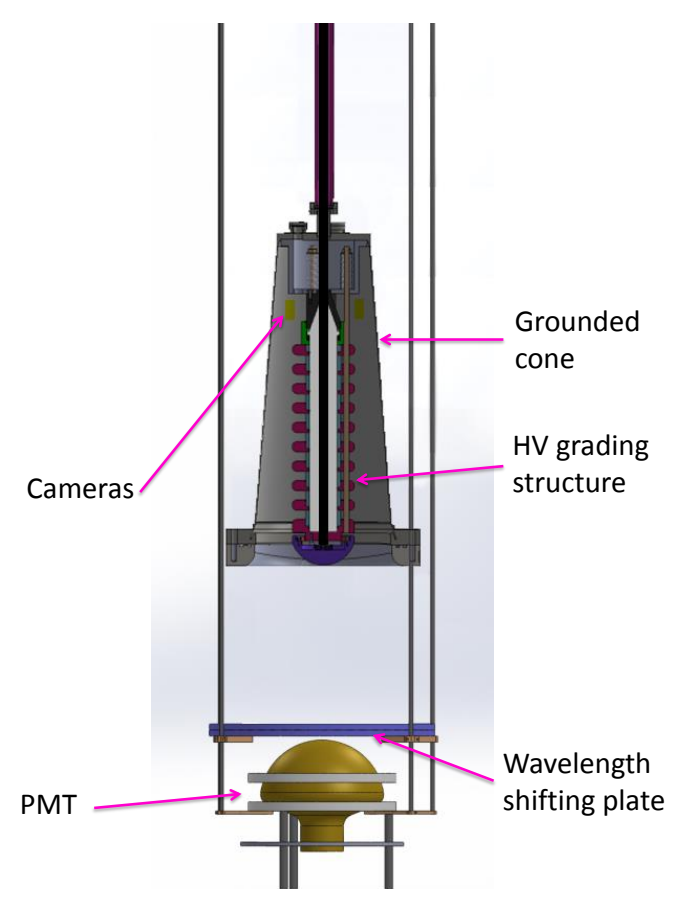

Figure 3.10.8: Schematic of the planned HV test of the cathode connection region immersed in LAr.

elements of the circulation system planned for LZ: a weir reservoir, two-phase heat exchanger, gas-phase heat exchanger, and a "sub-cooling" thermosyphon head on the condensing stream, along with an extensive set of fluid level sensors and thermometers.

The Phase-I tests will continue at SLAC through the spring of 2017 and will allow the assurance of HV operation of the TPC under controlled conditions as well as other LZ subsystems.

\subsubsection{Full Scale Grid Testing at SLAC}

A second stage of testing will occur at the SLAC platform to test full LZ-scale grids in liquid xenon. Initially, prototype full scale grids will be tested to confirm the cleaning and handling procedures developed through the Phase-I tests, prior to the testing of the final LZ grids, including the full gate-anode assembly.

The cryostat for Phase-II is shown in Figure 3.10 .10 (right). It will hold $60 \mathrm{~kg}$ of LXe per $\mathrm{cm}$ of liquid height, and is expected to operate at approximately $6 \mathrm{~cm}$ of depth. The extraction region of the gate and anode grids will be tested as a full assembly to full operational voltage. A shortened reverse field region with the cathode and bottom grid will, as in the Phase-I tests, scale the surface fields to LZ values with a reduced cathode voltage.

Optical cameras and a limited number of PMTs will instrument the Phase-II vessel set to look for emission at operational fields. Other instrumentation in the xenon will also combine LZ prototypes, final hardware (such as temperature and level sensors), and some hardware used specifically in the SLAC tests (cathode feedthrough and digitizers).

For Phase-II the purification and circulation system developed for Phase-I will be used, with the exception of requiring a compressor-based recovery system for the larger quantity of xenon. We will recover into standard storage cylinders, as is planned for LZ. This requires a highly reliable system with generator-based backup power.

The Phase-II testing of the final LZ grids in liquid xenon will be the culmination of the system test program and the final assurance of the TPC meeting both the HV performance requirements and goals. 

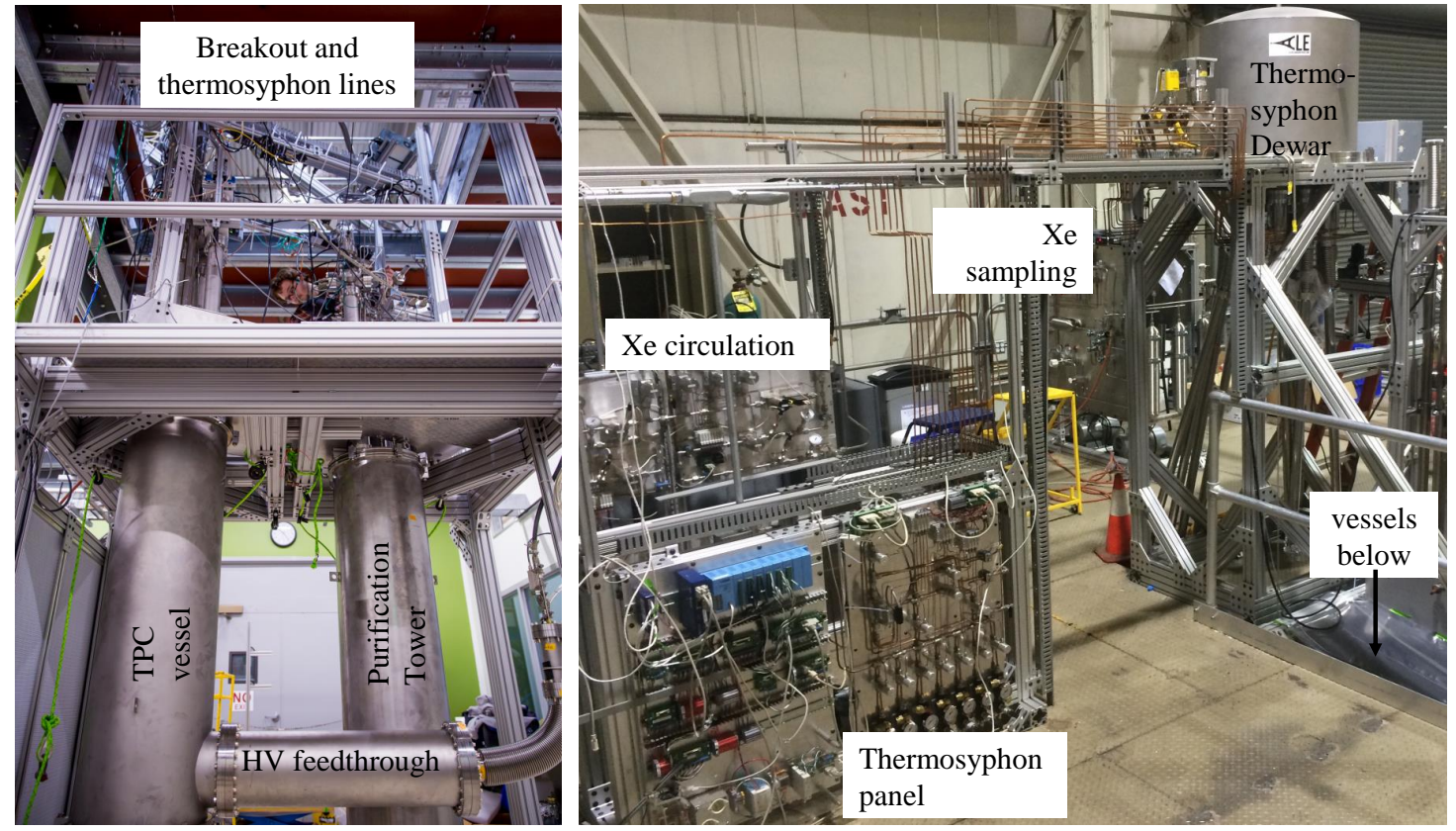

Figure 3.10.9: The LZ System Test platform at SLAC. Left: Phase-I system, consisting of the TPC vessel on the left with a side-entry cathode HV feedthrough visible at the bottom, purification tower on the right, and various PMT, other grid HV, plumbing and cryogenic breakouts above. Right: Support infrastructure housed above the vessels, consisting of thermosyphon system gas panel at front left, $\mathrm{Xe}$ circulation panel behind that, and the multi-port thermosyphon dewar at upper right. The cold-trap Xe assay system developed by the Maryland group is in the back right.
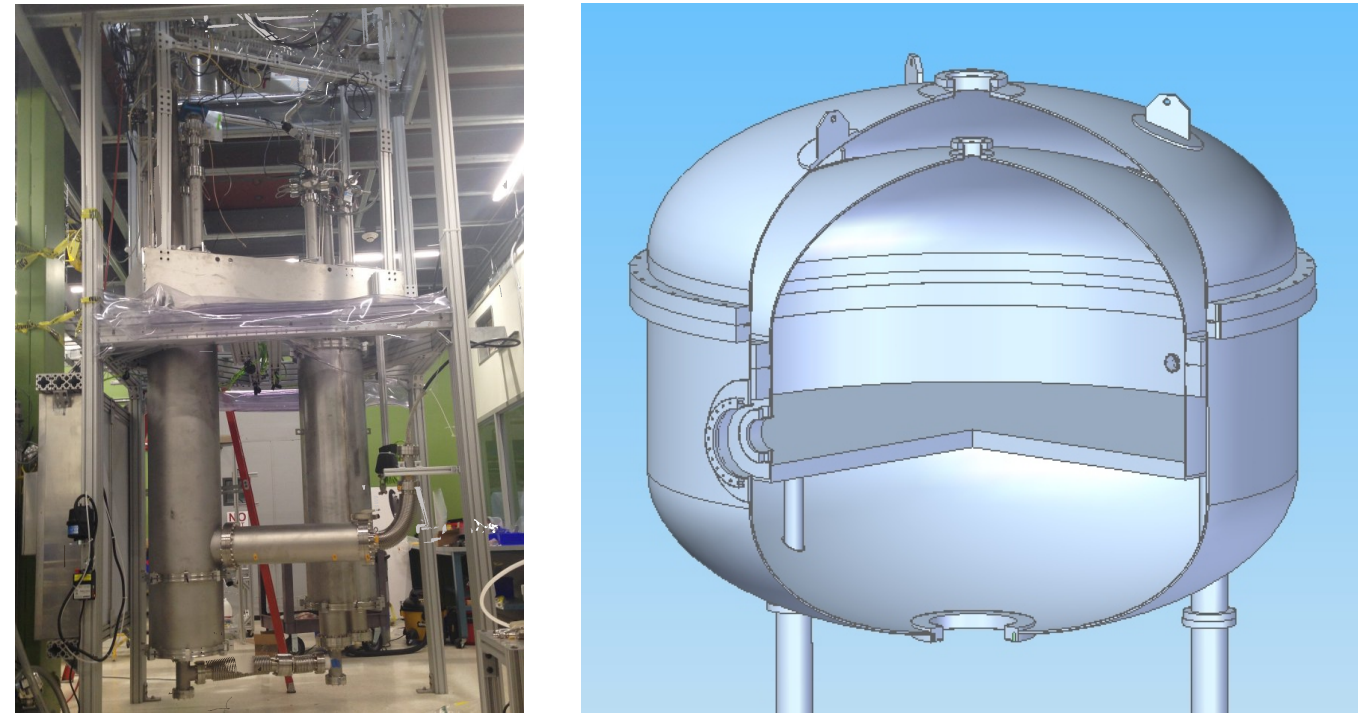

Figure 3.10.10: Left: SLAC Phase-I vessel set; On the left is the primary Xe vessel containing the TPC, and the HV feedthrough extends horizontally to the right. The purification tower is the vessel on the right, containing the weir reservoir, heat exchangers and sub-cooler. The HEPA units are visible on the left of the stand, and the cleanroom curtains are rolled up on the detector stand for the photograph. Right: Phase-II Xe vessel design, to test full-scale LZ grids. At the full diameter, $60 \mathrm{~kg}$ of xenon will be needed per $\mathrm{cm}$ of liquid height. 


\subsection{Bibliography}

[1] D. S. Akerib et al. (LZ), (2015), Conceptual Design Report; LBNL-190005, FERMILAB-TM-2621AE-E-PPD, arXiv:1509.02910 [physics.ins-det].

[2] W. Blum, W. Riegler, and L. Rolandi, Particle Detection with Drift Chambers, Particle Acceleration and Detection (Springer Berlin Heidelberg, Germany, 2008).

[3] K. McDonald, Notes on Electrostatic Wire Grids, Tech. Rep. (Joseph Henry Laboratories, Princeton University, Princeton, NJ 08544, 2003).

[4] Y. Mei, Direct Dark Matter Search with the XENON100 Experiment, Ph.D. thesis, Rice University (2011).

[5] E. Gushchin, A. Kruglov, V. Litskevich, A. Lebedev, and I. Obodovski, Sov. Phys. JETP 49, 856 (1979), [Zh. Eksp. Teor. Fiz. 76, 1685 (1979)].

[6] A. Fonseca, R. Meleiro, V. Chepel, A. Pereira, V. Solovov, and M. Lopes, in Nuclear Science Symposium Conference Record, 2004 IEEE, Vol. 1 (2004) pp. 572-576, Study of secondary scintillation in xenon vapour.

[7] C. F. P. da Silva, Study of the reflectance distributions of fluoropolymers and other rough surfaces with interest to scintillation detectors, Ph.D. thesis, Universidade de Coimbra, Faculdade de Ciências e Tecnologia (2009).

[8] C. Silva, J. Pinto da Cunha, A. Pereira, M. I. Lopes, V. Chepel, V. Solovov, and F. Neves, Nucl. Instrum. Meth. A619, 59 (2010), arXiv:0910.1058 [physics.ins-det].

[9] G. K. White and P. J. Meeson, Experimental Techniques in Low-Temperature Physics (Oxford University Press, 2002).

[10] DuPont Fluoroproducts, DuPont PTFE Properties Handbook (DuPont Fluoroproducts, 1996) P.O. Box 80713, Wilmington, DE 19880-0713.

[11] J. K. Miller, S. Howe, and W. E. Spear, Phys. Rev. 166, 871 (1968).

[12] U.S. Department of Energy, DOE Handbook: Electrical Safety, Tech. Rep. DOE-HDBK-1092-2013 (Forrestal Building, 1000 Independence Avenue, SW, Washington, DC 20585-0002, USA, 2013).

[13] D. S. Akerib et al. (LUX), Nucl. Instrum. Meth. A703, 1 (2013), arXiv:1205.2272 [physics.ins-det].

[14] K. Nakamura, Y. Hamana, Y. Ishigami, and T. Matsui, in $1^{\text {st }}$ International Conference on Technology and Instrumentation in Particle Physics, TIPP09, Tsukuba, Japan, March 12-17, 2009, Nucl. Instrum. Meth., Vol. A623 (2010) pp. 276-278, Latest bialkali photocathode with ultra high sensitivity.

[15] H. M. Araújo et al., Nucl. Instrum. Meth. A521, 407 (2004).

[16] D. C. Malling, Measurement and Analysis of WIMP Detection Backgrounds, and Characterization and Performance of the Large Underground Xenon Dark Matter Search Experiment, Ph.D. thesis, Brown University (2014).

[17] E. Aprile et al. (XENON1T), Eur. Phys. J. C75, 546 (2015), arXiv:1503.07698 [astro-ph.IM].

[18] T. Haruyama, AIP Conf. Proc. 613, 1499 (2002). 
[19] Z. Liu, J. Chen, P. Zhu, Y. Li, and G. Zhang, Appl. Rad. Isot. 65, 1318 (2007).

[20] C. H. Faham, Prototype, Surface Commissioning and Photomultiplier Tube Characterization for the Large Underground Xenon (LUX) Direct Dark Matter Search Experiment, Ph.D. thesis, Brown University (2014).

[21] C. H. Faham, V. M. Gehman, A. Currie, A. Dobi, P. Sorensen, and R. J. Gaitskell, J. Instrum. 10, P09010 (2015), arXiv:1506.08748 [physics.ins-det].

[22] J. Jortner, L. Meyer, S. A. Rice, and E. G. Wilson, J. Chem. Phys. 42, 4250 (1965).

[23] V. Chepel and H. Araújo, J. Instrum. 8, R04001 (2013), arXiv:1207.2292 [physics.ins-det].

[24] V. N. Solovov, V. Chepel, M. I. Lopes, and A. Hitachi, Nucl. Instrum. Meth. A516, 462 (2004), arXiv:physics/0307044 [physics.optics].

[25] D. S. Akerib et al. (LUX), Phys. Rev. Lett. 112, 091303 (2014), arXiv:1310.8214 [astro-ph].

[26] E. Aprile et al. (XENON10), Astropart. Phys. 34, 679 (2011), arXiv:1001.2834 [astro-ph].

[27] P. F. Sorensen, A Position-Sensitive Liquid Xenon Time-Projection Chamber for Direct Detection of Dark Matter: The XENON10 Experiment, Ph.D. thesis, Brown U., Phys. Dept. (2008).

[28] X. Xiao et al. (PandaX), Phys. Rev. D92, 052004 (2015), arXiv:1505.00771 [hep-ex].

[29] V. N. Lebedenko et al. (ZEPLIN-III), Phys. Rev. D80, 052010 (2009), arXiv:0812.1150 [astro-ph].

[30] E. Aprile et al. (XENON100), Astropart. Phys. 35, 573 (2012), arXiv:1107.2155 [astro-ph].

[31] G. J. Alner et al. (ZEPLIN-II), Astropart. Phys. 28, 287 (2007), arXiv:astro-ph/0701858 [astro-ph].

[32] F. P. Santos, T. H. V. T. Dias, A. D. Stauffer, and C. A. N. Conde, J. Phys. D. Appl Phys. 27, 42 (1994).

[33] S. F. Biagi, Nucl. Instrum. Meth. A421, 234 (1999), (Magboltz).

[34] A. Bailey, Dark matter searches and study of electrode design in LUX and LZ, Ph.D. thesis, Imperial Coll., London (2016).

[35] M. Lyly, J. Ruokolainen, and E. Järvinen, ELMER - A finite element solver for multiphysics, Tech. Rep. (CSC - IT Center for Sciene Ltd., P. O. Box 405, FI-02101 Espoo, Finland, 1999-2000) (Elmer).

[36] C. Geuzaine and J.-F. Remacle, Int. J. Numer. Meth. Eng. 79, 1309 (2009), (Gmsh).

[37] R. Veenhof, in Programming and mathematical techniques in physics. Proceedings, International Conference on programming and mathematical methods for solving physical problems, June 14-19, 1993, Dubna, Russia, Conf. Proc., Vol. C9306149 (1993) pp. 66-71, (Garfield++).

[38] A. Morozov, V. Solovov, R. Martins, F. Neves, V. Domingos, and V. Chepel, JINST 11, P04022 (2016), ANTS Package Downloads, arXiv:1602.07247 [physics.ins-det].

[39] V. N. Solovov et al. (ZEPLIN-III), in Nuclear Science Symposium and Medical Imaging Conference (NSS/MIC), 2011 IEEE (2011) pp. 1226-1233, Position Reconstruction in a Dual Phase Xenon Scintillation Detector. 
[40] D. W. Lynch and W. Hunter, in Handbook of Optical Constants of Solids, edited by E. D. Palik (Academic Press, Burlington, 1997) pp. 233-286.

[41] S. Stephenson, J. Haefner, Q. Lin, K. Ni, K. Pushkin, R. Raymond, M. Schubnell, N. Shutty, G. Tarlé, C. Weaverdyck, and W. Lorenzon, JINST 10, P10040 (2015), arXiv:1507.01310 [physics.ins-det].

[42] F. Neves et al. (ZEPLIN-III), J. Instrum. 6, P11004 (2011), arXiv:1106.0808 [astro-ph].

[43] E. Aprile, H. Contreras, L. W. Goetzke, A. J. Melgarejo Fernandez, M. Messina, J. Naganoma, G. Plante, A. Rizzo, P. Shagin, and R. Wall, J. Instrum. 9, P11012 (2014), arXiv:1408.6206 [physics.ins-det]. 



\section{Outer Detector}

\subsection{Introduction}

In this chapter, we describe the performance and design of the outer detector system for LZ. The principal signal we seek, that of a WIMP scatter depositing $5 \mathrm{keV}_{\mathrm{nr}}$ to $50 \mathrm{keV}_{\mathrm{nr}}$ of energy in the central $5.6 \mathrm{t}$ volume of LXe, will never be accompanied by deposited energy in the surrounding detector components. In contrast, many dominant backgrounds that might fake a WIMP signal will deposit energy not only in the central Xe detector but also in the material surrounding it. If we are able to detect these secondary interactions, we can veto the background event. Table 1.6.1 shows the major backgrounds in LZ, which include signals from gammas with energies in the few-MeV range and neutrons from $(\alpha, n)$ reactions or created by cosmic-ray interactions.

To reduce these backgrounds, we surround the large active Xe volume with an integrated detector capable of tagging gamma rays and neutrons with high efficiency. Three detector elements are used to achieve this performance:

- The instrumented "skin" of the Xe, the region outside the LXe TPC,

- The gadolinium-loaded liquid scintillator (Gd-LS), and

- The portion of the surrounding water that is instrumented as a muon veto.

The outer detector system comprises the scintillator and water systems. In addition to the performance of the integrated veto system, this chapter describes the design of the outer detector system and modifications needed in the water tank to accommodate the LZ experiment.

\subsection{Function and Performance}

The outer detector serves two critical functions:

1. To veto neutron and gamma backgrounds with high efficiency. Although the outer region of the LXe shields the inner region very efficiently, the outer half of the Xe could not be used as part of the fiducial mass without an external veto. By instrumenting the outer skin of the LXe and adding the outer liquid scintillator detector, we are able to nearly double the fraction of the Xe in which very-low backgrounds are achievable. The outer liquid scintillator detector is essential for vetoing neutrons, the background that most closely mimics dark-matter scattering.

One risk to the performance of the LZ detector is that some material very close to the LXe could have a concentration of radioactive impurities higher than expected. The combination of the LXe skin and the outer detector serves to mitigate this risk. The integrated veto can suppress most backgrounds even if they are significantly higher than the design goals, with only a slight reduction in fiducial volume.

2. To help characterize and measure the background. A claim of a WIMP signal would require extraordinary supporting evidence. The outer detector will provide crucial supporting evidence necessary to establish a discovery. In particular, the NR background to a WIMP signal can be estimated by measuring the number of low-energy deposit scatters in the TPC which are followed by neutron captures in the outer detector. 
The major non-neutrino background sources in LZ are neutrons and gammas from components within the cryostat and beta decays from radon and krypton distributed throughout the Xe. The principal goal of the integrated veto system is to reduce the effect of neutron and gamma backgrounds to a level smaller than that caused by radon and krypton over a very large fraction of the active LXe.

Neutrons represent a particularly troublesome background in the absence of an external veto. A neutron scatter produces a nuclear recoil (NR), as does a WIMP scatter, and after scattering they can escape the TPC and skin more easily than a gamma. The neutron background, which is principally produced in $(\alpha, n)$ reactions in materials near the LXe, is more difficult to predict than the gamma background. If a possible WIMP signal is seen, the outer detector will contribute to the identification and estimation of the neutron background.

The design requirement for NR background is stringent (see Chapter 9). Without the outer detector, the neutron background would exceed our requirements, so meeting the sensitivity target requires a veto efficiency of greater than $90 \%$ for neutrons escaping the TPC.
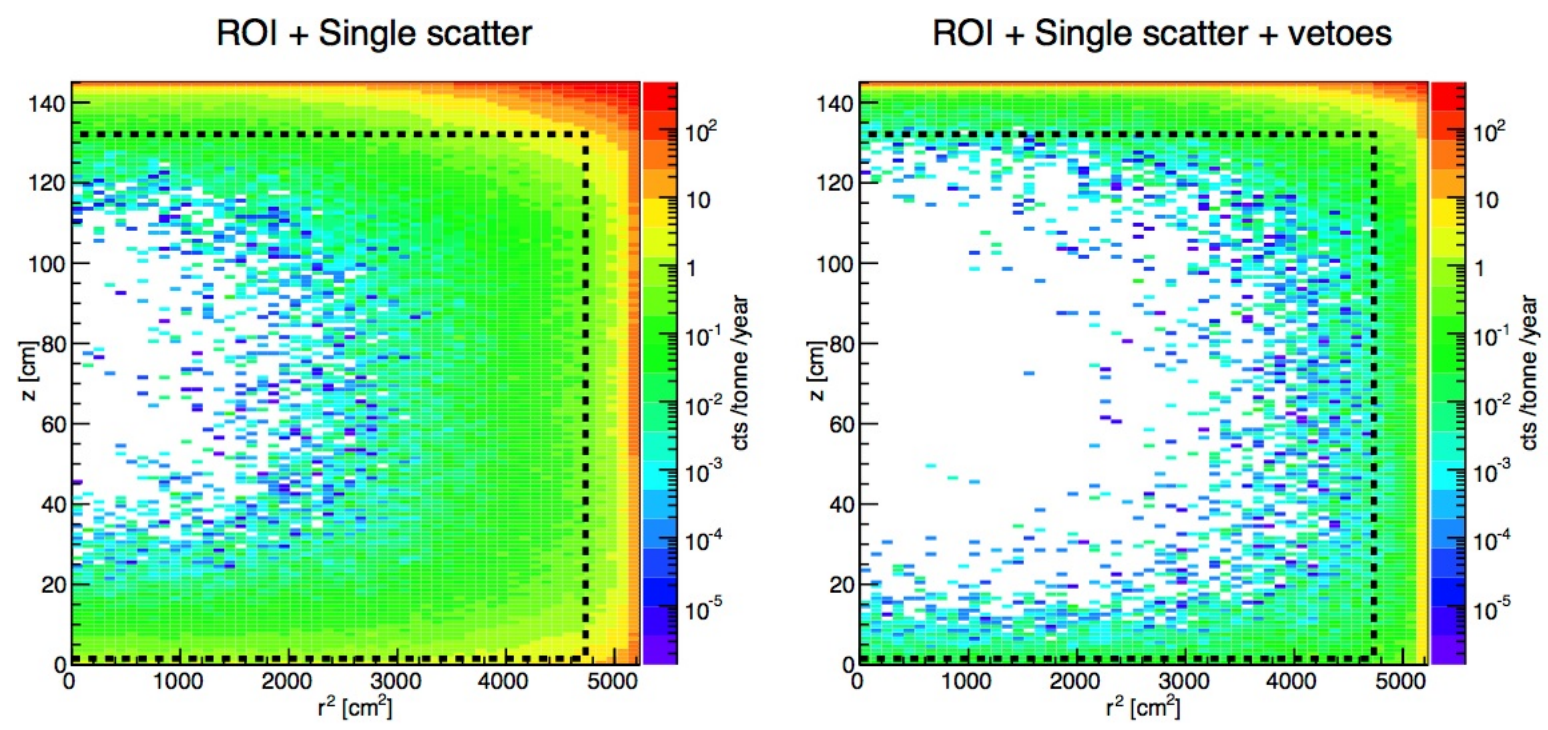

Figure 4.2.1: Total NR background plus ER leakage from material radioactivity for sources external to the LXe in the TPC, counted over a $6 \mathrm{keV}$ to $30 \mathrm{keV}$ acceptance region; a discrimination efficiency of $99.5 \%$ is applied to ERs from beta decays and gamma rays. Left: Single scatters only, no vetoing by the anti-coincidence systems. Right: Adding the combination of both the skin veto and the outer detector. The dashed line shows the boundary of the 5.6 tonnes fiducial mass.

The principal sources of gamma background are components in direct contact with the LXe volume, such as the PMTs, the PTFE reflectors, and the titanium inner vessel. Gammas in the few-MeV range can scatter at small angles in the outer region of the TPC, depositing $0.5 \mathrm{keV}$ to $6.5 \mathrm{keV}$ of energy corresponding to the WIMP signal region, and then exit the TPC without a second scatter. Meeting our requirement (see Chapter 12) requires a veto efficiency of $>70 \%$ for such gammas.

We have carried out simulations to characterize the impact of the outer detector on the background characteristics of the detector. The results of these simulations are captured in Figure 4.2.1, which shows the spatial distribution of the neutron backgrounds that scatter only once in the LXe volume.

The left panel of Figure 4.2.1 shows the distribution of single scatters from backgrounds originating from fixed contamination, such as uranium and thorium, which generate neutrons that scatter in the Xe TPC, as well as gamma rays which produce ERs that leak into the NR band. The figures plot depth ( $z$ ) versus radiussquared, so that the area on the plot is proportional to the volume of LXe. The central region is, as expected, 
extraordinarily free of background. But the neutron background is much higher within $\approx 20 \mathrm{~cm}$ of the outer structures. The dashed black line indicates the 5.6t fiducial volume. Without using information from the LXe skin or the outer detector, the fiducial mass is a bit less than half of the active LXe. Most of the active LXe itself in this case is used as a veto rather than as target material for WIMPs. If one of the component materials in the cryostat were to have a larger amount of radioactivity than the design target, even less of the active LXe would be in the fiducial volume.

The right panel of Figure 4.2.1 shows the performance when vetoing events that also deposit energy in either the instrumented LXe skin or the outer detector. The dashed line shows that the fiducial volume can be extended to within a few centimeters of the edge of the active LXe. The integrated veto system enables a fiducial volume of 5.6 t, almost twice as large as for a stand-alone LXe TPC. Even if the neutron backgrounds were significantly higher than assumed in this study, the very-low background needed for effective operation of LZ could be maintained by reducing the $5.6 \mathrm{t}$ fiducial volume by only a very small amount.

Because of the large surface area of the LXe vessel, the fiducial volume increases by about $270 \mathrm{~kg}$ for every additional centimeter thickness of LXe at the boundary. To meet the LZ background requirements for $>5.6 \mathrm{t}$ fiducial volume without using an external veto would require a TPC containing $11 \mathrm{t}, 4 \mathrm{t}$ more than the LZ design value.

\subsection{Outer Detector Overview}
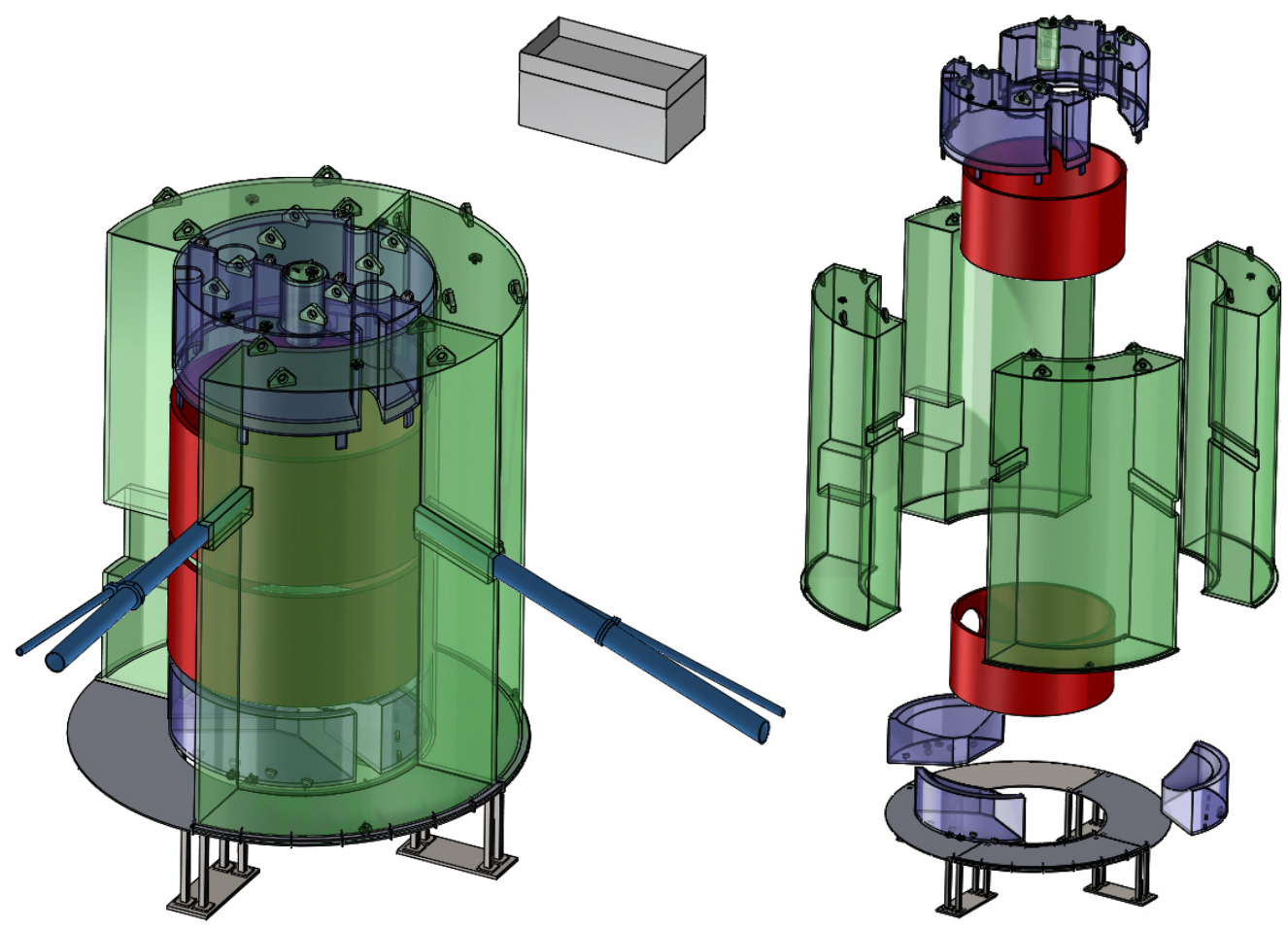

Figure 4.3.1: Layout of the LZ outer detector system, assembled on the left, and exploded to display the ten acrylic tanks, water displacers (in red), and stand, on the right. The tanks will be filled with Gd-LS, and the largest are the four quarter-tanks on the sides. Two tanks cover the top, and three the bottom. A small cylindrical vessel fits into a cutout at the center of the top tanks, replacing a YBe source for most running. The grey box is a reservoir for liquid scintillator. 
The proposed layout of the LZ outer detector is shown in Figure 4.3.1. A hermetic detector is built from ten vessels fabricated from UVT acrylic. The use of segmented vessels allows fabrication to take place at the manufacturer's facility at considerable cost savings. The sizes of the vessels are chosen to allow straightforward insertion into the water tank and assembly of the full detector inside the water tank. Structural finite element analyses (FEAs) of the vessels have been performed to validate the design without introducing more inert material than is needed for safe operation. The acrylic for the side vessels is 1 inch thick; for the top and bottom vessels, the acrylic is 0.5 inch thick except for the top wall of the top vessel and the bottom wall of the bottom vessel.

The vessels will be viewed by 1208 inch Hamamatsu R5912 PMTs. The PMTs are mounted on stainless steel frames in the water tank, separated from the Gd-LS vessels by $84 \mathrm{~cm}$ of ultrapure water. This arrangement gives a light-collection efficiency of about $7 \%$ averaged over the volume of the outer detector, corresponding to a light yield of about 130 photo-electrons for a $1 \mathrm{MeV}$ energy deposit. The water shields the Gd-LS from gammas that originate in the R5912 tubes. A low-density water displacer will be used to fill in the gaps between the cryostat and the acrylic vessels and the gaps around the penetrations, to reduce the probability of absorption in water. White diffuse reflector will be placed on all available surfaces to improve collection of the scintillation light.

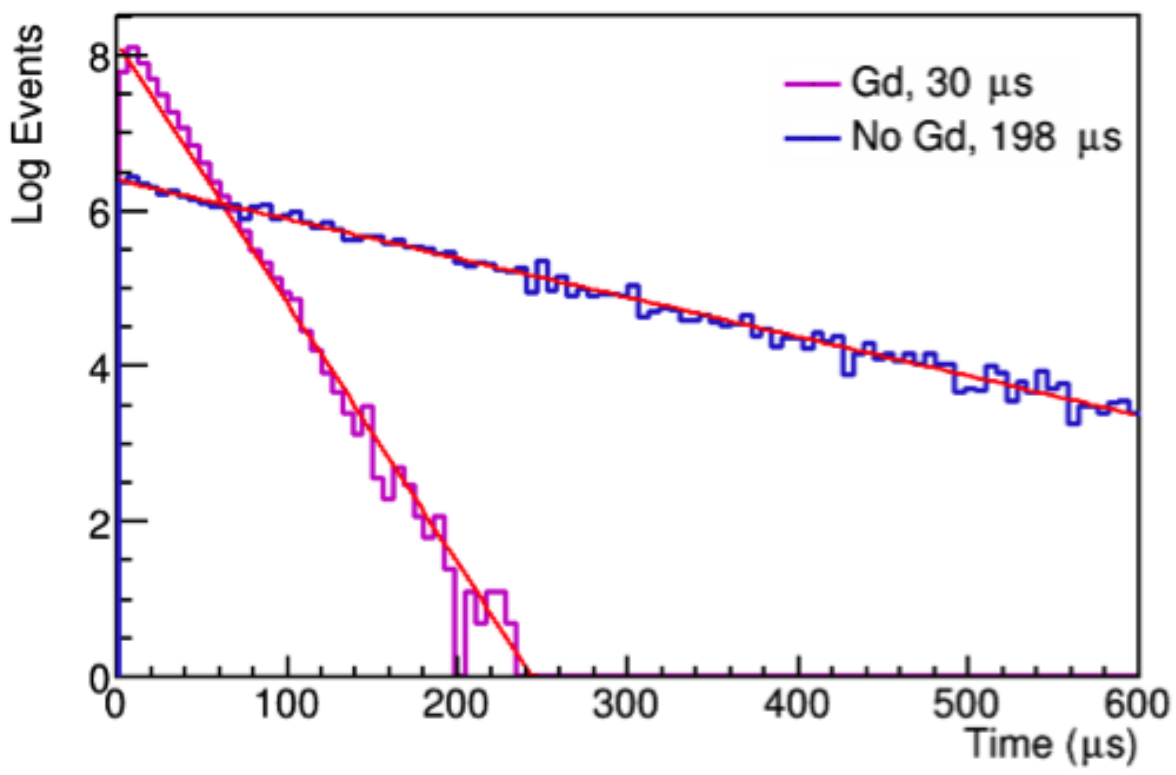

Figure 4.3.2: The simulated distribution of capture times for thermal neutrons in the outer detector for liquid scintillator with and without $0.1 \% \mathrm{Gd}$.

Simulation of the veto performance showed that the veto efficiency varies slowly with the thickness of the scintillator in the outer detector over the range 50 to $80 \mathrm{~cm}$. This thickness is therefore chosen to reduce the risk of problems during fabrication and assembly. To insert the side vessels into the water tank easily, the scintillator thickness needs to be no greater than $70 \mathrm{~cm}$. Cleaning the side vessels during fabrication requires that the thickness be no less than $61 \mathrm{~cm}$, which fixed the side tank scintillator thickness.

The radioactivity in the mine walls causes a flux of gamma rays to impinge on the water tank. The thinnest portions of water are at the top and bottom of the tank. The top and bottom tanks, as well as the vertical extent of the side tanks, were adjusted to reduce the rate caused by penetrating gamma rays at the top and bottom, without significant loss in neutron veto efficiency. Our simulation indicates a surviving rate of order 
$100 \mathrm{~Hz}$, however, simulation of the tiny fraction of surviving penetrating gammas is challenging. The "LS screener" campaign in late 2016, described briefly in Section 4.5, will move a small LS detector through a variety of vertical positions in the water, to test the simulation.

The liquid scintillator is based upon a linear-alkylbenzene (LAB) solvent, which is a hydrocarbon chain with one benzene ring attached. LAB has a flashpoint that exceeds that of diesel fuel, and the safety aspects of diesel fuel in an underground facility have been explored and defined. The LAB is loaded with Gd, $0.1 \%$ by mass, via an organic chelating agent, trimethyl hexanoic acid (TMHA). This scintillator mix with $0.1 \%$ Gd doping was used by Chooz [1], Palo Verde [2], and Daya Bay [3]. The specific approach adopted by LZ is very similar to that used in the Daya Bay neutrino experiment, but with additional purification to achieve a lower radioactivity levels from uranium/thorium/potassium $(\mathrm{U} / \mathrm{Th} / \mathrm{K})$ impurities.

Gadolinium is added to the scintillator to increase the efficiency for tagging neutrons while maintaining low veto deadtime. The benefit of using $\mathrm{Gd}$ and scintillator for this purpose was demonstrated in the ZEPLIN series of experiments. About $90 \%$ of the neutrons which moderate to thermal energies in the scintillator are captured on ${ }^{157} \mathrm{Gd}$ or ${ }^{155} \mathrm{Gd}$, releasing 3-4 gammas with total energy of $7.9 \mathrm{MeV}\left({ }^{157} \mathrm{Gd}\right)$ or $8.5 \mathrm{MeV}\left({ }^{155} \mathrm{Gd}\right)$; the remaining $10 \%$ of these neutrons are captured on hydrogen, producing a single $2.2 \mathrm{MeV}$ gamma. The Gd captures are tagged with higher efficiency because of the multiple gammas produced and the high energy of those gammas. The Gd capture also reduces the neutron capture time to about $30 \mu$ s, compared with about $200 \mu$ s in scintillator without Gd. Figure 4.3.2 shows the simulated capture time for low-energy neutrons entering the outer detector. The fast capture helps reduce deadtime for the veto system.

A veto window of $125 \mu$ s after a WIMP candidate is matched to the capture time with Gd. However, our detailed simulations have shown that $5 \%$ to $10 \%$ of neutrons actually capture in the acrylic, and can be accomodated by a longer veto window. Our goal is to use a veto window of $500 \mu$ s, which we use in our simulation studies. To maintain a deadtime $<5 \%$ for a veto window $500 \mu$ s, we must achieve our goal of a total OD rate below $100 \mathrm{~Hz}$. We can simultaneously achieve an inefficiency $<5 \%$ and deadtime $<5 \%$ with if the OD rate is $300 \mathrm{~Hz}$, and we reduce our veto window to $170 \mu \mathrm{s}$.

\subsection{Mechanical Systems}

\subsubsection{Acrylic Vessels}

The 17.2 $\mathrm{t}$ of scintillator liquid are contained in nine permanent acrylic vessels, as shown in Figure 4.3.1: four tall vessels on the sides, two vessels that form a plug on the top, and three vessels that form a plug at the bottom. A small tenth vessel is used when the YBe calibration source is not in use. Taken together, the LS system forms a $\approx 61 \mathrm{~cm}$ thickness of Gd-LS surrounding the LXe vessel, with several penetrations for connections to the Xe detector and for calibration systems. Similar acrylic vessels were used for the Daya Bay Antineutrino Detectors [3].

The masses and volumes of the ten vessels are shown in Table 4.4.1. The side vessels represent the largest part of the veto mass, holding about $89 \%$ of the scintillator. Each of the four side vessels is $375 \mathrm{~cm}$ high, extends in radius from 97.5 to $163.5 \mathrm{~cm}$, and covers one-quarter of the full azimuth. A vessel is supported and anchored to a stainless steel base frame, which is in turn anchored to a base plate installed on the floor of the water tank. The net upward force on each side vessel when filled is $4,940 \mathrm{~N}$. 
Table 4.4.1: The volume and masses of the scintillator vessels

\begin{tabular}{|l|c|c|c|c|}
\hline & $\begin{array}{c}\text { Acrylic Mass } \\
\mathrm{kg}\end{array}$ & $\begin{array}{c}\text { LS Volume } \\
\mathrm{m}^{3}\end{array}$ & $\begin{array}{c}\text { LS Mass } \\
\mathrm{kg}\end{array}$ & $\begin{array}{c}\text { Net buoyant force } \\
\mathrm{N}\end{array}$ \\
\hline YBe source plug (1) & 8 & 0.025 & 22 & 21 \\
\hline Side Tank (4) & 684 & 4.431 & 3,823 & 4,940 \\
\hline Top Tank (2) & 116 & 0.648 & 559 & 699 \\
\hline Bottom Tank (3) & 59 & 0.313 & 270 & 333 \\
\hline TOTAL & 3,154 & 19.984 & 17,242 & 22,178 \\
\hline
\end{tabular}

The two top vessels and the YBe plug form cylinder that fits inside the side vessels. At the thinnest point, the top(bottom) LS thickness is $40 \mathrm{~cm}(34.5 \mathrm{~cm}$ )-thick, and at the thickest, the top(bottom) LS thickness is $62 \mathrm{~cm}(57 \mathrm{~cm})$-thick. These tanks will be anchored from the top of the outer cryostat vessel. The three bottom vessels form a cylinder of the similar thickness at the bottom. The penetrations for services and calibrations are positioned within the gaps between the acrylic vessel and in cutouts in the acrylic vessels.

Filling the acrylic vessel with LS and the water tank with water at the same time makes it possible to engineer the vessels with acrylic 1 inch thick, much thinner than the 4 inch thickness that would be required if the vessels were ever filled with LS and just air surrounding them. The level of scintillator in each side tank will be kept near the optimum level for minimum stress, about 10 inch above the height of water in the tank. Figure 4.4.1 shows the results of a finite element analysis of the stress on the side vessels during the filling process. During filling the stresses will be kept below 1,000 psi, well below the short-term maximum stress of 3,000 psi. Once the final state has been reached, the maximum stress is 153 psi, well below the long-term crazing limit of 700 psi. The maximum deformation at that stage will be 0.05 inch.

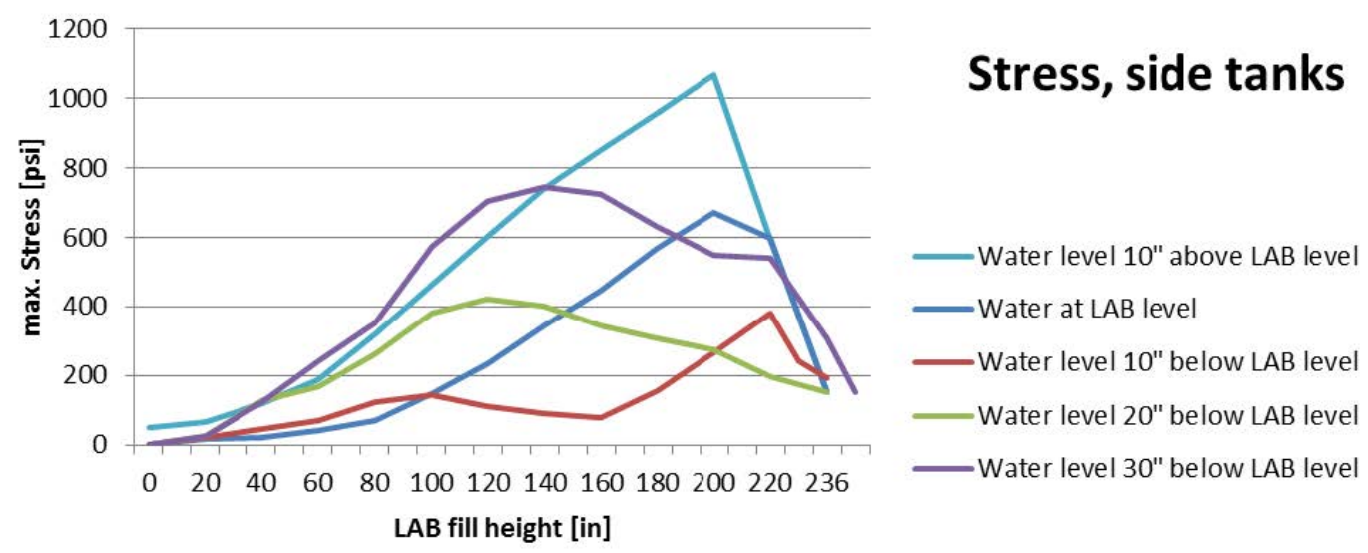

Figure 4.4.1: Stress on the side scintillator tanks during filling, from a finite element analysis. The scintillator height in each vessel will be maintained within the range shown in these five plots. The maximum stress will be maintained well below the short term maximum stress of 3,000 psi.

The flanges on the detector cryostat protrude about 2 inch outside the cylindrical surface. To avoid building recesses in the acrylic vessels to accommodate these protrusions, a low-density closed-cell foam will 
be installed as a water displacer around the outer vessel of the cryostat. This maintains low absorption of gammas between the scintillator and the LXe skin detector.

The vessels will be cleaned inside and leak-checked at the fabrication vendor. They will be wrapped in protective sheets at that time, and placed in double bags before being crated for shipping. The protective sheets will be removed after they are installed in the water tank. The final cleaning of the outside of the vessel will be done at that time.

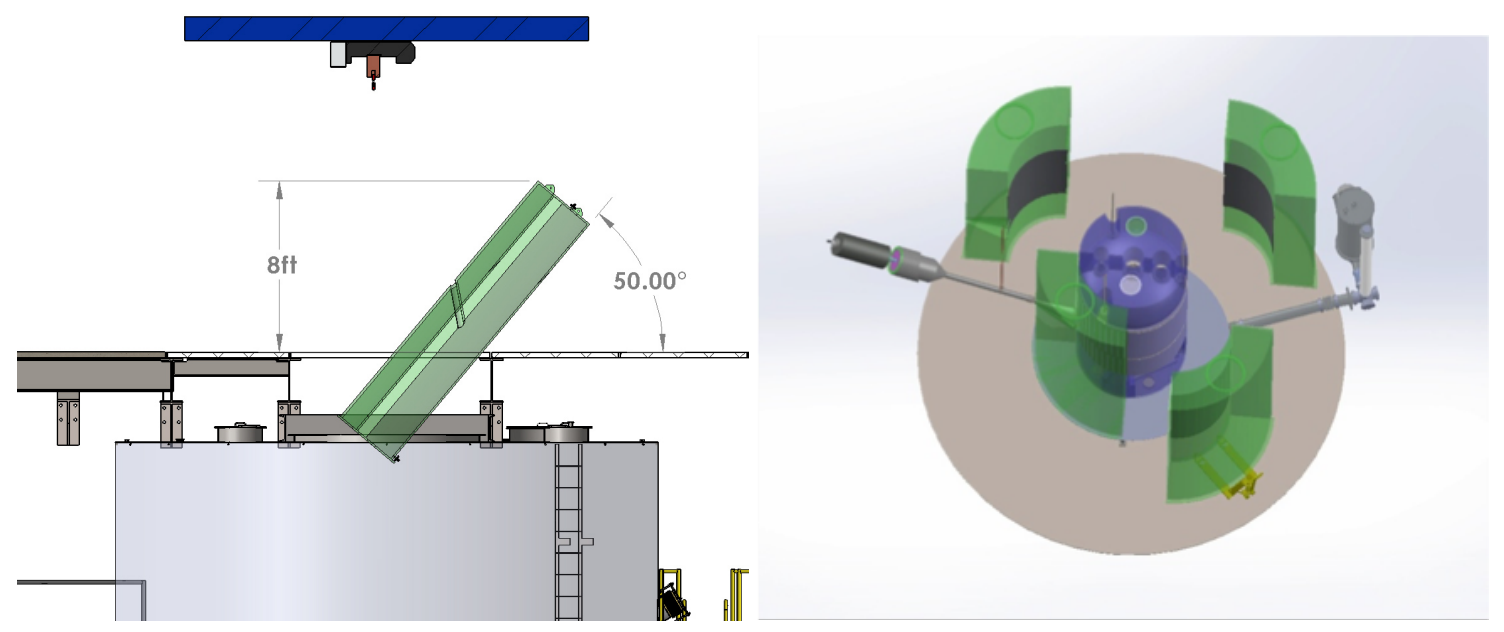

Figure 4.4.2: Two steps in the assembly sequence. The figure on the left shows one of the quadrant vessels at the point of maximum height above the water tank. The one on the right slows the four quadrant vessels in the tank, with one already moved into final location.

As a feasibility study, a mock side vessel was slung under the Yates cage, taken down the shaft, and transported to the cart-wash area just outside the LUX experimental hall. We have studied the process of installing the acrylic vessels into the LUX/LZ water tank using a detailed computer model. The acrylic vessel will be transported in a horizontal position to the deck immediately above the water tank. The vessel will then be rotated using lifting eyes at the top and bottom. The left-hand drawing in Figure 4.4.2 demonstrates one step of this process, near the point that requires maximum clearance above the deck. The vessel is lowered in vertical position into the water tank and then transported radially outward to near the wall of the tank.

The right-hand drawing in Figure 4.4.2 shows the assembly step at which all of the quadrant vessels are in the tank, and the first one is being brought into place around the cryostat. A white diffuse reflector, Tyvek, is strapped with the foam around the cryostat.

The vessels will be fabricated by Reynolds Polymer Technology of Grand Junction, Colorado. Fabrication will take place during calendar 2017.

\subsubsection{PMT Supports}

The LAB scintillation light is viewed by 1208 inch PMTs in a cylindrical array of 20 ladders with six PMTs each. Figure 4.4.3 shows the plan view of the PMT support system. The PMT faces are positioned $84 \mathrm{~cm}$ from the outer-detector tank wall. The water between the PMTs and the scintillator vessel shields the active detector elements from radioactivity in the PMT assemblies. In this location, the PMTs also see the Cherenkov light from cosmic-ray muons passing through the water.

The PMT ladders are attached to the top and bottom of the water tank, at a radius of $282 \mathrm{~cm}$. The PMT frame has been adapted from from the Daya Bay desin. A FINEMET magnetic shield to isolate the 
performance from the local magnetic field can be deployed The PMT cables run directly from the PMTs through one of the ports in the top of the water tank to an electronics rack outside. Strain relief is applied at the port and on the ladders.

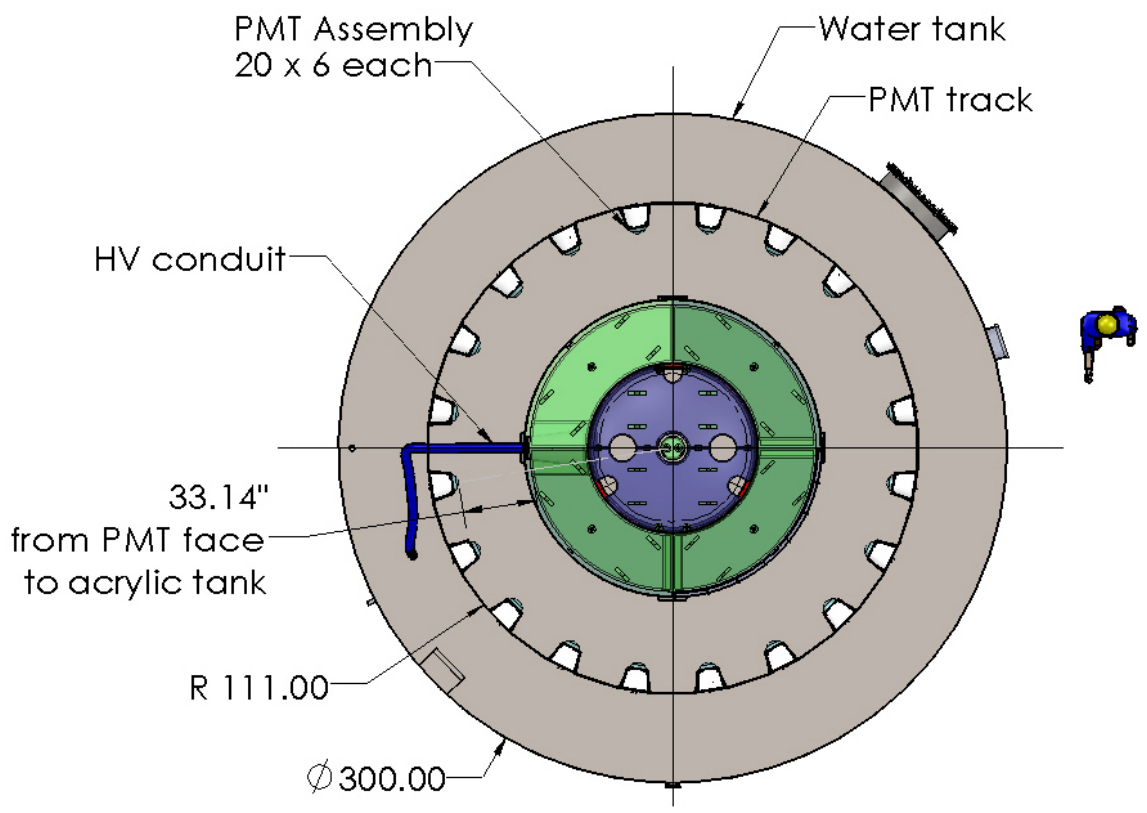

Figure 4.4.3: Plan view of the PMT support system. The 20 PMT ladders are mounted to a circular track on the roof of the water tank.

An optical calibration system (OCS) will be deployed on the support structure, and consists of 35 duplex optical fibers driven by LEDs. This system will enable monitoring of the response of the PMT system, and is described in Section 4.7.

\subsubsection{Scintillator Distribution System}

Each of the scintillator vessels has one input and one output line. The lines are Teflon ${ }^{\circledR}$ tubing, with strain relief to the PMT ladders. All lines terminate at a feedthrough panel in the 2 foot flange on the north side of the lid to the water tank. To reduce pressure on the acrylic tanks, a 100 gallon reservoir will be suspended from the floor beams above and next to the north flange of the water tank.

The Gd-LS will be taken underground in 55 gallon drums. Secondary containment will be provided for all of the lines carrying scintillator. All volumes to be filled with Gd-LS will first be purged with dry nitrogen, and all unfilled gas volumes will be kept purged with dry nitrogen to reduce contamination from atmospheric ${ }^{85} \mathrm{Kr}$.

To reduce the differential pressure inside and outside the vessels, they will be co-filled with the water tank that encloses them. The hydrostatic pressure on the outer surface of the side vessels varies during the filling process from 1 psi to $7.8 \mathrm{psi}$, while the pressure on the inside from the scintillator varies from $1.7 \mathrm{psi}$ to 7.6 psi. The 100 gallon reservoir is connected to each vessel and filled to match the internal pressure to the outside pressure. 


\subsection{Liquid Scintillator}

We chose Gd-LS for the detection medium to achieve excellent efficiency for neutrons and gammas that reach the outer detector. Various organic liquid scintillators have been used for neutron tagging due to their production of relatively large numbers of photons at low energies of a few $\mathrm{MeV}$. The neutron-capture reaction occurs on the hydrogen in the organic scintillator, $n+p \rightarrow d+\gamma$, but the cross section is only $0.33 \mathrm{~b}$, with a neutron-capture time of about $200 \mu \mathrm{s}$. The single $2.2 \mathrm{MeV} \gamma$ is also in the energy range of natural radioactivity.

Gadolinium-loaded scintillators have been used in several experiments designed to detect neutrons produced by inverse beta decay from reactor antineutrinos. There are several compelling advantages of adding Gd to the liquid scintillator (LS):

- The $(n, \gamma)$ cross section for natural Gd is very high, $49 \mathrm{~kb}$, with major contributions from ${ }^{155} \mathrm{Gd}$ and ${ }^{157} \mathrm{Gd}$ isotopes. Because of this high cross section, only a small concentration of $\mathrm{Gd}, 0.1 \%$ by mass, is needed to dominate over neutron capture on protons.

- The neutron-capture reaction on Gd releases $8 \mathrm{MeV}$ of energy in a cascade of 3-4 $\gamma$ rays. The efficiency for detecting at least one of these gammas is very high.

- The time delay for the neutron capture is also significantly shortened to $30 \mu$ s in scintillator doped with $0.1 \% \mathrm{Gd}$, compared to a time of $200 \mu$ in undoped scintillator. This means the veto window can be reduced by a factor of 7 , reducing the deadtime of the outer detector by the same factor.

To detect the low-neutron/gamma backgrounds with high efficiency, the Gd-LS must have the following key properties:

1. Long optical attenuation length, $>10 \mathrm{~m}$ at $430 \mathrm{~nm}$;

2. High light yield, $9,000 / \mathrm{MeV}$;

3. Ultralow impurity content, mainly of the natural radioactive contaminants, such as $\mathrm{U}$, Th, and $\mathrm{K}$; and

4. Long-term chemical stability, over the lifetime of the experiment.

All of these properties have been nearly achieved on a large scale for the Daya Bay experiment. We are adopting the same formulation and fabrication techniques to take advantage of this proven performance

It is necessary to avoid any chemical decomposition, hydrolysis, formation of colloids, or polymerization, which over time can lead to development of color, cloudy suspensions, or formation of gels or precipitates in the scintillator, all of which can degrade the scintillator. Recent successful demonstration of the abovementioned key items has been done by reactor electron antineutrino experiments using LAB-based, $0.1 \%$ Gd-loaded scintillator.

Assessments of the U/Th contaminations in the Daya Bay scintillator show that Daya Bay has already achieved levels nearly acceptable for the LZ experiment. The LZ goal is based upon limiting rate from gamma rays, betas, and alphas in the LXe fiducial volume to a rate lower by a factor of 8 than that expected from other sources, to achieve deadtime below 5 percent. The dominant source of rate in the OD system is the leakage of gamma rays from the walls of the SURF cavern through the top and bottom of the water shield, and will contribute of order $100 \mathrm{~Hz}$ to the OD rates.

The U/Th contamination goals for the scintillator are $<1.3 \mathrm{ppt}$ and $<4.5 \mathrm{ppt}$ by mass, respectively. Four of the five scintillator ingredients- $\mathrm{GdCl}_{3}, \mathrm{LAB}, \mathrm{PPO}$, and bis-MSB-of the scintillator mixture have already been analyzed for U/Th contamination by LZ, and met the goals for Gd-LS. Since the measurements exceeded the original goals, we have changed the goals to be equal to the measurements where measurements have been performed. 
These goals for each component are summarized in Table 4.5.1. The goal for ${ }^{238} \mathrm{U}$ is a factor of 15 below the level achieved by the Daya Bay collaboration. In addition, a level of $0.8 \mathrm{ppt}$ of ${ }^{40} \mathrm{~K}$ is the required, which is a factor of 9 lower than achieved by Daya Bay. KAMLAND and Borexino reached contamination levels orders of magnitude lower by filtering and stripping the scintillator solvent. We plan to meet the targets by inserting a second pass of purification into the production process, one step beyond that applied by the Daya Bay collaboration.

The goal for ${ }^{14} \mathrm{C}$ is aggressive, and to meet it, underground sources must be used for all organics. Should the ${ }^{14} \mathrm{C}$ level be higher than expected, the scintillator threshold will be raised from $100 \mathrm{keV}_{\mathrm{ee}}$ to above the ${ }^{14} \mathrm{C}$ endpoint of $156 \mathrm{keV}_{\mathrm{ee}}$. The scintillator must be kept out of contact with the atmosphere to avoid ${ }^{85} \mathrm{Kr}$, and the levels of ${ }^{85} \mathrm{Kr}$ observed in both KAMLAND and Borexino would contribute a negligible rate to LZ.

Table 4.5.1: Radioactive impurities in LAB-based Gd-LS. The left side contamination goals for various components of the Gd-LS. Cells shaded yellow are based on screening measurements. Propagation of these contributions to the proposed Gd-LS mixture is summarized in the last four columns. The goals (requirements) keep the rate contribution to the OD below $15 \mathrm{~Hz}(80 \mathrm{~Hz})$ from these sources.

\begin{tabular}{|c|c|c|c|c|c|c|c|c|c|}
\hline \multirow[b]{2}{*}{ Item } & \multicolumn{4}{|c|}{ Raw values (ppt) } & \multirow{2}{*}{$\begin{array}{l}\text { Gms/liter } \\
\text { in Gd-LS }\end{array}$} & \multicolumn{4}{|c|}{ Values in $0.1 \% \mathrm{Gd}-\mathrm{LS}$ (ppt) } \\
\hline & ${ }^{238} \mathrm{U}$ & ${ }^{232} \mathrm{Th}$ & ${ }^{40} \mathrm{~K}$ & ${ }^{14} \mathrm{C}$ & & ${ }^{238} \mathrm{U}$ & ${ }^{232} \mathrm{Th}$ & ${ }^{40} \mathrm{~K}$ & ${ }^{14} \mathrm{C}$ \\
\hline LAB & 0.004 & 0.007 & 0.5 & $12 \times 10^{-6}$ & 860 & 0.004 & 0.007 & 0.5 & $12 \times 10^{-6}$ \\
\hline Gd & 100 & 100 & 52 & & 0.86 & 0.17 & 0.17 & 0.09 & \\
\hline PPO & 150 & 640 & 27 & $110 \times 10^{-6}$ & 3 & 0.5 & 2.2 & 0.09 & $0.37 \times 10^{-6}$ \\
\hline TMHA & 180 & 650 & 27 & $140 \times 10^{-6}$ & 3 & 0.6 & 2.1 & 0.09 & $0.44 \times 10^{-6}$ \\
\hline bis-MSB & 210 & 190 & 50 & $19 \times 10^{-3}$ & 0.015 & 0.004 & 0.003 & 0.001 & $0.32 \times 10^{-6}$ \\
\hline \multicolumn{5}{|l|}{ Total } & 867.2 & 1.3 & 4.5 & 0.8 & $13 \times 10^{-6}$ \\
\hline \multicolumn{6}{|c|}{ Requirement } & 10 & 20 & 3 & $15 \times 10^{-6}$ \\
\hline \multicolumn{6}{|c|}{ Daya Bay } & 20 & 4 & 7 & \\
\hline
\end{tabular}

The production of the Gd-LS is being led by the Brookhaven National Laboratory (BNL) group. The manager of the LS production effort has considerable relevant experience, including supervision of the development and production of the same scintillator for the Daya Bay experiment. The development of LZ scintillator will be undertaken in two phases: a demonstration phase (to reach ppt levels), followed by a production phase for deployment. The BNL group has a state-of-the-art Liquid Scintillator Development Facility equipped with a variety of instruments (e.g., UV, IR, XRF, LC-MS, medium-scale mixing reactor, thin-film distillatory, $2 \mathrm{~m}$ attenuation length system, etc.) for quality assurance that are essential to quality control of the scintillator.

To test whether Gd-LS from the demonstration phase meets all the goals in Table 4.5.1, we have built a "LS screener," consisting of an acrylic vessel that can hold about $24 \mathrm{~kg}$ of Gd-LS viewed by three very lowbackground PMTs. We deploy the LS screener in the LZ water tank in November, 2016 through January 2017.

The most cost-effective plan for the 17.2 t of LZ low-background Gd-LS production is to carry out the production at the BNL facility and ship the synthesized scintillator to SURF for filling. The scintillator will be produced at a rate of $0.5 \mathrm{t}$ per week and will be stored in 55-gallon PTFE-lined drums, which are then shipped to SURF for storage. The purification methods for all components of the Gd-doped scintillator are developed and will be applied to each component before synthesis. 


\subsection{Light Detection}

\subsubsection{Photomultipliers}

Building on the successful use of the Hamamatsu R5912 PMTs in experiments such as MILAGRO, AMANDA, and most recently Daya Bay, the LZ outer detector will use 120 of these same PMTs. The two existing models for this PMT are R5912 and R5912-02. The latter possesses four more dynode stages and provides higher gain, at the cost of higher dark current and slightly degraded timing characteristics. As LZ does not need the additional gain, the less-expensive model R5912 was chosen. Even for this model, several subcategories exist that represent various quality levels of glass window and photocathode materials. Our assessment of radioactivity levels concluded that the basic model was sufficient for LZ needs and requirements.

Table 4.6.1: Characteristics of the R5912 PMTs, from the Hamamatsu data sheets.

\begin{tabular}{|l|c|}
\hline Characteristic & Value \\
\hline Number of dynode stages & 10 \\
\hline Window material & Borosilicate glass \\
\hline Photocathode material & Bialkali \\
\hline Minimum photocathode effective area & $284 \mathrm{~cm}^{2}$ \\
\hline Typical bias voltage for gain of $1 \times 10^{7}$ & $1,500 \mathrm{~V}$ \\
\hline Maximum voltage & $2,000 \mathrm{~V}$ \\
\hline Mean QE at $390 \mathrm{~nm}$ & $25 \%$ \\
\hline Pressure rating & $0.7 \mathrm{MPa}$ \\
\hline & ${ }^{238} \mathrm{U}: 1,200 \mathrm{mBq}$ \\
Radioactivity levels per PMT (all components) & ${ }^{232} \mathrm{Th}: 680 \mathrm{mBq}$ \\
& ${ }^{40} \mathrm{~K}: 920 \mathrm{mBq}$ \\
\hline
\end{tabular}

The R5912 PMTs are suitable for the outer detector for the following reasons:

1. The spectral response ranges from $300 \mathrm{~nm}$ to $650 \mathrm{~nm}$, with a peak wavelength at $420 \mathrm{~nm}$. This matches well the scintillation light from the LAB mix between $390 \mathrm{~nm}$ to $440 \mathrm{~nm}$. The quantum efficiency also covers the relevant range, with an average expected value of $\sim 25 \%$ at $430 \mathrm{~nm}$.

2. The PMTs will be submerged in up to $6 \mathrm{~m}$ water and must be able to operate in this environment. Daya Bay has been able to demonstrate successful operation of the R5912 assembly at higher pressures than those required for LZ. This experiment used the same assembly that LZ will use.

3. The radioactivity levels of the PMTs and assembly are a fairly weak constraint, thanks to the minimum $84 \mathrm{~cm}$ of water that separate them from any active detector volume. The water buffer typically reduces the integrated flux of incoming gammas by more than 2 orders of magnitude, before taking into account geometric effects. For the event rate in the Xe target, the $80 \mathrm{~cm}$ of water plus the thickness of the scintillator make the R5912 contribution largely subdominant to internal sources, for both gammas and neutrons. In the scintillator itself, the simulated event rate from PMT radioactivity is $<4 \mathrm{~Hz}$ ( $<4 \%$ deadtime would be caused by $100 \mathrm{~Hz}$ ). 
The R5912 PMTs and waterproof assemblies will undergo rigorous individual testing to fully characterize the response and to validate uniform operation and long-term stability for the lifetime of the detector. These tests will include individual electrical behavior, gain measurements, linearity, after-pulsing, dark current, and dark count. In addition, the PMTs will be radioactively screened to make sure the activities are consistent with the values listed in Table 4.6.1.

\subsection{Optical Calibration System}

An optical calibration system (OCS) will be used to monitor the performance of the outer detector and to maintain calibration. The light will be emitted from 30 LED-driven duplex optical fibers mounted on the PMT support system walls, and 5 duplex fibers routed underneath the bottom tanks. The system on the PMT walls will monitor overall system response, including attenuation in the Gd-LS and in the water. Some fibers under the bottom tanks will use light wavelengths tuned to monitor acrylic transmission.

The OCS will enable the monitoring of the response of the OD system at the level of 3 percent, in time and in space. Systems will monitor the stability of the OCS light sources and supporting light detectors. The range of light outputs covered by the OCS focus on the threshold of $\approx 100 \mathrm{keV}_{\text {ee }}$ up to the expectation of the largest signals from a neutron capture on gadolinium, about $10 \mathrm{MeV}_{\mathrm{ee}}$. The OCS will also enable the monitoring and calibration of large signals from penetrating muons.

\subsection{Threshold, singles rate, deadtime}

As described above, a neutron capture in the Gd-LS releases either a few gammas of total energy $8 \mathrm{MeV}$ or a single gamma of energy $2.2 \mathrm{MeV}$. The neutron-detection efficiency is therefore quite high with the $100 \mathrm{keV}_{\text {ee }}$ threshold planned for the outer detector, which corresponds to about 13 photoelectrons observed. Figure 4.8.1 shows the simulated inefficiency for vetoing $1 \mathrm{MeV}$ background neutrons that scatter once in the LXe TPC as a function of threshold in $\mathrm{keV}_{\text {ee }}$. These simulations indicate that the efficiency of the combined veto for neutrons escaping the LXe TPC is about $97 \%$.

We will read out the outerdetector PMTs each time the LXe TPC produces a trigger, without need of a hardware threshold. We will therefore be able to apply the veto offline, with parameters carefully selected to optimize the veto efficiency. Our goal is to apply neutron veto over a time window of $500 \mu$ s with a threshold of $100 \mathrm{keV}_{\text {ee }}$. In 
addition, we will apply a gamma veto over a much tighter time window of $1 \mu$ s with a threshold of $100 \mathrm{keV}_{\text {ee }}$. There will be an outer-detector hardware trigger to calibrate and monitor the background environment.

The sensitivity of the outer detector is estimated to be about 130 photoelectrons/MeV from simulation of $\mathrm{LZ}$ and this agrees with benchmarks against similar detectors. Thus, a $100 \mathrm{keV}_{\mathrm{ee}}$ threshold corresponds to about 13 photoelectrons, and the trigger rate will be dominated by alphas, gammas, and betas from the $\mathrm{U}$ and Th chains.

Because the time of the low-energy scatter in Xe is determined well by measuring the prompt S1 light, the neutron veto window will be determined by the capture time for neutrons. Our goal is a $500 \mu$ seto window. Our formal requirement is on deadtime, of $<5 \%$. This requirement can be met with a variety of combinations of thresholds and veto windows. Our goal is to keep the rate in the outer detector below $100 \mathrm{~Hz}$, and use a veto window of $500 \mu$ s, but currently our simulations show that we could achieve our deadtime and ineffiency requirements with an outer detector rate as high as $300 \mathrm{~Hz}$ and a veto window of $170 \mu \mathrm{s}$. The radiopurity goals in Table 4.5.1 keep the overall background rate in the OD below $100 \mathrm{~Hz}$.

Ultimately, the of the veto window and threshold needed for high-efficiency neutron tagging will be determined by studying it with calibration data.

\subsection{Environment, Safety, and Health Considerations}

The most important safety issue to consider underground is flammability. The flashpoint for LAB is $120^{\circ} \mathrm{C}$ to $140{ }^{\circ} \mathrm{C}$, which makes it a Class IIIB liquid, the lowest hazard category. A Class IIIB liquid is a combustible liquid with a flashpoint at or above $200^{\circ} \mathrm{C}\left(93^{\circ} \mathrm{C}\right)$. OSHA flammable and combustible liquid regulations do not apply to IIIB liquids. The boiling point is greater than $250{ }^{\circ} \mathrm{C}$ and the melting point is below $-70{ }^{\circ} \mathrm{C}$, so it is very stable as a liquid. The density of LAB is $0.86 \mathrm{gm} / \mathrm{cm}^{3}$, significantly less than water. In addition, it has very low solubility in water.

The primary risk to consider is a crack in one of the acrylic vessels. The first preventive step is to thoroughly check the integrity of the vessel before introducing the scintillator liquid into it. The second is to monitor the vessel carefully during the filling process. The water tank serves as a secondary containment system for the scintillator. We will also be able to separate LAB from the water in the water treatment. If a significant leak is observed, we will skim the surface of the water to recover most of the scintillator, and then remove the residual scintillator by distillation. 


\subsection{Bibliography}

[1] M. Apollonio et al. (CHOOZ), Eur. Phys. J. C27, 331 (2003), arXiv:hep-ex/0301017 [hep-ex].

[2] F. Boehm et al., Phys. Rev. Lett. 84, 3764 (2000), arXiv:hep-ex/9912050 [hep-ex]; A. G. Piepke, S. W. Moser, and V. M. Novikov, Nucl. Instrum. Meth. A432, 392 (1999), arXiv:nucl-ex/9904002 [nucl-ex].

[3] F. P. An et al. (Daya Bay), Nucl. Instrum. Meth. A685, 78 (2012), arXiv:1202.6181 [physics.ins-det]; $\mathrm{X}$. Guo et al. (Daya Bay), "A Precision measurement of the neutrino mixing angle $\theta_{13}$ using reactor antineutrinos at Daya-Bay,” (2007), proposal, arXiv:hep-ex/0701029 [hep-ex]. 


\section{Cryostat}

This chapter discusses the cryostat of the LZ detector. In particular it describes: the results of an extensive screening campaign and the simulations of associated backgrounds that lead to the cryostat material choice, cryostat technical specifications, design with key features, fabrication and tests requirements, and finally transportation and installation underground at SURF.

The cryostat will be fabricated by a specializing in titanium U-stamped pressure vessel manufacturer selected in the European Union tender process. The fabricator will be in charge of the supplied raw material processing including rolling and forging, cryostat manufacture, tests and certifications, cleaning, packaging and transportation to SURF.

\subsection{Material Search Campaign}

The LZ cryostat is one of the major contributors to the experiment's background budget due to the mass of material required and the vicinity to the LXe target. This imposes stringent radiopurity requirements on its materials, and essentially limits the choices to copper, stainless steel and titanium - although copper is rejected due to mechanical considerations. Titanium (Ti) has a high strength-to-weight ratio, low density and atomic number, and was previously sourced with low radioactivity by LUX [1]. The level of this radioactivity content would meet LZ requirements and the lower particle stopping power combined with considerably lower ${ }^{60} \mathrm{Co}$ content make it advantageous over stainless steel. The material studies and searches have therefore been focused on titanium. As a mitigation strategy against failing to source the material with adequate radiopurity and in sufficient quantity, on a sensible timescale, parallel studies have been conducted of stainless steel activity and a full cryostat design in that material has been developed.

Neutron and gamma-ray radioactivity from the cryostat is due to largely to uranium and thorium content in the construction material, as well as ${ }^{40} \mathrm{~K}$ and ${ }^{60} \mathrm{Co}$. To assess the typical concentration of these isotopes in titanium and stainless steel the complementary techniques of direct gamma-ray spectroscopy and and mass-spectrometry were employed. Samples were procured from various stages of production to inform typical activity, reproducibility of particular suppliers and points of inclusion of contamination - particularly for titanium.

\subsubsection{Titanium}

The production of Ti metal is a complex procedure that involves a number of stages in which additives and inclusions are deliberately introduced. Several such points in the production cycle may contribute to contamination of the final product with elements containing high concentration of radioactive isotopes such as ${ }^{238} \mathrm{U},{ }^{232} \mathrm{Th},{ }^{40} \mathrm{~K}$ and ${ }^{60} \mathrm{Co}$ which are of particular concern. The refinement of the mineral concentrates, particularly for ilmenite, involves the addition of or exposure to coke, coal, oil, and tar prior to the chlorination process. It is not uncommon for such materials to contain relatively high levels of $\mathrm{U}$ and Th. However, the $\mathrm{TiCl}_{4}$ produced at this stage undergoes chemical treatment and filtering to remove chlorides and sludge before pure liquid $\mathrm{TiCl}_{4}$ is created, carrying away most impurities, including $\mathrm{U}$ and $\mathrm{Th}$. Ultra-pure $\mathrm{TiCl}_{4}$ is commercially available, as are titanium hydride and titanium nitride powders that are produced through plasmochemical processing from the $\mathrm{TiCl}_{4}$. Beyond this stage, lack of sufficient contact controls with surfaces during the Kroll process and metallothermy present other potential sources of $U$ and Th. However, 
the elements introduced, largely $\mathrm{Mg}$ and Ar, will probably not be problematic. The Ti sponge post-Kroll processing is exposed to several stages in which $\mathrm{U}$ and $\mathrm{Th}$ can enter the chain. Ti ingots and slabs are produced by pressing and melting the Ti sponge, yet often Ti alloy and Ti scrap is added at this stage. Other alloys such as aluminum and vanadium may also be included. Radioactive contamination contained within the scrap and alloys is then carried through to the Ti ingot and into the roll stock. The major stages of this production process, indicating inclusion points, are depicted in Figure 5.1.1 [2].

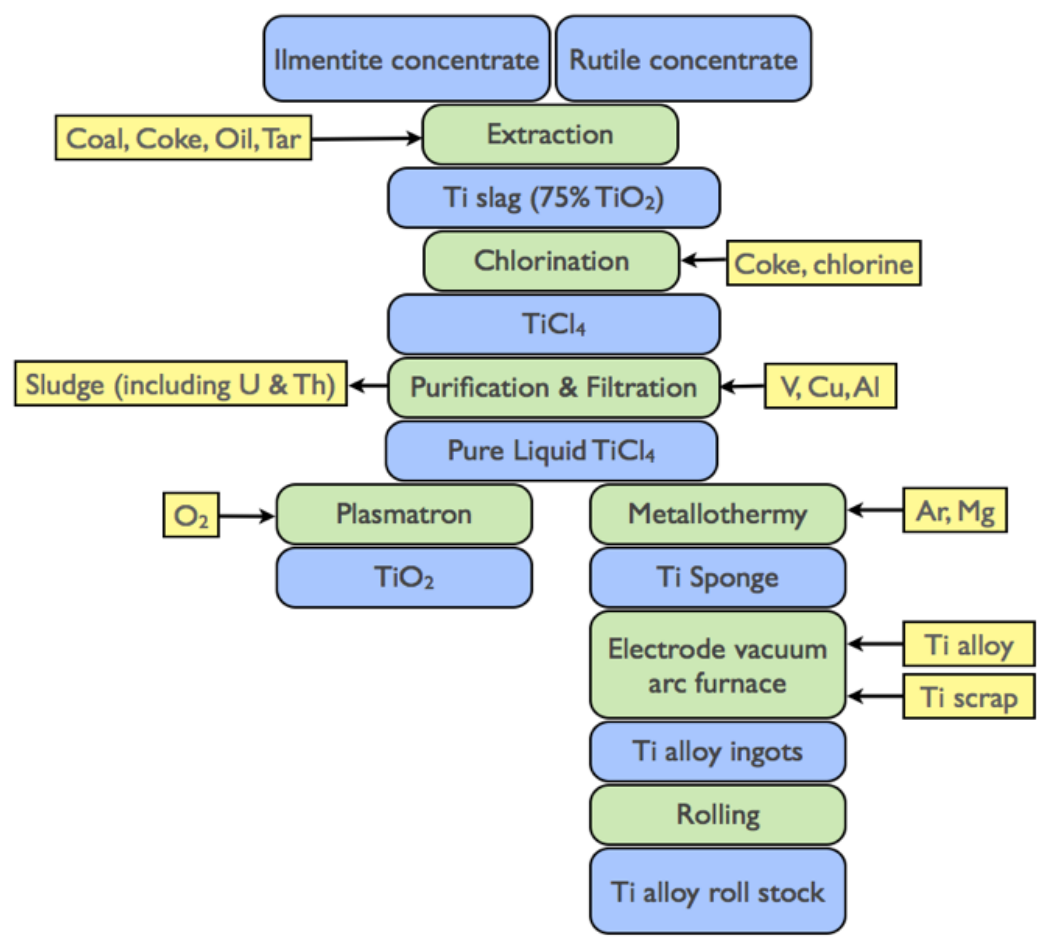

Figure 5.1.1: The commercial production of Ti metal, indicating the major stages (green boxes), the post- processing products (blue boxes), and the additives, as well as reductions during the procedure (yellow boxes). Figure adapted from [2]

In the material search campaign we have engaged several titanium providers including VSMPO [3], TIMET [4], Supra Alloy [5], Honeywell [6] and PTG [7] to provide sample material, taken from various stages along the production process, in order to determine where radioactivity, particularly $\mathrm{U}$ and $\mathrm{Th}$, enters the chain. In our campaign we have received 23 samples including: eight sponges (TIMET), one sample of very high purity Ti (Honeywell), one Gr-1 with 10\% scrap (VSMPO), two Gr-2 sheets (Supra Alloy and PTG), seven Gr-1 sheets (Supra Alloy, PTG and TIMET) - and also Ti bolts and nuts. Table 5.1.1 summarizes all the Ti samples that have been radio-assayed in this campaign. Upper screening limits are indicated in italics.

\subsubsection{Stainless Steel}

Low background experiments searching for dark matter or neutrino-less double beta decay, such as XENON 1T [8] and PANDA-X [9], or GERDA [10] and NEXT [11], respectively, use stainless steel for their cryostats, with the majority of materials coming from German stockholder NIRONIT [12]. 13 samples were procured from NIRONIT for radio-assay. Independent assays of samples received directly from the GERDA and NEXT experiments were conducted to cross-check published results $[13,14]$ and to measure 
Table 5.1.1: Summary of the 22 titanium samples assayed for LZ cryostat, including various grades and types from multiple suppliers.

\begin{tabular}{|c|c|c|c|c|c|c|c|c|}
\hline$\#$ & Supplier & Sample name & ${ }^{238} \mathrm{U}[\mathrm{mB}$ & $q / k g]$ & ${ }^{232} \mathrm{Th}$ & $\mathrm{mBq} / \mathrm{kg}]$ & ${ }^{40} \mathrm{~K}[\mathrm{mBq} / \mathrm{kg}]$ & Titanium \\
\hline & & & early & late & early & late & & grade/type \\
\hline 1 & Supra Alloy & Carlson 8J10 & 31.00 & 4.10 & 0 & 2.80 & 1.80 & Gr-1 Sheet \\
\hline 2 & TIMET & $\begin{array}{c}\text { Osaka } \\
\text { 26-29461 }\end{array}$ & 2.50 & 248 & 0 & 4.10 & 12.0 & Sponge \\
\hline 3 & TIMET & $\begin{array}{c}\text { Tanghsan } \\
\text { TX027594 }\end{array}$ & 2.50 & 6200 & 0 & 2.50 & 15.0 & Sponge \\
\hline 4 & TIMET & $\begin{array}{c}\text { Toho } \\
\text { C } 12009 \mathrm{C}\end{array}$ & 2.50 & 62 & 0 & 1.60 & 12.10 & Sponge \\
\hline 5 & TIMET & $\begin{array}{c}\text { Toho } \\
\text { W 112266W }\end{array}$ & 2.50 & 124 & 0 & 1.60 & 12.00 & Sponge \\
\hline 6 & TIMET & $\begin{array}{c}\text { Zaporozhye } \\
6680-12 \\
\end{array}$ & 2.50 & 744 & 0 & 1.60 & 12.00 & Sponge \\
\hline 7 & TIMET & $\begin{array}{c}\text { Zuny } \\
\text { TX027641 }\end{array}$ & 25.00 & 2480 & 0 & 4.1 & 12.00 & Sponge \\
\hline 8 & TIMET & HN0021-B/1 & 11.00 & 0.60 & 0 & 0.60 & 2.50 & Gr-1 Sheet \\
\hline 9 & TIMET & HN0021-B/2 & 4.90 & 3.33 & 2.85 & 0.80 & 1.50 & Gr-1 Sheet \\
\hline 10 & PTG & ATI W74M & 46.00 & 2.80 & 0 & 2.80 & 1.80 & Gr-1 Sheet \\
\hline 11 & Supra Alloy & $\begin{array}{c}\text { Timet } \\
\text { BN3672(2) RMI } \\
404666(9) \\
\end{array}$ & 110.00 & 2.40 & 0 & 170.00 & 2.40 & Gr-2 \\
\hline 12 & PTG & $\begin{array}{c}\text { Thyssen Krupp } \\
611292 \\
\end{array}$ & 9.60 & 3.60 & 0 & 2.40 & 2.10 & Gr-2 \\
\hline 13 & TIMET & $\begin{array}{c}\text { Henderson } \\
22-49312 \\
\end{array}$ & 3.70 & 2480 & 0 & 12.30 & 18.00 & Sponge \\
\hline 14 & S6MB annulus & Bolts & 13000.00 & 6.00 & 0 & 160.00 & 60.00 & Bolts \\
\hline 15 & EE-33 full & Nuts & 500.00 & 8.40 & 0 & 80.00 & 60.00 & Nuts \\
\hline 16 & Honeywell & T149858991 & 3.70 & 4.69 & 0 & 1.63 & 1.50 & Gr-1 Sheet \\
\hline 17 & VSMPO & $528 \mathrm{~g}$ & 61.70 & 6.20 & 0 & 4.10 & 31.00 & $\begin{array}{l}\text { Gr-1 Metal } \\
10 \% \text { scrap }\end{array}$ \\
\hline 18 & VSMPO & $996 \mathrm{~g}$ & 17.28 & 12.35 & 0 & 4.10 & 6.20 & Gr-1 Sponge \\
\hline 19 & TIMET & HN2470 & 8.51 & 0.37 & 0 & 0.61 & 0.52 & Gr-1 Sheet \\
\hline 20 & TIMET & Master ID \#46 & 8.00 & 0.124 & 0 & 0.12 & 0.62 & Gr-1 Sheet \\
\hline 21 & TIMET & HN3469-T & 1.6 & 0.1 & 0.31 & 0.30 & 0.62 & Gr-1 Slab \\
\hline 22 & TIMET & HN3469-M & 2.90 & 0.10 & 0.2 & 0.25 & 0.68 & Gr-1 Slab \\
\hline
\end{tabular}


radioisotopes not reported, as well as early $U$ and Th activity. The 13 samples (a total of $152 \mathrm{~kg}$ ) originated from different heats and were made by different mills: 7 samples at Thyssen Krupp Nirosta (Germany) and 6 samples at Aperam (Belgium). All samples were electro-polished at LBNL and pre-screened with a surface HPGe counter (MERLIN), particularly for excessive ${ }^{60} \mathrm{Co}$. Samples with $<20 \mathrm{mBq} / \mathrm{kg}$ of ${ }^{60} \mathrm{Co}$ were forwarded for more sensitive tests underground at SURF and at the University of Alabama. Stainless steel radio-assays are summarized in Table 5.1.2.

Table 5.1.2: Summary of the 13 stainless steel samples radio-assayed for $L Z$. Due to a high ${ }^{60}$ Co content detected in samples 10 to 13 during pre-screening, these were not assayed further and as such early-chain contents were not measured

\begin{tabular}{|c|c|c|c|c|c|c|c|}
\hline$\#$ & Sample name & \multicolumn{2}{|c|}{${ }^{238} \mathrm{U}[\mathrm{mBq} / \mathrm{kg}]$} & \multicolumn{2}{|c|}{${ }^{232} \mathrm{Th}[\mathrm{mBq} / \mathrm{kg}]$} & ${ }^{60} \mathrm{Co}$ & ${ }^{40} \mathrm{~K}$ \\
\hline & & early & late & early & late & & \\
\hline 1 & NIRONIT 311113 & 7.3 & 0.35 & 1.1 & 4 & 14.5 & 0.53 \\
\hline 2 & NIRONIT 511803 & 1.2 & 0.27 & 0.33 & 0.49 & 1.6 & 0.4 \\
\hline 3 & NIRONIT 512006 & 1 & 0.54 & 0.49 & 1.1 & 1.7 & 0.59 \\
\hline 4 & NIRONIT 512844 & 1.4 & 0.5 & 0.5 & 0.32 & 2.6 & 0.5 \\
\hline 5 & NIRONIT 521663 & 1.9 & 0.38 & 0.81 & 0.73 & 5.6 & 0.46 \\
\hline 6 & NIRONIT 521994 & 0.5 & 1.9 & 1.7 & 1.5 & 4.5 & 0.5 \\
\hline 7 & NIRONIT 124113 & 0 & 1.1 & 0 & 4.1 & 8.2 & 3.0 \\
\hline & NIRONIT (Alab) 124113 & $0 \pm 22$ & 4.89 & 0 & 5.37 & 14.6 & 1.7 \\
\hline 8 & NIRONIT 211093 & 0 & 0.6 & 0 & 0.8 & 7.4 & 3 \\
\hline & NIRONIT (Alab) 211093 & $0 \pm 11$ & 2.46 & 0.0 & 0.37 & 14.0 & 0 \\
\hline 9 & NIRONIT 528292 & 0 & 0.6 & 0.0 & 0.9 & 6.5 & 3 \\
\hline & NIRONIT (Alab) 528292 & $0 \pm 22$ & 2.22 & 0 & 0.67 & 9.69 & 0 \\
\hline 10 & NIRONIT 832090 & 0 & 4 & 0 & 2.2 & 26 & 4 \\
\hline 11 & NIRONIT 407156 & 0 & 0.6 & 0 & 4.8 & 32 & 2 \\
\hline 12 & NIRONIT 528194 & 0 & 0.8 & 0 & 2.1 & 32 & 5 \\
\hline 13 & NIRONIT 828660 & 0 & 1.4 & 0 & 1.5 & 335 & 4 \\
\hline
\end{tabular}

After screening $23 \mathrm{Ti}$ and 13 Stainless Steel samples in the material search campaign for the LZ cryostat, we have concluded that the highest and reproducible radiopurity is achieved with Commercially Pure Grade1, as per ASTM B256, Ti without added scrap material and using Cold Hearth Electron Beam (CHEB) refining technology. CHEB used for production of commercially pure Ti provides an important purification mechanism in which high density contaminants are removed by gravity separation. By contrast Vacuum Remelting Technology (VAR) does not have such material refining capabilities. All samples of the finished material supplied by TIMET were produced with such recipe. Their radioactivity levels were consistent and always below the LZ acceptance criteria. Samples HN3469-T and -M were from a 15,000 kg Ti jumbo slab that TIMET produced at its mill in Morgantown (Pennsylvania) and made available for the LZ cryostat. We note that the screening results of those samples represent the lowest radio-impurity contamination ever reported for a titanium sample. 


\subsection{Monte Carlo simulations of background from the cryostat}

Radioactive background due to the cryostat vessels has been evaluated by means of the detailed Monte Carlo simulations as described in 12.1, which is an evolution of the simulation developed for the LUX [15], based on the GEANT4 simulation toolkit [16]. Whilst sharing the same physics models and software structure, the package developed for LZ has been implemented with the detailed geometry of the LZ detector including shielding, cryostat (inner and outer vessels, flanges and bolts), inner detector, photomultiplier tubes (PMTs), PTFE reflectors, the cathode and anode grids, field shaping rings, and outer detectors comprising the LXe skin, and the Gd-doped liquid scintillator. The outer detectors are implemented appropriately as veto systems in these simulations.

For neutrons, $(\alpha, n)$ reactions and spontaneous fission neutron energy spectra were generated using the SOURCE software [17]. These spectra were then embedded into the simulation framework, which propagates neutrons isotropically emitted from the cryostat. Only neutrons from $(\alpha, n)$ reactions contribute to the nuclear recoil (NR) events background, thanks to the the very high efficiency of the LZ detector in vetoing neutrons from the spontaneous fission process, as described in Section 12.1.2.1. We evaluate separately the early and late part of the ${ }^{238} \mathrm{U}$ enabling us to properly consider samples in which the secular equilibrium is broken.

For electron recoil (ER) events from ${ }^{238} \mathrm{U}$ and ${ }^{232} \mathrm{Th}$ decay chains, and ${ }^{40} \mathrm{~K}$ and ${ }^{60} \mathrm{Co}$, we used a new particle generator, described in Section 12.1.2.3, based on the standard GEANT4 process.

To achieve the required sensitivity of $3 \times 10^{-48} \mathrm{~cm}^{2}$, we set as a goal the total ER event rate from detector materials to be below $10 \%$ of the rate from pp solar neutrinos in the WIMP search region, $1.5 \mathrm{keV}_{\text {ee }}$ to $6.5 \mathrm{keV}_{\text {ee }}$, and the NR event counts to be below 0.2 events after $\mathrm{S} 2 / \mathrm{S} 1$ discrimination in the full run time of 1,000 days, within $6 \mathrm{keV}_{\mathrm{nr}}$ to $30 \mathrm{keV}_{\mathrm{nr}}$.

The impact of the cryostat on the background counts has been estimated by the full detector and physics simulation, and is presented in Figure 9.2.1. The plot shows the results for Ti (TIMET) and stainless steel (NIRONIT) samples for LZ in the 1,000 d exposure with a 5.6 tonne LXe fiducial volume and after all the veto systems are applied, for ER events within $1.5 \mathrm{keV}_{\text {ee }}$ to $6.5 \mathrm{keV}_{\mathrm{ee}}$, with $99.5 \%$ rejection, and within (6-30) $\mathrm{keV}_{\mathrm{nr}}$, and $50 \%$ acceptance, for NR events. The Ti identified by the assay program, indicated by the star corresponding to 0.53 counts for ER and 0.01 counts for NR, is well below the requirements for LZ, for a specific detector component.

\subsection{Cryostat technical specification}

The intended purpose of the cryostat is to hold $10,000 \mathrm{~kg}$ of liquid xenon at $-100{ }^{\circ} \mathrm{C}$ with an immersed Time Projection Chamber in it. The cryostat consists of three main sub-assemblies: an Inner Cryostat Vessel (ICV), an Outer Cryostat Vessel (OCV) separated by a vacuum space and a Cryostat Support (CS). The cryostat is submerged in a water tank and surrounded by the outer detector acrylic vessels filled with a liquid scintillator. A general view of the cryostat with the acrylic vessels below (supported by the CS shelves) and above (supported by the OCV head and flange) is shown in Figure 5.3.1.

The material for the LZ cryostat is commercially pure Ti, Grade 1 per ASME SB-265, with additional low-radioactivity background requirements as presented in 5.1. The design of the vessels complies with the following codes: ASME BPVC [18], 2012 Int. Building Code, and ASCE 7 [19], with site soil classification Class B (Rock) for seismic conditions. The 2008 U.S. Geological Survey hazard data for this location are: $\mathrm{S}_{\mathrm{S}}=0.121 \mathrm{~g}, \mathrm{~S}_{\mathrm{MS}}=0.121 \mathrm{~g}$, and $\mathrm{S}_{\mathrm{DS}}=0.081 \mathrm{~g}$. The Seismic Design Force for LZ is $0.054 \mathrm{~g}$, which imposes a force of $6350 \mathrm{~N}$ at the center of mass of the cryostat during a seismic event. The LZ cryostat operational temperatures $\left({ }^{\circ} \mathrm{C}\right)$ and pressures (bar absolute) are summarized in Table 5.3.1 


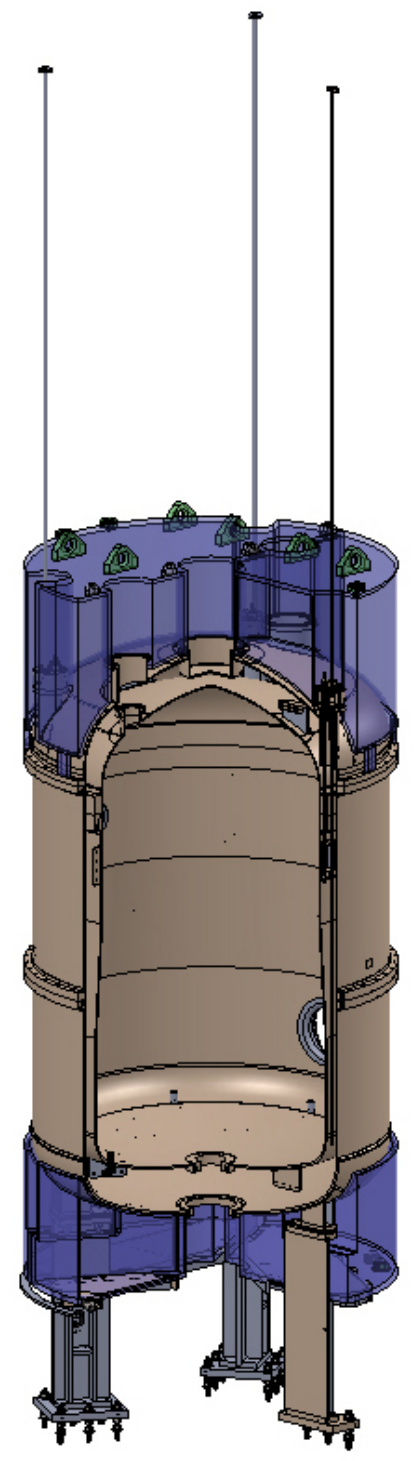

Figure 5.3.1: LZ cryostat assembly with the ICV nested in the OCV supported by the three legs of the CS. Acrylic vessels of the Outer Detector are shown at the top and bottom of the OCV. Three equally spaced vertical tubes will be used to deliver calibration sources into the vacuum space between the vessels. 
Table 5.3.1: Pressures and temperatures for cryostat operational conditions: normal, bake out and most severe failures.

\begin{tabular}{|c|c|c|c|c|}
\hline \multirow[t]{2}{*}{ Vessel } & \multicolumn{2}{|c|}{$\begin{array}{l}\text { Pressure } \\
\text { [bar absolute] }\end{array}$} & \multirow[t]{2}{*}{$\begin{array}{l}\text { Temperature } \\
\qquad\left[{ }^{\circ} \mathrm{C}\right]\end{array}$} & \multirow[t]{2}{*}{ Condition } \\
\hline & Internal & External & & \\
\hline \multirow{3}{*}{ Inner } & $\leq 4.0$ & Vacuum & -112 to 37 & Normal \\
\hline & Vacuum & 1.01 & $\leq 100$ & $\begin{array}{c}\text { Bake out } \\
\text { dry, no water in water tank }\end{array}$ \\
\hline & Vacuum & 1.48 & -112 to 37 & $\begin{array}{l}\text { Failure mode } \\
\text { water flooded between inner and outer vessels }\end{array}$ \\
\hline \multirow{3}{*}{ Outer } & Vacuum & 1.48 & 0 to 37 & Normal \\
\hline & Vacuum & 1.01 & $\leq 100$ & $\begin{array}{c}\text { Bake out } \\
\text { dry, no water in water tank }\end{array}$ \\
\hline & 1.48 & 1.01 & 0 to 37 & $\begin{array}{c}\text { Failure mode } \\
\text { Xe gas leak between inner and outer vessels } \\
\text { and no water in water tank }\end{array}$ \\
\hline
\end{tabular}

\subsection{Inner Cryostat Vessel}

The ICV has conventional cylindrical geometry with ellipsoidal heads, as shown in Figure 5.4.1. The inner vessel is split once near the top head with a flange pair. To minimize the passive volume filled with LXe, the diameter of the inner vessel is tapered near its half-height and the bottom head has an ellipsoidal shape with a 3:1 aspect ratio (the top head has the more common 2:1 aspect ratio). The 3:1 aspect ratio head requires greater material thickness compared to the 2:1 head, but this trade-off is well worth the savings in LXe.

For internal pressure, the thickness of the vessel walls in the cylindrical section is governed by a straightforward formula in the pressure vessel code. The minimum wall thickness is a function of the material, vessel diameter, pressure, and quality control measures. At the required internal pressure, the minimum wall thicknesses in the cylindrical section is $5.5 \mathrm{~mm}$. A number of ports are necessary to carry fluids and electrical signals to and from the inner detector. These are added to the top and bottom heads, as well as a side penetration for cathode high voltage. The latter is discussed further in Sections 3.3. Buckling is an important failure mode to consider for vessels that see external pressure (vacuum in this case). The ASME BPVC specifies safe external working pressures based on material, temperature, wall thickness, diameter, and length. If the allowable external pressure is insufficient, a vessel designer has a couple of options. The first is to increase the wall thickness. In the case of LZ, this is undesirable for a number of reasons: Most notably, it creates more background radiation and reduces veto efficiency. The other option is to add reinforcing rings. Reinforcing rings essentially shorten the length of the vessel from a buckling perspective. To comply with the ASME code, a stiffening ring is located at the top of the tapered (conical) section of the inner vessel. This ring acts as a line of support to increase buckling resistance, and therefore allows a thinner wall to be used, e.g. $6 \mathrm{~mm}$ instead of $8 \mathrm{~mm}$. Comparing the values for internal pressure 4 bar versus external pressure 1.48 bar, it is evident that the vessel design is driven by external pressure. It should also be noted that the minimum wall thickness is the minimum as-built, not the nominal. During the head-forming process, for instance, flat material is drawn or spun into shape, and in that process thinned from its original 


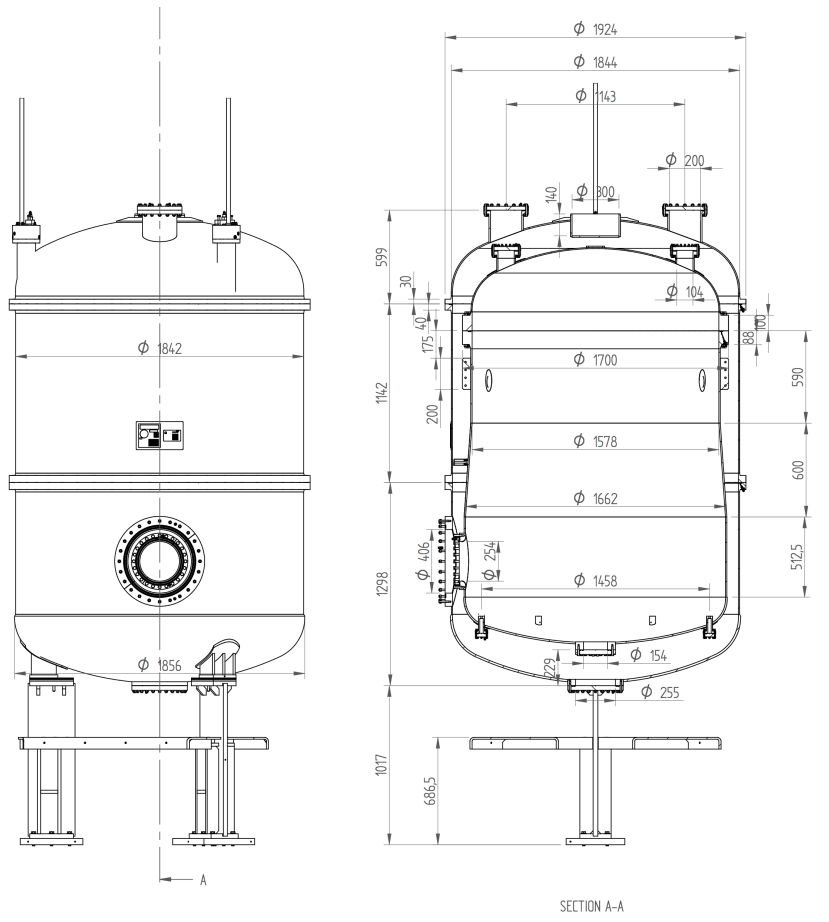

Figure 5.4.1: LZ cryostat assembly. Dimensions of major components are shown.

thickness. It should also be remembered that material is commercially available in discrete increments as opposed to infinitely variable thickness. Total mass and minimum thicknesses required by the ASME code for each segment of the inner and outer vessel made of Ti are summarized in Table 5.4.1.

Table 5.4.1: Vessel-wall thicknesses $[\mathrm{mm}]$ and total mass $[\mathrm{kg}]$ imposed by the external pressure at the normal, bakeout and failure conditions.

\begin{tabular}{|c|c|c|c|c|c|c|c|c|c|}
\hline \multicolumn{1}{|c|}{ Inner Vessel } & \multicolumn{4}{c|}{ Outer Vessel } \\
\hline $\begin{array}{c}\text { Top } \\
\text { head }\end{array}$ & $\begin{array}{c}\text { Upper } \\
\text { wall }\end{array}$ & $\begin{array}{c}\text { Conical } \\
\text { section }\end{array}$ & $\begin{array}{c}\text { Lower } \\
\text { wall }\end{array}$ & $\begin{array}{c}\text { Dished } \\
\text { end }\end{array}$ & $\begin{array}{c}\text { Total } \\
\text { mass }\end{array}$ & $\begin{array}{c}\text { Top } \\
\text { head }\end{array}$ & $\begin{array}{c}\text { Side } \\
\text { wall }\end{array}$ & $\begin{array}{c}\text { Dished } \\
\text { end }\end{array}$ & $\begin{array}{c}\text { Total } \\
\text { mass }\end{array}$ \\
\hline 7 & 9 & 9 & 9 & 11 & 950 & 8 & 7 & 14 & 1115 \\
\hline
\end{tabular}

To minimize the amount of LXe between the TPC and the inner cryostat, the shape of the inner vessel is tapered at its half-height. Studies of the electric-field distribution show that the electric field is below the maximum allowed value of $50 \mathrm{kV} / \mathrm{cm}$. The inner vessel will be sealed with a sprung metal C-seal because at the experimental temperature, O-ring seals with typical elastomeric materials are not suitable. Additionally, the large diameter prohibits the use of a knife-edge flange. Smaller ports on the vessels will be sealed with sprung metal C-seals as well. Inner-vessel flanges with C-seal gaskets for cryogenic service will also feature a secondary O-ring seal to facilitate room-temperature leak detection. 


\subsection{Outer Cryostat Vessel}

The OCV supports the ICV through three tie bar assemblies situated in the top head. The ICV contains the TPC and LXe. The ICV can be leveled by adjustment of the tie bars externally from above the water tank.

The OCV also provides support for top and bottom Liquid Scintillator tanks, access to the vacuum space for calibration purposes and ports in the top head for cryogenic and cable services. At the bottom a LXe recirculating system of pipes connects to the lower port. The OCV base interfaces with the CS and its position in the water tank determines the orientation of the HV port in the base of the vessel. The HV ports of both vessels are aligned for the umbilical connection.

The OCV, as shown in Figure 5.4.1, has been designed in three pieces for ease of transport to the Davis cavern and assembly in the water tank it is joined together around the circumference by two flange pairs. The top head comprises a flange, 2:1 ellipsoidal head, three ports for the ICV suspension, two conduit ports for cryogenic and cabling services and a large central recess for a calibration source called "YBe". The middle section of the OCV is a straight cylinder with two flanges that match the head and base flanges. The flanges are double-sealed with two elastomer O-rings and a pump path between the two to facilitate leak detection. Both O-rings are expected to seal at experimental temperatures since the OCV operates close to room temperature. The bottom head comprises a flange that matches the middle section, a HV port that enables the umbilical to be connected to the vessel, three support feet welded to the 2:1 ellipsoidal head and a heat exchange port flange centrally situated in the head. Each foot has a $6.3 \mathrm{~mm}$ diameter survey hole in the $20 \mathrm{~mm}$-thick plate at the outer surface. The flanges are joined with stainless steel bolts and nuts (M12, 48) (same grade as for the ICV and supplied by the LZ project). All flanges must be integral design. To facilitate assembly, tapered pins have been incorporated into the design. During assembly and leak testing, the conduit ports, tie bar ports, HV and HX ports will be sealed with blanking plates. The sealing plate for the tie bar ports on the OCV head has the feature of a hoist ring to lift the head or vessel assembly. The tie bar assemblies themselves are used for lowering the ICV into the OCV during final assembly.

The ICV is suspended from three tie bar assemblies situated in the OCV head. Cross section of the tie bar is shown in Figure 5.5.1. They incorporate an adjustment feature for leveling the ICV and the tie bar is sealed into the assembly with a metal bellows that facilitates vertical movement with a small angular offset.
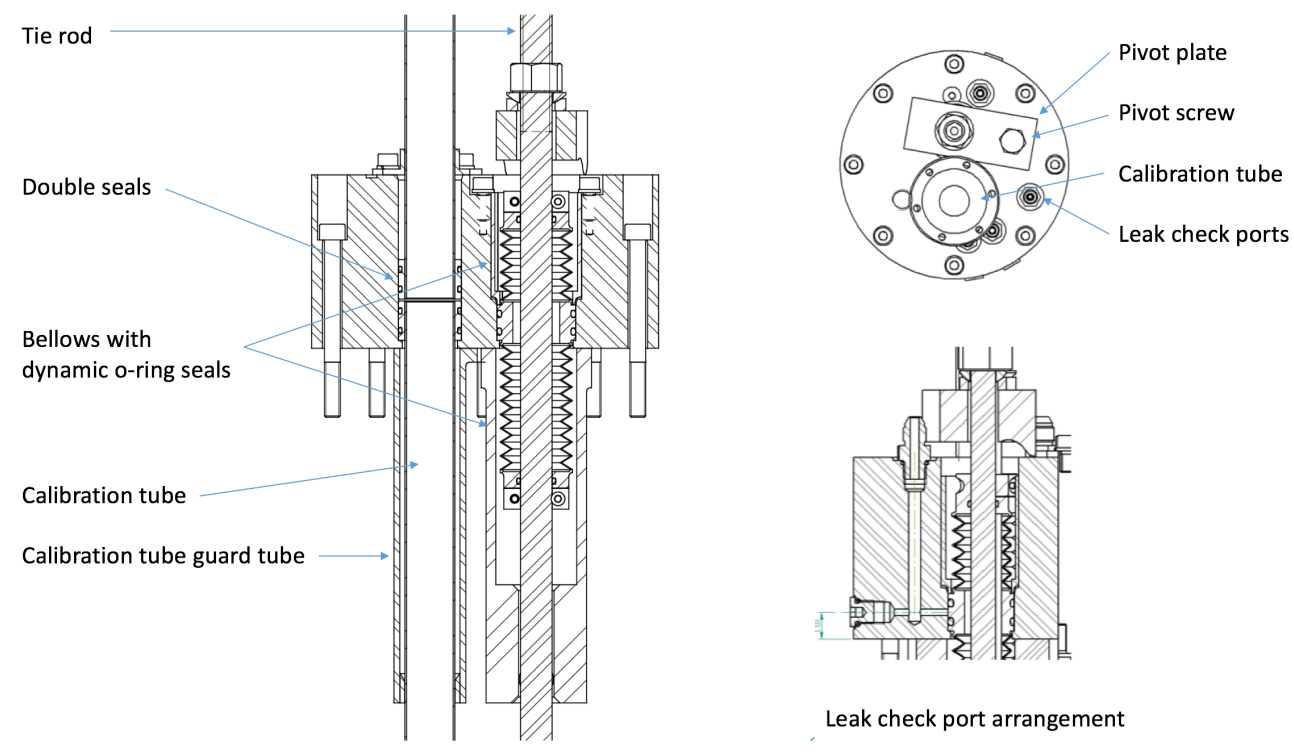

Leak check port arrangement

Figure 5.5.1: OCV tie rod port assembly 
There is a coarse and fine adjustment the fine adjustment uses a pivot plate giving a 3:1 advantage. Also housed within the assembly is a calibration tube that facilitates access to the vacuum space between the vessels and enables a calibration source to be lowered down to within a few $\mathrm{mm}$ of the ICV lower head. The inner diameter and the wall thickness of the tubes are 25.4 and $0.94 \mathrm{~mm}$, respectively. The length of the tube spans from the deck above the cryostat to the tangent line of the OCV dished end.

Double seals are used throughout the LZ cryostat and the volume between the grooves is accessed with a sealed nipple that is used to detect leaks between seals. The tie bar assemblies have four of these items to access the seals. The tie bars are manufactured from high strength stainless steel giving a factor of safety of $3: 1$.

\subsection{Cryostat Support}

The CS structure has been designed to support the operating load and resist a horizontal force due to a seismic event. The CS interfaces with three base plates mounted on studs protruding from the water tank base these plates will be surveyed and adjusted flat and level at the correct height for the umbilical. Holes for survey targets have been included in the relevant faces of the legs for this purpose. The support has been designed with stability, strength and installation in mind. The cryostat interfaces with flange plates welded to the top of the legs and is shimmed to the correct height. The support has also been designed with the requirements of the veto counter in mind and maximum coverage by the LS tanks has been achieved with a three leg flat plate design. The three segmented tanks fit between the legs and are supported on shelves between the legs.

\subsection{Cryostat Interfaces}

The cryostat interfaces with many other subsystems of the experiment. Some of them are physically connected to its ports creating additional loads which must be considered in the cryostat design. External loads imposed by the other subsystems on the cryostat ports are listed in Table 5.7.1. In addition, the feet of the ICV and OCV shall withstand the load of the vessels when filled with water for the hydrostatic tests.

\subsection{Thermal Insulation}

It is important to limit the heat transfer between the inner vessel and the environment to minimize the amount of refrigeration needed underground. In addition, reducing heat transfer helps to prevent unwanted convection currents in the LXe fluid. The vacuum between nested inner and outer vessels essentially eliminates thermal conduction and convection, in typical Dewar fashion. The dominant mode of heat transfer is therefore radiation, and with a large surface area $\left(\sim 16 \mathrm{~m}^{2}\right)$ and a large temperature difference, it is potentially substantial. Multilayer insulation (MLI or superinsulation) is proposed as the baseline solution to reduce this thermal load during normal operation. MLI is a well-known insulating material for in-vacuum cryogenic service. The thermal load with and without this material varies by approximately an order of magnitude. In this case, the expected heat load with bare vessels would be several hundred watts, and with MLI several tens of watts. In LZ, the proposed amount of total refrigeration is about a kilowatt, so MLI is the clear choice. A 3-parts MLI blanket design for the ICV is shown in Figure 5.8.1.

MLI works well during normal operation, but is ineffective in the event of a failure in which a gross amount of liquid water enters the volume normally occupied by vacuum between the vessels. To mitigate this failure mode, a closed-cell polyurethane foam will be applied to the outer surface of the inner vessel anywhere it is in contact with LXe (basically everywhere below the main seal flange). The proposed foam 
Table 5.7.1: External loads to the cryostat in addition to any loads exerted by vessel internal and external pressures. ${ }^{*}$ Combined load from cryostat, Outer Detector tanks and seismic load. ${ }^{* *}$ The moment due to the seismic load at $2 / 3$ height.

\begin{tabular}{|c|c|c|c|}
\hline Cryostat Item & $\begin{array}{c}\text { Number } \\
\text { of items }\end{array}$ & $\begin{array}{c}\text { Maximum allowable } \\
\text { load on a single item [N] }\end{array}$ & $\begin{array}{c}\text { Maximum allowable moment } \\
\text { in any direction to the flange face [Nm] }\end{array}$ \\
\hline \multicolumn{3}{|c|}{ Inner Cryostat Vessel } \\
\hline Top head port & 2 & 200 & 200 \\
Weir port & 3 & 100 & 100 \\
HV port & 1 & 2000 & 2000 \\
TPC port & 6 & 3000 & 200 \\
Dished end port & 1 & 1000 & 1000 \\
\hline \multicolumn{2}{|c|}{ Outer Cryostat Vessel } \\
\hline Top head port & 2 & 1000 & 1000 \\
YBe source port & 1 & 3200 & N/A \\
Top flange & 2 & 10000 & N/A \\
Tie bar port & 3 & 33000 & N/A \\
HV port & 1 & 2000 & 1000 \\
Bottom port & 1 & 1000 & 1000 \\
\hline \multicolumn{2}{|c|}{ Cryostat Support } \\
\hline Leg & 3 & N/A & $15265^{* *}$ \\
\hline Shelf & 3500 & \multicolumn{2}{|c|}{} \\
\hline
\end{tabular}

thickness is $2 \mathrm{~cm}$ over the bottom head, and $1 \mathrm{~cm}$ over the remainder. MLI will be wrapped over the foam, and covers the entire inner vessel and its cold appendages. With the foam in place, the maximum heat transfer in this failure mode is expected to be 3,600 W, which corresponds to a Xe boil-off of 450 standard liters per minute (slpm). This rate is within the Xe-recovery capacity of the system.

A dedicated thermal analysis has been carried out in order to investigate possible distortions in the calibration tubes from asymmetric thermal gradients caused by thermal radiation from the inner face of the tube onto the cooler cryostat. The steady state thermal simulation was carried out in Ansys CFX [20], and consisted of basic tubular geometries representing the outer and the inner vessels, with a nitrogen filled calibration tube geometry located between the two vessels in a vacuum interspace. Thermal radiation was modeled between the warmer OCV $\left(20^{\circ} \mathrm{C}\right)$ and the cooler ICV $\left(-100^{\circ} \mathrm{C}\right)$. For our preferred MLI product, (Coolcat $2 \mathrm{NW}$ ) the supplier - Ruag [21] specify that 10 layers at $300 \mathrm{~K}$ to $77 \mathrm{~K}$ give heat loss of $<1 \mathrm{~W} / \mathrm{m}^{2}$. Using the equation $\mathrm{E}_{e f f}=\mathrm{P} / \mathrm{S} \times\left(\mathrm{T}_{h}^{4}-\mathrm{T}_{c}^{4}\right)$ the average effective emissivity over this range is $0.002,2 \mathrm{~W} / \mathrm{mK}$. A more conservative emissivity value of $0.025 \mathrm{~W} / \mathrm{mK}$ has been used for the simulation and this value was backed up by wider research of similar MLI products, and also by data used for the cryostat vessel heat transfer calculations. Based on these boundary conditions, the maximum thermal gradient over the length of the calibration tubes was found to be negligible, resulting in a maximum deflection at the end of the tube of less than $0.1 \mathrm{~mm}$. 


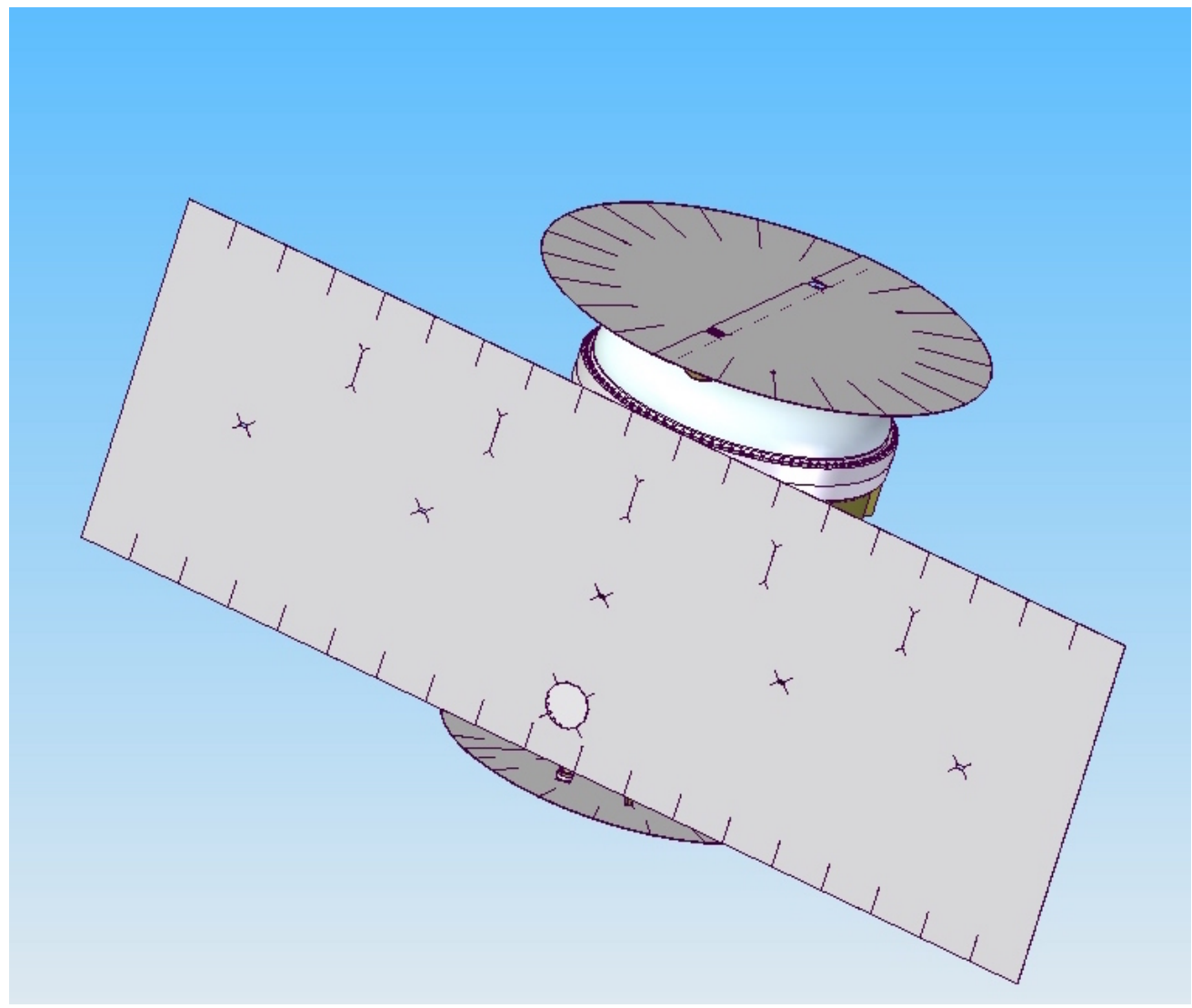

Figure 5.8.1: Three parts of the MLI blanket for the ICV thermal insulation. The design with only three parts has been chosen to effectively cover the whole ICV surface with a minimum work required for its assembly. Only final sewing will be needed. Several holes have been provisioned for ICV features such as: ports, fins, tie rods and seismic limiters. Cuts along the blanket's edges are to facilitate wrapping on curved surfaces of the vessel heads.

\subsection{Fabrication, Cleanliness, Tests and Certifications}

The titanium for the cryostat is extremely rare and difficult to obtain and as such contingency provision shall be made for spare material. Potential fabricators are required to demonstrate that they have built in an acceptable level of contingency and that they will take the necessary manufacturing precautions in order to minimize waste and ensure that they do no not exceed their allocation.

Together with the cryostat design, a comprehensive set of drawings has been made which contain toleranced geometric specifications. The general tolerance on machined parts of the cryostat such as flanges and mating surfaces is $1 \mathrm{~mm}$. This tolerance has been relaxed on the form of the barrels in most cases, and wherever possible in order to aid the ease of manufacture. Where squareness, flatness, position of holes and other features are important for full assembly these are generally tightened to $0.5 \mathrm{~mm}$. Several key features of the cryostat assembly are highlighted below:

- The ICV top head conduit ports must align with those in the OCV with a tolerance of $2 \mathrm{~mm}$.

- The HV port in the ICV has a squareness tolerance of $1 \mathrm{~mm}$ relative to top flange and can be positioned $\pm 1 \mathrm{~mm}$ from that flange. There is also a $\pm 1 \mathrm{~mm}$ tolerance applied to the face from the vessel wall. 
The face has a positional tolerance of $0.8 \mathrm{~mm}$ from the tie rod holes. The required flatness is $0.5 \mathrm{~mm}$ and the positional tolerance of the tie rod holes is $\pm 0.8 \mathrm{~mm}$.

- The HV port in the OCV has a squareness tolerance of $1 \mathrm{~mm}$ relative to the flange in the base and a linear tolerance of $\pm 1 \mathrm{~mm}$ to the flange tangent line. The required flatness is $0.5 \mathrm{~mm}$. The tie rod ports in the OCV head have a positional tolerance of $\pm 1 \mathrm{~mm}$.

- For helicoflex seals, the manufacturer's [22] specifications have been used, and for the O-ring grooves, BS1806 standard tolerances have been used.

Meeting the geometric requirements of form and alignment of key features can be particularly challenging when producing titanium weldments, where components are prone to distortion during machining and welding procedures. The cryostat shall be welded according to ASME IX BPVC [23]. GMAW or MIG welding should be used and the consumable electrode/welding rod shall be the same titanium material as the vessel raw material. Welding of titanium is a specialist process and the manufacturing contractor is required to demonstrate they are titanium welding specialists with particular emphasis on pre-weld preparation/cleaning, and on protection of the heat affected zone from oxidation during welding. They are required to outline their weld acceptance criteria such that it may be assessed by LZ.

A very high level of cleanliness is required for any component of the cryostat vessels throughout production. Every reasonable effort shall be made to minimize cross-contamination with swarf and loose dust of foreign material as well as environmental radon plate-out on the cryostat surfaces. The project goal is to achieve a dust level for the internal surface of the cleaned and packaged ICV of $10 \mathrm{ng} / \mathrm{cm}^{2}$. For all other surfaces the dust level shall not exceed $1 \mu \mathrm{g} / \mathrm{cm}^{2}$ at delivery. The following procedures shall be applied: the cryostat raw material shall be stored separately from other material in the workshop; parts shall be individually bagged in the bags provided by LZ to avoid any dust accumulation on the surfaces. Bagged components shall be placed on wooden pallets inside the pallet footprint, safe from the risk of impact or damage to the bag. The cleaning process for stock material at the mill shall follow the ASTM B 600 standard. Grease, oil and lubricants are to be removed with alkaline or emulsion type cleaners only. No mechanical abrasion type cleaning is allowed. A surface layer of at least 5 microns shall be removed from all stock material by pickling/etching with virgin chemicals. Chemicals shall be rinsed off after etching with deionized water. Prior to machining, rolling and spinning all surfaces in direct contact with cryostat components shall be cleared of dust and swarf with a stiff non-metallic brush, and cleaned with solvent using a lint-free cloth. All machines shall be flushed of coolant, and coolant replaced with fresh, water soluble coolant, approved by LZ. During machining, cryostat components shall be separated from other materials in the workshop, and the work area kept clean and tidy. Post manufacture, all components awaiting further processing shall be hand de-greased with solvent and a lint-free cloth before being bagged using bags provided by LZ, and stored in a clean place.

Prior to welding, components shall be comprehensively cleaned. Dirt, oil and dust shall be removed from the weld area, and oxide/scale removed by etching. All surface tables and tools in contact with any part of the cryostat shall be cleaned by hand with fresh solvents. Upon completion of welding, components shall be bagged using LZ provided bags and stored in a clean place. Heavy abrading, grinding or wire brushing is prohibited both before and after welding. For final cleaning, all faces of the cryostat vessels shall be etched using LZ approved virgin chemical solution, and in accordance with ASTM B 600 standard, removing at least 5 microns from all surfaces. Parts shall then be rinsed with deionized water and thoroughly dried. All parts shall then be bagged, purged with nitrogen and sealed.

With cooperation from the manufacturing contractor, LZ will carry out the following cleanliness and radioactivity testing: after etching of raw material, a representative $10 \mathrm{~kg}$ coupon shall be cut from the $8 \mathrm{~mm}$ stock plate along with all off-cuts from vessel ports and reinforcement pads, and swarf from bolt holes in large flanges shall be retained and radio-assayed at SURF. In addition, $10 \mathrm{~kg}$ of Ti welding rods and $10 \mathrm{~kg}$ 
of sample welds shall be sent to SURF for radio-assay. The impact of etching on the dimensions of critical features such as grooves shall be assessed prior to machining the real grooves. LZ shall measure the levels of radon present at various fabrication and cleaning locations.

The following tests will be conducted by the manufacturing contractor and witnessed by designated persons from RAL/STFC:

- A dimensional check of all components of the cryostat as well as its assemblies, including a localization of the survey markers in 3D space relative to the key features.

- Provide proof by mechanical assembly and documentation that the sequence of assembly at SURF shown in Figure 5.10.1 is achievable.

- Test and record ICV and OCV leak rate - demonstrate that the leak rate does not exceed $1 \times 10^{-6} \mathrm{mbar} 1 / \mathrm{s}$ (helium).

- Conduct a pressure test ICV and OCV for the internal pressure case per the ASME BPVC, as required to achieve certification.

- Conduct a suspension full load/leak test of ICV with tie bars assembled. The ICV shall be loaded to $1.5 \times 33,000 \mathrm{~N}=49,500 \mathrm{~N}$ and the upper part external to the vessel shall be exposed to water at the operating pressure of 1.48 bar absolute. The lower part of the vessel shall be evacuated and vacuum leak rate measured and certified to be below $1 \times 10^{-7} \mathrm{mbar} 1 / \mathrm{s}$. Further details are contained in the cryostat specification document.

- The cryostat support shall be full load tested with 12T applied in increments of 4T, with lateral deflection of the legs as well as vertical displacement of the support recorded. Measured deflection for each leg shall be smaller than $1 \mathrm{~mm}$.

- The vessel shall be stamped/marked relating to the testing and approval. This shall not impart dirt into the system or be etched away during cleaning.

\subsection{Transportation and Installation}

The cryostat inner vessel along with its contents will be moved as an assembly down the Yates shaft at SURF. The width of the shaft is nominally $1.85 \mathrm{~m}$, and the maximum payload width is $1.70 \mathrm{~m}$ to clear features in the shaft cross section and provide some margin of safety. The outer vessel in contrast will be moved down the Yates shaft in three pieces, and assembled once in the Davis Cavern.

The CS will be placed onto three base plates that are independently supported inside the water tank. The plates will have been leveled and surveyed prior to the LZ installation. An assembly procedure will be carried out at the manufacturers which will as closely as possible reproduce what will be carried out in the tank at SURF. Having placed the CS onto the plates and loosely assembled the fixing bolts the base of the outer vessel will be assembled onto the top supporting flanges with bolts and shims in place. The shelves between the legs for supporting the LS tanks must be included in this assembly procedure. A preliminary inspection will be carried out to check that there are no gaps between the mating interfaces greater than $0.5 \mathrm{~mm}$. Any gaps greater than $0.5 \mathrm{~mm}$ will be shimmed with appropriate thicknesses of shim stock. When this has been completed and a dimensional check has established that the CS support and base are in the correct position the fixing bolts will be tightened sequentially to ensure the geometry is preserved. A trial assembly can now proceed following the sequence shown in Figure 5.10.1. Care must be taken at all times to protect sealing surfaces, the seals will will not be in place when this procedure is carried out at the manufacturer. The middle section of the outer vessel is assembled next onto the base flange using 

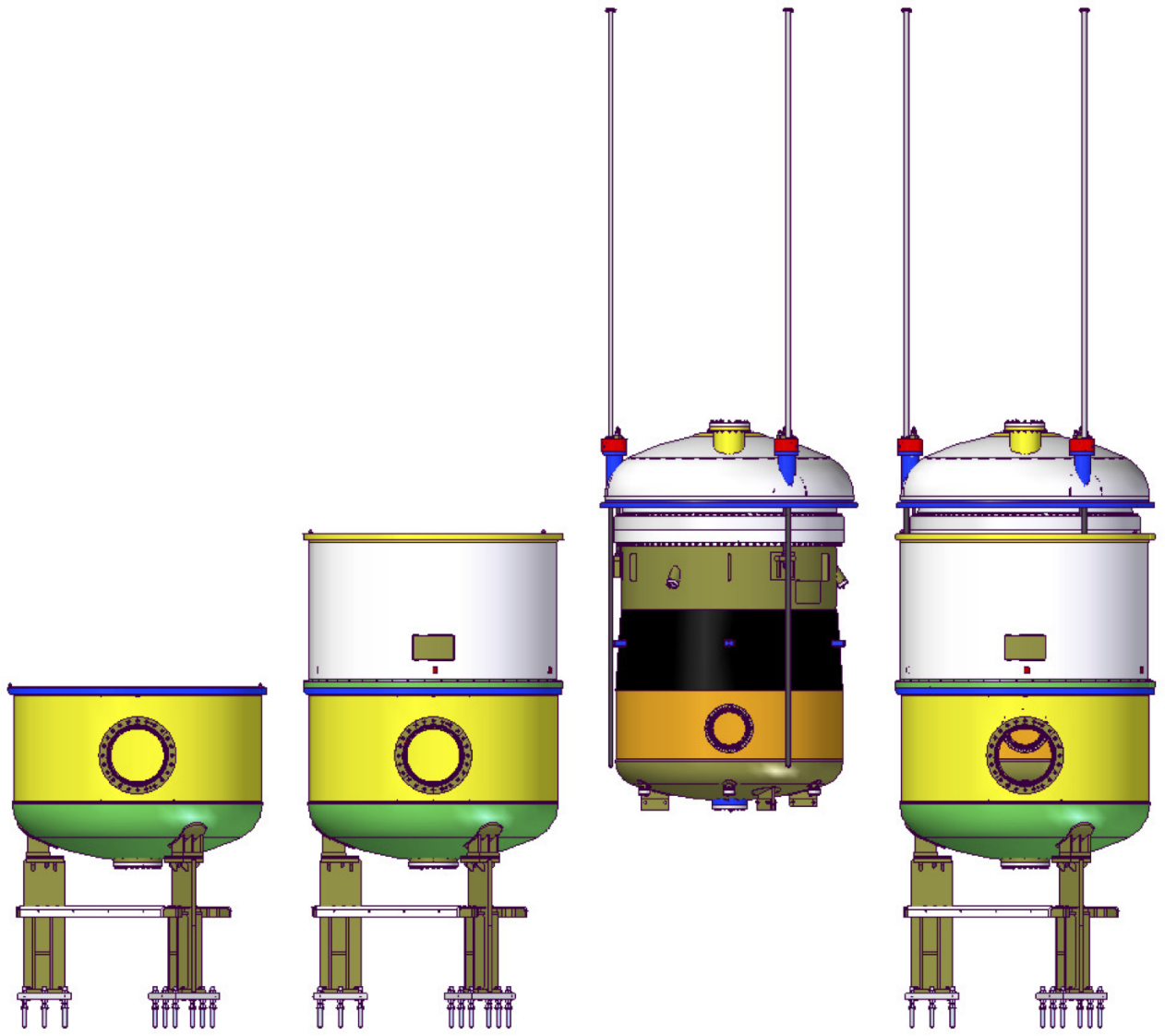

Figure 5.10.1: Major steps of the cryostat assembly underground.

the assembly dowels to locate it in the correct orientation at this stage a minimum of six bolts can be used to hold the assembly together. Next in the sequence is the assembly of the ICV into the OCV using a combined lift with the OCV head, tie bar assemblies and ICV. The OCV head and tie bars will have been assembled prior to the start of this procedure, a convenient supporting frame or gantry will be required to set up this lifting operation. Prior to the tie bars being used they must have been assembled and tested using the test equipment. With the ICV in a position accessible for the crane the OCV head complete with tie bars will be lifted over the ICV and the tie bars aligned with the three access holes in the ICV top head flange. Orientation is important as the HV ports of both vessels have to align with adjustment of the tie bars. There is a supporting CS leg at the 0 degree position and a tie bar assembly aligned with this. The HV ports are at $90^{\circ}$ to this position. Lower the head and tie bars through the access holes, make sure the top M16 nut and washer is in place before carrying this out. When the tie bar is through its support on the upper part of the ICV assemble the lower M16 nut and washer ensuring that at least twice the diameter is protruding through the nut. Take up the slack with the crane and check dimension of HV port to head flanges and parallelism adjust as necessary. Tighten the M16 nuts and lock tie bars they must not rotate. Lift ICV into OCV with crane, there should be a minimum of three people ensuring that the ICV enters the OCV without hitting the sides, when the head approaches the middle section upper flange place three spacers between the flanges and bring the head into contact with them. Rest the head at this position and check that the $\mathrm{HV}$ ports are aligned and that when the spacers are removed the ICV will not hit the bottom of the OCV 
vessel adjust as necessary. Remove the spacers and lower the OCV head and ICV until the flange engages with the location dowels and mates with the upper flange of the middle section. Secure with $6 \times$ M12 bolts and check alignment of HV ports. At this stage the alignment check can be visual if adjustment is needed then use the tie bars but no more than $10 \mathrm{~mm}$ of adjustment should be used. This assembly procedure at the manufacturers is an assessment of how easy or difficult it is to assemble the vessels and achieve HV port and axis alignment, the foam insulation, MLI, weir piping and bump stops will not be in place but will be for the assembly at SURF.

\subsection{Bibliography}

[1] D. S. Akerib et al. (LUX), "Radio-assay of Titanium samples for the LUX Experiment," (2011), (unpublished), arXiv:1112.1376 [physics.ins-det].

[2] A. Chepurnov, S. Nisi, M. L. di Vacri, and Y. Suvorov, in LOW RADIOACTIVITY TECHNIQUES 2013 (LRT 2013): Proceedings of the IV International Workshop in Low Radioactivity Technique, AIP Conf. Proc., Vol. 1549, edited by L. Miramonti and L. Pandola (2013) pp. 161-164, The ultra-pure Ti for the low background experiments, Conference Presentation.

[3] VSMPO-AVISMA, (2015), Parkovaya St. 1, Verkhnaya Salda, Sverdlovsk Region, 624760, Russia.

[4] TIMET, (2015), 224 Valley Creek Blvd. Suite 200, Exton, PA 19341-2300.

[5] Supra Alloys, (2015), 351 Cortez Circle, Camarillo, CA 93012-8638, USA.

[6] "Honeywell Electronic Materials," (2015), 15128 E Euclid Ave, Spokane Valley, WA 99216-1801, USA.

[7] PTG - Performance Titanium Group, (2015), 8400 Miramar Rd Suite 200-248C San Diego, CA 921264387, USA.

[8] E. Aprile (XENON1T), in Proceedings, 10th UCLA Symposium on Sources and Detection of Dark Matter and Dark Energy in the Universe, Springer Proc. Phys., Vol. 148 (2013) pp. 93-96, The XENONIT Dark Matter Search Experiment, Conference Presentation, arXiv:1206.6288 [astro-ph].

[9] X. Cao et al. (PandaX), Sci. China Phys. Mech. Astron. 57, 1476 (2014), arXiv:1405.2882 [physics.insdet].

[10] K. H. Ackermann et al. (GERDA), Eur. Phys. J. C73, 2330 (2013), arXiv:1212.4067 [physics.ins-det].

[11] J. J. Gomez-Cadenas (NEXT), Proceedings, 37th International Conference on High Energy Physics (ICHEP 2014): Valencia, Spain, July 2-9, 2014, Nucl. Part. Phys. Proc. 273-275, 1732 (2016), arXiv:1411.2433 [physics.ins-det].

[12] NIRONIT Edelstahlhandel GmbH \& Co. KG, (2015), Eckeler Strasse 10, D-21224 Rosengarten, Germany.

[13] W. Maneschg, M. Laubenstein, D. Budjas, W. Hampel, G. Heusser, K. T. Knopfle, B. Schwingenheuer, and H. Simgen (GERDA), Nucl. Instrum. Meth. A593, 448 (2008).

[14] V. Álvarez et al. (NEXT), J. Instrum. 8, T01002 (2013), arXiv:1211.3961 [physics.ins-det].

[15] D. S. Akerib et al. (LUX), Nucl. Instrum. Meth. A675, 63 (2012), arXiv:1111.2074 [physics.data-an]. 
[16] S. Agostinelli et al. (GEANT4), Nucl. Instrum. Meth. A506, 250 (2003).

[17] W. B. Wilson, R. T. Perry, W. S. Charlton, and T. A. Parish, Prog. Nucl. Energy 51, 608 (2009), (SOURCES-4C).

[18] Boiler and Pressure Vessel Committee, ASME boiler and pressure vessel code, Section VIII, Division 1 (American Society of Mechanical Engineers, Two Park Avenue New York, NY 10016-59905, 2015).

[19] United States Geological Survey, "Seismic Design Maps \& Tools," (2015), 12201 Sunrise Valley Dr, Reston, VA 20192-0003.

[20] “ANSYS CFX," (2015), ANSYS, Inc., Southpointe, 2600 ANSYS Drive, Canonsburg, PA 153170404; Accessed 12-10-2015.

[21] RUAG, "Cryogenic Insulation Coolcat,” (2015), RUAG Holding AG, Stauffacherstrasse 65, 3000 Bern 22 Switzerland.

[22] Technetics Group, (2015), Unit 3 Smitham Bridge Road Hungerford, Berkshire RG17 0QP UK.

[23] Boiler and Pressure Vessel Committee, ASME boiler and pressure vessel code, Section IX (American Society of Mechanical Engineers, Two Park Avenue New York, NY 10016-59905, 2015). 



\section{Xenon and Cryogenics Systems}

\subsection{Introduction and overview of requirements}

The functions of the xenon handling and cryogenics systems are to purify the xenon of the relevant contaminants, to condense it and to maintain it the liquefied state for the duration of the experiment, and to safely recover the xenon to permanent storage at the conclusion of the experiment.

Two classes of impurities drive the purification strategy.

1. Electronegatives such as oxygen or water limit the free electron lifetime and degrade the operation of the TPC. We require that the charge attenuation length of the LXe be 1.46 meters or larger, which is the maximum drift length of the detector. This corresponds to a free electron lifetime of $806 \mu \mathrm{s}$ at the drift field baseline of $310 \mathrm{~V} / \mathrm{cm}$ (see Table 3.3.1), or an oxygen-equivalent concentration of $0.37 \mathrm{ppb}$. Electronegatives are removed from the xenon by continuously circulating the xenon in gas phase through a hot zirconium getter during operations.

2. Radioactive noble gases require special measures because they are not removed from the getter and because they create ER background events which can not be mitigated with self-shielding. Our primary concerns are ${ }^{222} \mathrm{Rn}$ and ${ }^{85} \mathrm{Kr}$, but ${ }^{39} \mathrm{Ar},{ }^{37} \mathrm{Ar},{ }^{220} \mathrm{Rn}$, and ${ }^{127} \mathrm{Xe}$ also play a role.

As detailed in Sections 9.7.1 and 9.7.2 background considerations imply that the vendor supplied xenon should be purified of ${ }^{\text {nat }} \mathrm{Kr}$ and ${ }^{\text {nat }} \mathrm{Ar}$ to the levels of $0.015 \mathrm{ppt}(\mathrm{g} / \mathrm{g})$ and $0.45 \mathrm{ppb}(\mathrm{g} / \mathrm{g})^{1}$ respectively in order to suppress ER events from ${ }^{85} \mathrm{Kr}$ and ${ }^{39} \mathrm{Ar}$. The krypton requirement is particularly demanding, and the need to control this species drives much of the xenon handling strategy.

At $0.015 \mathrm{ppt}$, the amount of krypton in the ten-tonne xenon stockpile is equivalent to $40 \mathrm{std} \cdot \mathrm{cc}$ of $\mathrm{air}^{2}$. To meet this requirement the xenon will be purified of both krypton and argon with a custom gas chromatography system at SLAC (see Section 6.3). Because no krypton removal technology will be employed during detector operations, it is critically important that krypton not be re-introduced once it has been removed. Air leaks and detector outgassing are two mechanisms of particular concern. This places additional burdens on the performance of the gas handling infrastructure and the operational protocols.

This chapter is organized as follows. In Section 6.2 we review the air leaks specifications of the purification and storage systems. In Section 6.3 we describe the SLAC chromatography system for removing krypton and argon. The online purification system and the xenon recovery systems are described in Section 6.4 and Section 6.5. The long-term xenon storage system is described in Section 6.6. The purity monitoring program is described in Section 6.7, and the cryogenics systems are described in Section 6.8. Xe procurement is described in Section 6.9, and some gas species diffusion and solubility measurements are reported in Section 6.10.

\footnotetext{
${ }^{1}$ Unless otherwise specified, throughout this chapter we report concentration in units of $(\mathrm{g} / \mathrm{g})$. Concentrations of krypton and argon always refer to ${ }^{\text {nat }} \mathrm{Kr}$ and ${ }^{\text {nat }} \mathrm{Ar}$.

${ }^{2}$ The krypton concentration of air is $\left.2.9 \times 10^{-6}(\mathrm{~g} / \mathrm{g})\right)$
} 


\subsection{Xenon purification and storage specifications}

In this section we describe the purification and storage specifications that bare upon the designs of the various xenon handling systems. Many of these specifications are driven by the the need to control the krypton concentration in the xenon. We adopt the following policy:

- the krypton removal system should produce xenon that has no more than $0.015 \mathrm{ppt}(\mathrm{g} / \mathrm{g})$ of krypton;

- the concentration should not increase more than $0.005 \mathrm{ppt} / \mathrm{year}$ while the xenon is contained in the storage system;

- the time-averaged increase in the concentration during five years of detector operations at SURF should be no more than $0.015 \mathrm{ppt}$ (equivalent to $0.006 \mathrm{ppt} / \mathrm{year}$ ).

\subsubsection{Air leaks during storage}

We estimate that the xenon will spend on average about six months in storage between krypton removal and delivery into the detector. If the above requirement is met, then the krypton content will not rise by more than about $0.0025 \mathrm{ppt}$ from its initial value during this time. From the krypton concentration in air, we calculate that the allowed air leak rate into 10 tonnes is $0.017,3 \mathrm{~g} / \mathrm{y}$, equivalent to a helium leak rate of $1.15 \times 10^{-6} \mathrm{mbar} 1 / \mathrm{s}$ (helium). ${ }^{3}{ }^{39}$ Ar will also contribute beta decay backgrounds in this scenario, however, due to its small abundance ${ }^{4}$ and longer half-life (269 vs. 10.7 years), its background burden is only $1.5 \%$ of that of ${ }^{85} \mathrm{Kr}$ and is not constraining. Isotopes such as ${ }^{222} \mathrm{Rn}$ and ${ }^{37} \mathrm{Ar}$ are relatively short-lived (3.8 d and $35 \mathrm{~d}$ half-lives, respectively) and have a small enough concentration in air such that they are not a concern during long-term storage.

\subsubsection{One-time air contamination during operations.}

If a one-time xenon handling mistake introduces a small volume of air into the detector while running, the ${ }^{85} \mathrm{Kr}$ and ${ }^{39} \mathrm{Ar}$ decay rates will permanently increase, while ${ }^{222} \mathrm{Rn}$ and ${ }^{37}$ Ar will also contribute unsupported ER backgrounds. The background burden from these species is as follows. We take the nominal ${ }^{37} \mathrm{Ar}$ concentration in air to be $1.2 \mathrm{mBq} / \mathrm{m}^{3}$ [3] , and we take the Davis campus radon concentration as $300 \mathrm{~Bq} / \mathrm{m}^{3}$. We assume that the quantity of dissolved air is $40 \mathrm{cc}$, and that the air is introduced near the beginning of the run. Then the number of ER events between 1.5 and $6.5 \mathrm{keV}$ in the 5.6 tonnes fiducial volume in 1,000 days is $24.3,2.3,0.36$ and 0.11 for ${ }^{85} \mathrm{Kr},{ }^{222} \mathrm{Rn},{ }^{39} \mathrm{Ar}$, and ${ }^{37} \mathrm{Ar}$, respectively. Here we have taken the branching fraction of the ${ }^{214} \mathrm{~Pb}$ beta decay in the ${ }^{222} \mathrm{Rn}$ decay chain as being $9.2 \% .{ }^{37}$ Ar contributes $\mathrm{X}$-rays and Auger electrons to the ER band between 2 and $3 \mathrm{keV}$ at a branching fraction of $90 \%$.

\subsubsection{Continuous air leak during operations.}

Here we assume that a continuous air leak exists in the Xe handling system during five years of underground operations, such that the long-lived isotopes ${ }^{85} \mathrm{Kr}$ and ${ }^{39}$ Ar integrate continuously into the ten tonnes of Xe, and such that activity of ${ }^{222} \mathrm{Rn}$ and ${ }^{37} \mathrm{Ar}$ is now supported. We require that the average concentration of ${ }^{85} \mathrm{Kr}$ due to the leak lead to no more than $24.3{ }^{85} \mathrm{Kr}$ events, equivalent to the requirements on the krypton removal system. The average ${ }^{85} \mathrm{Kr}$ concentration is half the final concentration, and under these circumstances we find that the equivalent helium leak rate is $1.4 \times 10^{-6} \mathrm{mbar} 1 / \mathrm{s}$ (helium), and the number of events due to

${ }^{3}$ We have used a conversion factor of one std cc.atm $/ \mathrm{sec}$ (air) $=0.3722 \mathrm{std} \mathrm{cc} \cdot \mathrm{atm} / \mathrm{sec}$ (helium).

$\left.{ }^{4}\left({ }^{39} \mathrm{Ar} /{ }^{\text {nat }} \mathrm{Ar}\right)=8 \times 10^{-16}(\mathrm{~g} / \mathrm{g})\right)[1]$, while $\left({ }^{85} \mathrm{Kr} /{ }^{\mathrm{nat}} \mathrm{Kr}\right)=4-23 \times 10^{-12}[2]$. 
${ }^{85} \mathrm{Kr},{ }^{222} \mathrm{Rn},{ }^{39} \mathrm{Ar}$, and ${ }^{37} \mathrm{Ar}$ is $24.3,0.51,0.36$ and 0.13 respectively (again taking the ${ }^{222} \mathrm{Rn}$ and ${ }^{37} \mathrm{Ar}$ concentrations in air as above).

\subsubsection{Cosmogenic activation of the Xe}

As detailed in Section 9.8.2, cosmogenic activation of the xenon during surface handling will result in tritium and ${ }^{127} \mathrm{Xe}$ activity in the Xe. After infinite exposure at sea level, the tritium decay rate would be about 30 decays $/ \mathrm{kg}$-day, or $1.7 \times 10^{8}$ decays in 5,600 tonne-days. Tritium is efficiently removed by the hot zirconium getter and is not expected to be problematic. GEANT4 predicts that the ${ }^{127} \mathrm{Xe}$ activity will be $2.6 \mathrm{mBq} / \mathrm{kg}$ in equilibrium at sea level, and detector simulations show that the rejection factor of all selection cuts will be $1.1 \times 10^{-5}$. Therefore we require that the initial activity be reduced to $88 \mathrm{mBq} / \mathrm{kg}$ at the start of physics data taking so that no more than 23 ER events remain after cuts. This implies a 178 day cool-down period underground, which is comparable to the detector commissioning time and is acceptable.

\subsection{Krypton Removal via Chromatography}
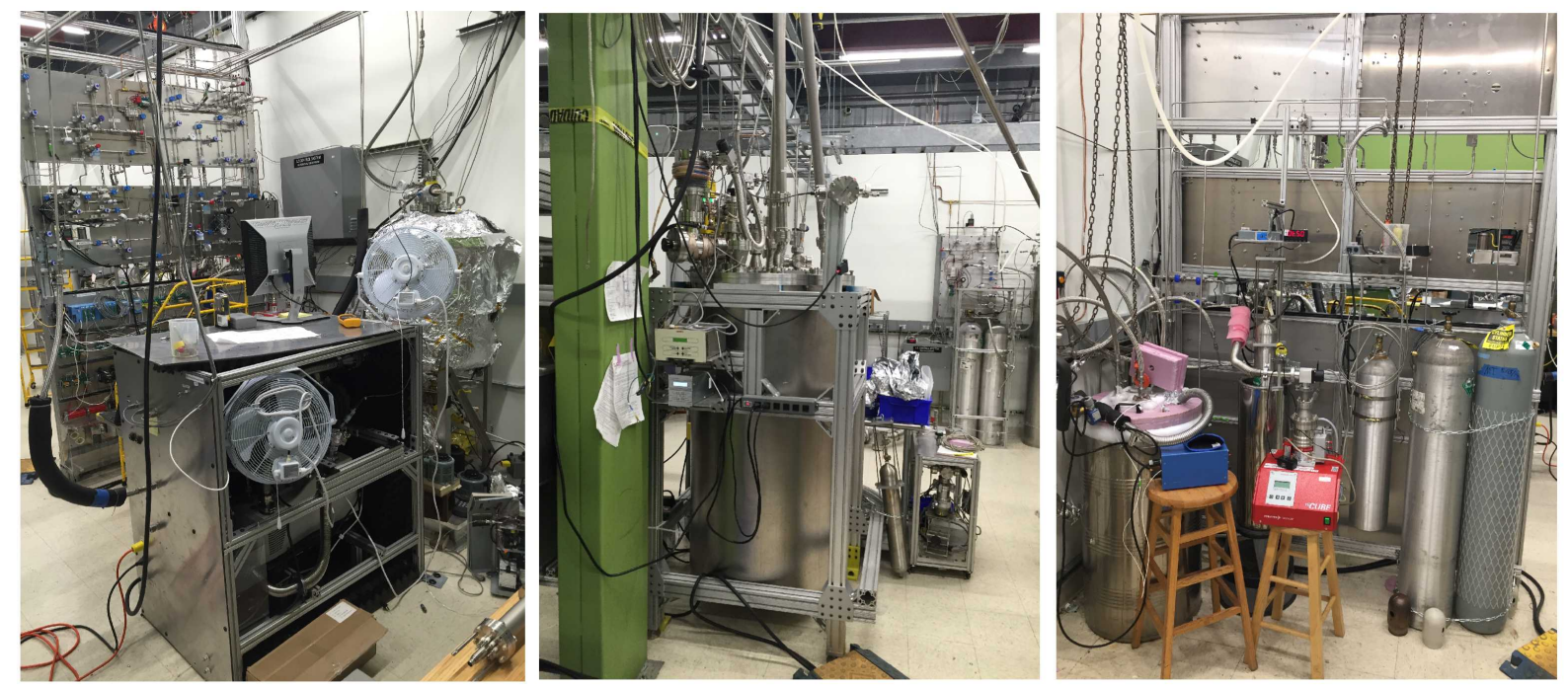

Figure 6.3.1: The three panels show the $\mathrm{Kr}$ removal development platform. Left: the gas control manifold, pumps, and charcoal column. Center: LN thermosyphon cooled condenser for xenon recovery. Right: krypton traps and xenon feed and storage located on the back side of the manifold.

Commercially available research-grade Xe typically contains trace $\mathrm{Kr}$ at a concentration up to $100 \mathrm{ppb}$ (parts per billion). ${ }^{85} \mathrm{Kr}$ is a $\beta$-emitter with an isotopic abundance of $\sim 2 \times 10^{-11}$ in natural $\mathrm{Kr}$ [1], an endpoint energy of $687 \mathrm{keV}$, and a half-life of 10.8 years. To reduce the rate of ${ }^{85} \mathrm{Kr}$ ER backgrounds to $10 \%$ of the $p p$ solar neutrino ER rate, we require that the total concentration of $\mathrm{Kr}$ be no more than $0.015 \mathrm{ppt}$ (parts per trillion), equal to a reduction of up to $\sim 10^{7}$ below that of research-grade Xe. Since the $\mathrm{Kr}$ is dissolved throughout the $\mathrm{Xe},{ }^{85} \mathrm{Kr}$ decays cannot be rejected via self-shielding, nor does the getter remove Kr during in situ purification, due to its inert nature. Gas vendors have indicated that they could guarantee Xe with a $\mathrm{Kr}$ concentration as small as $1 \mathrm{ppb}$ at additional cost, however this would still far exceed the LZ background goal. As noted in Section 6.9, 20\% of the Xe for LZ has been received and assayed, and all of it meets or exceeds spec. 


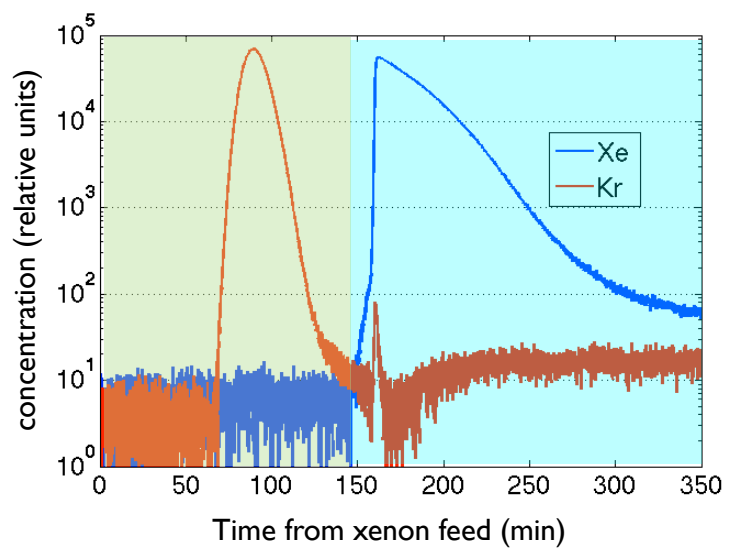

Figure 6.3.2: Test data taken with the LUX Kr removal system illustrate the time profiles for $\mathrm{Kr}$ and $\mathrm{Xe}$ as they exit the column for a sample of xenon spiked with approximately $1 \%$ krypton. Data are acquired with a sampling RGA. During production, the $\sim 100 \mathrm{ppb} \mathrm{Kr}$ in the raw $\mathrm{Xe}$ is below the RGA sensitivity so spiked samples are used to tune the chromatography parameters.

Trace Ar is also a concern due to the presence of the $\beta$-emitter ${ }^{39} \mathrm{Ar}$ (endpoint energy of $565 \mathrm{keV}$ and halflife of 269 years), and we require that the background rate due to ${ }^{39} \mathrm{Ar}$ be no more than $10 \%$ of that of ${ }^{85} \mathrm{Kr}$. Due to the low isotopic abundance of ${ }^{39} \mathrm{Ar}\left(8 \times 10^{-16}\right)$ [1], this implies an Ar concentration requirement of $4.5 \times 10^{-10}(\mathrm{~g} / \mathrm{g})$, substantially less demanding than the krypton requirement. Furthermore, because $\mathrm{Ar}$ is amenable to the same removal techniques as $\mathrm{Kr}$, no special measures are required to satisfy the $\mathrm{Ar}$ goal. Likewise, no special assays are required, since our technique simultaneously measures the residual Ar concentration along with the $\mathrm{Kr}$.

LZ will employ gas charcoal chromatography to remove $\mathrm{Kr}$ and Ar from Xe using the process that we successfully developed and executed for XENON-10 [4] and LUX [5]. The method employs a charcoal column with a continuously circulating He carrier gas and exploits the different transit time through the column for the various gas species. The weaker van der Waals binding of $\mathrm{Kr}$ to activated charcoal relative to Xe causes $\mathrm{Kr}$ atoms to flow through the charcoal column more rapidly than Xe. This property allows for a separation cycle by feeding $\mathrm{Xe}$ (with trace $\mathrm{Kr}$ ) into the column at a fixed rate and duration under the influence of the fixed carrier flow. The rate and duration are tuned so that the last of the Kr exits the column prior to the earliest $\mathrm{Xe}$. This permits the $\mathrm{Kr}$ to be purged by directing the $\mathrm{Kr}$-He stream to a coldtrap that captures the Kr. This feed-purge cycle is followed by the Xe recovery cycle, in which pumping parameters are altered to accelerate the rate at which the Xe exits the column.

Figure 6.3.2 illustrates this process by showing the time profiles for the two species as they exit the column. The Kr-He exits first during the chromatography cycle to achieve $\mathrm{Xe}-\mathrm{Kr}$ separation. Then, as the purified Xe-He stream exits the column, the Xe is frozen out from the stream using a cryogenic condenser, while the He circulates until recovery is complete. After a number of feed-purge-recovery cycles, the He is pumped away, the condenser is warmed, and the Xe is transferred to a storage cylinder. The purity of the $\mathrm{Xe}$ is measured using the coldtrap/mass-spectrometry technique developed at Maryland [6] and described in Section 6.7.

Using this method, the SLAC group, while still at Case Western, processed the $400 \mathrm{~kg}$ of LUX Xe at a rate of $50 \mathrm{~kg} /$ week down to a $\mathrm{Kr}$ concentration of $4 \mathrm{ppt}$, exceeding the LUX goal of $5 \mathrm{ppt}$ and rendering this background sub-dominant to the leading background by a factor of 10 during the 2013 LUX WIMP search [5,7]. The starting concentration was $130 \mathrm{ppb}$ of $\mathrm{Kr}$ and the typical batch reduction factor was $3 \times 10^{4}$. In addition, one $50 \mathrm{~kg}$ batch of Xe that was "spiked" to approximately $0.01 \% \mathrm{Kr}$ was processed 
twice, resulting in an upper limit on the concentration of $0.2 \mathrm{ppt}$, which was our assay sensitivity at that time. This powerful reduction of 9 orders of magnitude resulted in Xe with a $\mathrm{Kr}$ concentration only a factor of 13 higher than the ultimate LZ goal, and shows that sequential processing gives multiplicative reduction and is a suitable method to achieve clean Xe for LZ.

While this result is encouraging, it demonstrates only that cross-contamination of feedstock to recovered Xe can be mitigated by multi-pass processing. However, reaching the LZ goal also requires that sources of contamination and ingress of Kr-laden air also be controlled down to the ultimate required concentration. To ensure that our method is sound, we reconfigured the LUX production system as a development platform, which is shown in Figure 6.3.1. We have been using that system to refine our process and have learned several valuable lessons that are being incorporated into the production system including: the pre-scrub of the He carrier gas through a cold-trap to remove trace $\mathrm{Kr}$ prior to use for purging of the primary traps; the placement of the $\mathrm{Kr}$ trap as close as possible to the charcoal column to mitigate contamination from upstream components; the gradual turn-on of Xe into the feed cycle to avoid pressure spikes and backflow into the output side of the $\mathrm{Kr}$ trap, which otherwise results in cross-contamination; the importance of all-metal seals throughout the system; and the critical nature of the Xe recovery pump, which is the most complex component through which the purified Xe passes. In addition, we have used the assay system to prepare 100-gram samples of highly-purified xenon that we backflow into various parts of the system, recover, and assay to see if the xenon remains pure. We expect to make significant use of this powerful diagnostic tool to isolate, identify and mitigate deficiencies in the production system, as we have done in the R\&D system. Each of the lessons learned has resulted in progress in bringing down the level of the Kr, which in our best batches to date is $0.075 \mathrm{ppt}$. At the time of this writing, we await the arrival of an improved Xe recovery pump with better isolation between the process space, the internal gearbox, and the external environment.

In addition to purity requirements, the processing rate and duration of a production run also influence the design of the full LZ system. Figure 6.3.3 provides a simplified schematic of our design for the production system, which will process $200 \mathrm{~kg}$ of Xe per day, or 10 tonnes in 60 days at $85 \%$ uptime. This design has better isolation between the chromatography loop and the recovery loop than did the LUX design, which achieved $3 \times 10^{4}$ reduction per pass and was likely limited by cross-contamination. While we may expect some improvement of the $\mathrm{Kr}$ rejection factor as the system is tuned during initial operations, we conservatively plan for two complete passes of the Xe (120 days), which is more than sufficient to reduce the $\mathrm{Kr}$ concentration in the raw stock by a factor of $10^{7}$. Fast processing also provides schedule insurance in the event of an unintentional contamination event. We note that the introduction of just $40 \mathrm{~cm}^{3}$ of air at standard temperature and pressure (STP) corresponds to $0.015 \mathrm{ppt}$ of $\mathrm{Kr}$ in 10 tonnes of $\mathrm{Xe}$. A single pass through the chromatographic system could easily correct an error as large as hundreds of liters. (The corresponding requirement for argon is less demanding: $270 \mathrm{~cm}^{3}$ of air at STP.)

As illustrated in Figure 6.3.3, the three principal processing stages are: (1) chromatography to separate $\mathrm{Xe}$ and $\mathrm{Kr}$, and capture the $\mathrm{Kr}$ in a cooled charcoal trap; (2) recovery of the Xe from the column into a cooled condenser to strip the Xe from the carrier gas; and (3) storage and analytical sampling of the purified Xe into LZ storage packs for transport to the experiment. The system architecture follows two general principles. First, we minimize the components exposed to both the raw and purified Xe streams to minimize cross-contamination, which leads to the two mostly separate loops in the figure (green and blue). Second, the arrangement matches the feed-purge cycle time to the Xe recovery time, so that by alternating between the two columns, we achieve twice the duty cycle of single-column operation. Two condensers are also used so that one is always available in the recovery path when the other is in the storage phase. The double-swing system with two columns and two condensers gives a factor-of- 4 increase in production rate relative to a single-column-single-condenser system at modest additional cost and complexity. Moreover, reduced labor for the shorter production run more than offsets the incremental hardware cost of the second condenser and column. 


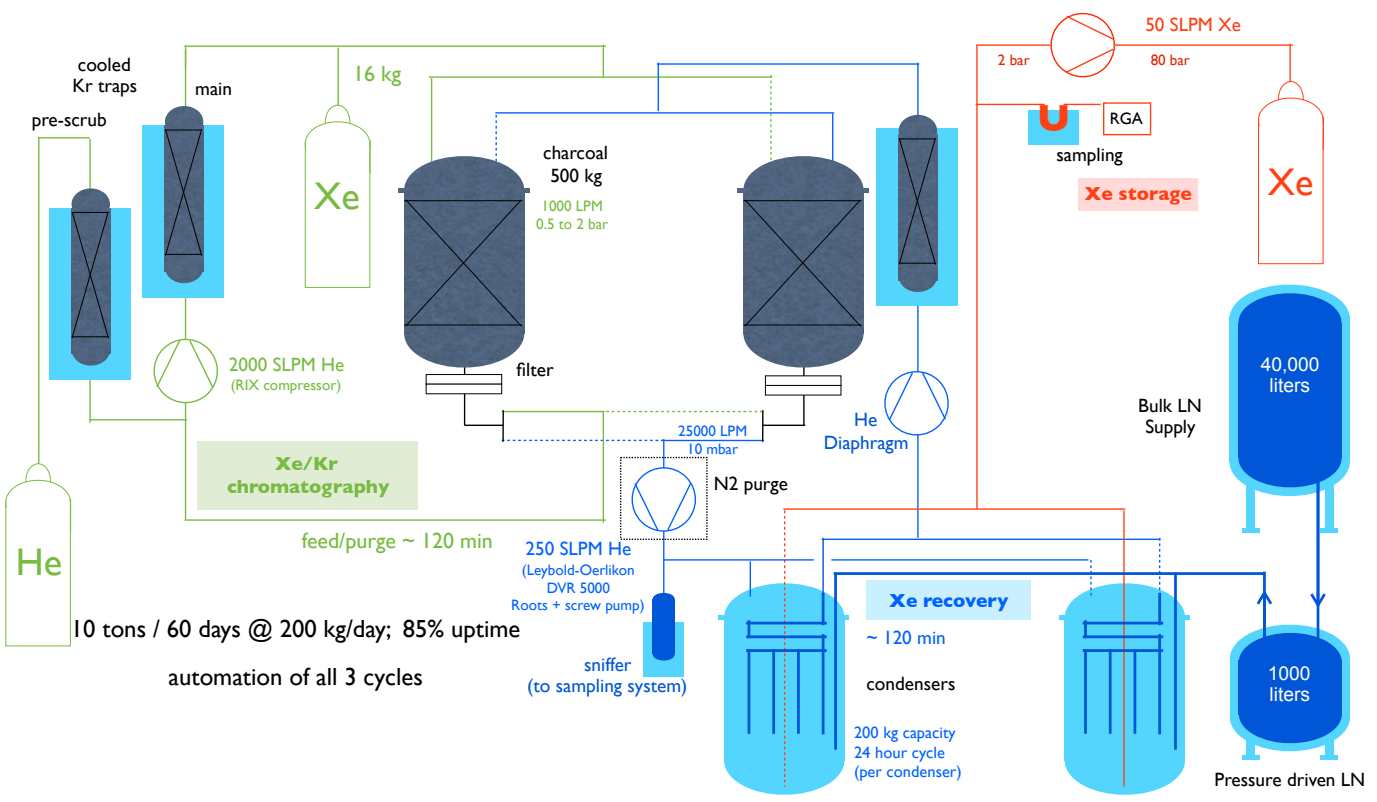

Figure 6.3.3: This figure shows the main components and flow paths of the LZ Kr removal system. Colored lines indicate the path for a given process. The green line shows the left-hand charcoal column in the chromatography feed-purge separation cycle with $\mathrm{Kr}$ being collected in the right trap; a second trap is valved out for cleaning (dashed green line). The blue line shows the subsequent part of the cycle in which $\mathrm{Xe}$ is being recovered from the column and collected in the condenser. The red line shows $\mathrm{Xe}$ from the condenser being stored and analyzed. Key pressures and flow rates are indicated.

The overall processing rate is governed by the differential transit time of the $\mathrm{Kr}$ and $\mathrm{Xe}$ in the column, whereas all other components are sized to match this rate. In the LUX system, each feed cycle consisted of a $2 \mathrm{~kg}$ slug of Xe into a column of $60 \mathrm{~kg}$ of charcoal at $50 \mathrm{slpm}^{5} \mathrm{He}$ flow rate. The $\mathrm{Kr}$ purge was completed 120 minutes after the start of the feed, at which time recovery commenced. Straightforward scaling to reach $200 \mathrm{~kg}$ per day calls for a $16 \mathrm{~kg}$-slug every two hours, which in turn requires an approximate eight-fold scaling in charcoal mass and flow rate. Some additional tuning will be required based on further studies of chromatography and cross-contamination using the LUX system. The remaining parameters, as shown in Figure 6.3.3, are appropriately scaled from the existing system.

The system architecture uses computer-controlled pneumatic valves for changing all of the process states between the various cycles. Pumps, mass flow controllers, heaters, and diagnostic sensors (pressure, temperature, etc.) are controlled and/or read out by this system as well. We will use programmable logic controllers (PLCs) for process control since they are a standard industrial solution that is robust and gives low latency for reliable control and system interlocks. The PLC system will interface with the SCADA software package Ignition, by Inductive Automation, which we have been using in the R\&D system. The three primary cycles (chromatography, recovery, storage), as well as $\mathrm{Kr}$ trap cleaning and analytic sampling, will be fully automated, minimizing the chance of operator error.

The Kr-removal system uses a cryogenic condenser system to freeze the purified Xe out of the Xe-He stream at LN temperature. A schematic of the combined condenser and LN system is shown in Figure 6.3.4. The design of the condenser is based on the LUX approach with several new features that will simplify construction and operation, and reduce cost. A set of LN-cooled surfaces mounted in a pressure vessel accumulates the purified xenon. After a number of runs, the He is evacuated, the system is warmed to LXe

${ }^{5}$ standard liters per minute 


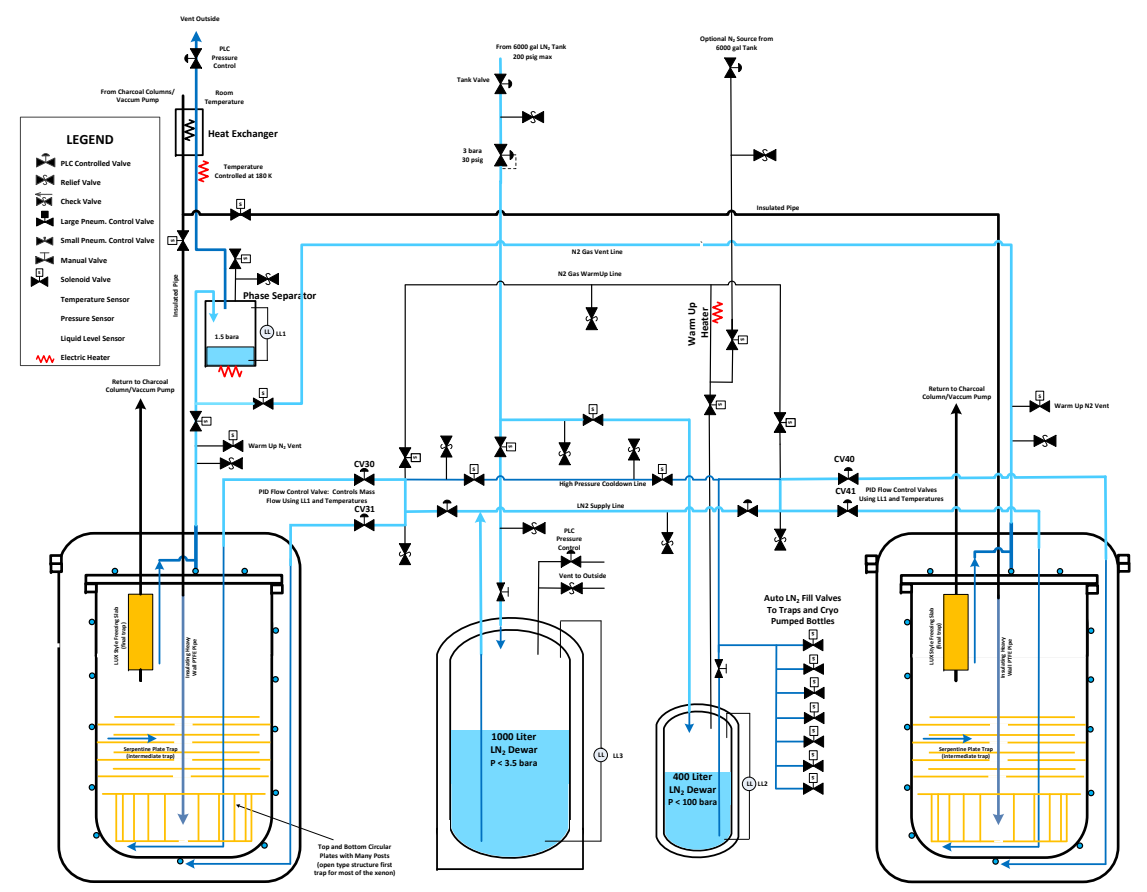

Figure 6.3.4: A schematic of the condenser and LN distribution system. One condenser is alternately coupled to the two charcoal columns, with LN supplied by the 1000-liter pressurized storage dewar, 1-2 days of recovery cycles. During that time, the other condenser is in the warming, storage, and re-cooling cycle. The 400-liter LN dewar supplies cryogens for rapid recooling as well as the various LN-cooled traps. The two LN dewars are resupplied by the 9,000 gallon tank at IR2 (not shown).

temperature, and the xenon is compressed into LZ storage packs for either a second pass or transport to the experiment. The $\mathrm{Kr}$ removal system will use one of the storage compressor skids described in Section 6.5, which will then travel to South Dakota with the processed xenon. Since we don't require precision temperature control as in a LXe detector, rather than using a more complex thermosyphon system to provide cooling we have designed a simpler force-flow system. This system will deliver LN through a system of copper tubes that are brazed to the vessel walls and internal structures. The direct LN cooling will easily meet peak load and rapidly recool the vessels after storage of a previous batch. The pressure-driven LN loops will consist of long circuits of copper piping that will be coupled to a series of copper plates in the condenser space and to the outer wall of the vessel. Because cooling power can be better distributed than with individual thermosyphon cold heads, the design will have a favorable ratio of xenon capacity to vessel size. Finally, the pressure-driven LN system has fewer control points than the thermosyphon and will be more straightforward to program and operate. A detailed engineering note contains a through analysis of the time-dependent heatload defined by the variable rate of the Xe recovery (Figure 6.3.2), as well as the reduction in cooling power as frozen Xe accumulates (Xe ice is a poor thermal conductor).

In Figure 6.3.5, we show a physical layout of the major system components, including pumps, columns, condensers, traps, pressure-driven LN supply, and sampling subsystems. The system will be installed in Building 624 at the IR2 complex at SLAC. The Xe, which is a high-value asset, will be stored in a locked "sea container" in front of the pad. Not shown is the nearby LN storage tank, which is refilled by truck under SLAC contract. 


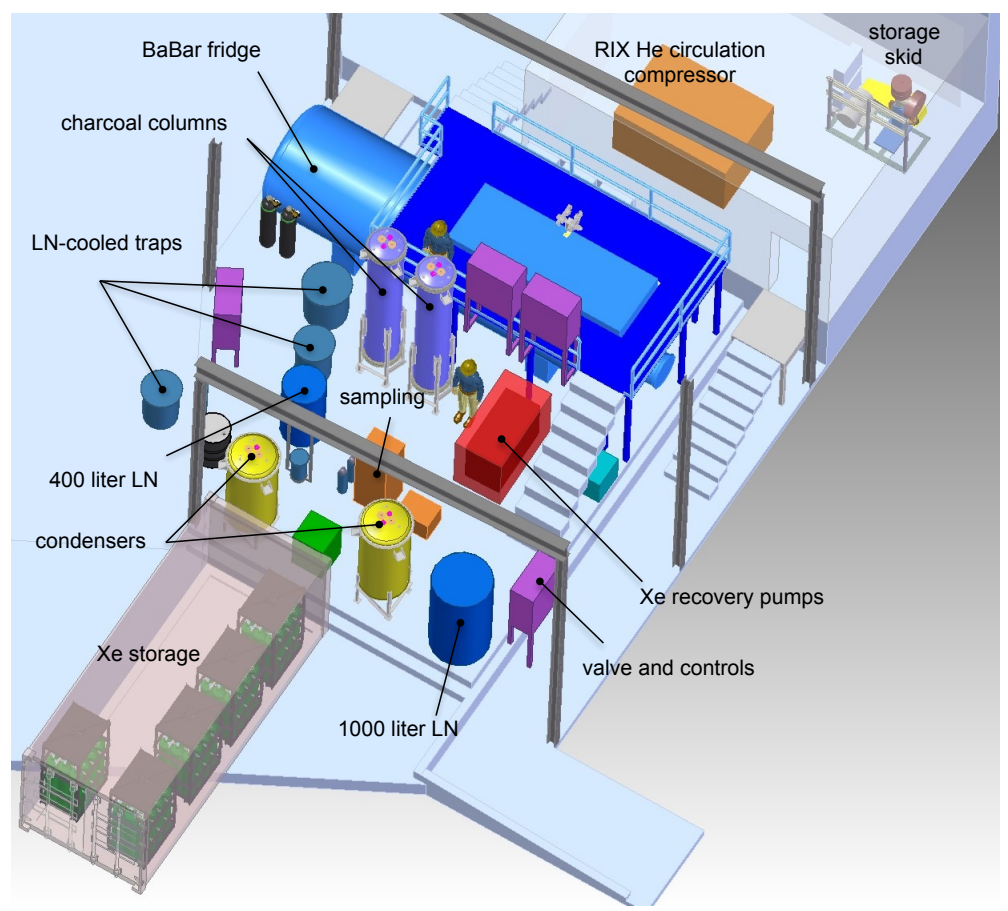

Figure 6.3.5: A layout of the LZ Kr removal system shows all major components located at the former BaBar LHe cryopad in building 624 at the SLAC IR2 complex. If a buyer for the refrigerator is not found, this layout shows the adequacy of the remaining space. The partial soft-wall exterior is well suited to mitigate oxygen deficiency hazard with the large quantity of cryogens in use. A 20-foot sea container at the front of pad will provide secure storage for Xe cylinder packs and ease in handling.

A high-flow-rate RIX 2-stage piston pump for circulating the carrier gas during chromatography has been identified at SLAC and is available for LZ. The purity requirements of this pump are modest because the He will pass through the LN-cooled charcoal trap prior to the Xe feed branch and the chromatography columns.

As noted in the R\&D discussion, the Xe recovery pump system is the most complex component that sees the purified xenon. We have worked closely with Leybold-Oerlikon to evaluate commercially available dry pumping systems. We provided the manufacturer with a fully-detailed data set rescaled from our LUX slow control data, including $\mathrm{He}$ and Xe pressures and mass flow rates for the full recovery cycle. The manufacturer has used their proprietary software to make a detailed a assessment of pump performance, and determined that a stock version of the DVR 5000, which combines a dry Roots booster backed by a dry screw pump will meet our requirements.

During processing, several hundred kilograms of Xe will be in various parts of the system. To protect against loss of Xe we will rely on backup systems to maintain cooling power. Short-term outages are protected by UPS units. For longer outages, the SLAC operations team has deployed a standby generator that will come online within 1 to 2 minutes. These capabilities will allow the control systems to replenish the $\mathrm{LN}_{2}$ reservoirs in the Kr removal system from the nearby 9,000 gallon LN storage tank at the IR2 complex.

A fully-detailed $\mathrm{P} \& \mathrm{ID}^{6}$ has been developed for the production system. The design is based on the principles of the LUX system that was reconfigured for R\&D, while following the overall architecture shown in Figure 6.3.3. The production system P\&ID is shown in Figure 6.3.6.

${ }^{6}$ Piping and Instrumentation Diagram 


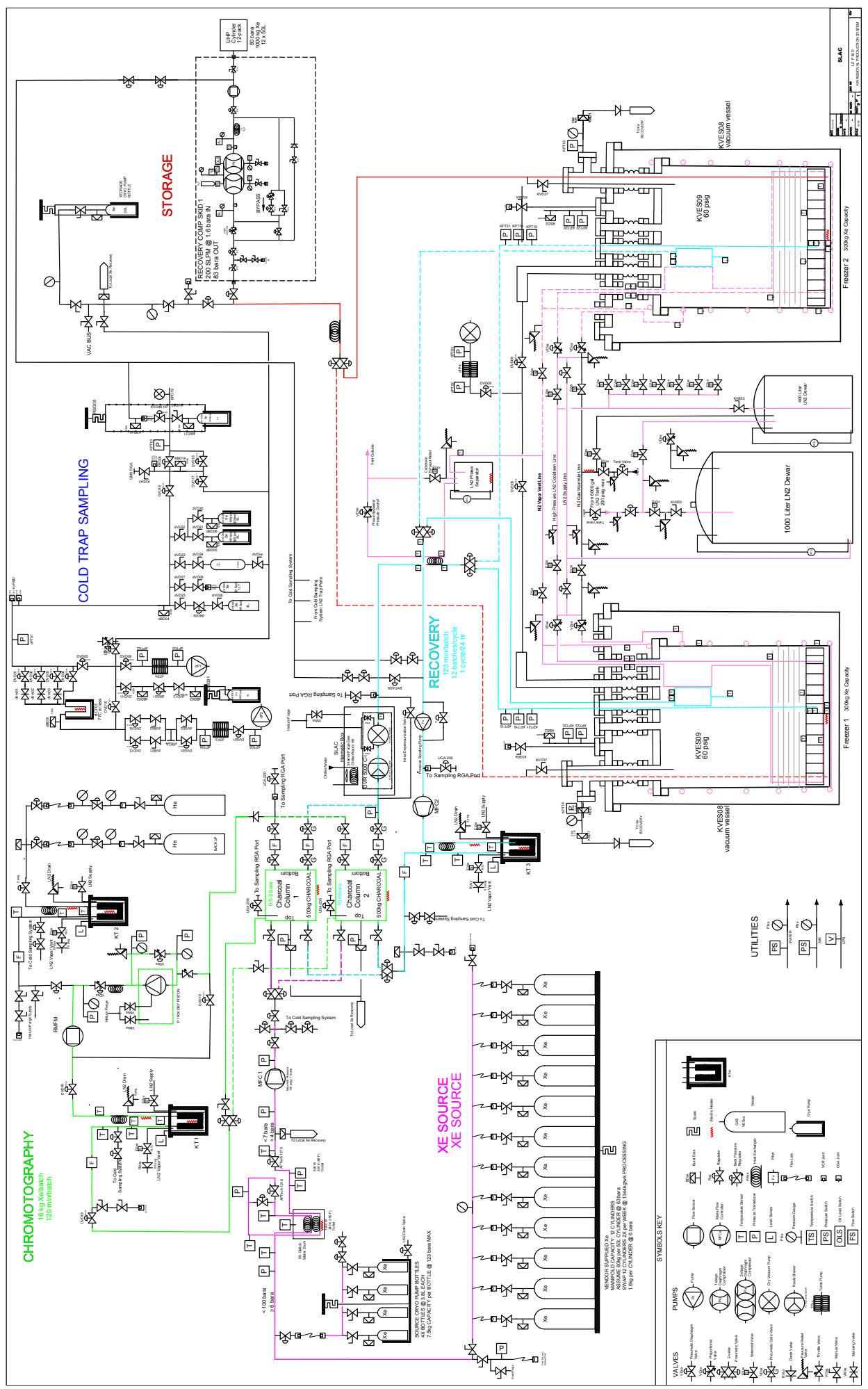

Figure 6.3.6: The P\&ID for the LZ Kr removal system. 


\subsection{Online Xenon Purification System}

This section describes the online Xe purification system, which controls the concentration of electronegative impurities such as $\mathrm{O}_{2}$ and $\mathrm{H}_{2} \mathrm{O}$ during detector operations through continuous purification. The system is shown schematically in Figure 6.4.1. Liquid xenon is removed from the detector at the weir trough near the top of the TPC. The design flow rate is $500 \mathrm{slpm}(2.8 \mathrm{~kg} / \mathrm{min})$, or about $1.01 / \mathrm{min}$. The liquid exits the cryostat and flows to the LXe tower through vacuum insulated transfer lines that penetrate the water tank. In the LXe tower it is evaporated, compressed to higher pressure, is purified, and then recondensed and returned to the detector.

A central feature of this design is a set of two heat exchangers located in the LXe tower that make the phase and temperature change thermally efficient. Without these heat exchangers, $4.3 \mathrm{~kW}$ would be required in each flow direction at 500 slpm for the phase change alone.

This section is organized as follows. The gaseous portion of the circulation system is described in Section 6.4.1. The LXe tower and the vacuum insulated transfer lines are described in Sections 6.4.2 and 6.4.3. We report on results from a prototype circulation system that implements the LZ architecture in Section 6.4.4. A radon removal system that treats the gaseous xenon in the breakout box and cable conduits is described in Section 6.4.5, and the slow controls is described in Section 6.4.6.

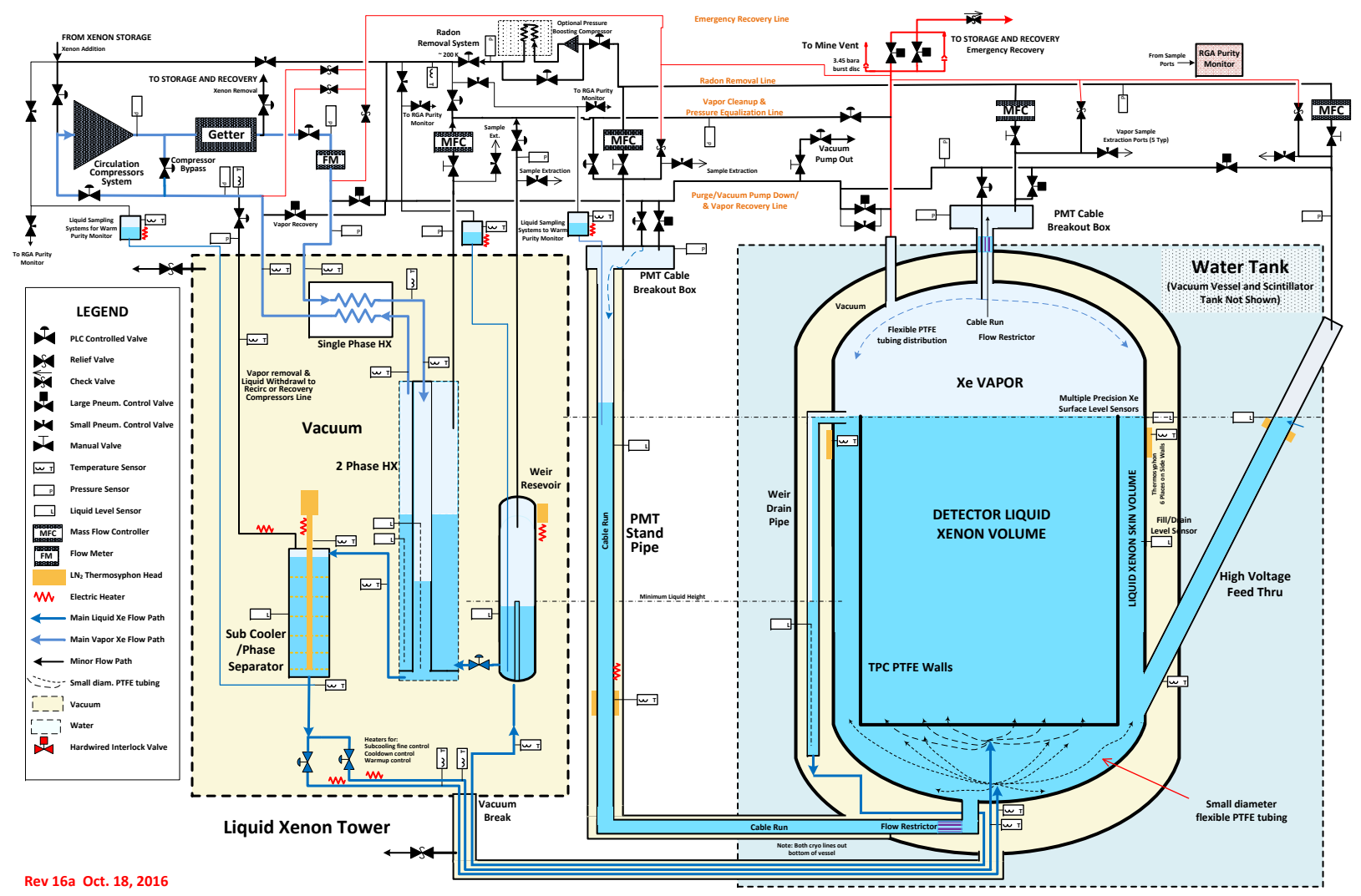

Figure 6.4.1: Overall flow diagram of the $L Z$ online purification system. The shaded box on the left represents the LXe Tower, where the phase changes between LXe and GXe occurs. The top of the diagram represents the gas recirculation system. Note that this figure captures the design at a high level, but the master P\&ID should be relied upon for details. 


\subsubsection{Gas recirculation system}

The Xe gas recirculation system receives gaseous xenon from the detector via the LXe Tower (Section 6.4.2), circulates the xenon through the hot zirconium getter to remove electronegative impurities, and returns the purified Xe to the LXe Tower. This system also handles Xe purge flow and Rn-Removal (Section 6.4.5) to/from the detector cable conduits, as well as calibration source injection. A complete P\&ID for this subsystem is provided in Figure 6.4.2.

The centerpiece of the LZ online purification system is a hot zirconium getter (model PS5-MGT100 from SAES), a purifier technology capable of achieving $>99 \%$ one-pass removal efficiency for virtually all non-inert impurities of interest, including hard-to-remove species such as $\mathrm{N}_{2}$ and $\mathrm{CH}_{4}$ [8]. Impurities chemically bond to the surface of zirconium pellets, irreversibly removing them from the Xe. The getter operates at elevated temperature $\left(450{ }^{\circ} \mathrm{C}\right)$ to allow the captured impurities to diffuse into the bulk of the getter pellets, leaving the surface free for additional gettering. The getter material must be replaced when it becomes saturated with impurities. The SAES getter comes equipped with valves, plumbing, controls, and an engineered integrated safety interlock system that will disable the getter power and isolate the getter from the process gas in the event that excessive heat is detected.

The Xe circulates through the purification system at a rate of $500 \mathrm{slpm}(2.8 \mathrm{~kg} / \mathrm{min})$, a value that was chosen based upon previous experience with the LUX system and based on economic and space constraints. At this flow rate, the 10 tonnes of Xe can be purified in 2.3 days, comparable to the 1.7-day turnover time of the LUX recirculation system. The recirculation rate and the charge attenuation goal constrains the allowed outgassing rate of the detector, and this drives the outgassing plan of the detector. Prior experience with LUX and other liquid noble detectors indicates that the scintillation absorption length goal $(>15 \mathrm{~m})$ is less demanding than the charge attenuation goal and will be satisfied by the same requirements.

Circulation through the purification system is provided by a set of all-metal triple-diaphragm two-stage compressors. This type of compressor uses an electric motor to drive single-piston oil pumps that pressurize the bottom diaphragms of each stage, transmitting force to the top diaphragms to compress the gas. Middle diaphragms with grooves create a space that is monitored for leakage of either oil or Xe. The all-metal design (including metal main head seals) minimizes the ingress of krypton and radon into the Xe. A 100 slpm Fluitron compressor of this type and utilizing this all-metal seal technology has been operating at SLAC as part of the System Test (Figure 6.4.3), allowing us to begin building a strong understanding of operating performance, characteristics, leak-tightness, and maintenance. For LZ, two compressors, each rated for 300 slpm at 1 bara suction pressure and capable of 10 bara output pressure, operate in parallel to provide the full system flow. Together these compressors nominally deliver $600 \mathrm{slpm}$ of flow, so a proportional control valve modulates excess flow back to compressor suction to maintain the nominal 500 slpm circulation through the purification system. Scheduled maintenance (every 5,000 hours) and unexpected repairs on these machines will require periodic operations at reduced circulation rate (approximately 300 slpm from the remaining online compressor).

After-cooling of the compressed gas may be adjusted so preheated gas can be delivered to the getter. Hot gas exiting the getter is cooled back to room temperature by a heat exchanger provided with the getter. The purified gas is then returned to the LXe Tower. The online sampling system (Section 6.7) has taps to draw samples from upstream of the compressor, and upstream/downstream of the getter. Xenon that has already been sampled reenters the circulation loop downstream of these taps.

The gas plumbing for the recirculation system is pre-cleaned stainless steel (SS) tubing connected with lanthanated or ceriated orbital welds wherever possible. VCR fittings with metal seals will be used where necessary to open a connection. Valves will be of the bellows- or diaphragm-seal type to keep the system hermetic. Gas panels will be sealed to allow for panel-wide He leak checking during testing and commissioning, as well as continued $\mathrm{N}_{2}$ purge during operations. 


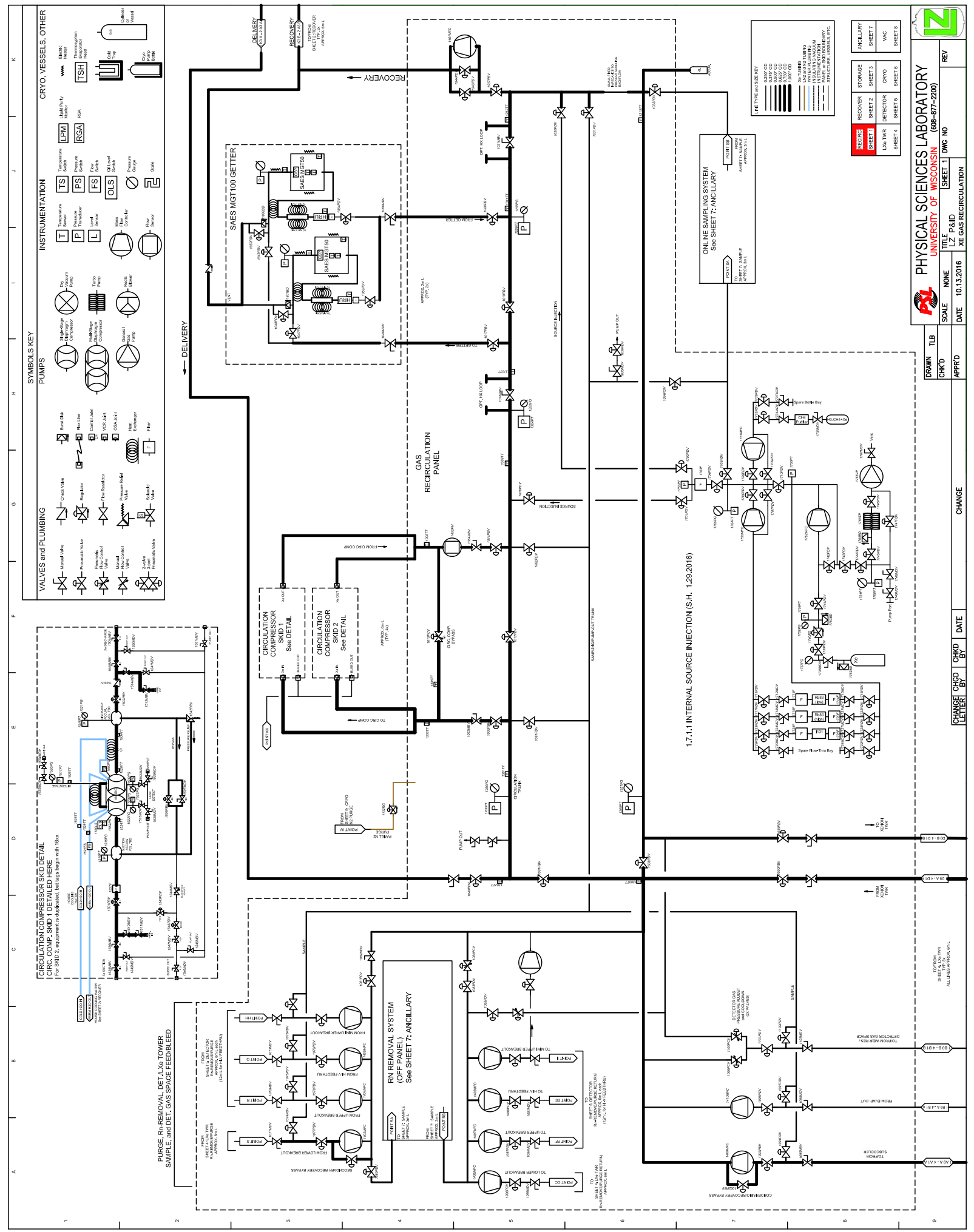

Figure 6.4.2: Xe Gas Recirculation system P\&ID. 


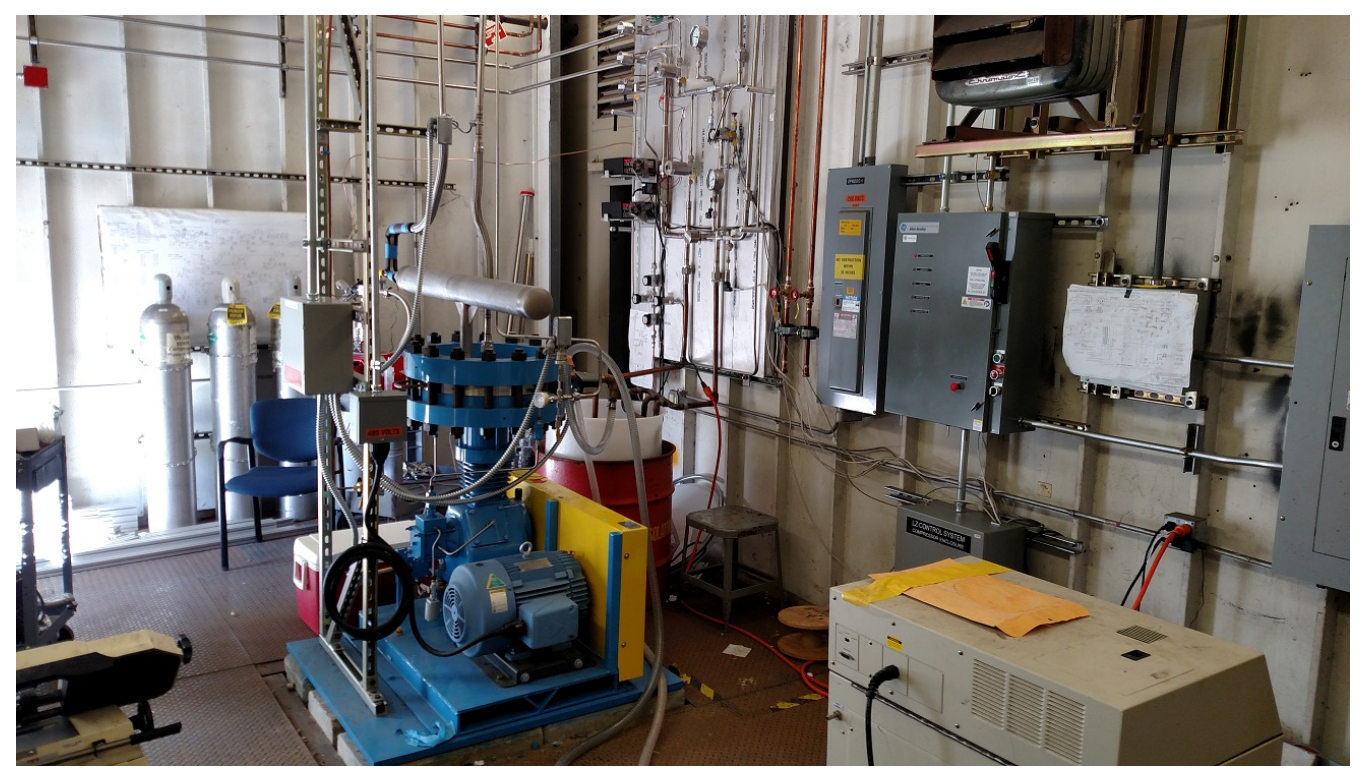

Figure 6.4.3: Fluitron $100 \mathrm{slpm}$ all-metal single-stage diaphragm compressor operating as part of SLAC System Test.

In addition to the primary Xe recirculation path, four secondary gas-only recirculation loops act on the detector conduits that house the cables for the PMTs and instrumentation cables (from Xe vessel top and the PMT standpipe). These cables are immersed in LXe on one end, but they penetrate the liquid surface and terminate at room-temperature feedthroughs filled with Xe gas at the other end. These cable bundles are a potential source of problematic outgassing, particularly the warm ends where the diffusion constants of the insulating plastics are the largest. We manage this outgassing by purging these conduits with a continuous flow of Xe gas away from the TPC at a modest flow rate. This purge gas flow merges at the input of the online Rn-removal system (Section 6.4.5), and then returns back to the conduits, bypassing the LXe tower. Purge flow is driven by a small independent gas compressor in the Rn-Removal system. Plumbing provisions also allow for a portion of the Rn-Removal system output flow to be directed into the main circulation loop to create a purge flow imbalance and provide evaporative cooling to the conduits if desired. The purge-gas flow rate required to ensure that back-diffusion is negligible is characterized by the unitless Peclet number, $P=V L / D$, where $V$ is the linear velocity of the gas in the conduit, $L$ is the diffusion distance of interest, and $D$ is the diffusion constant of the impurity species. For a 4 inch diameter conduit, a gas flow rate of $0.5 \mathrm{slpm}$ per conduit, a diffusion distance of $10 \mathrm{~cm}$, and a diffusion constant of $0.086 \mathrm{~cm}^{2} / \mathrm{s}$ (valid for $\mathrm{O}_{2}$ diffusion in $\mathrm{Xe}$ gas at room temperature), the Peclet number is 8.2, indicating that back diffusion is negligible. We plan for a total of $2.0 \mathrm{slpm}$ of flow across all conduits (three cable conduits and one high voltage feedthrough conduit). Figure 6.4.4 is a portion of the P\&ID detailing the detector, which shows how these purge paths are tied into the detector conduits. LXe paths are on bottom, GXe taps are above the LXe level.

The circulation system must also accommodate detector calibration and purity assays. As discussed in Chapter 7, radioactive sources such as ${ }^{83 \mathrm{~m}} \mathrm{Kr}$ and tritiated methane will be introduced into the Xe to calibrate the central regions of the TPC, and the online purification system has a valve and port system to allow for this. Due to its inert nature, ${ }^{83 \mathrm{~m}} \mathrm{Kr}$ may be injected into the circulation stream upstream of the getter, whereas tritiated methane must be injected downstream. There are also valves to allow for the sampling of the LXe in the tower and gaseous Xe before and after the getter, as described in Section 6.7. Details of the source injection plumbing and how it ties into the gas recirculation system can be found lower-center in Figure 6.4.2. 


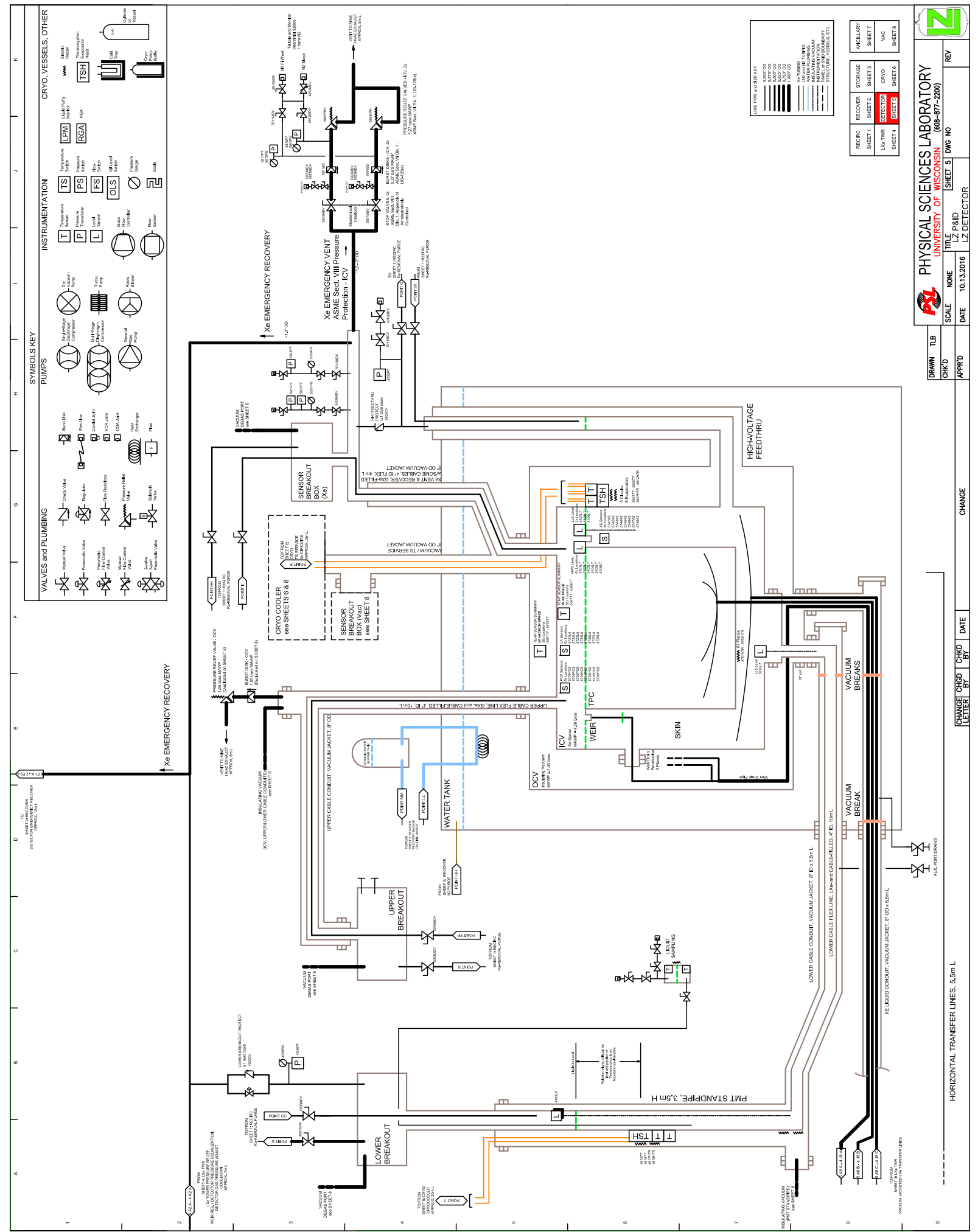

Figure 6.4.4: P\&ID of Detector and Xenon Handling Tie-Ins. 


\subsubsection{Liquid xenon tower}

Because the hot getter in the purification system (Section 6.4.1) operates on gaseous Xe (GXe) only, the purification system must vaporize and re-condense the liquid $\mathrm{Xe}(\mathrm{LXe})$ to create a continuous purification circuit. This process is made thermally efficient by using counter flowing single- and two-phase heat exchangers in series with a sub-cooler/phase-separator within the LXe Tower assembly. The Xe is transferred in liquid form between the LXe Tower and the detector through vacuum-insulated transfer lines that penetrate through the wall of the water shield and connect to the bottom of the TPC vessel.

The LXe Tower is shown in detail in Figure 6.4.5 and contains the following major components: 1) a valve block that houses LXe flow control and LXe Tower isolation valves; 2) a weir reservoir for collecting the liquid returning from the detector and preparing it for evaporation; 3) a counter flow serial two-phase and single-phase heat exchanger assembly for evaporating the LXe and bringing the exiting GXe to room temperature while pre-cooling the returning GXe and liquefying the xenon gas; 4) a subcooler/phase separator for cooling the return liquid and removing gas bubbles; and 5) three thermosyphon heads; three liquid sampling ports. The LXe Tower P\&ID is shown in Figure 6.4.6.

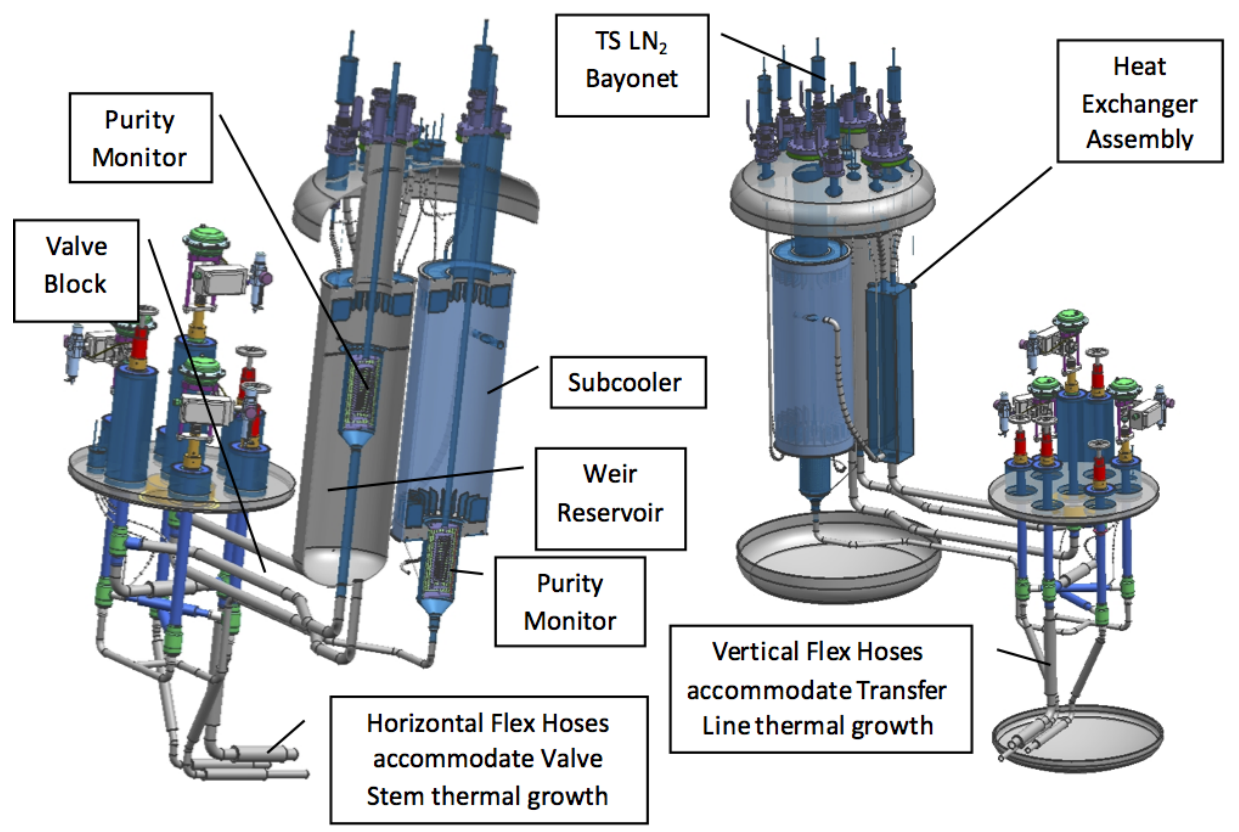

Figure 6.4.5: Two views of the LXe tower assembly. The heat exchanger assembly is visible in the right image and the subcooler and weir reservoir components can be seen at the left. The cryogenic valves are consolidated in a separate valve block (visible in both views) to provide easy access to the actuators and also to allow for isolation between the LXe tower and the detector.

In addition, the tower design includes space for two optional liquid purity monitors, one located in the inlet to the weir reservoir, to measure the purity of the xenon on the way out of the detector, and the other located in the subcooler, to measure the purity of the xenon entering the detector. Cryogenic components will hang from the top of the vacuum jacket to allow for fabrication with the vacuum jacket removed. When the internal structure is complete, the vacuum jacket is welded shut on the sides and bottom. There is a vacuum break at the bottom of the xenon tower isolating its vacuum from the detector for safety considerations and for LN2 checkout. 


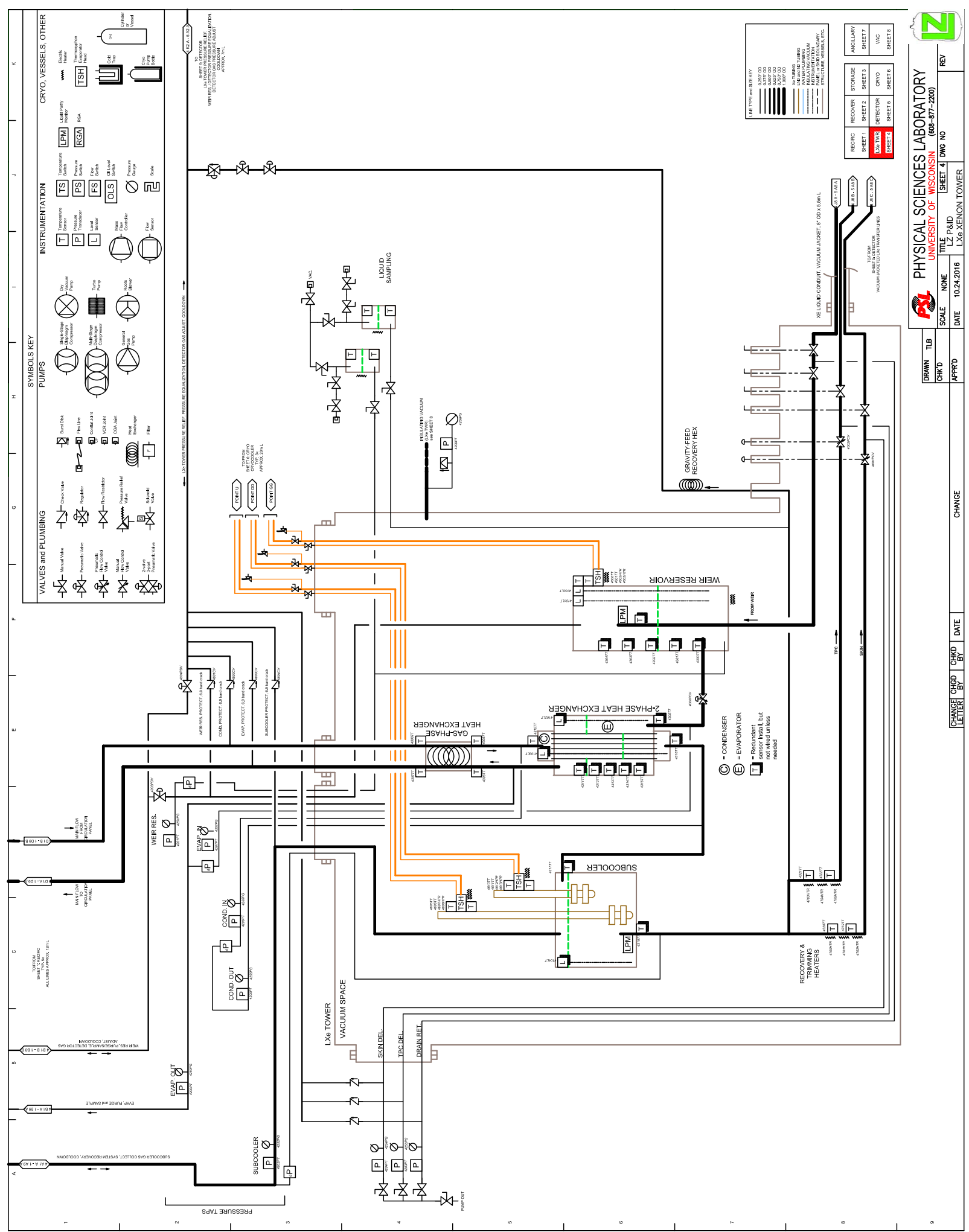

Figure 6.4.6: P\&ID of the LXe Tower. 
Liquid flow from the subcooler passes through two sets of two cold control valves that partition the flow between the TPC volume and the skin volume. Each valve set is paired to allow the LXe tower to be isolated from the detector. These valves will be used during normal operation, for vessel cool-down, and for changing flow rates and patterns during injection of radioactive $\mathrm{Kr}$ and methane. Both of these flow streams have an electric heater with thermometer for cool-down and warm-up control. These valves are also used for liquid extraction to compressor suction during warm-up. A third pair of cold control valve is placed between the weir reservoir and the evaporator side of the two-phase heat exchanger. This valve set works in conjunction with the compressor suction control to establish the height of the boiling LXe. It will also provide a small amount of Joule-Thomson cooling by using the energy supplied from the recirculation compressor. All three of these cold control valve sets are bellows sealed and designed for use in helium liquefiers.

The weir reservoir is a simple $35.6 \mathrm{~cm}$ diameter ASME stamped vessel that acts as a volume buffer between the weir drainpipe and the two-phase heat exchanger. LXe flows down the weir drainpipe in the detector, through the transfer line, and up the weir reservoir standpipe. Besides establishing the liquid level in the weir drainpipe in the detector, the weir reservoir also provides a location to collect nonvolatile impurity species that may be present in the LXe. Such species, if they exist, would become harmlessly trapped in the weir reservoir. A liquid sample tube from the weir reservoir liquid sampling system to the bottom of the weir reservoir makes it possible in principle to remove contamination.

Liquid flows from the bottom of the weir reservoir into the bottom (evaporator) of the two-phase heat exchanger where it is vaporized, while Xe from the getter is condensed. The bottom of the weir reservoir is placed at a relative height above the bottom of the two-phase heat exchanger to provide a small liquid pressure head to push fluid into the heat exchanger. Liquid is also drawn up into the evaporator side of the two-phase exchanger by the lower pressure of the suction side of the gas compressor. As LXe flows from the bottom of the weir reservoir into the bottom of the heat exchanger assembly, the LXe undergoes a phase change (vaporization) into GXe on the evaporator side while GXe from the getter changes to LXe in the condenser side of the assembly. The mass flow rates are the same for each circuit of the heat exchange assembly, during steady state operation. The condensing side operates at a higher pressure then the evaporator side, and because the heat of vaporization is higher at higher pressure, any excess energy released during the phase change evaporation process can be used to sub-cool the LXe after condensation and before entering the sub-cooler/phase separator.

Both the single-phase and two-phase heat exchangers will be commercial plate heat exchangers which have a high heat transfer efficiency relative to their physical footprint. Because the single-phase and twophase heat exchangers are of similar style, they are combined into a single heat exchanger package which houses both the gas to gas and phase change heat transfer. As discussed in Section 6.4.4, data from the LZ System Test are being used to ensure that the system conditions that affect heat exchanger design are understood. These system conditions include the relative liquid heights within the system and the inlet and outlet temperatures and pressures.

Any additional heat exchange assembly inefficiencies and thermal loads on the LXe are removed before the liquid enters the detector in the subcooler, the last major component of the LXe tower. The subcooler, or phase separator, is made with perforated copper plates attached to a central copper rod with thermosyphon heads at top or bottom. Copper mesh or similar will be placed between the copper plates (depending upon analysis) to ensure a more than adequate heat transfer area. During normal detector operation the subcooler serves two purposes. Any xenon vapor not condensed by the two phase heat exchanger can separate from the liquid flow in the ullage of the subcooler and be condensed, and the liquid itself can be sub-cooled before it enters the detector cryostat as required. The refrigeration of the cryostat can be optimized by adjusting the power of the subcooler thermosyphons and the detector cryostat thermosyphons. Using the nominal detector vapor pressure of 1.8 bara, the bottom of the detector has a pressure of 2.25 bara, translating to a 
saturation temperature of $180.3 \mathrm{~K}$. A thermosyphon is able to reduce the liquid xenon temperature close to the freezing point $161.5 \mathrm{~K}$. The temperature of the sub-cooled xenon can therefore be adjusted to make up any heat loads on the TPC space. The skin volume is heated from radiation heat through the vessel wall, detector support structure and PMT heat loads. Sub-cooling of the xenon returning to the skin will therefore need to account for the increased heat loads in the skin region.

The subcooler also plays an important role during cooldown of the detector. During cooldown operations, xenon gas will be circulated through the system and the subcooler thermosyphons and their copper fins will gradually cool the circulating gas. Once the detector has reached base temperature, the subcooler thermosyphons liquefy xenon gas to fill the detector.

\subsubsection{LXe transfer lines}

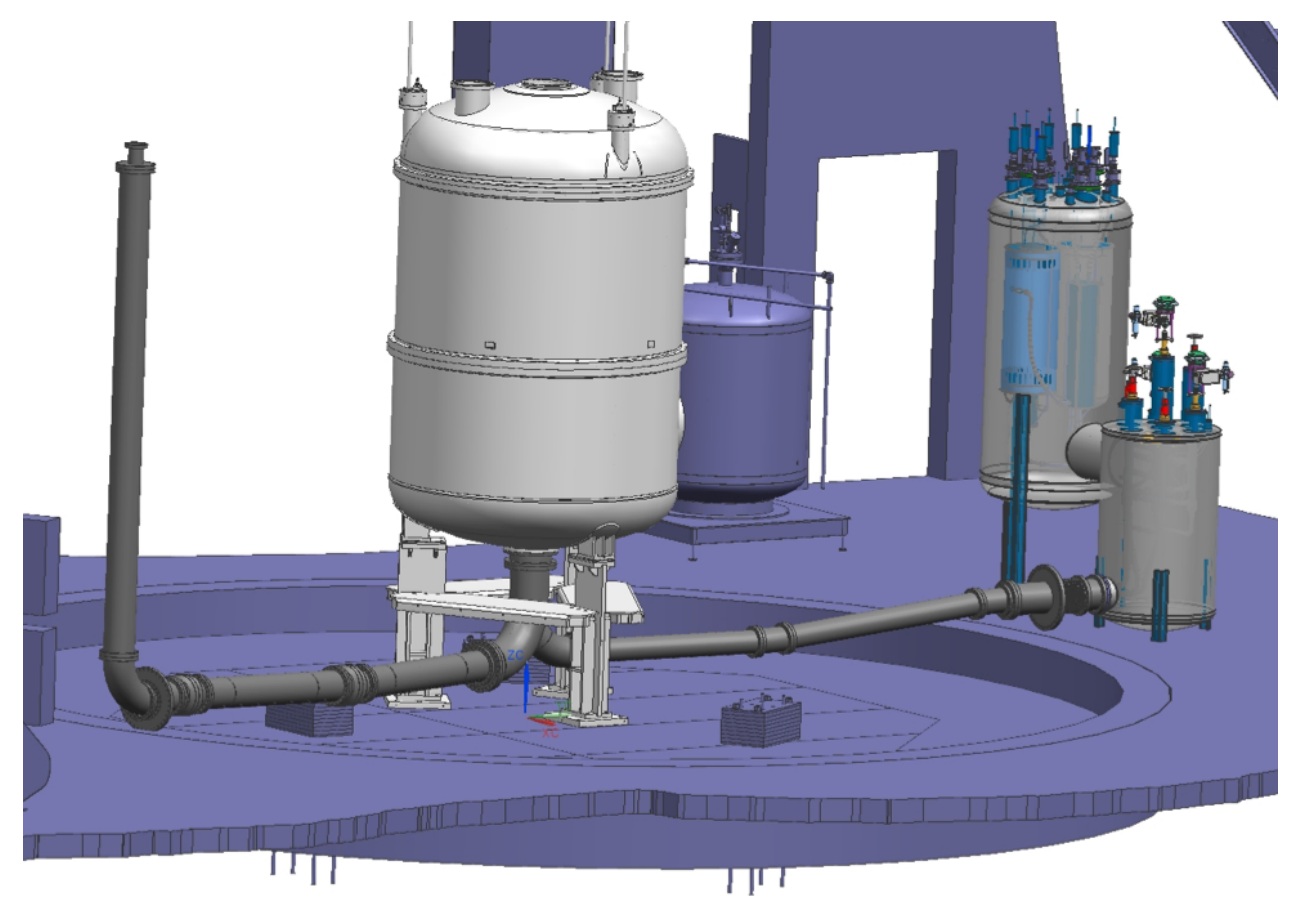

Figure 6.4.7: The TPC cryostat (center), the PMT cable standpipe (left), the LXe Tower (right), and the two vacuum insulation assemblies that house the $L X e$ transfer lines.

As shown in Figure 6.4.7, two vacuum insulated assemblies host cryogenic lines that service liquid xenon to and from the cryostat at its lower flange. These two assemblies meet at a center Tee directly underneath the cryostat. Both are composed of sections of 8 inch tube and are assembled inside the water tank and sealed to its wall with flanged connections on opposite sides of the tank.

One of these assemblies, on the right side of Figure 6.4.7, houses the LXe supply and return for the primary circulation path of the online purification system. This assembly hosts two lines that route purified LXe from the subcooler to the skin and TPC active region, and a third line that connects the weir drain lines to the weir reservoir. These three lines are individually insulated with multilayer insulation because they can be at different temperatures. Connections will need to be made in these lines during assembly. While it would be ideal to make welded connections with an orbital welder, there may need to be VCR fittings to allow these connections to be made due to space constraints. A vacuum break is planned just after the center Tee below the cryostat. This helps provide protection in case of a leak and would also allow softening the vacuum in the transfer line region to add heat during Xe recovery. The weir drain line will 
be actively cooled by a conductive connection to a thermosyphon. This will sub-cool the returning liquid below saturation temperature to decrease the possibility of vapor lock.

The second assembly, seen on the left side of Figure 6.4.7, houses the PMT and sensor cables from the TPC bottom PMT array. These cables exit the bottom of the detector cryostat, and are housed in a 4 inch ID bellows reinforced with a braid. The flange at the bottom of the inner cryostat has a connection for both the bellows housing the cables and the LXe lines. The cables are pulled into the bellows in the reduced radon clean room of the surface assembly lab and the bellows is sealed for transport underground to keep the cable clean. During lowering of the inner cryostat into the outer cryostat the lower cable bellows is pulled through the tee in the center bottom of the water tank. The shape of the Tee is designed to help guide the cable bellows. The multilayer insulation for this line is wrapped around segments of $180 \mathrm{~mm}$ OD polyethylene tube that is supported from the inside of the 8 inch SS tube with plastic thermal standoffs.

The final section of the cable path is the vertical PMT standpipe that is located outside the water tank and connects to the bottom of the breakout box. The standpipe is shown on the left side of Figure 6.4.7. The liquid level in the PMT stand pipe is about the same as the liquid level in the detector. The liquid is cooled with evaporation by pumping xenon out of the gas space above the liquid surface. The xenon gas between the liquid and the bottom of the breakout box has a temperature transition to room temperature.

As noted in Section 6.4.5, the upper and lower PMT conduits and the high voltage conduit will extract a total of $2.0 \mathrm{slpm}$ of xenon gas through the PMT cable breakout boxes. This ensures that flow with the higher level of contamination from the plastic is always out of the detector where it is cleaned in a radon trap before it is returned to compressor suction. Both the liquid and vapor PMT cable runs will have a flow restrictor as close as possible to the outer flange of the cryostat. The restrictor is designed such that the Peclet Number (advection/diffusion) $\gg 1$. For the liquid line this means a collar around the cables for several inches reducing the size of the big gaps.

\subsubsection{SLAC System Test prototype of the circulation architecture}

Previous experience with the LUX cryogenics and its xenon handling system is only partially applicable to LZ due to the increased complexity of the LZ design. To exercise the new architecture, a large System Test platform containing over $100 \mathrm{~kg}$ of LXe has been developed at SLAC, including an extensive xenon handling capacity to purify LXe to realistic levels. As shown in Figure 6.4.8, the platform emulates most of the critical design features of the LZ architecture. It implements a separated LXe tower, containing a weir reservoir, a subcooler, and two-phase and single-phase heat exchangers, and it is connected to the TPC by vacuum insulated LXe transfer lines. These elements perform the same functions that their counterparts will in LZ, although their designs are not identical. Purified liquid is delivered to the bottom of the TPC and is recovered from the top via a weir spillover. That liquid is routed to the LXe tower by draining into a vertical pipe that penetrates the inner cryostat and descends to the bottom of the TPC vessel in the insulating vacuum space.

Several test runs of the circulation system have been carried out, and after some exploration of the thermodynamic parameter space and some hardware fixes, uniform flow at a rate of up to $75 \mathrm{slpm}$ has been achieved for more than 80 consecutive hours. This provides a proof of principle that the LZ circulation architecture is viable. Under the conditions of uniform flow, the liquid level in the TPC is set by the weir spillover as desired, and the reservoir and sub-coolers are filled with a constant (but flowing) volume of buffer liquid. Data from these circulation tests provide a benchmark for engineering models of the two-phase heat exchangers in both the System Test and LZ.

The System Test has also highlighted some challenges presented by the LZ design, particularly the vacuum insulated weir drain line. We have found that, under some conditions, the flow of the saturated LXe in this line can be interrupted. Both the geometry of the line and the heat leak into it may be responsible 

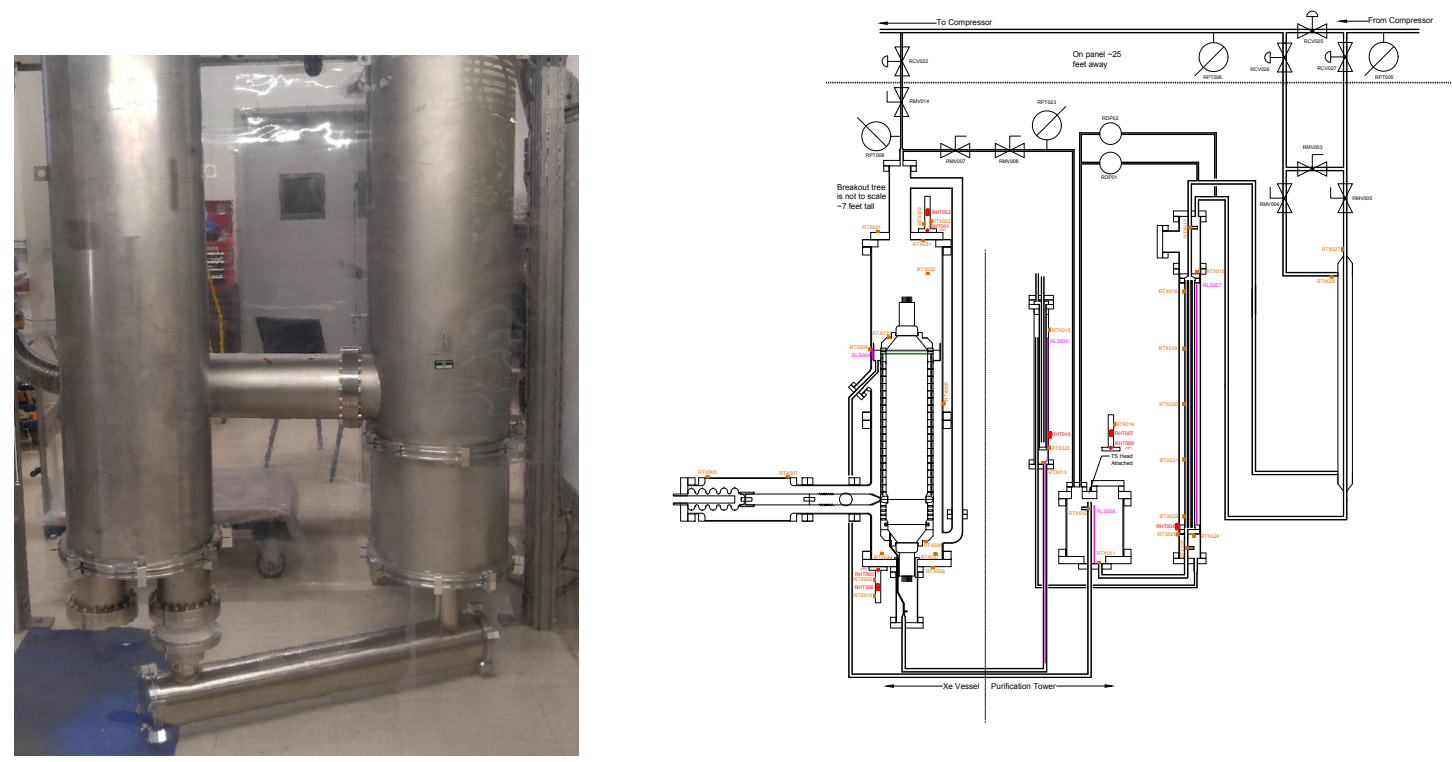

Figure 6.4.8: Left: System Test LXe tower vessel (left vertical vessel) and TPC cryostat (right vertical vessel), connected by vacuum insulated LXe transfer lines housed inside the lower horizontal vessel. Right: Schematic diagram of the internal elements. At left is the TPC, serviced by a large horizontal HV feedthru, and instrumented with one PMT on top and on bottom. The small vessel in the lower middle is the weir reservoir. It is surrounded by the sub-cooler and the two-phase and single-phase heat exchangers. The vacuum insulated transfer lines are below.

for this behavior. We are exploring the addition of active cooling to the line to counter this effect, so that uniform circulation can be stably achieved under a variety of pressure and temperature conditions.

\subsubsection{Radon removal}

Material screening is the primary route to control the ER and NR backgrounds resulting from radioactivity in the experiment. Recent estimates of radon emanation from PMT HV cables, cable feedthroughs and cable conduits, shown in Table 6.4.1, however, suggest that ${ }^{222} \mathrm{Rn}$ backgrounds may be up to $37 \mathrm{mBq}$, which is substantially above required levels. It is therefore required to mitigate radon with a combination of cable cladding and radon removal for the xenon gas circulation system. Neither action alone is sufficient. Adding $150 \mu \mathrm{m}$ FEP cladding to the braided PMT HV cables will greatly reduce the risk of dust or other surface contamination in the steel braiding of the cables and essentially eliminate emanation from the cables in LXe, but will have no effect on PMT feedthroughs. Adding a radon trap with a volumetric gas flow of $\phi=2$ slpm in the (warm) gas circulation system will eliminate $90 \%$ of radon from cables and feedthrough, but will have no effect on the PMT HV cables immersed in LXe.

Removal of radon from xenon is challenging due to the similar atomic diameters of these two species (120 pm vs. $108 \mathrm{pm}$, respectively [9]). However a pioneering demonstration of such removal at $\phi=1 \mathrm{slpm}$ has been performed by the XMASS collaboration using a cooled column of activated charcoal [10]. This result has been reproduced in Ref. [11] at $\phi=0.25 \mathrm{slpm}$.

To determine feasibility of radon removal in xenon gas for LZ, a series of elution curve measurements were performed to determine the breakthrough time, $\tau$, (i.e. adsorption coefficient) of radon in xenon carrier gas at various temperatures with a $30 \mathrm{~g}$ activated charcoal trap, since studies [12] of ${ }^{222} \mathrm{Rn}$ adsorption in 
Table 6.4.1: Radon emanation estimates for PMT HV cables, cable conduits and cable feedthroughs. Also shown are estimates for including only cladding or a charcoal trap, as well as combining both mitigating methods. With only initial measurements of PMT feedthroughs available at this point, estimates for the other components are based on similar items of materials from other experiments. All estimates are in $\mathrm{mBq}$.

\begin{tabular}{|lr|c|c|c|c|}
\hline \multirow{2}{|l|}{ Item } & Component & estimated & $\begin{array}{c}\text { w/ cladding } \\
\text { only }\end{array}$ & $\begin{array}{c}\text { w/ trap } \\
\text { only }\end{array}$ & $\begin{array}{c}\text { w/ cladding \& trap } \\
\text { combined }\end{array}$ \\
\hline PMT HV Cables & warm insulation & 0.27 & 0.41 & 0.027 & 0.04 \\
& warm braiding & 1.88 & 0.47 & 0.19 & 0.05 \\
& warm dust & 13 & 0 & 1.30 & 0 \\
& cold insulation & $2.7 \times 10^{-4}$ & $4.1 \times 10^{-4}$ & $2.7 \times 10^{-4}$ & $4.1 \times 10^{-4}$ \\
& cold braiding & 1.88 & 0 & 1.88 & 0 \\
& cold dust & 13 & 0 & 13 & 0 \\
\hline PMT HV Cables & Subtotal & 30.0 & 0.88 & 16.4 & 0.09 \\
Cabling Conduits & warm \& cold & 0.1 & 0.1 & 0.055 & 0.055 \\
PMT Feedthroughs & warm & 7.3 & 7.3 & 0.73 & 0.73 \\
\hline \multicolumn{2}{r|r|}{ Total } & $\mathbf{3 7 . 4}$ & $\mathbf{8 . 3}$ & $\mathbf{1 7 . 2}$ & $\mathbf{0 . 8 7}$ \\
\hline
\end{tabular}

gases (other than air [13]) are scarce. Breakthrough time is given by $\tau=\frac{m \cdot k_{a}}{\phi}$, where $k_{a}$ is the dynamic adsorption coefficient, $m$ the mass of charcoal, and $\phi$ the volumetric gas flow.

Figure 6.4.9 (left panel) shows breakthrough time versus inverse temperature of radon in xenon carrier gas with a $30 \mathrm{~g}$ activated charcoal trap. Although a linear relationship is expected over the entire temperature range in the semi-logarithmic representation shown in Figure 6.4.9, the deviation from linearity may reflect Xe saturation on the charcoal surface.

Based on the elution curve measurements, a $90 \%$-efficient radon removal element for the xenon gas circulation system was designed as shown in Figure 6.4.10. The size of the charcoal trap was determined to be $8.6 \mathrm{~kg}$ (i.e. 19 liters) by scaling up the elution curve measurements with the small trap ( $\mathrm{m}=30 \mathrm{~g}$, $\tau=156 \mathrm{~min}, \phi=0.5 \mathrm{slpm}$ at $-68^{\circ} \mathrm{C}$ ) to $\tau=12.7$ days (i.e. 3.3 half-lives), $\phi=2 \mathrm{slpm}$ and $-85^{\circ} \mathrm{C}$. This can be accomplished with two $1 \mathrm{~m}$-long columns of 3 inch diameter connected in series.

Gaseous xenon from the purge-gas line conduits will be introduced into the radon removal system through a SAES high temperature getter before it passes through the charcoal trap. The charcoal trap will be immersed in a cryostat vessel that is filled with HFE-7100 coolant and kept at a temperature of $-85^{\circ} \mathrm{C}$ using a EK-90 refrigerator or a thermosyphon. The system will allow transmission of xenon gas with flow rates ranging from 0 slpm to 2 slpm using a diaphragm circulation pump.

To measure the amount of radon in the carrier gases, a Si PIN photodiode (ORTEC) made from low radioactivity components will be used. The electronics readout will consist of a 142AH ORTEC preamplifier with low noise and fast timing characteristics as well as little temperature dependence, and a shaping preamplifier. A multichannel analyzer (ORTEC MCA-2K) will process the signals from the preamplifier. The radon system will be fully automated using high pressure pneumatic valves, and pressure and temperature sensors that are integrated into the LZ slow control system.

To validate the design and perform studies with different trap geometries using various adsorbents at various flow rates, a separate full-scale prototype system has been designed and built at the University of Michigan that makes use of the final cryogenics system. Initial measurements with the prototype system 

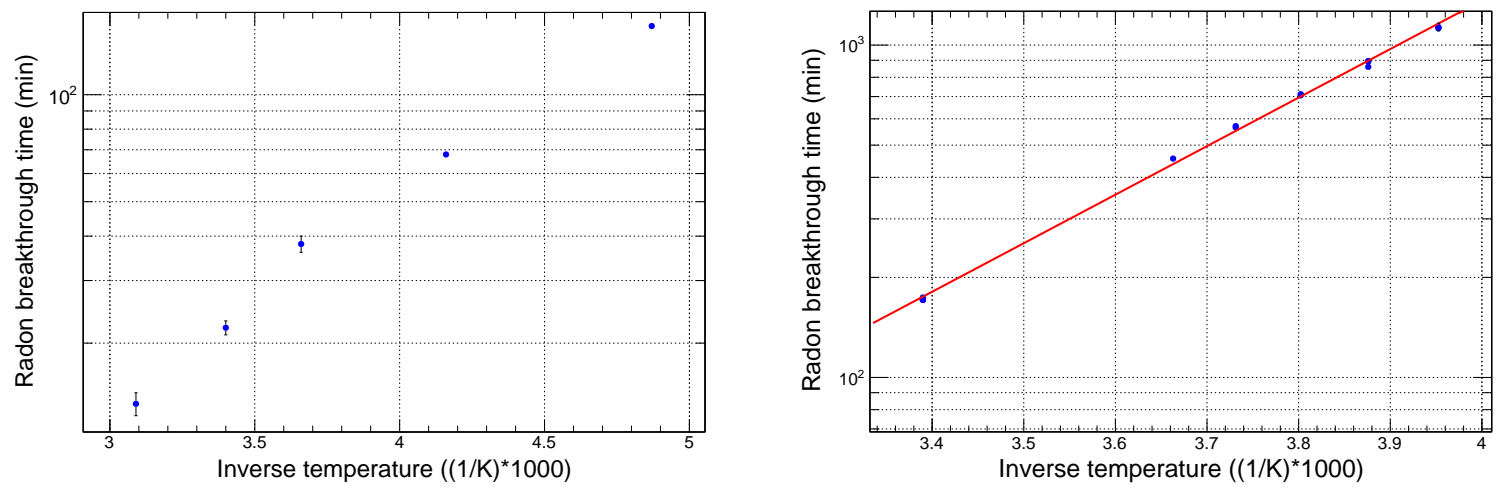

Figure 6.4.9: Radon breakthrough time versus inverse temperature for two different configurations. Left panel: Breakthrough time in a $30 \mathrm{~g}$ charcoal trap with a Xe carrier gas at a flow rate of $0.5 \mathrm{slpm}$. Right panel: Breakthrough time in a $50 \mathrm{~g}$ charcoal trap with nitrogen carrier gas at a flow rate of $2.0 \mathrm{slpm}$. The red line is a fit of the data to the Arrhenius equation $r=A \exp \left(\frac{E_{a}}{R T}\right)$, with $r$ the inverse breakthrough time, $A$ the collision frequency factor, $T$ the temperature, $E_{a}$ the activation energy, and $R$ the universal gas constant. The fit values are $A=0.00187 \pm 0.00044 \mathrm{~min}^{-1}$ and $E_{a} / R=3375 \pm 65(\mathrm{~K})$. Note that the temperatures in the traps are known to about $\pm 1 \mathrm{~K}$.

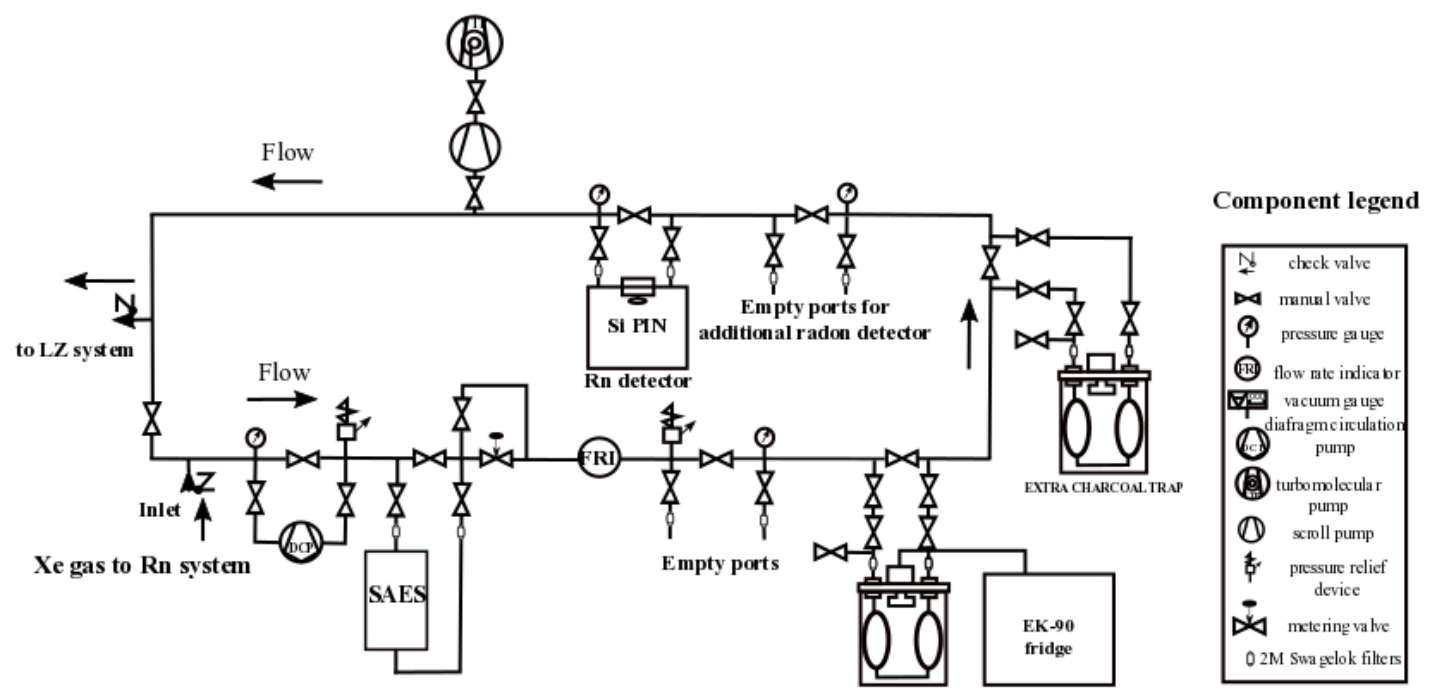

Figure 6.4.10: Schematic diagram of the radon removal system for LZ.

are underway. The right panel of Figure 6.4.9 shows the breakthrough time of radon in nitrogen carrier gas versus inverse temperature in a $50 \mathrm{~g}$ charcoal trap. The system will soon be operated with argon or helium gas before expensive xenon gas is used as carrier gas. It contains a radon calibration source to study the adsorption efficiency of the traps with different adsorbents besides natural carbon and different amounts of the adsorption materials. The test system can be used to study the adsorption efficiency of radon traps in a broad range of temperatures and trap geometries. The traps can be swapped and regenerated by baking and pumping at high temperatures. 


\subsubsection{Slow controls}

A set of industrial Programmable Logic Controllers (PLCs) will handle instrument read-out and control for the xenon purification and recovery systems, as well as the cryogenic and systems and TPC high voltages (excluding PMTs). The PLC logic will include interlocks protecting the integrity of the xenon system, control loops and automated procedures for the various subsystems, and automated emergency response. All PLC programming will follow IEC 61131-3 standards.

The overall PLC system architecture includes one top-level PLC plus smaller dedicated PLC's for large equipment items, namely the cryogenerator system and four xenon compressors ( $2 \mathrm{x}$ recovery and $2 \mathrm{x}$ circulation), see Figure 6.4.11. The top-level controller is a Siemens S7-410-H Redundant Hot Backup PLC, which includes redundant processors with independent links to I/O modules. The redundant processors guarantee bumpless switchover in the case of processor failure, and also allow for zero-downtime when updating PLC logic and I/O mapping. The Siemens S7 PLC system will have a dedicated internally redundant APC Symmetra UPS as well as parallel DC power units for the S7 cpu's and I/O power, ensuring uninterrupted control when switching to generator power.

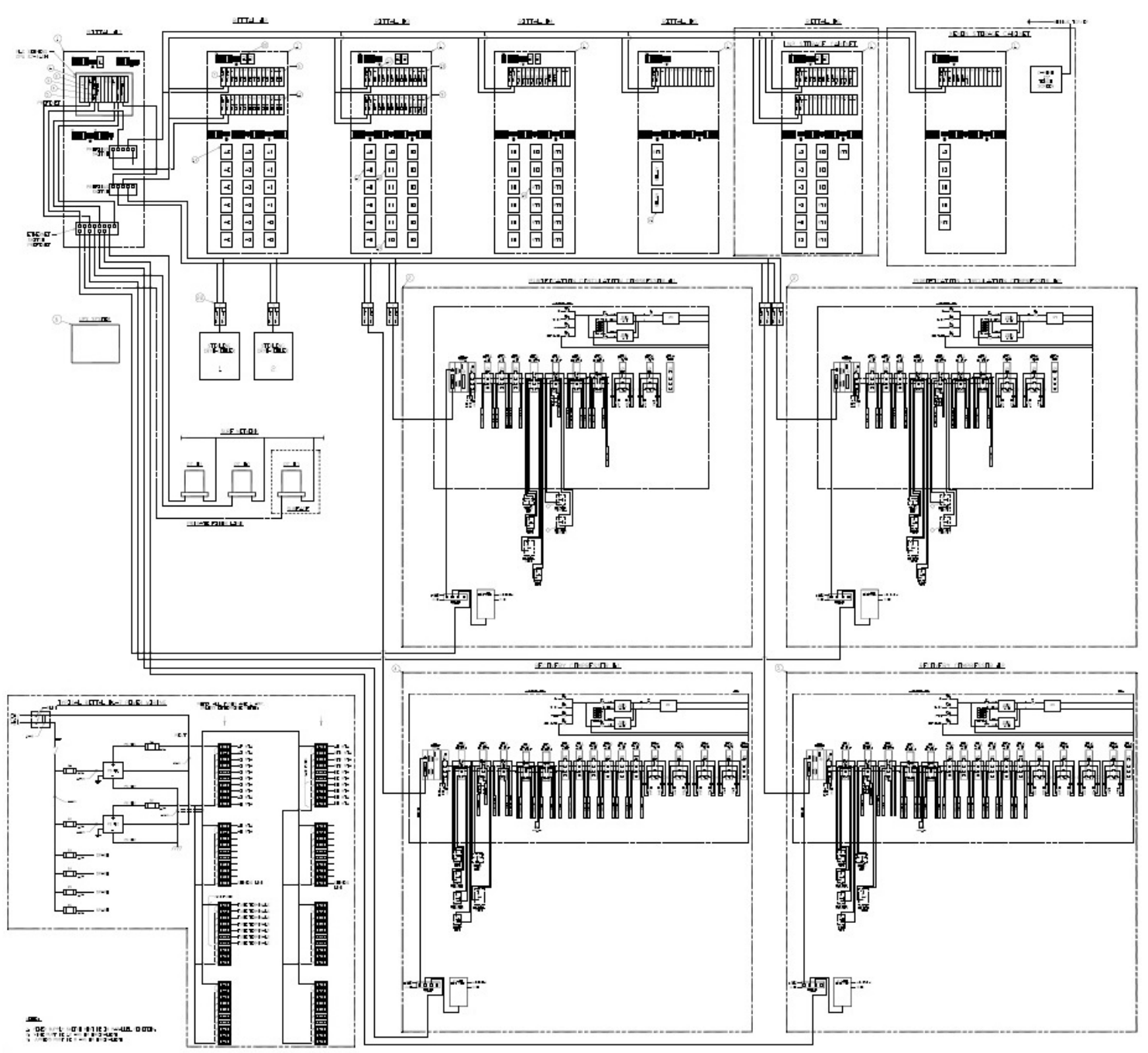

Figure 6.4.11: Block diagram for the PLC System. 
All instruments not directly related to compressor or cryogenerator control will be read-out/controlled-by the S7 PLC. This amounts to an estimated 832 channels of I/O, including 144 thermometers, 192 other Analog Inputs, 80 Analog Outputs, 80 Discrete Inputs, and 336 Discrete Outputs. The S7 logic will handle all interlocks and control loops relating to these instruments, in addition to top-level decision making for the compressor and cryogenerator systems. The S7 PLC will also be responsible for accomplishing xenon recovery in the event of a prolonged power outage (see Section 6.5). The S7 PLC will connect to the LZ Slow Control system via an Ignition Server on the PLC private network (see Section 8.8). The PLC network will receive power from the PLC UPS system and will have a direct, redundant fiber link to a backup server in the surface facility, ensuring monitoring and control capability in the event of power and network failures (see Section 8.12).

Each compressor skid will have a dedicated Beckhoff CX8031 PLC that will handle interlocks protecting that compressor, automation of compressor start-up and shut-down, and control loops to regulate flow rate and pressure at the compressor inlet. The Beckhoff PLCs on the recovery compressors will have the additional ability to independently initiate emergency xenon recovery if the TPC pressure exceeds a set threshold (See 6.5). All variables in the Beckhoff PLC logic will be exposed to the Siemens S7 via Profibus, and in normal operations the Beckhoff PLCs will receive high-level commands (start/load/unload/stop) from the S7 over the Profibus connection. In this way the S7 also serves as the gateway between the compressor PLCs and the LZ Slow Control system. A touch-screen human-machine-interface (HMI) on each skid will allow each compressor to be operated as an independent unit when disconnected Siemens S7.

The liquid nitrogen generation system will have a vendor-supplied Siemens S7-300 Series PLC with local HMI to control the Stirling cryogenerators. As with the compressor PLC's, all variables in the PLC logic will be exposed to the main Siemens PLC via Profibus, providing the same monitoring and controls as are available through the vendor-supplied HMI.

\subsection{Xenon Recovery}

The Xe recovery system removes the Xe from the detector and returns it to the storage cylinder packs. There is also a regulator-based Xe delivery function in the opposing direction to support detector cooldown and condensing.

The xenon recovery system must support three functions: 1) Normal recovery, such as a planned full recovery at the end of the experiment or for small operational adjustments of xenon quantity in the system, 2) Emergency recovery, where the xenon is automatically removed from the system and safely stored during an unexpected sustained pressure rise or extended power failure, and 3) Venting xenon as a last resort to provide detector $\mathrm{MAWP}^{7}$ pressure protection. With the exception of function 3 , in all scenarios the xenon asset must be retained and its purity kept intact. The system also offers online cryopumping and cryogenic buffer volume that can absorb small pressure spikes, xenon feed and bleed to various points throughout the system, and redistribution of trace xenon.

At the heart of the recovery system is a pair of recovery compressor skids. The skids are redundant replicates, each one containing all the hardware necessary for autonomous recovery, including valves, sensors and interlocks, Beckhoff PLC controller, and the compressor itself. Both compressors are commanded to run when activated in an emergency. Compressor suction is connected directly to the detector ullage, isolated by a pneumatic shutoff valve to allow for independent compressor startup routines. A discharge-to-suction proportional bypass valve provides variable flow, using compressor suction pressure as feedback. Once operating, the compressor is controlled to maintain constant suction pressure - this way the compressors only transfer the xenon being fed to them. The compressors themselves are all-metal triple-diaphragm two-stage

\footnotetext{
${ }^{7}$ Max Allowable Working pressure
} 


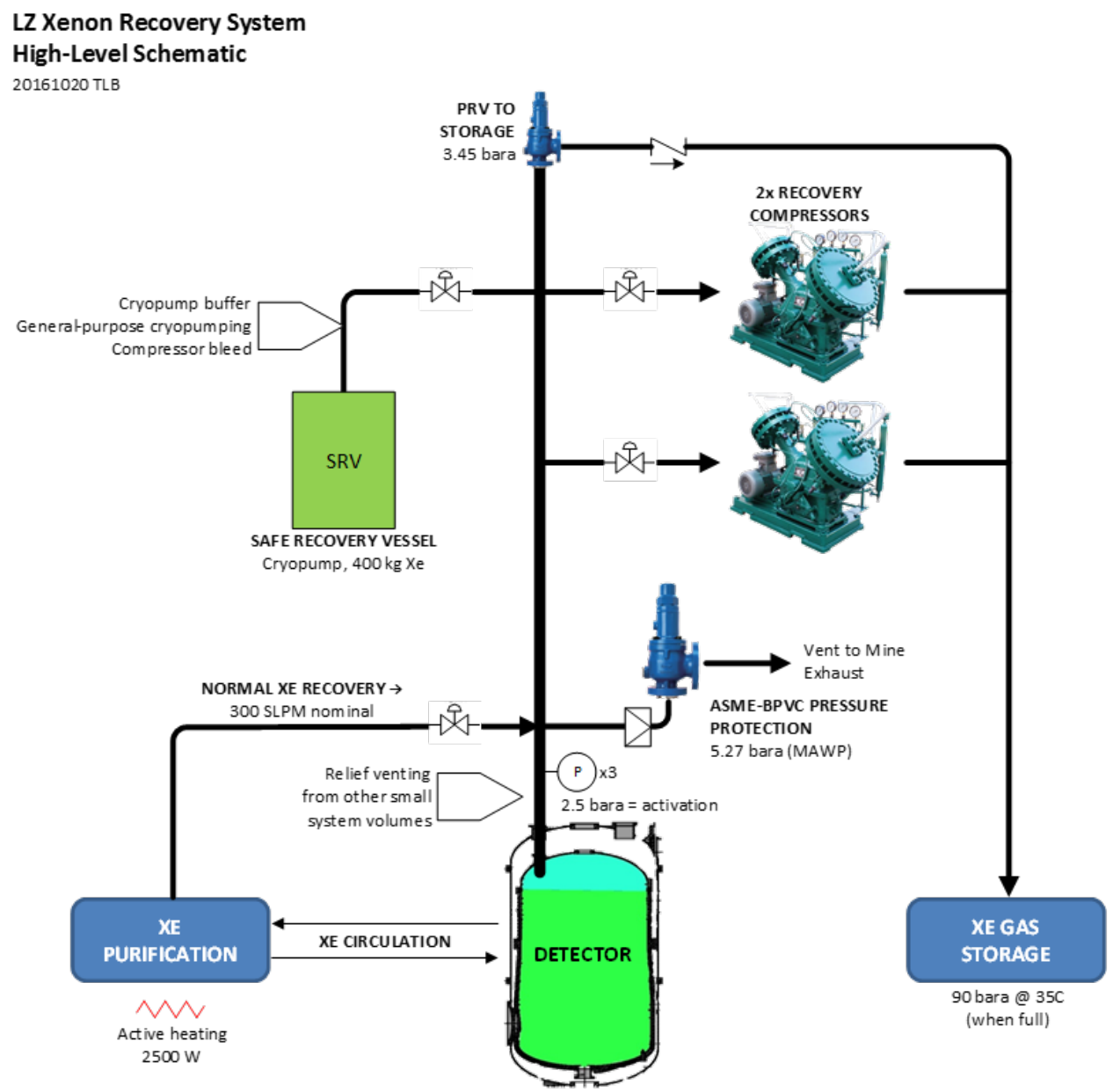

Figure 6.5.1: Simplified flow schematic of LZ Xe Recovery system.

compressors, each rated for $350 \mathrm{slpm}$ at 2.5 bara suction pressure (automatic recovery activation pressure), and 100 bara discharge. A simplified flow diagram of the recovery system is shown in Figure 6.5.1.

All pressure relief mechanisms throughout the xenon handling system are vented into the detector ullage, which acts as a gaseous buffer. Here the vented gas either condenses in the detector or can be stored/removed by the recovery system. A set of cryo vessels tap off the main delivery and recovery gas trunks. The larger vessel is the SRV (Safe Recovery Vessel) repurposed from LUX, and has a xenon capacity of $400 \mathrm{~kg}$ and maximum pressure rating of 83 bara (1190 psig). This vessel is nominally maintained cold at xenon ice vapor pressure, $1.0 \times 10^{-3} \mathrm{mbar}$, allowing it to act as an online cryopump buffer to absorb small pressure spikes within the detector and also bleeding of xenon from various points throughout the xenon handling system. A smaller cryopump consists of two $4 \mathrm{~L}$ research bottles with a combined xenon capacity of $15 \mathrm{~kg}$, and can be used in conjunction with the SRV to redistribute trace xenon by way of volume sharing.

Automatic emergency xenon recovery is a layered approach and illustrated in Figure 6.5.2. The automatic recovery trigger is keyed to the detector ullage pressure, which is monitored by three pressure transducers. All three communicate to the main Siemens PLC, two of them via the dedicated compressor skid Beckhoff PLCs. Detector operating range is 1.6 to 2.2 bara. A set of alerts are issued from the control system once detector pressure reaches 2.2 bara. At 2.5 bara, and a 2/3 vote between the three pressure transducers, automatic recovery is activated. If the pressure continues to rise to 2.8 bara, each Beckhoff controller assumes 


\section{LZ INNER CRYOSTAT VESSEL (ICV) PRESSURES and SAFETIES}

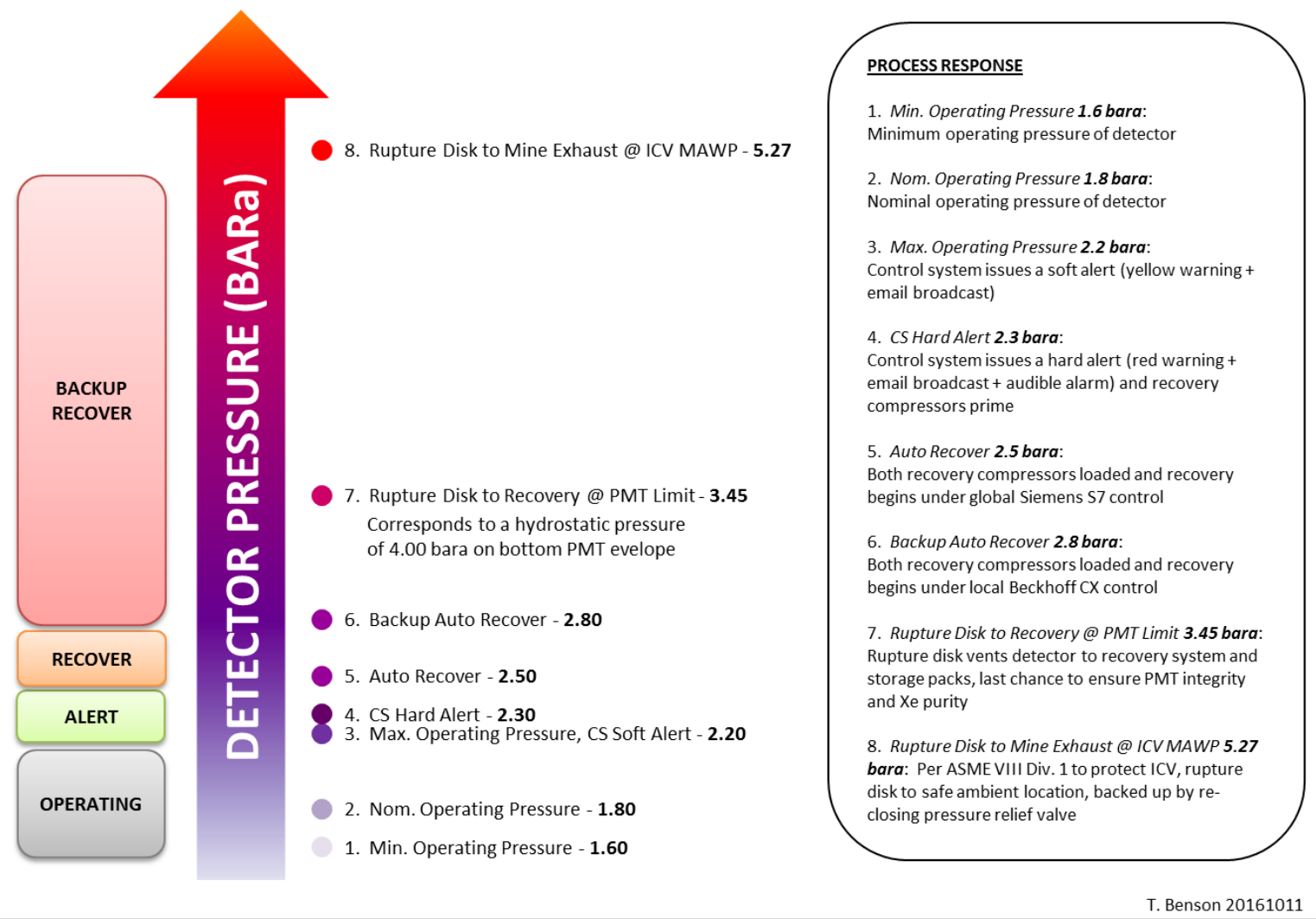

Figure 6.5.2: Xe system pressure threshold plot.

the main PLC has failed to initiate recovery and commands its own skid to begin recovering autonomously. At 3.45 bara the bottom PMTs are at risk of failure from hydrostatic pressure. At this point it is assumed the compressors are not functioning, or not functioning fast enough, and a pressure relief valve vents the detector to the evacuated storage cylinders in hopes that this is a transient spike and not a continuous rise in pressure. Finally, as a last resort to protect the cryostat against rupture, a rupture disk vents xenon to mine exhaust at 5.27 bara (detector MAWP). This burst disk is backed up by a re-closing pressure relief valve so all remaining xenon can be retained after the pressure event is resolved. In this case some xenon is lost, the system purity is compromised, and some PMTs have possibly mechanically failed.

See Figure 6.5.3 for P\&ID details of how the recovery system elements and detector tie together. The full P\&ID for the core system is shown in Figure 6.5.4.

An analysis of various failure scenarios drives the recovery system design and sizes the compressors. Specifically, our worst case scenarios would be an air or water breach of the vacuum jacket insulating the detector and LXe transfer lines, an air-breach being the more probable of the two. We first address these failures by specifying a layer of foam insulation to be applied to the outside of the inner cryostat vessel wall and bottom head (all vessel surfaces wetted by liquid xenon on the other side). The insulation is specified to have a maximum heat transfer coefficient $(\mathrm{k} / \mathrm{t})$ of $3 \mathrm{~W} / \mathrm{m} 2$ and $1.5 \mathrm{~W} / \mathrm{m} 2$ for the wall and bottom, respectively. Second, we include vacuum breaks on the lower transfer lines to isolate such a failure and minimize the effected heat transfer surface area. 


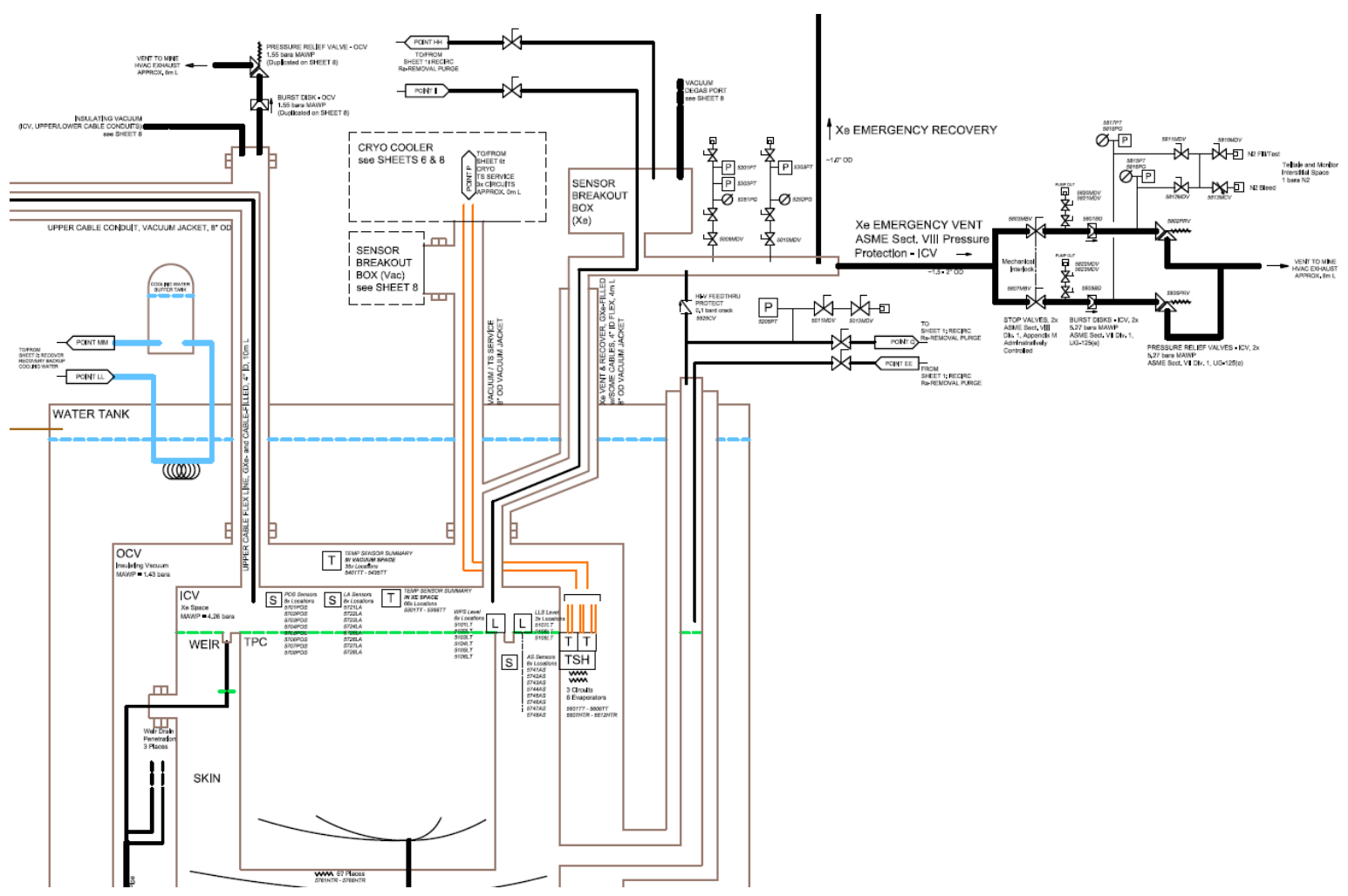

Figure 6.5.3: P\&ID detail of how the recovery system ties into the detector. The main recovery path out of the detector is via a 4 inch ID bellows containing a small bundle of sensor cables. This path is instrumented with redundant pressure transducers, and both the main recovery line and the ASME ICV pressure protection tap into this line.

With the above design provisions in place, a conservative dynamic model was developed to estimate heat loads for the air and water breach cases. From these, initial boiloff rates are in the range of $400 \mathrm{slpm}$ and 2,500 slpm for the air and water cases, respectively. Boiloff tapers as the LXe level in the detector drops, and significantly tapers in the water case as a layer of ice forms and grows. Then, by looking at the detector pressure response versus time for various sizes of compressors, and making sure it always stays below safe limits, we are able to choose an optimal compressor size. Note that compressor throughput is a strong function of suction pressure, so at higher suction pressure the compressors will move more gas, and vice versa. On a first-order this means the compressors self-regulate to match boiloff, and detector pressure is a function of how much xenon is being removed, or in other words what size the compressors are. Physical size is also a factor since underground space is extremely limited. From this analysis, one $350 \mathrm{slpm}$ compressor (at 2.5 bara suction pressure) can handle the more probable air-breach case, and two of these same compressors running in parallel can handle the water-breach case. Since the analysis is conservative, these compressors will be slightly over-sized for the application, which makes it important to have variable flow capability by way of the proportional bypass valves on the compressor skids.

Normal recovery is driven by electric heaters that actively heat and boil the xenon in a controlled manner. These heaters are located in the bottom of the LXe Tower on the LXe supply lines leading to the detector (Figure 6.4.6), and are sized to deliver a combined 2,500 W to boil off xenon at a rate of $300 \mathrm{slpm}$. Once normal recovery is initiated, main circulation stops and the recovery heaters begin boiling off xenon at the bottom of the LXe Tower. Flow through the supply lines is reversed from normal operations: LXe is draining out of the detector and flowing towards the heaters while xenon boiloff travels up through the subcooler and 


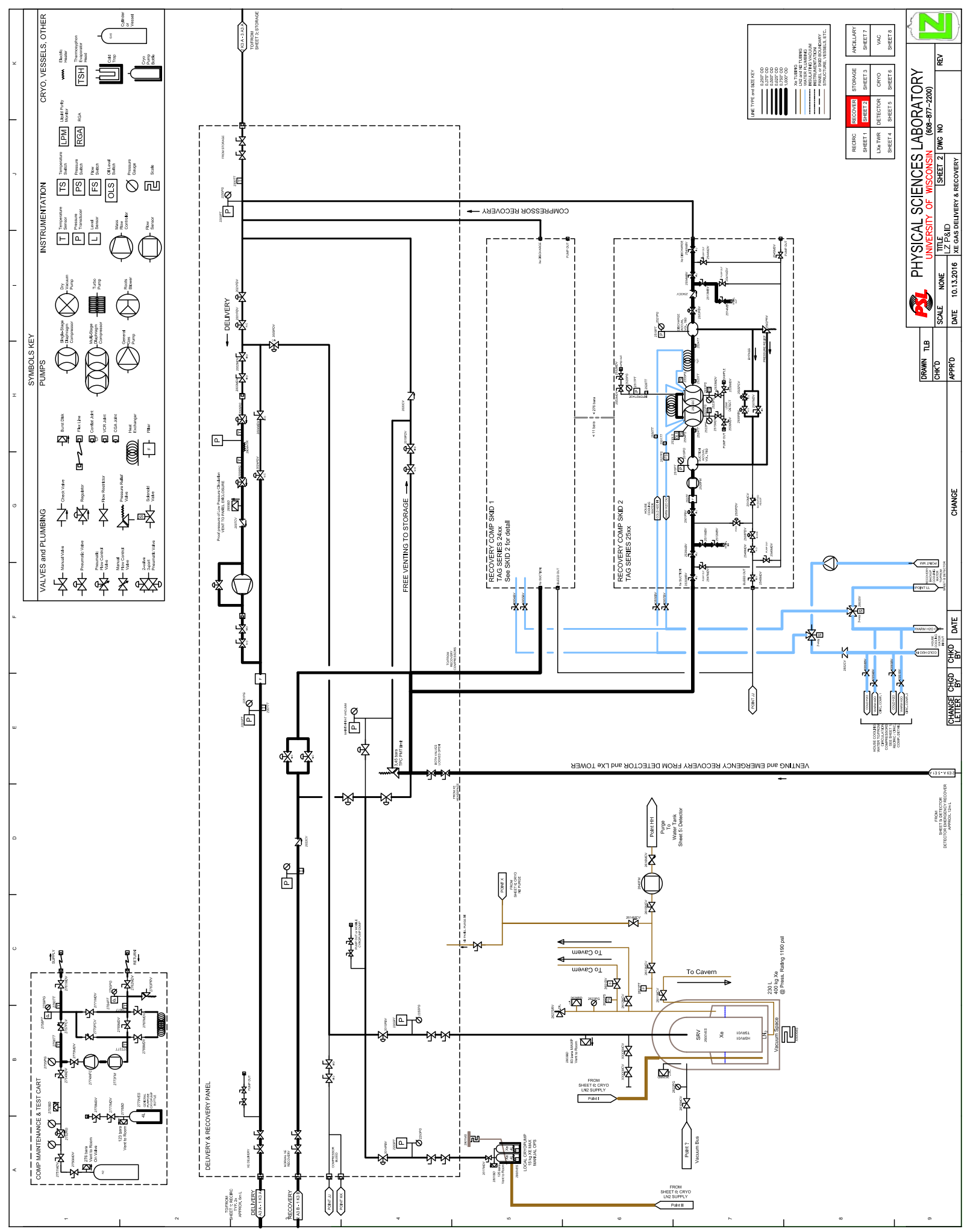

Figure 6.5.4: Xe Delivery \& Recovery core system P\&ID. 
out a gas extraction line. This extraction line plugs into the input of the gas recirculation loop. From there, the xenon can be routed directly to the recovery compressor inputs, or can first be pushed through the getter one last time before storage (this requires the circulation compressors to be operating to overcome the getter's impedance). Again, the recovery compressors operate on constant suction pressure control, and as more gas is liberated into the gas plumbing the recovery compressors transfer it out. Assuming a constant 300 slpm removal rate, full recovery would take approximately 4.2 days.

There are also design provisions for a more passive recovery strategy. Here, a low-elevation cold-to-warm liquid tap at the bottom of the LXe Tower is normally blocked off by redundant shutoff valves. Upstream of the valves (but at lower elevation) there is a room temperature heat exchanger. With this approach, LXe drains out of the detector and into the heat exchanger where it is boiled off. The boiloff gas is routed back into the detector ullage, where it both recondenses onto the liquid surface and helps raise the bulk xenon temperature over time, and raises detector pressure as gas builds up. The pressure buildup will eventually trigger the emergency recovery routine. This backup approach may be helpful to supplement and/or kickstart a full xenon recovery if there is a need to recover faster.

Two underground generators provide backup emergency power to the experiment: 1) A SURF $300 \mathrm{~kW}$ generator, and 2) An LZ $40 \mathrm{~kW}$ generator. Within one minute of a power outage, the large generator automatically starts and sustains ventilation and critical experimental operations in the Davis Campus. At this time, non-critical LZ loads are systematically shed while critical loads are maintained, including backup cryogenic cooling and any functions relating to xenon recovery. The large generator is coupled with a 1,000 gallon fuel tank and is able to sustain ventilation and critical experiment loads for approximately 3 days. During this time, a high-level decision can be made whether to initiate a full xenon recovery or wait. If a decision is not made, or if outside communications have been severed, the recovery system will automatically initiate a full recovery after a predetermined amount of time after the power loss. This is to ensure the xenon is safely stored before there is no fuel left to do so. When a low fuel condition is detected on the large generator, and power has still not been restored, the smaller LZ generator (coupled with a 270 gallon fuel tank) begins warming up. The large generator then runs out of fuel, and within 10-20 ms an automatic transfer switch transfers the load to the LZ generator. The recovery system plans ahead for the switchover by briefly shutting down the compressors and then restarting after the switch is complete. On the other hand, the control system is carried through all switchovers by their UPS units. Assuming all recovery loads are active while the LZ generator is running, and that it started with a full 270 gallons of fuel, it will continue running for approximately 4 days. The combined run time of both generators is 7 days, and it takes 4 days for a normal full recovery.

\subsection{Long-term xenon storage and transportation}

The LZ Xe will be sourced from multiple gas suppliers (Section 6.9), shipped to SLAC for krypton removal (Section 6.3), shipped to SURF to be moved underground, and finally liquefied into the LZ detector. Vendorgrade Xe arrives at SLAC in vendor-supplied gas cylinders. Once krypton has been removed, the Xe is stored and transported in special high-integrity gas cylinder packs specifically engineered for $\mathrm{LZ}$. The Xe storage, transport, and transfer must be done without any loss of the high-asset xenon, and must also meet or exceed the overall storage inbound leak rate requirement of $1.15 \times 10^{-6} \mathrm{mbar} 1 / \mathrm{s}$ (helium) as specified in Section 6.2.1.

The LZ cylinder packs conform to DOT 49 CFR Part 178, Subpart C, Specification for Cylinders. Base packs will be purchased as turnkey items from Praxair, with modification and final integration and testing occurring at the University of Wisconsin's Physical Sciences Laboratory (UW-PSL). There will be 12 gas cylinder packs available to contain the full 10 tonnes of Xe for LZ (see Figure 6.6.1 for some features of the LZ cylinder pack design). Each pack contains 12 DOT-3AA-2400 49.1 liter cylinders, each having a 


\section{LZXENONSTORAGE PACK}

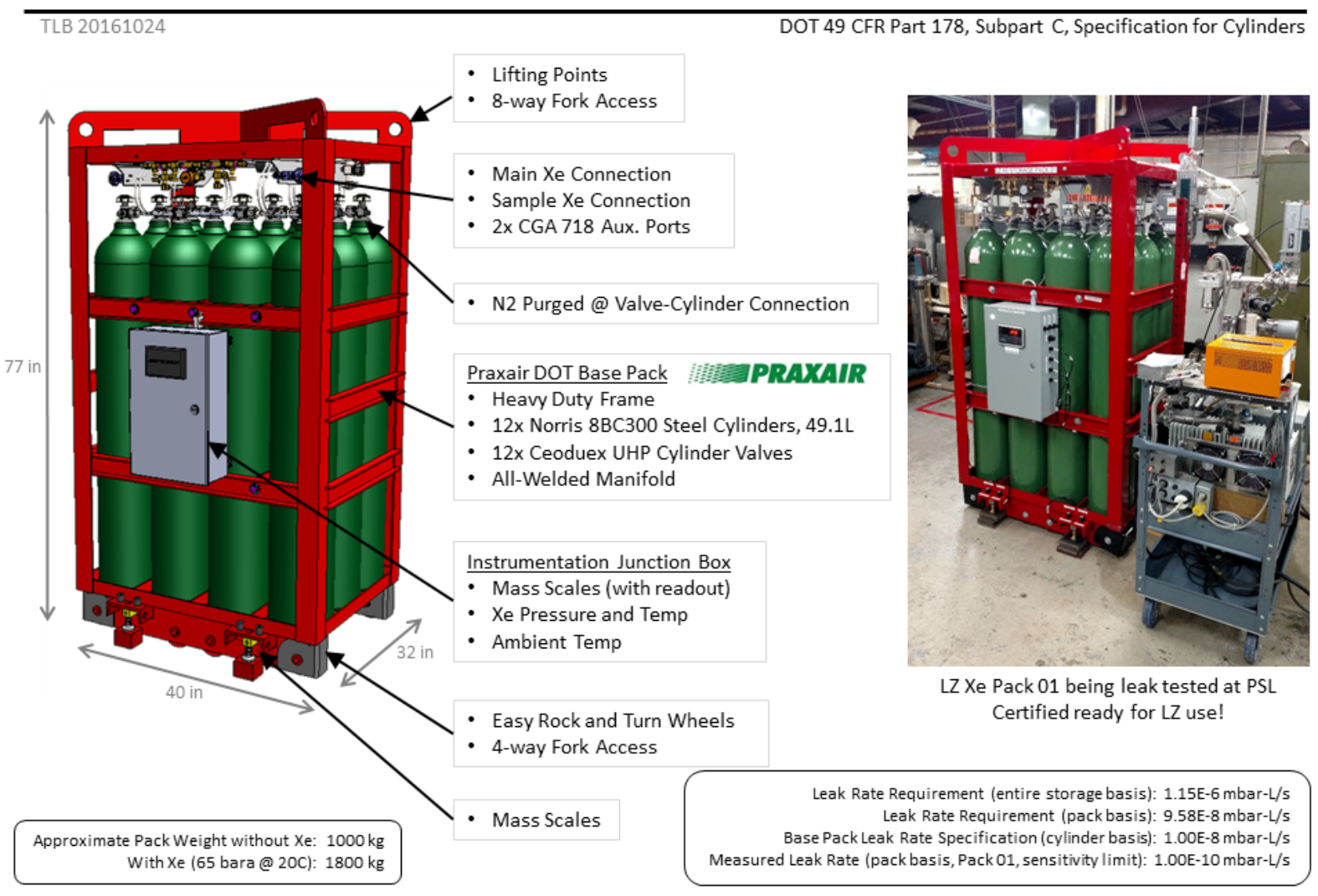

Figure 6.6.1: Overview of LZ cylinder gas pack.

working pressure of 166 bara (2,400 psig). The full quantity of Xe can be stored in all 144 cylinders in a supercritical state at a pressure of 65 bara $(928 \mathrm{psig})$ at $20^{\circ} \mathrm{C}$. Each cylinder then contains approximately $69 \mathrm{~kg}(12,700$ standard liters) of Xe and each full pack weighs a total of 1,800 kg. The maximum allowable Xe charge into these cylinders for transport per DOT regulations (pressure must be below 5/4 working pressure at $55^{\circ} \mathrm{C}$ ) is $91 \mathrm{~kg}$ (this would require at minimum 110 cylinders). The 65 bara target storage pressure offers a favorable packing density on the Xe density curve near its critical point $\left(16.6{ }^{\circ} \mathrm{C}, 58\right.$ bara $)$ without placing excessive output pressure requirements on the compressors needed to fill the packs.

The cylinder valves are equipped with a pressure burst disk rated at 277 bara (4,000 psig) per DOT specification. In the event of a fire, with $69 \mathrm{~kg}$ of Xe charge, this pressure would be reached at a temperature of $136{ }^{\circ} \mathrm{C}$. If the burst disk failed to vent, the cylinder burst pressure of 398 bara $(5,760 \mathrm{psig})$ would be reached at a temperature of $202^{\circ} \mathrm{C}$.

Particular attention is given to the seal between the valve and the cylinder. Economics drives us to use a standard NGT (National Gas Taper) thread that is readily available from DOT cylinder manufacturers. This seal can represent the weakest link in the system from a leak-tightness perspective. The requirement that the total leak rake be no more than $1.15 \times 10^{-6} \mathrm{mbar} 1 / \mathrm{s}$ implies that the average inbound leak should be less than $9.6 \times 10^{-8} \mathrm{mbar} 1 / \mathrm{s}$ into each of the 12 packs, and less than $8.0 \times 10^{-9} \mathrm{mbar} 1 / \mathrm{s}$ into each of the 144 cylinders. To achieve this we set a target inbound helium leak rate of $1 \times 10^{-8} \mathrm{mbar} 1 / \mathrm{s}$ for each of the cylinders, while we further reduce $\mathrm{Kr}$ ingress by flushing the volume immediately surrounding the NGT seals with boil-off nitrogen from the cryogenic system. We believe the flushing can reduce the Kr ingress 
rate of $\mathrm{Kr}$ by a factor of 10 over ambient air. During storage we also benefit from a favorable high pressure gradient between the stored Xe and outside ambient conditions, however this effect is not present during operations when the packs are empty. After integrating the base pack into the final LZ design at UW-PSL, a leak check is performed on the entire pack, including the manifold, to certify it ready for LZ use. For these tests, the leak checking system is first calibrated with a calibrated helium leak.

Considerable R\&D has been carried out in collaboration with Praxair's R\&D Division on achieving our target specification. Initially we tested two valve/cylinder pairs, and have since received and tested the first complete LZ storage pack. Initial factory leak rates range from $9.0 \mathrm{e}-8$ to $4.1 \mathrm{e}-9 \mathrm{mbar}-\mathrm{L} / \mathrm{s}$, but then after pumping down for a few days to reduce helium background in the cylinders, measured leak rates drop dramatically. In particular, for the first LZ pack, Storage Pack 01, the helium leak rate was measured to be less than 1.0e-10 mbar-L/s, the sensitivity limit of the leak test equipment. Two valve thread preparation techniques were compared: 1) standard practice of PTFE tape with Krytox applied above the 5th thread, and 2) thin layer of indium plating, 0.002 inch and 0.004 inch thicknesses were both trialed. A metal-metal seal must be achieved with the NGT thread in order to meet LZ's allowable leak rate requirement, and the purpose of the valve thread preparation is to allow full engagement and deformation of the NGT threads without onset of galling. The indium seemingly has the advantage of flowing to fill any gaps with metal, however results of all 14 cylinders ( 2 R\&D cylinders +12 cylinders in first pack) suggest both techniques work, and in fact the PTFE results were slightly better. The most important factors for achieving a good seal is careful thread inspection and gauging prior to installation, and that installation be performed by an experienced technician. All valves for the LZ storage packs will be installed by experts at Praxair, and the remaining valves will be prepared using the standard PTFE tape technique per Praxair's procedures.

We have selected Ceoduex D304 UHP tied diaphragm valves with CGA 718 (DISS) output connection type. The cylinders in a pack are manifolded together with an all-welded stainless steel manifold with VCR xenon connection taps and instrumentation ports for a pressure gauge and transducer. Xe gas line temperature is monitored and also ambient temperature within each pack. The packs are supported by scales that monitor xenon mass to an accuracy of $+/-0.23 \mathrm{~kg}$. Xe temperature and pressure, ambient temperature, and weight of each pack is read out to the PLC system. The pack frame is a rugged steel frame equipped with fork-access both on bottom and top, rigging pick points on top, and retractable wheels.

Transport of the Xe packs will be divided into approximately 3 to 4 separate shipments, both to match the Kr-removal production schedule and to reduce risk of total xenon loss in the event of a serious road accident. The shipments will be insured, and will travel in secured air-ride trucks.

Once at SURF, the packs will be moved underground as soon as possible to limit cosmogenic activation of ${ }^{127} \mathrm{Xe}$. The packs will be loaded on a small rail car in the Yates headhouse, loaded into the cage, lowered to the Davis Campus level, and rolled into the Davis Campus on a rail car. Part of the Davis Campus infrastructure work is to prepare a Xe Storage Room for the Xe using an existing excavation in the LN storage room access drift, as shown in Figure 6.6.2. In order to maintain clearance beneath the HVAC and sprinkler utilities in the access drift, the cylinder packs may need to travel horizontally. In this case, a specially-designed pack rigging fixture will assist in the rotation and handling of each pack from the Yates headhouse to the underground Xe Storage Room. We are investigating an alternative and preferred option to instead modify the utility services so that the packs may travel in their upright configuration. The Xe Storage Room will be sealed from the access drift with a block wall and secured double doorway.

The Xe Storage Room will have a boil-off nitrogen supply to purge the valve seal volumes, a sprinkler system to control temperature during a fire (activates at $68^{\circ} \mathrm{C}$ ), and an emergency ventilation and oxygen monitoring system in case of an accident. Supply and return plumbing from the Xe storage area will connect the packs to the LZ detector plumbing via a gas panel equipped with a sampling/pump-out bus and local cryopump (15 kg Xe capacity) for consolidating xenon locally. Xe Storage Room controls are supported by a local PLC control rack on UPS and backup power. Portable provisions for clean sampling of various 


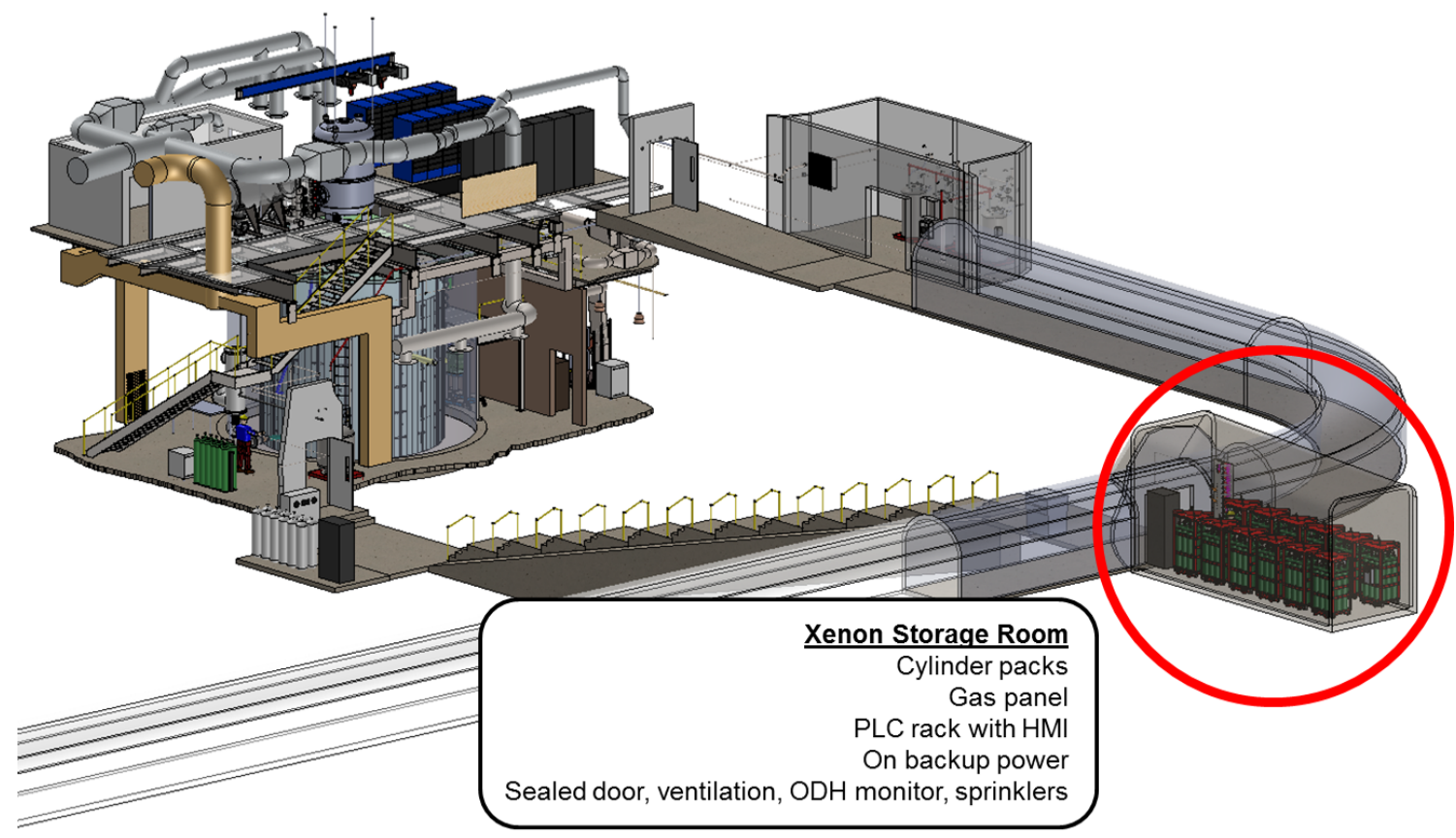

Figure 6.6.2: Location of the Xe Storage Room in the Davis campus, along the access drift to the LN2 Storage Room.

locations within the Storage Room will also be provided. A complete P\&ID of the integrated Xe storage plumbing is provided in Figure 6.6.3.

Once the Xe has been fully transferred into the LZ detector and Xe handling system, any xenon remaining will be consolidated to a single pack and the remaining packs will be maintained under vacuum and ready to accept incoming $\mathrm{Xe}$ from any recovery action.

\subsection{Xenon Sampling and Assay}

Sensitive Xe purity monitoring is integrated into the Xe handling plan. The basic monitoring methodology that we employ is the coldtrap/mass-spectrometry method developed for LUX and EXO-200 [6, 14]. The LUX experience is reviewed in Ref. [15]. In this section we describe the LZ implementation.

The method works as follows. A gaseous Xe sample flows through a precision vacuum leak valve or MFC to an RGA, where the partial pressures of the various species are measured. Once a uniform flow is established, these partial pressures are proportional both to the abundances in the sample and to the gas flow rate, and inversely proportional to the volumetric pumping speed of the vacuum system at the RGA. The partial pressures can be interpreted as absolute concentrations by calibrating the measurement under specified flow and pumping conditions with Xe gas samples prepared with known impurity content.

Good sensitivity may be achieved by maximizing the gas flow rate and minimizing the pumping speed, however the total pressure cannot exceed about $10^{-5}$ torr if the RGA and its electron multiplier are to function well. Without additional measures the Xe partial pressure would severely limit the flow rate, so the Xe is selectively removed by passing the gas sample through an LN coldtrap where it forms Xe ice. Under these conditions the Xe partial pressure at the coldtrap outlet is held at $1.8 \times 10^{-3}$ torr, the vapor pressure of Xe ice at $77 \mathrm{~K}$. This is still too high by a factor of 180 , however the Xe pressure may be further reduced at 


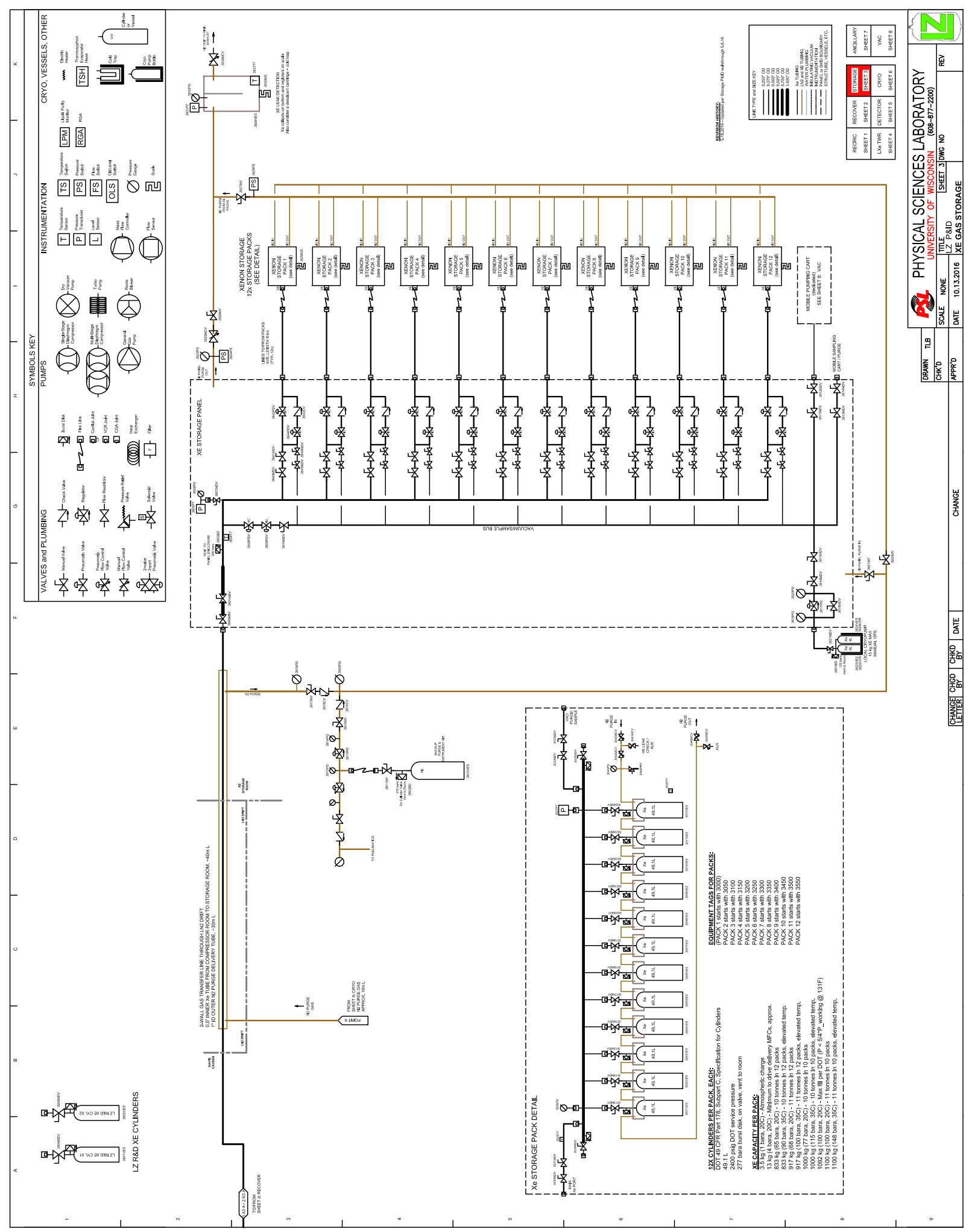

Figure 6.6.3: Xe Storage system P\&ID. 
the RGA by inserting an appropriate impedance into the plumbing. In the presence of the coldtrap the Xe partial pressure at the RGA does not depend on the gas flow rate into the trap.

Some impurity species (such as water) are removed from the gas stream by the coldtrap and are thus unobservable at the RGA. However, many species of interest pass through the trap with good efficiency, including $\mathrm{O}_{2}, \mathrm{~N}_{2}, \mathrm{CH}_{4}, \mathrm{Ar}, \mathrm{He}$, and, most importantly, $\mathrm{Kr}$. Studies have shown that the partial pressures of these species continue to be a good measure of their concentrations as long as an appropriate calibration is done at a similar flow rate and vacuum pumping speed.

In contrast to $\mathrm{Xe}$, the partial pressure of $\mathrm{Kr}$ at the RGA is found to be much less sensitive to the vacuum impedance between the coldtrap outlet and the RGA. It decreases only moderately as the impedance increases, allowing Xe to be selectively suppressed relative to Kr. The origin of this unusual behavior is that the Xe signal is a pressure source created by the Xe ice in the coldtrap, while the $\mathrm{Kr}$ signal is a current source created by the gas flow into the coldtrap.

This distinction in the nature of the $\mathrm{Xe}$ and $\mathrm{Kr}$ signals is advantageous because $\mathrm{Xe}$ dominates the total pressure and thereby limits how low the pumping speed may be set without exceeding the RGA's pressure limit. After Xe suppression, however, the pumping speed may be further reduced, increasing the partial pressures of all species, including $\mathrm{Kr}$ and $\mathrm{Xe}$, until $\mathrm{Xe}$ once again reaches the RGA's practical limit of $10^{-5}$ torr. In practice, vacuum parameters such as the impedance and pumping speed are not modified during a run but are instead chosen for optimal sensitivity in prior test runs. The resulting system configuration may be calibrated as usual with specially prepared Xe gas samples with known krypton content.

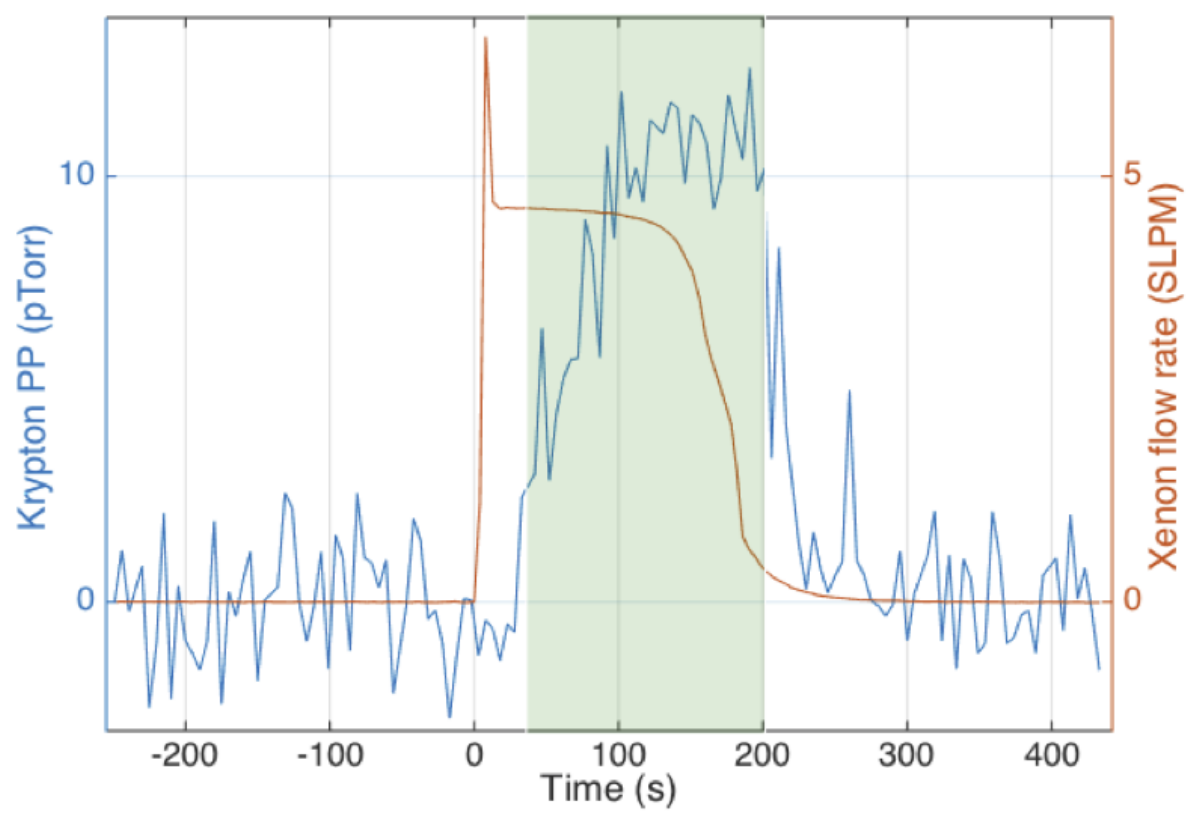

Figure 6.7.1: Blue: $\mathrm{Kr}$ partial pressure trace at a concentration of $0.34 \mathrm{ppt}(\mathrm{Kr} / \mathrm{Xe})(\mathrm{g} / \mathrm{g})$ (left vertical axis). Orange: Flow rate into the cold trap in (slpm, right vertical axis). The green shaded region is the signal integration window.

Empirical studies have shown that the efficiency to pass $\mathrm{Kr}$ through the coldtrap depends upon its tubing diameter, with smaller diameter tubing generally performing better. Another important factor is the total amount of Xe ice that can be collected before the flow path becomes blocked. Larger ice capacity implies 
that a larger flow rate may be sustained for the same time period, further enhancing the Kr signal. For LZ we have adopted a 0.5 inch OD tube as a baseline compromise between these competing needs.

To improve the $\mathrm{Kr}$ sensitivity of the method beyond the $0.2 \mathrm{ppt}$ achieved for the LUX Kr removal campaign, we have increased the mass flow rate by a factor of four from $\sim 1.5 \mathrm{slpm}$ to $6.5 \mathrm{slpm}$ and decreased the pumping speed by a factor of five from $\sim 5$ to 1 liters/sec. We've also implemented an MFC for better programmable flow control. Results are presented in Figure 6.7.1, where a prominent Kr partial pressure signal is shown for a $\mathrm{Kr}$ concentration of $0.34 \mathrm{ppt}(\mathrm{Kr} / \mathrm{Xe})(\mathrm{g} / \mathrm{g})$. Studies of the sensitivity of the method, performed with $\mathrm{Kr}$-free xenon gas samples, have found that the limit of detection is $0.007 \mathrm{ppt}(\mathrm{g} / \mathrm{g})(\mathrm{Kr} / \mathrm{Xe})$ at $90 \%$ C.L.

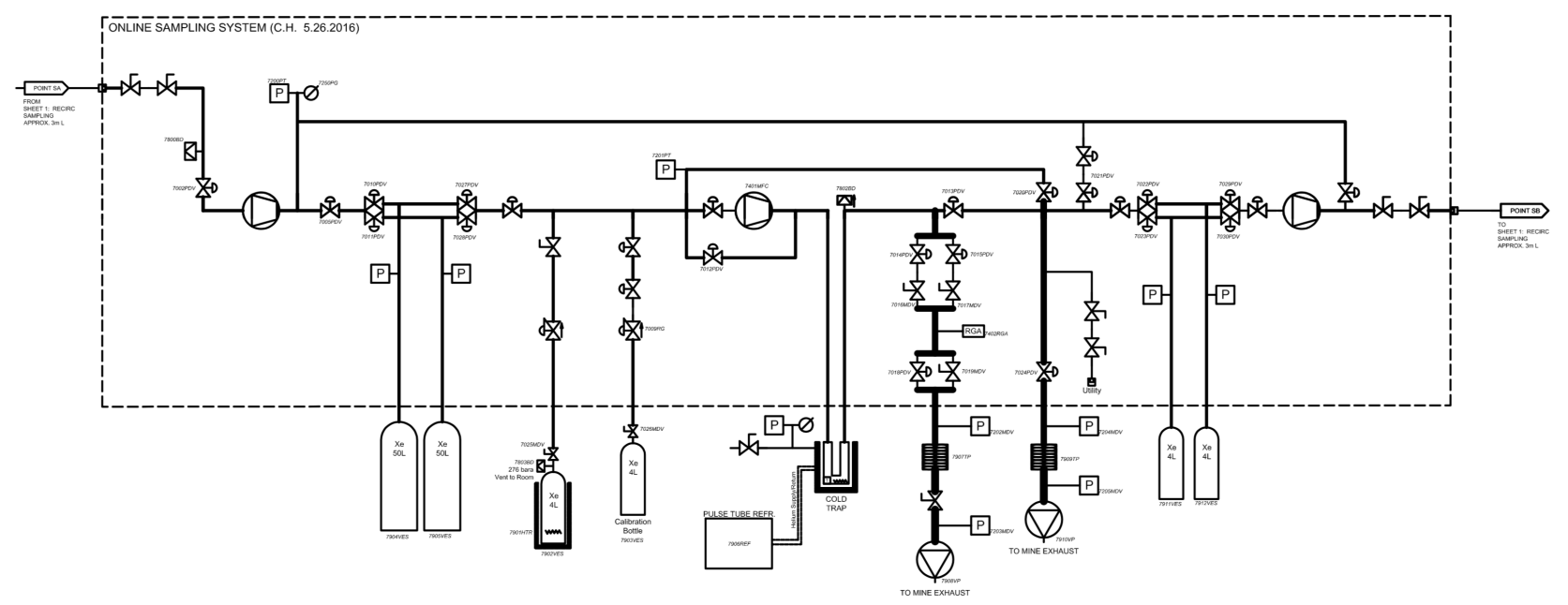

Figure 6.7.2: P\&ID of the LZ online Xe sampling system.

An analytical system incorporating these design features has been constructed at SLAC to aid the $\mathrm{Kr}$ removal campaign described in Section 6.3, and to perform quality assurance on the vendor-supplied xenon as it is acquired. The SLAC system is currently integrated into the R\&D chromatography system, and will be re-purposed for use in the production system in 2018.

At the conclusion of krypton removal operations, the SLAC sampling system will be shipped to SURF and be permanently integrated into the LZ xenon circulation system at the Davis campus. A P\&ID of this system is shown in Figure 6.7.2. To simplify continuous operations at SURF, the coldtrap will be cooled by a pulse-tube refrigerator as shown in Figure 6.7.3, and accumulation bottles on the input and output will allow xenon gas to flow continuously through the system. The LUX sampling system will also be re-built and re-purposed at SURF as a mobile utility sampling system for use during detector commissioning and operations.

\subsection{Cryogenics, vacuum services, and breakout boxes}

This section describes the design of the liquid nitrogen cryogenic systems. We also describe here the vacuum pumping systems and the breakout-box feedthroughs for the internal PMT and instrumentation cables.

Cooling power to maintain $\mathrm{Xe}$ in the liquid phase is provided by a cryogenic system that distributes nitrogen in gas and liquid phases. By utilizing the approximate $100 \mathrm{~K}$ temperature difference between the nitrogen and Xe evaporation temperatures, sufficient gradient exists to provide for thermal control and 

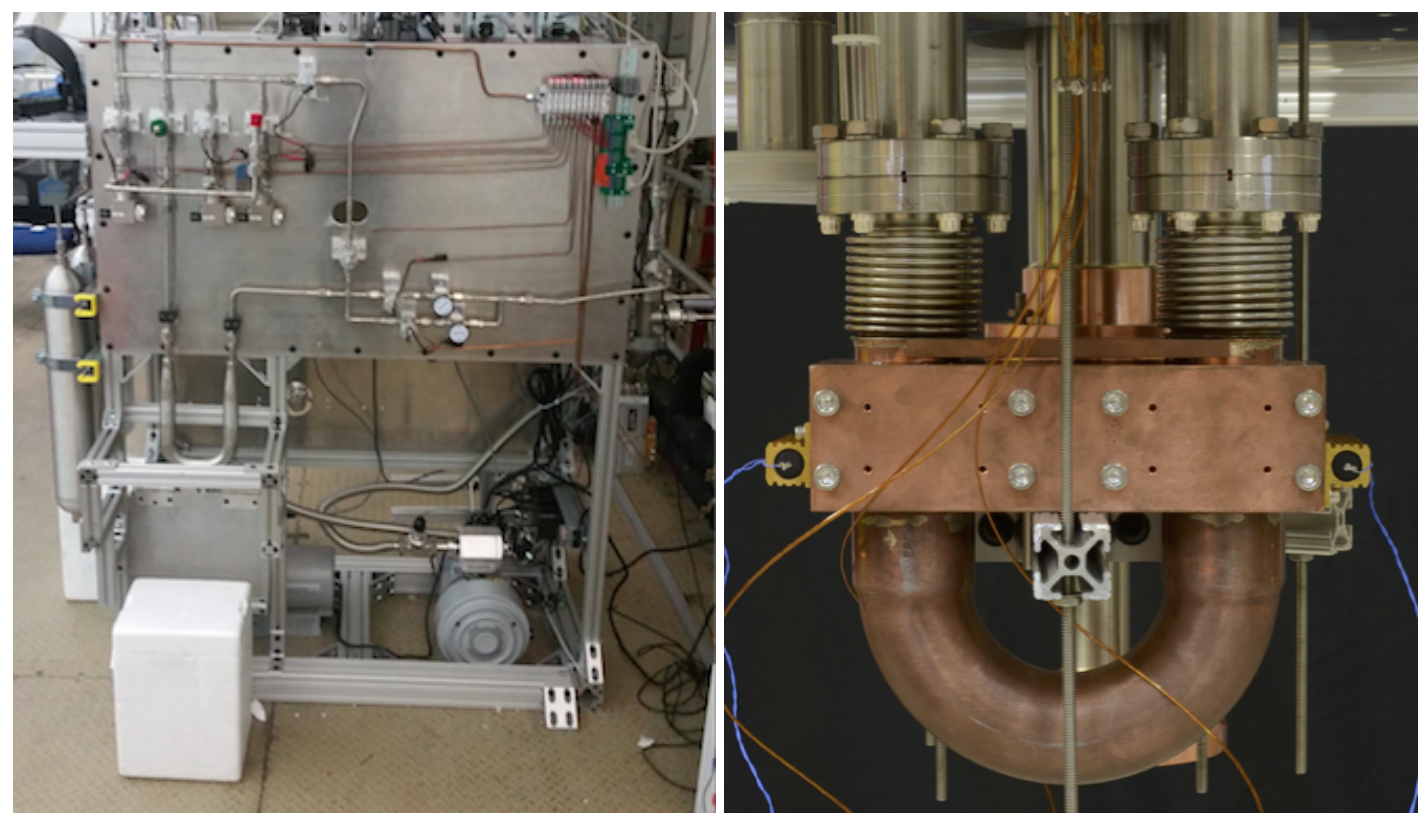

Figure 6.7.3: Left: Xe sampling system constructed at SLAC in 2015 for monitoring $\mathrm{Kr}$ removal activities and screening the commercially procured Xe stockpile. Right: Prototype copper coldtrap with temperature control provided by a pulse tube refrigerator coldhead.

temperature modulation. Existing infrastructure from LUX, including 450-liter LN storage tanks; vacuumjacketed (VJ) pipe; and miscellaneous valves, sensors, and fittings are modified and re-utilized to provide a front end to the primary cooling equipment for the experiment while providing supplemental LN storage. During operations, distribution of $\mathrm{LN}$ is from a VJ central 750-liter distribution tank that is co-located with a Stirling cycle cryocooler. The cryocooler liquefies cold evaporated nitrogen gas in a closed-loop cycle operating at near atmospheric pressure. Multiple thermosyphon heat pipes, cooled by the LN in the storage tank, are used as heat sinks to provide for heat removal from the Xenon system. Primary heat removal locations are the detector, high-voltage feedthrough, and the LXe tower. The underground installation is shown in Figure 6.8.1

Exterior to the Davis Cavern is an LN storage room that contains four 450-liter LN storage tanks installed on mass scales that monitor LN consumption. A commercially purchased vacuum-jacketed (VJ) piping system that has a complete implementation of control valves, relief valves, and pressure-monitoring equipment connects the tanks to equipment in the Davis Cavern. Separate small-diameter tubing connected to the storage tanks distributes boil-off nitrogen purge gas for the freeboard of the water tank, the water-purification system vacuum pump, the scintillator reservoir, radioactive source deployment systems, and Xe equipment protected from radon leakage. The four storage tanks are required to provide an initial liquid volume for startup of the cryocooler, meet high load transient conditions, and provide backup to the cryocooler until a second cryocooler procured at a future date. Alterations to the VJ piping system are required to allow for rerouting of LN to new destinations in the Cavern that will differ from the LUX LN use locations. The railmounted 1,100-liter LN storage tank utilized by LUX will provide for the resupply of LN from the surface during brief cryocooler operation interruptions and periodic replenishment of consumed purge gas.

A cryocooler based on the Stirling thermal cycle is selected for cooling of LZ. It is depicted schematically in Figure 6.8.2. Included in the cryocooler design is a cryogenerator, a work platform sized for two cryogenerators, a 750-liter LN storage tank to provide a distribution reservoir, and a complete monitoring and control system. The design of the cryocooler is closed cycle. Nitrogen inside the storage tank is re-liquefied 


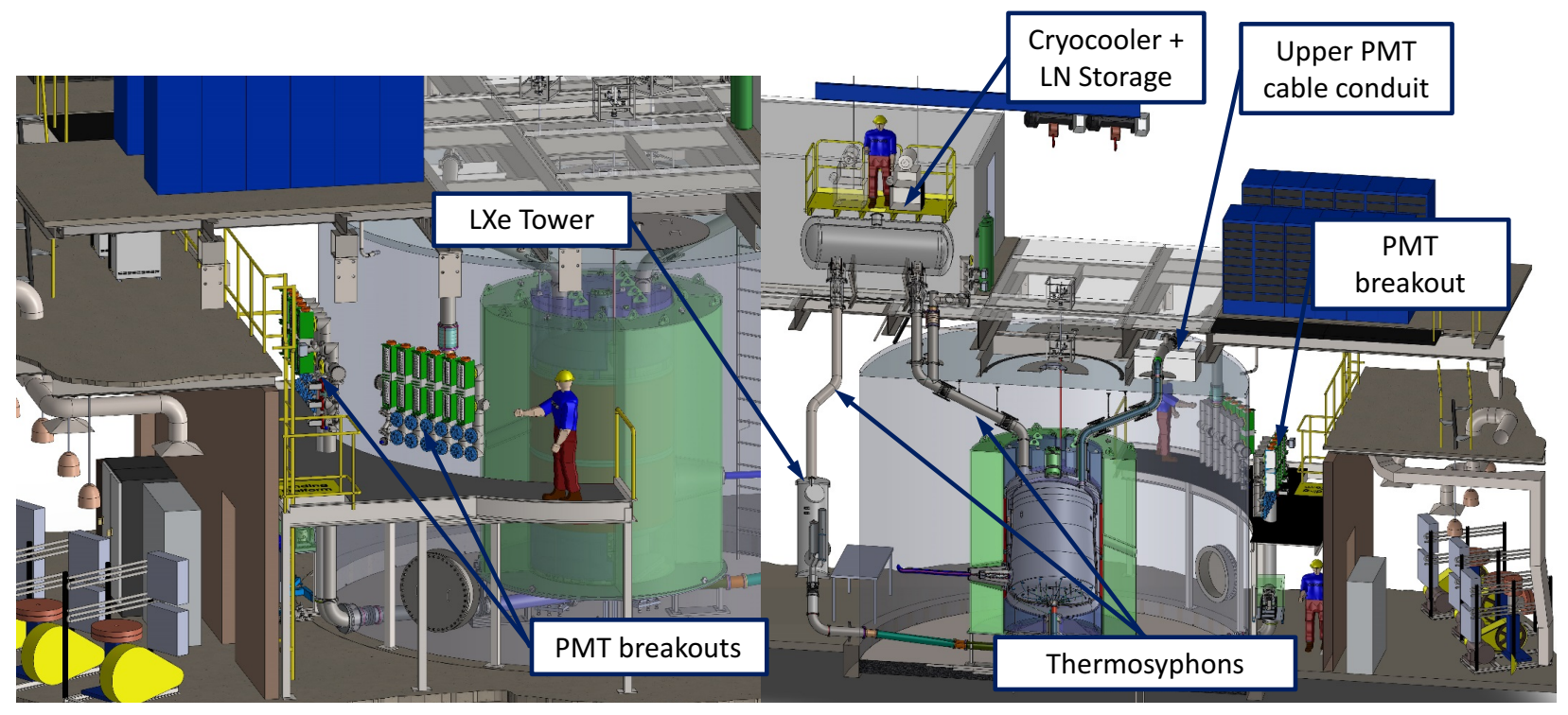

Figure 6.8.1: Cryogenics installation in the Davis Cavern.

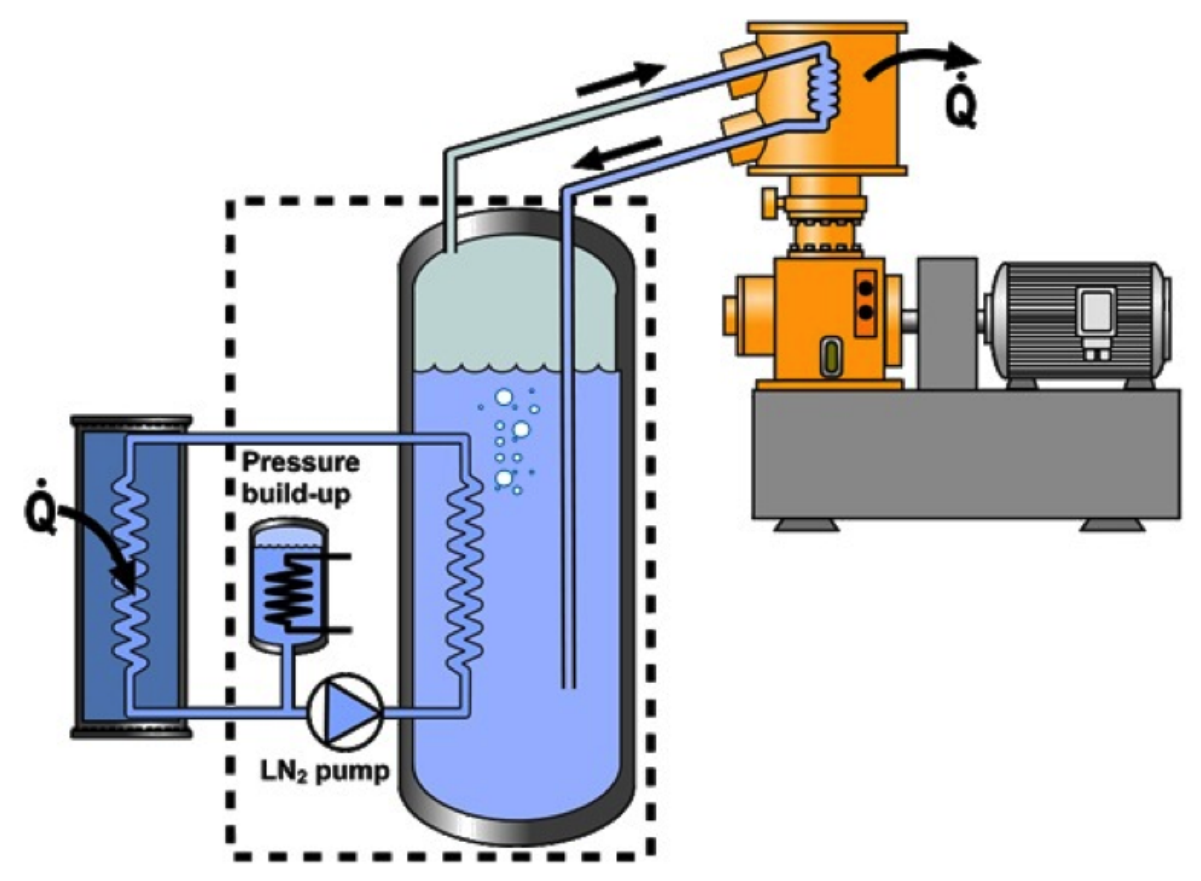

Figure 6.8.2: Cryocooler schematic. A cryogenerator removes heat, Q, from a closed liquid nitrogen storage reservoir. Other devices requiring heat removal are thermally connected to that reservoir. All of the systems are closed-loop. 
rather than being vented to the cavern as was done during LUX operations. The cryocooler is capable of $1,000 \mathrm{~W}$ of cooling power at the $77 \mathrm{~K}$ boiling temperature of $\mathrm{LN}$ at atmospheric pressure. Preliminary estimate of total heat load on the cryogenic system is $838 \mathrm{~W}$ including $20 \%$ contingency. The heat load rollup is shown in Table 6.8.1. The $1,000 \mathrm{~W}$ configuration provides a margin against unanticipated heat load.

Table 6.8.1: Heat load rollup.

\begin{tabular}{|c|c|l|}
\hline System/Component & Heat (W) & Notes \\
\hline LZ cryostat & 115 & $\begin{array}{l}\text { Assumes } 10 \text { liters of MLI; value con- } \\
\text { firmed by independent analysis in U.K. }\end{array}$ \\
\hline PMT Conduit & 34 & \\
\hline HV Conduit & 1 & \\
\hline Cryocooler Storage Tank & 28 & $\begin{array}{l}\text { Calculated for 1000-liter tank; value } \\
\text { diminishes for smaller Dewars. }\end{array}$ \\
\hline Heat Exchanger & 349 & $\begin{array}{l}\text { Assumed at 94\% efficient (LUX expe- } \\
\text { rience) @ 500 slpm Xe flow. }\end{array}$ \\
\hline Xenon Purge & 56 & Estimate for 5 slpm flow rate. \\
\hline Thermosyphons & 116 & $\begin{array}{l}\text { Dominated by heaters; could go up for } \\
\text { higher thermosyphon count. }\end{array}$ \\
\hline Contingency @ 20\% & 140 & \\
\hline Total & 838 & \\
\hline
\end{tabular}

Cryocoolers based on the Stirling cycle have the additional advantages: quick startup, low energy consumption (each unit draws $11 \mathrm{~kW}$ of electricity), and variable drive motors that allow adjustable cooling levels below 1,000 W. These units have been deployed at many institutions worldwide, including installations at SNOLAB, Gran Sasso (Icarus), and multiple university laboratories in the United States, Russia, and Asia. Maintenance of the cryocooler is required after 6,000 hours of continuous use, so the system is designed for the future addition of a second cryocooler (likely purchased as a component of operations). Transport of LN from the surface to the Davis Campus can sustain operations during short-term maintenance that is expected to have a duration not exceeding 8-hours.

LUX has received deliveries of LN two to three times per week. Even though LZ is better insulated, scaling indicates that LZ requires one LN delivery every 24 to 36 hours to sustain operation. For longterm operations, that would increase the inherent transport risk and manpower requirements related to LN transfer. Therefore, the addition of a second cryogenerator at some point would significantly reduce the risk to long-term operations.

Distribution of cooling power from the cryocooler to the Xe-containing experimental systems is accomplished by use of thermosyphons. A thermosyphon is a type of heat pipe. Thermosyphon technology was successfully applied to LUX following development at Case Western Reserve University (now SLAC). Thermosyphons comprise condenser and evaporator heads connected by small-diameter tubing wrapped with multilayer insulation (MLI) and charged with nitrogen gas at modest pressure. In this application, the condenser is immersed in an LN bath, causing the nitrogen gas in the thermosyphon to liquefy. The liquid flows in the small-diameter tubing by gravity to the evaporator that is physically attached to the device that requires cooling. Heat from the evaporator causes the LN pooled in the evaporator to change phase, removing heat via the nitrogen latent heat of vaporization. The warmed nitrogen gas then returns to the condenser by buoyancy effect. 
Cooling of the Xe relies upon the temperature difference between the $\mathrm{LN}$ and Xe vaporization temperatures as well as the adjustable cooling power of the thermosyphon. Changing the mass of circulating nitrogen can modulate the thermosyphon cooling power. As the mass is increased, pressure in the condenser rises, effectively increasing the boiling temperature in the thermosyphon and inducing an increased heat flux to the $\mathrm{LN}$ bath that remains at atmospheric pressure and $77 \mathrm{~K}$.

Thermosyphon cooling is completely passive, with a fixed amount of nitrogen in the secondary cooling loop. Therefore no pumps are required and there is no direct path for large quantities of LN (typically stored in Dewars) to get into the Xe via a leak. As the thermosyphon transport tubing may be $9.5 \mathrm{~mm}$ or $12.7 \mathrm{~mm}$ OD, a minimal amount of nitrogen can be placed into the tubes, on the order of $15 \mathrm{~g}$ to reach $10 \mathrm{bara}$. This mass gradually increases as the nitrogen condenses but the mass remains modest. Over-pressure protection is by relief valves and burst disks. The thermosyphons are charged with nitrogen via high-pressure cylinders. Control of the gas mass (and cooling power) is accomplished with control valves, mass flow controllers, sensors, and processing devices connected to slow control via Ethernet. Thermosyphons deployed in LUX were easily capable of delivering more than $200 \mathrm{~W}$ cooling for each deployed evaporator head. The single largest device needing cooling is the LXe tower, in which two-phase, sub-cooler, and gas-gas heat exchangers are anticipated to require $349 \mathrm{~W}$ of cooling to recondense Xe returning to the detector. Three thermosyphon evaporators are able to provide that energy removal.

Vacuum-pumping systems take advantage of tubing in place to route thermosyphons, Xe transfer lines, and PMT cables. Packages of combined scroll pumps and turbo-molecular pumps are deployed on the decking of upper Davis Cavern and near the Xe heat exchanger tower in lower Davis Cavern. These pump combinations evacuate: the annulus between the inner and outer vessels, detector internals, Xe heat-exchanger tower, and the entire Xe gas system. Two vacuum leak check carts are provided so that there is a minimum of one cart available at any time in the Surface Assembly Laboratory and the underground Davis Campus. A variety of gauges are deployed, including manual gauges (that can show vacuum in power outage), thermocouple gauges, and ion gauges.

A critical element of vacuum pumping is removal of residual gases from detector internals that are either attached to metal surfaces or dissolved into plastic volumes. Empirical data developed at U. Maryland for gas solubility and diffusion in plastics is used along PTFE volume / thickness measurements from SolidWorks CAD models to predict the required vacuum pumping to achieve acceptable levels of $\mathrm{Kr}, \mathrm{N}_{2}$, and $\mathrm{O}_{2}$. Predicted steps for vacuum pumping are as follows:

1. Pump detector to $1 \times 10^{-4}$ torr and hold for one day;

2. Backfill with 10 torr Xe gas;

3. Circulate $\mathrm{Xe}$ gas that is heated to $40{ }^{\circ} \mathrm{C}$ for 45 days;

4. Pump out $\mathrm{Xe}$ gas and achieve $1 \times 10^{-6}$ torr predicted to take 65 days;

5. Maintain that pressure at room temperature for one additional week.

By that time residual $\mathrm{Kr}$ in the detector is predicted to be less than $0.03 \mathrm{ppt}$ with a pumping duration of 3.3 months.

PMT, sensor, and grid power supply cables from both the inner and outer cryostats must be routed to locations where they can transition from either a pure Xe or high-vacuum environment to atmospheric conditions in the Davis Cavern. These cables are routed through VJ conduits. Two conduits exit the cryostat from the top and one from the bottom. One upper and one lower conduit, primarily carrying PMT cables, are routed to a SURF-installed mezzanine level. Sensor and grid cables exiting the top of the detector are routed to the main Davis Cavern deck. The inner tube of the VJ is braided flexible conduit sized to allow 
pulling of all cables during installation. The outer jacket has "tee" connections to the vacuum-pumping system. Stainless steel breakout boxes are installed on the conduits at the mezzanine and main Davis Cavern deck for installation of hermetic feedthroughs at the point where cables must be terminated in order to exit the Xe and vacuum spaces. Breakout boxes and inner conduits are compatible with the 3.4 bara maximum $\mathrm{Xe}$ pressure and meet Xe cleanliness requirements.

Control of temperature, pressure, and liquid levels within the cryogen system is needed both for the correct operation of detector systems and for safe operation. Control of these parameters is via connection to the slow control system described in Chapter 8. A detailed P\&ID along with a related instrument list exists. Each thermosyphon utilizes multiple thermometers, pressure transducers, a vacuum gauge, a mass flow controller, and controller boxes to digitize signals and transmit via Ethernet. Similarly, the LN storage room, VJ distribution piping, cryocooler, and vacuum pumping system need active monitoring and control of temperature and pressure.

\subsection{Xenon Procurement}

Approximately 10 tonnes $\left(1.8 \times 10^{6}\right.$ gas liters $)$ of Xe must be acquired for the LZ detector. The purity requirement is an industry standard of $99.999 \% \mathrm{Xe}$. The Xe currently in the LUX detector and from small previous purchases will be reused $(\sim 90,000$ liters $)$. SDSTA initiated a bid and procure process for $\sim 500,000$ liters in the second half of 2015. Bids were requested and received from all major producers in the world and a few other sources with existing Xe stock. Delivery has started and will continue into 2017. A second bid cycle for up to about $1.2 \times 10^{6}$ liters was completed in February 2016 and deliveries have started. A fixed price and delivery schedule consistent with the LZ need to complete delivery by the end of 2018 was achieved. The University of Alabama has procured about 36,000 liters that have been delivered to SLAC. All Xe will be delivered to SLAC by the fall of 2018 for later removal of Kr. Approximately $20 \%$ of the LZ Xe has been received and assayed at SLAC, and all of it has met or exceeded purity specs.

\subsection{Solubility and diffusion constants of common impurity gas species}

To quantify the outgassing burden of the LZ TPC materials, the LZ R\&D program produced a set of measurements of the diffusion and solubility constants of several common impurity species in various plastic and elastomer materials. Some result from that campaign were reported in Table 9.7.1 of Ref. [15], however, due to an editing error, portions of that table are incorrect. Table 6.10.1 presents the corrected values. 
Table 6.10.1: Solubility (K) and diffusion constants (D) of common impurity species. $K$ is defined as the ratio of impurity mass per unit volume inside and outside of the material sample. The PTFE sample is from the LUX TPC. PE1 is a polyethylene sample from LUX. The viton is an off-the-shelf sample from McMaster. PE2 is a polyethylene sample from a candidate cathode high voltage cable that was under consideration for $L Z$ during its $R \& D$ phase. All measurements were performed at room temperature.

\begin{tabular}{|c|c|c|c|c|}
\hline \multicolumn{5}{|c|}{ Solubility (K) (\%) } \\
\hline & PTFE & PE1 & viton & PE2 \\
\hline $\mathrm{N}_{2}$ & $10.7 \pm 0.7$ & $2.1 \pm 0.3$ & $51 \pm 10$ & \\
\hline $\mathrm{O}_{2}$ & $22 \pm 2$ & $1.8 \pm 0.6$ & $22 \pm 1$ & \\
\hline $\mathrm{Kr}$ & $58 \pm 5$ & $9.3 \pm 1.1$ & $23 \pm 2$ & $11 \pm 2$ \\
\hline $\mathrm{Xe}$ & $89 \pm 8$ & $55 \pm 6$ & $15 \pm 2$ & \\
\hline $\mathrm{Ar}$ & $8.8 \pm 0.8$ & $4.7 \pm 1.5$ & $8.3 \pm 2.6$ & \\
\hline $\mathrm{He}$ & $3.3 \pm 0.3$ & $0.64 \pm 0.07$ & $9.3 \pm 0.8$ & \\
\hline $\mathrm{CH}_{4}$ & & $16 \pm 3$ & & \\
\hline \multicolumn{5}{|c|}{ Diffusion constant (D) $\left(10^{-8} \mathrm{~cm}^{2} / \mathrm{s}\right)$} \\
\hline & PTFE & PE1 & viton & PE2 \\
\hline $\mathrm{N}_{2}$ & $15.1 \pm 0.3$ & $16 \pm 1$ & $2.2 \pm 0.1$ & \\
\hline $\mathrm{O}_{2}$ & $31.4 \pm 0.6$ & $39 \pm 3$ & $6.8 \pm 0.1$ & \\
\hline $\mathrm{Kr}$ & $5.6 \pm 0.1$ & $6.4 \pm 0.4$ & $1.25 \pm 0.02$ & $11.2 \pm 0.8$ \\
\hline $\mathrm{Xe}$ & $0.80 \pm 0.02$ & $2.0 \pm 0.1$ & $1.7 \pm 0.1$ & \\
\hline $\mathrm{Ar}$ & $16.8 \pm 0.3$ & $20 \pm 1$ & $4.0 \pm 0.1$ & \\
\hline $\mathrm{He}$ & $1270 \pm 25$ & $435 \pm 30$ & $436 \pm 5$ & \\
\hline $\mathrm{CH}_{4}$ & & $6.6 \pm 0.9$ & & \\
\hline
\end{tabular}




\subsection{Bibliography}

[1] H. H. Loosli, B. E. Lehmann, and W. M. Smethie Jr., in Environmental Tracers in Subsurface Hydrology, edited by P. G. Cook and A. L. Herczeg (Springer US, 2000) pp. 379-396, Noble Gas Radioisotopes: ${ }^{37} \mathrm{Ar},{ }^{85} \mathrm{Kr},{ }^{39} \mathrm{Ar},{ }^{81} \mathrm{Kr}$, ISBN 978-1-4613-7057-4.

[2] X. Du, R. Purtschert, K. Bailey, B. E. Lehmann, R. Lorenzo, Z.-T. Lu, P. Mueller, T. P. O’Connor, N. C. Sturchio, and L. Young, Geophys. Res. Lett. 30, 2068 (2003).

[3] R. A. Riedmann and R. Purtschert, Environ. Sci. Technol. 45, 8656 (2011).

[4] A. I. Bolozdynya, P. P. Brusov, T. Shutt, C. E. Dahl, and J. Kwong, in Proceedings of the 11th Symposium on Radiation Measurements and Applications Ann Arbor, MI, USA 23-26 May 2006, Nucl. Instrum. Meth., Vol. A579 (2007) pp. 50-53, A chromatographic system for removal of radioactive ${ }^{85} \mathrm{Kr}$ from xenon.

[5] D. S. Akerib et al. (LUX), "Chromatographic separation of radioactive noble gases from xenon," (2016), submitted to Astropart. Phys., arXiv:1605.03844 [physics.ins-det].

[6] A. Dobi, C. G. Davis, C. Hall, T. Langford, S. Slutsky, and Y.-R. Yen, Nucl. Instrum. Meth. A665, 1 (2011), arXiv:1103.2714 [astro-ph].

[7] D. S. Akerib et al. (LUX), Phys. Rev. Lett. 112, 091303 (2014), arXiv:1310.8214 [astro-ph].

[8] A. Dobi, D. S. Leonard, C. Hall, L. Kaufman, T. Langford, S. Slutsky, and Y. R. Yen, Nucl. Instrum. Meth. A620, 594 (2010), arXiv:1002.2791 [physics.ins-det].

[9] E. Clementi, D. L. Raimondi, and W. P. Reinhardt, J. Chem. Phys. 47, 1300 (1967).

[10] K. Abe et al. (XMASS), Nucl. Instrum. Meth. A 661, 50 (2012).

[11] S. Lindemann, Intrinsic ${ }^{85} \mathrm{Kr}$ and ${ }^{222} \mathrm{Rn}$ Backgrounds in the XENON Dark Matter Search, Ph.D. thesis, University of Heidelberg (2013).

[12] K. Munakata, T. Fukumatsu, S. Yamatsuki, K. Tanaka, and M. Nishikawa, J. Nucl. Sci. Technol. (Tokyo, Jpn.) 36, 818 (1999).

[13] Y. Fukuda et al. (Super-Kamiokande), Advanced computing and analysis techniques in physics research. Proceedings, 8th International Workshop, ACAT 2002, Moscow, Russia, June 24-28, 2002, Nucl. Instrum. Meth. A501, 418 (2003).

[14] D. S. Leonard, A. Dobi, C. Hall, L. Kaufman, T. Langford, S. Slutsky, and Y.-R. Yen, Nucl. Instrum. Meth. A621, 678 (2010), arXiv:1002.2742 [physics.ins-det].

[15] D. S. Akerib et al. (LZ), (2015), Conceptual Design Report; LBNL-190005, FERMILAB-TM-2621AE-E-PPD, arXiv:1509.02910 [physics.ins-det]. 


\section{Calibration Systems}

A rigorous calibration strategy is a prerequisite for the unambiguous direct detection of hypothetical dark matter interactions in the LZ detector. The basic questions about any event are: (1) how did the particle interact, and (2) how much energy did it deposit? But before these questions can be answered, the $(x, y, z, t)$ response of the LZ TPC must be understood.

The LZ calibration strategy is designed to accurately answer these questions, achieve the LZ science goals, and be ready to address the widest possible range of predicted dark-matter signatures. Basic requirements (referred to in this chapter as R-17nnnn for the different requirements) have been defined. The process for capturing requirements is described in Chapter 12. The principal calibration techniques planned for LZ have been used successfully in previous experiments, especially LUX and Zeplin. A summary of all sources to be used in LZ is included in Table 7.0.1.

Table 7.0.1: Baseline calibration sources for LZ.

\begin{tabular}{|c|c|c|c|c|}
\hline Isotope & What & Purpose & Deployment & Custom? \\
\hline $\begin{array}{c}\text { Tritium } \\
{ }^{83 \mathrm{~m}} \mathrm{Kr}\end{array}$ & beta, $\mathrm{Q}=18.6 \mathrm{keV}$ & ER band & Internal & $\mathrm{N}$ \\
${ }^{131 \mathrm{~m}} \mathrm{Xe}$ & $164 \mathrm{keV} \gamma$ & TPC $(x, y, z)$ & Internal & $\mathrm{Y}$ \\
${ }^{220} \mathrm{Rn}$ & various $\alpha$ 's & TPC $(x, y, z)$, Xe skin & Internal & $\mathrm{Y}$ \\
\hline${ }^{\mathrm{Am}} \mathrm{Li}$ & $(\alpha, n)$ & xenon skin & Internal & $\mathrm{N}$ \\
${ }^{252} \mathrm{Cf}$ & spontaneous fission & NR band & $\mathrm{CSD}$ & $\mathrm{Y}$ \\
${ }^{57} \mathrm{Co}$ & $122 \mathrm{keV} \gamma$ & NR efficiency & $\mathrm{CSD}$ & $\mathrm{N}$ \\
${ }^{228} \mathrm{Th}$ & $2.615 \mathrm{MeV} \gamma$, various others & OD energy scale & $\mathrm{CSD}$ & $\mathrm{N}$ \\
${ }^{22} \mathrm{Na}$ & back-to-back $511 \mathrm{keV} \gamma$ 's & TPC and OD sync & CSD & $\mathrm{N}$ \\
\hline${ }^{88} \mathrm{Y} \mathrm{Be}$ & $152 \mathrm{keV}$ neutron & low-energy NR response & External & $\mathrm{N}$ \\
${ }^{205} \mathrm{Bi} \mathrm{Be}$ & $88.5 \mathrm{keV}$ neutron & low-energy NR response & External & $\mathrm{Y}$ \\
${ }^{206} \mathrm{Bi} \mathrm{Be}$ & $47 \mathrm{keV}$ neutron & low-energy NR response & External & $\mathrm{Y}$ \\
\hline $\mathrm{DD}$ & $2,450 \mathrm{keV}$ neutron & NR light and charge yields & External & $\mathrm{N}$ \\
$\mathrm{DD}$ & $272 \mathrm{keV}$ neutron & NR light and charge yields & External & $\mathrm{Y}$ \\
\hline
\end{tabular}

As described in Chapter 1.3.1, signals in LZ consist of scintillation photons (S1) and ionized electrons, read-out as proportional scintillation $(\mathrm{S} 2)$. Variation in the $(x, y, z)$ response of $\mathrm{S} 1$ and the $(x, y)$ of $\mathrm{S} 2$ arise, for example, from detector geometry, light collection and other factors. Variation in the $(z, t)$ response of $\mathrm{S} 2$ could arise from changes in xenon purity, via attachment of ionized electrons by impurities. These calibrations will be addressed by mono-energetic internal radioisotope sources (Section 7.1).

Once these variations have been calibrated, it is possible to address the two questions posed in the first paragraph. The answer is contained in a plot like Figure 1.3.11 from Section 1.3.4, which shows the distri- 
bution of background-like events (top) and expected signal-like events (bottom). These bands were obtained from in-situ calibration of the LUX detector. Similar techniques will be used for LZ, as described in detail in the following sections. In Figure 1.3.11, the overlaid curves of approximate event energy are obtained from a knowledge of the absolute photon and electron detection efficiency of the instrument. This in turn is obtained from mono-energetic photon interactions in the detector.

In addition to the broad spectrum nuclear recoil response, $\mathrm{LZ}$ will measure the absolute response of LXe to nuclear recoils. It is critical to make this calibration in-situ and LZ will be able to make more precise measurements than are presently available. This is due to its large target mass and extremely low background count rate. These measurements are discussed in Section 7.3 and Section 7.4.

\subsection{Internal Radioisotope Sources}

Liquid Xenon's strong self-shielding ability, of great benefit to the dark matter search by largely eliminating external backgrounds from the central fiducial volume, conversely makes low-energy electron recoil calibrations of this central volume practically impossible using external radioisotope sources. Rather than rely on extremely long duration ( $\gg 1 \mathrm{~d}$ ) exposures to high-rate high-energy $(>1 \mathrm{MeV})$ sources, gaseous radioisotopes will be mixed into the LXe itself. This 'internal' calibration source strategy has been welldemonstrated in LUX.

\subsubsection{Metastable Krypton $83\left({ }^{83 \mathrm{~m}} \mathrm{Kr}\right)$}

Metastable ${ }^{83 \mathrm{~m}} \mathrm{Kr}$ is a low-energy $(41 \mathrm{keV})$ monoenergetic source that is easy to produce and exhibits a conveniently short decay time (half-life of $1.8 \mathrm{~h}$ ). The monoenergetic peak enables the production of highresolution 3-D maps of S1 and S2 detector response, producing a calibration of spatially-varying detector efficiency effects. Electron drift efficiency, extraction efficiency, and S2 light production efficiency are each individually position-dependent, and can combine to produce a larger spatial variation in S2 response, one that can also be time-varying with changes in liquid purity. This motivates requirements R-170002 and R170007, which necessitate a mono-energetic dispersed throughout the xenon. For all these reasons, regular ( $\sim$ weekly) 3-D detector response maps are essential to attaining the needs of the WIMP search.

It should be noted that ${ }^{83 \mathrm{~m}} \mathrm{Kr}$ 's decay occurs in two steps, separated by a short decay half-life ( $\left.\tau=154 \mathrm{~ns}\right)$. This timing variation from decay to decay produces a variation from event to event in the overall electronion recombination probability, meaning that while ${ }^{83 \mathrm{~m}} \mathrm{Kr}$ is quite useful as a monoenergetic peak source, its unusual recombination physics means it is not useful for mimicking typical electron recoil backgrounds.

${ }^{83 \mathrm{~m}} \mathrm{Kr}$ production and handling for liquid noble detectors is now a mature process: a radon-pure charcoal is infused with a ${ }^{83} \mathrm{Rb}$-containing aqueous solution, baked, and then placed in standard VCR plumbing (contained by particulate filters) to produce a ${ }^{83 \mathrm{~m}} \mathrm{Kr}$-generating source. To 'inject' ${ }^{83 \mathrm{~m}} \mathrm{Kr}$ into the detector, a xenon carrier gas flows over the charcoal, carrying some ${ }^{83 \mathrm{~m}} \mathrm{Kr}$ activity into the main xenon circulation path, with the dosed activity controllable by carrier gas flow rate and flow duration. As a noble gas, ${ }^{83 \mathrm{~m}} \mathrm{Kr}$ has the practical advantage of first passing through the circulation path getter, for added protection against the incidental injection of impurities. ${ }^{83} \mathrm{Rb}$ has a conveniently long half-life of 86.2 days, meaning a single ${ }^{83 \mathrm{~m}} \mathrm{Kr}$ source can sustain a usable activity for approximately one year.

\subsubsection{Metastable Xenon $131\left({ }^{131 \mathrm{~m}} \mathrm{Xe}\right)$}

Calibrations of the position reconstruction itself, essential to rejecting spatially-varying external backgrounds and to precisely defining a fiducial volume, are easiest if the calibration source activity is homogeneously distributed throughout the active LXe. The liquid mixing timescale in $\mathrm{LZ}$ may not allow ${ }^{83 \mathrm{~m}} \mathrm{Kr}$ 

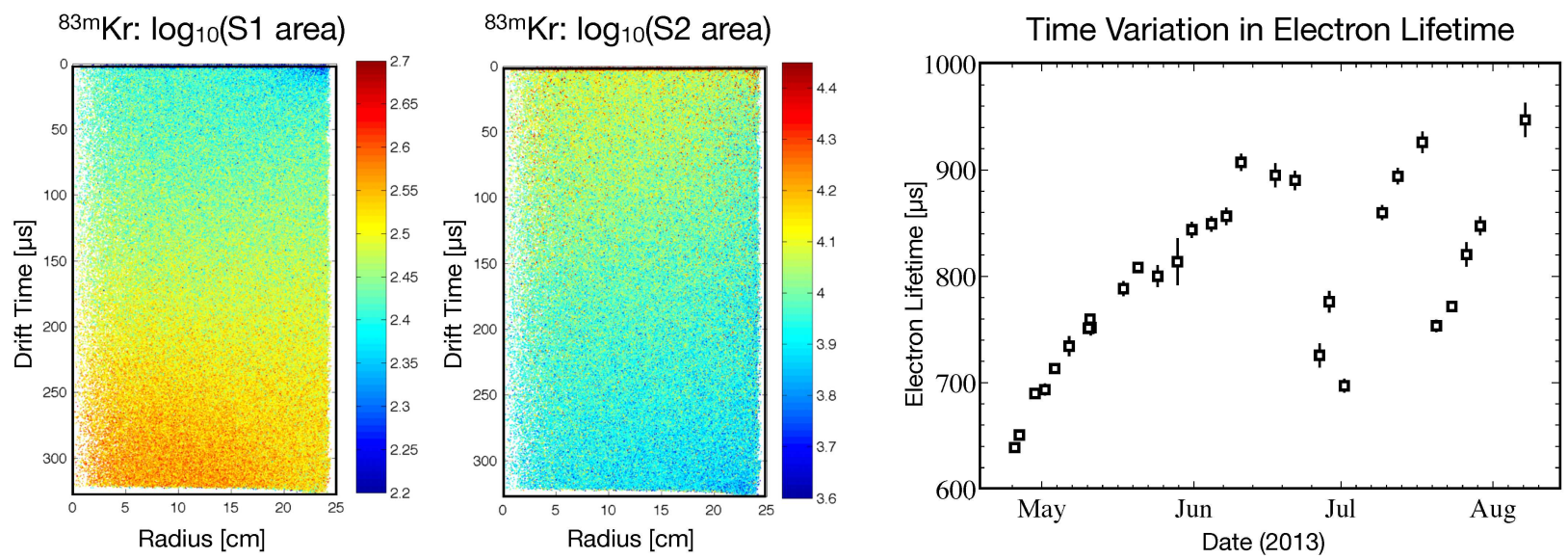

Figure 7.1.1: Several illustrations of ${ }^{83 \mathrm{~m}} \mathrm{Kr}$ calibrations in LUX: (left) $\mathrm{S} 1$ area in the radius vs. drift time plane, (middle) S2 area in the same projection (on a particular date), and (right) a time-dependent measurement of electron lifetime in the drift region.

to become fully homogeneous given its short decay timescale, and for this reason a ${ }^{131 \mathrm{~m}} \mathrm{Xe}$ source is being developed as a long-lived alternative for the specific goal of achieving homogeneity (cf. requirements R-170002 and R-170007). Compared with ${ }^{83 \mathrm{~m}} \mathrm{Kr},{ }^{131 \mathrm{~m}}$ Xe decays at a higher energy (164 keV) and with a longer half-life (11.9 d). The higher energy may result in the partial saturation of the central PMT of each S2 pulse, but the long half-life will guarantee homogeneity for the purposes of calibrating the position reconstruction metrics. An additional use of this comparatively higher-energy internal source is in calibrating the xenon skin region (cf. requirement R-170008), which will possess a position-dependent detection threshold of roughly the same $\sim 100 \mathrm{keV}$ scale.

The hardware for ${ }^{131 \mathrm{~m}} \mathrm{Xe}$ is nearly identical to the ${ }^{83 \mathrm{~m}} \mathrm{Kr}$ source: xenon carrier gas is flowed over a parent isotope that is emanating the calibration isotope. In this case, the parent isotope is ${ }^{131} \mathrm{I}$, which decays entirely to ${ }^{131} \mathrm{Xe}$ with a half-life of 8 days. A small fraction of these ${ }^{131}$ I decays $(\sim 0.39 \%)$ are to the metastable ${ }^{131 \mathrm{~m}} \mathrm{Xe}$ state rather than the ground state. ${ }^{131} \mathrm{I}$ is readily available from the medical isotope industry in a variety of forms. The most convenient form for our purposes is a solid pill, in which ${ }^{131} \mathrm{I}$ is doped into a solid matrix of anhydrous sodium phosphate.

The $164 \mathrm{keV}$ energy of ${ }^{131 \mathrm{~m}} \mathrm{Xe}$ decays is significantly larger than typical expected dark matter signals, and we estimate that a single PMT in the top array will experience mild analog saturation of its output signal at this energy. The saturation is a combination of exhausting the reserve charge in the coupling capacitors, and of anode saturation [1]. Nevertheless, the result of this saturation is expected to be a negligible impact on the utility of the source for $(x, y, z)$ map-making.

\subsubsection{Tritium-Labeled Methane}

In addition to calibrating for position-dependent detector efficiency effects, an internal calibration source is necessary when measuring the low-energy electron recoil physics of the combined S1 and S2 energy scale, and the S2/S1 discrimination ratio (cf requirement R-170003). For this essential calibration, LUX has demonstrated the use of ${ }^{3} \mathrm{H}$ beta decay, exhibiting a broad energy spectrum with an $18.6 \mathrm{keV}$ endpoint.

${ }^{3} \mathrm{H}$ decays with a 12.3 -year half-life, this necessitates the ability to remove it from the xenon. This is way tritium-labeled methane is used, as it will be removed by the LZ getter (cf. requirement R-170114). LUX demonstrated that ${ }^{3} \mathrm{H}$-labeled $\mathrm{CH}_{4}$ could overcome the paired challenges of a long half life and the polymer 
diffusion. $\mathrm{CH}_{4}$ exhibits a very small absorption into the PTFE, and is efficiency removed by the getter. The $\mathrm{CH}_{4}$ purification timescale in LZ may be somewhat different from the general Xe re-circulation timescale ( $\sim 2 \mathrm{~d}$ ), a result of the methane's differing solubility in the liquid and gas phases of Xe. In LUX, the $\mathrm{CH}_{4}$ purification timescale was shorter than the xenon re-circulation timescale by a significant factor, and we expect the $\mathrm{CH}_{4}$ purification timescale in $\mathrm{LZ}$ to be similarly shortened.

\subsubsection{Radon $220\left({ }^{220} \mathrm{Rn}\right)$}

${ }^{220} \mathrm{Rn}$ is being considered as a potential large-S1 calibration source for the Xe skin region. ${ }^{220} \mathrm{Rn}$ should not be mistaken for ${ }^{222} \mathrm{Rn} ;{ }^{220} \mathrm{Rn}$ has no long-lived radioactive daughters, thereby making it a compatible with the stringent low-background requirements of LZ. The longest-lived daughter is ${ }^{212} \mathrm{~Pb}$, with a half-life of 10.6 hours.

${ }^{220} \mathrm{Rn}$ decays through alpha decay at $6.3 \mathrm{MeV}$, followed shortly after $\left(\mathrm{t}_{1 / 2}=150 \mathrm{~ms}\right)$ by a second alpha at 6.8 MeV. Alpha emission produces a highly localized and proportionally high-recombination (high-lightyield) Xe response, ideal for calibrating the skin region. Depending on the liquid mixing timescales, ${ }^{220} \mathrm{Rn}$ 's daughter isotopes $\left({ }^{212} \mathrm{~Pb},{ }^{212} \mathrm{Bi},{ }^{212} \mathrm{Po}\right.$, and $\left.{ }^{208} \mathrm{Tl}\right)$ are expected to accumulate on surfaces before their decay, providing a unique tool for understanding light and charge yield at xenon-surface interfaces.

Like ${ }^{83 \mathrm{~m}} \mathrm{Kr}$ and ${ }^{131 \mathrm{~m}} \mathrm{Xe},{ }^{220} \mathrm{Rn}$ is most conveniently introduced by flowing a Xe carrier gas over a material containing the parent isotope, in this case ${ }^{228} \mathrm{Th}\left(\tau_{1 / 2}=1.9 \mathrm{y}\right) .{ }^{228} \mathrm{Th}$ can be purchased in an electroplated form, which both maximizes ${ }^{220} \mathrm{Rn}$ emanation probability and minimizes the total material (and any possible impurity content).

\subsubsection{Internal Radioisotope Source Delivery}

An important component of the internal calibration strategy is an effective and precise system for injecting controlled amounts of activity into the Xe circulation flow path (cf requirement R-170102). Fundamentally, the source injection system is a specialized extension of the Xe circulation system, and shares the basic design fundamentals (including the use of stainless steel VCR plumbing standards) and the basic system risks, the most important being the risk of ${ }^{222} \mathrm{Rn}$ and ${ }^{85} \mathrm{Kr}$ leakage from underground air into the circulating $\mathrm{Xe}$ gas. The mitigation for this radon concern is shared with the circulation system: rigorous leak testing standards during assembly, paired with enveloping the plumbing by a radon-free purge gas rather than underground air.

A schematic of the source injection system is shown in Figure 7.1.2. The source injection system will be entirely automated, both to reduce necessary onsite shift burden, and to reduce the risk to the xenon's purity from human operator error. This automation will be made possible through the general slow control system (described in Section 8.8). A connection will be in place between the source injection system and the Xe sampling system (described in Section 6.7), making it possible to perform careful checks of injection material purity before introducing any material to the flow path.

The trace amounts of radioactive gas are transported into the main circulation flow path by a small flow of Xe carrier gas, supplied by a system-specific supply of high-pressure Xe. This Xe will have gone through the same ${ }^{85} \mathrm{Kr}$-removal process as the bulk Xe (discussed in Section 6.3), introducing no new radiopurity concerns. This small Xe flow will pass through emanation sources $\left({ }^{83 \mathrm{~m}} \mathrm{Kr},{ }^{131 \mathrm{~m}} \mathrm{Xe}\right.$, etc. $)$, regulated using a mass flow controller for a total mass flow roughly proportional to desired injected activity. ${ }^{3} \mathrm{H}-$ labeled $\mathrm{CH}_{4}$ is injected according to a slightly different recipe; this activity is stored in a pressurized bottle, which is allowed to fill a small evacuated 'dosing region' volume to a precisely-measured pressure. This dose volume will then be flushed by the Xe carrier gas into circulation, as with the flow-through sources. The internal source injection system is being designed to be general and flexible, allowing for alternative injection procedures and for the possible use of internal sources not yet developed. 


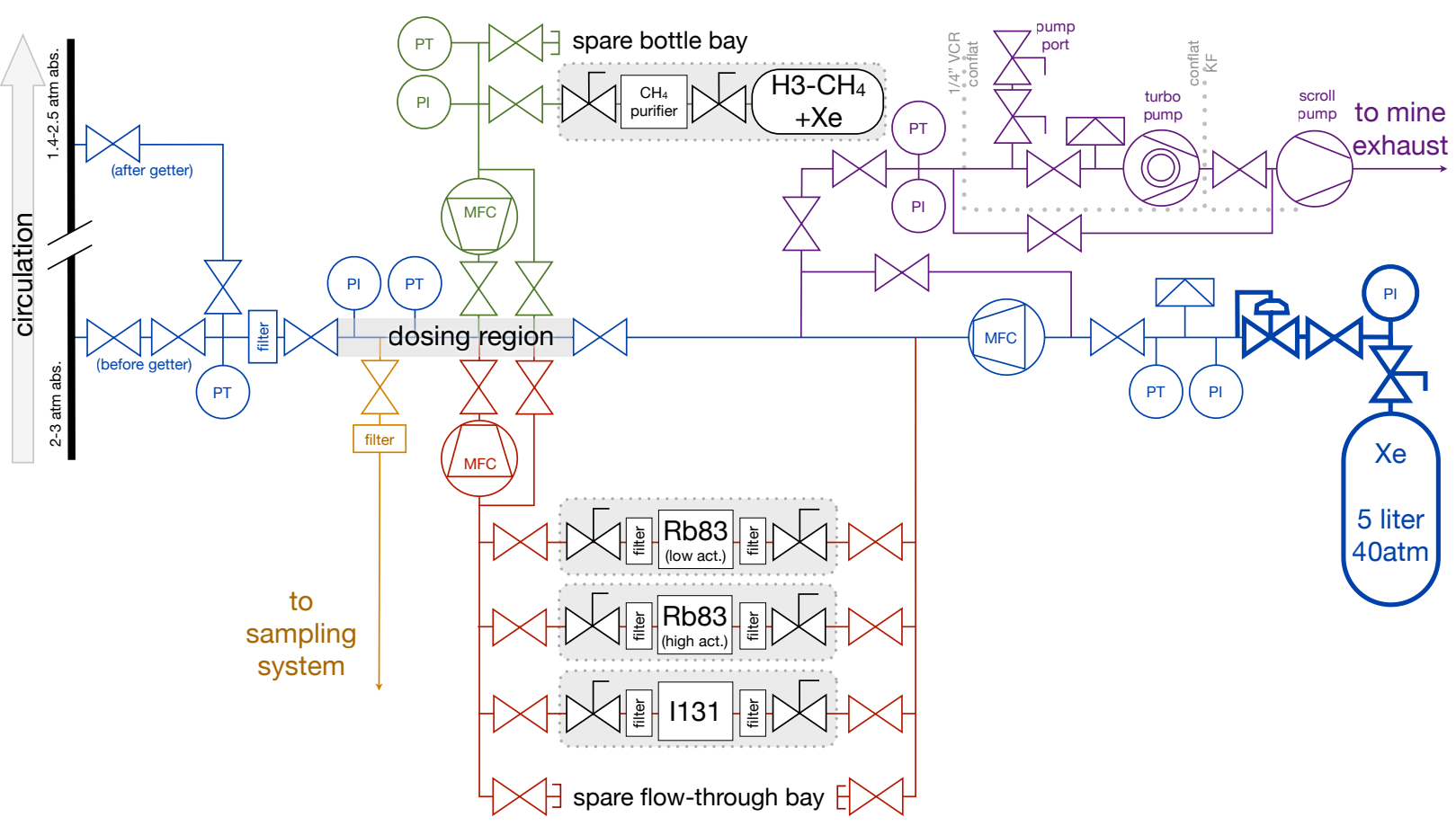

Figure 7.1.2: The internal radioisotope source delivery system is a portion of Xe plumbing with the goal of releasing a precise portion of various gaseous radioactive isotopes into the main xenon circulation path. This control is achieved through a combination of Mass Flow Controllers (MFCs) and Pressure Transducers (PTs), with flow from the dosing region into the circulation path (far left) motivated by a high-pressure Xe supply bottle (far right).

\subsection{External Radioisotope Source Delivery}

LZ will have three stainless steel vertical source tubes in the vacuum space between the inner and outer titanium cryostats, as shown in Figure 5.3.1. The calibration source deployment (CSD) system will position neutron and gamma calibration sources in the source tubes. The source tubes will have an inner diameter of $23.6 \mathrm{~mm}$, large enough to accommodate deployment of commercial sources in nearly all cases. The necessary source strengths are well within the available range. Sources that cannot be obtained commercially in our requisite dimensions and rate will be fabricated by LZ (University of Alabama). The source tubes will be sealed at both ends and kept in a nitrogen atmosphere at a pressure of 1.1 bar to suppress contaminants entering the system and prevent the plating of radon daughters. The photoneutron and DD neutron sources require dedicated deployments and are discussed separately below.

\subsubsection{Neutron sources}

A suite of four neutron sources will provide a broad-spectrum NR calibration, with the additional benefit of four distinct kinematic endpoints, as shown in Figure 7.2.1. In particular, these broadband sources are useful for measuring the NR band (cf. requirement R-170004). Also shown in the figure are the nuclear recoil detection efficiencies for 2- and 3-fold PMT coincidences. 3-fold coincidence is the baseline for 
WIMP search analysis, but in calibrations, where the total data taking time is much shorter, 2-fold PMT coincidence thresholds may be more appropriate for understanding low-energy response of the detector.

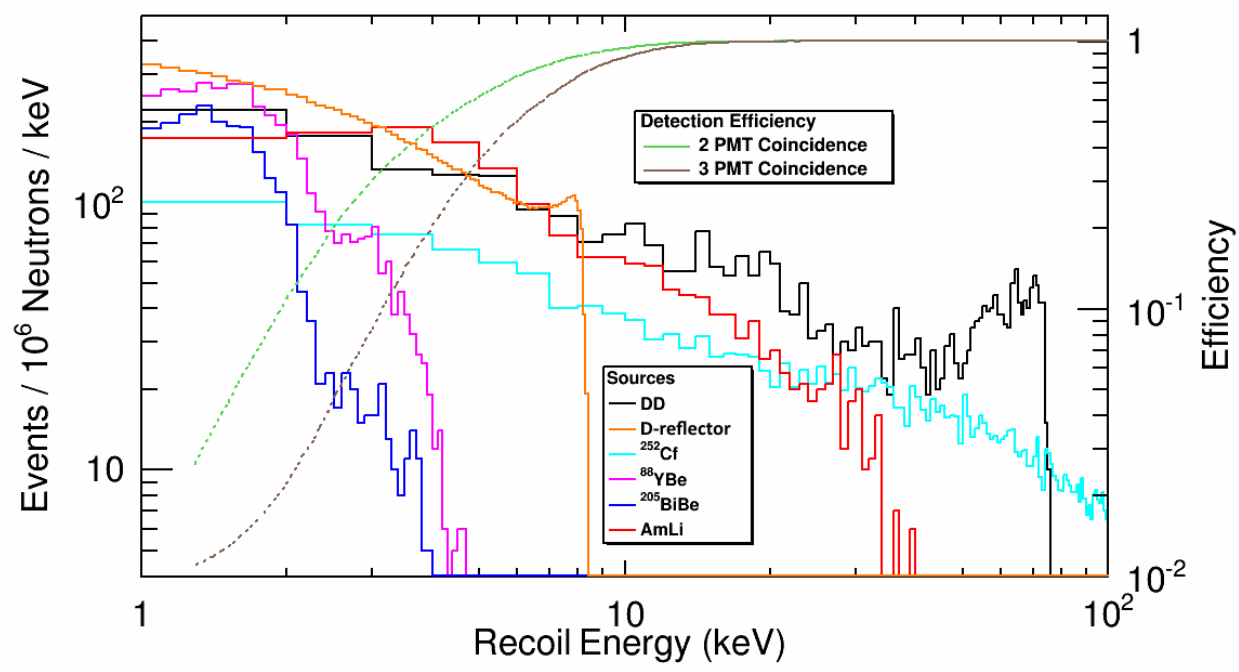

Figure 7.2.1: Recoil spectra obtained from each of the primary neutron sources, DD (black), DD back-scatter off deuterium (orange), ${ }^{252} \mathrm{Cf}$ (cyan), YBe (red), BiBe (blue), and AmLi (magenta). 1 million neutrons corresponds to roughly three hours of calibration with a 100 neutron/s source. The scalings for DD and DD deuterium back-scatter ("D-reflector" above) are arbitrary. These calibrations can easily achieve the single-scatter statistics required and their main goal is to use the double-scatter events. Shown on the $y$-axis on the right is the nuclear recoil detection efficiency for 2 and 3 fold PMT coincidences.

AmBe $(\alpha, n)$ neutron sources have typically been the broad-spectrum neutron source of choice, as they cover the range from threshold to in excess of $300 \mathrm{keV}$ recoil energy. The motivation to also use an AmLi source is the lower maximum neutron energy of about $1.5 \mathrm{MeV}$, which results in a fairly distinct endpoint at about $40 \mathrm{keV}$. Simulations show that the yield of single-scatter NR candidates with energy less than $25 \mathrm{keV}$ is comparable to AmBe but with an enhanced fraction of events at low recoil energy (less than $10 \mathrm{keV}$ ). The rates shown in Figure 7.2.1 would be obtained in about three hours of live time, assuming 100 neutron/s source strength. It is notable that the $(\alpha, n)$ yield is lower for AmLi than for AmBe, so that a higher americium activity of about $2.5 \mathrm{mCi}$ is required to obtain a source strength of 100 neutron/s. The sources will be encapsulated and there are no additional safety concerns.

A generic result of the $(\alpha, n)$ reaction is that the product nucleus may be in an excited state, leading to the release of a gamma. With AmBe, the most common gamma energy is $4.4 \mathrm{MeV}$ and it occurs in $58 \%$ of decays. No literature exists for this process with AmLi sources. Our own preliminary measurements indicate that final state gammas occur at the percent level.

\subsubsection{Gamma Sources for Calibration of the Active Xe TPC}

External gamma sources are not required for any of the primary calibrations of the active Xe TPC. This is by design, as the active region is self-shielded against external gammas. Nevertheless, several important calibrations will be obtained from external gammas. These include studies of higher-energy backgrounds 
and signal fidelity near the edge of the TPC. A single ${ }^{228} \mathrm{Th}(2,615 \mathrm{keV})$ source serves this purpose and, in addition, helps provide a calibration of the outer detector.

\subsubsection{Gamma Sources for Calibration of Xenon Skin and the Scintillator Veto}

The detection thresholds for the Xe skin veto and the organic scintillator veto are $100 \mathrm{keV}$ and $200 \mathrm{keV}$ respectively. Various sources (cf. Table 7.0.1) will be deployed in the source tubes to verify this performance (cf. requirement R-170008 an R-170009). Calibration of the outer detector energy scale (cf. requirement $\mathrm{R}-170009$ ) is achieved with a ${ }^{228} \mathrm{Th}$ source as shown in Figure 7.2.2. The simulated response as a function of $\mathrm{Z}$ in the $\mathrm{Xe}$ skin is also shown in Figure 7.2.2. For the timing synchronicity calibration between the outer detector and the skin, a ${ }^{22} \mathrm{Na}$ source will be deployed (cf. requirement R-170010). The source produces simultaneous back-to-back $511 \mathrm{keV}$ gammas which can be observed in the outer detector and the TPC to look for any difference in timing.
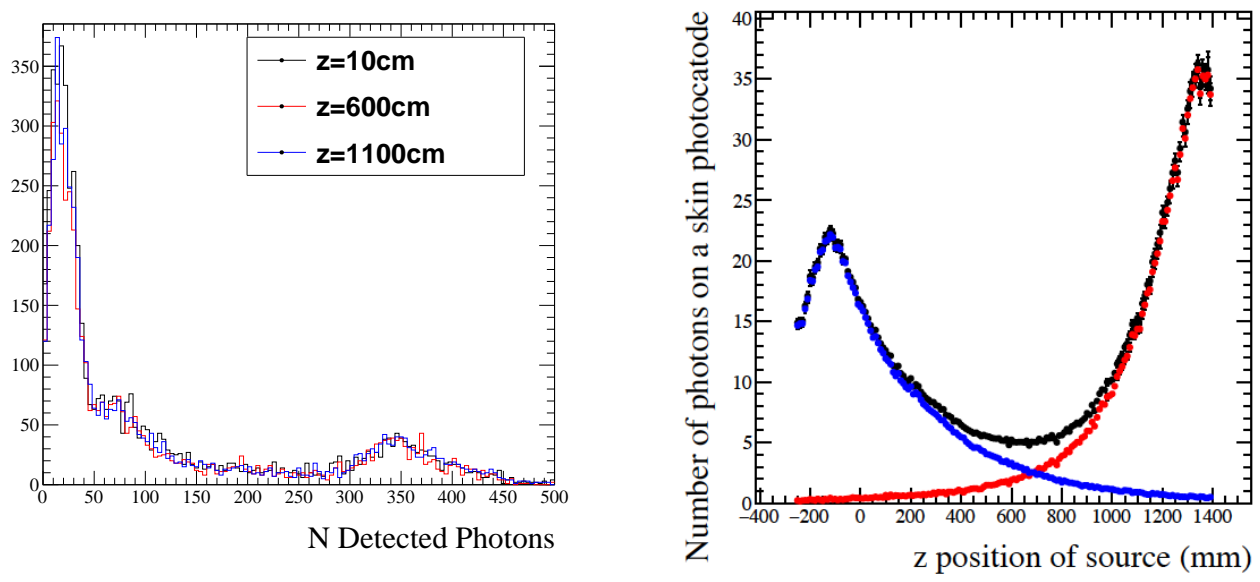

Figure 7.2.2: Number of measured photons detected versus Z-position in the outer detector (left panel, ${ }^{228}$ Th source) and the xenon skin (right panel, ${ }^{57} \mathrm{Co}$ source). The ability to study the performance of the Xe skin as a function of the Z-coordinate motivates the need to be able to locate a source to within $5 \mathrm{~mm}$ (cf. requirement R-170201)

\subsubsection{Calibration Source Deployment (CSD)}

Figure 7.2.3 shows the design of a prototype assembly developed to define the core design and components of the CSD. The source deployment system consists of a stepper motor (a SANMOTION F2 2-phase stepping motor model SH2141-5511 with a resolution of $1.8^{\circ}$ manufactured by SANYO DENKI Co) coupled to the planetary gear-head GP 22A with a 19:1 reduction (manufactured by Maxon). The gear unit couples the stepper motor to a drum holding the deployment filament and source. The deployment filament is a strong thin nylon composite $(\sim 0.1 \mathrm{~mm}$ diameter, maximum load $12 \mathrm{~kg})$ that carries the load of the source assemblies $(\sim 100 \mathrm{~g})$ with a $4 \times$ safety factor. The CSD covers the full $6 \mathrm{~m}$ length of the upper and lower calibration tube from the top of the water tank to beyond the cathode level. The CSD mechanics is housed in a K50 Tee-piece that couples via a connection chamber sideways to the calibration source tube making it a very compact system. The stepper motor and gear are held in a support structure clamped to the T-piece. A wheel on a lever feeds the filament from the drum into the calibration tube. Each calibration tube will 
be fitted with its individual deployment system; the three systems are configured to enable simultaneous operations. A prototype using the same design has been tested at RAL to ensure that thousands of source deployments are possible without failure (cf. requirement R-170202).

As additional control on the z-position of the calibration sources, each deployment system will be fitted with a laser based position monitoring system. For this an ILR1181-30 Micro-Epsilon laser ranger will be coupled to the top of the connection chamber allowing an accurate (better than $2 \mathrm{~mm}$ precision) reading of the source position. The monitoring system will be incorporated in a feedback protocol that drives the CSD system and thus ensures the z-position accuracy of $\pm 5 \mathrm{~mm}$ is met (cf requirement R-170201).

Each system is driven by a ZYBO FPGA board and a PMODSTEP daughter board both manufactured by Digilent. This system allows computer control of the deployment, provides feedback of the operational parameters and will be fully integrated in the Run Control structure.

An unacceptable failure mode of this system would be a source detached from the deployment filament dropped to the bottom of the tube. As a control against this eventuality, the sources are fitted with a ferromagnetic component which allows retrieving sources with a magnet. A full scale mock-up of the design of this system is constructed and extensively tested.

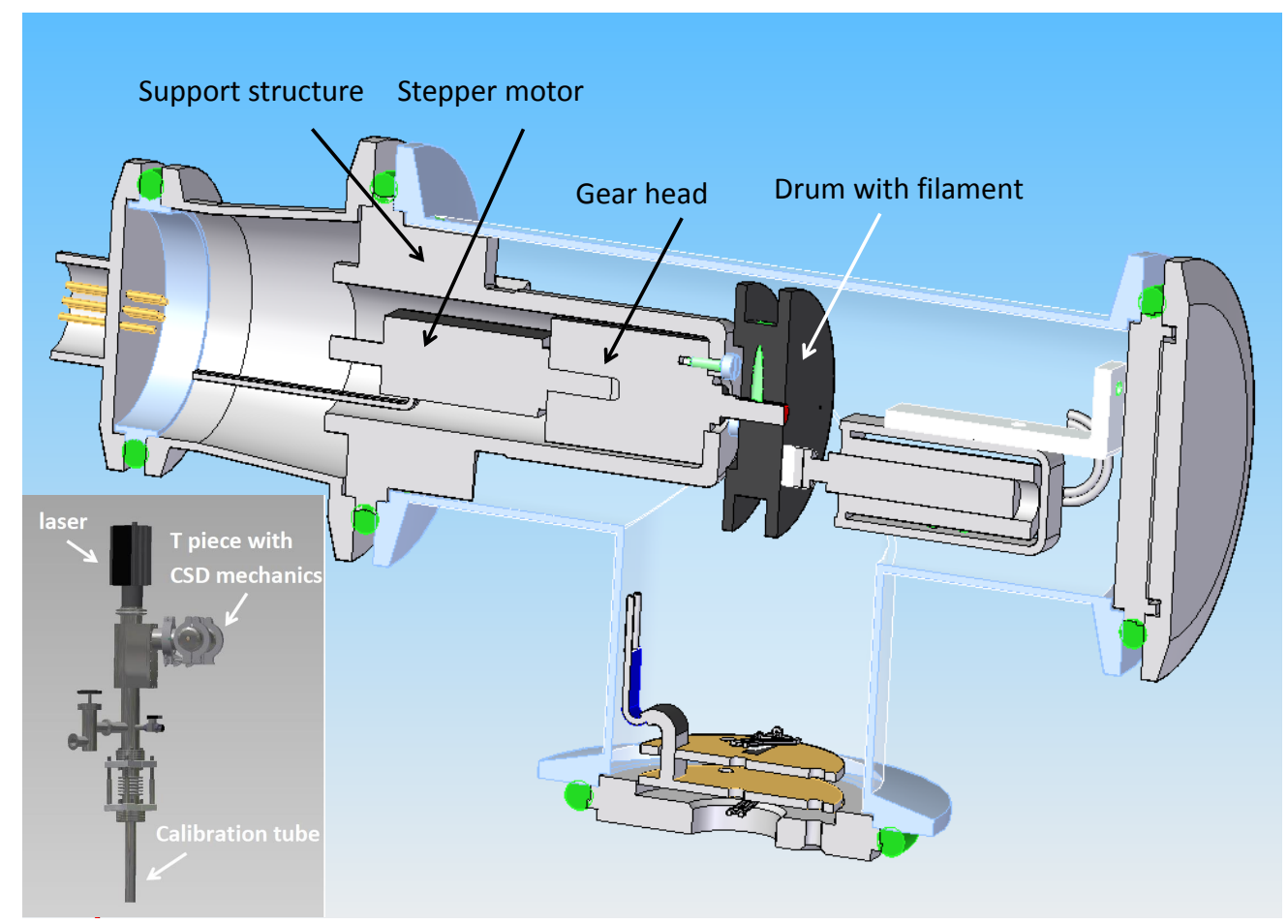

Figure 7.2.3: The prototype assembly developed to define the core design of the CSD system: the stepper motor and gear are held in a support structure clamped to the T-piece. The T-piece couples sideways via a connection chamber to the calibration tube as shown in the inset.

\subsubsection{Radioisotope Capsule Design}

Most sources for use in the CSD will be commercial Eckert \& Ziegler (E\&Z) type R sources. The source form factor is a $5 "$ long X 0.625 " diameter acrylic cylinders with the source epoxied and encapsulated at one end. The bare acrylic end of the cylinder (the end without the source) will be tapped with an M4 
hole, in order to connect to the CSD. The monofilament is captured in a vented screw, which itself secures a martensitic stainless steel (410SS) ring collar to the top of the acrylic cylinder. The use of this ring allows a simple, robust magnetic recovery system can be deployed in the unlikely event of a break in the monofilament which suspends the source.

The AmLi source will be custom fabricated at the University of Alabama since commercial versions are not available in our requisite form factor. Custom sources will have the same form factor and mating structure as commercial sources. Preliminary activities of these sources have been determined. Simulation effort to validate the choices are on-going (cf. requirement R170301).

\subsection{Photoneutron Sources}

As discussed in Section 2.2.1.2, coherent scatters from ${ }^{8} \mathrm{~B}$ neutrinos pose a potential background in $\mathrm{LZ}$ as they are difficult to distinguish from a light mass WIMP. However, higher WIMP masses are unaffected by this issue assuming the signal from such recoils is well understood. Having a calibration targeted at understanding the response of LZ to these low-energy scatters is critical. Because of their well-defined kinematic endpoint, photoneutron sources are the most important tool for this purpose and help to fulfill requirement R-170005.

\subsubsection{Physics of Photoneutron Sources}

Photoneutron sources exploit $(\gamma, n)$ reactions on nuclei to produce neutrons. Suppose there is an incident gamma of energy $\mathrm{E}_{\gamma}$ incident on a nucleus. That nucleus has some threshold, $\mathrm{Q}$, for emission of a neutron by $(\gamma, \mathrm{n})$. If $\mathrm{E}_{\gamma}>\mathrm{Q}$ there is some finite probability for the nucleus to absorb the gamma and emit a neutron with energy [2]

$$
E_{n}(\theta)=\frac{M\left(E_{\gamma}-Q\right)}{m+M}+E_{\gamma} \cos (\theta) \sqrt{\frac{(2 m M)\left(E_{\gamma}-Q\right)}{(m+M)^{3}}}
$$

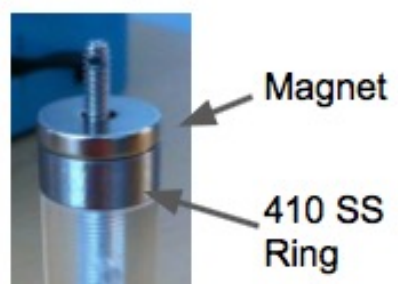

Figure 7.2.4: Prototype dummy source capsule showing 410SS ring with magnetic recovery system attached.

where $\mathrm{M}$ is the mass of the nucleus and $\mathrm{m}$ is the mass of the neutron. The second term, which has an angular dependence, as $\theta$ is defined as the emission angle of the photoneutron relative to the direction of the incident gamma ray. The angular dependence causes a relative difference of $4.6 \%$ in the emitted neutron energy between $\theta=0^{\circ}$ and $\theta=180^{\circ}$ for ${ }^{88} \mathrm{Y}$ Be source (described below). This energy difference is similar to the end point energy difference caused by isotopic spread (5\% between ${ }^{129} \mathrm{Xe}$ and ${ }^{136} \mathrm{Xe}$ ). Thus, photoneutron sources can be considered to produce mono-energetic neutrons. One example of a photoneutron source used by [3] was a ${ }^{88} \mathrm{Y}$ Be source, with $\mathrm{E}_{\gamma}=1.836 \mathrm{MeV}$ and $\mathrm{Q}=1.666 \mathrm{MeV}$, producing $153 \mathrm{keV}$ neutrons. A technical challenge of deploying a photoneutron source is that the cross sections for $(\gamma, n)$ are generally very low. For example, in [3], a $1.85 \mathrm{MBq}^{88} \mathrm{Y}$ source was used and $\sim 330$ neutrons/second were generated. That is $>5,000$ gammas per neutron. For practical purposes such sources need a large amount of lead or tungsten shielding to reduce the gamma rate in the detector, while preserving a useful neutron flux.

Two sources of interest for $\mathrm{LZ}$ are ${ }^{88} \mathrm{Y}$ Be and ${ }^{205} \mathrm{Bi} \mathrm{Be} .{ }^{205} \mathrm{Bi}$ Be creates neutrons of three different energies, the dominant one having an energy of $88.5 \mathrm{keV}$, with a nuclear recoil end point of $2.7 \mathrm{keV}_{\mathrm{nr}}$ in $\mathrm{Xe}$. The nuclear recoil end point from a $152 \mathrm{keV}$ neutron from ${ }^{88} \mathrm{Y} \mathrm{Be}$ is $4.6 \mathrm{keV}_{\mathrm{nr}}$. Figure 7.3.1 shows a 

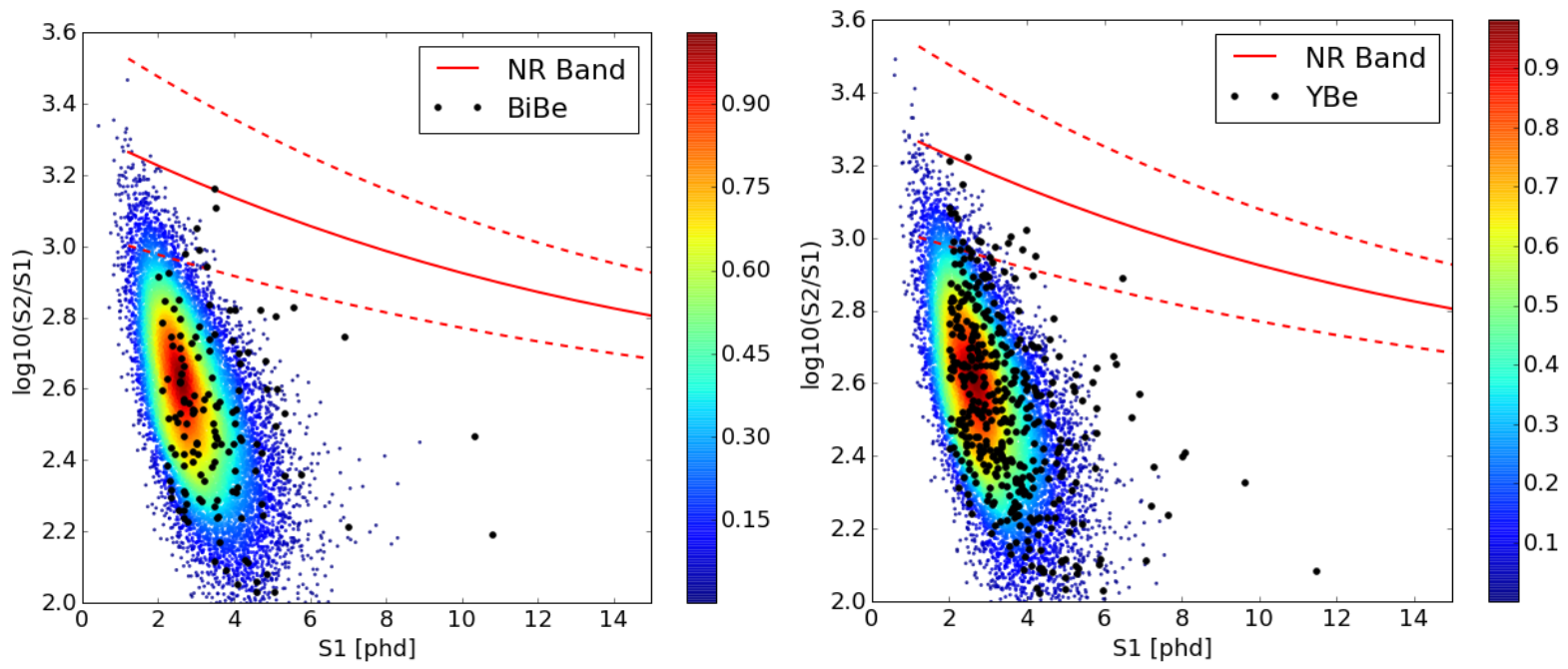

Figure 7.3.1: Comparison of $\mathrm{S} 1$ versus $\mathrm{S} 2$ spectra for ${ }^{8} \mathrm{~B}$ solar neutrino coherent scatters versus ${ }^{205} \mathrm{Bi}$ $\mathrm{Be}$ (top) and ${ }^{88} \mathrm{Y} \mathrm{Be}$ (bottom) in LZ. The ${ }^{8} \mathrm{~B}$ spectrum is a two dimensional PDF represented by the arbitrary color scale. The black dots are the neutron events and the red curves are the nuclear recoil band. The neutron events come from simulation. Events that appear to be at higher energy are the result of multiple scatters, some of which would pass all cuts in a real analysis. Here a 2-fold PMT coincidence cut is assumed, as the live time of these calibrations will be short compared to WIMP search and all the real events will have short drift times. This results in a very low rate of accidental S1+S2 coincidences. The above plot corresponds to 30 hours of calibration with a 100 neutron / s, indicating that with a reasonable activity source, one can perform this calibrations in a few days (cf. requirement R-170404)

comparison of the signals in $\mathrm{LZ}$ from ${ }^{8} \mathrm{~B}$ to the photoneutron sources. ${ }^{88} \mathrm{Y}$ Be bounds the end point for the ${ }^{8} \mathrm{~B}$ spectrum while ${ }^{205} \mathrm{Bi} \mathrm{Be}$ is more similar to the spectrum which would be observed from a 1,000-day exposure. Note that because the source is above the detector, most of the neutron scatters occur near the gate and have short drift times. Although the calibrations will contain a large number of gamma interactions, they are easily separated from the nuclear recoils by their apparent energy. The ER events are dominated by neutron capture gammas, as there is enough tungsten shielding in the design to make the gammas directly from the ${ }^{88} \mathrm{Y}$ or ${ }^{205} \mathrm{Bi}$ decay negligible.

\subsubsection{Photoneutron Source Deployment}

Photoneutron sources will be deployed in a $20 \mathrm{~cm}$ diameter $\times 20 \mathrm{~cm}$ tall cylindrical tungsten alloy pig from above LZ. The pig will weigh $116 \mathrm{~kg}$ and the cryostat is rated to hold this weight (cf. requirement R170401). During calibrations the pig will sit on top of the outer cryostat vessel, on axis. Figure 7.3.2 shows the individual components and the overall deployment scheme. The pig will lowered through a stainless steel guide tube from three points. The guide tube has a funnel top to accept the pig and holes in the side to displace water. There must be minimal water trapped underneath the tungsten (cf. requirement R-170402) and this is achieved by keeping the relevant surfaces relatively flat. There are two critical safety measures designed into this process to protect the cryostat and the experiment. First, the water above the cryostat acts as a cushion, slowing the descent of the pig. Second, the three lifting points are redundant, so even if one cable breaks, the other two can support the weight of the pig. In the unlikely event that the pig were to 
get stuck in the guide tube, the source is separately removable. Furthermore, the entire guide tube can be removed with the pig in it.

\subsection{Deuterium-Deuterium Neutron Source}

A deuterium-deuterium (DD) neutron source, the Adelphi Technologies DD109 neutron generator, will be deployed in the LZ experiment for nuclear recoil calibrations. The generator that will be set up outside of the LZ water tank can produce up to $10^{9}$ mono-energetic $2.45 \mathrm{MeV}$ neutrons per second into $4 \pi$. Conduits in place within the LZ water tank provide a path for neutrons to travel through the water tank and outer detector to reach the Xe volume within the detector (Figure 7.4.1). So as not to degrade the energy of the neutrons, the amount of water in their path needs to be kept small (cf. requirement R-170501). This novel in-situ NR calibration technique has been successfully implemented in the LUX experiment to calibrate the absolute energy response of $\mathrm{NR}$ in $\mathrm{LXe}$ down to $1.1 \mathrm{keV}_{\mathrm{nr}}$ for light yield (Ly) and $0.7 \mathrm{keV}_{\mathrm{nr}}$ for charge yield (Qy) with significant improvements to the calibration uncertainty[4] and has helped improve the LUX detector sensitivity to low-mass WIMPs by orders of magnitude[5].

\subsubsection{Physics motivation of Deuterium-Deuterium Neutron Sources}

This technique will be further upgraded in LZ to explore even lower energy NR response to reduce calibration uncertainty. Four types of measurements are planned to be carried out in LZ using the D-D neutron source as described in the following subsections.
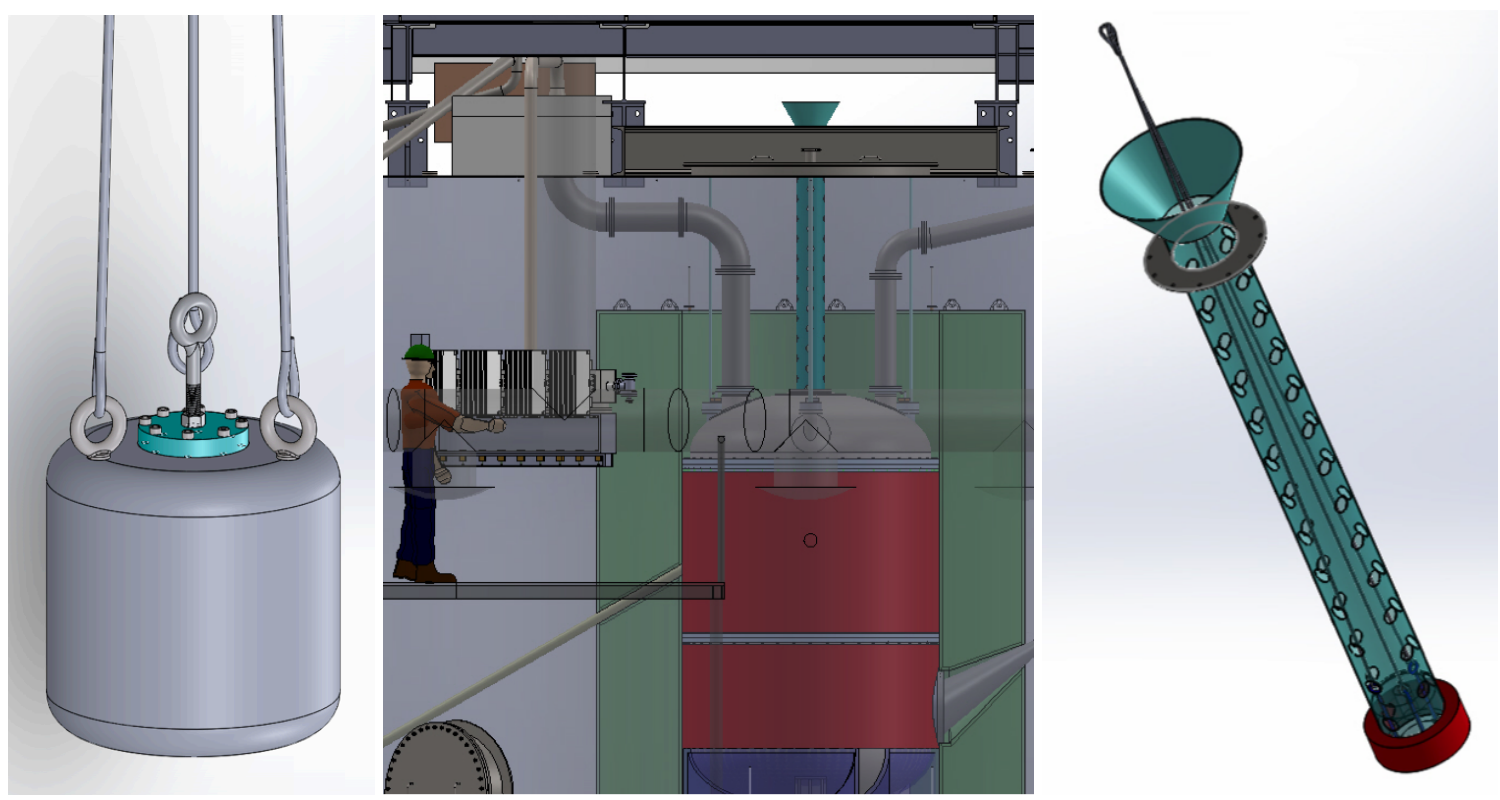

Figure 7.3.2: From left to right: Photoneutron pig $(20 \mathrm{~cm}$ outer diameter $\times 20 \mathrm{~cm}$ height tungsten) suspended from three points, side view of the guide tube in-place on top of the cryostat, guide tube ( 8.25 inch outer diameter $\times 8.0$ inch inner diameter stainless steel) on its own with the funnel top to accept the pig. 


\subsubsection{NR Qy / Ly calibration of the LZ detector}

In the DD calibrations implemented in LUX, double-scatter events in LXe due to mono-energetic $2.45 \mathrm{MeV}$ neutrons are used to calibrate the NR charge yield (S2) response[4]. The absolute deposited energy in the first scatter can be determined by measuring the neutron scattering angle between it and a second scatter. Given the deposited energy, the Qy can be determined by estimating the number of electrons produced at the first scatter. The light yield (S1) can then be inferred by using the calibrated S2 signal to assign an energy deposited to single-scatter events[4]. This calibration technique using $2.45 \mathrm{MeV}$ neutrons covers WIMP search energy range (requirement R-170010) from threshold (6 keV) up to $30 \mathrm{keV}$ (Figure 7.2.1) with an additional endpoint at $74 \mathrm{keV}$.

\subsubsection{Calibrating LZ detector using Reflected Neutrons from D2(O) Target}

As discussed in Section 7.3, an understanding of the LZ response to recoil energies at the threshold (requirement R-170005) is critical to probe low mass WIMP and ${ }^{8} \mathrm{~B}$ solar neutrino signals. By placing a deuteriumloaded reflector behind the DD generator and collecting the neutrons that are reflected at a near-180 degree angle (Figure 7.4.1), the generator's direct $2.45 \mathrm{MeV}$ neutron flux can be converted into a quasi-monoenergetic neutron beam with a minimum energy of $272 \mathrm{keV}$. These lower energy neutrons can be used to calibrate in a new energy regime. Lower energy neutrons provide smaller uncertainty, because the angles are more favorable. In addition, the recoil spectrum endpoint in $\mathrm{Xe}$ is reduced from $74 \mathrm{keV}_{\mathrm{nr}}$ to $8.2 \mathrm{keV}_{\mathrm{nr}}$, thus confining the neutron scatters to within this lower energy region of interest ( 1 to $8 \mathrm{keV}$ ). In addition, the slower incident neutron speed would provide greater separation in S1 times for double scatters, which would assist in the direct Ly calibrations planned for LZ (Section 7.4.1.4).
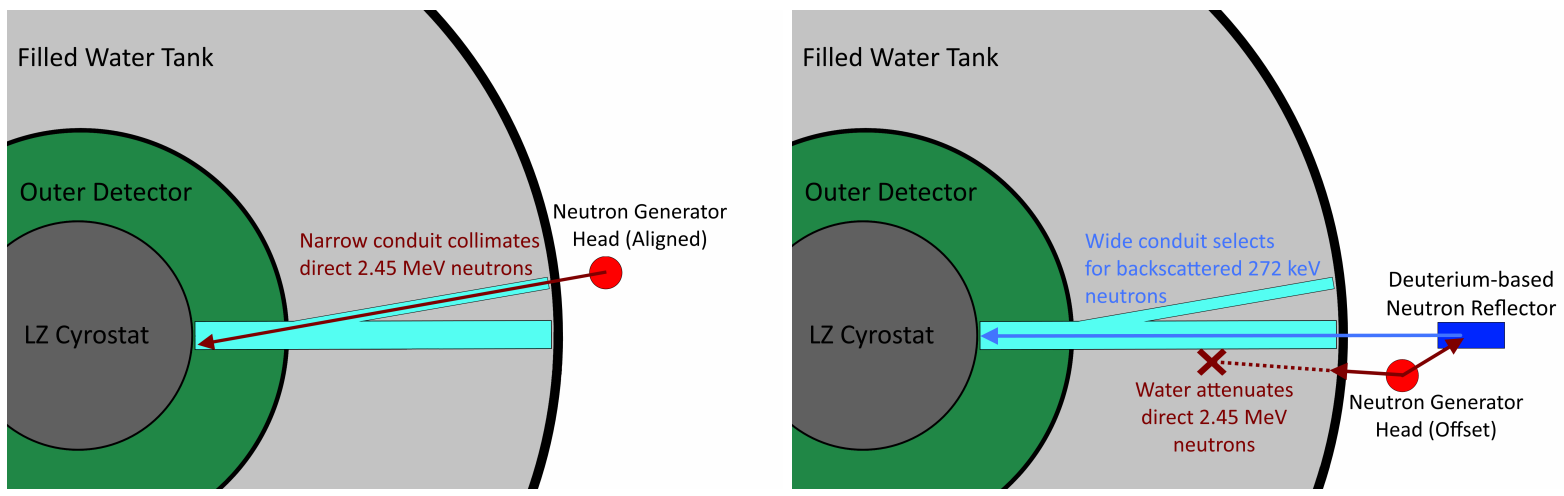

Figure 7.4.1: Approximate setup of the DD generator and neutron conduits. The y-shaped conjoined tube allows for a choice between finer collimation with lower flux by using the smaller, $5.25 \mathrm{~cm}$ inner diameter tube and broader collimation but greater flux by using the larger $15.4 \mathrm{~cm}$ inner diameter tube. The smaller tube joins the larger one in a bonded joint just outside the outer detector, though the path of the neutrons from the smaller tube continues up to the cryostat wall. (Left) The configuration for $2.45 \mathrm{MeV}$ calibration. The neutrons go directly from the generator to the detector through the narrow conduit. (Right) The configuration for $272 \mathrm{keV}$ calibration. The generator head (red) is offset from the neutron conduit to prevent direct neutron flux into the xenon via water attenuation by the water tank, while neutrons reflected off the deuterium reflector (blue) may enter via the air-filled neutron conduit (cyan). 


\subsubsection{Calibrating LZ Detector with Neutron Events with no S1 Light}

Another technique to calibrate LZ response at threshold (requirement R-170005) is to explore neutron events with no scintillation light. This requires an additional tool to establish $\mathrm{t}_{0}$ for the electron drift of a neutron event, which is usually determined by $\mathrm{S} 1$. This additional tool is given by shorter neutron production pulses, resulting in tighter bunches of neutrons. In LZ, our plan is to upgrade the Adelphi Neutron Generator such that it is capable of reducing the neutron bunch width down to the few-microsecond level(as shown in Figure 7.4.2). The neutron trigger pulse will be digitized by one of the DAQ channels to synchronize with other channels that digitize PMT signals. This allows identification of $t_{0}$. There are two direct benefits of implementing this technique. First, it provides an additional timing cut that helps significantly to reduce neutron analysis background events. Second, it allows probing no-S1 neutron events; the measured number of DD scatters with a known number of zero-collected-photon S1 pulses provides considerable statistical leverage and provides a stronger constraint on Ly because the number of no-S1 events can serve as a normalization for the observed number of $S 1>0$ events.

\subsubsection{Direct Ly Measurement Using Double (Multiple) Scatter Events}

A direct light yield measurement will significantly reduce the systematics in the existing technique (Section 7.4.1.1). A direct light yield measurement in LUX is not feasible because S1 light from two scatters overlaps in time. However, given that the LZ LXe volume is at the meter scale, it is possible to separate $\mathrm{S} 1$ signals from the first two scatters in a double (multiple) scatter D-D neutron event by leveraging the time of flight of neutrons in greater path lengths in the LZ LXe volume, especially for $272 \mathrm{keV}$ neutrons, whose speed is a factor of 3 lower than $2.45 \mathrm{MeV}$ neutrons but have similar scattering cross sections, and thus take significantly longer for the second scatter to occur on average Figure 7.4.3. This will thus allow a direct light yield measurement by using double (multiple) scatter events in a similar way to measuring Qy in the standard calibration, helping eliminate the systematics due to S2-energy mapping in the indirect Ly measurement.

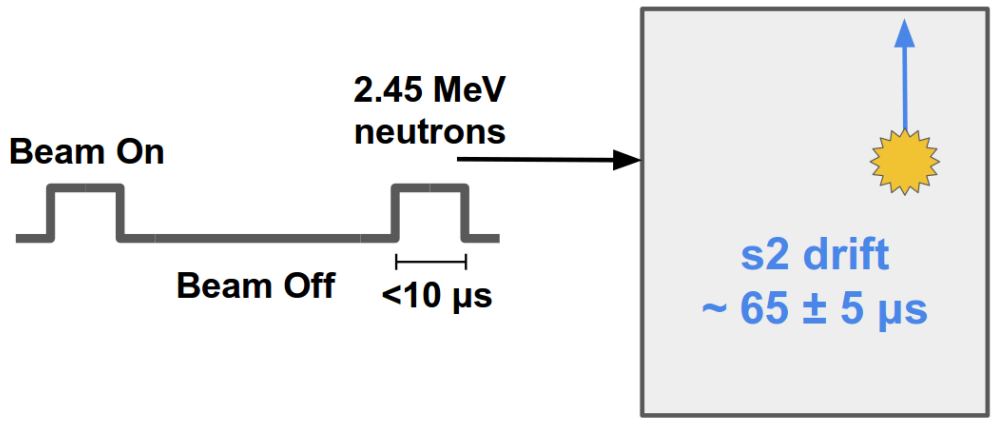

Figure 7.4.2: The square wave on the left indicates the DD trigger pulse. The schematic on the right indicates a no-S1 single-scatter neutron event based on a tube centered $12.5 \mathrm{~cm}$ from the $\mathrm{LXe}$ surface. The $z$ position resolution from the DD trigger is comparable to the $x, y$ position of such events. As described in the text, this technique gives additional capability to probe nuclear recoil response at threshold. 

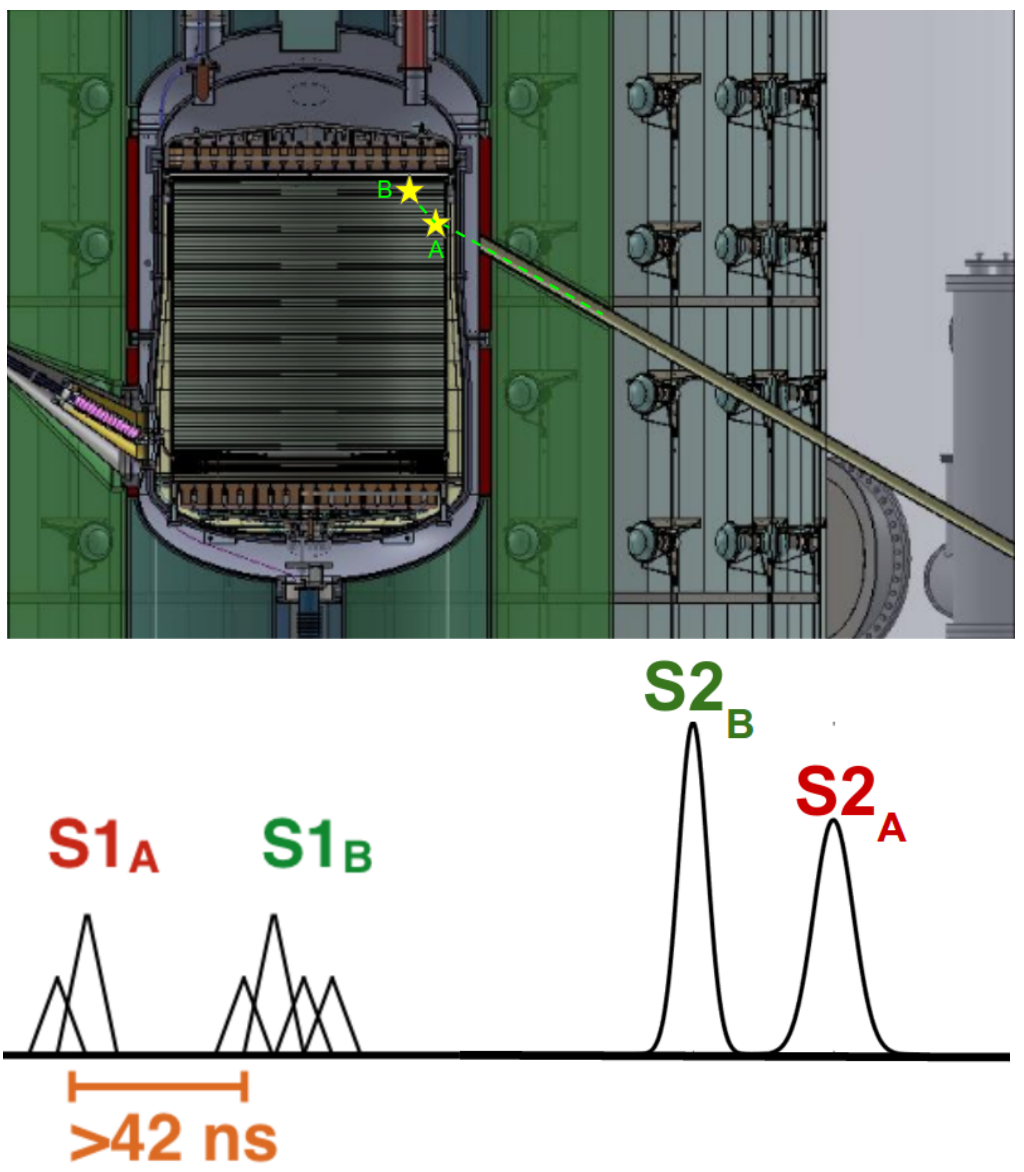

Figure 7.4.3: Top shows the solid model of $L Z$ detector with angled neutron tube. The leveled tube is not shown in this figure. A cartoon neutron double scatter event in the scenario of separated S1s is also shown in the figure. Bottom shows the expected waveform of a neutron double scatter event with two separated S1s.

\subsubsection{DD Source Deployment and Neutron Rates}

The generator, manufactured by Adelphi Technology Inc, uses deuterium-deuterium (DD) fusion to generate neutrons at $2.45 \mathrm{MeV}$. D2 gas is pumped into a chamber where it is ionized via RF induction discharge. A titanium plate is biased using a negative high voltage between -80 and $-125 \mathrm{kV}$, which draws the D+ ions. The ions embed in the titanium plate, forming titanium hydrate. Subsequent ions strike the embedded ions and eject neutrons via the fusion reaction $\mathrm{D}+\mathrm{D} \rightarrow{ }^{3} \mathrm{He}+n$. At maximum operating parameters, the generator is designed to output $10^{9} \mathrm{n} / \mathrm{s}$ into $4 \pi$. [6]

The DD generator is located outside the LZ water tank. The neutrons will be collimated via two sets of tubes inside the water tank, each of which has two options for the diameter: a $16.8 \mathrm{~cm}$ outer diameter tube and a $6.03 \mathrm{~cm}$ outer diameter tube. (See Figure 7.4.1 for a drawing of the two-diameter approach and Figure 7.4.3 for a depiction of the angled tube.) During WIMP search, the tubes are filled with water, thus maintaining the water shielding around the detector. During calibrations, the tubes are drained of water and filled with a nitrogen purge, which allows the neutrons to pass into the detector. One tube is horizontal and will be centered $12.5 \mathrm{~cm}$ below the LXe surface, while one is angled 20 degrees to the horizontal, the center of which enters the LXe volume $40.5 \mathrm{~cm}$ below the LXe surface. The horizontal tube mimics the 
implementation of the LUX DD tube, which has been used to great effect, while the angled tube seeks to take advantage of the preferential forward scattering via the higher precision in $z$-coordinate reconstruction.

The generator itself will be mounted on a platform supported by a modified SLA-10 (Super Lift Advantage) Genie lift. This lift can be moved to either neutron conduit site and will be capable of raising the generator head to the entrance of both neutron conduits. Form-fitting generator shielding will adequately reduce neutron flux in the Davis Cavern, and plugs in the shielding at both conduit angles will be added or removed to allow for maximum flux into the desired conduit.

For the DD backscatter calibrations, the generator head is placed off-axis of the neutron conduit while a backscatter target is aligned with the conduit. As the neutrons are produced by the generator head, those hitting the deuterium backscatter target scatter at a near $180^{\circ}$ angle and are sent down the conduit to the the interior of LZ, while direct neutron flux is attenuated by the water tank. Kinematically, the $180^{\circ}$ backscatter results in a reduction in neutron energy from $2.45 \mathrm{MeV}$ to $272 \mathrm{keV}$. Losses in neutron flux due to backscattering will be compensated by a heightened generator neutron flux (up from $4 \times 10^{6} \mathrm{n} / \mathrm{s}$ to $1 \times 10^{9}$ ). The diameter of the neutron conduit $(15.4 \mathrm{~cm}$ ID) is driven by the need to get enough flux in deuterium backscatter calibration.

\subsection{Calibration Rates}

In general, event rates in the LZ detector of course depend on source location, type and energy spectrum. For neutron calibrations, a number of generally applicable conclusions can be obtained from the representative case of $2.45 \mathrm{MeV}$ neutrons emanating from the wall of the outer Ti cryostat vessel. The scenario is identical to what would be realized during a DD neutron calibration, assuming neutrons that strayed out of the conduit do not contribute appreciably to the event rate in the Xe TPC. The scenario is also qualitatively similar to the foreseen AmLi calibration, obtained from a source located in one of the three external source tubes.

\subsubsection{Effect of Gd doping in liquid scintillator}

A generic concern for neutron calibrations of the Xe TPC is that Gd doping of the liquid scintillator in the outer detector will cause a large rate of gamma events in the Xe TPC. Monte carlo simulations suggest that this concern is not warranted. As shown in Figure 7.5.1, the event rate due to neutron captures on Gd is only slightly larger than it would have been with undoped liquid scintillator, and only in the energy window $2.2 \mathrm{MeV} \lesssim E \lesssim 4.0 \mathrm{MeV}$.

\subsubsection{Effect of gamma captures on useful neutron event rates}

A key number for neutron calibrations is the fraction of useful events. As mentioned above, this will depend on the geometry and energy of the source. Table 7.5.1 provides numbers for the simplified, representative case described at the beginning of Section 7.5. Shown are fractions of neutrons events for various scenarios. Single scatters with gamma veto provide the relevant useful event fractions for defining the NR band. $\sim 3 \%$ of neutrons result in a useful single-scatter in the detector, after removing events that contain any ER component. Double scatters with gamma veto provide the relevant useful event fractions for most DD studies.

\subsubsection{Maximum useful calibration rates}

The maximum useful calibration rate will in most cases be driven by the drift time of the TPC. This limitation can be circumvented by delivering events near the top of the TPC, in order to shorten the drift time. However, 


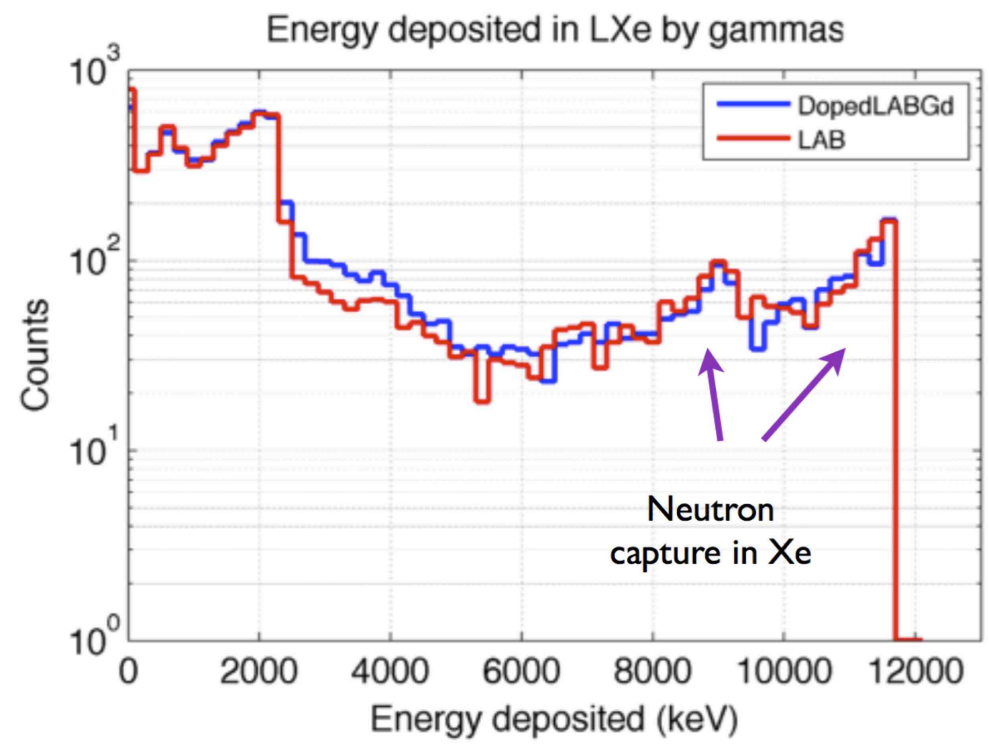

Figure 7.5.1: Event rate due to gammas in the xenon TPC from neutron captures on Gd.

Table 7.5.1: Fraction of neutron scatters in the Xe TPC for various assumptions about the outer detector material in the acrylic vessels. Gd doped LAB is the baseline choice. LUX, which has only $\mathrm{H}_{2} \mathrm{O}$ outside the TPC, is shown for reference. Also shown for comparison to make the impact of LAB and Gd doping clear are LZ with LAB only (no Gd doping) and water instead of scintillator.

\begin{tabular}{|l|c|c|c|c|}
\hline & \multicolumn{3}{|c|}{ LZ } & LUX \\
\hline OD material & Gd-doped LAB & LAB & $\mathrm{H}_{2} \mathrm{O}$ & - \\
\hline single scatters (no cuts) & 0.129 & 0.123 & 0.115 & 0.266 \\
\hline single scatters ( $\gamma$ veto) & 0.032 & 0.031 & 0.033 & 0.164 \\
\hline double scatters (no cuts) & 0.106 & 0.107 & 0.114 & 0.073 \\
\hline double scatters ( $\gamma$ veto) & 0.020 & 0.025 & 0.026 & 0.042 \\
\hline
\end{tabular}

in the interest of understanding the most general case first, the projections shown in Figure 7.5.2 assume an event window defined by twice the maximum drift time.

For an average event rate $\lambda$ in a time window $t_{w}$, the probability to obtain $n$ or more random events in any particular window is given by

$$
P=e^{-\lambda} \sum_{n=1}^{\infty} \frac{\lambda^{n}}{n !},
$$

from which Figure 7.5.2 is derived. A $10 \%$ pileup rate could be achieved by keeping the calibration rate below 50 to $70 \mathrm{~Hz}$, depending on the drift time. 


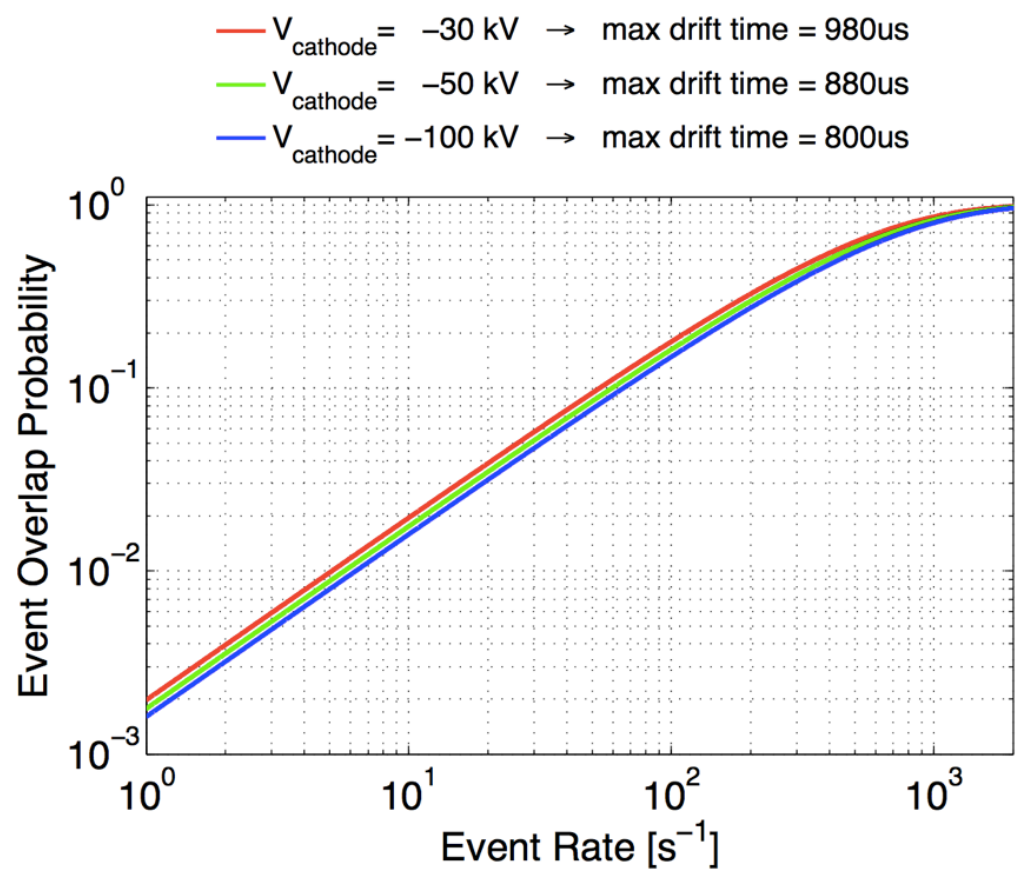

Figure 7.5.2: The electron drift time produces a time window in which an unrelated event could produce S1-S2 pairing confusion. The Poisson probability of event overlap is plotted here, and provides an estimate as to the maximum calibration rate in LZ for a desired maximum event overlap probability, for a specified field configuration (electron drift velocity), and a specified event distribution in $z$. Here, we assume all calibration events are at the position of longest drift time, and vary the field configuration according to several possible running modes. If a $10 \%$ overlap probability is acceptable, then the bottom of the detector could be calibrated at 50 to $70 \mathrm{~Hz}$.

\subsection{Environment, Health and Safety Concerns}

Safety concerns related to calibrations mainly relate to the use of radioactive sources. Most sources are either internal, and therefore don't expose users to radioactivity, or are NRC exempt. The most active source to be used in LZ is the DD generator, which will be capable of producing $10^{9}$ neutrons/second. This source was also used in LUX and the safety concerns were mitigated by having an exclusion zone around the generator while it is operating. Because the generator can be operated remotely, no one needs to be inside this exclusion zone during operation. For LZ there is a planned $9 \mathrm{~m}$ exclusion zone. Points inside and outside this exclusion zone will be monitored with neutron detectors to ensure the areas where work will continue to take place are still safe.

Photoneutron calibrations require gamma sources with activities up to $3 \mathrm{MBq}(81 \mu \mathrm{Ci})$ which are not NRC-exempt. However because these sources will always be deployed inside of a tungsten shield, there is no reason they can't generally live inside an insert with a small amount of shielding to protect the users. Only briefly upon initial receipt of a source does a user need to handle one outside of such shielding. Specific safe handling procedures are under development. 


\subsection{Bibliography}

[1] Hamamatsu Photonics K. K., Photomultiplier Tubes: Basics and Applications (2007), Third Edition (Edition 3a).

[2] G. F. Knoll, Radiation Detection and Measurement (John Wiley \& Sons, Hoboken, NJ, USA, 1989).

[3] J. I. Collar, Phys. Rev. Lett. 110, 211101 (2013), arXiv:1303.2686 [physics.ins-det].

[4] D. S. Akerib et al. (LUX), "Low-energy (0.7-74 keV) nuclear recoil calibration of the LUX dark matter experiment using D-D neutron scattering kinematics," (2016), submitted to Phys. Rev. C, arXiv:1608.05381 [physics.ins-det].

[5] D. S. Akerib et al. (LUX), Phys. Rev. Lett. 116, 161301 (2016), arXiv:1512.03506 [astro-ph].

[6] DD-108M Neutron Generator Operation Manual, Adelphi Technologies Inc., 2003 E. Bayshore Rd., Redwood City, CA 94603 (2012). 


\section{Electronics, DAQ, Controls, and Online Computing}

This chapter describes the LZ signal processing electronics, data acquisition, detector-control system, and online data processing.

\subsection{Signal Processing}

The processing of the signals generated by the TPC PMTs is schematically shown in Figure 8.1.1. The TPC PMTs operate at a negative HV supplied by the LZ HV system, described in more detail in Section 8.6. HV filters are installed at the HV flange on the breakout box. The PMT signals leave the breakout box via a different flange and are processed by the analog front-end electronics, described in more detail in Section 8.3. The amplified and shaped signals are connected to the data acquisition system (DAQ), described in more detail in Section 8.5. The digitized data are sent to Data Collectors and stored on local disks. The PMTs of the outer-detector system operate at positive HV. The processing of the signals from these PMTs, shown schematically in Figure 8.1.2. The same type of amplifier used for the Xe PMTs is used for the outerdetector PMTs. Gain and shaping parameters of these amplifier will be fixed once the operating conditions of the outer-detector PMTs have been finalized.

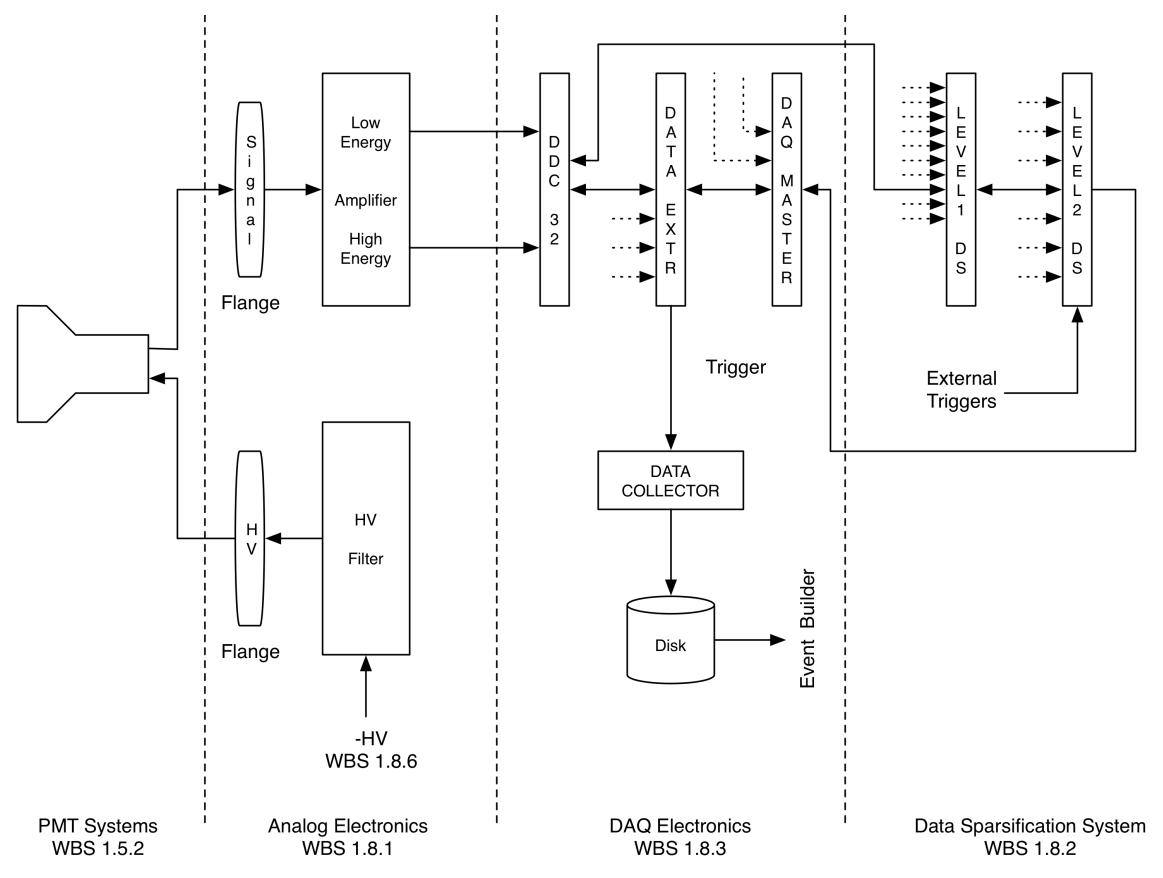

Figure 8.1.1: A schematic of the signal processing of the TPC PMTs. The TPC PMTs use dual-gain signal processing. The skin PMTs only utilize the low-energy section of the amplifiers. 


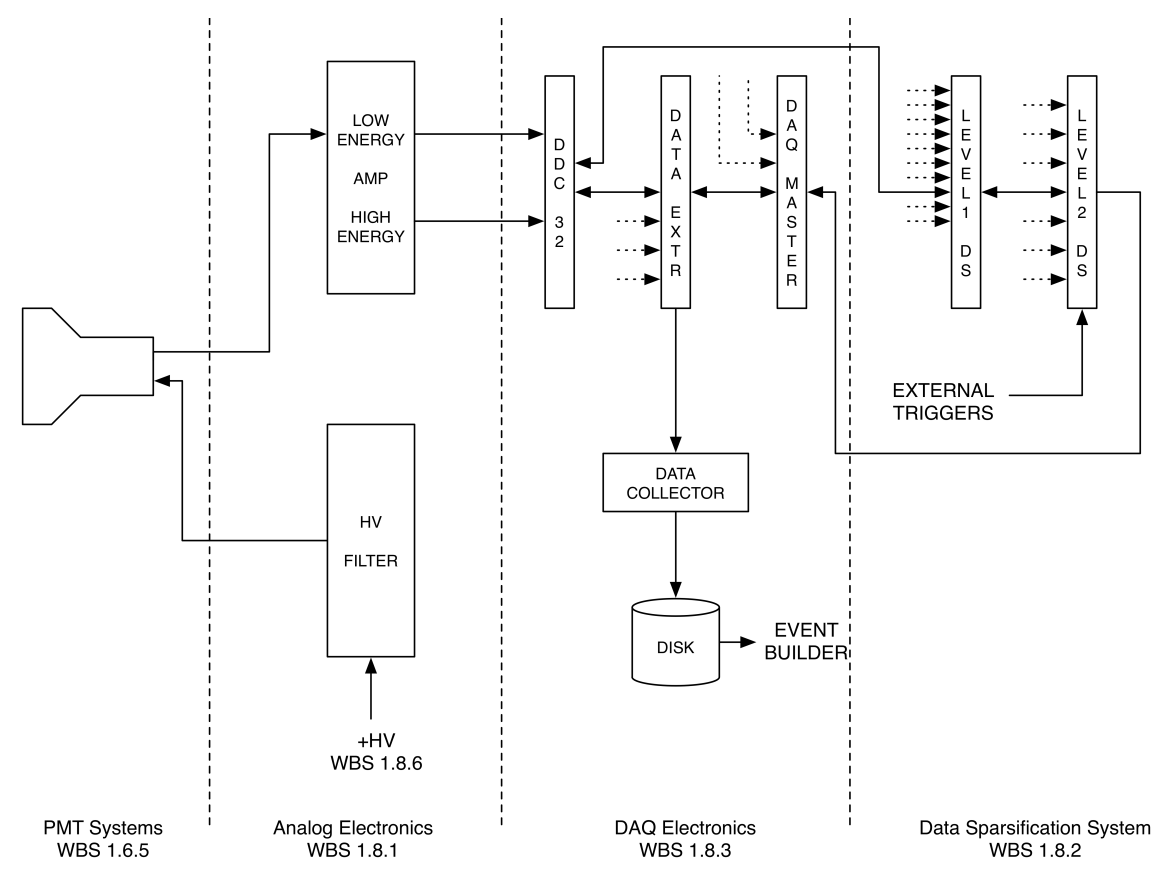

Figure 8.1.2: A schematic of the signal processing of the outer-detector PMTs. The outer detector PMTs use dual-gain signal processing.

The data flow is schematically shown in Figure 8.1.3. The event builder assembles the events by extracting the relevant information from the Data Collector disks, DAQ1 - 15. This step is discussed in more detail in Section 8.9. The event files are stored on local RAID arrays, RAID 1 and RAID 2, before being distributed to the data-processing centers for offline data processing and analysis.

\subsection{Requirements}

The parameters of the analog and digital electronics are defined based on the properties of the PMT signals, the required dynamic range of the different PMTs, and the expected calibration rates.

Three different PMTs are used in LZ. The TPC PMTs will see S1- and S2-type signals. The skin and outer-detector PMTs will only see S1-type signals. The relevant properties are listed in Table 8.2.1.

The cables that connect the xenon PMTs to the analog electronics are 40 to $50 \mathrm{ft}$ long; the actual length depends on the final design of the conduits and the location of the area in which the analog electronics will be installed. The internal cables will be similar to the type used for LUX (Gore). Measurements with 45-ft-long LUX cables have shown an area reduction of S1 signals by approximately 13 to $23 \%$.

The design of the analog electronics is constrained by the required dynamic range of the LZ signals. Our design relies on the assumption that a single photoelectron (SPHE) detected in a TPC PMT generates a $11.5 \mathrm{mV}$ ns pulse at the input of the amplifiers. The required dynamic range is defined by the sources used to calibrate LZ and the desire to detect high-energy events for background studies. Chapter 7 provides details on the LZ calibrations.

The DAQ system design is based on our experience with LUX and the required LZ calibration rates. Typical calibration rates are listed in Table 8.2.2. The TPC source calibration rates are limited by the maximum drift time of $700 \mu$ s in LZ. During source calibrations of the TPC, a $150 \mathrm{~Hz}$ calibration rate results in a $10 \%$ probability of detecting a second calibration event within the drift time of the previous calibration 


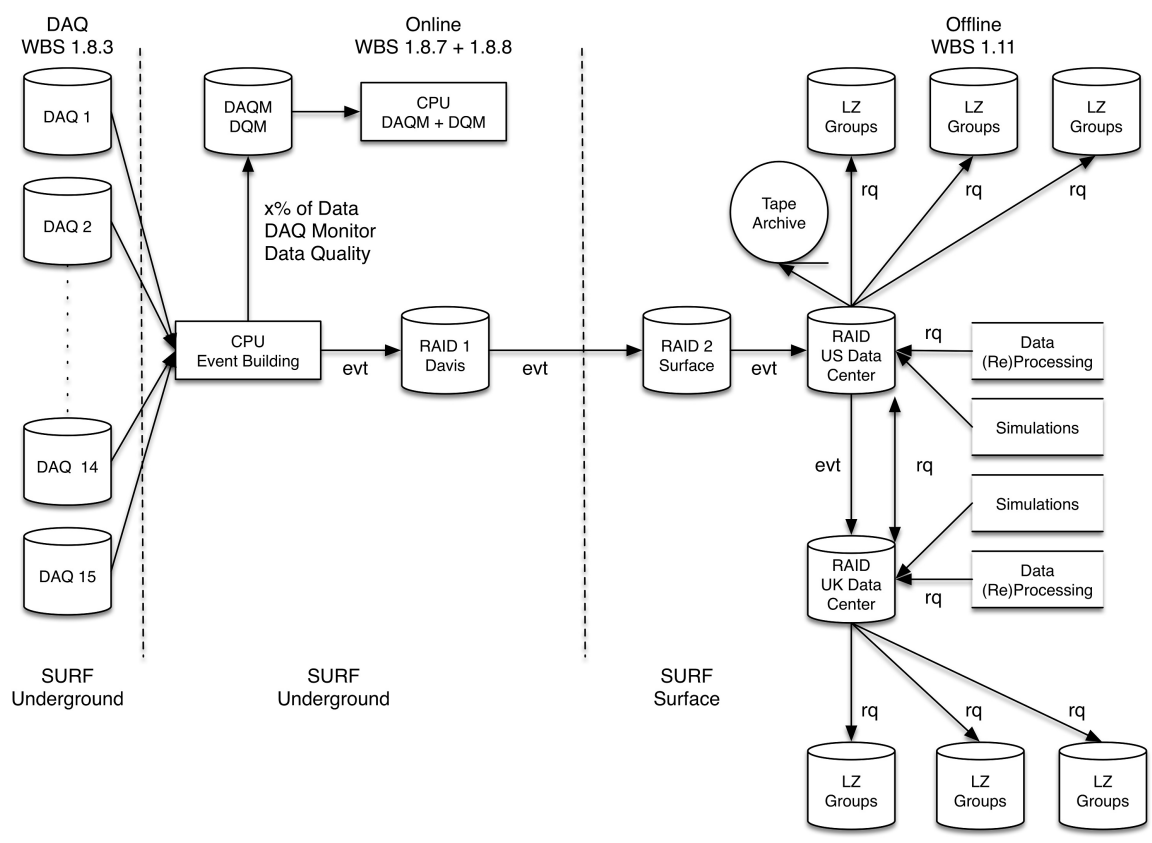

Figure 8.1.3: A schematic of the data flow.

event. Neutron calibrations are carried out to define the NR band in the TPC. These calibrations utilize external neutron sources and a neutron generator. External sources, inserted into the source tubes around the central cryostat, are used to calibrate the skin and the outer-detector PMTs. The count rates for these calibrations are not limited by the drift time in the TPC and they can be carried out at substantially higher rates. Weekly LED calibrations are done to examine the SPHE response of the PMTs and to monitor the PMT response. These calibrations can be carried out with rates as high as $4 \mathrm{kHz}$.

Table 8.2.1: Properties of the PMTs

\begin{tabular}{|l|c|c|c|r|}
\hline System & Type PMT & Number & Gain & HV \\
\hline TPC & R11410-20 & 494 & $<5 \times 10^{6}$ & $<1,750 \mathrm{~V}$ \\
\hline Skin & R8520 & 93 & $<1 \times 10^{6}$ & $<900 \mathrm{~V}$ \\
\hline Skin & R8778 & 38 & $<5 \times 10^{6}$ & $<1,750 \mathrm{~V}$ \\
\hline Outer Detector & R5912 & 120 & $<1 \times 10^{7}$ & $<1,750 \mathrm{~V}$ \\
\hline
\end{tabular}

The data volume to be handled by the DAQ system can be estimated on the basis of our experience with LUX. In WIMP search mode, we will focus on events with energy depositions below $40 \mathrm{keV}$. During krypton calibrations, in which the total energy deposition is $41.6 \mathrm{keV}$, the average LUX event size was $203 \mathrm{kB}$ (or $1.7 \mathrm{kB} / \mathrm{channel}$ ). The size of each event was dominated by the width of the $\mathrm{S} 2$ signals. The event size of LZ can be estimated by scaling the LUX event size by the ratio of the number of PMT channels. Because LZ has four times as many TPC PMTs as LUX, and taking into account the dual-gains, we expect that the event size in LZ will be roughly 8 times as large as the LUX event size, or 1.6 MB. This estimate does not include the data volume associated with the outer-detector and the skin PMTs. If each outerdetector and skin PMT detects a single S1-like pulse, that increases the event size by about $45 \mathrm{kB}$. With 
compression, the typical event size for $\mathrm{LZ}$ is estimated to be $0.53 \mathrm{MB}$. Monte Carlo simulations show that the total background rate in $\mathrm{LZ}$ will be about $40 \mathrm{~Hz}$. The background rate in the WIMP search region $(0$ to $40 \mathrm{keV}$ ) will be about $0.4 \mathrm{~Hz}$. At $40 \mathrm{~Hz}$, the data rate is $21 \mathrm{MB} / \mathrm{s}$. In 1,000 days, LZ will thus collect 1.9 PB of WIMP-search data. Other estimates, including energy depositions above $41.6 \mathrm{keV}$, result in an estimated total data volume of $2.8 \mathrm{~PB}$. By optimizing the event selection, we expect to be able to reduce the total volume of WIMP-search data by a factor of 2 (see Section 8.5.2).

Table 8.2.2: Calibrations and expected count rates.

\begin{tabular}{|l|c|c|c|}
\hline Calibration Type & System & Typical Count Rate & Frequency \\
\hline Internal sources & TPC & $<150 \mathrm{~Hz}$ & Twice a week (Kr) \\
\hline External gamma sources & Skin and outer detector & TBD & TBD \\
\hline Neutrons & TPC & $<150 \mathrm{~Hz}$ & TBD \\
\hline LEDs & TPC and outer detector & $4 \mathrm{kHz}$ & weekly \\
\hline
\end{tabular}

\subsection{Analog Electronics}

\subsubsection{Design Criteria}

The analog front-end design for the LZ experiment has benefited immensely from the experience with the LUX detector. In what follows, we have retained all the features of LUX electronics that performed well, while improving in some areas. The most important figure of merit of the LUX analog front end was its low noise characteristics, which allowed us to set our thresholds well below the SPHE level. Assuming the LZ noise characteristics will be the same as those for LUX, we expect to be able to run comfortably with a threshold of 0.25 PHE (photoelectron) for each PMT. Figure 8.3.1 shows a simulated distribution of total S1 pulse area for events in which one PHE each is detected in two PMTs (left) and in three PMTs (right). A threshold of $>0.25 \mathrm{PHE}$ is set for each PMT. The distributions are governed primarily by the $\sim 34 \% \mathrm{rms}$ width of the PMT signal (gain variation) and receives almost no contribution from electronic noise. Setting a threshold at 1 PHE for the total S1 signal yields an efficiency of $\sim 93 \%$ for events that produce at least two PHEs in the PMTs. For the case of three PHEs, the corresponding efficiency is approaching $\sim 100 \%$. These electronics thresholds correspond to the lowest energy threshold achievable in such a detector, and hence drive the noise specifications for the front end. The choice between two- or three-fold coincidence will be governed by the dark currents in the PMTs, which have been specified to be in the 50 to $200 \mathrm{~Hz}$ range.

The second key design parameter for the LZ front end is the dynamic range, which is defined by the energy range of the sources used to calibrate LZ. Isotopes such as ${ }^{83 \mathrm{~m}} \mathrm{Kr}$ (32.1 and $9.4 \mathrm{keV}$ transitions), activated $\mathrm{Xe}(236 \mathrm{keV}$ and $164 \mathrm{keV}$ transitions), and tritium (endpoint at $18.2 \mathrm{keV}$ ) will be present or injected directly into the LXe volume and will be used to calibrate the detector periodically. LZ will retain the ability to detect high-energy events that saturate the PMTs of the top array by using only the light collected by the bottom array to determine the total $\mathrm{S} 2$ area.

The LZ electronics will provide excellent resolution for single liquid electrons, which are expected to yield at most 50 PHEs, depending on the height of the gas gap and the strength of the electric field in that region of the TPC. The typical duration of such pulses will be about 0.5 to $1.1 \mu \mathrm{s}$. At the same time, we will need to provide extremely clean measurements of SPHEs in order to have a sharp turn-on of the S1 efficiency. SPHE spectra also help with maintaining an in situ calibration of the PMT gains. To meet these 
requirements, the analog electronics provides one low-energy (high-gain) and one high-energy (low-gain) output for each PMT.

Figure 8.3.2 shows this concept. The high-energy channel has low gain and a 30-ns full width at tenth maximum (FWTM) shaping-time constant. Its dynamic range is defined by the 236-keV Xenon activation line. The low-energy channel has a 10 times higher area gain and wider shaping of $60 \mathrm{~ns}$ FWTM. It is optimized for an excellent SPHE response and dynamic range. The shaping times and gains are derived from one assumption: the DAQ will have a usable dynamic range of $1.8 \mathrm{~V}$ at the input and will sample the pulses at $100 \mathrm{MHz}$ with 14-bit accuracy. A $0.2 \mathrm{~V}$ offset is applied to the digitizer channels in order to measure signal undershoots of up to $0.2 \mathrm{~V}$. In summary, the relevant parameters are:

- Typical SPHE response of the PMTs: $11.5 \mathrm{mV}$ ns pulse. The performance should also be verified if this value is as low as $6.5 \mathrm{mV}$ ns for some PMTs

- The dark current for the PMTs at operating voltage has been specified to be in the 50 to $200 \mathrm{~Hz}$ range. The distribution within this range is not yet known.

- S1 light yield at $236 \mathrm{keV}$ : 620 PHEs (for $650 \mathrm{~V} / \mathrm{cm}$ ).

- S1 light distribution: evenly distributed over all PMTs. The bottom array receives $80 \%$ of the total S1 light.

- S2 light yield for ERs at 236 keV: 42 liquid electrons / keV and 70 PHEs / extracted liquid electron (conservative maximum).

- S2 light distribution: $22 \%$ of the S2 light is in a single top PMT. The S2 light is distributed evenly over all bottom PMTs; the bottom array receives $45 \%$ of the total S2 light.

The op-amps indicated in Figure 8.3.2 (left) are very similar to those used in LUX. The gain and shapingtime constants of the amplifiers were optimized using simulations. Figure 8.3.3 and 8.3.4 shows the results of simulations of $\mathrm{S} 2$ pulses associated with the 236-keV transition in activated Xe (top) and a 3-MeV energy deposition (bottom). Figure 8.3.3 shows that $\mathrm{S} 2$ saturation in the top PMT array for the 236-keV transition is not a problem if the amplitude of a single PHE is less than $1.5 \mathrm{mV}$ (12 ADCC). If we allow one PMT to saturate, single PHEs of up to $3.0 \mathrm{mV}$ can be accommodated. Figure 8.3.4 shows that the $\mathrm{S} 2$ associated with larger energy depositions will not start to saturate the PMTs of the bottom array if the amplitude of a
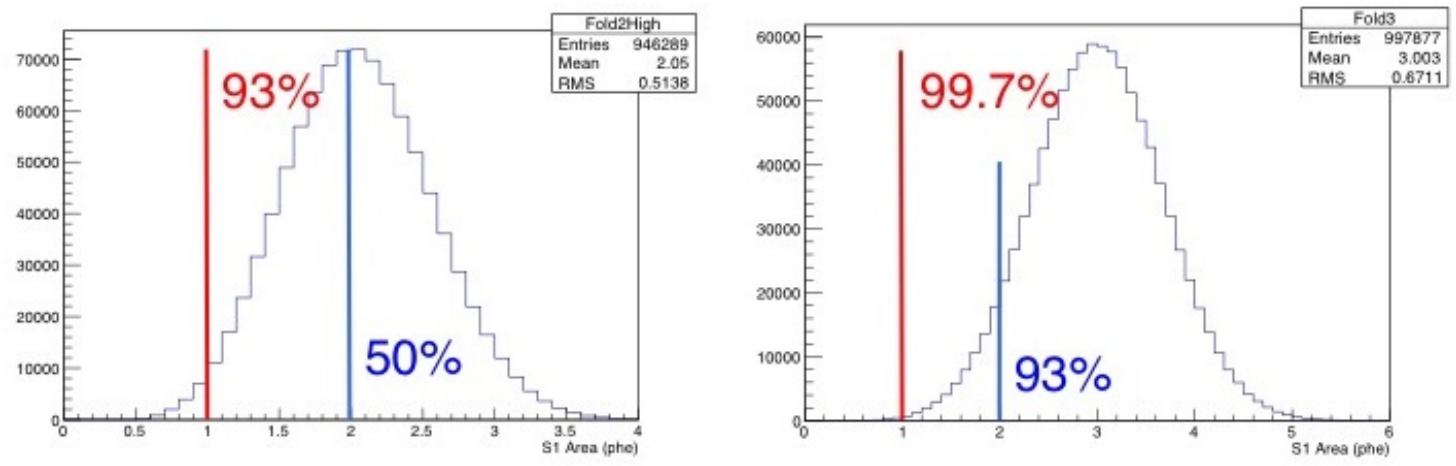

Figure 8.3.1: A simulated distribution of total pulse area for $\mathrm{S} 1$ pulses with two (left) and three (right) photoelectrons (left). Requiring a total area larger than 1 PHE provides nearly $100 \%$ efficiency for events with 3 or more S1 photoelectrons detected. 

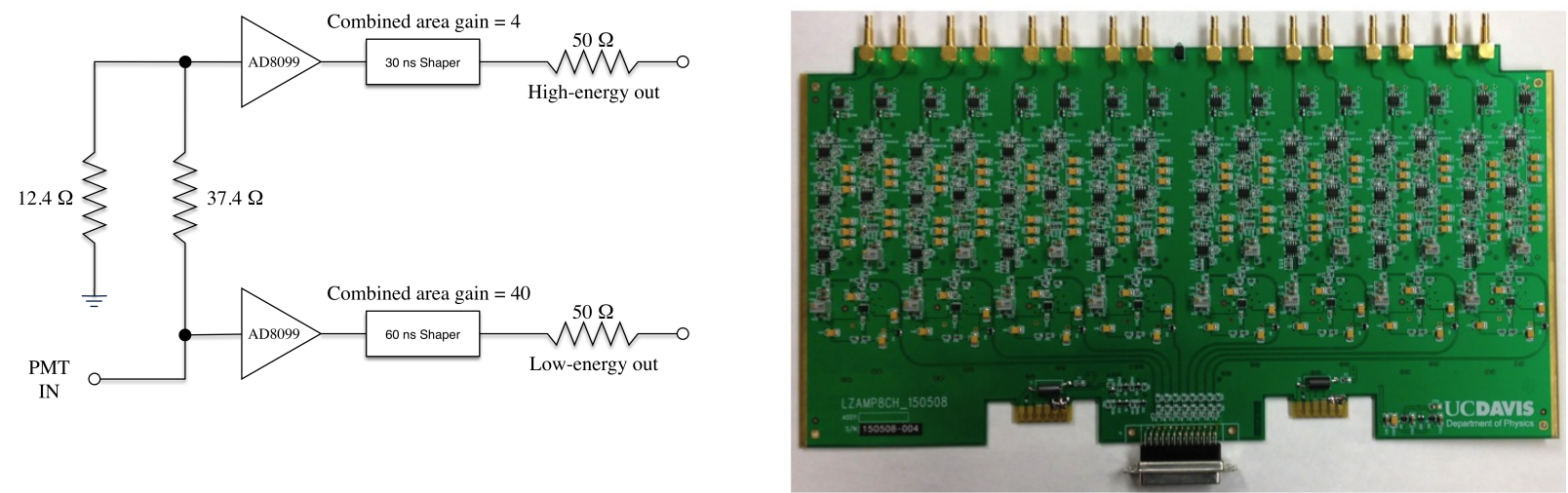

Figure 8.3.2: Left: A schematic diagram of a single channel of the LZ amplifier. Right: A photograph of the 8-channel prototype board.

single PHE is less than $15 \mathrm{mV}$ (120 ADCC). The two outputs of the amplifiers have the following properties (assuming a $11.5 \mathrm{mV}$ ns SPHE response of the PMT):

- Low-energy output: Area gain $=40,1 \mathrm{PHE}=105$ ADCC (amplitude), S1 dynamic range: 140 PHEs $(1,700 \mathrm{keV}$ ), S2 dynamic range 12 to $26 \mathrm{keV}$ (top) and 1,300 to $2,800 \mathrm{keV}$ (bottom) for 0.5 to $1.1 \mu \mathrm{s}$ wide pulses $(1 \sigma)$ with no saturation.

- High-energy output: Area gain = 4, 1 PHE = 21 ADCC (amplitude), S1 dynamic range: 700 PHEs, S2 dynamic range 120 to $260 \mathrm{keV}$ (top) and 13,000 to $27,000 \mathrm{keV}$ (bottom) for 0.5 to $1.1 \mu$ s wide pulses $(1 \sigma)$ with no saturation.

The dynamic range for S2 signals is shown in Figure 8.3.5 [1]. The low-energy channel of the amplifier provides the dynamic range required for the tritium and krypton calibrations. The high-energy channel is required to provide the dynamic range required to measure the activated Xe lines. S2 signals due to $0 v \beta \beta$

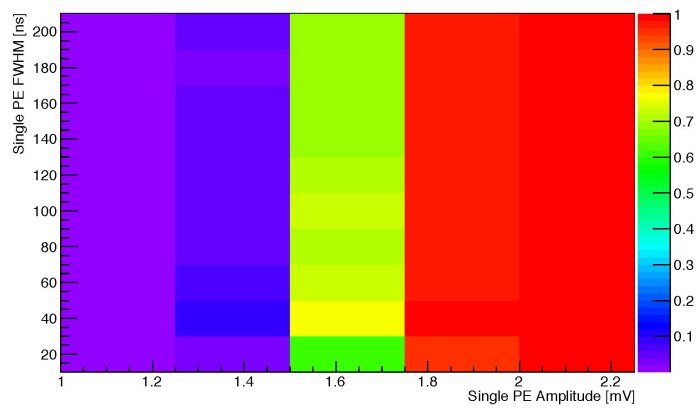

Figure 8.3.3: A simulation study of the S2 response for a 236-keV Xe transition in the center of the detector, as seen by the top PMTs. The gain and the shaping width of the SPHE response are varied. The color code shows the fraction of events in which the peak PMT saturates.

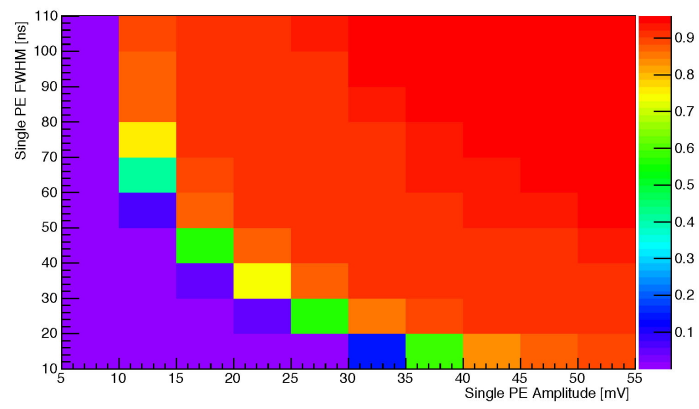

Figure 8.3.4: A simulation study of the S2 response for a 3-MeV energy deposition in the center of the detector, as seen by the bottom PMTs. The color code shows the fraction of PMTs of the bottom array that saturate. 
will saturate one or more channels of the top array, but the S2 pulse area can still be reconstructed using the low-energy channels of the bottom PMTs.

The dynamic range for S1 signals is shown in Figure 8.3.6 [1]. The figure shows the dynamic range of a bottom PMT. Also shown are the number of PHEs associated with the full-energy deposition of the 236$\mathrm{keV} \mathrm{Xe}$ activation line and $0 v \beta \beta$ decay of ${ }^{136} \mathrm{Xe}$. The dynamic range provided by the dual-gain channels is sufficient for all LZ calibrations.

Minor changes to the gain and shaping parameters of the amplifiers can be easily accommodated via changes in resistor and capacitor values. This will be done if more detailed simulations, including the electronics response and the noise of all components of the electronics chain, indicate a need for modifications.

The same amplifier design will be used for the skin and outer-detector PMTs although the gain and shaping may be adjusted slightly, if needed. Dual gain amplifiers will be used for the outer-detector PMTs, in order to make measurements of cosmic muons. For the skin PMTs, only the low-energy channel will be instrumented. A summary of the number and type of analog signals is shown in Table 8.3.1.

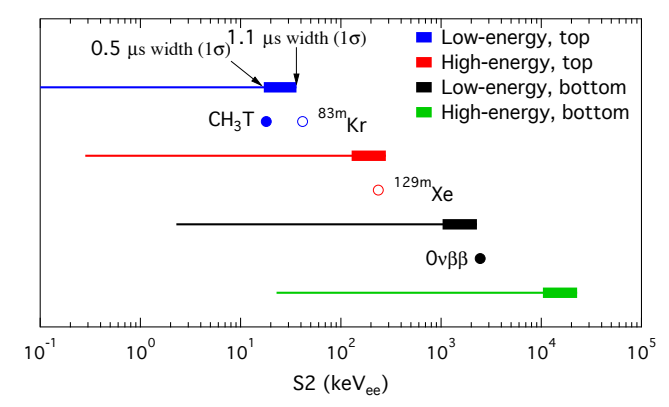

Figure 8.3.5: S2 dynamic range, expressed in terms of electron-recoil energy depositions for the lowand high-energy channels of the top and bottom PMT arrays [1]. The bars indicate variations in the upper level of the dynamic range due to the variations in the width of the S2. The lower and upper ends of these bars show the dynamic range for 0.5$\mu$ s-wide and 1.1- $\mu$ s-wide pulses, respectively. Energy depositions for various calibration sources are indicated by the circles.

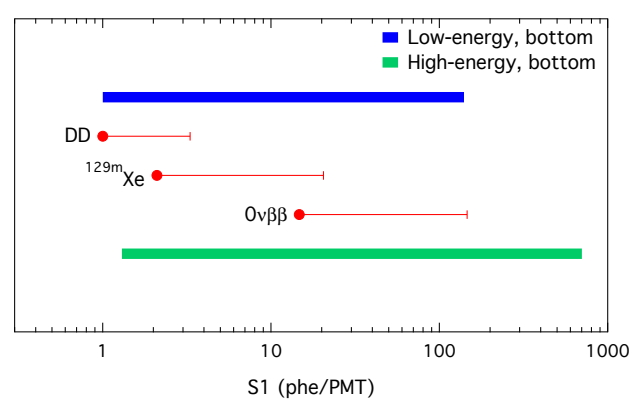

Figure 8.3.6: Dynamic range for $\mathrm{S} 1$ signals detected in the bottom PMTs [1]. The range required for DD neutrons, the ${ }^{129 m} \mathrm{Xe}$ activation line, and $0 v \beta \beta$ decay of ${ }^{136} \mathrm{Xe}$ are also shown. The leftend of each source line corresponds to an energy deposition in the center of the TPC; the right-end corresponds to an energy deposition $1 \mathrm{~cm}$ above the cathode. 
Table 8.3.1: Summary of the number and type of the 1,359 analog signals.

\begin{tabular}{|l|c|c|c|c|c|c|}
\hline & \multicolumn{3}{|c|}{ High-gain Signals } & \multicolumn{3}{|c|}{ Low-gain Signals } \\
\hline PMT Type & $\#$ & Area Gain & $\begin{array}{c}\text { Shaping } \\
\text { (FWTM) }\end{array}$ & $\#$ & Area Gain & $\begin{array}{c}\text { Shaping } \\
\text { (FWTM) }\end{array}$ \\
\hline Top TPC & 253 & 40 & $60 \mathrm{~ns}$ & 253 & 4 & $30 \mathrm{~ns}$ \\
\hline Bottom TPC & 241 & 40 & $60 \mathrm{~ns}$ & 241 & 4 & $30 \mathrm{~ns}$ \\
\hline Skin (1") & 93 & 100 & $60 \mathrm{~ns}$ & 0 & NA & NA \\
\hline Skin (2") & 38 & 40 & $60 \mathrm{~ns}$ & 0 & NA & NA \\
\hline Outer Detector & 120 & 40 & $60 \mathrm{~ns}$ & 120 & 4 & $30 \mathrm{~ns}$ \\
\hline Total & 745 & & & 614 & & \\
\hline
\end{tabular}

\subsubsection{LZ Amplifier Prototype}

Figure 8.3.2 (right) shows a photograph of the first amplifier prototype with eight input channels. The amplifiers connect to the PMT signal lines using the DB-25 connector visible at the bottom of the figure. The DB-25 connector allows the signal lines to be interleaved with two ground lines for minimizing crosstalk. The 32-channel flange is shown in Figure 8.3.7 (middle). The amplifiers will be housed a 5-card mini crate that mounts on the signal flange, as shown in Figure 8.3.7 (right). It houses four amplifier cards and a fifth card for power distribution and monitoring.

Waveforms captured with an oscilloscope at various stages of the analog chain are shown in Figure 8.3.8. The response of a PMT, operating at $1,300 \mathrm{~V}$, to an SPHE at the start and end of the cable in the vacuum space is shown. Also shown are the outputs of the LE and HE channels of the amplifier, at its output and at the end of external co-axial LMR-100 cables. The table at the bottom of the figure shows the losses and gains at each stage.

Figure 8.3.9 shows the measurements of the noise power in both LE and HE channels. The RMS ADC noise of the free-running DDC-32 digitizer channels was measured to be $(1.19 \pm 0.01)$ ADCC. In the spectra
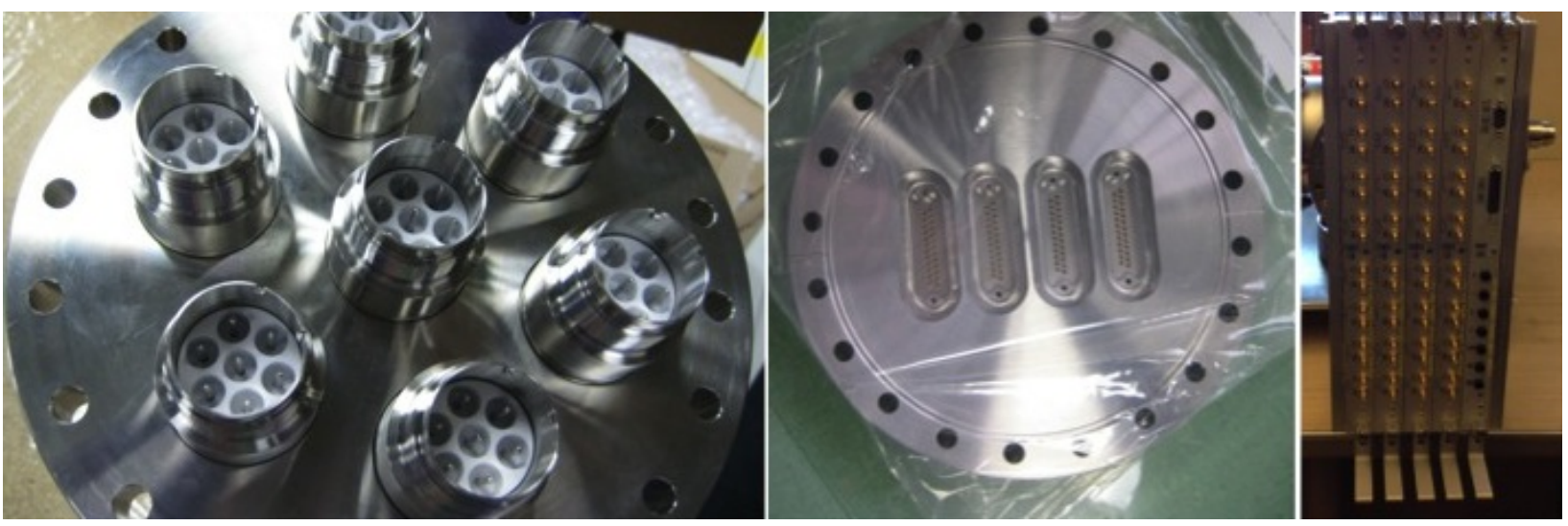

Figure 8.3.7: Photographs of the PMT HV flange (left), the 32-channel PMT signal flange (middle) and a 32-channel crate that mounts on the signal flange. 


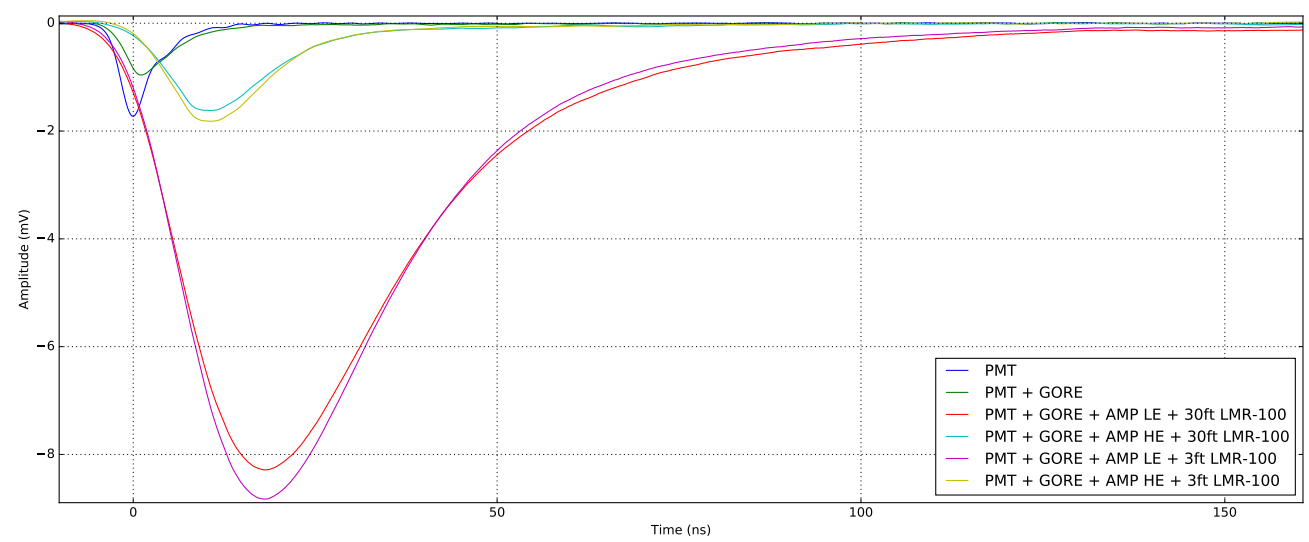

\begin{tabular}{|r|r|r|}
\hline Configuration & Area from -7 to 150 ns (mVns) & Area from -7 to 20 ns (mVns) \\
\hline PMT & -10.88 & -10.65 \\
\hline PMT + GORE & -9.46 & -8.22 \\
\hline PMT + GORE + AMP LE + 30ft LMR-100 & -336.77 & -117.62 \\
\hline PMT + GORE + AMP HE + 30ft LMR-100 & -32.71 & -22.82 \\
\hline PMT + GORE + AMP LE + 3ft LMR-100 & -338.00 & -122.77 \\
\hline PMT + GORE + AMP HE + 3ft LMR-100 & -34.09 & -25.03 \\
\hline
\end{tabular}

Figure 8.3.8: Waveforms for an SPHE at various stages of the analog chain. The PMT was operated at $-1,300 \mathrm{~V}$. The effect of the internal Gore cable and the external LMR-100 cables is shown. The desired shaping and gain specifications have been achieved.

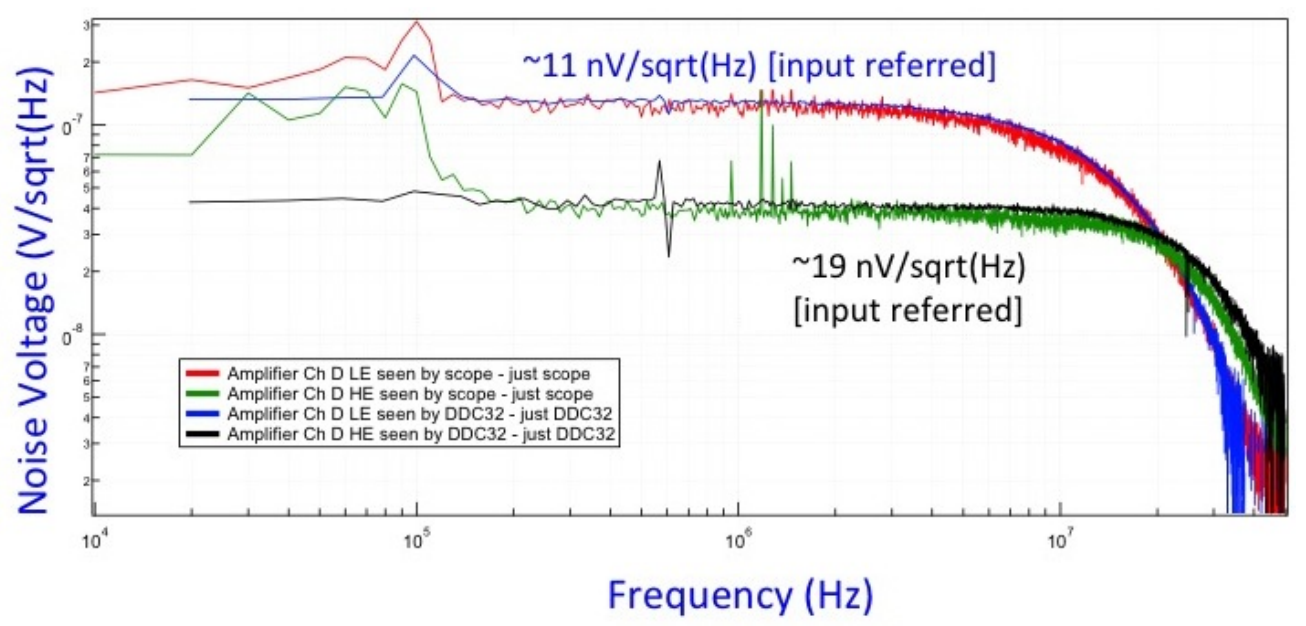

Figure 8.3.9: The measured spectrum of noise power in the amplifier channels. 
shown, the measurements were made using a DDC-32 and also using an oscilloscope. In both cases the contributions from the digitization have been subtracted in quadrature. The resulting input referred noise voltage in the "white" part of the spectrum were measured to be $11 \mathrm{nV} / \sqrt{\mathrm{Hz}}$ and $19 \mathrm{nV} / \sqrt{\mathrm{Hz}}$ respectively, for the LE and HE channels. These values are in excellent agreement with the circuit simulation model.

Figure 8.3.10 (left) shows measurements of the power dissipation. A fully loaded crate was powered up in a lab with an ambient temperature of $80 \mathrm{~F}$, without any active cooling or forced air through the crate. Within 30 minutes the crate reached a temperature of $100 \mathrm{~F}$ and stabilized at that value. This is well within the operating range of the electronics.

Figure 8.3.10 (right) shows measurements of crosstalk between the individual amplifier channels. The central channel was pulsed such that it had an output of $240 \mathrm{mV}$. The neighboring channels shown in cyan and green had bipolar induced pulses with a pulse height of $0.6 \mathrm{mV}$, or $0.25 \%$ crosstalk. The next-neighbor channel, shown in yellow, had a pulse height of $0.3 \mathrm{mV}$. These small values of crosstalk are completely acceptable. Moreover, bipolar pulses contribute very little area when integrated.

The linearity of the amplifier was studied using S2-like test pulses with a width of $1 \mu$ s (FWHM). The test pulses were propagated through $45 \mathrm{ft}$ of gore cable before reaching the amplifier. The output signals saturate when their amplitude exceeds $2.6 \mathrm{~V}$. Examples of the results of these linearity studies with S2-like pulses are shown in Figure 8.3.11 The low-energy and high-energy outputs becomes nonlinear when the area of
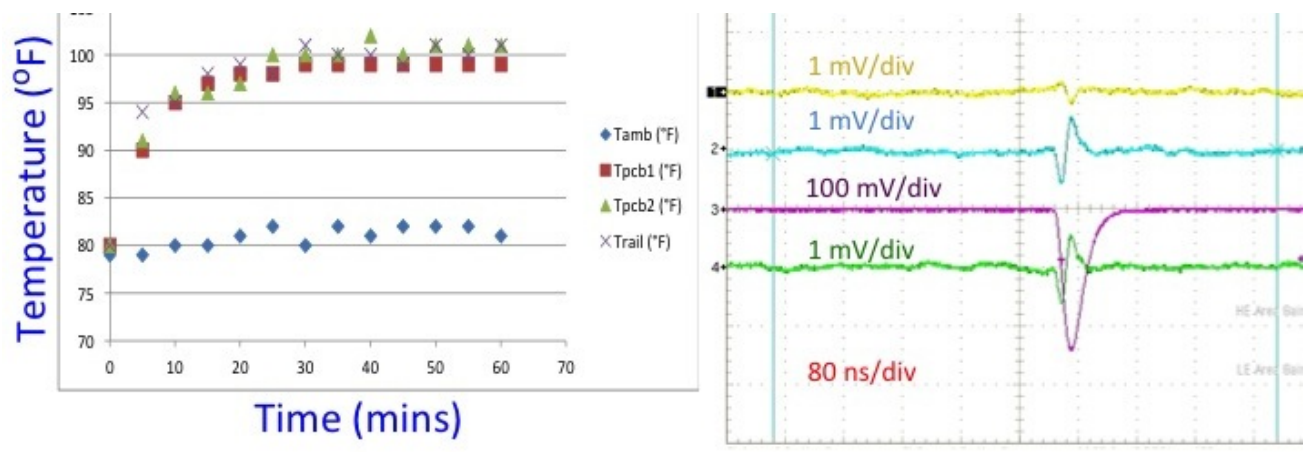

Figure 8.3.10: Left: The power dissipation and equilibrium operating temperature of a crate without any active cooling. Right: Measurements of the cross-talk for two neighboring and one next-neighbor channels.
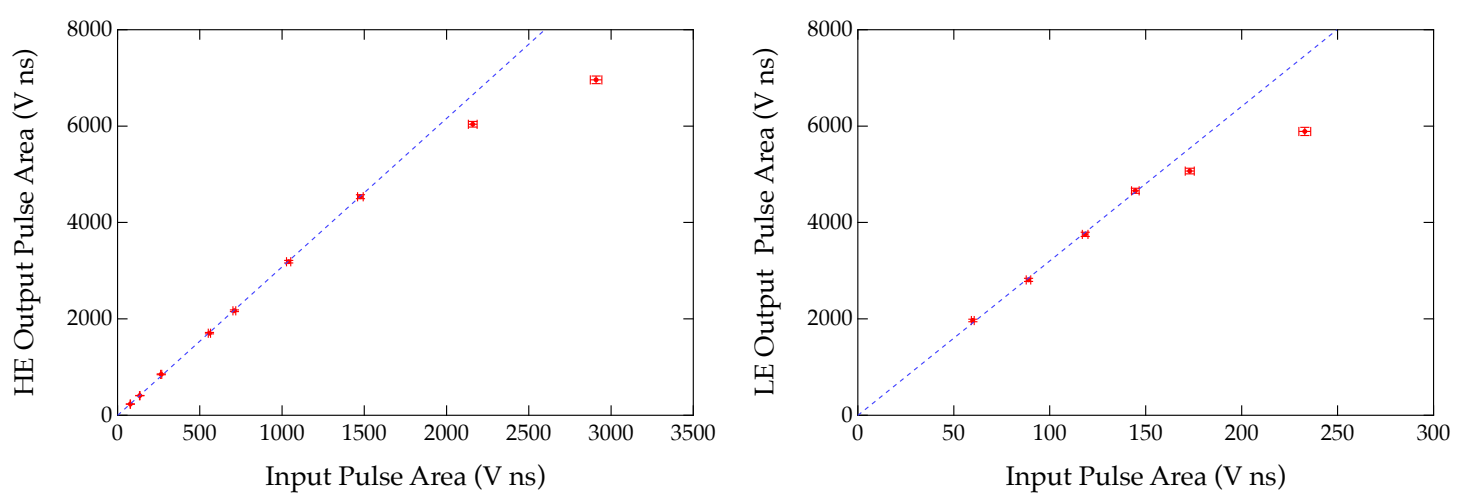

Figure 8.3.11: Results of linearity measurements. The area of the output pulse is plotted as function of the area on the input pulse. The results obtained for the high-energy (low-gain) channel are shown on the left while the data collected for the low-energy (high-gain) channel are shown on the right. 
the input signals exceed $150 \mathrm{Vns}$ and $1,500 \mathrm{Vns}$, respectively. Although the response is nonlinear in this region, there is still a one-to-one correlation between the area of the output pulse and the area of the input pulse.

Finally, the response of the amplifiers was measured for the case of PMTs that fail to provide the nominal gain. For this test, the HV on the PMT was lowered to $-1,220 \mathrm{~V}$, corresponding to a gain of $1.9 \times 10^{6}$. Figure 8.3.12 shows a histogram of the $\mathrm{S} 1$ filter output for a three-sample wide filter for signals from the LE channel. The SPHE peak can be seen to have a peak value of $84.2 \mathrm{mV}$ ns and an rms width of $28 \%$. The noise peak is also shown. Placing a cut at $50 \mathrm{mV}$ ns results in an SPHE detection efficiency of $92 \%$, which meets specifications. The singles rate of $1 \mathrm{~Hz}$ due to noise at this cut is negligible compared to the 50 to $200 \mathrm{~Hz}$ dark noise expected from the PMTs.

\subsection{Digital Electronics}

The LZ digital electronics is based on a digital platform (a motherboard); a prototype of this platform is shown in Figure 8.4.1. The final LZ motherboard will be based on this design, but will operate with a more powerful Series-7 Kintex field-programmable gate array (FPGA) from Xilinx [2]. The final motherboard will provide gigabit Ethernet, RS-232, and low-voltage differential signaling (LVDS) interfaces, and four logic outputs, either TTL or NIM. Waveform memory $(3,578 \mathrm{kB})$ will be provided by the FPGA. A large event-buffer memory of up to $128 \mathrm{MB}$ will be provided by the dual-core processor. The onboard clock can be driven externally in order to synchronize multiple boards to the same clock source. Very high processing power, nominally 52 giga-operations per second, will be provided by the onboard FPGA. Two daughter card connectors can host two separate daughter cards, or one daughter card of twice the size. The I/O pins of these connectors are arranged as differential pairs, supporting either the differential or single-ended signals. A dual-core processor will be connected to the FPGA with the 32-bit memory bus, as well as two dedicated 16-bit-wide FIFOs. Readout of the FPGA data can be performed either via the memory bus or via the FIFOs, depending on the application. The board can be hosted in a $6 \mathrm{U}$ VME crate, or it can be powered with a tabletop power supply. Power consumption is minimized by using low-voltage chips.

The onboard processing power and multiple interfaces provide flexibility that can be applied to almost any project. The LVDS links enable custom communication architectures. The Ethernet provides support for distributed experiments and/or standalone remote applications. The processor is running Linux, which is popular, free, and fully customizable, allowing each board to perform on-the-fly data processing and online diagnostics.

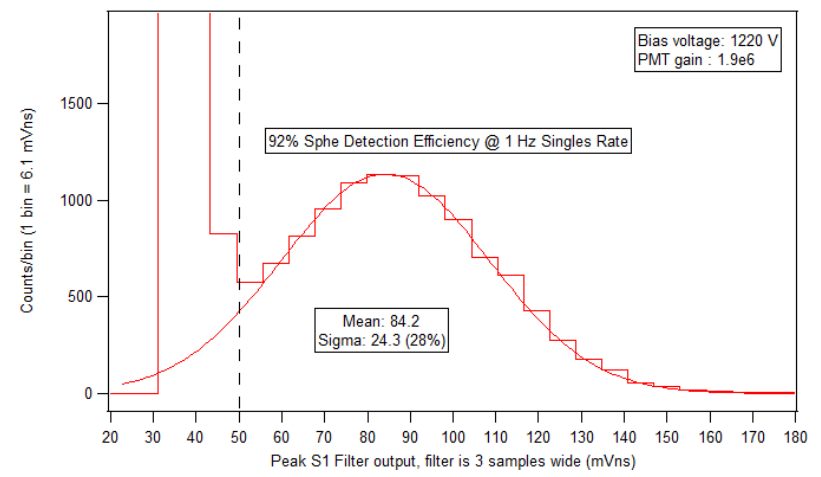

Figure 8.3.12: Measurements of the PMT response to SPHE at a gain much lower than the nominal value. 


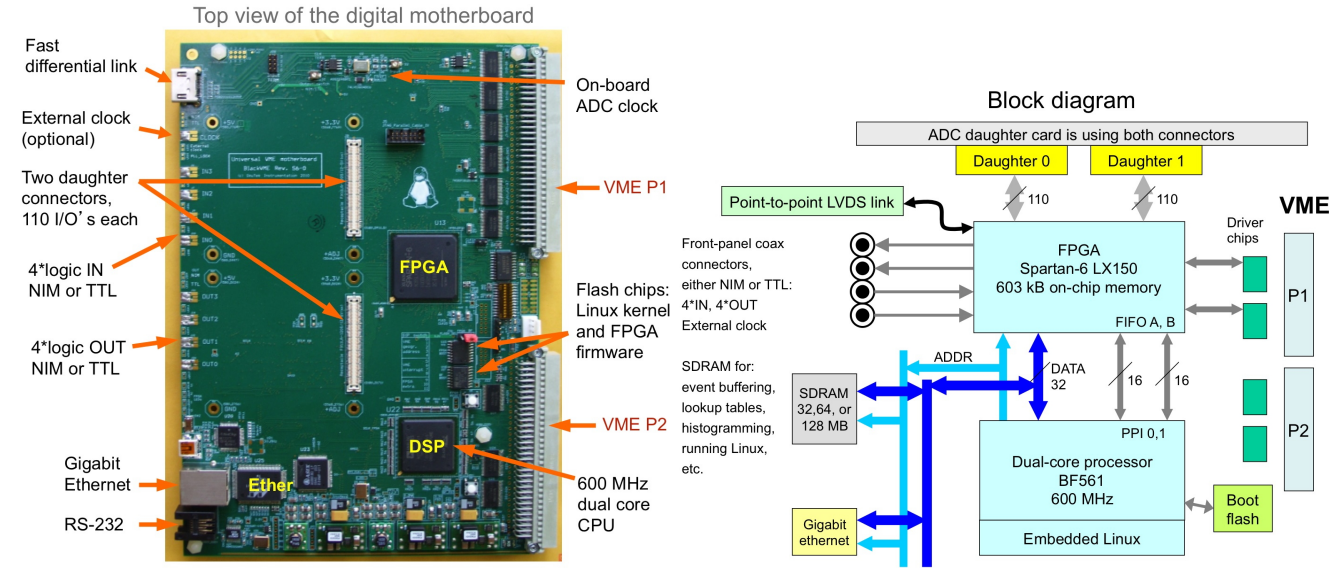

Figure 8.4.1: The digital motherboard used to develop the LZ digitizers. It provides gigabit Ethernet, RS-232, USB-2, VME, and LVDS interfaces. The FPGA and the dual-core processor are rated at 52 and 2.4 giga-operations per second, respectively.

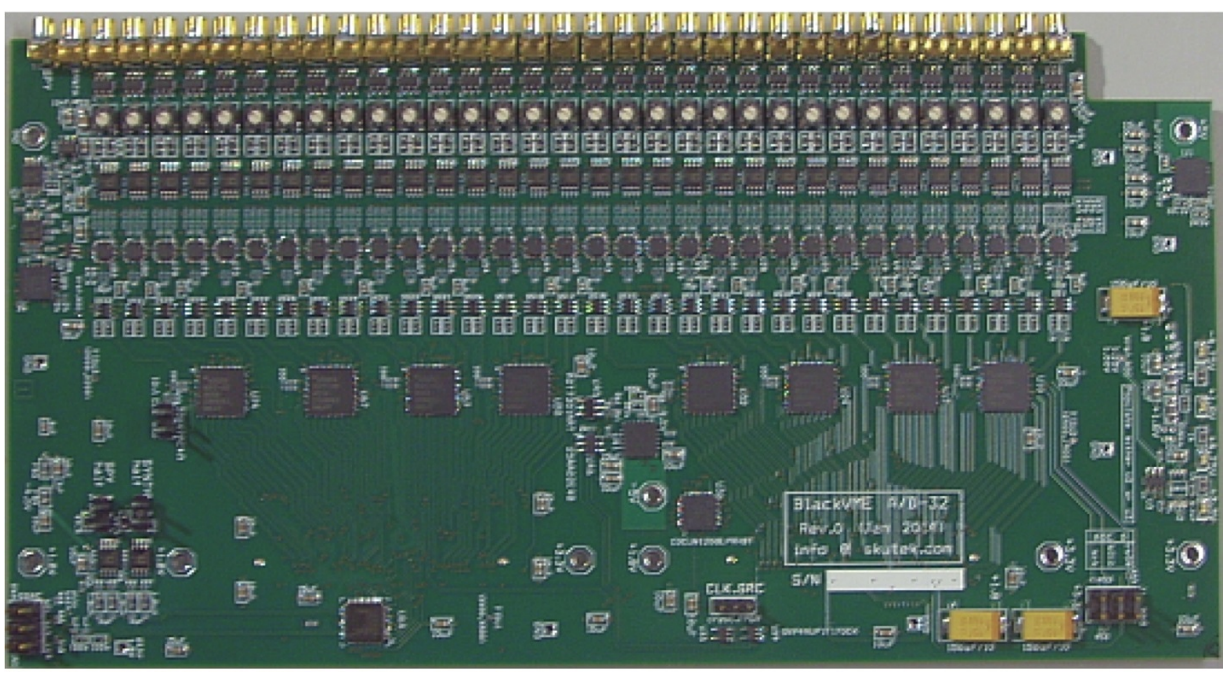

Figure 8.4.2: Prototype ADC daughter card with 32 channels. 
The 32-channel ADC card shown in Figure 8.4.2 implements the digitizer front end. It provides 32 channels of digitization and two waveform reconstruction outputs for diagnostic. The ADC channels feature remote DC offset control. The card is connected to the two daughter connectors of the digital baseboard that provide the control signals and power. The printed circuit-board layout can accommodate quad A/D chips with sampling frequency up to $125 \mathrm{MHz}$. This card, installed on the digital motherboard, is referred to as the DDC-32 in the remainder of this chapter.

\subsection{DAQ}

The top-level architecture of the LZ DAQ system is shown schematically in Figure 8.5.1 [3]. The DDC-32s continuously digitize the incoming PMT signals and store them in circular buffers. When an interesting event is detected, the Data Extractor (DE) collects the information of interest from the DDC-32s. The DEs compress and stack the extracted data using their FPGAs and send the data to Data Collectors (DCs) for temporary storage. The Event Builder (EB) takes the data organized by channels and assembles the buffers into full event structures for online and offline analysis. The DAQ operation is controlled by the DAQ Master (DM) for high-speed operations such as system synchronization and waveform selection, and by the DAQ Expert Control/Monitoring (DECM) system for slow operations such as running setup/control and operator diagnostics. The entire system runs synchronously with one global clock.

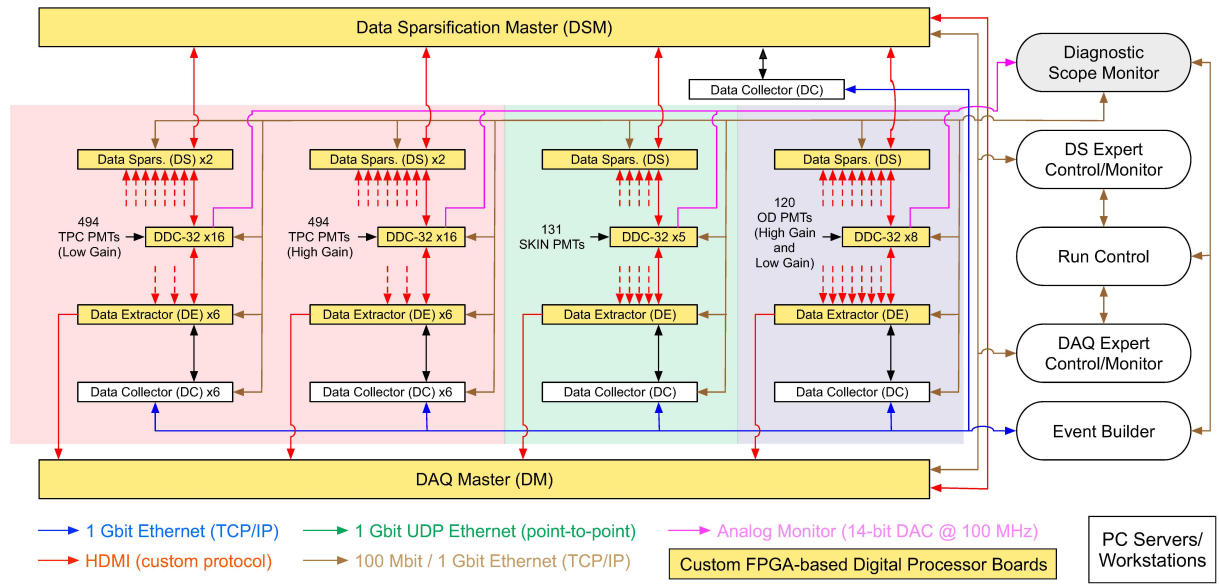

Figure 8.5.1: Diagram of the DAQ architecture [3]. Groups of digitizers (DDC-32) capture the amplified and shaped signals from the Xe, skin, and outer-detector PMTs. The waveforms of interest are extracted from the DDC-32s and compressed by the Data Extractors (DEs) before they are passed to Data Collectors (DCs) for temporary storage. One additional Data Collector will capture reduced sparsification quantities to be merged by the Event Builder with the waveform data in full event files. The DAQ Master Board (DM) coordinates the high-speed operation of the entire DAQ system when the Data Sparsification Master (DSM) signals the detection of waveforms to be preserved. The global clock will be distributed over the shown HDMI links or dedicated NIM clock inputs that are not shown in this diagram.

\subsubsection{Data Extraction}

Figure 8.5.2 shows a more detailed overview of the different key elements of the DAQ system. The digitizers are sampling at $100 \mathrm{MHz}$ with 14-bit resolution over a 2-V range. During normal operation, the boards will collect waveforms in a Pulse Only Digitization (POD) mode, which is expected to effectively reduce the raw 
waveform volume by a factor of 50 [4]. The amount of memory assigned to each channel is set so that no data truncation is expected even if the POD mode reduces the data volume by only a factor of 20. The POD waveforms are stored in dual-buffered memory that is divided into sections that hold the header information and the actual POD samples, as shown in Figure 8.5.3. Separate POD header and payload memories will improve the performance of extracting waveform data when the Data Sparsification Master (DSM) detects an event of interest [5].

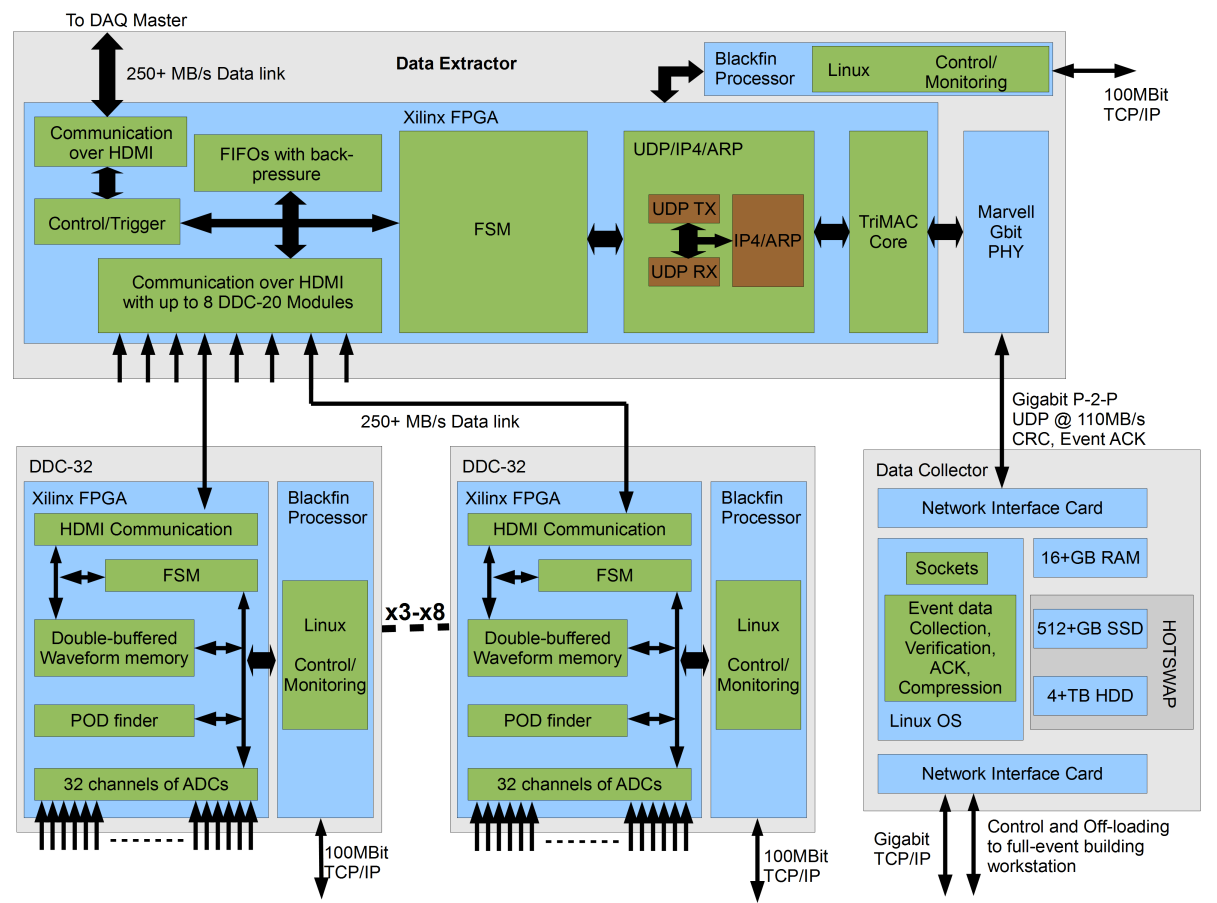

Figure 8.5.2: Detailed depiction of the inside of and interaction between the key elements of the proposed DAQ system.

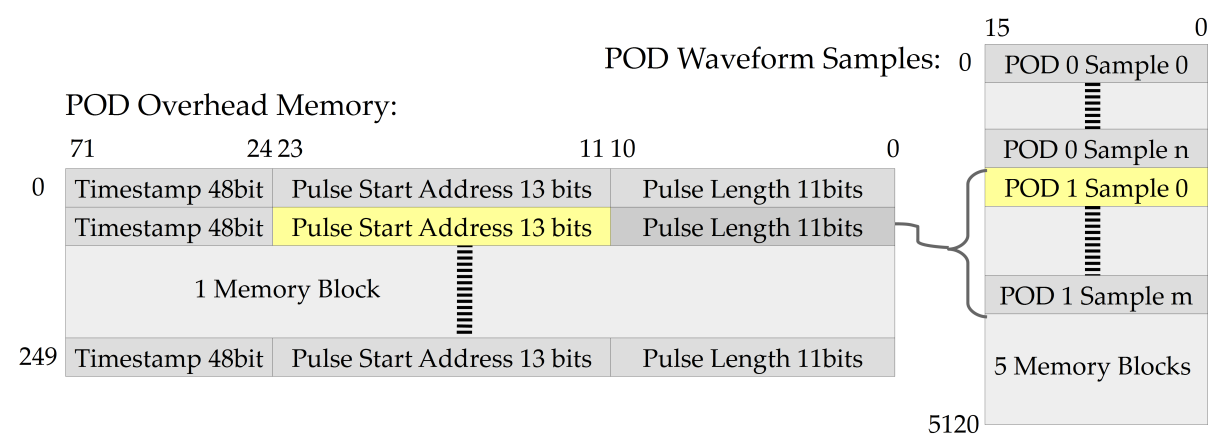

Figure 8.5.3: Depiction of the proposed memory organization of POD waveform storage in the FPGA for a single buffer (out of two) of a single channel. Separation of POD overhead and POD samples will improve the performance and ease of extracting information from memory.

The extreme flexibility that comes with using FPGAs and their internal memories allows us to assign the entire on-chip memory to just one specific channel when needed. This feature will be used for system diagnostics and noise measurements where capturing long, continuous (non-POD) waveforms is important. 
In such a mode, the DDC-32 will be able to capture 10-ms-long waveforms, suitable for power-spectraldensity analysis.

The DEs and the DM use the same hardware but different firmware. They use the same motherboard as the digitizer boards, with different daughter cards that enable communication with multiple DDC-32 modules and the DCs. Each daughter card can serve up to 14 DDC-32s over HDMI links and one DC over a dedicated gigabit Ethernet connection.

The HDMI link has seven single-ended lanes used for communicating states of the finite state machines (FSMs) between boards and four LVDS lanes used for fast offloading of the waveforms from the digitizers. On the current motherboard, we have used input/output serial/deserializer (IOSERDES) elements, offered in the Spartan-6 FPGA series, and have confirmed the advertised 1-Gbit throughput per LVDS lane.

Table 8.5.1: Key parameters of the prototype Data Collector.

\begin{tabular}{|l|l|l|l|}
\hline Processor: & $\begin{array}{l}\text { Intel Xeon E3-1270V3 } \\
\text { 3.5GHz Quad-Core }\end{array}$ & HDD 1: & $\begin{array}{l}\text { SAMSUNG 840 Pro Se- } \\
\text { ries 256GB SSD }\end{array}$ \\
\hline Motherboard: & ASUS P9D-V ATX & HDD 2: & $\begin{array}{l}\text { Western Digital RE 4TB } \\
7200 \text { RPM }\end{array}$ \\
\hline Memory: & $\begin{array}{l}\text { 16GB Kingston DDR3 } \\
\text { SDRAM ECC }\end{array}$ & Case:Case: & $\begin{array}{l}\text { NORCO RPC-270 2U } \\
\text { Server Case }\end{array}$ \\
\hline NIC: & $\begin{array}{l}\text { Intel Ethernet Server } \\
\text { Adapter I350-T2 }\end{array}$ & Hot Swap: & $\begin{array}{l}\text { ICY DOCK 3.5" and 2.5" } \\
\text { SATAlll 6Gps HDD Rack } \\
\text { Tray }\end{array}$ \\
\hline
\end{tabular}

Table 8.5.2: Summary of the performances of the DAQ links and their expected utilization levels.

\begin{tabular}{|c|c|c|c|}
\hline \multicolumn{1}{|c|}{ Link } & $\begin{array}{c}\text { Expected } \\
\text { Performance }\end{array}$ & $\begin{array}{c}\text { Maximum } \\
\text { Expected Usage }\end{array}$ & Usage \\
\hline LVDS over HDMI & $250+\mathrm{MB} / \mathrm{s}$ & $8.6 \mathrm{MB} / \mathrm{s}$ & $<3.5 \%$ \\
\hline $\mathrm{DE} \rightarrow$ Gigabit UDP $\rightarrow \mathrm{DC}$ & $109 \mathrm{MB} / \mathrm{s}$ & $34.4 \mathrm{MB} / \mathrm{s}$ & $<33 \%$ \\
\hline
\end{tabular}

Table 8.5.3: Summary of the expected storage and buffering capabilities on the Data Collectors during calibrations.

\begin{tabular}{|c|c|c|c|}
\hline Source & $\begin{array}{c}\text { Data Rate per Data Collector } \\
\text { (uncompressed) }\end{array}$ & $\begin{array}{c}\text { Solid-state Drive } \\
(512 \mathrm{~GB})\end{array}$ & $\begin{array}{c}\text { Hard-disk Drive } \\
(1 \mathrm{~TB})\end{array}$ \\
\hline${ }^{83} \mathrm{Kr}$ & $\sim 5.2 \mathrm{MB} / \mathrm{s}$ & $\sim 1.1$ days $(\mathrm{raw})$ & $\sim 9$ days $(\mathrm{raw})$ \\
& & $\sim 4.4$ days $(7 \mathrm{z})$ & $\sim 35$ days $(7 \mathrm{z})$ \\
\hline \multirow{2}{*}{$\mathrm{LED}$} & $\sim 34.4 \mathrm{MB} / \mathrm{s}$ & $\sim 4$ hours $(\mathrm{raw})$ & $\sim 1.4$ days $(\mathrm{raw})$ \\
& & $\sim 16$ hours $(7 \mathrm{z})$ & $\sim 5.3$ days $(7 \mathrm{z})$ \\
\hline
\end{tabular}


The gigabit Ethernet link between the DE and the DC utilizes the User Datagram Protocol (UDP). To mitigate the limitations of UDP, we will add for each event a cyclic redundancy check (CRC) and acknowledgment, and also use the dedicated Ethernet link in a point-to-point topology to eliminate congestion and packet loss. All UDP packets formed by the DE's FPGA will be sent over a single gigabit Ethernet cable to a dedicated network port on the DC.

The DCs will be implemented using server-grade, rack-mountable (2U) workstations. We have tested a prototype DC with the parameters shown in Table 8.5.1. We were able to confirm reliable data transfer from the DE to the solid-state drive (SSD) in the DC. With appropriate modifications to the network interface card (NIC) drivers, we were able to transfer data at a constant rate of $109.8 \mathrm{MB} / \mathrm{s}$, with no data loss or corruption. Table 8.5.2 summarizes the tested link performances and their expected utilization.

The data stored on the DCs are processed by the Event Builder (EB). The EB builds events by sampling the different DC disks and collecting all information associated with a given event. The resulting event (EVT) files are compressed in order to achieve the smallest possible file size for storage and transmission. The EVT files are written to RAID array \#1, located in the Davis Cavern, before being transferred to the RAID array \#2, located on the surface at SURF. The RAID arrays have sufficient storage capacity for a full month of data taking.

The DAQ system is designed to be able to allow LED calibrations of the TPC PMTs in about 10 minutes. This requires an event rate of $4 \mathrm{kHz}$, resulting in a $\sim 340 \mathrm{MB} / \mathrm{s}$ total waveform data rate. The fast (400+MB/s for SSDs) storage drives in the individual DCs allow for sustaining such collection rates, and the amount of space offered by each DC permits significant buffering in case of connection problems to the off-site permanent storage, as shown in Table 8.5.3.

We are planning to use $7 \mathrm{z}$ compression. Nominally, $7 \mathrm{z}$ uses the LZMA algorithm, but we have found that the PPMd algorithm [6] is better suited for the waveforms we are going to collect. Based on comparisons made using LUX data, the 7z PPMd compression offered an additional $33 \%$ data reduction for waveforms and a $15 \%$ data reduction for reduced quantities over gzip, which was used in LUX.

Because the POD mode relies on time stamping, the entire DAQ system will run with a global $100 \mathrm{MHz}$ clock. We are considering two ways of distributing the clock, either by using a proven method of dedicated NIM logic clock FAN-IN/OUTs or using clock recovery from the serial links of the HDMI cables; the latter method is currently being evaluated. We are also looking at the possibility of using one of the single-ended channels of the HDMI cable for a dedicated clock signal.

The configuration, acquisition control, and monitoring will be done using the DECM workstation. For improved robustness, the DECM will communicate with all DAQ elements using an isolated 100/1000 LAN; the run-control (RC) system, described in Section 8.9, will not have direct access to the front-end digitizers. We have successfully tested and are planning to use ICE middleware as our communication framework [7].

\subsubsection{Data Sparsification}

The design of the LZ data sparsification system is based on our experience with event selection in the LUX trigger system. The top architecture of the LZ data sparsification system is shown schematically in Figure 8.5.1. The DAQ and data sparsification firmware operates in parallel on the FPGAs of the DDC-32 digitizers.

The DDC-32s continually process the incoming pulses and extract specific required quantities such as pulse area, pulse height, and time of occurrence. By using digital filters with various filter lengths, the system can distinguish S1 and S2 pulses. The parameters extracted from the waveforms by the DDC-32s are sent to the Data Sparsifiers (DSs) for further processing and generation of secondary quantities such as the multiplicity vectors of groups of PMTs. These secondary quantities are sent to the Data Sparsification Master (DSM), where the final waveform selection decision is made. The decision to preserve the current waveforms is sent to the DAQ Master. It is important to reiterate that much of the information based on 
which a given event selection was made is going to be off-loaded alongside event waveform data and stored in the full event files. This will allow for simpler cross-checking and verification of the system performance off-line.

A new feature under development is the creation of a total waveform digital sum that can be created at the DSM from all or subset of DDC-32 channels as shown in Figure 8.5.4. This allows for including total area cuts in the event selection decision process.

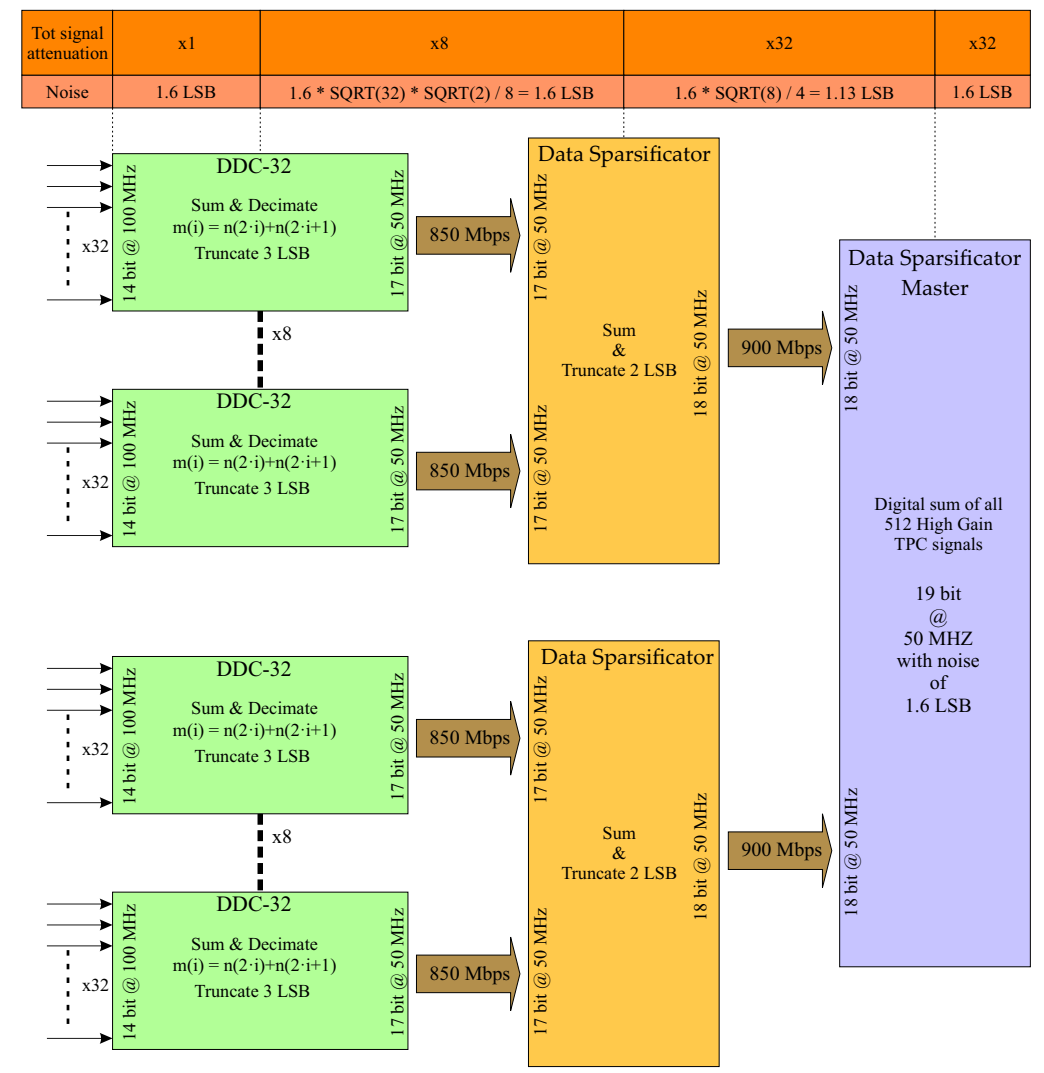

Figure 8.5.4: Schematic of digital waveform sum creation from all or subset of DDC-32 channels that allows the total pulse area to be a part of the sparsification decision making.

The LZ DAQ/Sparsification system is capable of handling event rates up to $\sim 250 \mathrm{kHz}$. This is much higher than the highest event rates expected in the LZ detector, which will occur during LED calibrations $(\sim 4 \mathrm{kHz})$.

As in LUX, the PMT signals will be processed by parallel digital integrating filters, allowing for discrimination against area of the incoming pulses. The filter with an integration width of about $60 \mathrm{~ns}$ to $100 \mathrm{~ns}$ is tailored to S1-like pulses; the second filter with a few-microsecond width is optimal for wider S2-like pulses. The filters are designed to also perform automatic baseline subtraction.

As shown in Figure 8.5.1, the TPC, skin, and outer-detector PMTs will use separate DDC-32s and DSs because these PMTs have different purposes and the firmware will be tailored to their specific needs. Such separation also makes it easier to apply different scaling factors for the digitization of signals from different PMT groups. The three major waveform-selection modes for the central TPC PMTs are summarized in Table 8.5.4. 
Table 8.5.4: Summary of three major waveform-selection modes for the central TPC PMTs.

\begin{tabular}{|l|l|}
\hline Trigger Mode & Summary \\
\hline S1 Mode & $\begin{array}{l}\text { Detection of coincident S1-like signals across selected channels. No fidu- } \\
\text { cialization. }\end{array}$ \\
\hline S2 Mode & $\begin{array}{l}\text { Detection of coincident S2-like signals across selected channels. Fidu- } \\
\text { cialization in the } x, y \text { plane. }\end{array}$ \\
\hline S1 and S2 Mode & $\begin{array}{l}\text { Detection of S1-like signals followed by S2-like signals within a selected } \\
\text { drift time range. Fiducialization in } x, y \text { and } z \text { planes. }\end{array}$ \\
\hline
\end{tabular}

If a waveform selection condition is met, the DSM sends a signal to the DM. At the same time, a packet containing the selection parameters used to make the decision is merged with the captured waveform data. This allows for offline evaluation of the selection decision for every individual event. This feature has proved extremely valuable in monitoring LUX data quality.

Similarly to the DAQ system, the DS system will be controlled and monitored by a dedicated DS expert control/monitoring (DSECM) workstation over an isolated 100/1000 LAN network. The DS system will operate on the same $100 \mathrm{MHz}$ global clock as the DAQ.

The LZ DS system will adopt and expand on the monitoring capabilities of the LUX system. Capabilities such as performing continuous noise sweeps (monitoring S1 and S2 filter crossing rates as a function of threshold) or monitoring channel hit distributions of the selected events, all in parallel to regular uninterrupted data-sparsification operation, have been invaluable in LUX and surely will be in LZ.

Each of the DDC-32s has a dual 14-bit, $100 \mathrm{MHz}$ analog reconstruction output (SPY) allowing for diagnostics and scope monitoring. The SPYs can be sourced with individual incoming channels or their digital sum. They can also be sourced with S1 and S2 filter outputs, individual or summed, or any other signal internal to the FPGA. This feature will be very useful, especially in development and deployment stages, as proven in the LUX project.

\subsection{PMT HV}

LZ will use the Wiener Mpod LX HV system to bias the xenon and outer-detector PMTs. This same system is currently being used for LUX. The system will provide negative HV to the xenon PMTs and positive HV to the outer-detector PMTs. The HV modules are part of the EDS 201-30x 504 series. Each module provides $32 \mathrm{HV}$ channels and uses a common floating ground. The voltage ripple is less than $5 \mathrm{mV}$. The $\mathrm{HV}$ can be set with a resolution of $10 \mathrm{mV}$, and the current on individual channels can be measured with a resolution of $50 \mathrm{nA}$.

HV connections to the HV filters are made using Kerpen cable with Redel connectors on both ends. Each Kerpen cable carries HV for 32 channels. Important properties of the HV system are listed in Table 8.6.1.

Table 8.6.1: Details of the PMT HV system.

\begin{tabular}{|c|c|c|c|c|c|}
\hline HV Module & $\begin{array}{c}\text { Maximum HV } \\
\text { HV }\end{array}$ & $\begin{array}{c}\text { Maximum } \\
\text { Current }\end{array}$ & PMTs & Channels & $\begin{array}{c}\text { Number } \\
\text { of Modules }\end{array}$ \\
\hline EDS 201 30n & $-3,000 \mathrm{~V}$ & $500 \mu \mathrm{A} / \mathrm{ch}$ & TPC/Skin & 625 & 21 \\
\hline EDS 201 30p & $+3,000 \mathrm{~V}$ & $500 \mu \mathrm{A} / \mathrm{ch}$ & Outer-detector & 120 & 4 \\
\hline
\end{tabular}




\subsection{Cables}

All network, signal, and HV cables are low smoke zero halogen (LSZH) cables. LZ uses the same cables that have been approved for LUX use in the Davis Laboratory. The exact lengths of the HV and the signal cables will be fixed once the location of the analog amplifiers is fixed and the route of the cable trays has been finalized.

The types of cable and their lengths are listed in Table 8.7.1. The networking cables will have various lengths; individual lengths will vary based on the location of the network switched and the location of the devices to be connected. About half of the network cables are used by the slow-control system; the other half will be used for the networks associated with the DAQ, trigger, and online systems.

We have not been able to identify a suitable HDMI cable that provides good performance and uses LSZH materials. The total length of these cables is small and the electronics racks are enclosed and vented directly into the exhaust system of the Davis Campus. This arrangement will be assessed by the SURF safety team. Note that rack enclosures and connections to the exhaust system are also used in LUX for this same reason.

Table 8.7.1: Information on LZ signal, logic, HV, power, and network cables.

\begin{tabular}{|c|c|c|c|c|}
\hline Cable Type & Type & Length (ft) & Number of Cables & Notes \\
\hline Network & Belden 7936A & $\begin{array}{l}10,000 \\
\text { (total) }\end{array}$ & 750 & $\begin{array}{c}\text { Cat 6, LSZH } \\
\text { Various lengths }\end{array}$ \\
\hline Signal & LMR-100A-FR & 56 & $\begin{array}{c}143 \text { bundles } \\
\text { (8 cables/bundle) }\end{array}$ & LSZH \\
\hline Logic & LMR-100A-FR & 2 & 500 & LSZH \\
\hline HV & Kerpen & 56 & 25 & $\begin{array}{c}\text { LSZH } \\
32 \text { channels per cable }\end{array}$ \\
\hline Power cords & CordMaster & 6 & 100 & LSZH \\
\hline HDMI & TBD & TBD & 99 & \\
\hline
\end{tabular}

\subsection{Slow Control}

\subsubsection{Functions of the slow control}

The slow control system performs supervisory control and monitoring of all the major subsystems of the experiment. The functions of slow control can be classified in several categories as described below:

- Experiment parameter monitoring and logging. This includes periodic readout of various sensors distributed throughout the xenon circulation, storage and recovery systems (temperature, pressure, gas flow etc.) and of advanced sensors installed in the detector (capacitive level and distance sensors, acoustic sensors and loop antennas). For the electronic racks the status of the uninterruptible power supplies (UPS) as well as temperature and current consumption of the front-end amplifiers will be monitored. Also, the environmental data, such as ambient temperature and pressure and radon concentration in the air, will be collected.

- Control over experiment subsystems. This includes controlling (by means of electrically-triggered pneumatic valves) pathways for performing operations of xenon circulation, storage and recovery, 
controlling the detector temperature via thermosiphons and heaters, and controlling distribution of liquid nitrogen required for thermosiphon operation. The slow control will also serve as a frontend to the calibration systems (krypton injection, radioactive source delivery, optical calibration) to guarantee that they are not interfering with normal detector operation. Finally, high voltage power supplies for the field-shaping grids and the PMTs in the detector will be programmed and monitored through slow control.

- Safety. One of the most important functions of the slow control system is to ensure safe operation of the detector and all the experiment subsystems in order to prevent equipment damage and xenon loss. This is achieved by implementing a set of interlocks that would protect the system from erroneous operator command. Additionally, the alarm system will be programmed to alert on-site crew and/or system experts when certain key parameters are out of the predefined range. The emergency xenon recovery, activated by pressure rise in the detector, will safely transfer xenon to the storage facility if the thermal control of the detector fails.

- User interface. The slow control system will provide the GUI to display all relevant telemetry information and controls organized in pages by subsystem. The plots of parameter history with be available as well. The controls available to the user will depend on privilege and expertise levels as determined by querying the centralized access control and privilege management facility provided by the experiment IT infrastructure. All control changes performed by users will be logged.

- Automation and interfaces with run control and offline systems. In addition to the set of scripts (procedures) directly accessible to slow control, a set of standard high-level operating procedures will be made compatible with remote procedure calls from run-control or to the offline systems. Programming these scripts on the slow-control (server side) will make these sequences faster, more reliable, and will eliminate possibility of an operator error. In some cases these scripts will include break points requiring explicit feedback from the run control client to advance to the next step, further ensuring system safety and accuracy.

\subsubsection{Requirements}

The main requirement to the slow control system is to provide the monitoring and control infrastructure that will guarantee safety of the experiment subsystems and xenon supply in all modes of operation. The Level 2 requirement R-180004 states: Guarantee the safety of xenon supply and the xenon circulation system. Use Programmable Logic Controllers (PLCS) to control and monitor the xenon purification, circulation, and storage systems. The corresponding Level 3 requirements specify the necessary parameters of the monitoring and control infrastructure:

- R-180501: Monitor state of detector. Monitor up to 2,500 channels.

- R-180502: Provide access to detector controls. Control up to 1,400 channels.

- R-180503: Provide an alarm system. Allow for alarm priorities, alarm profiles, and alarm pipelines.

- R-180504: Record system operations and system state. Provide a history of all monitored parameters and changes in the status of controls as well as a log of user access and actions.

\subsubsection{Overview}

The functional diagram of the slow control system and its interaction with the experiment subsystems and infrastructure is shown in Figure 8.8.1. The core of the slow control system is composed of two compo- 


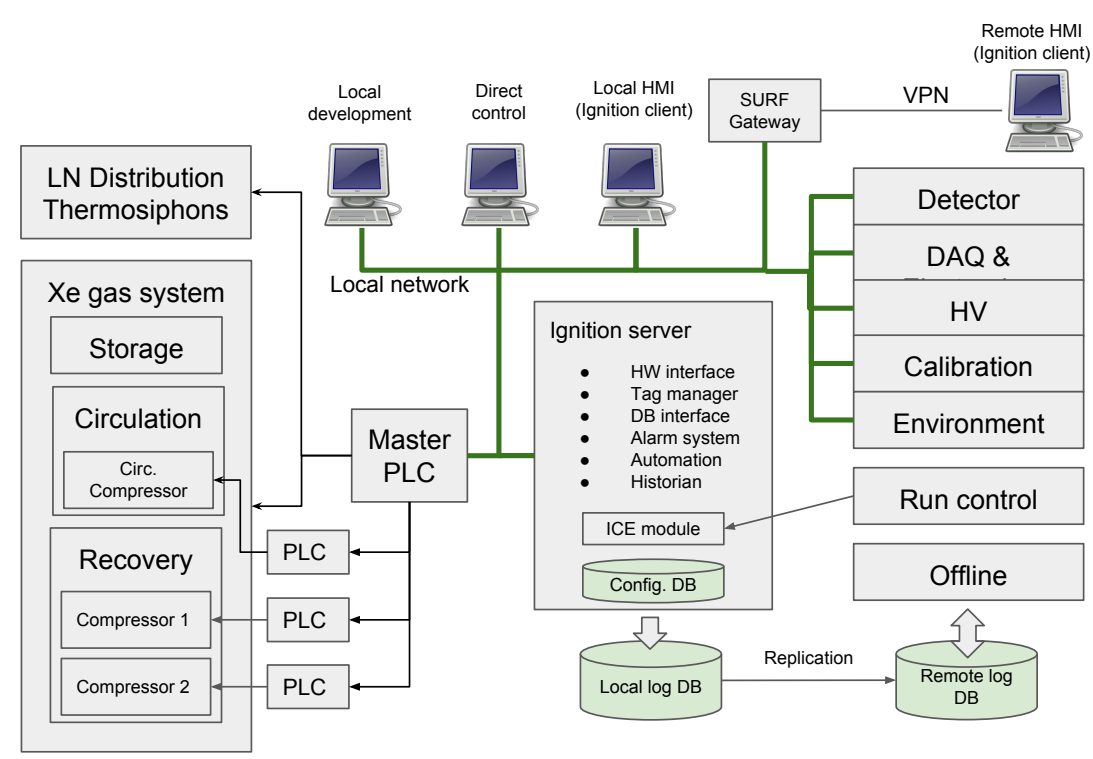

Figure 8.8.1: Slow control functional diagram.

nents: (1) the integrated supervisory control and data acquisition (SCADA) software platform Ignition from Inductive Automation [8], and (2) the Siemens SIMATIC S7-410H programmable logic controller (PLC) with associated I/O modules [9]. The Ignition server works as the main hub of the slow control system and is responsible for collecting and archiving the sensor data and providing the authorized users with access to the controls. Additionally, the Ignition server runs the alarm system, provides the scripting engine for experiment automation and provides the ICE server for interfacing with run control. It also provides a GUI for accessing historical data in the form of plots by local and remote clients. The configuration data (properties of sensors and controls, alarms, user preferences etc.) are stored in the local configuration database.

The PLC component is designed to guarantee safety of the critical subsystems (xenon circulation, storage and recovery, detector temperature control and liquid nitrogen distribution) by implementing necessary interlocks to safeguard their correct operation. It is also programmed to ensure safety of the xenon supply even in the case of a major infrastructure failure, for example a prolonged power or network outage with no access underground. The data flow between the Ignition server and the PLC passes through a dedicated TCP/IP network. The Ignition server directly communicates with the non-critical experiment subsystems through the same network. The computers used for local programming and control of both PLC and Ignition server are connected to this network as well. Communication between the Ignition server, the PLC, other non-critical experiment subsystems, operator consoles, and PLC software development workstations uses Ethernet. Multiple TCP/IP subnets in conjunction with router access control lists will be used to separate the slow control network into security zones with appropriate access restrictions.

The historical data log is stored in a local MySQL slow control database mirrored to a replica database from which these data can be accessed by clients on the LZ experiment network including the offline system. 


\subsubsection{PLC System}

The PLC system is the core component of the slow control which is expected to maintain the detector and xenon system in a safe state even in the case of failure or shutdown of the rest of the slow control infrastructure. It is designed to provide continuous real time monitoring and control of the critical subsystems, including:

- Xenon purification, circulation and storage systems,

- recovery compressors,

- detector temperature control and liquid nitrogen distribution systems,

- vacuum pumps.

The master control of the the critical subsystems is performed by a Siemens S7-410H Redundant Hot Backup PLC with S7-300 associated I/O modules. This PLC allows bumpless transfer from one active CPU to a backup CPU, with synchronization and self-testing of both CPUs. Each CPU is connected to a separate interface module (IM), allowing fault tolerant process control and bumpless programming and hardware changes in run mode. Members of the LZ run control team have extensive positive experience with the Siemens S7-410. This PLC system will be programmed using the Siemens PCS7 engineering programming software (meeting IEC 61131-3 standard).

Four compressor units (primary and secondary recovery compressors and two circulation compressors) are controlled by local Beckhoff PLCs, sharing data with the master PLC via Profibus. The Master PLC provides system data and interlocks to these subordinate PLC systems. Compressor-related instruments and valves are connected directly to Beckhoff modules. In the unlikely event of the failure of the master PLC, local PLCs can operate independently. Control logic resides in both master and locals PLCs. Local control of PLCs is also provided via touch screen so the autonomous units can be controlled directly by the on-site operator in case of an emergency. All PLCs are connected to a single dedicated private network with TCP/IP connectivity to 3 development machines ( 2 underground and one at the surface). The development machines will be remotely accessible through a secured connection (e.g. VPN).

More information on the PLC system and its support of xenon recovery operations is given in section 6.4.6.

\subsubsection{Ignition}

The choice of Ignition as the software platform for LZ slow control is based on the wide range of highly customizable, easy to use core functions and tools for development of efficient and robust SCADA systems. This set of tools includes: the tag management system, scripting and automation facilities, a versatile alarm system, a user management interface, a historian function, a database interface, a versatile toolbox for GUI desgin as well as a number of other functions and utilities. Ignition comes with a comprehensive library of device drivers supporting most of the hardware used in LZ.

Ignition follows a web-based client-server model where the server is deployed on site and takes care of communication with all the hardware while GUI clients can run both locally and remotely. It has a modular, easily expandable architecture: an extensive SDK allows developers to build their own custom modules and device drivers in Java or Python. At a higher level, Sequential Function Charts (SFC) or Python scripts can be used for development of automation procedures.

A high level of system availability is maintained even in the case of a system fault through the automatic swap to a mirror server to which configuration is constantly backed up (only a few minutes are lost in such an event). If even higher system availability is determined to be necessary, virtually zero downtime can be 
achieved by running two redundant servers in parallel with automatic hot swap (for the cost of the second server license). Security is provided by support of secure communication with clients, user authentication (site-wide authentication is possible through an Active Directory), user role management and action logging.

Ignition is also expected to be more cost-efficient for scientific automation than most commercial SCADA solutions as it is licensed by server with no limitation imposed on number of devices, tags or clients. Also, due to its multi-platform nature, it does not limit the developers or users to a single operating system.

\subsubsection{Tags and device interfaces}

A tag is the term used for an elementary unit of information in industrial automation. A tag can be bound to a device (made readable for monitoring or writable for control), a record in a database or a variable in control software. Ignition tags follow an object oriented philosophy. User Defined Tags (UDT), equivalent to classes used in programming, allow the user to define a set of generic tags each associated with a certain device type from which all the tags for individual devices are later derived. A well-structured UDT scheme speeds up the setup, configuration and modification of large groups of sensors. UDTs can be associated with GUI templates with positive impact on the GUI usability as well as a reduction in the development time and effort. A UDT library developed for the System Test slow control system will be used as the base for LZ slow control UDT.

The information transfer between the tags and the associated devices can be handled in several different ways. The preferred one is by using the OPC-UA protocol supported by many PLCs either directly or through middleware solutions (for example the Kepware server). In addition, Ignition has its own OPC-UA server with drivers for many well-known PLC brands, including Siemens PLCs. For the rest of the devices the preferred protocol will be MODBUS which is also supported by the Ignition OPC-UA server. The devices not supporting MODBUS (e.g. RGA units) will be interfaced via custom made Java drivers written in the Ignition SDK. All these interfacing schemes were successfully implemented and field-tested for the System Test slow control system.

\subsubsection{Historian, alarms and automation}

Ignition offers highly customizable historian features for logging the values of the selected tags in a database. There are options for both periodic logs and those on an evaluate-on-change basis. It is also possible to have different timing parameters for different tag classes or temporarily increase the readout rate for a particular tag. The GUI history explorer module developed for the System Test provides tools for rapid historical data visualization and analysis.

Ignition also provides a powerful alarm framework whereby each tag can have multiple alarm levels associated with it. Alarms can be configured with different profiles that include priority, escalation scheme and notification pipeline. Notifications can be both local (client GUI, speaker, siren) and remote (email, VoIP or text message). The configuration of these alarm profiles is done by the developers in the Designer. Individual alarm activation/deactivation, setpoint configuration and acknowledgments can be done in the client GUI by a user with adequate privilege level. This allows developing a unified interface for setting alarms and storing pre-defined alarm configurations on a per-user basis; for example, a gas system expert may have a particular set of alarms that they want to be active during a troubleshooting exercise.

For further customization, Ignition provides a Python scripting engine. Python scripts can run both in the client or gateway and can be attached to event handlers (e.g., mouse clicked, key pressed, a tag changing state events), a timer or run inside an SFC. Since Python scripts running on the client block the client execution, these are only suited for GUI customization or very simple automation routines. On the other hand, scripts running in the SFC which are executed in the gateway, are intended to control complex automation routines and procedures. SFCs use the IEC61131-3 programming specification (also used for PLCs) and can be 
developed in the designer using a drag and drop programming tool. SFCs can be started and monitored both from gateway or Clients GUI but they always run on the gateway. Several SFCs can run in parallel and can be programmed to run for extended periods (even surviving a gateway restart).

\subsubsection{Graphical User Interface}

Ignition offers an efficient and intuitive environment for high performance GUI development. The GUI design concepts were extensively tested and refined during the development of slow control for the System Test until the GUI configuration met with the approval of both project scientists and engineers. The LZ slow control GUI will be based on this configuration and will offer different levels of supervision and control of the system.

At a higher level, several panels will show the real-time status of the system and subsystems from which an operator will be able to tell the overall health of the system. These panels will allow the authorized user to verify and control key system parameters and assess high level information, for example alarm status, current operation mode, status of automation scripts, etc. These panels will be designed so that even a novice operator could recognize an abnormal situation. Furthermore, access to the solutions for the common problems and tasks will be provided.

At a lower GUI level, detailed information about every sensor and actuator in the system will be provided for debugging and development purposes. The panels will be organized in form of complete but simple P\&IDs, as shown in the example in Figure 8.8.2. Upon a click on a P\&ID element, a new window will open and the system experts will be able to see and change the sensor configuration or status of the control, allowing complete access and control for the run control system experts.

At a very high level, a single summary plot of the entire system can be prepared by slow control and sent to run control for display in a single status pane on the run control GUI. This high level display can ensure that key information is available to any shift personnel regardless of run-control expertise, and regardless of the specific arrangement of windows, dialog boxes or other information on the slow-control system.

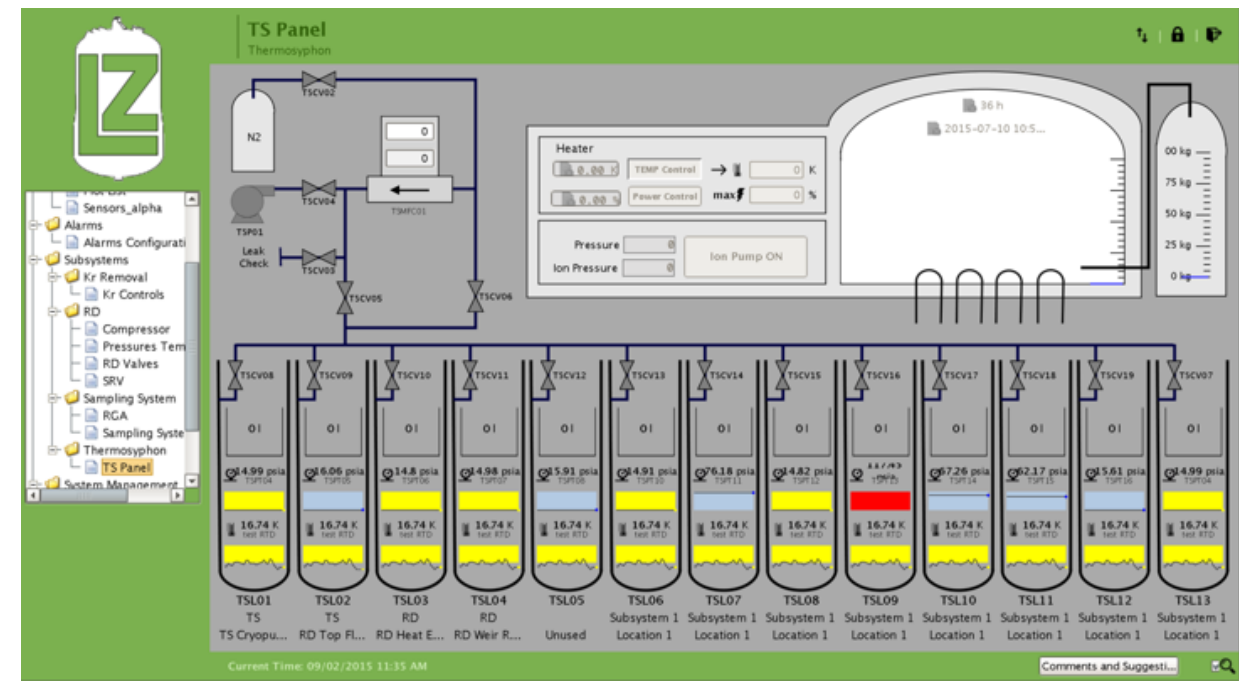

Figure 8.8.2: An example of a low-level panel in the System Test slow control GUI.

In a addition, the GUI will provide panels for historical data plotting (individual, multi- and scatter-plots) and analysis. The lists of sensors, controls and alarms similar to those used in the LUX slow control system will be provided as well. For increased usability, all the viewing panels will be fully customizable so that 
each user can save personalized views between usages. Finally, administrative panels will give access to advanced system configuration such as tag metadata, user management, etc.

\subsubsection{Integration into experiment online IT}

The slow control system will seamlessly integrate into the LZ experiment online IT and take advantage of the network security architecture and the authentication/authorization infrastructure provided for LZ online systems (see 8.12). The main servers for slow control (Ignition and the MySQL database) will be run as virtual machines, allowing for an additional layer of redundancy, fault tolerance and easy backup/restore.

With the possible exception of operator consoles where physical access is restricted, all users will authenticate to the slow control system with their own credentials (no shared accounts). All roles and permissions for slow control are maintained in the central directory and made available to Ignition through LDAP.

\subsection{Online System}

The core of the online system is the run-control system (RC), schematically shown in Figure 8.9.1. It comprises the software and hardware required to allow the operator to define and initiate data collection runs of different types, control and monitor subsystems (DAQ, slow control, the event builder (EB), and offline software), and log key information to the database. The physical system is hosted on a single rackmount computer, identical to the hardware used for the event builder. The use of identical hardware with an on-site spare minimizes any downtime of this critical system. RC software is committed to the LZ GIT repository and is maintained on a mirror system throughout the project and operation periods to allow off-line debugging and code development.

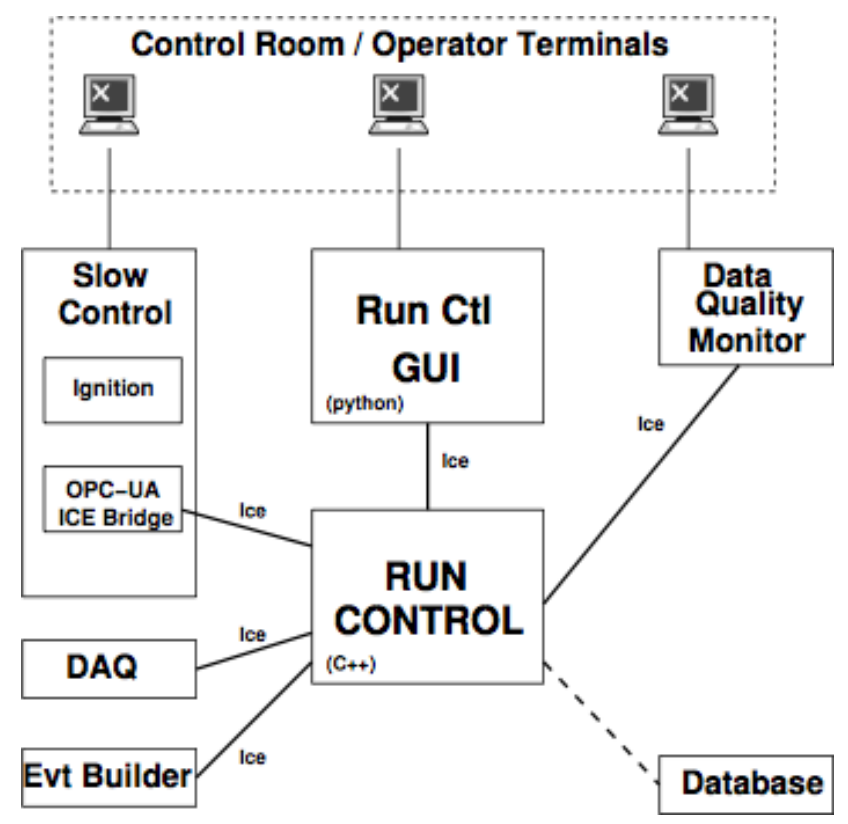

Figure 8.9.1: Block diagram showing the primary interfaces between the RC system and other LZ subsystems. The users use a GUI to interact with the RC system.

The work on the run-control software development started with a definition of the architecture (adopting various software standards), the development of detailed specifications for the software and the identification of key interfaces with the various subsystems. The interplay of run control with slow control is of central 
importance: Where slow control provides detailed control and monitoring of the detector hardware, run control both initiates high-level slow control scripts (to configure the instrument for various run types) and is the sole entity that configures and controls the data acquisition subsystems (DAQ, EB and DQM). OPC/UA (and similar) standards are used by much of the hardware for slow control, but the middleware (communication protocol) needed for coordinating other systems has different requirements. Thus one critical decision in determining the RC architecture was the selection of the middleware to be used for communications between RC and the subsystems. We have past experience with a number of middleware packages, including the industry standard CORBA (Common Object Request Broker Architecture) system. Given the limitations of CORBA (e.g., scalability, throughput, reliability, documentation and thread-safety) a new solution was considered with similar functionality but improved performance. A recent study [10] to identify middleware to run CERN accelerators ranked ICE (Internet Communications Engine) and ZeroMQ as the top two evaluated systems. The criteria for this evaluation included reliability and speed, and the ability of the system to handle a large number of messages per second (both small and large messages) and publish to a large number of clients in minimal time. Based on our comparisons of different Middleware options, ICE has been identified as the best choice for the LZ run control system. ICE is a remote-procedurecall (RPC) system with open source C++ and Python implementations. In ICE there is no clear distinction of client or server, but it is convenient to think of the subsystem ICE nodes as acting primarily as servers, while the RC process is the main ICE Client. The RC process also acts as a server for the RC GUI. The low-level RC program is implemented in $\mathrm{C}++$, while the RC GUI is implemented in Python. An API is being developed to allow LZ subsystems to implement ICE communications either through Python or C++. The RC Gui is being developed in PyQt [11] with Qwt for real-time displays.

To provide a seamless interface to the DAQ system, each data collection mode (e.g., calibration runs, data runs) is associated with a corresponding set of command sequences to the various subsystems. The list of data collection modes includes normal data-taking modes (S1 only, S2 only, S1 and S2; see Table 8.5.4 for more details) and a number of calibration modes, including the LED, krypton injection, tritium, and neutron calibration (see Chapter 7 for more details). The RC group interacts closely with the groups responsible for the various subsystems to determine the required interfaces, including defining what status information is shown, what commands are provided, and how status information should be displayed. Based on this information, the RC group has been developing skeleton (server/daemon) program for each subsystem, including the appropriate handles for communication with $\mathrm{RC}$, covering commands and sharing of status information. These skeleton server programs provide the starting point for further development by each group for their specific subsystem, working in close consultation with the RC programmer. This model has already been used to develop the DAQ interface, where the Washington University RC engineer provided a simple DAQ ICE server skeleton program to the DAQ system developer, who subsequently used this code to quickly develop the DAQ server side communications. The initial RC DAQ system is now complete, and was used for instantiating runs in the LZ electronics chain test. The run control/slow control interface is currently under development. As before, the RC engineer is providing code to the SC system programmer to be used in the development of an ICE/OPC-UA bridge to be used for the RC-SC command and communication link.

Throughout the course of the development, the run-control and slow-control groups have worked together closely and try to adhere to common standards for coding, code-management, and common libraries for communication and interfaces. Most commands and status data communication will be handled by the ICE/OPC-UA bridge, providing a bridge between the RC ICE RPC mechanism (optimized for data acquisition), and a tag-based interface primarily using OPC-UA (the standard for slow hardware interface) for Ignition. As with other subsystems, data sharing between run-control and various subsystems can occur through the database, but commands and critical data communication will occur only through the ICE connection between slow-control server computers and the run-control system. 
The final RC system provides the user with a simple GUI to the DAQ, data quality monitoring, and the slow-control systems. The GUI starts and stops all subsystems, and displays key status information and alerts if communication links are lost. The system will guide the user through setting up and starting/stopping various data-collection modes and starting runs, making sure all settings, data, and operator comments are logged into the database. The GUI includes key alerts for out-of-bound values, as determined by the slow-control system or other subsystems, and provides a small number of user-selected plots that allow the user to quickly monitor the health of the LZ system.

A skeleton version of the RC software is already complete and was used for the electronics chain test. See below for a screen shot of the RC GUI as of mid-January 2016. During this initial development period, the options for the basic system framework were determined. In the next R\& D stage, system hardware will be purchased. The RC group will work with software developers for the other subsystems to develop interface control documents (ICDs) specifying command sequences, settings, and critical data to be exchanged directly through an ICE link or indirectly through the database. Lessons learned from the electronic system test and the feedback from early users will provide input for the final design phase, during which ICDs will be finalized and the full set of interfaces between RC and the subsystem implemented.

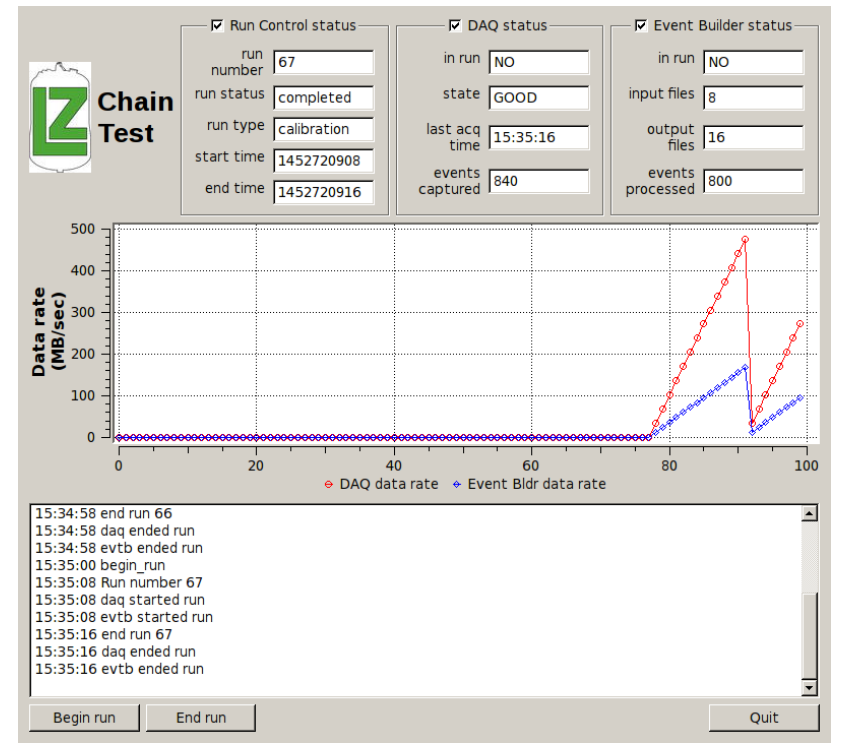

Figure 8.9.2: Run Control GUI for for electronics chain test.

The other major component of the online system is the Event Builder (EB). The Event Builder reads the binary files from the 15 individual data collectors (14 for the TPC, skin and outer detector channels, and one for the sparsification/trigger system), assembles the events, and writes out the raw data files on the underground RAID array.

The format and naming convention for both binary input and raw output files is documented in an ICD between WBS 1.8 and 1.11, i.e., between the online and offline systems. Based on the experience from the Daya Bay experiment, it has been decided that the size of the raw output files should be kept at about $1 \mathrm{~GB} /$ file. The choice of the raw data format was based on three factors, namely a self-defining data structure, machine independence, and the availability of a generic event viewer (which allows a quick inspection of the data without relying on an experiment-specific framework). This narrowed down the choices to HDF5 and root. The raw output format was chosen to be based on root. An HDF5-based EB was also developed, but its performance (both in speed and internal compression) was shown to be inferior to that of the root-based EB. The EB needs to be able to keep up with the highest data rate expected in the LZ detector, 
e.g., about $140 \mathrm{~Hz}$ during ${ }^{83} \mathrm{Kr}$ calibrations. Therefore, assuming a safety factor of 2 to 3 , the EB must be able to operate at $280 \mathrm{~Hz}$ to $420 \mathrm{~Hz}$. The internal compression is defined to be acceptable if it is comparable to the level achieved by the standard gzip compression algorithm on the binary files.

The EB is controlled by Run Control, which initiates the start of the EB for a particular run. The EB reports back to Run Control the current event and file number with a frequency that can be adjusted through a parameter. The end of run (of predetermined duration or number of events) is propagated to the EB directly through a flag included in the binary files, but can also be initiated directly by Run Control if the DAQ is non-responsive. Once a run has been successfully completed by the EB, Run Control is notified by the EB, which in turn releases the binary files on the data collectors to be deleted. All communications between Run Control and the EB occur through ICE.

Because the RC code will be developed and maintained by an experienced professional programmer, it is expected that the RC system (and other subsystem communication links) will be well maintained throughout the LZ project and operation periods. The RC software engineer will ensure that other online systems adhere to the ICDs, and use the software development standards decided by the WBS 1.8 group. The RC software engineer will assist with other online programming tasks as needed, lending support and responding to user requests for changes in the online system as needed.

\subsection{Electronics Chain Test Facility}

\subsubsection{Description and goals of the Electronics chain test facility}

To test all the components of the signal processing chain, an electronics chain test facility (ECT) has been set up at the University of Rochester [12]. The main goals of the ECT are:

\section{Evaluate Analog Electronics:}

- Noise and cross-talk. To measure the noise and cross-talk of the amplifiers, digitizers, and PMT bases.

- Signal propagation. To measure and optimize shaping, gain, linearity, and saturation.

\section{Evaluate Digital Electronics:}

- Firmware and software development. The ECT provides a direct hardware testing platform minimizing the need for time-consuming simulations.

- Clock distribution. Determine if we can distribute the clock over the HDMI links or if we have to use dedicated clock distribution resources.

- System performance. Verify that the system is capable of handling the expected data rates:

- SerDes links utilization.

- P-2-P Gigabit UDP link utilization.

- Data Collector CPU/disk speed utilization (storing on local disks and providing the data to the event builder).

\section{Develop Online Systems:}

- Run Control. Optimize interactions between run control and DAQ and DS systems.

- Event Building. Optimize event building with data collected with the entire electronics chain.

- Data Quality Monitor. Develop software tools to monitor the quality of the data collected. 
In the first phase of the ECT, we have a single chain setup shown in Figure 8.10.1. This stage allows us to concentrate on the quality of signal propagation from PMT to disk. The first results from phase 1 are described in more detail in the remainder of this Section.

Figure 8.10.2 shows the chain test setup in its second phase. The second phase is geared towards developing and scaling the DAQ system.

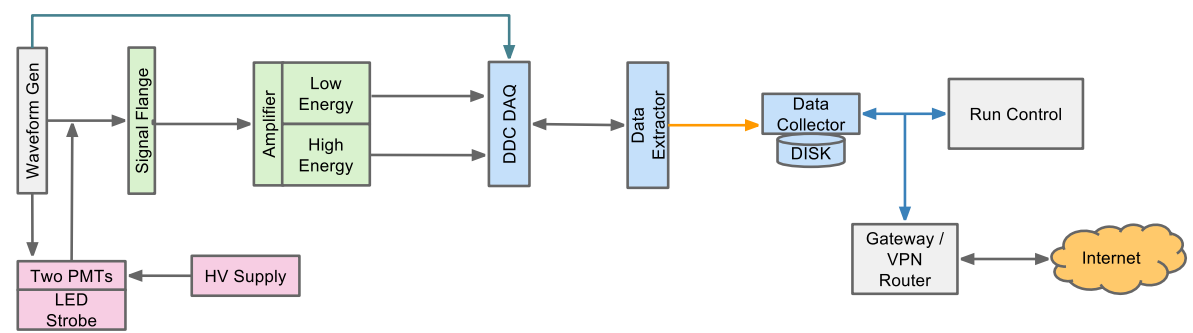

Figure 8.10.1: Diagram of the first stage of the Chain Test setup. The setup includes: two LZ PMTs, LZ internal signal and HV cable, LZ signal flange, LZ amplifier cage with one LZ amplifier board, one digitization chain, Run Control, and OpenVPN remote access.

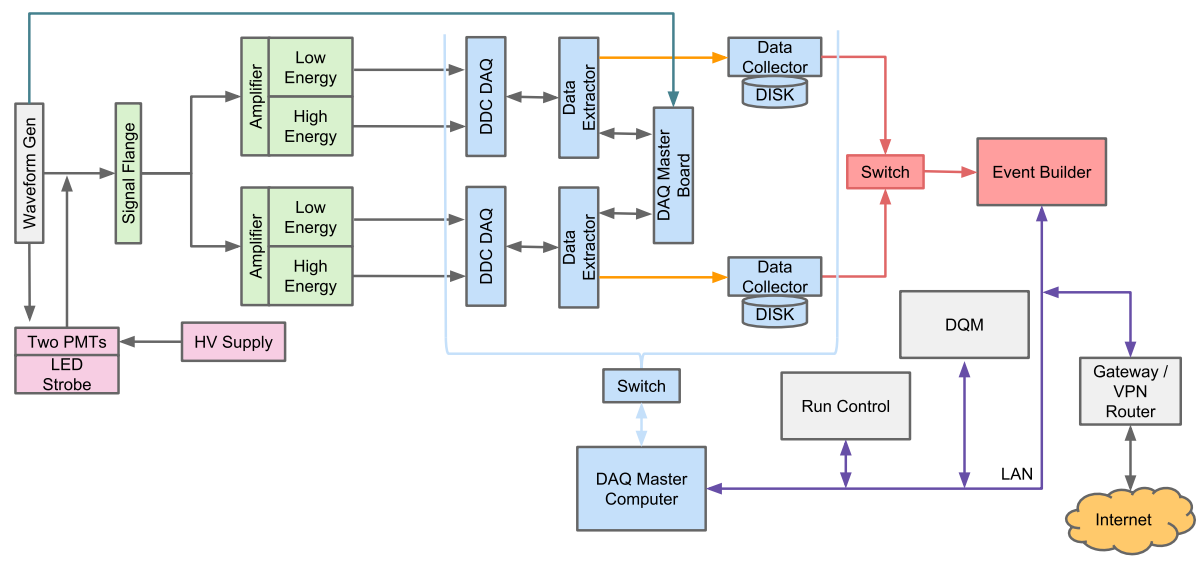

Figure 8.10.2: Diagram of the second phase of the Electronics Chain Test Facility [12]. The setup includes: two LZ PMTs, LZ internal signal and HV cable, LZ signal flange, LZ amplifier cage with two LZ amplifier boards, two parallel digitization chains, Event Builder, Run Control, DQM, DAQ Master Computer and OpenVPN remote access.

\subsubsection{Single photoelectron pulse propagation}

The initial measurements carried out at the ECT focused on SPHE pulses from a single R11410-20 PMT. These studies allowed use characterize signal-to-noise ratios and timing resolution of the electronics chain.

Figure 8.10.3a shows an intensity map and an average of 1000 SPHE pulses captured at the signal flange (before amplifiers), using a Tektronix MSO4104 oscilloscope with $250 \mathrm{MHz}$ set bandwidth and $2.5 \mathrm{GHz}$ sampling. Figure 8.10.3b shows a corresponding SPHE pulse captured at the output of the LE amplifier channel.

The R11410-20 are expected to operate at $3.5 \times 10^{6}$ gain, but since the manufacturer guarantees them to $2 \times 10^{6}$, we measured the SPHE response for both scenarios. Figure 8.10.4 shows two SPHE spectra 

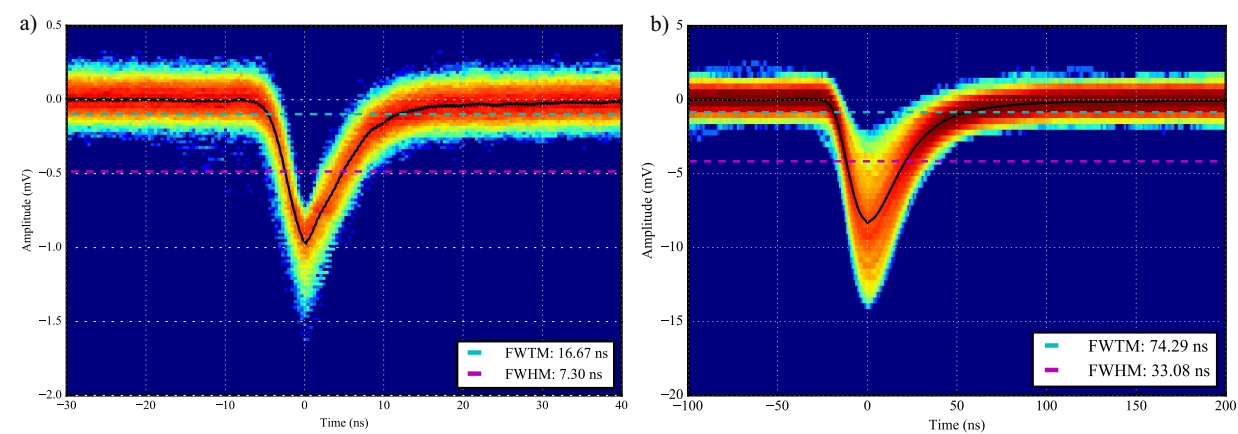

Figure 8.10.3: Single photoelectron intensity plots. (a) LZ PMT @ 1,300 $\rightarrow 45 \mathrm{ft}$ GORE cable $\rightarrow$ Signal Flange $\rightarrow 6 \mathrm{ft}$ LMR-100 $\rightarrow$ Scope, (b) LZ PMT @ 1,300 V $\rightarrow 45 \mathrm{ft}$ GORE cable $\rightarrow$ Signal Flange $\rightarrow$ LZ Amplifier LE output $\rightarrow 30 \mathrm{ft}$ LMR-100 cable $\rightarrow$ Scope

obtained at PMT gains of $3.25 \times 10^{6}$ and $1.9 \times 10^{6}$. The signals were propagated through the entire analog chain composed of 45 feet of gore cable, the amplifiers, and 30 feet of LMR-100 cable, before being digitized on the DDC-32. The waveforms were passed through an S1 filter and the filters' peak output (proportional to area) was histogrammed. The filters' width was set to 3 samples to optimize the peak to baseline noise ratio. The measured detection efficiency of $98 \%$ (at $3.25 \times 10^{6}$ ) and $92 \%$ (at $1.9 \times 10^{6}$ ) are obtained with only $1 \mathrm{~Hz}$ of singles rate (where the $\mathrm{S} 1$ filter captures a fluctuation in the baseline noise).

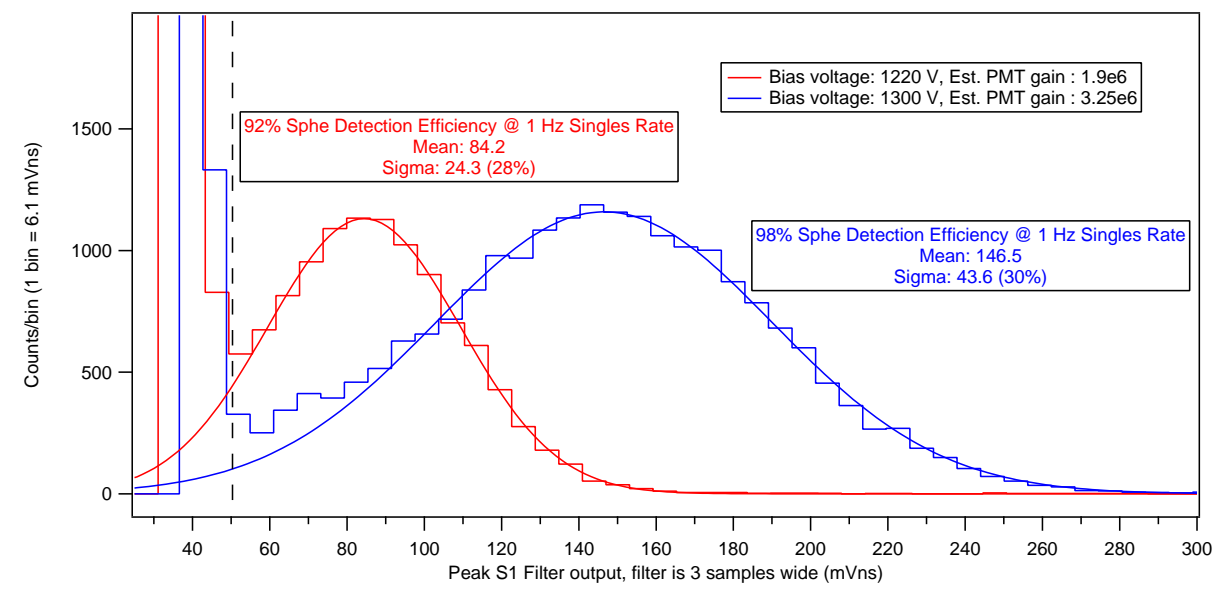

Figure 8.10.4: Single photoelectron spectrum at estimated $1.9 \times 10^{6}$ and $3.25 \times 10^{6}$ gain. Detection efficiency at $1 \mathrm{~Hz}$ singles rate is $92 \%$ and $98 \%$, respectively.

In order to determine how well we can reconstruct the relative timing between SPHE signals with a $60 \mathrm{~ns}$ shaping time (FWTM), digitized at $100 \mathrm{MHz}$, we carried our a series of measurements with short light pulses from an LED. As light source we used the CAEN SP5601 LED pulser, which has a measured resolution of $0.44 \mathrm{~ns}(1 \sigma)$ [13]. To limit the impact of the PMT's transit time spread (TTS) which has been measured to be $2.55 \mathrm{~ns}(1 \sigma)$ [14] for photons distributed across the entire photocathode, an aperture $(1 \mathrm{~cm}$ diameter) was placed in front of the PMT. The PMT was operated with a gain of $3.25 \times 10^{6}$. Figure 8.10.5a shows that measured reconstructed time difference between the LE SPHE signals and the LED pulser start signal has a resolution of $1.65 \mathrm{~ns}(1 \sigma)$. It should be noted that the resolution obtained between two LED pulser start signals, also digitized with the DDC-32, is $54 \mathrm{ps}(1 \sigma)$ (Fig. 8.10.5b). 
a)

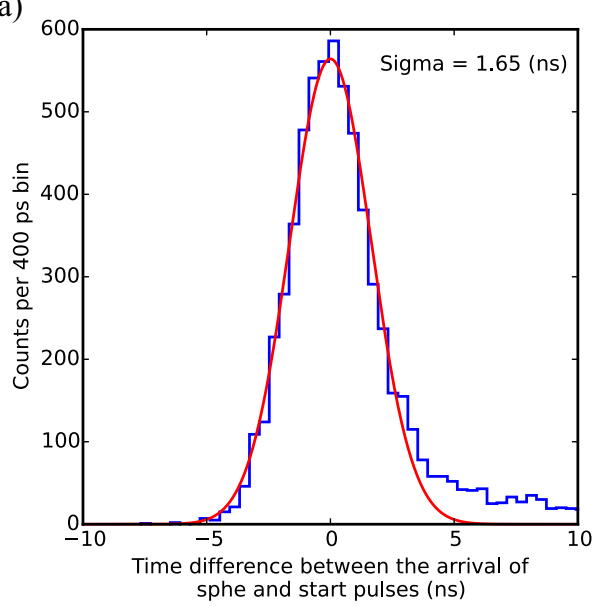

b)

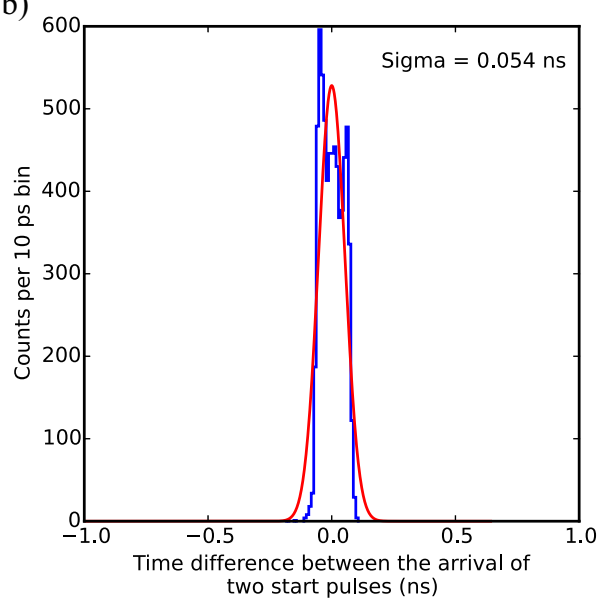

Figure 8.10.5: Time difference distributions for: a) LE SPHE signals obtained with a gain of $3.25 \times 10^{6}$, b) two LED start pulses. The time range in b) is ten times smaller than the time range in a).

\subsubsection{PMT Base Saturation}

The PMT base was evaluated for saturation with S2-like pulses. Wide pulses were sent to a $425 \mathrm{~nm}$ LED and varied in amplitude to produce S2-like responses in the PMT with a width of $0.5 \mu$ s $(1 \sigma)$. To determine the amount of light emitted by the LED, we collected data in two modes: 1) with a $1 \mathrm{~cm}$ diameter aperture in front of the PMT to limit the amount of incident light in order to calibrate the LED, and 2) without the aperture where we expect PMT saturation for large LED signals. Signals from the PMT are propagated through 45 feet of Gore cable to the DB25 connector on the signal flange and digitized on the Tektronix MSO4104 oscilloscope along with the signals sent to the LED. Figure 8.10.6 shows the averaged PMT pulses collected without the aperture for different LED pulses.

With the aperture, the PMT pulse area increases linearly with the LED pulse area, demonstrating that as the amplitude of the pulse to the LED increases the amount of light being generated increases. This allows us to calibrate the amount of light incident onto the PMT. In the measurements without the aperture, we know that the LED pulse area is proportional to the light emitted by the LED. Figure 8.10.7 shows a plot of the measured PMT pulse area when the aperture is removed versus the PMT pulse area we would have obtained if the aperture would have been in front of the PMT. This figure shows that for the two largest LED amplitudes, the PMT base saturates.

In the region of saturation, the shape of the response of the PMT changes, as can be see in Figure 8.10.6. The saturation manifests itself in the form of narrowing the shape of the pulse for larger light input to the PMT and no increase in pulse area. Saturation occurs when the amplitude of the signal from the PMT at the input of the oscilloscope exceeds $0.6 \mathrm{~V}$.

\subsubsection{DAQ Code and Performance}

The main Data Collector acquisition code is named DCCore and has been rewritten in C++ using Qt based multithreading framework. It is responsible for receiving UDP datagrams from the Data Extractors, performing consistency checks on it and storing it to a local SSD drive. The DCCore successfully communicates with the Run Control over ICE by responding to run start/stop commands and reporting the acquisition status and progress.

During active data collection, at the rate of $70 \mathrm{MB} / \mathrm{s}$, the DCCore has been shown to utilize a single CPU core at only $\sim 15 \%$ level. Currently the measurement was done with the peak rate of $70 \mathrm{MB} / \mathrm{s}$, because the 


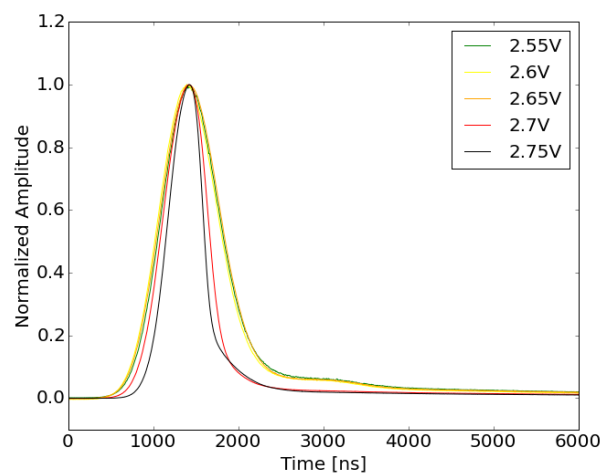

Figure 8.10.6: Average peak aligned and normalized pulses showing the S2-like response of the PMT. The legend corresponds to the peak amplitude of the pulse used to drive the LED. The first signs of saturation begin to manifest itself for an LED amplitude of $2.65 \mathrm{~V}$.

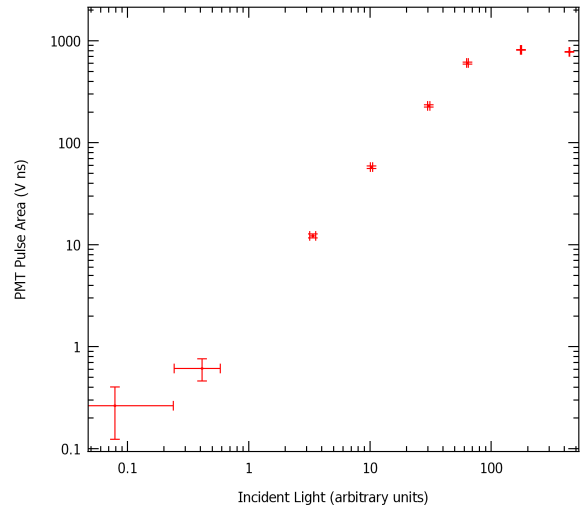

Figure 8.10.7: Result of measured pulse area detected by the PMT without a hole versus the expected pulse area of the PMT with a hole. As the expected amount of light increase the PMT's measured response increases till it begins to saturate for the last two points.

FPGA waveform capture firmware is implemented in a single-buffering mode and thus not push the Gigabit UDP link to its maximum of $109 \mathrm{MB} / \mathrm{s}$. The single-buffered rate is understood and agrees perfectly with what we estimate in this scenario.

Additionally a Python based DAQScope code has been developed. It communicates with the DCCore using ICE. It is a GUI tool that allows for real-time preview of the waveforms incoming into the Data Collector. It also allows for continuous calculation of the waveforms' power spectral density (PSD) plots. The screenshot of the tool in operation is shown in Figure 8.10.8

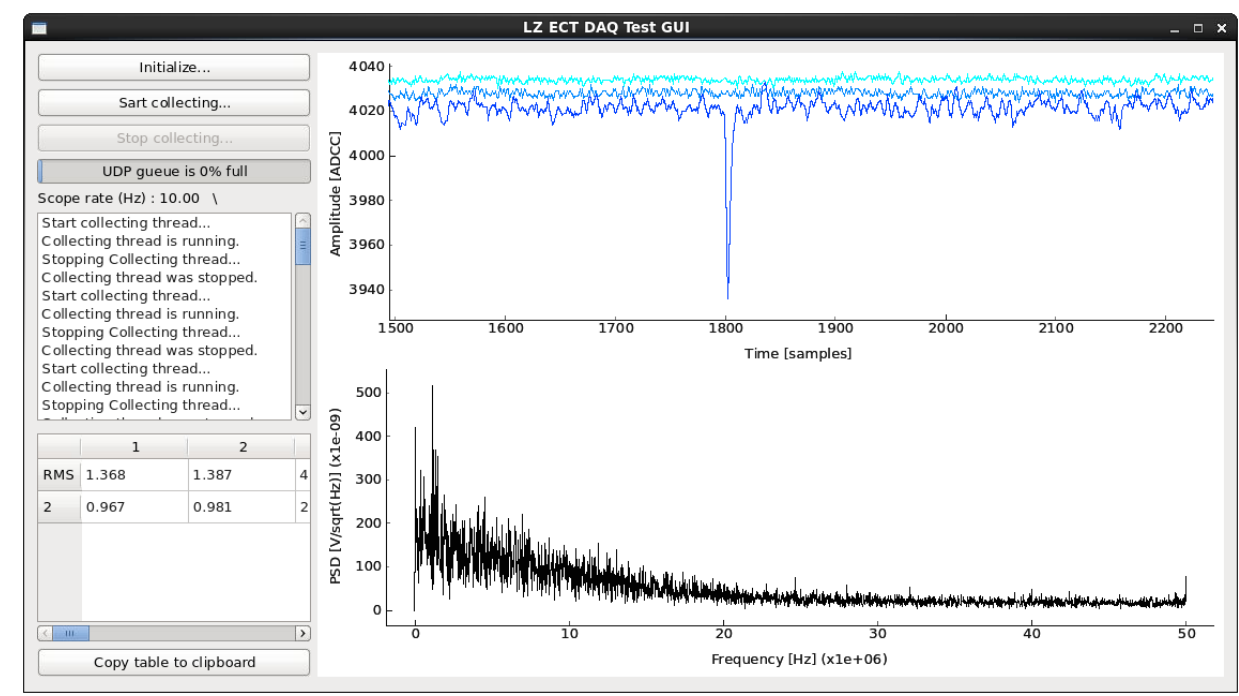

Figure 8.10.8: A screenshot of the DAQScope tool displaying a subsample of the waveforms incoming into a given Data Collector and showing a continuously calculated PSD spectrum for one of the channels. 


\subsubsection{Real Signals from LUX}

At the end of LUX, we had the opportunity to replace the existing signal chain with the LZ signal chain from amplifier to disk. This allowed us to benchmark the performance of our electronics against those used in an active dark matter detector and to look at real signals. For these tests, two LZ amplifiers were mounted on a LUX signal flange that had signals originating from both the top and bottom PMT arrays. Dual gain signals from the amplifier were routed through 45 feet of LMR-100 cable to be digitized on a prototype DDC32. Data was extracted from the DDC32 via and HDMI link to a DE board and then pushed to a DC where it was stored in a partial event file format. The LUX grounding scheme was used for the electronics chain.

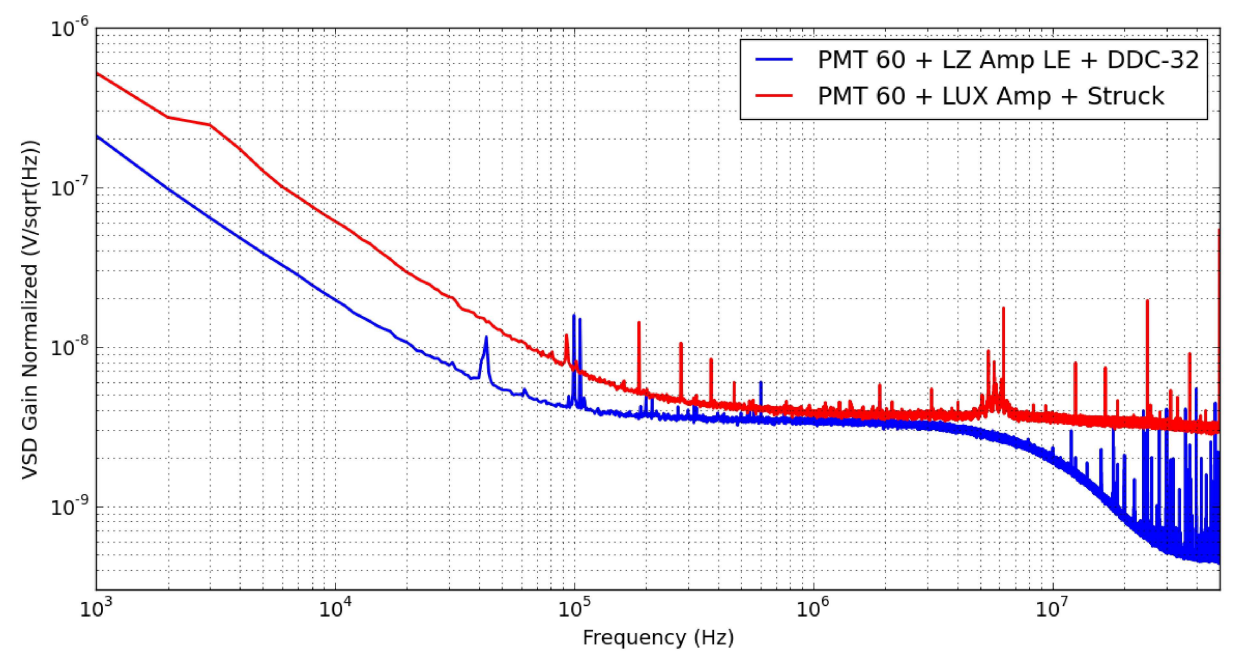

Figure 8.10.9: Gain normalized noise spectra compared between the LUX and LZ DAQ chains.

Noise measurements, SPHE calibrations, and real event signals from LUX were collected during these tests. The gain corrected noise measurements are shown in Figure 8.10.9. These measurements show that, even though no special consideration was given to optimize the grounding scheme, the LZ system performs as well as the LUX systems. From SPHE calibrations we measure a SPHE detection efficiency greater than 90\%. An example of the S1 filter output for SPHEs is shown in Figure 8.10.10.

\subsection{Grounding}

The LZ experiment comprises many electrical and electronic sub-systems that may be adversely affected by electrical noise. Each sub-system in the LZ experiment will be guarded from noise induced by other sub-systems. Guarding will also reduce noise introduced into other sub-systems. The PMT signal collection chain is one of the most sensitive and most critical sub-systems in LZ. PMT signals are in the sub-mV range before amplification so even a small amount of noise has a potentially large impact. PMT signal quality directly affects the quality of the physics data therefore signal integrity is of great concern.

Unwanted signals or noise can be coupled from an "aggressor" circuit into a "victim" circuit through capacitive coupling, inductive coupling and conduction. Proper grounding can be critical to reducing interference through these mechanisms. Grounding plans for experiments of the size of LZ are complex and require significant engineering effort.

The LUX grounding system serves as the starting point for the LZ grounding scheme. The main lesson learned in implementing the LUX grounding system was the importance of a single-point or "star" ground configuration (Figure 8.11.1 a). All of the equipment in the electronics racks was grounded to the rack 


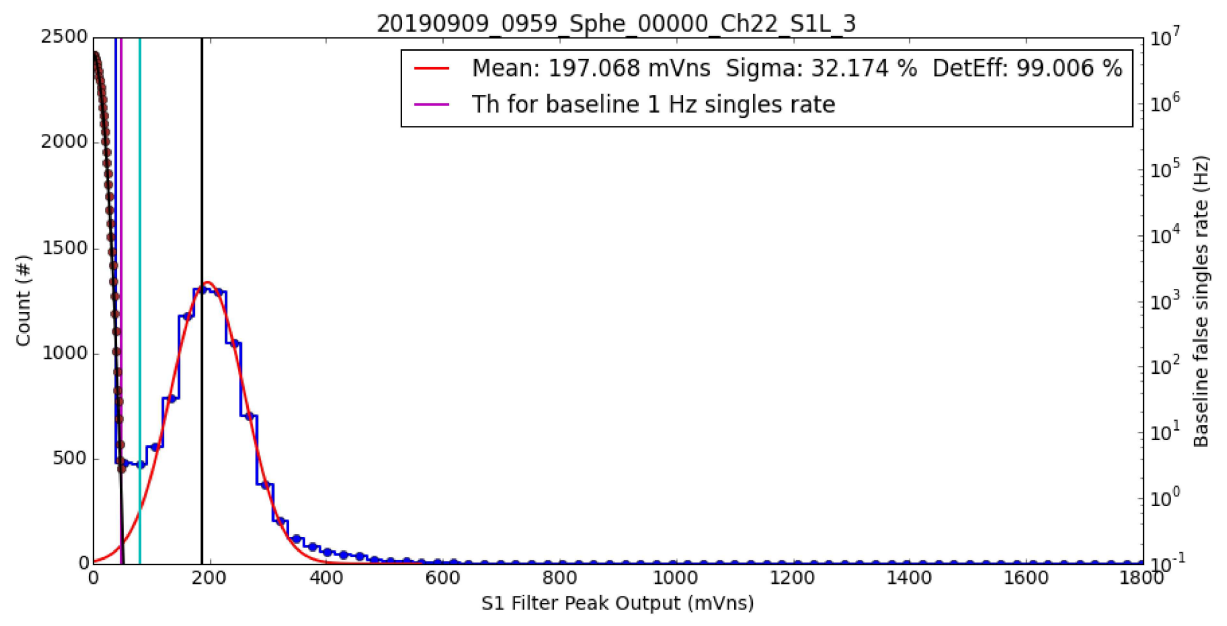

Figure 8.10.10: Maximum S1 filter output for SPHEs obtained with the LZ DAQ electronics installed on the LUX detector.

grounding bars using flat braided cable (Figure 8.11.1 b) and all of the grounding rods have dedicated grounding paths to the central star point using 2 AWG copper welding cable.
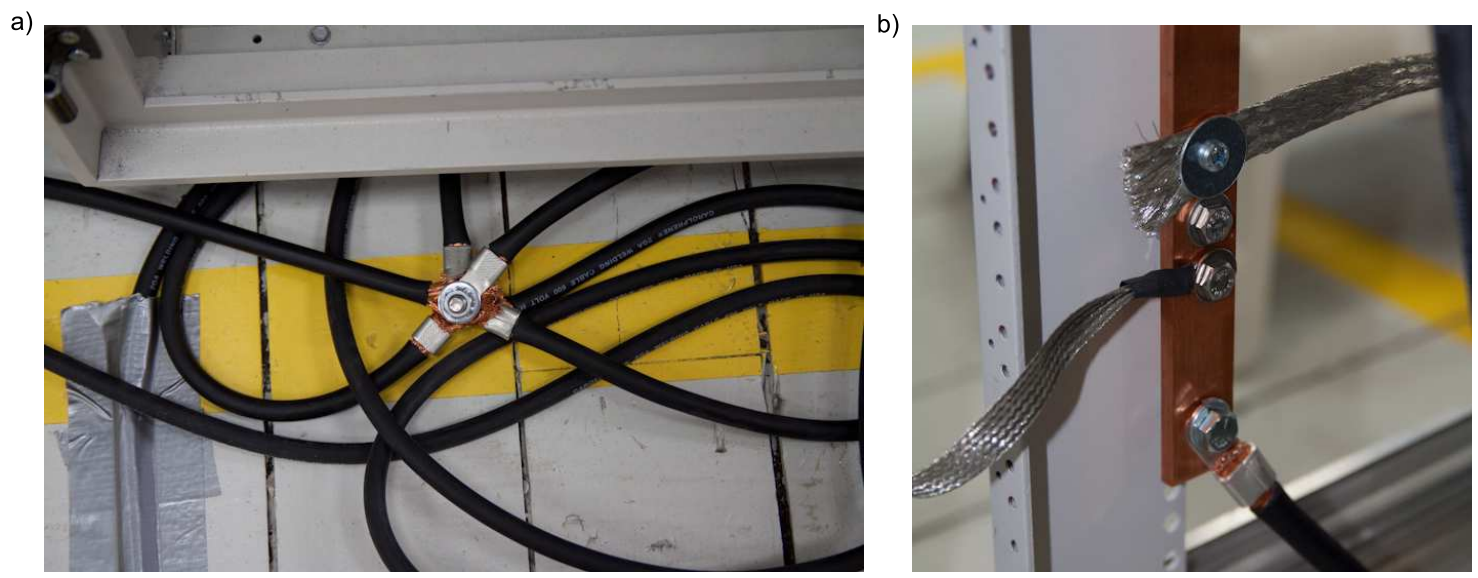

Figure 8.11.1: a) Star ground central point to all the rack grounds. b) Sample grounding connections within one of the racks, where individual equipment and the rack door are grounded with steel braid to the rack grounding rod. The grounding rod is connected to the center star grounding point using a high gauge copper welding cable.

For many of the circuits in LZ, the signal cable shield also acts as the signal return path. In order for the shield to be effective, it must not conduct significant amounts of current. If excess current does flow in the shield, it will show up as noise in the signal. This can occur when the "ground" potential is different at each end of the cable. In situations such as this, galvanic isolation can break the flow of current and eliminate the induced noise. We are currently testing the feasibility of using isolation transformers on the signal lines between the amplifier outputs (at the breakout) and the digitizer inputs ( $30 \mathrm{ft}$ of cable away in the electronic racks above). We are testing two candidates from Mini-Circuits: FTB-1-6+ and FTB-1-1+. As these are typically meant for RF applications, we need to quantify how these transformers impact the pulse time characteristics of typical amplified LZ PMT signals. If the transformers do not significantly degrade 
the signal quality they should allow for minimizing potential ground loops between the breakout area and the upper electronics racks.

Significant noise can also be generated by electrical equipment such as pumps, chillers, and blowers that draw significant current or have switched loads that might cause electrical transients. Interference from these sources will be eliminated or greatly reduced by isolating them on separate electrical circuits and by physically locating them as far from the sensitive PMT signal cables as practical. Capacitive and inductive coupling to PMT signal cables can occur if cables from other sub-systems run in close proximity to the PMT cables for significant distances. Again, by placing the PMT signal cables as far as is practical from other sub-system cables, we will greatly reduce the effects of capacitive and inductive coupling.

Ideally we will be able to identify all sources of potential noise and eliminate them during the design stages. But experience shows that it is unlikely that every noise source will be identified prior to the installation and commissioning of the system. During deployment/construction of the LZ experiment we will, early on, have a subset of the DAQ system installed such that we can constantly monitor PMT signals for any noise pickup as equipment is installed. If we see any noise being picked up from other systems during the installation of equipment for the project, the data we gather from the DAQ system will greatly simplify pinpointing the source and allow us to put in place fixes to ensure excellent signal integrity.

\subsection{Network Infrastructure, Security, Remote Access}

The LZ network and computing infrastructure will be designed according to best practices to meet the LZ requirements for performance, security and availability / operational reliability and uptime. A prototype has been developed and successfully demonstrated at the SLAC LZ System Test Facility, discussed in Section 3.10 .

\subsubsection{Local Area Network}

The LZ experiment LAN in the Davis Campus will be implemented with a single central router/switch that acts as communication hub for the experiment, provides separate network zones using Virtual LANs (VLANs) and IP subnets and enforces inter-zone network restrictions using simple access control lists (ACLs). Network zoning will be used to implement a standard network security architecture with "Demilitarized Zones" (DMZ) and restricted networks. If necessary, enhanced network security controls can be implemented with host-based firewalls or a dedicated firewall appliance. Figure 8.12.1 shows a logical diagram of the LZ experiment LAN.

\subsubsection{Reliability and Availability}

Connection to the surface will use dedicated and redundant fiber connections on physically separate paths (one fiber path through Ross shaft, one fiber path through Yates shaft). The central LZ router/switch located in the Davis Campus will be implemented as redundant stack of two switches with full power, uplink and control redundancy. All critical devices such as main PLCs and critical computing infrastructure will have redundant Ethernet connections to both stack elements. A separate, dedicated network connection to the surface will allow emergency out-of-band access to the PLCs and the network and IT infrastructure underground.

\subsubsection{Information Security}

The LZ computing architecture will implement a defense-in-depth security design based on a zoned network, central authentication and authorization (Kerberos and LDAP) with appropriate account-lockout policies, 


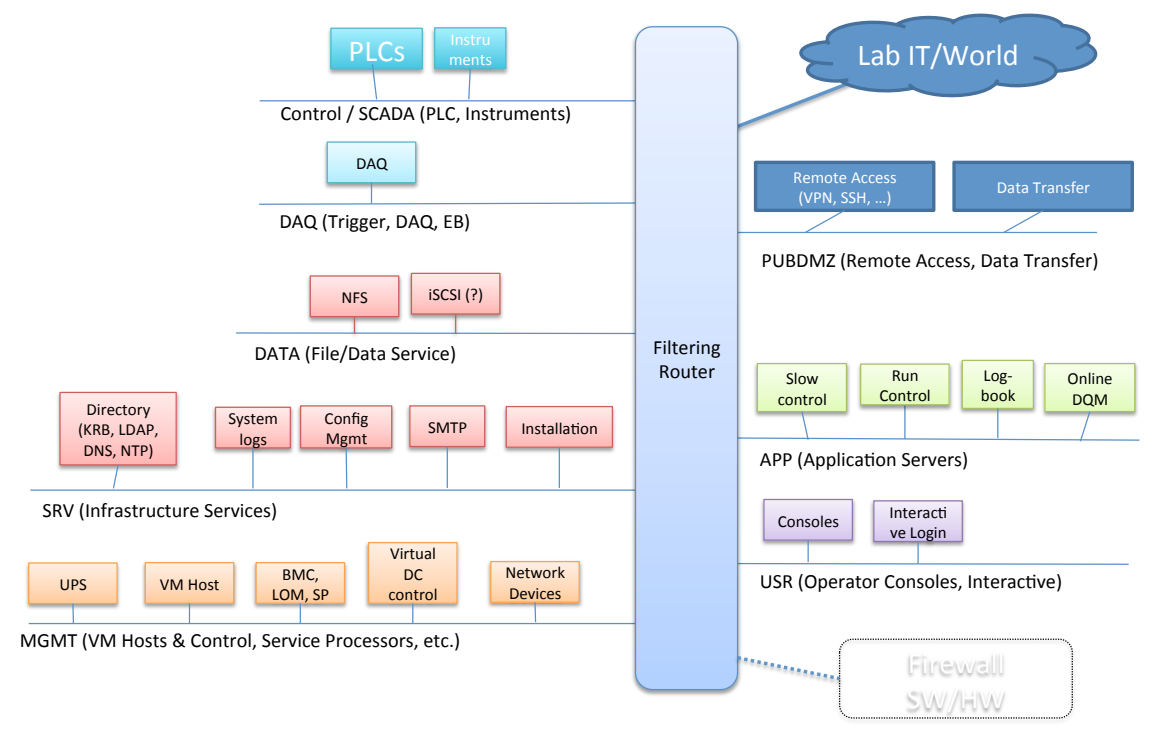

Figure 8.12.1: The LZ Online Network

a configuration management system for provisioning, managing and patching hosts, central logging and monitoring, and frequent backups for recovery from a wide class of problems. Advanced firewalls, networkor host-based intrusion detection, or other security controls can be easily added if deemed necessary.

Network zoning will be used to create a layered configuration where devices with weak security controls or network stacks (such as embedded systems, sensors, PLCs) are only allowed to communicate with trusted and managed systems.

Central management of authentication and authorization in a "Directory" based on Kerberos and LDAP will be used to manage user accounts and all privileges in the LZ system, including host access privileges and slow control roles. This will allow for easy enforcement of credential requirements (such as minimum password complexity or password expiration), secure auditing and logging of user privileges and lockout of user accounts if necessary. It will also allow to securely implement user self-service (e.g. for password reset or contact information change) and delegation of privilege management to subsystem managers (e.g. the slow control manager can assign roles to users without having to ask a system administrator to make the changes).

A configuration management system (such as e.g. SaltStack [15]) will be used to provision hosts with a secure baseline configuration and to maintain and update the host configuration as necessary. Using such a system minimizes the amount of effort required for system management and provides a way to detect unauthorized changes relative to the baseline configuration.

Central logging and monitoring provides another avenue to detect unauthorized activities, especially when combined with simple keyword-based automated log analysis, but is also a valuable tool for general nonsecurity related health monitoring, diagnosis and debugging of the system.

Where possible, frequent downtime-less file system snapshots and backups of all important system configuration and user/application data will be performed. Access to backup files will be restricted so that even a system compromise will not allow the attacker to modify historical backup data. 
Virtualization technologies may be used to reduce the effort required for system management and to provide virtual machines that can be easily controlled remotely by authorized users and that can be easily backed up.

\subsubsection{Remote Access}

The implementation of secure remote access will depend on the applications that need to be accessed and the client devices (desktop, laptop, tablet/phone) accessing them. All remote access protocols must use secure and encrypted communication and strong authentication mechanisms. While the details are still under study, we foresee some combination of web, command line, remote desktop and application client access over encrypted communication channels provided by a VPN and/or SSH.

\subsection{Installation and Commissioning}

The electronics will be installed in two locations. The analog electronics for the Xe PMTs will be installed on the mezzanine level, as shown schematically in Figure 8.13.1. The amplifiers will be arranged in 23 racks, with 4 amplifier cards installed in each rack. The racks will be located horizontally on the wall of the water tank in order to improve cooling and provide better access to each amplifier crate. Exhaust ducts may must be installed at this location to facilitate cooling of the electronics. Because it will be difficult to enclose the electronics crates at this location, it is important the air flowing over the electronics is directed into the exhaust system. This will prevent any smoke generation as a result of an electrical problem, e.g., overheating of components, from spreading into the Davis Campus. The outputs of the amplifiers are routed in cable trays to the DAQ and trigger systems installed in the electronics racks installed at deck level (see Figures 8.13.1 and 8.13.2).

A schematic of one element of the breakout box is shown in Figure 8.13.3. The internal signal and HV cables enter this section of the breakout box through the ports in he center. The signal cables are routed to the two flanges, shown in yellow, with four DB-25 connectors on which the amplifier crates mount. The HV cables are routed to the HV flange, shown in blue, visible at the bottom of this breakout section.

The amplifiers and the HV pickoffs for the outer-detector system will be installed in the electronics racks shown in Figure 8.13.1. The HV cables from the outer-detector PMTs are routed via one of the flanges on top of the water tank to this location. The design of the flange is such that no connectors are required to ensure a light-tight and radon-tight connection. Not using connectors on the flange improves signal quality.

A total of 11 electronics racks are installed at the deck level, as shown in Figure 8.13.1, to provide space for all electronics systems, except the xenon amplifiers. The racks are fully enclosed and the forced airflow across the electronics is directed into the exhaust system of the Davis Laboratory. Figure 8.13.4 shows the layout of the components installed in these 11 racks.

The power requirements of the electronics system in the Davis Laboratory have been estimated based on the measured power consumption of the various components of the system. The power required by the amplifiers, the DAQ, the computer systems, and the PMT HV systems is $26 \mathrm{~kW}$. The power required for the various calibration systems for the outer detector and the xenon PMTs remains to be determined. The power required by the electronics is provided by uninterruptible power supplies, installed at the bottom of each electronics rack. The total maximum load of the electronics is $94 \mathrm{~kW}$. This is the load of the equipment at startup. 


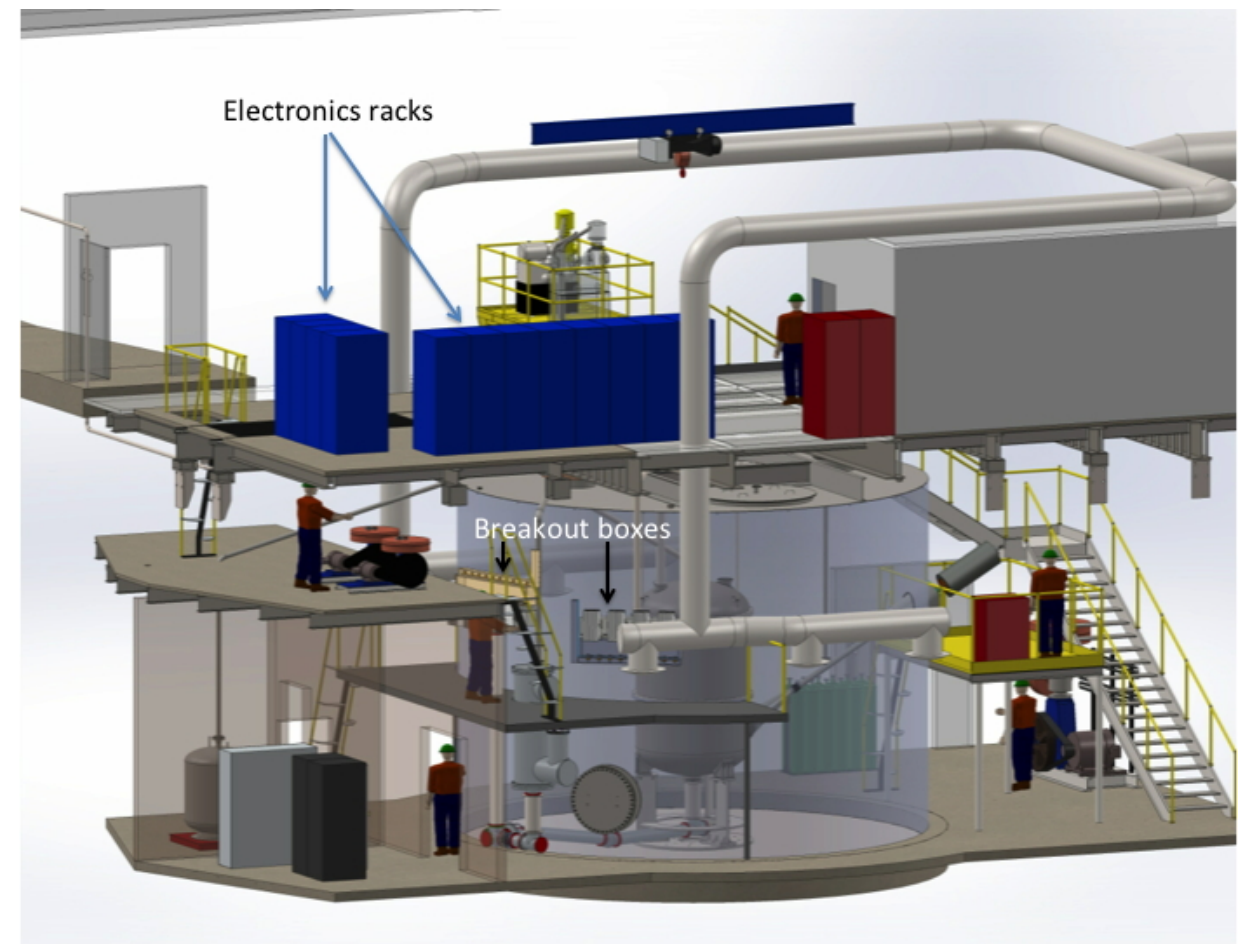

Figure 8.13.1: Installation of the analog electronics for the xenon PMTs at the mezzanine level and the electronics racks at the deck level.

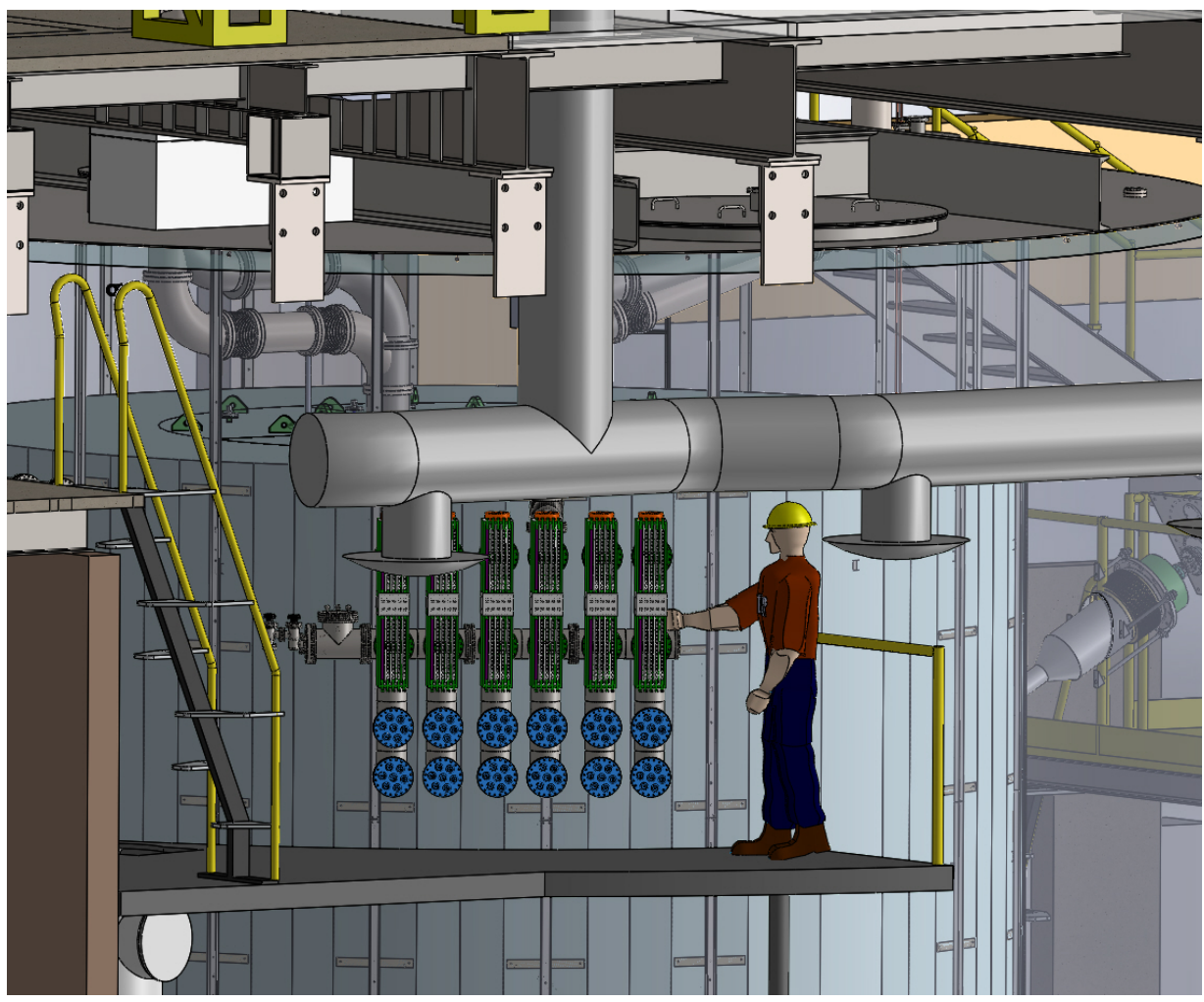

Figure 8.13.2: Close-up of the mezzanine level showing the amplifier racks installed on the top breakout box. The HV filter boxes are not shown. 

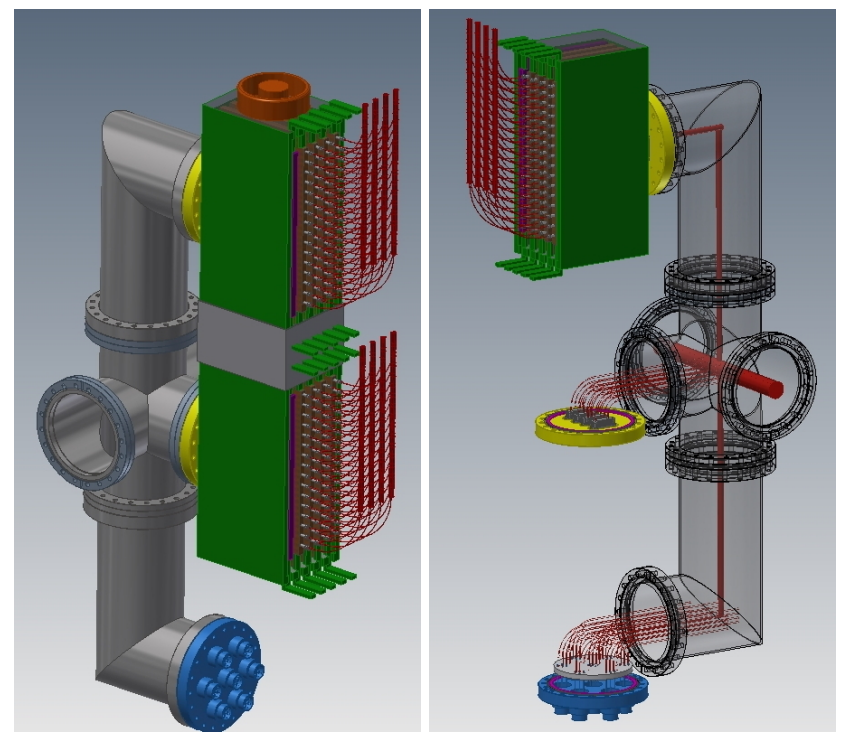

Figure 8.13.3: Close-up of one element of the breakout box. The figure on the left shows two amplifier cages installed on the top two signal flanges (yellow) of this section. The HV flange is visible at the bottom (blue). The figure on the right shows the routing of the signal and HV cables in this breakout section.

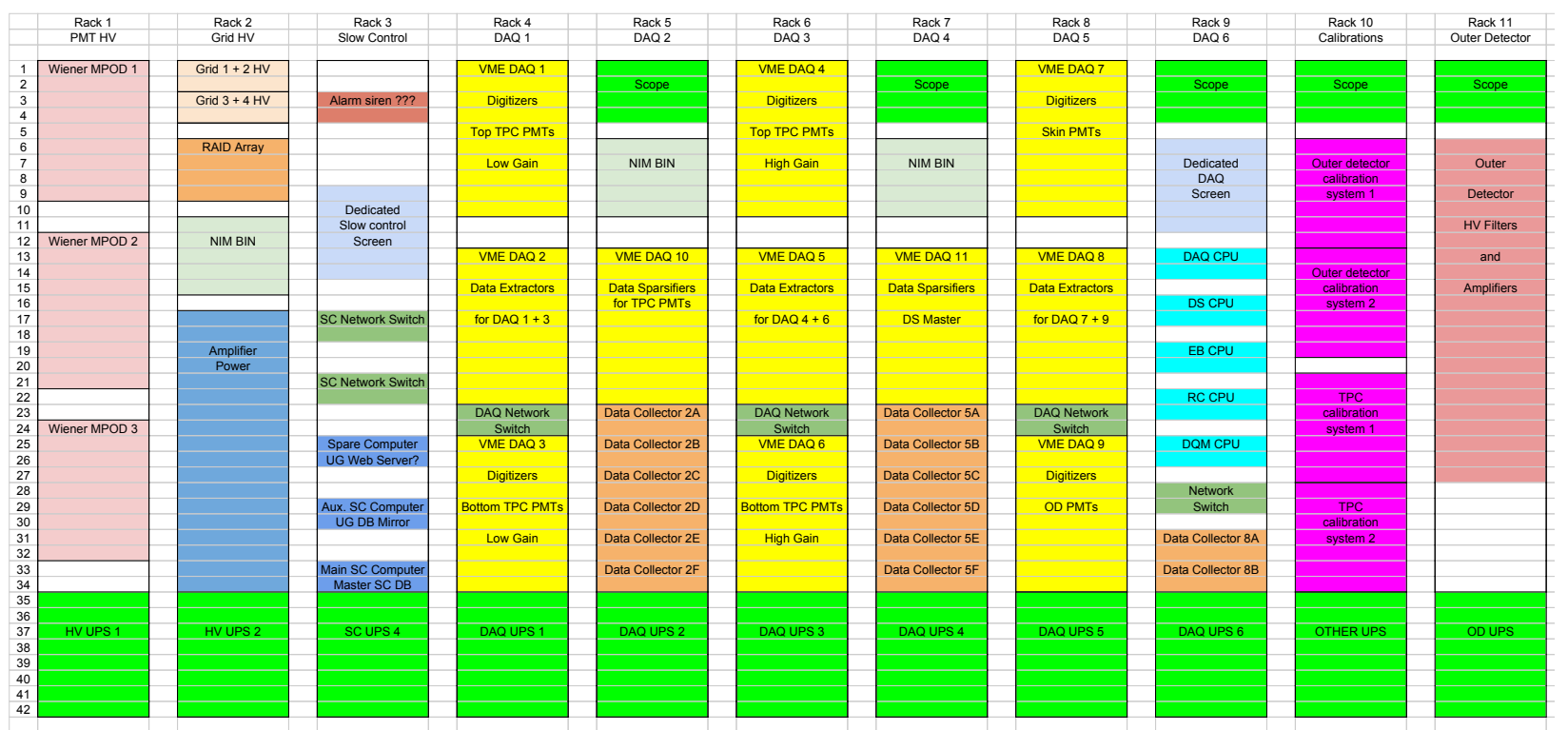

Figure 8.13.4: Detailed layout of the LZ electronics racks, installed at the deck level. 


\subsection{Bibliography}

[1] J. Yin (LZ), Proceedings, Light Detection in Noble Elements (LIDINE 2015): Albany, NY, USA, August 28-30, 2015, JINST 11, C02054 (2016), arXiv:1511.00260 [physics.ins-det].

[2] All Programmable FPGAs and 3D ICs, Xilinx Inc., 2100 Logic Drive, San Jose, CA 95124-3400, USA (2016).

[3] E. Druszkiewicz (LZ), Proceedings, Light Detection in Noble Elements (LIDINE 2015): Albany, NY, USA, August 28-30, 2015, JINST 11, C02072 (2016), arXiv:1511.08385 [physics.ins-det].

[4] D. S. Akerib et al. (LUX), Nucl. Instrum. Meth. A668, 1 (2012), arXiv:1108.1836 [astro-ph].

[5] E. Druszkiewicz, Digital Advances in Triggering and Data Acquisition Systems for Large Scale Dark Matter Search Experiments, Ph.D. thesis, University of Rochester (2017), [manuscript in prepration].

[6] D. Salomon and G. Motta, Handbook of Data Compression (Springer London, 2010) ISBN 978-184882-902-2.

[7] M. Henning, IEEE Internet Computing 8, 66 (2004).

[8] Ignition SQL Bridge, Inductive Automation, 340 Palladio Parkway, Suite 540 (4th Floor) Folsom, CA 95630-8833, USA (2015).

[9] Siemens Simatic PCS 7 CPU 410-5H Contoller (2016).

[10] A. Dworak, P. Charrue, F. Ehm, W. Sliwinski, and M. Sobczak, in 13th International Conference on Accelerator and Large Experimental Physics Control Systems, 10-14 ${ }^{\text {th }}$ October 2011, WTC Grenoble, France (2011) pp. 1334-1337, Middleware Trends And Market Leaders 2011.

[11] Introduction to PyQt, Riverbank Computing Limited, Redcotts House, 1 Redcotts Lane, Wimborne, Dorset BH21 1JX, UK (2016).

[12] D. Khaitan (LZ), Proceedings, Light Detection in Noble Elements (LIDINE 2015): Albany, NY, USA, August 28-30, 2015, JINST 11, C03029 (2016), arXiv:1511.07752 [physics.ins-det].

[13] SP5601 Led Driver - Data Sheet (2014), CAEN S.p.A., Via Vetraia, 1155049 Viareggio (LU), Italy.

[14] K. Lung et al., Nucl. Instrum. Meth. A696, 32 (2012), arXiv:1202.2628 [physics.ins-det].

[15] SaltStack system management software (2016). 


\section{Material Screening}

\subsection{Introduction}

The LZ sensitivity is assessed using a profile likelihood ratio (PLR) method, as described in Section 12. For material screening purposes, however, we evaluate conservative tolerable count rates by considering the residual events in a WIMP search region of about 0-20 detected photons (phd), equivalent to approximately $1.5 \mathrm{keV}_{\mathrm{ee}}$ to $6.5 \mathrm{keV}_{\mathrm{ee}}$ or $6 \mathrm{keV}_{\mathrm{nr}}$ to $30 \mathrm{keV}_{\mathrm{nr}}$. This energy window is functionally equivalent to the one employed in the full PLR method when considering the experiment's optimum sensitivity at about $40 \mathrm{GeV} / c^{2}$ WIMP mass. Events are considered within the 5.6 tonne fiducial mass, with vetoes from the liquid xenon (LXe) skin and outer detector (OD) applied, but before discrimination through S2/S1 and NR selection efficiency. In order for LZ to realize a sensitivity to a WIMP-nucleon cross section below $3 \times 10^{-48} \mathrm{~cm}^{2}$ within three years of data taking, the maximum tolerable electron recoil (ER) background from non-astrophysical sources is approximately $37 \times 10^{-6}$ events $/ \mathrm{keV} / \mathrm{kg} / \mathrm{d}(37 \mu \mathrm{dru})$. The nuclear recoil (NR) background must also be low, with $\sim 1$ count in the exposure.

Although this represents an unprecedented low background rate for dark-matter detectors, it can be achieved through the use of several proven low-background assay techniques that have been successfully employed in recent rare-event searches for dark matter, as well as in neutrinoless double-beta decay and neutrino experiments [1-9]. The collaboration maintains significantly stricter goals for all the detector elements to reduce the risk of any one element violating the required levels: $\sim 37 \mu$ dru and an NR rate of $\sim 1$ count in the exposure. In this section we frequently refer to the goals, which are between $1-10 \%$ of the levels specified as project requirements. The techniques LZ uses to monitor and control these backgrounds include:

- A comprehensive material-screening campaign to select components that satisfy stringent intrinsic radioactivity goals such that the single scatter rate within the fiducial volume and WIMP search energy range is less than $0.4 \mathrm{NR}$ counts and below $1 \mu \mathrm{dru}$ of ER background resulting from fixed contamination in the detector components;

- Direct measurements of radon emanation from construction materials for a maximum activity of $10 \mathrm{mBq}$ throughout the liquid xenon. The goals for emanation are $1 \mathrm{mBq}$;

- Adherence to cleanliness protocols for control of airborne radioactivity and particulates to limit background at the level of that from materials to contact the LXe, contributing $10 \mathrm{mBq}$ from radon emanation. This same level of surface contamination, in addition, contributes ER and NR backgrounds from the radioactive decays in the dust in the TPC and $(\alpha, n)$ reactions on the TPC components of $1 \mu \mathrm{dru}$ ER and $0.4 \mathrm{NR}$ counts, respectively. The goals for emanation from dust are $<1 \mathrm{mBq}$ with a corresponding reduction in ER and NR;

- Removal of radioactive elements such as $\mathrm{Ar}$ and $\mathrm{Kr}$ from the liquid xenon to limit their single scatter ER backgrounds in the WIMP search energy range to below $1 \mu \mathrm{dru}$.

Material screening is the primary route to controlling the ER and NR backgrounds resulting from radioactivity in the experiment. The measurement of radioactive isotopes in and on detector materials is required. These are primarily the $\gamma$-ray emitting isotopes ${ }^{40} \mathrm{~K} ;{ }^{137} \mathrm{Cs}$; and ${ }^{60} \mathrm{Co}$, as well as ${ }^{238} \mathrm{U},{ }^{235} \mathrm{U},{ }^{232} \mathrm{Th}$, and their 
progeny. The $\mathrm{U}$ and $\mathrm{Th}$ chains are also responsible for neutron production following spontaneous fission and $(\alpha, n)$ reactions. $\mathrm{Kr}$ and $\mathrm{Rn}$ outgassing from materials into the Xe also results in ER backgrounds, and $\alpha$-emitting $\mathrm{Rn}$ daughters can contribute to neutron backgrounds when deposited on certain materials.

Generally, radioactive contaminants in massive components or those closer to or within the central volume of Xe present more stringent cleanliness and radio-purity requirements. These include the PMTs, PMT bases and cables; the TPC components, including the PTFE sheets and support structures; and the Ti cryostat. Our screening campaign includes several mature techniques for the identification and characterization of radioactive species within these bulk detector materials, namely $\gamma$-ray spectroscopy with High Purity Germanium detectors (HPGe), Inductively-Coupled Plasma Mass Spectrometry (ICP-MS), Neutron Activation Analysis (NAA), and measurement of Rn emanation from components before their integration into LZ. These complementary techniques collectively produce a complete picture of the radiological contaminants. LZ has all of these available with sufficient sensitivity and throughput to meet the project goals, described in Section 9.3.

Detector components are matched with the appropriate assay technique depending on the material and requisite sensitivity, defined by Monte Carlo simulations described in Section 11.4. In some cases, the final detector material or components are assayed. When manufacturing or fabrication processes are complex, raw components as well as final components will be assayed to assist in maintaining purity through the manufacturing process and to assist in selecting those processes that do not introduce additional contamination.

Stringent constraints are also applied to "intrinsic" contamination of the Xe by radon, argon and krypton. The LZ Xe purification program will remove ${ }^{85} \mathrm{Kr}$ from the Xe down to the level of $0.015 \mathrm{ppt}$ using chromatographic techniques (where concentration is for $\mathrm{g} / \mathrm{g}$ here and throughput this section). The Xe purification program is detailed in Section 6. All components that come into contact with any Xe in the experiment, whether within the primary instrument or in the gas storage, circulation, or recovery pipework, are screened for fixed contaminants, $\mathrm{Rn}$ emanation, and $\mathrm{Kr}$ outgassing to ensure that the intrinsic background remains within defined limits. These emanation and outgassing measurements are performed in dedicated chambers built and operated by LZ institutions. Similarly, techniques to measure bulk contamination of materials with alpha-emitting radon progeny that are not readily identified using traditional HPGe, ICP-MS, or NAA have been developed by the collaboration. This is particularly important for the PTFE reflector panels within the TPC where prolonged exposure to radon-contaminated air during manufacture will result in the presence of alpha-emitting daughter of ${ }^{210} \mathrm{~Pb}$. The high cross section for $(\alpha, n)$ reactions on the fluorine in the PTFE will result in neutron emission, setting stringent constraints on ${ }^{210} \mathrm{~Pb}$ content in the bulk material.

The results of screening measurements are entered into an LZ materials database and build on the existing LUX screening campaign data [10]. The database collates assay results from materials selected for use and identifies the components that contain them. These are referenced to results from Monte Carlo simulations that detail the background from the components in LZ. The contributions from several other sources are included in the database, where each contributes no more than materials radioactivity ER and NR goals. The first is the contribution from dust and radon-daughter plate-out on components, especially during component transport, storage, and assembly. This is controlled through use of dedicated cleanrooms available to the project at SURF (especially the radon-reduced Surface Assembly Laboratory), active monitoring of the environment for radon, and following established cleanliness, handling, and storage procedures. Selected lightweight plastics and rubbers with low radon diffusion coefficients are used to enclose materials in transit and during temporary storage. Coupons and witness plates are used for measurements of surface contamination with high sensitivity alpha-screening devices and cameras. The second contribution comes from cosmogenic activation of components before they are moved underground, such as ${ }^{46} \mathrm{Sc}$ production from $\mathrm{Ti}$ activation or ${ }^{127} \mathrm{Xe}$ and radioactivity from the laboratory environment. While contribution to the background from local radioactivity and potential activation products including radioactive isotopes of Xe 
are assessed with a number of simulation toolkits, data from the LUX experiment in particular is able to provide considerable input to reduce the systematic uncertainty for such calculations.

Although the sensitivity goals for LZ require unparalleled low-background contamination control for dark matter experiments and consequent severe constraints on material contamination, the screening campaign outlined here builds on the demonstrated substantial experience of the collaboration and established procedures or techniques employed by rare-event searches for background mitigation to meet these challenges with confidence. The LZ collaboration has accomplished important steps in developing necessary infrastructure for our assay and cleanliness program, and in identifying appropriately low-activity materials for the five most critical detector components or materials including Ti for the cryostat, PTFE used in several locations in the inner TPC, PMT base materials, PMT raw materials, and stainless steel for large mass support structures. In the following subsections we define the goals and requirements for our screening program, present the details of assays associated with the major components together with our collected data for all materials, present the impact of the background sources in LZ as determined through Monte Carlo simulations, and summarize the techniques and resources available to the project to meet our assay and cleanliness requirements on schedule.

\subsection{Goals, Requirements and Estimates}

The LZSim Monte Carlo simulation package, described in detail in Section 11.4, has been constructed to model the experiment and inform the design, determine optimal performance parameters, and define tolerable rates from background sources. Developed using the GEANT4 toolkit [11], the framework inherits from, and closely follows, the successful implementation of the LUX model [12], with evolving design of all parts of the experiment, including the inner detector, Xe skin, cryostat, and the OD, reflected in appropriate changes to the model geometry.

Simulations are performed to assess the contribution from all expected background sources, including intrinsic radioactivity in the $\mathrm{Xe}$, emission from every component in the experiment, as well as estimates of neutrino interactions. This extends to subcomponents, with the model accurately representing the physical distribution of contaminants, particularly since $(\alpha, n)$ neutron yields vary by many orders of magnitude depending on the primary constituents of the materials containing the alpha-emitting uranium and thorium and decay products. Similarly, the physical distribution of $\gamma$-ray, alpha, and beta particle emitters are modeled, as electrons created by these may produce detectable photons through Cherenkov and Bremsstrahlung processes, particularly in quartz or plastics close to or in contact with Xe. Evaluations of material radioactivity include contributions from spontaneous fission of heavy nuclei. Spontaneous fission of ${ }^{238} \mathrm{U},{ }^{235} \mathrm{U},{ }^{234} \mathrm{U}$, ${ }^{231} \mathrm{~Pa},{ }^{230} \mathrm{Th}$, or ${ }^{232} \mathrm{Th}$, present in the $\mathrm{U}$ and $\mathrm{Th}$ decay chains generates multiple prompt and delayed neutrons and $\gamma$-rays per decay. LZSim models these products with accurate energy, multiplicity, timing, and angular distributions. The single scatter identification capability in the TPC coupled to the high vetoing efficiency of the Xe skin and OD systems allow $>99.99 \%$ rejection of all spontaneous fission events, and effective removal from our background models. The performance of the veto skin and OD veto systems are presented in Section 3.7 and Chapter 4, respectively. Events in the TPC with associated energy depositions identified in the Xe skin or OD with signal equivalent to approximately equal to or more than $100 \mathrm{keV}$ in $\mathrm{Xe}$ skin and $200 \mathrm{keV}$ in OD are rejected.

All energy depositions from interactions in the Xe and OD are recorded in LZSim. Where necessary, optical tracking is performed following scintillation and ionization generation implemented using NEST [13]. LZSim models photon hit patterns and timing to mimic S1 and S2 signal generation in LZ, and allows for accurate studies of rare mechanisms that might produce backgrounds such as MSSI (multiple-scintillation single-ionization) events or background pile-up. Such detailed characterization and quantification of all background sources and their impacts are necessary to assign confidence to expected background event 
rates, their spectra, and their physical distribution in the detector. As a discovery instrument, the expected background in LZ must be well understood and quantified before significance can be ascribed to observation of any potential signal and WIMP discovery.

Version controlled release management of LZSim includes validation of materials and geometries against CAD drawings, physics lists implementation and detector response using pre-defined macros, and impact tests from radio-activity released from major components as well as from specific benchmark locations distributed throughout the detector volumes. Details of LZSim and the simulations are given in Section 11.4.

With the exception of astrophysical neutrinos discussed in Section 2.2, the major sources of background in LZ will be radioactivity from construction materials surrounding the central fiducial volume, and radon daughters and krypton distributed throughout the xenon. The goal for the maximum unvetoed differential single-scatter ER rate from each of these non-astrophysical sources after cuts in the WIMP search energy range has been set to about $1 \mu$ dru. This is approximately $10 \%$ of that expected from irreducible pp solar neutrinos deduced from our Monte Carlo simulations of the detector. Similarly, an upper limit of 0.4 unvetoed WIMP-like single-scatter NRs due to neutron emission from material radioactivity is the goal for a 1,000-day exposure, reduced to 0.2 after a $50 \%$ NR efficiency is applied. Goals for maintaining cleanliness throughout the experiment construction, for parts manufacture, transport, storage, assembly and integration, allow for contribution to ER and NR background equivalent to that from materials. Table 9.2.7 presents the estimate of the backgrounds in the experiment. Material assay results in hand and achieved by similar collaborations form the input for material activity, and these meet project goals. Values for radon emanation are based on conservative estimates, as described in Section 9.5, that match our requirements, but are currently estimated to exceed our goals. As we assay our materials we anticipate a reduction from this contribution to Table 9.2.7. The project requirements are discussed in Section 12, where the sensitivity of LZ to WIMP dark matter is presented. The LZ collaboration maintains more aggressive goals for its radioactive backgrounds for several reasons. The first is to provide contingency for measured radio-content in materials and for intrinsic contributions, invoking safety factors that mitigate large variations in expected and final radioactivity. Second, the goals facilitate simplified analyses, with total material and intrinsic background contributions sub-dominant to the ER backgrounds from astrophysical neutrinos that will be uniformly distributed across the active volume. Finally, our goals enhance physics capability beyond the primary WIMP search, as described in Section 2, particularly for neutrino interactions, neutrinoless double-beta decay, and alternative dark matter models including axion-like particles (ALPs).

Acceptance of screened materials for use in LZ depends on the Monte Carlo simulations and the overall radioactive background budget. When a component is identified as required in LZ, it is incorporated into the LZSim model and a preliminary estimate of maximum tolerable activity from that component is calculated. This requirement necessarily depends on activity from other components and the overall budget, and initial inputs to LZSim for detector-related backgrounds are based on measured values, or from screening results from previous experiments. Initially the background budget is evenly divided among the major components, such that if there are ten components, the allowable background for each component is assigned 1/10 of the allowable contamination. The maximum tolerable activity for the new component, including contingency for dominant materials such as the PMTs, is then translated to a required screening sensitivity for radioassaying a material sample. This in turn informs the screening technique and facility that will be employed for the assay. Screening results are then fed back into LZSim to produce an accurate assessment of ER and NR background and overall impact. The acceptance of the component depends on whether it meets requirements, or if it can be accommodated given related constraints and achieved radio-purity in other components and materials. In some cases, and as is justified by our assay experience, we may employ sampling of complete components. As the materials are assayed, this screening provides "as-built" input for the LZ background model. 
The ER and NR background calculations take into account radioactivity from all the components. Requirements on different materials and components vary, with their impact depending on material and position in the experiment. The simulations inform the necessity for screening $\mathrm{U}$ and $\mathrm{Th}$ in materials at the order of tens of ppt levels, tens to hundreds of ppb for K, and $0.05 \mathrm{mBq} / \mathrm{kg}$ for ${ }^{60} \mathrm{Co}$. Sensitivity at $10 \mathrm{mBq} / \mathrm{kg}$ to ${ }^{210} \mathrm{~Pb}$ in bulk materials is required. Radon emanation systems are required to meet sensitivity to ${ }^{222} \mathrm{Rn}$ at the level of $0.3 \mathrm{mBq}$ from materials. Cleanliness-maintenance procedures that will be applied to all materials to guarantee adequate background control and accurate modeling set requirements on maximum dust or radon exposure, calculated taking into account assembly and integration duration. We require less than a $10 \mathrm{mBq}$ contribution to ER background from dust, conservatively equivalent to less than $1 \mathrm{~g}$ of dust in total on all wetted surfaces. We require a maximum ${ }^{210} \mathrm{~Pb}$ activity from radon progeny on surfaces of $10 \mathrm{mBq} / \mathrm{m}^{2}$, reduced to $0.5 \mathrm{mBq} / \mathrm{m}^{2}$ for the inner surfaces of the TPC reflectors.

Materials of sufficient radio-purity have been successfully deployed in rare-event search experiments and will be procured and incorporated in the LZ project following sample/component measurements with available technology and facilities that incorporate screening, cleanliness maintenance, and outputs from the completed R\&D program. These measurements and procedures are well on the way to reliably identify clean materials and maintain their purity throughout the chain, from fabrication to installation and operation. The assaying program to achieve these minimum limits and estimates adopted are detailed below.

\subsubsection{Materials Table}

Table 9.2.1 presents the justification for the assumed values of ${ }^{238} \mathrm{U},{ }^{232} \mathrm{Th},{ }^{60} \mathrm{Co}$, and ${ }^{40} \mathrm{~K}$ content used as initial input to LZSim for the major components. Throughout this document, we refer to early and late values for ${ }^{238} \mathrm{U}$ and ${ }^{232} \mathrm{Th}$. In the case of ${ }^{238} \mathrm{U}$, we define the early part of the chain $\left({ }^{238} \mathrm{U}_{\mathrm{e}}\right)$ as containing any isotopes above ${ }^{226} \mathrm{Ra}$ since chemical processes may induce a break of secular equilibrium at this point and it will take many years to be restored. The late part of the chain $\left({ }^{238} \mathrm{U}_{1}\right)$ is counted from ${ }^{226} \mathrm{Ra}$ and below. In practice, standard HPGe detectors are not sensitive to the low-energy $\gamma$-ray lines from ${ }^{210} \mathrm{~Pb}$ at the bottom of the chain but the BEGe and well-type detectors available to LZ are (see Section 9.4.1.2). We populate the table with values for ${ }^{210} \mathrm{~Pb}$ where appropriate for our materials and assays. For the ${ }^{232} \mathrm{Th}$ chain, we define the early part of the chain $\left({ }^{232} \mathrm{Th}_{\mathrm{e}}\right)$ as coming from isotopes above ${ }^{224} \mathrm{Ra}$ and the late part of the chain $\left({ }^{232} \mathrm{Th}_{1}\right)$ as coming from isotopes below as chemical processes may, again, break the secular equilibrium. The relatively long half-life of ${ }^{228} \mathrm{Ra}(5.7 \mathrm{y})$ means that, for example, with $100 \%$ removal of $\mathrm{Ra}$ isotopes it would take $\sim 50 \mathrm{y}$ for ${ }^{228} \mathrm{Ra}$ and ${ }^{224} \mathrm{Ra}$ to re-equilibrate. LZ and LUX assays are maintained in our dedicated database but for those items which we are yet to screen, XENON100 assays are taken from [14]; EXO-200 from [1]; XENON1T [15]; MAJORANA, GERDA, and SuperNEMO by private communication or conference presentations; SNO from [16].

Our assay campaign has made good progress in measuring the main contributors to the experiment's ER and NR backgrounds and those that represent challenging requirements. LZ has performed over 250 material assays from April 2015 to October 2016, and have already identified five critical components or materials that collectively account for $60 \%$ of the NR and $50 \%$ of ER background from intrinsic material radioactivity in LZ. These are the Ti used for the cryostat, field shaping rings, and PMT support structures; PMTs including all PMT raw materials; PMT base components; stainless steel for OD supports; and PTFE. All these materials satisfy the LZ goals that are well below the project requirements. Below we summarize the assay campaigns and results from these components and materials.

Titanium: The two cryostat vessels and flanges will be constructed with $\mathrm{CP}-1$ grade titanium. The selected material has measured ${ }^{238} \mathrm{U}$ and ${ }^{232} \mathrm{Th}$ chain activities of $<0.09 \mathrm{mBq} / \mathrm{kg}$ and $0.23 \mathrm{mBq} / \mathrm{kg}$, respectively, representing the lowest reported to-date worldwide and substantially below the LZ goals. Two billets of $\mathrm{Ti}$ from the same production lot have been assayed and material procured to provide the raw material for 
Table 9.2.1: Materials in the $L Z$ design listed with radioactivity $\mathrm{mBq} / \mathrm{kg}$ as determined by direct assay data from the LZ screening program, and from other published experimental results. Reference values are from $\mathrm{LZ}^{[1]}$, EXO-200 ${ }^{[2]}$, XENON100 ${ }^{[3]}$, SuperNEMO ${ }^{[4]}, \mathrm{SNO}^{[5]}$ and GERDA ${ }^{[6]}$. The activities shown represent error weighted averaged values for $\gamma$-ray detection or $68 \%$ C.L. upper limits (italicized).

\begin{tabular}{|c|c|c|c|c|c|c|c|}
\hline Material & ${ }^{238} U_{e}$ & ${ }^{238} U_{1}$ & ${ }^{232} \mathrm{Th}_{\mathrm{e}}$ & ${ }^{232} \mathrm{Th}_{1}$ & ${ }^{60} \mathrm{Co}$ & ${ }^{40} \mathrm{~K}$ & ${ }^{210} \mathrm{~Pb}$ \\
\hline \multicolumn{8}{|c|}{ Values in $(\mathrm{mBq} / \mathrm{kg})$} \\
\hline \multicolumn{8}{|c|}{ General Materials } \\
\hline Titanium $^{1}$ & 1.60 & 0.09 & 0.28 & 0.23 & 0.02 & 0.54 & - \\
\hline PTFE $^{2}$ & 0.02 & 0.02 & 0.03 & 0.03 & - & 0.12 & - \\
\hline PEEK $^{1}$ & 17.0 & 16.6 & 16.1 & 8.50 & 0.52 & 47.8 & - \\
\hline LED $^{1}$ & 2000 & 100.0 & 200 & 100.0 & - & 1000 & - \\
\hline Copper ${ }^{1}$ & 1.30 & 1.20 & 14.5 & 1.70 & 0.40 & 7.00 & - \\
\hline Cable (RG174) ${ }^{3}$ & 29.8 & 1.47 & 3.31 & 3.15 & 0.65 & 33.1 & - \\
\hline Stainless Steel ${ }^{1}$ & 1.20 & 0.27 & 0.33 & 0.49 & 1.60 & 0.40 & - \\
\hline Epoxy $^{2}$ & 0.55 & 0.55 & 0.10 & 0.10 & - & 0.63 & - \\
\hline Viton O-ring $^{1}$ & 2630 & 2490 & 220 & 220 & 10.0 & 2150 & - \\
\hline Tyvek $^{4}$ & 6.00 & 6.00 & 2.20 & 2.20 & - & 5100 & - \\
\hline HDPE $^{5}$ & 5.96 & 0.37 & 0.63 & 0.62 & - & 3.40 & - \\
\hline Rubber O-ring $^{2}$ & 124 & 124 & 41.0 & 41.0 & - & 24.5 & - \\
\hline Mini-HV cable ${ }^{1}$ & 40.0 & 2.10 & 1.50 & 1.30 & - & 0.17 & - \\
\hline \multicolumn{8}{|c|}{ Liquid Scintillator } \\
\hline $\mathrm{LAB}^{1}$ & 0.00005 & 0.00005 & 0.00003 & 0.00003 & - & 0.00001 & - \\
\hline $\mathrm{GdCl}_{3} \cdot 6 \mathrm{H}_{2} \mathrm{O}^{1}$ & 1.24 & 1.24 & 0.41 & 0.41 & - & 0.0006 & - \\
\hline $\mathrm{PPO}^{1}$ & 1.85 & 1.85 & 2.60 & 2.60 & - & 0.0008 & - \\
\hline $\mathrm{TMHA}^{1}$ & 0.25 & 0.25 & 0.29 & 0.29 & - & 0.0003 & - \\
\hline bis-MSB ${ }^{1}$ & 2.60 & 2.60 & 0.78 & 0.78 & - & 0.0009 & - \\
\hline \multicolumn{8}{|c|}{ Outer Detector } \\
\hline Glass bulb $^{1}$ & 1507 & 1507 & 1065 & 1065 & - & 3900 & - \\
\hline LS Tanks ${ }^{2,5}$ & 0.01 & 0.01 & 0.005 & 0.005 & - & 0.07 & - \\
\hline Displacement foam ${ }^{1}$ & 20.0 & 57.0 & 2.60 & 9.00 & 6.00 & 80.0 & - \\
\hline \multicolumn{8}{|c|}{ PMT Base Materials } \\
\hline Resistors $(4.99 \mathrm{M})^{1}$ & 965 & 284 & 240 & 172 & 18.0 & 3249 & 35475 \\
\hline Capacitors ${ }^{1}$ & 4540 & 11715 & 4035 & 4035 & 10.0 & 350 & 11590 \\
\hline Cirlex Board ${ }^{1}$ & 23.9 & 19.1 & 3.19 & 3.19 & 0.63 & 15.1 & 25.5 \\
\hline Solder ${ }^{1}$ & 58.2 & 11.8 & 10.7 & 10.7 & 2.24 & 31.8 & - \\
\hline Receptacles: PMT pins ${ }^{1}$ & 1178 & 7.00 & 22.0 & 15.0 & - & 110 & 22393 \\
\hline Receptacles: Signal/HV ${ }^{1}$ & 833 & 7.50 & 13.2 & 15.4 & - & 77.3 & 21171 \\
\hline Receptacles: Ground ${ }^{1}$ & 568 & 20.5 & 16.7 & 8.20 & - & 19.0 & 29112 \\
\hline Resistors $(7.5 \mathrm{M})^{1}$ & 1097 & 389 & 106 & 106 & 16.3 & 3082 & 41600 \\
\hline Resistors $(10.0 \mathrm{M})^{1}$ & 2420 & 414 & 226 & 226 & 11.6 & 987 & 21613 \\
\hline Resistors $(2.49 \mathrm{M})^{1}$ & 1787 & 571 & 645 & 150 & 65.0 & 7069 & 52702 \\
\hline Resistors $(100 k)^{1}$ & 3460 & 1036 & 510 & 144 & 169 & 6118 & 97516 \\
\hline \multicolumn{8}{|c|}{ (continued on next page) } \\
\hline
\end{tabular}




\begin{tabular}{|c|c|c|c|c|c|c|c|}
\hline Material & ${ }^{238} U_{\mathrm{e}}$ & ${ }^{238} U_{1}$ & ${ }^{232} \mathrm{Th}_{\mathrm{e}}$ & ${ }^{232} \mathrm{Th}_{1}$ & ${ }^{60} \mathrm{Co}$ & ${ }^{40} \mathrm{~K}$ & ${ }^{210} \mathrm{~Pb}$ \\
\hline \multicolumn{8}{|c|}{ Values in $(\mathrm{mBq} / \mathrm{kg})$} \\
\hline Resistors (50) ${ }^{1}$ & 3430 & 670 & 314 & 264 & 29.0 & 5425 & 61140 \\
\hline Receptacles: Spring ${ }^{1}$ & 4758 & 38.0 & 50.0 & 50.0 & 7.00 & 131 & 1364 \\
\hline \multicolumn{8}{|c|}{ R11410 PMTs } \\
\hline Metal Bulb ${ }^{1}$ & 17.9 & 0.90 & 1.67 & 1.28 & - & 6.41 & - \\
\hline Dynode $^{1}$ & 216 & 2.02 & 4.10 & 3.40 & 4.00 & 4.60 & - \\
\hline Shield Plate ${ }^{1}$ & 77.0 & 3.10 & 5.00 & 3.20 & 4.60 & 6.00 & - \\
\hline Faceplate $^{1}$ & 11.0 & 0.67 & 1.00 & 0.80 & - & 4.00 & - \\
\hline Insulator plate ${ }^{1}$ & 20.9 & 1.05 & 1.63 & 1.16 & - & 6.28 & - \\
\hline Electrode Disk ${ }^{1}$ & 203 & 9.50 & 4.30 & 14.0 & 8.50 & 9.60 & - \\
\hline Faceplate Flange $^{1}$ & 162 & 2.75 & 3.80 & 4.20 & 12.5 & 14.4 & - \\
\hline Ceramic Stem ${ }^{1}$ & 105 & 20.0 & 12.9 & 9.60 & - & 110 & 5.60 \\
\hline Ceramic Stem Flange $^{1}$ & 198 & 0.63 & 2.32 & 0.84 & 12.0 & 3.30 & - \\
\hline Aluminium Ring ${ }^{1}$ & 62.0 & 1.23 & 2.33 & 0.94 & 0.34 & 8.50 & - \\
\hline Getter $^{1}$ & 2508 & 39.0 & 133 & 102 & 9.40 & 173 & - \\
\hline Stem Coating $^{1}$ & 22.0 & 178 & 9.00 & 7.50 & - & 61.0 & 539 \\
\hline \multicolumn{8}{|c|}{ R8520 Skin PMTs } \\
\hline Metal Package ${ }^{3}$ & 19.0 & 19.0 & 13.0 & 13.0 & 40.0 & 90.0 & - \\
\hline Glass in Stem ${ }^{3}$ & 970 & 970 & 340 & 340 & 10.0 & 2300 & - \\
\hline Spacer ${ }^{3}$ & 780 & 780 & 260 & 260 & 12.0 & 800 & - \\
\hline Seal ${ }^{3}$ & 17.0 & 17.0 & 370 & 370 & 27.0 & 5.00 & - \\
\hline Electrodes $^{3}$ & 19.0 & 19.0 & 18.0 & 18.0 & 12.0 & 0.15 & - \\
\hline Window ${ }^{3}$ & 0.50 & 0.50 & 1.80 & 1.80 & 0.10 & 18.0 & - \\
\hline \multicolumn{8}{|c|}{ TPC Items } \\
\hline Loop Antenna Wire ${ }^{2}$ & 0.20 & 0.20 & 0.12 & 0.12 & - & 1.86 & - \\
\hline Thermometers ${ }^{1}$ & 4038 & 3433 & 1439 & 1042 & 6.6 & 156416 & 1364 \\
\hline Acoustic Sensor PVDF ${ }^{1}$ & 1710 & 595 & 2179 & 1951 & - & 1389 & - \\
\hline Field Shaping Resistor ${ }^{1}$ & 5679 & 1.73 & 0.57 & 0.57 & - & 0.19 & - \\
\hline \multicolumn{8}{|c|}{ HV Conduits } \\
\hline Tivar $^{1}$ & 6.20 & 22.2 & 1.22 & 1.22 & - & 9.30 & - \\
\hline Delrin $^{4}$ & 4.00 & 0.70 & 0.18 & 0.18 & 0.30 & 18.0 & - \\
\hline \multicolumn{8}{|c|}{ Cryostat Insulation } \\
\hline SI Aluminium ${ }^{6}$ & 1.13 & 1.13 & 0.37 & 0.37 & - & 25.5 & - \\
\hline SI PTFE ${ }^{6}$ & 0.34 & 0.34 & 0.75 & 0.75 & - & 38.0 & - \\
\hline Foam-insulation ${ }^{1}$ & 57.0 & 57.0 & 9.00 & 9.00 & 6.00 & 80.0 & - \\
\hline Helicoil $^{2}$ & 2.40 & 2.40 & 1.30 & 1.30 & 41.0 & 13.5 & - \\
\hline
\end{tabular}

all Ti components. The field-shaping rings, constructed from $260 \mathrm{~kg}$ of Ti, and the PMT support structures, constructed from $104 \mathrm{~kg}$ of $\mathrm{Ti}$, will use the same raw material as the cryostat.

A long campaign in assaying titanium from various sources and states of processing has been underway for a couple years, including investigation of alternative options such as stainless steel. The cryostat material 
assays have primarily been carried out by the Berkeley Low Background Facility using its surface screening detectors at LBNL and the underground HPGe system MAEVE both before and after its relocation from the Oroville Dam to the 4850L of SURF (see Section 9.4.1.1). Moreover, there is consistency in multiple assays of similar geometries with this HPGe counter which was also used to assay titanium samples ultimately selected for use in LUX. Dozens of titanium and stainless steel samples were assayed for the LZ experiment. As seen in Figure 9.2.1 a wide range of radioactivity values have been covered and that, in general, titanium has been more consistently clean as compared to stainless steel, which varies in both the natural radionuclides as well as ${ }^{60} \mathrm{Co}$.

The selected titanium sample from TIMET, Heat Number (HN) 3469 was initially screened at the Berkeley Low Background Facility using MAEVE in May of 2015. This initial sample consisted of $10.1 \mathrm{~kg}$ of plates selected from the top portion of the single slab that comprised this heat (HN3460-T). A second sample from this slab, taken from the middle (HN3469-M), was acquired and assayed in September of 2015. Both samples of this titanium stock were found to be consistent with one another with their contained radioactivity. The samples were analyzed using a GEANT4 model of the MAEVE detector with an exact sample geometry of the titanium placed around the detector on four sides and the top to simulate detection efficiency of $\gamma$-rays in the regions of interest for the detector. The simulation was first compared to calibrated samples of known activity to benchmark the detector geometry, then modeled with the titanium geometry. As a check, the titanium sample was also directly compared to a calibration sample in a similar geometry, which agreed well with the analysis performed with a simulated efficiency.

In both of the samples, each counted for approximately three weeks, the early uranium chain was nondetectable or barely detectable within the limited abilities of HPGe detectors to assay this portion of the decay chain via $\gamma$-ray spectroscopy. The late portion of the chain (at ${ }^{226} \mathrm{Ra}$ and below) however, is quite accessible via $\gamma$-ray spectroscopy due to both the branching ratio and detection efficiency for $\gamma$-rays emitted during the decay of constituent isotopes, registered no detectable activity above background down to the few ppt level. The late uranium value is based upon the $609 \mathrm{keV}$ peak from ${ }^{214} \mathrm{Bi}$ and is consistent when compared to upper limits from other useful peaks in the late uranium chain (such as 295, 352, and 1,764 keV). Both samples had detectable levels of the thorium series in both the early and late portions of the chain which implied equilibrium in both samples. The late series measurement is based upon the $238 \mathrm{keV} \gamma$-ray from ${ }^{212} \mathrm{~Pb}$, which is the strongest peak based upon the product of the detection efficiency of that $\gamma$-ray line and its branching ratio in the thorium chain for assay with MAEVE. The contributions of the uranium, thorium, and potassium concentrations varies across the chains and their corresponding decays. The assays are summarized in Table 9.2.2. The late uranium chain in the titanium cryostat, for example, is a larger overall source of NR backgrounds within the detector than the early uranium chain because there is a rapid succession of several high energy alpha decays which induce $(\alpha, n)$ reactions. These various contributions from the chains towards the detector background are totaled and accounted for within Figure 9.2.1.

In terms of cosmogenic activation there were several isotopes of scandium present, most of which are the result of cosmic ray-induced reactions with the several stable isotopes of titanium. Detected in the sample was ${ }^{46} \mathrm{Sc}(889,1,120 \mathrm{keV})$; as well as small amounts of ${ }^{47} \mathrm{Sc}(159 \mathrm{keV}),{ }^{48} \mathrm{Sc}(983,1,037,1,312 \mathrm{keV})$, and ${ }^{44,44 \mathrm{~m}} \mathrm{Sc}(271,1,157 \mathrm{keV})$. None of the scandium isotopes are a cause of concern as they are all short-lived, the longest of which is ${ }^{46} \mathrm{Sc}$ with a half live of $84 \mathrm{~d}$ is listed in Table 9.2.2. The ${ }^{47} \mathrm{Sc},{ }^{48} \mathrm{Sc}$, and ${ }^{44,44 \mathrm{~m}} \mathrm{Sc}$ activities were not listed as they have short half lives of a few days or less so essentially disappear over the course of the measurement. The reported value for ${ }^{46} \mathrm{Sc}$ was decay corrected to the start of counting for each of the samples. All limits are one sigma upper limits and uncertainties are statistical only. A conservative systematic uncertainty of up to $10 \%$ should also be assumed. The assays have being confirmed by ICP-MS and with additional direct counting assays with the Chaloner detector at Boulby.

R11410 PMTs: The activity for early production models of the 3-inch R11410 PMTs has been measured by the LZ collaboration [17]. Multiple batches of PMTs (25 total) were assayed in groups of five. The 
Table 9.2.2: Results from $\gamma$-ray spectroscopy of TIMET titanium HN3469 for both Top and Middle samples. All limits are one sigma upper limits and uncertainties are statistical only. A systematic uncertainty of up to $10 \%$ should also be assumed. The results are shown below as activities in the ${ }^{238} \mathrm{U}$, ${ }^{232} \mathrm{Th}$, and ${ }^{40} \mathrm{~K}$ chains - all in units of $\mathrm{mBq} / \mathrm{kg}$. Results were obtained with the MAEvE detector and confirmed with ICP-MS and the Chaloner detector.

\begin{tabular}{|l|c|c|}
\hline & Top & Middle \\
\hline date & $5-2015$ & $9-2015$ \\
sample mass & $10.07 \mathrm{~kg}$ & $8.58 \mathrm{~kg}$ \\
livetime & $23.9 \mathrm{~d}$ & $20.8 \mathrm{~d}$ \\
${ }^{238} \mathrm{U}_{\mathrm{e}}$ & $<1.6$ & $2.9(15)$ \\
${ }^{238} \mathrm{U}_{1}$ & $<0.09$ & $<0.10$ \\
${ }^{232} \mathrm{Th}_{\mathrm{e}}$ & $0.28(3)$ & $<0.20$ \\
${ }^{232} \mathrm{Th}_{1}$ & $0.23(2)$ & $0.25(2)$ \\
${ }^{40} \mathrm{~K}$ & $<0.54$ & $<0.68$ \\
${ }^{60} \mathrm{Co}$ & $<0.02$ & $<0.03$ \\
${ }^{46} \mathrm{Sc}$ & $2.0(1)$ & $2.7(1)$ \\
\hline
\end{tabular}

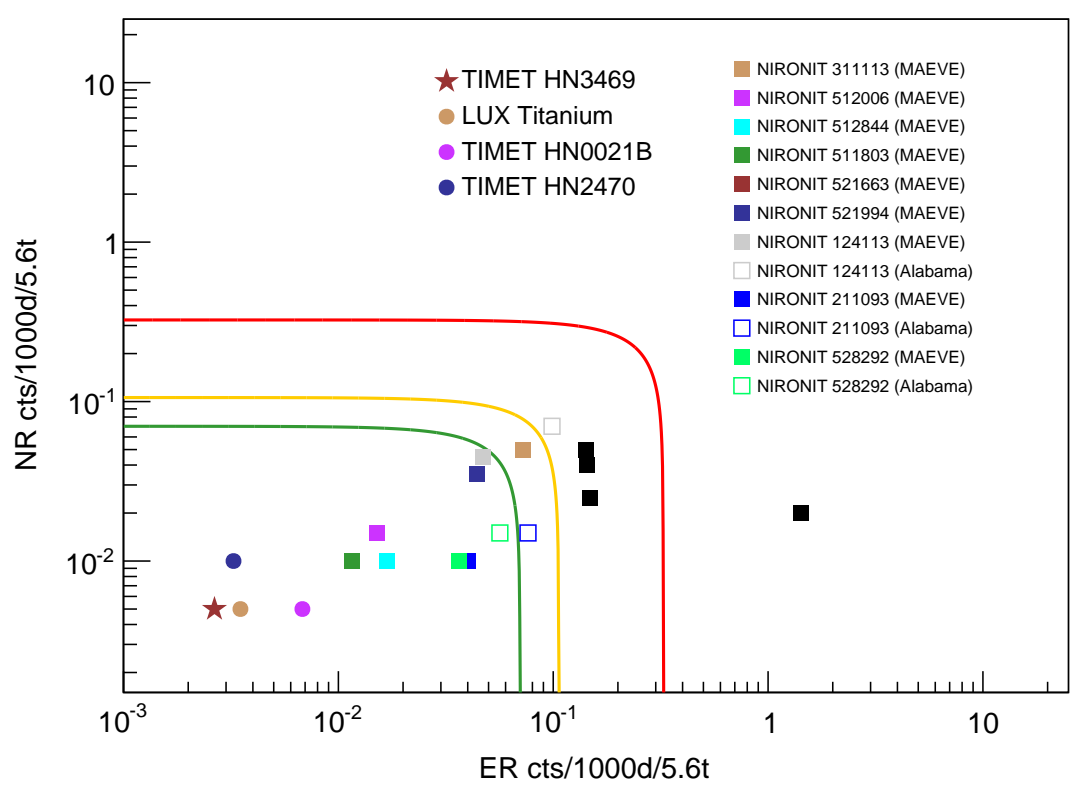

Figure 9.2.1: The plot shows the background counts resulting from LZ's Ti (TIMET) and stainless steel (NIRONIT) samples from the LXe cryostat in the 1,000 d-exposure with a 5.6 tonne-fiducial volume and after all the veto systems are applied, for ER events within $1.5 \mathrm{keV}_{\text {ee }}$ to $6.5 \mathrm{keV}_{\text {ee }}$, with $99.5 \%$ rejection, and within $6 \mathrm{keV}_{\mathrm{nr}}$ to $30 \mathrm{keV}_{\mathrm{nr}}$, and $50 \%$ acceptance, for NR events. The red curve corresponds to the sum of $10 \%$ of the pp solar neutrinos background for ERs and of $0.2 \mathrm{NR}$ events. The yellow curve is for the sum of $5 \%$ of the pp neutrino background and $0.05 \mathrm{NR}$ events. The green curve corresponds to the sum of $3.3 \%$ of the pp neutron background and $0.03 \mathrm{NR}$ events, the requirement for the LZ cryostat. The $\mathrm{Ti}$ identified by the assay program, indicated by the star, is well below requirements of $L Z$. 
raw material used to produce the experiment's PMTs were also assayed. The component materials used to produce the final LZ PMTs have now been assessed for radio-purity. The results are presented in Table 9.2.3, and are generally consistent with results from the XENON1T collaboration (albeit using $68 \%$ rather than $90 \%$ CL upper limits) following a similar campaign of screening of their R11410 PMTs and constituent materials [15]. The LZ assays of PMT materials have been performed with $\gamma$-ray spectroscopy principally with the SOLO detector and coordinated by the Brown University group, with the MAEVE detector at SURF and Chaloner detector at Boulby providing additional throughput and low energy sensitivity, particularly for early U chain content. Details of these detectors are given in Section 9.4.1. The materials have been returned to Hamamatsu and PMT production is underway. Screening of tubes that will be installed in LZ has begun at Boulby using the Chaloner and Lunehead detectors and will continue through 2017 when the screening will also be supported by the BHUC detectors.

PMT Bases: The activity of the components used to produce the bases for the R11410 TPC and R8520 Xe skin PMTs have been assayed at Boulby, principally with the Chaloner detector. These items include the resistors, capacitors, connectors and substrate material. We have also performed assays to measure ${ }^{210} \mathrm{~Pb}$ content in these materials, exploiting the capability of Boulby's Lumpsey detector (described in Section 9.4.1.2). This contamination is challenging to assay and usually overlooked, despite it being present in large quantity in components such as resistors and capacitors, with the potential to contribute significantly to neutron production. The ${ }^{210} \mathrm{~Pb}$ is accounted for in our background model. The radioactivity of the base

Table 9.2.3: Intrinsic radioactivity of the component materials which will be used in the manufacture of the R11410-20 PMTs to be used in the LZ experiment. All values are presented in $\mathrm{mBq} / \mathrm{kg}$ of material. Measurements are performed using the SOLO detector ${ }^{[1]}$, MAEvE ${ }^{[2]}$, and Chaloner ${ }^{[3]}$. Upper limits are italicized

\begin{tabular}{|c|c|c|c|c|c|c|c|c|c|}
\hline \multicolumn{10}{|c|}{ Values in $(\mathrm{mBq} / \mathrm{kg})$} \\
\hline Material & $\begin{array}{l}\text { Mass } \\
\text { screened } \\
\text { (g) }\end{array}$ & $\begin{array}{l}\text { Mass } \\
\text { /PMT } \\
(\mathrm{g})\end{array}$ & ${ }^{238} \mathrm{U}_{\mathrm{e}}$ & ${ }^{238} U_{1}$ & ${ }^{232} \mathrm{Th}_{\mathrm{e}}$ & ${ }^{232} \mathrm{Th}_{1}$ & ${ }^{60} \mathrm{Co}$ & ${ }^{40} \mathrm{~K}$ & ${ }^{210} \mathrm{~Pb}$ \\
\hline Metal Bulb ${ }^{2}$ & 506 & 78 & 17.9 & 0.90 & 1.67 & 1.28 & - & 6.41 & - \\
\hline Dynode $^{1}$ & 530 & 7.2 & 216 & 2.02 & 4.10 & 3.40 & 4.00 & 4.60 & - \\
\hline Shield Plate ${ }^{1}$ & 519 & 4 & 77.0 & 3.10 & 5.00 & 3.20 & 4.60 & 6.00 & - \\
\hline Faceplate ${ }^{2}$ & 1168 & 30 & 11.0 & 0.67 & 1.00 & 0.80 & - & 4.00 & - \\
\hline Insulator plate ${ }^{2}$ & 838 & 8.6 & 20.9 & 1.05 & 1.63 & 1.16 & - & 6.28 & - \\
\hline Electrode Disk $^{1}$ & 517 & 9.9 & 203 & 9.50 & 4.30 & 14.0 & 8.50 & 9.60 & - \\
\hline $\begin{array}{l}\text { Faceplate } \\
\text { Flange }^{1}\end{array}$ & 532 & 18 & 162 & 2.75 & 3.80 & 4.20 & 12.5 & 14.4 & - \\
\hline Ceramic Stem ${ }^{1,3}$ & 757.5 & 15.7 & 105 & 20.0 & 12.9 & 9.60 & - & 110 & 5.60 \\
\hline $\begin{array}{l}\text { Ceramic Stem } \\
\text { Flange }^{1}\end{array}$ & 1568 & 14 & 198 & 0.63 & 2.32 & 0.84 & 12.0 & 3.30 & - \\
\hline Aluminium Ring ${ }^{1}$ & 506 & 0.6 & 62.0 & 1.23 & 2.33 & 0.94 & 0.34 & 8.50 & - \\
\hline Getter $^{1}$ & 7 & 0.07 & 2508 & 39.0 & 133 & 102 & 9.40 & 173 & - \\
\hline Stem Coating & 100 & 0.00012 & 22.0 & 178 & 9.00 & 7.50 & - & 61.0 & 539 \\
\hline \multirow{2}{*}{\multicolumn{2}{|c|}{$\begin{array}{l}\text { Mass Weighted Ave } \\
\text { Total (mBq/PMT) }\end{array}$}} & 186.1 & 71.6 & 3.20 & 3.12 & 2.99 & 2.82 & 15.4 & 0.47 \\
\hline & & 186.1 & 13.3 & 0.60 & 0.58 & 0.56 & 0.53 & 2.87 & 0.09 \\
\hline
\end{tabular}


components meet LZ goals and are presented for the TPC PMTs in Table 9.2.4, and for the Xe skin in Table 9.2.5.

Stainless Steel: The base of the OD support stand and water PMT stands total some $770 \mathrm{~kg}$ of material and are constructed from stainless steel. The assay values are taken from the LZ determination of NIRONIT raw material using the MAEVE detector. These samples were identified as part of our R\&D campaign to select and procure sufficiently low background material for the detector cryostat. Though there is large variation across the sample activities, activities as low as $<1.60 \mathrm{mBq} / \mathrm{kg}{ }^{238} \mathrm{U}$ and $0.23 \mathrm{mBq} / \mathrm{kg}{ }^{232} \mathrm{Th}$ have been realized. Goals for OD support radioactivity are easily met by all of the samples assayed. In addition to the OD supports stainless steel will be used for several internal structures such as grid supports and pipework. Several NIRONIT samples meet the radioactivity goals and all meet the requirements for these components. Candidate materials for procurement and the materials used for the final fabrication will be assayed.

PTFE: The LZ collaboration has assayed four samples of PTFE in September 2015 using NAA at the MIT Research Reactor with surface $\gamma$-ray counters at Alabama. The NAA technique is described in Section 9.4.3. The samples were prepared at Alabama together with NIST certified fly ash used to calibrate the neutron flux, and measured in port 2PH1 with 5.5 MW of thermal reactor power. The samples include material from Flontech and DuPont, where the latter is the supplier used by the EXO experiment, employing sintering techniques developed with Applied Plastics Technology Inc. (APT) in Bristol, Rhode Island. The assayed contamination levels are presented in Table 9.2.6. While these are slightly higher than those obtained by EXO, they satisfy the radioactive contamination goals of $\mathrm{LZ}$.

LZ has developed assay capability to determine the ${ }^{210} \mathrm{~Pb}$ content of the bulk materials used to fabricate the PTFE. The Lumpsey detector at Boulby, described in Section 9.4.1.2, has sensitivity meeting the requirement of $10 \mathrm{mBq} / \mathrm{kg}$ to ${ }^{210} \mathrm{~Pb}$ and will be used to measure the PTFE.

The collaboration will continue assaying all materials used in the detector as they are designed and procured. We anticipate completing assays for all these items with LZ-specified materials and assembling a

Table 9.2.4: Assay results from 3-inch R11410 PMT base component materials. All values are presented in $\mathrm{mBq} /$ base.

\begin{tabular}{|c|c|c|c|c|c|c|c|c|c|}
\hline \multicolumn{10}{|c|}{ Values in $(\mu \mathrm{Bq} /$ base $)$} \\
\hline \multicolumn{2}{|c|}{ Material } & $\begin{array}{c}\text { Mass } \\
\text { per base }\end{array}$ & ${ }^{238} \mathrm{U}_{\mathrm{e}}$ & ${ }^{238} U_{1}$ & ${ }^{232} \mathrm{Th}_{\mathrm{e}}$ & ${ }^{232} \mathrm{Th}_{\mathrm{l}}$ & ${ }^{60} \mathrm{Co}$ & ${ }^{40} \mathrm{~K}$ & ${ }^{210} \mathrm{~Pb}$ \\
\hline \multirow{4}{*}{ Resistors } & $4.99 \mathrm{M}$ & 34.4 & 32.8 & 14.1 & 7.3 & 5.9 & 0.6 & 130 & 1290 \\
\hline & $7.5 \mathrm{M}$ & 14.1 & 15.3 & 5.4 & 1.5 & 1.5 & 0.3 & 43.2 & 584 \\
\hline & $10.0 \mathrm{M}$ & 12.9 & 31.5 & 5.3 & 2.9 & 2.9 & 0.2 & 12.8 & 280 \\
\hline & $50 \mathrm{R}$ & 4.3 & 12.9 & 2.9 & 1.4 & 1.1 & 0.1 & 23.5 & 265 \\
\hline Capacitors & $10 \mathrm{nF}$ & 24.5 & 111 & 286 & 98.5 & 98.5 & 0.1 & 8.5 & 283 \\
\hline \multirow{3}{*}{ Receptacles } & PMT Pins & 1170 & 775 & 6.5 & 25.8 & 12.8 & 6.0 & 129.0 & 26200 \\
\hline & Signal/HV & 156 & 103.4 & 0.9 & 3.4 & 1.7 & 0.8 & 17.2 & 3495 \\
\hline & Ground & 244.2 & 103.4 & 0.9 & 2.9 & 1.7 & 0.8 & 15.2 & 5780 \\
\hline Base & Cirlex & 3300 & 94.8 & 66.8 & 13.8 & 13.8 & 2.0 & 40.5 & 56.8 \\
\hline Solder & $\mathrm{Pb}$ Free & 180 & 10.5 & 2.1 & 1.9 & 1.9 & 0.4 & 5.7 & 63.0 \\
\hline \multicolumn{2}{|c|}{ Total } & 5140 & 1291 & 390 & 159 & 143 & 11 & 426 & 38300 \\
\hline
\end{tabular}


Table 9.2.5: Assay results from 1-inch R8520 skin component materials. All values are presented in $\mathrm{mBq} /$ base.

\begin{tabular}{|c|c|c|c|c|c|c|c|c|c|}
\hline \multicolumn{10}{|c|}{ Values in $(\mu \mathrm{Bq} /$ base $)$} \\
\hline \multicolumn{2}{|c|}{ Material } & \multirow{2}{*}{$\begin{array}{l}\text { Mass } \\
\text { per base } \\
(\mathrm{mg}) \\
34.4\end{array}$} & \multirow{2}{*}{$\frac{{ }^{238} U_{\mathrm{e}}}{32.8}$} & \multirow{2}{*}{$\frac{{ }^{238} \mathrm{U}_{1}}{14.1}$} & \multirow{2}{*}{$\frac{{ }^{232} \mathrm{Th}_{\mathrm{e}}}{7.3}$} & \multirow{2}{*}{$\frac{{ }^{232} \mathrm{Th}_{1}}{7.3}$} & \multirow{2}{*}{$\frac{{ }^{60} \mathrm{Co}}{0.6}$} & \multirow{2}{*}{$\frac{{ }^{40} \mathrm{~K}}{130}$} & \multirow{2}{*}{$\begin{array}{l}{ }^{210} \mathrm{~Pb} \\
1292\end{array}$} \\
\hline \multirow{5}{*}{ Resistors } & $4.99 \mathrm{M}$ & & & & & & & & \\
\hline & $7.5 \mathrm{M}$ & 4.7 & 5.1 & 1.8 & 0.5 & 0.5 & 0.1 & 14.4 & 195 \\
\hline & $10.0 \mathrm{M}$ & 4.3 & 10.5 & 1.8 & 0.1 & 0.1 & 0.1 & 4.28 & 93.4 \\
\hline & $2.49 \mathrm{M}$ & 8.4 & 15 & 4.8 & 1.2 & 1.2 & 0.5 & 59.4 & 443 \\
\hline & $100 k$ & 3.6 & 12.3 & 3.7 & 0.5 & 0.5 & 0.6 & 21.8 & 347 \\
\hline Capacitors & $10 \mathrm{nF}$ & 14.7 & 66.3 & 171 & 59.1 & 59.1 & 0.2 & 5.1 & 170 \\
\hline \multirow{2}{*}{ Receptacles } & Signal/HV & 156 & 103 & 0.9 & 1.7 & 1.7 & 0.8 & 17.2 & 3495 \\
\hline & Ground & 244 & 103 & 0.9 & 1.7 & 1.7 & 0.8 & 15.2 & 5780 \\
\hline Base & Cirlex & 1400 & 40.2 & 28.3 & 5.8 & 5.8 & 0.8 & 17.2 & 24.1 \\
\hline Solder & $\mathrm{Pb}$ Free & 250 & 10.5 & 2.1 & 1.9 & 1.9 & 0.4 & 5.7 & 63.0 \\
\hline \multicolumn{2}{|c|}{ Total } & 2120 & 400 & 230 & 81 & 81 & 5 & 291 & 11900 \\
\hline
\end{tabular}

Table 9.2.6: NAA measurements of ${ }^{40} \mathrm{~K},{ }^{238} \mathrm{U},{ }^{232} \mathrm{Th}$, and ${ }^{138} \mathrm{La}$ activities in PTFE samples.

\begin{tabular}{|c|c|c|c|c|}
\hline \multicolumn{5}{|c|}{ Values in $(\mathrm{mBq} / \mathrm{kg})$} \\
& $\begin{array}{c}{ }^{238} \mathrm{U} \\
\left(\times 10^{-3}\right)\end{array}$ & $\begin{array}{c}{ }^{232} \mathrm{Th} \\
\left(\times 10^{-3}\right)\end{array}$ & $\begin{array}{c}{ }^{40} \mathrm{~K} \\
\left(\times 10^{-3}\right)\end{array}$ & $\begin{array}{c}{ }^{138} \mathrm{La} \\
\left(\times 10^{-6}\right)\end{array}$ \\
\hline PTFE8764 & $<42$ & $13 \pm 3$ & $66 \pm 4$ & $\begin{array}{c}2.8 \pm \\
0.2\end{array}$ \\
DuPont 807NX & $38 \pm 9$ & $29 \pm 2$ & $96 \pm 5$ & $\begin{array}{c}9.4 \pm \\
0.5\end{array}$ \\
DuPont NXT85 & $<21$ & $28 \pm 2$ & $122 \pm 10$ & $6.4 \pm$ \\
Flontech FLON008 & $<27$ & $51 \pm 3$ & $329 \pm 17$ & $25 \pm 1$ \\
PTFE8764 sheet & $18 \pm 4$ & $29 \pm 1$ & $76 \pm 1$ & $8.5 \pm$ \\
\end{tabular}


detailed background model prior to the start of integration and assembly. The impact of these backgrounds is presented in Table 9.2.7.

\subsubsection{Backgrounds Table}

Table 9.2.7 presents the impact of the background sources assembled in Table 9.2.1 as determined through Monte Carlo simulations with LZSim and by adopting the radio-activities as assayed by LZ or as expected based on literature values. The activities for the material components easily satisfies the LZ requirements and are also well below the goals, with a total contribution to background of under $0.1 \mathrm{NR}$ events and $0.3 \mu \mathrm{dru}$ in $1,000 \mathrm{~d}$ with a fiducial volume of 5.6 tonne of Xe. This is for a WIMP search region defined by a 3-fold PMT coincidence lower bound and 20 phd upper bound, equivalent to approximately $1.5 \mathrm{keV}_{\text {ee }}$ to $6.5 \mathrm{keV}_{\text {ee }}$, before discrimination or NR efficiency is applied, but employing the spontaneous fission rejection power of LZ, OD and Xe skin vetoes, and single scatter requirements. This energy region, selected for evaluation of trial components, is functionally equivalent to that employed in the full PLR when considering the experiment's optimum sensitivity.

Most of the entries in Table 9.2.7 are formed from composite materials, where more than 130 components or subcomponents contribute to make up the different elements. A comprehensive table that includes separate sections for each detector element detailing each subcomponent is maintained in a version-controlled archive; Table 9.2.7 is the summary from the complete table. The assayed contamination values are used to generate mass-weighted average activities for Monte Carlo simulations for ER backgrounds, and in major components all the individual materials are used to generate NR rates from $(\alpha, n)$ and spontaneous fission neutrons. Neutron emission rates in the simulations include contributions from ${ }^{210} \mathrm{~Pb}$ at measured values for both the TPC PMT bases and skin PMT bases. For several components $90 \%$ C.L. upper limits have been set for ER rates where simulations are statistics limited given the lack of any recoils in the xenon that pass selection criteria. Where background is generated by some isotopes, such as ${ }^{238} \mathrm{U}$ or ${ }^{235} \mathrm{U}$, but not others, such as ${ }^{40} \mathrm{~K}$, mean values and upper limits are combined. This occurs in cases where either components are located far from the xenon target, are situated where radiation released is vetoed with very high efficiency, or where contamination levels are so low that no background is generated on timescales several times the LZ exposure. The components in Table 9.2.7 where this is the case, and reported ER counts include a significant rate from an upper limit, are the R8520 skin PMT bases and the HV Conduit and Cables.

There are several contributors to background in addition to the ER and NR counts induced by fixed radioactivity in materials. Radioactivity associated with radon emanated into the instrument is expected to contribute approximately 840 counts to the ER events, where half of this comes from materials and the rest from radon released from dust on material surfaces. This level of background, discussed in Section 9.5, is based on conservative estimates for emanation from materials and from dust. The activity, $2 \mu \mathrm{Bq} / \mathrm{kg}$, corresponds to a total of approximately $20.2 \mathrm{mBq}$ from ${ }^{222} \mathrm{Rn}$ dispersed throughout the xenon target, which is at the level of our requirements $(20 \mathrm{mBq})$. A total of $20 \mathrm{mBq}$ in the xenon corresponds to $13.4 \mathrm{mBq}$ from

${ }^{222} \mathrm{Rn}$ in the active volume and $11.2 \mathrm{mBq}$ in the fiducial target. We do not expect a contribution from ${ }^{220} \mathrm{Rn}$ in LZ. However, due to the observation of a population of alphas attributed to ${ }^{220} \mathrm{Rn}$ in LUX, where radon mitigation was minimal, we nonetheless conservatively include a rate from a $0.1 \mu \mathrm{Bq} / \mathrm{kg}$ concentration of ${ }^{220} \mathrm{Rn}$ in our background estimates.

We anticipate the decay-daughters from ${ }^{222} \mathrm{Rn}$ to accumulate on the surfaces of the detector during manufacturing and assembly. LZ has taken steps to minimize this plate-out deploying clean rooms and Rn-reduced air for some of the critical assembly tasks. Several large volume liquid scintillator experiments $[18,19]$ reported observing mobility of these products, in particular ${ }^{210} \mathrm{Bi}$. The untagged beta decay would present an ER background similar to the ${ }^{222} \mathrm{Rn}$. Studies were conducted to look for the mobility of ${ }^{210} \mathrm{Bi}$ in LUX data. Severe limits obtained from LUX, when applied to the LZ plate out requirement, place 
Table 9.2.7: Estimated background counts in the WIMP search region of interest, as discussed in the text, from all significant sources in the $L Z 1,000 \mathrm{~d}$ exposure. Mass-weighted average activities obtained from Table 9.2.1 are shown for composite materials. Solar ${ }^{8} \mathrm{~B}$ neutrinos are expected to contribute $(7 \pm 3)$ NR but only at very low energies and are excluded from the table.

\begin{tabular}{|c|c|c|c|c|c|c|c|c|c|c|}
\hline & $\begin{array}{c}\text { Mass } \\
(\mathrm{kg})\end{array}$ & ${ }^{238} U_{\mathrm{e}}$ & ${ }^{238} \mathrm{U}_{1}$ & $\begin{array}{r}{ }^{232} \mathrm{Th}_{\mathrm{e}} \\
\mathrm{mBg}\end{array}$ & $\begin{array}{l}{ }^{232} \mathrm{Th}_{1} \\
\mathrm{~kg}\end{array}$ & ${ }^{60} \mathrm{Co}$ & ${ }^{40} \mathrm{~K}$ & $\mathrm{n} / \mathrm{yr}$ & $\begin{array}{c}\text { ER } \\
\text { (cts) }\end{array}$ & $\begin{array}{l}\text { NR } \\
\text { (cts) }\end{array}$ \\
\hline Upper PMT Structure & 40.5 & 3.90 & 0.23 & 0.49 & 0.38 & 0.00 & 1.46 & 2.53 & 0.05 & 0.000 \\
\hline Lower PMT Structure & 69.9 & 2.40 & 0.13 & 0.30 & 0.24 & 0.00 & 0.91 & 6.06 & 0.05 & 0.001 \\
\hline R11410 3" PMTs & 91.9 & 71.6 & 3.20 & 3.12 & 2.99 & 2.82 & 15.4 & 81.8 & 1.46 & 0.013 \\
\hline R11410 PMT Bases & 2.8 & 288 & 75.8 & 28.4 & 27.9 & 1.43 & 69.4 & 34.7 & 0.36 & 0.004 \\
\hline R8778 2" Skin PMTs & 6.1 & 138 & 59.4 & 16.9 & 16.9 & 16.3 & 413 & 52.8 & 0.13 & 0.008 \\
\hline R8520 Skin 1" PMTs & 2.2 & 60.5 & 5.19 & 4.75 & 4.75 & 24.2 & 333 & 4.60 & 0.02 & 0.001 \\
\hline R8520 PMT Bases & 0.2 & 213 & 108 & 42.2 & 37.6 & 2.23 & 124 & 3.62 & 0.00 & 0.000 \\
\hline PMT Cabling & 104 & 29.8 & 1.47 & 3.31 & 3.15 & 0.65 & 33.1 & 2.65 & 1.43 & 0.000 \\
\hline TPC PTFE & 184 & 0.02 & 0.02 & 0.03 & 0.03 & 0.00 & 0.12 & 22.5 & 0.06 & 0.008 \\
\hline Grid Wires & 0.8 & 1.20 & 0.27 & 0.33 & 0.49 & 1.60 & 0.40 & 0.02 & 0.00 & 0.000 \\
\hline Grid Holders & 62.2 & 1.20 & 0.27 & 0.33 & 0.49 & 1.60 & 0.40 & 6.33 & 0.27 & 0.002 \\
\hline Field Shaping Rings & 91.6 & 5.41 & 0.09 & 0.28 & 0.23 & 0.00 & 0.54 & 10.8 & 0.23 & 0.004 \\
\hline TPC Sensors & 0.90 & 21.1 & 13.5 & 22.9 & 14.2 & 0.50 & 26.3 & 24.8 & 0.01 & 0.002 \\
\hline TPC Thermometers & 0.06 & 336 & 90.5 & 38.5 & 25.0 & 7.26 & 3360 & 1.49 & 0.05 & 0.000 \\
\hline Xe Tubing & 15.1 & 0.79 & 0.18 & 0.23 & 0.33 & 1.05 & 0.30 & 0.64 & 0.00 & 0.000 \\
\hline HV Components & 138 & 1.90 & 2.00 & 0.50 & 0.60 & 1.40 & 1.20 & 4.90 & 0.04 & 0.001 \\
\hline Conduits & 200 & 1.25 & 0.40 & 2.59 & 0.66 & 1.24 & 1.47 & 5.33 & 0.06 & 0.001 \\
\hline Cryostat Vessel & 2410 & 1.59 & 0.11 & 0.29 & 0.25 & 0.07 & 0.56 & 124 & 0.63 & 0.013 \\
\hline Cryostat Seals & 33.7 & 73.9 & 26.2 & 3.22 & 4.24 & 10.0 & 69.1 & 38.8 & 0.45 & 0.002 \\
\hline Cryostat Insulation & 23.8 & 18.9 & 18.9 & 3.45 & 3.45 & 1.97 & 51.7 & 69.8 & 0.43 & 0.007 \\
\hline Cryostat Teflon Liner & 26 & 0.02 & 0.02 & 0.03 & 0.03 & 0.00 & 0.12 & 3.18 & 0.00 & 0.000 \\
\hline Outer Detector Tanks & 3200 & 0.16 & 0.39 & 0.02 & 0.06 & 0.04 & 5.36 & 78.0 & 0.45 & 0.001 \\
\hline Liquid Scintillator & 17600 & 0.01 & 0.01 & 0.01 & 0.01 & 0.00 & 0.00 & 14.3 & 0.03 & 0.000 \\
\hline Outer Detector PMTs & 205 & 570 & 470 & 395 & 388 & 0.00 & 534 & 7590 & 0.01 & 0.000 \\
\hline OD PMT Supports & 770 & 1.20 & 0.27 & 0.33 & 0.49 & 1.60 & 0.40 & 14.3 & 0.00 & 0.000 \\
\hline \multicolumn{9}{|c|}{ Subtotal (Detector Components) } & 6.20 & 0.070 \\
\hline \multicolumn{9}{|c|}{${ }^{222} \mathrm{Rn}(2.0 \mu \mathrm{Bq} / \mathrm{kg})$} & 722 & - \\
\hline \multicolumn{9}{|l|}{${ }^{220} \mathrm{Rn}(0.1 \mu \mathrm{Bq} / \mathrm{kg})$} & 122 & - \\
\hline \multicolumn{9}{|l|}{${ }^{n a t} \mathrm{Kr}(0.015 \mathrm{ppt}(\mathrm{g} / \mathrm{g}))$} & 24.5 & - \\
\hline \multicolumn{9}{|l|}{${ }^{\text {nat }} \operatorname{Ar}(0.45 \mathrm{ppt}(\mathrm{g} / \mathrm{g}))$} & 2.47 & - \\
\hline \multicolumn{9}{|l|}{${ }^{210} \mathrm{Bi}(0.1 \mu \mathrm{Bq} / \mathrm{kg})$} & 40 & - \\
\hline \multicolumn{9}{|c|}{ Laboratory and Cosmogenics } & 4.3 & 0.06 \\
\hline \multicolumn{9}{|c|}{ Fixed Surface Contamination } & 0.19 & 0.37 \\
\hline \multicolumn{9}{|c|}{ Subtotal (Non- $v$ counts) } & 922 & 0.50 \\
\hline \multicolumn{9}{|c|}{${ }^{136} \mathrm{Xe} 2 v \beta \beta$} & 67 & 0.00 \\
\hline \multicolumn{9}{|c|}{ Astrophysical $v$ counts $\left(\mathrm{pp}+{ }^{7} \mathrm{Be}+{ }^{13} \mathrm{~N}\right)$} & 255 & 0.00 \\
\hline \multicolumn{9}{|c|}{ Astrophysical $v$ counts $\left({ }^{8} \mathrm{~B}\right)$} & 0.00 & 0.00 \\
\hline \multicolumn{9}{|c|}{ Astrophysical $v$ counts (hep) } & 0.00 & 0.21 \\
\hline \multicolumn{11}{|c|}{ (continued on next page) } \\
\hline
\end{tabular}




\begin{tabular}{|c|c|c|c|c|c|c|c|c|c|c|}
\hline & $\begin{array}{c}\text { Mass } \\
(\mathrm{kg})\end{array}$ & ${ }^{238} \mathrm{U}_{\mathrm{e}}$ & ${ }^{238} \mathrm{U}_{1}$ & $\begin{array}{c}{ }^{232} \mathrm{Th}_{\mathrm{e}} \\
\mathrm{mBq}\end{array}$ & ${ }^{232} \mathrm{Th}_{1}$ & ${ }^{60} \mathrm{Co}$ & ${ }^{40} \mathrm{~K}$ & $\mathrm{n} / \mathrm{yr}$ & $\begin{array}{c}\text { ER } \\
\text { (cts) }\end{array}$ & $\begin{array}{l}\text { NR } \\
\text { (cts) }\end{array}$ \\
\hline \multicolumn{9}{|c|}{ Astrophysical $v$ counts (diffuse supernova) } & 0.00 & 0.05 \\
\hline \multicolumn{9}{|c|}{ Astrophysical $v$ counts (atmospheric) } & 0.00 & 0.46 \\
\hline \multicolumn{9}{|c|}{ Subtotal (Physics backgrounds) } & 322 & 0.72 \\
\hline \multirow{2}{*}{\multicolumn{9}{|c|}{$\begin{array}{l}\text { Total } \\
\text { Total (with } 99.5 \% \text { ER discrimination, } 50 \% \text { NR efficiency) }\end{array}$}} & 1,244 & 1.22 \\
\hline & & & & & & & & & 6.22 & 0.61 \\
\hline \multicolumn{9}{|c|}{ Sum of ER and NR in LZ for $1,000 \mathrm{~d}, 5.6$ tonne FV, with all analysis cuts } & \multicolumn{2}{|c|}{6.83} \\
\hline
\end{tabular}

an upper limit on the ${ }^{210} \mathrm{Bi}$ of $0.1 \mu \mathrm{Bq} / \mathrm{kg}$, resulting in a limit of $40 \mathrm{ER}$ counts. We have explicitly added an entry in the table for this background source.

As discussed in Section 9.2, we maintain a goal for total activity from radon emanation in the xenon of $1 \mathrm{mBq}$. This would lower the contribution from radon emanation to $1 \mu$ dru, matching goals for krypton and from fixed material radioactivity. Contributions from $\mathrm{Kr}$ and Ar, with 27 counts, are discussed in Section 9.7. Laboratory and cosmogenic backgrounds contribute just 4.3 ER and 0.06 NR background events, well below goals for fixed material radioactivity; these backgrounds are discussed in Section 9.8. Finally, surface contamination on materials is described in Section 9.6. This generates both ER and NR counts at the level of 0.19 and 0.37 , respectively, from intrinsic radioactivity in the dust and backgrounds due to radon plateout.

Contributions from the irreducible physics sources are discussed in Section 2. These include ER contributions from the double beta decay of ${ }^{136} \mathrm{Xe}$ and astrophysical neutrinos, in particular pp and ${ }^{7} \mathrm{Be}$ solar neutrinos. Solar ${ }^{8} \mathrm{~B}$ and hep neutrinos, together with atmospheric and diffuse supernovae neutrinos contribute to NR background through coherent neutrino-nucleus scattering. As described in Section 2, (7士3) NR are expected from Solar ${ }^{8} \mathrm{~B}$ neutrinos. However, these are at very low energies and are therefore excluded from Table 9.2.7.

The total expected background to the WIMP search in LZ under the assumption of $99.5 \%$ discrimination against ERs and $50 \% \mathrm{NR}$ acceptance is 6.8 counts.

\subsection{Techniques and Sensitivities}

The LZ project will require approximately 500 materials assays, determined by counting materials, parts and components detailed in the project's CAD drawings. This is consistent with expectations based on our experience from LUX, EXO, and other similar low-background experiments when including multiple techniques and confirmation measurements. As already discussed we have achieved good success for major items through completed assays. ICP-MS, HPGe, NAA and Radon Emanation systems will all be utilized, as no single technique has sensitivity to all radioactive isotopes within all materials, nor can any single technique at present provide sensitivity to the full ${ }^{238} \mathrm{U}$ and ${ }^{232} \mathrm{Th}$ decay chains. Use of all four techniques will provide the required accurate model of a material's full $\gamma$-ray, neutron and beta emission, and the subsequent impact on the radiation budget and sensitivity of the experiment. Any rare-event search would benefit from the availability of all techniques, which vary in their sample throughput and screening duration, requirements for sample size, access to instruments, ability to do bulk screening of complete components, and preservation or destruction of samples in the assaying process. Table 9.3.1 provides a summary of these assay techniques.

Sensitivity to $\mathrm{U}$ and Th decay chain species down to $\approx 10 \mathrm{ppt}$ has been demonstrated using ultralowbackground HPGe detectors. HPGe can also assay ${ }^{60} \mathrm{Co},{ }^{40} \mathrm{~K}$, and other radioactive species emitting $\gamma$-rays. 
Table 9.3.1: Primary material radio-assay techniques, indicating isotopic sensitivity and detection limits, as well as typical throughput or single-sample measurement duration.

\begin{tabular}{|c|c|c|c|c|c|}
\hline Technique & $\begin{array}{l}\text { Isotopic } \\
\text { Sensitivity }\end{array}$ & $\begin{array}{l}\text { Typical } \\
\text { Sensitivity } \\
\text { Limits }\end{array}$ & $\begin{array}{l}\text { Sample } \\
\text { Mass }\end{array}$ & $\begin{array}{l}\text { Sampling } \\
\text { Duration }\end{array}$ & $\begin{array}{l}\text { Destructive/Non- } \\
\text { destructive and Notes }\end{array}$ \\
\hline HPGe & $\begin{array}{c}{ }^{238} \mathrm{U},{ }^{235} \mathrm{U}, \\
{ }^{232} \mathrm{Th} \\
\text { chains, }{ }^{40} \mathrm{~K}, \\
{ }^{60} \mathrm{Co},{ }^{137} \mathrm{Cs} \\
\text { any } \gamma \text {-ray } \\
\text { emitter }\end{array}$ & $\begin{array}{l}50 \text { ppt U, } \\
100 \text { ppt Th }\end{array}$ & $\mathrm{kg}$ & $\begin{array}{l}\text { Up to } 2 \\
\text { weeks }\end{array}$ & $\begin{array}{l}\text { Non-destructive, very versa- } \\
\text { tile, not as sensitive as other } \\
\text { techniques, large samples }\end{array}$ \\
\hline ICP-MS & $\begin{array}{l}{ }^{238} \mathrm{U},{ }^{235} \mathrm{U}, \\
{ }^{232} \mathrm{Th} \text { (top } \\
\text { of chain) }\end{array}$ & $10^{-12} \mathrm{~g} / \mathrm{g}$ & $\mathrm{mg}$ to $\mathrm{g}$ & Days & $\begin{array}{l}\text { Destructive, requires sample } \\
\text { digestion, preparation critical }\end{array}$ \\
\hline NAA & $\begin{array}{l}{ }^{238} \mathrm{U},{ }^{235} \mathrm{U} \\
{ }^{232} \mathrm{Th} \text { (top } \\
\text { of chain), } \mathrm{K}\end{array}$ & $\begin{array}{l}10^{-12} \mathrm{~g} / \mathrm{g} \\
\text { to } \\
10^{-14} \mathrm{~g} / \mathrm{g}\end{array}$ & g & $\begin{array}{l}\text { Days to } \\
\text { weeks }\end{array}$ & $\begin{array}{l}\text { Destructive, sensitive to some } \\
\text { contaminants }\end{array}$ \\
\hline GD-MS & $\begin{array}{l}{ }^{238} \mathrm{U},{ }^{235} \mathrm{U}, \\
{ }^{232} \mathrm{Th} \text { (top } \\
\text { of chain) }\end{array}$ & $10^{-10} \mathrm{~g} / \mathrm{g}$ & $\mathrm{mg}$ to $\mathrm{g}$ & Days & $\begin{array}{l}\text { Destructive, minimal matrix } \\
\text { effects, cannot analyze ceram- } \\
\text { ics and other insulators }\end{array}$ \\
\hline $\begin{array}{l}\text { Radon } \\
\text { Emanation }\end{array}$ & ${ }^{222} \mathrm{Rn},{ }^{220} \mathrm{Rn}$ & $0.1 \mathrm{mBq}$ & $\mathrm{kg}$ & $\begin{array}{l}1 \text { to } 3 \\
\text { weeks }\end{array}$ & $\begin{array}{l}\text { Non-destructive, large sam- } \\
\text { ples, limited by size of emana- } \\
\text { tion chamber }\end{array}$ \\
\hline
\end{tabular}

This technique is nondestructive and, in addition to sample screening, finished components can be assayed prior to installation. Under the assumption of secular equilibrium, with all isotopic decays remaining within the same volume, the activity and concentration for any particular isotope in the chain may be inferred from the measured $\mathrm{U}$ and $\mathrm{Th}$ content, assuming natural terrestrial abundance ratios. However, secular equilibrium can be broken through removal of radioactive isotopes during chemical processing or through emanation and outgassing. HPGe readily identifies the concentrations of isotopes from mid- to late-chain isotopes from the early chain decays of ${ }^{238} \mathrm{U}$ and ${ }^{232} \mathrm{Th}$, particularly those with energies in excess of several hundred keV. Background-subtracted $\gamma$-ray counting is performed around specific energy ranges to identify radioactive isotopes. Taking into account the detector efficiency at that energy for the specific sample geometry allows calculation of isotopic concentrations. A typical assay lasts 1 to 2 weeks per sample to accrue statistics at the sensitivities required for the LZ assays. These direct $\gamma$-ray assays probe the bulk of the radioactivity from any material, including identification of equilibrium states. The instruments available to the project are described in Section 9.4.1, and will be used to assay all materials. The HPGe technique is less sensitive, however, to the progenitor isotopes or the low-energy or low-probability $\gamma$-ray emission from decays in the early chain [20]. 
ICP-MS offers very precise determination of elemental contamination with potentially up to $100 \times$ better sensitivity for the progenitor $U$ and Th concentrations compared to $\gamma$-ray spectroscopy. Since ICP-MS directly assays the ${ }^{238} \mathrm{U},{ }^{235} \mathrm{U}$, and ${ }^{232} \mathrm{Th}$ progenitor activity it informs the contribution to neutron flux from $(\alpha, n)$ in low-Z materials, but also the contribution from spontaneous fission, which in specific materials can dominate. However, it is limited in identifying daughter isotopes in the $U$ and $T h$ decay chains that are better probed by HPGe. The ICP-MS technique assays very small samples that are atomized and measured with a mass spectrometer. As a destructive technique, it is not used on finished components. The limitation of ICP-MS is that the sample must be soluble - typically in mixtures of $\mathrm{HF}, \mathrm{HCl}$, and $\mathrm{HNO}_{3}$ - and that several samples from materials must be screened to probe contamination distribution and homogeneity. Assays take $1 \mathrm{~d}$ to $2 \mathrm{~d}$ per material, dominated by the sample preparation time, where extreme care must be taken to avoid contamination of solvents and reactants. ICP-MS will be used to provide confirmation of the direct $\gamma$-ray counting assays, and inform the background model with potentially significant contributions from the tops of the U/Th chain inaccessible to $\gamma$-ray measurements; particularly important for constraining systematic uncertainties in the PLR for signal identification. It will also provide chemical compositions to improve calculations of neutron emission yields from materials. Finally, ICP-MS available to the LZ project mitigates potential throughput limitations in long duration underground assays, with rapid direct U/Th measurements that can be used to establish background contributions under assumptions of secular equilibrium through the decay chains. This may be particularly useful for small/low-mass components that need rapid assessment, since they can be performed and material cleared for use before the $\gamma$-ray assays that will determine the bulk of the radio-content with sensitivity to equilibrium states are performed. The LZ ICP-MS systems are presented in Section 9.4.2.

Neutron activation analysis (NAA) achieves sensitivities up to $1,000 \times$ better than direct counting. Samples are irradiated with neutrons from the reactor to activate some of the stable isotopes, which subsequently emit $\gamma$-rays of well-known energy that are detected through $\gamma$-ray spectroscopy. Elemental concentrations are then inferred, using tabulated neutron-capture cross sections convoluted with the reactor neutron spectra. Samples must be specially prepared and compatible with neutron irradiation in a reactor, and then measured with surface $\gamma$-ray counters. NAA probes the bulk contamination simultaneously and is not limited by the composition of the material since no sample digestions or ablations are required. Indeed, of all known techniques, NAA can provide the best sensitivity to $U$ and Th concentration. However, concurrent activation of trace contaminants of little interest or from the primary constituents of the sample can produce high $\gamma$-ray fluxes that present a background to the $U$ and Th measurements, severely compromising sensitivity. Counting can be timed to allow interfering species to decay, allowing identification of the isotopes of interest with high accuracy. As with ICP-MS, this technique requires small sample masses, does not assay finished components, and assumptions of secular equilibrium need to be made since this technique measures the top of the $U$ and Th chains. NAA is particularly useful for materials with very low activities difficult to assay directly with $\gamma$-ray counting, and also difficult to digest for ICP-MS. The PTFE in the LZ detector represents one such material. The proximity of the PTFE to the active Xe, both in defining the TPC and around the PMTs, coupled to a high $(\alpha, n)$ cross section, sets stringent constraints on the acceptable $\mathrm{U}$ and Th content. As described in the previous subsection, NAA has been successfully utilized to identify suitable material for the LZ project, with details of the facility presented in Section 9.4.3.

Particular attention must be paid to radon, as it is a noble gas consisting solely of radioactive isotopes, is produced in the decay chains of uranium and thorium, and has the ability to enter Xe volumes due to its chemical inertness and subsequent long diffusion lengths through solids. Outgassing of radon from a material in which it has been produced is commonly termed "radon emanation". Especially for materials in contact with or in close proximity to Xe, radon emanation must be taken into account in setting the levels of U/Th that can be tolerated (with stringent limits particularly on $U$ ) due to the presence of ${ }^{222} \mathrm{Rn}$ in the ${ }^{238} \mathrm{U}$ decay chain, as well as ${ }^{220} \mathrm{Rn}$ from ${ }^{232} \mathrm{Th}$ decay. LXe cannot provide self-shielding against the 
dispersed Rn, unlike radioactivity from fixed contaminants. Radon emanation from the bulk of a material may be estimated once its U/Th decay chain content (in particular its ${ }^{226} \mathrm{Ra}$ content) has been assayed using HPGe detectors. However, such estimates must be supplemented by direct screening for radon emanation for critical materials due to limited sensitivity in HPGe, systematic error from assumptions on equilibrium chain states, uncertainties in describing temperature-dependent radon transport in materials, and uncertainties on the amount of contaminants near surfaces. Direct measurements are performed by allowing bulk materials, either large samples or finished components, to emanate radon within a sealed chamber for a period of one or more weeks. The radon is then transferred using a carrier gas such as $\mathrm{He}$ or $\mathrm{N}_{2}$ with high efficiency to a detector system, where radon progeny is detected through its radioactive decay, either using alpha counting or $\gamma$-rays. The four radon emanation systems available to $\mathrm{LZ}$ all meet requirements, with sensitivity ranging from $0.1 \mathrm{mBq}$ to $0.3 \mathrm{mBq}$. These systems are presented in Section 9.5.

Most of the facilities for the direct assaying measurements are operated directly by LZ groups or exist at LZ institutes, allowing us to maintain control of sample preparation, measurements, analysis, and interpretation of data necessary to ensure sufficient sensitivity with reliable reproducibility and control of systematics. Commercial facilities that provide Glow Discharge Mass Spectrometry (GD-MS) are available to the collaboration. GD-MS has poorer sensitivity $(\approx 0.1 \mathrm{ppb}$ U/Th) than ICP-MS, can only be used with conductive or semiconductive solids, and commercial service providers are typically limited in sensitivity due to regular exposure of their instruments and sample preparation infrastructure to materials with high concentrations of contaminants. However, it may be exploited for additional throughput or rapid confirmation of measurements if necessary.

\subsection{Intrinsic Contamination Techniques and Devices}

Identification of key materials presented in Section 9.2.1 with ultra-low activity assays for intrinsic contamination demonstrate existing instrument sensitivity and capabilities within the project. Here we present the instruments and facilities used to perform the fixed contaminant assays used already and available to the project to meet the both throughput and sensitivity requirements to schedule. The materials assay schedule includes all instruments described below, and is managed through the project to allow flexibility by assigning resources depending on sample type, size, required sensitivity, timescale, and risk. The project has performed cross-calibration campaigns of our instruments that have included circulating standard sources, blind samples, and exchanging data and analysis routines, to ensure reliability across our assays.

\subsubsection{HPGe}

Eleven HPGe detectors located in facilities both above- and underground are available to the LZ collaboration, with differences in detector types and shielding configuration providing useful dynamic range both in terms of sensitivity to particular isotopes and physical sample geometries. All of the instruments, with several previously used for LUX or ZEPLIN, are managed and operated by LZ collaborating institutes and are already in use for the LZ material screening campaign. The detectors are typically several hundreds of grams to several kilograms in mass, with a mixture of n-type, p-type, and broad energy Ge (BEGe) crystals, providing relative efficiencies at the tens of percent through to in excess of $100 \%$ (as compared to the detection efficiency of a $(3 \times 3)$-inch $\mathrm{NaI}$ crystal for $1.33 \mathrm{MeV} \gamma$-rays from a ${ }^{60} \mathrm{Co}$ source placed $25 \mathrm{~cm}$ from the detector face). While p-type crystals can be grown to larger sizes and hence require less counting time due to their high efficiency, the low energy performance of the n-type and broad energy crystals is superior due to less intervening material between source and active Ge. Clean samples are placed close to the Ge crystal and sealed for several days to weeks in order to accrue sufficient statistics, depending on the minimum detectable activity (MDA). The detectors are generally shielded with low-activity $\mathrm{Pb}$ and $\mathrm{Cu}$, flushed with dry 
nitrogen to displace the Rn-carrying air, and sometimes are surrounded by veto detectors to suppress background from Compton scattering that dominates the MDA for low-energy $\gamma$-rays. To reduce backgrounds further, most of the detectors are operated in underground sites, at SURF within the Black Hills State University Underground Campus (BHUC), and at the U.K. Boulby Underground Laboratory within the Boulby Germanium Suite (BUGS), lowering the muon flux by several orders of magnitude. We also retain a number of surface counters that are particularly useful for pre-screenings before more sensitive underground assays. All of the HPGe detectors available to LZ are shown in Table 9.4.1.

\subsubsection{BHUC}

The BHUC is a dedicated facility for low-background counting at SURF and was completed in late 2015. Four ultra-low background HPGe detectors are operational: MAEVE, MorgAN, MordRed, and SOLO. MAEVE, formerly situated at Oroville before operations in the Davis campus at SURF and final re-location in the BHUC, is an $85 \%$ p-type HPGe detector in a low-activity $\mathrm{Pb}$ - and $\mathrm{Cu}$-shielded and $\mathrm{Rn}$-flushed chamber. The MAEVE shield was recently upgraded with the addition of a layer of very old $\mathrm{Pb}$ shielding. For several $\mathrm{kg}$ sized samples, the sensitivity after a week of counting reaches approximately $10 \mathrm{ppt} \mathrm{U}(\approx 0.1 \mathrm{mBq} / \mathrm{kg}$ $\left.{ }^{238} \mathrm{U}\right)$ and $25 \mathrm{ppt}$ Th $\left(\approx 0.1 \mathrm{mBq} / \mathrm{kg}{ }^{232} \mathrm{Th}\right), 20 \mathrm{ppb}$ for $\mathrm{K}\left(\approx 0.7 \mathrm{mBq} / \mathrm{kg}{ }^{40} \mathrm{~K}\right)$ and $\approx 0.03 \mathrm{mBq} / \mathrm{kg}$ for ${ }^{60} \mathrm{Co}$. Nearly an identical detector, MORGAN, was paired with MAEVE in 2015, and is now fully operational.

LBNL upgraded the CUBED detector, now called MORDRED with a low background cryostat and improved design. MORDRED has a $1.2 \mathrm{~kg}$ ORTEC n-type coaxial HPGe detector with a $254 \mathrm{~cm}^{3}$ active volume and a relative efficiency of $60 \%$. The sample chamber, with a dimension of $8,000 \mathrm{~cm}^{3}$, is surrounded by a $10 \mathrm{~cm}$-thick $99.9 \%$ OFHC copper shield, enclosed in a stainless steel box that is itself sealed by $10 \mathrm{~cm}$ of lead. MORDRED achieves similar performance to MAEVE and MORGAN, and as an n-type detector will aid in measuring early $\mathrm{U}$ chain and ${ }^{210} \mathrm{~Pb}$.

The SOLO detector, formerly operated at Soudan Mine, has also been moved to BHUC. The SOLO shielding houses a nitrogen-flushed $\mathrm{Pb}$ shield that has a minimum thickness of $30 \mathrm{~cm} 50 \mathrm{~Bq} / \mathrm{kg}{ }^{210} \mathrm{~Pb}$ activity) with a $5 \mathrm{~cm}$ inner liner of 150 y-old low-activity $\mathrm{Pb}\left(50 \mathrm{mBq} / \mathrm{kg}{ }^{210} \mathrm{~Pb}\right.$ activity $)$. A counting chamber of

Table 9.4.1: $\gamma$-ray-counting facilities available for LZ material radio-assays. Sensitivities shown are approximate detectable activities after 2 weeks of counting and samples of order-kg mass. Typical cavity size within the shielding of these detectors within which samples may be placed is $0.03 \mathrm{~m}^{3}$.

\begin{tabular}{|c|c|c|c|c|c|c|}
\hline Detector & Site & $\begin{array}{c}\text { Site Depth } \\
(\mathbf{m w e})\end{array}$ & $\begin{array}{c}\text { Crystal } \\
\text { Type }\end{array}$ & $\begin{array}{c}\text { Crystal Mass } \\
\text { (Relative Eff) }\end{array}$ & $\begin{array}{c}\text { Sensitivity, } \\
\text { U }(\mathrm{mBq} / \mathrm{kg})\end{array}$ & $\begin{array}{c}\text { Sensitivity, } \\
\text { Th }(\mathrm{mBq} / \mathrm{kg})\end{array}$ \\
\hline Chaloner & Boulby & 2805 & BEGe & $0.8 \mathrm{~kg}(48 \%)$ & 0.6 & 0.2 \\
Ge-II & Alabama & 0 & p-type & $1.4 \mathrm{~kg}(60 \%)$ & 4.0 & 1.2 \\
Ge-III & Alabama & 0 & p-type & $2.2 \mathrm{~kg}(100 \%)$ & 4.0 & 1.2 \\
Lumpsey & Boulby & 2805 & Well & $1.5 \mathrm{~kg}(67 \%)$ & 0.4 & 0.3 \\
Lunehead & Boulby & 2805 & p-type & $2.0 \mathrm{~kg}(92 \%)$ & 0.7 & 0.2 \\
Maeve & SURF & 4300 & p-type & $1.7 \mathrm{~kg}(85 \%)$ & 0.1 & 0.1 \\
Merlin & LBNL & 180 & n-type & $2.3 \mathrm{~kg}(115 \%)$ & 6.0 & 8.0 \\
Mordred & SURF & 4300 & n-type & $1.2 \mathrm{~kg}(60 \%)$ & 0.7 & 0.7 \\
Morgan & SURF & 4300 & p-type & $2.1 \mathrm{~kg}(85 \%)$ & 0.2 & 0.2 \\
SOLO & SURF & 4300 & p-type & $0.6 \mathrm{~kg}(30 \%)$ & 0.5 & 0.2 \\
Wilton & Boulby & 2805 & BEGe & $0.4 \mathrm{~kg}(18 \%)$ & 7.0 & 4.0 \\
\hline
\end{tabular}


$8,000 \mathrm{~cm}^{3}$ contains the $0.6 \mathrm{~kg}$ "Diode M" HPGe detector. This detector approaches sensitivities at the $50 \mathrm{ppt}$ level for ${ }^{238} \mathrm{U}$ and ${ }^{232} \mathrm{Th}$ and $25 \mathrm{ppb}$ for ${ }^{40} \mathrm{~K}$ for multi-kg samples.

The LBNL group manage and operate the BHUC counters for the LZ project. MAEVE delivers the best sensitivity of all the project's detectors, and MORDRED will extend the facilities low-energy sensitivity as an n-type detector. The SOLO and MORGAN detectors are reserved with $100 \%$ live-time screening of LZ PMTs following delivery in 2016. The BHUC detectors, together with surface and Boulby counters, meet the LZ project's expected throughput demands with MDA's sufficient for most materials used in the construction of LZ. However, it is anticipated that additional counters will be added to the array in 2016 from the South Dakota School of Mines and Technology (SDSMT), UC Berkeley, and the University of South Dakota (USD).

\subsubsection{BUGS}

The Boulby Underground Facility, at 2,805 mwe, has been upgraded through 2015 to now include a dedicated low-background counting area that is operated as an ISO Class 6 cleanroom [21]. The Boulby Underground Germanium Suite (BUGS) is housed in this area and includes three primary high sensitivity ultra-low background detectors: Chaloner, Lunehead, and Lumpsey, and a fourth pre-screener: Wilton. The primary detectors are each housed in custom-built lead and copper shields using existing Boulby stock material. The shields feature a retractable roof to aid the reproducibility of backgrounds. The shield cavities range between 301 to 401 , and are purged with $\mathrm{N}_{2}$ gas fed directly into the inner cavity to reduce the ambient radon activity from $3 \mathrm{~Bq} / \mathrm{m}^{3}$ to negligible levels. The shields allow detectors to be retracted and interchanged, allowing detector maintenance without dismantling, and matching of instruments to castles to optimize sensitivity across the suite.

The Chaloner BEGe detector is a Canberra BE5030 (0.8 kg Ge, $48 \%$ relative efficiency) installed in 2014. The crystal is configured in a unique planar geometry, yielding greater peak-to-Compton ratios at the $\gamma$-ray energies of interest, and improved energy resolution (by $\approx 30 \%$ over typical p-type detectors at $122 \mathrm{keV}$ ). BEGe detectors also have considerably lower energy thresholds due to a factor-70 reduction in dead layer thickness on the front face of the Ge crystal and the utilization of a carbon-fiber end-cap window. These factors provide useful efficiency down to $10 \mathrm{keV}$ (as opposed to $\approx 80 \mathrm{keV}$ for p-type HPGe detectors) and consequently can directly measure ${ }^{210} \mathrm{~Pb}-$ a problematic source of background that is particularly difficult to quantify with other techniques. The BE5030 achieves $<50 \mathrm{ppt}$ sensitivity to ${ }^{238} \mathrm{U}$ and ${ }^{232} \mathrm{Th}$ for typical samples despite the low relative efficiency given the geometry and high resolution of the crystal. The energy range of the BEGe also gives us (unique to such a screening program) information about the elemental content of some materials through x-ray fluorescence. This has proved particularly useful in verifying composition of sample of candidate capacitors, found to contain $\mathrm{BaTiO}_{3}$, as the detector is sensitive to fluorescence $\mathrm{x}$-rays from barium, rather than $\mathrm{Al}_{2} \mathrm{O}_{3}$.

The Lunehead detector is a GEM-XX240-S p-type HPGe of $92 \%$ relative efficiency. Used extensively for the ZEPLIN-III experiment [22], this detector has undergone complete refurbishment. With the exception of the Ge crystal, the detector has been overhauled and retrofitted with ultra-low-background components in 2014 to become a GEMXX-95-LB-C-HJ model with J-type neck and carbon fiber entrance window. Lunehead achieves sensitivity to about 50 ppt of ${ }^{238} \mathrm{U}$ and ${ }^{232} \mathrm{Th}$.

The Lumpsey detector is a Canberra SAGe Well-type ultra-low-background GSW275L7950-30U-ULB instrument. Lumpsey has a $1.5 \mathrm{~kg}$ crystal that operates as a conventional co-axial (p-type) detector for large samples, with approximately $40 \mathrm{keV}$ threshold, and sensitivity equivalent to the Chaloner and Lunehead detectors. However, the crystal includes a $28 \mathrm{~mm}$ diameter, $40 \mathrm{~mm}$ cavity ('well') where small samples can be placed. This provides unique capability with high sensitivity rapid screening of small components or materials by enveloping the sample with $>3 \pi$ coverage. The thin lithium contact inside the well allows low energy threshold at $20 \mathrm{keV}$, similar to the BEGe detectors. This is particularly important for measurement of 
the $46.5 \mathrm{keV} \gamma$-ray from the decay of ${ }^{210} \mathrm{~Pb}$. As described earlier, detection and mitigation of ${ }^{210} \mathrm{~Pb}$ in bulk PTFE is required to mitigate $(\alpha, n)$ production on fluorine by ${ }^{210} \mathrm{Po}, \mathrm{a}{ }^{210} \mathrm{~Pb}$ daughter nucleus. Traditional HPGe $\gamma$-ray counting, NAA, and ICP-MS cannot readily be applied to detect ${ }^{210} \mathrm{~Pb}$ or its daughters at the $\mathrm{mBq} / \mathrm{kg}$ level. Lumpsey demonstrates sensitivity of $35 \mathrm{mBq} / \mathrm{kg}$ for a small $(30 \mathrm{~g})$ sample after $21 \mathrm{~d}$ of counting and $10 \mathrm{mBq} / \mathrm{kg}$ for more massive samples after $50 \mathrm{~d}$, meeting the project requirement for ${ }^{210} \mathrm{~Pb}$ in bulk material screening. The Lumpsey well-detector will be used for the assays of PTFE for ${ }^{210} \mathrm{~Pb}$ content.

The Wilton detector is a Canberra BE2825 with low mass $(0.4 \mathrm{~kg})$ BEGe crystal. Though Wilton is an ultralow-background instrument it has a straight neck from dewar to crystal, and is housed in a smaller fliplid lead shield with tin and copper liner. Wilton is used as a pre-screener, where samples are first assessed in a 1 to 2 day measurement. Wilton's sensitivity is at the level of $7 \mathrm{mBq} / \mathrm{kg}$ to ${ }^{238} \mathrm{U}$ and $4 \mathrm{mBq} / \mathrm{kg}$ to ${ }^{232} \mathrm{Th}$. This allows materials with high radioactivity to be immediately rejected if requirements are not met, without disturbing the primary high-sensitivity instruments. Where samples are to be passed to the primary detectors, the pre-screener can inform screening times required to meet sensitivity for the assay.

BUGS is managed by the LZ groups at Oxford and UCL, through the DMUK consortium, with dedicated effort from Boulby providing significant operational and infrastructure support. The instruments have already been used extensively through the project's R\&D phase and beyond to perform critical assays in particular to identify PMT base materials, cryostat materials, and support PMT construction material assays with SOLO and MAEVE. The Chaloner and Lunehead detectors are reserved exclusively for screening of PMTs, begun in mid-2016.

\subsubsection{Surface HPGe Detectors}

The MERLIN detector, as with Wilton at Boulby, is used to pre-screen materials and determine suitability for underground assaying and required sampling livetime. Materials identified as exceeding requirements by MERLIN are not assayed with the high sensitivity counters in the BHUC. MERLIN is a $115 \%$ relative efficiency n-type low-background HPGe operated at the surface Berkeley Low Background Facility (BLBF) within a $4 \pi$ shielded room with $1.5 \mathrm{~m}$-thick low-activity serpentine rock concrete walls. The HPGe detector head is mounted on a J-hook to reduce line-of-sight for background from electronics and the cryostat, and is shielded in $\mathrm{a} \mathrm{Pb}$ and OFHC Cu castle. It has an MDA of approximately $0.5 \mathrm{ppb}(6 \mathrm{mBq} / \mathrm{kg})$ to ${ }^{238} \mathrm{U}$ for $\mathrm{O}(\mathrm{kg})$ samples from $1 \mathrm{~d}$ of counting, and $2 \mathrm{ppb}(8 \mathrm{mBq} / \mathrm{kg})$ for ${ }^{232} \mathrm{Th}$. Sensitivity to $\mathrm{K}$ and ${ }^{60} \mathrm{Co}$ is at the level of $1 \mathrm{ppm}$ and $0.04 \mathrm{pCi} / \mathrm{kg}$, respectively.

Finally, the University of Alabama operates two high sensitivity low-background HPGe detectors at its surface screening facility: the Ge-II and Ge-III detectors. Each detector is housed in a lead-copper-shield equipped with an active cosmic-ray veto systems. The shield cavities are suitable for large samples. These devices can reach 0.3 ppb MDA for ${ }^{238} \mathrm{U}$ and ${ }^{232}$ Th with two weeks of counting, useful for many LZ components, and also provide pre-screening. These detectors are essential for the NAA screening program for LZ, described in Section 9.4.3.

\subsubsection{ICP-MS}

The UCL group operate an ICP-MS facility dedicated to the LZ project [21]. At UCL, An ISO Class 6 cleanroom, erected in late 2015, contains all instruments and the sample preparation areas. The primary instrument is an Agilent 7900 ICP-MS, with sensitivity to U and Th below $10^{-12} \mathrm{~g} / \mathrm{g}$, allowing assay of materials with $\mathrm{U}$ and Th content at the level of a several ppt. The ICP-MS has an integrated auto-sampler for high speed discrete sample uptake with low-flow, Peltier-cooled sample introduction system. The system octopole can provide species discrimination through interference removal either with kinetic energy discrimination (KED) in helium collision mode, or in reaction mode using $\mathrm{H}_{2}$, in addition to running with no gas at all, which in some cases is sufficient for $\mathrm{U}$ and Th measurements. High-purity $\mathrm{Ar}, \mathrm{He}$, and $\mathrm{H}_{2}$ gases 
(5N grade) are introduced to the system as carrier gas, collision cell gas, or reaction gas, respectively. The system has been fitted with an inert sample introduction kit such that a micro flow nebuliser and platinum skimmer and sampling cones are used to allow up to $20 \%$ concentration of acid in samples introduced to the ICP-MS, including HF. Calibration of the system is performed using tuning solutions containing Li, $\mathrm{Y}, \mathrm{Tl}, \mathrm{Co}$, and $\mathrm{Ce}$, in $2 \% \mathrm{HNO}_{3}$. The mass numbers 7 (Li), $89(\mathrm{Y})$ and $205(\mathrm{Tl})$ are tracked for stability, sensitivity, resolution and linearity for the integrated system across the mass range. The backgrounds at these masses allow sub-ppt sensitivity, $<3 \%$ relative standard deviation (RSD) in count rate over a $20 \mathrm{~min}$ stability measurement period, and detector resolutions of $<1 \%$.

The limiting factors in realizing reproducible high throughput ppt sensitivity is clean sample preparation, requiring digestion apparatus and procedures, as well as cleanliness of acids, that do not contaminate samples. Sample preparation infrastructure has been procured to address these issues and mitigate risks. A Milestone EthosUP microwave digestion system is able to digest solid samples at high temperatures in closed containers in minutes; this cannot readily be achieved using open hot-plate technology since volatiles are lost, the open vials may be contaminated depending on the ambient environment and handling, and low temperatures limit the digestion efficiency, further increasing exposure to background. The digestion oven also allows for the use of high quantities of HF that greatly simplifies digestion and, crucially, allows measurement of virtually all materials that are being considered for use in LZ. The quality of the reagents used in the sample material and, more importantly, analytical blank digestions further limit sensitivity through variations in contamination levels, even for ultra-pure commercial products. Milestone acid distillation and reflux systems have been installed for control of acid purity and reproducibility, and the laboratory includes a Veloia $18 \mathrm{M} \Omega$ water purifier. Finally, a Pyro-260 microwave ashing system allows the possibility of digestion of materials such as PTFE and acrylics that are not otherwise digested easily, and are required with low radioactivity levels inaccessible to HPGe, necessitating the need for NAA.

Protocols and methodologies for operations, sample handling, and digestions are largely based on established protocols and methodologies developed that demonstrate sub-ppt measurements [1, 23, 24] The cleanroom contains an ISO Class 4 laminar flow unit, and a fume cupboard with H+ filtration for sample handling. Digestion protocols with specific acid chemistries and heating profiles with microwave digestion on a material-by-material basis are also available for the EthosUP system following our R\&D phase and microwave digestion routines developed in partnership with Milestone and U.K. operators Analytix Limited. Microwave energy couples directly to ions, rotating around the dipole to cause friction and release heat, such that acids with higher dipole moments absorb microwaves readily for fast and even heating of reactant solutions. The vessels used in these high pressure reactors, in contrast, are constructed from materials with low or no dipole moment, such as tetra-fluoromethoxy (TFM), making them transparent to microwaves. High pressures in these closed vessels allow acids to be heated beyond their boiling points, further aiding material sample digestion. Protocols for digestion of all materials within the R11410 PMT, for example, have been developed and successfully tested already. A mixture of $\mathrm{HNO}_{3}, \mathrm{HCl}$ and $\mathrm{HF}$ at 220 degrees Celsius (reaching 30 bar) over 45 minutes is sufficient for complete dissolution of all component materials. The nitric acid is commonly used to digest organic material, $\mathrm{HCl}$ for Fe-based alloys due to ability to hold chloro-complex in solution, and HF used for decomposing silicates.

The UCL ICP-MS facility became fully operational in December 2015, and material assays began in early 2016 following the establishment of QC and QA procedures for consistency checks, calibrations, optimizing of cleaning procedures, and assessment of systematics. Sample assays for the LZ project have begun with initial assays of plastics and foams for the cryostat sub-system, achieving results consistent with HPGe assays with the BUGS detectors.

The Center for Underground Physics (CUP), S. Korea, operates an ICP-MS laboratory supporting a number low-background experiments, including LZ. The facility and operations are largely the same as those described for UCL above, including ISO 6 cleanroom, Agilent 7900, and identical microwave digestion 
system now on order. The instrument was made operational and staffed its $20 \mathrm{~m}^{2}$ unidirectional-flow cleanroom (about 180 air changes per hour) in October of 2015. The ICP-MS is equipped for He collision mode with potential to add a Hydrogen line at a later date if a strong need arises. It is has a UHMI (gas-diltuion) introduction system which can help to optimize sample introduction load into the plasma and reduce water and reagent backgrounds. It also was purchased with cold-plasma capability allowing removal of particular interferences with high ionization potentials. This is particularly useful for measurement of ${ }^{39} \mathrm{~K}$ which is severely limited by interference from ${ }^{38} \mathrm{ArH}^{+}$. The lab contains an in-house acid distillation system and water is supplied from a $500 \mathrm{~L} / \mathrm{hr} 18 \mathrm{M} \Omega$ building-wide supply as input to a Millipore Advantage A10 water-purifier.

Protocols for cleaning are generally very similar to those stated above, derived largely from the same sources of experience, but sample preparation has so far been restricted only to un-assisted open digestion. Particular attention has been paid to quantifying and understanding the ultimate limits of measurement backgrounds, based largely on previous work performed at the University of Seoul. Using Savillex brand digestion vessels, cleaning procedures consistent with descriptions above, and statistical subtraction of sample-dependent continuous backgrounds described by Ref. [25], CUP achieves blank contaminants of U and Th statistically consistent with zero (in the blanks this level can be at or below a few fg/g but increases for high concentration samples), and with sample limits significantly below the background equivalent level. A figure of merit using the calibration response, the measured background rate, and simple propagation of statistical errors is used to accurately predict sensitivities in a particular sample based on results of initial in-matrix tuning. Three sigma detection capability below $2 \mathrm{ppt}$ has been demonstrated, but not yet pursued for a sufficiently pure material. Details depend on the sample chemistry, but similar levels are reasonable to expect for most easily acid-dissolvable samples such as metals.

These measurements take significant time owing to the need for high statistics, standard addition calibration of high-concentration samples, and frequent machine cleaning. For more routine measurements, the backgrounds are simply monitored and reported as an equivalent concentration, and lower concentration samples allow for external calibrations and a significantly relaxed cleaning schedule, allowing to measure less demanding samples at the rate of several per day with sensitivities to $U$ and Th on the order of a few hundred ppt. Finally some initial work has been done to develop measurements of potassium using the cold plasma configuration, leading to measurements of a few hundred $\mathrm{ppb}$ of $\mathrm{K}$, and to indications of potential sensitivity much lower, but not yet explored.

ICP-MS facilities are also available Black Hills University Campus, and at the University of Alabama. BHUC also operate an Agilent 7900, with additional laser ablation sample introduction capability with an ESI NWR 213 system. The system is housed in an ISO 7 clean area within a dedicated ICP-MS laboratory. The University of Alabama group have access to a Perkin-Elmer SCIEX-ELAN 6000 ICP-MS within the Geology department that has demonstrated capability to detect U/Th down to the level of tens of ppt and has availability to screen tens of samples on the timescale of a few months. This facility does not, however, provide services for sample digestion and separation/concentration of U/Th content. The Alabama group has carried out a systematic program to certify the capability of the facility, develop protocols to prepare samples for analysis without risk of cross-contamination, and set up a laboratory where common digestion and separation/concentration procedures can be performed.

\subsubsection{NAA}

The University of Alabama group within the LZ collaboration utilizes the 5.5 $\mathrm{MW}_{t h}$ MIT Reactor II (MITRII) to perform neutron activation of samples and subsequent measurements with the Ge-II and Ge-III surface HPGe detectors described in Section 9.4.1.3. MITR-II is a double-tank reactor with an inner tank for lightwater coolant moderator and an outer one serving as heavy-water reflector [26]. Two pneumatic sample insertion facilities are available. Steady-state thermal neutron fluxes of up to $5.5 \times 10^{13}$ neutrons $/ \mathrm{s} / \mathrm{cm}^{2}$ can 
be achieved. The sample insertion facilities can accommodate multiple samples that range in size but are typically a few $\mathrm{mm}$ in diameter and several $\mathrm{cm}$ in length. The two sample insertion facilities offer differing thermal over fast neutron flux ratios. Sample irradiations ranging from minutes to days can be performed, allowing accumulation of very large neutron fluences, which is key for reaching high analysis sensitivity.

LZ samples are prepared, cleaned, and hermetically sealed at the University of Alabama in a cleanroom prior to activation. Polyethylene irradiation vials and samples are separately soaked in ultrapure $\mathrm{HNO}_{3}$, rinsed and dried in a vacuum oven. Vials are welded shut with the clean samples within, and leak tested in a heated water bath. Irradiation at MITR-II is typically for about $10 \mathrm{~h}$, with a NIST certified fly ash sample used to calibrate the neutron flux also irradiated for about $5 \mathrm{~min}$. After activation, the samples can be recovered cleanly and safely to avoid any carry-over contamination problems from the activation vials or other instruments. The counting of the activated samples at Alabama utilizes a double differential timeenergy analysis over a period of about 2 weeks. The typical shipping delay of $24 \mathrm{~h}$ is acceptable compared with the half-lives of the activation products of interest (such as ${ }^{42} \mathrm{~K},{ }^{233} \mathrm{~Pa}$, and ${ }^{239} \mathrm{~Np}$ ). An exponential decay is fit to each time series of activity, for each radionuclide over multiple time intervals. The activity is reported relative to an earlier reference time, by fitting the decay of each radionuclide with a known half-life for determining the composition of the sample. Elemental concentrations are inferred using tabulated energy dependent radiative neutron capture cross sections folded with a standard reactor neutron spectrum, where the model of the neutron flux assumes a thermal Maxwell-Boltzmann neutron energy distribution plus an epi-thermal tail.

The Alabama group routinely achieved $10^{-12} \mathrm{~g} / \mathrm{g}$ sensitivity for Th and U using these techniques, as reported in [1], appropriate for the LZ material screening campaign. Indeed, NAA results obtained by the same group for the KamLAND experiment reached sensitivity to $U$ and Th at the $10^{-14} \mathrm{~g} / \mathrm{g}$ to $10^{-15} \mathrm{~g} / \mathrm{g}$ level [1] for liquid scintillator. Requirements for assaying PTFE for the LZ project have already been met using NAA, as described in Section 9.2.1.

\subsection{Radon Emanation}

The background from radon emanation is dominated by the "naked" beta emission from ${ }^{214} \mathrm{~Pb}$ in the ${ }^{222} \mathrm{Rn}$ sub-chain as it decays to ${ }^{214} \mathrm{Bi}$, whereas the ${ }^{214} \mathrm{Bi}$ beta decay itself is readily identified by the subsequent ${ }^{214} \mathrm{Po}$ alpha decay that would be observed within an $\mathrm{LZ}$ event timeline $\left(\mathrm{T}_{1 / 2}=160 \mu \mathrm{s}\right)$. Similar coincidence rejection also occurs where beta decay is accompanied by a high-energy $\gamma$-ray, which may still be tagged by the LXe skin or external Gd-LS vetoes even if it leaves the active Xe volume. Radon-220 generates ${ }^{212} \mathrm{~Pb}$, which decays with a short-timescale $\mathrm{Bi}-\mathrm{Po}$ (beta-alpha delayed coincidence) scheme similar to ${ }^{214} \mathrm{~Pb}$. Radon daughters are readily identified through their alpha decay signatures, as demonstrated in LUX, and can be used to characterize the ${ }^{222} \mathrm{Rn}$ and ${ }^{220} \mathrm{Rn}$ decay chain rates and distributions in the active region, providing a useful complement to estimating radon concentration from the beta decay contribution to the ER background. Indeed, these isotopes were the only sources of alpha decay identified in LUX [10]. As detailed in Table 9.5.2, there are multiple potential sources of radon emanation (e.g., PTFE reflectors, PTFE skin, PMT glass, PMT and HV cables, grid resistors, components in the circulation system), and radon emanation screening must be sensitive to sources that individually sustain smaller populations. We use $0.67 \mathrm{mBq}$ in the active target within the TPC as the goal for ${ }^{222} \mathrm{Rn}$, which equates to a steady-state population of approximately 300 atoms. This activity corresponds to $0.56 \mathrm{mBq}$ in the fiducial $\mathrm{LXe}$, and $1 \mathrm{mBq}$ in the total amount of LXe. This activity results in a background contribution that matches those from $\mathrm{Kr}$ and from intrinsic material radioactivity, and is about $10 \%$ of the irreducible pp solar neutrino background. Requirements for $\mathrm{LZ}$ are twenty times higher than these goals, with $20 \mathrm{mBq}{ }^{222} \mathrm{Rn}$ total, of which $13.4 \mathrm{mBq}$ is in the active $\mathrm{LXe}$, and $11.2 \mathrm{mBq}$ in the fiducial LXe. This requirement ensures that the background due to radon does not dominate significantly over the irreducible pp solar neutrino background, so that the WIMP- 
search reach of the experiment is not significantly reduced. Background from ${ }^{220} \mathrm{Rn}$ is not expected given its very short half-life. Due to observation of an alpha population ascribed to ${ }^{220} \mathrm{Rn}$ in LUX, we conservatively include a contribution in our estimates. ${ }^{220} \mathrm{Rn}$ is required to contribute no more than than $20 \%$ of the ER counts from ${ }^{222} \mathrm{Rn}$.

All components that may contribute to the radon load within the LXe will be screened for radon emanation. These components include all that are within the inner cryostat or that come into direct contact with Xe during experimental operation. Some materials are screened to inform material selection. These assays are scheduled carefully in conjunction with material procurements. In other cases (such as the PMTs), the materials cannot be changed due to finite resources and schedule, but the assay is performed in order to inform the background model.

The LZ collaboration has extensive access to four radon-emanation screening stations that meet the sensitivity requirement for our assays. These are summarized in Table 9.5.1. In one of the stations, at Alabama, the radon atoms are collected by passing the radon-bearing gas through liquid scintillator, with the Bi-Po coincidence detected through gated coincidence logic using one PMT viewing the scintillator. In the other three stations, radon atoms and daughters are collected electrostatically onto silicon PIN diode detectors to detect ${ }^{218} \mathrm{Po}$ and ${ }^{214} \mathrm{Po}$ alpha decays. One of these stations was developed at Case Western Reserve University and has been commissioned at the University of Maryland. Work at this system is focusing on emanation of systems that act as their own emanation chambers, such as plumbing for the LZ Xe recirculation system. The third station, at SDSM\&T, has two emanation chambers including one large, 300-liter chamber that will be used to emanate large amounts of materials to achieve the best sensitivity. Finally, there is additional capability and throughput available in the U.K. using the system employed by the SuperNEMO group at UCL [27]. This system was used extensively in the R\&D phase and LZ is expected to have access sufficient to measure emanation from six samples per year with it.

All stations were initially evaluated using calibrated sources of radon, with a cross-calibration program performed to ensure the accuracy of each system's overall efficiency and ability to estimate and subtract backgrounds. The first cross-calibration sample had a relatively high emanation rate, so that system efficiencies could be determined without possible interference from backgrounds. The second cross-calibration

Table 9.5.1: Radon-emanation facilities available for LZ material radio-assays. Sensitivities shown are approximate detectable activities after 2 weeks of emanation and counting. With the exception of the UCL system, all are managed by LZ groups. The UMd and Alabama systems are dedicated to LZ, while the UCL system is primarily for SuperNEMO, and the SDSM\&T system will be shared (at $<25 \%$ time) with SuperCDMS.

\begin{tabular}{|c|c|c|c|c|c|c|c|}
\hline Detector & Type & $\begin{array}{c}\text { Detector } \\
\text { Efficiency }\end{array}$ & $\begin{array}{c}\text { Detector } \\
\text { Back- } \\
\text { ground } \\
(\mathrm{mBq})\end{array}$ & $\begin{array}{c}\text { Samples/ } \\
\text { Year }\end{array}$ & $\begin{array}{c}\text { Chamber } \\
\text { Volume } \\
(\mathrm{I})\end{array}$ & $\begin{array}{c}\text { Transfer } \\
\text { Effi- } \\
\text { ciency }\end{array}$ & $\begin{array}{c}\text { Blank } \\
\text { Rate } \\
(\mathrm{mBq})\end{array}$ \\
\hline UCL & PIN-diode & $30 \%$ & 0.2 & 6 & $\begin{array}{c}2.6 \\
2.6\end{array}$ & $\begin{array}{c}97 \% \\
97 \%\end{array}$ & $\begin{array}{c}0.2 \\
0.4\end{array}$ \\
\hline Maryland & PIN-diode & $24 \%$ & 0.2 & 12 & 4.7 & $96 \%$ & 0.2 \\
\hline SDSM\&T & PIN-diode & $20 \%$ & 0.15 & $\begin{array}{c}12 \\
12\end{array}$ & $\begin{array}{c}13 \\
300\end{array}$ & $\begin{array}{c}94 \% \\
80 \%\end{array}$ & $<0.3$ \\
\hline Alabama & Liquid & $40 \%$ & $<0.15$ & 12 & 2.6 & $30 \%$ & $<0.4$ \\
\hline
\end{tabular}


sample has a rate close to the quoted system sensitivities, to check the accuracy of background subtraction with the systems.

Screening a single sample for LZ takes about two weeks, including emanation and collection/detection times. Taking into consideration the desire for repeated measurements to check reproducibility and improve sensitivity, as well as runs to ensure stability of background rates and transfer efficiencies, no more than one sample per month per emanation chamber is planned for the scheduling of radon emanation screening.

In advance of the radon emanation screening, estimates were made of expected radon emanation from all materials in contact with the $\mathrm{Xe}$, based on previous measurements of radon emanation from similar materials, or based on the combination of HPGe measurements of the bulk ${ }^{226} \mathrm{Ra}$ contamination together with measurements or estimates of Rn diffusion in the material. It must be noted that surface contamination may cause a higher rate of radon emanation from a material than is estimated from its bulk contamination, especially if the material's bulk is relatively radiopure or if the material has a very low radon diffusion constant. Table 9.5.2 summarizes the resulting radon budget from the critical materials. In cases where the dominant radon emanation is expected due to diffusion of radon from the material bulk, the expected radon emanation is based on room-temperature assays, conservatively ignoring the expected reduction of radon diffusion at LXe temperatures for all materials except the cables. For materials with low radon diffusion, such as metals, whose radon emanation is expected to be dominated by recoil punch-out, no emanation suppression is expected at reduced temperatures.

Without remediation, the cables would have been expected to be a major source of radon, emanating up to $30 \mathrm{mBq}$ based on previous measurements [28]. This total would likely be dominated by the large surface area of the stainless steel braiding and contamination, such as dust, that is caught in this braiding. Mitigation of this large radon source will be achieved by adding a thin FEP cladding to the outside of the cables and by the installation of a carbon trap (described in Section 6.4.5) to filter radon out of the Xe gas from the conduits before it enters the main re-circulation. The cladding should make it easier to minimize contamination of the cables with dust. Furthermore, radon diffuses very slowly through the cold FEP cladding, nearly eliminating the emanation into the liquid Xe by the cables, while the trap will reduce the radon emanation into the gas portion of the conduits by at least $90 \%$. The combination of these two strategies reduces the expected emanation by the cables to $0.09 \mathrm{mBq}$. The carbon trap should significantly reduce the radon load from other room-temperature materials such as the PMT cable feedthroughs and conduits. Table 9.5.2 includes the expected reduction from the carbon trap.

Other potentially significant contributors were identified. Early assays of the components of the PMT bases indicate sufficiently low emanation even at room temperature that mitigation strategies, such as potting, need not be explored. The Xe purification getter material has been identified as a source of radon [29]. In order to minimize the radon emanation from the getter while allowing the high throughput needed by LZ, the baseline plan is to purchase a full-size getter cabinet with a partially-loaded getter cartridge (see Section 6.4.1). In addition, a screening program to identify a clean $\mathrm{Zr}$ substitute is underway, along with an investigation of other material options.

The largest single contributor to radon backgrounds in the experiment is expected to be due to dust on material surfaces. As described in Section 9.6.1, certified protocols for assembly and cleaning will provide the means to achieve a low enough dust concentration on materials in LZ. The dust is conservatively estimated to generate $10 \mathrm{mBq}$ of activity. We assume that $25 \%$ of ${ }^{222} \mathrm{Rn}$ from the dust on material surfaces escapes into the xenon; however preliminary measurements of of radon emanation from dust support fractions considerably lower (see Section 9.6.1).

The radon-emanation screening campaign, coordinated through dedicated management in the screening working group, extends beyond initial material selection. The system from SDSM\&T will be relocated underground to SURF in order to screen large-scale assembled detector elements and plumbing lines. As pieces or sections are completed during installation of gas pipework for the $\mathrm{LZ}$ experiment, they will be 
Table 9.5.2: List of materials in contact with $\mathrm{Xe}$, indicating the quantity of the material and the requirement for radon emanation from the material. This requirement is set by dividing the maximum activity from the materials, $10 \mathrm{mBq}$ in the full 10 tons of $\mathrm{LXe}$, evenly among the 9 major systems. A further $10 \mathrm{mBq}$ from dust makes up the full requirement of $20 \mathrm{mBq}$ from radon. The estimates of radon emanation are based either on direct assays (listed in boldface) or on the most similar object or material for which emanation rates are available in the literature. Some materials (labeled with ${ }^{*}$ ) are expected to emanate less radon when cold, but the estimate listed conservatively does not take such reduction into account. Only the estimate for PMT cables, as described in the text, takes this into account. We also list the quantity of material planned for screening. Expected reduction of radon by the carbon trap described in Section 6.4.5 is included in estimates for those components affected (labeled with ${ }^{\dagger}$ ).

\begin{tabular}{|c|c|c|c|c|c|c|}
\hline Material & Component(s) & Quantity & Unit & $\begin{array}{l}\text { Require- } \\
\text { ment } \\
(\mathrm{mBq})\end{array}$ & $\begin{array}{c}\text { Estimate } \\
(\mathrm{mBq})\end{array}$ & $\begin{array}{c}\text { Screening } \\
\text { Quantity }\end{array}$ \\
\hline $\mathrm{Al}_{2} \mathrm{O}_{3}$ resistor & PMT Bases & 9790 & $\#$ & 0.66 & $0.58^{*}$ & 3,650 \\
\hline $\mathrm{BaTiO}_{3}$ capacitor & PMT Bases & 3010 & $\#$ & 0.66 & $0.016^{*}$ & 100,000 \\
\hline Cirlex & PMT Bases & 6000 & $\mathrm{~cm}^{2}$ & 0.11 & $0.37^{*}$ & 668 \\
\hline Titanium & $\begin{array}{c}\text { Cryostat, PMT } \\
\text { Mounts, Field } \\
\text { Rings, Grid } \\
\text { Supports }\end{array}$ & 412,000 & $\mathrm{~cm}^{2}$ & 1.70 & 0.41 & 550 \\
\hline PTFE & $\begin{array}{l}\text { Reflectors, HV } \\
\text { Umbilical }\end{array}$ & 840,000 & $\mathrm{~cm}^{2}$ & 0.66 & $<1.3^{*}$ & 205,000 \\
\hline PMT Cabling $^{\dagger}$ & PMT Cabling & 17,000 & $\mathrm{~m}$ & 0.55 & 0.09 & 3,000 \\
\hline $\mathrm{PMT}$ Feedthrough ${ }^{\dagger}$ & PMT HV Flange & 122 & $\#$ & 0.11 & 0.49 & 5 \\
\hline PMT Feedthrough ${ }^{\dagger}$ & Signal Flange & 88 & $\#$ & 0.11 & $<0.24$ & 5 \\
\hline Steel Conduit ${ }^{\dagger}$ & Cabling Conduit & 100,000 & $\mathrm{~cm}^{2}$ & 0.22 & 0.055 & 100,000 \\
\hline R11410 PMT & R11410 PMT & 488 & $\#$ & 1.10 & 1.26 & 488 \\
\hline R8520 PMT & R8520 PMT & 90 & $\#$ & 0.47 & 0.15 & 90 \\
\hline R8778 PMT & R8778 PMT & 36 & $\#$ & 0.08 & 0.09 & 36 \\
\hline Polyethylene & HV Umbilical & 4200 & $\mathrm{~cm}^{2}$ & 0.11 & 0.10 & 42,000 \\
\hline Tin-coated copper & HV Umbilical & 11,000 & $\mathrm{~cm}^{2}$ & 0.11 & 0.002 & 110,000 \\
\hline Tivar & HV Umbilical & 3894 & $\mathrm{~cm}^{2}$ & 0.22 & $0.004^{*}$ & 20,000 \\
\hline Acetal & HV Umbilical & 195 & $\mathrm{~cm}^{2}$ & 0.11 & $0.0002^{*}$ & 2000 \\
\hline Copper & HV Umbilical & 39 & $\mathrm{~cm}^{2}$ & 0.11 & 0.000007 & 400 \\
\hline Epoxy & HV Umbilical & 1000 & $\mathrm{~cm}^{2}$ & 0.11 & $0.0001^{*}$ & 10,000 \\
\hline Steel & $\begin{array}{l}\text { Cryostat Seals, Xe } \\
\text { Recirculation }\end{array}$ & 135,000 & $\mathrm{~cm}^{2}$ & 0.77 & 0.104 & 135,000 \\
\hline $\begin{array}{l}\text { Recirculation } \\
\text { Pump }\end{array}$ & Xe Recirculation & 1 & $\#$ & 0.22 & 0.1 & 1 \\
\hline Purification Getter & Xe Recirculation & 2.5 & $\mathrm{~kg}$ & 1.10 & 1.34 & 2.5 \\
\hline $\begin{array}{c}\text { Transducers \& } \\
\text { Valves }\end{array}$ & Xe Recirculation & 30 & $\#$ & 0.44 & 0.17 & 30 \\
\hline Welds & $\begin{array}{c}\text { Recirculation } \\
\text { System, Cryostat }\end{array}$ & 32.3 & $\mathrm{~m}$ & 0.22 & 0.11 & 18.3 \\
\hline Dust & & & & 10.0 & 10.0 & \\
\hline Total & & & & 20.0 & $<16.9$ & \\
\hline
\end{tabular}


isolated and assessed for Rn emanation and outgassing for early identification of problematic seals or components that require replacement, cleaning, or correction.

\subsection{Surface Cleanliness}

Once materials and components have met screening requirements and components have been procured, they will be kept clean during fabrication, storage, transport, and final assembly and integration into the experiment. We refer to this task as cleanliness. The major sources of contamination that must be addressed by the LZ cleanliness program are radon diffusion and daughter-nuclei plate-out, and dust and debris. Radondaughter plate-out onto material surfaces may generate NR backgrounds through two mechanisms: $(\alpha, n)$ processes that release neutrons into the xenon; and ions from the ${ }^{210} \mathrm{~Pb}$ sub-chain originating at the edge of the TPC being mis-reconstructed as NRs within the fiducial volume. ${ }^{210} \mathrm{~Pb}$ on surfaces may also present ER background if it or daughters become mobile and enter the fiducial volume, with ${ }^{210} \mathrm{Bi}$ of note as a beta-emitter. Dust on component surfaces carried into the xenon may produce ER and NR from intrinsic activity, as well as contributing significantly to the radon emanation background. We require that the effects of radon plate-out and dust deposition do not contribute to the background in LZ any more than the material components. Other sources of contamination must also be addressed, for example residual chemicals from fabrication processes, removal of which should be effected at the same time as cleaning to remove dust.

LZ draws on extensive cleanliness experience from earlier involvement in LUX, EXO, SNO, KamLAND, MAJORANA Demonstrator, and other low-background experiments to develop cleanliness protocols and assay techniques for dust and surface contamination. LZ is developing experiment-specific techniques for validating protocols and establishing a rigorous and comprehensive Quality Control (QC) and Quality Assurance (QA) program. All protocols and documentation are stored in the LZ information repository where they are linked to the specific material or component. No material or component will be integrated into the experiment without adequate documentation demonstrating handling following these protocols and successfully passing quality control tests.

In the following subsections we present details on the origins of these background sources; requirements and goals for both radon plate-out and dust depositions and equivalent maximum exposure times; controls and mitigation protocols; and assay techniques to ensure QC and QA certification.

\subsubsection{Dust}

Dust is typically characterized as fine-grained $(<100 \mu \mathrm{m})$ material that results from the breaking/grinding of materials in the local environment. The specific content will reflect the location, but in general will include minerals from rock and soil, small amounts of plant pollen, human and animal hairs, textile fibers, paper fibers, human skin cells and even burnt meteorite particles. The contents and their relative proportions will determine the type and level of radioactivity, but in general the major contributors are ${ }^{40} \mathrm{~K},{ }^{238} \mathrm{U}$ and ${ }^{232} \mathrm{Th}$, all with activity levels around $\sim 10 \mathrm{mBq} / \mathrm{g}$. Fine grains may easily become airborne, to be transported over significant distances, and then deposit on local surfaces. Electrostatic attraction allows vertical as well as horizontal surfaces to become contaminated. We expect to encounter dust in all locations where work is conducted, including the sourcing of materials where dust may become internalized to components, fabrication and construction phases, and in assembly and deployment. A detailed understanding of the radiological content of the dusts to be encountered has been developed, primarily through HPGe screening of floor sweepings and HEPA filter samples from the SURF underground areas. Measurements of the dustparticle count density, size, and deposition rate at SURF have also been completed [30] and will be extended to other assembly locations at LZ institutes and external sites where appropriate, such as the Ti-cryostat manufacturer. 
The quantity of airborne dust in a specific location is found to vary significantly, with strong dependence on the local environment, geometry of any buildings, rate of airflow, and the use of air filtration to reduce the presence of dust, for example through the use of cleanrooms. The effectiveness of a cleanroom is highly dependent on associated procedures, for example the level of use, gowning procedures of users, and supplementary cleaning activities. The rate at which dust settles out on surfaces depends on the dust level in the air, the particle size distribution and density, and the rate of airflow. Where possible dust levels and dust deposition rates have been measured directly, but also modeling of these factors has been performed under a number of reasonable assumptions and model bases. We have developed two web-based calculators, denoted "SNO" and "ASML-Delft", respectively, that give allowed exposure times based on model and environmental inputs. The "SNO" model is based on the mass fallout model developed by R. Stokstad for SNO [31]. Input parameters include fresh air dust content, volume exchange rate, recirculation fraction, filter efficiency, and mass carry-in rate. "ASML-Delft" [32] is an empirical model derived from test measurements and validated by monitoring particle fallout rates as a function of air particle concentrations in operational cleanrooms in the semiconductor industry. Test measurements and operational cleanroom observations are consistent with parameterization to within an order of magnitude. As an example, the SNO model predicts that a volume of fresh air (ISO class 8 ) that has 120 volume exchanges per hour including $0.8 \%$ fresh air, and a carry-in rate of dust of 0.25 grams $/ \mathrm{d}$, would accumulate $500 \mathrm{ng} / \mathrm{cm}^{2}$ of dust in a period of 2.3 days. The dust fallout rate is sensitive to the carry-in rate: for example, reducing the carry-in rate to $0.025 \mathrm{grams} / \mathrm{d}$ increases the accumulation time to $22 \mathrm{~d}$. The carry-in rate is a factor that we control by implementation of cleanroom protocols. We are conducting a program of dust fall-out measurements within the LZ cleanrooms to determine actually realized rates under particular LZ conditions and locations. Our results indicate that fall-out rates are below those expected from the ASML-Delft models.

There are a number of mechanisms by which the radiological content of dust that is present on the surface of a component may be problematic. The relative importance of these varies depending on the properties and location of the component in the experiment. Contributions to ER and NR rates have been estimated based on the estimated levels of dust, known nuclear properties, and simulations propagating the decay products to the LZ experiment.

The most significant radiological concern for LZ from dust, and the one that drives our requirements, is radon emanation from dust within the inner cryostat and conduits. As previously described in Section 9.5, this contributes ERs via the naked beta decay of ${ }^{214} \mathrm{~Pb}$. The requirement for the total activity from radon emanation in the xenon is $20 \mathrm{mBq}$, with a goal of $1 \mathrm{mBq}$. Dust contributes half the rate in the requirement. Our present estimate is that the radon emanation from dust is equivalent to $10 \mathrm{mBq} / \mathrm{g}$. This estimate is based on measured rates of radon emanation from naturally-occurring materials [33] and SNO reports on radon emanation from mine dust [34]. This sets the requirement on maximum amount of dust within the xenon at $1 \mathrm{~g}$. We anticipate the contribution from dust will be significantly less given conservative estimates that define our requirements. Direct HPGe measurements we have performed to infer radon emanation from debris collected at SURF has indicated a $12 \%$ radon emanation fraction, suppressed to $4 \%$ at cryogenic temperatures due to reduced radon mobility. This is considerably lower than the $25 \%$ we assume in defining our requirements. Direct radon emanation measurements of dust at room temperature will be performed end2016. The goal for dust is a total of $10 \mathrm{mg}$ within the xenon, corresponding to an activity of $0.1 \mathrm{mBq}$ (based on the assumed $25 \%$ emanation fraction). The total surface area from all components within and including the ICV is about $1.6 \times 10^{6} \mathrm{~cm}^{2}$ and we conservatively set the required limit on dust surface mass density at $0.5 \mu \mathrm{g} / \mathrm{cm}^{2}$. Our goal defines a density limit of $5 \mathrm{ng} / \mathrm{cm}^{2}$. Dust on all other components is constrained to be at the same level, driven by the need to avoid cross contamination during assembly, however radon emitted from dust on non-wetted surfaces not in contact with any xenon is not a concern.

Beyond radon emanation, the intrinsic activity of dust generates both ER and NR background, and this contribution has also been evaluated in detail. Dust concentrations that meet requirements are assumed for 
the surfaces of all components within the ICV, taking into account the appropriate surface areas from the LZ CAD model. LZSim is used to generate radioactivity from these surfaces by propagating $\gamma$-rays and neutrons and estimating impact in LZ. These calculations account for $(\alpha, n)$ reactions on the appropriate targets. The range of few-MeV alpha particles in dust is on the order of $100 \mu \mathrm{m}$ and thus in most decays it is reasonable to expect the alpha particle to escape. For a surface deposit, a simple geometric consideration predicts half of these to then impact the surface. Of particular concern is dust upon the surface of PTFE components, since the number of neutrons emitted for each incident alpha particle is particularly high for fluorine, 9.48 neutrons per $10^{6} \alpha$-particles [35]. Aluminum also has a moderately high $(\alpha, n)$ cross section resulting in 0.63 neutrons per $10^{6} \alpha$-particles. The $(\alpha, n)$ cross sections of other elements used in LZ all have significantly smaller cross sections. Of note, the design for the internal PTFE reflectors has them attached by pins to the inner cryostat, deliberately allowing a thin layer of Xe to penetrate between the titanium and the PTFE, thus mitigating against alpha particles emitted from $U$ and Th contaminants in dust on the cryostat surface from inducing $(\alpha, n)$ reactions on the adjacent PTFE reflector. Between the inner and outer cryostats is the vacuum region. Dust here has been considered on the titanium surfaces, the MLI superinsulation, which is aluminized mylar, and displacer foam. Beyond the cryostats, there is a layer of displacer foam for mechanical stability, the outer detector comprised of acrylic vessels holding gadolinium loaded scintillator, mechanical supports, the water tank and its PMTs and reflective liners. While some of these have large areas, our calculations suggest that their distance from the fiducial volume prevent them from posing significant concern under any reasonable assumption of dust level on their surfaces. Dust contributes a negligible 0.19 ER counts to the background in LZ before any discrimination is applied. The total NR contribution from dust is similarly low, at 0.05 counts before application of NR efficiency. These contributions are included in Table 9.2.7.

Detailed cleanliness protocols to clean components and maintain purity are developed for each subsystem, specific to the materials and adopting industry standard cleaning procedures. Each protocol is defined to satisfy the cleanliness goal rather than just the requirement. Each subsystem develops its own cleanliness protocols that are reviewed by the cleanliness working group before being put into effect. The protocols describe how the subsystem components are to be cleaned and kept clean through fabrication, assembly, integration and installation, and describe the validation of cleaning and assay methods. Documentation with each component demonstrates that protocols have been followed step-by-step, and includes results from assay of witness plates and coupons, and from environmental monitoring during fabrication and storage. Upon delivery to SURF each component's documentation pack will include a requirement for signoff for dust cleanliness at the required level. The protocols and documentation are stored in the LZ Information Repository. No part is to be accepted for final assembly and integration on-site without QA and requisite documentation that protocols having been successfully followed.

Final shipping of clean components will involve the use of sealed bags made from films of certified cleanliness. Vendors have been identified for procurement of 50 micron thick Nylon bags certified to be dust free at Level 50 of U.S. MIL-SPEC 1246C, equivalent to there being less than one $50 \mu \mathrm{m}$ sized particle per square foot. For large components, custom-fabricated bags meeting cleanliness requirements will be used. The standard packaging procedure will employ three layers of packaging: outermost will be "bug bag" which will be removed just before transferring the package from the loading dock to an inside climate-controlled area; the next layer of packaging will be removed in an anteroom to the cleanroom assembly/integration area; and the innermost package will be removed inside the cleanroom just before the part is integrated. Dust deposition rates of $1 \mathrm{ng} / \mathrm{cm}^{2} / \mathrm{h}$ have been achieved previously by the SNO experiment, and by the LZ SDSM\&T group.

Essential to evaluating cleanliness is the ability to assay at the required levels to maintain QC and perform QA before components are accepted for integration. Several methods for the assay of dust have been developed for the LZ project. At SDSM\&T, an optical system has been used. Glass witness slides are imaged, 
revealing dust, fibers and hairs that have been deposited onto the slides. An automated software process analyses these images and determines the size and mass of each particle. Sensitivity at the level of $5 \mathrm{ng} / \mathrm{cm}^{2}$ has been demonstrated, sufficient not only for our requirement but also for our goal. In parallel, tape lifts are also being used on non-transparent surfaces, such as Cirlex that is to be used in the PMT bases. Acetate tape is first immersed in acetone and then placed on the surface of the material to be assayed. The tape secures the dust and, once dry, is removed along with the dust. This tape is then optically imaged using the same method as for the glass witness slides. The size distributions for all surfaces assayed broadly follows the expected logarithmic behavior, with the majority of particles measuring below $10 \mu \mathrm{m}$ and only a few larger. However, these few larger particles dominate the mass of the sample. For large areas of titanium sweeping of dust from the surface and then measurement of the weight of that material is possible with sensitive scales. An area of $1 \mathrm{~m}^{2}$ with dust deposition at the level of $5 \mathrm{ng} / \mathrm{cm}^{2}$ equates to a mass of $50 \mu \mathrm{g}$, an amount that may be collected and measured with a standard microbalance.

\subsubsection{Radon Plate-out}

Radon plate-out is a phenomenon in which charged radon progeny are deposited onto the surfaces of materials exposed to air that typically contains concentrations of ${ }^{222} \mathrm{Rn}\left(\mathrm{T}_{1 / 2}=3.82 \mathrm{~d}\right)$ ranging from tens to hundreds of $\mathrm{Bq} / \mathrm{m}^{3}[36,37]$. The decay daughters can be embedded into material as they recoil due to subsequent decays. Beyond radon concentration and surface area, the susceptibility to plate-out depends on the material and factors such as air-flow rates, which are difficult to predict and therefore must be measured. In the cases where measurements are not available, conservative estimates are used to predict contamination risk from plate-out. Plate-out may be further enhanced in the presence of an electric field, since positively charged radon daughters are deposited on negatively charged surfaces such as electrodes within the TPC. The background due to radon daughters on the surfaces arises predominantly from neutron production. In particular the long-lived ${ }^{210} \mathrm{~Pb}\left(\mathrm{~T}_{1 / 2}=22.3 \mathrm{y}\right)$ in the decay series decays to ${ }^{210} \mathrm{Po}$ (via ${ }^{210} \mathrm{Bi}$ ), which emits an $\alpha$ that feeds $(\alpha, n)$ reactions. In addition to neutron background, progeny from $\mathrm{Rn}$ plated onto the inner surfaces of the TPC, particularly ${ }^{206} \mathrm{~Pb}$, can lead to spatial leakage of mis-reconstructed events at the TPC walls, rapidly reducing the fiducial mass. Furthermore, incomplete charge collection of these recoils at the edges of the TPC can cause them to overlap with the low-energy NR band. We have conducted detailed simulations, utilizing the position reconstruction algorithm successfully deployed in both LUX and ZEPLIN-III and adapted for the TPC, extraction electrodes, and top PMT array configurations of LZ, to study position reconstruction of such edge events. In the following we describe in detail our estimate of the level of background produced by plate-out, our approach to estimating the rate at which plate-out could occur in LZ, and the measures we will take to adequately mitigate background risk.

The first background due to $(\alpha, n)$ depends on the area and material composition of component surfaces. It also depends on the degree of surface contact between two components. As described for $\alpha$ 's from dust above, if two components are in close contact, $\alpha$ 's emitted from the surface of one material could induce $(\alpha, n)$ in the other material. Of the LZ detector components, the only ones with a significant product of surface area and $(\alpha, n)$ reaction yield are the teflon components, due to the fluorine, and the 10 to 20 layers of superinsulation containing aluminum between the inner and outer vessels of the cryostat. Using results on the simulation of the detector response to neutrons and application of analysis cuts, the expected NR background counts from the $(\alpha, n)$ reaction on teflon and aluminum has been estimated, not only for plateout activity directly on these surfaces but also for plate-out activity on surfaces of other components (e.g. PMTs) in tight contact with teflon or aluminum. The results are shown in Table 9.6.1, with a low total of only 0.05 NR counts before NR efficiency is applied. This is based on a required surface activity density of $10 \mathrm{mBq} / \mathrm{m}^{2}$. 
The second background is the mis-identification of ion daughters which recoil into the TPC active volume from the decays of radon progeny on the surface of the teflon cylindrical reflectors. In the long-lived ${ }^{210} \mathrm{~Pb}$ sub-chain, the only decay capable of producing an ion recoil of sufficient energy is the ${ }^{210}$ Po decay into an alpha and ${ }^{206} \mathrm{~Pb}$ ion. Using a fiducial volume enclosing 5.6 tonne on $\mathrm{LXe}$, the probability that an ion recoil is mis-identified as an NR event has been estimated to be $10^{-6}$. At the minimum ${ }^{210}$ Po activity LZ expects to be able to detect, namely, $0.5 \mathrm{mBq} / \mathrm{m}^{2}$, the expected $\mathrm{NR}$ background is 0.16 counts. This background is expected to be mitigated with straightforward measures since it affects only small S2 signals, and its rate falls as a steep function of the distance of the fiducial volume boundary from the physical reflector surface. It therefore does not affect the peak sensitivity of LZ, and may in any case be removed efficiently through fiducialization. Nonetheless, we include this background in Table 9.2.7, and derive a requirement on plate-out activity on the inside of the TPC walls at $0.5 \mathrm{mBq} / \mathrm{m}^{2}$.

Radon plate-out may also generate ER background if the ${ }^{210} \mathrm{~Pb}$ or any of its daughters become mobile and detach from surfaces to enter the fiducial volume. ${ }^{210} \mathrm{Bi}$ in particular would generate single site ER background if present in the target. As discussed in Section 9.2.2, LUX data has been assessed to place upper limits on the mobility of ${ }^{210} \mathrm{~Pb}$ daughters from the TPC walls. For an activity of $0.5 \mathrm{mBq} / \mathrm{m}^{2}$ on the PTFE panels of the TPC in LZ, an upper limit of 40 ER counts may be expected; a contribution similar to the fixed radioactivity or intrinsic contamination goals.

For evaluating measures to control backgrounds due to plate-out, one must estimate the rate at which plate-out occurs. As already mentioned, plate-out is a complex process depending not only on the concentration of radon in the air and the area of exposed surfaces but also on environmental conditions and surface properties and conditions. We have developed three rate calculators, one ("Guiseppe") that parameterizes the daughter deposition rate in terms of the radon concentration in air, surface area, and a "deposition velocity" that has been measured for different radon daughters under different conditions [36]; a second ("Borexino") based on a model used by the Borexino experiment [38] that parameterizes the daughter deposition rate in terms of radon concentration, a column height (e.g. the height of a cleanroom above a work surface), and plate-out fraction; and a third ("dead-air") based on a pessimistic model in which all of the radon decays in a defined volume result in ${ }^{210} \mathrm{~Pb}$ daughters deposited on the enclosing surface of the volume. Plate-out measurements carried out within the LZ collaboration to validate this model under conditions in which radon-laden air was circulated very slowly (one volume exchange every 12 hours) over aluminum, teflon, and glass plates set at the bottom of a 8-liter rectangular purge box gave results consistent with the dead-air model.

Numerical results for the estimated exposure time required for ${ }^{210} \mathrm{~Pb}$ activity to reach a level of $10 \mathrm{mBq} / \mathrm{m}^{2}$ are presented in Table 9.6.2. For the first calculator, the largest measured value for the deposition velocity was used as a conservative value, corresponding to the measured plate-out of ${ }^{218} \mathrm{Po}$ on acrylic; for the

Table 9.6.1: Expected NR background due to $(\alpha, n)$ reaction from plate-out activity at the level of $10 \mathrm{mBq} / \mathrm{m}^{2}$.

\begin{tabular}{|l|c|}
\hline Component & NR Background \\
\hline TPC PMTs (3-inch) & 0.002 \\
Teflon & 0.021 \\
PMT cables (teflon jacket) & 0.017 \\
Field-shaping rings & 0.004 \\
Superinsulation & 0.007 \\
\hline Total & 0.051 \\
\hline
\end{tabular}


second calculator a column height of $2.5 \mathrm{~m}$ and a plate-out fraction of 0.01 was used, compatible with radon daughter plate-out onto nylon as measured by Borexino; for the third calculator, the area of the enclosing surface was set to $8.0 \mathrm{~m}^{2}$ and the volume was set to $3.5 \mathrm{~m}^{3}$, corresponding approximately to the volume of the TPC.

The estimates from the Guiseppe and Borexino models, in qualitative agreement with each other, indicate that detector fabrication and assembly in well-ventilated areas significantly reduces the plate-out rate. Even with good ventilation, however, the allowed exposure times under typical laboratory conditions $(\mathrm{Rn}$ concentration on the level of $20 \mathrm{~Bq} / \mathrm{m}^{3}$ ) are short for a maximum surface activity of $0.5 \mathrm{mBq} / \mathrm{m}^{2}$ allowed for the TPC cylindrical reflectors. To assure that radon plate-out is controlled during fabrication, assembly, and integration, the following measures will be taken:

1. Final assembly and integration at SURF of the inner cryostat vessel and all interior components, projected to take approximately 6 months, will be carried out in radon-scrubbed clean room with a design radon concentration of $0.3 \mathrm{~Bq} / \mathrm{m}^{3}$. This dramatically increases the acceptable exposure for the TPC reflectors to meet installation and integration timescales, as shown in Table 9.6.2.

2. During storage and transport, parts will be sealed in at least three layers of nylon or metalized mylar bags backfilled with nitrogen or argon. Metallized mylar and nylon are known to be excellent radon barriers although use of nylon in high-humidity environments significantly degrades its effectiveness. Tests within the LZ collaboration using commercially-supplied 2-mil nylon bags and 2.5-mil aluminized mylar bags have shown that the radon concentration reached inside a single bag after one month is at least two orders of magnitude below external levels in the case of nylon and at least three orders of magnitude less for the case of aluminized mylar.

3. In the case of critical parts for which the storage time is long compared to a month, nitrogen purge boxes will be used.

4. Assembly and integration procedures for the TPC cylindrical reflectors will assure good ventilation to avoid higher plate-out rates which would be expected to occur in a dead-air environment.

5. Witness plates and coupons will be assayed at critical points to assure that plate-out is being controlled at the required level.

6. The detailed prescription for implementing the above measures and documenting that they have been successfully followed will be incorporated into the cleanliness protocol for each subsystem.

Within LZ, two sensitive detectors will be used to carry out the assays to determine surface activity and plate-out. The first is the commercial XIA Ultralow 1800 surface alpha detector system, suitable for routine screening of small samples including our witness plates and coupons. Recently installed and commissioned

Table 9.6.2: Allowable exposure times (days) to reach radon daughter plate-out requirements

\begin{tabular}{|c|c|c|c|c|}
\hline $\begin{array}{c}\text { Maximum Activity } \\
\left(\mathbf{m B q} / \mathbf{m}^{\mathbf{2}}\right)\end{array}$ & $\begin{array}{c}\text { Radon Concentration } \\
\left(\mathrm{Bq} / \mathbf{m}^{\mathbf{3}}\right)\end{array}$ & Guiseppe & Borexino & Dead-Air \\
\hline 10 & 20 & 235 & 168 & 13.4 \\
\hline 10 & 0.3 & 15700 & 11200 & 895 \\
\hline 0.5 & 20 & 11.8 & 8.4 & 0.7 \\
\hline 0.5 & 0.3 & 783 & 560 & 44.8 \\
\hline
\end{tabular}


at Brown University, the detector has already achieved a sensitivity to ${ }^{210} \mathrm{Po}$ at the level of $1 \mathrm{mBq} / \mathrm{m}^{2}$, easily meeting the requirements for the materials contributing to the $(\alpha, n)$ backgrounds. Based on the experience of operating this instrument at other experiments, we expect to ultimately achieve a sensitivity below $0.5 \mathrm{mBq} / \mathrm{m}^{2}$ that will match requirements for assaying the inner reflector panels of the TPC to determine an upper limit on ion recoil mis-reconstruction backgrounds. The second detector has been developed by the LZ group at SDSM\&T, deploying a panel of large-area Si detectors installed in a large vacuum chamber. This detector allows assays of bulky detector components as well as planar witness plates and coupons. With a single Si detector, a sensitivity to ${ }^{210}$ Po surface activity at the level of a few $\mathrm{mBq} / \mathrm{m}^{2}$ has already been achieved, meeting requirements for the materials generating $(\alpha, n)$ backgrounds. The full system will be online for routine LZ assays by early-2017. For materials such as Teflon, which are produced in granular form before being sintered in molds, plate-out comprises an additional dimension of risk because surface contamination of the granular form becomes contamination in bulk when the granules are poured into molds. Measurements of ${ }^{210} \mathrm{~Pb} /{ }^{210} \mathrm{Po}$ in bulk PTFE to the required sensitivity of $10 \mathrm{mBq} / \mathrm{kg}$ will be performed using the Lumpsey detector as previously described in Section 9.4.1.2. At this activity, the ${ }^{210} \mathrm{~Pb}$ in the bulk PTFE will contribute a background of 0.1 NR counts and is included in Tab. 9.2.7.

\subsection{Liquid Xenon Contamination}

Krypton and argon present in the xenon will generate backgrounds that, as with radon emanation, cannot be self-shielded against. Here we outline the strategies for limiting the total background contribution from these elements to a relatively low level of less than $1 \mu$ dru total activity from both, equivalent to the ER background from material radioactivity.

\subsubsection{Krypton}

Krypton contamination dispersed throughout the fiducial volume can generate ER background. ${ }^{85} \mathrm{Kr}$ is a beta-emitter with a half-life of $10.8 \mathrm{y}$ and a dominant ( $99.6 \%$ branching ratio) bare beta decay mode of endpoint energy $687 \mathrm{keV}$. Its presence in the atmosphere is largely anthropogenic, resulting from nuclear fuel reprocessing and testing of nuclear weapons [39, 40]. Coupled with a long half-life and diffusion properties as a noble gas, it can become a significant contaminant in the course of the production and storage of Xe.

The isotopic abundance of ${ }^{85} \mathrm{Kr}$ in air is nominally $\sim 2 \times 10^{-11}$ [40, 41]. It is present in some air samples at levels that are $10 \%$ to $20 \%$ higher than this. The XMASS experiment found the ${ }^{85} \mathrm{Kr}$ isotopic abundance in its distilled xenon to be $(0.6 \pm 0.2) \times 10^{-11}$ [42]. For the purpose of calculating the LZ requirement we use an abundance of $2 \times 10^{-11}$.

The research-grade Xe used in LUX contained an average $130 \mathrm{ppb}{ }^{\text {nat }} \mathrm{Kr} / \mathrm{Xe}$ upon procurement and was reduced to $(3.5 \pm 1.0) \mathrm{ppt}$ in LUX, resulting in a measured event rate of $(0.17 \pm 0.10)$ mdru [10]. Recent measurements of the ${ }^{\text {nat }} \mathrm{Kr}$ content of the first 100,000 liters of Xe purchased for LZ from Praxair found an average concentration of $1.2 \mathrm{ppb}$.

The allowed ${ }^{\text {nat }} \mathrm{Kr}$ concentration in $\mathrm{LZ}$ is determined by requiring that ER backgrounds from ${ }^{85} \mathrm{Kr}$ contribute no more than $10 \%$ of the solar pp neutrino rate. To achieve this, the ${ }^{\text {nat }} \mathrm{Kr}$ concentration in the LXe must be less than $0.015 \mathrm{ppt}$, accounting for the ${ }^{85} \mathrm{Kr}$ isotopic abundance and the beta decay spectrum and branching ratio. This concentration will be accomplished with chromatographic separation at SLAC prior to physics operations (see Section 6.3), followed by a comprehensive program to limit the ingress of air from leaks during storage (see Section 6.2.1). Additional requirements are placed on the acceptable leak rate of the Xe handling system during operations (see Section 6.4), and on the acceptable level of $\mathrm{Kr}$ outgassing from detector components (see Chapter 9 of Ref. [43]). There is a corollary requirement of 
$0.015 \mathrm{ppt}^{\text {nat }} \mathrm{Kr}$ detection sensitivity to confirm that the removal and storage programs have been successful (see Section 6.7.)

${ }^{\text {nat }} \mathrm{Kr}$ concentration levels of $<0.2 \mathrm{ppt}$ have been demonstrated during the LUX production run by doubleprocessing a $50 \mathrm{~kg}$ LXe batch.

\subsubsection{Argon}

Trace quantities of argon are also a concern due to beta-emitting ${ }^{39} \mathrm{Ar}$, with a 269 y half-life, a $565 \mathrm{keV}$ endpoint energy, and a $100 \%$ branching fraction to the ground state. The isotopic abundance is $8 \times 10^{-16}$ [44]. This background is constrained to be less than $10 \%$ of ${ }^{85} \mathrm{Kr}$, resulting in a specification of $4.5 \times 10^{-10} \mathrm{~g} / \mathrm{g}$ or $2.6 \mu \mathrm{Bq}$. The $\mathrm{Kr}$ removal system, which also removes Ar, should easily achieve this goal.

${ }^{37} \mathrm{Ar}$ is another isotope of concern. It decays via electron capture, producing bare $\mathrm{x}$-rays and Auger electrons between 2 and $3 \mathrm{keV}$ with a $90 \%$ branching ratio. Its half-life is 35 days, similar to ${ }^{127} \mathrm{Xe}$, but since it is not cosmogenically produced while the Xe is in storage, it is not expected to be present in Xe stockpile at the start of physics operations. Its concentration in air is typically $1.2 \mathrm{mBq} / \mathrm{m}^{3}$ [45], and at this level it is of secondary importance to the leak rate tolerance of the experiment compared to ${ }^{85} \mathrm{Kr}$ (see Section 6.4). However its local concentration in air may be elevated depending on soil conditions, being produced via neutron capture on calcium. We are pursuing a measurement of the ${ }^{37}$ Ar activity of the Davis campus air in collaboration with the Pacific Northwest National Laboratory to confirm that it is not a driving factor for the leak rate tolerance of the experiment.

\subsection{Laboratory and Cosmogenic Backgrounds}

Mitigation of external radioactivity arising from the laboratory environment or from cosmic-ray muons is largely derived from the LZ water shielding and OD. Use of the LXe skin and fiducial volume definition in the TPC for single scatter events also reduces this background, as well as radioactivity arising from cosmogenic activation of materials in the LZ project during manufacture, transport, or storage. Cosmogenic activation of the Xe target also produces radioisotopes but these are intrinsic to the LXe target and cannot be shielded against. While such cosmogenic production of radioisotopes underground in LXe may be neglected due to the low cosmic-ray flux, it is surface activation during production and storage that will generate background in LZ. These backgrounds are shown in Table 9.8.1, and are discussed in the following subsections.

Table 9.8.1: Events expected from different external or activation sources in 1,000 d live exposure of 5.6 tonne fiducial mass in LZ. Rates are given before expected $99.5 \%$ discrimination for ER events or $50 \%$ efficiency for NRs, with the exception of the last row where the expected count rate in the WIMP search region is quoted.

\begin{tabular}{|l|l|c|l|}
\hline Source & Recoils & Number of events & Systematic uncertainty \\
\hline Walls & ER & $4.1 \pm 0.8$ & Factor of 2 \\
Walls & NR & $<0.001$ & Included in the limit \\
Muons & NR & $<0.056$ & $30 \%$ \\
${ }^{127}$ Xe & ER & 0.11 & Factor of 5 \\
${ }^{46} \mathrm{Sc}$ & ER & $<0.1$ & Included in the limit \\
\hline Total & $(99.5 \%$ discr., 50\% NR) & $0.01-0.09$ & Included in the range \\
\hline
\end{tabular}




\subsubsection{Laboratory Backgrounds}

The background in the underground SURF facility outside the water tank is dominated by $\gamma$-rays and neutrons produced in the cavern walls. The sources of these $\gamma$-rays and neutrons are the uranium and thorium decay chains with a contribution of ${ }^{40} \mathrm{~K}$ decay to high-energy $\gamma$-rays. Many samples of rock have been screened to get $U$ and Th contamination varying from $0.1 \mathrm{ppm}$ to $7.5 \mathrm{ppm} \mathrm{U}$, from $1.9 \mathrm{ppm}$ to $47 \mathrm{ppm}$ Th and from $0.04 \%$ to $3.3 \%$ of natural potassium, depending on a specific sample and rock formation $[46,47]$. At the 4850 level, the $\gamma$-ray flux has been measured as $(2.16 \pm 0.06) / \mathrm{cm}^{2} / \mathrm{s}$ above $0.1 \mathrm{MeV}$ and $(0.632 \pm 0.019) / \mathrm{cm}^{2} / \mathrm{s}$ above $1 \mathrm{MeV}$ [48]. This agrees well with the simulations of $\gamma$-ray flux based on the average values of contamination in the Homestake formation: $1.51 \mathrm{ppm} \mathrm{U}, 7.38 \mathrm{ppm}$ Th and $0.96 \%$ of natural potassium [48]. Some other sources indicate a lower contamination of U/Th in rock around the lab (down to $0.22 \mathrm{ppm} \mathrm{U}$ and $0.33 \mathrm{ppm} \mathrm{Th}$ ) but the shotcrete and concrete on the walls may give the radioactivity levels an order of magnitude higher and similar to (or even higher than) those quoted above for the Homestake formation (see for instance [49] and references therein). Recently the flux and spectrum of $\gamma$ rays in the laboratory hall (East Counting Room) have been measured with a bare HPGe detector [50]. The intensities of $\gamma$-ray lines from $\mathrm{U}$, Th and $\mathrm{K}$ have been found to be higher than previously reported, giving the integrated flux of $2.19 / \mathrm{cm}^{2} / \mathrm{s}$ for energies $>1 \mathrm{MeV}$ with a large uncertainty (about a factor of 2 towards lower flux values) due to the fact that the flux in the continuum comprises Compton scattering events in the detector itself, with no on-site calibration of the detector. Recent measurements of a sample of gravel from beneath the LZ water tank [51] have revealed concentrations of $1.65 \mathrm{ppm} \mathrm{U,} 0.30 \mathrm{ppm}$ Th and $0.066 \%$ of natural potassium, significantly smaller than previous reports. In our estimates we have used the results from [50] as a conservative estimate.

The $\gamma$-ray and neutron fluxes from rock have been shown to be attenuated by many orders of magnitude by the water shielding and then by the LUX background rejection strategy so they do not contribute to the measured number of events in LUX. LZ, however, is expected to achieve $100 \times$ better sensitivity to WIMPs and the thickness of shielding is slightly reduced compared to LUX due to a larger cryostat. We have simulated $\gamma$-ray production in $\mathrm{U}$ and Th decay chains and from ${ }^{40} \mathrm{~K}$, and transport of these $\gamma$-rays from lab walls through the shielding down the LXe detector in several steps and applied the same event selection algorithms as for other background sources. The decay chain of Th should give the highest contribution because of the presence of the most energetic $2.62 \mathrm{MeV} \gamma$-ray line in the Th decay chain. The simulation results have been normalized to high-energy line intensities as measured in [50] which suggested the concentrations of $6.42 \mathrm{ppm}(26.1 \mathrm{~Bq} / \mathrm{kg}) \mathrm{Th}, 5.95 \mathrm{ppm} \mathrm{U}(73.4 \mathrm{~Bq} / \mathrm{kg})$ and $2.31 \%$ of natural $\mathrm{K}\left(716 \mathrm{~Bq} / \mathrm{kg}\right.$ of $\left.{ }^{40} \mathrm{~K}\right)$. Our simulations have shown that the number of single ER events in 5.6 tonne of fiducial volume and a live-time of $1,000 \mathrm{~d}$ after all cuts is $4.1 \pm 0.8$ dominated by the $2.6 \mathrm{MeV}$ gammas from Th decay chain. The errors are purely statistical and have been calculated from the number of observed events in simulations. The systematic uncertainty is about a factor of 2. Assuming discrimination power of $99.5 \%$ the limit on the number of events in the NR band will be $<0.05$ events, much less than the background from internal sources and neutrinos. Neutrons from the laboratory walls are attenuated efficiently by water and scintillator that will surround the LZ cryostat with a minimum thickness of hydrogenous shielding to be $70 \mathrm{~cm}$ and a reduction of the neutron flux by more than 6 orders of magnitude. We have carried out simulations of neutron transport through rock and shielding beneath the detector where one of the conduits is located and have shown that no neutrons are likely to pass through this bottom conduit to the LXe target. Other conduits are smaller in diameter and longer which, together with a big thickness of water and scintillator make other sides of the detector better protected against neutrons from rock. This will bring the event rate from rock neutrons to less than 0.001 events in 5.6 tonne fiducial mass for a live time of $1,000 \mathrm{~d}$, well below the contribution from detector components.

Muon-induced neutron production and impact in LZ has been assessed using full Monte Carlo. Muon simulations for $\mathrm{LZ}$ were carried out using accurate surface profile for the Davis campus at the 4850 level at 
SURF. Initially, muons with different energies at the surface were transported through various thicknesses of rock using MUSIC $[52,53]$. The resulting spectra of surviving muons have been convolved with muon spectra at the surface for different zenith angles and slant depths. Underground muons have been sampled on the surface of a box which contained the cavern and $5 \mathrm{~m}$ to $7 \mathrm{~m}$ of rock around the cavern using the MUSUN code [52] and transported through the rock and the detector using LZSim. All particles have been tracked and their interactions in LXe and OD recorded. Selection of surviving events has been based on energy deposition, hit multiplicity, fiducial volume and the anti-coincidence with LXe skin and the Outer Detector; identical to all other event selection criteria in LZSim background simulations. No candidate events has survived the cuts following $118.5 \mathrm{y}$ of equivalent live time. The rejection efficiency of muoninduced neutron background benefits from the large multiplicity of particles produced in cascades initiated by muons. The absence of observed events after cuts allows us to set a $90 \%$ CL upper limit on the muoninduced background rate in LZ of $0.056 \mathrm{NR}$ events for a live-time of 1,000 d, reduced to 0.023 events after applying the $50 \%$ NR efficiency.

\subsubsection{Cosmogenic Activation}

The irradiation of xenon by cosmic rays at the surface produces a number of radioactive isotopes including those of Xe, tritium, ${ }^{134} \mathrm{Cs},{ }^{125} \mathrm{I},{ }^{121 \mathrm{~m}} \mathrm{Te},{ }^{123 \mathrm{~m}} \mathrm{Te}$. These cannot be shielded against but non-xenon isotopes will be efficiently removed from the xenon during purification. Radioisotopes of Xe that can be produced through cosmogenic or neutron activation are ${ }^{127} \mathrm{Xe}\left(\mathrm{T}_{1 / 2}=36.4 \mathrm{~d}\right),{ }^{129 \mathrm{~m}} \mathrm{Xe}\left(\mathrm{T}_{1 / 2}=8.9 \mathrm{~d}\right),{ }^{131 \mathrm{~m}} \mathrm{Xe}\left(\mathrm{T}_{1 / 2}\right.$ $=11.9 \mathrm{~d})$, and ${ }^{133} \mathrm{Xe}\left(\mathrm{T}_{1 / 2}=5.3 \mathrm{~d}\right)$. Most of these will decay quickly and will not pose any threat after a few months of commissioning and calibration runs. Decaying xenon isotopes, in particular ${ }^{131 \mathrm{~m}} \mathrm{Xe}$, and ${ }^{129 \mathrm{~m}} \mathrm{Xe}$, will also serve as important calibration sources in these early runs. The exception will be ${ }^{127} \mathrm{Xe}$, with a half-life of $36.4 \mathrm{~d}$. LUX measurements [10] showed a decay rate of $(2.7 \pm 0.5) \mathrm{mBq} / \mathrm{kg}$ of ${ }^{127} \mathrm{Xe}$ after xenon was exposed to cosmic rays at the Earth's surface. This can be a factor of 3-4 higher if xenon is stored on the surface at SURF (at about 1,600 m elevation).

${ }^{127} \mathrm{Xe}$ produces energy depositions within the WIMP search region of interest and also poses a background for axion searches. ${ }^{127} \mathrm{Xe}$ undergoes electron capture that results in an orbital vacancy that is filled by electron transitions from higher orbitals, resulting in an X-ray or Auger electron cascade. An $85 \%$ probability for the capture electron coming from the $\mathrm{K}$ shell results in a cascade with a total energy of $33 \mathrm{keV}$. A further $12 \%$ of captures from the $\mathrm{L}$ shell generate cascades of $5.2 \mathrm{keV}$ total energy deposition, and the remaining $3 \%$ of decays come from higher shells ( $\mathrm{M}$ and $\mathrm{N}$ ) to deposit up to $1.2 \mathrm{keV}$. For a WIMP search energy window of $1.5 \mathrm{keV}_{\text {ee }}$ to $6.5 \mathrm{keV}_{\text {ee }}$, significant numbers of $\mathrm{L}, \mathrm{M}$, and $\mathrm{N}$ shell decays will generate background. However, the daughter ${ }^{127} \mathrm{I}$ nucleus is left in either a $619 \mathrm{keV}, 375 \mathrm{keV}$, or $203 \mathrm{keV}$ excited state (with no direct population of the ground state as part of the electron capture). The subsequent decay of the ${ }^{127} \mathrm{I}$ to the ground state emits internal conversion electrons or $\gamma$-rays that permit almost all of the ${ }^{127} \mathrm{Xe}$ background to be rejected by coincidence tagging — only those X-ray/Auger events for which the associated $\gamma$-rays are not detected contribute to the low-energy ER background in the Xe active region. This effect is expected predominantly at the edge of the Xe target. For example, with a mean free path of $2.6 \mathrm{~cm}$ in LXe, the $375 \mathrm{keV} \gamma$-ray can potentially escape the active region, reducing the efficiency of coincidence rejection further for events at the edges of the LXe, as seen in LUX [10]. LUX data showed that, after a cooling down period, $0.115 \mathrm{mBq} / \mathrm{kg}$ of ${ }^{127} \mathrm{Xe}$ produced a background single hit rate of $0.5 \times 10^{-3}$ events $/ \mathrm{keV} / \mathrm{kg} / \mathrm{d}$ in the ROI. In LZ, the skin and external veto systems significantly aid rejection and characterization of this background. Our simulations with LZSim show that the initial ER background in LZ after cuts at $1.5 \mathrm{keV}_{\text {ee }}$ to $6.5 \mathrm{keV}_{\mathrm{ee}}$ is 0.22 events per day (assuming no exposure to cosmic rays at $1,600 \mathrm{~m}$ elevation). After 8 months of cooling down time underground this rate drops by two orders of magnitude to 0.0022 events/day 
or about 0.11 events for a live time of $1,000 \mathrm{~d}$ (before discrimination and taking into account the continuous decay of the isotope). The early storage of xenon underground mitigates this background further.

The most dangerous isotope due to activation of titanium, used to manufacture the cryostat and many internal detector components, is ${ }^{46} \mathrm{Sc}\left(\mathrm{T}_{1 / 2}=83.8 \mathrm{~d}\right)$. Using GEANT4 and ACTIVIA [54] codes, we have determined that after 6 months of activation of titanium at sea level, the decay rate of ${ }^{46} \mathrm{Sc}$ will be $2.4 \mathrm{mBq} / \mathrm{kg}$. This is consistent with measurements from LUX [10]. Assembling the TPC within the inner vessel at the surface at SURF will increase this rate by a factor of 2 . The beta-decay of ${ }^{46} \mathrm{Sc}$ is almost always followed by the emission of two $\gamma$-rays, of energies $1,120 \mathrm{keV}$ and $889 \mathrm{keV}$. We expect that, after 8 months of cooling, activated ${ }^{46} \mathrm{Sc}$ will contribute less than 0.1 event for a live-time of $1,000 \mathrm{~d}$ of $\mathrm{LZ}$ operation before $99.5 \%$ discrimination, rendering this background negligible. Effects from activation of other materials are small compared to possible background rates from activated xenon and titanium due to either smaller masses of materials in the vicinity of the LXe target (copper, stainless steel) or absence of long-lived 'dangerous' radioactive isotopes (PTFE). Activation of various materials underground has also been evaluated using GEANT4 and found to be negligible. 


\subsection{Bibliography}

[1] D. S. Leonard et al., Nucl. Instrum. Meth. A591, 490 (2008), arXiv:0709.4524 [physics.ins-det].

[2] D. S. Akerib et al. (LUX), Nucl. Instrum. Meth. A704, 111 (2013), arXiv:1211.3788 [physics.ins-det].

[3] H. M. Araújo et al. (ZEPLIN-III), Astropart. Phys. 35, 495 (2012), arXiv:1104.3538 [physics.ins-det].

[4] E. Aprile et al. (XENON100), Phys. Rev. D83, 082001 (2011), [Erratum: Phys. Rev. D85, 029904 (2012)], arXiv:1101.3866 [astro-ph].

[5] E. Aprile et al. (XENON100), J. Phys. G40, 115201 (2013), arXiv:1306.2303 [astro-ph].

[6] C. Ghag et al. (ZEPLIN-III), Astropart. Phys. 35, 76 (2011), arXiv:1103.0393 [astro-ph].

[7] N. Abgrall et al. (Majorana), Adv. High Energy Phys. 2014, 365432 (2014), arXiv:1308.1633 [physics.ins-det].

[8] A. Gando et al. (KamLAND-Zen), Phys. Rev. C85, 045504 (2012), arXiv:1201.4664 [hep-ex].

[9] F. P. An et al. (Daya Bay), Phys. Rev. Lett. 112, 061801 (2014), arXiv:1310.6732 [hep-ex].

[10] D. S. Akerib et al. (LUX), Astropart. Phys. 62, 33 (2015), arXiv:1403.1299 [astro-ph].

[11] S. Agostinelli et al. (GEANT4), Nucl. Instrum. Meth. A506, 250 (2003).

[12] D. S. Akerib et al. (LUX), Nucl. Instrum. Meth. A675, 63 (2012), arXiv:1111.2074 [physics.data-an].

[13] M. Szydagis, N. Barry, K. Kazkaz, J. Mock, D. Stolp, M. Sweany, M. Tripathi, S. Uvarov, N. Walsh, and M. Woods (NEST), J. Instrum. 6, P10002 (2011), arXiv:1106.1613 [physics.ins-det].

[14] E. Aprile et al. (XENON100), Astropart. Phys. 35, 43 (2011), arXiv:1103.5831 [physics.ins-det].

[15] E. Aprile et al. (XENON1T), Eur. Phys. J. C75, 546 (2015), arXiv:1503.07698 [astro-ph.IM].

[16] J. Boger et al. (SNO), Nucl. Instrum. Meth. A449, 172 (2000), arXiv:nucl-ex/9910016 [nucl-ex].

[17] D. S. Akerib et al. (LUX), Nucl. Instrum. Meth. A703, 1 (2013), arXiv:1205.2272 [physics.ins-det].

[18] A. Gando et al. (KamLAND-Zen), Phys. Rev. Lett. 117, 082503 (2016), [Addendum: Phys. Rev. Lett.117,no.10,109903(2016)], arXiv:1605.02889 [hep-ex].

[19] G. Alimonti et al. (Borexino), Nucl. Instrum. Meth. A600, 568 (2009), arXiv:0806.2400 [physics.insdet].

[20] D. C. Malling, S. Fiorucci, M. Pangilinan, J. J. Chapman, C. H. Faham, J. R. Verbus, and R. J. Gaitskell, "Dark Matter Search Backgrounds from Primordial Radionuclide Chain Disequilibrium," (2013), (unpublished), arXiv:1305.5183 [astro-ph].

[21] C. Ghag, Proceedings, 5th Topical Workshop on Low Radioactivity Techniques (LRT 2015): Seattle, WA, USA, March 18-20, 2015, AIP Conf. Proc. 1672, 020003 (2015).

[22] D. Yu. Akimov et al. (ZEPLIN-III), Astropart. Phys. 34, 151 (2010), arXiv:1004.4207 [hep-ex].

[23] B. D. LaFerriere, T. C. Maiti, I. J. Arnquist, and E. W. Hoppe, Nucl. Instrum. Meth. A775, 93 (2015). 
[24] P. Grinberg, S. Willie, and R. Sturgeon, Anal. Chem. 77, 2432-2436 (2005).

[25] D. S. Leonard, J. Korean Phys. Soc. 64, 1878 (2014).

[26] The MIT Nuclear Reactor Laboratory, (2015), 138 Albany Street, Cambridge, MA 02139-4201.

[27] J. Mott, in Low Radioactivity Techniques 2013 (LRT 2013): Proceedings of the IV International Workshop in Low Radioactivity Techniques, AIP Conf. Proc., Vol. 1549, edited by L. Miramonti and L. Pandola (2013) pp. 152-155, Low-background tracker development for the SuperNEMO experiment, Conference Presentation.

[28] H. Richter, H. Simgen, and G. Zuzel (GERDA), “222 Rn Measurements,” (2008), Presented at GERDA Collaboration Meeting: Crakow, Poland, USA, Feb. 18-20, 2008.

[29] S. Lindemann, Intrinsic ${ }^{85} \mathrm{Kr}$ and ${ }^{222} \mathrm{Rn}$ Backgrounds in the XENON Dark Matter Search, Ph.D. thesis, University of Heidelberg (2013).

[30] D. Tiedt, Radioactive Background Simulation and Cleanliness Standards Analysis for the Long Baseline Neutrino Experiment Located located at the Sanford Underground Research Facility, Master's thesis, South Dakota School of Mines and Technology (2013).

[31] E. D. Hallman and R. G. Stokstad, Establishing a Cleanliness Program and Specifications for the Sudbury Neutrino Observatory, Tech. Rep. SNO-STR-91-009 (Sudbury Neutrino Observatory, Department of Physics, Stirling Hall, Queen's University at Kingston, Kingston, Ontario, Canada K7L 3N6, 1991).

[32] D. Parasuraman, A. Kemps, H. Veeke, and G. Lodewijks, J. IEST 55, 1 (2012).

[33] R. R. Schumann and L. C. Gundersen, Environ. Int. 22, 439 (1996).

[34] H. W. Lee, Radon in the Acrylic Vessel, Tech. Rep. SNO-STR-94-028 (Sudbury Neutrino Observatory, Department of Physics, Stirling Hall, Queen's University at Kingston, Kingston, Ontario, Canada K7L 3N6, 1994).

[35] R. Heaton, H. Lee, P. Skensved, and B. C. Robertson, Nucl. Instrum. Meth. A276, 529 (1989).

[36] V. E. Guiseppe, S. R. Elliott, A. Hime, K. Rielage, and S. Westerdale, in Topical Workshop on Low Radioactivity Techniques: LRT-2010, AIP Conf. Proc., Vol. 1338, edited by R. Ford (2011) pp. 95-100, A Radon Progeny Deposition Model, Conference Presentation, arXiv:1101.0126 [nucl-ex].

[37] C. Arpesella et al. (Borexino), Astropart. Phys. 18, 1 (2002), arXiv:hep-ex/0109031 [hep-ex].

[38] T. Shutt, (2016), Private Communication.

[39] J. Ahlswede, S. Hebel, J. O. Ross, R. Schoetter, and M. B. Kalinowski, J. Environ. Radioact. 115, 34 (2013).

[40] P. Collon, W. Kutschera, and Z.-T. Lu, Ann. Rev. Nucl. Part. Sci. 54, 39 (2004), arXiv:nucl-ex/0402013 [nucl-ex].

[41] X. Du, R. Purtschert, K. Bailey, B. E. Lehmann, R. Lorenzo, Z.-T. Lu, P. Mueller, T. P. O’Connor, N. C. Sturchio, and L. Young, Geophys. Res. Lett. 30, 2068 (2003).

[42] K. Abe et al. (XMASS), Nucl. Instrum. Meth. A716, 78 (2013), arXiv:1301.2815 [physics.ins-det]. 
[43] D. S. Akerib et al. (LZ), (2015), Conceptual Design Report; LBNL-190005, FERMILAB-TM-2621AE-E-PPD, arXiv:1509.02910 [physics.ins-det].

[44] H. H. Loosli, B. E. Lehmann, and W. M. Smethie Jr., in Environmental Tracers in Subsurface Hydrology, edited by P. G. Cook and A. L. Herczeg (Springer US, 2000) pp. 379-396, Noble Gas Radioisotopes: ${ }^{37} \mathrm{Ar},{ }^{85} \mathrm{Kr},{ }^{39} \mathrm{Ar},{ }^{81} \mathrm{Kr}$, ISBN 978-1-4613-7057-4.

[45] R. A. Riedmann and R. Purtschert, Environ. Sci. Technol. 45, 8656 (2011).

[46] K. T. Lesko et al., (2011), DUSEL Preliminary Design Report, p. 3-138, arXiv:1108.0959 [hep-ex].

[47] W. Roggenthen and A. R. Smith, "Uranium, Thorium, Potassium Contents of Materials in the Homestake Underground, Lead SD,” (2008), AGU Fall Meeting, San Francisco, CA.

[48] D.-M. Mei, C. Zhang, K. Thomas, and F. Gray, Astropart. Phys. 34, 33 (2010), arXiv:0912.0211 [nucl-ex].

[49] K. T. Lesko, Proceedings, 13th International Conference on Topics in Astroparticle and Underground Physics (TAUP 2013): Asilomar, California, September 8-13, 2013, Phys. Procedia 61, 542 (2015).

[50] K. J. Thomas (LZ), An Estimate of the Gamma Flux in the East Counting Room of the Davis Cavern, Tech. Rep. (Lawrence Berkeley National Laboratory (LBNL), 1 Cyclotron Road, Berkeley, CA 947208099, USA, 2014).

[51] D. Harvey, K. Thomas, and A. R. Smith (LZ), LUX Water Tank Gravel, Tech. Rep. (Lawrence Berkeley National Laboratory (LBNL), 1 Cyclotron Road, Berkeley, CA 94720-8099, USA, 2016).

[52] V. A. Kudryavtsev, Comput. Phys. Commun. 180, 339 (2009), (MUSIC), arXiv:0810.4635 [physics.comp-ph].

[53] P. Antonioli, C. Ghetti, E. V. Korolkova, V. A. Kudryavtsev, and G. Sartorelli, Astropart. Phys. 7, 357 (1997), (MUSIC), arXiv:hep-ph/9705408 [hep-ph].

[54] J. J. Back and Y. A. Ramachers, Nucl. Instrum. Meth. A586, 286 (2008), (ACTIVIA), arXiv:0709.3472 [nucl-ex]. 



\section{SURF Infrastructure, Assembly, and Integration}

This section describes the surface and underground infrastructure improvements and additions needed at SURF in order to facilitate LZ assembly and installation. A detailed assembly and installation sequence for LZ is also presented here. Infrastructure at SURF includes the following: (1) surface laboratory space for assembly of the Xe detector (WBS 1.5) into the inner cryostat vessel (WBS 1.2); (2) staging space for scintillator veto tanks and scintillator (WBS 1.6), for the outer cryostat subcomponents (WBS 1.2), and other detector components; (3) secure storage space for Xe; (4) custom tooling for lowering the fully assembled Xe detector sealed inside the inner cryostat down the Yates shaft; and (5) modifications to the Davis Campus at the 4850L of SURF. These infrastructure elements are described below. Surface and infrastructure improvements are being funded primarily by the South Dakota Science and Technology Authority (SDSTA). The design of the infrastructure improvements has been a joint effort between the SDSTA engineering and technical staff, an external contractor and the LZ collaboration. The firm Leo A. Daly [1] is under contract to SDSTA for the detailed design of the surface and underground infrastructure improvements. The final design is complete.

\subsection{Surface Infrastructure}

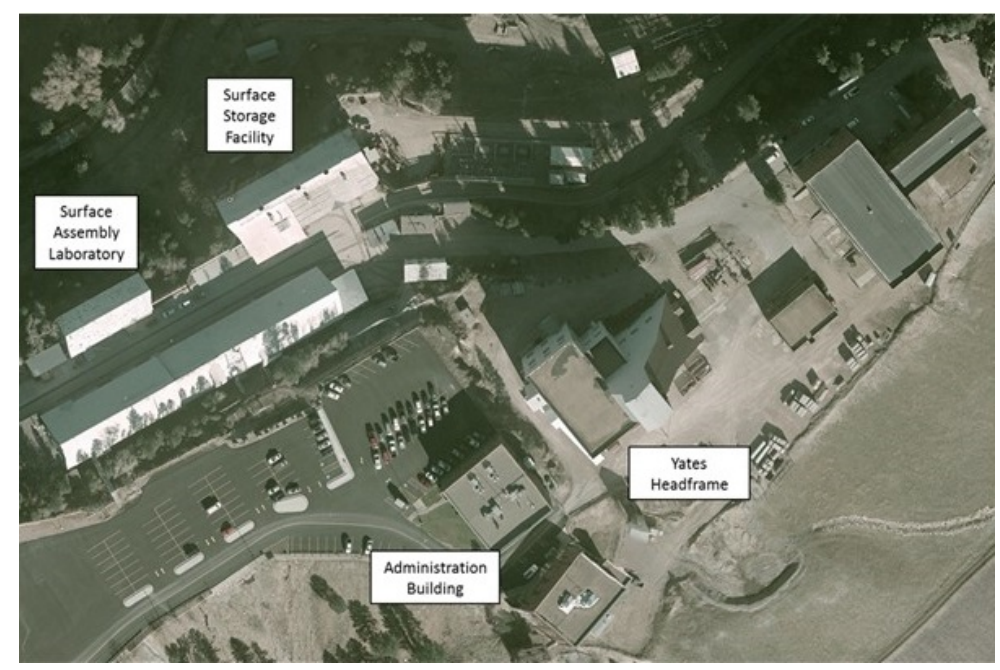

Figure 10.1.1: Aerial view of the SURF site showing the locations of the Surface Assembly Laboratory and the Surface Storage Facility.

The principal components of the surface infrastructure specific to LZ are: (a) the Surface Assembly Laboratory (SAL), (b) the Surface Storage Facility (SSF), and (c) office and meeting space at the SDSTA administration and education buildings. Office and meeting space already exists for ongoing experiments at SDSTA; general improvements to these capabilities will serve the broader experimental community at 
SURF, and are not described here. The locations of the SAL and the SSF at SURF are shown in Figure 10.1.1

The SAL was utilized for the assembly of the LUX detector and for operation of LUX in a water tank located in the SAL. Significant upgrades to the SAL are planned for assembly of the LZ detector and are described later. The most significant improvement to the SAL is the addition of radon-reduced airhandling capability. A new building to house a system that provides reduced-radon air to the SAL will be constructed between the SAL and the SSF. The SSF is currently used for general storage and modest interior improvements were made in order to be used for storage and disposition of components during LUX decommissioning, which began in September 2016. These improvements will also provide staging space for LZ components.

\subsubsection{Surface Assembly Laboratory (SAL)}

The SAL building is a wood-frame structure with four levels: the surface level and levels $-1,-2$, and -3 , which are successively deeper below the surface. This building was renovated for LUX assembly and testing and has a sprinkler system, HVAC, and $220 \mathrm{kVA}$ installed electrical capacity. An existing cleanroom of $2,900 \mathrm{ft}^{3}$ will be used for cleaning and staging metal parts and bagged PTFE parts prior to Xe detector assembly in the low-radon cleanroom, and for other assembly tasks not requiring a low-radon environment. A new low-radon cleanroom will provide workspace beneath an existing monorail and over a pit in Level -1 . The new cleanroom will be connected, but separated by doors, from the existing cleanroom and will have a separate air-handling system. Transport of the detector will be facilitated by rolling it to the building shipping dock. The layout of the SAL is shown in Figure 10.1.2. The design of the new cleanroom is based in part on the design of a low-radon cleanroom in place at SDSM\&T [2]. The design goal for this new cleanroom is $<1 \mathrm{~Bq} / \mathrm{m}^{3}$ (unoccupied). The cleanroom is under fabrication by a commercial vendor to specifications set by SDSTA and the LZ Project.

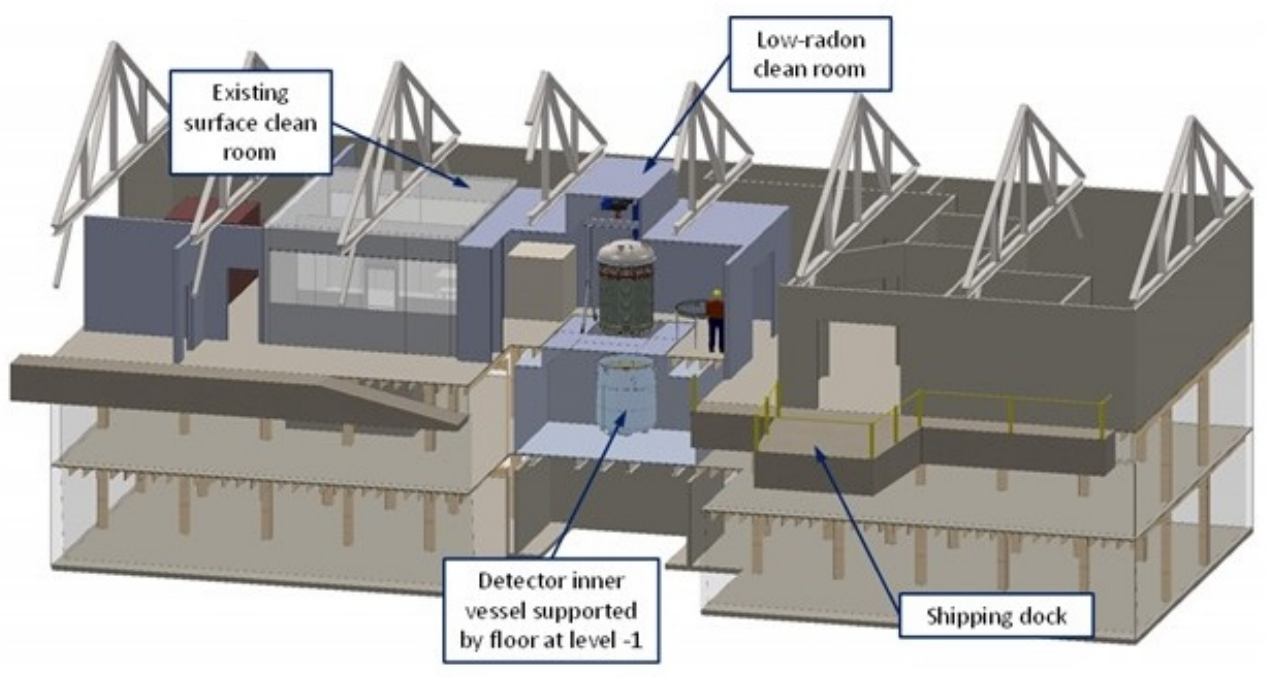

Figure 10.1.2: The Surface Assembly Laboratory.

\subsubsection{Radon-reduced Air System}

Radon is one the highest-risk contaminants for low-background experiments because it can easily escape from bulk material and quickly diffuse into active parts of the detectors. The innermost materials and parts 
of ultralow-background experiments must be manufactured or assembled in a radon-suppressed atmosphere. Final assembly of the LZ time projection chamber (TPC) will occur in a cleanroom in the SAL, which has a radon-reduced air system.

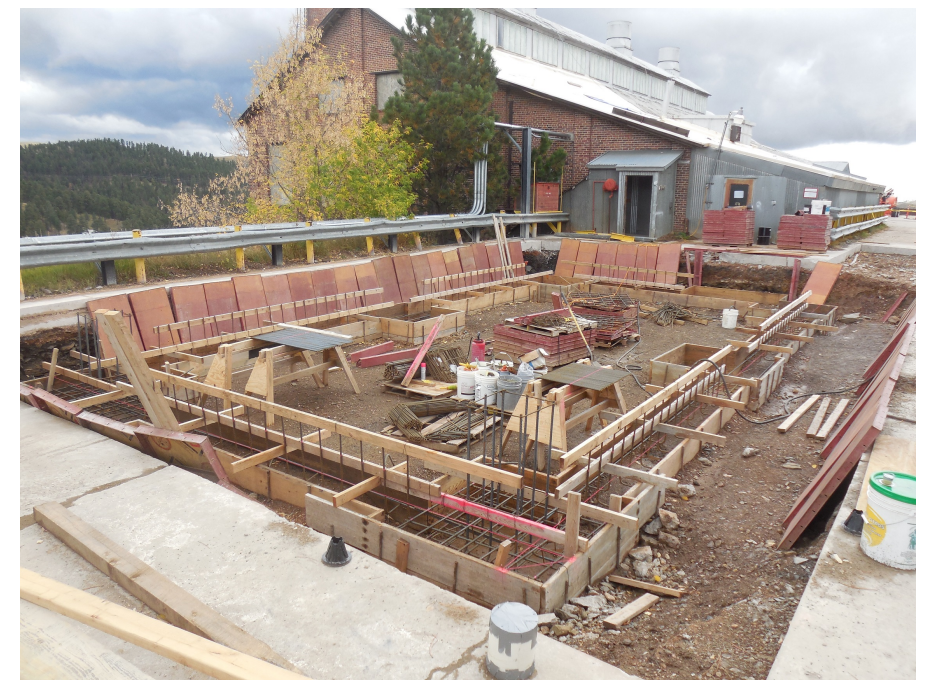

Figure 10.1.3: A picture (October 2016) of the additional building being constructed to house the radon-removal system (RRS).

Carbon adsorption is the preferred technique for removing radon from air and has been effective for varying degrees of radon contamination, from as low as a few $\mathrm{mBq} / \mathrm{m}^{3}$ for low- background experiments to levels as high as $\sim 100 \mathrm{~Bq} / \mathrm{m}^{3}$. The surface air radon level at SURF is expected to be in the range of $\sim 20 \mathrm{~Bq} / \mathrm{m}^{3}$. The LZ detector assembly cleanroom will be designed with a radon-reduction factor of greater than 1,000. It will be flushed with radon-reduced air at a flowrate of about $250 \mathrm{~m}^{3} / \mathrm{h}$, which is produced by compressing, drying, cooling, and pushing the air through two $1,600 \mathrm{~kg}$ activated-

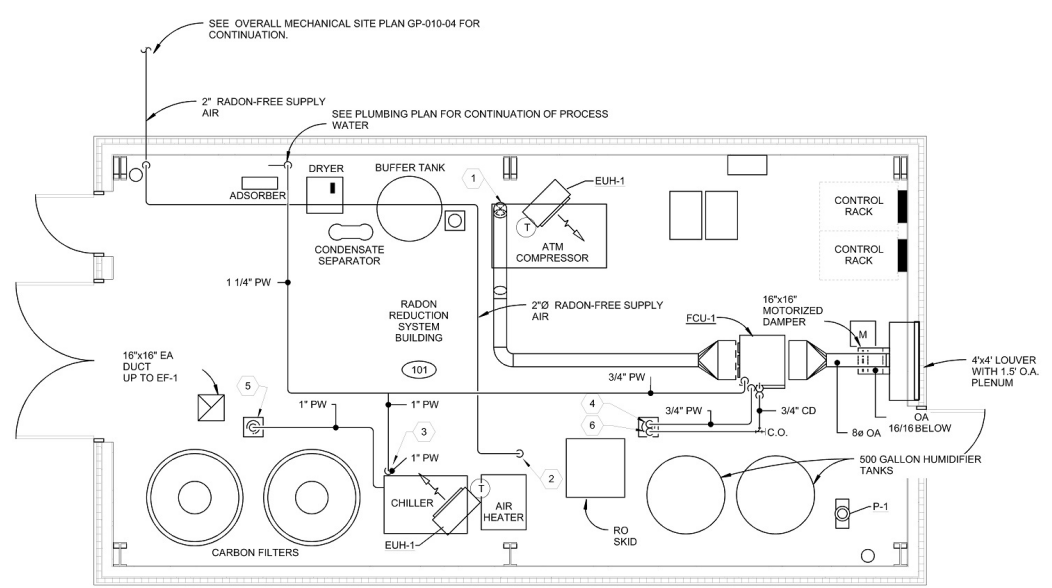
carbon towers. Our plan is to use

Figure 10.1.4: Plan view of RRS Building. a commercial unit provided by ATEKO, which has developed and built large commercial radon-removal systems for other sensitive underground experiments. These units are capable of reducing radon concentration in the air by a factor of greater than 1,000. The fabrication of the LZ unit has begun for delivery in Spring 2017.

The process is based on compression, cleaning, and drying (dew point $-70{ }^{\circ} \mathrm{C} \mathrm{min}$.) of air, cooling to $-55^{\circ} \mathrm{C}$, adsorption of radon from air on activated carbon at $-50^{\circ} \mathrm{C}$ at approximately 8 bars of pressure, 
followed by heating and pressure reduction of air to ambient pressure and temperature. A new building is being constructed to specifically house this system, and will be located between the SAL and SSF on an existing concrete pad illustrated in Figure 10.1.3.

A plan view of the building, showing the location of key components, is illustrated in Figure 10.1.4.

\subsubsection{Surface Storage Facility (SSF)}

Short-term storage and staging of equipment needed for experiment assembly - including the large scintillator tanks - will be facilitated by upgrades to an existing building next to the SAL. The SSF has a large high bay with approximately $445 \mathrm{~m}^{2}\left(4,800 \mathrm{ft}^{2}\right)$ of lay-down space under a 40 -ft-wide 10 -ton bridge crane. Hook height on the bridge crane reaches $7.3 \mathrm{~m}(24 \mathrm{ft})$. Handling of equipment will also be facilitated by three wall-mounted jib cranes rated at 1 ton, 2 tons, and 3 tons, respectively. Truck entry to the building is via a 4-m (13-ft)-wide $\times 5$-m (16-ft)-tall entry rollup door. A structure within the SSF has been built to first house materials and equipment resulting from the removal of LUX from the Davis Cavern and decommissioning, and then LZ components. A view of this building showing scintillator barrels (about $50 \%$ of the total) and transport boxes containing the outer detector acrylic vessels is shown in Figure 10.1.5.

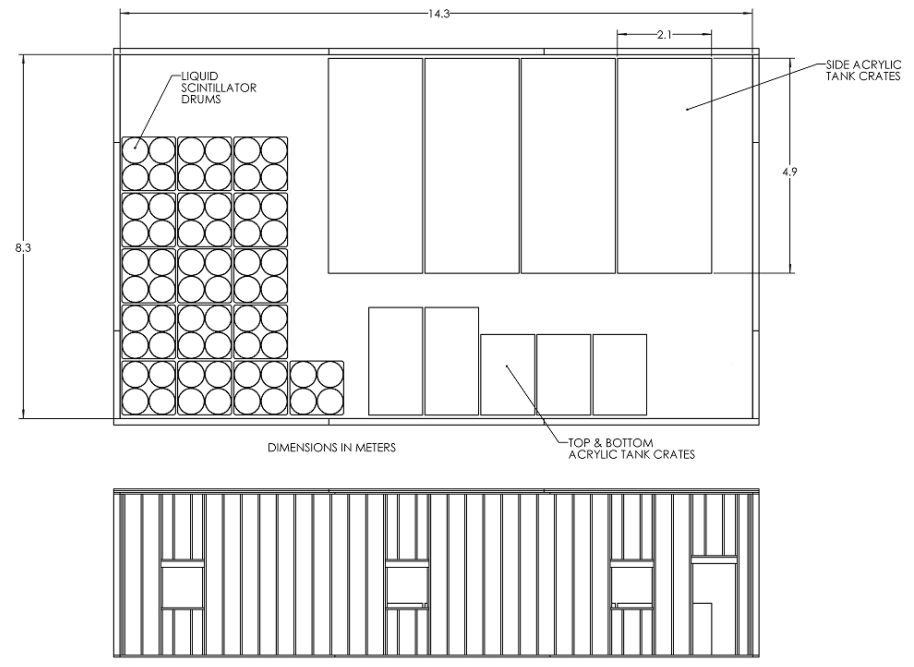

Figure 10.1.5: Building constructed for staging LZ detector components.

\subsection{Yates Shaft Infrastructure and Custom Transport}

The LZ TPC inside the inner cryostat will be transported from the SAL to the Yates headframe in a horizontal orientation. The transport method will be similar to the successful transport of LUX from the same building to the headframe. A large telehandler was used, along with continuous monitor of orientation and G-forces. LUX was transported in the Yates cage but the LZ assembly will be slung under the cage, given its larger size. The LZ detector/inner cryostat will be placed on a custom transport frame. 

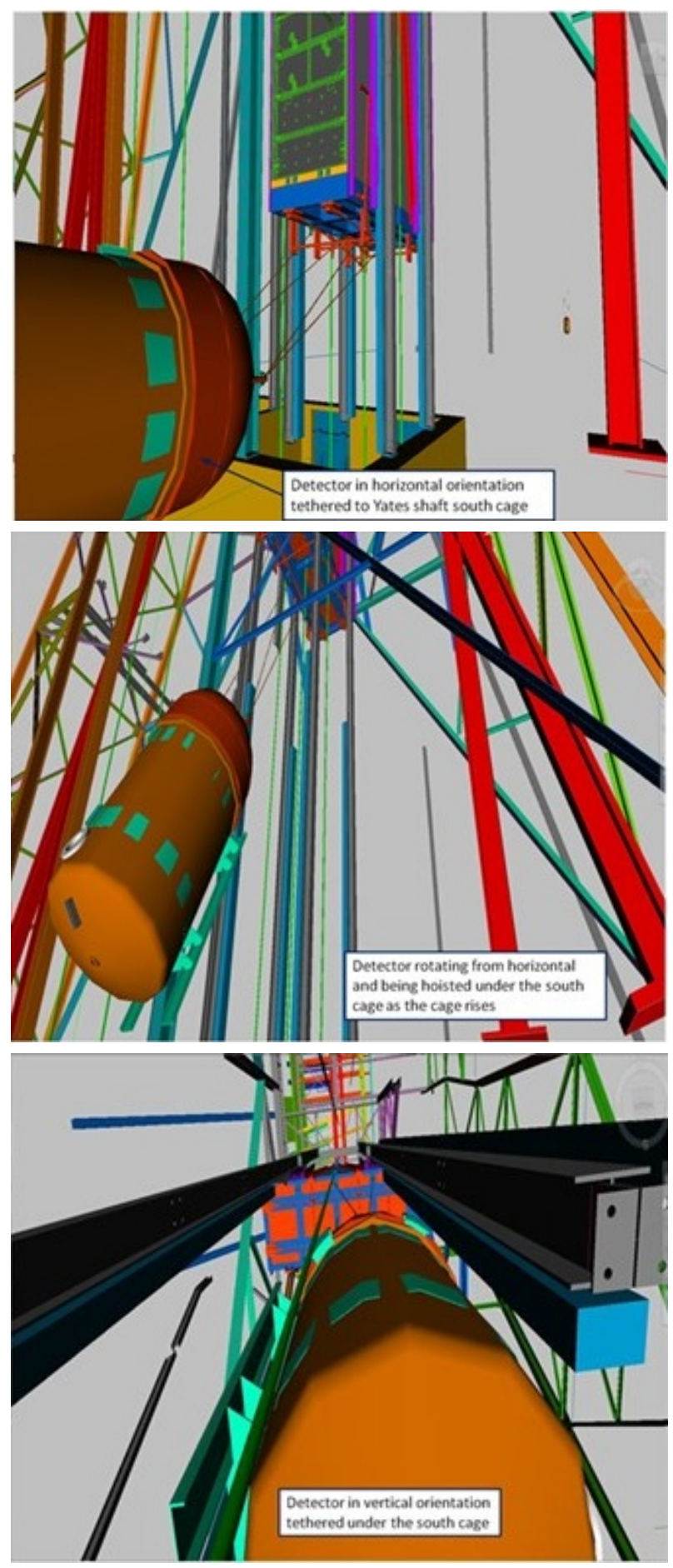

In the first image, the LZ detector lies horizontally at the shaft entrance and the cage bottom is 6 to 7 feet above the floor. Crews will cover the shaft by installing a work platform and attach the slings to the bottom of the cage. These are also attached to the top of the LZ detector. The platform will be removed and the cage hoisted to remove slack. Another wire rope control line from a winch is attached to the bottom of the detector.

In the second image, the hoist is lifting the LZ detector in the shaft to its vertical position. The control line mounted on the detector bottom will maintain tension in order to control the swing into the shaft. Even though not fully in the shaft, the bottom of the custom transport frame is off the ground. Once hoisting is in process, it will not be stopped unless a difficulty arises. The entire procedure is short-lived.

In the third image, looking almost vertically. Here, the detector is hanging vertically. It will be positioned properly in order for the crew to attach the bumpers or guide shoes for clearance and centering during shaft transit. After inspections, a crew will man the cage and watch as it travels underground, being lowered at a rate of $0.7 \mathrm{ft} / \mathrm{s}$.

Figure 10.2.1: The transport method of the LZ TPC inside the inner cryostat will be similar to the successful transport of LUX from the same building to the headframe. 
Once the detector/inner cryostat assembly arrives at the Yates headframe, the assembly on the transport frame will be lowered in the Yates shaft, as shown in Figure 10.2.1. Lowering the assembly on its transport cart slung under the Yates cage will take some hours. A crew member will accompany the assembly in the Yates conveyance, as was done for LUX. The assembly and transport cart will be extracted from the Yates shaft at the 4850L and rotated back to the horizontal orientation. A trial lowering in the Yates shaft of a mockup of the transport frame and the cryostat has been completed successfully.

\subsection{Underground Infrastructure}

A plan view of the Davis Campus is shown in Figure 10.3.1. After arriving on the 4850L, the detector will be transported to the Davis Cavern via the Primary Access Drift, passing by the room housing the MAJORANA detector, as depicted in Figure 10.3.1. That is the same access-way utilized for transport of the LUX detector. The larger LZ inner cryostat can be moved through the same space by temporarily removing short segments of the Davis Campus HVAC air-supply ducts to allow for sufficient clearance. All access doorways are sufficiently large to allow for detector passage. Entry to the Davis Cavern via the Primary Access Drift allows the transporter design to take advantage of the 8,100-lb maximum floor loading afforded by design of the Davis Cavern structural steel. It would also be possible to transport the inner detector in the drift that passes by the LN storage room, but this would require the removal (and later replacement) of a wall between the drift and the Davis Cavern.

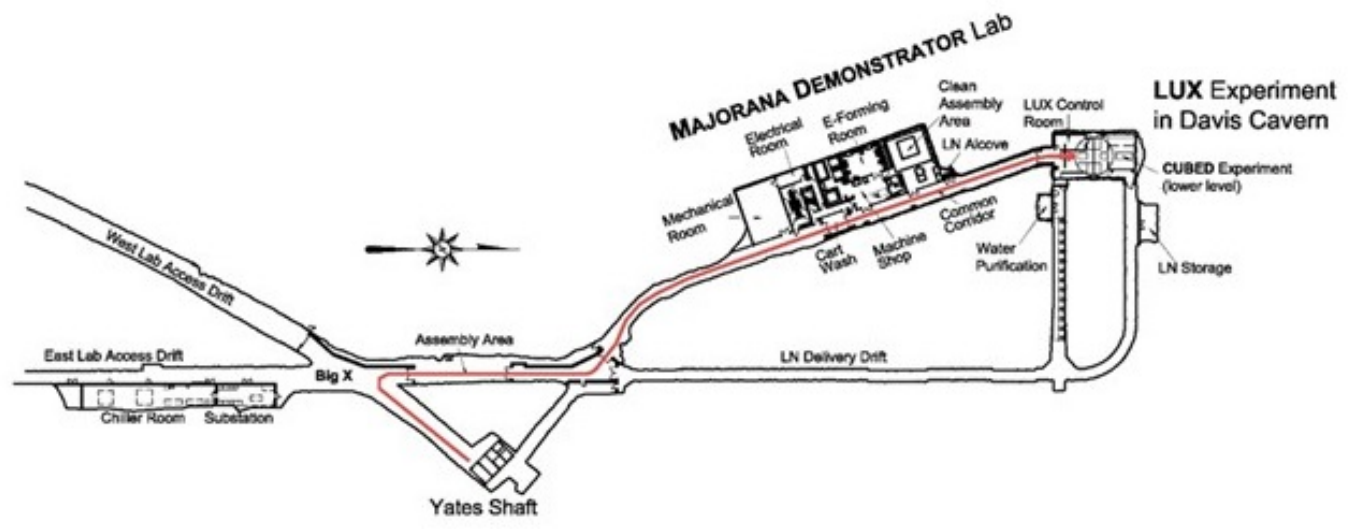

Figure 10.3.1: Plan view of the Davis Campus at the 4850L.

LZ will take advantage of design features built into the Davis Cavern to allow for deployment of a larger detector than LUX. A 102-inch-diameter flange is built into the 70,000-gallon water tank. Additionally, the redundant 50-ton water chillers and the Davis Campus 1,500 kVA substation were implemented with a large future detector in mind.

Figure 10.3.2 shows an isometric view of the LZ deployment in the Davis Campus. Xenon storage cylinders will be securely deployed in a newly upgraded portion of the present $\mathrm{LN}$ storage room access drift (shown in the lower-right corner). Currently, this is an unfinished area that has been used for rock- moving equipment and storage. SURF will upgrade this area by providing concrete floors and covered walls while also closing off the space to allow for flow-through ventilation. Ventilation will be adequate to mitigate potential oxygen - deficiency hazards that could occur in the event of a release from one of the Xe storage cylinders. The Xe cylinders are more thoroughly described in Chapter 6.

The LN storage rooms and control rooms used for LUX will be largely reused. LZ will utilize a similar scheme for LN storage tanks until a second cryocooler is secured for long-term operational flexibility. These 
tanks will supply makeup nitrogen to the cryocooler, assist with transient startup effects, purge gas for the water purification system, and provide some buffer against short-term power interruptions. The control room is expected to remain unchanged, providing minimal office space for underground workers during assembly, commissioning, and data taking.

The ventilation duct that exhausts the LN storage room will be modified to also ventilate the Xe storage room. New supplemental ducting and a fan added to the existing ventilation will provide fresh air flow.

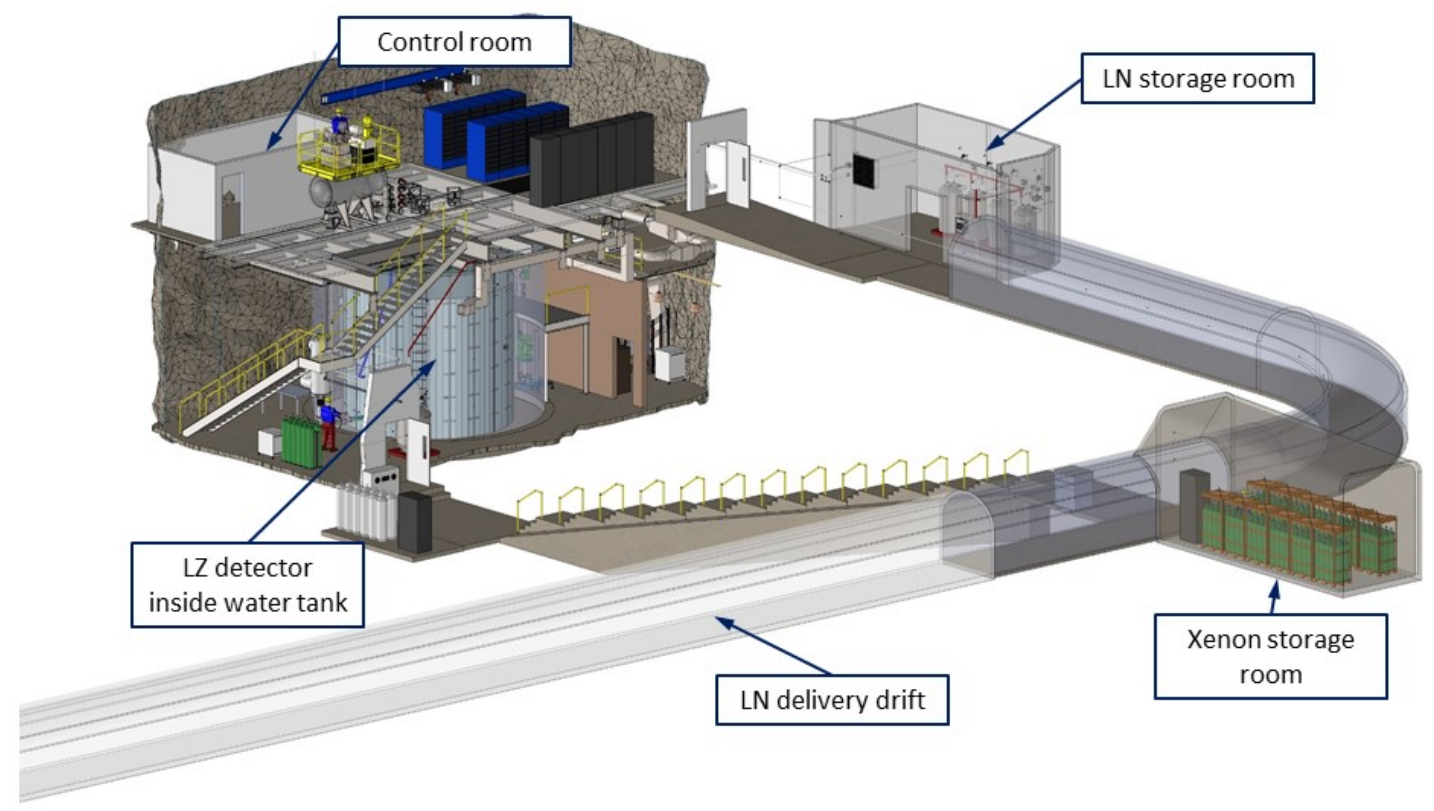

Figure 10.3.2: Overall layout of LZ in the Davis Campus.

Figure 10.3.3 depicts a close-up view of the Davis Cavern showing supporting infrastructure installed. Key elements include data acquisition (DAQ) cabinets, a cryocooler to support cooling thermosyphon lines, a platform that interfaces to the PMT and sensor cable breakout, a XE purification tower for circulation heat exchange, and Xe circulation/storage equipment deployed both in and above the current low-background counting rooms in the lower Davis Cavern.

To facilitate deployment of a larger number of DAQ racks and cryocoolers on the deck of the upper Davis Cavern, SURF will decommission and remove the existing cleanroom to open up floor space. A new platform deployed near the PMT cable breakouts will also serve to significantly increase the effective floor space in the cavern.

All other Xe circulation, storage, and recovery equipment is co- located in space behind block walls previously utilized for low- background experiments. Included are the Xe recovery compressors. These large $480 \mathrm{~V}$ units are positioned near existing in-cavern power panels and also the low-pressure/higher-loss suction side of the Xe gas system. 
The Davis Campus is fed from a 1,500 kVA substation. The substation is presently loaded at approximately $60 \%$ during worst-case conditions, and has ample reserve capacity to accommodate the projected LZ electrical load of about $115 \mathrm{~kW}$ (an increase of about $\sim 75 \mathrm{~kW}$ from LUX). The campus has a $300 \mathrm{~kW}$ backup generator that supplies the air- handling systems, communications, facility control and alarm systems, and egress lighting for up to 48 hours. An additional $40 \mathrm{~kW}$ generator will be installed to provide backup power for LZ.

A system to provide reduced-radon air during critical assembly steps inside the water tank will be located underground just outside the entrance to the clean area of the Davis Campus in an existing space. This system will provide reduced-radon air for the few months of critical connections to the Xe detector and possibly later. Air will be piped from this system to the water tank. The baseline plan for this system follows closely from a similar system constructed at the South Dakota School of Mines and Technology [2].

Because the water tank installed for the LUX experiment was designed to be able to accommodate a much larger experiment, the modifications required for $\mathrm{LZ}$ are modest.

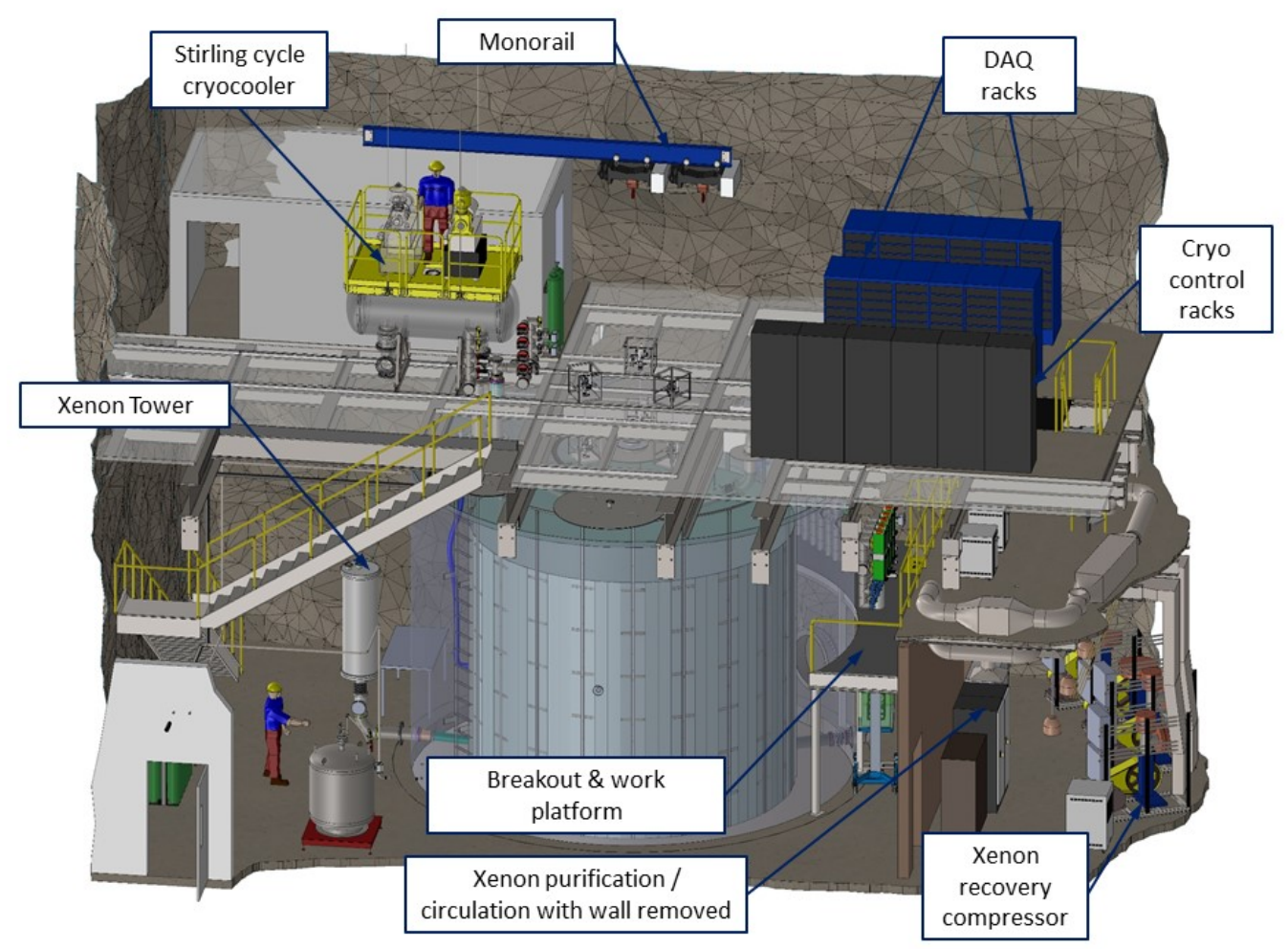

Figure 10.3.3: LZ and related support systems in the Davis Cavern.

Mounting plates will be added to the tank floor for the LZ detector along with mounting points for the four side scintillator vessels. At larger radius, mounting points will be installed for the outer detector PMT ladders. In addition, a support for the lower conduit that connects the detector to the LXe tower and PMT cable breakout will be installed.

The detector HV system for LZ requires a feedthrough in the top of the water tank. In addition, two penetrations of the tank wall are needed for routing the conduits that connect the detector to the LXe tower and the PMT cable breakout. Two neutron tubes will be installed for the neutron calibration system, and that tube will be filled with water for normal running and nitrogen for calibration. The top of the water tank will 
also have several feedthroughs to accommodate thermosyphons, detector cables, source tubes, scintillator lines, water PMT cables, LED flasher cables, and vacuum pumping.

\subsection{Integration and Assembly}

This section describes the effort to integrate work at the subsystem level into a coherent design that meets the science requirements and that will result in an operational detector at SURF. A detailed assembly sequence is presented. While the overall scope of integration and assembly is contained in WBS 1.9, significant resources from every subsystem are required. Much of this effort comes from the distributed pool of engineers working for subsystems at many institutions. Coordination of this engineering effort is part of the integration task.

\subsubsection{Integration}

For LZ to achieve its science goals, it uses primary requirements that define what the subsystems must do; subsystem requirements drive more specific design specifications (see Chapter 12). The subsystems must be safe, affordable, timely, compatible with the other subsystems, and possible to assemble and operate with the available infrastructure. The Integration Group provides the necessary management, engineering, design, organizational tools, and administrative effort to assist all subsystems in completing the LZ design. Phone meetings and technical workshops help identify interface issues and hidden constraints created by design choices in other subsystems. An interface matrix is used to record and highlight interfaces between WBS areas. Interface Control Documents (ICDs) are authored by the key driver of the interface and reviewed and approved by all stakeholders. The Integration Group maintains CAD models of the overall LZ detector and of the Davis Campus. This helps define physical interferences and design gaps that are not adequately covered. At integration meetings, engineers from each subsystem, and many scientists, share ideas to help with some of the more difficult design challenges.

The Integration Group develops general standards and controls, such as engineering document standards to be used project-wide for CAD file exchange, engineering drawings and design notes, specifications and procedures, controled Technical Documents (CTDs), technical change (TCs) documents, and a document numbering and organization system. The Integration Group defines component reference names and an overall coordinate system for the experiment. The group maintains a cable and feed-through list with the help of the subsystem management. A key parameters list is maintained as a reference for design, modeling, and science.

As designs mature, they must be documented and reviewed. The Integration Group works with project and subsystem management to arrange and execute design reviews at appropriate times. Conceptual Design Reviews evaluate whether the design meets the requirements, interfaces have been identified and successfully coordinated, and engineering details are sufficiently developed to proceed. Preliminary Design Reviews focus on manufacturability, cost, schedule, risk, and safety. Final Design Reviews ensure that documentation and drawings are complete and the fabrication plan fits within project budgets and timelines. All LZ systems have completed Final Design Reviews. Production Readiness Reviews for WBS 1.2, WBS 1.5 (PMTs), and parts of WBS 1.6 (liquid scintillator production and acrylic vessels) have taken place.

LZ is being assembled and installed in an underground area administered by SURF. Integration includes working with SURF to be sure the infrastructure is adequate to support assembly, installation, and operation of the LZ experiment. SURF engineers are tasked with design and execution of infrastructure projects to support the LZ project. The Integration Group coordinates communication of requirements with SURF and the detector subsystems. 
The overall planning of the on-site assembly and installation of the detector at the Davis Campus is part of the integration effort. This work includes defining the sequence of steps to put the detector together, creating a schedule for this work, and developing an understanding of the resources needed to accomplish it. Subsystems support this effort by providing details for handling components and aiding in resource and schedule development. Subsystems also develop detailed work procedures that technicians can follow for cleaning, assembly, and testing.

\subsubsection{Assembly}

The LZ assembly will happen in three stages-off-site subassembly, surface assembly in the Surface Assembly Lab (SAL), and underground assembly in the Davis Campus-with transportation between stages. The general strategy is to do as much work as practical off site at universities and national laboratories, where highly skilled, specialized labor can easily work with students and scientists. This also reduces travel costs. Parts delivered to the site will be tested, clean, and ready to use, with a few exceptions. The development of detailed delivery requirements and procedures, particularly cleanliness relevant to achieve the low-radioactivity needed, is under active development. A database and electronics logging tools will be used to track the location and status of parts and subassemblies, from initial fabrication through delivery to SURF, and up to assembly into the final detector. Assembly workers will check that the part has been approved for radioactivity, cleanliness, and function before using it on the detector.

Xe PMTs will be assembled, tested, and characterized prior to delivery to SURF. The PMT vendor will do some QA testing, but burn -in, final electrical characterization, and cold testing of each PMT will be done by LZ. Assembly includes connecting a PMT base to the PMT, attaching polytetrafluoroethylene (PTFE) reflectors, and final cleaning. Because they have PTFE as a reflector, the assemblies must be kept under a nitrogen purge during storage and shipping to prevent radon contamination. Internal PMT cables will have a pin-and-socket connection at both ends. They will ship separately and be routed to the PMTs after the PMTs are installed on the support arrays. The warm end of the cables will be connected to the feedthroughs mounted in flanges after the cables are routed. The cables must be kept clean and under nitrogen purge during storage and shipping because they contain fluorinated ethylene propylene (FEP) as the primary insulator.

The tested PMTs will be placed into the titanium PMT support structure, including preliminary placement and routing of the cables. The baseline plan is to do this work in a cleanroom at Brown University. The titanium arrays will be mounted in the two "PMT Array Lifting And Commissioning Enclosures" (PALACE - see Section 3.4.5), a multipurpose light- and gas-tight enclosure. The PALACE will be opened for PMT, reflector, and cable placement, and then closed and purged with nitrogen between operations and during testing. This work will create an upper and lower populated PMT array. The PALACEs will also be used to protect the arrays during shipping. The upper skin PMTs will be mounted into teflon trays that attach to the sides of the TPC. This work will be done in the reduced radon environment of the SAL clean room at SURF. The lower skin PMTs will be attached to a titanium support that is mounted to the bottom of the ICV. This work will also be done in the SAL.

The wire grids for cathode, gate, anode, and PMT shields will also be manufactured off site. The grids will be cleaned, inspected, packaged, and shipped to SURF. The packaging must keep the wires clean from any debris and protect the fragile wires from shock during shipping. Boxes of the same design as the PALACEs will be used for grid storage, shipment, and testing to save on design and manufacturing cost. Inspection procedures after fabrication and after arrival at SURF are under development and will be guided by experience in the system test at SLAC (see Chapter 3). These could involve automated optical inspection and voltage testing in gas.

The field-grading region of the TPC is made from conductive metal rings, insulating PTFE spacers, and resistors. These parts will be fabricated by vendors and then inspected and cleaned off site at LBNL before 
shipping to SURF. Radon exposure of completed PTFE parts will be minimized after final machining. They can be stored and shipped in nitrogen-filled metalized bags.

The cryostat will be manufactured in Italy as fully tested and cleaned code-stamped vessels under contract with the Imperial College. The fabrication vendor has the responsibility for final design, fabrication, testing, cleaning, and shipping to SURF. The inner and outer vessel will be shipped separately (not nested) in nitrogen-filled sealed bags from the cleaning facility. Receiving acceptance for the cryostat will include visual inspection for any shipping damage, separate assembly of each vessel, and vacuum leak-checking with helium. Rate of rise leak checking of the double O-ring seals will also be performed to get a baseline rate of rise for each joint. The reflective PTFE liner for the inner vessel will be attached to the inner cryostat vessel wall in the SAL. The plan is to suspend the bottom of the inner cryostat upside down in the vesselassembly area and to access the inside of the vessel from underneath with a manlift. This will allow a worker to tile the inside while standing on a stable surface. The bottom skin PMT array will be installed into the bottom of the ICV. The ICV will be sealed after the line is installed. A radon emanation test of the lined ICV can be performed both to discover if there are any large radon issues and to establish the emanation from this part of the assembly so it can be compared to the emanation of the full assembly later.

The HV umbilical will be delivered from LBNL as a clean, tested, and sealed assembly. The heatexchanger tower subassembly will be built, cleaned, and tested at the University of Wisconsin Physical Sciences Lab, and transported as a sealed assembly. The Gd-LS acrylic tanks will be manufactured, cleaned internally, tested off site, and shipped in protective packaging. They will be stored in the SSF. The outside of the Gd-LS tanks will be cleaned on site, underground. The ladders, PMTs, and Tyvek reflectors of the outer detector will be shipped separately.

It is unlikely that the timing of delivery can match the time when each piece is needed. The SSF is the identified storage location at SURF. A temperature-controlled storage room has been built in the SSF to store the Gd-LS and the acrylic tanks.

The subassembly work and transportation described above will primarily be the responsibility of the subsystems. Once things arrive at SURF, primary responsibility shifts to the Integration and Installation Team (WBS 1.9). The plan is to work with SURF to hire a pool of local technicians to perform much of the work. This pool will include experts in cleaning, vacuum, rigging, and mechanical assembly. A lead technician will handle managerial supervision and work direction, but technical supervision will be supplied by engineering and scientific staff. An LZ engineer will be on site to coordinate the work and provide technical oversight. Experts from subsystems will be on site during assembly of their subsystems. Existing SURF engineering and technical personnel will also provide support. Worker training and work control will be performed as part of the SURF safety program.

On-site assembly starts above ground with assembly of the TPC. Cleanliness is critical for this assembly work, both to reduce radioactivity backgrounds in the detector and to control particles that could cause field enhancements and reduce operating voltage. This work will be done in the SAL at SURF with a large reduced-radon cleanroom, described previously. Figure 10.4.1 shows the major steps of assembly in the SAL. The first step is receiving and inspection of the parts and subassemblies. Clean parts destined to enter the cleanroom will be shipped in triple bags. The first bag will be removed in the receiving area to keep dust and dirt out of the cleanroom area. The second bag will be removed in a soft-walled semi-cleanroom at the entrance to the cleanroom. The third bag will be removed inside the cleanroom after the particle count in the room has returned to normal after opening the door. The goal of the inspection is to ensure that the parts have not been damaged in shipment and that they will meet functional requirements. This includes cleanliness. The PMTs in the arrays will be tested to ensure they were not damaged in shipment. The grids will also be inspected.

The next several subsections describe the various stages of assembly. Each subsection concludes with a description of the suite of checkouts that will be performed prior to declaring the stage complete. Final 
definition of these checkouts is a crucial aspect of the assembly and will be made by WBS 1.9 in close consultation with the relevant subsystem owners.

\subsubsection{TPC Assembly (Steps SA2 - SA6)}

The assembly will start with the lower PMT array (see Chapter 3 for descriptions of the components referenced here) The lower PMT array will be set upside down on a ring shaped cart for initial assembly. The cart will have telescoping legs and locking wheels that allow adjustment to a comfortable working height and easy movement into an extra-clean storage garage. Eighteen skin 2-in PMTs will be connected to the titanium truss of the lower array. Xe return liquid distribution tubing and manifolds will be mounted and dressed. Loop antennae and temperature sensors will then be placed and cabled. The fully populated bottom subassembly will be rotated $180^{\circ}$ and set back on the ring cart. A layer of arc shaped PTFE segments is screwed to the titanium support plate and then the PMT guard grid is placed on top of the PTFE. The reverse field-grading assembly composed of arc shaped PTFE segments and complete metal rings will be assembled onto the PMT array next. The parts are held together with axial PEEK screws that pass through a counterbored hole in the top PTFE part, a through hole in the titanium field shaping ring, and into a PEEK nut press fit into a coutnerbored hole (farside) in the bottom PTFE part. Leads from resistors that fit into pockets in the PTFE will be attached with screws to electrically connect the metal rings as they are stacked. When the reverse field region has been stacked to the correct height, The cathode grid is installed on the top of the stack. An additional layer of PTFE segments is placed on top of the cathode to allow connection to the forward field shaping subassemblies. The completed bottom subassembly will be stored in the garage when it is completed. Subassemblies are also stored in the garage whenever active work is not being done on them. This will reduce contamination of surfaces with dust. Sections of the forward field-grading assembly will be built on similar carts with ring shaped tops. The open support structure is designed to allow airflow through the pieces being assembled so less dust will accumulate on the work pieces. The forward field PTFE segments and rings are held together with radial pins. This allows the assemblies to be joined with only access from the outside. There will be four forward field subassemblies. The extraction region will be assembled at LBNL and shipped as a subassembly consisting of the weir trough, anode grid, gate grid, and a few layers of field shaping rings. The extraction region sub assembly will be mounted to a similar cart and the upper PMT array will be rigged in place over it. The extraction sub-assembly will be by connecting to the titanium plate of the upper array assembly. The 93 upper skin PMTs will be attached to the outside of this assembly. The cables from these PMTs will be carefully dressed with the other PMT cables. Voltagecontrol cables will be connected to the grids. Loop antennas, temperature sensors, and position sensors will then be placed and cabled. The completed extraction region subassembly will then be stored in the garage.

The full TPC will be assembled by moving carts holding sections under one side of the monorail, lifting the sections off the carts with the monorail crane, transporting them to the other side of the monorail, and lowering them onto the stack. The sections will be connected with PEEK radial pins. Before a subassembly is lifted, it will be inspected and tested for dust accumulation. This test and possible cleaning mitigations are under development. The assembled TPC will undergo a series of tests to ensure light-tightness of the field-grading cylinder, and function of all PMTs, HV grids, resistor network, and sensors. 

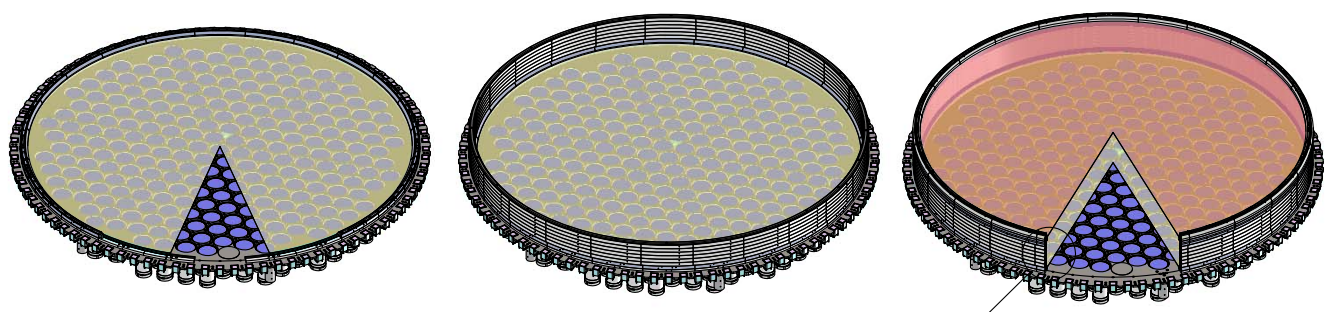

$\frac{\text { SA1 - BOTIOM PMTARRAY }}{\text { WTH GUARD GRID }}$

SA2 - REVERSE RED

SHAPING REGION

SA3 - CATHODE GRD CONNEC TEDWTIH SCREWS CONNEC TED WTH SCREWS
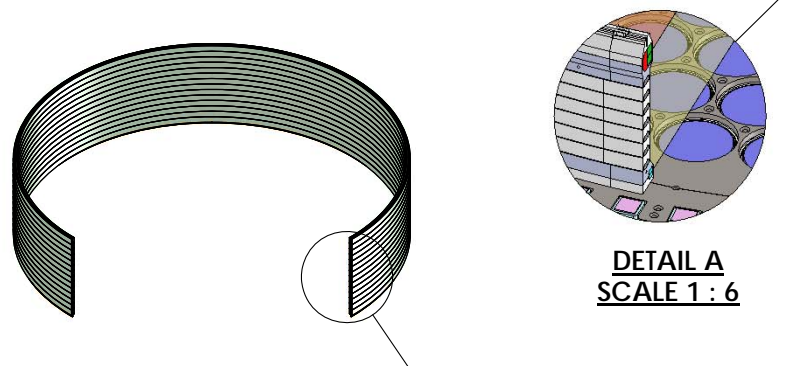

DETAILA SCALE 1:6

SA4 - FORWARD REID SHAPING
SUB-ASSEMBUES OF METALRINGS PINNED TO TERLN SEGMENIS

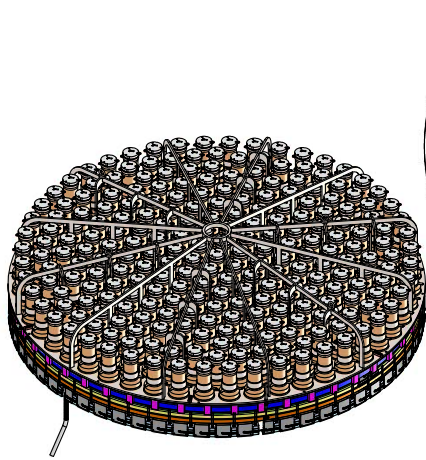

SA5 - TOP PMTARRAY ASSEMBLED WTH ANODE GRI, WER TROUGH AND GATE GRID

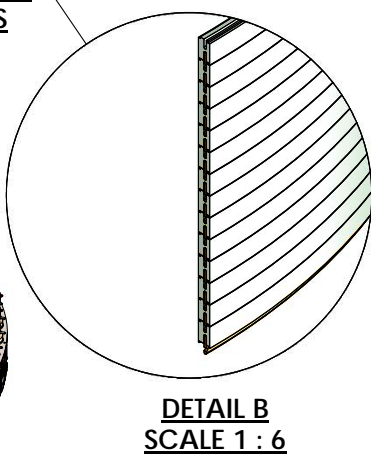

SCALE 1: 6

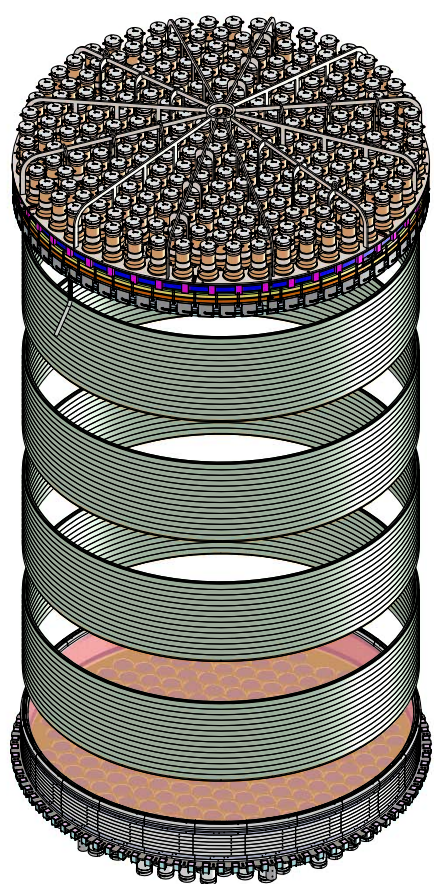

SA6 - TPC ASSEMBYY RADIALPINS CONNECT SUB-ASSEMBUES

Figure 10.4.1: TPC assembly forms subassemblies of the lower PMT array with reverse field region and cathode grid; the upper PMT array, anode grid, weir trough and gate grid; and four short stacks of field shaping grids. These are then combined into a complete assembly 


\subsubsection{TPC Insertion into Cryostat with Fluid and Electrical Final Routing}

The inner cryostat vessel (ICV) will be staged in the lower level of the cleanroom under a removable grated floor. The first step of insertion is to remove the lid of the ICV and raise it so the top flange is a few inches above the floor. The TPC will then be lifted with the hoist on the monorail, transported on the monorail over the ICV, and lowered into the ICV. Guide bars will be attached to the sides of the ICV and go vertically up to the top of the TPC to help guide the TPC as it is lowered. During this process, the PMT and sensor cables coming from the lower PMT array will be routed through the central port in the bottom of the inner cryostat. Xe fluid circulation lines also routed through this port will have been placed into position earlier, but may need adjusting and securing as part of the cable routing. Access for this operation will be through the HV connection port and the bottom port of the vessel. The three Xe tubes from the weir trough must be routed to the ports in the inner cryostat wall. These tubes are PTFE bellows that will initially be pointing straight down and then guided into the ports as the TPC is lowered. The TPC is supported on six posts projecting upward from the bottom head of the inner cryostat. Tapered guide pins will be installed in the bottom mounting holes of the TPC to engage the holes in the posts and guide the TPC into the correct position. Once the TPC is in the correct place, the guide pins will be removed and bolts will be installed to secure the TPC to the posts. The titanium plate for the upper PMT array will be guided from the inner cryostat near the main flange with tabs that allow vertical motion but constrain radial motion. Access for this work is from the top over the main flange. After these upper guides are secured, the TPC will be fixed in the vessel. The location of the weir surfaces that establish the LXe plane will be surveyed relative to known positions on the outside of the inner cryostat. This will allow rough leveling of the weir surface during future assembly steps. The next step is to stage the lid of the inner cryostat over the bottom and install a temporary safety support. The PMT cables, grid cables, sensor cables, and Xe gas lines coming from the upper PMT array need to be routed through a port in the inner cryostat lid. The sensors that monitor the position of the TPC relative to the inner cryostat wall will be installed and cables dressed. The lid can then be lowered onto the bottom of the inner cryostat and the large-diameter seal formed. This seal is designed as two seals (inner helicoflex metal seal and outer elastomer o-ring seal) to facilitate a check for leaks by pumping between the seals and looking at the rate of pressure rise. This rate can be comared to the baseline rate established during cryostat acceptance helium leak checking. All other ports on the inner cryostat must be sealed for transportation to keep the TPC clean. The cables will be wiped clean and tested for dust before they are pulled through a long bellows. The bellows will be sealed to the flanges on the ICV and leak-checked. The outer end of the bellows will be capped. The sealed inner cryostat will be pumped down to vacuum to ensure the seals are adequate. Other testing will be done at this point. This could include PMT functional tests, sensor tests, light-tightness tests between the skin and central TPC volume, and HV tests. A radon emanation test of the completed and closed vessel can be performed at this point. Closed-cell foam insulation and superinsulation blankets will be fit-tested on the outside of the inner cryostat for later underground installation. After these tests, the bellows will be dressed to the outside of the ICV and the ICV with TPC assembly will be doublebagged so it is ready to be moved underground. The special transport frame for this move will be brought into the clean room. The vessel will be rotated from vertical to horizontal using two hoists on the monorail and raised so the special transport frame can be placed beneath it. The vessel will be lowered on the special transport frame and secured for transport.

\subsubsection{Underground Outer Detector Tank Preparation / Staging (Steps U1 - U2)}

The first step of underground assembly is to prepare the water tank. All LUX components have been removed. Infrastructure work includes reorienting the overhead monorail crane so it runs North South. Some welding is needed in the water tank for attachment points for the cryostat support, the Gd-LS tanks, and the outer detector PMT ladders. New penetrations are needed for the HV umbilical, the heat exchanger 
(HX) conduit through the wall of the tank, and calibration tubes. After these welding operations, the tank will be passivated again to improve corrosion resistance to the pure water. The water tank will then be cleaned and made into a cleanroom with reduced-radon air delivered from the system located underground. The tank will be kept with slightly positive pressure to reduce air infiltration. The access door in the side of the tank will be outfitted with a temporary changing room and air lock.

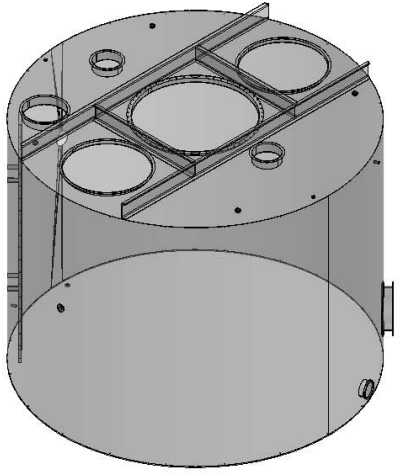

U1 - EMPTY WATER TANK

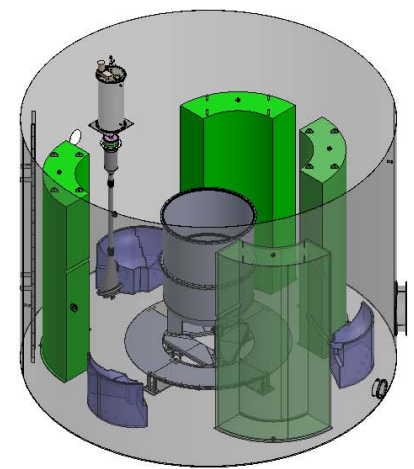

U4- OUIER VESSE

BOTIOM \& MIDDLF

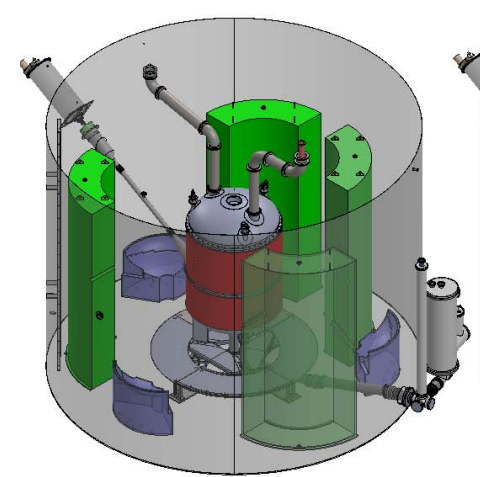

U7 - HN UMBUCAL\& HXCONDUIT

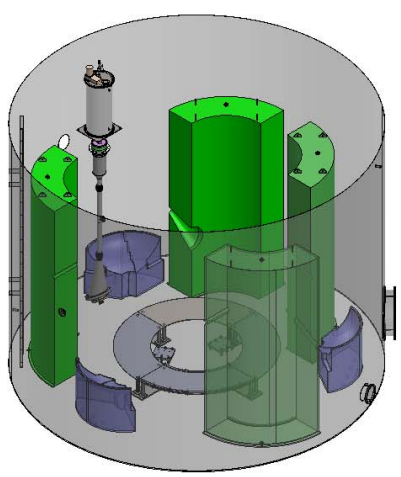

U2 - LSTANKS

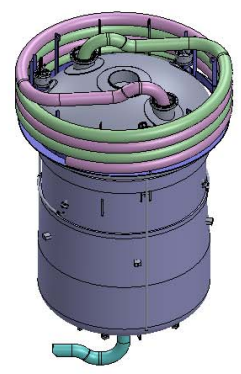

U5 - INNER VESSE OUIER UD, CABLES

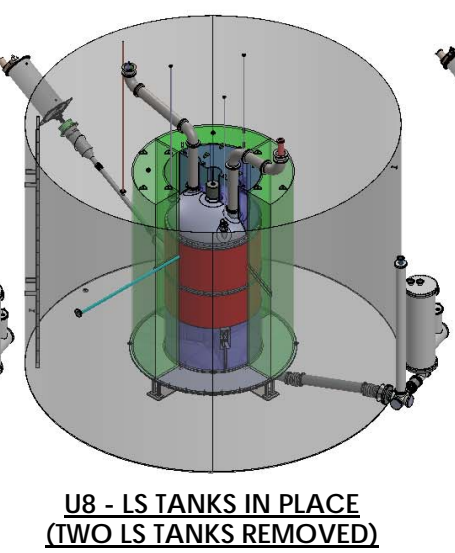

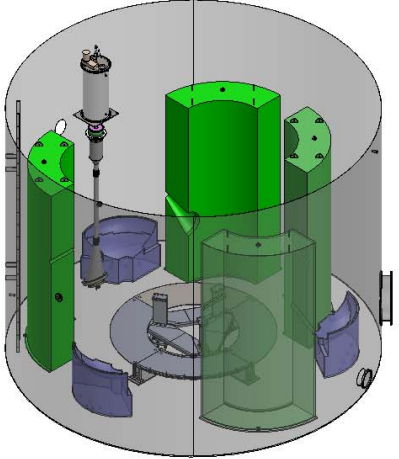

U3 - VESSEL SUPPORTLEGS

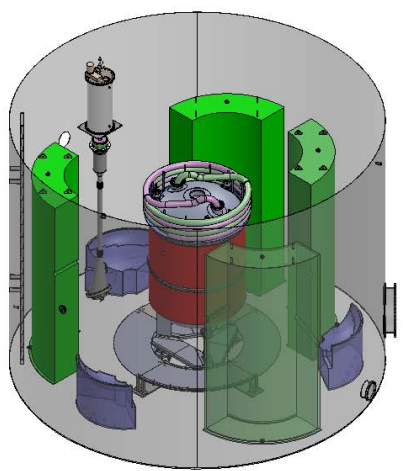

U6 - INNER VESSEI

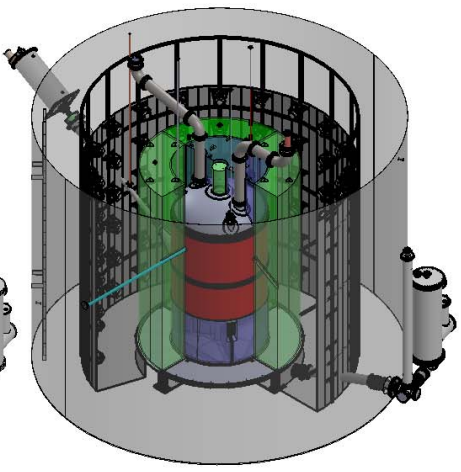

U9 - WATER PMTS (TWO LSTANKS REMOVED)

Figure 10.4.2: Underground installation sequence in water tank.

The central top port of the water tank is the only port big enough to allow installation of the Gd-LS tanks. Once the cryostat is installed, this path will be blocked. So the bottom and side Gd-LS tanks must 
be transported underground and staged in the water tank. Figure 10.4.2 shows the sequence of installation in the water tank. The Gd-LS tanks will arrive clean on the inside and covered with protective plastic and rigging frames. The acrylic tanks will be brought into the Yates headframe with a telehandler and set on a cart in front of the Yates cage. The four large side tanks will be moved first. Rigging will be used to attach the steel frame around the tank to the underside of the cage. The cage will be raised to lift the tank until it is vertically under the cage. A drag line will guide the lower end of the tank. The cage will be lowered slowly until the bottom of the tank is at the $4850 \mathrm{~L}$. A drag line will be reconnected to the bottom to pull the tank out as it is lowered further. The tank will be placed on a receiving cart, which will transport the tank to the entrance to the Davis Campus cleaning area (so-called cart wash). The external frame and external packaging around the tank will be cleaned to remove mine dust from transport. The bottom of the tank must enter the Davis area first. One hook from the monorail in the Davis Cavern will connect to two points near the bottom of one side of the rigging frame. A second hook from the same monorail will connect to the top of the tank's rigging frame. The motion of the hooks will be choreographed to lower the acrylic tank into the water tank, keeping the rigging vertical over the lifting points by moving the hooks along the monorail. Once the tank is vertical and set down on the floor of the water tank, it will receive a final inspection and leak test. The external packaging and protective plastic will be removed from the acrylic tank. A temporary beam with hoist will be installed to the inside top of the water tank to move the Gd-LS tanks inside the water tank and aid with unpackaging. The outside of the acrylic tanks will be cleaned with Alconox and water and visually inspected for any cracks. The three bottom tanks and the two top tanks will be transported inside the cage and unpackaged and cleaned in the cart-wash area. The three bottom tanks will be staged inside the water tank. The two top tanks will not be staged, as they will be installed from the top after the cryostat and cable-conduit installation is completed. Stainless steel support stands that hold the four tall outer Gd-LS tanks will also be staged into the water tank. The HV umbilical and parts for the HX conduit may also be staged in the water tank. A simple mockup that had the appropriate dimensions for the largest acrylic vessel transport frame was successfully lowered in the Yates shaft and partially transported underground.

\subsubsection{Cryostat Transportation and Underground Assembly (Steps U3 - U6)}

The installation of the cryostat starts with the cryostat support. The survey reference system for the detector will be established and a template for drilling the anchor bolts for the cryostat support leg will be located. Nine anchors will be installed into holes that are drilled and tapped into the thick steel shielding plates beneath the water tank. Once the anchor rods are installed, nuts will lock them to the floor. These nuts need to be seal welded to the bottom of the tank and the threaded rod so water can not leak out. This work will most likely be done during the modification to the water tank. Three machine leveling jacks will be place on the floor of the water tank temporarily. Three mounting plates will be installed onto the anchor plates and supported by the leveling jacks. The cryostat support legs, cleaned and triple-bagged, will be brought down in the cage. The outside bag will be removed at the entrance to the Davis Campus. The inside bag will be removed on the top deck near the opening to the water tank. The legs will be lowered into the water tank through the central port with a single hoist. The final bag is removed inside the water tank. The legs will be set into position on the mounting plated and loosely secured. The outer cryostat has been designed as three pieces so each piece can fit in the Yates cage. Each piece will be brought down in the cage cleaned and triple-bagged. The bottom head of the outer cryostat is lowered into the water tank with a single hoist and positioned over the three support legs. Once the bottom head is lowered, it will be bolted to the legs. The bottom section is then surveyed and located using the machine leveling jacks. Once it is in place, the other bolts and nuts are tightened. The final position is then surveyed for verification. The middle section will be brought in next, rigged into position above the bottom, sealed to the bottom, and leak-tested. The middle section will be surveyed to ensure it is level. Adjustment will be needed if it is out of range. A displacer around the outer cryostat cylindrical section reduces the amount of water between the Gd-LS tanks and 
the cryostat. This may be installed around the outer cryostat at this point or after the HV connection is completed. The top lid will be brought into the Davis Campus and staged on the top deck with its inner bag still in place.

The inner cryostat will not fit in the cage, so it must be hung under the cage. It will be horizontal for some of its journey from the SAL to the Davis Campus, and vertical for other parts. The TPC support system will be designed to accommodate support in both conditions and the transition between them. An external rigging company will be contracted to remove the ICV from the SAL and transport to the Yates headframe. It is anticipated that this move will also be done with a telehandler. There is a detailed plan for transport of the inner cryostat from the surface to the $4850 \mathrm{~L}$, described earlier in this chapter. On the $4850 \mathrm{~L}$, a special cart with air skates will be used to bring the inner cryostat from the Yates shaft to the Davis Cavern (this was the method used for LUX). The special transport frame must be cleaned. The outer bag around the cryostat will also be removed in the cleaning area. The transport frame will move the inner cryostat onto the deck near the entry hole to the water tank. The deck plates above the central hole of the water tank will have already been removed. A temporary platform will be built resting on the lower flanges of the beams that support the Davis deck. This temporary platform will be about 19 inches below the main floor. The platform is designed to roll along the flanges of the beam so it can be moved clear of the large port in the water tank. The two hooks on the monorail will be used to lift the inner cryostat off the transport frame, rotate it back to vertical, and rest it on the temporary platform. The bellows full of cables exiting the bottom of the cryostat will need to be managed during this lift. A temporary cleanroom will be built around the inner cryostat. Reduced-radon air from the underground system will be used to provide a clean atmosphere. The inner bag around the inner cryostat will be removed. Closed-cell foam insulation will be installed onto the sides and bottom of the cryostat. The LXe weir drain lines will be connected and routed. The cryostat cooling thermosyphon evaporators will be attached to the fins on the cryostat walls. Temporary supports for the outer cryostat lid will be installed onto the inner cryostat. The outer cryostat lid that was previously staged will be unbagged, rigged over the inner cryostat, and set on the temporary supports. The two upper bellows will need to be guided through the ports in the outer cryostat lid as it is staged. The permanent support rods that hold the inner cryostat from the outer cryostat will be installed. The supports will be adjusted to position the weir surface of the inner cryostat parallel to the sealing surface of the outer cryostat lid. Then prefabricated superinsulation blankets will be installed. The lower section of the three calibration ports will also be installed. The outer cryostat lid will then be lifted and the load from the inner cryostat will transfer from the temporary supports on the deck to the permanent supports from the lid. The temporary platform will be rolled along the flange of the beam until it is clear of the water tank opening. The assembly of the outer cryostat lid and inner cryostat will be lowered into the water tank and into the outer cryostat bottom. As the inner cryostat is lowered, the bottom bellows will need to be threaded through the central port of the outer cryostat bottom head. The reduced-radon air will again be flowing into the water tanks for this process. The crane will set the assembly down so the outer cryostat lid rests on the outer cryostat middle-section top flange. The inner cryostat will still be hanging from the lid. This flange can then be assembled and leak-checked.

\subsubsection{Utility Connection (Step U7)}

The lower cable bellows are routed to the edge of the water tank through a vacuum jacket. Xe transport lines have a separate vacuum jacket and connections will be made with an orbital welder whenever possible. Some connections may be made using VCR fittings. These lines continue through the outer wall of the water tank to the cryo tower. The lower PMT cable conduit continues vertically after penetration of the water tank wall and connects to the breakout box. The cables continue into the breakout boxes and connect to an array of flanges and hermetic feed-throughs. The upper bellows will be routed through vacuum jackets to the appropriate flanges on the top of the water tank. One bellows connects to vacuum pumps and Xe 
recovery system plumbing; the other connects to another breakout box with an array of flanges and hermetic feed- throughs. During the connection of the cables in the breakout boxes, reduced-radon air will be routed through the ICV to prevent back diffusion of mine air into the ICV. This area has many details that must be carefully planned.

The HV umbilical attaches to the large side port. To reduce krypton and radon absorption by the internal plastic components, this assembly will also be purged with nitrogen or reduced-radon air whenever it is opened. The HV umbilical is a flexible assembly that connects to the top of the water tank and the side of the cryostat. The central cable of the umbilical needs to be electrically connected to the cathode. The inner tube of the umbilical then seals against the inner cryostat. The flange will have a double seal (inner helicoflex and outer o-ring) so it can be leak-checked at this point. Then the outer vacuum jacket will slide down toward the detector and make a seal to the outer cryostat. This seal will have a double o-ring so it can be leak-checked. Sealing rings at the water tank wall are installed to seal the inner tube to the outer tube and the outer tube to the vacuum tank. There are no direct water-to-Xe seals.

The final step of cryostat installation is positioning and leveling. The connections for the conduit and HV umbilical add load and positional constraints to the hanging inner cryostat. The support rods will be adjusted using feedback from built-in electronic level sensors. We have designed in enough compliance to these connections so the inner vessel can be moved. The cryostat should now be sealed and the reducedradon air flow can be stopped. The inner cryostat will be pumped down to start long- term outgassing of the internal plastics.

\subsubsection{Outer Detector Assembly (Steps U8 - U9)}

The Gd-LS tanks can now be placed into final position. The three bottom tanks are set on platforms connected to the cryostat legs. The upper Gd-LS tanks are then installed through the top port of the water tank. They are lowered slightly radially outward from their final positions to clear the PMT cable conduit and thermosiphon conduits and, once they are low enough, translated under the conduits to the correct final position. The upper Gd-LS tanks are supported by the top flange of the outer cryostat. The four side tanks will be moved in adjacent to the displacer around the outer cryostat and rest on stainless steel supports. There are notches for the HV umbilical so the tanks have to come in from the proper direction. After positioning, the tops of the tanks are connected for stability. Each tank is secured so it will not float in the water or tip over in an earthquake. Each tank has a fill line and vent line that come to a common overflow reservoir on the top of the water-tank lid. The final system will be visually inspected and leak-checked with a low-pressure gas.

The outer detector PMTs will be installed onto half-ladders and lowered into the water tank through one of the larger off-axis ports in the lid. The half-ladders are assembled together and secured to the roof and floor of the water tank and cables are run up to one of the top water-tank ports. Cables are sealed at these ports. The Tyvek reflectors that direct light lie on the floor, are hung vertically from the top of the ladders, and are stretched across the top of the ladders.

The detector is now ready to be filled with Xe, Gd-LS, and water. Xe filling must wait until the inner cryostat has been at vacuum long enough to get the residual gas content of the plastics to an acceptable level. Warm low-pressure Xe gas may be circulated to heat the plastic and enhance diffusion. This Xe would be pumped out and repurified or sold. Once plastic outgassing is at an acceptable level, the vessel will be filled with Xe gas and slowly cooled with LN2 until the Xe starts to condense. Filling continues until the Xe liquid is at the desired level. Gd-LS is received on site ready to use in 55-gallon drums, temporarily stored in the SSF. The Gd-LS and water must be filled at the same time to minimize stress on the acrylic walls. The levels do not need to match exactly, so one drum of Gd-LS can be added to the tanks one at a time as the water level rises. It is added by pressurizing the drums with nitrogen gas to force Gd-LS through the filling tubes at the overflow reservoir. Water is purified before it is added, and covered with a nitrogen head once 
the tank is filled. A flowing nitrogen head is maintained over the Gd- LS to protect it from both radon and oxygen.

While the main detector installation and assembly sequence described above are occurring, the support equipment and utilities for the experiment will be installed in Davis. This includes cryogenic cooling equipment, vacuum pumps, $\mathrm{LN}_{2}$ thermosiphons, Xe purification and circulation equipment, TPC HV supplies, PMT readout electronics, PMT HV supplies, calibration source tubes, connections and hardware, the emergency Xe recovery system, DAQ, and control systems. Details of these items are covered in other chapters.

The duration of the work in the SAL from the start of the assembly of TPC to the inner cryostat being sealed and ready to move underground is expected to be about seven months. Before underground installation work can begin, LUX must be decommissioned and removed (by very early 2017) and Davis infrastructure work described earlier in this chapter will need to be completed. LZ installation underground has an estimated duration of seven months from staging of the Gd-LS tanks to being ready to fill. 


\subsection{Bibliography}

[1] Leo A. Daly, (2015), 8600 Indian Hills Drive, Omaha, NE 06114.

[2] J. Street, R. Bunker, C. Dunagan, X. Loose, R. W. Schnee, M. Stark, K. Sundarnath, and D. Tronstad, Proceedings, 5th Topical Workshop on Low Radioactivity Techniques (LRT 2015), AIP Conf. Proc. 1672, 150004 (2015), arXiv:1506.00929 [physics.ins-det]. 


\section{Offline Computing and Software}

\subsection{Introduction}

This section describes the LZ offline computing systems, including offline software for the LZ experiment, the definition of the computing environment, the provision of hardware and manpower resources, and the eventual operation of the offline computing systems.

The offline computing organization provides the software framework, computing infrastructure, datamanagement system, and analysis software as well as the hardware and networking required for offline processing and analysis of LZ data. The system will be designed to handle the data flow starting from the raw event data files (the so-called EVT files) on the SURF surface RAID array, all the way through to the data-analysis framework for physics analyses at collaborating institutions, as illustrated in Fig. 11.1.1.

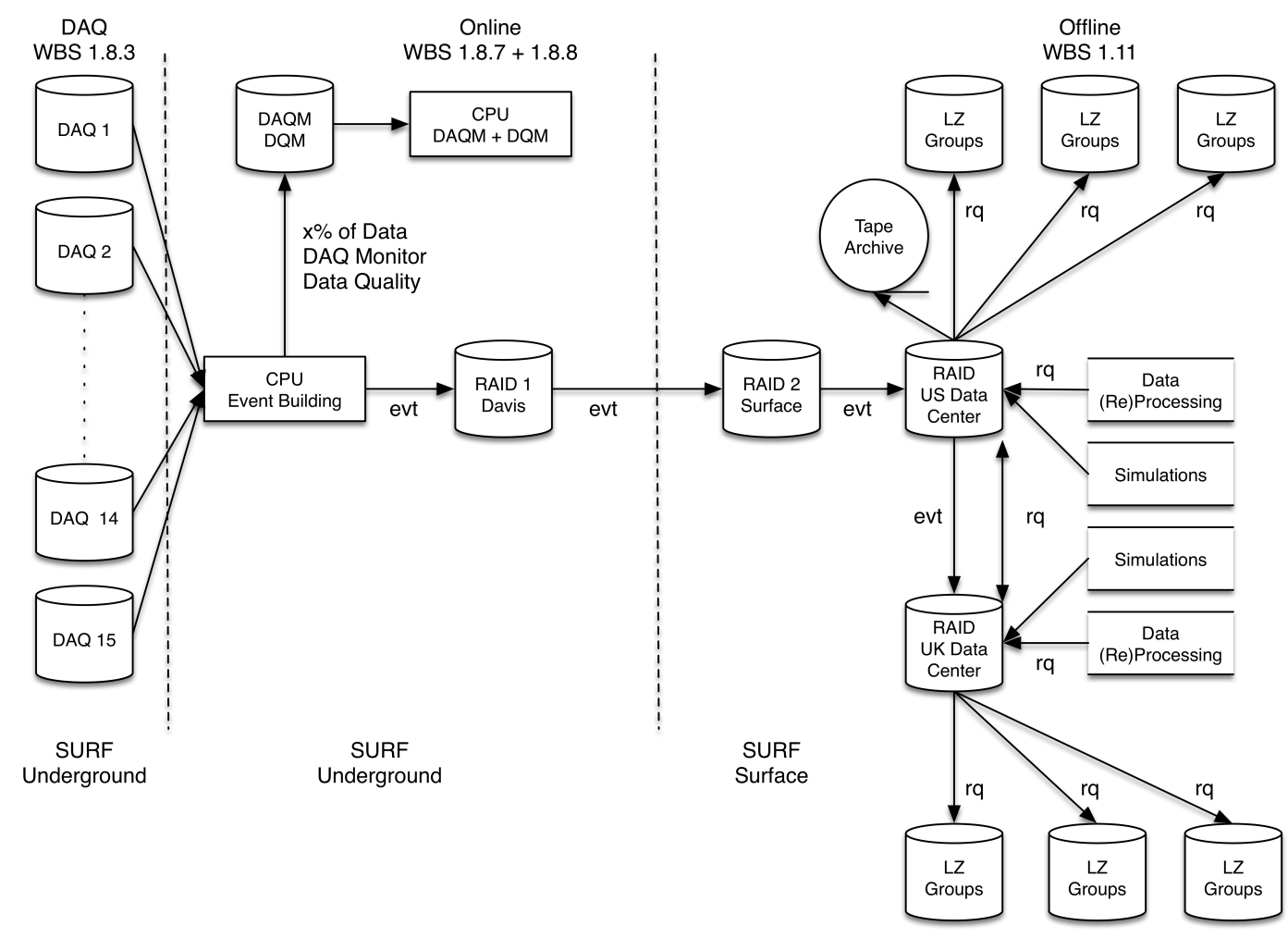

Figure 11.1.1: Schematic data-flow diagram for LZ. 


\subsection{Data Volume, Data Processing, and Data Centers}

The LZ data will be stored, processed and distributed using two data centers, one in the U.S. and one in the U.K. Both data centers will be capable of storing, processing, simulating and analyzing the LZ data in near real-time. The SURF surface staging computer ships the raw data files (EVT files) to the U.S. data center, which is expected to have sufficient CPU resources for initial processing. The National Energy Research Scientific Computing (NERSC) center at LBNL will contain the resources to act as the LZ U.S. data center.

The run processing software (LZ analysis package, or LZap for short) extracts the PMT charge and time information from the digitized signals, applies the calibrations, looks for S1 and S2 candidate events, performs the event reconstruction, and produces the so-called reduced quantity (RQ) files. The RQ files will be accessible to all groups in the collaboration and represent the primary input for the physics analyses.

The EVT and the RQ files are also mirrored from the U.S. data center to the U.K. data center (UKDC, located at Imperial College London) partly as a backup, and partly to share the load of file access/processing, giving better use of resources for all LZ collaborators. The EVT file transfer to the U.K. data center is done from the U.S. data center as opposed to directly from SURF, in order to avoid any reduction of the bandwidth available to ship the raw data from the experiment. Subsequent reprocessing of the data (following new calibrations, reconstruction and identification algorithms, etc.) is expected to take place at one or both centers, with the newly generated RQ files copied to the other center and made available to the collaboration.

From the hardware point of view, the system must be able to deal with the LZ data volume in terms of storage capacity and processing. Based on the LUX experience and appropriate scaling for LZ (in terms of number of channels, single/dual gains, event rates, etc.), the amount of WIMP search data generated in 1.000 days of $\mathrm{LZ}$ running is estimated to be $2.8 \mathrm{~PB}$. Including calibration runs, the total amount of LZ data produced per year is expected to be $1.3 \mathrm{~PB}$ to $1.4 \mathrm{~PB}$, depending on the amount and type of calibration data collected during yearly operation. This estimate assumes that about three hours of calibration data are collected each week. The breakdown of the different sources of data in LUX and their scaling to LZ is given in Table 11.2.1, which clearly shows that the data volume is dominated by the S2 signals.

Table 11.2.1: Daily raw and compressed data rates in LZ based on scaled LUX data. The scaling factors have been computed as follows: (a) PMT surface area ratio (2) times number of channels ratio - not including the low-gain channels (4), $2 \times 4=8$; (b) number of channels ratio (8) times rate ratio (13), $8 \times 13=104$; (c) liquid surface area ratio. The compression factor is taken to be 3 , as described in the text. (Abbreviations: $\mathrm{PE}=$ photoelectron, $\mathrm{SE}=$ single electron.)

\begin{tabular}{|l|r|r|r|r|}
\hline Source & LUX (GB/d) & Scaling factor & LZ (GB/d) & LZ compressed $(\mathrm{GB} / \mathrm{d})$ \\
\hline Single PE & 44.00 & $8^{(a)}$ & 352 & 117 \\
S1 & 0.24 & $104^{(b)}$ & 25 & 8 \\
S2 & 76.34 & $104^{(b)}$ & 7,939 & 2,646 \\
Uncorrelated SE & 20.00 & $9^{(c)}$ & 180 & 60 \\
\hline \hline Total & 140.58 & & 8,496 & 2,832 \\
\hline
\end{tabular}

The SURF staging computer will have a disk capacity of $192 \mathrm{~TB}$, enough storage for slightly more than two months (68 days) of LZ running in WIMP-search mode (at $2.8 \mathrm{~TB} /$ day), similar to its underground counterpart. The capacity of the staging arrays was based on the assumption that any network problems between SURF underground and the surface, or the surface to the outside, would take at most several weeks 
to be fully resolved. The remaining storage capacity can be used to store additional calibration data. The anticipated data rates imply that the network must be able to sustain a transfer rate of about $33 \mathrm{MB} / \mathrm{s}$. Such rates do not represent a particular challenge for the existing networks between SURF and LBNL or between LBNL and Imperial College. We note that the LUX experiment currently sends data from SURF to the primary data mirror at Brown University with an average throughput of $100 \mathrm{MB} / \mathrm{s}$.

From the current LUX experience, we expect that processing one LZ event should take no more than one second on one core (using a conservative estimate based on an Intel Xeon ES-2670 at $2.6 \mathrm{GHz}$ and $4 \mathrm{~GB}$ of RAM per core). Therefore, assuming a data-collection rate of $40 \mathrm{~Hz}, \mathrm{LZ}$ needs 40 cores to keep up with the incoming data stream in WIMP-search mode. For reprocessing, as analysis software and/or calibrations are refined, a larger number of cores will be needed to keep the processing time within reasonable limits (e.g., a factor of 10 more CPU cores allows reprocessing of a year's data in approximately one month).

Simulated data will also be created and stored. The top-level estimates in terms of storage capacity and CPU power for Monte Carlo simulations based on existing simulations are summarized in Table 11.4.1. They add up to a total data volume of about 85 to $100 \mathrm{~TB}$ and require approximately $10^{6} \mathrm{CPU}$ hours per year.

\subsubsection{The U.S. Data Center}

The U.S. data center will be located at NERSC/LBNL. Currently NERSC has three main systems: the Parallel Distributed Systems Facility (PDSF) and two CRAY systems. PDSF provides approximately 3,200 cores running Scientific Linux and is a dedicated system for astrophysics, high-energy and nuclear physics projects. The CRAY systems, Cori Phase 1 and Edison, provide approximately 52,000 and 153,000 cores, respectively. All systems can access the Global Parallel File System (GPFS) with a current capacity of about 8 PB, which is coupled to the High Performance Storage System (HPSS) with a 240 PB tape robot archive.

The LZ resources will be incorporated within the PDSF cluster. Our planning assumes modest needs for data storage and processing power for simulations, as described in Section 11.4, a rapid growth in preparation for commissioning and first operation, and then a steady growth of resources during LZ operations. The planned evolution of data storage and processing power at the U.S. data center is given in Table 11.2.2. The amounts of raw and calibration data per year are assumed to be 1,120 TB and $320 \mathrm{~TB}$, as described in the text, while the Monte Carlo data are ramped up to the maximum estimated capacity over the Project period ( 85 TB from Table 11.4.1, increased to $100 \mathrm{~TB}$ as a safety margin). Once the regular data-taking begins, the amount of simulations data is doubled in order to be able to accommodate both current and previous simulations. The processed data are assumed to be $50 \%$ of the Monte Carlo simulations and $10 \%$ of the data (assuming a slightly higher percentage of $10 \%$ in the size of the RQ-files compared to the $7 \%$ in LUX). The user data are assumed to be $50 \%$ of the Monte Carlo simulations in the years prior to experimental data, and $5 \%$ of the total data once LZ is running. The total disk space allocated includes a $20 \%$ safety margin with respect to the total amount of calculated data.

The CPU power is ramped up to reach the maximum of 350 cores needed by the simulations in two years (2016 and 2017) and is increased by 40 cores to 390 cores in the commissioning year (2020) in order to be able to continue Monte Carlo production in parallel with real-time data processing (which assumes $1 \mathrm{~s} /$ event at $40 \mathrm{~Hz}$ ). In the subsequent years of operation, the CPU power is increased by 440 cores per year, in order to be able to perform full data reprocessing in a reasonable time, in addition to the real-time data processing $\left(400+40\right.$ cores). The CPU estimates for simulations are based on the total number of $10^{6} \mathrm{CPU}$ hours from Table 11.4.1, which yields an average of about 115 cores/year in a steady state operation. Assuming that the simulations have a duty factor of about 1/6, i.e., run for 1 month and analyze/develop for 5 months, the total number of cores needed for simulations yields about 700, which is then equally divided between the U.S. and U.K. data centers. 
Table 11.2.2: Planned storage (in TB) and processing power by U.S. fiscal year at the U.S. and U.K. data centers.

\begin{tabular}{|l|r|r|r|r|r|r|r|r|r|r|r|}
\hline FY & 2015 & 2016 & 2017 & 2018 & 2019 & 2020 & 2021 & 2022 & 2023 & 2024 & 2025 \\
\hline Raw data & - & - & - & - & - & 560 & 1680 & 2800 & 3920 & 5040 & 6160 \\
Calibration data & - & - & - & - & - & 160 & 480 & 800 & 1120 & 1440 & 1760 \\
Simulation data & 40 & 80 & 80 & 100 & 100 & 200 & 200 & 200 & 200 & 200 & 200 \\
Processed data & 20 & 40 & 40 & 50 & 50 & 172 & 316 & 460 & 604 & 748 & 892 \\
User data & 20 & 40 & 40 & 50 & 50 & 55 & 134 & 213 & 292 & 371 & 451 \\
Total data & 80 & 160 & 160 & 200 & 200 & 1147 & 2810 & 4473 & 6136 & 7799 & 9463 \\
\hline \hline USDC: Disk space & 40 & 220 & 220 & 220 & 220 & 1360 & 3360 & 5360 & 7360 & 9360 & 11360 \\
\hline USDC: CPU cores & - & - & 175 & 350 & 350 & 390 & 830 & 1270 & 1710 & 2150 & 2590 \\
\hline \hline UKDC: Disk space & 150 & 220 & 220 & 270 & 650 & 1597 & 3260 & 4923 & 6586 & 8249 & 9913 \\
\hline UKDC: CPU cores & 150 & 175 & 350 & 350 & 350 & 390 & 830 & 1270 & 1710 & 2150 & 2590 \\
\hline
\end{tabular}

Data flow from the surface data cache onsite to NERSC is automated by use of the Spade system. Spade will transfer raw data files from SURF to NERSC within 15 minutes of file close by the DAQ. At NERSC, data will be written to NGF (NERSC Global File system) and automatically archived to HPSS (High Performance Storage System) tape. Data integrity will be verified by comparing checksums before and after transfer. From NERSC, data will automatically be transferred to the UKDC and verified by a second Spade data pipeline. Nominally (when network continuity is complete), all DAQ data files will be automatically replicated at the USDC and UKDC and backed up to HPSS within 30 minutes of DAQ file close. Although the U.S. data center will be located at NERSC, other U.S. computing resources are likely to be available to the collaboration. We will utilize resources available to the collaboration as appropriate.

\subsubsection{The U.K. Data Center}

The U.K. data center is led by Imperial College, and built on an infrastructure of GridPP [1,2] hardware and software. It currently runs Monte Carlo production and analysis jobs, providing the data products to the collaboration via GridFTP and XROOTD. In the remainder of the construction phase, it will run the end-to-end-simulation, event-reconstruction and analysis applications described in Sections 11.4 and 11.3. The output will be background and signal models which capture the full physics of detection and measurement with the precision needed to predict dark-matter sensitivity. Tests with LUX data, system-test data, and simulation in the mock data challenges will assure that a validated pipeline is in place ready for first underground data. Timely commissioning, calibration and performance monitoring, as well as evaluation of final physics results, will all be helped by the high throughput, high availability and flexible scaling afforded by a Grid computing model.

UKDC will provide redundancy and parallel capacity for carrying out the first level, near real-time processing of all LZ raw data (when needed), and carrying out reprocessing of the entire data set on timescales of several weeks. The U.S. and U.K. data centers will use the same analysis framework, access the same 
central databases, and run identical software. The single, central installation is distributed via CVMFS and the single, dedicated LZ virtual organization provides user authentication. Both are in place since June 2015, served from the University of Wisconsin.

Grid storage at UKDC was initiated in 2015 at $150 \mathrm{~TB}$, to store the results of background simulations informing this Design Report. As of October 2016 the storage is $220 \mathrm{~TB}$ and a gradual increase is planned in 2018 and 2019 to reach $650 \mathrm{~TB}$ at the time of detector deployment (see Table 11.2.2). A further $1 \mathrm{~PB}$ to 1.5 PB per year, dependent on realized background rates and trigger strategy, will be added to maintain a complete copy of acquired LZ data.

Production using GridPP nodes at Imperial started during the simulation campaign of autumn 2015. Since December 2015, LZ has been using CPU resources at 5 GridPP sites with up to 2,000 jobs running simultaneously. This proves that the UKDC can meet its requirement of reprocessing WIMP search and calibration from scratch in one-tenth of the acquisition time. Transfer rates from UKDC to USDC were tested during the same simulation campaign. Performance using the globus data transfer toolkit with no optimization comfortably exceeded the $80 \mathrm{MBps}$ required for a factor-of-two margin when mirroring acquired data.

The second large scale processing campaign was conducted in September 2016 for the validation of the background model. The simulations were performed for a modified geometry and for a larger number of detector components with increased statistics. A total of $5 \times 10^{10}$ events were generated within 411,000 CPU hours in one month. These files are using $85 \mathrm{~TB}$ of disk space. In addition, the UKDC has been used on request, for specific studies thorough the year using significant CPU hours $(283,000)$ and disk storage $(>50 \mathrm{~TB})$. The requests are currently managed via a custom job submission system using Google sheet documents. This system will be soon superseded by a production job submission system currently in development.

The DIRAC system of middleware [3] is used to manage LZ data and computation on GridPP, providing command line, web portal, and Python API interfaces for submission, monitoring and accounting. Development has begun of tools to streamline building, editing, and merging the output of LZ jobs, using the object-oriented Python package Ganga [4] (the same approach as the LHCb collaboration, which has long experience running the Gaudi framework on the Grid). The LZ-specific aspects of UKDC are handled by a researcher at $50 \%$ FTE and a programmer at $10 \%$ FTE. Support of non-LZ-specific systems, which can include development of Grid infrastructure software as required, is provided by the GridPP team at Imperial.

\subsection{Analysis Framework}

The key element for the LZ data processing is the analysis framework (AF), which will contain some standard processing modules and will also allow users to put together modular code for data analysis to automatically take care of the basic data handling (I/O, event/run selection, etc.). A dedicated task force was created at the end of July 2014 to evaluate various options for LZ. In terms of existing frameworks, two ROOTbased frameworks were considered: Gaudi (developed at CERN and used by ATLAS, LHCb, MINERvA, Daya Bay, etc.) [5] and art (developed at Fermilab and used by MicroBooNE, NOvA, LBNE, DarkSide50, etc.) [6]. In parallel, the possibility of evolving the framework developed for LUX, which is based on Python scripts and a MySQL database, and supports modules written in Python, C++/ROOT or MATLAB was also evaluated. For completeness, developing a new framework from scratch was also considered as another alternative. However, given the amount of effort this would have required (of the order of at least several FTE-years based on estimates from other experiments such as CMS, Double Chooz, MiniBooNE, T2K, etc.), this option was dismissed.

Input from the entire collaboration regarding the desired features for the LZ framework was collected and organized by the task force, and the three candidate frameworks were evaluated and ranked against this list. In addition, presentations and live demos for each of the three contenders were given during the regular 
task force meetings, while core frameworks were also installed on different test platforms to evaluate the respective installation processes.

The task force ranked unanimously Gaudi in first place, followed by a distant second by art and LUX in a close third position. The recommendation to adopt Gaudi as the LZ analysis framework was approved by the collaboration in April 2015. With the adoption of Gaudi as the foundation for the analysis framework, the first milestone (see Table 11.6.1) was the creation of a First Release, which was delivered to the collaboration in February 2016. This release was based on the Gaudi Hive branch of the Gaudi code, which is specially designed to support multi-threading within the framework itself. Goal of the release was to demonstrate end-to-end processing of mock (user-generated) events through the entire framework chain, and to support the development of different modules from a team of LZ collaborators.

A Physics Integration Release is currently under production and will be delivered to the collaboration in November 2016. The features of the framework needed for this release are:

- Definition of the transient data model for DAQ and Physics data;

- Creation of the necessary modules to read and write raw DAQ data based on the above transient model;

- Provision of a programming and build environment in which the physicists can adapt existing modules and develop new ones to run within the framework;

- Access to non-DAQ data, such as calibrations, slow control variables, etc., from a conditions database.

Before any of those can be achieved it is necessary to be able to build a version of Gaudi within the LZ infrastructure (see 11.5). This has been done by importing the Gaudi Hive codebase into our own GitLab in order to manage the impact of any changes made to Gaudi Hive in the future and building it from that source.

The definition of the transient data models are well underway. The DAQ one is a straightforward representation of the objects read out by the DAQ. The physics one requires more development as it has to capture the objects and relationships of derived quantities and these have not all yet been defined. The creation of the input and output modules has begun. Gaudi has a well established mechanism for extending its input and output modules to handle custom formats and we plan to use the experience of other experiments, such as Daya Bay, with this task to speed its development.

The Gaudi codebase presently supports two types of build systems, the legacy one based on CMT [7] and a new one base one CMake [8]. LZ has decided to use the CMake-based version for its build and is now using this as a model to develop the programming and build environment for developing modules for its framework. The creation of the input and output modules is being used as the test bed on which this mechanism can be developed.

Gaudi itself has a fully developed fault handling system so that any problems that arise during processing can be evaluated and either stop the processing altogether, stop the processing for a single event or allow the event to continue processing. This, together with its comprehensive logging system, means that is straight forward to decide which processings are successfully, which need to be redone once the cause of the fault has been addressed and which are irrecoverable.

Wherever possible, we anticipate that existing code from the successful LUX and ZEPLIN experiments will be adapted and optimized for use within the LZ analysis framework. The LZ processing and analysis codes will be written to be as portable as possible to ensure straightforward running on both Linux and OSX platforms for those groups who wish to do analysis in-house in addition to (or instead of) running codes on the data centers.

All non-DAQ data, i.e. any data recorded or developed that is not read out by the DAQ with each event, will be stored in a database (DB) known as the "conditions database". The challenges of this database are 
that it not only needs to understand the interval of validity for each piece of data, but also needs to support versioning of that data for instances such as when better calibrations are available. This problem is not unique to LZ and therefore it was decided to used the DBI package developed for the MINOS experiment. Not only does this allow for intervals of validity and versioning, it also supports a hierarchy of data sources. This means that during development of code or calibrations it is possible to specify an alternate DB to be used for certain values which supersede the values in the main underlying DB. This also allows for the validation of new entries before they are inserted into the main conditions DB.

\subsection{Simulations}

Detailed, accurate simulations of the LZ detector response and backgrounds are necessary, both at the detector design phase and during data analysis. Current LZ simulations use the LZSim package, which in turn is based on the existing LUXSim software package [9], originally developed for the LUX experiment. The LZSim codebase is entirely separated from LUXSim, although several of the developers contribute to both. It is managed through the collaboration's git repository. This software provides object-oriented coding capability specifically tuned for noble liquid detectors, working on top of the Geant 4 engine [10]. LZ intends to further update its simulation software in 2017 with two significant changes: first a switch to the most recent version of Geant4, 10.2, in order to take advantage of a number of critical improvements to the code and physics lists. Second, a recast of the LZSim framework into the more generalized BACCARAT framework, which is also based on LUXSim but not tied to any detector-specific or legacy code.

All LZ simulations will be integrated into the broader LZ analysis framework, from production to validation and analysis. The framework will in fact largely be developed using simulation output until detector data is available. Two output formats are supported, a raw simulation output at the GEANT interaction level, and a reduced tree format at the event level. Both use the ROOT format.

The simulations group is organized into several distinct areas of technical expertise, a structure reflected in the organization of this task. In addition to the computing-centric approach described here, physics output coordination is managed within a working group of the entire scientific collaboration. Tasks include:

(a) Simulation software packages maintenance, development, and collaboration support;

(b) Definition, maintenance, and implementation of an accurate detector geometry;

(c) Maintenance and continued improvement of the micro-physics model of particle interactions in liquid xenon, as captured in the NEST package [11];

(d) Detector response implementation - which transforms the ensemble of individual GEANT4 photon hits at the PMTs to produce an event file of the same format and structure as in the data.

(e) Generators for relevant event sources in LZ for both backgrounds and signal;

Table 11.4.1 shows the computing needs estimates for all simulation tasks, based on recent production data. These needs are on the order of about $100 \mathrm{~TB}$ of disk storage and a total of $10^{6} \mathrm{CPU}$ hours per year, mostly concentrated in short 1-to-4-week periods of burst activity. Both hard disk storage and CPU needs are dominated by background simulations, including a $50 \%$ contingency to account for the need to repeat and/or compare some studies. There is also a provision for very-high statistics data sets for three major detector components, in order to produce a high-granularity map of the background spatial profile inside the TPC. These data sets are only kept in their most reduced format in order to conserve storage resources. For all other simulations, both reduced files and raw GEANT4 output files (translated to ROOT format) are kept. The total also includes optical simulations, which only provide photon collection results and are less demanding in terms of resources, calibration simulations, necessary to validate calibration data and early 
R\&D efforts, and a number of ad-hoc small simulation tasks all grouped together. Resource time profiles for different simulation categories feed into the higher level computing estimates.

Table 11.4.1: High-level summary table of LZ simulations projections for computing power and storage needs, based on the simulation campaign of autumn 2015. The dominant part of the resources is required for detailed background simulations of detector components, including $50 \%$ contingency for updates and comparative studies. Storage needs are contained within a 100 TB envelope for the duration of the project through commissioning (another 100 TB is allocated for processed and user data). Corresponding computing power is relatively modest as a yearly average, however most of the needs occur in short bursts with a 1-4 week timescale.

\begin{tabular}{|l|r|r|r|}
\hline Simulation type & $\begin{array}{r}\text { Raw ROOT } \\
\text { files (TB) }\end{array}$ & $\begin{array}{r}\text { Reduced ROOT } \\
\text { files (TB) }\end{array}$ & $\begin{array}{r}\text { CPU needs } \\
\text { (core-hours) }\end{array}$ \\
\hline Background simulations & 30 & 2 & $1.0 \times 10^{5}$ \\
High-statistics background map & 0 & 17 & $8.0 \times 10^{5}$ \\
Contingency / repeats & 15 & 2 & $5.0 \times 10^{4}$ \\
\hline Optical simulations & 2 & 0 & $5.0 \times 10^{3}$ \\
Calibration simulations & 10 & 1 & $3.3 \times 10^{4}$ \\
Other misc. simulations & 5 & 0.5 & $1.7 \times 10^{4}$ \\
\hline \hline Totals & 62 & 23 & $1.0 \times 10^{6}$ \\
\hline
\end{tabular}

\subsection{Software Infrastructure}

All LZ software is centrally maintained through a software repository based on GitLab [12], which is currently operating at the University of Alabama. The repository is backed up daily and the snapshots are retained for 15 days. GitLab implements excellent tools for release management and code review. A GitLab snapshot from a recent developement cycle of LZSim is shown in Figure 11.5.1: different developers were working simultaneously on a round of updates to the detector geometry. After extensive testing, the updates were eventually merged into the master branch and folded into a tagged release. GitLab also offers a continuous integration tool, allowing for automatic testing and installation of the offline codebase on the U.S. and U.K. data center servers. Build automation is inherited from the Gaudi infrastructure and supported via CMake and cmt.

Release Management and Version Control standards were strictly enforced from a very early stage of the project to ensure sharing, verifiability and reproducibility of the results. Each code release undergoes a battery of tests before being deployed to production. For every update cycle, the code is inspected by a moderator, who checks its integrity and reports possible inconsistencies or conflicts. The package is then installed and executed on each data center and checked for functionality. If one test fails, the code update is rejected. Release management also acts as a bridge between development and production: this ensures that all the changes are properly communicated and documented, to achieve full reproducibility.

A comprehensive validation suite is being developed, and each class of software update has a well-defined set of tests, depending on the nature of the change and on its magnitude. For example, when the detector 


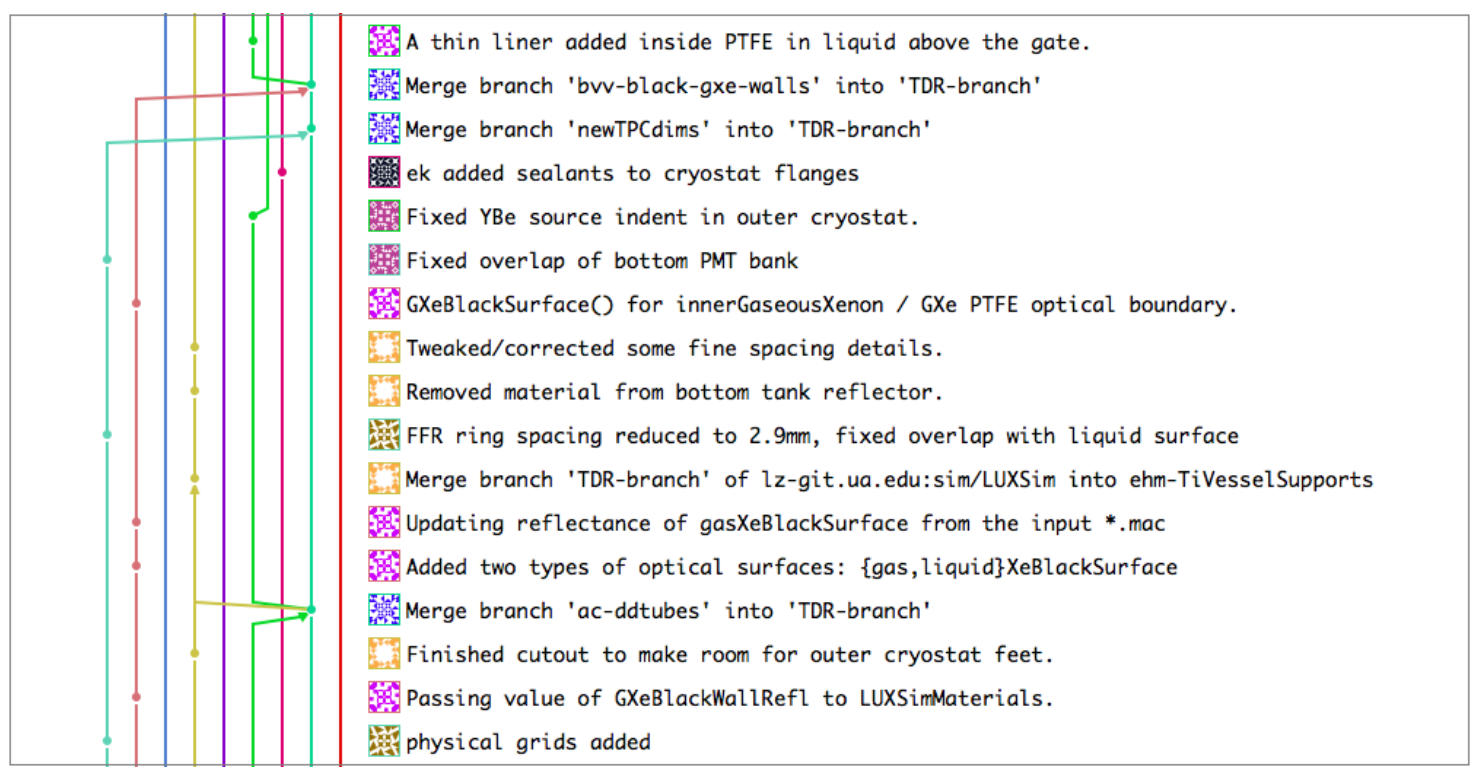

Figure 11.5.1: Parallel development workflow in GitLab: several contributors were updating the LZSim detector geometry simultaneously. Each vertical line corresponds to a different development branch. After extensive testing, every update was eventually merged to the master branch.

geometry in LZSim is updated, the standard overlap checks and geantino tests provided by Geant 4 are always performed. If the update leads to a tagged release, a large statistics of photons is simulated in every part of the detector and the light collection carefully examined (light collection is the parameter most sensitive to geometry changes). Based on the successful experience from the Fermi-LAT software validation suite [13], the results from these simulations are compared via a mono-dimensional Kolmogorov-Smirnov test. If discrepancies are found, they are reported back to the developers, who are tasked with explaining the changes or fixing the underlying errors before the code is deployed to production.

Software distribution is achieved via CernVM File System (CVMFS) [14]. CVMFS is a CERN-developed network file system based on HTTP and optimized to deliver experiment software in a fast, scalable, and reliable way. Files and file metadata are aggressively cached and downloaded on demand. Thereby the CernVM-FS decouples the life cycle management of the application software releases from the operating system. The LZ CVMFS server is hosted at University of Wisconsin, Madison and is visible to all the machines in the U.S. and U.K. data centers, and to most computing centers available to the collaboration (Wisconsin, SLAC, Edinburgh, Sheffield, etc.). It can be loaded to each collaborator's personal laptop by installing a FUSE client. All the LZ software releases and external packages are currently delivered via CVMFS: this ensures a unified data production and analysis stream, because the data centers access exactly the same versions of the same executables, removing possible dependencies on platform and configuration.

CERN software is also made available to the collaboration via CVMFS. Besides the above-mentioned Geant4 and Gaudi, we use several external packages, including CLHEP, ROOT and AIDA. The environment defining a specific combination of external packages is inherited via LCGCMT [15] and shares a build automation infrastructure with Gaudi. Again, delivering the external packages via CVMFS removes unwanted dependencies on architecture and environment. However, in order to maximize software availability to each collaborator, we also plan to deliver precompiled tarballs of LZ tagged releases and external packages for individual download. 
Cybersecurity risks posed to the offline computing systems relate to the experiment's data and information systems. Much of the LZ computing and data will be housed at major computing facilities in the United States (NERSC/LBNL) and U.K. (Imperial College), which have excellent cybersecurity experience and records. Specific risks posed to the LZ project relate to data transfer (in terms of data loss or corruption during transfer) and malicious code insertion. File checksums will mitigate the danger of loss or corruption of data during transfer, while copies at both the U.S. and U.K. data centers provide added redundancy. Moreover, CVMFS features robust error handling and secure access over untrusted networks [16]. By requiring digital signatures and secure hashes on all distributed data, CVMFS also provides a strong security mechanisms for data integrity. Malicious code insertion can be mitigated by monitoring each commit to the code repository by the offline group and requiring username/password authentication unique to each contributor to the code repository. A comprehensive policy for release management and continuous testing will be the key factor in preventing malicious software from being deployed to production.

\subsection{Schedule and Organization}

Offline software by its nature is heavily front-loaded in the schedule. To enable the scientists to commission the LZ detector, the software for reading, assembling, transferring, and processing the data must be in place before detector installation. This implies, in particular, that the data transfer, offline framework, and analysis tools themselves will have been developed, tested, debugged, and deployed to the collaboration. We rely on the collaboration's existing experience with the LUX experiment and others (Daya Bay, Double Chooz, Fermi, LAT, etc.), which routinely handled similar challenges. Key offline computing milestones are summarized in Table 11.6.1.

The decision on the choice of analysis framework for LZ has been taken in April 2015. The first version of the framework with a minimal number of modules was released in early March 2016. This will be followed by the first physics integration release planned for November 2016. This version includes all necessary modules for real-time processing (i.e., hit-finding algorithms, calibration constants modules, S1/S2 identification, event reconstruction), as well as a fully integrated simulations package (i.e., from event generation through photon hits, digitization, trigger, and data-format output).

Table 11.6.1: Key offline computing milestones.

\begin{tabular}{|c|l|c|}
\hline Date & Milestone & Status \\
\hline Mar. 2015 & Analysis Framework decision & Done (Apr. 2015) \\
\hline Feb. 2016 & First Analysis Framework release & Done (Mar. 2016) \\
\hline Nov. 2016 & First physics integration release & Done (Nov. 2016) \\
\hline Sep. 2017 & First mock data challenge & \\
\hline Feb. 2018 & OCS FDR - Final Design Review & \\
\hline Aug. 2018 & Second mock data challenge & \\
\hline Nov. 2019 & Third mock data challenge & \\
\hline Dec. 2019 & Full software release for commissioning & \\
\hline
\end{tabular}


The first mock data challenge (September 2017) will test both the data flow (transfers, processing, distribution, and logging), as well as the full physics analysis functionality of the framework, separately. The first few weeks of LZ commissioning (calibrations included) will be simulated; participants should be able to quantify detector response and main backgrounds, based on simulated data. The second data challenge (July 2018) will be dedicated to testing the entire data chain. It will contain 6 months of simulated data with physics signals, including calibration data. Participants should be able to establish a detailed background model and perform low-energy calibrations (ER/NR). The third data challenge (October 2019) will test the complete analysis strategy and is expected to validate the readiness of the offline system just before the LZ cool-down phase. It will include 1,000 days of simulated data including physics signals. No MC truth will be available to participants and physics signals will be known only to a subset of organizers. It will simulate the analysis for the first LZ science paper, including possible blinding/salting plans.

Offline computing will be led by physicists experienced in software development and use and a computing professional from LBNL. The software professional will also liaise with NERSC for collaboration on providing LZ compute resources - in particular, provisioning and/or allocating of network, CPU, disk, and tape resources sufficient for LZ collaborators to transfer, manage, archive, and analyze all data for the experiment. The infrastructure software effort will also involve professional software engineering from LBNL. This person will provide technical leadership, oversight, and coordination of LZ collaboration efforts on infrastructure software as well as the design, implementation, testing, and deployment of critical LZ infrastructure components. LZ infrastructure software includes data management and processing, offline systems and monitoring, offline interfaces to LZ databases, and the analysis framework. The remainder, and bulk, of the software is a collaboration responsibility. Software for simulation, analysis, monitoring, and other tasks will be written and maintained by collaboration scientists. 


\subsection{Bibliography}

[1] P. J. W. Faulkner et al. (GridPP), J. Phys. G: Nucl. Part. Phys. 32, N1 (2006).

[2] D. Britton et al., Philos. Trans. R. Soc. London, Ser. A 367, 2447 (2009).

[3] A. Tsaregorodtsev et al., Journal of Physics: Conference Series 119, 062048 (2008).

[4] J. Mościcki et al., Comput. Phys. Commun. 180, 2303 (2009).

[5] G. Barrand et al., Comput. Phys. Commun. 140, 45 (2001).

[6] C. Green, J. Kowalkowski, M. Paterno, M. Fischler, L. Garren, and Q. Lu, Proceedings, 19th International Conference on Computing in High Energy and Nuclear Physics (CHEP 2012), J. Phys. Conf. Ser. 396, 022020 (2012).

[7] C. Arnault and V. Garonne, "Configuration and Management Tool," (2015), accessed: 2015-12-15.

[8] “CMake," (2015), accessed: 2015-12-15.

[9] D. S. Akerib et al. (LUX), Nucl. Instrum. Meth. A675, 63 (2012), arXiv:1111.2074 [physics.data-an].

[10] S. Agostinelli et al. (GEANT4), Nucl. Instrum. Meth. A506, 250 (2003).

[11] M. Szydagis, A. Fyhrie, D. Thorngren, and M. Tripathi (NEST), Proceedings, LIght Detection In Noble Elements (LIDINE2013), J. Instrum. 8, C10003 (2013), arXiv:1307.6601 [physics.ins-det].

[12] “Gitlab Inc.” (2015), accessed: 2015-12-07.

[13] W. B. Atwood et al. (Fermi-LAT), Astrophys. J. 697, 1071 (2009), arXiv:0902.1089 [astro-ph.IM].

[14] J. Blomer, C. Aguado Sanchez, P. Buncic, and A. Harutyunyan, Proceedings, 18th International Conference on Computing in High Energuy and Nuclear Physics (CHEP 2010), J. Phys.: Conf. Ser. 331, 042003 (2011).

[15] "LCG Software Elements," (2015), accessed: 2015-12-10.

[16] D. Dykstra and J. Blomer, Proceedings, 20th International Conference on Computing in High Energy and Nuclear Physics (CHEP 2013), J. Phys. Conf. Ser. 513, 042015 (2014). 


\section{Simulations, Requirements, and Detector Performance}

This chapter ties together the previous descriptive chapters and describes the performance we expect from the LZ apparatus. Our estimates of performance are based on detailed simulations, which have been substantially overhauled since completion of the LZ Conceptual Design Report [1]. The LUX collaboration has performed extensive high-statistics calibrations of ER background [2], NR signal [3], and implemented these calibrations in an improved analysis using the profile likelihood ratio technique [4] of the LUX WIMP search data [5].

We have incorporated the performance improvements made by LUX, which change the sensitivity in two principal manners:

1. The LZ sensitivity to low-mass WIMPs and to nuclear recoils from solar ${ }^{8} \mathrm{~B}$ neutrinos is much improved.

2. The LZ sensitivity to sources of ER background, including, prominently, the "quiet" beta decay of daughters that trace their parentage to radon impurities, is much reduced.

\subsection{Simulations}

In this section we describe in detail the simulations used to develop the background and sensitivity studies presented in this report. The simulations were performed using LZSim, an offshoot of the LUXSim package originally developed for the LUX experiment and based on the GEANT4 particle physics simulation software [6,7]. Designed specifically for low-background detector modeling, LZSim generates events and records particle interactions on a detector geometry component-by-component basis, but with an infrastructure independent of the detector geometry.

The LZSim infrastructure allows the user to define any detector component as a GEANT4 sensitive detector at run-time with a macro command. It incorporates internal bookkeeping to automate the generation of backgrounds arising from a variety of event generators, each with intensities set by the user at run time, and with a time-ordered stochastic primary event record. The geometry components are customized using coding techniques familiar to users of the base GEANT4 code. The event generators can be based either on the internal GEANT4 classes or created from scratch by the users. LZSim automatically records quality control information to a header in each output file to establish a record of how the data was generated.

We use GEANT4 version 4.9.5, the physics list QGSP_BIC_HP, and the libraries of CLHEP version 2.1.0.1. LZSim provides the option to incorporate the NEST model that describes ionization and scintillation formation for NRs and ERs [8,9]. Currently we instead pass the output from LZSim to a standalone version of the NEST model that incorporates the latest results from the LUX experiment $[2,5]$. 


\subsubsection{Geometry construction}

A detailed model of the LZ detector geometry was created within the LZSim framework according to CAD drawings of the detector. Components with significant mass or high amounts of radio-impurities as well as those located very close to the active xenon target are included. Elements that influence light collection and their respective optical properties are also described in the model.

Our model describes the detector from the outside in, with a few exceptions, nesting each successive volume or component within the one preceding it. The outermost volume is the steel water shielding tank. Placed within the water of the shielding tank are the major components of the outer detector of Chapter 4, including the segmented acrylic vessels, liquid scintillator, foam displacer, reflectors and R5912 PMTs. Services for the TPC such as the cryostat support stand, cathode high voltage conduit and thermosyphon and PMT cabling conduits require penetrations in the acrylic tanks that can impact veto performance and are therefore implemented in the detector model. Conduits that contain multiple or complex materials such as coaxial cable, gaseous or liquid xenon, and vacuum are modeled by a single material which represents the average density and composition of all materials in that conduit. Angled and horizontal neutron calibration tubes and a port for the YBe source are included for calibration source studies.

Located within the outer detector is the titanium cryostat, built according to the engineering models, as shown in Fig. 12.1.1. An inner cryostat vessel is contained within the outer cryostat vessel, also made of titanium and built to engineering specifications. The model includes multi-layer insulation between these two volumes, in addition to vacuum. No optical properties are defined between these regions as no photons are expected to be produced here.
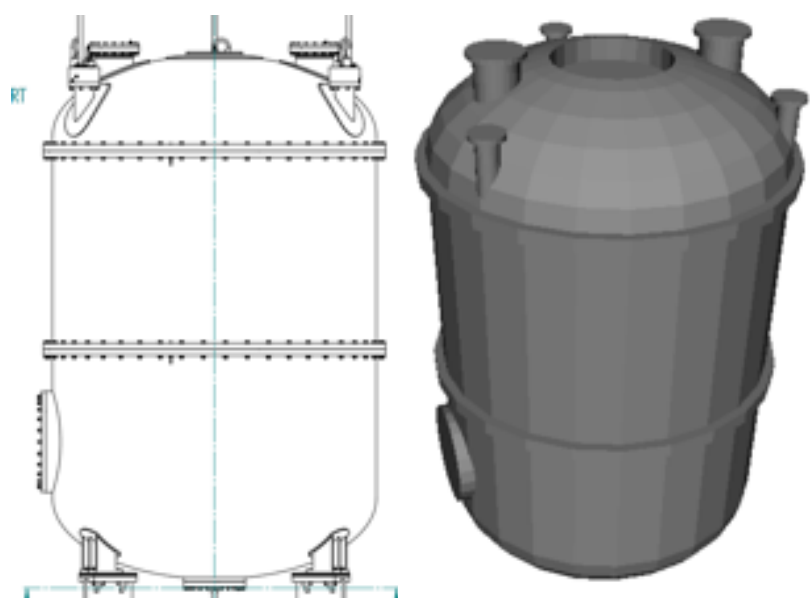

Figure 12.1.1: Engineering drawing and simulation geometry of the outer cryostat. tion from ionization (S2) signals would be caused by a WIMP, is nested inside the skin volume. A rendering of the TPC is shown Fig. 12.1.2. The liquid xenon in the TPC is divided into two volumes. The active volume contains all the liquid xenon above the cathode, where well-reconstructed S1/S2 events occur. The second volume is the reverse field region (RFR), below the TPC cathode. Energy depositions in this volume cause an S1 signal, but no S2. Nevertheless, sometimes an RFR S1 signal becomes associated with an unrelated S2 signal, resulting in a class of events known as "gamma-X". A third xenon volume, the gaseous xenon above the liquid where electroluminescence develops for the S2 signal, is also defined.

A cylinder of PTFE with extremely high diffuse reflectivity forms the TPC wall. Field shaping rings and grading resistors in the walls are represented. TPC components including the bottom shield grid, cathode 
grid, gate grid, and anode grid, according to the design described in Table 3.6.3, are represented. Optical boundaries between the liquid xenon and the PTFE walls as well as between the liquid xenon and the stainless steel of the grid wires are included to describe the light collection properties of the detector.

At the top and bottom end of the TPC cylinder are the PMT arrays, containing representations of each R11410 PMT that will be used in the LZ experiment. The PMTs are described as a stainless steel shell with vacuum inside. Materials representing the dynode chain are included to model the radioactivity of those components.

The face of the PMT is a quartz window with an additional thin layer of quartz buried within to represent the photocathode. The window and photocathode volumes are divided to model cases where a photon reflects off the front face of the PMT and fails to pen-

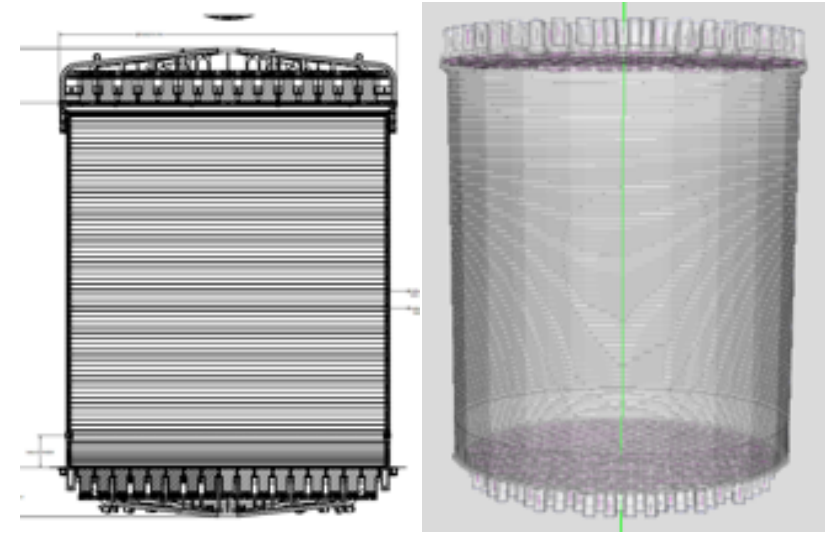

Figure 12.1.2: Engineering drawing and simulation geometry of the TPC etrate to the photocathode.

Optical interfaces at the top of the TPC between the PMT windows and gaseous xenon in the extraction region, and at the bottom array between the PMT windows in liquid xenon, are described so GEANT4 appropriately handles the reflection and transmission of photons. The PMT photocathode is defined as a GEANT4 sensitive detector to collect optical photons. The PMT array volumes also contain the support plates and support trusses that provide the mechanical support for the PMTs. Figure 12.1.3 shows a comparison between the current engineering design and the simulated geometry of the bottom PMT array.
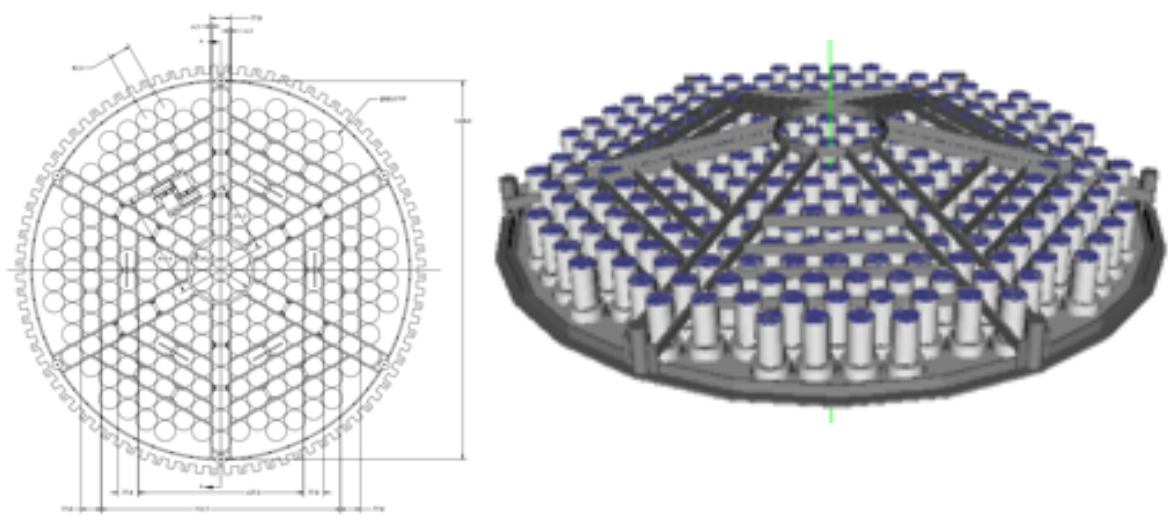

Figure 12.1.3: Engineering drawing and simulation geometry of the bottom PMT array and support truss. The skin PMTs that mount to this truss are not shown.

\subsubsection{Event Generators}

A variety of software packages are employed to simulate the physics of signals and backgrounds that induce responses in the LZ detector. The event generators which describe the WIMP signal and neutrino physics are derived from the references cited in Chapter 2. Here we discuss the event generators employed to describe various types of background phenomena in the $\mathrm{LZ}$ detectors. 


\subsubsection{Neutron production in detector materials}

Neutrons emitted from radioactive processes in the material near to the LZ liquid xenon detector can create isolated nuclear recoils that might fake the recoils expected from WIMPs. To simulate neutron backgrounds from radioactivity (the ${ }^{238} \mathrm{U},{ }^{235} \mathrm{U}$ and ${ }^{230} \mathrm{Th}$ decay chains), LZSim uses input neutron spectra as calculated with the SOURCES-4A [10] package.

The SOURCES-4A code calculates neutron yields and spectra from spontaneous fission, $(\alpha, n)$ reactions and delayed neutron emission due to the decay of radionuclides. Its library contains all alpha emission lines from known radioactive isotopes. The code takes into account the energy losses of alphas, cross-sections of $(\alpha, n)$ reactions and the probabilities of nuclear transition to different excited states. We use an option for a thick target neutron yield allowing for calculation of neutron yields and spectra under the assumption that the size of a material sample exceeds significantly the range of alphas.

The original SOURCES-4A code has been modified [11-13] to extend the energy range of alpha particles to $10 \mathrm{MeV}$ and to include $(\alpha, n)$ cross-sections and transition probabilities to excited states for most isotopes relevant to underground rare event experiments, based either on measurements or on EMPIRE1.19 [14].

The neutron spectra from SOURCES-4A are implemented as generators in LZSim, allowing any detector component to become a source of neutrons. For each component of interest, we simulate concentrations of $10 \mathrm{ppb}$ of uranium or $10 \mathrm{ppb}$ of thorium; these concentrations are then scaled to match the results of the materials screening. The uranium decay chains are split into early and late branches, and the ${ }^{210} \mathrm{~Pb}$ sub-chain is calculated separately.

The spontaneous fission (s.f.) process is treated separately to exploit the ability of LZ to reject decays producing multiple neutrons and gammas, such as those produced in s.f. events. The SOURCES-4A package generates individual neutron spectra without accounting for simultaneous multiple neutrons and gamma rays. A special generator was developed to accurately simulate multiple neutrons $\left(2.01\right.$ on average for $\left.{ }^{238} \mathrm{U}\right)$ and gammas ( 6.44 on average for ${ }^{238} \mathrm{U}$ ) in s.f. events. Accounting for multiple neutrons and gammas permits accurate description of multiple simultaneous signals in the LZ detector, and accurate accounting of the rejection of events with multiple signals. Most spontaneous fission events, particularly those in materials close to the LXe target, are rejected through their tendency to produce multiple hits.

\subsubsection{Muon-induced neutrons}

Energetic neutrons can be produced by atmospheric muons that penetrate to the rock around the Davis Cavern. Simulations of this process commence with the selection of muons with positions, directions, and energies sampled according to the MUSUN code [15] after transport using standalone MUSIC code [16]. MUSUN has been integrated into LZSim as a particle generator in such a way that events are generated from the surface of a cuboid surrounding the detector.

Muons sampled with the MUSUN code are then passed to GEANT4 which transports them and their secondaries including neutrons to the detector. All processes relevant to muon, photon, electron, and hadron interactions are included and the models are equivalent to those used in the GEANT4 physics list called Shielding, recommended by the GEANT4 developers for this application.

\subsubsection{Gamma activity in large detector components}

Background ER events from ${ }^{40} \mathrm{~K},{ }^{60} \mathrm{Co}$, and the ${ }^{238} \mathrm{U}$ and ${ }^{232} \mathrm{Th}$ decay chains are also modeled using a particle generator developed in LZSim. Ions of the parent isotopes are positioned in a component and the full decay chain is simulated according to the physics described by the GEANT 4 radioactive decay data libraries. Crucially, the decay chain is produced in equilibrium, which allows splitting of the chain by individual isotopes during analysis. Thus, only one simulation is required, independent of the relative decay 
rates of individual radioactive isotopes within the chain. As for the neutron studies, fixed activities are simulated for each component, which are then renormalized based on materials screening results.

\subsubsection{Gammas from the cavern rock}

The external background from gammas in the rock walls of the cavern are also assessed using the radioactive decay chain generators described in the previous section. However, due to the large number of primary decays required to accumulate events in the active xenon, event biasing is used to boost statistics. This involves saving events at decreasing distances from the target and feeding them back into LZSim multiple times as primary particles. By default, LZSim records all hits within a detector component with a defined record level, but for these simulations the structure has been modified so that the output is recorded for tracks according to the distance from the center of the TPC. Additionally, modifications were made to allow the LZSim binary output files to be input as primary particles.

\subsubsection{Benchmark points}

A new generator has been developed to allow the assessment of the impact of the radioactivity of small size components (e.g. sensors) quickly. LZSim was designed to primarily distribute primary events throughout a given volume. Our new generator can associate radioactive events with a specific geometric location (the 'Benchmark Point') within the LZSim geometry.

\subsubsection{From energy deposition to signals}

The event generators described in the previous section are used to generate different radioactive decay products. These particles are tracked by GEANT4 as they deposit their energy in different volumes of the geometry. LZSim allows any part of the volume to be a sensitive detector, and for these simulations, we record all depositions in the TPC, as well as associated energy depositions in the skin region and the outer detector. Among the data saved in each volume for each event are the locations, times, and magnitudes of energy deposits made by various particle types.

In the skin and TPC liquid xenon volumes, energy depositions within $400 \mu \mathrm{m}$ are clustered (to encompass the largest possible ER and NR track sizes) and categorized as either ER or NR depending on the interaction. The result is a list of energy-deposition clusters from a given particle type (ER or NR). The energy and associated particle type of each cluster as well as the local electric field are then fed into the NEST (Noble Element Simulation Technique) $[8,9,17]$ package which stochastically computes the number of expected photons and electrons produced at each cluster.

NEST models the scintillation light and ionization charge yields of nuclear and electron recoils as a function of electric field and energy or $\mathrm{d} E / \mathrm{d} x$. NEST also models the drift, diffusion, absorption, extraction, and electroluminescence of the electrons as they move through the liquid and gas. "NEST" refers both to a collection of microscopic models for energy deposition in noble elements and to the Monte Carlo simulation code that implements these models. NEST provides mean yields and intrinsic fluctuations due to the physics of excitation, ionization, and recombination, including both Gaussian and non-Gaussian components of the energy resolution.

The NEST methodology was initially trained on data from a small dual-phase detector from Case Western Reserve University (Xed), which yielded comprehensive data sets in terms of energy range and field sweep [18]. The NEST model used in this simulation has been updated to incorporate the latest calibration results from the LUX experiment $[2,3,5]$. 
After the clustered energy depositions have been converted from energy to quanta (scintillation photons and ionization electrons) via the NEST models, a detector model is applied to convert these raw quanta into the detector observables, that is, into S1 and S2.

The primary scintillation light from the NEST models is propagated to the faces of the PMTs, accounting for binomial fluctuations, using the light collection model, including Fresnel transmission and reflection, described in Section 3.5. We refer to the average light collection efficiency as $\alpha_{1}$, which the optical model currently predicts to be $8.5 \%$ (better than our baseline value and requirement of $7.5 \%$ ), and we correct the raw signal (denoted S1) for the variation of light collection with position in the detector (denoted S2). Photoelectron production at the photocathode accounts for the double-photoelectron phenomenon described in [19]. A trigger requirement of three-fold coincidence of PMT hits is applied prior to subsequent analysis. Both S1 and S1c are reported to the user.

The S2 signal is produced from the number of ionization electrons that drift away from the interaction site. The LZ extraction efficiency (see Table 3.6.1) is applied to determine how many electrons are extracted to the gas phase. The extracted electrons are converted to a number of luminescent photons with NEST, accounting for LZ parameters, and these photons are then converted into photoelectrons at the PMTs. The S2 signal is also corrected for the position of the event in the detector, including the effect of non-infinite electron lifetime presumed in LZ to be $850 \mu$ s (corresponding to an absorption length of greater than $1.5 \mathrm{~m}$ ). The raw and corrected signals, denoted S2 and S2c, are reported to the user.

In the central TPC the energy-weighted position and variance of all energy clusters is computed. The total $\mathrm{S} 1$ signal in the central xenon volume includes the $\mathrm{S} 1$ signal created by energy deposits in the reverse field region (RFR) below the cathode. Here, the electric field drifts electrons downward, making no contribution to the S2 signal.

A similar procedure is followed to model the S1 signal observed in the skin region (excluding S2 as no charge is collected in the skin). A light collection model is determined by simulating photons throughout the skin. Each energy deposit in the skin is converted into photon quanta using an electric field model of the skin and the NEST package. These photons are then converted to detected photoelectrons, and the final skin $\mathrm{S} 1$ is reported to the user.

The process that translates energy depositions into observed S1c and S2c signals does not currently account for PMT or DAQ electronics noise. A more complete model incorporating all known sources of noise leading to the generation of simulated waveforms is under development.

A substantial effort has gone into simulating the light collection for the outer detector liquid scintillator system. Currently, however, for the primary TDR physics simulations, only energy depositions from GEANT4 in the outer detector are used. The transport, capture, and conversion to gamma rays and nuclear recoils of neutrons are simulated in GEANT4.

\subsubsection{Analysis cuts}

For each event, we have a tree containing the energy and location of interactions in the TPC, the skin region, and the outer detector, and energy depositions in liquid xenon have been translated into raw and corrected $\mathrm{S} 1$ and $\mathrm{S} 2$ as described in the previous section. We apply a set of cuts to the simulation data to determine the backgrounds produced by a given radioactive decay chain in a given detector component. The baseline cuts that are used to produce the numbers in Table 9.2.7 are as follows:

- Region of Interest: $0<\mathrm{S} 1 \mathrm{c}<20$ detected photoelectrons, but assuming 3-fold coincidence in the TPC PMTs. In other words, three PMTs have to have observed light, but the total sum of the signal can be arbitrarily small. For ER, this range is approximately 1.5 to $6.5 \mathrm{keV}_{\text {ee }}$, and for NR, this range is approximately 6 to $30 \mathrm{keV}_{\mathrm{nr}}$. In addition, the uncorrected $\mathrm{S} 2$ signal is required to be $>350$ detected photoelectrons ( 5 emitted electrons) ensuring adequate signal size for position reconstruction. 
- Single scatter event: $\sigma_{Z}<0.2 \mathrm{~cm}$ and $\sigma_{r}<3.0 \mathrm{~cm}$. The energy-weighted variance in position must be less than the expected spatial resolution of the detector for an event to be classified as a single scattering event. The given values are based on the LUX performance with an estimated scaling to LZ. Gamma-X events are treated as single scatters, as no S2 can be observed from the second interaction vertex.

- Skin cut: $<3$ detected photoelectrons in the skin veto region, to ensure that no visible energy is deposited in the skin within the $800 \mu$ s coincidence window.

- Outer detector cut: $<200 \mathrm{keV}_{\mathrm{ee}}$ deposited in the outer detector, to ensure that no visible energy is observed in the outer detector within the $500 \mu$ s coincidence window.

- Fiducial volume cut: the fiducial volume is defined as $4 \mathrm{~cm}$ from the TPC walls, $1.5 \mathrm{~cm}$ from the cathode grid and $13.5 \mathrm{~cm}$ from the gate grid, corresponding to 5.6 tonnes of LXe.

In some cases, particularly for the simulation of gamma backgrounds, adequate statistics could not be generated due to limitations in time and available disk space. In these cases, the upper bound of the region of interest was increased to $100 \mathrm{keV}_{\mathrm{ee}}$ to increase the statistics in the analysis. This scaling is only valid if the spectrum of background events is roughly flat below $100 \mathrm{keV}_{\mathrm{ee}}$; in the fiducial volume used here, this condition was met.

\subsubsection{Validation}

To ensure that the simulations are an accurate reflection of the detector design, the simulation code and outputs are validated in several ways. First, because the LZSim simulation package shares a code base with LUXSim, the extensive validations of LUXSim that have been performed using LUX data can be incorporated directly into LZSim. Therefore, the GEANT4 physics list, event generators, and the NEST models are vetted against LUX data before being applied to the LZ detector model.

Where possible, specific predictions of LZSim are validated against external, independent models. For example, the light collection studies described in Sec. 3.5.1 are produced using the full LZSim model, but are checked against an independent, MATLAB-based ray tracing code package developed by collaborators within LZ. Similarly, the parameters that drive the S2 photon detection described in Sec 3.6 are compared with results from independent electron transport models that are validated against LUX S2 pulses.

To validate the detector geometry, all components are checked against engineering drawings by at least two people. Given the high level of confidence in the optical model, based on the agreement with LUX data and the independent checks, the light collection models are used as a second validation step. The majority of mistakes in implementing the geometry become immediately apparent when looking at the predicted light collection. Before a modification to the geometry can be accepted into the repository, any changes to the light collection output that result from that modification are studied and understood.

Finally, a set of high-level cross-checks provides additional quality assurance for the key simulation results used for background rate estimates. These include comparisons to back-of-the-envelope calculations for the dominant sources of background, comparison of the SOURCES-4A neutron yields to those from an alternative simulation package, and sanity checks of neutron and gamma attenuation lengths along critical paths throughout the LZ geometry. All cross-checks are consistent with the full simulations output at a level that does not impact the $\mathrm{LZ}$ sensitivity requirement. 


\subsection{Requirements}

In this section, we summarize the key requirements for the LZ experiment. The LZ collaboration has established a small number of requirements to guide and evaluate the design and fabrication of the detector systems.

The top-level scientific requirement is the sensitivity to WIMP dark matter, via the spin-independent scattering process. Subsidiary high-level science requirements and the flow-down from the overall sensitivity are shown in Fig. 12.2.1. The high-level requirements, including the key infrastructure requirements, are summarized in Table 12.2.1. These requirements flow down to the detector subsystems and are captured in a concise form available to the collaboration. There are two practical high-level requirements. First, all equipment and subassemblies must be transported via the Yates shaft (see Chapter 10), which imposes dimensional and weight limits. Second, the existing water tank now housing the LUX detector will be reused. The collaboration has also captured the requirements for detector subsystems at WBS Level 2.

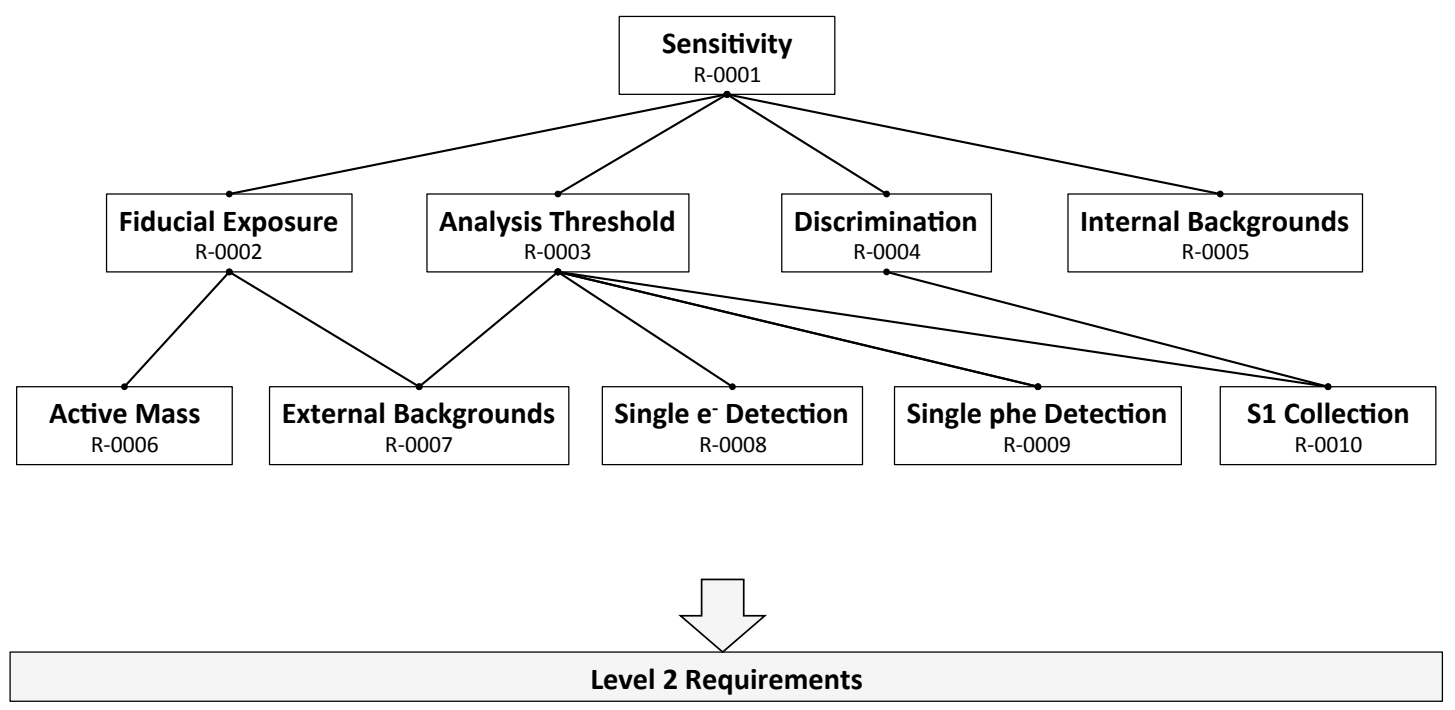

Figure 12.2.1: Flow down of the top level scientific requirements.

Requirements development and explication have been key elements of internal reviews of LZ detector systems and will be an important aspect of configuration control. All top-level and Level-2 requirements have been developed and reviewed in dedicated meetings. The requirements are captured in a Google document, along with additional material and documentation for each relevant WBS Level-2 element. The LZ instrument scientist and chief and deputy chief engineers (see Chapter 13) are primarily responsible for the maintenance of the requirements and their further development, if required, in close collaboration with the Level 2 managers. 
Table 12.2.1: LZ Top Level Science Requirements

\begin{tabular}{|c|c|c|c|c|}
\hline Number & WBS & $\begin{array}{l}\text { Requirement } \\
\text { Name }\end{array}$ & $\begin{array}{l}\text { Requirement Descrip- } \\
\text { tion }\end{array}$ & Rationale \\
\hline \multicolumn{5}{|c|}{ Primary } \\
\hline R-0001 & Science & $\begin{array}{l}\text { WIMP Sensitiv- } \\
\text { ity }\end{array}$ & $\begin{array}{l}\text { Sensitivity to } \\
40 \mathrm{GeV} / \mathrm{c}^{2} \text { WIMPs } \\
\text { is } 3 \times 10^{-48} \mathrm{~cm}^{2} \text { or } \\
\text { better }\end{array}$ & $\begin{array}{l}\text { Probe limit of liquid xenon tech- } \\
\text { nology set by solar neutrino back- } \\
\text { ground. Approach sensitivity to } \\
\text { atmospheric neutrinos. Test su- } \\
\text { persymmetric and extra-dimension } \\
\text { models of dark matter }\end{array}$ \\
\hline \multicolumn{5}{|c|}{ Secondary } \\
\hline R-0002 & Science & $\begin{array}{l}\text { Fiducial Expo- } \\
\text { sure }\end{array}$ & $\begin{array}{l}\text { Minimum of } \\
5,600 \text { tonne-days }\end{array}$ & $\begin{array}{l}\text { Needed to achieve sensitivity re- } \\
\text { quirement. Achievable with fiducial } \\
\text { mass of } 5.6 \text { tonnes and assumed } \\
\text { running period of } 1,000 \text { live days or } \\
\text { less fiducial mass and longer run- } \\
\text { ning time }\end{array}$ \\
\hline R-0003 & Science & $\begin{array}{l}\text { Analysis thresh- } \\
\text { old }\end{array}$ & $\begin{array}{l}50 \% \text { efficiency at } \\
6 \mathrm{keV}_{\mathrm{nr}}\end{array}$ & $\begin{array}{l}\text { Probe WIMP mass range down to } \\
5 \mathrm{GeV} / c^{2} \text { with non-negligible sensi- } \\
\text { tivity }\end{array}$ \\
\hline R-0004 & Science & $\begin{array}{l}\text { ER Discrimina- } \\
\text { tion }\end{array}$ & $\begin{array}{l}99.5 \% \text { ER discrimi- } \\
\text { nation for } 50 \% \text { NR } \\
\text { acceptance }\end{array}$ & $\begin{array}{l}\text { Limit background from ERs so as } \\
\text { to reach WIMP sensitivity require- } \\
\text { ment }\end{array}$ \\
\hline R-0005 & Science & $\begin{array}{l}\text { Internal back- } \\
\text { grounds }\end{array}$ & $\begin{array}{l}\text { Internal backgrounds } \\
\text { from radioactive no- } \\
\text { ble gases (Rn, Kr, Ar) } \\
\text { not to exceed four } \\
\text { times the solar neu- } \\
\text { trino ER background }\end{array}$ & $\begin{array}{l}\text { Limit ERs from internal back- } \\
\text { grounds to an acceptable level. So- } \\
\text { lar neutrino rate does not include } \\
{ }^{8} \mathrm{~B}\end{array}$ \\
\hline \multicolumn{5}{|c|}{ Tertiary } \\
\hline R-0006 & Science & Active mass & 7.0 tonnes & Required to reach fiducial exposure \\
\hline R-0007 & Science & $\begin{array}{l}\text { External back- } \\
\text { grounds }\end{array}$ & $\begin{array}{l}\text { Backgrounds from } \\
\text { radioactivity of the } \\
\text { detector components } \\
\text { (not including in- } \\
\text { ternal backgrounds, } \\
\text { R-0005). ER counts } \\
\text { before discrimination } \\
<25 \text { and NR counts } \\
\text { before discrimination } \\
<0.7\end{array}$ & $\begin{array}{l}\text { ER counts constrained to be }<10 \% \\
\text { of ERs from solar neutrinos (not in- } \\
\text { cluding }{ }^{8} \mathrm{~B} \text { ), including uncertainty } \\
\text { in this rate. NR events to be con- } \\
\text { strained to be comparable to neu- } \\
\text { trino rate. We rely on veto effi- } \\
\text { ciency to reduce the NR rate con- } \\
\text { tribution. This rate, and to a lesser } \\
\text { extent external ER contributions, } \\
\text { define the fiducial mass. Analysis } \\
\text { threshold also depends on size of } \\
\text { these backgrounds. }\end{array}$ \\
\hline \multicolumn{5}{|c|}{ (continued on next page) } \\
\hline
\end{tabular}


Table 12.2.1: (continued)

\begin{tabular}{|c|c|c|c|c|}
\hline Number & WBS & $\begin{array}{l}\text { Requirement } \\
\text { Name }\end{array}$ & $\begin{array}{l}\text { Requirement Descrip- } \\
\text { tion }\end{array}$ & Rationale \\
\hline R-0008 & Science & $\begin{array}{l}\text { Single electron } \\
\text { detection }\end{array}$ & $\begin{array}{l}50 \text { S2 photoelectrons } \\
\text { detected per emitted } \\
\text { electron }\end{array}$ & $\begin{array}{l}\text { Sufficiently large S2 signal for ac- } \\
\text { curate reconstruction of peripheral } \\
\text { interactions, such as those arising } \\
\text { from contamination on the TPC } \\
\text { walls. }\end{array}$ \\
\hline R-0009 & Science & $\begin{array}{l}\text { Single pho- } \\
\text { toelectron } \\
\text { detection }\end{array}$ & $\begin{array}{l}\text { Single S1 photoelec- } \\
\text { tron detection with } \\
>90 \% \text { efficiency, so } \\
\text { as to reach }>70 \% \text { ef- } \\
\text { ficiency for } 3 \text { phe }\end{array}$ & $\begin{array}{l}\text { Main determinant of analysis } \\
\text { threshold. }\end{array}$ \\
\hline R-0010 & Science & $\begin{array}{l}\text { S1 light collec- } \\
\text { tion }\end{array}$ & $\begin{array}{l}\text { Volume-averaged S1 } \\
\text { photon detection } \\
\text { efficiency (geometric } \\
\text { light collection times } \\
\text { effective PMT quan- } \\
\text { tum efficiency) of } \\
>7.5 \%\end{array}$ & $\begin{array}{l}\text { Good discrimination and low en- } \\
\text { ergy threshold, equal or better than } \\
\text { past Xe experiments. Exponentially } \\
\text { falling (in recoil energy) WIMP } \\
\text { spectrum means more recoils at } \\
\text { lower energies, and low-energy re- } \\
\text { coils produce less S1 (both total } \\
\text { and per-unit-energy) driving the S1 } \\
\text { light collection efficiency require- } \\
\text { ment. }\end{array}$ \\
\hline \multicolumn{5}{|c|}{ Infrastructure } \\
\hline R-0100 & General & $\begin{array}{l}\text { All parts fit } \\
\text { down Yates } \\
\text { shaft }\end{array}$ & $\begin{array}{l}\text { All detector elements } \\
\text { must be sized so that } \\
\text { they can be lowered } \\
\text { via the Yates shaft }\end{array}$ & $\begin{array}{l}\text { Yates shaft is primary access to the } \\
\text { Davis campus }\end{array}$ \\
\hline R-0110 & General & $\begin{array}{l}\text { Reuse Davis wa- } \\
\text { ter tank }\end{array}$ & $\begin{array}{l}\text { Existing Davis water } \\
\text { tank is reused. In- } \\
\text { clude minor modifi- } \\
\text { cations and refurbish- } \\
\text { ment. }\end{array}$ & $\begin{array}{l}\text { Not practical or cost effective to } \\
\text { replace water tank. Insufficient } \\
\text { underground space to make larger } \\
\text { tank. }\end{array}$ \\
\hline
\end{tabular}

The baseline design described in this Technical Design Report meets or exceeds all requirements. We briefly summarize the key requirements by WBS element in the subsections below. Linkage among the various requirements has been part of the requirements review process. 


\subsubsection{WBS 1.1 Xenon Procurement}

There is a single requirement for WBS 1.1 Xenon Procurement, that being to procure 10 total tonnes of xenon. This requirement flows down from R-0006, as 10 total tonnes are adequate to result in 7 active tonnes in the detector. 


\subsubsection{WBS 1.2 Xenon Vessel}

The primary requirements for the cryostat vessels are shown in Table 12.2.2. The size of the cryostat determines the amount of target that can be deployed and flows down from the top level science requirement but also the infrastructure requirement that all parts must fit in the Yates shaft. The cryostat vessels must also be low radioactivity. Safety considerations dictate that the vessels are compliant with standard engineering codes.

Table 12.2.2: Level 2 Requirements for WBS 1.2 Xenon Vessel

\begin{tabular}{|c|c|c|c|c|}
\hline Number & WBS & Requirement Name & Requirement Description & Rationale \\
\hline R-120001 & 1.2 & $\begin{array}{l}\text { Inner Cryostat Ves- } \\
\text { sel (ICV) Maximum } \\
\text { Outer Diameter }\end{array}$ & $\begin{array}{l}\text { Inner cryostat outside di- } \\
\text { ameter less than } 1.702 \mathrm{~m} \\
(67.0 \text { inch). Ports must be } \\
\text { at angles where there is suf- } \\
\text { ficient clearance. }\end{array}$ & $\begin{array}{l}\text { Must fit within Yates } \\
\text { shaft. }\end{array}$ \\
\hline R-120002 & 1.2 & $\begin{array}{l}\text { Inner cryostat ves- } \\
\text { sel (ICV) compact } \\
\text { geometry }\end{array}$ & $\begin{array}{l}\text { Design compact ICV to min- } \\
\text { imize use of passive xenon: } \\
\text { ICV conical section and el- } \\
\text { lipsoidal } 3: 1 \text { dished end }\end{array}$ & Saving xenon \\
\hline R-120003 & 1.2 & $\begin{array}{l}\text { Outer Cryostat } \\
\text { Vessel segmented }\end{array}$ & No fabrication underground & $\begin{array}{l}\text { Segmented OCV fits into } \\
\text { Yates shaft }\end{array}$ \\
\hline R-120004 & 1.2 & $\begin{array}{l}\text { Low radioactivity of } \\
\text { the cryostat mate- } \\
\text { rial }\end{array}$ & $\begin{array}{l}\text { Radioactivity budget from } \\
\text { background simulations. } \\
\text { Maximum contribution to } \\
\text { the overall background: } \\
3.3 \% \text { of pp solar neutrinos } \\
\text { and } 0.03 \mathrm{NR} \text { event }\end{array}$ & $\begin{array}{l}\text { Low radioactivity, low } \\
\text { density minimizing, effi- } \\
\text { ciency of Outer Detector }\end{array}$ \\
\hline R-120005 & 1.2 & $\begin{array}{l}\text { Outer Cryostat } \\
\text { Vessel (OCV) and } \\
\text { Inner Cryostat Ves- } \\
\text { sel (ICV) designed } \\
\text { for } 1.48 \text { bar exter- } \\
\text { nal pressure and } \\
\text { vacuum internal }\end{array}$ & $\begin{array}{l}\text { Working conditions for OCV } \\
\text { and most severe failure } \\
\text { mode for ICV }\end{array}$ & $\begin{array}{l}\text { Vacuum inside and water } \\
\text { outside }\end{array}$ \\
\hline R-120006 & 1.2 & $\begin{array}{l}\text { Inner Cryostat Ves- } \\
\text { sel (ICV) designed } \\
\text { for } 4 \text { bar inner pres- } \\
\text { sure and vacuum } \\
\text { external }\end{array}$ & ICV working conditions & $\begin{array}{l}\text { Xe gas inside with maxi- } \\
\text { mum pressure - including } \\
\text { hydrostatic pressure at } \\
\text { the bottom dished end; } \\
3.4 \text { bar top head }\end{array}$ \\
\hline R-120007 & 1.2 & $\begin{array}{l}\text { Design compliance } \\
\text { to codes }\end{array}$ & $\begin{array}{l}\text { Compliance to ASME BPVC } \\
\text { code VIII, } 2012 \text { Int. Building } \\
\text { Code, ASCE } 7 \text { with soil clas- } \\
\text { sification Cals B for seismic } \\
\text { conditions, Fabricator holds } \\
\text { U-stamp certificate }\end{array}$ & $\begin{array}{l}\text { Required by SURF SD } \\
\text { regulations }\end{array}$ \\
\hline
\end{tabular}




\subsubsection{WBS 1.3 Cryogenic System}

The primary requirements for the Cryogenic System are shown in Table 12.2.3. The main objective of this system is to enable safe and efficient cooling of the LXe target volume. In particular, the cooling power must be adequate to enable rapid circulation of the Xe volume for purification to satisfy the Level-2 requirements in WBS 1.4, which is an example of linkage between WBS requirements.

Table 12.2.3: Level 2 Requirements for WBS 1.3 Cryogenic System

\begin{tabular}{|c|c|c|c|c|}
\hline Number & WBS & $\begin{array}{l}\text { Requirement } \\
\text { Name }\end{array}$ & Requirement Description & Rationale \\
\hline R-130001 & 1.3 & $\begin{array}{l}\text { Sufficient cool- } \\
\text { ing power }\end{array}$ & $\begin{array}{l}\text { Cryogen cooling systems shall } \\
\text { be sufficient to remove heat for } \\
500 \text { slpm of Xenon circulation; } \\
\text { purge of the detector Xenon gas } \\
\text { space; and thermal losses of all } \\
\text { the system components. }\end{array}$ & $\begin{array}{l}\text { There must be adequate } \\
\text { cooling to liquefy Xenon } \\
\text { within the parameters of } \\
\text { flow required to attain } \\
\text { purity. }\end{array}$ \\
\hline R-130002 & 1.3 & $\begin{array}{l}\text { Oxygen defi- } \\
\text { ciency hazard } \\
\text { safety }\end{array}$ & $\begin{array}{l}\text { Engineered controls shall be } \\
\text { implemented to achieve ODH } \\
\text { Class } 2 \text { or better. }\end{array}$ & $\begin{array}{l}\text { SURF requires ODH } \\
\text { Class } 2 \text { or better for all } \\
\text { experimental implemen- } \\
\text { tations }\end{array}$ \\
\hline R-130003 & 1.3 & $\begin{array}{l}\text { Pressure safety } \\
\text { - valves }\end{array}$ & $\begin{array}{l}\text { Redundant relief devices shall } \\
\text { be employed for pressure safety. } \\
\text { Relief devices shall be sized per } \\
\text { CGA S-1.3. }\end{array}$ & $\begin{array}{l}\text { Properly sized redundant } \\
\text { relief devices are a pri- } \\
\text { mary engineered control } \\
\text { for pressure systems. }\end{array}$ \\
\hline R-130004 & 1.3 & $\begin{array}{l}\text { Pressure safety } \\
\text { - monitoring }\end{array}$ & $\begin{array}{l}\text { Active pressure monitoring shall } \\
\text { be utilized such that alarms pro- } \\
\text { vide warning of pressure going } \\
\text { higher than the planned operat- } \\
\text { ing pressure range. }\end{array}$ & $\begin{array}{l}\text { Monitoring of pressure } \\
\text { may provide an opportu- } \\
\text { nity to act on elevated } \\
\text { pressure before one of } \\
\text { the primary safety de- } \\
\text { vices is activated. }\end{array}$ \\
\hline R-130005 & 1.3 & Materials & $\begin{array}{l}\text { Materials exposed to LN tem- } \\
\text { peratures shall be: a) low car- } \\
\text { bon stainless steel; b) aluminum } \\
\text { based alloys; c) nickel based al- } \\
\text { loys; d) copper / copper based } \\
\text { alloys; or e) pure titanium. }\end{array}$ & $\begin{array}{l}\text { These materials will re- } \\
\text { main ductile at } 77 \mathrm{~K} \text {. }\end{array}$ \\
\hline R-130006 & 1.3 & $\begin{array}{l}\text { Thermal insula- } \\
\text { tion }\end{array}$ & $\begin{array}{l}\text { Equipment at } 175 \mathrm{~K} \text { shall have } \\
\text { a minimum of } 10 \text { layers and } \\
\text { equipment at } 77 \mathrm{~K} \text { shall have a } \\
\text { minimum of } 25 \text { layers of multi- } \\
\text { layer insulation }(\mathrm{MLI}) \text {. }\end{array}$ & $\begin{array}{l}\text { MLI is the most effec- } \\
\text { tive method of minimiz- } \\
\text { ing radiative heat load. } \\
\text { Lower operating temper- } \\
\text { atures require additional } \\
\text { layers of MLI. }\end{array}$ \\
\hline
\end{tabular}




\subsubsection{WBS 1.4 Xenon Purification}

The primary requirements for WBS 1.4 Xenon Purification are shown in Table 12.2.4. These requirements primarily flow down from R-0003 and R-0005, as the Xe must be pure enough to enable efficient extraction of signals to satisfy the analysis threshold requirement but also low enough in internal radioactive sources like $\mathrm{Kr}$ and $\mathrm{Rn}$ to satisfy the internal backgrounds requirement.

Table 12.2.4: Level 2 requirements for WBS 1.4 Xenon Purification

\begin{tabular}{|c|c|c|c|c|}
\hline Number & WBS & Requirement Name & Requirement Description & Rationale \\
\hline R-140001 & 1.4 & $\begin{array}{l}\text { Xe electronegative } \\
\text { purity }\end{array}$ & $\begin{array}{l}\text { Charge absorption length } \\
>1.5 \mathrm{~m}, \quad \mathrm{O}_{2} \text { equivalent } \\
=0.4 \mathrm{ppb} .\end{array}$ & $\begin{array}{l}\text { Collect charge and scin- } \\
\text { tillation throughout the } \\
\text { entire volume of the } \\
\text { TPC. }\end{array}$ \\
\hline R-140002 & 1.4 & $\begin{array}{l}\text { Removal of } \mathrm{Kr} \text { and } \\
\text { Ar from } \mathrm{Xe} .\end{array}$ & $\begin{array}{l}\text { Ability to remove natural } \mathrm{Kr} \\
\text { from the } \mathrm{Xe} \text { to a concen- } \\
\text { tration of }<0.015 \mathrm{ppt} \mathrm{g} / \mathrm{g} \text {. } \\
\text { Ability to remove natural } \mathrm{Ar} \\
\text { from the } \mathrm{Xe} \text { to a concentra- } \\
\text { tion of }<0.45 \mathrm{ppb} \mathrm{g} / \mathrm{g} \text {. }\end{array}$ & $\begin{array}{l}\text { Limit ER Background } \\
\text { from }{ }^{85} \mathrm{Kr} \text { and }{ }^{39} \mathrm{Ar} \text {. }\end{array}$ \\
\hline R-140003 & 1.4 & $\begin{array}{l}\text { Control the ingress } \\
\text { of }{ }^{222} \mathrm{Rn} \text { into the } \\
\mathrm{Xe}\end{array}$ & $\begin{array}{l}\text { Control the decay rate of } \\
{ }^{222} \mathrm{Rn} \text { in the } \mathrm{Xe} \text {. }\end{array}$ & $\begin{array}{l}\text { ER background from } \\
{ }^{214} \mathrm{~Pb} \text {. Limit the }{ }^{222} \mathrm{Rn} \\
\text { decay rate in the active } \\
\text { Xe to } 13.4 \mathrm{mBq} .\end{array}$ \\
\hline R-140004 & 1.4 & ${ }^{127}$ Xe activity & $\begin{array}{l}88 \mu \mathrm{Bq} / \mathrm{kg} \text { activity in the } \\
5.6 \text { tonnes fiducial volume at } \\
\text { the start of physics data tak- } \\
\text { ing. }\end{array}$ & $\begin{array}{l}\text { No more than } 23 \text { single- } \\
\text { scatter events below } \\
6 \mathrm{keV}_{\text {ee from }{ }^{127} \mathrm{Xe}} \\
\text { in the lifespan of the } \\
\text { experiment. }\end{array}$ \\
\hline R-140005 & 1.4 & Safe $X e$ recovery & $\begin{array}{l}\text { Safe recovery of the Xe dur- } \\
\text { ing normal operations and } \\
\text { during an emergency }\end{array}$ & $\begin{array}{l}\text { Protection of the Xe in- } \\
\text { vestment. }\end{array}$ \\
\hline
\end{tabular}




\subsubsection{WBS 1.5 Xenon Detector System}

There are several requirements in WBS 1.5 Xenon Detector System. For example, the TPC must be large enough to accommodate the required target mass, the electric fields need to be adequate to achieve efficient single electron detection and to achieve the required $99.5 \% \mathrm{ER} / \mathrm{NR}$ discrimination, the TPC must have adequate light collection, and the detector components must be made from clean materials to limit the external backgrounds seen by the LXe. R-150009 is another good example of the linkages between different WBS systems, as the power dissipated in the detector must be low enough that the cooling power specified in R-130001 is adequate. The primary requirements for WBS 1.5 Xenon Detector System are listed in Table 12.2.5.

Table 12.2.5: Level 2 Requirements for WBS 1.5 Xenon Detector System

\begin{tabular}{|c|c|c|c|c|}
\hline Number & WBS & Requirement Name & $\begin{array}{l}\text { Requirement } \\
\text { Description }\end{array}$ & Rationale \\
\hline R-150001 & 1.5 & $\begin{array}{l}\text { TPC inner dimen- } \\
\text { sions }\end{array}$ & $\begin{array}{l}\text { Dia. }=1,456 \mathrm{~mm} / \\
\text { length }=1,456 \mathrm{~mm}\end{array}$ & $\begin{array}{l}7 \text { tonne active mass, optimal self- } \\
\text { shielding }\end{array}$ \\
\hline R-150002 & 1.5 & TPC drift field & $300 \mathrm{~V} / \mathrm{cm}$ & $\begin{array}{l}99.5 \% \text { discrimination; drift time } \\
<1 \mathrm{~ms}\end{array}$ \\
\hline R-150003 & 1.5 & $\begin{array}{l}\text { Electroluminescence } \\
\text { field }(G X e)\end{array}$ & $10 \mathrm{kV} / \mathrm{cm}$ & $\begin{array}{l}95 \% \text { emission; } 50 \text { phe } / e^{-} ; \quad \mathrm{e}- \\
\text { trains; wall events }\end{array}$ \\
\hline R-150004 & 1.5 & Energy resolution & $\begin{array}{l}2.0 \% \text { at } 2.5 \mathrm{MeV} \\
(\mathrm{S} 1+\mathrm{S} 2)\end{array}$ & $\begin{array}{l}\text { Gamma spectroscopy for back- } \\
\text { ground model }\end{array}$ \\
\hline R-150005 & 1.5 & LXe Skin threshold & $\begin{array}{l}100 \mathrm{keV}_{\text {ee }} \text { with } \\
3 \text { phe in }>95 \% \text { of } \\
\text { skin volume }\end{array}$ & $\begin{array}{l}\text { Veto efficiency required for fiducial } \\
\text { mass }\end{array}$ \\
\hline R-150006 & 1.5 & $\begin{array}{l}\text { Component ra- } \\
\text { dioactivities }\end{array}$ & $\begin{array}{l}< \\
250 / 240 / 240 / 540 \\
\mathrm{mBq} \mathrm{U} / \mathrm{Th} / \mathrm{Co} / \mathrm{K}\end{array}$ & $\begin{array}{l}<0.4 \mathrm{NR} \text { cts, }<10 \% \text { pp neutrino } \\
\text { ER cts (matches R-200114 to R- } \\
200117 \text { ) }\end{array}$ \\
\hline R-150007 & 1.5 & $\begin{array}{l}\text { Photocathode cov- } \\
\text { erage }\end{array}$ & $\begin{array}{l}38 \% \text { top and bot- } \\
\text { tom arrays }\end{array}$ & $\begin{array}{l}\text { S1 Photon detection efficiency, } \\
\text { vertex resolution, discrimination }\end{array}$ \\
\hline R-150008 & 1.5 & PTFE reflectivity & $>95 \%$ in $\mathrm{LXe}$ & $\begin{array}{l}\text { Light collection efficiency in TPC } \\
\text { and Skin }\end{array}$ \\
\hline R-150009 & 1.5 & $\begin{array}{l}\text { Total heat in or on } \\
\text { cryostat }\end{array}$ & $140 \mathrm{~W}$ & $\begin{array}{l}\text { Cooling power requirement, fluid } \\
\text { model }\end{array}$ \\
\hline R-150010 & 1.5 & $\begin{array}{l}\text { Max field for ca- } \\
\text { thodic surfaces }\end{array}$ & $50 \mathrm{kV} / \mathrm{cm}$ & $\begin{array}{l}\text { Limit spurious photon/electron } \\
\text { emission }\end{array}$ \\
\hline
\end{tabular}




\subsubsection{WBS 1.6 Outer Detector}

WBS 1.6, the Outer Detector, acts as the main neutron veto, and therefore most requirements in this WBS flow down from R-0007. The Level 2 Requirements for WBS 1.6 Outer Detector are shown in Table 12.2.6.

Table 12.2.6: Level 2 Requirements for WBS 1.6 Outer Detector

\begin{tabular}{|c|c|c|c|c|}
\hline Number & WBS & Requirement Name & Requirement Description & Rationale \\
\hline R-160001 & 1.6 & $\begin{array}{l}\text { Neutron veto effi- } \\
\text { ciency }\end{array}$ & $\begin{array}{l}\text { Detection efficiency of } 95 \% \\
\text { for a } 1 \mathrm{MeV} \text { neutron that } \\
\text { scatters once in the xenon }\end{array}$ & $\begin{array}{l}\text { Needed to meet the neu- } \\
\text { tron part of requirement } \\
\text { on external backgrounds }\end{array}$ \\
\hline R-160002 & 1.6 & $\begin{array}{l}\text { Outer Detector } \\
\text { threshold }\end{array}$ & $\begin{array}{l}\text { Threshold of } 200 \mathrm{keV} \text { ( } 50 \% \\
\text { efficiency) on energy deposit } \\
\text { in liquid scintillator }\end{array}$ & $\begin{array}{l}\text { Highest achievable veto } \\
\text { efficiency with accept- } \\
\text { able deadtime }\end{array}$ \\
\hline R-160003 & 1.6 & $\begin{array}{l}\text { Number of photo- } \\
\text { electrons detected } \\
\text { per energy deposit }\end{array}$ & $>80$ phe/MeV & $\begin{array}{l}\text { Corresponds to about } \\
15 \text { phe at threshold of } \\
200 \mathrm{keV}\end{array}$ \\
\hline R-160004 & 1.6 & $\begin{array}{l}\text { Light collection ef- } \\
\text { ficiency }\end{array}$ & $\begin{array}{l}\text { Efficiency of }>5 \% \text { for having } \\
\text { VUV photons strike a PMT }\end{array}$ & $\begin{array}{l}\text { Light detection is pro- } \\
\text { portional to light collec- } \\
\text { tion efficiency. }\end{array}$ \\
\hline R-160005 & 1.6 & Deadtime & $\begin{array}{l}\text { OD must not veto more than } \\
5 \% \text { of the WIMP search live- } \\
\text { time }\end{array}$ & $\begin{array}{l}\text { Keep overall activity low } \\
\text { to limit deadtime im- } \\
\text { posed by OD veto trig- } \\
\text { gers }\end{array}$ \\
\hline
\end{tabular}

\subsubsection{WBS 1.7 Calibration System}

The Calibration System must provide accurate calibrations of the light and charge yields, position dependencies, time variations, energy threshold, resolution, and discrimination parameters of the central TPC without taking an undue amount of running time away from the primary dark matter search. The Calibration System must also provide an accurate picture of the performance of the veto systems: the Xe skin and Outer Detector. The L2 requirements for WBS 1.7 Calibration System are listed in Table 12.2.7.

Table 12.2.7: Level 2 Requirements for WBS 1.7 Calibration System

\begin{tabular}{|c|c|l|l|l|}
\hline Number & WBS & Requirement Name & Requirement Description & Rationale \\
\hline R-170001 & 1.7 & Calibration Times & $\begin{array}{l}<12 \mathrm{hrs} / \text { week for periodic } \\
\text { calibrations and }<100 \mathrm{~d} \text { to- } \\
\text { tal for infrequent calibrations }\end{array}$ & $\begin{array}{l}\text { gle day of SURF opera- } \\
\text { tions (2 shifts). 100 days } \\
\text { is } 10 \% \text { of total exposure } \\
\text { target. }\end{array}$ \\
\hline \multicolumn{2}{|c|}{ (continued on next page) } \\
\hline
\end{tabular}


Table 12.2.7: (continued)

\begin{tabular}{|c|c|c|c|c|}
\hline Number & WBS & Requirement Name & Requirement Description & Rationale \\
\hline R-170002 & 1.7 & $\begin{array}{l}\text { Calibrate } \quad \text { TPC } \\
(x, y, z) \text { variation }\end{array}$ & $\begin{array}{l}<2 \% \text { uncertainty on S1 and } \\
\mathrm{S} 2 \text { mean area (of a mo- } \\
\text { noenergetic peak) in bins of } \\
5 \times 5 \times 5 \mathrm{~cm}\end{array}$ & $\begin{array}{l}\text { Understand detection ef- } \\
\text { ficiencies and gains at } \\
\text { scales relevant to varia- } \\
\text { tion }\end{array}$ \\
\hline R-170003 & 1.7 & Calibrate ER band & $\begin{array}{l}<2 \% \text { uncertainty on the } \\
\text { measured ER band mean } \\
\text { and } \pm 1 \sigma \text { contours (in } 1 \text { phd } \\
\text { bins of } S 1 \text { ) }\end{array}$ & $\begin{array}{l}\text { Clearly define ER back- } \\
\text { ground region }\end{array}$ \\
\hline R-170004 & 1.7 & Calibrate NR band & $\begin{array}{l}<2 \% \text { uncertainty on the } \\
\text { measured NR band mean } \\
\text { and } \pm 1 \sigma \text { contours (in } 1 \text { phd } \\
\text { bins of } S 1 \text { ) }\end{array}$ & $\begin{array}{l}\text { Clearly define signal re- } \\
\text { gion }\end{array}$ \\
\hline R-170005 & 1.7 & $\begin{array}{l}\text { Calibrate NR re- } \\
\text { sponse at threshold }\end{array}$ & $\begin{array}{l}<5 \% \text { uncertainty on energy } \\
\text { at } 50 \% \text { acceptance }\end{array}$ & $\begin{array}{l}\text { Clearly define threshold } \\
\text { and map out }{ }^{8} B \text { neutrino } \\
\text { response }\end{array}$ \\
\hline R-170006 & 1.7 & $\begin{array}{l}\text { Calibrate TPC, skin } \\
\text { and LS signal time } \\
\text { offsets }\end{array}$ & $\begin{array}{l}< \pm 1 \text { sample uncertainty in } \\
\text { primary scintillation rise time } \\
\text { jitter across the three analog } \\
\text { electronics chains }\end{array}$ & $\begin{array}{l}\text { Necessary for optimal } \\
\text { veto efficiency }\end{array}$ \\
\hline R-170007 & 1.7 & $\begin{array}{l}\text { Calibrate temporal } \\
\text { response }\end{array}$ & $\begin{array}{l}<2 \% \text { uncertainty in S2 } \\
\text { mean area (of a monoen- } \\
\text { ergetic peak) in z-direction } \\
\text { bins of } 5 \mathrm{~cm}\end{array}$ & $\begin{array}{l}\text { To ensure stable energy } \\
\text { reconstruction }\end{array}$ \\
\hline R-170008 & 1.7 & $\begin{array}{l}\text { Calibrate Xe skin } \\
\text { energy response }\end{array}$ & $\begin{array}{l}<2 \% \text { uncertainty on ER } \\
\mathrm{phd} / \mathrm{keV}(1 \mathrm{~cm} \text { z bins }) \text { in } \mathrm{Xe} \\
\text { side skin }\end{array}$ & $\begin{array}{l}\text { Understand skin thresh- } \\
\text { old }\end{array}$ \\
\hline R-170009 & 1.7 & $\begin{array}{l}\text { Calibrate energy } \\
\text { scale of LS }\end{array}$ & $\begin{array}{l}<2 \% \text { uncertainty on ER } \\
\text { phd } / \mathrm{keV} \text { (volume-averaged) } \\
\text { at } 2.2 \mathrm{MeV}\end{array}$ & $\begin{array}{l}\text { Verify outer detector } \\
\text { performance }\end{array}$ \\
\hline R-170010 & 1.7 & $\begin{array}{l}\text { Calibrate NR re- } \\
\text { sponse }\end{array}$ & $\begin{array}{l}<5 \% \text { uncertainty on energy } \\
\text { at about } 30 \mathrm{keV}\end{array}$ & $\begin{array}{l}\text { Bound the upper end of } \\
\text { the WIMP-search range }\end{array}$ \\
\hline R-170011 & 1.7 & $\begin{array}{l}\text { Calibrate fiducial } \\
\text { volume fraction }\end{array}$ & $\begin{array}{l}<5 \% \text { uncertainty on frac- } \\
\text { tion of TPC LXe mass sat- } \\
\text { isfying fiducial selection cri- } \\
\text { teria }\end{array}$ & $\begin{array}{l}\text { Directly scales exposure } \\
\text { and sensitivity }\end{array}$ \\
\hline
\end{tabular}




\subsubsection{WBS 1.8 Electronics, DAQ, Controls, and Computing}

The primary requirements for WBS 1.8 Electronics, DAQ, Controls, and Computing are shown in Table 12.2.8. The main requirement here is to enable a low energy threshold in the detector. However, there are two requirements that flow across from WBS 1.7, as the data acquisition must be robust enough to handle the event rates listed in Table 12.2.7 needed to effectively calibrate the detector. On the controls side, this subsystem is responsible for providing monitoring and control during emergencies, and in particular the control of the xenon recovery system of WBS 1.4.

Table 12.2.8: Level 2 Requirements for WBS 1.8 Electronics, DAQ, Controls, and Computing

\begin{tabular}{|c|c|c|c|c|}
\hline Number & WBS & Requirement Name & $\begin{array}{l}\text { Requirement } \\
\text { Description }\end{array}$ & Rationale \\
\hline R-180001 & 1.8 & Energy threshold & $\begin{array}{l}90 \% \text { efficiency for } \\
\text { a single phe }\end{array}$ & $\begin{array}{l}\text { The S1 analysis threshold is de- } \\
\text { fined by our efficiency to capture } \\
\text { single-photoelectron signals. For } \\
\text { a specific gain, this requirement } \\
\text { fixes the noise requirements of the } \\
\text { electronic chain. This calcula- } \\
\text { tion depends sensitively on the as- } \\
\text { sumed } 35 \% \text { variations in the PMT } \\
\text { response. }\end{array}$ \\
\hline R-180002 & 1.8 & Source count rate & $150 \mathrm{~Hz}$ & $\begin{array}{l}\text { Able to handle source calibrations. } \\
\text { Rates are limited by the drift time. }\end{array}$ \\
\hline R-180003 & 1.8 & LED count rate & $4 \mathrm{kHz}$ & $\begin{array}{l}\text { Able to handle LED calibration } \\
\text { rates. }\end{array}$ \\
\hline R-180004 & 1.8 & $\begin{array}{l}\text { Guarantee the } \\
\text { safety of xenon } \\
\text { supply and the } \\
\text { xenon circulation } \\
\text { system }\end{array}$ & $\begin{array}{l}\text { Use Programmable } \\
\text { Logic Controllers } \\
\text { (PLCs) to control } \\
\text { and monitor the } \\
\text { xenon purification, } \\
\text { circulation, and } \\
\text { storage systems }\end{array}$ & $\begin{array}{l}\text { PLCs are used to ensure that the } \\
\text { detector and xenon system are } \\
\text { maintained in a safe state during } \\
\text { emergencies that result in a shut- } \\
\text { down of the slow-control infras- } \\
\text { tructure. The PLC system pro- } \\
\text { vides continuous real-time moni- } \\
\text { toring and control of the critical } \\
\text { subsystems and will initiate auto- } \\
\text { matic recovery of the xenon to } \\
\text { the storage facility during an emer- } \\
\text { gency. }\end{array}$ \\
\hline
\end{tabular}




\subsubsection{WBS 1.9 Integration and Installation}

The primary requirements for WBS 1.9 Integration and Installation are shown in Table 12.2.9. There are two main requirements in this WBS. The first is that all parts must be installed correctly, and the second is that the detector must be installed with clean parts in such a way to minimize exposure to radon and dust. These requirements flow down from the top level science requirements, particularly R-0005 and R-0007.

Table 12.2.9: Level 2 Requirements for WBS 1.9 Integration and Installation

\begin{tabular}{|c|c|c|c|c|}
\hline Number & WBS & $\begin{array}{l}\text { Requirement } \\
\text { Name }\end{array}$ & Requirement Description & Rationale \\
\hline R-190001 & 1.9 & $\begin{array}{l}\text { Parts used } \\
\text { have acceptable } \\
\text { radioactivity }\end{array}$ & $\begin{array}{l}\text { All parts have a traceable history } \\
\text { that shows they have low enough } \\
\text { radioactivity to be used. This will } \\
\text { be documented in a database and } \\
\text { there will be an acceptance sheet } \\
\text { with sign off that the part is usable. }\end{array}$ & $\begin{array}{l}\text { Control background } \\
\text { level in the experi- } \\
\text { ment }\end{array}$ \\
\hline R-190002 & 1.9 & $\begin{array}{l}\text { Parts used are } \\
\text { clean }\end{array}$ & $\begin{array}{l}\text { All parts have a cleaning procedure } \\
\text { to ensure surface contamination is } \\
\text { at an acceptable level. This will } \\
\text { be documented in a database and } \\
\text { there will be an acceptance sheet } \\
\text { with sign off that the part is usable. }\end{array}$ & $\begin{array}{l}\text { Control background } \\
\text { in the experiment. } \\
\text { Control contami- } \\
\text { nation of Xenon } \\
\text { that would reduce } \\
\text { electron drift length } \\
\text { and/or increase light } \\
\text { absorption }\end{array}$ \\
\hline R-190003 & 1.9 & $\begin{array}{l}\text { Parts are moved } \\
\text { and lifted with- } \\
\text { out damage }\end{array}$ & $\begin{array}{l}\text { Moving and lifting will be done to } \\
\text { procedures written and approved by } \\
\text { subsystem experts. This will in- } \\
\text { clude analysis of rigging attachment } \\
\text { and support loads where applicable. } \\
\text { Workers will review the procedures } \\
\text { and have rigging experience }\end{array}$ & $\begin{array}{l}\text { Detector is assem- } \\
\text { bled without damag- } \\
\text { ing parts }\end{array}$ \\
\hline R-190004 & 1.9 & $\begin{array}{l}\text { Parts are cor- } \\
\text { rectly assembled }\end{array}$ & $\begin{array}{l}\text { Assembly work will be done to pro- } \\
\text { cedure written and approved by } \\
\text { subsystem experts. Workers will re- } \\
\text { view the procedure and have train- } \\
\text { ing where necessary. }\end{array}$ & $\begin{array}{l}\text { Detector is assembled } \\
\text { correctly }\end{array}$ \\
\hline R-190005 & 1.9 & $\begin{array}{l}\text { Control Part ex- } \\
\text { posure to Radon }\end{array}$ & $\begin{array}{l}\text { Parts have an allowable total expo- } \\
\text { sure to Radon, based on material } \\
\text { and location. Monitored time of ex- } \\
\text { posure and Radon level of air are } \\
\text { recorded in a database. For critical } \\
\text { parts, may use samples. }\end{array}$ & $\begin{array}{l}\text { Control background } \\
\text { level in the experi- } \\
\text { ment }\end{array}$ \\
\hline
\end{tabular}




\subsubsection{WBS 1.10 Cleanliness and Screening}

The primary requirements for WBS 1.10 Cleanliness and Screening are shown in Table 12.2.10. At Level 2 , the WBS 1.10 requirements dictate that the project has the sensitivity and capacity to screen all detector components at the level needed to ensure that the radioactivity requirements are satisfied. WBS 1.10 also keeps track of allowed radioactivity levels for individual components, and more details can be found in Chapter 9.

Table 12.2.10: Level 2 Requirements for WBS 1.10 Cleanliness and Screening

\begin{tabular}{|c|c|c|c|c|}
\hline Number & WBS & Requirement Name & $\begin{array}{l}\text { Requirement } \\
\text { Description }\end{array}$ & Rationale \\
\hline R-200001 & 1.10 & $\begin{array}{l}\text { Assay Sensitivity for } U \text { and } \\
\text { Th by Direct Counting }\end{array}$ & $10 \mathrm{ppt}$ & $\begin{array}{l}\text { Sensitivity needed to assess to- } \\
\text { tal radioactive background }\end{array}$ \\
\hline R-200002 & 1.10 & $\begin{array}{l}\text { Assay Sensitivity for } U \text { and } \\
\text { Th by Neutron Activation } \\
\text { Analysis }\end{array}$ & $1.5 \mathrm{ppt}$ & $\begin{array}{l}\text { Sensitivity needed to assess to- } \\
\text { tal radioactive background }\end{array}$ \\
\hline R-200003 & 1.10 & $\begin{array}{l}\text { Assay Sensitivity for } U \text { and } \\
\text { Th by ICP-MS }\end{array}$ & $10 \mathrm{ppt}$ & $\begin{array}{l}\text { Sensitivity needed to assess to- } \\
\text { tal radioactive background }\end{array}$ \\
\hline R-200004 & 1.10 & $\begin{array}{l}\text { Assay Sensitivity for Radon } \\
\text { Emanation }\end{array}$ & $0.3 \mathrm{mBq}$ & $\begin{array}{l}\text { Sensitivity needed to assess to- } \\
\text { tal radioactive background }\end{array}$ \\
\hline R-200005 & 1.10 & $\begin{array}{l}\text { Assay Sensitivity for }{ }^{210} \mathrm{~Pb} \\
\text { in the bulk }\end{array}$ & $10 \mathrm{mBq} / \mathrm{kg}$ & $\begin{array}{l}\text { Sensitivity needed to assess to- } \\
\text { tal radioactive background }\end{array}$ \\
\hline R-200006 & 1.10 & $\begin{array}{l}\text { Assay Sensitivity for }{ }^{210} \mathrm{~Pb} \\
\text { Plateout }\end{array}$ & $60 \mathrm{nBq} / \mathrm{cm}^{2}$ & $\begin{array}{l}\text { Sensitivity needed to assess to- } \\
\text { tal radioactive background }\end{array}$ \\
\hline R-200007 & 1.10 & $\begin{array}{l}\text { Assay Sensitivity for Dust } \\
\text { Accumulation }\end{array}$ & $10 \mathrm{ng} / \mathrm{cm}^{2}$ & $\begin{array}{l}\text { Sensitivity needed to assess to- } \\
\text { tal radioactive background }\end{array}$ \\
\hline
\end{tabular}




\subsubsection{WBS 1.11 Offline Computing}

The primary requirements for WBS 1.11 Offline Computing are shown in Table 12.2.11, and are primarily the amount of disk and CPU to enable adequately detailed studies of the detector and background levels to be performed. Without sufficient computing resources, the project is unable to determine whether it is satisfying the top level science requirements. WBS 1.11 is also critically important in coordinating computing and software tasks with other WBS elements, particularly in simulations and analysis tools.

Table 12.2.11: Level 2 Requirements for WBS 1.11 Offline Computing

\begin{tabular}{|l|l|l|l|l|}
\hline Number & WBS & Requirement Name & $\begin{array}{l}\text { Requirement } \\
\text { Description }\end{array}$ & Rationale \\
\hline R-210001 & 1.11 & $\begin{array}{l}\text { US Disk Costs } \\
\text { FY15-FY18 }\end{array}$ & $\begin{array}{l}\text { Procure NERSC } \\
\text { GPF disk for } \\
\text { simulation, devel- } \\
\text { opment, and initial } \\
\text { data taking }\end{array}$ & $\begin{array}{l}\text { Handle simulation data, derived } \\
\text { data, and user data volumes as de- } \\
\text { fined in Table 11.2.2 }\end{array}$ \\
\hline R-210002 & 1.11 & $\begin{array}{l}\text { US CPU Costs } \\
\text { FY15-FY18 }\end{array}$ & $\begin{array}{l}\text { Procure NERSC } \\
\text { PDSF CPU for } \\
\text { simulation, devel- } \\
\text { opment, and initial } \\
\text { data taking }\end{array}$ & $\begin{array}{l}\text { Handle simulation data, derived } \\
\text { data, and user data volumes as de- } \\
\text { fined in Table 11.2.2 }\end{array}$ \\
\hline R-210003 & 1.11 & $\begin{array}{l}\text { US Server Costs } \\
\text { FY15-FY18 }\end{array}$ & $\begin{array}{l}\text { Procure and main- } \\
\text { tain servers for } \\
\text { simulation and } \\
\text { development }\end{array}$ & $\begin{array}{l}\text { Handle simulation data, derived } \\
\text { data, and user data volumes as de- } \\
\text { fined in Table 11.2.2 }\end{array}$ \\
\hline R-210004 & 1.11 & UK Data Center & $\begin{array}{l}\text { Design, deploy, } \\
\text { test, and maintain } \\
\text { UK Data Center } \\
\text { at Imperial }\end{array}$ & $\begin{array}{l}\text { Handle simulation data, derived } \\
\text { data, and user data volumes as de- } \\
\text { scribed in Sec. 11.2.2 }\end{array}$ \\
\hline
\end{tabular}




\subsection{Sensitivity and Detector Performance}

We employ the simulation tools described in Section 12.1 to evaluate the sensitivity of LZ, as defined by the requirements in Section 12.2, to a WIMP signal. Additionally, we evaluate the impact of variations in the parameters that define the LZ apparatus on its sensitivity.

The high-statistics calibrations performed by LUX and their incorporation into NEST have driven a conversion from the simple cut-and-count statistical methods used in the CDR to more advanced likelihood methods. The use of likelihood methods has markedly reduced the sensitivity of LZ to the dominant electron recoil backgrounds. Table 12.3.1 shows the number of counts with S1 signals between 0 and 20 photoelectrons coming from different background sources in a 1,000 d run with no discrimination applied, analogous to Table 9.2.7 (see Sec. 12.1.4 for further discussion of the cuts used to generate these numbers). For the majority of the chapter, we use the PLR method described in Sec. 12.3.1 to exploit the ER/NR discrimination power of liquid xenon to calculate our sensitivity to dark matter.

Table 12.3.1: Backgrounds described by PDFs in the profile likelihood analysis, with the counts expected with $\mathrm{S} 1$ signal between 0 and 20 photoelectrons in a 1,000 d run, with no discrimination applied, analogous to Table 9.2.7 (see Sec. 12.1.4 for further discussion of the cuts used to generate these numbers).

\begin{tabular}{|l|l|l|}
\hline Background & Type & Counts \\
\hline${ }^{8} \mathrm{~B}$ solar $v$ & $\mathrm{NR}$ & 7 \\
\hline hep $v$ & $\mathrm{NR}$ & 0.21 \\
\hline $\mathrm{DSN} v$ & $\mathrm{NR}$ & 0.05 \\
\hline $\mathrm{ATM} v$ & $\mathrm{NR}$ & 0.46 \\
\hline $\mathrm{pp}+{ }^{7} \mathrm{Be}+{ }^{14} \mathrm{~N}$ solar $v$ & $\mathrm{ER}$ & 255 \\
\hline${ }^{136} \mathrm{Xe}(2 v \beta \beta)$ & $\mathrm{ER}$ & 67 \\
\hline${ }^{85} \mathrm{Kr}$ & $\mathrm{ER}$ & 24.5 \\
\hline${ }^{222} \mathrm{Rn}$ & $\mathrm{ER}$ & 722 \\
\hline${ }^{220} \mathrm{Rn}$ & $\mathrm{ER}$ & 122 \\
\hline Detector components + Environmental & $\mathrm{ER}$ & 11.3 \\
\hline Detector components + Environmental & $\mathrm{NR}$ & 0.5 \\
\hline
\end{tabular}

Our evaluation of the LZ sensitivity should be considered as a snapshot in time. The field of direct dark matter detection with liquid xenon TPCs continues to achieve increasing sensitivity. Since the completion of the LZ CDR [1], the LUX Collaboration has substantially advanced the understanding of the response of liquid xenon to both background and signal [3,5], knowledge which has been incorporated in this report. As the LZ construction project progresses, key response and radioactive background properties will be measured and our sensitivity evaluation will adapt. We endeavor to bound this evolution by providing a "baseline" parameter set as well as "goal" and "reduced" sets, summarized in Table 12.3.2. The variation of sensitivity with excursions in many LZ parameters is captured in Section 12.3.3.

\subsubsection{Profile Likelihood Ratio Method}

The sensitivity projections in this report are based on a profile likelihood ratio (PLR) method [4], which allows near-optimum exploitation of the differences between signal and background in the key parameters that are reconstructed by the LZ apparatus. The parameters with the highest sensitivity to these differences are the position-corrected S1 (primary scintillation light) and S2 (secondary luminescence from ionization) signals. The position of events in radius $r$ and height $z$ also allows distinction between signal and the 
background events which originate from radioactive impurities in the material in the vicinity of the liquid xenon TPC of LZ; these background events cluster near the edges of the TPC, while the WIMP signal is uniform in the liquid xenon mass. In this report, we apply a simple fiducial volume cut, where 5.6 tonnes of the inner liquid xenon is retained as the sensitive volume. In the future, our sensitivity studies will exploit the distinct spatial distributions of signal and background just as the LUX experiment has done, and the fiducial requirement will become unnecessary.

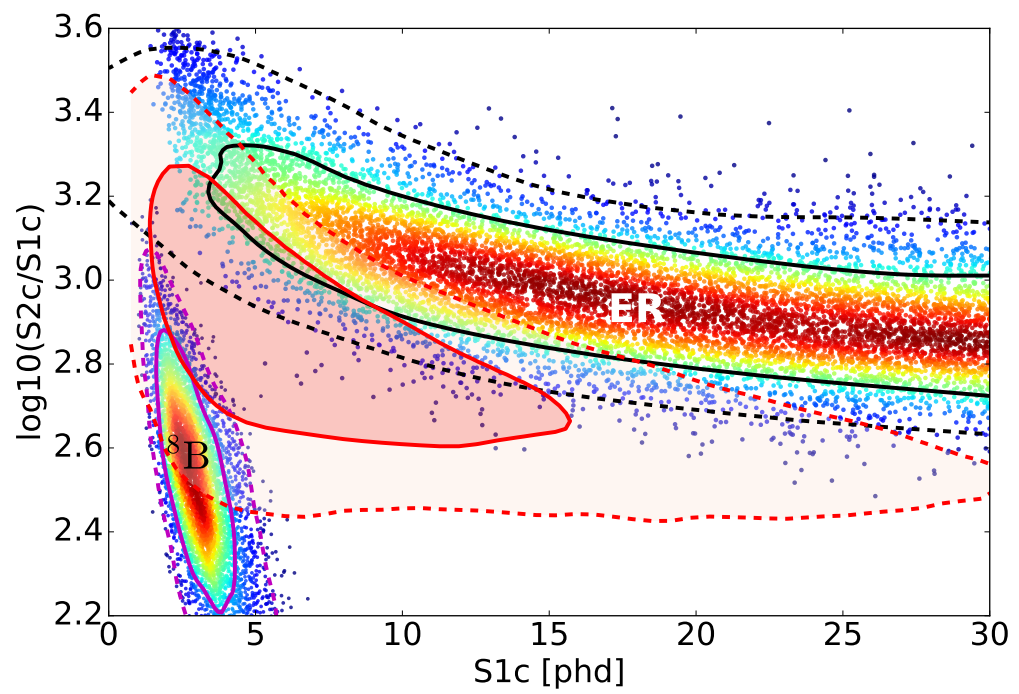

Figure 12.3.1: Simulations of the most prominent ER and NR (from ${ }^{8} \mathrm{~B}$ ) backgrounds are plotted in the $\log _{10}(\mathrm{~S} 2 \mathrm{c} / \mathrm{S} 1 \mathrm{c})-\mathrm{S} 1 \mathrm{c}$ plane. The statistics shown represent $5 \mathrm{x}$ the expected ER background and $500 \mathrm{x}$ the expected NR background in the nominal LZ exposure. The red tinted area shows the expectation for events from a $40 \mathrm{GeV} / c^{2}$-mass WIMP, falling between the two background populations with the region enclosed by the solid(dashed) line representing the $1 \sigma(2 \sigma)$ band.

To execute the PLR sensitivity estimate, signal and background probability distribution functions (PDFs) are created in S1 and S2 after application of the fiducial cut in $r$ and $z$. The signal PDF for each WIMP mass is generated by converting the differential energy spectrum calculated from [20] to S1 and S2 signals in the LZ detector using NEST and the parameterization of detector response described in Section 12.1.3.

The background PDFs are broken into the eleven individual components listed in Table 12.3.1. The simulations for detector components and environmental backgrounds are summed together into a single PDF each for ER and NR events. For each WIMP mass, we scan over the cross section to set a $90 \%$ confidence interval (CI) for the expected number of signal events, evaluated using RooStats [21]. In the PLR technique we use the unbinned likelihood computed in the plane of $\log _{10}(\mathrm{~S} 2 / \mathrm{S} 1)$ versus S1. Poisson fluctuations are innate to the technique.

The power of the PLR technique arises from an optimal weighting of the background-free and background-rich regions in the $\log _{10}(\mathrm{~S} 2 / \mathrm{S} 1)-\mathrm{S} 1$ plane. Figure 12.3.1 shows high-statistics simulations of the most prominent backgrounds (ER events from pp solar neutrinos, ${ }^{222} \mathrm{Rn}$, and ${ }^{220} \mathrm{Rn}$, and NR events from ${ }^{8} \mathrm{~B}$ neutrinos) in the $\log _{10}(\mathrm{~S} 2 / \mathrm{S} 1)-\mathrm{S} 1$ plane, representing $5 \mathrm{x}$ and 500x the count rates expected in the nominal LZ exposure for ER and NR, respectively. Also shown is the region that would be populated by events from a $40 \mathrm{GeV} / \mathrm{c}^{2}$ WIMP signal, which falls between the ${ }^{8} \mathrm{~B}$ and ER background areas. The PLR technique optimally combines the background-free and background-rich regions. The intense calibrations 
recently conducted by the LUX collaboration provide confidence in the knowledge of the shapes of the backgrounds and the signal.

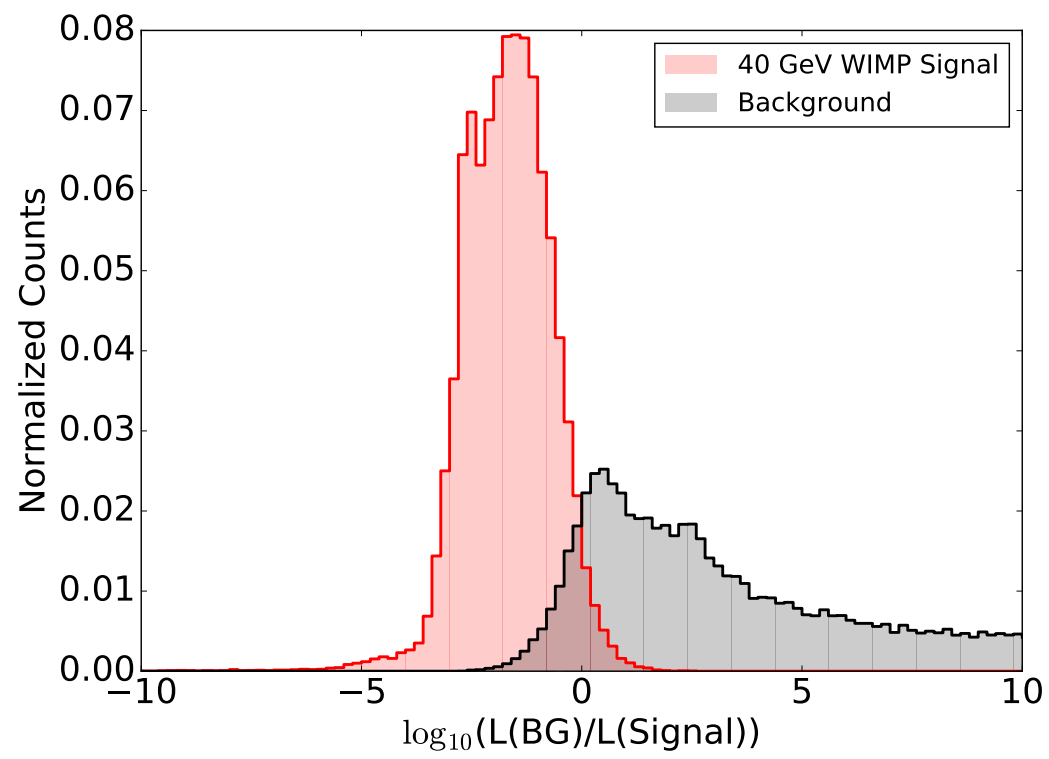

Figure 12.3.2: A one-dimensional projection of the PLR discrimination statistic. Two ensembles of points in the $\log _{10}(\mathrm{~S} 2 / \mathrm{S} 1)-\mathrm{S} 1$ plane are considered, one distributed like a $40 \mathrm{GeV} / c^{2} \mathrm{WIMP}$ signal, and the other like the expected background (combining both the ER and ${ }^{8} \mathrm{~B}$ bands of Fig. 12.3.1).
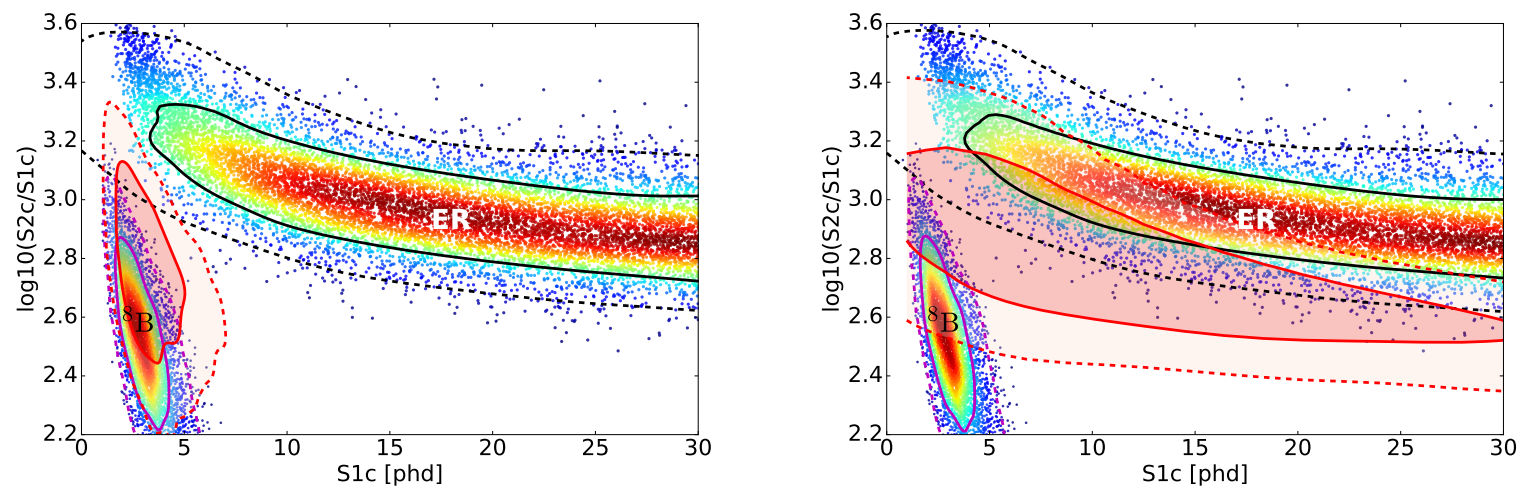

Figure 12.3.3: PLR technique for different masses. The background distributions are the same as in Figure 12.3.1, but the expected signal regions are for a $10 \mathrm{GeV} / c^{2}$ WIMP (left) and a $1,000 \mathrm{GeV} / c^{2}$ WIMP (right). The signal regions merge, respectively, into the ${ }^{8} \mathrm{~B}$ and $\mathrm{ER}$ background regions. The expected signal regions are tinted red, with the darker(lighter) color $1 \sigma(2 \sigma)$.

The discrimination statistic that quantifies whether each point in the respective ensemble more resembles background or signal is evaluated in one dimension as the difference between logarithms of the likelihoods that a point in the ensemble is background or signal. Low values correspond to poor likelihood to be background, and high values to a good likelihood to be background. Figure 12.3.2 shows the PLR discrimination statistic integrated over the full energy range for a $40 \mathrm{GeV} / c^{2}$ WIMP signal and the expected background 
(combining both the ER and ${ }^{8} \mathrm{~B}$ bands of Fig. 12.3.1). Comparison of the two ensembles shows the considerable separation between signal and background available using this method. This plot and similar plots constructed for different WIMP masses are used to describe the discrimination power of the LZ apparatus against background.

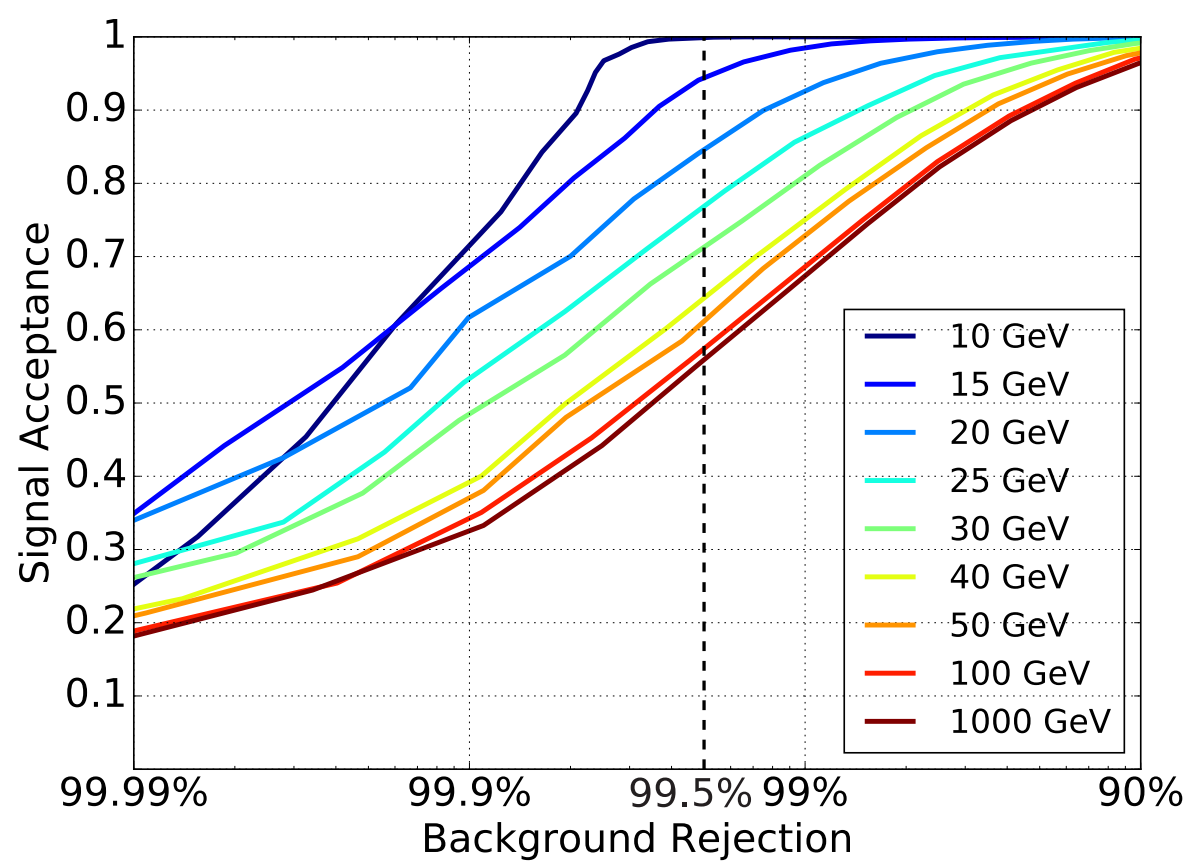

Figure 12.3.4: Acceptance and rejection for WIMP signals in LZ. For a variety of WIMP masses, histograms like that shown in the Fig. 12.3.2 are integrated to derive the curves shown. Backgrounds from both ${ }^{8} \mathrm{~B}$ and $\mathrm{ER}$ events are included. The requirement of $99.5 \%$ rejection at $50 \%$ acceptance is projected for all WIMP masses.

The expected signal region varies according to the WIMP mass, and Figure 12.3.3 shows the signal regions for WIMP masses of $10 \mathrm{GeV} / c^{2}$ and $1,000 \mathrm{GeV} / c^{2}$ compared to the same ER and NR simulations of Fig. 12.3.1. The PLR method naturally takes into account the shape of the expected WIMP signal, and the achievable discrimination at all WIMP masses is thus significantly better than that attainable in the "cut and count' technique utilized in the LZ CDR. Figure 12.3.4 shows the signal acceptance for WIMPs of a variety of masses as a function of the fraction of the background rejected. For all WIMP masses, a background rejection that exceeds $99.5 \%$ for signal acceptance of $50 \%$ is projected.

\subsubsection{LZ Sensitivity Projection}

To evaluate the projected sensitivity for LZ, we consider a run of 1,000 live days and a 5.6 tonne fiducial mass. We assume the same background models of Table 12.3.1, where background counts were shown for a signal region encompassing 0 to 20 phd, effectively the search region for $100 \mathrm{GeV} / \mathrm{c}^{2}$ WIMP masses and below. To increase the sensitivity over the entire WIMP mass range, we consider an expanded S1 signal region of 0 to $50 \mathrm{phd}$, corresponding to 1.5 to $16 \mathrm{keV}_{\mathrm{ee}}$ for $\mathrm{ER}$ and 6 to $60 \mathrm{keV}_{\mathrm{nr}}$ for NR. Although the expanded search region brings with it a higher absolute count of backgrounds than those listed in the table, the use of the PLR method smoothly accounts for the profiles of these backgrounds relative to each WIMP 
mass as described in the previous section. We include the requirement of a 3-fold coincidence for the PMTs.

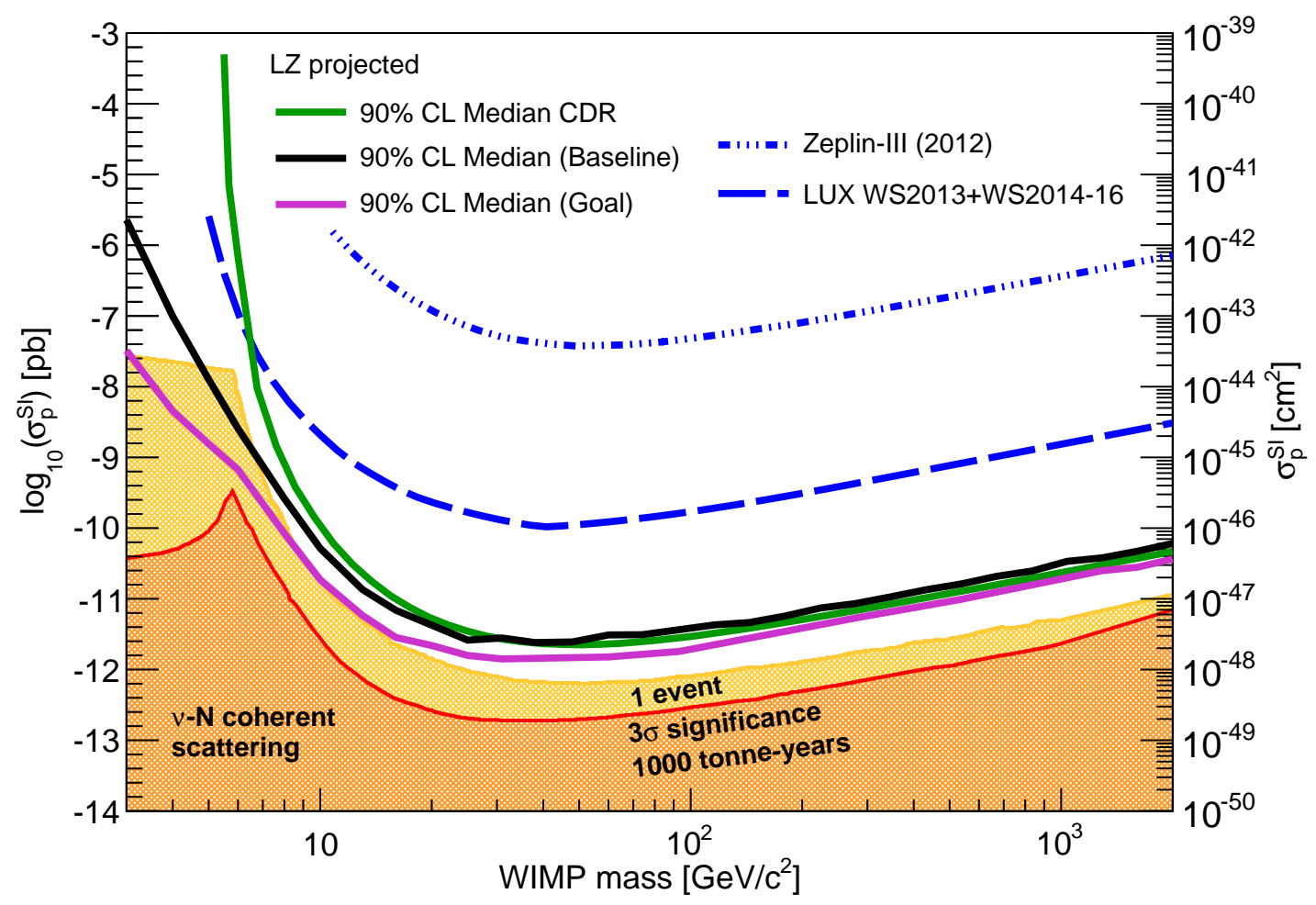

Figure 12.3.5: LZ sensitivity projection. The baseline LZ assumptions in this report give the solid black curve. LUX and ZEPLIN results are shown in broken blue lines. If LZ achieves the design goals listed in Table 12.3.2, the sensitivity would improve, resulting in the pink sensitivity curve. The green line shows the projected sensitivity in the LZ Conceptual Design Report (CDR) [1] (see text for details of the changes from the CDR to this report). Lastly, the shaded regions show where coherent scattering neutrino backgrounds emerge.

The projected sensitivity curve for LZ is shown in Figure 12.3 .5 . The best sensitivity is $2.3 \times 10^{-48} \mathrm{~cm}^{2}$ for a $40 \mathrm{GeV} / c^{2}$ WIMP mass, which satisfies our top level science requirement.

Figure 12.3.5 also shows the projected sensitivity from the LZ Conceptual Design Report (CDR) [1]. There are three main differences in this projection with respect to the CDR:

1. We have increased the assumed level of ${ }^{222} \mathrm{Rn}$ and ${ }^{220} \mathrm{Rn}$ by a factor of twenty, so radon is now the dominant source of ER events in the detector.

2. The PLR technique as described in the previous section is used to evaluate the dark matter sensitivity, instead of a simple cut and count approach. These two changes effectively offset: there is a higher overall background level but those backgrounds are more effectively rejected by the analysis technique. Our confidence in the ER background shape, most important for using the PLR technique, rests on the high-statistics LUX tritium ER calibration [2]. 
3. The LUX neutron calibration results, which allow LZ to more confidently project the response of the detector to very low energy nuclear recoils. Use of the neutron calibration provides the greatly increased sensitivity to very low-mass WIMPs in the new projection.

Figure 12.3.6 shows the discovery potential for LZ under the baseline assumptions, calculated using a cut-and-count technique via the TRolke package $[22,23]$. With the baseline parameters, LZ will have $3 \sigma$ significance for $40 \mathrm{GeV} / c^{2}$ WIMP mass at a cross section of $6.0 \times 10^{-48} \mathrm{~cm}^{2}$. We also show the $5 \sigma$ significance curve, which falls below the projections from the XENON1T experiment at all WIMP masses. Figure 12.3.7 shows the background and signal events populating the $\log _{10}(\mathrm{~S} 2 / \mathrm{S} 1)-\mathrm{S} 1$ plane in an example LZ experiment with 1000 days and $5600 \mathrm{~kg}$ exposure for the $3 \sigma$ example combination of WIMP parameters.

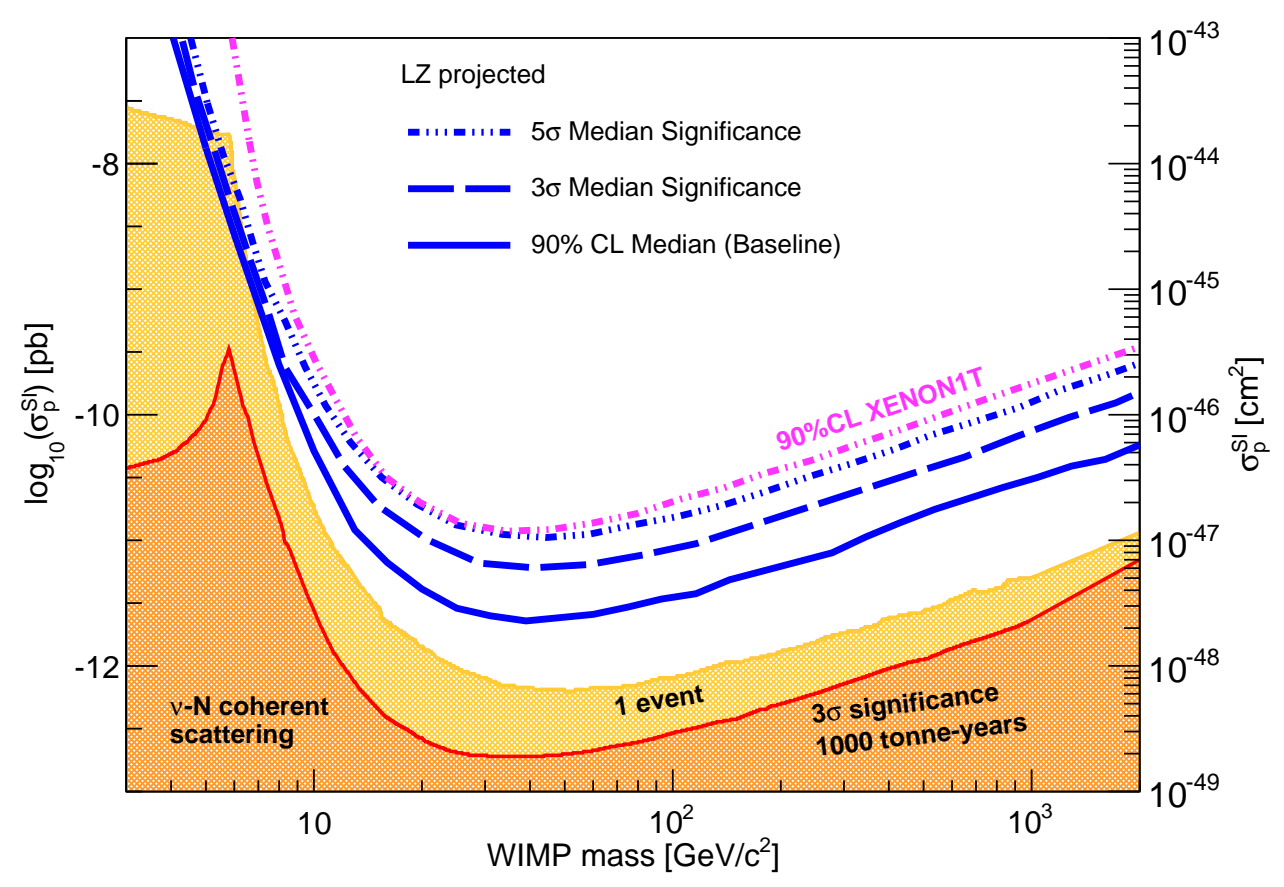

Figure 12.3.6: The discovery potential for LZ under the baseline assumptions, calculated using a cutand-count technique via the TRolke package $[22,23]$. With the baseline parameters, LZ will have $3 \sigma$ significance for $40 \mathrm{GeV} / c^{2}$ WIMP mass at a cross section of $6.0 \times 10^{-48} \mathrm{~cm}^{2}$. The $5 \sigma$ significance expectation is just below the expected $90 \% \mathrm{CL}$ limit from a two year run of XENON1T at all WIMP masses.

\subsubsection{Parameter Scans of LZ Sensitivity}

We have explored the dependency of the LZ sensitivity to spin-independent interactions of WIMPs upon critical detector performance assumptions. Our baseline parameters lead to the sensitivity shown by the solid blue curve in Fig. 12.3.5

One such study defines "goals" as a set of parameters that are likely to be achieved during the fabrication of LZ, but which are somewhat less conservative than the baseline. We define a "reduced" set of parameters as an unlikely worst-case. Both the goal and baseline parameter sets meet all requirements in Section 12.2. 


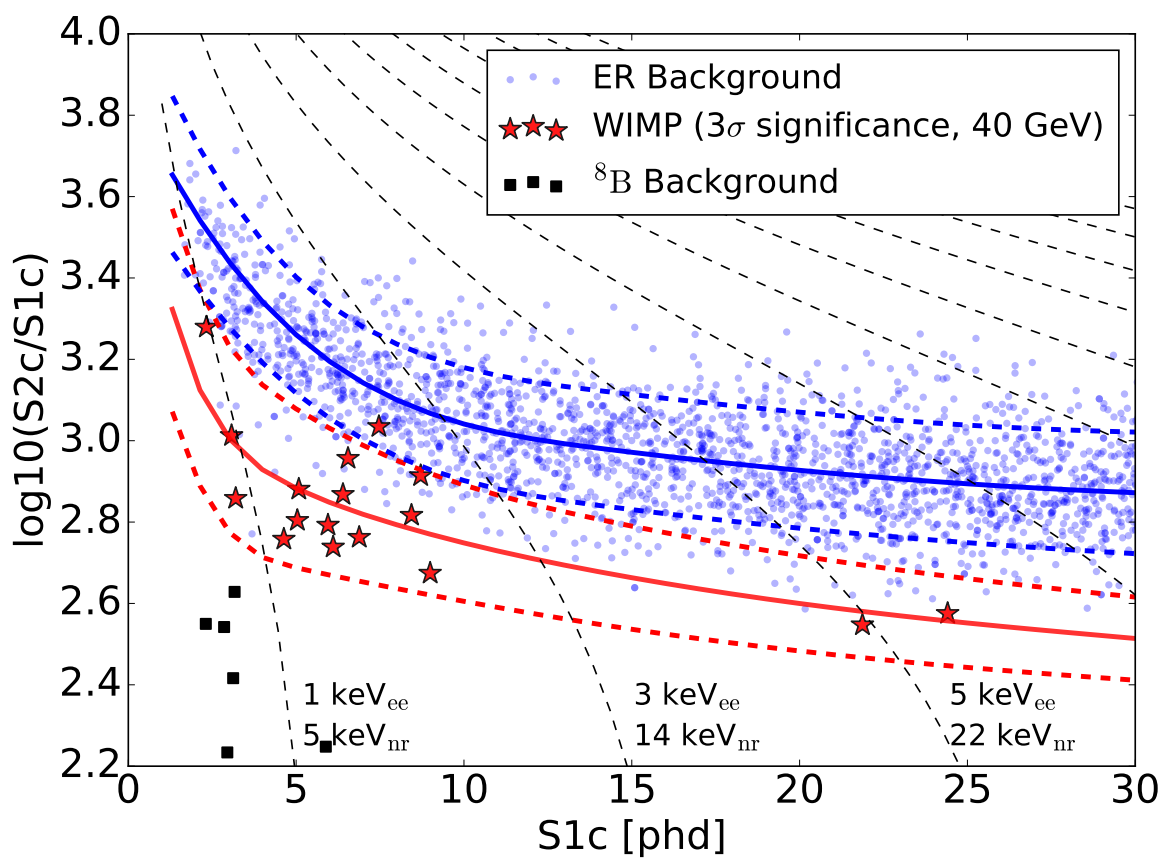

Figure 12.3.7: A sample LZ exposure under the baseline assumptions where the signal represents a $40 \mathrm{GeV} / c^{2}$ WIMP mass with a cross section of $6.0 \times 10^{-48} \mathrm{~cm}^{2}$. LZ will observe several ${ }^{8} \mathrm{~B}$ events under these assumptions, along with the nominal ER backgrounds. For these WIMP parameters, LZ will have a $3 \sigma$ median significance for WIMP discovery. The solid blue and red lines represent the median of the ER and NR bands, the dashed blue and red lines indicate the $10 \%$ to $90 \%$ intervals for each population. The dashed lines running from the top left corner down to the $\mathrm{x}$-axis show lines of constant recoil energy.

These three parameters sets are listed in Table 12.3.2, and their projected performance is shown in Figure 12.3.8. Reaching the LZ goals achieves a sensitivity of $1.1 \times 10^{-48} \mathrm{~cm}^{2}$ at $40 \mathrm{GeV} / c^{2}$. The reduced parameter set degrades the sensitivity to $5.1 \times 10^{-48} \mathrm{~cm}^{2}$ at $40 \mathrm{GeV} / c^{2}$.

Table 12.3.2: Key parameters for reduced, baseline and goal detector performance as explained in the text.

\begin{tabular}{|l|c|c|c|}
\hline Detector Parameter & Reduced & Baseline & Goal \\
\hline Light collection (PDE) & 0.05 & 0.075 & 0.12 \\
\hline Drift field $(\mathrm{V} / \mathrm{cm})$ & 160 & 310 & 650 \\
\hline Electron lifetime $(\mu \mathrm{s})$ & 850 & 850 & 2800 \\
\hline PMT phe detection & 0.8 & 0.9 & 1.0 \\
\hline $\mathrm{N}$-fold trigger coincidence & 4 & 3 & 2 \\
\hline${ }^{222} \mathrm{Rn}(\mathrm{mBq}$ in active region) & 13.4 & 13.4 & 0.67 \\
\hline Live days & 1000 & 1000 & 1000 \\
\hline
\end{tabular}

A notable observation that would accompany the achievement of the "goal" set of parameters: over 300 ${ }^{8} \mathrm{~B}$ neutrino events would be observed in a 1,000 live days $\mathrm{LZ}$ run. While these events would slow the 
discovery of a WIMP in the $7 \mathrm{GeV} / c^{2}$ mass range, they would also demonstrate a physical process not yet observed in nature, the coherent scattering of neutrinos from nuclei.

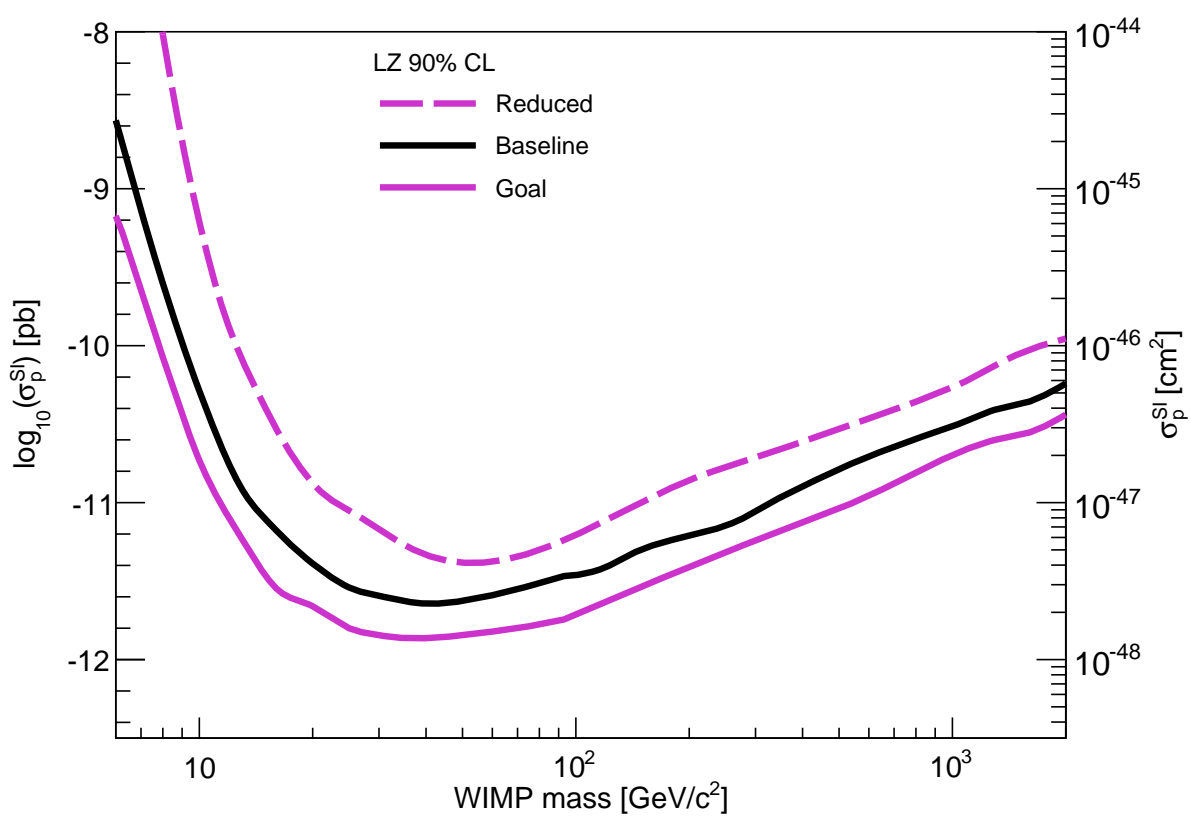

Figure 12.3.8: LZ sensitivity projections for goal, baseline, and reduced parameters. The respective best sensitivities are $1.1 \times 10^{-48} \mathrm{~cm}^{2}, 2.3 \times 10^{-48} \mathrm{~cm}^{2}$ (which both satisfy the primary science requirement), and $5.1 \times 10^{-48} \mathrm{~cm}^{2}$ at a WIMP mass of $40 \mathrm{GeV} / c^{2}$.

In the next several figures, we vary key detector performance parameters to gauge their impact on the median $90 \% \mathrm{CL}$ of the upper limit for WIMP cross-section. Figure 12.3.9 shows the impact of ${ }^{222} \mathrm{Rn}$, the dominant ER background, on LZ sensitivity. Assuming that the goal of $0.67 \mathrm{mBq}$ is achieved, the sensitivity (to a $40 \mathrm{GeV} / c^{2}$ WIMP) improves by $20 \%$ over the baseline case. If the Rn background is increased another 10x over the nominal case then the sensitivity would degrade $20 \%$. The ${ }^{222} \mathrm{Rn}$ rate is representative of a generic flat ER background in LZ. Even a factor of 10 increase in the radon has marginal impact on sensitivity, demonstrating the power of using the PLR to separate ER backgrounds from signal like events. The effect of radon on the discovery potential is more significant, as shown in Fig. 12.3.10, where a 10x increase in the radon rate degrades the discovery potential of the detector by a factor of two.

Figure 12.3.11 shows the impact on sensitivity of scaling the atmospheric (ATM) coherent scattering neutrino background. ATM neutrinos produce nuclear recoils similar to that of a $40 \mathrm{GeV} / \mathrm{c}^{2} \mathrm{WIMP}$, and their PDF has a high degree of overlap with the WIMP signal. Adjusting the ATM rate serves as a proxy for changing the overall NR count. When the ATM rate is turned up by a factor of 10 , it is equivalent to having roughly 5 extra NR counts, degrading the sensitivity (to a $40 \mathrm{GeV} / c^{2}$ WIMP) by $25 \%$.

Figure 12.3.12 shows the effect of changes in the S1 photon detection efficiency alone. Greater light collection leads to better S1 resolution, tightening the distribution of NR and ER events and leading to improved discrimination. Better light collection also improves the low energy threshold of LZ, enhancing sensitivity to low-mass dark matter, although this effect is somewhat countered by also seeing a higher ${ }^{8} \mathrm{~B}$ background at low energy. Figure 12.3.13 shows the effect of changes in the purity of the LXe, as represented by the electron lifetime. There is significant margin until the drift time drops below half the baseline value. Figure 12.3.14 gives the dependence on electron extraction efficiency. With only $50 \%$ extraction efficiency, 
larger fluctuations in the S2 signal lead to a reduction in discrimination power and a corresponding loss of sensitivity. Figure 12.3.15 shows that the sensitivity depends weakly on the drift field. Figure 12.3.16 shows the dependence of the sensitivity on the coincidence trigger requirement, where the baseline design assumes a 3-PMT coincidence trigger. Going to 2-PMT coincidence reduces the threshold and makes a significant impact for low-mass WIMPs and similarly the ${ }^{8} \mathrm{~B}$ neutrino signal.

Lastly, Figure 12.3.17 shows how extending the run from 1,000 to 3,000 live days would improve the sensitivity of the experiment. The plot shows the median $90 \%$ confidence level upper limit on the cross section for a $40 \mathrm{GeV} / \mathrm{c}^{2}$ WIMP. In the baseline case, the sensitivity can be improved from $2.3 \times 10^{-48} \mathrm{~cm}^{2}$ to $1.3 \times 10^{-48} \mathrm{~cm}^{2}$. If all the design goals are achieved, the sensitivity can be improved from $1.1 \times 10^{-48} \mathrm{~cm}^{2}$ to $6 \times 10^{-49} \mathrm{~cm}^{2}$ with 3,000 live days. Figure 12.3 .17 also shows the $1 \sigma$ bands on the expected sensitivity for both the baseline and goal parameter sets in a given exposure of LZ.

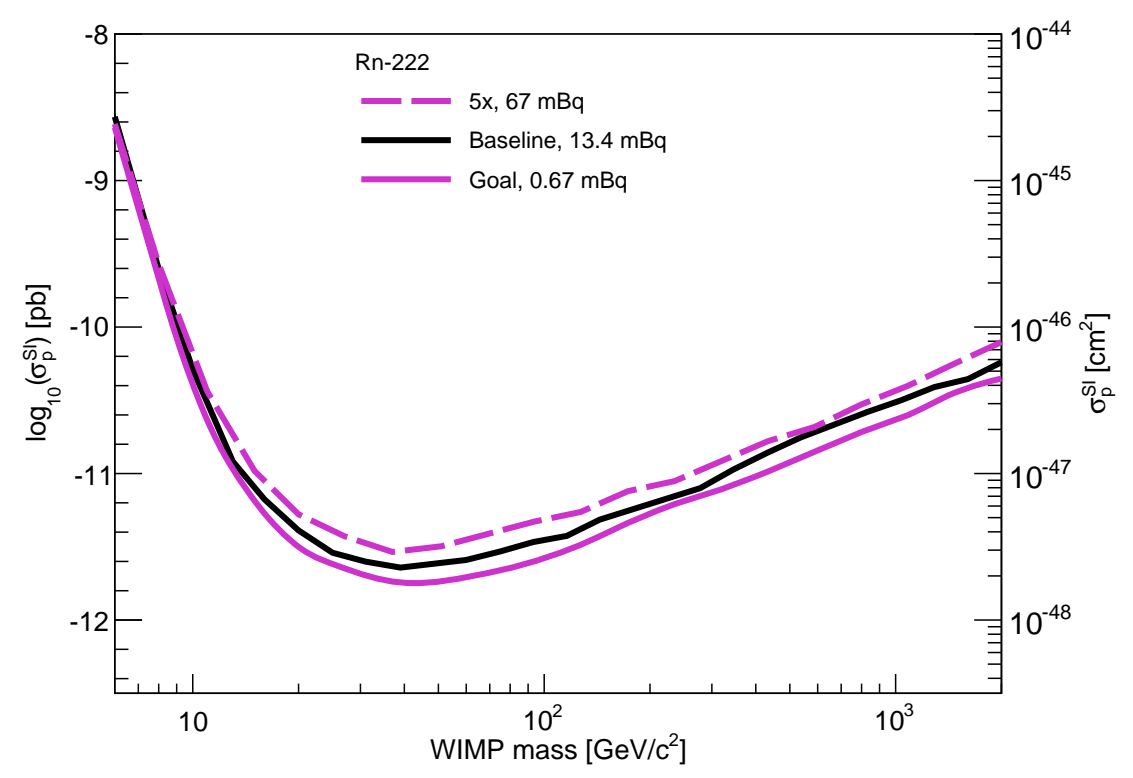

Figure 12.3.9: LZ sensitivity projections for three different assumptions on the concentration of radon in the active volume. 


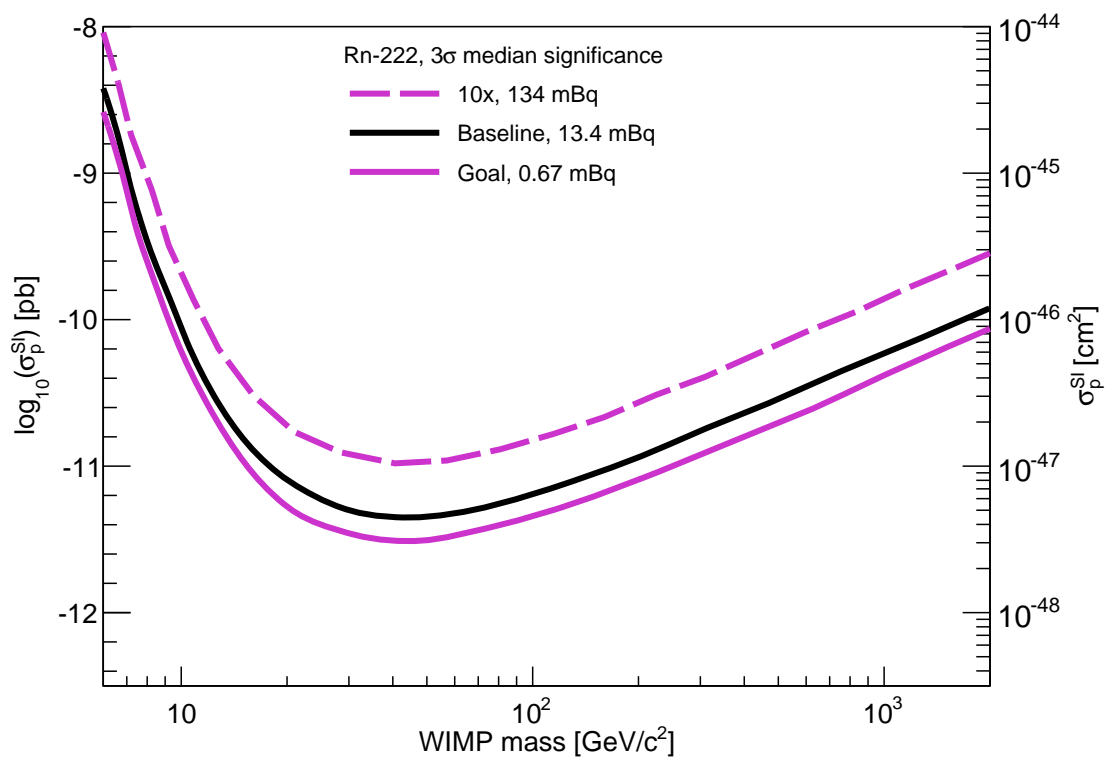

Figure 12.3.10: LZ $3 \sigma$ median significance projections for three different assumptions on the concentration of radon in the active volume.

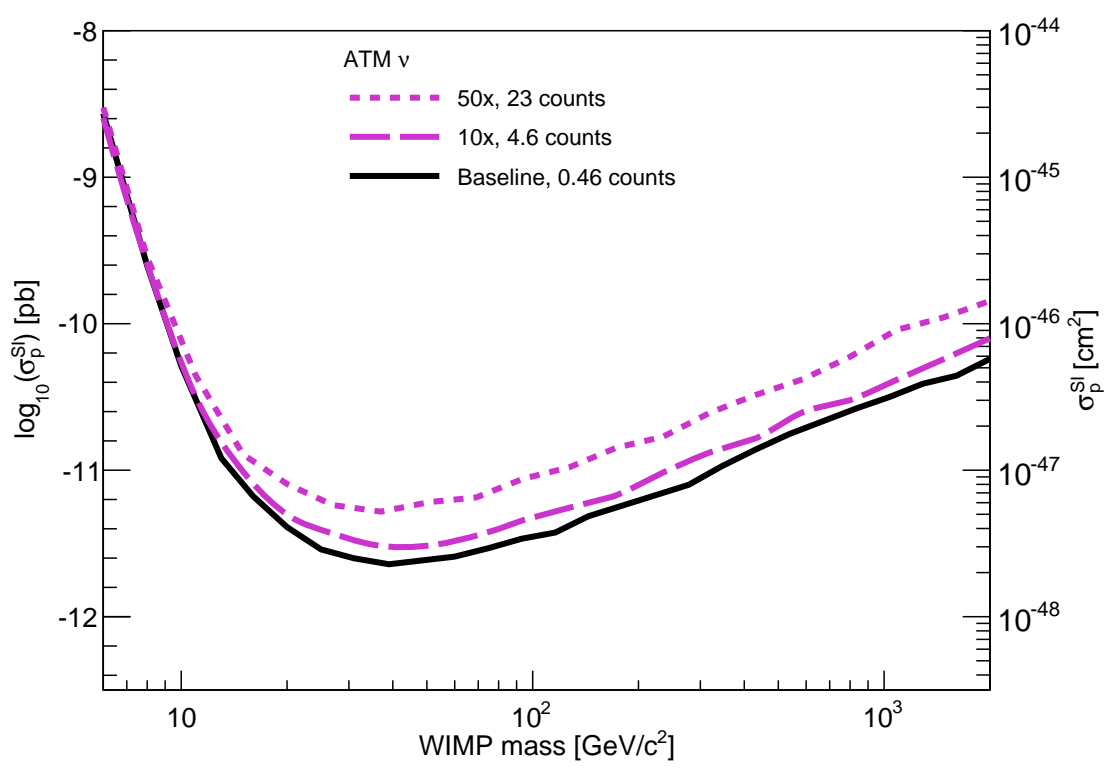

Figure 12.3.11: LZ sensitivity projections vs. scaled atmospheric neutrino flux. Scaling the atmospheric neutrino rate is a proxy for scaling the overall NR backgrounds in LZ. 


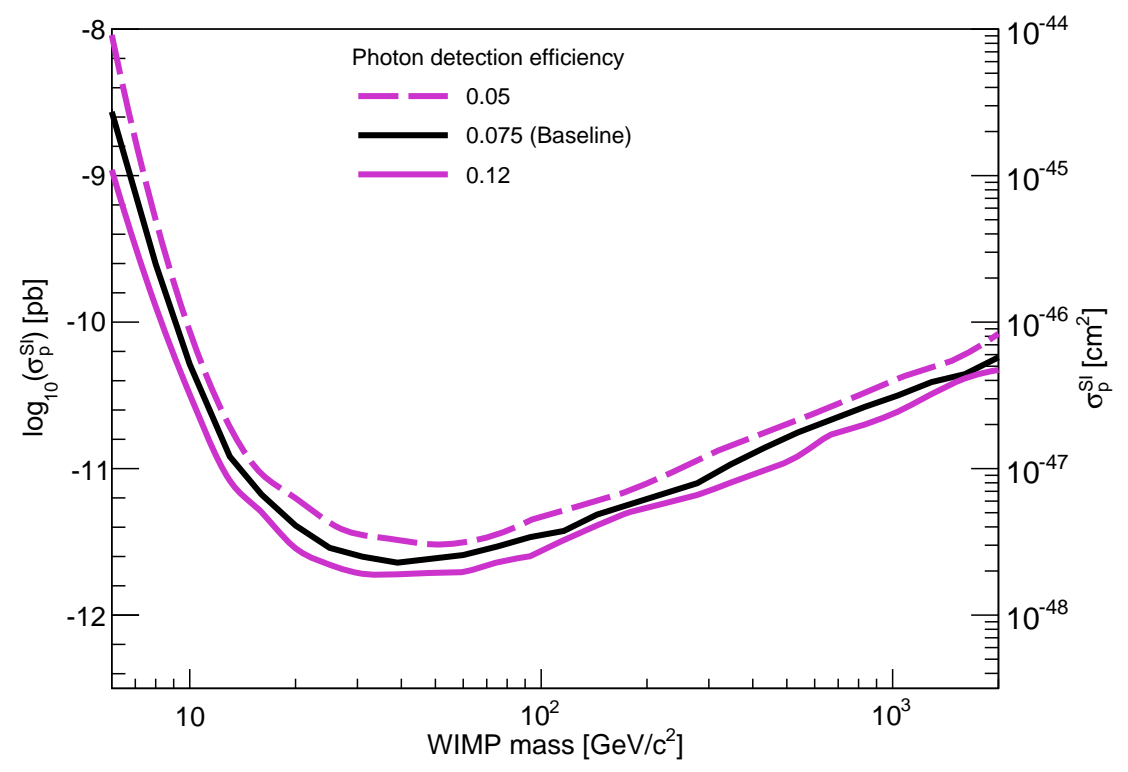

Figure 12.3.12: LZ sensitivity projections vs. S1 photon detection efficiency. We assume a photon detection efficiency of 0.075 for the baseline sensitivity, matching the requirement. The current model of the detector predicts a value of 0.085 (see Sec. 3.5.1).

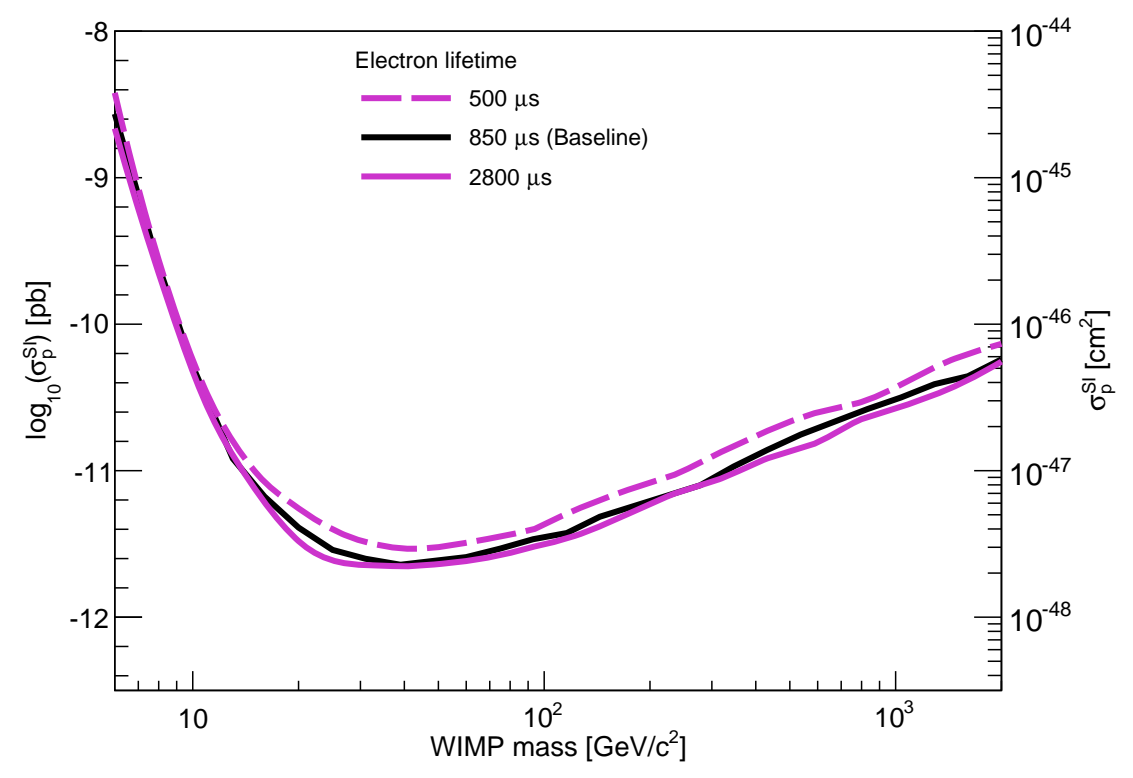

Figure 12.3.13: LZ sensitivity projections vs. electron lifetime (for the nominal electric field value of $310 \mathrm{~V} / \mathrm{cm}$ ). LZ does not lose significant sensitivity until the lifetime drops below half the nominal value of $850 \mu \mathrm{s}$. 


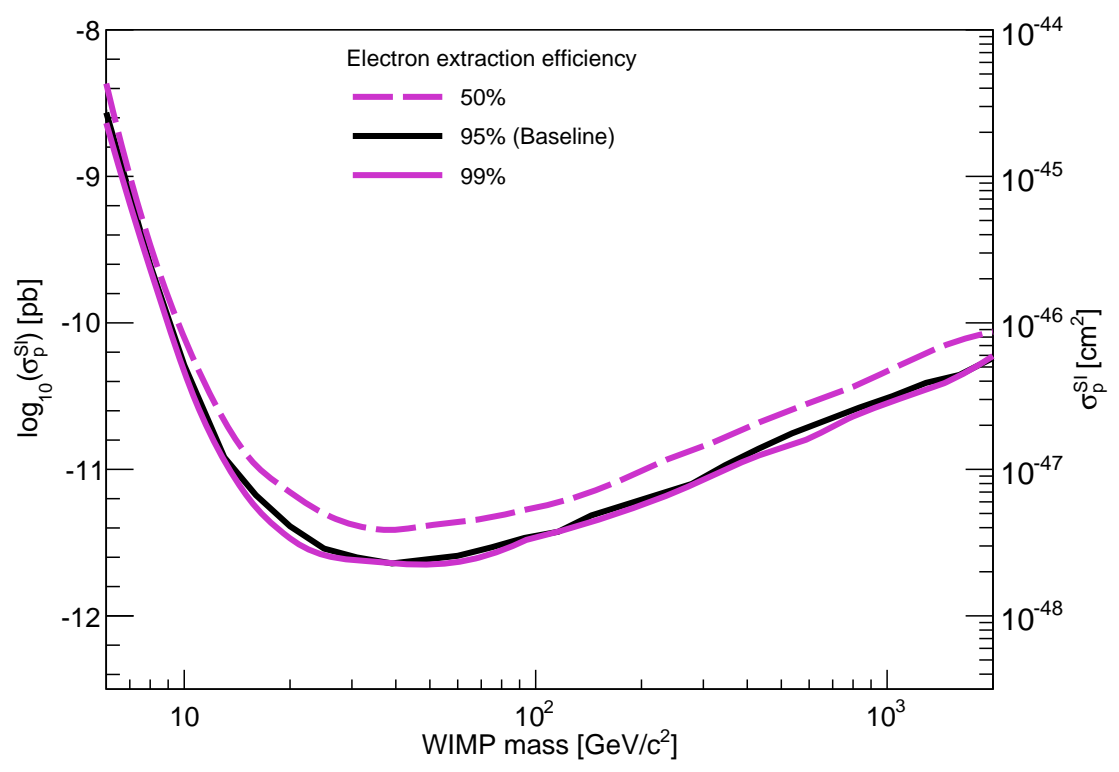

Figure 12.3.14: LZ sensitivity projections vs. electron extraction efficiency. With only $50 \%$ extraction efficiency, larger fluctuations in the S2 signal lead to a reduction in discrimination power and a corresponding loss of sensitivity.

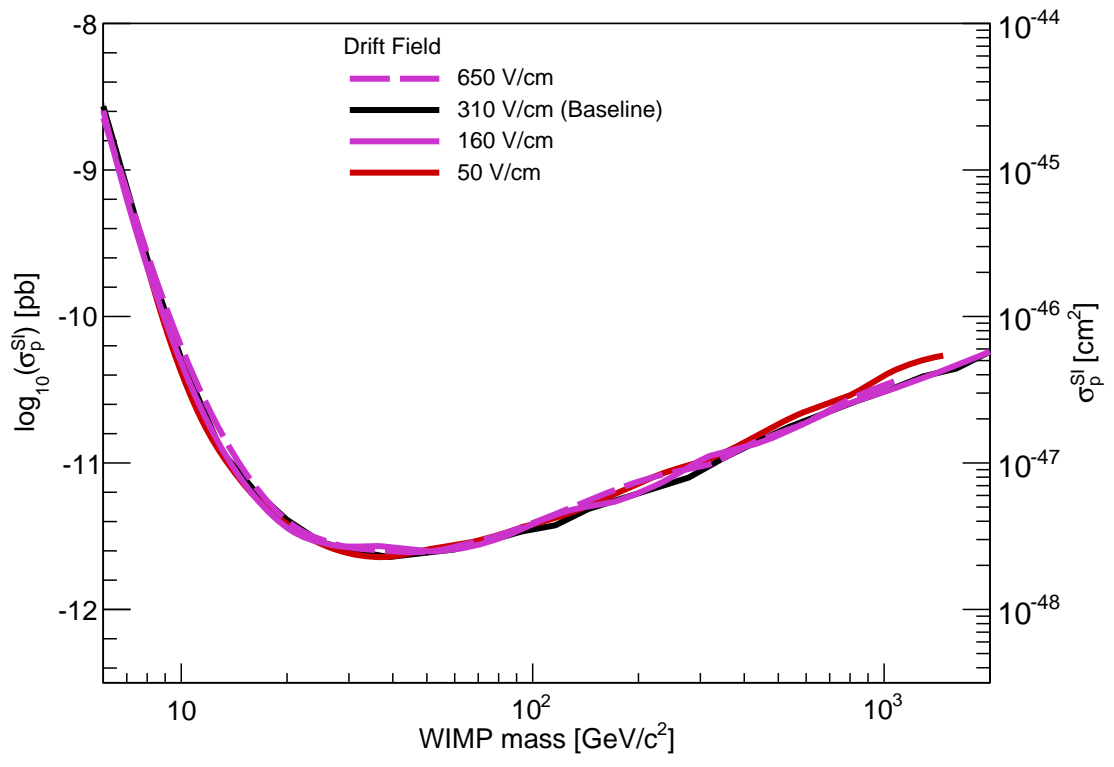

Figure 12.3.15: LZ sensitivity projections vs. electric field. In this regime, the ER/NR discrimination is robust to changes in the drift field, and the effect on sensitivity is minor. 


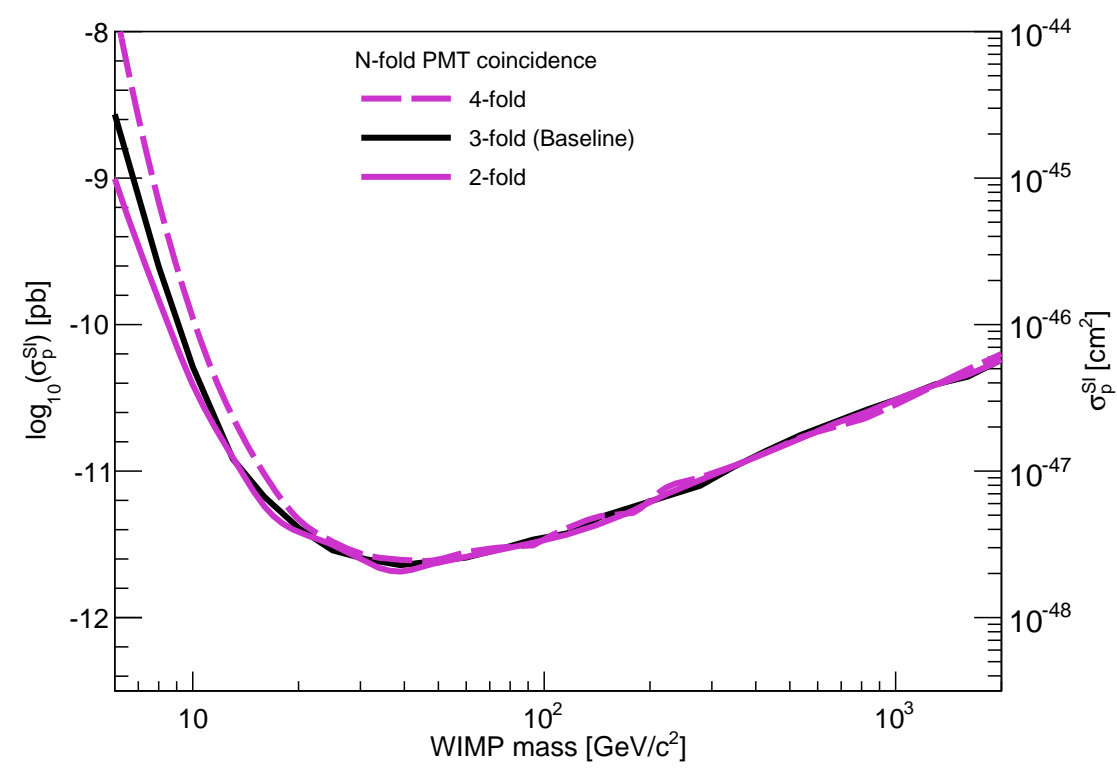

Figure 12.3.16: LZ sensitivity projections vs. trigger coincidence level. The primary effect of the coincidence requirement is on the detection of very low energy events, with direct consequences for sensitivity to low WIMP masses. For comparison, the LUX detector operates with a 2-fold trigger coincidence.

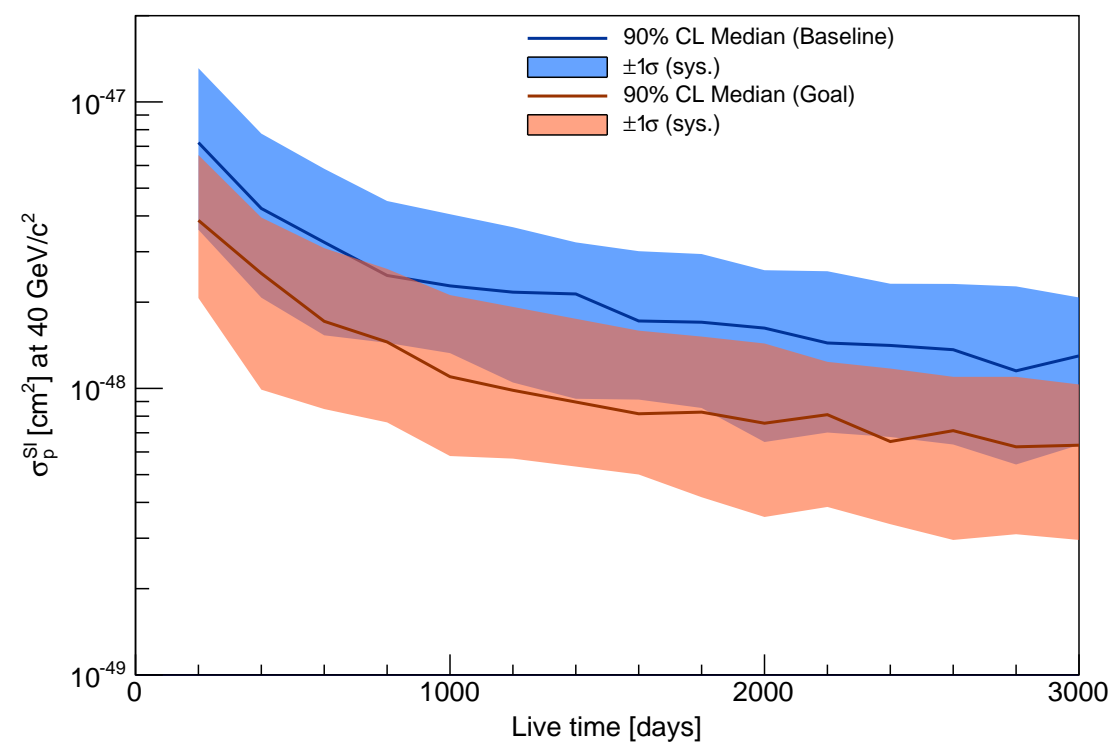

Figure 12.3.17: LZ sensitivity projections vs. exposure for the baseline (blue) and goal (orange). The impact of running the experiment for an additional 2000 days is to improve the sensitivity to $1.2 \times 10^{-48} \mathrm{~cm}^{2}$ and $6 \times 10^{-49} \mathrm{~cm}^{2}$ for the baseline and goal scenarios, respectively. Also shown are the $1 \sigma$ bands showing the range of possible sensitivities for a given exposure. 


\subsection{Bibliography}

[1] D. S. Akerib et al. (LZ), (2015), Conceptual Design Report; LBNL-190005, FERMILAB-TM-2621AE-E-PPD, arXiv:1509.02910 [physics.ins-det].

[2] D. S. Akerib et al. (LUX), Phys. Rev. D93, 072009 (2016), arXiv:1512.03133 [physics.ins-det].

[3] D. S. Akerib et al. (LUX), "Low-energy (0.7-74 keV) nuclear recoil calibration of the LUX dark matter experiment using D-D neutron scattering kinematics," (2016), submitted to Phys. Rev. C, arXiv:1608.05381 [physics.ins-det].

[4] G. Cowan, K. Cranmer, E. Gross, and O. Vitells, Eur. Phys. J. C71, 1554 (2011), [Erratum: Eur. Phys. J.C73,2501(2013)], arXiv:1007.1727 [physics.data-an].

[5] D. S. Akerib et al. (LUX), Phys. Rev. Lett. 116, 161301 (2016), arXiv:1512.03506 [astro-ph].

[6] D. S. Akerib et al. (LUX), Nucl. Instrum. Meth. A675, 63 (2012), arXiv:1111.2074 [physics.data-an].

[7] S. Agostinelli et al. (GEANT4), Nucl. Instrum. Meth. A506, 250 (2003).

[8] M. Szydagis, N. Barry, K. Kazkaz, J. Mock, D. Stolp, M. Sweany, M. Tripathi, S. Uvarov, N. Walsh, and M. Woods (NEST), J. Instrum. 6, P10002 (2011), arXiv:1106.1613 [physics.ins-det].

[9] M. Szydagis, A. Fyhrie, D. Thorngren, and M. Tripathi (NEST), Proceedings, LIght Detection In Noble Elements (LIDINE2013), J. Instrum. 8, C10003 (2013), arXiv:1307.6601 [physics.ins-det].

[10] W. B. Wilson et al., SOURCES4A: A Code for Calculating (alpha,n), Spontaneous Fission, and Delayed Neutron Sources and Spectra, Tech. Rep. LA-13639-MS (Los Alamos, 1999).

[11] M. J. Carson et al., Astropart. Phys. 21, 667 (2004), arXiv:hep-ex/0404042 [hep-ex].

[12] R. Lemrani, M. Robinson, V. A. Kudryavtsev, M. De Jesus, G. Gerbier, and N. J. C. Spooner, Nucl. Instrum. Meth. A560, 454 (2006), arXiv:hep-ex/0601030 [hep-ex].

[13] V. Tomasello, M. Robinson, and V. A. Kudryavtsev, Astropart. Phys. 34, 70 (2010).

[14] M. Herman, R. Capote, B. Carlson, P. Oblozinsky, M. Sin, A. Trkov, H. Wienke, and V. Zerkin, Nucl. Data Sheets 108, 2655 (2007).

[15] V. A. Kudryavtsev, Comput. Phys. Commun. 180, 339 (2009), (MUSIC), arXiv:0810.4635 [physics.comp-ph].

[16] P. Antonioli, C. Ghetti, E. V. Korolkova, V. A. Kudryavtsev, and G. Sartorelli, Astropart. Phys. 7, 357 (1997), (MUSIC), arXiv:hep-ph/9705408 [hep-ph].

[17] B. Lenardo, K. Kazkaz, A. Manalaysay, J. Mock, M. Szydagis, and M. Tripathi (NEST), IEEE Trans. Nucl. Sci. 62, 3387 (2015), arXiv:1412.4417 [astro-ph.IM].

[18] C. E. Dahl, The physics of background discrimination in liquid xenon, and first results from Xenon10 in the hunt for WIMP dark matter, Ph.D. thesis, Princeton U. (2009).

[19] C. H. Faham, V. M. Gehman, A. Currie, A. Dobi, P. Sorensen, and R. J. Gaitskell, J. Instrum. 10, P09010 (2015), arXiv:1506.08748 [physics.ins-det].

[20] C. McCabe, Phys. Rev. D82, 023530 (2010), arXiv:1005.0579 [hep-ph]. 
[21] L. Moneta, K. Belasco, K. S. Cranmer, S. Kreiss, A. Lazzaro, D. Piparo, G. Schott, W. Verkerke, and M. Wolf, Proceedings, 13th International Workshop on Advanced computing and analysis techniques in physics research (ACAT2010), PoS ACAT2010, 057 (2010), arXiv:1009.1003 [physics.data-an].

[22] J. Lundberg, J. Conrad, W. Rolke, and A. Lopez, Comput. Phys. Commun. 181, 683 (2010), arXiv:0907.3450 [physics.data-an].

[23] W. A. Rolke, A. M. Lopez, and J. Conrad, Nucl. Instrum. Meth. A551, 493 (2005), arXiv:physics/0403059 [physics]. 


\section{Management, Cost, and Schedule Summary}

The LZ Project (the Project) is international in scope, funding, and organization. This chapter presents an overview of the overall Project organization and a summary of the Project cost and schedule. The integrated overall Project Management organization is also described here. This Project organization has authority and responsibility over all aspects of the Project, including those funded by DOE, SDSTA, and non-U.S. agencies: the U.K.'s Science \& Technology Facilities Council (STFC); Portugal's Fundação para a Ciência e a Tecnologia (FCT); and Korean-funded scope. The functions of the Project Advisory Board (PAB) are also described. A detailed discussion of Project Management, management systems, and approaches is described separately in the LZ Project Execution Plan (PEP) document.

\subsection{LZ Project Organization}

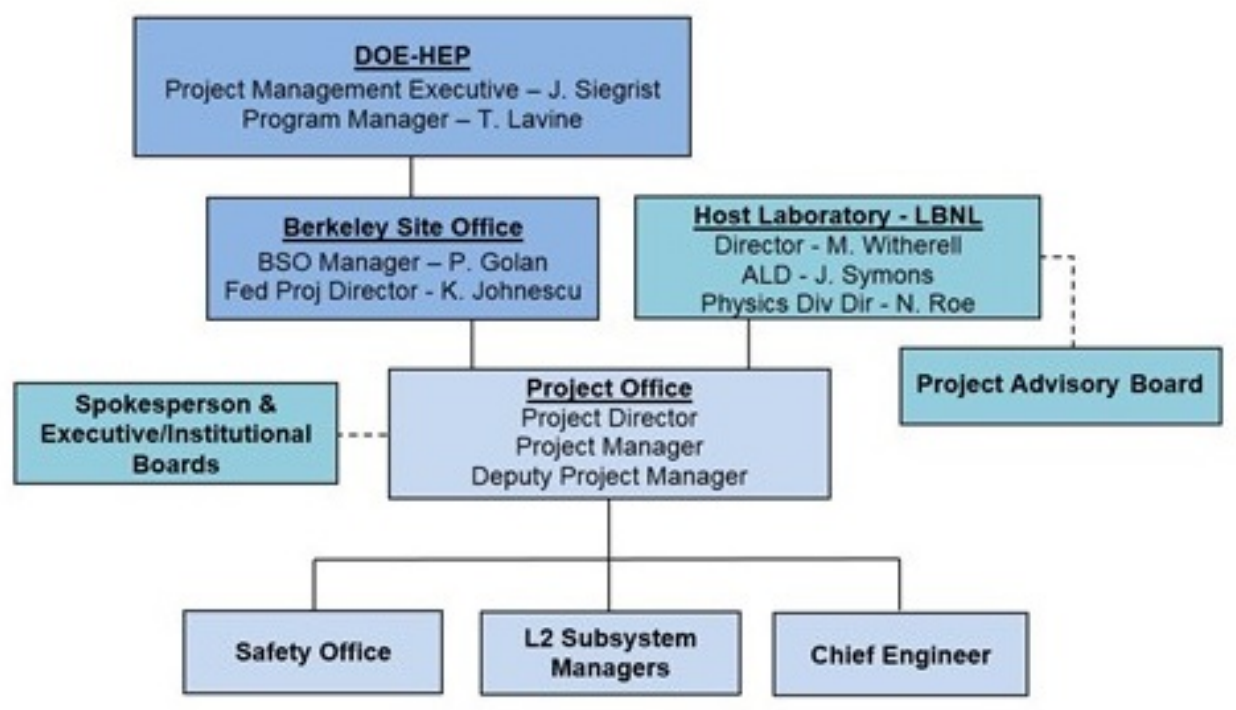

Figure 13.1.1: LZ Project reporting and responsibility organization chart, with an emphasis on the relationship to $\mathrm{DOE}$.

The Project's organization from the perspective of DOE is summarized in Figure 13.1.1. LBNL is the DOE lead laboratory for LZ. As lead laboratory, LBNL will be responsible for Project management and funding from DOE and for ensuring that essential manpower and necessary infrastructure are provided to the Project during the R\&D, construction, and operations phases. The Project Director and Project Manager will be from the lead laboratory and will report to the Physics Division Director of LBNL. These two Project positions must be jointly approved by LBNL and by the collaboration Executive Board (EB). The LBNL 
Project Management Office (PMO) will review and provide oversight of the Project and its management systems to ensure that all DOE project guidelines and procedures are followed.

Figure 13.1.2 presents the internal organization of the Project. The Spokesperson is elected by the collaborating institutes to represent the scientific interests of the collaboration. The roles and term of the Spokesperson are defined in a governance document. The current Spokesperson is Prof. Harry Nelson (University of California, Santa Barbara). The Spokesperson chairs the EB, which is a representative, elected body of senior collaboration members. The EB will help guide the Project organization in its goal of delivering the experimental apparatus and software that will meet the scientific requirements of the LZ collaboration. An Institutional Board (IB), with representatives from each collaborating institution, meets regularly with the Spokesperson and Project team.

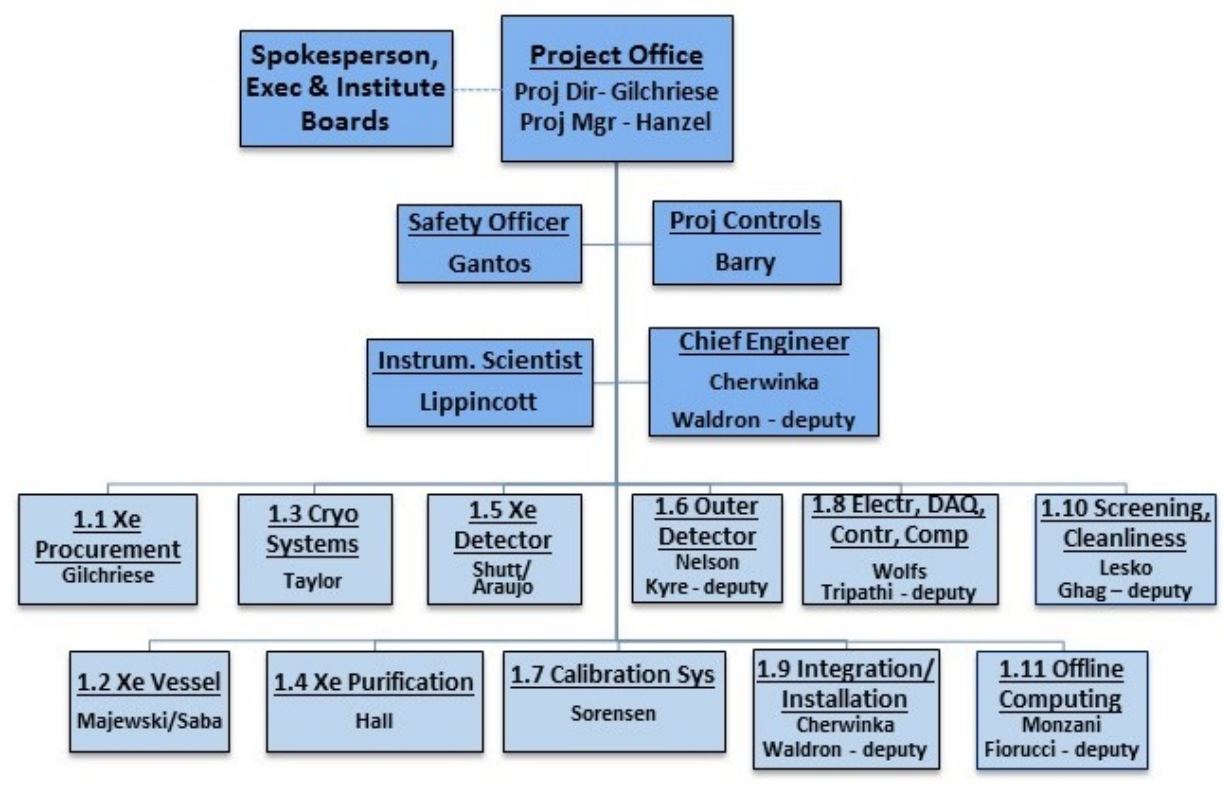

Figure 13.1.2: LZ Project organization.

\subsubsection{Project Advisory Board}

The PAB is an external board, gathered from the U.S. and non-U.S. scientific communities, that has expertise in large scientific projects. This board will provide valuable guidance and advice to the Project over the course of the construction life cycle. The PAB is charged by, and reports to, the Physics Division Director of LBNL. The current members are: David McFarlane (SLAC-chair), Jay Marx (Caltech-retired), Chris Bebek (LBNL), Elaine McCluskey (Fermi National Accelerator Laboratory [FNAL]), Bob McKeown (Jefferson National Accelerator Facility [J-LAB]), Mark Thomson (Cambridge), and Dan Dwyer (LBNL). The PAB will be supplemented as required to provide advice on a specific subject and for specific reviews.

\subsubsection{Project Management Office}

PMO personnel include Project Director M. Gilchriese (LBNL), Project Manager K. Hanzel (LBNL), Deputy Project Manager W. Edwards (LBNL), Instrument Scientist H. Lippincott (FNAL), Chief Engineer J. Cherwinka (Physical Sciences Laboratory, University of Wisconsin), Deputy Chief Engineer W. Waldron (LBNL), Safety Officer J. Gantos (LBNL), and Project Controls Officer M. Barry (LBNL). Systems engineering and QC/QA functions are also under the direction of the PMO. 


\subsubsection{Project Work Breakdown Structure}

The LZ Work Breakdown Structure (WBS) has 12 major categories, as shown in Table 13.1.1.

Table 13.1.1: LZ Work Breakdown Structure (WBS) shown at L2 and description of what each element covers

\begin{tabular}{|c|c|c|}
\hline WBS & WBS Title & WBS Description \\
\hline 1.1 & $\begin{array}{l}\text { Xenon } \\
\text { Procurement }\end{array}$ & $\begin{array}{l}\text { Specification and procurement of the Xe necessary for the LZ experiment. Xe storage } \\
\& \text { transportation vessels are covered in WBS } 1.4, \text { Xe Purification \& Handling. }\end{array}$ \\
\hline 1.2 & $\begin{array}{l}\text { Xenon Vessel } \\
\text { (Cryostat) }\end{array}$ & $\begin{array}{l}\text { Labor, materials, and equipment associated with the design, prototyping, materials } \\
\text { selection, construction, certification, and delivery, as well as planning and oversight } \\
\text { of assembly and testing efforts on site, for the cryostat vessel system, its tanks, } \\
\text { connecting flanges, insulation, and support structures. }\end{array}$ \\
\hline 1.3 & $\begin{array}{l}\text { Cryogenic } \\
\text { Systems }\end{array}$ & $\begin{array}{l}\text { Labor, materials, and equipment associated with the design, prototyping, procure- } \\
\text { ment, construction, assembly, testing, and delivery of the liquid nitrogen cryogenic } \\
\text { system and nitrogen purge system. }\end{array}$ \\
\hline 1.4 & $\begin{array}{l}\text { Xenon } \\
\text { Purification \& } \\
\text { Handling }\end{array}$ & $\begin{array}{l}\text { Labor, materials, and equipment associated with the production of high-purity LXe, } \\
\text { its storage, delivery to, and recovery from the TPC. This element covers the online } \\
\text { purification system, the Xe purity analysis systems, the automated fail-safe Xe re- } \\
\text { covery system, and selected radon-reduction systems. A major subcomponent of this } \\
\text { element is the stand-alone krypton-removal system, which will be used to purify the } \\
\text { Xe prior to experimental operations. }\end{array}$ \\
\hline 1.5 & $\begin{array}{l}\text { Xenon } \\
\text { Detector }\end{array}$ & $\begin{array}{l}\text { Labor, materials, and equipment associated with the design, prototyping, fabrication, } \\
\text { testing, and assembly planning for the central Xe detector. This element covers the } \\
\text { central detector region with its PMTs and the accompanying field-shaping electrodes } \\
\text { and reflecting walls. It includes the "skin" veto region outside the main TPC volume } \\
\text { and its PMTs. Included are the cathode, anode, and gate HV power supplies and the } \\
\text { cathode HV umbilical connection to the TPC cathode and the grid structures, as well } \\
\text { as the internal Xe liquid fluid system that brings liquid into the TPC region, providing } \\
\text { cooling surfaces for temperature control. Also included is monitoring equipment for } \\
\text { temperature, pressure, fluid flow, and other necessary measurements. }\end{array}$ \\
\hline 1.6 & $\begin{array}{l}\text { Outer } \\
\text { Detector } \\
\text { System }\end{array}$ & $\begin{array}{l}\text { Labor, materials, and equipment associated with the design, fabrication, testing, } \\
\text { and assembly planning for the outer detector system. This includes the acquisition } \\
\text { of the acrylic vessels, construction of the scintillator filling system, the acquisition } \\
\text { and testing of the outer detector PMTs, the mixing and handling of the gadolinium- } \\
\text { loaded liquid scintillator, procurement of reflector materials, as well as all the support } \\
\text { infrastructure required. It also includes the planning, procedures, and oversight, plus } \\
\text { the installation tooling required during the assembly of the system inside the water } \\
\text { tank. }\end{array}$ \\
\hline 1.7 & Calibration & $\begin{array}{l}\text { Labor, materials, and equipment associated with the design, prototyping, construc- } \\
\text { tion, delivery, assembly, and testing of the calibration system for the Xe detector } \\
\text { and the outer detector system, along with the mechanisms, plumbing, valves, and } \\
\text { radiation sources required to implement the calibration systems. Included are safety } \\
\text { and administrative custodial requirements for source security, handling, and shipping. }\end{array}$ \\
\hline & & (continued on next page) \\
\hline
\end{tabular}


Table 13.1.1: (continued)

\begin{tabular}{|c|c|c|}
\hline WBS & WBS Title & WBS Description \\
\hline 1.8 & $\begin{array}{l}\text { Electronics, } \\
\text { DAQ, } \\
\text { Controls, } \\
\text { Computing }\end{array}$ & $\begin{array}{l}\text { Labor, materials, and equipment associated with the design, prototyping, construc- } \\
\text { tion, delivery, assembly, and testing of the analog and digital electronics for the Xe } \\
\text { and outer detector PMTs, the DAQ and trigger systems, the PMT HV system, the } \\
\text { detector control system, and the online and offline hardware and software. This ele- } \\
\text { ment includes the external signal, PMT HV, and network cables. Not included are the } \\
\text { internal HV and signal cables for the PMTs (covered by WBS 1.5) and the detector } \\
\text { sensors/instruments required for detector control. This element provides standard } \\
\text { interfaces for detector sensors/instruments; custom interfaces required to connect } \\
\text { custom sensors/instruments to the detector control system will not be provided by } \\
\text { this element. }\end{array}$ \\
\hline 1.9 & $\begin{array}{l}\text { Integration \& } \\
\text { Installation }\end{array}$ & $\begin{array}{l}\text { Labor and materials necessary to integrate the design effort of the subsystems into an } \\
\text { overall detector design, maintain CAD models of the LZ detector and Davis Campus, } \\
\text { upgrade the SURF infrastructure to support the detector assembly and operation, } \\
\text { and perform on-site surface-level assembly of the detector and installation into the } \\
\text { Davis Campus underground. Other subsystem elements maintain the responsibility } \\
\text { to support integration by communicating design requirements, interface issues, sub- } \\
\text { system CAD models, infrastructure needs, and assembly and operation needs. WBS } \\
1.9 \text { supplies planning, management, and skilled labor for assembly and installation, } \\
\text { and the subsystems supplies experts on site to support this as needed. }\end{array}$ \\
\hline 1.10 & $\begin{array}{l}\text { Cleanliness \& } \\
\text { Screening }\end{array}$ & $\begin{array}{l}\text { Labor, materials, and equipment associated with specification of radioactive } \\
\text { background-level tolerances in the experiment; material radioassaying and control } \\
\text { of radioactive background contaminants in the Xe resulting from component out- } \\
\text { gassing; control of ambient radioactivity; and establishing cleanliness controls, mon- } \\
\text { itoring, and maintenance procedures for manufacture, transport, storage, handling, } \\
\text { assembly, and integration of detector components. }\end{array}$ \\
\hline 1.11 & $\begin{array}{l}\text { Offline } \\
\text { Computing }\end{array}$ & $\begin{array}{l}\text { Software professional labor and computing hardware needed to begin operations of } \\
\text { the LZ experiment. Interface to collaboration responsibilities for data processing, } \\
\text { analysis, and simulation software. }\end{array}$ \\
\hline 1.12 & $\begin{array}{l}\text { Project } \\
\text { Management }\end{array}$ & $\begin{array}{l}\text { The cost of labor, travel, and materials necessary to plan, track, organize, manage, } \\
\text { maintain communications, conduct reviews, and perform necessary safety, risk, and } \\
\text { QA tasks during all phases of the Project. Subsystem-related management and } \\
\text { support activities for planning, estimating, tracking, and reporting as well as their } \\
\text { specific EH\&S and QA tasks are included in each of the subsystems. }\end{array}$ \\
\hline
\end{tabular}

\subsubsection{Project Subsystem Organization}

The current Subsystem Managers (at Level 2 of the WBS and selected Level 3 Managers) and lead engineers are listed in Table 13.1.2. The LZ Technical Board comprises the WBS Level 2 Managers (Bold), their deputies, and the Project Office.

The Level 2 or Subsystem Managers, in addition to being members of the LZ Technical Board, are responsible for overseeing the development of the Project baseline with regard to their subsystems. They work with the Project Office to establish a level (L3) organization, helping to ensure that adequate technical resources have been identified, and defining the subsystem-specific requirements as they flow down from the overall Project. The L2 Managers oversee the development of the technical design as well as the schedule and cost estimates associated with design, fabrication/execution, assembly, and test of their subsystems. 
They are also responsible for producing design reports and internal and external reviews. Ultimately, they are responsible for executing the Project Plan with respect to their subsystems.

Design reviews are held for all relevant subsystems and are organized by the PMO. Each major subsystem and procurement undergoes multiple reviews (typically preliminary, final, and production readiness) as the design of the particular subsystem matures and reaches readiness for construction.

Table 13.1.2: The LZ Project Level 2 and 3 managers and lead engineers.

\begin{tabular}{|c|c|c|c|c|}
\hline WBS & Description & L2/3 Manager & Deputy or Co-mgr. & Lead engineer \\
\hline 1.1 & Xe Procurement & M. Gilchriese (LBNL) & & \\
\hline 1.2 & Xe Vessel & P. Majewski (RAL) & J. Saba (LBNL) & $\begin{array}{l}\text { E. Holtom (RAL) / } \\
\text { J. ODell (RAL) }\end{array}$ \\
\hline 1.2 .1 & Design & E. Holtom (RAL) & & \\
\hline 1.2 .2 & Material Selection & P. Majewski (RAL) & & \\
\hline 1.2 .3 & Fabrication & J. ODell (RAL) & & \\
\hline 1.2 .4 & Cleaning & J. ODell (RAL) & & \\
\hline 1.2 .5 & Transportation & J. ODell (RAL) & & \\
\hline 1.2 .6 & $\begin{array}{l}\text { Acceptance, } \\
\text { Assembly, } \\
\text { Installation }\end{array}$ & J. ODell (RAL) & & \\
\hline 1.2 .7 & $\begin{array}{l}\text { Subsystem } \\
\text { Management }\end{array}$ & $\begin{array}{l}\text { P. Majewski (RAL)/J. Saba } \\
\text { (LBNL) }\end{array}$ & & \\
\hline 1.3 & Cryogenic Systems & D. Taylor (SDSTA) & & \\
\hline 1.3 .1 & $\begin{array}{l}\text { Nitrogen } \\
\text { Distribution }\end{array}$ & D. Taylor (SDSTA) & & \\
\hline 1.3 .2 & Thermosyphons & D. Taylor (SDSTA) & & \\
\hline 1.3 .3 & Vacuum System & C. Maupin (SDSTA) & & \\
\hline 1.3 .4 & Breakout & C. Maupin (SDSTA) & & \\
\hline 1.3 .5 & Controls and Power & C. Maupin (SDSTA) & & \\
\hline 1.3 .6 & $\begin{array}{l}\text { Subsystem } \\
\text { Management }\end{array}$ & D. Taylor (SDSTA) & & \\
\hline 1.4 & $\begin{array}{l}\text { Xe Purifica- } \\
\text { tion/Handling }\end{array}$ & C. Hall (UMd) & & \\
\hline 1.4 .1 & Xenon Sampling & C. Hall (UMd) & & \\
\hline 1.4 .2 & Kr Removal & D. Akerib (SLAC) & & \\
\hline 1.4 .3 & $\begin{array}{l}\text { Xe Storage \& } \\
\text { Transport }\end{array}$ & T. Benson (UW-PSL) & & \\
\hline 1.4 .4 & $\begin{array}{l}\text { Xe Gas Delivery \& } \\
\text { Recovery }\end{array}$ & T. Benson (UW-PSL) & & \\
\hline 1.4 .5 & $\begin{array}{l}\text { Xe Gas } \\
\text { Recirculation }\end{array}$ & J. Cherwinka (UW-PSL) & & \\
\hline 1.4.6 & Liquid Xe Tower & H. Lippincott (FNAL) & & \\
\hline 1.4 .7 & LXe Transfer Lines & J. Cherwinka (UW-PSL) & & \\
\hline 1.4 .8 & $\begin{array}{l}\text { Undergnd Instal. } \\
\text { Planning }\end{array}$ & C. Hall (UMd) & & $\begin{array}{l}\text { J. Cherwinka } \\
\text { (UW-PSL) }\end{array}$ \\
\hline \multicolumn{5}{|c|}{ (continued on next page) } \\
\hline
\end{tabular}


Table 13.1.2: (continued)

\begin{tabular}{|c|c|c|c|c|}
\hline WBS & Description & L2/3 Manager & Deputy or Co-mgr. & Lead engineer \\
\hline 1.4 .9 & $\begin{array}{l}\text { Subsystem } \\
\text { Management }\end{array}$ & C. Hall (UMd) & & \\
\hline 1.4 .10 & $\begin{array}{l}\text { Xe Recovery \& } \\
\text { Cont. Man. }\end{array}$ & E. Dahl (Northwestern) & & \\
\hline 1.5 & Detector & T. Shutt (SLAC) & $\begin{array}{l}\text { H. Araujo } \\
\text { (Imperial) }\end{array}$ & J. Saba (LBNL) \\
\hline 1.5 .1 & $\begin{array}{l}\text { Cathode High } \\
\text { Voltage }\end{array}$ & D. McKinsey (UCBerkeley) & & W. Waldron (LBNL) \\
\hline 1.5 .2 & U.S. PMT Systems & R. Gaitskell (Brown) & & \\
\hline 1.5 .3 & U.K. PMT Systems & H. Araujo (Imperial) & & \\
\hline 1.5 .4 & Field Cage & J. Saba (LBNL) & & \\
\hline 1.5 .5 & Grids & W. Wisniewski (SLAC) & & K. Skarpaas \\
\hline 1.5 .6 & $\begin{array}{l}\text { Xe Monitoring } \\
\text { System }\end{array}$ & H. Kraus (Oxford) & & \\
\hline 1.5 .7 & $\begin{array}{l}\text { Internal Fluid } \\
\text { System }\end{array}$ & T. Shutt (SLAC) & & \\
\hline 1.5 .8 & Skin System & H. Lippincott (FNAL) & & \\
\hline 1.5 .9 & $\begin{array}{l}\text { Assembly and } \\
\text { Installation }\end{array}$ & J. Saba (LBNL) & & \\
\hline 1.5 .10 & $\begin{array}{l}\text { Screening } \\
\text { Coordination }\end{array}$ & R. Webb (TAMU) & & \\
\hline 1.5 .11 & System Test & K. Palladino (SLAC) & & \\
\hline 1.5 .12 & $\begin{array}{l}\text { Subsystem } \\
\text { Management }\end{array}$ & T. Shutt & H. Araujo & \\
\hline 1.6 & $\begin{array}{l}\text { Outer Detector } \\
\text { System }\end{array}$ & H. Nelson (UCSB) & S. Kyre (UCSB) & D.White (UCSB) \\
\hline 1.6 .1 & Scint. Vessels & S. Kyre (UCSB) & & \\
\hline 1.6 .2 & Reflector System & S. Kyre (UCSB) & & \\
\hline 1.6 .3 & LS Filling System & D. White (UCSB) & & \\
\hline 1.6 .4 & Liquid Scint. & M. Yeh (BNL) & & \\
\hline 1.6 .5 & Water Tank & D. White (UCSB) & & \\
\hline 1.6 .6 & PMT Supports & S. Burdin (Liverpool) & & D. White (UCSB) \\
\hline 1.6 .7 & PMTs & S. Fiorucci (LBNL) & & \\
\hline 1.7 & $\begin{array}{l}\text { Calibration } \\
\text { System }\end{array}$ & P. Sorensen (LBNL) & & \\
\hline 1.7 .1 & $\begin{array}{l}\text { Internal Radioact. } \\
\text { Sources }\end{array}$ & S. Hertel (UCBerkeley) & & \\
\hline 1.7 .2 & $\begin{array}{l}\text { Calibration Source } \\
\text { Delivery }\end{array}$ & M. van der Grinten (RAL) & & \\
\hline 1.7 .3 & $\begin{array}{l}\text { Radioisotope } \\
\text { Sources }\end{array}$ & P. Sorensen (LBNL) & & \\
\hline 1.7.4 & $\begin{array}{l}\text { Photoneutron } \\
\text { Sources }\end{array}$ & P. Sorensen (LBNL) & & \\
\hline \multicolumn{5}{|c|}{ (continued on next page) } \\
\hline
\end{tabular}


Table 13.1.2: (continued)

\begin{tabular}{|c|c|c|c|c|}
\hline WBS & Description & L2/3 Manager & Deputy or Co-mgr. & Lead engineer \\
\hline 1.7 .5 & DD Neutron Source & D. Huang (BrownU) & & \\
\hline 1.7 .6 & $\begin{array}{l}\text { Assembly and } \\
\text { Installation Planning }\end{array}$ & P. Sorensen (LBNL) & & \\
\hline 1.7.7 & $\begin{array}{l}\text { Subsystem } \\
\text { Management }\end{array}$ & P. Sorensen (LBNL) & & \\
\hline 1.8 & $\begin{array}{l}\text { Electr., DAQ, } \\
\text { Controls, } \\
\text { Computing }\end{array}$ & F. Wolfs (URochester) & $\begin{array}{l}\text { M. Tripathi } \\
\text { (UCDavis) }\end{array}$ & \\
\hline 1.8 .1 & Analog Electronics & M. Tripathi (UCDavis) & & $\begin{array}{l}\text { R. Gerhard } \\
\text { (UCDavis) }\end{array}$ \\
\hline 1.8 .2 & $\begin{array}{l}\text { Data Sparsification } \\
\text { System }\end{array}$ & $\begin{array}{l}\text { E. Druszkiewicz } \\
\text { (URochester) }\end{array}$ & & $\begin{array}{l}\text { E. Druszkiewicz } \\
\text { (URoch) }\end{array}$ \\
\hline 1.8 .3 & $\begin{array}{l}\text { Data Acquisition } \\
\text { System }\end{array}$ & W. Skulski (URochester) & & W. Skulski (URoch) \\
\hline 1.8 .4 & $\begin{array}{l}\text { External PMT HV, } \\
\text { Signal }\end{array}$ & F. Wolfs (URochester) & & \\
\hline 1.8 .5 & Slow Control & V. Solovov (Coimbra) & & TBD (FNAL) \\
\hline 1.8 .6 & PMT HV Supplies & M. Tripathi (UCDavis) & & R. Gerhard (UDavis) \\
\hline 1.8 .7 & Online HW & J. Buckley (Washington U) & & $\begin{array}{l}\text { M. Olevitch } \\
\text { (WashingtonU), P. } \\
\text { Zarzhitsky } \\
\text { (UAlabama) }\end{array}$ \\
\hline 1.8 .8 & Online SW & J. Buckley (Washington U) & & \\
\hline 1.8 .9 & $\begin{array}{l}\text { Assembly \& } \\
\text { Installation Planning }\end{array}$ & F. Wolfs (URochester) & & $\begin{array}{l}\text { R. Gerhard } \\
\text { (UCDavis), E. } \\
\text { Druszkiewicz } \\
\text { (URoch) }\end{array}$ \\
\hline 1.8 .10 & $\begin{array}{l}\text { Subsystem } \\
\text { Management }\end{array}$ & F. Wolfs (URoch) & & \\
\hline 1.8 .11 & $\begin{array}{l}\text { Detector Response } \\
\text { Simulations }\end{array}$ & H. Kraus (Oxford) & & \\
\hline 1.9 & $\begin{array}{l}\text { Integration \& } \\
\text { Installation }\end{array}$ & J. Cherwinka (UW-PSL) & & \\
\hline 1.9 .1 & SURF Infrastructure & S. DeVries (LBNL) & & \\
\hline 1.10 & $\begin{array}{l}\text { Cleanliness \& } \\
\text { Screening }\end{array}$ & K. Lesko (LBNL) & C. Ghag (UCL) & \\
\hline 1.10 .1 & $\begin{array}{l}\text { Screening for Fixed } \\
\text { Contaminants }\end{array}$ & $\begin{array}{l}\text { P. Scovell (Oxford)/A. Cole } \\
\text { (LBNL) }\end{array}$ & & \\
\hline 1.10 .2 & $\begin{array}{l}\text { Radon Emanation } \\
\text { Screening }\end{array}$ & R. Schnee (SDSMT) & & \\
\hline 1.10 .3 & $\begin{array}{l}\text { Cleanliness } \\
\text { Maintenance }\end{array}$ & A. Manalaysay (UCDavis) & & \\
\hline 1.10 .4 & $\begin{array}{l}\text { Background } \\
\text { Simulations }\end{array}$ & A. Lindote (Coimbra) & & \\
\hline \multicolumn{5}{|c|}{ (continued on next page) } \\
\hline
\end{tabular}


Table 13.1.2: (continued)

\begin{tabular}{|c|c|c|c|c|}
\hline WBS & Description & L2/3 Manager & Deputy or Co-mgr. & Lead engineer \\
\hline 1.10 .5 & $\begin{array}{l}\text { Information } \\
\text { Repository }\end{array}$ & J. Busenitz (UAlabama) & & \\
\hline 1.11 & Offline Computing & M.E. Monzani (SLAC) & $\begin{array}{l}\text { S. Fiorucci } \\
\text { (LBNL) }\end{array}$ & \\
\hline 1.11 .1 & U.S. Data Center & C. Tull (LBNL) & & \\
\hline 1.11 .2 & U.K. Data Center & A. Vacheret (Imperial) & & \\
\hline 1.11 .3 & $\begin{array}{l}\text { Infrastructure } \\
\text { Software }\end{array}$ & S. Patton (LBNL) & & \\
\hline 1.11 .4 & Simulations & J. Dobson (UCL) & & \\
\hline 1.11 .5 & Analysis Software & C. Carmona (Penn State) & & \\
\hline 1.11 .6 & $\begin{array}{l}\text { Subsystem } \\
\text { Integration and } \\
\text { Validation }\end{array}$ & M.E. Monzani (SLAC) & & \\
\hline 1.11 .7 & $\begin{array}{l}\text { Subsystem } \\
\text { Management }\end{array}$ & M.E. Monzani (SLAC) & S. Fiorucci (LBNL) & \\
\hline 1.12 & $\begin{array}{l}\text { Project } \\
\text { Management }\end{array}$ & M. Gilchriese (LBNL) & K. Hanzel (LBNL) & $\begin{array}{l}\text { J. Cherwinka } \\
\text { (UW-PSL) }\end{array}$ \\
\hline
\end{tabular}

\subsection{Safety}

Personnel safety, protecting the environment, and equipment safety are high priorities for the LZ Project. Its scientific goals cannot be achieved without an effective safety and environmental protection program that is integrated into the overall management of the experiment. The details of the EH\&S organization are described in an Integrated Safety Management document. A separate Hazard Analysis Report (HAR) describes the hazards that will be encountered and their associated controls during the execution of the Project. The HAR received significant input from the L2 Subsystem Managers who are, and will remain, closely involved in identifying and mitigating these hazards. Many hazards will be similar to those found in past operation of similar experiments (e.g., LUX and Daya Bay). The LZ Project work will take place at multiple institutions in addition to LBNL. Safety of the work at each institution will be the responsibility of the institution and work will be performed in accordance with the requirements and management systems of the home institutions. A sharing of lessons learned for the various locations is expected. Additionally, the LZ Project team will assist collaborating institutions as requested to address any hazard concerns.

Final assembly of the LZ experiment and its operation will take place at SURF, where integrated safety management is well established and will be employed in all phases. SURF EH\&S rules and responsibilities will apply to all LZ activity at the SURF site, and SURF will provide relevant safety training for all members of LZ who work on the site. SURF has established an external EH\&S panel that will review the LZ Project during the construction period and prior to commissioning and operations. This panel has completed the first review of $\mathrm{LZ}$ and provided review of the HAR.

\subsection{Risk}

The LZ risk program has several key aspects. The first is the early identification of potential risks in each of the detector elements as well as the system as a whole. Second, an early R\&D program focuses on understanding, reducing, or eliminating the identified risks. Third is the formal tracking of the remaining risks 
and mitigation strategies throughout the life of the experiment's construction phase. Last is an accounting for technical, cost, and schedule risk in developing the contingency analysis for the cost of the experiment. These first three components (ID, R\&D, tracking) will be discussed in this chapter. A Risk Registry for LZ has been assembled and is updated and reviewed regularly. A Risk Management Plan has also been completed.

Subsystem Managers have performed a risk assessment of their technical systems. These have been gathered by the Project Office and disseminated back out to the Subsystem Managers, key engineering leads, and the rest of the Project leadership team. The Risk Registry will be reviewed and discussed regularly in subsystem and overall Project meetings. Updates to the Risk Registry are considered monthly as the Project proceeds, as more information and experience are gathered and risk status changes.

\subsection{Operations}

Experiment Operations will be managed centrally from an Operations Project office in much the same way the construction phase of the Project is managed. Our plan is based on the successful experience operating the LUX detector at SURF and other projects. The collaboration will provide much of the necessary resources for shifts and on-call experts. A small engineering and technical group will provide maintenance planning, oversight, and the resources for achieving them. A small computing and software maintenance group will ensure high availability of computing hardware and software and will support the collaboration's data production and analysis activities.

The elements of the LZ operations support are:

- LBNL operations manager. Provides oversight of budget and EH\&S matters.

- Engineering support. Provides engineering oversight during operations, particularly during the early phases of operations.

- On-site EH\&S officer. Provides on-site EH\&S oversight. This is an SDSTA employee under contract to LBNL.

- On-site operations technical staff. Provides on-site maintenance, support, and interface to SURF staff. This will involve both operations aspects and technical support. Multiple positions are planned and these will be SDSTA staff under contract to LBNL.

- Procurement. Obtains materials, supplies, consumables (e.g. liquid nitrogen) and equipment necessary for operations and maintenance. Under the direction of the LBNL operations manager.

- University travel support. Provides support of travel to the site. Under the direction of the LBNL operations manager.

- Computing support. Provides support for professional services for computing hardware and software.

Transition-to-operations support for LZ-related travel of scientific staff began in FY 2016 and will necessarily expand as work on site increases. 


\subsection{Cost and Schedule Summary}

The overall LZ Project plan is summarized in this chapter, including an overview of the Project schedule and the concept for the division of scope and cost among the various funding sources. The planned contributions supported by DOE, SDSTA, the U.K.'s Science \& Technology Facilities Council (STFC), Korea, and Portugal are outlined. This is a joint project with an international collaboration, and the cost-accounting approaches differ. Therefore, we have attempted to utilize the U.S. DOE cost-accounting approach for the costs summarized in this chapter.

\subsubsection{Project Schedule}

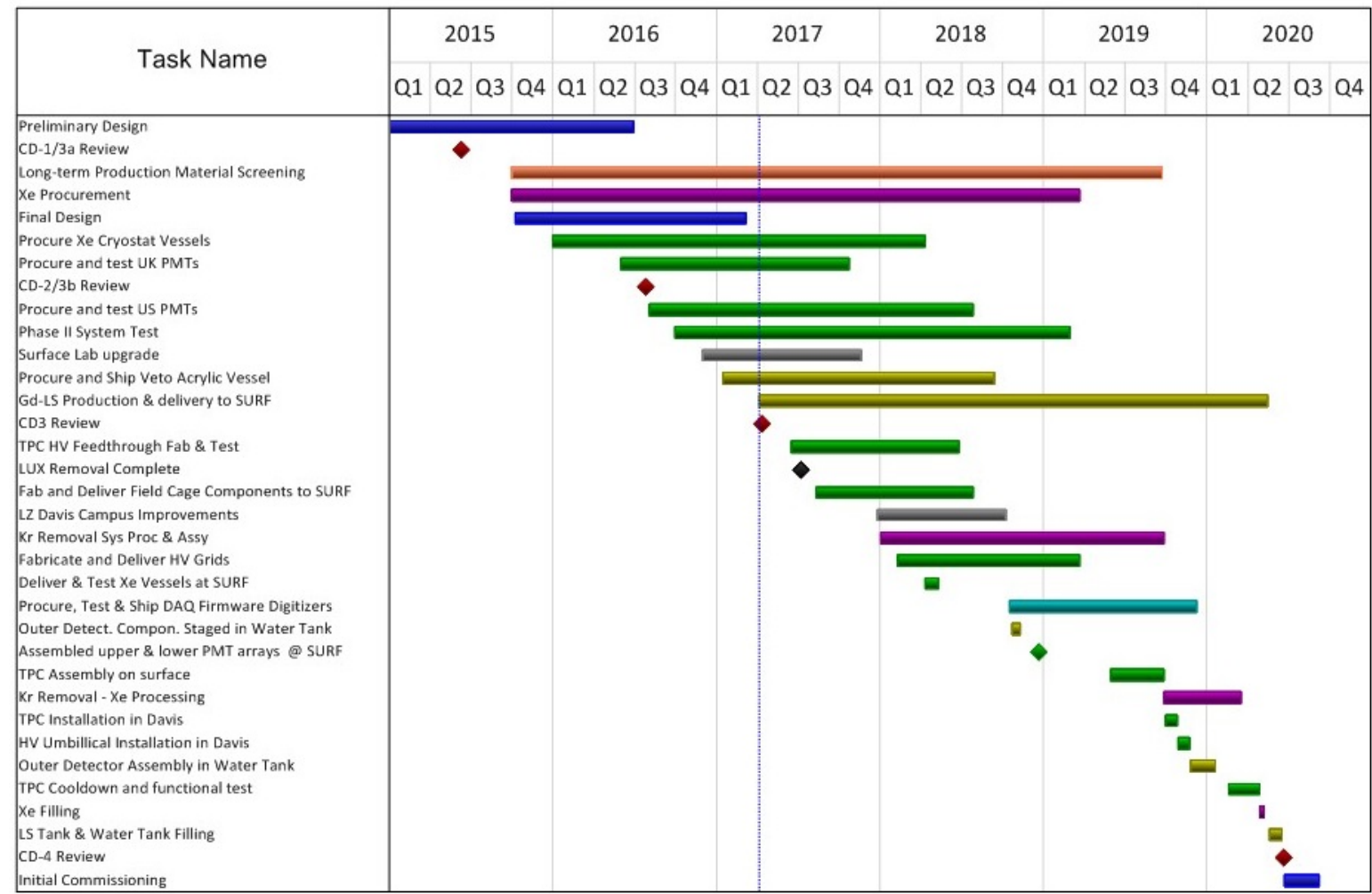

Figure 13.5.1: Summary of LZ schedule. The dates are in U.S. fiscal year quarters.

The goal is to begin commissioning and early operations by early 2020. Infrastructure improvements (to surface laboratories) began in September 2016. Components begin to arrive at SURF in mid-2017. Underground installation begins in mid-2018. The DOE critical decision milestones in this plan are CD-1/3a obtained in April 2015, CD-2/3b obtained in August 2016 and a CD-3(all remaining items) review in January 2017. The procurement of long lead items (Xe, the cryostat vessels, PMTs, outer detector acrylic vessels and others) has already started. A more complete view of the Project schedule is shown in Figure 13.5.1. The dates shown in this schedule correspond to early-finish milestones. 


\subsection{Project Scope}

The Project's technical scope has been described in previous chapters. The complete LZ Project includes the detector elements - purified Xe, cryogenic systems, Xe detector, cryostat, veto system, calibration system, electronics, DAQ, trigger, online and offline software - as well as all the integrating activities - system tests, system integration, assembly/installation, on-site infrastructure, and project management.

The planned scope division among the various U.S. and non-U.S. agencies is summarized briefly here.

- The major elements of U.K. scope deliverables include about one-third of the low-background PMTs for the Xe detector, the cryostat set (inner and outer), elements of the low-background counting capability, contributions to integrated system tests, the source calibration delivery mechanism, and extensive contributions to computing and software.

- Korea is expected to contribute PMTs for the outer detector, low-background assay capability, and software.

- Portugal is expected to contribute to control systems, software, and a measurement system for TPC quality control.

- The SDSTA scope includes above- and belowground modifications to required facilities and much of the Xe needed for the experiment.

- The NSF is assumed to support scientific efforts for those U.S. collaborating institutions funded by NSF but will not contribute to the Project scope.

- The DOE is assumed to fund all remaining Project scope. 


\subsection{Cost Summary}

The U.S.-based cost estimate associated with the above scope is shown in Table 13.7.1 in at-year dollars without contingency. U.S. contingency amounts to about $25 \%$ of the DOE - funded amount.

Table 13.7.1: LZ Project U.S.-equivalent base cost (without contingency) in at-year kilo-dollars. The equivalent U.S. costs for non - DOE items are also shown.

\begin{tabular}{|c|c|c|c|c|}
\hline WBS (2) & \multicolumn{2}{|c|}{ DOE Funded } & \multicolumn{2}{|c|}{ NONDOE Funded } \\
\hline $1.01-$ Xe PROCUREMENT & \$k & 420 & \$k & 9846 \\
\hline 1.02 - Xe VESSEL SUBSYSTEM & \$k & 5 & $\$ k$ & 1466 \\
\hline 1.03 - CRYOGENIC SUBSYSTEM & \$k & 1881 & \$k & - \\
\hline 1.04 - Xe PURIFICATION SUBSYSTEM & \$k & 7399 & \$k & - \\
\hline 1.05 - Xe DETECTOR SUBSYSTEM & \$k & 10332 & \$k & 2838 \\
\hline 1.06 - OUTER DETECTOR SUBSYSTEM & \$k & 4191 & \$k & 661 \\
\hline 1.07 - CALIBRATION SUBSYSTEM & \$k & 727 & \$k & 98 \\
\hline $\begin{array}{ll}1.08 \text { - } & \text { ELECTRONICS, DAQ, CONTROLS \& } \\
\text { COMPUTING SUBSYSTEM }\end{array}$ & \$k & 5274 & \$k & 34 \\
\hline 1.09 - INTEGRATION \& INSTALLATION SUBSYSTEM & \$k & 5379 & \$k & 1971 \\
\hline 1.10 - CLEANLINESS AND SCREENING SUBSYSTEM & $\$ k$ & 1153 & \$k & 650 \\
\hline 1.11 - OFFLINE COMPUTING SUBSYSTEM & \$k & 2322 & \$k & - \\
\hline 1.12 - PROJECT MANAGEMENT & \$k & 4733 & \$k & - \\
\hline Total & \$k & 44,817 & \$k & 17,661 \\
\hline
\end{tabular}

